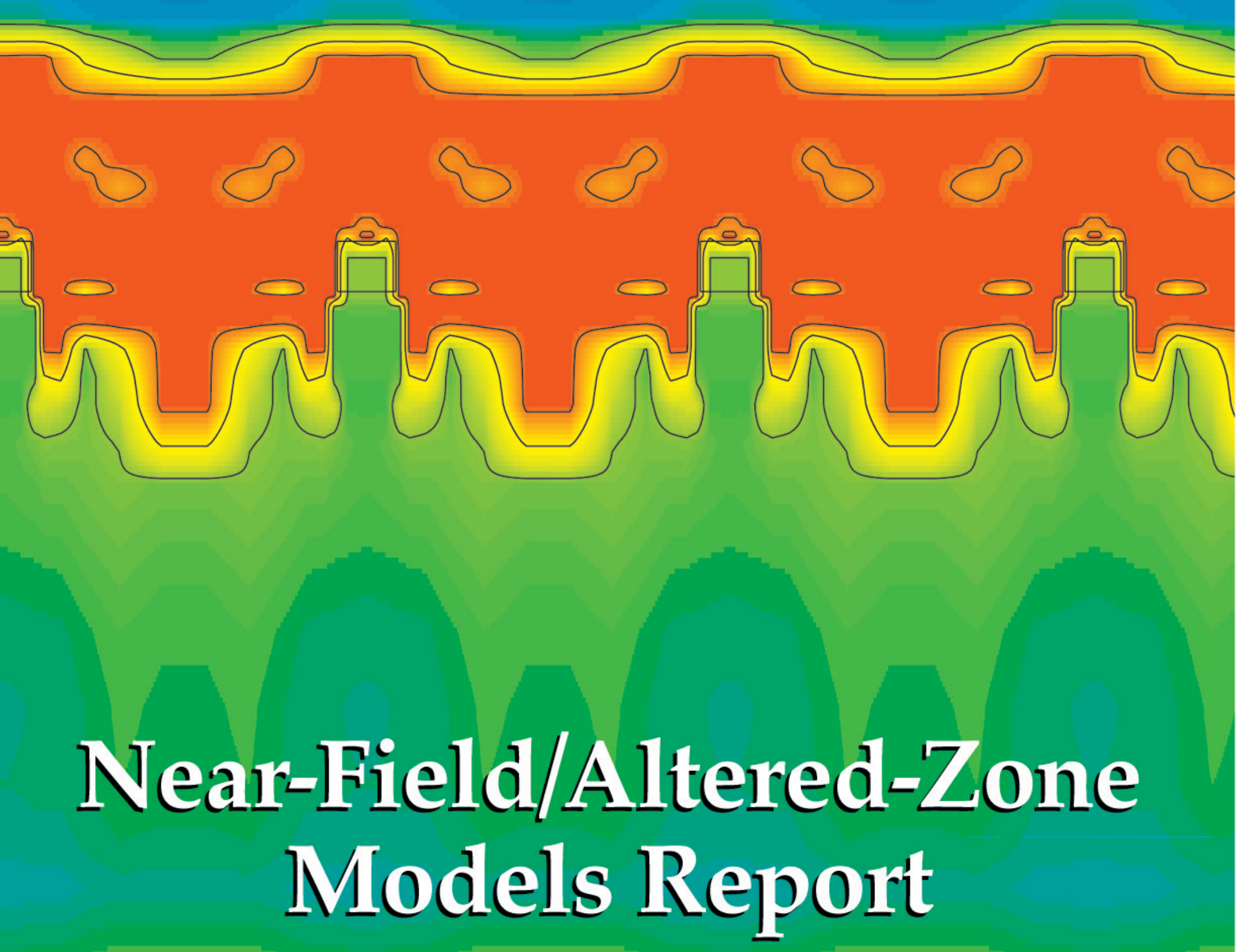

Lawrence Livermore National Laboratory 


\section{DISCLAIMER}

This document was prepared as an account of work sponsored by an agency of the United States Government. Neither the United States Government nor the University of California nor any of their employees, makes any warranty, express or implied, or assumes any legal liability or responsibility for the accuracy, completeness, or usefulness of any information, apparatus, product, or process disclosed, or represents that its use would not infringe privately owned rights. Reference herein to any specific commercial product, process, or service by trade name, trademark, manufacturer, or otherwise, does not necessarily constitute or imply its endorsement, recommendation, or favoring by the United States Government or the University of California. The views and opinions of authors expressed herein do not necessarily state or reflect those of the United States Government or the University of California, and shall not be used for advertising or product endorsement purposes.

This report has been reproduced directly from the best available copy.

Available to DOE and DOE contractors from the Office of Scientific and Technical Information P.O. Box 62, Oak Ridge, TN 37831

Prices available from (423) 576-8401

Available to the public from the National Technical Information Service

U.S. Department of Commerce 5285 Port Royal Rd. Springfield, VA 22161 


\title{
Near-Field/Altered-Zone Models Report
}

\author{
by \\ Ernest L. Hardin
}

with contributions from

Stephen C. Blair

William L. Bourcier Thomas A. Buscheck

Dwayne A. Chesnut

Laura D. DeLoach

William E. Glassley

James W. Johnson

Richard B. Knapp

Kevin G. Knauss
Kenrick Lee

Annemarie Meike

Katherine Myers

John J. Nitao

Cynthia E. Palmer

Leah L. Rogers

Nina D. Rosenberg

Brian E. Viani

C. Wittwer

Thomas J. Wolery

March 1998

SP3100M3 WBS 1.2.3

Lawrence Livermore National Laboratory

Livermore, CA 94550 
This work was performed under the auspices of the U.S. Department of Energy by the Lawrence Livermore National Laboratory under contract W-7405-ENG-48 and was supported specifically by the Yucca Mountain Site Characterization Project at LLNL. 


\section{Contents}

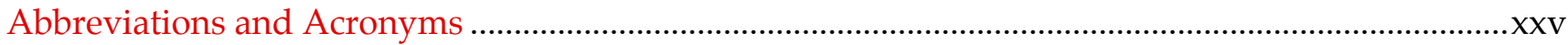

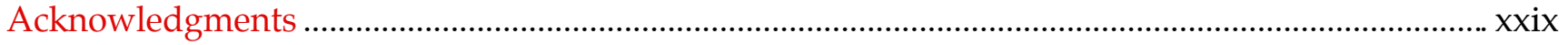

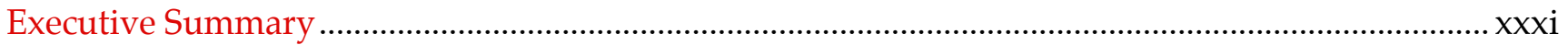

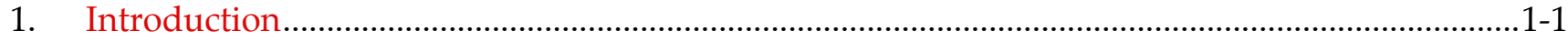

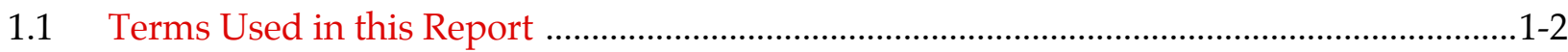

1.2 Relevance of Near-Field / Altered-Zone Models to the Yucca Mountain

Site Characterization Project ................................................................................................. 1-3

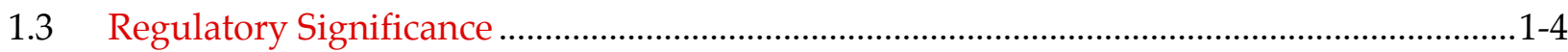

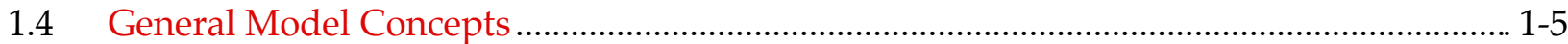

1.4.1 Spatial Heterogeneity …......................................................................................... 1-5

1.4.2 Heterogeneity vs. Uncertainty ……..................................................................... 1-6

1.4.3 Modeling, Characterization, and Dampening...................................................... 1-6

1.5 Building Confidence in Models ..........................................................................................

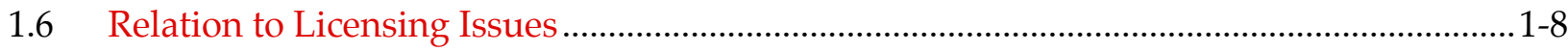

1.6.1 Evolution of the Near-Field Environment ............................................................. 1-8

1.6.2 Thermal Effects on Flow ...................................................................................... 1-10

1.7 Relation to Guidance From Peer Review and Oversight Groups ................................... 1-11

1.7.1 Performance Assessment Peer Review ................................................................ 1-11

1.7.2 Thermohydrology Peer Review ...................................................................... 1-12

1.7.3 Nuclear Waste Technical Review Board ............................................................. 1-13

1.8 Scope and Organization of the Near-Field/Altered-Zone Models Report................................1-14

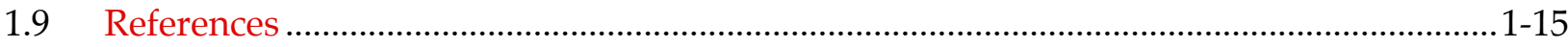

2. Conceptual Model for Evolution of Coupled Processes in the

Near Field and Altered Zone..................................................................................................... 2-1

2.1 Application of Near-Field and Altered-Zone Models.......................................................... 2-3

2.2 Evolution of the Near-Field Environment and Altered Zone............................................2-4

2.2.1 Time Period A: Drift Closure to Repository Closure ............................................... 2-4

2.2.2 Time Period B: Development of the Superheated Dryout Zone (Repository Closure to a Few Hundred Years) ........................................................2-6 
2.2.3 Time Period C: Quasi-Stable Reflux Zone (500 to 1500 yr) ..................................... 2-7

2.2.4 Time Period D: Collapse of the Superheated Dryout Zone (1000 to 3000 yr) ........ 2-9

2.2.5 Time Period E: Cool-Down (3000 to 10,000 yr)..................................................... 2-10

2.2.6 Time Period F: Post-Thermal Period (10,000 to 100,000 yr) .................................. 2-11

2.3 Uncertainties in the Conceptual Model for Near-Field / Altered-Zone

Coupled Processes............................................................................................................. 2-12

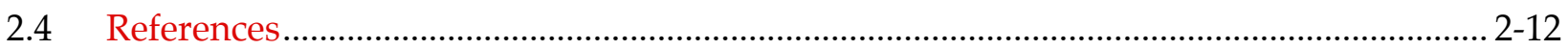

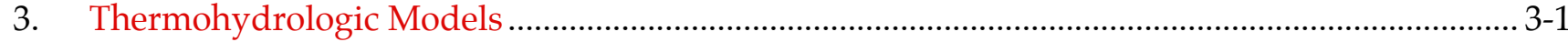

3.1 Impact of Thermohydrology on the Proposed Yucca Mountain Repository ...................... 3-1

3.1.1 Effects of Design Alternatives …........................................................................... 3-1

3.1.2 Identification of Users for Thermohydrologic Models .......................................... 3-2

3.2 Qualitative Description of Unsaturated-Zone Conditions, Processes,

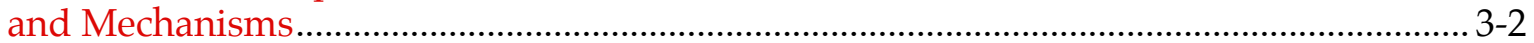

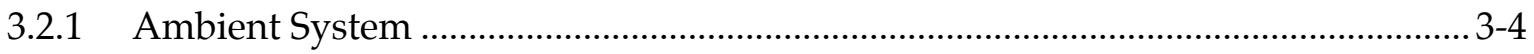

3.2.2 Thermally Disturbed System ............................................................................. 3-7

3.2.3 Final System State ................................................................................................ 3-8

3.3 Heat and Mass Flow in Fractured Permeable Media..............................................................3-8

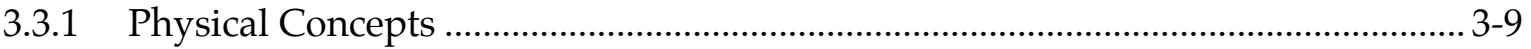

3.3.2 Heat Conduction Models ......................................................................................... 3-9

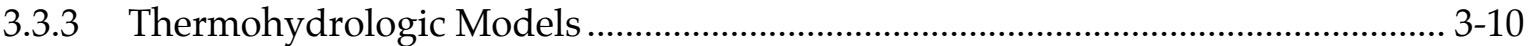

3.3.4 Mathematical Model Description …..................................................................... 3-13

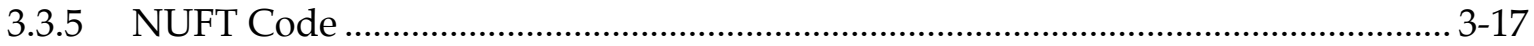

3.3.6 Model Limitations: Scale and Dimensionality ................................................... 3-18

3.4 Modeling Field Experiments …......................................................................................... 3-19

3.4.1 Purpose of Thermal Tests.................................................................................... 3-19

3.4.2 Simulation of the Single-Heater Test..................................................................... 3-21

3.4.3 Simulation of Large-Block Test .......................................................................... 3-29

3.5 Reference Calculations for Drift-Scale Coupled Processes …............................................ 3-33

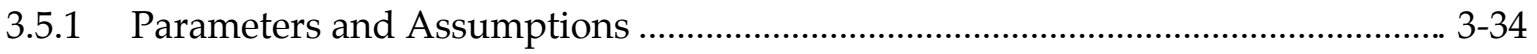

3.5.2 TH Model Results for Analysis of Coupled Effects ............................................. 3-44 


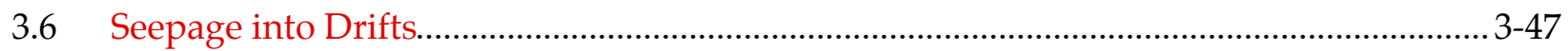

3.6.1 Physics of Seepage into an Open Cavity ….......................................................... 3-47

3.6.2 Scales of Heterogeneity and their Influence on Seepage ...................................... 3-48

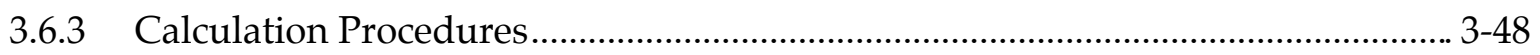

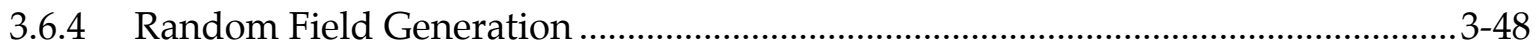

3.6.5 Steady-State Isothermal Seepage ....................................................................... 3-49

3.6.6 Transient Isothermal Models ............................................................................ 3-51

3.6.7 Nonisothermal Models .................................................................................. 3-56

3.7 Multi-Scale Models and Assumptions for TSPA-VA Support ...........................................3-61

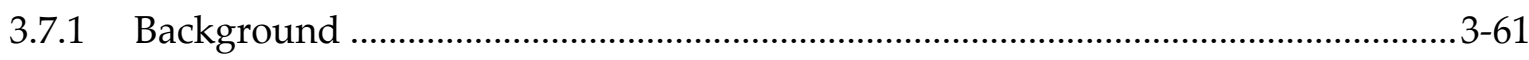

3.7.2 Calculated Performance Measures ....................................................................... 3-61

3.7.3 Location-Specific Data for Calculation of TSPA-VA Performance Measures ....... 3-63

3.7.4 Thermal-Loading Criteria Used in Multi-Scale Approach.................................... 3-65

3.7.5 Description of Submodels Used in Multi-Scale Approach..................................... 3-66

3.7.6 Procedure for Calculating EBS TH Conditions ....................................................... 3-73

3.7.7 Multi-Scale Model Results and Analysis ............................................................ 3-80

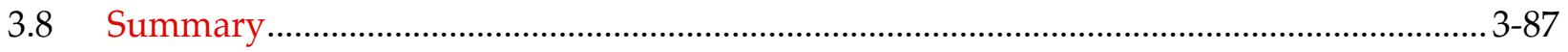

3.8.1 Conceptual Models and Codes.............................................................................

3.8.2 Process Model Applications .................................................................................... 3-89

3.8.3 TSPA Multi-Scale Abstraction ........................................................................... 3-91

3.8.4 Improving TH Models to Reduce Uncertainty ….............................................. 3-92

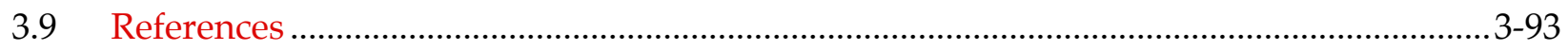

4. Thermomechanical and Thermohydromechanical Models .......................................................4-1

4.1 Introduction to Thermomechanical and Thermohydromechanical Models....................... 4-1

4.1.1 Identification of Users for Thermomechanical and

Thermohydromechanical Models ......................................................................... 4-2

4.2 Conceptual Basis for Rock-Mass Thermomechanical Models .............................................4-3

4.2.1 Geomechanical Aspects of the Near-Field Environment ......................................4-3

4.2.2 Available Model Approaches ................................................................................. 4-3

4.2.3 Approach to Study of Coupled Behavior ............................................................ 4-7 


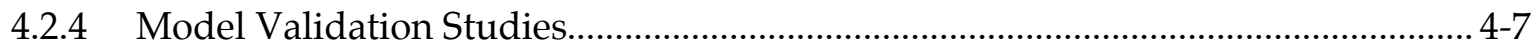

4.2.5 Testing Model Predictions for the Large-Block Test .............................................. 4-8

4.3 Initial Conditions and Rock-Mass Rheological Behavior at Ambient

and Elevated Temperature ............................................................................................. 4-10

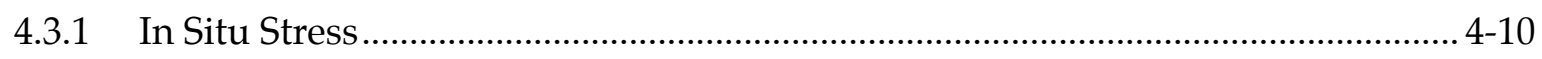

4.3.2 Mechanical Properties of Topopah Spring Tuff .................................................. 4-11

4.3.3 Thermal Properties of Topopah Spring Tuff......................................................... 4-14

4.3.4 Fracture Mechanical Properties …........................................................................ 4-16

4.4 Simulation of Thermomechanical Responses to Thermal Loading .................................. 4-20

4.4.1 Thermomechanical Simulation of Drift Scale Test ............................................... 4-20

4.4.2 Thermomechanical Modeling ............................................................................. 4-20

4.4.3 FLAC Simulation of Reference Case 1-Total System Performance Assessment-Viability Assessment (TSPA-VA) Base Case ..................................... 4-22

4.4.4 FRACROCK Simulation of Reference Cases 1 and 5 .......................................... 4-27

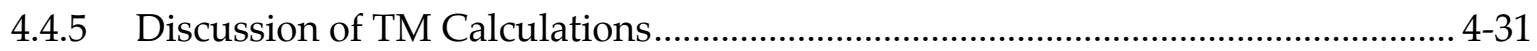

4.5 Conceptual Models of Fracture-Permeability Change in Response

to Stress and Deformation...................................................................................................... 4-32

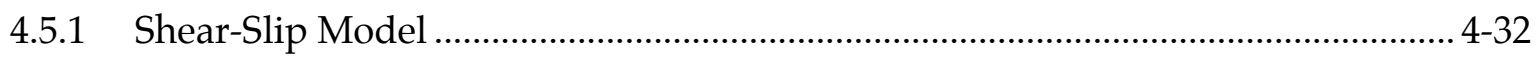

4.6 Thermohydromechanical Changes Predicted for the Drift-Scale Test ............................. 4-36

4.6.1 Predicted Changes ......................................................................................... 4-36

4.7 Thermohydromechanical Changes Predicted for Reference Drift-Scale

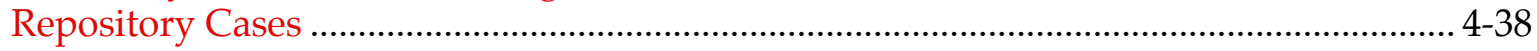

4.8 Summary of Thermomechanical Models............................................................................ 4-38

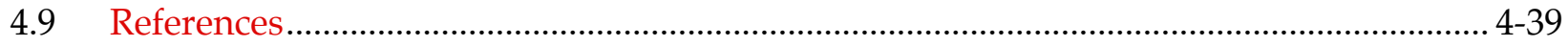

5. Thermohydrochemical Models of the Altered Zone and the Near Field .................................... 5-1

5.1 Introduction to Thermohydrochemical Models ................................................................. 5-1

5.1.1 Identification of Users for Thermohydrochemical Models................................... 5-2

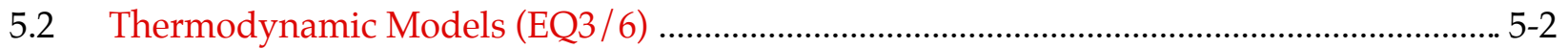

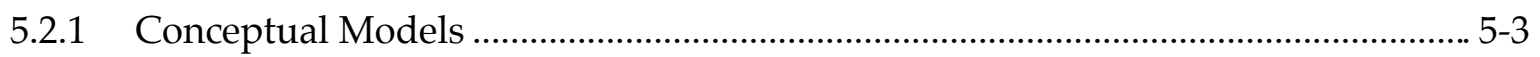

5.2.2 Supporting Thermodynamic Data...................................................................... 5-13

5.2.3 Numerical Approach and Description of EQ3 / 6 ............................................ 5-16 
5.3 Reactive-Transport Models .............................................................................................. 5-24

5.3.1 Mathematical and Numerical Approaches to Reactive-Transport Modeling ...... 5-25

5.3.2 Supporting Kinetic Data ................................................................................. 5-37

5.3.3 Conceptual Model for Reactive Transport in the Altered Zone ........................... 5-42

5.4 Reaction-Path Model for Water Chemistry and Mineral Evolution

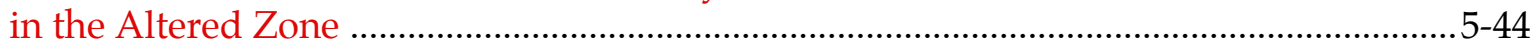

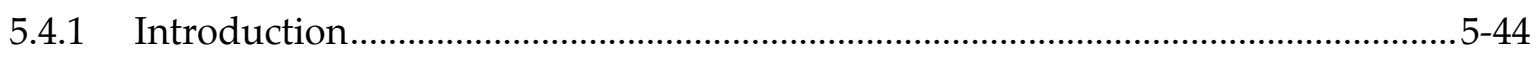

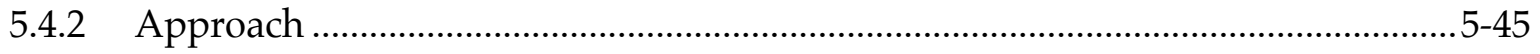

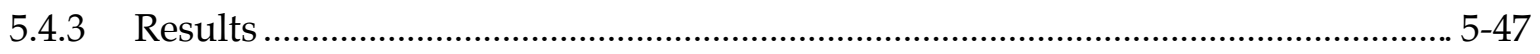

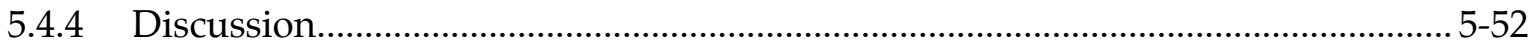

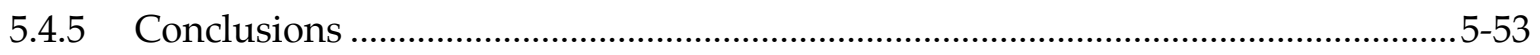

5.5 Repository-Scale Thermohydrochemical Scoping Calculations Involving Silica ............. 5-53

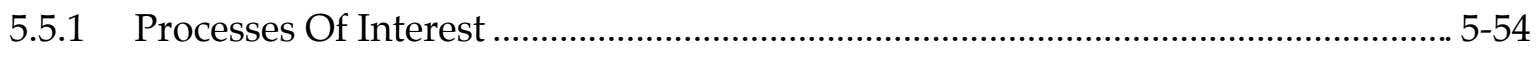

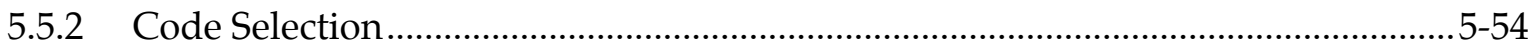

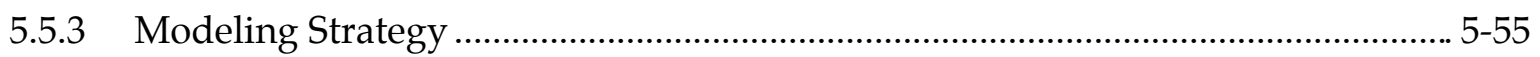

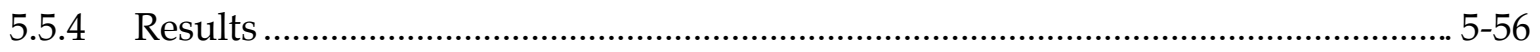

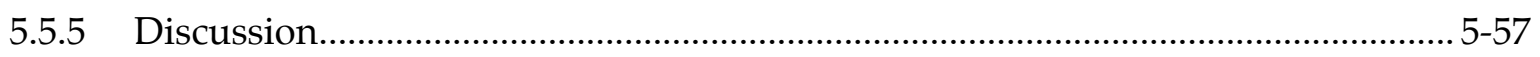

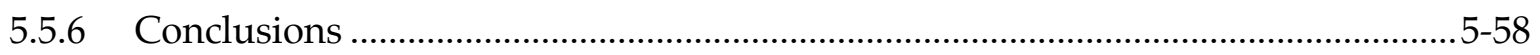

5.6 Thermohydrochemical Alteration of Flow Pathways Above and Below

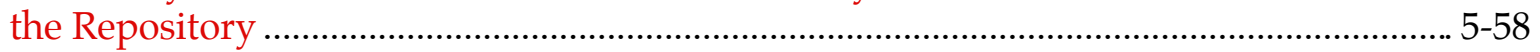

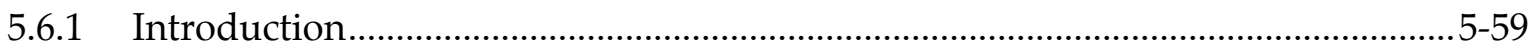

5.6.2 Description of Coupled-Process Phenomena ........................................................5-60

5.6.3 Mathematical Model for Reactive Transport ......................................................... 5-61

5.6.4 Numerical Solution of Coupled Equations..........................................................5-64

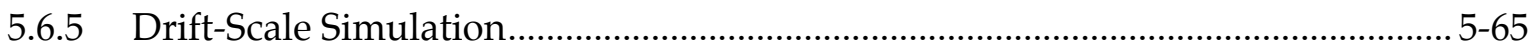

5.7 Drift-Scale THC Scoping Calculations and Alteration of Flow Pathways

Above and Below Repository Drifts ..................................................................................... 5-70

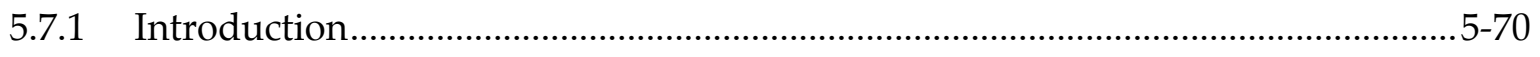

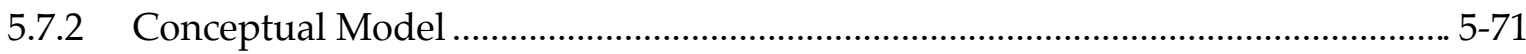

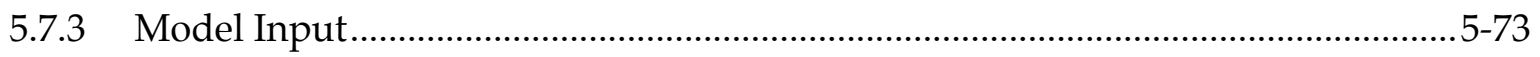

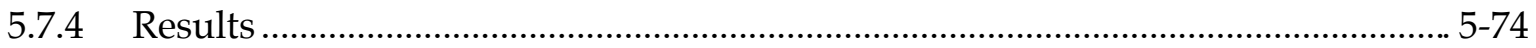


5.7.5 Discussion …………………………………………………………………. 5-79

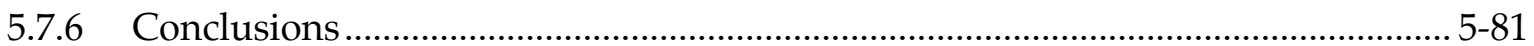

5.8 Summary of Thermohydrochemical Models ………........................................................... 5-81

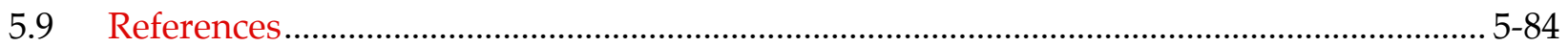

6. Near-Field Geochemical Environment …………………........................................................... 6-1

6.1 Introduction to Models of the Near-Field Geochemical Environment .................................. 6-1

6.1.1 Previous Description of Conditions in the Near Field/ Altered Zone ..................... 6-2

6.1.2 Identification of Users for Models of the Near-Field Geochemical

Environment ........................................................................................................... 6-2

6.2 Chemical Composition of Water in the Altered Zone Before Entering

the Near-Field Environment.............................................................................................. 6-3

6.2.1 Fracture-Water Composition in the Absence of Evaporation Effects ...................... 6-4

6.2.2 Water Composition Affected by Evaporative Concentration..................................... 6-9

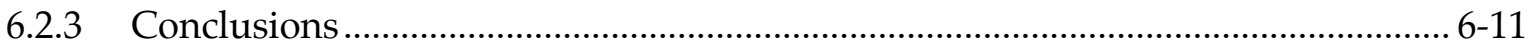

6.3 Chemistry of Water in the Near-Field Environment ......................................................... 6-12

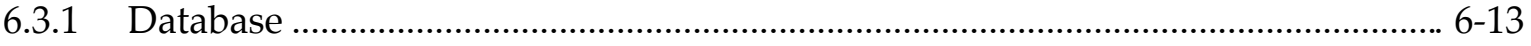

6.3.2 EQ3 Calculations: Speciation of Incoming Water and Additional Constraints............................................................................................... 6-14

6.3.3 EQ6: Interaction of Water with Solid Phases............................................................ 6-14

6.3.4 Results and Discussion....................................................................................... 6-18

6.4 Concentrations of Radionuclides in Water Leaving the Near Field ................................... 6-20

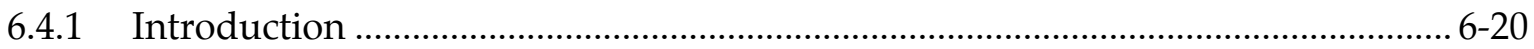

6.4.2 Phases that Will Occur along Transport Pathways................................................... 6-21

6.4.3 Iron Corrosion Products...................................................................................... 6-21

6.4.4 Cementitious Materials …………………………………………………………. 6-37

6.5 Radionuclide Transport in the Altered Zone ...................................................................... 6-44

6.5.1 Thermodynamic Constraints on Water-Borne Radionuclide Concentrations ..... 6-45

6.5.2 Alternative Modes of Radionuclide Transport in the NF / AZ ............................... 6-59

6.6 Summary of Geochemical Conditions in the Near Field / Altered Zone............................ 6-69

6.6.1 Altered Zone Water Composition ………………………………………………...... 6-69

6.6.2 Water Composition in Contact with Cement............................................................. 6-70 
6.6.3 Concentrations of Radionuclides Leaving the Repository …................................. 6-70

6.6.4 Radionuclide Transport in the Altered Zone .......................................................6-71

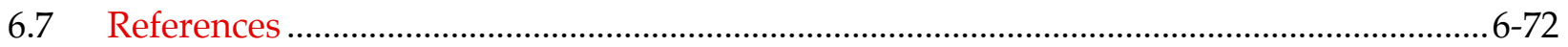

7. Modeling the Effects of Introduced Materials and Microbial Activity …....................................7-1

7.1 Chemical Composition of Materials, Their Evolution, and Processes Operating in the Emplacement Drifts ......................................................................................................

7.1.1 Range of Introduced Materials That May Be Used in the NFE .............................7-1

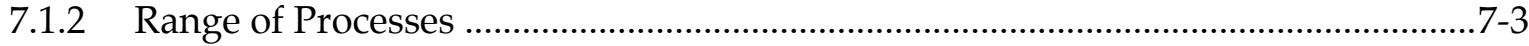

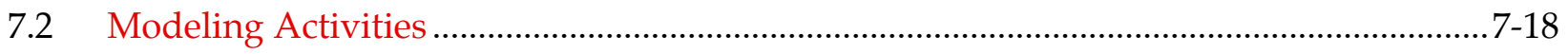

7.2.1 Abiotic Chemical Models ....................................................................................... 7-18

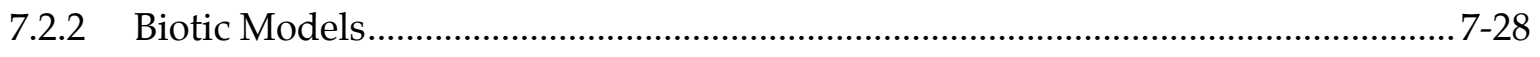

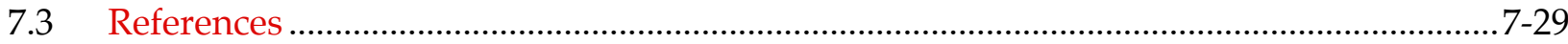

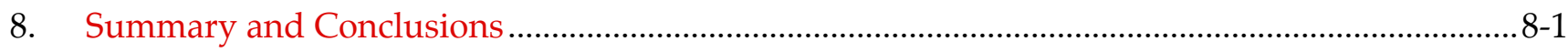

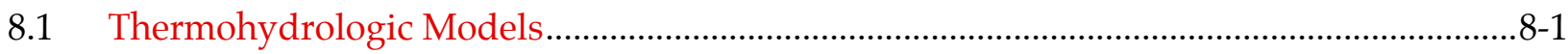

8.1.1 Activities for Building Confidence in Models........................................................ 8-2

8.1.2 Reference Calculations for Evaluation of Coupled Processes ................................8-2

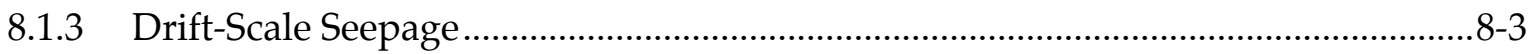

8.1.4 Total System Performance Assessment Multi-Scale Abstraction............................ 8-3

8.1.5 Improving Thermohydrologic Models to Reduce Uncertainty.............................. 8-4

8.1.6 Conclusions Regarding Thermohydrologic Models ..............................................8-4

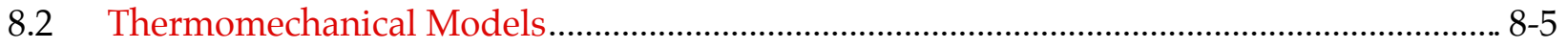

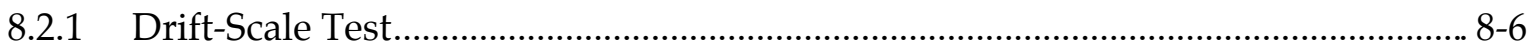

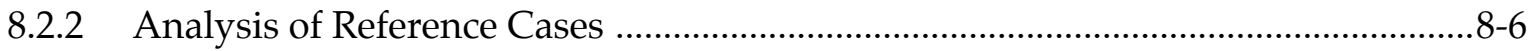

8.2.3 Improving Thermomechanical Models to Reduce Uncertainty …..........................8-7

8.2.4 Conclusions Regarding Thermomechanical Models .............................................. 8-7

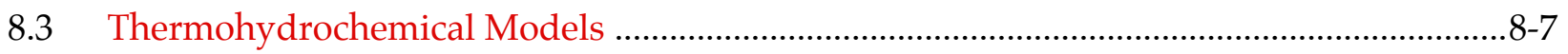

8.3.1 Reaction-Path Study ........................................................................................... 8-8

8.3.2 Coupled Repository-Scale Calculation with Silica ................................................ 8-8

8.3.3 Drift-Scale TH Simulations with Silica.................................................................. 8-9

8.3.4 Reactive-Transport Calculations …...................................................................... 8-10 
8.3.5 Conclusions Regarding THC Models................................................................... 8-11

8.4 Geochemical Conditions in the Near Field / Altered Zone................................................. 8-12

8.4.1 Altered-Zone Water Composition ........................................................................ 8-13

8.4.2 Water Composition in Contact With Cement .................................................... 8-13

8.4.3 Concentrations of Radionuclides Leaving the Repository................................... 8-14

8.4.4 Radionuclide Transport in the Altered Zone ..................................................... 8-14

8.5 General Conclusions for the Near-Field / Altered-Zone Models Report........................... 8-15

8.5.1 Understanding the Precipitation Cap ............................................................. 8-16

8.5.2 Thermohydrology Issues for Repository Design................................................... 8-16

8.5.3 Near-Field / Altered-Zone Models .................................................................... 8-17

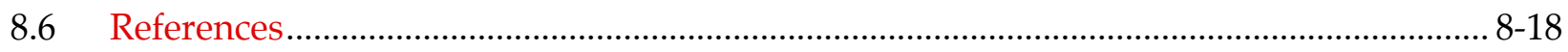

Appendix A: Quality Assurance Information ……...................................................................... A-1

Appendix B: Technical Data Submissions.........................................................................................

\section{Tables}

Table 1-1 Issue resolution for Key Technical Issue: Evolution of the

Near-Field Environment........................................................................................... 1-9

Table 1-2 Issue resolution for Key Technical Issue: Thermal Effects on Flow ..................................1-11

Table 2-1 Direct and indirect Onsager-type coupled processes.................................................. 2-2

Table 3-1 Relations among model hydrogeologic units and geologic formations, geologic framework model (DTN LB970601233129.001) ............................................................ 3-3

Table 3-2 Summary of thermal and hydrologic properties of host rock for the SHT area (tsw34 unit).

Table 3-3 Matrix and fracture capillary properties: comparison among property sets and

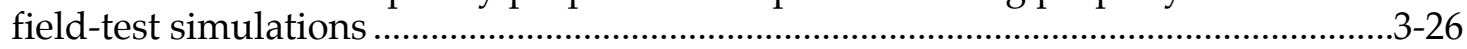

Table 3-4 Model parameters for reference calculations ….........................................................3-33

Table 3-5 TSPA-VA thermal properties for the rock matrix .......................................................3-36

Table 3-6 Matrix property values in the July 1997 TSPA-VA base-case hydrologic parameter set for $\mathrm{I} \times 1$, where I stands for the nominal infiltration-flux map

Table 3-7 Fracture property values in the July 1997 TSPA-VA base-case hydrologic parameter set for $\mathrm{I} \times 1$, where I stands for the nominal infiltration-flux map 3-38 
Table 3-8 Matrix property values for the July 1997 TSPA-VA base-case hydrologic parameter set for I/5, where I stands for the nominal infiltration-flux map

Table 3-9 Fracture property values for the July 1997 TSPA-VA base-case

hydrologic parameter set for I/5, where I stands for the nominal infiltration-flux map

Table 3-10 Matrix property values for the November $1997 \mathrm{TH}$ hydrologic parameter set calibrated against the SHT.

Table 3-11 Fracture property values for the November $1997 \mathrm{TH}$ hydrologic parameter set that was calibrated against the SHT.

Table 3-12 Matrix property values for the December 1997 "modified-TH" hydrologic parameter set for only the property values that differ from the November 1997 TH hydrologic parameter set

Table 3-13 The value of matrix wetting diffusivity $D_{\text {imb }}$ for the host-rock units and neighboring units, for various hydrologic parameter sets $\left(\mathrm{m}^{2} / \mathrm{sec}\right)$

Table 3-14 The magnitude of liquid-phase flux qliq $(\mathrm{mm} / \mathrm{yr})$ in fractures in heat-pipe zone above repository horizon

Table 3-15 The vertical length Lhp (m) of the heat-pipe-zone..

Table 3-16 Reference hydrologic properties for steady-state isothermal seepage calculations

Table 3-17 Hydrologic properties for the TSw35 unit, from the 1997 TSPA-VA property set (DKM)

Table 3-18 Hydrologic properties for the TSw35 unit, from the 1997 TSPA-VA property set $(\mathrm{ECM})$.

Table 3-19 Hydrologic properties of the TSw1 unit, based on

Klavetter and Peters

Table 3-20 Thermohydrologic measures estimated by the multi-scale

TH modeling approach ...

Table 3-21 Summary of model types used in the multi-scale

TH modeling approach

Table 3-22 Drift-scale model column locations 3-63

Table 3-23 Infiltration $q$ imp ( $\mathrm{mm} / \mathrm{yr})$ and liquid-phase saturation Sliq.rep at the repository depth, for the columns modeled for TSPA-VA

Table 3-24 Summary of the WP heat sources used in submodels for the multi-scale approach 3-66

Table 4-1 Values and ranges of principal stresses in the potential repository

horizon at Yucca Mountain (Stock et al., 1984, 1985). $4-11$

Table 4-2 Mechanical properties of Topopah Spring tuff and fractures..... 4-11 
Table 4-3 Thermal properties of Topopah Spring tuff. $4-14$

Table 4-4 Coefficient of thermal expansion in TSw2 unit at different temperatures, from Brodsky et al. (1997)

Table 4-5 Young's modulus values for Experiment 7195 (SB-3 fracture parallel to rock fabric)

Table 4-6 FLAC required properties and sources

Table 4-7 Input parameters for FRACROCK and HEAT

Table 5-1 The calculated speciation of uranium, neptunium, and plutonium present in trace amounts in spiked J-13 well water

Table 5-2 The calculated speciation of $\mathrm{U}, \mathrm{Np}$, and $\mathrm{Pu}$ present in trace amounts in theoretically spiked J-13 well water $(\mathrm{Eh}=100,200,340,500$, and $778 \mathrm{mV})$

Table 5-3 Calculated saturation indices $(\log Q / K)$ for selected solids in theoretically spiked J-13 well water $(\mathrm{Eh}=340 \mathrm{mV})$.

Table 5-4 Some of the EQ3 / 6 data files in the Revision 6 set and their characteristics.

Table 5-5 Provisional dissolution rate constants and activation energies for important minerals in the Topopah Spring member of the Paintbrush Tuff

Table 5-6 Distribution of selected mineralogical, chemical, and mechanical processes that are likely to develop within Regimes A through D and to affect TH conditions....

Table 5-7 Cristobalite dissolution base-case parameter values..... $5-63$

Table 5-8 Quartz dissolution base-case parameter values. $.5-64$

Table 5-9 Carbon dioxide extraction yield from distillation of condensable gases from preserved Yucca Mountain core samples

Table 5-10 Minerals considered in the simulations, their initial abundances and surfaces along the fracture surfaces, and the controlling rate constants and respective activation energies for the applicable dissolution reactions

Table 6-1 Fracture-water compositions from field samples, experiments, and models

Table 6-2 Solid reactants used in EQ6 simulations at $25^{\circ} \mathrm{C}$; Identical simulations have been conducted at $60^{\circ}$ and $150^{\circ} \mathrm{C}$.

Table 6-3 Parameters used for modeling sorption (DLM) and transport of uranium using REACT and X1t codes.....

Table 6-4

Summary of parameters used to simulate transport of $U$ through hematite corrosion layer.

Table 6-5 Composition of five hypothetical U-bearing groundwaters used to simulate transport of $U$ through hematite corrosion products. 
Table 6-6 Composition of fluids used for assessing the effect of a high pH cementitious material on transport of $U$ through a hematite corrosion product layer.

Table 6-7 Phases identified by X-ray diffraction of untreated and hydrothermally altered concrete

Table 6-8 Tracer experiments with fractured, altered concrete core* 6-42

Table 6-9 Cation concentrations $(\mathrm{mM})$ in the reference water $6-46$

Table 6-10 Anion concentrations (mM) in the reference water $6-47$

Table 6-11 Steady-state results from Ollila et al. (1996).

Table 6-12 Solubility-controlling solids from Nitsche, 1991; Nitsche et al., 1993a, 1995, $1993 b$ $6-50$

Table 6-13 Summary of solubility product constants for $\mathrm{Ni}(\mathrm{OH})_{2}$ 6-55

Table 6-14 Solubility products for $\mathrm{Zr}(\mathrm{OH})_{4}$ and $\mathrm{ZrO}_{2}$ 6-56

Table 6-15 Summary of expected solubility-controlling solids 6-58

Table 7-1 Calculation of the chemical composition of starting materials: grout per linear meter of repository emplacement drift.

Table 7-2 Calculation of mineral assemblage per linear meter in young grout $7-8$

Table 7-3 Calculation of mineral assemblage per linear meter in thermally treated concrete, $\mathrm{O}_{2}$-rich environment $\left(\mathrm{O}_{2}=\right.$ close to or above atmospheric)

Table 7-4 Calculation of mineral assemblage per linear meter in thermally treated concrete, $\mathrm{CO}_{2}$-rich environment ( $\mathrm{CO}_{2}$ above atmospheric)

Table 7-5 Thermally treated concrete, $\mathrm{CO}_{2}$-rich environment $\left(\mathrm{CO}_{2}\right.$ only) 7-11

Table 7-6 Experimental compositions investigated by selected authors $7-22$

Table 7-7

Sources of the Berner and Glasser et al. models.....

Table 7-8

Selected aspects of the Berner and Atkins et al.-Bennett et al. models

Table 7-9 List of the cement-related minerals present in the EQ3 / 6 COM data file (included in cem.R27): extrapolation algorithm, specific heat ( $\mathrm{C}_{\mathrm{p}}$ coefficients), and corresponding bibliographic references $7-25$

Table A-1 "Roadmap" table identifying where the deliverable (SP3100M4) acceptance criteria are met in the Near-Field/Altered-Zone Models Report

Table A-2 Simulation codes and chemical databases used in the Near-Field/Altered-Zone Models Report

Table A-3 Summary of significant data used in Chapter 3 A-8

Table A-4

Summary of significant data used in Chapter 4 A-10

Table A-5

Summary of significant data used in Chapter 5. A-11

Table A-6

Summary of significant data used in Chapter 6 . A-12 
Table A-7 Summary of significant data used in Chapter 7 ....................................................... A-13

Table A-8 Summary of models presented in the Near-Field/Altered-Zone Models Report ...............................................................................13

Table A-9 List of Yucca Mountain Site Characterization Project deliverables used in the Near-Field/Altered-Zone Models Report ..................................................... A-14 


\section{Figures}

Figure 1-1 Distribution of inflow rates per each 2- $\mathrm{m}^{2}$ collector in the Stripa experiment

Figure 2-1 Schematic of Near-Field Environment and Altered Zone showing TSPA reaction cells, annotated with section numbers from this report

Figure 2-2 Schematic of emplacement drift conditions at repository closure

Figure 2-3 Schematic of emplacement drift conditions at a few hundred years after repository closure (after dryout zone development)

Figure 2-4 Schematic of emplacement drift conditions during the period of a quasi-stable reflux zone

Figure 2-5 Schematic of emplacement drift conditions during collapse of the superheated dryout zone

Figure 2-6 Schematic of emplacement drift conditions during the cool-down period

Figure 2-7 Schematic of emplacement drift conditions during the post-thermal period

Figure 3-1 Surface topography, shallow infiltration, repository footprint, and model domain for site-scale thermal modeling at Yucca Mountain

Figure 3-2 Drift-scale schematic showing decay-heat-driven TH flow and transport processes

Figure 3-3 Mountain-scale schematic showing decay-heat-driven TH flow and transport processes that influence moisture redistribution and the moisture balance in the UZ

Figure 3-4 SHT layout, including dimensions and SHT-model coordinate system, in plan view and vertical Section A-A'

Figure 3-5 Temperature and liquid-phase saturation distributions in a vertical $(x-z)$ plane transverse to the midpoint of the heater for Case 2

Figure 3-6 Temperature and liquid-phase saturation distributions in a vertical $(y-z)$ plane along the axis of the heater for Case 2

Figure 3-7 Calculated and measured temperature histories for selected thermocouples and boreholes in the SHT

Figure 3-8 Plan view of the host-rock distribution

Figure 3-9 Schematic of the LBT showing the location of the five heater boreholes and the LBTmodel coordinate system

Figure 3-10 Temperature and liquid-phase saturation distributions along different profiles through the large block

Figure 3-11 Calculated and measured temperature and liquid-phase saturation histories at thermocouple TT-114

Figure 3-12 Vertical temperature and liquid-phase saturation profiles at the center of the LBT

Figure 3-13 Vertical distribution of hydrostratigraphic model units shown for drift-scale model locations (in Nevada-State coordinates)

Figure 3-14 Vertical cross-section of the emplacement drift in the DDT and LDTH model showing the thermal radiation connections among the surfaces in the drift that are used in thermal simulations

Figure 3-15 Temperature and liquid-phase saturation distributions for Case 1 
Figure 3-16 Temperature and liquid-phase saturation distributions for Case 2

Figure 3-17 Temperature and liquid-phase saturation distributions for Case 3

Figure 3-18 Temperature and liquid-phase saturation distributions for Case 4

Figure 3-19 Temperature and liquid-phase saturation distributions for Case 5

Figure 3-20 Vertical distribution of liquid-phase flux $q_{\text {liq }}$ for Cases 3 and 5

Figure 3-21 Vertical temperature and liquid-phase saturation distributions for Cases 3 and 5

Figure 3-22 Front and side views of 3-D base-10 logarithm of hydraulic conductivity (m/s) field for the matrix continuum

Figure 3-23 Front and side views of the 3-D grid

Figure 3-24 Seepage flux into drift vs. average percolation flux into top of model

Figure 3-25 Ratio of seepage flux into the drift divided by ambient percolation flux vs. time

Figure 3-26 Steady-state isosurfaces of 50\% fracture saturation for a 3-D simulation

Figure 3-27 Seepage flux into the drift vs. percolation flux into the top of the model for the heterogeneous dual-permeability base case, heterogeneous equivalent continuum case, and homogeneous dual-permeability case

Figure 3-28 Seepage flux vs. percolation flux for the July 1997 TSPA-VA DKM case

Figure 3-29 Seepage flux vs. percolation flux is $1.2 \mathrm{~m}$ around the drift for the base case and for the increased-fracture-permeability case

Figure 3-30 Total flux into drift vs. percolation flux for the base case and the case with $50 \% \mathrm{RH}$ in the drift to simulate ventilation

Figure 3-31 Seepage flux vs. time for cases with and without ventilation at $50 \%$ relative from time 0 to $100 \mathrm{yr}$

Figure 3-32 Seepage flux vs. time for the case with 10,000 mm/yr pulse every year, lasting over a 2-day period, imposed on a background flux of $5 \mathrm{~mm} / \mathrm{yr}$

Figure 3-33 Liquid fracture saturation during the start of an infiltration event with a flux into the top of the model of $10,000 \mathrm{~mm} / \mathrm{yr}$ over a 2-day period

Figure 3-34 Seepage flux vs. time from beginning of each year for the case with $100 \mathrm{~mm} / \mathrm{yr}$ pulse every year

Figure 3-35 Seepage flux vs. time from beginning of each year for the case with $1000 \mathrm{~mm} / \mathrm{yr}$ pulse every year

Figure 3-36 Seepage flux vs. time from beginning of each year for the case with 10,000 mm/yr pulse every year

Figure 3-37 Seepage flux vs. time from beginning of each year for the case with 100,000 mm/yr pulse every year

Figure 3-38 Close-up of the nested grid and the $\log _{10}$ bulk permeability field around the drift

Figure 3-39 Seepage flux into the drift for the homogeneous, 5-mm/yr case under point-loading with the TSPA-VA

Figure 3-40 Seepage flux into the drift for heterogeneous, 5-mm/ yr case under point-loading with TSPA-VA property set

Figure 3-41 Seepage flux into the drift for the homogeneous, 5-mm/yr case under point-loading with the Klavetter and Peters (1986) property set 
Figure 3-42 Seepage flux into the drift for the heterogeneous, 5-mm/yr case under point-loading with the Klavetter and Peters (1986) property set

Figure 3-43 Liquid saturation in the drift at 3 yr for 5-mm/yr percolation flux using the Klavetter and Peters (1986) property set with heterogeneous various

Figure 3-44 Seepage flux into the drift for the heterogeneous, 5-mm/yr case with the Klavetter and Peters (1986) property set-comparison between line- and point-loading

Figure 3-45 Seepage flux into the drift for the heterogeneous, 5-mm/yr case under point-loading with the Klavetter and Peters (1986) property set-effect of altering the degree of fracturing around the drift

Figure 3-46 Schematic of conceptual models used by the multi-scale TH modeling approach

Figure 3-47 Vertical distribution of hydrostratigraphic model units for several drift-scale model locations (in Nevada-State coordinates) (columns 11c1-11c5)

Figure 3-48 Vertical distribution of hydrostratigraphic model units for drift-scale model locations (in Nevada-State coordinates) (columns 12c1-12c5)

Figure 3-49 Vertical distribution of hydrostratigraphic model units for drift-scale model locations (in Nevada-State coordinates) (columns 13c1-13c5)

Figure 3-50 Vertical distribution of hydrostratigraphic model units for drift-scale model locations (in Nevada-State coordinates) (columns 15c1-15c5)

Figure 3-51 Vertical distribution of hydrostratigraphic model units for drift-scale model locations (in Nevada-State coordinates) (columns 16c1-16c5)

Figure 3-52 Vertical distribution of hydrostratigraphic model units for drift-scale model locations (in Nevada-State coordinates) (columns 17c1-17c5)

Figure 3-53 Plan view of the WP layout represented in the DDT models

Figure 3-54 Flow chart for the multi-scale TH modeling approach

Figure 3-55 Functional relationships plotted for the process-model inputs to the multi-scale $\mathrm{TH}$ modeling approach

Figure 3-56 Functional relationships plotted for the process-model inputs to the multi-scale TH modeling approach as functions of time

Figure 3-57 The six infiltration-flux subdomains and the infiltration-flux $q_{\text {inf }}$ distribution shown for the SMT-model representation of the repository area

Figure 3-58 Temperature distribution in the repository host rock predicted by the SMT model

Figure 3-59 Mountain-scale temperature distribution

Figure 3-60 Relative humidity $R H$ on the surface of "average" 21-PWR medium-heat CSNF WPs plotted for the 7/97 TSPA-VA "I $\times 1$ " hydrologic-parameter set and for the 12/97 modified-TH parameter set

Figure 3-61 Relative humidity RH on the surface of "average" 21-PWR medium-heat CSNF WPS plotted for three infiltration-flux $q_{\text {inf }}$ cases

Figure 3-62 Summary of TH condiions for three WP types

Figure 3-63 RH on the surface of "average" 21-PWR medium-heat CSNF WPs plotted for the point-load design

Figure 3-64 Temperature and $R H$ on the drift wall and on the WP for the point-load and designload designs with no backfill 
Figure 3-65 Temperature and RH on the drift wall and on the WP for the point-load and designload designs with backfill at $100 \mathrm{yr}$

Figure 4-1 Illustration of small-block experiment for flow through horizontal fracture

Figure 4-2 History of fluid flow and axial stress on sample SB-3 during first $600 \mathrm{hr}$ of testing

Figure 4-3 Axial load (uniaxial stress) vs. elapsed time for Test 7195

Figure 4-4 Cumulative flow data for Test 7195

Figure 4-5 Spatial representation of total flow in plane of fracture for sample SB-3 during Test 7195

Figure 4-6 Flow rate at four different fluid inlet pressures, plotted as a function of axial stress for Test 7195

Figure 4-7 Schematic of the Drift-Scale Test

Figure 4-8 Total FLAC grid

Figure 4-9 Enlarged portion of grid between heated drift and access drift

Figure 4-10 Portion of mesh used for FLAC simulations of reference Case 1

Figure 4-11 Principal stresses computed for excavation only using FLAC for Case 1

Figure 4-12 Vertical stress field computed using FLAC for excavation only, Case 1

Figure 4-13 Horizontal stress field computed using FLAC for excavation only, Case 1

Figure 4-14 Shear stress field computed using FLAC for excavation only, Case 1

Figure 4-15 Temperature field for Case 1 at $50 \mathrm{yr}$

Figure 4-16 Principal stress field computed using FLAC, Case 1 at $50 \mathrm{yr}$

Figure 4-17 Vertical stress field computed using FLAC, Case 1 at $50 \mathrm{yr}$

Figure 4-18 Vertical displacement field computed using FLAC, Case 1 at $50 \mathrm{yr}$

Figure 4-19 Horizontal stress field computed using FLAC, Case 1 at $50 \mathrm{yr}$

Figure 4-20 Horizontal displacement field computed using FLAC, Case 1 at $50 \mathrm{yr}$

Figure 4-21 Shear stress field computed using FLAC, Case 1 at $50 \mathrm{yr}$

Figure 4-22 Temperature field for Case 1 at $100 \mathrm{yr}$

Figure 4-23 Principal stress field computed using FLAC, Case 1 at $100 \mathrm{yr}$

Figure 4-24 Vertical stress field computed using FLAC, Case 1 at $100 \mathrm{yr}$

Figure 4-25 Vertical displacement field computed using FLAC, Case 1 at $100 \mathrm{yr}$

Figure 4-26 Horizontal stress field computed using FLAC, Case 1 at $100 \mathrm{yr}$

Figure 4-27 Horizontal displacement field computed using FLAC, Case 1 at $100 \mathrm{yr}$

Figure 4-28 Shear stress field computed using FLAC, Case 1 at $100 \mathrm{yr}$

Figure 4-29 Temperature field for Case 1 at $1000 \mathrm{yr}$

Figure 4-30 Principal stress field for 1000 yr computed using FLAC

Figure 4-31 Vertical stress field for 1000 yr computed using FLAC

Figure 4-32 Vertical displacement field for 1000 yr computed using FLAC

Figure 4-33 Horizontal stress field for 1000 yr computed using FLAC

Figure 4-34 Horizontal displacement field for $1000 \mathrm{yr}$ computed using FLAC

Figure 4-35 Shear stress field for 1000 yr computed using FLAC 
Figure 4-36 Zone used in FRACROCK for Case 1 stress analysis

Figure 4-37 Temperature field for Case 1 at $50 \mathrm{yr}$, as computed by NUFT

Figure 4-38 Simulation of 50-yr temperature field for Case 1 for input to FRACROCK

Figure 4-39 Vertical stress field for Case 1 at $50 \mathrm{yr}$, computed using FRACROCK

Figure 4-40 Horizontal stress field for Case 1 at $50 \mathrm{yr}$, computed using FRACROCK

Figure 4-41 Shear stress field for Case 1 at $50 \mathrm{yr}$, computed using FRACROCK

Figure 4-42 Temperature field for Case 1 at $100 \mathrm{yr}$, as computed by NUFT

Figure 4-43 Simulation of 100-yr temperature field for Case 1 for input to FRACROCK

Figure 4-44 Vertical stress field for Case 1 at $100 \mathrm{yr}$, computed using FRACROCK

Figure 4-45 Horizontal stress field for Case 1 at $100 \mathrm{yr}$, computed using FRACROCK

Figure 4-46 Shear stress field for Case 1 at $100 \mathrm{yr}$, computed using FRACROCK

Figure 4-47 Temperature field for Case 1 at $1000 \mathrm{yr}$, as computed by NUFT

Figure 4-48 Simulation of 1000-yr temperature field for Case 1 for input to FRACROCK

Figure 4-49 Vertical stress field for Case 1 at $1000 \mathrm{yr}$, computed using FRACROCK

Figure 4-50 Horizontal stress field for Case 1 at $1000 \mathrm{yr}$, computed using FRACROCK

Figure 4-51 Shear stress field for Case 1 at $1000 \mathrm{yr}$, computed using FRACROCK

Figure 4-52 Temperature field for Case 5 at $50 \mathrm{yr}$, as computed by NUFT

Figure 4-53 Simulation of 50-yr temperature field for Case 5 for input to FRACROCK

Figure 4-54 Vertical stress field for Case 5 at $50 \mathrm{yr}$, computed using FRACROCK

Figure 4-55 Horizontal stress field for Case 5 at $50 \mathrm{yr}$, computed using FRACROCK

Figure 4-56 Shear stress field for Case 5 at $50 \mathrm{yr}$, computed using FRACROCK

Figure 4-57 Temperature field for Case 5 at $100 \mathrm{yr}$, as computed by NUFT

Figure 4-58 Simulation of 100-yr temperature field for Case 5 for input to FRACROCK

Figure 4-59 Vertical stress field for Case 5 at $100 \mathrm{yr}$, computed using FRACROCK

Figure 4-60 Horizontal stress field for Case 5 at $100 \mathrm{yr}$, computed using FRACROCK

Figure 4-61 Shear stress field for Case 5 at $100 \mathrm{yr}$, computed using FRACROCK

Figure 4-62 Zones of enhanced permeability predicted for the DST before heating

Figure 4-63 Zones of enhanced permeability predicted for the DST at $0.5 \mathrm{yr}$

Figure 4-64 Zones of enhanced permeability predicted for the DST at $1.0 \mathrm{yr}$

Figure 4-65 Zones of enhanced permeability predicted for the DST at $2.0 \mathrm{yr}$

Figure 4-66 Zones of enhanced permeability predicted for the DST at $3.0 \mathrm{yr}$

Figure 4-67 Zones of enhanced permeability predicted for the DST at $4.0 \mathrm{yr}$

Figure 4-68 Ratio of shear stress to frictional strength for vertical fractures for Case 1 after $50 \mathrm{yr}$ heating, computed using FLAC

Figure 4-69 Ratio of shear stress to frictional strength for horizontal fractures for Case 1 after $50 \mathrm{yr}$ heating, computed using FLAC

Figure 4-70 Ratio of shear stress to frictional strength for vertical fractures for Case 1 after $100 \mathrm{yr}$ heating, computed using FLAC 
Figure 4-71 Ratio of shear stress to frictional strength for horizontal fractures for Case 1 after 100 yr heating, computed using FLAC

Figure 5-1 Changes in the total molalities of dissolved aluminum, potassium, and silica as a function of reaction progress $\xi$ in closed-system reaction of microcline with $\mathrm{pH} 4.0$ $\mathrm{HCl}$ solution

Figure 5-2 Changes in the $\mathrm{pH}$ as a function of reaction progress $\xi$ in closed-system reaction of microcline with $\mathrm{pH} 4.0 \mathrm{HCl}$ solution

Figure 5-3 The number of moles of secondary minerals $n_{\phi}$ as a function of reaction progress $\xi$ in closed-system reaction of microcline with $\mathrm{pH} 4.0 \mathrm{HCl}$ solution

Figure 5-4 The affinity of microcline to dissolve as a function of reaction progress $\xi$ in closedsystem reaction of microcline with $\mathrm{pH} 4.0 \mathrm{HCl}$ solution

Figure 5-5 The precipitation kinetics of quartz in the system $\mathrm{SiO}_{2}-\mathrm{H}_{2} \mathrm{O}$ as a function of time

Figure 5-6 Comparison of simulated (EQ3/6) and experimental aqueous solution compositions for the $150^{\circ} \mathrm{C}$ reaction between Topopah Spring tuff (Tpt) (core wafer) and J-13 well water

Figure 5-7 Major secondary mineralogy as a function of time for the reaction of devitrified TSw2 tuff and glassy TSw3 tuff with J-13 well water

Figure 5-8 Changes in the $\mathrm{pH}$ of J-13 well water during reaction with devitrified TSw2 tuff

Figure 5-9 Changes in the chemistry (Si) of J-13 well water during reaction with devitrified TSw2 tuff

Figure 5-10 Changes in the chemistry $(\mathrm{Na})$ of J-13 well water during reaction with devitrified TSw2 tuff

Figure 5-11 Changes in the chemistry (Al) of J-13 well water during reaction with devitrified TSw2 tuff

Figure 5-12 Changes in the $\mathrm{pH}$ of J-13 well water during reaction with glassy TSw3 tuff

Figure 5-13 Changes in the chemistry (Si) of J-13 well water during reaction with glassy TSw3 tuff

Figure 5-14 Changes in the chemistry $(\mathrm{Na})$ of J-13 well water during reaction with glassy TSw3 tuff

Figure 5-15 Changes in the chemistry (Ca) of J-13 well water during reaction with glassy TSw3 tuff

Figure 5-16 Changes in the chemistry $(\mathrm{Mg})$ of J-13 well water during reaction with glassy TSw3 tuff

Figure 5-17 Changes in the chemistry (Al) of J-13 well water during reaction with glassy TSw3 tuff

Figure 5-18 Changes in sanidine abundance resulting from a plus or minor one order of magnitude change in the dissolution rate of this mineral

Figure 5-19 Estimates of sanidine abundance, assuming the dissolution rates used to construct Figure 5-18

Figure 5-20 Hydrothermal Regimes A-C at 100 and 1000 yr after repository closure

Figure 5-21 Temperature contours at 10,000 yr after waste emplacement

Figure 5-22 Saturation contours at 10,000 yr for the case with no coupling between silica transport and permeability change 
Figure 5-23 Saturation contours at 10,000 yr for the case with coupling between silica transport and permeability change

Figure 5-24 Distribution of silica at 10,000 yr with no coupling between silica transport and permeability change

Figure 5-25 Distribution of silica at 10,000 yr with coupling between silica transport and permeability change

Figure 5-26 Regions of net precipitation of silica at 10,000 yr with no coupling between silica transport and permeability change

Figure 5-27 Regions of net precipitation of silica at 10,000 yr with coupling between silica transport and permeability change

Figure 5-28 Regions of net dissolution of silica at 10,000 yr with no coupling between silica transport and permeability change

Figure 5-29 Regions of net dissolution of silica at 10,000 yr with coupling between silica transport and permeability change

Figure 5-30 Passing of parameter fields during the sequential solution of the TH and reactivetransport models

Figure 5-31 Relative change in fracture porosity (ratio of the porosity to the initial porosity) at $1000 \mathrm{yr}$ for the base case

Figure 5-32 Relative change in matrix porosity at $1000 \mathrm{yr}$ for the base case

Figure 5-33 Mineral volumetric fractions of quartz and cristobalite in the fracture plane at $1000 \mathrm{yr}$ for the base case

Figure 5-34 Base-case conditions at $1000 \mathrm{yr}$

Figure 5-35 Temperature history of the WP with and without silica reactive transport, relative humidity on the WP, and liquid saturation in the rock above the drift crown

Figure 5-36 Base-case conditions at $100 \mathrm{yr}$

Figure 5-37 Base-case conditions at $500 \mathrm{yr}$

Figure 5-38 Base-case conditions at $1000 \mathrm{yr}$

Figure 5-39 Base-case conditions at $5000 \mathrm{yr}$

Figure 5-40 Base-case conditions at 900,000 yr

Figure 5-41 Relative change to fracture porosity at $1000 \mathrm{yr}$ for the base case with infiltration reduced by a factor of $1 / 5$

Figure 5-42 Relative change to fracture porosity for the base case using hydrologic properties from the December 1997 "Modified TH" property set

Figure 5-43 Relative change to fracture porosity at $1000 \mathrm{yr}$ for the line-load case

Figure 5-44 Relative change to fracture porosity for the base case only, allowing precipitation of amorphous silica

Figure 5-45 Relative change to fracture porosity for the line-load only, allowing precipitation of amorphous silica

Figure 5-46 Calcite abundance as a function of location for Case 1 after 10 yr heating

Figure 5-47 Calcite abundance as a function of location for Case 1 after 100 yr heating

Figure 5-48 Calcite abundance as a function of location for Case 1 after 1000 yr heating 
Figure 5-49 Calcite abundance as a function of location for Case 1 after 10,000 yr heating

Figure 5-50 Calcite abundance as a function of location for Case 3 after 10 yr heating

Figure 5-51 Calcite abundance as a function of location for Case 3 after 100 yr heating

Figure 5-52 Calcite abundance as a function of location for Case 3 after 1000 yr heating

Figure 5-53 Calcite abundance as a function of location for Case 3 after 10,000 yr heating

Figure 5-54 Production rate of stilbite (a zeolite) for each case examined as a function of time

Figure 5-55 Porosity rate of change for each case examined as a function of time

Figure 5-56 Calcite abundance as a function of location for Cases 2 and 5 after $1000 \mathrm{yr}$ heating

Figure 5-57 Temperature distribution for Case 1 after $1000 \mathrm{yr}$ heating

Figure 5-58 Temperature distribution for Case 2 after 1000 yr heating

Figure 5-59 Temperature distribution for Case 3 after $1000 \mathrm{yr}$ heating

Figure 5-60 Temperature distribution for Case 5 after $1000 \mathrm{yr}$ heating

Figure 5-61 Flux vector distribution for Case 1 after 1000 yr heating

Figure 5-62 Flux vector distribution for Case 2 after $1000 \mathrm{yr}$ heating

Figure 5-63 Flux vector distribution for Case 3 after 1000 yr heating

Figure 5-64 Flux vector distribution for Case 5 after 1000 yr heating

Figure 5-65 Porosity, calcite, stilbite, and diaspore distributions for Case 1 after 1000 yr thermal evolution

Figure 5-66 Porosity, calcite, stilbite, and diaspore distributions for Case 2 after 1000 yr thermal evolution

Figure 5-67 Porosity, calcite, stilbite, and diaspore distributions for Case 3 after 1000 yr thermal evolution

Figure 5-68 Porosity, calcite, stilbite, and diaspore distributions for Case 5 after 1000 yr thermal evolution

Figure 5-69 Mesolite distributions for the cases represented in the preceding four figures

Figure 5-70 Porosity distribution for Case 2 after 1000 yr thermal evolution, under the pillar flow constraints

Figure 5-71 Stilbite distribution for Case 2 after $1000 \mathrm{yr}$ thermal evolution, under the pillar flow constraints

Figure 5-72 Mesolite distribution for Case 2 after 1000 yr thermal evolution, under the pillar flow constraints

Figure 5-73 Porosity distribution for Case 5 after $1000 \mathrm{yr}$ thermal evolution, under the pillar flow constraints

Figure 5-74 Stilbite distribution for Case 5 after $1000 \mathrm{yr}$ thermal evolution, under the pillar flow constraints

Figure 5-75 Mesolite distribution for Case 5 after $1000 \mathrm{yr}$ thermal evolution, under the pillar flow constraints

Figure 5-76 Porosity, calcite, stilbite, and mesolite distributions for Case 1 after 1000 yr thermal evolution

Figure 5-77 Porosity, calcite, stilbite, and mesolite distributions for Case 5 after 1000 yr thermal evolution 
Figure 5-78 Porosity, calcite, stilbite, and mesolite distributions for Case 1 after $1000 \mathrm{yr}$ thermal evolution

Figure 6-1 Bar graphs of water compositions from various fracture studies

Figure 6-2 Temperature $\left({ }^{\circ} \mathrm{C}\right)$, outlet Si concentration $(\mathrm{mg} / \mathrm{L})$, and temperature-dependent theoretical saturation Si concentration values for amorphous silica and cristobalite as a function of time for the fracture flow-through experiment )

Figure 6-3 Temperature $\left({ }^{\circ} \mathrm{C}\right)$, outlet Si concentration $(\mathrm{mg} / \mathrm{L})$, and temperature-dependent theoretical saturation Si concentration values for amorphous silica and cristobalite as a function of time for the fracture flow-through experiment

Figure 6-4 The log molality [log $m(i)]$ of selected dissolved species in the outlet solution of the $95^{\circ} \mathrm{C}, 15-\mathrm{m}$ fracture flow model, as a function of time

Figure 6-5 Porosity and mineralogy (all in volume percent) at the inlet position of the $95^{\circ} \mathrm{C}$, 15-m fracture-flow model, as a function of time

Figure 6-6 Porosity and mineralogy (all in volume percent) at the outlet position of the $95^{\circ} \mathrm{C}$, 15-m fracture flow model, as a function of time

Figure 6-7 The $\mathrm{pH}$ as a function of moles of water evaporated

Figure 6-8 Eh as a function of moles of water evaporated

Figure 6-9 Na as a function of moles of water evaporated

Figure 6-10 $\quad \mathrm{Cl}$ as a function of moles of water evaporated

Figure 6-11 $\quad \mathrm{F}$ as a function of moles of water evaporated

Figure 6-12 Si as a function of moles of water evaporated

Figure 6-13 Ca as a function of moles of water evaporated

Figure 6-14 Log of the number of moles of calcite precipitated, as a function of moles of water evaporated

Figure 6-15 Log of the number of moles of dolomite precipitated, as a function of moles of water evaporated

Figure 6-16 Log of the number of moles of saponite precipitated, as a function of moles of water evaporated

Figure 6-17 Log of the number of moles of stilbite precipitated, as a function of moles of water evaporated

Figure 6-18 Schematic diagram of conditions under which mineral assemblages described in Table 6-2 might develop from grout of an original Type $\mathrm{V}$ composition

Figure 6-19 Plot of concentration of dissolved elements as a function of reaction progress for a simulation conducted at $60^{\circ} \mathrm{C}$, using a young concrete mineral assemblage and tuff aggregate composition and fixed $\mathrm{O}_{2}$ and $\mathrm{CO}_{2}$ compositions at atmospheric values

Figure 6-20 Plot of precipitated mineral phases as a function of reaction progress for the simulation described in Figure 6-19

Figure 6-21 Plot of concentration of dissolved elements as a function of reaction progress for a simulation conducted at $60^{\circ} \mathrm{C}$, using a young concrete mineral assemblage and tuff aggregate composition and fixed $\mathrm{O}_{2}$ and $\mathrm{CO}_{2}$ compositions where $\mathrm{CO}_{2}$ is set at a low value

Figure 6-22 Plot of precipitated mineral phases as a function of reaction progress for the simulation described in Figure 6-21 
Figure 6-23 Plot of concentration of dissolved elements as a function of reaction progress for a simulation conducted at $60^{\circ} \mathrm{C}$, using a young concrete mineral assemblage and tuff aggregate composition and unfixed $\mathrm{O}_{2}$ and $\mathrm{CO}_{2}$ compositions

Figure 6-24 Plot of precipitated mineral phases as a function of reaction progress for the simulation described in Figure 6-23

Figure 6-25 Variation in percent sorbed and concentration of $U$ in solution vs. final pH in four-day batch experiments

Figure 6-26 Comparison of DLM model (single-site) prediction

Figure 6-27 Comparison of DLM model (two-site) prediction

Figure 6-28 Percent $\mathrm{U}$ and $\mathrm{Np}$ sorbed vs. final $\mathrm{pH}$ in four-day batch experiments

Figure 6-29 Variation in concentration of $\mathrm{I}^{-}$and $\mathrm{U}$ vs. volume of effluent at $26^{\circ} \mathrm{C}$ for hematite:quartz columns at four $\mathrm{pH}$ values

Figure 6-30 Variation in concentration of $\mathrm{I}^{-}$and $\mathrm{U}$ vs. volume of effluent in $\mathrm{pH} 8$ column subsequent to the input of $\mathrm{pH} 4$ tracer-free solution

Figure 6-31 Comparison of X1t transport model (single- and two-site DLM) and observed I ${ }^{-}$and $\mathrm{U}$ concentrations in effluent from $\mathrm{pH} 4$ column

Figure 6-32 Comparison of X1t transport model (two-site DLM) and observed $\mathrm{I}^{-}$and $\mathrm{U}$ concentrations in effluent from $\mathrm{pH} 8$ column

Figure 6-33 Calculated years to breakthrough of radionuclide-bearing groundwater through corrosion layer as a function of $K_{\mathrm{d}}$ and volume fraction of the corrosion product from one waste canister $(\sim 30,000 \mathrm{~kg})$ contacted by the groundwater

Figure 6-34 Variation in relative concentration of $U\left(C / C_{0}\right)$ with time at node $1,10 \mathrm{~cm}$ into hematite corrosion layer

Figure 6-35 Variation in $K_{\mathrm{d}}$ vs. time at node $1(10 \mathrm{~cm})$ and concentration of $U$ vs. time on strong and weak sites at node 1, $10 \mathrm{~cm}$ into hematite corrosion layer

Figure 6-36 Variation in relative concentration of $U\left(C / C_{0}\right)$ with time at node $1,10 \mathrm{~cm}$ into hematite corrosion layer, for $\mathrm{pH} 10$ and no $\mathrm{CO}_{2}$

Figure 6-37 Variation in concentration of $U$ and Ca surface species with time at node 1, $10 \mathrm{~cm}$ into hematite corrosion layer, for $\mathrm{pH} 10\left(\mathrm{no} \mathrm{CO}_{2}\right)$ and cement-derived groundwaters

Figure 6-38 Flow chart showing the treatments applied to ESF concrete samples

Figure 6-39 Batch sorption isotherms $\left(26^{\circ} \mathrm{C} ; \mathrm{pH} 10.8\right)$ based on analysis of filtered and unfiltered supernatants for $\mathrm{U}$ and $\mathrm{Np}$ on hydrothermally altered crushed concrete

Figure 6-40 Partition coefficient $K_{d}$ vs. $\mathrm{pH}$ for $U$ and $\mathrm{Np}$ on hydrothermally altered concrete

Figure 6-41 Schematic drawing of apparatus employed for experiments using intact, fractured, hydrothermally altered concrete core samples

Figure 6-42 Relative concentration of iodide $\left(\mathrm{C} / \mathrm{C}_{0}\right)$ in $0.01-\mathrm{M} \mathrm{NaCl}$ effluent from fractured, hydrothermally altered concrete core for two pulses of $\mathrm{NaI}$

Figure 6-43 Maximum attached population density $\left(c_{\mathrm{s}}^{\max }\right)$ at various ionic strengths

Figure 6-44 Measures and predicted bacterial recovery in column effluent

Figure 6-45 Two types of fractures, a rough-walled fracture and a parallel-plate fracture, used in the laboratory experiments 
Figure 6-46 A reconstructed image of the apertures and the aperture-probability plot for the fractured core

Figure 6-47 Particle recovery curves for the parallel plate core

Figure 6-48 Particle recovery curves for the fractured core

Figure 6-49 All fractures mapped on the five exposed surfaces of the large block

Figure 6-50 The major fractures of the large block fracture system

Figure 7-1 Idealized, cumulative water loss based on simplified mineral assemblages calculated in Table 7-2 through Table 7-5 


\section{Abbreviations and Acronyms}

2-D

two-dimensional

3-D

three-dimensional

AECL

Atomic Energy of Canada Limited

AML

areal mass loading

ATP

adenosine triphosphate

AZ

altered zone

BEM

boundary-element method

BWR

boiling-water reactor

C-H-S

calcium-silicate-hydrate

CAM

corrosion-allowance material

CRM

corrosion-resistant material

CSNF

commercial spent nuclear fuel

DDT

discrete-heat-source, drift-scale thermal conduction

DDTH

discrete-heat-source, drift-scale thermohydrologic

DEF

delayed ettringite formation

DFM

discrete-fracture method

DHLW

defense high-level waste

DIC

dissolved inorganic carbon

DKM

dual-permeability method

DLM

double-layer model

DMTH

discrete-heat source, mountain-scale thermohydrologic

DOE

U.S. Department of Energy

DST

Drift-Scale Test

DTN

data tracking number

EBS

engineered barrier system

ECM

equivalent-continuum method

ENFE

evolution of the near-field environment

EPA

U.S. Environmental Protection Agency

ESF

Exploratory Studies Facility

FLAC

Fast Lagrangian Analysis of Continua

FMX

fracture-matrix interaction factor

GWB

Geochemist's Workbench ${ }^{\mathrm{TM}}$

GWTT

groundwater travel time

IAP

ion activity product 
LBNL Lawrence Berkeley National Laboratory

LBT Large-Block Test

LDTH line-averaged-heat-source, drift-scale thermohydrologic

LDTH-S line-averaged heat source drift-scale thermohydrologic seepage

LLNL Lawrence Livermore National Laboratory

LML lineal mass loading

$\mathrm{M} \& \mathrm{O} \quad$ management and operating

MIC microbially induced corrosion

MMX matrix-to-adjacent matrix

MPBX multiple-point borehole extensometer

MTU metric tons uranium

NEA Nuclear Energy Agency

NEA-TDB Nuclear Energy Agency Thermochemical Data Base

NF/AZ near field and altered zone

NFE near-field environment

NFGE near-field geochemical environment

NRC U.S. Nuclear Regulatory Commission

NUFT Nonisothermal Unsaturated-Saturated Flow and Transport

NWTRB Nuclear Waste Technical Review Board

ODE ordinary differential equation

OPC ordinary Portland cement

PA performance assessment

PWR pressurized water reactor

QA quality assurance

REV representative elementary volume

$\mathrm{RH}$ relative humidity

RIB Reference Information Base

SDT smeared-heat-source, drift-scale thermal-conduction

SDTH smeared-heat-source, drift-scale thermohydrologic

SHT Single-Heater Test

SI saturation index

SKB Swedish Nuclear Fuel Co.

SMT smeared-heat-source, mountain-scale thermal-conduction

SNF spent nuclear fuel

SZ saturated zone

TC thermochemical 
TDIF technical data information form

TDMS Technical Data Management System

TEF

thermal effects on flow

$\mathrm{TH}$

thermohydrologic

THC

thermohydrochemical

THCM

thermal-hydrologic-chemical-mechanical

THM

thermohydromechanical

$\mathrm{TM}$ thermomechanical

TSPA

total system performance assessment

$\mathrm{UZ}$

unsaturated zone

VA viability assessment

WIS Waste Isolation Strategy

WP waste packag

WPS waste package size

YMSCP Yucca Mountain Site Characterization Project 


\section{Acknowledgments}

We acknowledge the reviews of Charles Carrigan, Richard Knapp, Ken Jackson, Leah Rogers, and Dwayne Chesnut; and the technical contributions of Patricia A. Berge, Matthew J. Cordery, Truc Ho DeLorenzo, James Gansemer, Wunan Lin, Ronald J. Shaffer, Jeffrey L. Wagoner, and Herbert Fan Wang.

This report would not be possible without the editing of Karen L. Lew, Elaine Price, Cynthia Talaber, Bob Kirvel, and Jason Carpenter; proofing by Steve Peterson; graphic-design support of Paul Harding, Sabrina Fletcher, Treva Carey, Dennis Chan, Amy Henke, Cyndi Brandt, and Ray Marazzi; CD-ROM production by Sabrina Fletcher, with assistance from Kirk Hadley; support from compositors Sharlene Markow, Beverly Chamberlain, Sherry Emmons, and Louisa Cardoza; and the document coordination of Karen L. Lew. 



\section{Executive Summary}

This Near-Field/Altered-Zone Models Report describes the conceptual and numerical models available for predicting conditions in the near-field and altered-zone environments for the potential high-level radioactive-waste repository at Yucca Mountain. The term near field describes the region of the host-rock mass in which response to repository-induced conditions is critical to engineered barrier performance. The altered zone is the region in which the temperature increases significantly (i.e., relative to repository system performance) as a result of repository heating. The altered zone (AZ) extends beyond the near field and may extend throughout the unsaturated zone (UZ) above and below the repository. The near field and $\mathrm{AZ}$ are regions where hydrologic flow and radionuclide transport behavior will be changed by repository construction and operation, ventilation, and the thermal effects of waste emplacement.

The need for detailed predictions of chemical conditions in the near-field environment (NFE) has been elevated to a primary concern within the past year because of changes in the waste-isolation strategy and related changes in the reference repository design (CRWMS $\mathrm{M} \& O, 1997 ; \mathrm{DOE}, 1998)$. Characterization of the site, natural processes, and conditions, especially those related to estimates of increased flux, has led to better understanding of how engineered barriers can be designed to assist the geologic setting in meeting post-closure performance objectives. At the same time, incorporation of large amounts of concrete into the reference repository design (where steel was previously designated) has significantly changed the conceptual model for geochemistry of the NFE; therefore, a different set of chemical data is required.

The extent of detailed predictions that can be made concerning long-term evolution of thermohydrologic (TH) and chemical conditions in the NFE is limited by the availability of experimental data to support predictive models. Nevertheless, over the past several years, considerable progress has been made toward developing reliable models for predicting nearfield conditions. One purpose of this report is to identify how these models can be improved through additional effort.

This report includes example calculations to demonstrate the types of results that can be obtained using the available models. To compare results from different types of models and to evaluate coupled processes, a set of reference TH cases was developed. The calculated TH results were used as input for thermohydromechanical (THM) and thermohydrochemical (THC) models. The reference cases were also used for fully coupled THC simulations that show the effects from redistribution of silica in the near field and AZ.

Organized around the types of coupled models (i.e., TH, THM, and THC), this report also presents models used to predict geochemical conditions in the NFE. Among the major conclusions in this report are those relating to the following:

- Thermohydrology issues for repository design

- Understanding of the "precipitation cap" effect

- Significant retardation of $\mathrm{Np}$ and other radionuclides caused by introduced materials

\section{Thermohydrology Issues for Repository Design}

The TH simulators described in this report actually simulate a spatially and temporally averaged response at each waste package (WP) location in the model. Laboratory and field tests discussed in this report show that liquid reflux behavior in heat pipes is considerably more complex than the average response. These experiments evidently produce behavior that is not part of the conceptual models on which the simulations are based. By extrapolating 
these observations, it is reasonable to infer that a heat pipe in the repository host rock could produce episodic reflux events capable of penetrating the dryout zone, thus causing drift seepage at elevated temperature during the peak thermal period.

The repository design can be optimized to limit the possible effects from penetration of thermally driven reflux. By increasing the lineal power loading within each drift, the dryout zone thickness is increased. Also, by increasing the spacing between drifts, the pillars remain at sub-boiling temperature, and drainage of reflux and the ambient percolation flux can occur. Heat pipes will be smaller and function less efficiently with loss of mass and enthalpy due to drainage through pillars.

\section{Understanding the Precipitation Cap}

The precipitation cap is very likely to form and will decrease fracture permeability while increasing fracture capillarity in the affected region. Although these simulations depend on coupling relationships that have not generally been tested experimentally, it is certain that heat pipes will form, that dissolution will occur, and that solute will be precipitated at the boiling zones. Preliminary simulations performed for this report show the effect on permeability to be significant for a wide range of key input parameters.

The question of whether the precipitation cap will persist for thousands of years depends on whether the precipitates are dissolved by the ambient percolation flux and on whether vertical fractures will reopen when the rock mass cools. Dissolution can be addressed using the same THC simulator used to predict formation of the precipitation cap. Evaluation of changes in the permeability of vertical fractures will require additional information on fracture rheology and on the actual mode of fracture displacement that accompanies heating.

\section{Radionuclide Retardation by Introduced Materials}

Experiments show that iron oxides and altered concrete immobilize $\mathrm{U}$ and $\mathrm{Np}$ under oxidizing conditions. Both batch-sorption and transport experiments have been conducted, and partition coefficients of $10^{4} \mathrm{~mL} / \mathrm{g}$ or greater have been observed. A model of onedimensional transport through a layer of degraded iron or cementitious materials shows that the retardation performance of such a layer could be equivalent to hundreds of meters of the undisturbed host rock. These results are described subsequently in more detail.

\section{Thermohydrologic Models}

TH models are used to calculate the evolution of liquid-water seepage flux, temperature, relative humidity $(R H)$, and air mass-fraction within the drifts as functions of time after repository closure. In assessing total system performance, these are used directly as NFE performance measures. TH models also serve as the starting point for other process model calculations that describe water chemistry, engineered barrier performance, and radionuclide transport.

The physical principles of heat and mass balance and conceptual models of flow in fractured porous media are well understood and can easily be expressed mathematically and solved numerically. Available TH simulator codes (principally NUFT, VTOUGH, and TOUGH 2) have been thoroughly tested and qualified, or qualification is under progress, under the Yucca Mountain Site Characterization Project quality assurance (QA) program. All of these codes can perform calculations using any of several means for treating the coupling of heat and mass flow between matrix and fracture elements. 


\section{Model Confidence-Building Activities}

Field tests in unsaturated tuffs are the principal means for building confidence in $\mathrm{TH}$ models. The Single-Heater Test (SHT) in the Exploratory Studies Facility (ESF) at Yucca Mountain was conducted in welded tuff near both the repository horizon and the planned location of emplacement drifts. Modeling results for the SHT have focused on the extent to which capillary forces appear to control the distributions of temperature and liquid water. Results using six property sets have been compared, and those that produce temperature profiles that most closely resemble observed data also have the least capillarity.

The Large-Block Test (LBT) is still in progress. It uses a partially excavated 3- $\mathrm{m} \times 3-\mathrm{m} \times$ 4 -m block in an outcrop of the repository host rock at Fran Ridge near Yucca Mountain. One of the goals for this test is to produce a heat-pipe zone to observe TH and chemical effects. TH simulations have been performed with the same property sets used in modeling the SHT and with similar results. The LBT field-temperature data show many short-duration episodes in which measured temperatures within the superheated region suddenly decreased by $10^{\circ}$ to $40^{\circ} \mathrm{C}$, reaching the boiling point in much less than one hour. These events probably resulted from episodic flow of condensate in fractures, which has never been modeled by the available $\mathrm{TH}$ codes because they only simulate temporally and spatially averaged behavior. (An animation of the LBT, which profiles temperature phases during the test, is included in the CD-ROM accompanying this report.)

\section{Reference Calculations for the Evaluation of Coupled Processes}

The NUFT code was used in a series of two-dimensional (2-D) models, which served as the basis for chemical and mechanical calculations used in evaluating coupled processes for this report. The infiltration conditions and stratigraphy chosen for these simulations correspond to a location near the center of the repository layout, where the ambient percolation flux is estimated to be $16 \mathrm{~mm} / \mathrm{yr}$. The calculations have been repeated using two sets of hydrologic properties: a smaller infiltration rate and two thermal loading options (point load and line load).

The reference calculations indicate that the thermally driven liquid flux near the emplacement drifts (1) initially will be much greater than the ambient percolation flux and (2) will gradually decline to the ambient flux in 100 to $500 \mathrm{yr}$. Comparing the point-load and line-load cases, a larger dryout zone will develop for the line load because of greater condensate drainage through the wider pillars.

\section{Drift-Scale Seepage}

Estimation of the temporal and spatial distributions of seepage into the emplacement drifts is arguably the most important problem for ambient and thermally driven, unsaturated-zone (UZ) hydrology at Yucca Mountain. Models have been developed, using the NUFT code, to estimate seepage into drifts under both isothermal and nonisothermal conditions. One of most important results from seepage models is that the predicted average seepage flux at ambient temperature is only a fraction of the ambient percolation flux in the host rock. By combining this with the likelihood of only part of the seepage flow actually dripping onto the WPs, a case can be made that very little water will contact the waste. It should be noted that, although the spacing between fractures may be a significant portion of the drift radius, this result is obtained using a continuum representation of the fracture network. This result is an approximation, and there is considerable opportunity for reduction of uncertainty in this area. 


\section{Total System Performance Assessment Multi-Scale Abstraction}

A multi-scale TH abstraction methodology has been developed to provide important input to total system performance assessment (TSPA). The abstraction tool calculates performance measures including the WP temperature and drift-air $R H$ at the WP surface. The need for abstracting from TH simulations, rather than using TH simulations directly, arises from the limited number of computationally intensive, full TH simulation runs that can reasonably be conducted.

The abstraction tool exploits several aspects of the repository $\mathrm{TH}$ problem to reduce computational effort. Scaling relationships are used in which the repository is modeled as a "smeared-heat source" for calculating large-scale effects such as three-dimensional (3-D) repository-edge effects. For regions of the host rock that are dried out or are beyond the influence of heat-pipe activity, heat-transfer during the thermal period can be represented by thermal-conduction-only models. This abstraction tool has been tested by comparing its results to computationally intensive, full $\mathrm{TH}$ simulations.

The abstraction methodology has made it possible (1) to compare the effects of different $\mathrm{TH}$ property sets and percolation flux maps and (2) to compare the performance of point-load and line-load repository designs. Comparisons are facilitated by calculating the TSPA performance measures over the entire repository layout. Results show that, depending on the values selected for capillary properties and infiltration flux, rewetting behavior can significantly prolong or shorten the period of reduced $R H$ in the emplacement drifts. The magnitude of estimated infiltration is arguably the strongest influence on the calculated performance measures.

The abstraction methodology has been constructed so that refinements in the repository design can be readily analyzed. Backfill has already been incorporated as an option. Seepage models using spatial heterogeneity of fracture properties can also be incorporated, although the computational effort will greatly increase.

\section{Improving Thermohydrologic Models to Reduce Uncertainty}

TH modeling has led the way in predicting NFE conditions and will continue to do so. Improved accuracy, flexibility, and reliability of $\mathrm{TH}$ predictions will contribute directly to success in licensing and to potential cost-saving features in the repository design. To continue making these contributions, the following specific areas need improvement:

- The multi-scale abstraction methodology is a significant accomplishment in modeling for NFE studies because it consolidates what is known about variability of infiltration and rock properties and optimizes the use of the available modeling tools. The methodology has the potential to be applied for TSPA-VA in ways beyond those presented here.

- The usefulness of TH simulations critically depends on the property sets. Problems with these properties have been identified in the simulation of field-scale heater tests and have been corrected. TH constraints are important for estimating hydrologic properties of the host rock.

- In the future, more emphasis will be given to TH models, including modeling of drip shield and backfill options, for evaluating EBS design alternatives that will control indrift flow processes. 


\section{Conclusions Regarding Thermohydrologic Models}

Evolution of temperature and matrix saturation calculated for the reference cases, and for other cases discussed in Chapter 3, make possible some conclusions regarding the TH regime:

- The TH system has a tendency to achieve quasi-steady conditions because the duration and scale of repository heating are large compared with the characteristic time and length scales for thermal diffusion and convective heat transport.

- Major features of the TH regime are determined by macroscopic factors such as heat output, repository depth, and the water-table boundary condition. Temperatures in the NFE and throughout the UZ depend on the type of thermal boundary condition imposed at the water table.

- Conduction-only problems are similar enough to full-TH problems that useful information can be abstracted for estimating TSPA performance measures, while substantially decreasing computational effort.

- Coalescence of dryout zones results if the sustained thermal output of the repository during the early thermal period exceeds the power required to evaporate the average ambient percolation flux at the boiling zone and, at the same time, to evaporate the matrix pore water in the region between drifts. Once the coalesced dryout region forms, maintenance of the quasi-steady state requires thermal power sufficient to evaporate the ambient flux.

- Results from the LBT show that the behavior of liquid condensate can produce shortterm episodic reflux events. Extrapolated to a thick heat-pipe zone, such episodic reflux may be capable of penetrating the emplacement drifts in significant quantity. Evaluation of short-term episodic reflux events appears to be beyond the capabilities of current TH simulators.

\section{Thermomechanical Models}

An approach is presented for estimating changes in fracture permeability caused by thermomechanical (TM) stress changes. The method is based on published approaches (Barton et al., 1985) and considers only effects from shear displacement, which has greater potential to permanently change permeability than does normal displacement. This approach can be tested by comparing its results to changes in permeability associated with the DriftScale Test (DST). For this report, regions in which permeability changes could occur in the repository host rock are evaluated using an elastic continuum model of rock-mass response. The simulators available (e.g., FRACROCK, FLAC, and UDEC) also support the use of ubiquitous-fracture or discrete-element models.

\section{Drift-Scale Test}

For the DST, TM effects may cause significant enhancement of permeability in a significant volume of the rock mass. Permeability is predicted to increase where temperature gradients and the associated thermal-stress gradients develop. Permeability in these parts of the rock mass is predicted to increase by a factor of 2 to 4 . Permanent changes in fracture apertures should be detectable after the affected region dries out. However, during the test and before dryout, such permanent changes could be difficult to discern from changes in fracture saturation.

The guard heater geometry used in the DST introduces TM effects that may differ significantly from repository drift-scale effects. The DST heater arrays were designed primarily to achieve representative TH conditions. 


\section{Analysis of Reference Cases}

Smaller changes in permeability are projected for the reference cases used to assess coupled processes for this report than are for the DST. Slip on fractures resulting from induced shear stresses is expected to be limited to a few small zones that are within a few drift diameters of the drift wall. The zones of shear slip calculated for the DST are not present in the repository models because adjacent drifts tend to produce canceling stress fields when heated at the same time.

\section{Improving Thermomechanical Models to Reduce Uncertainty}

The uncertainties that attend predicting rock-mass response to the construction and performance of a potential Yucca Mountain repository are comparable to the uncertainties typically encountered in mine development. The following additions could significantly enhance confidence in TM models for the NFE:

- Interpret microseismic monitoring performed for the DST, and any future heater tests, to confirm joint slip events that are inferred from other data sets such as rock-mass displacement, stress, and hydrologic testing

- Perform ubiquitous fracture modeling (FLAC) and discrete-element modeling (UDEC) to examine the relative contributions from different joint sets, sensitivity to fracture parameters, etc.

- Measure additional laboratory-scale properties of fractures, particularly the shear deformation parameters, including dilation angle and internal friction

- Assess creep and pressure-solution effects to determine whether THM effects are reversed during cool-down

\section{Conclusions Regarding Thermomechanical Models}

Evaluation of the stress fields calculated for the reference cases makes possible some conclusions regarding important THM effects in the AZ around the repository:

- Permeability decreases resulting from THM effects will generally be smaller than increases.

- Decreases will be limited to approximately an order of magnitude and may be partly reversed on cool-down. Permeability increases could be as many as 3 orders of magnitude locally; large increases will generally be caused by shear slip on fractures and will not be reversed on cool-down.

- The heat-pipe zone is isothermal; thus, THM changes associated with shear deformation in this zone are limited to effects that occur during growth and collapse of the temperature field.

- Conditions favoring shear displacement of fractures are predicted to be much less prevalent in the repository host rock than in the rock mass above and below the DST.

\section{Thermohydrochemical Models}

Thermodynamic models calculate the state of a chemical reaction based on equilibrium behavior and may also include kinetic behavior and controls on reactant availability. These concepts are embodied in the EQ3/6 modeling software package, which is widely used in geochemistry and is qualified for use in licensing. Reactive-transport models include reaction rates and a time variable and represent a spatial domain. Several reactive-transport simulators are available in the public domain; these incorporate a wide range of chemical species and a limited range of mass-transport behaviors. 
Another category of reactive-transport simulators consists of TH codes such as FEHM and NUFT, which have been modified to incorporate the chemical behavior of a small number of components. This approach has been useful for studying the effects of boiling processes on mineral precipitation, using silica as a single component.

Four modeling studies represent considerable progress in THC modeling: Two of these are recent LLNL studies prepared for the Yucca Mountain Site Characterization Project, and two are new work reported here. Of the two new ones, the first is a reaction-path study presented for water evolution in contact with the host rock (EQ3/6); this work examines the impact of uncertainty in the reaction rate constants for water-rock interaction. The second one is reactive transport of silica movement simulated at the repository scale using FEHM. Fully coupled calculations (i.e., coupling porosity and permeability) are then presented and show (1) reactive transport of silica at the drift scale (NUFT) and (2) reactive-transport simulations involving aqueous mineral dissolution and alteration reactions in the AZ (OS3D/GIMRT).

\section{Reaction-Path Study}

The reaction-path study presented in this report analyzes the impact of uncertainty in the relative-reaction rates used in the EQ3/6 model on the capability to simulate the evolution of J-13 water composition in the potential repository host rock. Results show that evolution of recharge water to the J-13 composition depends on several rate reactions, principally for silicate dissolution, for which reaction-rate data are uncertain.

\section{Coupled Repository-Scale Calculation with Silica}

Fully coupled repository-scale THC calculations were performed previously using the FEHM finite-element code, which was modified to incorporate the behavior of a single chemical component and to couple changes in fracture porosity with changes in permeability. These calculations show that sufficient silica will be mobilized by repository $\mathrm{TH}$ processes to cause significant changes in local fracture porosity. Boiling occurs predominantly at the bottom of the isothermal heat-pipe zone, and the residual solute deposits from boiling accumulate there. In a simulation that couples fracture permeability and porosity, solute accumulation is not hindered, although fracture porosity decreases from precipitation (relative permeability and moisture characteristic relationships were not coupled). Repository-edge effects in the model are reflected in the distribution of silica dissolution and precipitation, and similar effects can be expected near the edge of the waste-emplacement area during repository development.

\section{Drift-Scale TH Simulations with Silica}

Fully coupled THC calculations were performed using the NUFT code, which was modified to incorporate the behavior of silica. The initial TH properties, 2-D geometry, and boundary conditions correspond to the reference cases developed for this report. Model input includes coupling relationships between porosity and permeability and between porosity and the water potential and relative permeability curves. Reaction rates and chemical equilibria are fully temperature-dependent. The dual-permeability-method (DKM) model for fracturematrix interaction is used for these calculations (see Chapter 3), and chemical dissolution/precipitation reactions are modeled in both the fracture and matrix continua. 
Formation of a precipitation cap above the emplacement drifts is predicted by this model for all conditions considered, including ranges of the specific surface area available for silica dissolution and reaction constants corresponding to different silica phases (e.g., quartz, cristobalite, and amorphous silica). Significant precipitate accumulation takes place within a few years, or tens of years, after the start of heating.

Calcite is common in fractures in the host rock and will dissolve because of hydrothermal activity associated with repository heating. Calcite dissolution and precipitation of related species in the boiling zone are not incorporated in this model. When included, precipitates will form more rapidly because dissolution occurs more rapidly with calcite than it does with silicates such as cristobalite.

A lower bound on permeability reduction in the boiling zone is incorporated in the model to reflect the relation between permeability and heat-pipe formation, which is well established in TH simulations (see Chapter 3). When the bulk permeability decreases to a threshold value (100 millidarcy), throttling inhibits the penetration of liquid reflux. The lower end of the heat-pipe zone then migrates away from the heat source. Thus, although precipitation is concentrated at the bottom of the heat-pipe zone where boiling occurs, the zone of permeability reduction migrates upward with time.

\section{Reactive-Transport Calculations}

Coupled reactive-transport simulations were performed with the OS3D/GIMRT code, using temperature fields and fracture flow fields taken directly from the TH reference cases. The GIMRT simulations are based only on fracture flow conditions extracted from DKM simulations, ignoring matrix processes.

There is substantial uncertainty about the rate constants for silicate dissolution and precipitation reactions and about the inventory of fracture minerals present in the host rock. Reference values for rate constants that are consistent with the latest available experimental data are assumed, but are far from complete insofar as the number of silicate minerals included, the representation of both dissolution and precipitation, and temperature effects. The OS3D/GIMRT simulations were undertaken to examine the implications of what is known with relative certainty about the chemical behavior, without generating results that could be heavily influenced by uncertain parameters and mineral distributions.

These simulations show that dissolution of calcite will occur rapidly. Aqueous dissolution and precipitation reactions involving silicates are likely to produce significant changes in fracture porosity, but will do so relatively slowly, over time periods on the order of decades. Later in the repository thermal evolution, $\mathrm{CO}_{2}$ will be displaced from the gas phase by water vapor as the air mass-fraction approaches zero in the host rock (see Chapter 3 ).

OS3D/GIMRT simulations show that decreased $\mathrm{CO}_{2}$ fugacity will have little impact on calcite and silicate reactions at near-neutral $\mathrm{pH}$. The presence of condensate that is less chemically aggressive to calcite will result in the physical spreading of precipitation and dissolution zones.

Comparing OS3D/GIMRT simulations with the silica distributions calculated using NUFT shows that the aqueous dissolution and boiling processes predicted by the two approaches may be incompatible above the emplacement drifts. Although the lower end of the heat-pipe zone is at a relatively high liquid saturation and elevated temperature, dissolution may still be hindered because the concentrations of solutes have been increased by evaporation. An integrated model that accommodates a full set of possible chemical reactions with evaporation, boiling, and gas-phase transport of reactive components, such as $\mathrm{CO}_{2}$, is the next step for improving the realism of THC simulations. 


\section{Conclusions Regarding Thermohydrochemical Models}

The conclusions drawn from THC modeling presented in this report may be summarized as follows:

- THC effects will definitely cause redistribution of soluble components such as calcite and silica and will very likely produce a precipitation cap over the emplacement drifts.

- The bulk permeability of the rock mass making up the precipitation cap should reach a quasi-uniform value for a range of different model conditions (e.g., ambient flux, reactive surface). The existence of a lower-bound bulk permeability for heat-pipe effects has been shown in previous TH simulations. The result of this limit is that the precipitation cap will tend to move outward with time, increasing the zone thickness.

- Simulations show that, at a later time, precipitation is likely to occur in the pillars, and, if capillary reflux is significant, precipitation also can occur below the drifts. This means that, depending on the TH properties, the emplacement drifts can become encircled by precipitation, an effect that can have adverse implications for repository performance because it implies that water that seeps into drifts may become trapped there and accumulate. Clearly, it is important to accurately represent the relative importance of gravity- and capillary-driven fracture and matrix flow imbibition and reflux processes when simulating THC effects.

- These simulations show that the TH properties of the fracture system directly above the drifts may change and that analyses of post-thermal seepage should take these changes into account.

- The $\mathrm{CO}_{2}$ fugacity is an important chemical uncertainty in the NFE throughout the thermal period and beyond. Existing TH or THC models need enhancement to represent the behavior of $\mathrm{CO}_{2}$ as it is mobilized in the host rock, is displaced by water vapor, then moves away from the repository, dissolves in condensate, and undergoes aqueous interaction with mineral phases.

- The different THC simulation approaches presented in this report have shown that conflicting results arise when simulations that consider only aqueous processes are compared with simulations that include boiling processes but do not incorporate a full set of chemical components and reactions. Needed is a single simulation code that incorporates a full set of chemical reactions, boiling processes, and realistic $\mathrm{TH}$ processes (e.g., dual-permeability conceptual model).

- Sensitivity studies on THC models that incorporate silica redistribution are needed to focus laboratory investigation of the properties and reaction-rate parameters critical to formation of the precipitation cap. Simulation should also be used to evaluate the regularizing behavior of mineral precipitation, whereby all fractures are eventually filled.

\section{Geochemical Conditions in the Near Field/Altered Zone}

The approach to modeling geochemical conditions in the NF / AZ emphasizes water composition, which is needed to assess the WP lifetime, the release of radionuclides from the waste form, and the mobility of released radionuclides. Modeling of water composition begins with water in the AZ above the repository at ambient and elevated temperatures. This water is then introduced to the NFE where it interacts with introduced materials. 
For this report, the near-field geochemical environment is assumed to be dominated by cementitious materials, although the WP materials will also contribute, and steel may be used instead of concrete for some ground-support components. The evolution of water composition in contact with cementitious materials is presented for young concrete, and additional calculations have been performed for alternative compositions that represent concrete alteration by heating in the presence of moisture and varying amounts of $\mathrm{CO}_{2}$.

If radionuclides are released to the NFE, interaction with introduced materials may be significant to transport. A series of laboratory experiments is presented that helps to define the behavior of $\mathrm{U}$ and $\mathrm{Np}$ in contact with iron corrosion products and with altered concrete. Preliminary results show that partition coefficients of at least $10^{4} \mathrm{~mL} / \mathrm{g}$, and probably higher, are likely. Modeling of the transport of dissolved radionuclides through a layer of degraded introduced materials shows that retardation could be equivalent to that of hundreds of meters of the host rock. This model does not incorporate colloidal modes of transport.

A conceptual model for radionuclide mobility in the AZ is presented as a discussion of the available thermodynamic data for predicting solubility controls and as a conceptual discussion of colloid transport and what is being done to bound alternative modes of transport.

\section{Altered-Zone Water Composition}

At ambient temperature, water in the host rock will have a likely composition closely resembling J-13 water, whereas condensate will essentially be distilled water. Water that evolves in the AZ will have a composition that represents a mixture of ambient water similar to that from well J-13 and condensate that has interacted with rock-fracture mineralogy. Evaporative concentration will produce different effects, depending on the $\mathrm{CO}_{2}$ fugacity and other factors. The range of water compositions that is expected in the $\mathrm{AZ}$ will be bounded by the following:

- During heat-up, the water composition will be dominated by evaporative processes, and the composition will be represented by J-13 water concentrated to some degree.

- During cool-down, the water composition will be dominated by condensate, and the actual composition will depend on the extent of rate-dependent water-rock interaction.

- At the repository edges and in the late stage of cool-down, the water composition will be dominated by ambient percolation. These waters are expected to be similar to J-13 well water.

Significant discrepancies exist for some chemical components in this analysis, indicating that model refinement is needed. Several specific problem areas identified in this report include chemical boundary conditions, dissolution rate kinetics, and treatment of minor mineral phases.

\section{Water Composition in Contact With Cement}

A chemical model has been developed for water composition in the NFE and represents interaction with cementitious materials at different stages of maturation. This model differs from previous calculations in that additional cement phases are included in a new thermodynamic database to represent the observed products of hydrothermal alteration of Portland-type cement in the presence of $\mathrm{CO}_{2}$ (see Chapter 7). Cement mineral assemblages have been developed to represent different types of cement alteration. This is a recently developed database; however, it will be assessed along with previous data to produce estimates of water composition in contact with the WPs and interacting with released radionuclides for TSPA-VA. 
Simulating the chemical environment as water first impinges on introduced materials after the thermal period is complicated by uncertainty about the nature and extent of cement alteration, the evolution of $\mathrm{CO}_{2}$ fugacity, and the chemical contributions from different concrete constituents. These simulations show that, depending on the $\mathrm{CO}_{2}$ pressure assumed, the alkaline $\mathrm{pH}$ effect from interaction with cement can be substantially moderated. When sufficient $\mathrm{CO}_{2}$ is present, the $\mathrm{pH}$ is in the near-neutral range; when the $\mathrm{CO}_{2}$ is exhausted by reaction with portlandite, the $\mathrm{pH}$ increases to 10 or greater. Further specification of water composition in the NFE will require (1) modeling the mobilization and transport of natural $\mathrm{CO}_{2}$ in the NF/ AZ during the thermal period and (2) redistributing the $\mathrm{CO}_{2}$ by gas-phase circulation processes in the post-thermal regime.

\section{Concentrations of Radionuclides Leaving the Repository}

Iron oxides derived from the WP corrosion-allowance material (CAM) or steel ground support can potentially provide a sorptive barrier to radionuclide transport. Partition coefficients greater than $10^{4} \mathrm{~mL} / \mathrm{g}$ have been measured for $\mathrm{U}(\mathrm{VI})$ and $\mathrm{Np}(\mathrm{V})$ species on hematite, and greater partitioning would probably be observed on the "lower" oxides of iron that have greater specific surface area. Consistent results have been obtained from transport experiments conducted using these materials. Retardation will be strongly affected by fluid chemistry, particularly $\mathrm{pH}$ and $\mathrm{CO}_{2}$ fugacity, by colloidal transport, and by the nature of the flow path through a layer of corrosion products.

Surface complexation modeling of U sorption to hematite indicates that, at alkaline $\mathrm{pH}$, the presence of significant $\mathrm{CO}_{2}$ can inhibit the sorption of $U$ and, by analogy, Np. Simulations indicate that retardation will be significant for two scenarios: (1) alkaline waters in which $\mathrm{CO}_{2}$ fugacity is controlled by calcite equilibrium (e.g., fluids within cementitious material) and (2) alkaline fluids that are neutralized by reaction with ambient $\mathrm{CO}_{2}$ (e.g., fluids that exit cementitious material).

A transport experiment was conducted to investigate $\mathrm{U}$ and $\mathrm{Np}$ behavior in a fractured core of concrete that had been hydrothermally altered to represent repository conditions. Altered concrete strongly retarded $\mathrm{U}$ and $\mathrm{Np}$, either by sorption or by causing precipitation of one or more solubility-limiting solid phases. The solubility hypothesis implies greater retardation performance than sorption, but additional experiments are needed to establish the mechanism. Measurable fractions of the $\mathrm{U}$ and $\mathrm{Np}$ in batch experiments were associated with particulate matter; but, in general, colloids did not retain a significant fraction of the radionuclides.

\section{Radionuclide Transport in the Altered Zone}

It is expected that transport processes in the AZ below the repository will depart significantly from the unaltered, ambient system. The current approach is to use chemical solubility to bound the aqueous concentrations of radionuclides in the AZ, while using an experimental approach to bound colloidal and microbial effects on transport.

An evaluation of the available thermochemical data for key elements shows that solubility-limiting solid phases have been identified for $\mathrm{U}, \mathrm{Np}, \mathrm{Pu}, \mathrm{Am}, \mathrm{Tc}, \mathrm{Ni}$, and $\mathrm{Zr}$ for both oxidizing and reducing conditions. Solubilities for a range of $\mathrm{U}, \mathrm{Zr}$, and $\mathrm{Ni}$ phases can be reliably predicted over the temperature range $20^{\circ}$ to $150^{\circ} \mathrm{C}$. For other radioelements, including $\mathrm{Np}$ and $\mathrm{Pu}$, the likely solubility-limiting phases in J-13 water have been identified, but there are no data for elevated temperature. The relative need for further solubility data is reported to be $\mathrm{Np}>\mathrm{U}>\mathrm{Pu}>\mathrm{Am}>\mathrm{Zr}>\mathrm{Tc}>\mathrm{Ni}$. Coprecipitation is potentially important for radionuclide retardation in the $\mathrm{NF} / \mathrm{AZ}$, and a model for predicting coprecipitation effects is under development, but virtually no experimental data exist for model testing. 
The formation, stability, and sorption of radionuclides by colloids in the NFE remain matters of considerable uncertainty. Introduced materials in the repository can serve as sources for colloidal particles with strong affinity for radionuclides. The chemical and physical environments are conducive to formation of colloids and biocolloids. Few data are available on colloid formation and mobility, particularly because colloidal processes are complex and difficult to control.

Laboratory experiments consistently show that bacteria and colloids are transported faster than conservative solute tracers in individual fractures. Preliminary results from the bacterial transport experiment associated with the LBT show a breakthrough of labeled bacteria near the base of the block, but additional information is needed to establish a model framework for interpreting this observation.

\section{Near-Field/Altered-Zone Models}

Several areas have been identified for future enhancement of predictive models used in NF / AZ studies. The discussion of TM models described various approaches available for modeling the effects of rock joints. Methods of analysis that permit inelastic slip along fractures were not used in this report, although it is recognized that two major differences would result if such slip behavior were incorporated: (1) more shear slip would be predicted, increasing the fracture permeability, and (2) the softening that would result from slip would cause the deformation response to be concentrated closer to the drifts. For significant slip to be predicted, calculations will probably require values for fracture-strength parameters that are somewhat different than those used in this report. These calculations are within the current capabilities of commercially available modeling software.

Existing chemical modeling codes such as EQ3/ 6 are capable of simulations involving redox reactions; however, with the exception of a scoping study on the use of iron in backfill, these have not yet been incorporated into studies of the NFE. With abundant metallic iron and reduced uranium $\left(\mathrm{UO}_{2}\right)$ in the waste form, there is potential for reducing conditions in the NFE. This would give rise to lower solubility limits for $\mathrm{Np}$ and $\mathrm{Pu}$. It is therefore conservative to ignore redox if the actual redox state is more reducing, and that is the approach currently taken. Plausible simulation of redox effects in the NFE would be difficult; but, if achieved, such simulation could have a positive impact on radionuclide mobility. This is a matter for consideration in future experiments and modeling efforts.

The THC models presented in this report are limited with respect to the range of phenomena considered, particularly gas-phase transport of reactive components and reactions involving boiling. These limitations are common to all TC and reactive-transport models surveyed for this report and to other models for which detailed descriptions were available. Incorporation of more chemical components, gas-phase transport, boiling reactions, etc., into an integrated model that will also simulate thermohydrology and may include coupled mechanical or TM effects is an active area of geochemical research. 


\section{References}

Barton, N.R., S. Bandis, and K. Bakhtar (1985). "Strength, deformation, and conductivity coupling of rock joints." Int. J. Rock Mech. Min. Sci. and Geomech. 22(3):121-140. [217135]

CRWMS M\&O (1997). "Reference Design Description for a Geological Repository." (B00000000-01717-5707-00002, Rev. 1). Civilian Radioactive Waste Management System Management and Operation Contractor: TRW Environmental Safety Systems, Inc. [MOL.19980127.0313]

DOE (1998). “Repository Safety Strategy: U.S. Department of Energy's Strategy to Protect Public Health and Safety After Closure of a Yucca Mountain Repository, Rev. 1." Washington, DC: U.S. Department of Energy, Office of Civilian Radioactive Waste Management. [MOL. 19980211.0250; MOL. 19980330.0239] 



\section{Introduction}

The U.S. Department of Energy is studying Yucca Mountain as the possible site for the first underground repository for permanent disposal of spent fuel from commercial nuclear reactors as well as for other types high-level nuclear waste. Emplacement of high-level radioactive waste, especially commercial spent nuclear fuel (CSNF), in Yucca Mountain will release a large amount of heat into the rock above and below the repository. The heating rate will decrease with time, creating a thermal pulse. Over a period of several thousand years, the rock temperature will rise initially, then drop when the production of decay heat falls below the rate at which heat escapes from the hot zone. Besides raising the rock temperature, much of this heat will vaporize water, which will then condense in cooler regions. The condensate is likely to form a gravity-driven heat pipe above the repository, creating the possibility that water may drain back onto the waste packages (WPs) or that it may "shed" through the pillars between emplacement drifts.

The long-term importance of these effects has been investigated through the development, testing, and application of thermohydrologic (TH) models. Other effects, such coupled chemical and mechanical processes, may also influence the movement of water above, within, and below the emplacement drifts. A recent report on thermally driven coupled processes (Hardin and Chesnut, 1997) provides a qualitative assessment of the probable significance of these processes for the Yucca Mountain Site Characterization Project (YMSCP) and is the phenomenological framework for the present report. This report describes the conceptual and numerical models that have been developed to predict the thermal, mechanical, hydrologic, and chemical responses to the cumulative heat production of the potential host rock at Yucca Mountain. (An introduction to the topic of coupled processes is provided in Chapter 2.)

As proposed, the repository horizon will be situated within the Topopah Spring tuff, in the adjacent middle nonlithophysal and lower lithophysal units. These units are made up of moderately to densely welded, devitrified, fractured tuff. The rock's chemical composition is comparable to that of typical granite, but has textural features and mineralogical characteristics of large-scale, silicic volcanism. Because the repository horizon will be approximately $300 \mathrm{~m}$ below the ground surface and $200 \mathrm{~m}$ above the water table, the repository will be partially saturated. The welded tuff matrix in the host units is highly impermeable, but water and gas flow readily through fractures. The degree of fracturing in these units is highly variable, and the hydrologic significance of fracturing is an important aspect of site investigation.

This report describes the characterization and modeling of a region around the potential repository - the altered zone-a region in which the temperature will be increased significantly by waste-generated heat. Numerical simulation has shown that, depending on the boundary conditions, rock properties, and repository design features incorporated in the models, the altered zone (AZ) may extend from the water table to the ground surface. This report also describes models of the near field, the region comprising the repository emplacement drifts and the surrounding rock, which are critical to the performance of engineered components.

Investigations of near-field and altered-zone (NF/AZ) processes support the design of underground repository facilities and engineered barriers and also provide constraint data for probabilistic calculations of waste-isolation performance (i.e., performance assessment). The approach to investigation, which is an iterative process involving hypothesis testing and experimentation, has relied on conceptualizing engineered barriers and on performance 
analysis. This report is a collection, emphasizing conceptual and numerical models, of the recent results contributed from studies of NF / AZ processes and of quantitative measures of $\mathrm{NF} / \mathrm{AZ}$ performance. The selection and presentation of contributions are intended to show the iterative development of understanding in this area.

\subsection{Terms Used in this Report}

Regulations promulgated by the U.S. Nuclear Regulatory Commission (NRC) for licensing of a geologic repository (10 CFR Part 60) place performance requirements on the WP and other components of the engineered barrier system (EBS). This system is required to provide "substantially complete containment" of radionuclides for a specified time period after repository closure and thereafter to provide "controlled release" of radionuclides at an annual rate not to exceed a specified fraction of the inventory remaining at the end of the time period. The EBS is thus seen to play a vital role in the ability of a potential repository to successfully contain radioactive waste. Its performance will be strongly coupled with physical and chemical conditions in the near-field region of host rock within a few meters of the underground openings.

The near field is the region in which response to repository-induced conditions is critical to EBS performance. The following features of the near field are favorable to demonstration of repository performance:

- The near field can be characterized in greater detail than can any other volume of rock associated with the potential repository. Spatial variability can be characterized with relative accuracy, provided that near-field phenomena can be adequately understood and modeled.

- Prior to repository closure, the temperature, liquid water and vapor fluxes, and chemical processes can be readily observed within the near field and compared with predictions.

- The near field can be engineered to enhance waste isolation. For example, decay heat can be used to dry out the host rock and delay the onset of water contacting WPs (Buscheck and Nitao, 1992). In the immediate vicinity of the WPs and mined openings, multiple engineered barriers to flow can be developed, and materials can be added to chemically inhibit radionuclide transport.

Within the altered zone, temperature is increased significantly by radioactive decay heating. The $A Z$ extends beyond the near field and may extend throughout the unsaturated zone (UZ) above and below the repository horizon. The term "altered zone" is not used in the NRC performance criteria.

The near field and altered zone can be defined as regions in which flow and transport processes will be changed significantly, and for a long time, by repository construction, ventilation during preclosure operations, and the thermal effects of waste emplacement. In contrast to what occurs in the near field and the AZ, flow and transport processes in the far field are, by definition, unaffected by the repository. Portions of the AZ that recover to prerepository conditions may be included in the far field after sufficient time has elapsed.

The source term is defined as the projected history of the rate of release of radionuclides. The source term may include the effects of interaction with the host rock in the near field, to the extent that this interaction affects EBS performance. 


\subsection{Relevance of Near-Field/Altered-Zone Models to the Yucca Mountain Site Characterization Project}

Demonstration of repository safety will involve predictions of EBS performance and radionuclide transport over periods of 40 kyr or more; the NF/ AZ models will provide important supporting information for these predictions. The drift, or near-field, environment evolves over time during the excavation, emplacement, monitoring, and post-closure phases of repository operation. Its evolution can be controlled to some degree by understanding how the natural system responds to different design and operating options such as drift layout and thermal-management strategy. Predicting this response is a principal reason for developing and using models.

The near-field environment (NFE) controls the radionuclide source term. Liquid water is the agent most likely to cause containment failure and release of radionuclides. Although minor releases of gaseous radionuclides may occur as soon as a WP is breached, major releases will occur only if liquid water does the following:

1. Enters the emplacement drifts

2. Penetrates failed WPs

3. Contacts the waste form

4. Mobilizes radionuclides in aqueous solution or in suspension as water-borne colloids

5. Carries its radionuclide load from the WP through degraded EBS components from the drift into the surrounding rock, where it may ultimately be transported by groundwater flowing through the unsaturated and saturated zones to the accessible environment

During the thermal period (see Chapter 2), the main cause of water (or moisture) entering the repository will be TH processes; natural percolation will be insignificant. However, after a few thousand years, natural percolation will be the principal cause for water entering emplacement drifts. Water flow from the host rock into the repository will vary with location and is expected to significantly increase the failure rate of a few WPs (compared to the failure rate of most packages, which will be exposed to water vapor and minor amounts of liquid water on an intermittent basis). The flow of natural percolation is likely to be altered by durable hydrologic, mechanical, and chemical changes in the host rock, which are caused by heating.

Within the emplacement drifts, the most important time-dependent NFE conditions will be the humidity and temperature at the WP surfaces and the amount of liquid water (and its chemical composition) contacting the WPs and waste forms. These depend primarily on design choices (e.g., the thermal-loading density, the choice of drift-liner materials, and the presence or absence of backfill) and secondarily on far-field boundary conditions (e.g., surface topography and infiltration flux and the pre-emplacement state of the mountain).

WPs will eventually fail from aqueous corrosion activated by the occurrence of specific NFE conditions. Corrosion will occur at a significant rate when a thin film of water covers WPs. Water film formation and its effect on corrosion rate depend on temperature, relative humidity, and the nature of salts deposited on the WP surface. Liquid water actually dripping or flowing onto the WP may be either more or less corrosive than humid air. The actual time to failure (breach of WPs) after the onset of these conditions depends on the choice of materials, the WP design, temperature, and the water chemistry at the WP surface. Note that WPs can fail even if no liquid water is present, but no significant release can occur until water contacts waste. 
Advective transport will be the principal mechanism for radionuclide release at the repository. Molecular diffusion may also contribute to release, but will be strongly ratelimited. Accordingly, movement of water through the NF/AZ-and coupled processes that affect hydrologic behavior-are important to predictions of long-term repository performance.

Properties of the natural system will be changed by decay-heat-driven coupled processes within the AZ extending tens to hundreds of meters above and below the repository. Thermomechanical (TM) effects (e.g., the opening and closing of fractures in response to stress changes caused by heating and cooling) will produce some alteration within a few tens of meters of the drifts. However, the changes will result from geochemical alteration, dissolution, and precipitation of minerals within the AZ. UZ groundwater flow pathways and fluxes will be permanently changed within this large volume of rock, and the chemistry and amount of water entering the drift will be dominated for tens of thousands of years by AZ processes.

Chemical interaction of water with altered minerals or with human-introduced materials in the NF/AZ may also significantly affect radionuclide transport. Concrete leachate will have a composition that is significantly different than that of background-formation waters. The leachate composition may adversely affect WP corrosion rates and produce a chemically altered plume along flowpaths downgradient from the repository. Distal parts of the AZ may be associated with accelerated alteration in vitric tuff units, or where perched water below the repository horizon interacts with rock at elevated temperatures.

Consequently, the AZ will control the NFE for most, if not all, of the postclosure period. It may also enhance or degrade radionuclide retardation in the UZ because mineral dissolution and precipitation will increase sorptive capacity in some regions and decrease it in others. The system will not return to its pre-emplacement state even if climatic conditions remain constant. Even though purely $\mathrm{TH}$ responses (redistribution of moisture by evaporation and condensation) will eventually vanish after a few thousands to a few tens of thousands of years, flow and transport calculations for long-term performance must include AZ effects if they are to have any credibility.

Ultimately, the purpose of near-field process models is to predict the evolution of the environmental variables affecting the integrity of the WPs and the mobilization and transport of radionuclides within and near the drifts.

\subsection{Regulatory Significance}

The concept of a disturbed zone surrounding the mined openings of a potential geologic repository for high-level radioactive waste was introduced by the NRC as a region to be excluded for determining groundwater travel time to the accessible environment. However, the disturbed zone was to be included for determining, for total system performance assessment (TSPA), the impact of underground construction and radioactive-decay heat on radionuclide transport.

The terms "near field" and "altered zone" are not used in 10 CFR Part 60, although the boundary of the disturbed zone is defined as the starting point for calculating groundwater travel time (GWTT) to the accessible environment. The regulation indicates that the highest overall objective for the postclosure period is waste-isolation performance by the total repository system (e.g., dose limitation at the accessible environment). To demonstrate that the repository will comply with the regulatory performance objectives, the Department of Energy must provide a technical justification that defines the interaction of the natural 
system with the repository. This will require definition and detailed analysis of both the disturbed zone and the thermally altered region beyond (i.e., the AZ) to account for the impact of altered conditions on the flow of water and on the transport of radionuclides.

The total system-release standard in 40 CFR 191 was remanded in 1987, and the U.S. Environmental Protection Agency (EPA) was directed to implement a dose-based standard. Subsequent changes in compliance strategy have significantly increased the importance ascribed to NF/ AZ processes in the design and performance of a Yucca Mountain repository. It is currently assumed that compliance will be based on the calculated probabilistic peak dose to a member of the public exposed to groundwater at some location downgradient from the repository. The postclosure performance period is extended from $10 \mathrm{kyr}$ to an indefinite term, with the peak dose calculated to occur at approximately 30 to $100 \mathrm{kyr}$. Additional waste-isolation performance is required to demonstrate compliance over the longer term, and the increment will greatly exceed that which can be provided by far-field natural barriers. Accordingly, more performance is required from the engineered barriers, and added emphasis is placed on NF/AZ models.

Significant changes in compliance strategy have also resulted from acceptance of recently published data on percolation flux at Yucca Mountain. Average flux was previously estimated to be on the order of $1 \mathrm{~mm} / \mathrm{yr}$ at Yucca Mountain; several independent lines of evidence indicate that a range from 5 to $10 \mathrm{~mm} / \mathrm{yr}$ is more appropriate (Flint et al., 1996). Whereas $1 \mathrm{~mm} / \mathrm{yr}$ allows much of the downward flux to be transmitted as porous flow in the tuff matrix, significantly greater values require predominantly fracture flow. This further reduces the contribution to waste-isolation performance that can be ascribed to natural barriers in the UZ. In addition, greater flux accelerates degradation of engineered barriers. Hence, added emphasis is placed on models of the NF/AZ, which must now include response to significantly greater percolation flux than previously recognized.

\subsection{General Model Concepts}

Within the AZ, signification changes will take place in thermodynamic-state variables, primarily temperature (e.g., within the $60^{\circ} \mathrm{C}$ isotherm). Permanent changes to rock characteristics may occur within the AZ. The innermost part of the AZ is the near field; while physically composed predominantly of geologic materials, the near field is conceptually part of a system of natural and engineered barriers.

The entire AZ, including the near field, combines some aspects of both the engineered components and the natural components of a multiple barrier system. AZ characteristics will depend on repository effects, which can be controlled through design. The geologic system can therefore be divided into a natural geologic barrier system, corresponding to the far field, and an altered geologic barrier system.

Coupled processes refers to thermal-hydrologic-chemical-mechanical (THCM) processes, in any combination, that have the potential to significantly degrade natural or engineered barriers. This concept is discussed in detail in Chapter 2.

\subsubsection{Spatial Heterogeneity}

A significant uncertainty in describing the NF/ AZ environment is the spatial distribution of liquid water flux entering from above and its variation, with time, throughout the history of the repository. This flux will strongly influence both the rate of rewetting of the host rock after the thermal period and the quantity of percolating water that reaches the engineered barriers. The distribution of percolation flux in fractured silicate rocks is strongly heterogeneous: most of the flow occurs in a small proportion of the fractures. This has been demonstrated by field experiments such as the Drift-Scale Test at Stripa (Neretnieks et al., 1987). 
The Stripa experiment produced direct experimental observations of the spatial and temporal variability of percolation flux crossing a plane parallel to the ground surface. Water inflow was collected in 375 plastic rectangles, each of which covered $2 \mathrm{~m}^{2}$ and was monitored for 3 yr. Approximately $70 \%$ of the collectors produced $1 \%$ of the total flow, while the remaining $30 \%$ exhibited a large coefficient of variation $\left(C_{v} \approx 10\right)$. Figure $1-1$ shows that the water accumulation data from the latter group are log-normal (Chesnut, 1992).

Figure 1-1 Distribution of inflow rates per each 2- $\mathrm{m}^{2}$ collector in the Stripa experiment (after Chesnut [1992]; data from Neretnieks et al. [1987])

The Stripa experiment showed that strongly heterogeneous distribution of percolation is probably typical for rocks in which flow is principally in fractures. Much of the rock mass apparently has little effect on hydraulic transport under partially saturated conditions, and similar behavior may be inferred for chemical transport. Whereas the effects of heterogeneity of hydrologic properties on seepage into drifts have been investigated for a Yucca Mountain repository (Nitao, 1997b; Tsang et al., 1997), the effects of chemical heterogeneity on the magnitude and chemistry of seepage have not. For coupled thermohydrochemical (THC) modeling, the effects of hydrologic and chemical heterogeneity are the focus of ongoing research.

\subsubsection{Heterogeneity vs. Uncertainty}

Heterogeneity is an intrinsic characteristic of geologic media. For example, the width of a concentration front as it reaches the outflow boundary of a flow system arises from variability in flow and transport properties, connectivity among possible flow paths, dispersion, diffusion, etc. The effects of heterogeneity on flow and transport cannot be removed by gathering more data on formation properties, although the accuracy with which these effects can be calculated may be improved.

Uncertainty arises from imperfect knowledge of the variables and parameters, and of the mathematical relations among them, that allow one to predict the value of any measurable property or the measurable response of a system. If the applicable conceptual and mathematical models are known explicitly for a particular system, the uncertainty lies entirely in the accuracy and precision with which the parameters or system behavior are known or measured. This is parametric uncertainty, and it can be reduced with additional data or better methods of interpolating between measured data.

However, all models are approximations in which some processes are neglected or approximated, generally for convenience in generating predictions. Model validation is a matter of determining whether the approximations are consistent with the intended use of model predictions. Until the approximations are shown to be adequate, there is conceptual uncertainty. Additional experiments must be designed to challenge models (i.e., test hypotheses) until the resulting conceptual models adequately represent the significant processes.

\subsubsection{Modeling, Characterization, and Dampening}

The state of the art of reactive-transport modeling is limited by the difficulty of coupling chemical and $\mathrm{TH}$ processes. Flow and transport calculations typically use approximations (e.g., local equilibrium) that tend to dampen the temporal and spatial system responses. These approximations are thought to be inadequate for modeling the EBS hydrochemical environment. For this environment, models must treat not only coupled fluid-flow, heattransfer, and chemical processes, but must also accommodate spatial heterogeneity and transient hydraulic and chemical interactions between fractures and the porous tuff matrix. 
Determining the temporal and spatial distribution of water contacting the EBS is the first step in understanding NF / AZ processes. Models of drift seepage should account for the heterogeneity of hydrologic properties representing fractures and also account for fluctuating boundary conditions. Numerical simulations using nonequilibrium (dual-permeability) models have shown that drift seepage in response to episodic percolation boundary conditions, for random spatially heterogeneous media, is enhanced relative to steady-state conditions (Nitao, 1997b; Tsang et al., 1997). These studies also showed that model dimensionality (two-dimensional vs. three-dimensional) is important for simulating drift seepage.

Developing models to determine the effects of heterogeneity on flow and transport, and developing the methods for characterizing the geologic systems to which they are applied, are important research topics in hydrology and petroleum engineering. Characterization refers to estimating values for model parameters, and the values of the parameters cannot be separated from model selection and formulation.

A traditional approach to modeling the effects of spatial heterogeneity on flow and transport is to determine values of formation properties at several locations and interpolate at intermediate points to construct a discrete array. Conceptual models are selected for the coupling relations between interacting forces and fluxes. Boundary and initial conditions are defined, and a computer is used to solve systems of interdependent equations. As complexity and nonlinearity increase, solving the system of equations can become dramatically more difficult; thus dampening in the solution method is needed to achieve convergence. Dampening produces artificial smoothing in the spatial and temporal variability of the calculated fields. Selection of conceptual models may also contribute to dampening by smoothing the variability of coupling coefficients (e.g., continuum approximation) and state variables (effective continuum method). Dampening is common to all coupled models, including the coupled process models described in this report, for which solutions are readily obtainable.

\subsection{Building Confidence in Models}

Assessing compliance with regulatory performance objectives for the Yucca Mountain potential repository site requires scientifically credible predictions of the movement of liquid water, solutes, gases, and colloids through the AZ to the EBS, and then from the EBS through the $A Z$ to its outer boundary. The predictions should apply to a reasonable range of designs and operating variables and for a reasonable range of uncertainty in hydrologic conditions.

Confidence in model results, expressed by the scientific community at large, can be ensured through parallel achievements in several areas:

- Incorporating spatial heterogeneity and realistic boundary conditions

- Comparing alternative conceptual models of coupled processes

- Comparing alternative numerical formulations, model grids, and numerical solution methods

- Employing effective methods for model visualization and analysis of results

- Performing sensitivity studies to establish the range of applicability to prediction of repository performance

- Presenting documentation that traces the progression of model development and application to repository performance assessment

- Designing experimentation to test model-generated hypotheses 
These principles were applied in developing the models presented in this report to the extent practicable, given the computationally intensive nature of nonisothermal, dualpermeability, multiply coupled model calculations. Experiments designed to test TH and hydrochemical models are currently in progress in the Exploratory Studies Facility (ESF) at Yucca Mountain.

\subsection{Relation to Licensing Issues}

A repository safety strategy identified by the Department of Energy (DOE, 1996) lists the following as required attributes of the geologic disposal system:

- $\quad$ Limited water contacting WPs

- Long duration of WP containment

- Slow rate of radionuclide release from breached WPs

- Concentration reduction for released radionuclides during transport through engineered and natural barriers

Each of these required attributes depends to some extent on conditions in the NFE and in the $\mathrm{AZ}$ and is addressed by this report. All but one pertain specifically to the NFE, indicating that NF/ AZ models will form an important part of the safety case to be evaluated in licensing.

The following discussion is based on two issue-resolution status reports published by the NRC technical staff (NRC, 1997, 1997b). The issues in Key Technical Issues: Evolution of the Near Field Environment and Thermal Effects on Flow were defined by the NRC staff. The status reports are organized around subissues, with discussion of technical background and importance to repository performance as well as proposed acceptance criteria for resolution at the subissue level.

\subsubsection{Evolution of the Near-Field Environment}

The NRC defined evolution of the near-field environment (ENFE) subissues in terms of coupled processes. This terminology is useful; it is noted, however, that, whereas every physical or chemical process can be considered coupled to some extent, the effects of elevated temperature on a particular physical or chemical process may be insignificant. The subissues for ENFE were defined as follows:

- The effects of coupled processes on the rate of seepage into the repository

- The effects of coupled processes on the WP lifetime

- The effects of coupled processes on the rate of release of radionuclides from breached WPs

- The effects of coupled processes on radionuclide transport through engineered and natural barriers

For repository licensing purposes, it has been proposed that each of these subissues be evaluated with respect to four aspects of coupled processes:

1. Potential effects must be identified.

2. Interaction of coupled processes with the natural system must be addressed.

3. Interaction of coupled processes with engineered materials must be evaluated.

4. The adequacy of the representation of coupled processes in TSPA must be evaluated. The first aspect is reported to have been resolved for the four subissues (Table 1-1). 
Table 1-1 Issue resolution for Key Technical Issue: Evolution of the Near-Field Environment*

\begin{tabular}{|c|c|c|c|c|}
\hline & & Subissues: Effec & f Coupled Proces & \\
\hline Aspect of Resolution & A. Seepage & B. WP Lifetime & C. Release Rate & D. Transport \\
\hline 1. Identify Effects & \multicolumn{4}{|c|}{ Resolved (NRC, 1997a) } \\
\hline 2. Natural System & & & & \\
\hline 3. Engineered Materials & & & & \\
\hline 4. TSPA Representation & & & & \\
\hline
\end{tabular}

\subsubsection{Seepage}

The effects of coupled processes on the rate of seepage are being investigated through TH and THC simulations of AZ processes that affect the hydrology of the host rock. This report describes the simulation approaches adopted for predicting repository performance and the experimental data that provide important support for numerical models.

One determinant of the nature of seepage into emplacement drifts is the relative importance, as the driving force for TH reflux, of gravity over capillarity. This report includes a current discussion of this question, which is based on results from the Large-Block Test (LBT) and the Single-Heater Test (SHT) in the ESF.

Numerical simulations of seepage at ambient temperatures and elevated temperatures (Nitao, 1997a, 1997b; Tsang et al., 1997) have shown that seepage is significantly enhanced in the presence of spatial heterogeneity of hydrologic properties, particularly fracture permeability. In addition, simulations based on nonequilibrium models of fracture-matrix interaction have shown that seepage is enhanced when percolation is episodic. These analyses are discussed in this report.

The reference repository design calls for a concrete liner, which will tend to promote shedding of reflux and ambient percolation flux for as long as the liner remains substantially intact. However, the liner will not be designed for mechanical stability after repository closure, and the liner is not considered for seepage calculations described in this report. To the extent that ambient percolation and $\mathrm{TH}$ reflux are driven primarily by gravity, the effects of coupled processes on seepage will be limited to changes in the AZ above the repository (i.e., changes in the natural system).

\subsubsection{WP Lifetime}

Two major determinants of WP lifetime are the projected TH conditions in the emplacement drifts and the chemical composition of water contacting the WP wall. These factors influence the timing and the rate of corrosion.

Low relative humidity in proximity to WPs can delay corrosion, and the duration of lowhumidity conditions is closely related to the extent of rock dryout around repository drifts. The extent and duration of dryout depend on rock properties and on the magnitude of the ambient percolation flux. Conceptual and numerical models of these effects and their variation throughout the repository are considered in detail in this report. Discussion also includes the amount of water evolved by heating concrete and zeolites in the NFE. 
When water contacts WPs at elevated temperatures, a residue of salts is expected to occur. In addition, particles of rock or concrete that fall on the WPs may contribute corrosive halides and other solutes. Models for the chemical composition of water in the AZ, and for the effects of evaporation, are presented in this report and are combined with projected seepage to estimate solute deposition in the near-field environment.

Eventually the support pedestals will fail, and the WPs will settle onto accumulated rock, degraded concrete, and corrosion products. Packages will be buried to some extent by rock fall. This report presents a bounding approach, based on a conceptual model for the composition of water in contact with natural and engineered materials, to estimate WP lifetime.

\subsubsection{Release Rate}

The rate of radionuclide release from WPs will depend on the flux of water entering (and leaving), the composition of the water, and the waste-form degradation processes occurring inside each package. Molecular diffusion may also transport radionuclides from the WPs, but the rate of release due to diffusion only will be relatively small. The flux of water entering each WP will depend on the magnitude and distribution of the incident flux. A conceptual bounding model for water entering the WP is presented in this report.

Composition of water in the NFE, before entry into a WP, is determined by chemical interaction with natural and engineered materials. As discussed previously, a conceptual model for the composition of water in contact with natural and engineered materials is presented in this report.

With respect to the issue of concentrations of radionuclides as dissolved species, colloids, and secondary precipitates inside the WP, parts of this complex issue are beyond the scope of this report. Conceptual models for these processes typically mix the influent water, using the composition discussed previously, with a prescribed total flux of radionuclides derived from waste-form degradation. Precipitation of stoichiometric minerals is then calculated using available laboratory solubility data, which are summarized in this report.

Where the flux of water into a WP is insignificant, the principal release mode will be diffusion through perforations in the package wall. Where this condition is associated with low flux in the adjacent NFE, the diffusive transport may also be operative in the accumulated layer of rock, degraded concrete, and corrosion products on the drift floor.

\subsubsection{Radionuclide Transport}

Eventually, radionuclides will be released to the NFE, either by diffusion or advection. Discussed in this report are conceptual models for interaction of key radionuclides (e.g., ${ }^{237} \mathrm{~Np}$ and ${ }^{129} \mathrm{I}$ ) with altered cementitious materials and iron corrosion products. Supporting experimental data are presented for interaction of $U$ and I with engineered materials. WP effluent water mixing with other water in the near field is addressed in these models.

\subsubsection{Thermal Effects on Flow}

The thermal-effects-on-flow (TEF) subissues were defined by the NRC in terms of technical emphasis on conceptual and mathematical models, on model parameters, and on the adequacy of field testing. The subissues for TEF were defined as follows:

- Determination that the TH testing program, including performance confirmation, is sufficient to assess the potential for thermal reflux to occur in the near field

- Determination that the TH modeling approach is sufficient to predict the nature and bounds of thermal effects on flow in the near field

- Representation of TEF in TSPA 
Discussion of TH principles, conceptual models, parameter values, and other aspects of the modeling approach subissue (Table 1- 2 is included in this report.

Table 1- 2 Issue resolution fo Krey Technical Issue: Thermal Effects on Flow*

\begin{tabular}{|l|l|l|}
\hline \multicolumn{1}{|c|}{ A. Testing Program } & \multicolumn{1}{|c|}{ B. Modeling Approach } & \multicolumn{1}{c|}{ C. Representation by TSPA } \\
\hline \hline $\begin{array}{l}\text { Test Design } \\
\text { (Hypothesis-testing, scale, } \\
\text { spatial heterogeneity, THC } \\
\text { coupling; the effects of thermal } \\
\text { reflux on WPs) }\end{array}$ & $\begin{array}{l}\text { Data Sufficiency } \\
\text { (Parameter values, conceptual } \\
\text { models, and consistency with } \\
\text { site characteristics) }\end{array}$ & $\begin{array}{l}\text { Modeling Approach Elements } \\
\text { (Data sufficiency, process-model } \\
\text { descriptions, coupled processes, } \\
\text { and dimensionality) }\end{array}$ \\
\hline \multirow{5}{*}{} & $\begin{array}{l}\text { Process Model Description } \\
\text { (Principles, design features, and } \\
\text { site characteristics, including } \\
\text { infiltration, conceptual } \\
\text { uncertainties, and model } \\
\text { redundancy) }\end{array}$ & $\begin{array}{l}\text { Model Completeness } \\
\text { (Thermal reflux, THC effects, design } \\
\text { features, site characteristics, } \\
\text { conceptual uncertainties, and } \\
\text { alternative models) }\end{array}$ \\
\cline { 2 - 3 } & Coupled Processes & Bounding Abstractions \\
\cline { 2 - 3 } & Dimensionality & Sensitivity Testing \\
\cline { 2 - 3 } & $\begin{array}{l}\text { Verification by Comparison to } \\
\text { Field Tests }\end{array}$ & $\begin{array}{l}\text { Verification of Consistency with } \\
\text { Process Models }\end{array}$ \\
\hline
\end{tabular}

* Items in the shaded areas of this table are discussed in this report.

\subsection{Relation to Guidance from Peer Review and Oversight Groups}

\subsubsection{Performance Assessment Peer Review}

A peer review of performance assessment strategy and calculations for the Yucca Mountain site characterization was recently convened and has produced two status reports (Witherspoon et al., 1997a, 1997b). The initial findings included the following statement: "There is at present no general conceptual description of the physical and chemical state of the near-field environment over time as a function of repository parameters (e.g., flow rate, physical and chemical effects of backfill, thermal regime, etc.)." This report includes a conceptual discussion of the evolution of the NFE and of the dependence on repository parameters.

In a related observation, the peer review panel indicated that evaluation of the THCM behavior of the repository is needed to correctly plan and interpret in situ tests and that the mechanical and chemical aspects were particularly underdeveloped. This report presents a series of model results based on TH cases, further analyzed for TM, thermohydromechanical (THM), and THC effects. The panel emphasized a need for process models that are amenable to probabilistic modeling.

The first interim report (Witherspoon et al., 1997a) also specified processes represented by TSPA, for which technical basis information is lacking either as experimental data or as conceptual and numerical models. Some of the processes identified include transport of radionuclides from partially failed WPs, microbially induced corrosion, and radionuclide precipitation. These topical areas are addressed in this report. In addition, the panel stated that tradeoffs are unclear between the increased duration of dry conditions associated with greater thermal loading and the greater uncertainty associated with NF/AZ processes at 
higher temperature. These tradeoffs are incorporated in the five cases that are discussed in Chapter 3, Chapter 4, Chapter 5, and Chapter 8 for comparative analysis of coupled processes.

The second interim report (Witherspoon et al., 1997b) identified technical issues, many of which pertain to modeling of repository effects in the NF/ AZ environment. The authors of the report observed that modeling and experimental studies have shown that significant changes in hydrologic properties are possible as a result of dissolution in the condensate zone, formation of secondary minerals, and fracture-matrix chemical interaction. They state that the extent of such changes is a major uncertainty in TSPA at present and has consequences that include great uncertainty in the nature of seepage that would flow into repository drifts. This report takes significant steps in specifying the causes and in evaluating the potential significance of these changes.

The second interim report also identified uncertainties in the extent and distribution of dryout caused by waste-heat generation and indicated that a conceptual description is needed for the effects of variations in WPs and their placement, repository edge effects, and unused areas in the repository vicinity. This report addresses variations in WP heat output and placement and variations in edge effects. Evaluation of repository-loading sequence and unused areas is beyond the scope of the modeling reported here.

Water chemistry in the NF/AZ is identified as a key area of uncertainty and one for which the response estimates generated using current models do not correlate well with limited available experimental data. Accordingly, there is significant uncertainty in current estimates of water composition in the NFE. This report summarizes the current status of conceptual and predictive modeling in this area and provides comparison to observations from the SHT in the ESF.

Further, the second interim report states that models for transport of radionuclides from the EBS are underdeveloped, particularly with respect to the nature and distribution of WP perforations, the form and distribution of degraded waste-form materials inside the WP, and the form and distribution of corrosion products or degraded cementitious materials outside the WP. This report pertains to the NFE and does not discuss WP nor waste-form degradation, except insofar as the degradation products of steel and common concrete are found to have potential to strongly retard U, I, and possibly $\mathrm{Np}$.

\subsubsection{Thermohydrology Peer Review}

An independent peer review of TH investigations for the YMSCP produced specific recommendations for investigation of NF / AZ processes (TRW Environmental Safety Systems Inc., 1996). Following are the recommendations that pertained to modeling compared with the contents of this report.

The peer review panel stated that enhancements are needed in existing TH codes to improve representation of the fundamental physics with respect to anisotropy in fracturematrix flow systems, two-phase flow in fracture-matrix systems, and channeling of flow due to heterogeneities in the fracture network. These enhancements are incorporated in the models described by this report. Base-case, dual-permeability TH simulations discussed in this report have incorporated transverse anisotropy of the continuum representing the fracture network. These simulations also use finite-matrix block approximations to incorporate two-phase nonequilibrium heat flow. This report also presents discussion of flow-channeling, which has been investigated using stochastic representations of fracture permeability (Nitao, 1997a, 1997b; Tsang et al., 1997). 
The peer reviewers recommended identifying conditions for which the equivalent continuum method (ECM) is valid and invalid and developing alternative approaches. This recommendation has been addressed in this report by comparison of base-case, dualpermeability models with ECM results and through general emphasis on dual-permeability models. The panel also recommended improvements in representation, in TH models, of infiltration and of the water table. This recommendation has been addressed through use of a surface-infiltration map based on analysis of field data and through sensitivity cases that explore the use of alternative water table boundary conditions.

The peer review panel indicated that 3-D TH simulations will be needed at the scale of in situ testing, but will not be feasible at the mountain scale, where 2-D simulations should suffice. The panel indicated that quasi-3-D and other hybrid modeling approaches should be evaluated. This recommendation has been implemented in the model abstraction process for drift-scale TH predictions, which is also discussed in this report.

The panel also stated that improved validation of TH models can be achieved using rigorous comparison of observations with prior predictions. Further, the panel said that uncertainty analysis of TH behavior using customary geostatistical methods to analyze parameter uncertainty is unfeasible and unwarranted and that reducing process uncertainty should have the highest priority; second priority should be given to geologic uncertainty and last priority given to parameter uncertainty. These recommendations have generally been adopted for the models and in situ thermal tests discussed in this report.

In addition, the $\mathrm{TH}$ peer review panel made other recommendations concerning the testing program and funding priorities. Some of these recommendations that are addressed in this report concern the LBT and modeling of in situ thermal tests.

\subsubsection{Nuclear Waste Technical Review Board}

The Nuclear Waste Technical Review Board (NWTRB) submits semiannual reports to the U.S. Congress and to the Secretary of Energy. These reports contain technical recommendations, many of which pertain to the investigation of conditions contributing to repository performance.

The most recent report (NWTRB, 1996) recommended designing precast or cast-in-place concrete liner and evaluation of its effects on waste isolation. This report presents recent information on chemical and mechanical testing of concrete samples and on the potential for significant sorption of radionuclides on degraded concrete.

The NWTRB also recommended comprehensive analyses, using a systematic approach to ensure accurate representation of costs and benefits, of additional engineered barriers in support of a defense-in-depth strategy. This recommendation implies that the NF/AZ environment will be understood well enough that performance benefits from additional barriers can be evaluated unequivocally.

The previous semiannual report (NWTRB, 1995) recommended that stronger emphasis be placed on predicting or bounding the release of important radionuclides from the EBS. In particular, it recommended that alternative models be evaluated for the rate and distribution of seepage flux in the NFE and for the concentration of radionuclides such as Np in the water leaving the EBS. This report includes current analysis of drift-scale seepage and discussion of recent experimental data on the potential for sorption of $U$ (as an analog for $\mathrm{Np}$ and similar actinides) in degraded EBS materials. 


\subsection{Scope and Organization of the Near-Field/Altered-Zone Models Report}

This report presents the status of conceptual and numerical models of NF / AZ processes in a Yucca Mountain repository. Some models require intensive numerical calculation, whereas others are based on experimental results and do not include intensive calculations. The chapters of this report address many of the issues raised by the NRC staff in its issue resolution status reports, issues raised by TH and TSPA peer review panels, and those raised by the NWTRB. The Near-Field/Altered-Zone Models Report is intended to describe and defend the development of NF / AZ models and their application in TSPA and design of a potential geologic repository at Yucca Mountain.

Timing of this report will allow its publication prior to completing the final TSPA for the viability assessment (VA) in 1998. It is anticipated that this report will be updated and revised accordingly in late 1999, prior to preparation of the repository license application.

This report is structured around the major topics for analysis of coupled effects:

- Thermohydrology

- Thermomechanics

- Thermohydromechanical coupling

- Thermohydrochemical coupling

A conceptual framework for evaluating these effects is presented in Chapter 2 and includes a conceptual sequence of significant processes affecting NF / AZ performance. Chapter 3, Chapter 4, and Chapter 5 summarize advances in the investigation and identify the major uncertainties in current understanding for each of these topics. Discussion in these chapters includes results from simulations representing partly coupled processes and comparisons with experimental observations.

Chapter 6 describes models pertaining to the chemical evolution of water that enters the NFE, its interaction with introduced materials, and transport of released radionuclides within these materials. The organizing principle for transport calculations is to follow the movement of liquid water from the AZ into the near field and back through the AZ. This chapter also identifies uncertainties in the chemical and hydrologic characterization of the NFE.

In Chapter 7, the fate of introduced materials-especially cementitious materials, which predominate in the reference repository design-is discussed. That discussion is based on a thorough inventory of artificial materials that will be introduced to the repository.

Conceptual models and supporting experimental data are presented for alteration of cement, interaction of cement with the host rock, effects of introduced organic materials, and microbial effects, including induced corrosion.

The nature and significance of multiply coupled processes are key uncertainties in predicting NFE conditions. The approach, described in this report, for evaluating THCM coupled processes represents the current state of the art in modeling multiply coupled processes, given limitations imposed by computationally intensive simulation. Five basic TH cases are selected, analyzed, and discussed in Chapter 3. These are drift-scale TH simulations for a representative location near the center of the reference repository layout. The TSPA-VA "base case" hydrologic properties are used in some of these cases. TM and THM analyses of these cases are presented in Chapter 4 . THC simulations of the cases, based on experience gained from previous THC simulations and supporting laboratory experiments, are presented in Chapter 5. In Chapter 8, the calculated results are compared, and the implications for repository performance are discussed. 
Reference cases for investigation of coupled processes that are discussed in Chapters 3, 4, and 5 are also visually depicted and linked in the "References Cases Presentation" on the accompanying CD-ROM. For review of laboratory and field data on thermally driven coupled processes that are likely to occur in the repository, the reader is referred to a recent synthesis report (Hardin and Chesnut, 1997).

Models of WP corrosion and waste-form release are not included in this report, but are discussed in other reports (McCright, 1997; Stout, 1996) Links between NF/AZ studies and performance predictions for the waste form and WP are provided in this report by predicted performance measures, including WP temperature and relative humidity, drift air mass fraction, and drift seepage.

\section{Return to Table of Contents}

\subsection{References}

Buscheck, T.A., and J.J. Nitao (1992). “The impact of thermal loading on repository performance at Yucca Mountain." In proceedings from Third International High-Level Radioactive Waste Management Conference. Las Vegas, NV: April 1216, 1992. Am. Nucl. Soc., La Grange, IL. pp. 1003-1017. (Also UCRL-JC-109232, Lawrence Livermore National Laboratory, Livermore, CA) [NNA.19920408.0008]

Chesnut, D.A. (1992). "Characterizing the altered zone at Yucca Mountain: The beginning of a testing strategy." In proceedings from Third Annual International High-Level Radioactive Waste Management Conference. Las Vegas, NV: Am. Nucl. Soc., La Grange Park, IL. (Also UCRL-JC-109231, Lawrence Livermore National Laboratory, Livermore, CA) [NNA.19920324.0014; 208832]

DOE (1996). “Draft Highlights of the U.S. Department of Energy's Updated Waste Containment and Isolation Strategy for the Yucca Mountain Site." U.S. Department of Energy: enclosure to a letter dated July 19, 1996, from S.J. Brocoum, U.S. DOE, to M.V. Federline, U.S. Nuclear Regulatory Commission.

Flint, A., J.A. Hevesi, and L.E. Flint (1996). “Conceptual and Numerical Model of Infiltration for the Yucca Mountain Area, Nevada." Water Resources Investigation Report. (3GUT623M). Denver, CO: U.S. Geological Survey. (U.S.G.S. Survey Milestone Report) [MOL.19970409.0087]

Hardin, E.L., and D.A. Chesnut (1997). "Synthesis Report on Thermally Driven Coupled Processes." Milestone report for the CRWMS Management and Operating Contractor, U.S. Department of Energy. (SPL8BM3). Livermore, CA: Lawrence Livermore National Laboratory. (Also UCRL-ID-128495, Lawrence Livermore National Laboratory, Livermore, CA) [MOL.19980120.0035]

McCright, R.D. (1997). “Engineered Materials Characterization Report.” Milestone report for the CRWMS Management and Operating Contractor, U.S. Department of Energy. (TR251FB9, Rev. 1). Livermore, CA: Lawrence Livermore National Laboratory. (Also UCRL-ID-119564, Rev. 1) [MOL.19980105.0616]

Neretnieks, I., H. Abelin, and L. Birgersson (1987). “Some recent observations of channeling in fractured rocks - its potential impact on radionuclide migration." In proceedings from GEOVAL 1987: A Symnposium on Verification and Validation of Geosphere Performance Assessment Models. Stockholm, Sweden: Swedish Nuclear Power Inspectorate. pp. 547-548. [210021] 
Nitao, J.J. (1997a). “Distribution of Post-Emplacement Seepage into the Repository Drifts with Parametric Variation of Intrinsic Properties: Models and Bounds for PostEmplacement Seepage into the Repository." Milestone report for the CRWMS Management and Operating Contractor, U.S. Department of Energy. (SPLC2M4 and SPLC3M4). Livermore, CA: Lawrence Livermore National Laboratory. [MOL.19980107.0400]

Nitao, J.J. (1997b). "Preliminary Bounds for the Drift-Scale Distribution of Percolation and Seepage at the Repository Level under Pre-Emplacement Conditions." Letter report to CRWMS Management and Operating Contractor, U.S. Department of Energy. Livermore, CA: Lawrence Livermore National Laboratory. [LLYMP 9703034; 234515]

NRC (1997a). “Issue Resolution Status Report-Key Technical Issue: Evolution of the Near Field Environment." Washington, DC: Division of Waste Management, Office of Nuclear Material Safety and Safeguards, U.S. Nuclear Regulatory Commission. (Rev. 0) [MOL.19971016.0549]

NRC (1997b). “Issue Resolution Status Report—Key Technical Issue: Thermal Effects on Flow." Washington, DC: Division of Waste Management, Office of Nuclear Material Safety and Safeguards, U.S. Nuclear Regulatory Commission. (Rev. 0) [MOL.19971016.0549]

NWTRB (1995). "Report to the U.S. Congress and The Secretary of Energy: 1995 Findings and Recommendations." Washington, DC: Nuclear Waste Technical Review Board. (April 1996) [HQO.19960613.0001]

NWTRB (1996). "Report to the U.S. Congress and The Secretary of Energy: 1996 Findings and Recommendations." Washington, DC: Nuclear Waste Technical Review Board. (March 1997) [232686]

Stout, R.B. (1996). “Waste Form Characteristics Report.” (UCRL-ID-108314, Rev. 1). Livermore, CA: Lawrence Livermore National Laboratory. [MOL.19971017.0266]

TRW Environmental Safety Systems Inc. (1996). “Thermohydrologic Modeling and Testing Program: Peer Review Record Memorandum." Office of Civilian Radioactive Waste Management Milestone Report. (TRWF1). Las Vegas, NV: U.S. Department of Energy. [MOL.19960618.0060]

Tsang, C.-F., J. Birkholzer, G. Li, and Y. Tsang (1997). “Drift Scale Modeling: Progress in Studies of Seepage into a Drift." Milestone report. (SP331CM4). Berkeley, CA: Lawrence Berkeley National Laboratory. [MOL.19971204.0420]

Witherspoon, P., C. Whipple, J. Payer, D. Moeller, R. Ewing, and R. Budnitz (1997a). “First Interim Report of the Total Systems Performance Assessment Peer Review Panel." Letter (with appendix) to J.L. Younker. June 20, 1997.

Witherspoon, P., C. Whipple, J. Payer, D. Moeller, R. Ewing, and R. Budnitz (1997b). “Second Interim Report of the Total Systems Performance Assessment Peer Review Panel." December 12, 1997. 


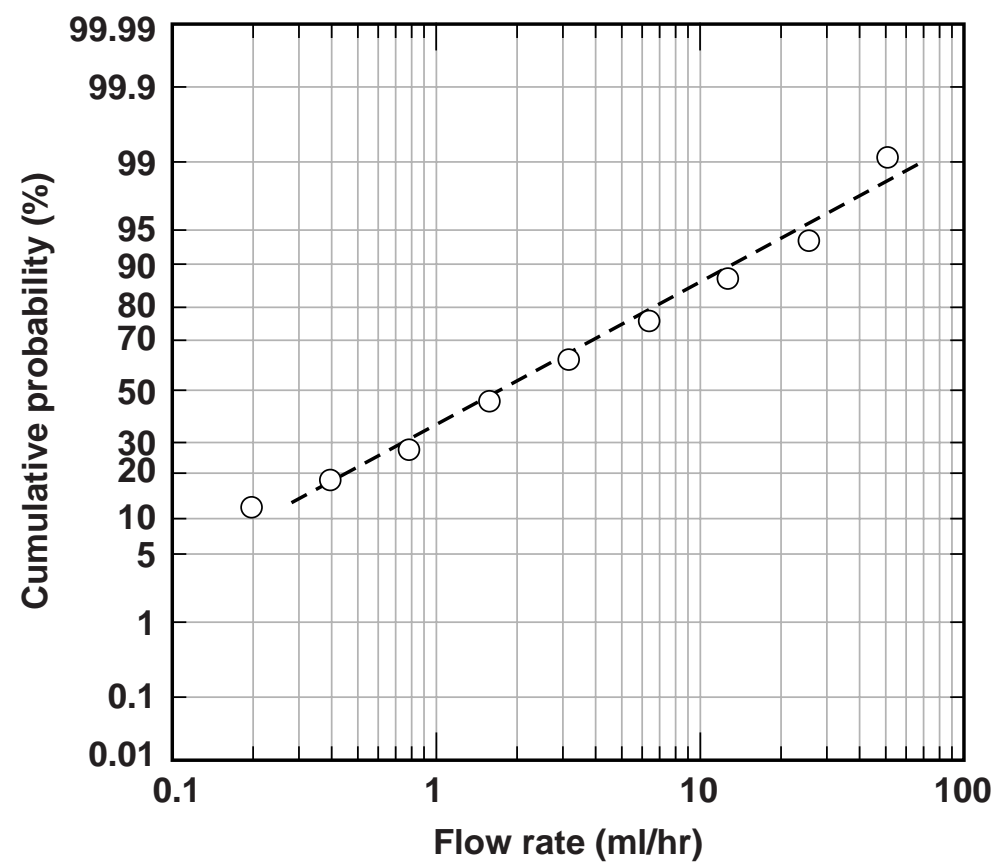

Figure 1-1. Distribution of inflow rates per each 2- $\mathrm{m}^{2}$ collector in the Stripa experiment (after Chesnut [1992] data from Neretnieks et al. [1987]. Circles represent measured data, and the dashed line is a least-squares fit to a log-normal distribution. Collectors with no reported flow were not included. 



\section{Conceptual Model for Evolution of Coupled Processes in the Near Field and Altered Zone}

Coupled processes may have beneficial or adverse effects on the performance of a repository at Yucca Mountain. Because large amounts of water will be redistributed by the heat of radioactive decay, it is believed that coupled effects will very likely occur. The redistributed water will dominate seepage into emplacement drifts for hundreds or thousands of years and will affect the temperature, humidity, and other measures that control performance of engineered barriers.

Based on the current repository safety strategy (DOE, 1998), the lifetime of the waste package (WP) is a key attribute of repository system performance. For WP lifetime to contribute significantly to isolation of long-lived, relatively mobile radionuclides, containment must continue to be effective many thousands of years after cool-down. The WP is one part of an engineered barrier system (EBS) that will perform during the thermal period, and in a post-thermal environment that will resemble present conditions at Yucca Mountain. The importance of coupled processes pertains to the potential for accelerated degradation of the EBS during the thermal period, which could significantly affect performance both during and after the thermal period.

Discussion of near-field and altered-zone (NF / AZ) performance in terms of coupled processes emphasizes differences between ambient conditions prior to waste emplacement, and an environment affected by excavation, heating, and introduced materials. For this report, "coupled processes" refers to thermal-hydrologic-chemical-mechanical (THCM) processes (in any combination) having the potential to significantly degrade natural or engineered barriers; this encompasses all modes of potentially significant degradation of engineered barriers, even for those of the so-called "cold repository"(Halsey, 1994).

Coupled processes figure prominently in current discussion of repository performance (NRC, 1997). The topic receives close attention because of uncertainty associated with the long-term changes in the host rock resulting from artificially imposed conditions, and the behavior of introduced materials. These uncertainties are poorly constrained by human experience or by the geologic record, so predictive models are relied upon to judge the effects. This Near-Field and Altered-Zone Environment Models Report is intended to summarize conceptual and numerical models that have been developed for predicting the effects of coupled processes in the NF/ AZ surrounding a Yucca Mountain repository.

In the consideration of repository performance, coupled processes can be classified in two general categories:

- Onsager-type processes driven by gradients of thermodynamic state variables

- Another type in which processes are affected indirectly by the magnitudes of state variables (Carnahan, 1987; Hardin and Chesnut, 1997)

The Onsager processes are fundamental and occur in response to thermodynamic potential gradients, even if the associated physical or chemical properties do not vary. Many of the indirect Onsager processes such as thermal osmosis, the Soret effect, chemical osmosis, the Seebeck effect, and electro-osmosis are probably insignificant because of overwhelming competition from direct Onsager processes such as Fourier heat flow, electrical conduction, Fickian diffusion, and Darcy flow (Hardin and Chesnut, 1997). Thus the off-diagonal (unshaded), indirect Onsager processes in Table 2-1 tend to be insignificant, compared with the direct Onsager processes on the main diagonal. It should be noted that none of the phenomenological coefficients for the indirect Onsager coupled processes (unshaded) have been measured or investigated in the repository host rock. 
Table 2-1 Direct and indirect Onsager-type coupled processes (after Carnahan, 1987)

Fluxes are driven by gradients of temperature $(T)$, pressure $(P)$, chemical potential $(\mu)$, and electrical potential $(V)$.

\begin{tabular}{|l|c|c|c|c|}
\hline \multicolumn{1}{|c|}{ Flux $\downarrow$ Force $\rightarrow$} & $\nabla \mathbf{T}$ & $\nabla \mathbf{P}$ & $\nabla \mu$ & $\nabla \mathbf{V}$ \\
\hline \hline Heat & Fourier's Law & Thermal filtration & Dufour effect & Peltier effect \\
\hline Volume & Thermal osmosis & Darcy's Law & Chemical osmosis & Electro-osmosis \\
\hline Mass diffusion & Soret effect & Reverse osmosis & Fick's Law & Electrophoresis \\
\hline Electrical current & Seebeck effect & Streaming current & Sedimentation current & Ohm's Law \\
\hline
\end{tabular}

Processes in the latter category, affected by the magnitudes of thermodynamic potentials, are of principal concern for repository performance. These processes generally occur because of changes in medium, fluid, or reactive properties. For example, the phenomenological coefficients for direct Onsager processes (shaded region in Table 2-1) may be changed through the effects of chemical potentials, mechanical state, or temperature, on liquid or solid medium properties.

Thermally driven coupled processes at a Yucca Mountain repository can also be categorized by longevity of the transient and residual effects:

- Transient effects are processes that produce reversible changes (e.g., drying and rewetting) that eventually return to pre-emplacement conditions, possibly altered by hysteresis or by changing boundary conditions such as the climate.

- Residual effects are processes that result in permanent changes to flow and transport pathways, changes that remain after cooling of the host rock. Important examples include thermohydrochemical (THC) coupling associated with the dissolution and precipitation of minerals, especially along fractures, and with microbial activity.

Both transient and residual effects are potentially important to EBS performance. Transient effects can expose the EBS to aggressive chemical conditions during the thermal period, whereas residual effects have the potential to act over the entire lifetime of EBS performance.

Every physical or chemical process that is likely to occur in the repository can be considered as thermally coupled in some way. However, all thermally coupled processes are not relevant to performance, and the occurrence of thermally coupled processes does not generally imply conditions adverse to waste isolation. Depending on repository thermal loading, the NF/ AZ may not reach temperatures sufficient to drive thermally coupled processes to produce significant effects. However, other types of coupled processes, such as the ambient temperature effects of alkaline leachate from Portland cement, are likely to be significant.

This chapter presents a discussion, using coupled-process terminology, of conceptual models for EBS performance. The discussion focuses on the NF / AZ processes likely to be important with a reference repository design that consists of in-drift emplacement of a bimetallic WP in a "point-loaded" repository layout without backfill (CRWMS M\&O, 1997b). Section 2.1 briefly describes how conceptual and numerical models for coupled processes will be used in performance assessment, and Section 2.2 provides a detailed discussion of the temporal evolution of coupled processes in the NF/AZ. 


\subsection{Application of Near-Field and Altered-Zone Models}

Thermohydrologic (TH) models have been used extensively to predict EBS performance measures, including WP surface temperature, relative humidity $(R H)$ at the WP surface, and air-mass fraction in the emplacement drifts. Corrosion of WP materials will occur in humid air as the thermal period declines, and the onset timing for humid-air corrosion can be estimated from calculated temperature and $R H$. Condensate reflux into emplacement drifts during the thermal period is of interest because aqueous chemical interactions with introduced materials are generally accelerated at elevated temperature (NRC, 1997). The average rate of reflux to the drifts can be predicted using available simulators. Calculations of reflux activity and drift-air humidity depend on properties and boundary conditions that have been shown to vary significantly throughout the repository (Bodvarsson et al., 1997). Models and parameter-estimation strategies used to predict TH conditions over the repository area are described in Chapter 3.

The available water for degradation of engineered barriers, and release and transport of radionuclides, depends on seepage into emplacement drifts. The occurrence of seepage as thermally driven reflux or natural ambient percolation, is sensitive to nonequilibrium fracture-matrix interaction, spatial heterogeneity of hydrologic properties, flow-field dimensionality, and episodic variation of boundary conditions (Nitao, 1997b; Tsang et al., 1997). Drift-seepage simulations based on assumed spatial heterogeneity and episodic boundary conditions are discussed in Section 3.8 of Chapter 3. Threshold values for incident flux, at or above which seepage into drifts is predicted by these models, are available for use in total system performance assessment (TSPA).

Coupled processes during the thermal period may significantly alter hydrologic properties that influence reflux activity and seepage. Thermomechanical (TM) effects will produce stress conditions in the host-rock mass that change fracture porosity and permeability. Thermal stresses, and the resulting changes in hydrologic properties based on alternative models of thermohydromechanical (THM) behavior during and after the thermal period, are discussed in Chapter 4. Results include predictions of thermal-stress magnitude and changes in fracture permeability during and after the thermal period.

Because of mineral dissolution and precipitation reactions, and precipitation in response to boiling or evaporation, THC effects will cause alteration of flowpaths above and below the repository emplacement drifts. Consequent changes in fracture hydraulic properties for the host rock have never been measured under controlled conditions and must be inferred from model results. Example calculations of THC effects, based on the TH predictions described previously are discussed in Chapter 5.

A flowpath paradigm is used in TSPA to represent processes controlling engineered barrier degradation and radionuclide transport in the altered zone (AZ) and the near-field environment (NFE). In this approach (CRWMS M\&O, 1996), percolation flux interacts chemically with the host rock in the $\mathrm{AZ}$, then water with altered composition enters the emplacement drifts (if hydrologic conditions are sufficient to cause seepage). The seepage interacts chemically with introduced materials such as cement and makes up the chemical boundary condition for modeling WP corrosion and waste-form degradation. Advective release of radionuclides from WPs mixes with drift seepage, interacts with introduced materials (including degraded cement and steel), and flows downgradient into the host rock.

Each of these steps is abstracted for TSPA as a reaction cell (Figure 2-1). The initial composition of fracture water in the host rock is probably similar to that from well J-13 (Harrar et al., 1990). Changes in water composition resulting from heating and partial evaporation of that water are discussed in Chapter 6. As condensate is produced during the 
thermal period, distilled water accumulates and interacts with the host rock. The condensate composition has been modeled and compared with water collected from the Single-Heater Test at Yucca Mountain (Section 6.2). As discussed previously, seepage of fracture water into the drifts will be controlled by capillary behavior (Richards barrier effect), and by the spatial variability of hydraulic properties in the host rock. Chemical interaction of seepage with introduced materials, and the effects of microbial activity, will influence the chemistry of water in contact with WPs (Sections 6.3 and 6.4). Released radionuclides will be retarded to some extent by introduced materials in the NFE (Section 6.5), and mineral alteration along flow pathways below the emplacement drifts will influence radionuclide mobility by means of changes in mineral composition, reactive surface area, and fracture-matrix hydraulic interaction (Section 6.6).

Figure 2-1 Schematic of NFE and AZ showing TSPA reaction cells, annotated with section numbers from this report

Evolution of the near-field geochemical environment (NFGE) is particularly important for predicting WP lifetime, but reliability of model predictions is limited by the experimental data available to represent important chemical reactions. The nature and extent of microbially mediated reactions are not well known. Experimental studies are the key to reducing uncertainty in predictive models for the NFGE. Laboratory studies to evaluate the fate of cement and other introduced materials, and to assess microbial effects, are described in detail in Chapter 7.

\subsection{Evolution of the Near-Field Environment and Altered Zone}

The following description of coupled processes is simplified from a sequence created for the Design-Basis Models effort, part of the Yucca Mountain Viability Assessment (VA; CRWMS M\&O, 1997a). The discussion is based on the reference design, which consists of the following:

- Point-load WP layout with areal mass loading of $85 \mathrm{MTU} /$ acre

- Drifts spaced $28 \mathrm{~m}$ apart

- Reinforced-concrete invert and pre-cast concrete liner

- Bimetallic WPs

- In-drift disposal

In the following sections, approximate time periods are given; more detailed predictions can be obtained using TH properties and boundary conditions appropriate for a particular location. Time ranges bracket the time at which the indicated events are predicted to occur in TH simulations using TSPA-VA base-case properties (Chapter 3). Typical conditions expected in the repository block are defined for this report as base-case TH properties and unit thicknesses and ambient percolation conditions near the center of the proposed repository area, based on the same stratigraphic model and surface infiltration model used for the TSPA-VA.

\subsubsection{Time Period A: Drift Closure to Repository Closure}

The repository's engineered systems will be actively maintained during this period, and the drift wall and liner will remain substantially intact through permanent closure (Figure 2-2). Waste package temperature will increase from $70^{\circ}$ to $200^{\circ} \mathrm{C}$, depending on local thermal output of WPs and on TH conditions, which will vary throughout the repository block. $R H$ of the drift air at the WP wall will decrease to approximately $20 \%$. The drifts will receive slight ventilation to facilitate monitoring of possible leaks from WPs. The RH will be limited by the total pressure in the gas phase, which will not significantly exceed one 
atmosphere in the drifts and in the fracture system although the temperature will exceed boiling. When drift maintenance or other service activities are required, ventilation will be increased for temperature control, dropping the partial pressure of water vapor so that the RH will approach $5 \%$.

\section{Figure 2-2 Schematic of emplacement drift conditions at repository closure}

During the first few years after waste emplacement, before the drift-wall temperature exceeds boiling, moisture may condense at cooler locations on the inside surface of the liner and drip onto WPs (see Section 3.6.7). Without use of backfill, radiative thermal coupling between the WP, the liner, and other exposed surfaces is expected to limit temperature differences to a few degrees. However, in-drift condensation could result from temperature differences associated with unheated intervals of the emplacement drifts or from adjacent, separated WPs with different power output. The relatively small quantity of water mobilized in this way will tend to be imbibed into the liner and removed by ventilation.

Vapor generated from evaporation of matrix pore water will move outward along fractures and condense, increasing fracture and matrix saturation in a surrounding zone. When matrix imbibition is satiated in regions of condensation, fracture flow will drain condensate downward under the influence of gravity. Reflux activity will result from ambient percolation and condensate drainage above the drifts, while drainage below the drifts will conduct condensate to cooler regions.

The rate of heat production will be maximal during this period. Temperature gradients in the NFE will be at their highest, and the local heat flux in the NFE may be much greater than the average repository thermal loading because of geometric concentration near WPs. The dryout zone will expand as long as the local total heat flux (conduction plus convection) exceeds the heat flux corresponding to the combined rates of reflux and ambient percolation.

During this period, the thermal regime will be in an early state of development, and reflux of condensate may penetrate episodically into the boiling zone, as shown from physical models of gravity-driven reflux (Hardin and Chesnut, 1997; Kneafsey and Pruess, 1997) and implied by nonisothermal dual-permeability models of reflux behavior in spatially heterogeneous media (Nitao, 1997a). Some reflux may contact the concrete drift liner, but there will be no significant penetration through the liner. Simulations show that matrix saturation in the NFE will decline from the ambient value of about $90 \%$ to $50 \%$ or less, depending on the extent to which evaporated water is replaced by capillarity (capillary reflux). During this period, a precipitation cap will begin to form in the host rock above the emplacement drifts.

During this period, the WP outer layer, which will be constructed of mild-steel corrosionallowance material (CAM), will be subject to minor oxidation because a small fraction of air will be introduced to the emplacement drifts by forced ventilation. Waste-emplacement areas will be maintained at lower absolute pressures than will occupied working areas, so air will migrate into the emplacement drifts through fractures. Radiolysis of nitrogen is expected to produce small amounts of oxidizing compounds.

The WP, and possibly the pedestal, invert, rails, and other components of the wastehandling system will remain structurally intact. Water movement may be complicated by the effects of thermal-load sequencing (i.e., the order in which drifts are constructed and loaded with WPs). Drift ventilation has the potential to remove substantial amounts of water, but ventilating a significant fraction of the emplacement drifts for this purpose would require greater ventilation capacity than that provided in the reference design. 
For high values of average ambient percolation flux, such as may exist in some parts of the repository area (Flint et al., 1996), a dryout zone may not form without the removal of vapor by active drift ventilation. Contributing causes include low areal thermal loading and capillary reflux produced by significant wicking behavior in the matrix or fractures. It is important to note that, prior to permanent repository closure, emplacement drift conditions can be evaluated, until heat output has substantially decreased, to determine that dryout will prevail after closure.

\subsubsection{Time Period B: Development of the Superheated Dryout Zone (Repository Closure to a Few Hundred Years)}

Throughout much of the repository, the dryout zone will continue to expand, and in most areas of the repository this will lead to coalescence of the boiling isotherms projected from adjacent drifts (which are $28 \mathrm{~m}$ apart in the reference design). As the boiling isotherm expands, the rock temperature within the enclosed region will exceed the boiling temperature (i.e., the rock will be "superheated").

The occurrence and duration of coalescence will depend on areal thermal loading, local TH properties, and the ambient percolation flux (see Chapter 3). If the ambient flux is high or if reflux is predominantly caused by capillarity, the spatial extent of boiling will be limited, and coalescence may not occur. During this period, the repository heat output will decline, but the dryout zone will continue to expand.

Condensate will reflux in developing heat pipes, and some will drain through the pillars between drifts or through cooler regions of the repository, such as the gaps between widely spaced WPs (cold-trap effect). The concrete liner will provide protection against water contacting WPs for tens or hundreds of years after closure. Corrosion of the CAM will be minimal during this period because the NFE will be at super-boiling temperatures and will remain mostly dry.

For high ambient percolation flux such as may exist in some parts of the repository area (Flint et al., 1996), a dryout zone may not form or may persist for only a short time. Other contributing causes include low areal thermal loading and capillary reflux produced by significant wicking behavior in the matrix or fractures. Abundance of moisture at elevated temperature will have important consequences for EBS performance (e.g., early onset of humid-air corrosion of the WP).

In the reference repository design, there is no ventilation of emplacement drifts after permanent closure, and there is no backfill (Figure 2-3). Without ventilation, the drift atmosphere will consist of nearly pure water vapor during this period. The temperature at the WP surface will decrease to approximately $150^{\circ} \mathrm{C}$, and the $\mathrm{RH}$ will be approximately $25 \%$. As the host rock is heated, the $\mathrm{CO}_{2}$ contained in the pore water, or complexed at the rockwater interface, will be released to the gas phase. The $\mathrm{CO}_{2}$ fugacity in condensing regions of the NF/ AZ will be elevated, which will lower the condensate $\mathrm{pH}$ and increase calcite dissolution.

Figure 2-3 Schematic of emplacement drift conditions at a few hundred years after repository closure (after dryout zone development)

Natural fracture-lining calcite, along with primary and secondary silicate minerals, will be dissolved in the expanding condensate zone (see Chapter 5). Cristobalite and feldspars will be dissolved from the tuff matrix adjacent to fractures. The constituents will be transported into the boiling zone by reflux or will drain around the emplacement drifts into 
cooler regions. In the boiling zone, evaporation or boiling will cause precipitation; in cooler parts of the rock mass, cooling will cause precipitation. The precipitation cap will continue to develop during this period.

Within a few hundred years after repository closure, the temperature of the waste form inside the WPs will decline because of decreasing heat output and will cool safely through the threshold temperature value for "unzip" behavior of spent-fuel cladding. With the addition of backfill to the reference design, the WP and waste form peak temperatures will be considerably higher, delaying this stage of cool-down.

TM loading of the rock mass will approach maximum intensity as the temperature increases throughout an increasingly larger volume (see Chapter 4). Thermal loading will cause rotation of the principal normal stress from vertical to horizontal, with a magnitude of approximately twice the overburden stress. Stress concentration at the drift crown will produce average compressive stresses of four to six times the overburden stress, but the intact rock strength will not be exceeded. Displacement of rock joints will be significant and will contribute to collapse of the concrete liner.

Thermal alteration reactions in the concrete liner will proceed to completion during this period (see Chapter 7). Portland-type cements will recrystallize, forming more highly ordered, less hydrated crystalline forms. Inhomogeneous alteration will promote spalling of the liner. Where concrete is contacted by reflux water at elevated temperature, leaching may also contribute to strength degradation. Loading of the liner associated with joint displacement will eventually lead to collapse of the liner into the opening. With liner collapse, there will be potential for increased incidence of reflux water dripping onto WPs.

By the end of this period, the liner will collapse completely, and the reinforced concrete invert will crack. There will be some rock fall into the drifts and resulting minor impact damage to WPs (CRWMS M\&O, 1997b).

\subsubsection{Time Period C: Quasi-Stable Reflux Zone (500 to $1500 \mathrm{yr}$ )}

In this period, several effects will tend to stabilize the position of the boiling isotherm, as shown by TH simulations:

- Geometrical spreading of the boiling isotherm will decrease the heat flux at the boiling isotherm.

- The rate of change of heat production will decrease significantly as fission products decay.

- The average heat flux at the boiling isotherm will approach the ambient percolation flux (expressed as latent heat).

The relation between heat production and ambient percolation flux can be shown by a simple calculation. Repository areal thermal loading will decrease by an order of magnitude during the first $1000 \mathrm{yr}$, depending on the type of waste considered. For pressurized water reactor (PWR) spent fuel that is $10 \mathrm{yr}$ old at emplacement, heat production at 1,000 yr will have declined by a factor of approximately 15. For areal mass loading of 85 metric tons uranium (MTU)/ acre, the initial heat output will correspond to the enthalpy of vaporization associated with a moisture flux of $275 \mathrm{~mm} / \mathrm{yr}$ (considering only heat of vaporization). At $1000 \mathrm{yr}$, moisture flux will decay to approximately $20 \mathrm{~mm} / \mathrm{yr}$. Simulations show that during this period, up to half the repository heat output will be transported by conduction (especially downward), while the remainder will be transported by vapor-liquid movement in the heat pipe. Thus, the moisture reflux in a heat-pipe zone in this example will have a magnitude of 10 to $15 \mathrm{~mm} / \mathrm{yr}$. This is comparable to infiltration values reported for Yucca Mountain (Flint et al., 1996). As the rate of heat production declines further, or the ambient flux increases (e.g., climate change), the heat-pipe zone will begin to collapse. 
During this period, the thickness of the dryout zone above each emplacement drift will be directly related to thermal loading, and inversely related to the ambient percolation flux. The coalescence of dryout zones from adjacent drifts will change the dependence of system behavior on ambient flux, by limiting condensate drainage between drifts. The geometry of the heat-pipe zone will also be influenced by the spatial variability of $\mathrm{TH}$ properties and of the ambient percolation flux.

Temporal fluctuations in heat-pipe geometry will occur because of variations in ambient flux, but the average position of the boiling isotherm at the lower limit of the heat-pipe zone will tend to stabilize during this period. This is important for evaluation of THC processes for two reasons:

- The position of the condensate zone at the upper limit of the heat-pipe zone will also stabilize, concentrating the effects from aqueous dissolution and precipitation reactions at $96^{\circ} \mathrm{C}$.

- The zone of evaporation and boiling will tend to stabilize, concentrating the effects from solute precipitation.

Precipitated minerals will accumulate in fractures and could serve to divert future percolation from entering emplacement drifts (see Chapter 5). Condensate will dissolve natural fracture-filling minerals and primary minerals from the adjacent matrix and will flow downward under the influence of gravity. The precipitation cap will become fully developed during this period. The significance of THC effects on permeability depends on the abundance of readily dissolved fracture-lining minerals, relative to fracture porosity, the dissolution rates for feldspars and silica polymorphs, and the precipitation rates for secondary minerals such as clays and zeolites.

When condensate penetrates the boiling isotherm in fractures, it will tend to be imbibed into the adjacent tuff matrix. Because the sensible heat entering this zone is conducted through the matrix, the matrix will be hotter than the fractures, and the imbibed water will evaporate. The associated solute will be deposited in the matrix, thus decreasing matrix permeability. The cumulative effects of this process will inhibit fracture-matrix hydrologic interaction both above and below the emplacement drifts so that subsequent THC effects will tend to be limited to fractures.

During time periods A and B, the boiling isotherm will have expanded outward, leaving precipitates in the fractures and matrix of the dryout zone (Figure 2-4). Throughout the dryout zone, the potential for fracture-matrix interaction will decrease, and fracture permeability will be reduced. Within the quasi-stable boiling zone, these changes will be accentuated.

Figure 2-4 Schematic of emplacement drift conditions during the period of a quasi-stable reflux zone (for typical conditions expected in the repository block)

Temperatures at the WP surface and the drift wall will begin to decline, but will remain well above boiling throughout time period $\mathrm{C}$ (see Chapter 3). As the temperature falls, $\mathrm{RH}$ at the WP will start to rise, approaching values of $50 \%$ to $90 \%$, depending on thermal loading and TH conditions. The CAM will be $20 \%$ to $50 \%$ oxidized by the end of this period. The steel pedestals supporting the WPs will begin to collapse, which will cause the WPs to settle onto the invert materials. Matrix saturation in the NFE will remain near the residual value for the tuff matrix in the dryout zone ( $15 \%$ to $50 \%$, depending on the rock temperature).

The preceding discussion pertains to typical conditions in the repository. For high ambient percolation flux, direct reflux onto WPs at elevated temperature may commence during this period. Simulations show that buoyant gas-phase convection may transport water 
vapor upward from below the repository, whereupon it will condense above the repository, and become available for reflux and drainage (see Chapter 3). This may occur even if the NFE remains at sub-boiling temperatures.

The maximum TM stresses will occur during this period, and fracture deformation will be at maximum in the NF / AZ (see Chapter 4). This is likely to close radial fractures around the emplacement drifts and may cause fractures with certain orientations to open. Therefore, some effects of THC processes that redistribute soluble minerals in the condensate and boiling zones will be manifested in fractures that will be partly closed by TM stress. To the extent that the fractures reopen on cooling, THC effects on fracture permeability will be reversed.

\subsubsection{Time Period D: Collapse of the Superheated Dryout Zone (1000 to $3000 \mathrm{yr}$ )}

During this period, the dryout zone will no longer be supported by the repository heat output and will start to collapse. Eventually, the boiling isotherm will converge on the drift wall, demarking collapse of the superheated dryout zone. For TSPA-VA base-case TH properties, the drift wall temperature cools to boiling at about 2000 to $5000 \mathrm{yr}$, depending on the ambient flux (see Chapter 3). For greater ambient percolation, or at the edge of the repository, this happens sooner (e.g., $1000 \mathrm{yr}$ ). For smaller values of percolation flux, collapse will occur later (e.g., 3000 yr or beyond). Whereas expansion of the dryout zone will occur within a few tens or hundreds of years, collapse of the dryout will occur over a longer time period because the rate of change of heat production will have declined by an order of magnitude.

Simulations show that, with sufficient matrix imbibition and fracture-matrix interaction and with limited ambient percolation flux, the host rock can cool to temperatures less than boiling before liquid water penetrates to the emplacement drifts (Chapter 3). This performance will depend on fracture-matrix interaction, which will be irreversibly inhibited by the cumulative effects of THC activity. Because collapse of the dryout zone will occur relatively slowly, more time will be available for fracture-matrix interaction than during expansion of the dryout zone. The effects of THC activity on fracture-matrix interaction will be important during this period, even if other THC effects, such as reduction of fracture permeability, are minor. Because boiling is still going on, the precipitation cap continues to accumulate during this period.

The duration of the dryout zone will be prolonged at locations within the repository where the ambient percolation flux is sufficiently small. This will occur for several reasons:

- The extent of dryout will be greater in low-flux areas.

- The availability of water for rewetting will be smaller in low-flux areas.

- Significant amounts of water vapor may continue to be transported upward, through the waste emplacement areas, by buoyant gas-phase convection.

The geometry of rewetting will depend on spatial heterogeneity of fracture hydraulic properties. This dependence has been shown from simulation of isothermal and nonisothermal drift seepage (see Section 3.6). If coalescence of dryout zones occurs, the dryout zone thickness will be on the order of the drift spacing or greater, so that drainage will tend to occur at larger-scale heterogeneities (e.g., fracture zones) as well as near the repository edge.

Condensate drainage through fractures into the superheated dryout zone will cool the surrounding rock and depress the boiling isotherm. Eventually, the boiling isotherm will penetrate to the emplacement drifts at a few locations, and, at some locations, there may be liquid water in contact with engineered barriers at elevated temperatures approaching $96^{\circ} \mathrm{C}$. The collapsed concrete liner will not be a barrier to incursion of reflux during this period. 
$\mathrm{RH}$ will approach $100 \%$ at the emplacement drift wall as the rock cools to the boiling point, but RH will be lower ( 95\%) at the WP surface (see Chapter 3). Matrix saturation in the NFE will increase to $80 \%$ or more from rewetting by permeating water. The CAM will develop moderate corrosion (20\% or more of CAM mass will be affected), and corrosion will cause pedestals made from mild steel to collapse as depicted in Figure 2-5 (CRWMS M\&O, 1997b).

Figure 2-5 Schematic of emplacement drift conditions during collapse of the superheated dryout zone (for typical conditions expected in the repository block)

Significant incursion of liquid water into the NFE will occur at a few isolated locations, for the first time since repository closure. The water entering the NFE will have composition similar to that of water from the water table in well J-13. Minor changes in composition are calculated for water in contact with Topopah Spring welded tuff at elevated temperatures (see Section 6.2). This water will interact with the CAM and degraded cementitious materials in the NFE. With increased RH, humid-air corrosion will cause progressive degradation of the CAM. Pedestal failure will be complete during this period, and the WPs will rest directly on cementitious materials from the invert and collapsed liner. Depending on the concrete formulation, the presence of alkaline materials will determine the nature and rate of corrosion processes affecting the parts of the WPs exposed to detrital material. If drift backfill is used, chemical interaction of liquid water with the degraded concrete liner will affect corrosion processes over the upper, as well as the lower, parts of the WPs.

When the dryout zone collapses, heat-pipe activity will continue even as the boiling isotherm contracts around each emplacement drift. Minerals precipitated in fractures during expansion of the dryout zone will be reworked during collapse. Gradual collapse of the reflux zone will produce concentrative THC activity. Labile precipitates such as calcite and amorphous silica, which are precipitated during dryout zone expansion, will be readily redissolved by condensate and cycled back toward the drift. Consequently, the solute inventory available for deposition by THC coupled processes will increase closer to the drift. Concentrative activity will be limited by mineral solubility and by drainage of condensate around the emplacement drifts. The most soluble species, such as halides, will be most highly concentrated, which may be significant if they are deposited onto the WPs.

Maximum TM stresses will be extant at the start of time period D. As the superheated dryout zone collapses, the temperature in the NF/AZ will decrease, and thermal stresses will partially relax. Fractures that were closed by thermal stresses during earlier expansion of the dryout zone will reopen and will become available to participate in coupled THC processes. The temperature change during this period will be only a portion of the total change relative to pre-emplacement conditions, so relaxation will be incomplete, and further reopening of fractures can be expected to occur at a later time. Rock fall will occur in response to relaxation of thermal stress and liner collapse.

\subsubsection{Time Period E: Cool-Down (3000 to 10,000 yr)}

Thermally driven reflux will become much less important during this time period because the maximum temperature in the NFE will be less than boiling. The rate of seepage into drifts will decline as temperatures decrease, whereupon seepage will depend principally on ambient percolation.

Simulations show that capillary effects in the host rock may promote some diversion of ambient percolation around drift openings. The matrix hydraulic conductivity in the host rock is limited, so significant diversion will depend on permeability and capillary behavior in 
fractures. The durable effects of THCM coupled processes on fracture-lining minerals will determine the extent to which seepage conditions will be modified from pre-emplacement conditions. Slight dissolution of the precipitation cap will occur during this period, but will be limited by diminished solubility at lower temperatures and by prior equilibration of percolation flux with silicate minerals above the repository.

Simulations also show that seepage in response to episodic infiltration could be more than an order of magnitude greater than the response to steady-state percolation. As discussed previously, THC activity will irreversibly inhibit fracture-matrix interaction in the $\mathrm{NF} / \mathrm{AZ}$ to some extent. Accordingly, the response to episodic percolation is likely to be modified by THCM coupled processes.

WP temperature will decline to approximately $65^{\circ} \mathrm{C}$, and $\mathrm{RH}$ at the WP surface will be approximately 95\% (Figure 2-6). Matrix saturation in the NFE will approach preemplacement conditions $(>80 \%)$. Fractures in the NFE will open further in response to relaxation of TM stress. Fracture-lining precipitates in the NF/ AZ will interact with ambient percolation, and the system hydrologic response to THC effects will approach steady state.

Figure 2-6 Schematic of emplacement drift conditions during the cool-down period (for typical conditions expected in the repository block)

The CAM is likely to be at least half oxidized for WPs throughout the repository, and corrosion of the underlying corrosion-resistant material (CRM) will have begun under these conditions (CRWMS M\&O, 1997b). Roughly half of the WPs will have at least one penetration through the CRM. These estimates are based on extrapolation of measured corrosion rates for these materials. Penetration of the CRM will be caused by humid-air pitting processes in the upper part of each WP and by related processes acting externally on the lower part or from the inside of penetrated packages. Within WPs with penetrations, partial failure of internal structural components made from mild steel will occur.

Waste radionuclides will be released to the NFE, where retardation will occur by precipitation, coprecipitation, surface complexation, or ion-exchange reactions. A fraction of released radionuclides will be transported advectively as dissolved or colloidal species. Mixing of the gas phase with atmospheric air will increase during this period, and $\mathrm{CO}_{2}$ will be transported into the NFE.

Where there is little or no seepage into the NFE, advective transport of radionuclides will be minimal. The retardation capacity of materials in the NFE will be limited; thus, the fraction of released inventory that is transported advectively will increase with time.

\subsubsection{Time Period F: Post-Thermal Period (10,000 to 100,000 yr)}

Hydrologic conditions will be dominated by the magnitude and distribution of ambient percolation flux, as modified by the durable effects of THCM coupled processes. Important effects on the NF / AZ hydraulic structure will include fracture-matrix interaction and durable changes to fracture porosity and permeability.

Temperatures at the surface of WPs will approach the ambient, pre-emplacement rock mass temperature $\left(\sim 26^{\circ} \mathrm{C}\right)$. The CAM is likely to be fully oxidized throughout the repository, and at least half of all WPs will have one or more penetrations through the CRM (CRWMS $\mathrm{M} \& \mathrm{O}, 1997 \mathrm{~b})$. The CRM will be substantially intact throughout the repository, and penetrations will be limited to those caused by pitting and crevice corrosion. Failed WPs will suffer complete failure of internal structural components. 
During this period, radionuclides released advectively from failed WPs will produce a plume that will travel to the accessible environment (Figure 2-7). Diffusive transport from within the WPs to the NFE, and within the NFE, will not contribute significantly to this plume.

Figure 2-7 Schematic of emplacement drift conditions during the post-thermal period (for typical conditions expected in the repository block)

\subsection{Uncertainties in the Conceptual Model for Near-Field/Altered-Zone Coupled Processes}

The foregoing discussion has identified a series of stages for the evolution of coupled processes in the NF/AZ. The discussion was referred to a typical set of properties and conditions expected for the repository block. Actual properties and conditions may differ from those described. In particular, the discussion covered the effects of variation in $\mathrm{TH}$ properties, ambient percolation flux, local thermal output, repository ventilation, relative abundance of fracture-lining minerals, and repository edge effects. Key steps in the evolution of coupled processes (e.g., the occurrence of dryout) and the timing of these steps depend critically on combinations of these variables.

In the discussion, several types of uncertainties were identified, including the following:

- Uncertain property values and spatial heterogeneity of properties

- Uncertain temporal variation in boundary conditions

- Uncertain conceptual basis for prediction

In addition, elements of EBS design (e.g., the composition of concrete used in the liner and invert) may change relative to the current reference design. Questions of material property values, boundary conditions, spatial heterogeneity, and design changes are beyond the scope of this report. This report does describe the conceptual and numerical basis for prediction of NF / AZ coupled processes. Some of the fundamental process uncertainties identified in the foregoing discussion include fracture-system capillarity, buoyant convection, reworking of mineral precipitates during cool-down, fracture deformability and permeability coupling, and the nature of condensate drainage pathways.

The conceptual model presented in time periods A through F can be used as the basis for discussion of coupled processes, with acknowledgment that predictions may vary for different parts of the repository, that significant changes in repository design and planned operation may be developed, and that conceptual uncertainties in predictive models exist.

\section{Return to Table of Contents}

\subsection{References}

Bodvarsson, G.S., T.M. Bandurraga, and Y.S. Wu (1997). “The Site-Scale Unsaturated Zone Model of Yucca Mountain, Nevada, for the Viability Assessment." June 1997. (LBNL-40376, UC-814). Berkeley, CA: Lawrence Berkeley National Laboratory, in collaboration with the U.S. Geological Survey. [MOL.19971014.0232]

Carnahan, C.L. (1987). “Effects of Coupled Thermal, Hydrological and Chemical Processes on Nuclide Transport." (LBL-23186). Berkeley, CA: Lawrence Berkeley National Laboratory. [232514] 
CRWMS M\&O (1996). “Total System Performance Assessment-Viability Assessment (TSPAVA) Plan." September 13, 1996. (B00000000-01717-2200-00179). Las Vegas, NV: prepared by the Civilian Radioactive Waste Management System, Management and Operating Contractor, TRW Environmental Safety Systems, Inc. for the U.S. Department of Energy. [MOL.19970320.0078]

CRWMS M\&O (1997a). "Reference Design Description for a Geological Repository." (B00000000-01717-5707-00002, Rev. 1). Civilian Radioactive Waste Management System Management and Operation Contractor: TRW Environmental Safety Systems, Inc. [MOL.19980127.0313]

CRWMS M\&O (1997b). “Year-End Status Report on Design Basis Models.” September 15, 1997. (B00000000-01717-5703-00006). Las Vegas, NV: prepared by the Civilian Radioactive Waste Management System, Management and Operating Contractor, TRW Environmental Safety Systems, Inc. for the U.S. Department of Energy. [MOL.19980127.0257]

DOE (1998). “Repository Safety Strategy: U.S. Department of Energy's Strategy to Protect Public Health and Safety After Closure of a Yucca Mountain Repository, Rev. 1." Washington, DC: U.S. Department of Energy, Office of Civilian Radioactive Waste Management. [MOL. 19980211.0250; MOL. 19980330.0239]

Flint, A., J.A. Hevesi, and L.E. Flint (1996). “Conceptual and Numerical Model of Infiltration for the Yucca Mountain Area, Nevada." Water Resources Investigation Report. (3GUT623M). Denver, CO: U.S. Geological Survey. (U.S.G.S. Survey Milestone Report) [MOL.19970409.0087]

Halsey, W.G. (1994). "What do we mean by a 'cold' repository?" In proceedings from Fifth International High-Level Radioactive Waste Management Conference. Las Vegas, NV: 4:2183-2188. (Also UCRL-JC-116352, for Lawrence Livermore National Laboratory, Livermore, CA) [NNA.19940524.0039]

Hardin, E.L., and D.A. Chesnut (1997). "Synthesis Report on Thermally Driven Coupled Processes." Milestone report for the CRWMS Management and Operating Contractor, U.S. Department of Energy. (SPL8BM3). Livermore, CA: Lawrence Livermore National Laboratory. (Also UCRL-ID-128495, Lawrence Livermore National Laboratory, Livermore, CA) [MOL.19980120.0035]

Harrar, J., J.F. Carley, W.F. Isherwood, and E. Raber (1990). "Report of the Committee to Review the Use of J-13 Well Water in Nevada Nuclear Waste Storage Investigations." (UCRL-ID-21867). Livermore, CA: Lawrence Livermore National Laboratory. [NNA.910131.0274]

Kneafsey, T.J., and K. Pruess (1997). “Preferential Flow Paths and Heat Pipes: Results from Laboratory Experiments on Heat-Driven Flow in Natural and Artificial Rock Fractures." Milestone report for the CRWMS Management and Operating Contractor, U.S. Department of Energy. (SPL6A5M4). Berkeley, CA: Lawrence Berkeley National Laboratory. [MOL.19971204.0415]

Nitao, J.J. (1997a). “Distribution of Post-Emplacement Seepage into the Repository Drifts with Parametric Variation of Intrinsic Properties: Models and Bounds for PostEmplacement Seepage into the Repository." Milestone report for the CRWMS Management and Operating Contractor, U.S. Department of Energy. (SPLC2M4 and SPLC3M4). Livermore, CA: Lawrence Livermore National Laboratory. [MOL.19980107.0400] 
Nitao, J.J. (1997b). “Models for the Distribution of Percolation at the Repository Level and Seepage into Drifts under Pre-Emplacement Conditions." Milestone report for the CRWMS Management and Operating Contractor, U.S. Department of Energy. (SPLB2M4). Livermore, CA: Lawrence Livermore National Laboratory.

NRC (1997). "Issue Resolution Status Report-Key Technical Issue: Thermal Effects on Flow." Washington, DC: Division of Waste Management, Office of Nuclear Material Safety and Safeguards, U.S. Nuclear Regulatory Commission. (Rev. 0) [MOL.19971016.0549]

Tsang, C.-F., J. Birkholzer, G. Li, and Y. Tsang (1997). “Drift Scale Modeling: Progress in Studies of Seepage into a Drift." Milestone report. (SP331CM4). Berkeley, CA: Lawrence Berkeley National Laboratory. [MOL.19971204.0420] 


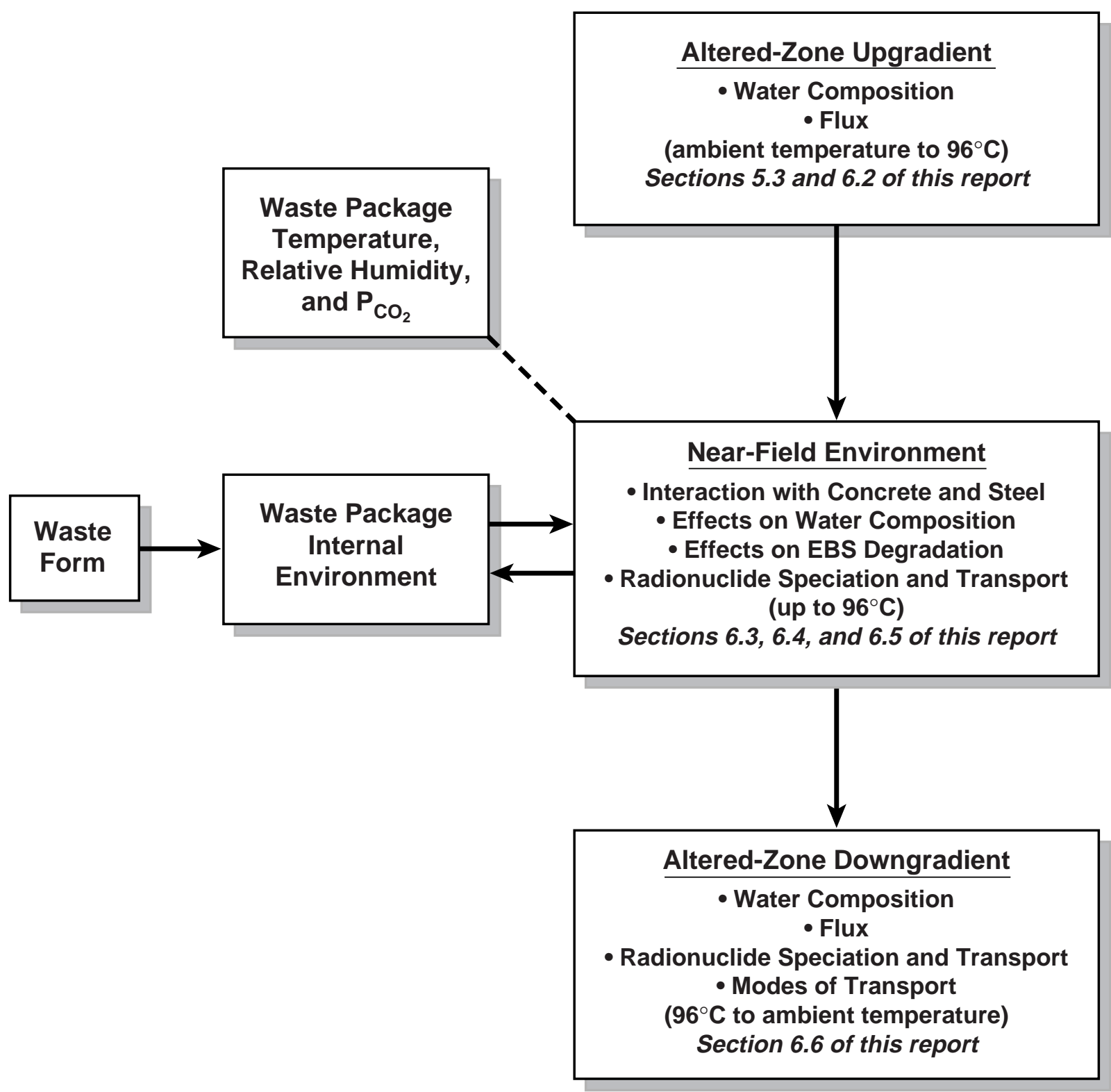

Figure 2-1. Simplified schematic of the conceptual model used in TSPA-VA to represent engineered barrier system (EBS) degradation, radionuclide release, and transport in the near-field environment (annotated with section numbers from this report) 


\section{Time Period A: Drift Closure to Repository Closure}

-WP temperature increases from $70^{\circ}$ to $200^{\circ} \mathrm{C}$

- $\mathrm{RH}$ at WP decreases to $20 \%$

- Matrix saturation decreases to $\sim 50 \%$ or less

- EBS structures, including liner-intact

- No reflux through liner

- CAM-minor oxidation

- Initial development of precipitation cap

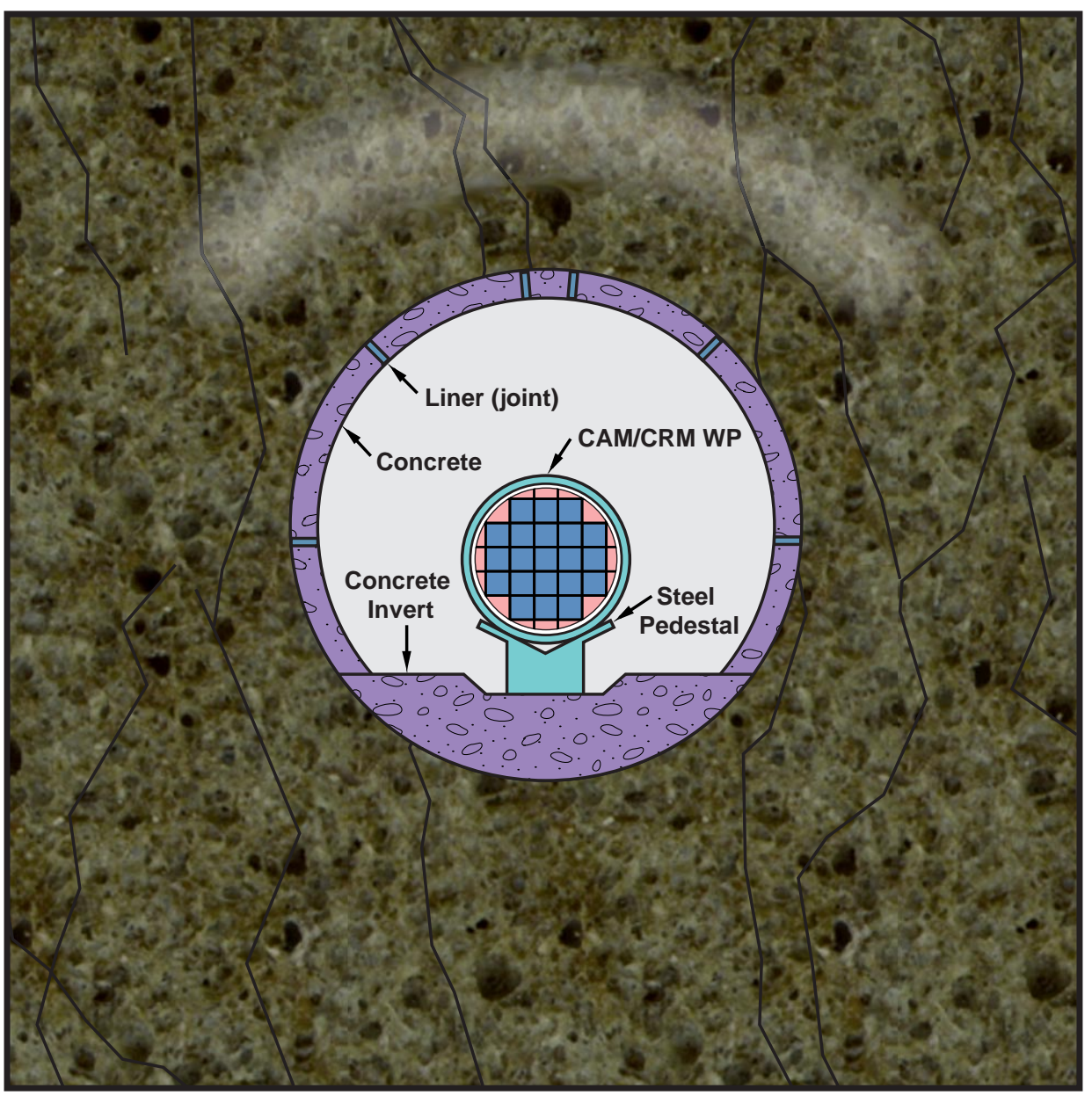

$A E=$ accessible environment

CAM = corrosion-allowance material

CRM = corrosion-resistant material

EBS = engineered barrier system

NFE = near-field environment

$\mathbf{R H}=$ relative humidity

$\mathrm{RN}$ = radionuclide

$\mathrm{TM}=$ thermomechanical

WF $=$ waste frame

WP = waste package

Figure 2-2. Schematic of emplacement drift conditions at repository closure 


\section{Time Period B: Development of the Superheated Dryout Zone (Repository Closure to a Few Hundred Years)}

-WP temperature decreases from $200^{\circ}$ to $150^{\circ} \mathrm{C}$

-WF temperature cools through "unzip" threshold (no backfill)

- RH at WP 25\%

- Matrix saturation $\sim 50 \%$ or less

- Concrete liner-complete mechanical failure by end of period

- Some rock fall

- CAM-minimal corrosion

- TM stress-loading-maximum intensity

- Possible episodic reflux into drifts after liner failure

- Development of precipitation cap

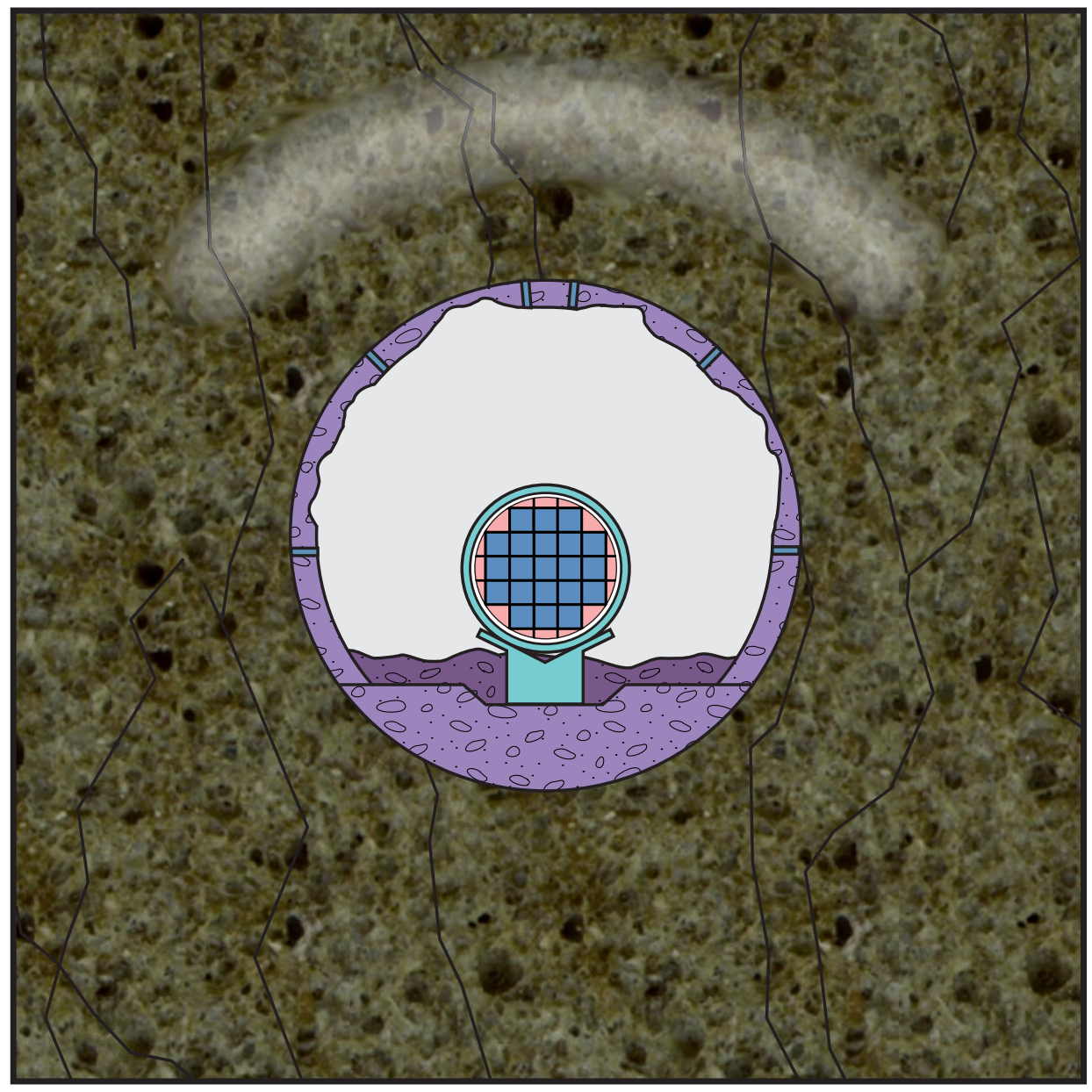

$\mathrm{AE}=$ accessible environment CAM = corrosion-allowance material

CRM = corrosion-resistant material

EBS = engineered barrier system

NFE = near-field environment $\mathbf{R H}=$ relative humidity

$\mathrm{RN}$ = radionuclide $\mathrm{TM}=$ thermomechanical WF = waste frame WP = waste package

Figure 2-3. Schematic of emplacement drift conditions at a few hundred years after repository closure (after dryout zone development) 


\section{Time Period C: Quasi-Stable Reflux Zone (500 to $1500 \mathrm{yr}$ )}

-WP temperature $>100^{\circ} \mathrm{C}$

- Drift-wall temperature $>100^{\circ} \mathrm{C}$

- RH at WP increases to $~ 50 \%$ or more

- Matrix saturation $\sim 50 \%$ or less

- Concrete liner-complete mechanical failure

- Invert-partial mechanical failure

- Pedestal-partial mechanical failure

- More rock fall; partly buries WPs

- CAM-20\% to $50 \%$ oxidized

- TM stress-loading-maximum intensity

- Possible episodic reflux into drifts

- Precipitation cap fully developed

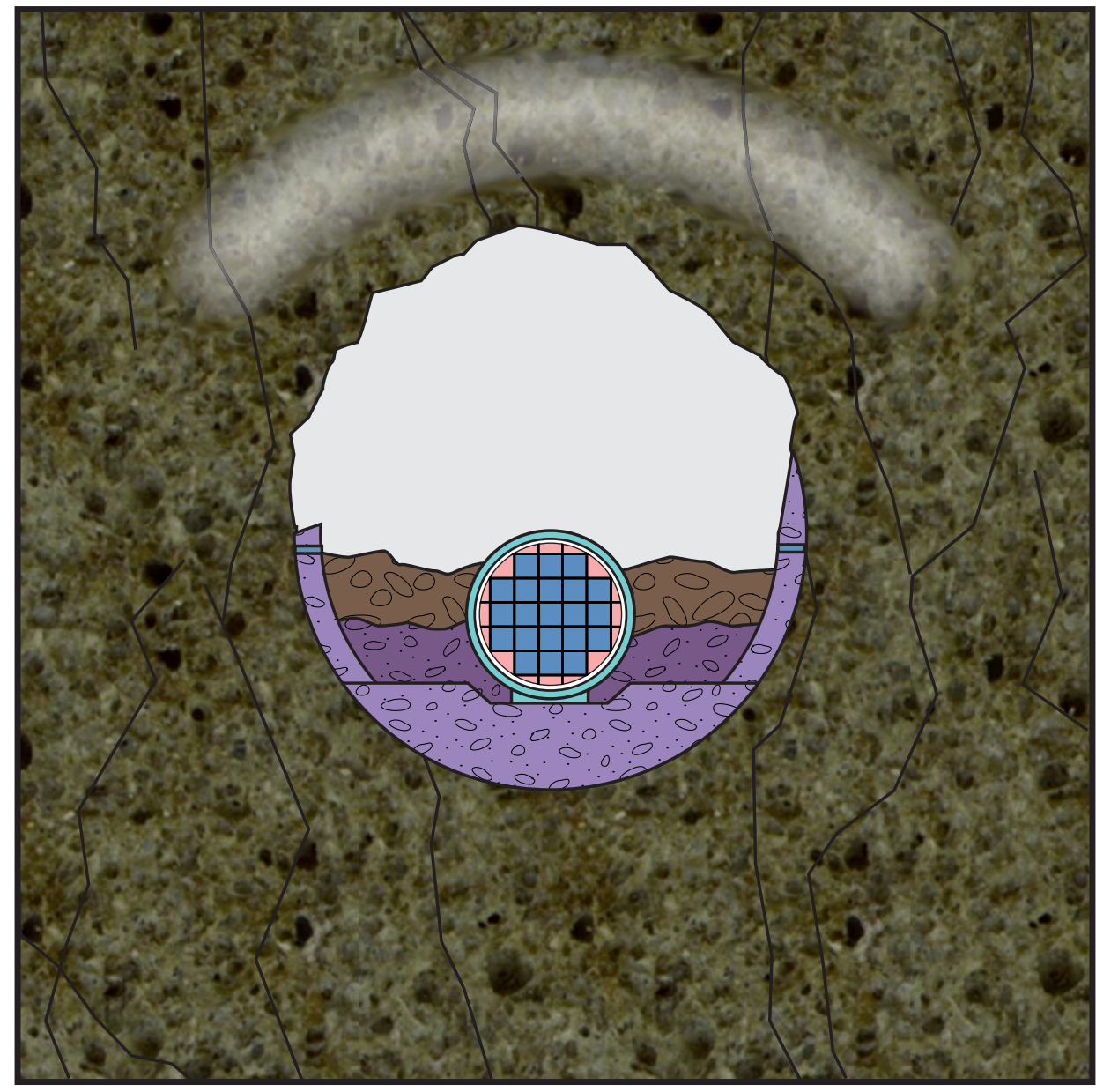

$A E=$ accessible environment CAM = corrosion-allowance material

CRM = corrosion-resistant material

EBS = engineered barrier system

NFE = near-field environment $\mathbf{R H}=$ relative humidity

$\mathrm{RN}=$ radionuclide

$\mathrm{TM}=$ thermomechanical

WF = waste frame

$\mathrm{WP}=$ waste package

Figure 2-4. Schematic of emplacement drift conditions during the period of a quasi-stable reflux zone (for typical conditions expected in the repository block) 
Time Period D: Collapse of the Superheated Dryout Zone (1000 to $3000 \mathrm{yr}$ )

- WP temperature $>96^{\circ} \mathrm{C}$

- Drift-wall temperature $\sim 96^{\circ} \mathrm{C}$ after dryout zone collapse

- RH at WP 95\%

- Matrix saturation $\sim 80 \%$ or more

- Invert-complete mechanical failure

- Pedestal-complete mechanical failure (WPs rest on cementitious material)

- CAM $>20 \%$ oxidized

- TM stress-loading-decreases from maximum intensity

- Solute-concentrative THC activity as dryout zone collapses

- Episodic reflux into drifts

- Precipitation cap stable

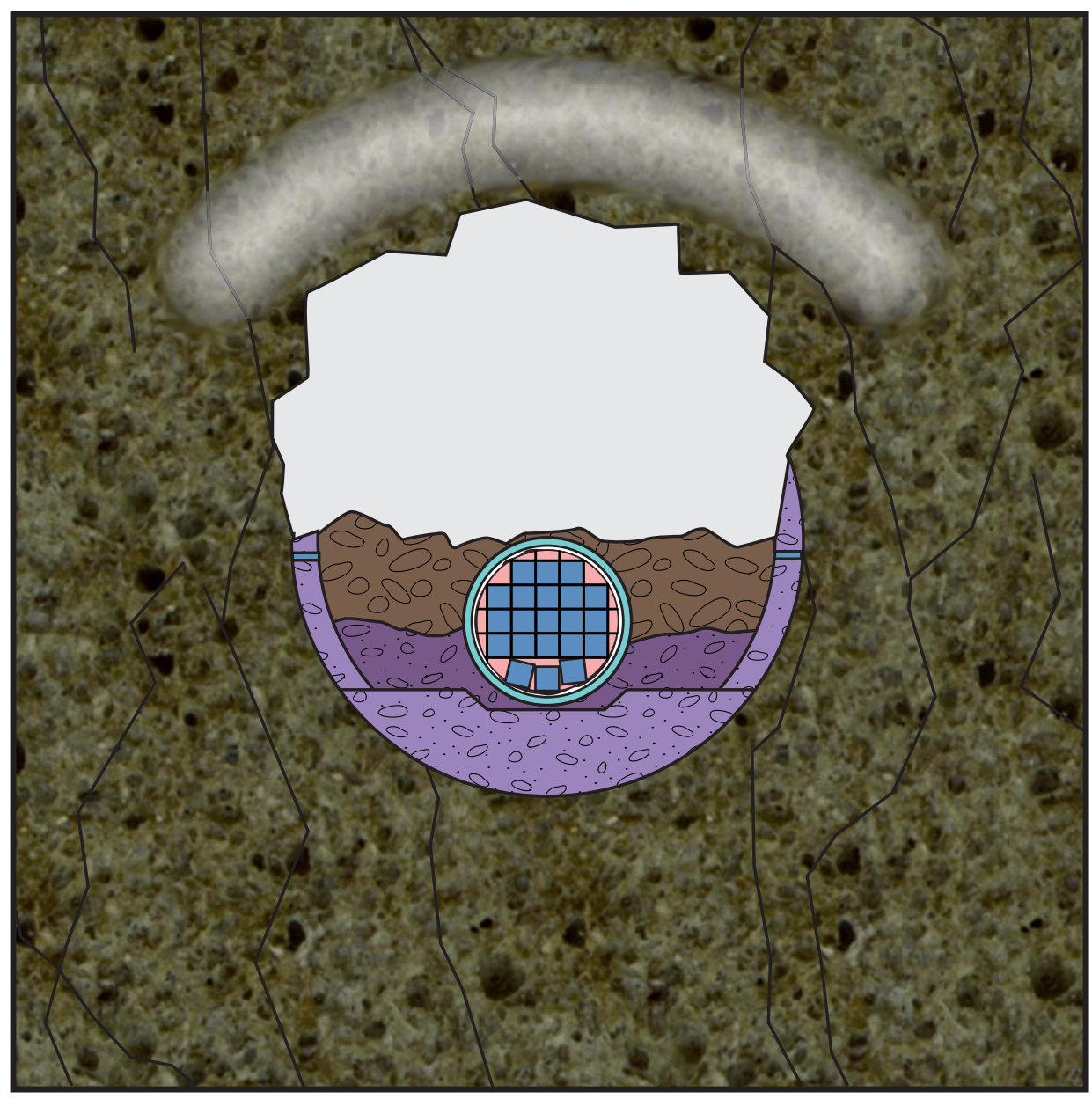

$\mathrm{AE}=$ accessible environment

CAM = corrosion-allowance material

CRM = corrosion-resistant material

EBS = engineered barrier system

NFE = near-field environment

$\mathbf{R H}=$ relative humidity

$\mathrm{RN}$ = radionuclide

TM = thermomechanical

WF = waste frame

WP = waste package

Figure 2-5. Schematic of emplacement drift conditions during collapse of the superheated dryout zone (for typical conditions expected in the repository block) 
Time Period E: Cool-down

(3000 to $10,000 \mathrm{yr}$ )

-WP temperature $\sim 65^{\circ} \mathrm{C}$

- Drift-wall temperature $<65^{\circ} \mathrm{C}$

- RH at WP 95\%

- Matrix saturation $>80 \%$

- CAM $50 \%$ oxidized

- CRM corrosion begins, and $~ 50 \%$ of WPs have $\geq 1$ CRM penetration

- Within failed WPs, partial failure of internal structural components occurs

- TM stress released-fractures reopen

- Episodic, ambient seepage into drifts

- Waste radionuclides are released to the NFE

- Slight dissolution of precipitation cap

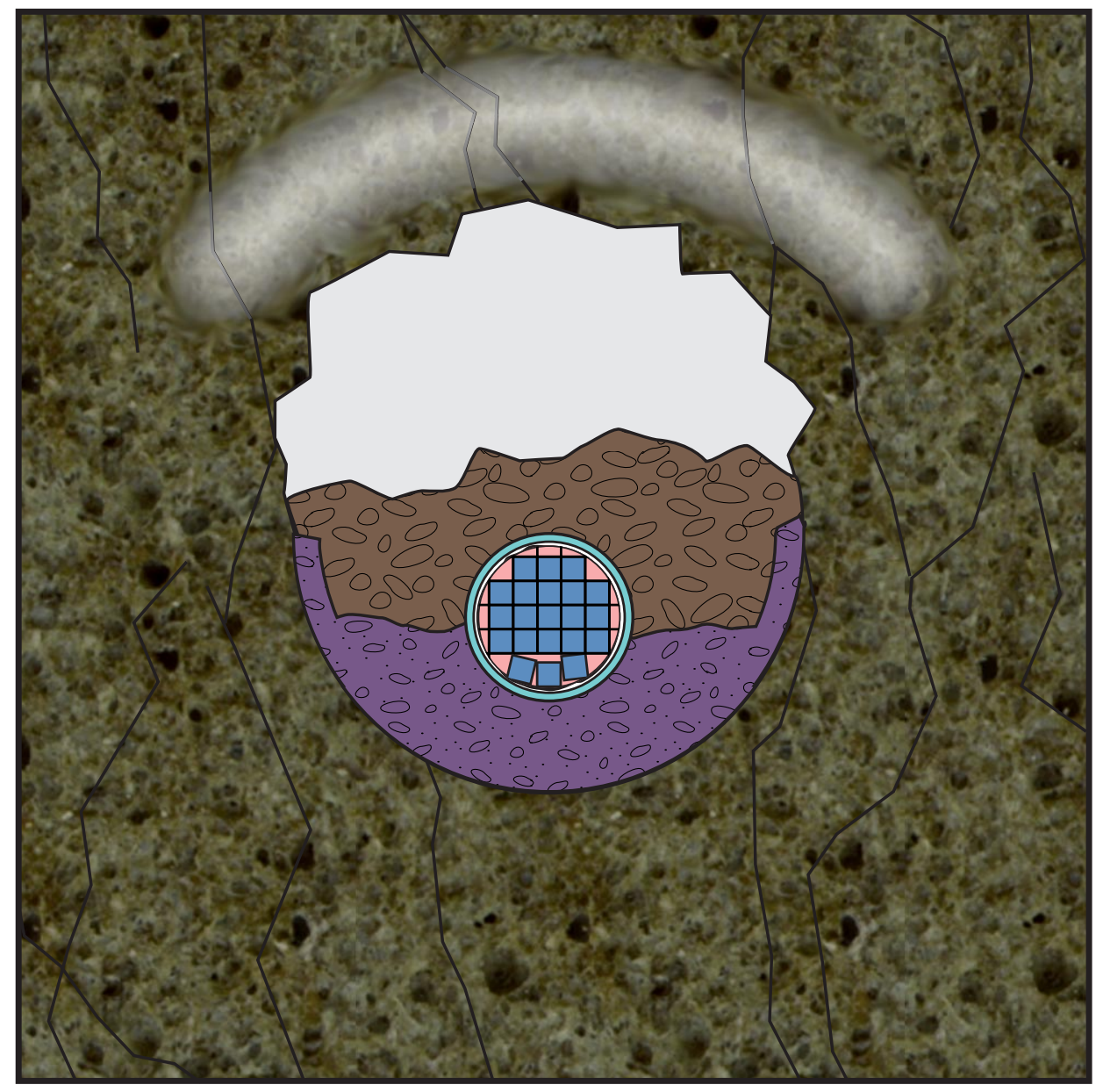

AE = accessible environment

CAM = corrosion-allowance material

CRM = corrosion-resistant material

EBS = engineered barrier system

NFE = near-field environment

$\mathbf{R H}=$ relative humidity

$\mathrm{RN}$ = radionuclide

TM = thermomechanical

WF = waste frame

WP = waste package

Figure 2-6. Schematic of emplacement drift conditions during the cool-down period (for typical conditions expected in the repository block) 
Time Period F: Post-Thermal Period $(10,000$ to $100,000 \mathrm{yr})$

-WP and drift wall approach ambient temperature

- RH and matrix saturation approach pre-emplacement conditions

- CAM $100 \%$ oxidized

- CRM mostly intact, with penetrations from pitting/crevice corrosion

- Complete failure of WP internal structural components

- Episodic, ambient seepage into drifts

- Waste RNs released to the NFE

- Advective releases produce plume to the AE

- Dose standard met at the AE

- Partial dissolution of precipitation cap

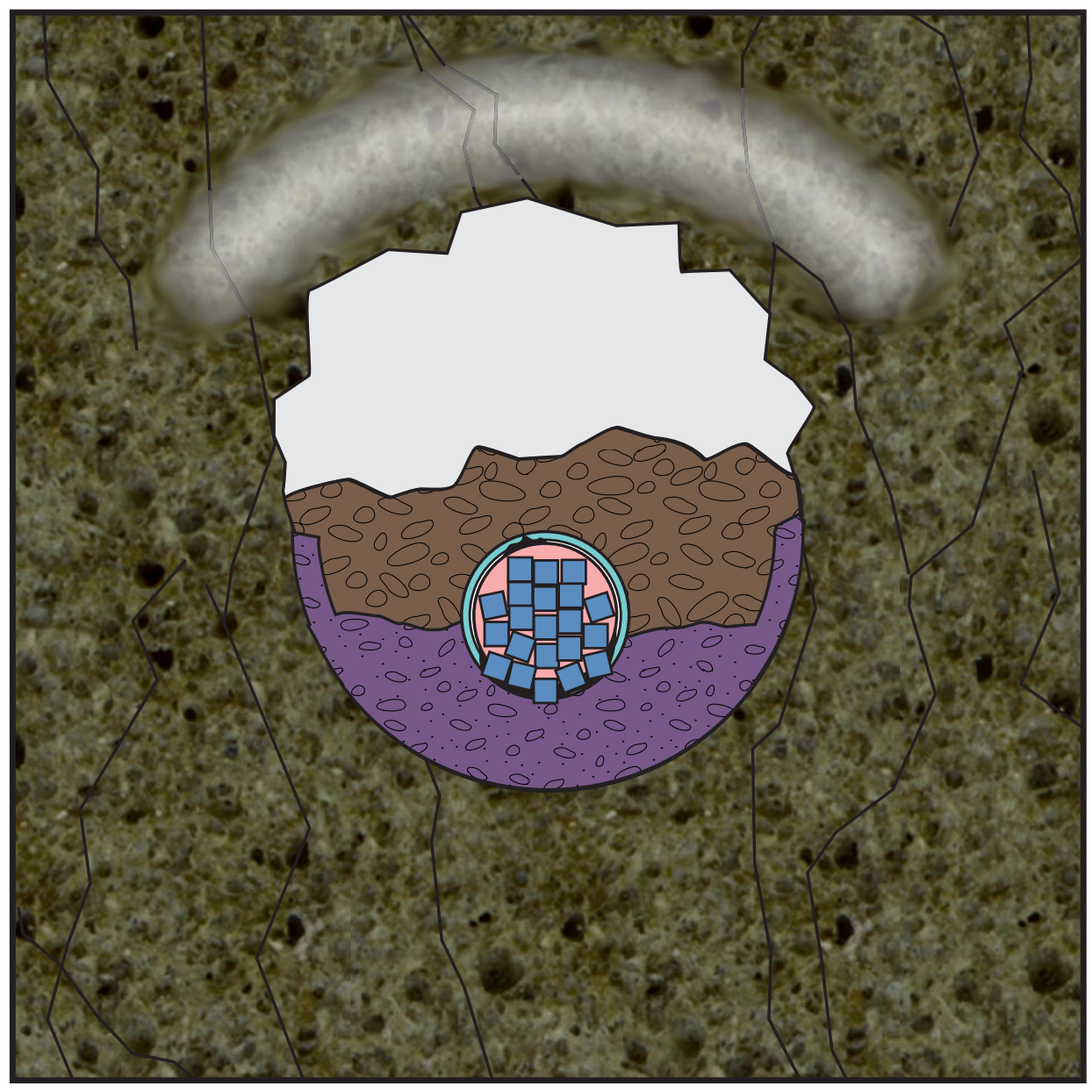

$\mathrm{AE}=$ accessible environment

CAM = corrosion-allowance material

CRM = corrosion-resistant material

EBS = engineered barrier system

NFE = near-field environment

$\mathbf{R H}=$ relative humidity

$\mathrm{RN}$ = radionuclide

$\mathrm{TM}=$ thermomechanical

WF = waste frame

WP = waste package

Figure 2-7. Schematic of emplacement drift conditions during the post-thermal period (for typical conditions expected in the repository block) 



\section{Thermohydrologic Models}

\section{by Thomas A. Buscheck}

Radioactive decay of high-level nuclear waste emplaced in a Yucca Mountain repository will produce an initial heat flux on the order of 50 times the heat flux in The Geysers geothermal reservoir in California. Even though the rate of heat production decreases rapidly with time after emplacement, thermal processes will dominate the distribution and movement of air, water vapor, and liquid water in the unsaturated zone (UZ) for hundreds to thousands of years after closure of the repository. The responses of the system to this heat load determine the near-field environment (NFE), or temperature, relative humidity $(R H)$, and the quantity and quality of liquid water present at different locations within the engineered barrier system (EBS), and also will produce changes in a large altered zone (AZ) of unexcavated rock surrounding the repository. Prediction of these responses requires thermohydrologic $(\mathrm{TH})$ models to analyze the simultaneous movement of heat and fluids in permeable media. TH models are inherently more complex than the conventional hydrologic models used for isothermal systems and require different kinds of experiments and data for their testing, development, and application.

This chapter describes a large number of models developed to predict changes, as functions of time after emplacement, in environmental conditions near the waste packages (WPs) and in the surrounding host rock.

\subsection{Impact of Thermohydrology on the Proposed Yucca Mountain Repository}

Although minor amounts of radioactivity may be released and transported in the vapor phase, the bulk of the radionuclide dose will be borne in aqueous solution or colloidal suspension. Hence, liquid water must contact waste before a breached package contributes any significant quantity of radionuclides. Models are required to estimate the time of first water contact and the temporal and spatial distribution of liquid flux.

The movement of water, driven by thermal processes and ambient percolation, controls both the WP failure time distribution and the rate of radionuclide release from failed WPs. Accordingly, TH modeling is a key element in making a credible total system performance assessment (TSPA), for viability assessment (VA), and for license application.

\subsubsection{Effects of Design Alternatives}

The project has selected a reference design for the repository, specifying the number and types of spent-fuel assemblies contained in each type of WP, the layout of the emplacement drifts, and the spacing of WPs along drifts. These three design features, along with the characteristics of the waste (type of reactor, burn-up, time out of core, etc.), determine the amount of heat released into the repository after emplacement of waste. When combined with a strategy for local "tailoring" of the heat load by controlling the placement of packages with different initial thermal power output, these design and operating variables determine the spatial and temporal distribution of heat sources driving thermally coupled phenomena in the surrounding host rock. Currently, the reference design envisions a drift spacing of $28 \mathrm{~m}$ and spacing along drifts between packages of comparable magnitude. This is called the "point-load" design. The spatially averaged time integral of heat output is closely related to the mass-loading density of commercial spent nuclear fuel, expressed as metric tons of uranium (MTU) per unit area (usually MTU per acre of the repository footprint). The reference design is based on $85 \mathrm{MTU} /$ ac. The reference design does not include backfilling the drifts at closure, but this option is to be evaluated as one of several design alternatives. 
The VA, including TSPA, will focus on the reference design. However, preliminary analyses of alternatives also will be conducted. One important option affecting the TH behavior of the system is the "line-load" design. In this design, packages are placed as close together within drifts as practical, with drift spacing adjusted to keep the mass-loading density constant. Also being considered is the addition of backfill, with either the point-load or the line-load design. Backfill will produce significant TH effects. Other design alternatives are actively being pursued by the project to select the most promising ones for possible use in the license application. Credible TH models will play a key role in performance assessment of design and operating alternatives and in the ultimate success or failure of licensing a repository at Yucca Mountain.

\subsubsection{Identification of Users for Thermohydrologic Models}

This chapter describes three types of TH models:

1. The drift-scale TH model

2. The drift-scale isothermal and nonisothermal seepage model

3. The multi-scale TH abstraction methodology for estimating performance measures for performance assessment (PA)

These models are listed in Appendix A (Table A-9) along with the other models that make up this report.

The drift-scale TH model is a basic tool for repository performance analysis and is used for PA and design. A set of two-dimensional (2-D) drift-scale calculations is presented for illustrating and evaluating the effects of coupled processes in this report (Section 3.5). The model is readily extended to a three-dimensional (3-D) version using the equivalentcontinuum or dual-permeability conceptual models. Temperature fields calculated from driftscale TH models are used as the basis for simulating thermomechanical (TM) effects for both PA and EBS Design (see Chapter 4).

The drift-scale isothermal and nonisothermal seepage model has been demonstrated in 2-D and 3-D calculations (Section 3.6). This model is used for PA to estimate the proportion of the ambient percolation flux that enters drift openings as seepage. The model also forms the basis for seepage estimates that will be used to evaluate the function of engineered barriers under consideration in repository design.

The multi-scale TH abstraction methodology is used by PA to estimate performance measures such as WP temperature and drift air $R H$ as a function of location in the repository block, thermal loading, infiltration flux, and other factors. The methodology combines full $\mathrm{TH}$ simulations with heat conduction-only models to estimate results that would be obtained from very large, finely gridded, 3-D TH models for which the computational effort would be prohibitive.

\subsection{Qualitative Description of Unsaturated Zone Conditions, Processes, and Mechanisms}

The potential repository is located in the UZ, approximately midway between the ground surface and the water table. Both the saturated and unsaturated zones comprise a sequence of variably fractured, densely welded to nonwelded volcanic tuffs (see Table 3-1 for a description of the major hydrostratigraphic units underlying Yucca Mountain). In general, the more densely welded units are the more highly fractured. Matrix porosity in the welded units typically ranges from about $10 \%$ to about $20 \%$, whereas matrix porosity in some nonwelded units exceeds $40 \%$. Fracture porosity is poorly known, but believed to be much less than $1 \%$. Under existing conditions, matrix pores are partially filled with liquid water; 
the rest of the matrix pore volume contains "rock gas" (predominantly air) and water vapor. The potential repository horizon is within the Topopah Springs welded unit, with about 10\% matrix porosity and $90 \%$ water saturation.

The welded tuffs at Yucca Mountain have characteristics similar to those of fractured petroleum and geothermal reservoirs, with high storage capacity and low permeability in the matrix and with very low storage capacity and high permeability in a network of connected fractures. Hence, most of the total fluid-storage capacity is contained in the matrix pores, but most of the bulk permeability is the result of fractures. A major problem in modeling such systems is to properly account for the properties of these interpenetrating continua, including the transfer of mass and energy between them, while retaining the ability to perform simulations within the limitations of computing resources. This problem will be discussed more fully in a later section of this chapter. With the possible exception of the nonwelded units, which are more permeable and behave like classical porous media, little movement of water, air, and water vapor will occur in the absence of connected fractures. Fractures are the primary conduits for large-scale transport of water, air, and water vapor in the repository host-rock units.

Table 3-1 Relations among model hydrogeologic units and geologic formations, geologic framework model (DTN LB970601233129.001)

\begin{tabular}{|c|c|c|c|}
\hline Geological Unit & $\begin{array}{c}\text { Welding } \\
\text { Intensity/Formation } \\
\text { Name } \\
\text { (Buesch et al., 1995) }\end{array}$ & $\begin{array}{l}\text { Model Layer } \\
\text { Name }\end{array}$ & $\begin{array}{c}\text { Hydrogeologic } \\
\text { Unit }\end{array}$ \\
\hline \multicolumn{4}{|c|}{ Paintbrush Group } \\
\hline \multirow[t]{3}{*}{ Tiva Canyon Tuff } & $\begin{array}{l}\text { M,D' (Tpcpll) } \\
\text { M,D' (Tpcpln) }\end{array}$ & $\begin{array}{l}\text { tcw11 } \\
\text { tcw12 }\end{array}$ & \multirow[t]{2}{*}{ Tiva Canyon (TCw) } \\
\hline & $\begin{array}{l}\text { D-Basal vitrophyre (Tpcpv3) } \\
\text { M (Tpcpv2) }\end{array}$ & tcw13 & \\
\hline & N,P (Tpcpv1) & & \multirow{7}{*}{ Paintbrush (PTn) } \\
\hline Bedded Tuff & N (Tpbt4) & ptn21 & \\
\hline Yucca Mountain Tuff & $\mathrm{N}, \mathrm{P}, \mathrm{M}$ (Tpy) & ptn22 & \\
\hline Bedded Tuff & $\mathrm{N}($ Tpbt3) & ptn23 & \\
\hline \multirow[t]{2}{*}{ Pah Canyon Tuff } & $\mathrm{N}, \mathrm{P}, \mathrm{M}(\mathrm{Tpp})$ & ptn24 & \\
\hline & $\mathrm{N}$ (Tpbt2) & ptn25 & \\
\hline Bedded Tuff & $\mathrm{N}, \mathrm{P}$ (Tptrv3) & & \\
\hline \multirow[t]{7}{*}{ Topopah Spring Tuff } & $\begin{array}{l}\text { M (Tptrv2) } \\
\text { D-Upper vitrophyre (Tptrv1) }\end{array}$ & tsw31 & \multirow[t]{7}{*}{ Topopah Spring (TSw) } \\
\hline & M,D (Tptrn) & tsw32 & \\
\hline & $\begin{array}{l}\text { M,D, } L^{2} \text { (Tptrl) } \\
\text { M,D,L (Tptpul) }\end{array}$ & tsw33 & \\
\hline & D (Tptpmn) & tsw34 & \\
\hline & M,D,L (Tptpll) & tsw35 & \\
\hline & D (Tptpln) & tsw36 & \\
\hline & D-Basal vitrophyre (Tptpv3) & tsw37 & \\
\hline
\end{tabular}




\begin{tabular}{|c|c|c|c|}
\hline \multirow[t]{2}{*}{ Geological Unit } & $\begin{array}{l}\text { Welding } \\
\text { Intensity/Formation } \\
\text { Name } \\
\text { (Buesch et al., 1995) }\end{array}$ & $\begin{array}{l}\text { Model Layer } \\
\quad \text { Name }\end{array}$ & $\begin{array}{l}\text { Hydrogeologic } \\
\text { Unit }\end{array}$ \\
\hline & $\begin{array}{l}\text { N,P,M; may be altered } \\
\text { (Tptpv1, Tptpv2) }\end{array}$ & $\operatorname{ch} 1(\mathrm{vc}$ or $\mathrm{zc})$ & \multirow[t]{9}{*}{ Calico Hills (CHn) } \\
\hline Bedded Tuff & $\mathrm{N}$; may be altered (Tpbt1) & & \\
\hline \multirow[t]{3}{*}{ Calico Hills Formation } & $\mathrm{N}$; unaltered (Tac-vitric) & ch2(vc or zc) & \\
\hline & N; altered (Tac-zeolitic) & $\mathrm{ch} 3(\mathrm{vc}$ or zc) & \\
\hline & $\mathrm{N}$; may be altered (Thtbt) & $\operatorname{ch} 4(\mathrm{vc}$ or $\mathrm{zc})$ & \\
\hline \multicolumn{2}{|c|}{ Crater Flat Group } & & \\
\hline \multirow[t]{3}{*}{ Prow Pass Tuff } & $\begin{array}{l}\mathrm{N} ; \text { may be altered }(\mathrm{T} c \mathrm{p}) \text { Unit } \\
43\end{array}$ & & \\
\hline & P,M Unit 3 & pp3vp & \\
\hline & $\begin{array}{l}\mathrm{N}, \mathrm{P} ; \text { generally altered Units } \\
2,1 \\
\mathrm{~N} ; \text { generally altered (Tcpbt) }\end{array}$ & pp2zp & \\
\hline \multicolumn{2}{|c|}{ Crater Flat Group } & & \\
\hline Upper Bullfrog Tuff & $\mathrm{N}, \mathrm{P} ;$ generally altered $(\mathrm{Tcb})$ & & \multirow{5}{*}{$\begin{array}{l}\text { Crater Flat } \\
\text { Undifferentiated (CFu) }\end{array}$} \\
\hline Middle Bullfrog Tuff & $\mathrm{P}, \mathrm{M}$ & bf3vp & \\
\hline $\begin{array}{l}\text { Lower Bullfrog Tuff } \\
\text { Bedded Tuff } \\
\text { Upper Tram Tuff }\end{array}$ & $\begin{array}{l}\mathrm{N}, \mathrm{P} ; \text { generally altered } \\
\mathrm{N} \text {; generally altered (Tcbbt) } \\
\mathrm{N}, \mathrm{P} ; \text { generally altered }(\mathrm{Tct})\end{array}$ & bf2zp & \\
\hline \multirow[t]{2}{*}{ Older tuffs and lavas } & \multirow[t]{2}{*}{ Generally altered (Tct) } & $\operatorname{tr} 3 z p$ & \\
\hline & & $\operatorname{tr} 2 z p$ & \\
\hline \multicolumn{4}{|c|}{$\begin{array}{l}{ }^{1} \text { Welding Intensity } N=\text { Non } \mathrm{P}=\text { Partially; } \mathrm{M}=\text { Moderately; } \mathrm{D}=\text { Densely } \\
{ }^{2} \mathrm{~L}=\text { Lithophysal Zone } \\
{ }^{3} \text { Units per Moyer and Geslin }\end{array}$} \\
\hline
\end{tabular}

\subsubsection{Ambient System}

The following description of the site hydrostratigraphy, topography, and repository geometry is drawn from the UZ site-scale model (Bodvarsson et al., 1997). Both liquid and gas phases flow in the UZ at Yucca Mountain. Depending on the conditions, both phases may flow through fractures and through rock matrix. As noted previously, liquid water saturation at the repository horizon is approximately $90 \%$, and the gas phase, comprising $98.5 \%$ air and $1.5 \%$ water vapor (by mass), fills the remaining $10 \%$ of the matrix pore space. The repository host-rock units (Tptpmn, Tptpll, and Tptplnl in Table 3-1) are among the most highly fractured hydrostratigraphic units in the UZ at Yucca Mountain. Consequently, they have bulk permeability values, $k_{\mathrm{b}}$, on the order of $10^{6}$ times the matrix permeability, $k_{\mathrm{m}}$. In this and subsequent discussion, bulk permeability means the permeability of a volume of rock large enough to contain a "representative" connected fracture network-at least a few meters in each dimension. Matrix permeability is typically measured on a small, unfractured sample and is generally much less than $k_{\mathrm{b}}$. This is significant because, without fractures, the rock throughout most of the UZ (including the repository horizon) would be nearly impermeable. 
Figure 3-1a shows the depth of the repository horizon below the ground surface. The summit of Yucca Mountain is parallel to (and approximately one-fourth of the way from) the western edge of the repository. Because the repository is close to being horizontal (with a northward dip of about $1.6 \%$ ), the repository depth contours correspond to the surface topography. The approximate depth to the water table is about $350 \mathrm{~m}$ more than the depth to the repository. The ground surface slopes steeply downward to the west of the summit and less steeply to the east of the summit. The depression in the northeast corner of the repository corresponds to drill-hole wash. The repository depth plays an important role in the thermal evolution of Yucca Mountain after the emplacement of heat-producing high-level nuclear waste.

\section{Figure 3-1 Surface topography, shallow infiltration, repository footprint, and model domain for site-scale thermal modeling at Yucca Mountain}

As outlined previously, the amount of water contacting waste is the principal variable controlling the radionuclide source term. Under ambient conditions, water infiltrates at the ground surface and percolates more-or-less vertically downward to the water table, with some degree of lateral diversion and the occasional occurrence of perched or semi-perched aquifers. Infiltration occurs episodically, mostly during wetter winters associated with El Niño events, based on interpretation of shallow neutron log data by Flint and Hevesi (1996a). According to their analysis, infiltration occurs only where the depth of alluvium is less than $2 \mathrm{~m}$ on top of fractured bedrock.

Figure $3-1 b$ is a contour map of the infiltration-flux $q_{\text {inf }}$ distribution over the repository area at Yucca Mountain based on the work of Flint and Hevesi (1996a). The boundary of the repository area shown in Figure 3-1b is approximated as a rectangle, which was used in the thermal and TH modeling described in later sections of this chapter. The actual repository area shown in Figure 3-1c deviates slightly from this idealized rectangle along the northern, western, and southern edges of the repository. This figure also shows an array of 5 dots E-W by 7 dots N-S, which represents the locations of 1-D and 2-D submodels described subsequently. These locations were chosen to sample the variability in depth to the repository from the ground surface and in the spatial variation in infiltration flux.

Infiltration flux varies from 0 to $26 \mathrm{~mm} / \mathrm{yr}$ over the repository area (Figure 3-1b). There is an apparent correlation between repository depth (directly related to ground-surface elevation) and $q_{\text {inf }}$ is higher when the depth is greater (i.e., at higher ground-surface elevation). The largest infiltration rates occur along the crest of Yucca Mountain, and the lowest rates occur along the flanks. In the absence of significant lateral diversion, percolation at the repository level would follow the same pattern. This distribution is governed more by the distribution of alluvium over the repository footprint than by the elevation itself: alluvial cover is generally thickest in washes and down the lower flanks of the mountain and thinnest near the crest. Areas of thicker alluvium, such as Drill-Hole Wash, receive little infiltration into the bedrock; areas with minimal alluvial cover and exposed fractured bedrock, such as the summit, receive more.

Figure $3-1 \mathrm{~d}$ is a histogram of the $q_{\text {inf }}$ distribution over the repository area. The $q_{\text {inf }}$ distribution is an important parameter in determining the TH response of the mountain. Nearly $25 \%$ of the repository area has $q_{\text {inf }}<2 \mathrm{~mm} / \mathrm{yr}$, whereas $25 \%$ of the area has a $q_{\text {inf }}>14$ $\mathrm{mm} / \mathrm{yr}$ (Figure 3-1d). Over the entire repository area, the mean infiltration flux is $7.8 \mathrm{~mm} / \mathrm{yr}$. Note that this flux distribution is an average for the present-day climate conditions; large year-to-year fluctuations about this average are expected. A shift in the average value to much higher values is believed possible under some future climate scenarios. 
Modeling and analytic studies have demonstrated the importance of nonequilibrium flow processes between the fractures and the matrix in the UZ (Buscheck et al., 1991; Nitao et al., 1993; Pruess and Tsang, 1994; Pruess et al., 1990). Except within a perched water body or during periods of transient infiltration, capillary forces cause most fractures to be drained of liquid water in the UZ. Because the matrix permeability is extremely low, matrix flow is much less likely than fracture flow to seep into emplacement drifts and contact WPs. The shallow infiltration of rainfall and snowmelt not removed from the evapotranspiration zone by gas-phase advection and diffusion to the atmosphere determines the net infiltration flux, which is also called percolation or recharge flux. Liquid-phase flow occurs in fractures when the net infiltration (or percolation) flux results in a combination of the following conditions:

- Steady-state percolation for which the liquid-phase drainage flux exceeds the hydraulic conductivity of the rock matrix, a condition not likely to occur at Yucca Mountain

- Episodic percolation events during which liquid-phase flow in the fracture is not in capillary-pressure equilibrium with the adjoining rock matrix, as may happen during periods of intense precipitation

- Lateral flow in perched water bodies, a condition that probably results when either steady-state or episodic percolation in fractures intersects a flow barrier such as an unfractured, low-permeability medium

The distribution of percolation flux in fractures is spatially heterogeneous as well as temporally variable.

Capillary pressure (also called matric or suction potential) in the UZ varies with height above the water table and with the local recharge flux. Under static gravity-capillary equilibrium (i.e., zero recharge flux), the suction potential at any point is determined by its height above the water table. For example, the gravity-capillary equilibrium potential $500 \mathrm{~m}$ above the water table is -50 bars, approximately. It increases in magnitude (becomes more negative) by approximately 10 bars for each $100 \mathrm{~m}$.

Near-zero values of gas- and liquid-phase flux through the UZ cause the suction potential to deviate from gravity-capillary equilibrium. At zero liquid recharge flux, the net transport of water vapor out of the UZ (by gas-phase advection and diffusion) will reduce $S_{\text {liq }}$ in the rock matrix, increasing the magnitude of suction potential. Under ambient conditions, advective drying may occur as a result of barometric pumping. Advective drying can also occur when decay heat from WPs drives a sufficiently large buoyant gas-phase convective flux.

Incoming liquid-phase flux will decrease the magnitude of suction potential as a result of any combination of the following conditions:

- An increase in steady-state percolation increases the $S_{\text {liq }}$ and correspondingly decreases the suction potential. Higher liquid permeability is required to accommodate an increase in flux.

- Episodic nonequilibrium fracture-flow percolation events cause imbibition in the adjoining rock matrix, increasing $S_{\text {liq }}$ and consequently decreasing the suction potential. The water in the matrix in excess of the gravity-capillary equilibrium level must eventually drain to the water table or be removed from the mountain by gasphase advection and diffusion.

- Perched water bodies decrease the effective height above the water table for overlying locations in the UZ.

Recent measurements indicate higher values of $S_{\text {liq }}$ in the repository host rock than previously estimated. Samples from the Single-Heater Test (SHT) in the Exploratory Studies Facility (ESF) indicate a $S_{\text {liq }}$ range of $80.5 \%$ to $99.0 \%$, which compares with values of 
$85 \% \pm 12 \%$ for the same interval from surface boreholes (CRWMS M\&O, 1996a). LLNL's version (Buscheck et al., 1997a) of the LBNL site-scale model (Bodvarsson and Bandurraga, 1996) yields $S_{\text {liq }}$ ranging from $88 \%$ to $96 \%$, with an average of $90.5 \%$. These recent measurements of higher $S_{\text {liq }}$ are consistent with measurements of suction potential in the UZ. Suction potentials are less (in some locations, much less) than previously anticipated (Bodvarsson and Bandurraga, 1996).

\subsubsection{Thermally Disturbed System}

Under ambient conditions, liquid-phase fracture flow arises as a result of the percolation of rainfall and snowmelt. After the emplacement of waste, the heat of radioactive decay will change the spatial distribution of in situ pore fluids (air, water, and water vapor) within a large volume of rock, extending from the ground surface to some distance below the water table, over an area larger than the repository footprint. Fluid redistribution and other important features of the thermally disturbed system are illustrated schematically in Figure 3-2 at the drift scale and in Figure 3-3 at the mountain scale.

Figure 3-2 Drift-scale schematic showing decay-heat-driven TH flow and transport processes

Figure 3-3 Mountain-scale schematic showing decay-heat-driven TH flow and transport processes that influence moisture redistribution and the moisture balance in the UZ

This thermally driven transport of water and water vapor away from the heat source (TH behavior) causes a redistribution of the pore fluids, including the following:

- Dryout zones with liquid saturation $S_{\text {liq }}$ decreased from its initial value are created around the emplacement drifts. $R H$ in dryout zones is also reduced, and, because the matric potential is mathematically related to $R H$ via the Kelvin equation, the capillary suction potential will be much greater than ambient. The vertical extent of the dryout zone increases with local decay-heat flux, distance from the repository edge, and decreasing percolation flux $q_{\text {perc }}$. For locations away from the repository edge and $q_{\text {perc }}$ less than $5 \mathrm{~mm} / \mathrm{yr}$, the vertical extent of dryout increases with repository depth below the ground surface.

- Condensation zones within $S_{\text {liq }}$ increase from its initial value are created above and below the dryout zones.

- A region of reduced air mass fraction in the vapor phase is created. The generation of steam replaces air within the boiling zone, reducing the vapor-phase mass fraction of air, $X_{\text {air,gas }}$ almost to zero. This reduction will occur as long as boiling liquid water is present, whether or not rock dryout occurs.

Decay heat flows away from the drifts by conductive and advective (also called convective) heat-transfer processes. For heat convection, latent-heat transport is much more important than sensible-heat transport; consequently, the flow of water vapor results in much greater heat transport than does liquid-phase flow. The following are the two primary mechanisms for gas-phase, advective heat transfer:

- Heat pipes result from countercurrent vapor (away from the heat source) and condensate flow (back towards the heat source). Because this mechanism requires gravity-driven condensate flow, it will primarily occur in zones with well-connected vertical fractures. The location of heat pipes is strongly affected by ambient 
percolation flux $q_{\text {perc }}$ high $q_{\text {perc }}(>10 \mathrm{~mm} /$ yr) suppresses rock dryout and increases the tendency for heat pipes to form in the vicinity of drifts; low $q_{\text {perc }}(<5 \mathrm{~mm} / \mathrm{yr})$ favors rock dryout and suppresses heat-pipe development near the drifts.

- Buoyant gas-phase convection results from mass-density gradients driven by temperature gradients in the rock mass. This mechanism is significant if the rock-mass bulk permeability $k_{\mathrm{b}}>1-10$ darcy and the fractures are ubiquitous and well-connected over large distances. This mechanism, which may occur at the drift and at mountain scales, can increase the buildup of condensate above the repository.

Heat flow in the rock causes a temperature buildup in the near field and AZ as well as in the EBS. The temperature buildup depends strongly on the thermal-loading conditions imposed by the emplacement of WPs. The overall repository thermal-loading conditions are best quantified using the areal mass loading (AML), expressed in MTU/acre. Details of the heat output from individual WPs strongly influence near-field TH behavior, particularly the TH conditions immediately adjacent to and within emplacement drifts, including the conditions on the WP surfaces.

Gas-phase, advective heat-transfer mechanisms increase the overall efficiency of heat transfer away from the drift; consequently, these mechanisms decrease near-field temperature buildup. If heat pipes extend from the boiling front all the way back to the repository horizon, near-field temperatures cannot increase much above the nominal boiling point $\left(\approx 96^{\circ} \mathrm{C}\right)$. If buoyant gas-phase convection (e.g., mountain-scale) is significant, near-field temperatures will be decreased, particularly at the edges of the repository. A key issue (or hypothesis) to be resolved through field-scale thermal testing and analysis is the following: Does conduction dominate heat flow or do heat pipes and buoyant gas-phase convection significantly influence heat flow around emplacement drifts and in the repository as a whole?

\subsubsection{Final System State}

The preceding section discusses long-term transient changes in the UZ associated with the redistribution of water by evaporation and condensation. The permeability distribution changes because of the associated saturation changes. In principle, the mountain would return to something resembling its initial state, except for the effects of climate change, after decay of the thermal pulse and rewetting of the dried-out regions. However, the $\mathrm{TH}$ disturbance will also generate thermochemical (TC) and thermomechanical (TM) alteration of hydrologic and transport properties, particularly in the fractures, in both the unsaturated and saturated zones. Some changes, such as fracture closure that results from TM effects, may be temporary; others, such as the filling of fractures that results from TC effects, may be permanent. It is possible that these changes are significant enough to require them to be included in long-term calculations of repository performance.

\subsection{Heat and Mass Flow in Fractured Permeable Media}

This section presents a conceptual and mathematical framework for modeling heat and mass flow in fractured media. A general framework is developed for modeling the effects of fractures, and some currently used approaches to this problem (e.g., equivalent-continuum method [ECM] and dual-permeability method [DKM]) are shown to be special cases. The mathematical development is sufficient to express this generality, and the reader is referred to other sources for further information (Nitao, 1993, 1995; Pruess, 1991).

In this discussion, the term "conceptual model" refers to a collection of physical relationships based on observation and theory. Conceptual models are expressed singly or in combinations as mathematical models, which are solved as numerical models, implemented as computer codes. The end product is often referred to as a process model, which generally 
can be used for mechanistic simulation of directly observable behavior. Through model confidence-building activities, process models are deemed suitable for prediction of repository performance (i.e., for calculation of behavior that cannot be directly observed).

Process models have an important role in repository risk assessment. However, there typically are significant limitations on what can be simulated with numerical process models. One reason is that, because of parameter uncertainty (see Chapter 1), process models must often be repeated many times with different input for parameter sensitivity studies. Where this is unfeasible, another approach is needed. One alternative is to develop abstracted models that capture major features of the predicted behavior with less computational effort. The conduction-only models discussed in this section (Section 3.3) are an important application of the latter abstraction approach.

\subsubsection{Physical Concepts}

Two ideas central to TH models are conservation of mass and conservation of energy. Components (principally water and air) must be conserved and fully accounted for within the model domain, at the domain boundaries, and at sources or sinks. The same holds true for thermal energy (heat). The balances are coupled because movement of components involves transport of sensible and latent heat.

Driving forces for heat and component fluxes in TH models are thermal potential (temperature), matric potential (includes water potential and osmolality), and pressure of the gas and liquid phases. The conceptual models that link potential gradients to fluxes include the following:

- Flow of heat in response to a temperature gradient

- Flow of the gas phase in response to gradients of pressure and temperature

- Flow of gas-phase components in response to gradients of concentration

- Flow of liquid in response to gradients of pressure and temperature

- Flow of liquid in response to a gradient of matric potential

In addition, the flow of heat is coupled to mass flow for each component, in each phase, as sensible and latent heat.

The flux laws used in the process models described in this section are based on macroscopic application of Fourier's Law for thermal conduction, Fick's Law for solute diffusion, and Darcy's Law for fluid flow. Darcy's Law is applied to both gas and liquid flow. These flux laws are applied in all of the TH models of the near field and AZ and to those of the field-scale thermal tests. Detailed exposition of flux laws for TH problems, including solute-transport behavior, are given by Nitao and Bear (1994).

Fluid-flow constitutive properties of the matrix and fractures impart significant nonlinearity to TH process models. The van Genuchten model for moisture potential as a function of saturation (van Genuchten, 1980) and the Mualem model for unsaturated hydraulic conductivity as a function of saturation (Mualem, 1976) are commonly used in application of the TH models discussed here. Other relations can be readily substituted.

\subsubsection{Heat Conduction Models}

Models incorporating heat transfer only by conduction and radiation (T-models) do not include the effects of fluid flow on heat transport or the effects of thermal gradients on fluid flow and solute transport. Conduction-only models are far less computationally intensive than TH models and are used in the multi-scale model hierarchy developed for TSPA in conjunction with the viability assessment (TSPA-VA). These multi-scale calculations are described in the following sections. In the multi-scale analysis, there are three applications for conduction-only calculations: 
1. Smeared-heat-source, mountain-scale thermal-conduction (SMT) models, in which the repository is represented by a panel of coarse grid blocks in which heat is generated

2. Smeared-heat-source, drift-scale thermal-conduction (SDT) models, in which WPs are represented by planar heat sources

3. Discrete-heat-source, drift-scale thermal-conduction (DDT) models, in which the local thermal effects associated with emplacement schemes involving WPs of different types are examined

Conduction-only models were also used for highly detailed calculations of temperature distributions for the Drift-Scale Test (DST; Buscheck et al., 1997d). With use of the Nonisothermal Unsaturated-Saturated Flow and Transport (NUFT) code discussed later, conduction-only models can be developed that represent the following thermal effects:

- Thermal conduction in the bulk rock and in the EBS materials-Thermal conduction can occur in both the unsaturated-zone (UZ) and saturated-zone (SZ) parts of the model. The dependence of rock thermal conductivity $K_{\mathrm{th}}$ on liquid-phase saturation $S_{\text {liqu }}$ which is discussed subsequently, is approximated by correlating temperature in the T-model to $S_{\text {liq }}$ in a corresponding TH model and developing a corresponding functional relationship between $K_{\text {th }}$ and temperature in the T-model. This $K_{\text {th }}$ versus $T$ relationship in the T-model mimics the $K_{\text {th }}$ versus $S_{\text {liq }}$ in the TH model.

- Liquid-phase convection in the SZ that arises from regional flow-Flux of groundwater removes heat at the lower boundary of the UZ. This heat-flow mechanism has been examined in mountain-scale models and is calculated using a specified regional groundwater flux in the SZ (Buscheck et al., 1997a).

- Thermal radiation between all surfaces in the emplacement drift-The primary component of thermal radiation occurs between the WP and adjacent, exposed surfaces such as the drift wall, invert, and other WPs. Radiative coupling between all exposed surfaces in the drift environment tends to smooth out temperature variations in drifts that are not backfilled. Where backfill partially fills the drift, thermal radiation is important in the cavity between the surface of the backfill and the drift crown. Thermal radiation is incorporated in models with discrete heat sources (e.g., the DDT model).

- Storage of thermal energy in the bulk rock and in the EBS materials such as the invert and backfill-In this approach, the heat capacity of each hydrostratigraphic unit is regarded as temperature-dependent to approximate, using a conduction-only model, the effects of water evaporation or condensation on the temperature field. The temperature dependence of the heat capacity for a given hydrostratigraphic unit is based on the initial bulk water content.

\subsubsection{Thermohydrologic Models}

The TH model class represents both heat flow and the multiphase (gas- and aqueousphase) flow and transport of chemical species, including air and water. This model class is used in all the model-geometry/scale types (see Section 3.7.1), including (1) line-averagedheat-source, drift-scale, TH (LDTH) models, (2) discrete-heat-source, drift-scale TH (DDTH) models, and (3) smeared-heat-source, drift-scale TH (SDTH) models, as well as various models of the field-scale thermal tests. The following are the mechanisms of heat flow that can be represented: 
- Thermal conduction in the bulk rock and in EBS materials can occur in both the UZ and SZ domains of the model. The dependence of rock thermal conductivity $K_{\text {th }}$ on liquid-phase saturation $S_{\text {liq }}$ is explicitly represented in the model. The large difference between wet and dry $K_{\text {th }}\left(K_{\text {th }}\right.$ decreases with $\left.S_{\text {liq }}\right)$ plays an important role in the nearfield and EBS temperature distributions, particularly in the lower lithophysal Tptpll unit (which is model unit tsw35). Rock dryout around the drift causes a large decrease in $K_{\text {th }}$ that results in a further increase in the temperature buildup around the emplacement drift.

- Liquid-phase convection in the SZ, including that arising from regional groundwater flow and decay-heat-driven, buoyant, liquid-phase convection is only applicable to mountain-scale models.

- Thermal radiation between all surfaces in the emplacement drift is represented in the $\mathrm{TH}$ models in the same fashion as in the T-models. Thermal radiation is represented in model-geometry/scale types, including the LDTH model and the DDTH model.

- Liquid-phase convection in the $\mathbf{U Z}$ arises from percolation flux and condensate drainage.

- Gas-phase convection in the UZ includes that arising from barometric pumping, decay-heat-driven, buoyant, gas-phase convection, and decay-heat-driven boiling

- Liquid-phase species diffusion in the UZ and SZ is included in the mathematical and numerical models but is not considered significant.

- Binary gas-phase diffusion of air and water vapor in the UZ includes the possibility of enhanced vapor diffusion. The most significant impact of this mechanism is its influence on the gas-phase air-mass fraction in the boiling zone and within the EBS.

- Latent-heat effects that arise from the changes in thermal energy from the condensation of water vapor and the evaporation of liquid water contribute to the extremely high heat-transfer efficiency of heat pipes.

- Storage of thermal energy in the bulk rock and in EBS materials is accounted for explicitly. The temperature-dependent, heat-capacity approach is not required in $\mathrm{TH}$ models because the influence of evaporation, latent-heat transport, and condensation are already accounted for.

Three principal classes of the mathematical treatment of fracture-matrix interaction are used in the TH models. These include the ECM, the DKM, and discrete-fracture method (DFM).

\subsubsection{Equivalent-Continuum Method}

The ECM assumes that the local matric potential (water potential plus osmotic potential) is equal in the fractures and the adjacent matrix. Local thermodynamic equilibrium is assumed between the fractures and matrix. Composite functions are derived to describe the equivalent behavior of a single continuum and to define the relations between unsaturated hydraulic conductivity and liquid saturation and between matric potential and liquid saturation (Klavetter and Peters, 1986). The ECM does not treat fractures as discrete features; instead fracture-flow effects are averaged over the whole spatial domain. The ECM involves less computational effort than do other models described subsequently because it uses a single continuum to represent the fractures and matrix.

The assumption of local equilibrium between fractures and matrix is appropriate if the liquid-phase flux in the fractures is sufficiently small (Buscheck et al., 1991; Nitao et al., 1993). Thus, the ECM is appropriate for modeling condensate drainage during periods of quasisteady moisture movement in thermally driven models, but may be less well suited for 
modeling the early stages of repository heating, when the rate of thermally driven moisture reflux will be near its peak. The ECM assumptions are poorly suited for modeling the effects of transient boundary conditions (e.g., episodic infiltration events).

This ECM approach has been applied in the following studies with direct bearing on prediction of conditions in the near field and altered zone (NF/AZ):

- Pre-test analysis of the DST (Buscheck et al., 1997d)

- Pre- and post-test analyses of the SHT, described in Section 3.4 (Buscheck et al., 1997c; Lee, 1996)

- $\quad$ Pre- and post-test analyses of the Large-Block Test (LBT), described in Section 3.4 (Lee, 1995; Wilder et al., 1997)

\subsubsection{Dual-Permeability Method}

The DKM treats the matrix and the fractures as two distinct porous continua, with transfer terms to represent the mass and heat flux between them. Because the DKM does not assume capillary-pressure equilibrium between fracture and matrix continua, it can handle much larger liquid-phase fluxes than can the ECM without producing conditions near 100\% liquid saturation in the matrix. The DKM also allows thermodynamic disequilibrium between matrix blocks and the adjoining fractures because of its capability to represent heat flow between these two continua.

Because the DKM allows for gas-phase pressure $P_{\text {gas }}$ disequilibrium between the matrix blocks and adjoining fractures, it can account for how $P_{\text {gas }}$ buildup in matrix blocks throttles the rate of rock dryout. This throttling occurs early during the dryout period, where there is boiling in the rock matrix. It will persist if the matrix permeability $k_{\mathrm{m}}$ is extremely small (e.g., $k_{\mathrm{m}}$ less than approximately 10 microdarcy) and the matrix blocks are large (e.g., greater than $3 \mathrm{~m})$. Compared to the DFM described next, the DKM tends to overpredict the $P_{\text {gas }}$ buildup during the early stage of dryout in the rock matrix and therefore overpredicts the throttling of dryout during this period. For large matrix blocks, the DKM continues to overpredict throttling after the early dryout period.

The DKM is more computationally intensive than is the ECM, but less intensive than the DFM. Typical transfer terms for heat and mass transfer between the fracture and matrix continua do not represent the diffusive nature of matrix imbibition in the matrix block. In other words, the DKM does not treat fractures as spatially discrete features; rather, the effects of fracture flow are distributed throughout the spatial domain. Consequently, the DKM underpredicts the fracture-to-matrix liquid flux during the early stages of matrix imbibition and thus tends to overpredict the magnitude of condensate drainage ("shedding") around emplacement drifts.

The DKM approach has been applied in drift-scale TH models supporting TSPA-VA, with direct bearing on prediction of conditions in the NF / AZ. The DKM is applied to all the LDTH model calculations used in the multi-scale TH modeling approach to predict NFE conditions for performance assessment. In this family of models, the fracture-to-matrix liquid flow is strongly influenced by the fracture-matrix interaction factor (FMX), which is specified for each hydrostratigraphic unit as a model input. This parameter varies between 0 and 1 , and quantifies the fraction of the fracture surfaces that are wetted by the liquid phase. This fraction, together with a specified value for the fracture spacing, quantifies the interfacial flow area per unit volume of the rock matrix available for fracture-to-matrix liquid transfer.

The FMX parameter for liquid-phase interaction accounts for channeling of flow as the liquid phase "fingers" through the fracture network. However, this factor probably underrepresents the wetted surface area of fractures that occurs during condensate drainage in TH models. Other approaches $(\mathrm{Ho}, 1997)$ attempt to account dynamically for changes in 
the influence of condensate drainage on the fracture-matrix interaction. In such approaches, the parameter that is analogous to FMX increases with the magnitude of liquid flux in the fracture continuum. Because repository decay heat will generally produce greater liquid flux than that which occurs at ambient conditions, and because condensate flow may be more ubiquitous than ambient percolation in fractures, this dynamic approach results in a larger value of the interaction factor where there is development of condensate flow. As the repository heat output declines, thermally driven reflux decreases asymptotically toward the ambient percolation, and the interaction factor decreases to its previous value. The drift-scale TH calculations supporting TSPA-VA assume a constant value for FMX rather than the dynamic FMX approach.

\subsubsection{Discrete Fracture-matrix Method}

The DFM spatially discretizes the space occupied by the fractures and the matrix blocks using finite-difference grid blocks (or elements). Because the location and morphology of individual fractures are not usually well known, their geometric characteristics are (1) interpolated from field data, (2) randomly generated, or (3) represented using an idealized geometry. The third approach is commonly used (Buscheck et al., 1991; Nitao and Buscheck, 1995; Nitao et al., 1993). The DFM is, by far, the most computationally intensive of the three approaches described here. Moreover, available data on the fracture networks may not justify using the DFM. The DFM more accurately represents fracture-to-matrix liquid flow and matrix-to-fracture gas flow, particularly at early time when the DKM tends to underpredict mass transfer between the fractures and matrix. The DFM has been used in the following modeling studies:

- Analysis of episodic nonequilibrium percolation events (Buscheck et al., 1991; Nitao et al., 1993)

- Analysis of the G-Tunnel heater test (Nitao and Buscheck, 1995)

The selection of an approach (ECM, DKM, or DFM) for representing fracture-matrix interaction must weigh the trade-off between computational complexity and physical accuracy. Relevant factors include the following:

- Magnitude of liquid flux in fractures

- Size of the domain being modeled

- Scale over which predicted results are desired

The DFM is probably not practical for repository-scale and mountain-scale calculations, but it can be used to determine phenomenological parameters needed for the DKM. The DFM can also be useful in interpreting the results of laboratory-scale experiments. Complementary use of these three approaches is an effective way of incorporating some of the effects of nonequilibrium fracture-matrix behavior at any practical scale of analysis.

\subsubsection{Mathematical Model Description}

The behavior of $\mathrm{TH}$ processes is predicted with the use of mathematical models. These models consist of partial differential equations describing the balance of energy and the balance of mass of each important chemical species, with the primary species being air and water. A species such as water can occur in both a gas and a liquid (or aqueous) phase. The mathematical models of TH processes are defined at the macroscopic level-that is, they are derived from microscopic balance laws through volume-averaging (Bear and Bachmat, 1990) over a representative elementary volume (REV) of the porous medium. Predicted quantities (e.g., temperature or liquid-phase saturation) are therefore averaged over the REV, and any variations in these quantities are lost over distances less than the size of the REV. 


\subsubsection{Model for Nonisothermal TH Behavior of Interpenetrating Continua}

The following is the mathematical description of the balance equations solved by the USNT model in NUFT (Nitao, 1993, 1995). Some features available in NUFT (e.g., kinetic chemical reactions) are not described here because they are not used in TH modeling.

The convention used in this section to describe variables and component species is as follows: Variable superscripts refer to the component species, and subscripts refer to the phase. In the following presentation of the mathematical model used for repository $\mathrm{TH}$ simulations, two components are assumed: air and water, represented by superscripts a and $\mathrm{w}$, respectively. Two phases are assumed: gas and liquid, represented by subscripts gas and liq, respectively.

The mass balance equations for the air and water components are given by

$$
\begin{aligned}
& \frac{\partial}{\partial t}\left(\rho_{\mathrm{B}} K_{\mathrm{d}}^{\gamma} \rho_{\alpha_{\mathrm{w}}} \omega_{\alpha_{\mathrm{w}}}^{\gamma}+\sum_{\alpha=\text { gas,liquid }} \phi \rho_{\alpha} S_{\alpha} \omega_{\alpha}^{\gamma}\right) \\
& \quad+\sum_{\alpha=\text { gas,liquid }} \nabla \cdot \phi \rho_{\alpha} S_{\alpha}\left(\omega_{\alpha}^{\gamma} \mathbf{v}_{\alpha}+\mathbf{J}_{\mathrm{h} \alpha}^{\gamma}+\mathrm{J}_{\alpha}^{*} \gamma\right)=Q_{\alpha}^{\gamma}+\varepsilon q_{\alpha}^{\gamma}
\end{aligned}
$$

where $\gamma=$ air, water, and where Fickian laws for dispersive and diffusive fluxes are given by

$$
\mathbf{J}_{\mathrm{h} \alpha}^{\gamma}=-D_{\mathrm{h} \alpha}^{\gamma} \nabla \omega_{\alpha}^{\gamma}
$$

and

$$
\mathrm{J}_{\alpha}^{* \gamma}=-D_{\alpha}^{* \gamma} \nabla \omega_{\alpha}^{\gamma}
$$

and Darcy's law gives

$$
S_{\alpha} \phi \mathbf{V}_{\alpha}=-\frac{\boldsymbol{k}_{\alpha}\left(S_{\alpha}\right)}{\mu_{\alpha}} \cdot\left(\nabla p_{\alpha}+\rho_{\alpha} g \nabla z\right)
$$

The retention pressure (capillary pressure) relations are given by

$$
p_{\alpha}=p_{\text {gas }}-p_{\text {c } \alpha}\left(S_{\delta}\right), \quad \alpha \neq g
$$

The variables used in Eq. 3-1 through Eq. 3-6 are defined as follows:

$$
\begin{aligned}
\omega_{\alpha}^{\gamma} & =\text { mass fraction of } \gamma \text { component in phase } \alpha \\
S_{\alpha} & =\text { liquid saturation of } \alpha \text { phase } \\
\phi & =\text { porosity } \\
\rho_{\alpha} & =\text { mass phase density } \\
\omega_{\alpha}^{\gamma} & =\text { mass fraction } \\
\mathbf{V}_{\alpha} & =\text { liquid phase velocity } \\
\mathbf{J}_{\mathrm{h} \alpha}^{\gamma} & =\text { hydrodynamic dispersive flux } \\
\mathrm{J}_{\alpha}^{* \gamma} & =\text { mass diffusive flux } \\
D_{\mathrm{h} \alpha} & =\text { dispersion tensor }
\end{aligned}
$$




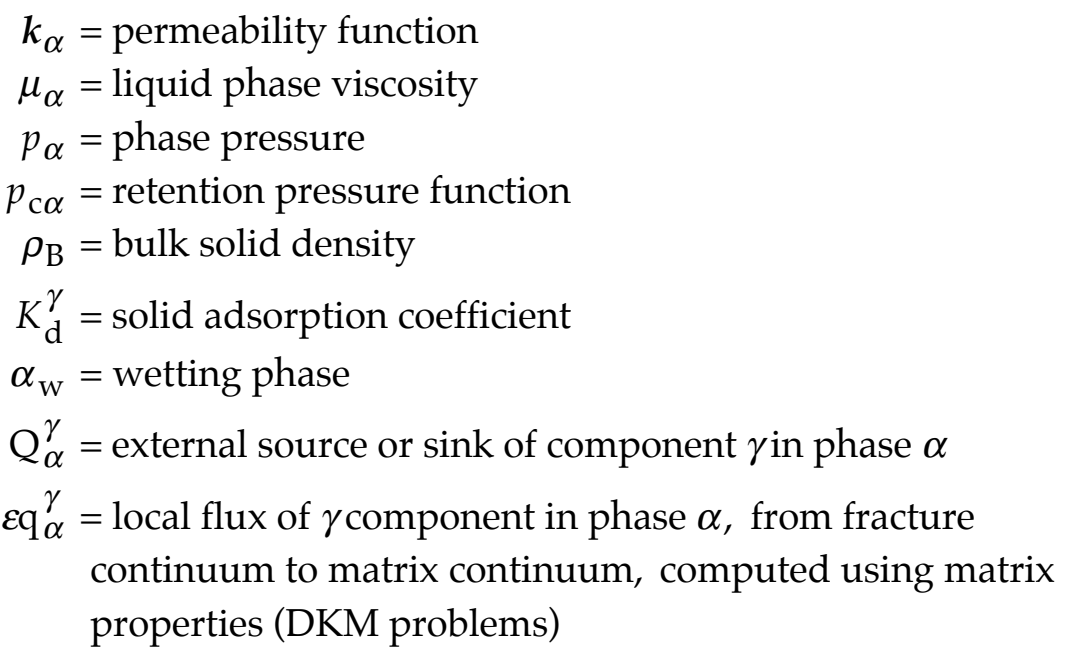

For nonisothermal problems, including all TH calculations, the following energy balance equation is solved in conjunction with the mass balance equations:

$$
\begin{aligned}
& \frac{\partial}{\partial t}\left[\sum_{\alpha=\text { gas, liquid }} \phi \rho_{\alpha} u_{\alpha} S_{\alpha}+(1-\phi) \rho_{\mathrm{s}} C_{\mathrm{p}}\left(T-T_{\text {ref }}\right)\right]= \\
& \quad-\sum_{\gamma=\alpha, \mathrm{w} \alpha=\text { gas,liquid }}\left[\nabla \cdot \phi h_{\alpha}^{\gamma} \rho_{\alpha} S_{\alpha}\left(\omega_{\alpha}^{\gamma} V_{\alpha}+\mathrm{J}_{\mathrm{h} \alpha}^{\gamma}+\mathrm{J}_{\alpha}^{* \gamma_{\alpha}}\right)\right]+\nabla \cdot K_{\mathrm{th}} \nabla T
\end{aligned}
$$

where

$T=$ temperature

$\mathrm{C}_{\mathrm{p}}=$ specific heat of solid

$\rho_{\mathrm{S}}=$ solid density

$u_{\alpha}=$ specific internal energy

$h_{\alpha}^{\gamma}=$ partial specific enthalpy

$K_{\mathrm{H}}=$ thermal conductivity

For typical TH modeling applications, the diffusion coefficients $\left(\mathbf{D}_{\alpha}{ }^{*} \eta\right)$, the viscosity parameters $\left(\mu_{\alpha}\right)$, and the phase densities $\left(\rho_{\alpha}\right)$ vary with temperature, whereas the retention pressure relations $\left(\mathrm{p}_{\mathrm{c} \alpha}\right)$, permeabilities $\left(k_{\alpha}\right)$, dispersion tensors $\left(\mathbf{D}_{\mathrm{h} \alpha}\right)$, and bulk solid density $\left(\rho_{\mathrm{B}}\right)$ are not coupled with temperature. For simulating the behavior of water, air, and water vapor in rock, the adsorption coefficients $\left(K_{\mathrm{d}}{ }^{\gamma}\right)$ are set to zero.

The balance equations Eq. 3-1 through Eq. 3-6 are discretized in space using the integrated finite-difference method and discretized in time using the fully implicit backward Euler method. All three partial differential equations are fully coupled. The resulting nonlinear system of equations is solved at each time step using the Newton-Raphson method.

The methods for solving the system of linear equations generally are (1) the directsolution method using D4 ordering or (2) the preconditioned, conjugate gradient method with various preconditioning schemes, including incomplete ILU with D4 or natural ordering. The primary solution variables predicted by the model are fluid pressure, mass fraction of water vapor in the gas phase, liquid-phase saturation, and temperature. 


\subsubsection{Extension to Alternative Conceptual Models}

The set of equations represented by Eq. 3-1 through Eq. 3-6 describes the behavior of water and air in nonisothermal porous media (with $\varepsilon=0$ to represent a single continuum). For isothermal models, Eq. 3-6 is not used, and the remaining parameters do not vary with temperature.

For ECM problems, a single continuum is assumed $(\varepsilon=0)$ with one set of equations, as represented by Eq. 3-1 through Eq. 3-5 for isothermal problems and including Eq. 3-6 for nonisothermal problems. The equivalent medium properties are calculated from a weighted average (e.g., volume average) composite of the fracture and matrix properties at each point (i.e., grid block). Equivalent-continuum properties representing behavior of the fractures and matrix, assuming local thermodynamic equilibrium, were derived by Klavetter and Peters (1986).

For DKM problems, the set of equations represented by Eq. 3-1 through Eq. 3-6 is repeated for each continuum, with simultaneous solution of the matrix and fracture sets. In Eq. 3-1, $\varepsilon=+1$ for the matrix continuum, and $\varepsilon=-1$ for the fracture continuum. The fracturematrix interaction flux is given by

$$
\begin{aligned}
q_{\alpha}^{\gamma}= & \frac{\mathrm{FMX} \mathrm{ak}_{\alpha, \text { matrix }}}{L \mu_{\alpha}}\left(\mathrm{P}_{\alpha, \text { fracture }}-\mathrm{P}_{\alpha, \text { matrix }}\right) \\
& +\frac{\mathrm{a} \phi \mathrm{S}_{\alpha} \tau_{\alpha} \mathrm{D}_{\alpha}^{\gamma}}{L}\left(\omega_{\alpha, \text { fracture }}^{\gamma}-\omega_{\alpha, \text { matrix }}^{\gamma}\right)
\end{aligned}
$$

where

$$
\begin{aligned}
\text { FMX } & =\text { fracture-matrix interaction } \\
\mathrm{a} & =\text { surface area of fracture walls per unit bulk volume } \\
\mathrm{t} & =\text { effective tortuosity factor } \\
\mathrm{L} & =\text { average radius of matrix blocks }
\end{aligned}
$$

The subscripts "fracture" and "matrix" are added to variables defined above to indicate the continuum to which they refer.

The FMX factor controls fracture-to-matrix imbibition of liquid water, as described in the foregoing discussion of DKM models. In addition, for DKM models used in TSPA-VA, matrix-to-fracture transfer of liquid water is modeled using an expression exactly analogous to Eq. 3-7. A factor, MFX, is defined by writing Eq. 3-7 with MFX in place of FMX and interchanging subscripts for "fracture" and "matrix". The MFX interaction-factor parameter specifies the relative availability of the fracture surface for seepage flow from the matrix and has a value ranging from 0 to 1 . For TSPA-VA calculations, the MFX parameter was assigned a value of unity.

It is also mathematically possible to specify matrix-to-adjacent-matrix (MMX) interaction factors, which also have values between 0 and 1. The MMX parameter can be used to represent restriction of flow across the intervening fracture between adjacent matrix blocks, which could be associated with focusing of liquid flow or transfer as vapor. The MMX transfer fluxes occur entirely within the matrix and are therefore implemented through application of the MMX factor in the flux laws and mass balance equation (Eq. 3-1 through Eq. 3-5) for each matrix grid block. 
For the TSPA-VA calculations described in this report, the MFX and MMX factors are assigned values of unity. Note that the MFX and MMX parameters are not coupled to the FMX parameter, although it is conceivable that the MMX parameter and the FMX parameter could both depend on fracture saturation.

The FMX, MFX, and MMX parameters described previously only affect liquid-phase flow. For gas-phase flow, it is also possible to independently specify FMX, MFX, and MMX parameter values between 0 and 1 . For the TSPA-VA TH calculations, these gas-phase parameters are all effectively equal to unity.

The effective tortuosity factor is the product of a tortuosity multiplier and a factor representing enhanced diffusion of water-vapor transport in the gas phase. Note that Eq. 3-7 incorporates an assumption of locally steady-state fracture-matrix interaction within the duration of a single simulation time step.

The DFM approach uses the mathematical model for porous media (Eq. 3-1 through Eq. 3-5) to represent flow processes in the rock matrix and uses the same mathematical model with different medium properties to represent flow in a fracture (Buscheck et al., 1991; Nitao and Buscheck, 1995; Nitao et al., 1993). Grid blocks representing the fracture, with fracture properties, are embedded in a domain representing the matrix.

\subsubsection{NUFT Code}

The NUFT code is a unified suite of multiphase, multicomponent codes for the numerical simulation of nonisothermal flow and transport in fractured porous media with application to geologic nuclear-waste storage and subsurface environmental contaminant-transport problems (Nitao, 1993, 1995). NUFT is an efficient and robust code that has been used to model a wide range of computationally demanding problems. NUFT contains the entire suite of alternative mathematical models described previously in a single source code instead of multiple source versions. As a result, NUFT is easily maintained. The model input format is user-friendly and flexible and is upwardly compatible (i.e., future versions of the code will accept earlier input files).

All of the TH calculations used in estimating NFE conditions for TSPA-VA (Buscheck et al., 1997a; Dunlap et al., 1997; Francis et al., 1997; Nitao, 1997a, 1997b, 1997c) and all of the calculations in support of the field-scale thermal tests (after 1995) have used the NUFT code (Nitao, 1993, 1995).

Many of the TH calculations conducted prior to 1995 were conducted with the VTOUGH code (Nitao, 1988b). UZ site-scale model simulations of the ambient hydrologic system at Yucca Mountain have been performed using the TOUGH2 code (Bodvarsson et al., 1997). All of the these codes (NUFT, VTOUGH, and TOUGH2) use the integrated finite-difference method and simulate the transport of air, water, and energy. They can compute the spatial and temporal distribution of temperature, gas pressure, liquid pressure, gas and liquid saturation, air mass fraction in gas and liquid phases, and water mass fraction in gas and liquid phases. NUFT also simulates the transport of any number of other components, as long as they behave independently and their retardation behavior can be described by linear sorption. NUFT can treat the hydrodynamic dispersion of transported components and can handle additional fluid phases, such as a nonaqueous hydrocarbon phase.

The VTOUGH code was qualified for quality-affecting work, according to the individual software plan for VTOUGH. The NUFT code has been successfully benchmarked against the VTOUGH code (which had already been qualified) and, as a result of that successful benchmarking, has also been qualified for quality-affecting work, according to the individual software plan for NUFT. 


\subsubsection{NUFT Features}

The following description pertains primarily to the USNT module of NUFT, which is used for solving the nonisothermal flow and transport equations of a multiphase system with multiple components. The NUFT code can solve 1-, 2-, and 3-D problems. The ECM and DKM options are used to represent fracture-matrix interaction. In general, any number of components and fluid phases is possible. For Yucca Mountain repository-related TH simulations, two components are generally assumed: air (a) and water (w); two phases are also assumed: gas (gas) and liquid (liq).

Vapor-pressure lowering is always applied for Yucca Mountain repository simulations. The solid phase is assumed to be nondeformable, although NUFT has the ability to handle isotropic solid compressibility in response to fluid pressure. Heat transfer by thermal radiation is also included in the code.

The preprocessing code YMESH generates the grid input files for NUFT drift-scale, mountain-scale, and multi-scale models. YMESH utilizes user-specified criteria to vertically size the grid blocks for these models, in accordance with hydrostratigraphy information from the UZ site-scale model (Bodvarsson et al., 1997). The vertical extent of each hydrostratigraphic unit is honored, and the areal geometry of the UZ site-scale model is honored in 3-D models. The drift-scale models typically contain 80 or 81 grid blocks in the vertical direction. YMESH is one of a family of pre- and post-processors associated with NUFT. The pre-processor code RADPRO (radiation processor) is used to prepare the radiation connectivity information for NUFT models that simulate in-drift conditions.

Where spatially heterogeneous TH properties are used, stochastic 3-D thermal property and hydrologic property fields are generated with spatial correlation from a specified autocorrelation function.

For solute transport calculations, local thermodynamic equilibrium is assumed, and partition coefficients are used for calculating the transfer of species between fluid phases and between fluid and solid phases.

The distinct models implemented by the NUFT code employ a common set of utility routines and a common input-file format. The various models (e.g., ECM, DKM) are essentially isolated from each other; additional models can be added without affecting existing ones, which facilitates code maintenance and revision. Global variables are virtually nonexistent. An embedded LISP language interpreter for the SCHEME dialect reads the input file, which is in LISP syntax, and performs data-checking. The NUFT code is written in the C language and runs under the UNIX MS-DOS operating systems.

\subsubsection{Model Limitations: Scale and Dimensionality}

NUFT and the other simulators mentioned previously can readily solve virtually any 1-D problem (subject to the limitations of the conceptual model), including transient, nonisothermal, DKM simulations with spatially varying medium properties, and layer interfaces. An important application of 1-D models was the UZ hydrostratigraphic parameter estimation approach of Bodvarsson et al. (1997).

For 2-D problems, significantly more computational effort is required. Several thousand 2-D, transient, nonisothermal, DKM simulations with homogeneous hydrologic properties were run on professional workstations. These models contained approximately 2000 grid blocks, and a single run requires up to an hour execution time. Run time in such models is determined by the time-step sizes, which are dynamically selected by NUFT to ensure numerical convergence. Accordingly, execution time is directly related to the heterogeneity of medium properties and initial conditions and also to the transient nature of boundary conditions imposed on the model. 
For drift-seepage problems, considerably greater computational effort was required for 2-D models because a much finer model grid was used, and thermal-radiation calculations were added at each time step. The ECM model was used in lieu of DKM calculations for nonisothermal problems, where appropriate, to reduce execution time.

Three-dimensional TH problems are run to investigate the effects of detailed geometry of heat sources and to realistically incorporate TH processes, such as thermally driven gasphase convection. Many tens of thousands of grid blocks can be required for 3-D simulations. When combined with spatial or temporal heterogeneity of medium properties and boundary conditions, the required computational effort can be prohibitive, with run times on the order of days or weeks using professional workstations.

For some problems, the phenomena of interest (e.g., gas-phase convection) can be modeled using simplified heat-source geometry, as was done for the smeared-heat-source models used with the multi-scale approach for TSPA-VA. For other problems, ECM has been used in lieu of DKM to reduce run time (e.g., for the 3-D drift-scale seepage calculations with spatially variable properties).

\subsection{Modeling Field Experiments}

Field experiments are essential steps in the development and testing of TH models. The earliest tests of interest to the Yucca Mountain Project were conducted in the Climax experiment, in which both electric heaters and actual heat-producing radioactive waste were placed in crystalline host rock below the water table. The selection of the UZ in the volcanic tuffs as the location of a potential repository at Yucca Mountain introduced more complex $\mathrm{TH}$ processes because the water content of the rock is higher than that of granite, and the unsaturated system already contains both liquid and vapor phases. Later experiments were conducted in G-Tunnel at Rainier Mesa, which is underlain by a sequence of welded and nonwelded tuffs similar to those at Yucca Mountain. These experiments provided the first field data showing that a dryout zone could be developed around a heat source in unsaturated, fractured porous rock and that condensate could drain through fractures.

TH modeling was used to help design the LBT at Fran Ridge in an outcrop of the Yucca Mountain repository host rock (Lin et al., 1998; Wilder et al., 1997). A major objective of this test was to determine how a condensate bank would actually develop above a planar heat source-a configuration intended to minimize the possibility of condensate drainage. The LBT is currently underway. Remaining work includes completing the ramp-down of heater power, post-test characterization, disassembly, and geochemical analysis to determine whether rock alteration has occurred.

The SHT, now in its cool-down phase, was the first thermal test in the ESF and is located in the actual host rock (the Tptpll hydrostratigraphic unit). Modeling was also a key part of designing this experiment as it was with the DST (Buscheck et al., 1997d). The DST is also in the repository host rock and is the first test to be conducted at a scale similar to an actual emplacement drift. The heating phase started in December 1997; therefore, the thermal response has just started. A second SHT is being considered in the E-W drift currently under construction.

\subsubsection{Purpose of Thermal Tests}

Historically, the primary role of field-scale thermal tests is to provide data for the effects of coupled thermal-hydrologic-chemical-mechanical (THCM) processes on the NF/AZ.

Because these processes involve the interaction of a relatively impermeable, but porous, rock matrix with a relatively low-porosity, but highly permeable, network of connected fractures, they cannot be studied adequately on a laboratory scale. 
To determine WP lifetime and the ultimate mobilization of waste in aqueous solution or suspension, thermal testing is needed to understand and model how water contacts WPs, including the following mechanisms:

- Liquid-phase seepage into drifts

- Condensation of water vapor on the WP surface

- The temperature and chemistry of the water contacting WPs

Thermal tests are also required for understanding how coupled processes influence radionuclide mobilization from the waste form, release from the $\mathrm{WP}$, transport in the EBS and near field, and transport through the AZ. Numerous studies (e.g., Buscheck and Nitao, 1993) have shown that significant temperature, saturation, and flux changes extend upward all the way to the ground surface and downward to deep within the SZ below the water table. Although currently there are no fully coupled models, laboratory experiments and reactive-flow models suggest that thermohydrochemical (THC) coupled effects may produce significant changes in flow pathways and adsorption capacity within the thermally disturbed region. These could permanently alter the flow and transport behavior of the mountain, even after decay of the thermal pulse.

Thermal testing is also required to address important hypotheses and issues about $\mathrm{TH}$ behavior, including the following:

- The dominant mode(s) of heat flow (conduction or convection)

- The major TH regime(s) that influence the magnitude and direction of vapor and condensate flow

- The key coupled THCM processes and site conditions that influence rock dryout and rewetting

- The influence of heterogeneity on the flow of heat, vapor, and condensate, with particular emphasis on rock dryout and rewetting

The DST (Buscheck and Nitao, 1995; Buscheck et al., 1997d) has been designed to be of sufficient size and duration to adequately address these TH hypotheses and THCM issues. However, results will not be available, particularly on THC coupling, for some time.

Field-scale thermal tests, particularly the SHT and LBT, are also needed to calibrate hydrologic properties for input to mountain-scale and drift-scale TH models supporting the TSPA-VA. Hydrologic parameter sets used for TH modeling were initially derived by inverse modeling of ambient hydrologic measurements. These inversions are subject to significant uncertainty with respect to the percolation flux $q_{\text {perc }}$. The magnitude of $q_{\text {perc }}($ e.g., $10 \mathrm{~mm} / \mathrm{yr})$ is smaller than the heat-pipe liquid-phase flux $q_{\text {liq }}$ generated by thermal processes above the repository horizon during the first $1000 \mathrm{yr}$. For the reference repository design, the maximum calculated liquid flux above the repository is about $300 \mathrm{~mm} / \mathrm{yr}$ immediately after emplacement. It declines to approximately $100 \mathrm{~mm} / \mathrm{yr}$ at $100 \mathrm{yr}$ and to $30 \mathrm{~mm} / \mathrm{yr}$ at $1000 \mathrm{yr}$. Hydrologic-parameter sets calibrated to particular values of infiltration flux (e.g., $10-\mathrm{mm} / \mathrm{yr}$ ) do not necessarily represent TH behavior when heat-driven liquid-phase fluxes are on the order of 30-300 mm/yr.

These tests are useful tools for calibration for several reasons:

- They generate a magnitude of liquid-phase flux $q_{\text {liq }}$ that can be much more accurately determined, using a heat-balance analysis, than can ambient percolation flux. Fluxes in thermal tests can be much more readily detected and quantified by direct physical means rather than inferred by the indirect means required under ambient conditions. 
- In fractured rock, it is not clear how much deep percolation occurs as steady flow and how much by episodic flow. In thermal tests, heat-driven condensate fluxes develop in a progressive, steady fashion. Thermal tests can be designed so that the fluxes are equal to or greater than those expected in the actual repository after waste emplacement. This removes the need for extrapolation between calibration flux conditions and long-term repository conditions.

- Finally, field-scale thermal tests are transient tests that include a "buildup" phase (during heat-up) and an "equilibration" phase (during cool-down) for testing alternative hydrologic inversion sets.

Field-scale thermal tests activate processes, such as capillary-driven liquid-phase flow, that are not observable under ambient conditions; these processes include matrix imbibition and capillary wicking in fractures. Models of ambient site conditions generally use information about capillary pressure vs. liquid-phase saturation under drainage conditions. However, the processes of vaporization, vapor flow, and rewetting depend strongly on the relations, for imbibition conditions, between capillary pressure and liquid-phase saturation. Therefore, it is crucial to use laboratory-scale imbibition tests and field-scale thermal tests to calibrate the property values that govern capillary-driven rewetting behavior under thermal gradients that are relevant to repository conditions.

As represented in the site-scale UZ flow model (Bodvarsson and Bandurraga, 1996), the repository host rock comprises three hydrologic model units: tsw34, tsw35, and tsw36 (Table 3-1). It is possible that further site investigation will reveal that the repository host rock should be divided into additional hydrologic subunits. It will then be important to calibrate hydrologic-parameter sets for each distinct subunit by conducting field-scale thermal tests for each of them. Ideally, to resolve key TH uncertainties and to observe and quantify the influence of coupled THCM processes under conditions relevant to WP emplacement, each test would be similar to the DST in size and duration. However, conducting multiple, largescale thermal tests is not feasible. Small-scale, short-duration tests such as the SHT are a valuable means of extending the knowledge of coupled THCM processes and calibration experience gained in the DST to other regions of the repository host rock that have hydrologic properties differing from those of the DST area. Modeling will be an important component for interpreting the DST and for designing and interpreting smaller-scale tests.

\subsubsection{Simulation of the Single-Heater Test}

This subsection describes the post-test modeling of the heating and cooling phases of the SHT in the ESF (Buscheck et al., 1997c). The model calculations were conducted with the NUFT code (Nitao, 1993, 1995). All of the calculations are 3-D and account for the geometric details of the heater borehole and the drifts in the SHT area. The heating phase of the SHT lasted nine months (between August 26, 1996, and May 28, 1997). The SHT model incorporates the temporal details, including the influence of power fluctuations and outages, of the heating history during the nine months of heating.

The primary purpose of the SHT model calculations is to test the adequacy of some of the hydrologic-parameter sets that have been used in TH model calculations supporting various Yucca Mountain Site Characterization Project (YMSCP) studies, including the waste isolation study (CRWMS M\&O, 1997) and TSPA-VA. This is accomplished by comparing the numerically predicted temporal and spatial temperature distribution with temperatures measured during the heating and cooling phases of the SHT. 


\subsubsection{Numerical Model and Assumptions}

A conceptual plan and overview of the SHT is described by Wagner (CRWMS M\&O, 1996a, 1996b). The test layout is shown in Figure 3-4. A small-diameter heater placed in a horizontal borehole was used to heat the rock. The THCM response of the rock was monitored by instrumentation placed in boreholes at various locations within the rock. The heated block is $12.86 \mathrm{~m}$ wide, bounded to the north by the access/observation drift, to the south by the TM alcove extension, and to the west by the TM alcove. The horizontal heater borehole is $9.6 \mathrm{~cm}$ in diameter, collared $6.59 \mathrm{~m}$ from the access/observation drift and $1.52 \mathrm{~m}$ above the floor of the TM alcove, and drilled parallel to the access/ observation drift. The heater is $5.0 \mathrm{~m}$ long, installed with its front (i.e., closest) end at a distance of $1.99 \mathrm{~m}$ from the borehole collar. The walls of the access / observation drift and alcoves that face the block are covered by a 15-cm-thick layer of fiberglass insulation with a thermal conductivity of 0.044 $\mathrm{W} / \mathrm{mK}^{\circ}$. The nominal electrical power supplied to the heater was $3.86 \mathrm{~kW}$. The actual deviations of power from $3.86 \mathrm{~kW}$, because of fluctuations and outages, is accounted for in all the model calculations.

\section{Figure 3-4 SHT layout, including dimensions and SHT-model coordinate system, in plan view and vertical Section $\mathbf{A}-\mathbf{A}^{\prime}$}

The 3-D SHT model takes into account the geometric details of the SHT area, including the heater hole, the access / observational drift, the TM alcove, and the TM alcove extension (Figure 3-4). The model uses a Cartesian $(x, y, z$ or $i, j, k)$ coordinate system. The $x$ direction is transverse to the heater borehole, with $x$ or $i$ increasing to the right (i.e., south). The $y$ direction is parallel to the heater borehole, with $y$ or $j$ increasing with distance from the borehole collar. The $z$ direction is vertical, with $z$ or $k$ increasing with distance below the ground surface. The overall grid-block dimensions of the model are $i=54, j=27$, and $k=44$. There are 2106 null blocks in the model, which results in a total of 62,046 active grid blocks.

The lateral model boundaries are adiabatic/no-mass flow boundaries. The northern and southern boundaries are $52.5 \mathrm{~m}$ from the heater axis, while the eastern boundary is $62.7 \mathrm{~m}$ from the eastern end of the heater (Figure 3-4). The distance to the ground surface is large enough $(250 \mathrm{~m})$ that it is accurately represented as a constant-temperature, constant-pressure, and constant-relative-humidity boundary. The water table, which is $572.3 \mathrm{~m}$ below the ground surface, is a constant-temperature, constant-pressure, and constant-liquid-saturation boundary. The models are initialized to account for the geothermal temperature gradient and static air-pressure gradient in the SHT area. The initial temperature at the heater horizon is $24.4^{\circ} \mathrm{C}$.

The western boundary is the inside surface of the insulation on the TM alcove wall. This boundary, along with the access / observation drift and the TM alcove extension, is maintained at a constant temperature $\left(24.4^{\circ} \mathrm{C}\right), R H(82.5 \%)$, and gas-phase pressure $\left(8.91 \times 10^{4} \mathrm{~Pa}\right)$. These values were chosen to account approximately for the influence of ventilation in the SHT area.

Table 3-2 describes the thermal and hydrologic property sets that were used to model the SHT and the LBT. Five of these property sets were used by Buscheck et al. (1997c). The TH model calculations were conducted for six different hydrologic-parameter sets for the host rock in the SHT area. The parameter sets give hydrologic properties for the fracture and matrix continua in the ECM. All six property sets were initialized with a value of ambient percolation flux $q_{\text {perc }}$ that results in a liquid-phase saturation $S_{\text {liq }}=0.92$ in the host rock, in agreement with experimental measurements. Property Sets 1 and 2 use $q_{\text {perc }}=0.21 \mathrm{~mm} / \mathrm{yr}$, whereas Property Sets 3-6 have $q_{\text {perc }}=0.36,3.6,6.2$, and $5.8 \mathrm{~mm} / \mathrm{yr}$, respectively. The host rock in the SHT (and DST) area is the tsw34 (Tptpmn) model unit of the UZ site-scale flow 
model. Because the TH perturbations from the SHT do not extend to the contacts of the overlying tsw33 (Tptpul) model unit and underlying tsw35 (Tptpll) model unit, it was not necessary to explicitly represent these contacts in the six property sets. Instead, the model represents a simplified UZ column consisting of the following units in the site-scale UZ sitescale model: ptn21, tsw34, and ch1vc. For all six property sets, the TH model is initialized for the assumed $q_{\text {perc }}$ and hydrologic-parameter set, and the distribution of thermal and hydrologic properties is assumed to be homogeneous.

Table 3-2 Summary of thermal and hydrologic properties of host rock for the SHT area (tsw34 unit)

\begin{tabular}{|c|c|c|c|c|c|c|}
\hline TH Property & Set 1 & Set 2 & Set 3 & Set 4 & Set 5 & Set 6 \\
\hline $\begin{array}{l}\text { Percolation flux } q_{\text {perc }} \\
(\mathrm{mm} / \mathrm{yr})\end{array}$ & 0.21 & 0.21 & 0.36 & 3.60 & 6.20 & 5.80 \\
\hline $\begin{array}{l}\text { Bulk permeability } k_{\mathrm{b}} \\
\text { (millidarcy) }\end{array}$ & 3.3 & 122 & 100 & 100 & 4000 & 1200 \\
\hline Matrix permeability $\left(\mathrm{m}^{2}\right)$ & $4.0 \times 10^{-18}$ & $4.0 \times 10^{-18}$ & $1.24 \times 10^{-17}$ & $9.14 \times 10^{-18}$ & $2.44 \times 10^{-16}$ & $8.91 \times 10^{-17}$ \\
\hline $\begin{array}{l}\text { Fracture permeability } \\
\left(\mathrm{m}^{2}\right)\end{array}$ & $8.33 \times 10^{-10}$ & $8.33 \times 10^{-10}$ & $4.12 \times 10^{-10}$ & $4.12 \times 10^{-10}$ & $1.65 \times 10^{-8}$ & $9.09 \times 10^{-9}$ \\
\hline Matrix porosity & 0.11 & 0.11 & 0.11 & 0.11 & 0.13 & 0.092 \\
\hline Fracture porosity & $3.96 \times 10^{-6}$ & $1.46 \times 10^{-4}$ & $2.43 \times 10^{-4}$ & $2.43 \times 10^{-4}$ & $2.43 \times 10^{-4}$ & $1.32 \times 10^{-4}$ \\
\hline $\begin{array}{l}\text { Matrix van Genuchten } \\
\alpha_{\mathrm{m}}(1 / \mathrm{Pa})\end{array}$ & $6.40 \times 10^{-7}$ & $6.40 \times 10^{-7}$ & $2.25 \times 10^{-6}$ & $1.71 \times 10^{-6}$ & $2.44 \times 10^{-6}$ & $7.41 \times 10^{-7}$ \\
\hline $\begin{array}{l}\text { Matrix van Genuchten } \\
\beta_{\mathrm{m}}\end{array}$ & 1.47 & 1.47 & 1.328 & 1.471 & 1.3337 & 1.7065 \\
\hline $\begin{array}{l}\text { Fracture van } \\
\text { Genuchten } \alpha_{\mathrm{f}}(1 / \mathrm{Pa})\end{array}$ & $1.34 \times 10^{-3}$ & $1.34 \times 10^{-3}$ & $9.73 \times 10^{-5}$ & $2.17 \times 10^{-5}$ & $1.22 \times 10^{-3}$ & $1.32 \times 10^{-4}$ \\
\hline $\begin{array}{l}\text { Fracture van } \\
\text { Genuchten } \beta_{\mathrm{f}}\end{array}$ & 1.9685 & 1.9685 & 1.9685 & 1.9685 & 1.2937 & 1.9685 \\
\hline $\begin{array}{l}\text { Fracture residual } \\
\text { saturation }\end{array}$ & $3.00 \times 10^{-2}$ & $3.00 \times 10^{-2}$ & $1.00 \times 10^{-2}$ & $1.00 \times 10^{-2}$ & $1.00 \times 10^{-2}$ & $1.00 \times 10^{-2}$ \\
\hline Initial liquid saturation & $92 \%$ & $92 \%$ & $92 \%$ & $92 \%$ & $92 \%$ & $92 \%$ \\
\hline $\begin{array}{l}\text { Dry thermal conductivity } \\
\left(\mathrm{W} / \mathrm{m}^{\circ} \mathrm{C}\right)\end{array}$ & 1.67 & 1.67 & 1.67 & 1.67 & 1.67 & 1.67 \\
\hline $\begin{array}{l}\text { Wet thermal } \\
\text { conductivity }\left(\mathrm{W} / \mathrm{m}^{\circ} \mathrm{C}\right)\end{array}$ & 2.00 & 2.00 & 2.00 & 2.00 & 2.00 & 2.00 \\
\hline Specific heat $\left(\mathrm{J} / \mathrm{kg}^{\circ} \mathrm{C}\right)$ & 928 & 928 & 953 & 953 & 953 & 865 \\
\hline Grain density $\left(\mathrm{kg} / \mathrm{m}^{3}\right)$ & 2526 & 2526 & 2480 & 2480 & 2480 & 2560 \\
\hline $\begin{array}{l}2 k_{\mathrm{m}} /\left(\alpha_{\mathrm{m}}\right)^{2} \sigma^{2}, \text { where } \sigma= \\
\text { surface tension of water }\end{array}$ & $3.79 \times 10^{-3}$ & $3.79 \times 10^{-3}$ & $9.53 \times 10^{-4}$ & $1.21 \times 10^{-3}$ & $1.59 \times 10^{-2}$ & $6.29 \times 10^{-2}$ \\
\hline $\begin{array}{l}\text { Notes (ECM, unless } \\
\text { specified) }\end{array}$ & $\begin{array}{l}\text { Pretest } \\
\text { analysis of } \\
\text { SHT 12/96 }\end{array}$ & $\begin{array}{l}\text { Pretest } \\
\text { analysis of } \\
\text { SHT } 12 / 96\end{array}$ & $\begin{array}{l}\text { Pretest } \\
\text { analysis of } \\
\text { DST 6/97 }\end{array}$ & $\begin{array}{l}\text { Pretest } \\
\text { analysis of } \\
\text { DST 6/97 }\end{array}$ & $\begin{array}{l}\text { WIS } \\
\text { parameter } \\
\text { set } 1 / 97\end{array}$ & $\begin{array}{c}\text { TSPA-VA } \\
\text { parameter } \\
\text { set } 7 / 97 \\
\text { (DKM) }\end{array}$ \\
\hline
\end{tabular}


Property Sets 1 and 2 utilize the same properties that were used to conduct the pretest TH calculations of the SHT (Lee, 1996). Both sets require $q_{\text {perc }}=0.21 \mathrm{~mm} / \mathrm{yr}$ to yield a liquidphase saturation $S_{\text {liq }}=0.92$ in model layer tsw34. These sets correspond, respectively, to the low bulk-permeability ( $k_{\mathrm{b}}=3.3$ millidarcy) set and medium- $k_{\mathrm{b}}$ (122 millidarcy) set considered by Lee (1996).

Property Sets 3 and 4 utilize hydrologic property sets that were used to conduct the pretest calculations of the DST (e.g., Buscheck et al., 1997d). Property Sets 3 and 4 incorporate $q_{\text {perc }}=0.36 \mathrm{~mm} / \mathrm{yr}$ and $3.6 \mathrm{~mm} / \mathrm{yr}$ and were based on a refined 1-D column-model ITOUGH inversion for borehole SD-9 (LBNL correspondence, M. Bandurraga to T.A. Buscheck, May $20,1997)$. The inversion was based on obtaining a liquid-phase saturation $S_{\text {liq }}=0.92$ in model layer tsw34.

Property Set 5 is based on an ITOUGH hydrologic-properties inversion that was released by Lawrence Berkeley National Laboratory (LBNL) on January 28, 1997 (LBNL memorandum, Y.S. Wu to Bryan Dunlap, January 28, 1997). This inversion set is a modification of parameter set \#4 (Table 8.5.4 of Bodvarsson and Bandurraga (1996). The hostrock properties for the SHT area are the same as the repository host-rock properties assumed for the drift-scale TH model calculations used in the FY97 Waste Isolation Strategy (WIS) study (CRWMS M\&O, 1997) and in the Waste Package Size (WPS) study (TRW, 1997). This case was modeled in the pretest analysis of the DST (Buscheck et al., 1997d) and in the posttest TH-model calculations of the SHT (Buscheck et al., 1997c). The TH models for the SHT, DST, WIS, and WPS studies all assumed the same $q_{\text {perc }}(6.2 \mathrm{~mm} / \mathrm{yr})$ and the same value of initial liquid-phase saturation $\left(S_{\text {liq }}=0.92\right)$.

Property Set 6 is a test that pertains directly to the July 1997 TSPA-VA base-case hydrologic-parameter set. The lowermost repository host-rock unit is the tsw36 (Tptpln) model unit, which has matrix hydrologic properties that result in much stronger matriximbibition flux than do the other two repository host-rock units (tsw34 and tsw35). Because the tsw36 unit is hydrogeologically similar to the tsw34, it is useful to examine the sensitivity of TH behavior around the SHT to the tsw36 properties in the TSPA-VA base-case assumptions. To obtain a liquid-phase saturation $\left(S_{\text {liq }}=0.92\right)$ requires $q_{\text {perc }}=5.8 \mathrm{~mm} / \mathrm{yr}$ for Case 6.

\subsubsection{Model Results and Analysis}

The temperature and liquid-phase saturation distributions at the end of the heating phase (275 days) of the SHT are given in Figure 3-5 and Figure 3-6 for Property Set 2 (Table 3-2), which is the medium bulk-permeability $\left(k_{\mathrm{b}}=122\right.$ millidarcy) set considered in the pretest SHT predictions (Lee, 1996). Air-injection measurements in the rock resulted in a $k_{\mathrm{b}}$ range of 3.3 millidarcy to 4.5 darcy for the SHT area (CRWMS M\&O, 1996a, 1996b). The log center of the extreme values is 122 millidarcy; therefore, $k_{\mathrm{b}}=122$ millidarcy is considered a representative value for TH models that assume a homogeneous $k_{\mathrm{b}}$ distribution.

Figure 3-5 Temperature and liquid-phase saturation distributions in a vertical $(x-z)$ plane transverse to the midpoint of the heater for Case 2

Figure 3-6 Temperature and liquid-phase saturation distributions in a vertical $(y-z)$ plane along the axis of the heater for Case 2

Because the 5-m-long heater effectively functions as a point heat source, the temperature and liquid-phase saturation distributions attain nearly steady-state profiles within nine months. Because of the relatively short distance between the heater and the TM alcove 
$(1.99 \mathrm{~m})$, the temperature field is influenced by heat loss to the TM alcove, which is clearly indicated by the flattening of the $40^{\circ} \mathrm{C}$ contour (Figure 3-6a). The model assumes the drifts and alcoves bounding three sides of the SHT area are maintained at a constant temperature $\left(24.4^{\circ} \mathrm{C}\right)$ to represent the influence of ventilation. Because the heater is $6.59 \mathrm{~m}$ from the access/observation drift and $6.26 \mathrm{~m}$ from the TM alcove, these rooms have a negligible influence on the temperature distribution around the heater, including the boiling and superheated zones. Figure 3-6a shows that there is no flattening of the $40^{\circ} \mathrm{C}$ contour in the vicinity of the drifts bounding the sides of the SHT area.

The liquid-phase saturation $S_{\text {liq }}$ distribution (Figure 3-5b and Figure 3-6b) has the same overall shape as does the temperature distribution; both distributions are essentially symmetric with respect to the heater axis. Dryout is seen to occur for temperatures in excess of $96^{\circ} \mathrm{C}$. The $96^{\circ} \mathrm{C}$ contour almost exactly coincides with the $S_{\text {liq }}=0.9$ contour, which is close to the initial (ambient) value $S_{\text {liq }}=0.92$ in the SHT area. Notice that the $150^{\circ} \mathrm{C}$ isotherm approximately coincides with the $S_{\text {liq }}=0.2$ contour; therefore, it is necessary to drive temperatures well above the nominal boiling point to significantly dry out the rock.

Figure 3-5b and Figure 3-6b indicate that the condensation zone, which is the area where $S_{\text {liq }}>0.92$, is symmetric about the heater axis. The calculations imply that the effects of gravity-driven condensate drainage are negligible for the SHT. However, this conclusion would be different if a model were used that represents fracture-matrix disequilibrium (e.g., the DKM model or the DFM model) and if the hydrologic properties of the host rock did not result in such strongly capillary-driven liquid-phase flow in the matrix and fractures. Calculations conducted for a SHT at G-Tunnel (Nitao and Buscheck, 1995) — which used the DFM model and hydrologic properties that did not result in strongly capillary-driven liquidphase flow-predicted significant gravity-driven drainage around the dryout zone.

\subsubsection{Comparison of Predicted and Measured Temperature Distributions}

The temporal and spatial evolution of temperatures in the SHT area is a useful indicator of $\mathrm{TH}$ behavior during the test. Of particular importance is the ability to use the temperature distribution as an indicator of the extent of rock dryout. The temperature distributions of Property Sets 1-6 are extremely indicative of the extent of rock dryout and the magnitude of rewetting (Figure 3-7). Rock dryout is the result of the balance between (a) the rate of vaporization and vapor transport away from the heat source and (b) the rate of return liquidphase flow to the dryout zone. Two mechanisms influence the rate of rewetting:

- Gravity-driven percolation and condensate flux in fractures

- Capillary-driven

- matrix imbibition, as quantified by the matrix wetting diffusivity $D_{\text {imb }}$ (Buscheck et al., 1997a, 1997c)

- wicking in fractures, as quantified by $1 / \alpha_{\mathrm{f}}$, where $\alpha_{\mathrm{f}}$ is the van Genuchten alpha parameter for fractures, which is equivalent to the air-entry (or bubble-point) pressure

Figure 3-7 Calculated and measured temperature histories for selected thermocouples and boreholes in the SHT 
Table 3-3 summarizes the capillary imbibition properties of the fracture and matrix continua for Property Sets 1-6, represented by the matrix wetting diffusivity $D_{i m b}$ and the fracture van Genuchten $\alpha$ parameter $\alpha_{f}$. The matrix wetting diffusivity $D_{\text {imb }}$ is a measure of the magnitude of matrix imbibition that contributes to rewetting the dryout zone back to ambient liquid-phase saturation conditions. The volumetric flux $q_{\mathrm{imb}}$ into a 1-D imbibition experiment is known to have the form

$$
q_{\mathrm{imb}}=\phi\left(S_{\mathrm{s}}-S_{\mathrm{i}}\right) \sqrt{D_{\mathrm{imb}} / \pi \mathrm{t}}
$$

where $\phi$ is porosity, $S_{\mathrm{s}}$ is satiated liquid-phase saturation, $S_{\mathrm{i}}$ is initial liquid-phase saturation, $t$ is time, and $D_{\text {imb }}$ is effective matrix imbibition diffusivity defined by Nitao (1991). Nitao used the results of Zimmerman and Bodvarsson (1989) to write the following approximate expression for the matrix diffusivity:

$$
D_{\mathrm{imb}} \approx \frac{\pi K_{\mathrm{m}}}{2 \phi \alpha(2-m)\left(S_{\mathrm{s}}-S_{\mathrm{i}}\right)}\left(\frac{1}{m}(1-\gamma)\right)^{1 / \mathrm{n}}
$$

where $k_{\mathrm{m}}$ is saturated matrix hydraulic conductivity, $\alpha$ and $m$ are the matrix van Genuchten parameters, $n=1 /(m-1)$, and $\gamma$ is the normalized initial saturation defined by

$$
\gamma=\left(S_{\mathrm{i}}-S_{\mathrm{r}}\right) /\left(S_{\mathrm{s}}-S_{\mathrm{r}}\right)
$$

Table 3-3 Matrix and fracture capillary properties: comparison among property sets and field-test simulations

\begin{tabular}{|l|c|c|c|}
\hline \multicolumn{1}{|c|}{ Hydrologic Property Set } & $\begin{array}{c}\text { Matrix Imbibition Diffusivity } \\
\boldsymbol{D}_{\text {imb }}\left(\mathbf{m}^{2} / \mathbf{s}\right)\end{array}$ & $\boldsymbol{D}_{\text {imb } / \boldsymbol{D}_{\text {imb }}}$ & $\left(\mathbf{1} / \mathbf{\alpha}_{\mathrm{f}}\right)(\mathbf{P a})$ \\
\hline \hline Q-tunnel tuff imbibition test & $3.44 \times 10^{-9}$ & 1 & $\mathrm{NA}$ \\
\hline Property Set 1: SHT pre-test 12/96 & $2.76 \times 10^{-7}$ & 80 & 750 \\
\hline Property Set 2: SHT pre-test 12/06 & $2.76 \times 10^{-7}$ & 80 & 750 \\
\hline Property Set 3: DST pre-test 6/97 & $2.65 \times 10^{-7}$ & 77 & $1.0 \times 10^{4}$ \\
\hline Property Set 4: DST pre-test 6/97 & $2.36 \times 10^{-7}$ & 69 & $4.6 \times 10^{4}$ \\
\hline Property Set 5: WIS 1/97 & $3.70 \times 10^{-6}$ & 1087 & 820 \\
\hline $\begin{array}{l}\text { Property Set 6: TSPA-VA base- } \\
\text { case IX1 7/97 }\end{array}$ & $2.17 \times 10^{-7}$ & 63 & $1.04 \times 10^{4}$ \\
\hline
\end{tabular}

Buscheck and Nitao, 1988

Table 3-3 also gives the value for $D_{\text {imb }}$ for an imbibition experiment on densely welded Grouse Canyon tuff taken from G-Tunnel (Buscheck and Nitao, 1988). For comparison, the $D_{\text {imb }}$ values for the six hydrologic-property sets are normalized by the experimentally determined value of $D_{\text {imb }}$. Notice that all values of $D_{\text {imb }}$ are larger than the measured value. With the exception of Cases 5 and 6, all of the values for $D_{\mathrm{imb}}$ fall within a narrow range. The value of $D_{\text {imb }}$ in Case 5 is significant because it applies to the major hydrologic host-rock unit (model unit tsw35, which corresponds to hydrostratigraphic unit Tptpll) in the WIS and WPS studies (CRWMS M\&O, 1997; TRW, 1997). Case 6 is significant because it corresponds to the hydrologic properties of the tsw36 (Tptpll)—which is lowermost repository host-rock unitused in the July 1997 TSPA-VA base-case hydrologic-parameter set. The much wetter and 
cooler conditions predicted for Cases 5 and 6 are entirely the result of its very large value of $D_{\text {imb; }}$ the magnitude of ambient percolation flux did not contribute to the wetter and cooler $\mathrm{TH}$ behavior for Cases 5 and 6 .

The parameter group $\frac{2 k_{m}}{\alpha_{m}^{2} \sigma^{2}}$ helps determine whether the hydrologic properties that determine $D_{\text {imb }}$ are internally consistent (Table 3-2). Assuming the classical Poisseuille equation for a single circular capillary tube, this parameter group can be expressed as $k_{\mathrm{m}} /\left(r_{\mathrm{c}, \mathrm{m}}\right)^{2} / 8$, where $r_{\mathrm{c}, \mathrm{m}}$ is the effective capillary radius applicable to air-entry value or bubbling pressure head. The capillary radius $r_{\mathrm{c}, \mathrm{m}}$, which is proportional to $\alpha_{\mathrm{m}}$, represents the pore radius in the matrix that is most easily drained during desaturation. Among various soil and rock media, the mean value of saturated permeability $k$ is approximately proportional to the square of the mean value of the capillary radius $r_{\mathrm{c}, \mathrm{m}}$. Therefore, $k_{\mathrm{m}}$ should be approximately proportional to $\left(\alpha_{\mathrm{m}}\right)^{2}$. Whereas $k_{\mathrm{m}}$ can span several orders of magnitude, $2 k_{\mathrm{m}} /\left(\alpha_{\mathrm{m}}\right)^{2} \sigma^{2}=$ $k_{\mathrm{m}} /\left(r_{\mathrm{c}, \mathrm{m}}\right)^{2} / 8$ is generally much less variable (Wang, 1992). Note that it was decided not to compare $2 k_{\mathrm{m}} /\left(\alpha_{\mathrm{m}}\right)^{2} \sigma^{2}$ for the G-Tunnel tuff imbibition experiment with Cases 1-6 because the capillary properties for the imbibition experiment are applicable to imbibition, whereas the values of $2 k_{\mathrm{m}} /\left(\alpha_{\mathrm{m}}\right)^{2} \sigma^{2}$ for the Cases 1-6 are applicable to drainage conditions.

For Property Sets $1-4,2 k_{\mathrm{m}} /\left(\alpha_{\mathrm{m}}\right)^{2} \sigma^{2}$ spans a factor of 4 , while Property Sets 5 and 6 have values of $2 k_{\mathrm{m}} /\left(\alpha_{\mathrm{m}}\right)^{2} \sigma^{2}$ that are 4 to 67 times greater than that of the other sets. This indicates that the values of $k_{\mathrm{m}}$ and $\alpha_{\mathrm{m}}$ for Property Sets 5 and 6 may be internally inconsistent, which may explain the anomalously large magnitude of matrix imbibition as compared with the other sets. As shown in Figure 3-7, Property Sets 5 and 6 yield, by far, the worst agreement with temperatures measured in the superheated zone of the SHT.

The second measure of capillary-driven, liquid-phase rewetting in Table 3-3 is associated with the fractures. The reciprocal van Genuchten alpha parameter for the fractures $1 / \alpha_{\mathrm{f}}$ is approximately equivalent to the height of capillary rise that is predicted to occur in fractures. A small value of $\alpha_{\mathrm{f}}$ is equivalent to having a very small capillary diameter, which results in a large capillary rise. The magnitude of capillary-driven flow in the fractures is proportional to $k_{\mathrm{b}} / \alpha_{\mathrm{f}}$. Because net dryout decreases with the magnitude of capillary-driven wicking in the fractures, predicted temperatures decrease with increasing $k_{\mathrm{b}} / \alpha_{\mathrm{f}}$. Note that Property Sets $2-4$ share nearly the same value of $k_{\mathrm{b}}$ (Table 3-2); therefore, the primary factor determining the magnitude of capillary-driven wicking in the fractures is $1 / \alpha_{\mathrm{f}}$.

Predicted temperatures for Property Sets 1-6 are compared with measured temperatures in Figure 3-7. Additional temperature comparisons are found in Buscheck et al (1997c). From these comparisons the following observations can be made:

- Predicted temperature rise in the superheated zone decreases with increasing $D_{\text {imb. }}$. Stronger matrix imbibition (associated with larger $D_{\text {imb }}$ ) results in a larger rewetting flux back to the dryout zone, which suppresses the spatial extent of dryout and thus limits temperature rise near the heater. For Property Sets 5 and 6, matrix imbibition is so strong that it completely thwarts rock dryout and prevents temperature from rising above the nominal boiling point $\left(96^{\circ} \mathrm{C}\right)$. Notice the distinct "plateau" at $96^{\circ} \mathrm{C}$ for Property Sets 5 and 6 (Figure 3-7b).

- Measured temperatures are not longitudinally symmetric about the heated interval (Figure 3-7b), with temperatures on the TM alcove side (i.e., $y<2 \mathrm{~m}$ ) of the heated interval being higher than those on the opposite side $(y>7 \mathrm{~m})$. This asymmetry probably resulted from inadequate sealing in the thermocouple boreholes, which allowed water vapor (and latent heat) to migrate back toward the TM alcove. 
- Measured temperatures are not vertically symmetric about the heater axis: temperatures above the heater are significantly greater than temperatures below the heater. The possible causes of this asymmetry include (1) buoyant gas-phase convection, (2) spatial variations in $k_{\mathrm{b}}$ (3) spatial variations in thermal conductivity, and (4) the larger number of (partially sealed) boreholes above (compared to below) the heater, which may increase the extent of dryout and temperature buildup above the heater.

- Property Set 1 gives the best apparent agreement between measured and predicted temperatures. However, even for Case 1, the predicted temperatures are lower than the measured temperatures in the superheated zone. Property Sets 5 and 6 give, by far, the worst agreement between measured and predicted temperatures in the superheated zone. Property Set 4 also predicts temperatures that are much less than the measured temperatures in the superheated zone.

Although Property Set 1 gives the best agreement with measured temperatures, it is difficult to conclude that Property Set 1 is the most representative of TH behavior in the SHT. Its assumed value of bulk permeability $\left(k_{\mathrm{b}}=3.3\right.$ millidarcy $)$ is much lower than the mean of the measured values ( $k_{\mathrm{b}}=122$ millidarcy).

During the cool-down period, predicted temperatures are higher than measured temperatures (Figure 3-7a and Figure 3-7c). The three rooms surrounding the SHT area are ventilated, causing them to function as heat sinks. Because it is closest to the heated interval of the heater, the TM alcove is the most significant of these three heat sinks. During the heatup period, the heat-sink effect at the TM alcove does not influence the axial temperature distribution around the heater (Figure 3-7b), as indicated by the axial symmetry about the heated interval. However, during the cool-down period, the axial temperature distribution is asymmetric, with cooler temperatures on the side closest to the TM alcove. Notice that the calculated axial temperature distribution is biased in the opposite direction, with higher temperatures on the side closest to the TM alcove; the calculated temperatures are also much higher than measured. This biasing of temperatures toward values that are too high is caused by the manner in which heat flow is treated at the western boundary of the model (Figure 3-6). There are two effects that are not accurately represented by the western boundary condition in the model and that cause predicted temperatures close to the TM alcove to be higher than measured values during the cool-down period:

- The treatment of the fiberglass insulation on the TM alcove wall (Figure 3-6), which is only $2 \mathrm{~m}$ from the heater, eventually influences heat flow in the SHT area during the cool-down period. In the model, the fiberglass insulation is assumed to (1) be in tight contact with the rock surface, effectively sealing the rock from the alcove, and (2) maintain its rated insulative (or R) value. Both assumptions are probably invalid. If the insulation did not lay tightly against the alcove wall, and the ventilated air were able to get between it and the rock face, this would reduce the effectiveness of the insulation. Furthermore, water vapor that is driven into the alcove by the heater will condense on the cooler insulation. Fiberglass insulation that becomes wet from condensate will lose much of its insulative value. (Incidentally, the fiberglass insulation covering the thermal bulkhead of the DST will also suffer from this effect.) To be more representative of actual conditions, the SHT model should (1) have a much larger value for the thermal conductivity of the fiberglass insulation and (2) account for the effect of air flowing between the insulation and the rock face. 
- The western model boundary condition above and below the TM alcove, which is treated as being adiabatic, will eventually significantly influence heat flow in the SHT area during the cool-down period. During the heating phase of the SHT, this boundary condition did not influence temperatures in the boiling and superheated zones. Eventually, heat flow around the top and bottom of the TM alcove will be significant. As a result of ventilation, the three rooms surrounding the SHT function as constant-temperature heat sinks. Because the ceiling and floor of the TM alcove are not insulated, these surfaces will strongly function as heat sinks, drawing heat around the top and bottom of the TM alcove. Ideally, the SHT model should be extended to the west of its current western boundary to adequately capture this heat flow.

\subsubsection{Implications of the SHT for TSPA-VA and Thermal-Loading Systems Studies}

The SHT results clearly show that the magnitude of matrix imbibition in the repository host rock is much too large in drift-scale TH model calculations supporting two very important YMSCP studies: the WIS (CRWMS M\&O, 1997) and the WPS (TRW, 1997). The SHT results also clearly show that the magnitude of calculated matrix-imbibition flux in the tsw36 unit (Tptpll) is much too high when the July 1997 TSPA-VA base-case hydrologicparameter (Property Set 6) set is used. This finding is very important because the magnitude of matrix imbibition in the tsw36 unit, which is the repository host at the western side of the repository (Figure 3-8), greatly influences near-field dryout around emplacement drifts in much of the repository area. This finding resulted in a joint effort between LLNL and LBNL to modify the TSPA-VA base-case hydrologic-parameter set to be in better agreement with the SHT temperature measurements (Lawrence Berkeley National Laboratory memorandum to G.S. Bodvarsson from J. Birkholzer, November 19, 1997; Buscheck et al., 1997b). The importance of properly representing matrix imbibition in the tsw36 and tsw37 units is discussed in other portions of this chapter.

\section{Figure 3-8 Plan view of the host-rock distribution}

\subsubsection{Simulation of Large-Block Test}

This section describes model calculations for the heating phase of the LBT. The model calculations were conducted with the NUFT code (Nitao, 1993, 1995). All of the calculations are 3-D, accounting for the geometric details of the five heater boreholes and the boundaries of the LBT. The heating phase of the LBT has lasted more than 300 days (starting on February $28,1997)$. As reported here, the LBT model incorporates the temporal details of the heating history during the first 300 days of the heating period, including the influence of power fluctuations and outages. An animation of the LBT, which profiles temperature phases in the large block during the test, is included in the CD-ROM accompanying this report.

An important purpose of the LBT model calculations is to assess the ability to model the TH behavior observed during the test. Another purpose is to test the adequacy of some of the hydrologic parameter sets that have been used in TH model calculations supporting various YMSCP studies, including the WIS (CRWMS M\&O, 1997) and the TSPA-VA. This is accomplished by comparing the numerically predicted temporal and spatial temperature distribution with temperatures measured during the heating phase of the LBT.

\subsubsection{Numerical Model and Assumptions}

A conceptual plan and overview of the LBT is described by Wilder et al (1997). The test layout and how it is represented in the LBT model are shown in Figure 3-9. The LBT consists of a 3-m $\times 3-\mathrm{m} \times 4.4-\mathrm{m}$-high block of densely welded tuff that was quarried out of the side of 
Fran Ridge. The hydrogeologic unit of the LBT area is equivalent to the tsw34 model unit (Tptpmn) of the site-scale UZ flow model (Bodvarsson and Bandurraga, 1996). The block is heated by 5 small-diameter heaters placed in 5 horizontal boreholes located $2.75 \mathrm{~m}$ from the top of the block and oriented east-west. For the first 6 months of the heating period, the heater power of each heater was about $0.41 \mathrm{~kW}$; after 6 months, the heater power was reduced in an attempt to limit the temperature rise in the rock and to maintain a constant temperature at the heater horizon. The THCM response of the rock is monitored by instrumentation placed in boreholes at various locations within the block (Wilder et al., 1997).

\section{Figure 3-9 Schematic of the LBT showing the location of the five heater boreholes and the LBT-model coordinate system}

The four sides of the block were sealed with an impervious liner to prevent moisture loss or gain and were insulated to limit the heat loss out. On July 3, 1997 (125 days after the start of heating), additional insulation was applied to the sides of the block. The effect of this additional insulation is accounted for in the model by decreasing the value of thermal conductivity for the insulation at 125 days. Outside of the insulation, the atmospheric temperature, gas-phase pressure, and $R H$ are assumed to be constant. In the test, a heat exchanger at the top of the block maintained the upper block boundary at a specified, timedependent temperature. The top of the block was gradually ramped to $60^{\circ} \mathrm{C}$ from the initial temperature of $12^{\circ} \mathrm{C}$ and maintained at $60^{\circ} \mathrm{C}$ for the remainder of the heating period. Heat, air, and water vapor were allowed to leave the top of the block. In the model, the atmosphere at the top of the block is maintained at a specified gas-phase pressure $P_{\text {gas }}$ and relative humidity $R H$. For the initial LBT calculations, it is assumed that the $P_{\text {gas }}$ and $R H$ at the top of the block are constant.

In the model, the lower boundary is $129 \mathrm{~m}$ below the ground surface, which is far enough away to not affect predicted TH behavior in the block. Below the ground surface, the model extends far enough horizontally in all four directions that the boundary conditions at the four sides of the model do not influence TH behavior in the block. The ground surface is not insulated; consequently, heat, air, and water vapor can leave the ground surface. Because an attempt was made to protect the top of the block from precipitation, the infiltration flux at the top of the model block is zero. The infiltration flux is also assumed equal to zero below the ground surfaces surrounding the block.

Most of the LBT model calculations use the ECM approximation to represent fracturematrix interaction; some use the DKM approximation. All LBT calculations assume homogeneous fracture and matrix properties. Because it assumes a homogeneous property distribution and uniform boundary conditions, the LBT model can take advantage of symmetry about the two vertical midplanes of the test (Figure 3-9). This makes it possible to represent the entire block with a quarter-symmetry model. The numerical grid has 25 rows in the lateral $(x)$ direction perpendicular to the heater axes, 21 columns in the axial $(y)$ direction parallel to the heater axes, and 55 layers in the vertical $(z)$ direction. Of the 28,875 grid blocks, 11,856 are null grid blocks, resulting in 17,019 active grid blocks. The grid-block size varies from a minimum of $5 \mathrm{~cm}$ at the heater boreholes to a maximum of $15 \mathrm{~cm}$ in the $x-y$ plane inside the block. In the $y$ direction, the maximum grid-block dimension is $20 \mathrm{~cm}$ in the block; in the $z$ direction, the maximum grid-block dimension is $12 \mathrm{~cm}$.

The model is initialized with a geothermal temperature gradient that is typical for the area, a static gas-phase pressure gradient, and a value of $R H$ at the top block that yields an initial liquid-phase saturation $S_{\mathrm{liq}}=0.92$ at the heater horizon. The six hydrologic-parameter sets used to model the SHT are also used to model the LBT. 


\subsubsection{Model Results and Analysis}

Figure 3-10 gives the temperature and liquid-phase saturation distributions at 100 and 300 days for Case 2 (Table 3-2). Note that 300 days corresponds to the last day that the detailed heater-power history was available for the LBT TH calculations presented in this section. Later in this section, it will be shown that Property Set 2 (Table 3-2) yields the best agreement with observed temperatures (Figure 3-11). The boiling and dryout zones coalesce between heaters within 100 days (Figure 3-10a and Figure 3-10c). Because the sides of the block allow for heat loss, a steep temperature gradient occurs at the four sides of the block. The liquid-phase saturation distribution bends down towards the edges of the block in response to this heat loss.

\section{Figure 3-10 Temperature and liquid-phase saturation distributions along different profiles through the large block}

\section{Figure 3-11 Calculated and measured temperature and liquid-phase saturation histories at thermocouple TT-114}

The coalescence of the dryout zones between the heaters makes it difficult for condensate to drain between the heaters after about 100 days. However, because of the lateral heat loss out of the sides of the block, condensate is able to continue to drain along the sides, particularly at the east and west sides of the block perpendicular to the heaters. At 100 days, the $96^{\circ} \mathrm{C}$ isotherm is inside the block in both the lateral $(x)$ and axial $(y)$ directions (Figure 3$10 \mathrm{a}$ and Figure 3-10c). At 300 days, the $120^{\circ} \mathrm{C}$ isotherm is at the edge of the north and south sides of the block (Figure 3-10b), whereas the $96^{\circ} \mathrm{C}$ isotherm is at the edge of the east and west sides of the block (Figure 3-10e). On the basis of the temperature distributions, it is easier for condensate to continue to drain along the east and west sides of the block than along the north and south sides.

Figure 3-12 gives the vertical temperature and liquid-phase saturation profiles at the center of the block at 100 days for the six property sets (Table 3-2) considered in the LBT and SHT model studies. As was found in the SHT modeling study, the vertical extent of rock dryout and magnitude of temperature rise in the superheated zone are inversely proportional to the matrix wetting diffusivity $D_{\text {imb }}$ (Table 3-3). A larger value of $D_{\text {imb }}$ corresponds to a larger matrix-imbibition rewetting flux that thwarts the extent of rock dryout and temperature rise in the superheated zone.

\section{Figure 3-12 Vertical temperature and liquid-phase saturation profiles at the center of the LBT}

Models employing Property Sets 1-4 show a reduction in liquid-phase saturation $S_{\text {liq }}$ at the top of the block, corresponding to moisture loss primarily as vapor (by advection and binary gas-phase diffusion) out of the top of the block (Figure 3-12b). Property Sets 5 and 6 do not show a reduction in $S_{\text {liq }}$ at the top of the block. This is not an indication that no moisture loss (by vapor transport) is occurring at the top of the block. The magnitude of matrix imbibition is so strong for Property Sets 5 and 6 that capillary-driven liquid-phase flow is able to immediately replenish the moisture transported out of the top of the block. Matrix imbibition is so strong for Property Sets 5 and 6 that it also prevents the buildup of $S_{\text {liq }}$ in the condensate zone lying above the dryout zone. For Property Sets 1-4, the upper condensate zone is a zone of increased $S_{\text {liq. }}$. Similarly, in the condensate zone below the dryout zone, 
capillary-driven liquid-phase flow is so strong for Property Sets 5 and 6 that it prevents the buildup of $S_{\text {liq }}$ in the condensate zone below the dryout zone. For Property Sets 1-4, the lower condensate zone is a zone of increased $S_{\text {liq. }}$.

Because of the inexorable transport of water vapor out of the top of the block, all property sets are predicted eventually to result in some rock dryout and temperature rise in the superheated zone Figure 3-11. This is different from what is shown in the SHT modeling study, where Property Sets 5 and 6 predicted negligible rock dryout and temperature rise. The difference between the LBT and SHT is that the moisture that leaves the top of the LBT is no longer available to rewet the dryout zone; the SHT did not have a test/model boundary where moisture could be lost from the problem domain.

\subsubsection{Comparison of Predicted and Measured Temperature Distributions}

Figure 3-11 compares the calculated temperature, at a location adjacent to the central heater, with measured temperatures. As was seen in the SHT modeling study, temperature rise is inversely proportional to the matrix wetting diffusivity $D_{\mathrm{imb}}$. The best agreement between calculated and measured temperatures is obtained with Case 2, which has a bulk permeability $k_{\mathrm{b}}$ value that is closest to measured values (Lee and Ueng, 1991; Wilder et al., 1997). Property Set 1 has a relatively low value of $k_{\mathrm{b}}$ (3.3 millidarcy) that results in gas-phase pressure $P_{\text {gas }}$ buildup in the boiling zone and an increase in $T_{\text {sat }}$ (which is the effective boiling temperature), causing higher temperatures than those in Property Set 2. Because of throttling (restricting liquid and vapor flow) due to restricted bulk permeability, Property Set 1 results in less rock dryout than do Property Sets 2-4 (Figure 3-12b and Figure 3-11b); Property Sets 2-4 have similar values of $D_{\mathrm{imb}}$, resulting in a similar extent of rock dryout. Property Sets 2 and 3 result in temperatures that are in good agreement with measured temperatures. As was true in the SHT modeling study, Property Sets 5 and 6 are in very poor agreement with measured temperatures in the superheated zone. The hydrologic properties in Property Sets 5 and 6 strongly overpredict matrix imbibition.

At 105 and 185 days, the measured temperatures at the heater horizon decreased abruptly from above boiling to the nominal boiling point. The first event required 15-20 days before temperatures climbed back up to the pre-event level. The second event required less than 5 days to restore to pre-event levels. These two events corresponded to intense rainstorms and power outages. The rapid reduction of temperatures corresponded to increased heatpipe behavior propagating all the way down to the heater horizon; this was probably brought on by two factors: (1) an unknown quantity of water was able to infiltrate into the block; (2) the power outage shut down the main heaters and the heat exchanger at the top of the block. While the effect of the power outage on the main heaters is accounted for in the model, it was not accounted for at the heat exchanger at the top of the block. Removal of the heat source at the top of the block caused a rapid cooling that quenched steam located in the upper boiling zone. The rapid introduction of additional condensate probably contributed to the heat-pipe behavior propagating all the way down to the heater horizon.

The agreement between calculated and measured temperatures for Property Sets 2 and 3 is not as good beyond 225 days as it was prior to 225 days (which corresponds to the middle of October 1997). The assumption of a constant atmospheric temperature in the model ignores seasonal temperature variations. Because of the significant heat loss out of the sides of the block, it would be useful to repeat the LBT model calculations with a time-dependent atmospheric temperature. Because of the decreasing atmospheric temperatures in October through December, the observed temperatures in the LBT probably reflect the increased heat loss out of the sides of the block. It is also possible that rainfall progressively wets the fiberglass insulation, which decreases its effective R-value. 


\subsubsection{Implications of the LBT for TSPA-VA and Thermal-Loading Systems Studies}

The most useful finding from the LBT concerns the importance of understanding heatpipe phenomena and its influence on rock dryout and temperatures around the heat source. The DST will provide additional insight into the heat-pipe effect and the implications on the NFE.

Another useful finding from the LBT concerns than manner in which matrix imbibition in the repository host rock is treated in various hydrologic parameter sets. The LBT results clearly show that the predicted magnitude of matrix imbibition in the repository host rock is much too large in the drift-scale TH model calculations for the WIS (CRWMS M\&O, 1997) and the WPS Study (TRW, 1997). The LBT results also clearly show that the predicted magnitude of matrix-imbibition flux in the tsw36 unit (Tptpll) is too large if the July 1997 TSPA-VA base-case hydrologic-parameter set is used.

\subsection{Reference Calculations for Drift-Scale Coupled Processes}

This section provides $\mathrm{TH}$ calculations for five reference cases. These reference cases are used to compare hydrologic property sets, infiltration conditions, and thermal-loading scenarios and to develop a basis for evaluating coupled processes. (Reference cases for investigation of coupled processes in the NF/AZ Models Report are visually depicted and linked in the presentation on the CD-ROM that accompanies this report.) Table 3-4 summarizes the parameters and design assumptions used for the five cases.

Table 3-4 Model parameters for reference calculations

\begin{tabular}{|c|c|c|c|}
\hline Case Number & Hydrologic Parameter Set & Infiltration Flux (mm/yr) & Design Option \\
\hline \hline 1 & 7/97 TSPA VA I $\times 1$ & 16.0 & Point load \\
\hline 2 & 7/97 TSPA VA I/5 & 3.2 & Point load \\
\hline 3 & Modified TH & 16.0 & Point load \\
\hline 4 & Modified TH & 3.2 & Point load \\
\hline 5 & Modified TH & 16.0 & Line load \\
\hline
\end{tabular}

Models for thermomechanically and thermochemically coupled processes require, as input, drift-scale spatial distributions of temperature $T$, liquid-phase saturation $S_{\text {liq, }}$ relative humidity $R H$, liquid-phase flux $q_{\text {liq }}$ and air-mass fraction $X_{\text {air,gas }}$ as functions of time. Twodimensional cross-sectional TH models were used to calculate these results for five cases, using two different sets of hydrologic properties, two different infiltration rates, and two different thermal-loading options. The Nevada State coordinates for the model hydrostratigraphic column are easting $170488 \mathrm{~m}$ and northing $233748 \mathrm{~m}$. This column (second from the left in Figure 3-13) is located on the crest of Yucca Mountain, halfway between the northern and southern boundaries of the repository footprint and one-third of the way from the western to the eastern boundary. The nominal infiltration flux $q_{\text {inf }}$ is $16.0 \mathrm{~mm} / \mathrm{yr}$ at this location (Flint et al., 1996a).

Figure 3-13 Vertical distribution of hydrostratigraphic model units shown for drift-scale model locations (in Nevada-State coordinates)

These 2-D drift-scale models are in the $x-z$ plane, oriented $N-S$ and perpendicular to the E-W emplacement-drift axis. In the $Z$ direction (depth), the hydrostratigraphic units correspond to those used in the site scale UZ model, with the ground surface at the top 
(model unit tcw11) and the water table at the bottom (model unit bf3vb). There is no lateral variation in hydrologic or thermal properties, and the $X$ domain is treated as an infinite repetition of identical drifts with uniform interdrift spacing (this is equivalent to periodic boundaries in the $x$ direction). By symmetry, an element from the drift centerline to the midpoint between drifts will have closed boundaries to both mass and heat flow. This basic symmetry element makes up the model domain. Mountain-scale lateral heat and mass flow are neglected. The error introduced is believed to be small for locations near the center of the repository, but it is probably significant for the edges of the repository. However, the main purpose of these reference calculations is to assess the importance of these coupled effects by providing reasonable magnitudes for $\mathrm{TH}$ variables as functions of time for input to the TM and TC models. For these purposes, the current approximations will suffice, with the understanding that any coupled effects found to be significant to repository performance will be investigated more rigorously in the future.

\subsubsection{Parameters and Assumptions}

\subsubsection{Repository Design}

Two repository designs were analyzed (Buscheck, 1996). In the point-load design, WP spacing within drifts is about the same as the spacing between drifts. The current reference point-load design has a drift spacing of $28 \mathrm{~m}$, at a lineal mass loading (LML) of 0.588 $\mathrm{MTU} / \mathrm{m}$. In the line-load design, WPs are placed as nearly end to end as practical. The lineload design analyzed has a drift spacing of $56.6 \mathrm{~m}$ and a LML of $1.189 \mathrm{MTU} / \mathrm{m}$. Drift-scale model calculations were conducted for cases without backfill and for cases in which an engineered backfill is emplaced at $100 \mathrm{yr}$.

\subsubsection{Boundary Conditions}

The water table is a constant-property boundary with specified fixed temperature, liquid saturation, and gas pressure. The ground surface is a constant-property boundary with specified fixed temperature, gas pressure, and $R H$. The $R H$ at the ground surface is assumed to be $100 \%$; this virtually eliminates vapor flux and is consistent with fixing the infiltration flux at this boundary.

The values of $T$ at the water table and ground-surface boundaries are taken from the sitescale UZ flow model (Bodvarsson et al., 1997). At the water table, $S_{\text {liq }}=100 \%$. The value of $P_{\text {gas }}$ at the ground surface is taken from the site-scale UZ flow model. The value of $P_{\text {gas }}$ at the water table is consistent with the value of $P_{\text {gas }}$ at the ground surface and the pressure profile of a static gas column from the ground surface to the water table.

Figure 3-14 is a vertical cross-section of the emplacement drift, orthogonal to the drift axis. By using periodic boundaries, the centerline of the drift becomes an axis of symmetry and, consequently, provides an adiabatic, no-flow boundary. The centerline of the pillar between drifts provides the other adiabatic, no-flow boundary in the $x$ direction.

Figure 3-14 Vertical cross-section of the emplacement drift in the DDT and LDTH model showing the thermal radiation connections among the surfaces in the drift that are used in thermal simulations

For the current design, the WP diameter is $1.67 \mathrm{~m}$, and the emplacement drift diameter is $5.1 \mathrm{~m}$. For earlier drift-scale-model calculations for TSPA-VA, the WP diameter was assumed to be 1.85 m (Buscheck et al., 1997a; Dunlap et al., 1997; Francis et al., 1997). The invert, which is at the bottom of the drift, is assumed to occupy $16.6 \%$ of the drift cross-section. As was demonstrated previously (Buscheck, 1996), a circular WP in a circular drift can be accurately 
represented by a square WP in a square drift, provided that the respective circular and square cross-sectional areas are equal. In the model, the WP dimensions are $1.445 \mathrm{~m} \times 1.445 \mathrm{~m}$, and the drift measures $4.52 \mathrm{~m} \times 4.52 \mathrm{~m}$. The invert is assumed to be filled with concrete, consisting of fractured and jointed matrix blocks.

Heat flux from the WP sources is averaged over the length of the drifts and coupled to the drift surfaces primarily by radiant heat transfer. Thermal radiation is also accounted for between different locations on the drift surface. An efficient heat-transfer mechanism distributes the heat flux uniformly to the perimeter surfaces of the emplacement drift. The drift-wall and drift-floor surfaces are assumed to be blackbodies $(\varepsilon=1)$, and the WPs have $\varepsilon=0.8$. This allows the use of a constant heat-flux boundary condition at the drift perimeter.

\subsubsection{Initial Conditions}

The value of $P_{\text {gas }}$ at the water table is established when the models are initialized at ambient conditions. The initialization model runs are continued until the $T, S_{\text {liq }}, P_{\text {gas }}$ and $X_{\text {air,gas }}$ distributions in the model attain steady-state distributions.

\subsubsection{Grid}

The DKM approximation is used to represent fracture-matrix coupling. Because different properties are assigned to fracture elements and matrix elements, two grid blocks have to be assigned to each spatial location (i.e., twice as many as would be required to give the same spatial resolution with the ECM.

Sixteen grid blocks are used in the $x$ direction for the point-load design, and 18 are used for the line-load design. In the vertical direction, 80 grid-block layers are used to represent the hydrostratigraphy; thus, the point-load design requires 1280 grid blocks, whereas the line-load design requires 1440 grid blocks.

\subsubsection{Thermal and Hydrologic Properties}

All five cases use the set of thermal properties summarized in Table 3-5 (Francis et al., 1997). Three sets of matrix and fracture hydrologic parameters were used. The first two were supplied for the 1997 TSPA-VA base case and are based on the use of 1-D inverse modeling to match field observations, including the vertical distribution of liquid saturation and matric potential (Francis et al., 1997). Because the infiltration flux is considered very uncertain, inverse modeling was applied for the nominal infiltration-flux map, I (Figure 3-1b), at specific locations, for $\mathrm{I} / 5$, and for $\mathrm{I} \times 5$. The resulting hydrologic properties depend on the assumed value of infiltration flux. Table 3-6 and Table 3-7 summarize matrix and fracture hydrologic properties, respectively, for the nominal infiltration case. Table 3-8 and Table 3-9 provide corresponding values for infiltration at $1 / 5$ the nominal rate (I/5).

In attempting to model the SHT and the LBT, it was found that capillary forces associated with these property values in the repository host rock were too strong, contrary to experimental data, to allow the formation of a superheated dryout zone. This difficulty was attributed to large values of matrix imbibition diffusivity $D_{\text {imb }}$ in the host-rock units and in the units immediately above and below the host-rock units, which led to rapid rewetting of the rock. The model hydrostratigraphic unit of particular importance is the tsw36 (Tptpln) unit.

This difficulty was partially overcome in November 1997 by the development of a TH parameter set calibrated by LLNL and LBNL with temperature measurements from the SHT (Lawrence Berkeley National Laboratory memorandum to G.S. Bodvarsson from J.

Birkholzer, November 19, 1997; Buscheck et al., 1997b). Analysis of the SHT in Section 3.4 (Buscheck et al., 1997c) shows that the value of $D_{\text {imb }}$ for the tsw36 in the July 1997 TSPA-VA parameter sets is too large to allow rock dryout and temperature rise in the nominally 
superheated zone. The reduced value of $D_{\text {imb }}$ for the tsw36 TH set produces more rock dryout in this unit and a closer match to temperatures observed in the SHT. These parameters are summarized in Table 3-10 and Table 3-11, respectively, for the matrix and fractures.

Table 3-5 TSPA-VA thermal properties for the rock matrix

\begin{tabular}{|c|c|c|c|c|c|}
\hline Geologic Unit & Model Unit ${ }^{\mathrm{a}}$ & $K_{\text {wet }}(\mathrm{W} / \mathrm{mK})$ & $K_{\mathrm{dry}}(\mathrm{W} / \mathrm{mK})$ & $\begin{array}{c}\text { Rock Density } \\
\left(\mathrm{kg} / \mathrm{m}^{3}\right)\end{array}$ & $\begin{array}{c}\text { Specific Heat } \\
(\mathrm{J} / \mathrm{kgK})\end{array}$ \\
\hline Tpсp11 & tcw11 & 1.76 & 1.02 & 2510 & 847 \\
\hline Tpcpln & tcw12 & 1.88 & 1.28 & 2510 & 837 \\
\hline Tpcpv & tcw13 & 0.98 & 0.54 & 2470 & 857 \\
\hline Tpcpv1 & ptn21 & 0.50 & 0.35 & 2340 & 1080 \\
\hline Tpy & ptn22 & 0.97 & 0.44 & 2400 & 849 \\
\hline Tpbt3 & ptn23 & 1.02 & 0.46 & 2370 & 1020 \\
\hline Tpp & ptn24 & 0.82 & 0.35 & 2260 & 1330 \\
\hline Tpbt2 & ptn25 & 0.67 & 0.23 & 2370 & 1220 \\
\hline Tptrv & tsw31 & 1.00 & 0.37 & 2510 & 834 \\
\hline Tptrn & tsw32 & 1.62 & 1.06 & 2550 & 866 \\
\hline Tptpul & tsw33 & 1.80 & 0.71 & 2510 & 883 \\
\hline Tptpmn & tsw34 & 2.33 & 1.56 & 2530 & 948 \\
\hline Tptp11 & tsw35 & 2.02 & 1.2 & 2540 & 900 \\
\hline Tptpln & tsw36 & 1.84 & 1.42 & 2560 & 865 \\
\hline Tptpv & tsw37 & 2.08 & 1.69 & 2360 & 984 \\
\hline Tpbt1 & ch1zc & 1.31 & 0.70 & 2310 & 1060 \\
\hline $\operatorname{Tac}(z)$ & $\operatorname{ch} 2 z c$ & 1.20 & 0.61 & 2350 & 1150 \\
\hline $\operatorname{Tac}(z)$ & $\operatorname{ch} 3 z \mathrm{c}$ & 1.20 & 0.61 & 2350 & 1150 \\
\hline Tacbt & $\operatorname{ch} 4 z c$ & 1.35 & 0.73 & 2440 & 1170 \\
\hline Tpbt1 & ch1vc & 1.31 & 0.70 & 2310 & 1060 \\
\hline $\operatorname{Tac}(\mathrm{v})$ & $\mathrm{ch} 2 \mathrm{vc}$ & 1.17 & 0.58 & 2240 & 1200 \\
\hline $\operatorname{Tac}(\mathrm{v})$ & ch3vc & 1.17 & 0.58 & 2240 & 1200 \\
\hline Tabt1 & $\mathrm{ch} 4 \mathrm{vc}$ & 1.17 & 0.58 & 2240 & 1200 \\
\hline $\operatorname{Tcp}(3)$ & pp3vp & 1.26 & 0.66 & 2580 & 841 \\
\hline $\operatorname{Tcb}(\mathrm{w})$ & bf3vb & 1.26 & 0.66 & 2580 & 841 \\
\hline $\operatorname{Tcb}(\mathbf{w})$ & $\mathrm{tm} 3 \mathrm{vt}$ & 1.26 & 0.66 & 2580 & 841 \\
\hline $\operatorname{Tcp}(2)$ & pp2zp & 1.35 & 0.74 & 2510 & 644 \\
\hline $\operatorname{Tcb}(n)$ & bf2zb & 1.35 & 0.74 & 2510 & 644 \\
\hline
\end{tabular}


Table 3-6 Matrix property values in the July 1997 TSPA-VA base-case hydrologic parameter set for $I \times 1$, where I stands for the nominal infiltration-flux map

\begin{tabular}{|c|c|c|c|c|c|c|}
\hline Geologic Unit & Model Unit & Porosity & $\begin{array}{c}\text { Permeability } \\
\left(\mathrm{m}^{2}\right)\end{array}$ & $S_{r} *$ & $\alpha_{m}\left(\mathbf{P a}^{-1}\right) * *$ & $\boldsymbol{m} * *$ \\
\hline Трср11 & tcw11 & 0.066 & 5.37E-18 & 0.13 & 1.17E-06 & 0.232 \\
\hline Tpcpln & tcw12 & 0.066 & 5.37E-18 & 0.13 & $1.32 \mathrm{E}-06$ & 0.236 \\
\hline Tpcpv & tcw13 & 0.14 & $4.90 \mathrm{E}-17$ & 0.33 & $6.46 \mathrm{E}-07$ & 0.427 \\
\hline Tpcpv1 & ptn21 & 0.369 & 3.09E-14 & 0.10 & 3.80E-05 & 0.231 \\
\hline Tpy & ptn22 & 0.234 & $3.02 \mathrm{E}-15$ & 0.14 & 8.71E-06 & 0.488 \\
\hline Tpbt3 & ptn23 & 0.353 & 8.32E-14 & 0.17 & 4.57E-05 & 0.287 \\
\hline Tpp & ptn24 & 0.469 & $1.15 \mathrm{E}-13$ & 0.10 & 4.27E-05 & 0.349 \\
\hline Tpbt2 & ptn25 & 0.464 & $2.45 \mathrm{E}-13$ & 0.10 & 1.95E-04 & 0.279 \\
\hline Tptrv & tsw31 & 0.042 & $4.90 \mathrm{E}-17$ & 0.11 & $1.00 \mathrm{E}-05$ & 0.237 \\
\hline Tptrn & tsw32 & 0.146 & $2.75 \mathrm{E}-16$ & 0.04 & 2.29E-05 & 0.273 \\
\hline Tptpul & tsw33 & 0.135 & $1.15 \mathrm{E}-17$ & 0.06 & $6.76 \mathrm{E}-06$ & 0.247 \\
\hline Tptpmn & tsw34 & 0.089 & 4.07E-18 & 0.18 & $1.02 \mathrm{E}-06$ & 0.322 \\
\hline Tptp11 & tsw35 & 0.115 & $1.55 \mathrm{E}-17$ & 0.08 & 3.31E-06 & 0.229 \\
\hline Tptpln & tsw36 & 0.092 & 8.91E-17 & 0.18 & 7.41E-07 & 0.414 \\
\hline Tptpv & tsw37 & 0.02 & $1.29 \mathrm{E}-17$ & 0.50 & $1.55 \mathrm{E}-06$ & 0.387 \\
\hline Tpbt1 & ch1zc & 0.193 & $1.38 \mathrm{E}-17$ & 0.36 & 8.32E-07 & 0.366 \\
\hline $\operatorname{Tac}(z)$ & ch2zc & 0.24 & $9.12 \mathrm{E}-18$ & 0.20 & 1.95E-06 & 0.220 \\
\hline $\operatorname{Tac}(z)$ & ch3zc & 0.24 & $9.12 \mathrm{E}-18$ & 0.20 & 1.95E-06 & 0.220 \\
\hline Tacbt & ch4zc & 0.169 & $1.55 \mathrm{E}-17$ & 0.33 & 7.76E-07 & 0.477 \\
\hline Tpbt1 & ch1ve & 0.265 & $1.32 \mathrm{E}-12$ & 0.04 & 6.61E-05 & 0.190 \\
\hline $\operatorname{Tac}(\mathrm{v})$ & ch2vc & 0.321 & $2.57 \mathrm{E}-13$ & 0.06 & 7.41E-05 & 0.224 \\
\hline $\operatorname{Tac}(\mathrm{v})$ & ch3vc & 0.321 & $2.57 \mathrm{E}-13$ & 0.06 & 7.41E-05 & 0.224 \\
\hline Tabt1 & ch4vc & 0.321 & $2.57 \mathrm{E}-13$ & 0.06 & 7.41E-05 & 0.224 \\
\hline Tcp(3) & pp3vp & 0.274 & $2.82 \mathrm{E}-15$ & 0.07 & 1.74E-05 & 0.311 \\
\hline $\operatorname{Tcb}(\mathrm{w})$ & bf3vb & 0.274 & $2.82 \mathrm{E}-15$ & 0.07 & 1.74E-05 & 0.311 \\
\hline $\mathrm{Tcb}(\mathrm{w})$ & tm3vt & 0.274 & $2.82 \mathrm{E}-15$ & 0.07 & 1.74E-05 & 0.311 \\
\hline $\operatorname{Tcp}(2)$ & pp2zp & 0.197 & $5.75 \mathrm{E}-17$ & 0.18 & $1.66 \mathrm{E}-06$ & 0.316 \\
\hline $\operatorname{Tcb}(n)$ & $b f 2 z b$ & 0.197 & $5.75 \mathrm{E}-17$ & 0.18 & 1.66E-06 & 0.316 \\
\hline
\end{tabular}

$S_{\mathrm{r}}$ is the residual liquid-phase saturation (not in situ saturation).

** $\quad \alpha$ and $m$ are fitting parameters for capillary pressure and relative permeability curves, respectively. 
Table 3-7 Fracture property values in the July 1997 TSPA-VA base-case hydrologic parameter set for $I \times 1$, where I stands for the nominal infiltration-flux map

\begin{tabular}{|c|c|c|c|c|c|c|c|c|}
\hline \multirow[t]{2}{*}{$\begin{array}{c}\text { Geologic } \\
\text { Unit }\end{array}$} & \multirow[t]{2}{*}{$\begin{array}{c}\text { Model } \\
\text { Unit }\end{array}$} & \multirow[t]{2}{*}{ Porosity } & \multicolumn{2}{|c|}{$\begin{array}{l}\text { Permeability } \\
\left(m^{2}\right)\end{array}$} & \multirow[t]{2}{*}{$S_{r} *$} & \multirow[t]{2}{*}{$\begin{array}{c}\alpha * * \\
\left(\mathrm{~Pa}^{-1}\right)\end{array}$} & \multirow[t]{2}{*}{$\boldsymbol{m} * *$} & \multirow[t]{2}{*}{ FMX } \\
\hline & & & Vertical & Horizontal & & & & \\
\hline Tрср11 & tcw11 & 2.33E-04 & $2.29 \mathrm{E}-11$ & 6.03E-12 & 0.01 & 2.95E-04 & 0.492 & 4.90E-04 \\
\hline Tpcpln & tcw12 & 2.99E-04 & $1.38 \mathrm{E}-11$ & 6.03E-12 & 0.01 & 2.95E-04 & 0.492 & 4.90E-04 \\
\hline Tpcpv & tcw13 & 7.05E-05 & 2.82E-12 & $2.40 \mathrm{E}-13$ & 0.01 & $9.12 \mathrm{E}-05$ & 0.492 & 4.90E-04 \\
\hline Tpcpv1 & ptn21 & 4.84E-05 & $5.25 \mathrm{E}-13$ & $5.25 E-13$ & 0.01 & 1.10E-03 & 0.492 & 1.10E-01 \\
\hline Tpy & ptn22 & 4.83E-05 & $1.95 \mathrm{E}-13$ & $1.95 \mathrm{E}-13$ & 0.01 & 1.82E-03 & 0.492 & 7.08E-01 \\
\hline Tpbt3 & ptn23 & 1.30E-04 & $2.57 \mathrm{E}-13$ & $2.57 \mathrm{E}-13$ & 0.01 & 3.39E-03 & 0.492 & 6.92E-01 \\
\hline Tpp & ptn24 & 6.94E-05 & $6.17 \mathrm{E}-14$ & $6.17 \mathrm{E}-14$ & 0.01 & 9.33E-04 & 0.492 & 4.79E-01 \\
\hline Tpbt2 & ptn25 & 3.86E-05 & $7.76 \mathrm{E}-14$ & $7.76 \mathrm{E}-14$ & 0.10 & 1.95E-04 & 0.279 & 4.79E-01 \\
\hline Tptrv & tsw31 & 8.92E-05 & $1.07 \mathrm{E}-11$ & $1.00 \mathrm{E}-12$ & 0.01 & 3.98E-05 & 0.481 & 5.01E-01 \\
\hline Tptrn & tsw32 & $1.29 \mathrm{E}-04$ & $1.51 \mathrm{E}-11$ & $7.08 \mathrm{E}-13$ & 0.01 & 9.33E-05 & 0.488 & 2.88E-05 \\
\hline Tptpul & tsw33 & $1.05 \mathrm{E}-04$ & $2.63 \mathrm{E}-11$ & $8.91 \mathrm{E}-13$ & 0.01 & $1.78 \mathrm{E}-04$ & 0.492 & 7.94E-05 \\
\hline Tptpmn & tsw34 & $1.24 \mathrm{E}-04$ & $6.76 \mathrm{E}-12$ & $4.27 \mathrm{E}-13$ & 0.01 & 9.77E-05 & 0.492 & $1.55 \mathrm{E}-04$ \\
\hline Tptp11 & tsw35 & 3.29E-04 & $3.80 \mathrm{E}-12$ & $9.12 \mathrm{E}-13$ & 0.01 & 1.10E-04 & 0.492 & 7.76E-02 \\
\hline Tptpln & tsw36 & 3.99E-04 & $1.20 \mathrm{E}-12$ & $1.20 \mathrm{E}-12$ & 0.01 & 1.32E-04 & 0.492 & 4.79E-05 \\
\hline Tptpv & tsw37 & 4.92E-04 & $1.20 \mathrm{E}-12$ & $1.20 \mathrm{E}-12$ & 0.01 & 1.17E-04 & 0.492 & 4.90E-04 \\
\hline Tpbt1 & ch1zc & $1.10 \mathrm{E}-05$ & $2.40 \mathrm{E}-14$ & $2.40 \mathrm{E}-14$ & 0.01 & 1.12E-03 & 0.492 & 1.82E-01 \\
\hline $\operatorname{Tac}(z)$ & ch2zc & $1.10 \mathrm{E}-05$ & 1.17E-14 & 1.17E-14 & 0.01 & $1.23 \mathrm{E}-03$ & 0.492 & $1.00 \mathrm{E}+0 \mathrm{C}$ \\
\hline $\operatorname{Tac}(z)$ & ch3zc & $1.10 \mathrm{E}-05$ & 1.17E-14 & 1.17E-14 & 0.01 & 1.23E-03 & 0.492 & $1.00 \mathrm{E}+0 \mathrm{C}$ \\
\hline Tacbt & ch4zc & $1.10 \mathrm{E}-05$ & $1.55 \mathrm{E}-14$ & $1.55 \mathrm{E}-14$ & 0.01 & $1.15 \mathrm{E}-03$ & 0.492 & $1.00 \mathrm{E}+0 \mathrm{C}$ \\
\hline Tpbt1 & ch1ve & 7.14E-05 & $1.74 \mathrm{E}-13$ & $1.74 \mathrm{E}-13$ & 0.01 & 1.17E-03 & 0.492 & 4.90E-01 \\
\hline $\operatorname{Tac}(\mathrm{v})$ & ch2vc & 7.14E-05 & $2.88 \mathrm{E}-13$ & $2.88 \mathrm{E}-13$ & 0.01 & 1.17E-03 & 0.492 & 4.89E-01 \\
\hline $\operatorname{Tac}(v)$ & ch3vc & 7.14E-05 & $2.88 \mathrm{E}-13$ & $2.88 \mathrm{E}-13$ & 0.01 & 1.17E-03 & 0.492 & 4.89E-01 \\
\hline Tabt1 & ch4vc & 7.14E-05 & $2.88 \mathrm{E}-13$ & $2.88 \mathrm{E}-13$ & 0.01 & 1.17E-03 & 0.492 & 4.89E-01 \\
\hline $\operatorname{Tcp}(3)$ & pp3vp & 7.14E-05 & $6.92 \mathrm{E}-13$ & $6.92 \mathrm{E}-13$ & 0.01 & $1.41 \mathrm{E}-03$ & 0.492 & 5.13E-04 \\
\hline $\operatorname{Tcb}(w)$ & bf3vb & 7.14E-05 & $6.92 \mathrm{E}-13$ & $6.92 \mathrm{E}-13$ & 0.01 & $1.41 \mathrm{E}-03$ & 0.492 & 5.13E-04 \\
\hline $\operatorname{Tcb}(w)$ & tm3vt & 7.14E-05 & $6.92 \mathrm{E}-13$ & $6.92 \mathrm{E}-13$ & 0.01 & $1.41 \mathrm{E}-03$ & 0.492 & 5.13E-04 \\
\hline $\operatorname{Tcp}(2)$ & pp2zp & $1.10 \mathrm{E}-05$ & $6.46 \mathrm{E}-14$ & $6.46 \mathrm{E}-14$ & 0.01 & $3.72 \mathrm{E}-04$ & 0.492 & 4.89E-01 \\
\hline $\operatorname{Tcb}(\mathrm{n})$ & bf2zb & $1.10 \mathrm{E}-05$ & $6.46 \mathrm{E}-14$ & $6.46 \mathrm{E}-14$ & 0.01 & $3.72 \mathrm{E}-04$ & 0.492 & 4.89E-01 \\
\hline
\end{tabular}

$\mathrm{S}_{\mathrm{r}}$ is the residual liquid-phase saturation (not in situ saturation).

** $\quad \alpha$ and $\mathrm{m}$ are fitting parameters for capillary pressure and relative permeability curves, respectively. 
Table 3-8 Matrix property values for the July 1997 TSPA-VA base-case hydrologic parameter set for $\mathrm{I} / 5$, where I stands for the nominal infiltration-flux map

\begin{tabular}{|c|c|c|c|c|c|c|}
\hline Geologic Unit & Model Unit & Porosity & Permeability $\left(m^{2}\right)$ & $S_{r}^{*}$ & $\alpha_{m}\left(\mathbf{P a}^{-1}\right) * *$ & $\boldsymbol{m} * *$ \\
\hline Трср11 & tcw11 & 0.066 & $5.40 \mathrm{E}-18$ & 0.13 & $1.15 \mathrm{E}-06$ & 0.232 \\
\hline Tpcpln & tcw12 & 0.066 & $5.40 \mathrm{E}-18$ & 0.13 & $1.29 \mathrm{E}-06$ & 0.231 \\
\hline Tpcpv & tcw13 & 0.140 & $5.00 \mathrm{E}-17$ & 0.33 & 7.30E-07 & 0.426 \\
\hline Tpcpv1 & ptn21 & 0.369 & $1.60 \mathrm{E}-14$ & 0.10 & $3.65 E-05$ & 0.228 \\
\hline Tpy & ptn22 & 0.234 & $3.30 \mathrm{E}-15$ & 0.14 & 7.56E-06 & 0.492 \\
\hline Tpbt3 & ptn23 & 0.353 & $5.40 \mathrm{E}-14$ & 0.17 & 3.66E-05 & 0.279 \\
\hline Tpp & ptn24 & 0.469 & $8.80 \mathrm{E}-14$ & 0.10 & $4.30 \mathrm{E}-05$ & 0.326 \\
\hline Tpbt2 & ptn25 & 0.464 & $1.27 \mathrm{E}-13$ & 0.10 & 1.96E-04 & 0.272 \\
\hline Tptrv & tsw31 & 0.042 & $6.87 \mathrm{E}-16$ & 0.11 & $1.33 \mathrm{E}-05$ & 0.230 \\
\hline Tptrn & tsw32 & 0.146 & $3.63 \mathrm{E}-16$ & 0.04 & $2.31 \mathrm{E}-05$ & 0.278 \\
\hline Tptpul & tsw33 & 0.135 & $2.11 \mathrm{E}-17$ & 0.06 & $6.44 \mathrm{E}-06$ & 0.248 \\
\hline Tptpmn & tsw34 & 0.089 & $6.75 \mathrm{E}-18$ & 0.18 & $1.14 \mathrm{E}-06$ & 0.323 \\
\hline Tptp11 & tsw35 & 0.115 & $7.96 \mathrm{E}-18$ & 0.08 & $3.16 \mathrm{E}-06$ & 0.232 \\
\hline Tptpln & tsw36 & 0.092 & $9.62 \mathrm{E}-17$ & 0.18 & $6.92 \mathrm{E}-07$ & 0.414 \\
\hline Tptpv & tsw37 & 0.020 & $6.69 \mathrm{E}-18$ & 0.50 & $1.34 \mathrm{E}-06$ & 0.372 \\
\hline Tpbt1 & ch1zc & 0.193 & $6.00 \mathrm{E}-17$ & 0.36 & $6.90 \mathrm{E}-07$ & 0.359 \\
\hline $\operatorname{Tac}(z)$ & ch2zc & 0.240 & $1.87 \mathrm{E}-18$ & 0.20 & $2.49 \mathrm{E}-06$ & 0.221 \\
\hline $\operatorname{Tac}(z)$ & ch3zc & 0.240 & $1.87 \mathrm{E}-18$ & 0.20 & $2.49 \mathrm{E}-06$ & 0.221 \\
\hline Tacbt & ch4zc & 0.169 & $1.33 \mathrm{E}-17$ & 0.33 & 7.83E-07 & 0.476 \\
\hline Tpbt1 & ch1vc & 0.265 & $1.60 \mathrm{E}-12$ & 0.04 & $9.80 \mathrm{E}-05$ & 0.187 \\
\hline $\operatorname{Tac}(\mathrm{v})$ & ch2vc & 0.321 & $5.50 \mathrm{E}-14$ & 0.06 & 8.65E-05 & 0.222 \\
\hline $\operatorname{Tac}(\mathrm{v})$ & ch3vc & 0.321 & $5.50 \mathrm{E}-14$ & 0.06 & 8.65E-05 & 0.222 \\
\hline Tabt1 & ch4vc & 0.321 & $5.50 \mathrm{E}-14$ & 0.06 & 8.65E-05 & 0.222 \\
\hline $\operatorname{Tcp}(3)$ & pp3vp & 0.274 & $1.24 \mathrm{E}-15$ & 0.07 & $1.81 \mathrm{E}-05$ & 0.310 \\
\hline $\operatorname{Tcb}(w)$ & bf3vb & 0.274 & $1.24 \mathrm{E}-15$ & 0.07 & $1.81 \mathrm{E}-05$ & 0.310 \\
\hline $\operatorname{Tcb}(w)$ & tm3vt & 0.274 & $1.24 \mathrm{E}-15$ & 0.07 & $1.81 \mathrm{E}-05$ & 0.310 \\
\hline $\operatorname{Tcp}(2)$ & pp2zp & 0.197 & $9.40 \mathrm{E}-18$ & 0.18 & $1.71 \mathrm{E}-06$ & 0.312 \\
\hline $\operatorname{Tcb}(n)$ & bf2zb & 0.197 & $9.40 \mathrm{E}-18$ & 0.18 & $1.71 \mathrm{E}-06$ & 0.312 \\
\hline
\end{tabular}

$S_{\mathrm{r}}$ is the residual liquid-phase saturation (not in situ saturation).

** $\quad \alpha$ and $m$ are fitting parameters for capillary pressure and relative permeability curves, respectively. 
Table 3-9 Fracture property values for the July 1997 TSPA-VA base-case hydrologic parameter set for $I / 5$, where I stands for the nominal infiltration-flux map

\begin{tabular}{|c|c|c|c|c|c|c|c|c|}
\hline \multirow{2}{*}{$\begin{array}{c}\text { Geologic } \\
\text { Unit }\end{array}$} & \multirow{2}{*}{$\begin{array}{l}\text { Model } \\
\text { Unit }\end{array}$} & \multirow[t]{2}{*}{ Porosity } & \multicolumn{2}{|c|}{ Permeability $\left(\mathbf{m}^{2}\right)$} & \multirow[t]{2}{*}{$S_{\mathrm{r}} *$} & \multirow{2}{*}{$\begin{array}{l}\alpha * * \\
\left(\mathrm{~Pa}^{-1}\right)\end{array}$} & \multirow[t]{2}{*}{$\boldsymbol{m} * *$} & \multirow[t]{2}{*}{ FMX } \\
\hline & & & Vertical & Horizontal & & & & \\
\hline Tpcp11 & tcw11 & 2.33E-04 & $2.29 \mathrm{E}-11$ & $6.03 \mathrm{E}-12$ & 0.01 & $2.95 \mathrm{E}-04$ & 0.492 & $4.90 \mathrm{E}-04$ \\
\hline Tpcpln & tcw12 & 2.99E-04 & $1.38 \mathrm{E}-11$ & $6.03 \mathrm{E}-12$ & 0.01 & 2.95E-04 & 0.492 & $4.90 \mathrm{E}-04$ \\
\hline Tpcpv & tcw13 & 7.05E-05 & $2.82 \mathrm{E}-12$ & $2.40 \mathrm{E}-13$ & 0.01 & $9.12 \mathrm{E}-05$ & 0.492 & $4.90 \mathrm{E}-04$ \\
\hline Tpcpv1 & ptn21 & 4.84E-05 & $5.25 \mathrm{E}-13$ & $5.25 \mathrm{E}-13$ & 0.01 & $1.10 \mathrm{E}-03$ & 0.492 & $1.10 \mathrm{E}-01$ \\
\hline Tpy & ptn22 & 4.83E-05 & $1.95 \mathrm{E}-13$ & $1.95 \mathrm{E}-13$ & 0.01 & $1.82 \mathrm{E}-03$ & 0.492 & 7.08E-01 \\
\hline Tpbt3 & ptn23 & $1.30 \mathrm{E}-04$ & $2.57 \mathrm{E}-13$ & $2.57 \mathrm{E}-13$ & 0.01 & 3.39E-03 & 0.492 & $6.92 \mathrm{E}-01$ \\
\hline Tpp & ptn24 & $6.94 \mathrm{E}-05$ & $6.17 \mathrm{E}-14$ & $6.17 \mathrm{E}-14$ & 0.01 & 9.33E-04 & 0.492 & 4.79E-01 \\
\hline Tpbt2 & ptn25 & 3.86E-05 & 7.76E-14 & $7.76 \mathrm{E}-14$ & 0.10 & $1.95 \mathrm{E}-04$ & 0.279 & 4.79E-01 \\
\hline Tptrv & tsw31 & 8.92E-05 & $1.07 \mathrm{E}-11$ & $1.00 \mathrm{E}-12$ & 0.01 & 3.98E-05 & 0.481 & 5.01E-01 \\
\hline Tptrn & tsw32 & $1.29 \mathrm{E}-04$ & $1.51 \mathrm{E}-11$ & $7.08 \mathrm{E}-13$ & 0.01 & 9.33E-05 & 0.488 & $2.88 \mathrm{E}-05$ \\
\hline Tptpul & tsw33 & $1.05 \mathrm{E}-04$ & 2.63E-11 & 8.91E-13 & 0.01 & $1.78 \mathrm{E}-04$ & 0.492 & 7.94E-05 \\
\hline Tptpmn & tsw34 & $1.24 \mathrm{E}-04$ & $6.76 \mathrm{E}-12$ & $4.27 \mathrm{E}-13$ & 0.01 & 9.77E-05 & 0.492 & $1.55 \mathrm{E}-04$ \\
\hline Tptp11 & tsw35 & $3.29 \mathrm{E}-04$ & $3.80 \mathrm{E}-12$ & $9.12 \mathrm{E}-13$ & 0.01 & 1.10E-04 & 0.492 & 7.76E-02 \\
\hline Tptpln & tsw36 & 3.99E-04 & $1.20 \mathrm{E}-12$ & 1.20E-12 & 0.01 & $1.32 \mathrm{E}-04$ & 0.492 & 4.79E-05 \\
\hline Tptpv & tsw37 & 4.92E-04 & $1.20 \mathrm{E}-12$ & $1.20 \mathrm{E}-12$ & 0.01 & 1.19E-04 & 0.492 & 4.90E-04 \\
\hline Tpbt1 & ch1zc & $1.10 \mathrm{E}-05$ & $2.51 \mathrm{E}-14$ & $2.40 \mathrm{E}-14$ & 0.01 & $1.14 \mathrm{E}-03$ & 0.492 & $1.82 \mathrm{E}-01$ \\
\hline $\operatorname{Tac}(z)$ & ch2zc & 1.10E-05 & $2.51 \mathrm{E}-14$ & 1.17E-14 & 0.01 & $1.14 \mathrm{E}-03$ & 0.492 & $1.00 \mathrm{E}+00$ \\
\hline $\operatorname{Tac}(z)$ & ch3zc & $1.10 \mathrm{E}-05$ & $2.51 \mathrm{E}-14$ & 1.17E-14 & 0.01 & $1.14 \mathrm{E}-03$ & 0.492 & $1.00 \mathrm{E}+00$ \\
\hline Tacbt & ch4zc & 1.10E-05 & $2.51 \mathrm{E}-14$ & $1.55 \mathrm{E}-14$ & 0.01 & $1.14 \mathrm{E}-03$ & 0.492 & $1.00 \mathrm{E}+00$ \\
\hline Tpbt1 & ch1ve & 7.14E-05 & $1.74 \mathrm{E}-13$ & $1.74 \mathrm{E}-13$ & 0.01 & $1.18 \mathrm{E}-03$ & 0.492 & $4.90 \mathrm{E}-01$ \\
\hline $\operatorname{Tac}(\mathrm{v})$ & $\mathrm{ch} 2 \mathrm{vc}$ & 7.14E-05 & $2.88 \mathrm{E}-13$ & $2.88 \mathrm{E}-13$ & 0.01 & $1.18 \mathrm{E}-03$ & 0.492 & 4.89E-01 \\
\hline $\operatorname{Tac}(v)$ & ch3vc & 7.14E-05 & $2.88 \mathrm{E}-13$ & $2.88 \mathrm{E}-13$ & 0.01 & $1.18 \mathrm{E}-03$ & 0.492 & 4.89E-01 \\
\hline Tabt1 & $\mathrm{ch} 4 \mathrm{vc}$ & 7.14E-05 & $2.88 \mathrm{E}-13$ & $2.88 \mathrm{E}-13$ & 0.01 & $1.18 \mathrm{E}-03$ & 0.492 & 4.89E-01 \\
\hline $\operatorname{Tcp}(3)$ & pp3vp & 7.14E-05 & $7.08 \mathrm{E}-13$ & $6.92 \mathrm{E}-13$ & 0.01 & $1.42 \mathrm{E}-03$ & 0.492 & $5.13 \mathrm{E}-04$ \\
\hline $\operatorname{Tcb}(w)$ & bf3vb & 7.14E-05 & $7.08 \mathrm{E}-13$ & $6.92 \mathrm{E}-13$ & 0.01 & $1.42 \mathrm{E}-03$ & 0.492 & $5.13 \mathrm{E}-04$ \\
\hline $\mathrm{Tcb}(\mathrm{w})$ & tm3vt & 7.14E-05 & $7.08 \mathrm{E}-13$ & $6.92 \mathrm{E}-13$ & 0.01 & $1.42 \mathrm{E}-03$ & 0.492 & $5.13 \mathrm{E}-04$ \\
\hline $\operatorname{Tcp}(2)$ & pp2zp & $1.10 \mathrm{E}-05$ & $2.51 \mathrm{E}-14$ & $6.46 \mathrm{E}-14$ & 0.01 & $1.14 \mathrm{E}-03$ & 0.492 & 4.89E-01 \\
\hline $\operatorname{Tcb}(\mathrm{n})$ & bf2zb & 1.10E-05 & $2.51 \mathrm{E}-14$ & $6.46 \mathrm{E}-14$ & 0.01 & 1.14E-03 & 0.492 & 4.89E-01 \\
\hline
\end{tabular}

$S_{\mathrm{r}}$ is the residual liquid-phase saturation (not in situ saturation).

** $\quad \alpha$ and $m$ are fitting parameters for capillary pressure and relative permeability curves, respectively. 
Table 3-10 Matrix property values for the November $1997 \mathrm{TH}$ hydrologic parameter set calibrated against the SHT

\begin{tabular}{|c|c|c|c|c|c|c|}
\hline Geologic Unit & Model Unit & Porosity & Permeability $\left(\mathrm{m}^{2}\right)$ & $S_{r} *$ & $\alpha_{m}\left(\mathbf{P a}^{-1}\right) * *$ & $\boldsymbol{m} * *$ \\
\hline Трср11 & tcw11 & 0.066 & $5.40 \mathrm{E}-18$ & 0.13 & 1.15E-06 & 0.2310 \\
\hline Tpcpln & tcw12 & 0.066 & $5.40 \mathrm{E}-18$ & 0.13 & 2.01E-06 & 0.2447 \\
\hline Tpcpv & tcw13 & 0.140 & $5.69 \mathrm{E}-17$ & 0.33 & $3.74 \mathrm{E}-06$ & 0.4548 \\
\hline Tpcpv1 & ptn21 & 0.369 & $1.61 \mathrm{E}-14$ & 0.10 & 3.98E-05 & 0.2531 \\
\hline Tpy & ptn22 & 0.234 & 3.30E-15 & 0.14 & 7.94E-06 & 0.4925 \\
\hline Tpbt3 & ptn23 & 0.353 & $5.40 \mathrm{E}-14$ & 0.17 & 5.44E-05 & 0.3002 \\
\hline Tpp & ptn24 & 0.469 & 8.80E-14 & 0.10 & 3.43E-05 & 0.3859 \\
\hline Tpbt2 & ptn25 & 0.464 & $3.18 \mathrm{E}-13$ & 0.10 & 1.81E-04 & 0.3195 \\
\hline Tptrv & tsw31 & 0.042 & 7.76E-17 & 0.11 & 5.84E-05 & 0.2304 \\
\hline Tptrn & tsw32 & 0.146 & $1.82 \mathrm{E}-16$ & 0.04 & 2.00E-05 & 0.2861 \\
\hline Tptpul & tsw33 & 0.135 & $2.04 \mathrm{E}-17$ & 0.06 & $6.21 \mathrm{E}-06$ & 0.2479 \\
\hline Tptpmn & tsw34 & 0.089 & $4.08 \mathrm{E}-18$ & 0.18 & 1.19E-06 & 0.3212 \\
\hline Tptp11 & tsw35 & 0.115 & $2.22 \mathrm{E}-17$ & 0.08 & 4.01E-06 & 0.1983 \\
\hline Tptpln & tsw36 & 0.092 & 8.70E-18 & 0.18 & 8.08E-07 & 0.5138 \\
\hline Tptpv & tsw37 & 0.020 & 8.39E-18 & 0.50 & 5.30E-07 & 0.3709 \\
\hline Tpbt1 & ch1zc & 0.193 & $1.36 \mathrm{E}-17$ & 0.36 & 4.29E-06 & 0.3489 \\
\hline $\operatorname{Tac}(z)$ & ch2zc & 0.240 & $2.50 \mathrm{E}-18$ & 0.20 & 2.16E-05 & 0.2119 \\
\hline $\operatorname{Tac}(z)$ & ch3zc & 0.240 & $2.50 \mathrm{E}-18$ & 0.20 & 2.16E-05 & 0.2119 \\
\hline Tacbt & ch4zc & 0.169 & $5.49 \mathrm{E}-18$ & 0.33 & 1.03E-06 & 0.4322 \\
\hline Tpbt1 & ch1vc & 0.265 & $1.60 \mathrm{E}-12$ & 0.04 & 7.60E-05 & 0.1592 \\
\hline $\operatorname{Tac}(\mathrm{v})$ & ch2vc & 0.321 & $5.50 \mathrm{E}-14$ & 0.06 & 4.12E-05 & 0.2291 \\
\hline $\operatorname{Tac}(\mathrm{v})$ & ch3vc & 0.321 & $5.50 \mathrm{E}-14$ & 0.06 & 4.12E-05 & 0.2291 \\
\hline Tabt1 & ch4vc & 0.321 & $5.50 \mathrm{E}-14$ & 0.06 & 4.12E-05 & 0.2291 \\
\hline Tср(3) & pp3vp & 0.274 & $1.91 \mathrm{E}-15$ & 0.07 & 1.66E-05 & 0.3142 \\
\hline $\operatorname{Tcb}(\mathrm{w})$ & bf3vb & 0.274 & $1.91 \mathrm{E}-15$ & 0.07 & 1.66E-05 & 0.3142 \\
\hline $\operatorname{Tcb}(w)$ & tm3vt & 0.274 & $1.91 \mathrm{E}-15$ & 0.07 & 1.66E-05 & 0.3142 \\
\hline Tcp(2) & pp2zp & 0.197 & $1.75 \mathrm{E}-17$ & 0.18 & 8.39E-06 & 0.3568 \\
\hline $\operatorname{Tcb}(\mathrm{n})$ & bf2zb & 0.197 & $1.75 \mathrm{E}-17$ & 0.18 & 8.39E-06 & 0.3568 \\
\hline
\end{tabular}

$S_{\mathrm{r}}$ is the residual liquid-phase saturation (not in situ saturation).

** $\quad \alpha$ and $m$ are fitting parameters for capillary pressure and relative permeability curves, respectively. 
Table 3-11 Fracture property values for the November $1997 \mathrm{TH}$ hydrologic parameter set that was calibrated against the SHT.

\begin{tabular}{|c|c|c|c|c|c|c|c|c|}
\hline \multirow[t]{2}{*}{$\begin{array}{c}\text { Geological } \\
\text { Unit }\end{array}$} & \multirow[t]{2}{*}{$\begin{array}{l}\text { Model } \\
\text { Unit }^{\mathrm{a}}\end{array}$} & \multirow[t]{2}{*}{ Porosity } & \multicolumn{2}{|c|}{$\begin{array}{c}\text { Permeability } \\
\left(\mathrm{m}^{2}\right)\end{array}$} & \multirow[t]{2}{*}{$S_{r}^{b}$} & \multirow[t]{2}{*}{$\begin{array}{c}\alpha^{c} \\
\left(\mathrm{~Pa}^{-1}\right)\end{array}$} & \multirow[t]{2}{*}{$m^{c}$} & \multirow[t]{2}{*}{ FMX } \\
\hline & & & Vertical & Horizontal & & & & \\
\hline Tpсp11 & tcw11 & 2.33E-04 & 2.29E-11 & $6.03 \mathrm{E}-12$ & 0.01 & 2.37E-03 & 0.667 & 5.00E-04 \\
\hline Tpcpln & tcw12 & 2.99E-04 & $1.38 \mathrm{E}-11$ & $6.03 \mathrm{E}-12$ & 0.01 & 2.37E-03 & 0.669 & 5.00E-04 \\
\hline Tpcpv & tcw13 & 7.05E-05 & 2.82E-12 & $2.40 \mathrm{E}-13$ & 0.01 & $9.12 \mathrm{E}-04$ & 0.669 & 5.00E-04 \\
\hline Tpcpv1 & ptn21 & 4.84E-05 & $5.25 \mathrm{E}-13$ & $5.25 \mathrm{E}-13$ & 0.01 & 1.10E-03 & 0.669 & 5.02E-01 \\
\hline Tpy & ptn22 & 4.83E-05 & $1.95 \mathrm{E}-13$ & $1.95 \mathrm{E}-13$ & 0.01 & $1.85 \mathrm{E}-03$ & 0.669 & 5.00E-01 \\
\hline Tpbt3 & ptn23 & $1.30 \mathrm{E}-04$ & $2.57 \mathrm{E}-13$ & $2.57 \mathrm{E}-13$ & 0.01 & $3.45 \mathrm{E}-03$ & 0.667 & $5.00 \mathrm{E}-01$ \\
\hline Tpp & ptn24 & 6.94E-05 & $6.17 \mathrm{E}-14$ & $6.17 \mathrm{E}-14$ & 0.01 & $9.13 \mathrm{E}-04$ & 0.667 & $5.00 \mathrm{E}-01$ \\
\hline Tpbt2 & ptn25 & 3.86E-05 & 7.76E-14 & $7.76 \mathrm{E}-14$ & 0.10 & $1.81 \mathrm{E}-04$ & 0.320 & 5.00E-01 \\
\hline Tptrv & tsw31 & 8.92E-05 & $1.07 \mathrm{E}-11$ & $1.00 \mathrm{E}-12$ & 0.01 & $1.44 \mathrm{E}-04$ & 0.566 & 4.68E-01 \\
\hline Tptrn & tsw32 & 1.29E-04 & $1.51 \mathrm{E}-11$ & $7.08 \mathrm{E}-13$ & 0.01 & 1.42E-03 & 0.667 & $5.00 \mathrm{E}-04$ \\
\hline Tptpul & tsw33 & $1.05 \mathrm{E}-04$ & 2.63E-11 & 8.91E-13 & 0.01 & 1.73E-03 & 0.667 & $5.00 \mathrm{E}-04$ \\
\hline Tptpmn & tsw34 & 1.24E-04 & $6.76 \mathrm{E}-12$ & $4.27 \mathrm{E}-13$ & 0.01 & 9.34E-04 & 0.643 & 1.23E-03 \\
\hline Tptp11 & tsw35 & $3.29 E-04$ & $3.80 \mathrm{E}-12$ & $9.12 \mathrm{E}-13$ & 0.01 & $1.26 \mathrm{E}-03$ & 0.667 & $5.00 \mathrm{E}-04$ \\
\hline Tptpln & tsw36 & 3.99E-04 & $1.20 \mathrm{E}-12$ & $1.20 \mathrm{E}-12$ & 0.01 & 1.32E-03 & 0.667 & $5.00 \mathrm{E}-04$ \\
\hline Tptpv & tsw37 & 4.92E-04 & $1.20 \mathrm{E}-12$ & $1.20 \mathrm{E}-12$ & 0.01 & 1.19E-03 & 0.659 & 5.00E-04 \\
\hline Tpbt1 & ch1zc & 1.10E-05 & $2.51 \mathrm{E}-14$ & $2.40 \mathrm{E}-14$ & 0.01 & 1.14E-03 & 0.667 & 5.00E-01 \\
\hline $\operatorname{Tac}(z)$ & ch2zc & 1.10E-05 & $2.51 \mathrm{E}-14$ & 1.17E-14 & 0.01 & 1.12E-03 & 0.654 & 9.22E-01 \\
\hline $\operatorname{Tac}(z)$ & ch3zc & 1.10E-05 & $2.51 \mathrm{E}-14$ & 1.17E-14 & 0.01 & 1.12E-03 & 0.654 & $9.22 \mathrm{E}-01$ \\
\hline Tacbt & ch4zc & 1.10E-05 & $2.51 \mathrm{E}-14$ & $1.55 \mathrm{E}-14$ & 0.01 & 1.14E-03 & 0.667 & 5.00E-01 \\
\hline Tpbt1 & ch1vc & 7.14E-05 & $1.74 \mathrm{E}-13$ & $1.74 \mathrm{E}-13$ & 0.01 & 1.18E-03 & 0.669 & 5.00E-01 \\
\hline $\mathrm{Tac}(\mathrm{v})$ & ch2vc & 7.14E-05 & $2.88 \mathrm{E}-13$ & $2.88 \mathrm{E}-13$ & 0.01 & $1.18 \mathrm{E}-03$ & 0.667 & $5.00 \mathrm{E}-01$ \\
\hline $\mathrm{Tac}(\mathrm{v})$ & ch3vc & 7.14E-05 & $2.88 \mathrm{E}-13$ & $2.88 \mathrm{E}-13$ & 0.01 & $1.18 \mathrm{E}-03$ & 0.667 & 5.00E-01 \\
\hline Tabt1 & ch4vc & 7.14E-05 & $2.88 \mathrm{E}-13$ & $2.88 \mathrm{E}-13$ & 0.01 & $1.18 \mathrm{E}-03$ & 0.667 & $5.00 \mathrm{E}-01$ \\
\hline $\mathrm{Tcp}(3)$ & pp3vp & 7.14E-05 & $7.08 \mathrm{E}-13$ & $6.92 \mathrm{E}-13$ & 0.01 & 1.42E-03 & 0.667 & $5.00 \mathrm{E}-04$ \\
\hline $\operatorname{Tcb}(\mathrm{w})$ & bf3vb & 7.14E-05 & $7.08 \mathrm{E}-13$ & $6.92 \mathrm{E}-13$ & 0.01 & 1.42E-03 & 0.667 & $5.00 \mathrm{E}-04$ \\
\hline $\mathrm{Tcb}(\mathrm{w})$ & tm3vt & 7.14E-05 & $7.08 \mathrm{E}-13$ & $6.92 \mathrm{E}-13$ & 0.01 & 1.42E-03 & 0.667 & $5.00 \mathrm{E}-04$ \\
\hline $\mathrm{Tcp}(2)$ & pp2zp & 1.10E-05 & $2.51 \mathrm{E}-14$ & $6.46 \mathrm{E}-14$ & 0.01 & $1.14 \mathrm{E}-03$ & 0.667 & 5.00E-01 \\
\hline $\operatorname{Tcb}(\mathrm{n})$ & bf2zb & 1.10E-05 & $2.51 \mathrm{E}-14$ & $6.46 \mathrm{E}-14$ & 0.01 & $1.14 \mathrm{E}-03$ & 0.667 & 5.00E-01 \\
\hline
\end{tabular}

$S_{\mathrm{r}} \mathrm{b}$ is the residual liquid-phase saturation (not in situ saturation).

$\alpha^{c}$ and $m$ are fitting parameters for capillary pressure and relative permeability curves, respectively. 
The parameter set actually used for the reference cases was developed at LLNL in December 1997 by modifying the TH set. Table 3-12 contains the matrix parameters in the tsw36 and tsw37 units for this "modified-TH" parameter set. Fracture properties, and matrix properties for other units, were not changed from values in the TH set. The modifications were made so that the value of $D_{\mathrm{imb}}$ for the tsw36 and tsw37 units are the same as those in the tsw34 unit. These changes were made because laboratory measurements from Flint et al. (1996b) indicate that the values of $D_{\text {imb }}$ for the tsw36 and tsw37 units are no greater than those for the tsw34 unit. The principal change was a reduction, by a factor of 28 , in the value of $D_{\text {imb }}$ for the basal vitrophyre (tsw37). The value for the tsw36 unit was reduced by a factor of 2.8 . Model calculations with these parameters indicate more rock dryout in both of these units than was obtained with the TH set.

Table 3-12 Matrix property values for the December 1997 "modified-TH" hydrologic parameter set for only the property values that differ from the November 1997 TH hydrologic parameter set

The December 1997 set was modified from the November 1997 TH set on the basis of the "modified-TH" measurements by Flint et al. (1996b).

\begin{tabular}{|c|c|c|c|c|c|c|}
\hline Geologic Unit & Model Unit & Porosity & Permeability $\left(\mathbf{m}^{2}\right)$ & $\mathbf{S}_{\mathbf{r}} *$ & $\alpha_{\mathbf{m}}\left(\mathbf{P a}^{-1}\right) * *$ & $\boldsymbol{m} * *$ \\
\hline \hline Tptpln & tsw36 & 0.092 & $8.70 \mathrm{E}-18$ & 0.18 & $2.272 \mathrm{E}-6$ & 0.5138 \\
\hline Tptpv & tsw37 & 0.02 & $4.080 \mathrm{E}-18$ & 0.5 & $7.3856 \mathrm{E}-6$ & 0.3709 \\
\hline
\end{tabular}

$S_{\mathrm{r}}$ is the residual liquid-phase saturation (not in situ saturation).

** $\quad \alpha$ and $m$ are fitting parameters for capillary pressure and relative permeability curves, respectively.

Table 3-13 summarizes the values of imbibition diffusivity for the host-rock and neighboring units based on the different hydrologic parameter sets discussed.

Table 3-13 The value of matrix wetting diffusivity $D_{\text {imb }}$ for the host-rock units and neighboring units, for various hydrologic parameter sets $\left(\mathrm{m}^{2} / \mathrm{sec}\right)$

The value of $D_{\text {imb }}$ is determined for an assumed $S_{\text {liq }}$ of $90 \%$.

\begin{tabular}{|c|c|c|c|c|c|}
\hline $\begin{array}{c}\text { Host-Rock } \\
\text { Model Unit }\end{array}$ & \multicolumn{2}{|c|}{$\mathbf{1 1 / 9 7}$ TSPA-VA Base-Case Parameter Set } & $\begin{array}{c}\mathbf{1 1 / 9 7} \text { TH } \\
\text { Parameter Set }\end{array}$ & $\begin{array}{c}\mathbf{1 2 / 9 7} \text { Modified-TH } \\
\text { Parameter Set }\end{array}$ \\
\hline \hline & $\mathbf{I} / \mathbf{5}$ & $\mathbf{I} \times \mathbf{1}$ & $\mathbf{I} \times \mathbf{5}$ & & \\
\hline tsw33 & $1.15 \mathrm{E}-07$ & $5.98 \mathrm{E}-08$ & $1.30 \mathrm{E}-07$ & $1.15 \mathrm{E}-07$ & $1.15 \mathrm{E}-07$ \\
\hline tsw34 & $3.22 \mathrm{E}-07$ & $2.17 \mathrm{E}-07$ & $2.18 \mathrm{E}-07$ & $1.86 \mathrm{E}-07$ & $1.86 \mathrm{E}-07$ \\
\hline tsw35 & $1.09 \mathrm{E}-07$ & $2.03 \mathrm{E}-07$ & $2.10 \mathrm{E}-07$ & $2.65 \mathrm{E}-07$ & $2.65 \mathrm{E}-07$ \\
\hline tsw36 & $7.31 \mathrm{E}-06$ & $6.32 \mathrm{E}-06$ & $3.56 \mathrm{E}-06$ & $5.33 \mathrm{E}-07$ & $1.86 \mathrm{E}-07$ \\
\hline tsw37 & $1.63 \mathrm{E}-06$ & $2.70 \mathrm{E}-06$ & $3.37 \mathrm{E}-06$ & $5.23 \mathrm{E}-06$ & $1.86 \mathrm{E}-07$ \\
\hline
\end{tabular}




\subsubsection{TH Model Results for Analysis of Coupled Effects}

Figure 3-15 (a through 1) shows contour plots around two drifts for temperature and liquid saturation in the $x-z$ plane for Case 1 at $t=50,100,500,1000,2000$, and $5000 \mathrm{yr}$, respectively. Two drifts are also depicted in similar plots for the other point-load cases (Cases 2-4) in Figure 3-16, Figure 3-17, and Figure 3-18. A single drift is shown in Figure 3-19 (a through 1) for the line-load design (Case 5).

Figure 3-15 Temperature and liquid-phase saturation distributions for Case 1

Figure 3-16 Temperature and liquid-phase saturation distributions for Case 2

Figure 3-17 Temperature and liquid-phase saturation distributions for Case 3

Figure 3-18 Temperature and liquid-phase saturation distributions for Case 4

\section{Figure 3-19 Temperature and liquid-phase saturation distributions for Case 5}

The evolution of the temperature distribution indicates the degree of coalescence of rock dryout zones between drifts for the point-load design option (Cases 1-4). Considerably more coalescence occurs for $q_{\text {inf }}=3.2$ than for $q_{\text {inf }}=16.0$ (compare, for example, the 500-yr isotherms: Figure 3-15f with Figure 3-16f and Figure 3-17f with Figure 3-18f) for both sets of hydrologic parameters. The $100^{\circ} \mathrm{C}$ isotherm coalesces between the drifts for both Case 2 and Case 4 . At the nominal infiltration rate of $16.0 \mathrm{~mm} / \mathrm{yr}$, coalescence is minimal. For Case 1 , the $100^{\circ} \mathrm{C}$ isotherms from adjacent drifts never coalesce (Figure 3-15); for Case 3, they coalesce briefly, as shown at $50 \mathrm{yr}$ in Figure 3-17b.

For large (56.6-m) drift spacing in the line-load design (Case 5), the dryout zones (and the $100^{\circ} \mathrm{C}$ isotherm) never coalesce between the drifts (Figure 3-19). The heat-flux density, which is greater by a factor of 2 along the line-load drifts (Case 5), as compared to the point-load drifts (Cases 1-4), results in much higher $T$ rise (and a much steeper $T$ gradient) in the vicinity of the drifts than it does in any of the point-load cases.

Flow of liquid mobilized by decay-heat flow results in a heat-pipe zone above the repository horizon and a condensate-drainage zone below. The vertical distribution of vertical liquid-phase flux $q_{\mathrm{liq}}$ in the fractures at the drift and pillar centerlines (Figure 3-20 and Table 3-14) clearly shows the vertical extent of these two zones and shows the ability of condensate to drain between the drifts. Within $100 \mathrm{yr}$, the condensate-drainage zone reaches the water table, $342 \mathrm{~m}$ below the repository horizon (Figure 3-20). The condensate drainage zone below the repository and the heat-pipe zone above the repository are manifested as sharply defined regions where $q_{\text {liq }}>q_{\text {perc }}$ where $q_{\text {perc }}$ is the ambient percolation flux.

Table 3-15 and Table 3-14 give the length of the heat-pipe zone in the fractures and the magnitude of $q_{\mathrm{liq}}$ in the fractures, respectively. The magnitude of $q_{\mathrm{liq}}$ in the heat-pipe and condensate-drainage zones is greatest immediately after WP emplacement and decays continually until it reaches the value of the background ambient percolation flux $q_{\text {perc }}$.

Figure 3-20 Vertical distribution of liquid-phase flux $q_{\text {liq }}$ for Cases 3 and 5 
Table 3-14 The magnitude of liquid-phase flux $q_{\mathrm{liq}}(\mathrm{mm} / \mathrm{yr})$ in fractures in heat-pipe zone above repository horizon

\begin{tabular}{|l|c|c|c|c|c|c|c|}
\cline { 2 - 9 } \multicolumn{1}{c|}{} & \multicolumn{7}{c|}{ Time (yr) } \\
\hline Design & $\mathbf{0}$ & $\mathbf{5 0}$ & $\mathbf{1 0 0}$ & $\mathbf{5 0 0}$ & $\mathbf{1 0 0 0}$ & $\mathbf{2 0 0 0}$ & $\mathbf{5 0 0 0}$ \\
\hline \hline point load & 16.0 & 118 & 82 & 35 & 29 & 22 & 16.0 \\
\hline line load & 16.0 & 118 & 82 & 35 & 29 & 22 & 16.0 \\
\hline
\end{tabular}

Table 3-15 The vertical length $\operatorname{Lhp}(\mathrm{m})$ of the heat-pipe-zone

\begin{tabular}{|l|c|c|c|c|c|c|c|}
\cline { 2 - 8 } \multicolumn{1}{c|}{} & \multicolumn{7}{c|}{ Time (yr) } \\
\hline \multicolumn{1}{c|}{ Design } & $\mathbf{0}$ & $\mathbf{5 0}$ & $\mathbf{1 0 0}$ & $\mathbf{5 0 0}$ & $\mathbf{1 0 0 0}$ & $\mathbf{2 0 0 0}$ & $\mathbf{5 0 0 0}$ \\
\hline \hline point load & 0 & 72 & 110 & 210 & 210 & 187 & 0 \\
\hline line load & 0 & 55 & 88 & 210 & 210 & 187 & 0 \\
\hline
\end{tabular}

Although the magnitude of $q_{\text {liq }}$ in the heat-pipe zone continually declines, the length of the heat-pipe zone continues to expand during the first $500 \mathrm{yr}$ (on average). The length of the heat-pipe zone and the time required to attain its maximum length are strongly influenced by the depth of the repository below the ground surface (i.e., overburden thickness). The vertical length of the heat-pipe zone, as well as the time required to attain its maximum length, increases with repository depth $Z_{\text {rep. }}$. Thus, regions of the repository that underlie the crest of Yucca Mountain have taller heat-pipe zones than do shallower regions such as at the eastern side of the repository. Once the heat-pipe zone reaches its maximum length, this length is maintained for about $1000 \mathrm{yr}$ (500 yr to $1500 \mathrm{yr}$ ); thereafter, the upper extent of the heat-pipe zone slowly recedes back to the repository horizon. At the repository edge, the maximum heat-pipe-zone length is considerably less than it is at locations near the center.

The magnitude of $q_{\mathrm{liq}}$ in the heat-pipe zone is the same for the point- and line-load designs (Figure 3-20 and Table 3-14). The magnitude of $q_{\mathrm{liq}}$ in the condensate-drainage zone below the repository horizon is greater for the line-load design than it is for the point-load design during the first $100 \mathrm{yr}$; thereafter, the magnitude of $q_{\text {liq }}$ below the repository horizon is the same for the point- and line-load designs. For the point-load design, $q_{\mathrm{liq}}$ decreases to zero at the pillar centerline of the repository horizon from 50 to $100 \mathrm{yr}$. Thereafter, it increases and remains above zero. For the line-load design, $q_{\text {liq }}$ is always nonzero at the pillar centerline. Therefore, the line-load design maintains more efficient condensate drainage between drifts than does the point-load design. The higher early-time $q_{\text {liq }}$ in the condensate-drainage zone below the line-load drifts is the result of more efficient condensate drainage between the more widely spaced drifts of the line-load design.

The vertical distribution of $T$ and $S_{\text {liq }}$ (Figure 3-21) clearly indicates the vertical extent of the heat-pipe zone. The upper extent of the heat-pipe zone is indicated by the upper extent of the $96^{\circ} \mathrm{C}$ isotherm, which reaches the contact between the ptn22 and ptn23 units. Recall that the upper extent of heat-mobilized $q_{\text {liq }}$ in fractures extends up to the contact between the ptn25 and tsw31 (Figure 3-20). Therefore, the heat-pipe zone extends into the ptn25, ptn24, ptn23, and ptn22 units as a result of matrix flow in the ptn22-25 units, which have large enough matrix permeability to accommodate the increase in liquid flux arising from thermally driven evaporation and condensation. Consequently, $q_{\mathrm{liq}}$ is zero in the fractures in 
the ptn22-25 interval (Figure 3-20). Immediately above the drift, the lower boundary of the heat-pipe zone is indicated by a superheated region $\left(T>96^{\circ} \mathrm{C}\right)$ as well as by the upper limit of the dryout front, with reduced liquid saturation (Figure 3-21).

\section{Figure 3-21 Vertical temperature and liquid-phase saturation distributions for Cases 3 and 5}

The relatively large percolation flux $\left(q_{\text {perc }}=16.0 \mathrm{~mm} / \mathrm{yr}\right)$ for Case 3 and Case 5 restricts the vertical extent of rock dryout (Figure 3-17, Figure 3-19, and Figure 3-21) above the drifts. Above the drifts, the line-load design maintains a larger dryout zone than does the point-load design. Below the drifts, both the point- and line-load designs result in partial rock dryout throughout the lower nonlithophysal (tsw36) unit and the entire basal vitrophyre (tsw37) unit. However, $S_{\text {liq }}$ is reduced more in the tsw36 and tsw37 for the line-load design than it is for the point-load design, particularly in the tsw36 (Figure 3-21).

The following general observations can be made about the TH behavior modeled in these reference calculations:

- $\quad \boldsymbol{q}_{\mathrm{liq}}>>\boldsymbol{q}_{\mathrm{perc}}$ for early time $(t<100-500 \mathrm{yr})$ and then gradually declines to the magnitude of $q_{\text {perc }}$.

- $\boldsymbol{q}_{\mathrm{liq}}$ is insensitive to $\boldsymbol{q}_{\text {perc }}$ for early time $(t<200 \mathrm{yr})$. At $100 \mathrm{yr}, q_{\mathrm{liq}}$ is nearly the same for all of the cases considered (ranging from 108 to $118 \mathrm{~mm} / \mathrm{yr}$ ). Therefore, when $q_{\text {liq }}$ is at its greatest (i.e., for early time), $q_{\text {liq }}$ does not depend on the magnitude of $q_{\text {perc }}$. For later time ( $t>200 \mathrm{yr}$ ), the magnitude of $q_{\text {liq }}$ does increase with $q_{\text {perc }}$.

- $q_{\mathrm{liq}}$ is the same for the point- and line-load designs. The magnitude of $q_{\text {liq }}$ depends only on the total heat flux that is available to mobilize water and not on how efficiently condensate sheds around the drifts. The more efficient condensate shedding for the line-load design results in less return flow of condensate to the drift from the previous refluxing cycle. The heat that is not required to reboil the condensate that has shed off to the sides is, in effect, "excess" heat, which is available to mobilize additional water vapor from the rock matrix. Thus, the total return flow of condensate toward the drift comprises water from the previous refluxing cycle and water vapor that has just been mobilized from the rock matrix and condensed in the fractures. The only difference between the point- and line-load designs is that a larger fraction (compared with the point load) of the heat for the line load is available for rock dryout, which allows the upper dryout front to advance more rapidly for the line load than for the point load.

- The vertical length of the heat-pipe zone is less for the line load than it is for the point load at early time. For low $q_{\text {perc }}$ the heat-pipe-zone length is also less for the line-load design for later time. The smaller heat-pipe-zone length for the line-load design arises from the more efficient condensate drainage between the line-load drifts, which results in less condensate buildup above the line-load drifts.

- The vertical length of the heat-pipe zone increases with repository depth below the ground surface (based on calculations not described in detail previously).

- The tendency for dryout zone coalescence decreases with increasing $\boldsymbol{q}_{\text {perc }}$ it is also less for the line-load design than it is for the point-load design. 


\subsection{Seepage into Drifts}

This section focuses on modeling results for water seepage into emplacement drifts under both pre-emplacement (isothermal) and post-emplacement (nonisothermal) conditions. The pre-emplacement results are also applicable to modeling the post-thermal period of the repository. The principal feature that distinguishes the seepage models from other drift-scale hydrologic models is the fineness of the model grid.

Following are some of the major findings from the modeling studies:

- Average rate of seepage increases with introduction of spatially heterogeneous fracture properties.

- Average rate of seepage increases when episodic infiltration is imposed on a nonequilibrium, dual-permeability model.

- Seepage decreases for 3-D models when compared with similar 2-D models.

- Seepage may occur immediately after emplacement as a result of condensation in the NFE.

The seepage calculations discussed in this section were performed using various property sets with model parameters corresponding to point-load and line-load repository designs, but the conclusions regarding factors contributing to seepage are general and apply for any reasonable hydrologic property set. Another study of seepage (Tsang et al., 1997) was conducted in the same time frame as the calculations described subsequently, and it reached similar conclusions.

\subsubsection{Physics of Seepage into an Open Cavity}

Flow in an unsaturated porous medium is governed by three forces: viscous, gravitational, and matric (capillary + osmotic). As long as the total fluid pressure in fracture or matrix pore water is maintained below atmospheric pressure by matric forces, seepage cannot flow into the drift. Matric forces are caused by the sum of capillary pressure and surface forces at or near the pore walls. At high saturation, or for large pore sizes, such as in fractures, gravitational forces predominate. If flow is sufficiently high, a combination of gravitational and viscous forces will eventually overcome matric forces, and fluid pressure will increase to atmospheric pressure; at that point, seepage into the drift can occur. In particular, as water flows around a drift, it has to travel a longer distance than does water following the flow lines farther away from the drift. From Darcy's law, the pressure must therefore be higher in the vicinity of the crown of the drift than at the sides. Because water is essentially incompressible, liquid flow diverted around a drift opening must flow faster than where there is no opening. The increased flux immediately above the opening also contributes to increased pressure. If the pressure increase is high enough to overcome the matric potential, seepage can enter the opening.

Natural heterogeneities in hydrologic properties can increase the likelihood for seepage through a variety of mechanisms. Low-permeability areas can obstruct flow and divert flow paths so they are longer, thus increasing the pressure above drift openings. Along preferential flow pathways, stream lines are concentrated, thus producing higher saturation than in the surrounding area. Because of spatial variability in hydrologic properties, there also may be localized regions with very low air-entry pressure (i.e., areas in which the fractures drain more readily). In some cases, heterogeneities may actually reduce the likelihood of seepage by diverting flow away from drift openings. However, reduction of seepage in a large area of the repository may increase seepage at a few locations. Further discussion of drift seepage can be found in Birkholzer et al. (1996). 


\subsubsection{Scales of Heterogeneity and their Influence on Seepage}

The emphasis for seepage calculations in this study was on the flow field within a few meters of drift openings. Therefore, the primary interest was permeability heterogeneities occurring within approximately one drift diameter of the opening. Simulation problems were posed that allow the possibility that heterogeneities actually focus flow from a wide area onto drift openings. The scale of such focusing would be equivalent to the scale length for spatial heterogeneity in the lateral direction, which is comparable to the model dimensions. To study focusing over a larger area, larger models are required; this was not undertaken in this study. To examine the effects from the variability in local flux caused by large-scale focusing, the model discussed here could be run for a wide range of local percolation fluxes. The following sections contain analyses with spatially variable hydrologic properties, using a single stochastic realization to examine the nature of the effects of heterogeneity on seepage.

The model domain used in the studies reported here was large enough to include local heterogeneities with scale length on the order of the drift diameter. With a model of this scale, the variability of calculated seepage along the drift axis was expected to be small because the integration of seepage fluxes at the drift wall acts as an averaging filter. The flux can still be highly variable, however, on a scale smaller than the drift diameter. Note that, if the scale of heterogeneity approaches the size of this model, each realization will resemble a homogeneous model that is one sample from an ensemble.

\subsubsection{Calculation Procedures}

The NUFT code (Nitao, 1993, 1995) can solve many different problems in a computationally efficient manner, retaining only the minimum required processes. In calculations performed for this study, the gas phase was assumed to be passive (i.e., more mobile than the liquid phase so that it does not significantly affect liquid flow). The primary variables in the NUFT code are the saturation $S$ for unsaturated elements and the pressure head $H$ for saturated elements. The model switches primary variables at any element when conditions change from saturated to unsaturated or vice versa. The liquid density is taken as constant for unsaturated elements and is equal to $\rho(H)=\rho_{0} \exp (c H)$ for saturated elements, where $c$ is the liquid compressibility.

The NUFT code solves the partial differential equations by the integrated finite-difference method and the backward Euler method. Newton-Raphson iteration is used to solve the system of nonlinear equations at each time step. Matrix inversion is performed using the conjugate gradient method, with incomplete ILU decomposition with matrix partitioning resulting from zebra ordering.

For this study, steady-state fracture behavior in 2-D and 3-D models was simulated using the ECM, which has been widely used in previous applications (Nitao, 1988a). For both isothermal (2-D and 3-D) and nonisothermal (2-D) steady-state problems, the ECM in NUFT was used. For steady-state problems, the ECM generally produces results that are comparable to DKM results. For transient isothermal problems, DKM simulations were used in 2-D and 3-D, also implemented in the NUFT code.

\subsubsection{Random Field Generation}

A random field is a set of random variables defined over either a continuous or a discrete portion of space (see Vanmarcke, 1988). The random variables are usually statistically correlated, with the degree of correlation depending on location and on the direction and separation distance between points. For the seepage calculations described in this section, the random field was generated by the Fourier spectral method in three dimensions (Nitao, 1997b). 
The value of the constant of proportionality used in the Fourier method is irrelevant because the resulting random field minus the mean is normalized by its standard deviation and then multiplied by the desired standard deviation. The random field algorithm is fully integrated into the NUFT code (Nitao, 1993, 1995). The problem domain can be divided into subdomains, each with its own random field structure. Any of the hydrologic parameters can be a function of as many as three independent random fields per subdomain. The randomfield generator works seamlessly with the dual permeability capability in NUFT to generate matrix and fracture properties that can be statistically independent or dependent. A single realization of a 3-D field for matrix hydraulic conductivity is shown in Figure 3-22.

Figure 3-22 Front and side views of 3-D base-10 logarithm of hydraulic conductivity $(\mathrm{m} / \mathrm{s})$ field for the matrix continuum

\subsubsection{Steady-State Isothermal Seepage}

\subsubsection{Model Geometry and Mesh}

A mesh was constructed to represent a drift with 5-m diameter and with a gravel invert that is $1.2 \mathrm{~m}$ deep at the deepest point. Both 2-D and 3-D models were run. The 2-D model domain was $10 \mathrm{~m}$ wide, with the drift at the midline, and $15.2 \mathrm{~m}$ tall, with the bottom of the invert $2.4 \mathrm{~m}$ from the bottom edge of the domain. The model grid was rectangular and had 50 subdivisions in the horizontal direction and 59 subdivisions in the vertical direction, with a nominal block size of $20 \mathrm{~cm}$ in the vicinity of the opening. Unnecessary elements in the drift were removed, except for those next to the drift wall, which were assigned very high permeability to simulate the open drift. Seepage was allowed to occur from the rock into these drift elements, and the total seepage flux was output by the model. The 3-D model had the same basic mesh as the 2-D model (Figure 3-23), with an axial dimension of $5 \mathrm{~m}$, and 25 equal grid subdivisions.

Figure 3-23 Front and side views of the 3-D grid

\subsubsection{Boundary Conditions and Initial Conditions}

For the steady-state problem, a constant uniform flux was applied to the top of the model domain. Drift elements were maintained at zero matric potential to represent an open void. The sides of the model were no-flow boundaries. The bottom was fixed at a constant matric potential corresponding to $90 \%$ saturation for the host rock, which was based on the matric potential curve for unit TSw35, in parameter set number 1, of Bodvarsson and Bandurraga (1996). The bottom acted as an outlet-flow boundary, so the matric potential value there did not affect the steady-state flow field, although the time to reach steady state was affected. Simulations performed at $70 \%$ saturation showed virtually no difference in the steady-state seepage flux. The initial condition elsewhere in the model was defined as the saturation for a steady-state system with zero percolation flux.

\subsubsection{Hydrologic Parameters}

The reference hydrologic parameters used in this study are shown in Table 3-16. They are assigned to the TSw34 unit, in parameter set 1 of Bodvarsson and Bandurraga (1996). For parameter sensitivity studies, the van Genuchten parameter $n_{\mathrm{f}}$ and saturated conductivity $K_{\mathrm{f}}$ for the fracture were varied (van Genuchten, 1980). When $K_{\mathrm{f}}$ was varied, the fracture porosity $\phi_{\mathrm{f}}$ and van Genuchten parameter $\alpha$ were scaled as the square root of $K_{\mathrm{f}}$. The bulk conductivity 
was scaled as $\phi_{\mathrm{f}} K_{\mathrm{f}}$. In the heterogeneous models, the $K_{\mathrm{m}}$ and $K_{\mathrm{f}}$ were independent, log-normal random fields with $\phi_{\mathrm{m}}$ and $\alpha_{\mathrm{m}}$ varying as the square root of $K_{\mathrm{m}}$ and $\phi_{\mathrm{f}}$ and $\alpha_{\mathrm{f}}$ varying as the square root of $K_{\mathrm{f}}$. In some simulations, $n_{\mathrm{f}}$ was a uniformly distributed variate, spatially uncorrelated.

Table 3-16 Reference hydrologic properties for steady-state isothermal seepage calculations

\begin{tabular}{|l|l|}
\hline Fracture permeability $K_{f}$ & $6.54 \times 10^{-7} \mathrm{~m}^{2}$ \\
\hline Fracture porosity $\phi_{f}$ & $2.43 \times 10^{-4}$ \\
\hline Fracture residual saturation $S_{r f}$ & 0.04 \\
\hline Fracture van Genuchten $\alpha_{f}$ & $6.86 \times 10^{-4} \mathrm{~Pa}^{-1}$ \\
\hline Fracture van Genuchten $n_{f}=1 /\left(1-m_{f}\right)$ & 1.481 \\
\hline \hline Matrix permeability $K_{m}$ & $1.0 \times 10^{-13} \mathrm{~m}^{2}$ \\
\hline Matrix porosity $\phi_{m}$ & 0.110 \\
\hline Matrix residual saturation $S_{r m}$ & 0.08 \\
\hline Matrix van Genuchten $\alpha_{m}$ & $7.54 \times 10^{-7} \mathrm{~Pa}^{-1}$ \\
\hline Matrix van Genuchten $n_{m}=1 /\left(1-m_{m}\right)$ & 1.473 \\
\hline
\end{tabular}

\subsubsection{Results for Steady-State Isothermal Models}

A model with uniform hydrologic properties has symmetry along the direction of the drift so that a 2-D model can be used. Various runs were made with 2-D models with different uniform property values. Also run were 2-D and 3-D heterogeneous models, including isotropic and anisotropic heterogeneities. Note that 2-D heterogeneous models can be viewed as approximations to 3-D heterogeneous models with large-scale statistical correlation in the direction of the third axis.

Figure 3-24 shows the seepage flux vs. average percolation flux on a semilog scale. The seepage flux is in units of flow per unit area of the footprint of the drift (i.e., the horizontal plane passing through the drift centerline). Case A shown on the plot is for a 2-D homogeneous model with the reference properties (Table 3-16).

\section{Figure 3-24 Seepage flux into drift vs. average percolation flux into top of model}

Case B is the same as $\mathrm{A}$, except that $n_{f}$ is increased in the reference case to 4.23 , a value more indicative of well-sorted material (i.e., instead of being widely distributed, the pore sizes are more uniform). With a larger value of $n_{\mathrm{f}}$ the matric potential curve has a more sharply defined air-entry pressure, at which water will flow from the fracture. As the value of $n_{\mathrm{f}}$ increases, the fracture becomes more like a parallel plate. The matric potential curve for smaller $n_{\mathrm{f}}$ has a more gradual curve, and the saturation remains closer to unity until the matric potential approaches a value small enough for the fracture to drain.

Case $\mathrm{C}$ on the plot is for a 2-D model with heterogeneous properties, and curve $\mathrm{D}$ is for a $3-\mathrm{D}$ heterogeneous model. Both $\mathrm{C}$ and $\mathrm{D}$ have mean hydrologic properties equal to the reference properties (Table 3-16), and both generated fields have an isotropic correlation length of $1 \mathrm{~m}$.

In Figure 3-25, the seepage-flux ratio is plotted against the simulation time, in days, for the initial value problem in which a steady-state percolation flux boundary condition is applied at time $t=0$. This ratio is the seepage flux divided by the ambient average 
percolation flux. A comparison of Figure 3-24 and Figure 3-25 shows the seepage-flux ratio vs. percolation flux for the same four cases: A, B, C, and D. For a homogeneous medium, the ratio must approach unity as the ambient percolation increases because the seepage flux, as defined, can never exceed the percolation flux. For a heterogeneous medium, this ratio can exceed one, but the effect is minor if the scale of heterogeneity is smaller than the drift diameter. Also, because of numerical discretization errors, the ratio for either medium can exceed unity by a small amount.

Seepage does not occur in the homogeneous case (A) until the ambient flux is approximately $200 \mathrm{~mm} / \mathrm{yr}$. In the 2-D heterogeneous case, a very small amount of seepage occurs when the ambient flux is approximately $4 \mathrm{~mm} / \mathrm{yr}$. In the 3-D heterogeneous case, seepage starts at $50 \mathrm{~mm} / \mathrm{yr}$. Seepage occurs in the homogeneous case with the increased $n_{f}$ at approximately $10 \mathrm{~mm} / \mathrm{yr}$. Compared to heterogeneous models, the homogeneous model (A) strongly underpredicts the onset of seepage flux. The relative amounts of seepage at the lower percolation fluxes for the heterogeneous cases are very small, indicating that there are only a few preferential flow pathways that are slowly seeping. Note that the effect of increasing $n_{f}$ is quite significant and has a stronger effect than does spatial heterogeneity.

The seepage flux ratio in the comparison of Figure 3-24 and Figure 3-25 indicates that the seepage flux does not equal the percolation flux until the percolation flux is on the order of at least several tens of $\mathrm{mm} / \mathrm{yr}$.

Figure 3-25 Ratio of seepage flux into the drift divided by ambient percolation flux vs. simulation time for the initial value problem

Figure 3-26 is a representative plot of the isosurfaces of 50\% saturation in the fractures for the 3-D heterogeneous case (D) and shows the impact of heterogeneity on the spatial distribution of water in the fractures. The fracture saturation in the ECM is computed from the ratio of the volume of water minus the volume in the matrix divided by the fracture volume, under the assumption that the matrix fills first.

Figure 3-26 Steady-state isosurfaces of 50\% fracture saturation for a 3-D simulation

\subsubsection{Summary of Steady-State Isothermal Models}

By examining the dependence of seepage flux on percolation flux for a range of cases, very small amounts of seepage were found able to occur at relatively small percolation fluxes. With plausible parameter values, small amounts of seepage could occur for ambient percolation fluxes of a few $\mathrm{mm} / \mathrm{yr}$ up to tens of $\mathrm{mm} / \mathrm{yr}$. In ventilated drifts, these fluxes are on the order of less than tenths of $\mathrm{mm} / \mathrm{yr}$ and were likely to be evaporated by ventilation. The van Genuchten parameter $n_{\mathrm{f}}$, which characterizes the pore-size distribution in the fractures, can have a significant impact on the seepage-percolation curve. Although one stochastic realization of spatially heterogeneous permeability was used in this study, the conclusion that heterogeneity increases seepage would very likely be reached with any other realization. Further simulations could be used to explore the relation between seepage magnitude and stochastic parameter values.

\subsubsection{Transient Isothermal Models}

\subsubsection{Model Geometry and Mesh}

The 3-D, transient, isothermal model grid was the same grid used for the 3-D steady-state isothermal problem (see Figure 3-53). Unnecessary elements in the drift were removed, except for those next to the drift wall, which were assigned very high permeability to 
simulate the open drift. Seepage was allowed to occur from the rock into these drift elements, and the total seepage flux was output by the model. The same grid layout was used for both the fracture and matrix continua.

\subsubsection{Boundary Conditions and Initial Conditions}

In the transient simulations, the initial conditions were assumed to be saturations from a steady-state system with steady percolation flux of $5 \mathrm{~mm} / \mathrm{yr}$. Drift elements were maintained at zero matric potential to represent an open void. The sides of the model were no-flow boundaries. The bottom was fixed at a constant matric potential corresponding to $90 \%$ saturation for the host rock, which was based on the matric potential curve for unit TSw35, in parameter set number 1, of Bodvarsson and Bandurraga (1996). The bottom acted as an outlet-flow boundary, so the matric potential value there did not affect the flow field. Simulations performed with a constant matrix potential corresponding to $70 \%$ saturation at the bottom of the model showed virtually no difference in the steady-state seepage flux. The initial condition elsewhere in the model was defined as the saturation for a steady-state system with zero percolation flux.

\subsubsection{Hydrologic Parameters}

The DKM hydrologic parameters used in transient isothermal simulations (see Table 3-17) are representative of properties at the repository horizon and were obtained for the TSw35 unit in the July 1997 property set for TSPA-VA. The $K_{\mathrm{m}}$ and $K_{\mathrm{f}}$ are independent log-normal random fields, with $\phi_{\mathrm{m}}$ and $\alpha_{\mathrm{m}}$ varying as the square root of $K_{\mathrm{m}}$, and $\phi_{\mathrm{f}}$ and $\alpha_{\mathrm{f}}$ varying as the cube root of $K_{\mathrm{f}}$. The parameters $m_{\mathrm{m}}$ and $m_{\mathrm{f}}$ are spatially uniform. Note that the definition of the symbol $K_{\mathrm{f}}$ used here is different from that used in previous reports (Nitao, 1997a, 1997c) where it was used as the permeability of the fracture itself and not the bulk continuum permeability (or hydraulic conductivity) as defined here. This explains why, in the previous reports, $\phi_{\mathrm{f}}$ and $\alpha_{\mathrm{f}}$ were varied as the square root of $K_{\mathrm{f}}$.

Table 3-17 Hydrologic properties for the TSw35 unit, from the 1997 TSPA-VA property set (DKM)

\begin{tabular}{|l|l|}
\hline Fracture continuum hydraulic conductivity $K_{f}$ & $3.72 \times 10^{-5} \mathrm{~m} / \mathrm{s}$ \\
\hline Fracture porosity $\phi_{f}$ & $3.29 \times 10^{-4}$ \\
\hline Fracture residual saturation $S_{r f}$ & 0.04 \\
\hline Fracture van Genuchten $\alpha_{f}$ & $1.10 \times 10^{-4} \mathrm{~Pa}^{-1}$ \\
\hline Fracture van Genuchten $m_{f}=\left(n_{f}-1\right) / n_{f}$ & 0.492 \\
\hline \hline Matrix continuum hydraulic conductivity $K_{m}$ & $1.55 \times 10^{-10} \mathrm{~m} / \mathrm{s}$ \\
\hline Matrix porosity $\phi_{m}$ & 0.115 \\
\hline Matrix residual saturation $S_{r m}$ & 0.08 \\
\hline Matrix van Genuchten $\alpha_{m}$ & $3.31 \times 10^{-6} \mathrm{~Pa}^{-1}$ \\
\hline Matrix van Genuchten $m_{m}=\left(n_{m}-1\right) / n_{m}$ & 0.229 \\
\hline
\end{tabular}




\subsubsection{Results for Equilibrated, Transient Isothermal System}

A preliminary steady-state case was run to compare seepage as a function of the ambient percolation flux. The preliminary run used a matrix block radius $L_{\mathrm{fm}}$ equal to 0.1667 and a fracture surface area density $a_{\mathrm{fm}}$ equal to 4.0 , which, for a parallel set of fractures, corresponds to three fractures per meter. An isotropic correlation length equal to $1 \mathrm{~m}$ was used for the random fields. The flux into the top of the model was introduced entirely to the fracture continuum; no flux was specified into the matrix continuum. The model was run until a steady-state seepage flux was attained.

Figure 3-27 shows the seepage flux into the drift versus the percolation flux into the top of the model. The seepage flux was normalized by the area of the footprint of the drift (i.e., it was equal to the total flux into the drift divided by the area of the horizontal plane segment within the drift passing through the drift centerline). Seepage occurred at a percolation flux of approximately $25 \mathrm{~mm} / \mathrm{yr}$, for the DKM (preliminary steady-state) problem. Steady-state conditions were attained in all cases within $3 \mathrm{yr}$.

Figure 3-27 Seepage flux into the drift vs. percolation flux into the top of the model for the heterogeneous dual-permeability base case, heterogeneous equivalent continuum case, and homogeneous dual-permeability case

A simulation using spatially homogeneous properties obtained from the mean property values of the heterogeneous case is also shown in Figure 3-27. A percolation flux of $300 \mathrm{~mm} / \mathrm{yr}$ was required for seepage to occur in the homogeneous case. Note that, as a result of the wider distribution of pore sizes in the latter, the curve is steeper for the homogeneous case than for the heterogeneous case. The heterogeneous case had a few large pores that, because of their weaker capillarity, could produce seepage under relatively low percolation flux conditions. The ECM used in a previous study (Nitao, 1997c) was adequate for steadystate conditions. To illustrate this fact, the heterogeneous case was rerun with the ECM. As shown in Figure 3-27, the results were almost identical with the DKM results using the same properties, as expected. Note that the ECM case represented in Figure 3-27 is different than that in Figure 3-24. There, the random field for the fracture permeability was generated, whereas here the random field for bulk fracture permeability was generated.

Figure 3-28 shows a transient run made using the July 1997 TSPA-VA DKM properties (Table 3-17). For this model, seepage does not occur until the percolation flux reaches a value greater than $1000 \mathrm{~mm} / \mathrm{yr}$. This case has a smaller fracture $\alpha_{\mathrm{f}}$ parameter, which means the capillarity in the fractures is greater, so a larger flux is needed for the gravitational head in the fracture to overcome the capillary potential. The $\alpha_{\mathrm{f}}$ parameter is approximately the same as the reciprocal air-entry pressure, which is the minimum suction pressure needed to remove water from a porous medium (in this case, the fracture continuum). The $\alpha_{\mathrm{f}}$ value in Table 3-16 gives a relatively low air-entry pressure of $826 \mathrm{~Pa}$, whereas the $\alpha_{\mathrm{f}}$ value in Table 3-17 corresponds to a much larger air-entry pressure of $9100 \mathrm{~Pa}$.

\section{Figure 3-28 Seepage flux vs. percolation flux for the July 1997 TSPA-VA DKM case}

The foregoing example illustrates the importance of the parameter $\alpha_{\mathrm{f}}$. Note that a smaller $\alpha_{\mathrm{f}}$ corresponds to fractures with smaller apertures. To model the zero-th order effect of fracture alteration due to geochemical or geomechanical processes, a calculation was run in which the region of rock was altered at a distance as much as $1.2 \mathrm{~m}$ from the drift wall. With the following changes, the altered region was treated as a random field distinct from the surrounding rock: (1) the mean bulk fracture permeability was increased by three orders of magnitude, and (2) the mean fracture porosity and $\alpha_{\mathrm{f}}$ parameters were increased by one order 
of magnitude. The results shown in Figure 3-29 indicate that the seepage flux for the altered case is slightly less than that for the base case. One would have expected the opposite to be true. However, the random field structure of the altered area is statistically independent of the rest of the rock, so the connectivity of pathways at the contact between the two regions is suppressed; this may not be realistic. Further simulations of this type should address what happens when the altered and surrounding rock are derived from the same underlying random field. Figure 3-29 does show the importance of the statistical correlation of geochemical and geomechanical alteration with hydrologic features in the surrounding rock.

Figure 3-29 Seepage flux vs. percolation flux is $\mathbf{1 . 2} \mathrm{m}$ around the drift for the base case and for the increased-fracture-permeability case

Evaporation of incoming water by ventilation during the pre-emplacement period can be significant when compared to seepage fluxes. During the construction of the ESF tunnel, evaporation was estimated by Wang (1996) to be on the order of hundreds of $\mathrm{mm} / \mathrm{yr}$ expressed as seepage. The base case run had no ventilation, and a zero potential boundary condition corresponding to a $\mathrm{RH}$ of $100 \%$ within the drift was imposed at the drift wall. Ventilation can be approximately treated within this model by specifying a matric suction as a boundary condition at the drift wall (Birkholzer et al., 1996) using Kelvin's law (Edlefsen and Anderson, 1943) for a porous medium. Figure 3-30 shows the flux into the drift (seepage plus evaporated) for a case with ventilation equal to a $\mathrm{RH}$ of $50 \%$ within the drift. The base case with no ventilation is also shown. The difference between the two curves is the amount that is evaporated from the drift wall and varies from 10 to $50 \mathrm{~mm} / \mathrm{y}$. The ventilation flux increases with increasing percolation flux. Note that, for a ventilation system to carry water away from seepage, it must also carry the water evaporating from the walls. The upper curve is the sum of these two contributions of evaporating water. Therefore, it is the total flux of water (per area of drift footprint) that a ventilation system has to carry away to evaporate the water produced from seepage. The effect of ventilation is transient, as shown from DKM runs which show the history of seepage flux in the repository, after ventilation for the first 100 yr (Figure 3-31).

Figure 3-30 Total flux into drift vs. percolation flux for the base case and the case with $50 \% R H$ in the drift to simulate ventilation

Figure 3-31 Seepage flux vs. time for cases with and without ventilation at $50 \%$ relative from time 0 to $100 \mathrm{yr}$

\subsubsection{Results for a Transient, Periodic Isothermal System}

Seepage flux in response to episodic percolation pulses into the top of the model was also investigated. The model was initialized to a percolation flux of $5 \mathrm{~mm} / \mathrm{yr}$ with no drift present. The drift was then placed into the model at time zero, and the percolation flux was held at $5 \mathrm{~mm} / \mathrm{yr}$ for $10 \mathrm{yr}$, at which point the flux was suddenly increased to a constant value that was held for 2 days and then returned to $5 \mathrm{~mm} / \mathrm{yr}$. This cycle was repeated every year for 4 yr.

Figure 3-32 shows the seepage flux for the case where the flux undergoes a 10,000-mm/yr percolation event. Development of the saturation front in a vertical plane within the fracture continuum is shown in Figure 3-33, for several time steps from 10 yr (after equilibration but prior to the percolation event) until $3.2 \mathrm{hr}$ after the start of the event. 
Figure 3-32 Seepage flux vs. time for the case with $10,000-\mathrm{mm} / \mathrm{yr}$ pulse every year, lasting over a 2-day period, imposed on a background flux of $5 \mathrm{~mm} / \mathrm{yr}$

Figure 3-33 Liquid fracture saturation during the start of an infiltration event with a flux into the top of the model of $10,000 \mathrm{~mm} / \mathrm{yr}$ over a 2-day period

Figure 3-34, Figure 3-35, Figure 3-36, and Figure 3-37 show the seepage flux versus time from the beginning of each year that the percolation pulse occurs. Seepage calculations are shown for percolation pulses corresponding to transient fluxes of 100,1000,10 ${ }^{4}$, and $10^{5}$ $\mathrm{mm} / \mathrm{yr}$. The case for $10 \mathrm{~mm} / \mathrm{yr}$ is not shown because no seepage occurred. Note that these fluxes are instantaneous and not annualized. For example, $10^{5} \mathrm{~mm} / \mathrm{yr}$ over a period of 2 days is $546 \mathrm{~mm}$ of water, which, on an annualized basis, is $546 \mathrm{~mm} / \mathrm{yr}$. Several observations can be made from these results. The lag time for the percolation pulse to affect the drift is on the order of one day for the 100-mm/yr case and on the order of hours, or less, for the higher flux cases. The duration of the pulse effect varies from a few days for the lower flux cases to tens of days for the higher flux cases.

Figure 3-34 Seepage flux vs. time from beginning of each year for the case with $100-\mathrm{mm} / \mathrm{yr}$ pulse every year

Figure 3-35 Seepage flux vs. time from beginning of each year for the case with $1000-\mathrm{mm} / \mathrm{yr}$ pulse every year

Figure 3-36 Seepage flux vs. time from beginning of each year for the case with 10,000-mm/yr pulse every year

Figure 3-37 Seepage flux vs. time from beginning of each year for the case with $100,000-\mathrm{mm} / \mathrm{yr}$ pulse every year

There appears to be very little memory between percolation pulses, at least over a time frame of 1 yr. (Note that models extending to the ground surface might show different time constants.) One important observation is that the peak seepage flux does not exceed the steady-state seepage flux in Figure 3-34 through Figure 3-37. (At approximately $200 \mathrm{~mm} / \mathrm{y}$ and above, the seepage flux is approximately equal to the percolation flux.) Therefore, in some cases it may be possible to estimate maximum transient fluxes using steady-state simulations.

\subsubsection{Summary of Transient Isothermal Models}

By examining the dependence of seepage flux on percolation flux for a range of cases, small rates of seepage were found to be possible for small percolation flux. Under realistic parameter values, small amounts of seepage could occur at percolation fluxes on the order of tens of $\mathrm{mm} / \mathrm{yr}$. It was shown that a homogeneous model can severely underpredict seepage fluxes. Also, it was confirmed that the ECM can be used to model steady-state seepage. The simulations showed that increases in fracture permeability due to geochemical or geomechanical changes can actually decrease seepage if there is lack of spatial correlation between the altered area and the surrounding rock. Evaporation from the drift wall due to ventilation at $50 \% \mathrm{RH}$ was found to be comparable to drift seepage fluxes. Evaporation fluxes equivalent to seepage fluxes on the order of 10 to $50 \mathrm{~mm} / \mathrm{yr}$ are possible. For drift-scale 
processes, the memory between short, episodic percolation pulses is on the order of $1 \mathrm{yr}$ or less. The simulations indicate that the peak seepage flux during short, transient percolation events does not exceed the seepage flux under steady-state conditions.

Although one stochastic realization of spatially heterogeneous permeability was used in this study, the conclusion that heterogeneity increases seepage would very likely be reached with any other realization. Further simulations could be used to explore the relations among seepage magnitude and stochastic parameter values.

\subsubsection{Nonisothermal Models}

A nonisothermal model was developed to evaluate seepage during the thermal evolution of the NFE. The ECM approach was used, and cases were run with homogeneous and spatially heterogeneous fracture-continuum properties. Results presented subsequently for a single set of properties indicate that heating of the rock by conduction has a regularizing effect on TH processes in the presence of spatial heterogeneity of hydrologic properties.

\subsubsection{Model Geometry and Mesh}

For the nonisothermal seepage problem, a 2-D, periodic drift-scale model was used with drift spacing of $28 \mathrm{~m}$, consistent with the reference point-load repository design. The diameter of the drift was specified as $5.5 \mathrm{~m}$, which assumed that the drift liner is not present (see Figure 3-38). Intact ground support in the model would divert seepage either behind or through joints in the lining. As is the case with the reference design, the nonisothermal model does not include backfill. The reference design for the invert was not finalized at the time this study was performed, so a gravel invert was assumed.

\section{Figure 3-38 Close-up of the nested grid and the $\log _{10}$ bulk permeability field around the drift}

The model domain was $694 \mathrm{~m}$ tall, representing the UZ from the water table to the ground surface. A grid with square elements $0.3 \mathrm{~m}$ by $0.3 \mathrm{~m}$ was placed around the drift and nested into a coarse main grid (Figure 3-38). Fine gridding was required to resolve the flow processes that can strongly affect drift seepage, especially around the crown of the drift. In general, the resolution of the grid should be smaller than the correlation length of the heterogeneous hydrologic properties. Fine gridding better defines the circular drift geometry for more accurate simulation of streamlines around the drift opening.

\subsubsection{Hydrologic Parameters}

The parameters used for nonisothermal, ECM simulations are shown in Table 3-18 and Table 3-19. These are distinct from the parameters in Table 3-17 because the two sets were generated for use with different mathematical models (ECM vs. DKM), according to the methodology presented by Bodvarsson et al. (1997). 
Table 3-18 Hydrologic properties for the TSw35 unit, from the 1997 TSPA-VA property set (ECM)

\begin{tabular}{|l|l|}
\hline Fracture permeability $K_{f}$ & $4.19 \times 10^{-9} \mathrm{~m}^{2}$ \\
\hline Fracture porosity $\phi_{f}$ & $3.29 \times 10^{-4}$ \\
\hline Fracture residual saturation $S_{r f}$ & 0.01 \\
\hline Fracture van Genuchten $\alpha_{f}$ & $1.10 \times 10^{-4} \mathrm{~Pa}^{-1}$ \\
\hline Fracture van Genuchten $n_{f}=1 /\left(1-m_{f}\right)$ & 1.969 \\
\hline \hline Matrix permeability $K_{m}$ & $1.55 \times 10^{-17} \mathrm{~m}^{2}$ \\
\hline Matrix porosity $\phi_{m}$ & 0.115 \\
\hline Matrix residual saturation $S_{r m}$ & 0.08 \\
\hline Matrix van Genuchten $\alpha_{m}$ & $3.31 \times 10^{-6} \mathrm{~Pa}^{-1}$ \\
\hline Matrix van Genuchten $n_{m}=1 /\left(1-m_{m}\right)$ & 1.297 \\
\hline \hline Bulk dry thermal conductivity $K_{H}$ & $2.1 \mathrm{~W} / \mathrm{m}-\mathrm{K}$ \\
\hline Bulk wet thermal conductivity $K_{H}$ & $2.1 \mathrm{~W} / \mathrm{m}-\mathrm{K}$ \\
\hline Grain heat capacity & $9.0 \times 10^{2} \mathrm{~J} / \mathrm{kg}$ \\
\hline Grain density & $2.54 \times 10^{3} \mathrm{~kg} / \mathrm{m}^{3}$ \\
\hline
\end{tabular}

Table 3-19 Hydrologic properties of the TSw1 unit, based on Klavetter and Peters (1986)

\begin{tabular}{|l|l|}
\hline Fracture permeability $K_{f}$ & $8.83 \times 10^{-10} \mathrm{~m}^{2}$ \\
\hline Fracture porosity $\phi_{f}$ & $3.330 \times 10^{-4}$ \\
\hline Fracture residual saturation $S_{r f}$ & 0.04 \\
\hline Fracture van Genuchten $\alpha_{f}$ & $1.315 \times 10^{-3} \mathrm{~Pa}^{-1}$ \\
\hline Fracture van Genuchten $n_{f}=1 /\left(1-m_{f}\right)$ & 4.23 \\
\hline \hline Matrix permeability $K_{m}$ & $1.9 \times 10^{-18} \mathrm{~m}^{2}$ \\
\hline Matrix porosity $\phi_{m}$ & 0.110 \\
\hline Matrix residual saturation $S_{r m}$ & 0.08 \\
\hline Matrix van Genuchten $\alpha_{m}$ & $5.8 \times 10^{-7} \mathrm{~Pa}-1$ \\
\hline Matrix van Genuchten $n_{m}=1 /\left(1-m_{m}\right)$ & 1.798 \\
\hline \hline Bulk dry thermal conductivity $K_{H}$ & $1.5 \mathrm{~W} / \mathrm{m}-\mathrm{K}$ \\
\hline Bulk wet thermal conductivity $K_{H}$ & $2.13 \mathrm{~W} / \mathrm{m}-\mathrm{K}$ \\
\hline Grain heat capacity & $9.0 \times 10^{2} \mathrm{~J} / \mathrm{kg}$ \\
\hline Grain density & $2.54 \times 10^{3} \mathrm{~kg} / \mathrm{m}^{3}$ \\
\hline
\end{tabular}




\subsubsection{Thermal Radiation}

Radiative heat transfer must be accounted for in nonisothermal problems containing void space (e.g., open drift). If radiative transfer is not included, temperature differences between various locations in the drift environment can be overestimated by an order of magnitude. Because of the fine model grid around the drift, there were many elements on the surface of the drift wall (see Figure 3-38) resulting in numerous radiation connections from drift-wall elements to other drift-wall elements, from drift wall to WP elements, and from drift wall to invert elements. These connections numbered on the order of several thousand, so a computer program (RADPRO) was developed to create the connections for 2-D and 3-D problems. It automatically verifies line-of-sight connection between elements and can also calculate radiation reflections from axial or transverse symmetry planes. For the nonisothermal model, only a single reflection stage was implemented. The RADPRO program removed the redundant connections associated with reflected elements.

In some applications, especially large 3-D problems, the number of radiation connections can be prohibitive. The RADPRO program eliminates those for which the contribution to the total view factor is smaller than a specified tolerance or for which the angle from the surface normal is larger than a specified threshold. Because of these approximations, the view factor of the WP may not total to unity, and the program can adjust the coefficients of the connections to normalize the view factor.

The RADPRO program can treat not only a single WP but multiple WPs. It also generates the specification in the NUFT input file for the location of the various material types in the model (e.g., the location of the drift, WP, and invert with respect to the grid indices). The program is intended for use with fine-grid models so that the size of the exposed surface area of the radiating elements is small compared to the distance between elements.

\subsubsection{Heat Load}

The heat load used in the 2-D nonisothermal model was expressed as a line heat load. The magnitude of the average linear power density was consistent with an assumed arrangement of three types of WPs from the reference repository design: 21 PWR (5.436 MTU), 21 PWR (8.148 MTU), and 21 PWR (9.051 MTU). Each of these WP design types contains 21 fuel assemblies from pressurized water reactors (PWR), with age and burn-up characteristics that determine heat output.

\subsubsection{Boundary Conditions and Initial Conditions}

A constant uniform flux was applied to the top of the nonisothermal model. The sides of the model were specified as no-flow boundaries. The bottom was fixed at constant saturation, pressure, air mass fraction, and temperature. The gas phase was not allowed to flow through the water table. The initial conditions were obtained by running the model at the specified percolation flux with no drift and no heat until steady-state conditions were achieved.

\subsubsection{Results}

Simulations were first run using the TSPA-VA property set under heat and no-heat conditions. Figure 3-39 and Figure 3-40 show the net liquid-phase seepage flux into the drift as a function of time for the cases with homogeneous and heterogeneous hydrologic properties, respectively. Negative flux was the result of water vapor condensing into the drift wall. As the air in the drift was heated, water vapor condensed onto the cooler drift wall and was imbibed by capillarity. The condensation stopped when the temperature of the drift wall reached the boiling point of water. The imbibition flux magnitude was slightly greater for the heterogeneous case. 
Figure 3-39 Seepage flux into the drift for the homogeneous, 5- $\mathrm{mm} / \mathrm{yr}$ case under point loading with the TSPA-VA

Figure 3-40 Seepage flux into the drift for heterogeneous, 5-mm/yr case under point loading with TSPA-VA property set

Figure 3-41 and Figure 3-42 show the same cases computed using the Klavetter and Peters (1986) ECM property set. For this situation, there was a net positive flux of liquid into the drift because the condensate that formed on the drift wall was not completely imbibed, so it drained back into the drift. The liquid saturation of the rock matrix in the NFE using the Klavetter and Peters properties was quite high, at an ambient percolation flux of $5 \mathrm{~mm} / \mathrm{yr}$, when compared to the TSPA-VA properties; hence the imbibition capacity was limited.

Figure 3-41 Seepage flux into the drift for the homogeneous, 5-mm/yr case under point loading with the Klavetter and Peters (1986) property set

Figure 3-42 Seepage flux into the drift for the heterogeneous, 5-mm/yr case under point loading with the Klavetter and Peters (1986) property set

Figure 3-43 shows the liquid saturation calculated for high-permeability elements inside the drift at 3 yr. It graphically depicts the liquid water seeping into the drift from the drift crown. In the simulation, the seepage evaporated in the drift before reaching the WP and invert because the high-permeability elements representing the drift were assigned an artificially small, liquid relative permeability at small saturations to eliminate the small time constant associated with the falling water. Otherwise, the resulting small time steps in the numerical solution algorithm would have made simulation impractical. The small drift relative permeability slows the velocity of the water and allows time for the heat in the drift to evaporate it while it is still falling. In actuality, the seepage would contact the hot WP or invert and boil upon contact.

Figure 3-43 Liquid saturation in the drift at $3 \mathrm{yr}$ for $5-\mathrm{mm} / \mathrm{yr}$ percolation flux using the Klavetter and Peters (1986) property set with heterogeneous various

Figure 3-44 compares a line-load and the reference point-load heating configuration for the Klavetter and Peters (1986) property set. The line load was simulated by preserving AML while shortening the axial spacing between WPs by a factor of 0.6 . The preboiling time period of condensate drainage (i.e., negative flux) was projected to last less than 1 yr. Note that a potential benefit of the line load is that there is no postboiling condensate drainage. Another benefit, which is not represented in the 2-D model, is that more uniform thermal conditions will result; this will reduce the likelihood of water vapor moving along the drift toward cooler regions where it can condense.

Figure 3-44 Seepage flux into the drift for the heterogeneous 5- $\mathrm{mm} / \mathrm{yr}$ case with the Klavetter and Peters (1986) property set-comparison between line and point loading

A parameter study was performed to make a zero-order estimate of the effects of possible alteration of fracture properties by geomechanical or geochemical processes. The fracture permeability and fracture porosity of a region around the drift were modified out to one drift radius $(2.75 \mathrm{~m})$ from the drift wall in all directions. Uniform hydrologic properties were used 
in the modified zone, with the fracture permeability decreased by a factor of 0.01 and fracture porosity decreased by 0.1 . A second case was run with fracture permeability increased by 100 and porosity by 10 .

The results shown in Figure 3-45 indicate that decreased fracture permeability around the drift could reduce the likelihood of seepage from the rock. (Water condensing onto the ceiling surface might also drip onto the WP, but the model did not include the details of this phenomenon.) Note that the case with increased fracture permeability had a higher seepage flux, especially during the postboiling period.

Figure 3-45 Seepage flux into the drift for the heterogeneous $5-\mathrm{mm} / \mathrm{yr}$ case under point loading with the Klavetter and Peters (1986) property set-effect of altering the degree of fracturing around the drift

\subsubsection{Summary of Nonisothermal Models}

The capability has been developed to model seepage of water into emplacement drifts from a heterogeneous formation under nonisothermal conditions using the NUFT code. A new program (RADPRO) was created to automatically generate, for 2-D and 3-D models, the radiation connections among elements of the model exposed to the drift void space. This includes the connections due to virtual reflections about symmetry planes.

Simulations showed that, before the temperature at the drift wall reaches the boiling point of water, radioactive decay heat from WPs could give rise to seepage of condensate back into the drift (i.e., thermally driven reflux within the drift). This phenomena could occur for either boiling or sub-boiling repository designs. Seepage fluxes during this period were projected to be significantly higher than percolation fluxes and could range from tens to hundreds of $\mathrm{mm} / \mathrm{yr}$. The likelihood of such fluxes depends strongly on the hydrologic properties and conditions of the surrounding rock mass.

For example, at a percolation flux of $5 \mathrm{~mm} / \mathrm{y}$, reflux within the drift was simulated using hydrologic properties from Klavetter and Peters (1986), but not using the 1997 TSPA-VA properties (ECM). The duration of this condensation period was relatively short, in simulations where it occurred, and seepage stopped after the rock reached boiling temperature. The simulations also indicated that reflux water could enter the drift after the temperature at the drift wall returned to boiling or sub-boiling conditions.

Calculations showed that the length of the condensate-drainage period was reduced from approximately $4 \mathrm{yr}$ for the reference point-load design to approximately $1 \mathrm{yr}$ for the line-load configuration. In exchange for the shorter seepage period, the amount of seepage flux was roughly doubled compared to that of the reference point-load design. Another advantage of the compared line load over the point load was the absence of seepage during the postboiling period. A simple sensitivity study was undertaken to estimate the effects of rock-mass alteration by geomechanical or geochemical processes. Decreased fracture permeability and porosity out to one drift radius from the drift wall were found to decrease condensation seepage, whereas increased values of these properties led to increased condensation seepage especially during the post-boiling period.

Although one stochastic realization of spatially heterogeneous permeability was used in this study, the conclusion that heterogeneity increases seepage would very likely be reached with any other realization. Further simulations could be used to explore the relations among seepage magnitude and stochastic parameter values. 


\subsection{Multi-Scale Models and Assumptions for TSPA-VA Support}

\subsubsection{Background}

The multi-scale approach is used to develop time-varying estimates of key NFE performance measures for locations throughout the repository. Performance predictions vary significantly for different locations because of differences in hydrostratigraphy and infiltration flux. The performance measures include drift-wall temperature, WP temperature, and drift-air $R H$ at the WP surface (see Table 3-20). The estimation procedure is fast enough to be conducted for many locations in the repository layout and repeated for TSPA-VA sensitivity cases and alternative model exercises.

Table 3-20 Thermohydrologic measures estimated by the multi-scale TH modeling approach

Measures calculated for TSPA-VA are indicated by bold, uppercase $X$. Other measures also calculated by the approach are indicated by lowercase $x$. The measures are temperature $(T)$, relative humidity $(R H)$, gas-phase air-mass fraction $\left(X_{\text {air,gas }}\right)$, liquid phase saturation $\left(S_{\text {liq }}\right)$, and liquid phase flux $\left(q_{\text {liq }}\right)$.

\begin{tabular}{|l|c|c|c|c|c|}
\hline \multicolumn{1}{|c|}{ Location } & $\boldsymbol{T}$ & $\boldsymbol{R H}$ & $\boldsymbol{X}_{\text {air,gas }}$ & $\boldsymbol{S}_{\text {liq }}$ & $\boldsymbol{q}_{\text {liq }}$ \\
\hline \hline Rock above drift wall & $\mathrm{x}$ & $\mathrm{x}$ & $\mathrm{x}$ & $\mathrm{x}$ & $\mathrm{X}$ \\
\hline Rock at drift wall & $\mathrm{X}$ & $\mathrm{X}$ & $\mathrm{x}$ & $\mathrm{x}$ & $\mathrm{x}$ \\
\hline Drift above WP & & & $\mathrm{X}$ & & \\
\hline WP surface & $\mathrm{X}$ & $\mathrm{X}$ & & & \\
\hline Invert & $\mathrm{x}$ & $\mathrm{x}$ & $\mathrm{x}$ & $\mathrm{X}$ & \\
\hline
\end{tabular}

The need for a multi-scale approach stems from the fact that the performance measures depend on TH behavior within a few meters of the emplacement drifts and also on thermal and TH behavior on a repository scale. A single numerical model cannot readily incorporate the required range of scales, for locations throughout the repository, without involving an unfeasible number (millions) of grid blocks. Consequently, a procedure has been developed for estimating the results that would be obtained using a full-scale, 3-D repository model with drift-scale resolution (i.e., millions of grid blocks).

A possible approach to the multi-scale prediction problem would be to embed a 3-D driftscale model with a relatively fine mesh into a 3-D repository-scale model with a coarse mesh. This approach is also too computationally intensive for the required number of cases. The multi-scale approach presented subsequently combines 1-D, 2-D, and 3-D thermalconduction-only models (T-models) and TH models at different scales to estimate the required performance measures.

\subsubsection{Calculated Performance Measures}

The multi-scale approach combines 1-D, 2-D, and 3-D drift-scale T-models and TH models with a conduction-only 3-D mountain-scale model (Figure 3-46). Output from this system provides estimates for the time evolution of the performance measures summarized in Table 3-20. These measures are used by TSPA-VA to estimate WP corrosion rates, wasteform dissolution rates, and transport of radionuclides in the NFE. 
The use of conduction-only models will introduce small errors in the abstracted performance measures. The magnitude of these errors can be estimated by comparison of abstracted results with direct simulations. Where possible, conduction-only models are used to calculate temperature differences instead of absolute temperatures; this minimizes the abstraction errors.

Figure 3-46 Schematic of conceptual models used by the multi-scale TH modeling approach

The TH performance measures (Table 3-20) are provided to TSPA-VA for various combinations of the following:

- Different locations in the repository

- Variations in heat production corresponding to different WP designs and types of waste such as commercial spent nuclear fuel (CSNF) vs. defense high-level waste (DHLW), CSNF age and burnup, WP spacing, and sequencing of WP types in the emplacement drifts

- Design options such as the line-load design or backfill

The TH performance measures are also provided for testing sensitivity of the TSPA-VA to variations such as the following:

- Alternative thermal and hydrologic properties

- Alternative distributions of infiltration flux, including the influence of climate change

- Alternative conceptual models of fracture-matrix interaction (e.g., comparing ECM with DKM results)

- Alternative conceptual models of heat-flow conditions at the water table, (e.g., a fixed temperature vs. explicit representation of heat loss to the SZ)

Given the number of required implementations of the calculation procedure for TSPA-VA performance measures, the multi-scale approach was developed to efficiently incorporate as much detail as is computationally feasible.

\subsubsection{Multi-Scale Submodel Nomenclature}

Four different submodel types are used by the multi-scale approach:

- Smeared-heat source, mountain-scale thermal conduction-only (SMT) model

- Smeared-heat source, drift-scale, thermal conduction-only (SDT) model

- Line-averaged heat source, drift-scale, TH (LDTH) models that use the DKM conceptual model

- Discrete-heat source, drift-scale, thermal conduction-only (DDT) models

These types are defined and discussed in the following subsections (see Table 3-21). In addition, the following other submodel types have been used during the course of sensitivity analysis and model-abstraction testing:

- $\quad$ Line-averaged heat source, drift-scale, TH, Seepage (LDTH-S) models

- Smeared-heat source, drift-scale, TH (SDTH) model

- Line-averaged heat source, drift-scale, TH (LDTH) model that uses the ECM conceptual model

- Line-averaged heat source, drift-scale, TH (LDTH) model that uses the DFM conceptual model

- Discrete-heat source, drift-scale, TH (DDTH) model 
Table 3-21 Summary of model types used in the multi-scale TH modeling approach Process types are thermal-conduction $(\mathrm{T})$ and thermal-hydrologic (TH).

\begin{tabular}{|c|c|c|c|c|c|}
\hline $\begin{array}{l}\text { Model } \\
\text { Type }\end{array}$ & Heat Source & Dimensionality & $\begin{array}{c}\text { Process } \\
\text { Type }\end{array}$ & $\begin{array}{c}\text { In-Drift } \\
\text { Thermal } \\
\text { Radiation }\end{array}$ & $\begin{array}{c}\text { Use in TSPA-VA } \\
\text { Multi-Scale TH } \\
\text { Modeling Approach }\end{array}$ \\
\hline SMT & smeared & 3-D mountain-scale & $\mathrm{T}$ & no & Process-model input \\
\hline SMTH & smeared & 3-D mountain-scale & $\mathrm{TH}$ & no & Process-model input $^{1}$ \\
\hline LMTH & line-averaged & 3-D mountain-scale & $\mathrm{TH}$ & yes & Abstracted result $^{2}$ \\
\hline DMTH & discrete & 3-D mountain-scale & $\mathrm{TH}$ & yes & Abstracted result $^{2}$ \\
\hline SDT & smeared & 1-D drift-scale & $\mathrm{T}$ & no & Process-model input \\
\hline SDTH & smeared & 1-D drift-scale & $\mathrm{TH}$ & no & Process-model input $^{1}$ \\
\hline LDTH & line-averaged & 2-D drift-scale & $\mathrm{TH}$ & yes & Process-model input \\
\hline DDT & discrete & 3-D drift-scale & $\mathrm{T}$ & yes & Process-model input \\
\hline DDTH & discrete & 3-D drift-scale & $\mathrm{TH}$ & yes & Model-abstraction testing \\
\hline
\end{tabular}

To be used in a future version of the multi-scale TH modeling approach

See Figure 3-54.

\subsubsection{Location-Specific Data for Calculation of TSPA-VA Performance Measures}

Drift-scale model calculations are made throughout the repository area (Table 3-22) in either a slightly irregular $3 \times 5$ grid or a uniform $5 \times 7$ grid (c). The $3 \times 5$ grid with 15 locations is used for sensitivity analysis (Buscheck et al., 1997a). The $5 \times 7$ grid with 35 drift locations is used for the TSPA-VA TH predictions. The vertical hydrostratigraphy for all of these locations, including the elevations of unit contacts, and unit properties, is consistent with the UZ site-scale model (Bodvarsson et al., 1997).

Table 3-22 Drift-scale model column locations

Northing and easting values are given in the Nevada State Plane coordinate system.

\begin{tabular}{|c|c|c|c|c|c|}
\hline $\begin{array}{c}\text { Column } \\
\text { Name }\end{array}$ & $\begin{array}{c}\text { Easting } \\
(\mathbf{m})\end{array}$ & $\begin{array}{c}\text { Northing } \\
(\mathbf{m})\end{array}$ & $\begin{array}{c}\text { Repository } \\
\text { elevation }(\mathbf{m})\end{array}$ & $\begin{array}{c}\text { Repository } \\
\text { depth }(\mathbf{m})\end{array}$ & $\begin{array}{c}\text { Repository } \\
\text { unit name }\end{array}$ \\
\hline \hline I1c1 & 170190 & 232406 & 1093.3 & 387.3 & Tsw36 \\
\hline I1c2 & 170417 & 232394 & 1092.5 & 365.3 & Tsw35 \\
\hline I1c3 & 170644 & 232382 & 1091.6 & 335.2 & Tsw35 \\
\hline I1c4 & 170871 & 232370 & 1090.8 & 288.1 & Tsw35 \\
\hline I1c5 & 171098 & 232358 & 1089.9 & 274.4 & Tsw34 \\
\hline I2c1 & 170214 & 232857 & 1087.0 & 296.2 & Tsw36 \\
\hline I2c2 & 170441 & 232845 & 1086.2 & 369.8 & Tsw35 \\
\hline I2c3 & 170668 & 232833 & 1085.4 & 341.5 & Tsw35 \\
\hline I2c4 & 170895 & 232821 & 1084.5 & 278.9 & Tsw35 \\
\hline I2c5 & 171122 & 232809 & 1083.7 & 230.6 & Tsw34 \\
\hline
\end{tabular}




\begin{tabular}{|c|c|c|c|c|c|}
\hline $\begin{array}{c}\text { Column } \\
\text { Name }\end{array}$ & $\begin{array}{c}\text { Easting } \\
(\mathrm{m})\end{array}$ & $\begin{array}{c}\text { Northing } \\
(\mathrm{m})\end{array}$ & $\begin{array}{c}\text { Repository } \\
\text { elevation }(\mathrm{m})\end{array}$ & $\begin{array}{c}\text { Repository } \\
\text { depth }(m)\end{array}$ & $\begin{array}{r}\text { Repository } \\
\text { unit name }\end{array}$ \\
\hline $13 c 1$ & 170238 & 233308 & 1080.8 & 329.9 & Tsw36 \\
\hline $13 c 2$ & 170464 & 233296 & 1079.9 & 382.6 & Tsw35 \\
\hline $13 c 3$ & 170691 & 233285 & 1079.1 & 298.8 & Tsw35 \\
\hline $13 c 4$ & 170918 & 233273 & 1078.2 & 258.5 & Tsw35 \\
\hline $13 c 5$ & 171145 & 233261 & 1077.4 & 256.0 & Tsw34 \\
\hline $14 c 1$ & 170261 & 233760 & 1074.5 & 369.8 & Tsw36 \\
\hline $14 \mathrm{c} 2$ & 170488 & 233748 & 1073.7 & 386.6 & Tsw35 \\
\hline $14 c 3$ & 170715 & 233736 & 1072.8 & 352.6 & Tsw35 \\
\hline $14 c 4$ & 170942 & 233724 & 1072.0 & 293.7 & Tsw35 \\
\hline $14 c 5$ & 171169 & 233712 & 1071.1 & 253.7 & Tsw34 \\
\hline $15 c 1$ & 170285 & 234211 & 1068.3 & 400.1 & Tsw35 \\
\hline $15 \mathrm{c} 2$ & 170512 & 234199 & 1067.4 & 384.3 & Tsw35 \\
\hline $15 c 3$ & 170739 & 234187 & 1066.6 & 357.0 & Tsw35 \\
\hline $15 c 4$ & 170966 & 234175 & 1065.7 & 303.2 & Tsw35 \\
\hline $15 \mathrm{c5}$ & 171193 & 234164 & 1064.9 & 281.9 & Tsw34 \\
\hline $16 \mathrm{c} 1$ & 170309 & 234663 & 1062.0 & 365.0 & Tsw36 \\
\hline $16 \mathrm{c} 2$ & 170535 & 234651 & 1061.2 & 396.9 & Tsw35 \\
\hline $16 \mathrm{c3}$ & 170762 & 234639 & 1060.3 & 340.2 & Tsw35 \\
\hline $16 c 4$ & 170989 & 234627 & 1059.5 & 295.7 & Tsw35 \\
\hline $16 c 5$ & 171216 & 234615 & 1058.6 & 243.0 & Tsw35 \\
\hline $17 c 1$ & 170332 & 235114 & 1055.7 & 408.8 & Tsw35 \\
\hline $17 \mathrm{c} 2$ & 170559 & 235102 & 1054.9 & 371.7 & Tsw35 \\
\hline $17 c 3$ & 170786 & 235090 & 1054.0 & 291.7 & Tsw35 \\
\hline $17 c 4$ & 171013 & 235078 & 1053.2 & 346.9 & Tsw35 \\
\hline $17 c 5$ & 171240 & 235066 & 1052.4 & 317.8 & Tsw35 \\
\hline
\end{tabular}

Local infiltration flux is taken from the Flint et al. (1996a) infiltration map. For the $3 \times 5$ grid, an exponential area weighting function is used with a length scale of $100 \mathrm{~m}$; for the $5 \times 7$ grid, the length scale is $50 \mathrm{~m}$ (horizontal). The assumed infiltration flux values for the $5 \times 7$ grid are given in Table 3-23.

Table 3-23 Infiltration $q_{\text {imp }}(\mathrm{mm} / \mathrm{yr})$ and liquid-phase saturation $S_{\text {liq.rep }}$ at the repository depth, for the columns modeled for TSPA-VA

\begin{tabular}{|c|c|c|c|c|c|c|}
\hline \multirow{2}{*}{$\begin{array}{c}\text { Column } \\
\text { Name }\end{array}$} & \multicolumn{3}{|c|}{$q_{\text {inf }}(\mathrm{mm} / \mathrm{yr})$} & \multicolumn{3}{|c|}{$S_{\text {liq.rep }}$} \\
\hline & $0.2 \times q_{\text {inf }}$ & $1 \times$ qinf & $5 \times q_{\text {inf }}$ & $0.2 \times q_{\text {inf }}$ & $1 \times q_{\text {inf }}$ & $5 \times q_{\text {int }}$ \\
\hline $\mid 1 \mathrm{c1}$ & 2.48 & 12.4 & 61.9 & 0.946 & 0.936 & 0.944 \\
\hline $11 \mathrm{c} 2$ & 2.69 & 13.4 & 67.2 & 0.875 & 0.866 & 0.898 \\
\hline $11 \mathrm{c3}$ & 1.41 & 7.0 & 35.2 & 0.850 & 0.841 & 0.869 \\
\hline $11 \mathrm{c} 4$ & 1.08 & 5.4 & 26.9 & 0.922 & 0.900 & 0.929 \\
\hline $11 \mathrm{c5}$ & 0.96 & 4.8 & 23.9 & 0.965 & 0.958 & 0.964 \\
\hline
\end{tabular}




\begin{tabular}{|c|c|c|c|c|c|c|}
\hline \multirow{2}{*}{$\begin{array}{l}\text { Column } \\
\text { Name }\end{array}$} & \multicolumn{3}{|c|}{$q_{\text {inf }}(\mathrm{mm} / \mathrm{yr})$} & \multicolumn{3}{|c|}{$S_{\text {liq,rep }}$} \\
\hline & $0.2 \times q_{\mathrm{inf}}$ & $1 \times$ qinf & $5 \times q_{\text {inf }}$ & $0.2 \times q_{\text {inf }}$ & $1 \times q_{\text {inf }}$ & $5 \times q_{\text {inf }}$ \\
\hline 12c1 & 0.67 & 3.3 & 16.6 & 0.936 & 0.926 & 0.931 \\
\hline 12c2 & 2.19 & 10.9 & 54.8 & 0.870 & 0.861 & 0.891 \\
\hline 12c3 & 3.46 & 17.3 & 86.5 & 0.933 & 0.908 & 0.933 \\
\hline 12c4 & 1.40 & 7.0 & 35.1 & 0.930 & 0.900 & 0.928 \\
\hline $12 \mathrm{c5}$ & 1.71 & 8.5 & 42.6 & 0.963 & 0.957 & 0.967 \\
\hline $13 \mathrm{c} 1$ & 1.19 & 5.9 & 29.7 & 0.930 & 0.918 & 0.924 \\
\hline $13 c 2$ & 3.47 & 17.4 & 86.8 & 0.887 & 0.877 & 0.913 \\
\hline $13 c 3$ & 1.49 & 7.5 & 37.3 & 0.927 & 0.904 & 0.925 \\
\hline $13 \mathrm{c} 4$ & 0.95 & 4.8 & 23.8 & 0.925 & 0.900 & 0.928 \\
\hline $13 c 5$ & 0.65 & 3.3 & 16.3 & 0.961 & 0.956 & 0.961 \\
\hline $14 c 1$ & 1.20 & 6.0 & 30.0 & 0.841 & 0.831 & 0.848 \\
\hline $14 \mathrm{c} 2$ & 3.20 & 16.0 & 80.1 & 0.884 & 0.874 & 0.907 \\
\hline $14 \mathrm{c3}$ & 2.18 & 10.9 & 54.5 & 0.933 & 0.908 & 0.933 \\
\hline $14 \mathrm{c} 4$ & 1.24 & 6.2 & 31.0 & 0.947 & 0.925 & 0.942 \\
\hline $14 c 5$ & 0.69 & 3.5 & 17.3 & 0.961 & 0.957 & 0.961 \\
\hline $15 c 1$ & 2.24 & 11.2 & 56.1 & 0.837 & 0.828 & 0.856 \\
\hline $15 \mathrm{c} 2$ & 1.71 & 8.5 & 42.6 & 0.934 & 0.908 & 0.931 \\
\hline $15 \mathrm{c3}$ & 3.05 & 15.3 & 76.3 & 0.937 & 0.911 & 0.936 \\
\hline $15 c 4$ & 0.40 & 2.0 & 10.1 & 0.906 & 0.897 & 0.919 \\
\hline $15 c 5$ & 0.13 & 0.7 & 3.4 & 0.942 & 0.949 & 0.950 \\
\hline $16 c 1$ & 1.87 & 9.4 & 46.8 & 0.857 & 0.847 & 0.864 \\
\hline $16 \mathrm{c} 2$ & 1.54 & 7.7 & 38.4 & 0.875 & 0.867 & 0.893 \\
\hline $16 \mathrm{c3}$ & 2.94 & 14.7 & 73.5 & 0.936 & 0.910 & 0.936 \\
\hline $16 \mathrm{c} 4$ & 0.51 & 2.6 & 12.8 & 0.911 & 0.898 & 0.924 \\
\hline $16 \mathrm{c5}$ & 0.73 & 3.7 & 18.3 & 0.936 & 0.922 & 0.938 \\
\hline $17 \mathrm{c} 1$ & 1.49 & 7.5 & 37.3 & 0.880 & 0.873 & 0.898 \\
\hline $17 \mathrm{c} 2$ & 0.59 & 2.9 & 14.6 & 0.866 & 0.864 & 0.887 \\
\hline $17 c 3$ & 0.83 & 4.2 & 20.8 & 0.918 & 0.898 & 0.917 \\
\hline $17 c 4$ & 1.19 & 6.0 & 29.9 & 0.925 & 0.902 & 0.927 \\
\hline $17 c 5$ & 1.07 & 5.3 & 26.7 & 0.927 & 0.900 & 0.926 \\
\hline
\end{tabular}

\subsubsection{Thermal-Loading Criteria Used in Multi-Scale Approach}

The TSPA-VA TH predictions are for the reference AML of $85 \mathrm{MTU} /$ acre, which is based on the total MTU of emplaced CSNF and which neglects the MTU of other waste forms such as DHLW. The inventory of WPs that is assumed to be emplaced at Yucca Mountain consists of several major WP types, including the following:

- CSNF WPs, roughly half of which contains PWR fuel assemblies and the other half containing boiling-water-reactor (BWR) fuel assemblies-The heat output from CSNF WPs varies widely from design-basis fuel WPs that have a high heat output to older WPs that have a relatively low heat output. 
- Co-disposal DHLW WPs that contain DHLW glass logs and spent nuclear fuel (SNF)—Co-disposal DHLW WPs produce relatively little heat.

- Direct-disposal DOE SNF WPs that contain Department of Energy (DOE) spent nuclear fuel-These WPs produce relatively little heat.

For mountain-scale model calculations, it is assumed that the repository-wide thermalloading conditions can be characterized by a uniform radioactive heat-of-decay curve (Table 3-24), which is a blend of the heat-of-decay curves from the entire inventory of WPs emplaced in the repository (Bahney, 1997). This curve is based on the total number of assemblies of the two major CSNF types (BWR and PWR), the total heat output from DHLW, and the major heat component of the DOE SNF. The repository-wide heat output assumes 63,000 MTU of CSNF, 4667 MTU of DHLW, and 2333 MTU of the DOE SNF waste. For a $85-\mathrm{MTU} /$ acre repository, this results in a repository area of 741 acres. The actual repository area is slightly larger because about $7.5 \%$ of the repository area does not contain WPs; therefore, the actual repository area is 798 acres.

Table 3-24 Summary of the WP heat sources used in submodels for the multi-scale approach

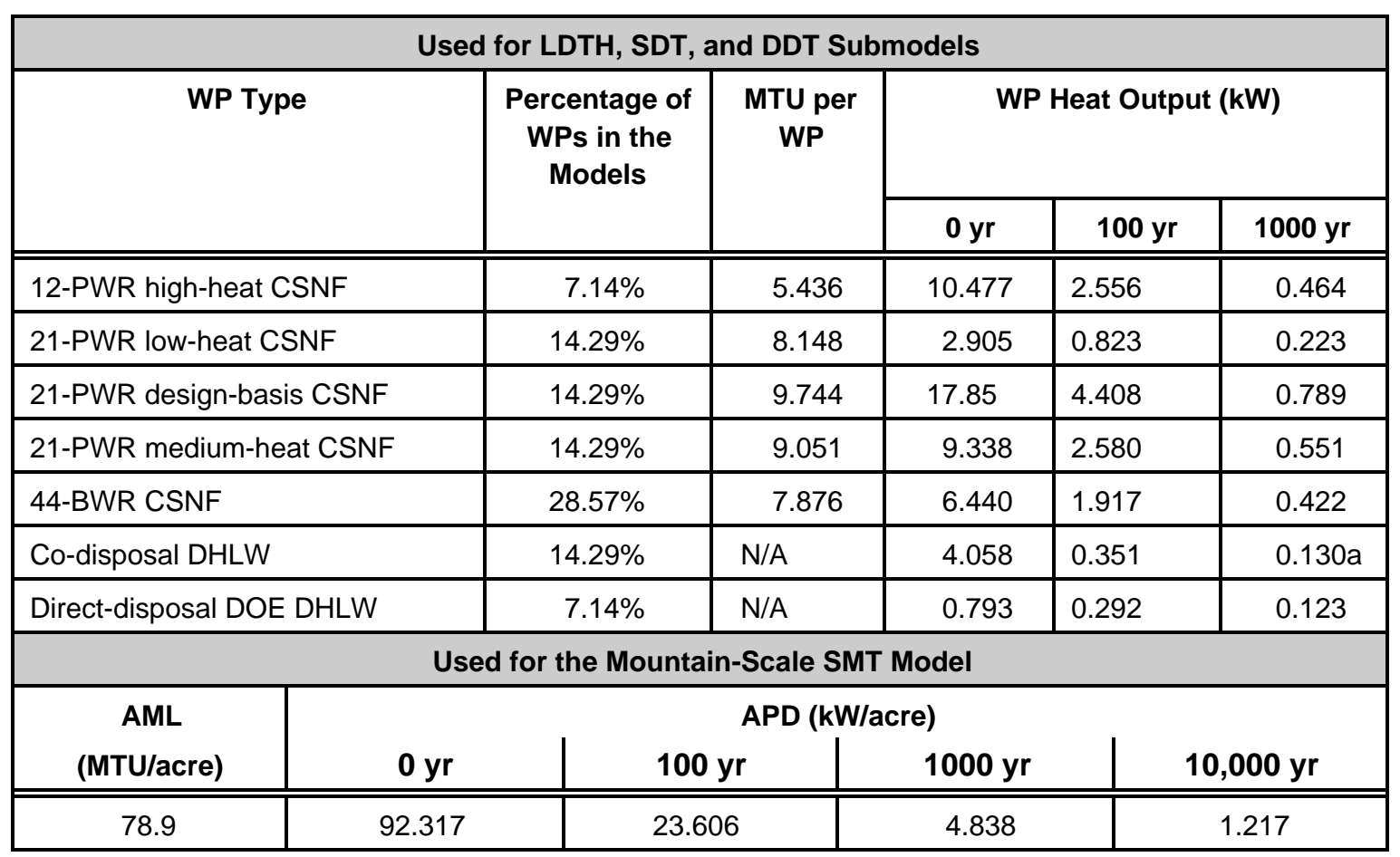

For drift-scale model calculations, it is assumed that the thermal-decay curves for seven WP types in Table 3-24 are representative of the wide range of WPs to be emplaced in the repository. These seven WP types span the range of heat output, from the high-output CSNF WPs to the low-output DHLW WPs.

\subsubsection{Description of Submodels Used in Multi-Scale Approach}

\subsubsection{Smeared-Heat-Source, Mountain-Scale, Thermal Model}

The 3-D SMT submodel enables the multi-scale TH modeling approach to capture the influence of surface topography and edge-cooling effects on repository temperature.

Temperature predictions are then used for interpolating other performance measures. The 
SMT model also captures the influence of the thermal-property distribution in the mountain on the overall temperature distribution. The specific SMT model input to the multi-scale TH modeling approach is the repository host rock temperature $T_{\text {rep }}(\mathrm{SMT})$. Because the SMT results are predicted with a thermal-conduction model, it is likely that temperatures above the boiling point will be overpredicted relative to those predicted by a TH model.

Transient, 3-D, conduction-only problems can be solved readily by NUFT, permitting the use of fine lateral and vertical grid-block spacing in the repository area. At the repository edges, a lateral grid-block spacing of $15 \mathrm{~m}$ is used. In the vertical interval that corresponds to the dryout zone in TH models, a vertical grid-block spacing of about $4 \mathrm{~m}$ is used.

The repository is represented as a rectangular approximation of the actual shape (Figure 3-1). The long axis of the repository is oriented three degrees from the north. The SMT model assumes the N-S length of the repository to be $2912 \mathrm{~m}$, which is obtained from the product of 104 emplacement drifts times the drift spacing of $28 \mathrm{~m}$. The SMT model assumes the E-W width of the repository to be $1109 \mathrm{~m}$, which is obtained by dividing the area of the overall heated repository footprint $\left(3.23 \mathrm{~km}^{2}\right.$ or 798 acres $)$ by $2912 \mathrm{~m}$.

For an AML of 85 MTU/acre (calculated on the basis of CSNF) and 63,000 MTU of CSNF, the repository area is calculated to be only 741 acres, which is $7.5 \%$ less than the 798 acre repository area assumed in the SMT model. However, 4 of the 104 emplacement drifts are left empty, thus about $4 \%$ of the repository drifts are not directly heated by WPs. Note that the bulkheads that will separate the emplacement drifts from the perimeter drifts are located about $30 \mathrm{~m}$ from the outermost WPs at the eastern and western ends of the emplacement drifts. Heat-transfer mechanisms, such as the cold-trap effect (Buscheck, 1996; Buscheck et al., 1996) and thermal radiation will efficiently distribute the decay heat well beyond the outermost WPs. The assumption is effectively made that decay heat is axially spread $18 \mathrm{~m}$ beyond the outermost WPs at either end of the drifts, thereby increasing the effective heated length of the emplacement drift by $36 \mathrm{~m}$. When these adjustments are made, the heated footprint of the repository becomes 798 acres, and the effective AML becomes 78.9 MTU/acre.

The SMT model represents the 1.6\% south-to-north dip of the repository. In the east-west direction, the repository is represented as being horizontal. For the actual repository, the eastern and western boundaries have the same elevation, whereas the center of the repository (in the east-west direction) is elevated by about $3 \mathrm{~m}$ relative to the eastern and western edges. The small rise at the center of the repository is neglected in the SMT model.

The numerical grid for the SMT consists of three concentric nests of grid blocks. The preprocessor YMESH determines the areal dimensions of the grid blocks on the basis of userspecified dimensions for each of the nests. YMESH determines the vertical grid-block spacing on the basis of user-specified criteria and honoring the unit contacts in the UZ site-scale model. The vertical spacing criteria are specified for each nest. The objective is to provide fine spatial resolution in the repository area, with the grid refinement increasing in proximity to the repository edges. Because of the large number of model units (Figure 3-47, Figure 3-48, Figure 3-49, Figure 3-50, Figure 3-51, Figure 3-52, and Figure 3-13), relatively fine vertical grid refinement occurs over the entire UZ, particularly within the Paintbrush Tuff (ptn21ptn25) units.

Figure 3-47 Vertical distribution of hydrostratigraphic model units for several driftscale model locations (in Nevada-State coordinates) (columns 11c1-11c5)

Figure 3-48 Vertical distribution of hydrostratigraphic model units for drift-scale model locations (in Nevada-State coordinates) (columns 12c1-12c5) 
Figure 3-49 Vertical distribution of hydrostratigraphic model units for drift-scale model locations (in Nevada-State coordinates) (columns 13c1-13c5)

Figure 3-50 Vertical distribution of hydrostratigraphic model units for drift-scale model locations (in Nevada-State coordinates) (columns 15c1-15c5)

Figure 3-51 Vertical distribution of hydrostratigraphic model units for drift-scale model locations (in Nevada-State coordinates) (columns 16c1-16c5)

Figure 3-52 Vertical distribution of hydrostratigraphic model units for drift-scale model locations (in Nevada-State coordinates) (columns 17c1-17c5)

Thermal-loading conditions of the reference 85-MTU/ acre repository are represented as a uniform, 4.52-m-thick, smeared heat source, which is a blend of the heat-decay curves (Table 3-24) for the entire waste inventory (Bahney, 1997). The blended heat-decay curve has a slightly different effect than the blending the seven different WP types that are included in the drift-scale models. The numerical grid of the SMT will be used in the future for the SMTH model.

The dependence of rock thermal conductivity $K_{\text {th }}$ on liquid-phase saturation $S_{\text {liq }}$ (discussed previously) is approximated by correlating temperature in the T-model to $S_{\text {liq }}$ in a corresponding TH model and developing a corresponding functional relations between $K_{\text {th }}$ and temperature in the T-model. This $K_{\mathrm{th}}$ vs. T relationship in the T-model mimics the $K_{\mathrm{th}}$ vs. $S_{\text {liq }}$ in the TH model. This effect is particularly important in the lower lithophysal tsw35 model unit because of the relatively low value of dry thermal conductivity in that unit.

The surface topography, hydrostratigraphy (including all of the model units), and T boundary conditions of the UZ site-scale model (Bodvarsson et al., 1997) are incorporated in the SMT model. The thermal load for the total water inventory (63,000 MTU of CSNF; 4667 MTU of DHLW; 2333 MTU of DOE SNF waste) is uniformly distributed over a heated repository footprint of $3.23 \mathrm{~km}^{2}$ (798 acres).

The 3-D SMT submodel (Figure 3-46d) incorporates the following processes, conditions, and assumptions:

- 3-D mountain-scale heat flow in the $\mathrm{UZ}$ and in the upper $1000 \mathrm{~m}$ of the SZ

- Convective heat flow is driven by regional groundwater flow in the upper $1000 \mathrm{~m}$ of the SZ. For the base-case TSPA-VA calculations, the influence of regional groundwater flow on heat flow is assumed to be negligible.

- Topography, stratigraphy, thermal properties, temperature boundary conditions, and initial temperature are consistent with the UZ site-scale model (Bodvarsson and Bandurraga, 1996; Bodvarsson et al., 1997). Because the SMT model extends below the lower boundary of the UZ site-scale model, it is necessary to vertically extrapolate the temperature distribution from the UZ site-scale model below the water table.

- The influence of dryout on rock thermal conductivity $K_{\text {th }}$ is approximated with the use of a $K_{\mathrm{th}}$ vs. temperature relations. The dependence of $K_{\mathrm{th}}$ on liquid-phase saturation $S_{\text {liq }}$ is approximated by correlating temperature computed with a 1-D SDT model, with $S_{\text {liq }}$ in a corresponding 1-D SDTH model, and developing a functional relations between $K_{\text {th }}$ and temperature predicted by the SDT model. The $K_{\mathrm{th}} \mathrm{vs}$. $T$ relations in the SDT model mimics the $K_{\mathrm{th}}$ vs. $S_{\mathrm{liq}}$ in the SDTH model.

- The vertical and areal geometry of the reference $85 \mathrm{MTU} /$ acre repository design is closely approximated in the SMT.

- Homogeneous thermal properties are assumed within each hydrostratigraphic unit. 
- Thermal-loading by the reference 85-MTU/acre repository is represented as a uniform, 4.52-m-thick, smeared heat source, based on a composite of the decay curves for the entire waste inventory. The SMT assumes the same heat-producing area as that in the reference repository (798 acres).

- Mountain-scale, buoyant, gas-phase convection in the UZ does not significantly influence the temperature distribution. This assumption is least-well suited to repository edge locations where buoyant gas-phase convection is most likely to be significant.

- Mountain-scale condensate flow in the UZ does not significantly influence the temperature distribution. Using the abstraction methodology, vertical flow of condensate is simulated explicitly. However, the lateral component of this flow is not, and it may be significant at repository edge locations.

- Mountain-scale, buoyant, liquid-phase convection in the SZ does not significantly influence the temperature distribution. The SZ will warm in response to repository heating, and liquid-phase convection will decrease the water table temperature. A constant water-table temperature is assumed in the abstraction methodology.

- The influence of regional groundwater flow does not significantly influence the temperature distribution. Groundwater flow will also decrease the water-table temperature.

In the multi-scale TH modeling approach, many of the assumptions just stated can be tested by replacing the SMT submodel with an equivalent SMTH submodel.

The SMT model was selected because its computational economy allows for the use of fine lateral and vertical grid-block spacing in the repository area. At the repository edges, a lateral grid-block spacing of $15 \mathrm{~m}$ was used. In the vertical interval that corresponds to the dryout and heat-pipe zones in the TH models, a vertical grid-block spacing of about 4-8 $\mathrm{m}$ was used. Thus, relatively fine gridding is used to capture the influence of edge-cooling effects and the temperature distribution in the vertical interval of the dryout and heat-pipe zones. An important test case to include during the model-abstraction testing phase of TSPAVA involves replacing the SMT model in the multi-scale TH modeling approach with a SMTH model. If mountain-scale, buoyant, gas-phase convection significantly affects the temperature distribution in the repository, it would be useful to use the SMTH model rather than the SMT model, which cannot capture convective heat-flow effects in the UZ.

\subsubsection{Smeared-Heat-Source, Drift-Scale, Thermal Model}

The 1-D SDT submodel (Figure 3-46a) incorporates the following processes, conditions, and assumptions:

- 1-D vertical heat flow in the UZ is equivalent to that in the LDTH and DDT models.

- For base-case TSPA-VA TH predictions, the water table is assumed to be a constant temperature boundary, consistent with the UZ site-scale flow model (Bodvarsson and Bandurraga, 1996; Bodvarsson et al., 1997).

- Local topography, stratigraphy, thermal properties, temperature boundary conditions, and initial temperature are consistent with the UZ site-scale model (Bodvarsson and Bandurraga, 1996; Bodvarsson et al., 1997). The SDT submodel calculations are made throughout the repository area in either a slightly irregular $3 \times 5$ grid or in a uniform $5 \times 7$ grid (Figure 3-1c and Table 3-21). The $3 \times 5$ grid with 15 drift-scale model locations was used for sensitivity analysis (Buscheck et al., 1997a). The $5 \times 7$ grid with 35 drift-scale model locations is used for the TSPA-VA EBS TH predictions. Figure 3-47 through Figure 3-52 and Figure 3-13 give the vertical hydrostratigraphy for the 35 drift-scale model locations. 
- The thermal properties within each hydrostratigraphic unit are homogeneous.

- The thermal load is represented as a 4.52-m-thick, smeared-heat source, just as in the SMT model; however, the SDT model does not use the same heat-of-decay curve as does the SMT model.

- The smeared-heat source in the SDT model uses a heat-of-decay curve that is an areal average of the heat-of-decay curves used in the LDTH and DDT models. The SDT, LDTH, and DDT models all assume the same seven WP types that are described in Table 3-24.

- AMLs of 85, 56.67, and 42.5 MTU/acre are assumed for SDT submodel calculations. The two smaller loads are used to represent cooler areas, such as those near the edges of the repository.

The primary role for the SDT submodel in the multi-scale approach is to provide a functional relations between repository host-rock temperature predicted with the SDT submodel and the perimeter-averaged drift-wall temperature predicted by a LDTH model. This functional relations allows estimation of the perimeter-averaged drift-wall temperature $T_{\mathrm{dw}}(\mathrm{LMTH})$ using the calculated conduction-only, mountain-scale temperature $T_{\text {rep }}(\mathrm{SMT})$.

The SDT submodel input to the multi-scale TH modeling approach is repository host-rock temperature $T_{\text {rep }}(\mathrm{SDT})$. The SDT submodel calculations are made in parallel with the LDTH model calculations.

\subsubsection{Line-Averaged-Heat-Source, Drift-Scale, TH Model}

The 2-D LDTH submodel (Figure 3-46b) incorporates the following processes, conditions, and assumptions:

- 1-D vertical heat flow in the UZ is equivalent to that of the SDT and DDT models.

- For base-case TSPA-VA TH predictions, the water table is assumed to be a constanttemperature boundary, consistent with the UZ site-scale model (Bodvarsson and Bandurraga, 1996; Bodvarsson et al., 1997).

- Point-load and line-load designs are considered by using different values for the linear power density of the line-heat source and different values of drift spacing.

- At the drift scale, 2-D heat flow (as well as mass flow) is represented in the near-field rock and in the emplacement drifts. Heat flow in and near the drifts is primarily radial.

- The cross-sectional, 2-D geometry of the emplacement drifts is represented, including the invert, WP, and the open drift below, above, and to the side of the WP. For drift backfill scenarios, the open space in the drift is filled with a uniform, porous material.

- As demonstrated in an earlier study (Buscheck, 1996), a circular WP in a circular drift opening can be accurately represented by a square WP in a square drift, insofar as prediction of the key performance measures, provided that the circular cross-sectional areas are equal to the equivalent square cross-sectional areas. Accordingly, for the LDTH submodels, the circular geometry of the WP and the drift is approximated by rectilinear geometry (Figure 3-14). The drift is represented as being $4.52 \mathrm{~m} \times 4.52 \mathrm{~m}$, and the WPs are represented as $1.445 \mathrm{~m} \times 1.445 \mathrm{~m}$ in cross-section. The invert is assumed to occupy $16.6 \%$ of the drift cross-section. Note that the same cross-sectional geometry is used for the LDTH and DDT models.

- Radiative heat transfer is represented between all surfaces in the emplacement drifts, resulting principally in heat transfer between the WPs and adjacent surfaces.

- Local topography, hydrostratigraphy, thermal properties, hydrologic properties, boundary conditions $\left(T, X_{\text {air,gas }} P_{\text {gas }}\right)$, and initial temperature are consistent with the UZ site-scale model (Bodvarsson and Bandurraga, 1996; Bodvarsson et al., 1997). 
- Local infiltration flux is taken from the Flint et al. (1996a) infiltration map (Table 3-23).

- The thermal and hydrologic properties within each hydrostratigraphic unit are homogeneous.

- The LDTH model uses a heat-source decay curve that is an average of the decay curves for the eight individual WPs (which includes seven WP types) represented in the DDT model. In other words, the LDTH model smears the heat output from a conduction-only model, which uses discrete heat sources, into a line-averaged heat source. The SDT, LDTH, and DDT models all assume the same seven WP types described in Table 3-24.

- The DKM is used to represent fracture-matrix interaction.

- AMLs of 85,56.67, and 42.5 MTU/ acre are assumed for LDTH submodel calculations. The two smaller values are used to represent cooler areas (e.g., near the edges of the repository).

One of the primary roles for the LDTH model in the multi-scale TH modeling approach is to provide a functional relations between repository host-rock temperatures predicted with a thermal-conduction-only model and the perimeter-averaged drift-wall temperature predicted by a TH model. This relation allows the mapping of the LDTH perimeter-averaged drift-wall temperature $T_{\mathrm{dw}}(\mathrm{LDTH})$ onto the SMT predicted repository host-rock temperature $T_{\text {rep }}(\mathrm{SMT})$. This approximates a drift-wall temperature prediction from a mountain-scale model with a finely resolved, line-averaged heat source $T_{\mathrm{dw}}(\mathrm{LMTH})$, which can be readily computed for locations throughout the repository area.

Another role for the LDTH model is to provide a functional relation between the driftwall temperature $T_{\mathrm{dw}}(\mathrm{LDTH})$ and other TH conditions in the NFE, including the perimeteraveraged relative humidity $R H_{\mathrm{dw}}(\mathrm{LDTH})$ at the drift wall, the liquid-phase flux $3 \mathrm{~m}$ above the drift, the gas-phase air mass fraction $X_{\text {air,gas }}($ LDTH $)$ in the drift, and the liquid-phase saturation in the invert $S_{\text {liq,inv }}(\mathrm{LDTH})$.

The LDTH submodel inputs to the multi-scale TH modeling approach include the following:

- Perimeter-averaged, drift-wall temperature $T_{\mathrm{dw}}(\mathrm{LDTH})$

- Perimeter-averaged, drift-wall relative humidity $R H_{\mathrm{dw}}(\mathrm{LDTH})$

- Perimeter-averaged, drift-wall liquid-phase saturation $S_{\text {liq }}($ LDTH)

- Gas-phase, air-mass fraction in the drift $X_{\text {air,gas }}($ LDTH)

- Liquid-phase flux $3 \mathrm{~m}$ above the drift $q_{\mathrm{liq}}(\mathrm{LDTH})$

- Liquid-phase saturation in the invert $S_{\text {liq,inv }}($ LDTH)

The LDTH submodel calculations are made in parallel with the SDT calculations.

\subsubsection{Discrete-Heat-Source, Drift-Scale T Model}

The 3-D conduction-only DDT model (Figure 3-46c) incorporates the following processes, conditions, and assumptions:

- 1-D vertical heat flow in the UZ is equivalent to that of the SDT and LDTH models.

- Point-load and line-load designs are considered as are backfill and no-backfill scenarios.

- At the drift scale, 3-D heat flow is represented in the near-field rock and in the emplacement drifts. For the point-load design, heat flow in and near the drifts is spherical at early time, becoming more radial at later time. For the line-load design, heat flow in and near the drifts is mostly radial. 
- The full 3-D geometry of the emplacement drifts is represented, including the invert; WPs; the open drift lying below, above, and to the side of the WPs; and the open drift lying axially between WPs (Figure 3-53). For drift backfill scenarios, the open space in the drift is filled with a uniform, porous material.

- The circular cross-sectional geometry of the WP and drift are approximated with squares having the same cross-sectional areas as their circular counterparts (Figure 3-14). The cross-sectional drift geometry is the same as that discussed previously for the LDTH and DDT models.

- Radiative heat transfer is represented between all surfaces in the emplacement drifts, resulting principally in heat transfer between the WPs and adjacent surfaces. For the line-load design, radiative heat flow between WPs is also important.

- Local topography, hydrostratigraphy, thermal properties, boundary conditions $(T)$, and initial temperature are consistent with the UZ site-scale model (Bodvarsson and Bandurraga, 1996; Bodvarsson et al., 1997). For the base-case TSPA-VA EBS TH predictions, DDT model calculations are conducted for the center location of the repository, which is the $13 \mathrm{c} 2$ location in the $3 \times 5$ grid and the $14 \mathrm{c} 3$ location in the $5 \times 7$ grid (Table 3-22).

- The thermal and TH properties within each hydrostratigraphic unit are homogeneous.

- The DDT model discretely represents decay-heat output from eight WPs, and different heat-decay curves corresponding to seven WP types are considered (Table 3-24). The DDT represents six full WPs and two half WPs. The SDT, LDTH, and DDT models all assume the same seven WP types, as described in Table 3-24.

- Repository AML of $85 \mathrm{MTU} /$ acre is assumed for the DDT submodel calculations for the base-case TSPA-VA TH predictions.

\section{Figure 3-53 Plan view of the WP layout represented in the DDT models}

An important role for the DDT model in the multi-scale TH modeling approach is to represent the drift-wall temperature deviations that occur between specific WP locations $\left(\Delta T_{\mathrm{dw}}\right)$, which are defined relative to the average drift-wall temperature along the drift. Another role is to determine the temperature difference between the WP and the average drift-wall temperature $\Delta T_{\mathrm{wp}}$.

The DDT submodel inputs to the multi-scale TH modeling approach include the following:

- WP-location-specific temperature deviations $\Delta T_{\mathrm{dw}}$ from the average drift-wall temperature along the drift.

- WP-location-specific temperature difference $\Delta T_{\text {wp }}$ between the WP and the average drift-wall temperature along the drift.

The DDT (conduction-only) model adequately determines drift-wall and WP temperatures differences, as determined from direct comparison with a DDTH model. The DDT model predicts slightly higher drift-wall and WP temperatures than does the DDTH model, but the two models produce very similar temperatures at each of the eight WP locations, and the differences are insignificant. This is important because it requires several days to conduct a single DDTH calculation on a Sun Ultra2 Sparc workstation, whereas it only takes about $1 \mathrm{hr}$ to conduct the corresponding DDT calculation.

The differences in temperature at hotter and cooler locations along the emplacement drift are related to the heat-flux distribution, which is controlled by thermal radiation for the nobackfill case or by thermal conduction for the backfill case. Advective heat transfer driven by 
$\mathrm{TH}$ behavior in the host rock has little effect on axial temperature variation in the drift. In other words, TH processes in the host rock do not contribute significantly to equalization of axial temperature variations in the drift.

\subsubsection{Line-Averaged Heat-Source, Drift-Scale, TH, Seepage Model}

The LDTH-S model is used to evaluate seepage (i.e., the proportion of liquid flux in the host rock) that is diverted around the drift vs. that which is projected to seep into the drift. The LDTH submodel is not gridded finely enough around the drift opening, to provide reasonable calculations of drift seepage.

The 2-D LDTH-S model is similar to the LDTH with respect to model geometry, boundary conditions, thermal properties, and TH properties. Numerically, the LDTH-S submodel contains a finely gridded region, nested inside of the LDTH model. The nested region is a more detailed representation of the emplacement drift, WP, and NFE. The fine gridding allows the LDTH-S to closely represent the circular cross-sectional geometry and also allows for investigation of the effects of spatial heterogeneity in $\mathrm{TH}$ properties.

The LDTH-S submodel incorporates the same processes, conditions, and assumptions detailed previously for the LDTH submodel, with the following distinctions:

- The DKM conceptual model approach is used to represent fracture-matrix interaction for isothermal-model calculations. For nonisothermal LDTH-S submodel calculations, the ECM approach is used to limit computational effort.

- AML of 85 MTU / acre is assumed for LDTH-S submodel calculations.

- Because of the finer gridding used, spatially heterogeneous TH properties can be assigned to the host rock near the drift (where the mesh is finely gridded).

Subdomains within this region can be specified, each with its own heterogeneous stochastic field. Each TH parameter in the near field, or each hydrostratigraphic unit making up the host rock, can have unique statistics of variation, and the fields may be correlated.

The purpose for the LDTH-S model is to evaluate seepage into drifts $\left(q_{\text {seep }}\right)$. This input is especially important during the thermal period, when thermally driven reflux may have magnitude well in excess of the ambient percolation flux. Seepage is also especially important if liquid water at elevated temperature is predicted to contact the engineered barriers.

The multi-scale approach uses the LDTH-S submodel to predict the liquid-phase flux $q_{\text {liq }}$ in the rock $3 \mathrm{~m}$ above emplacement drifts at locations throughout the repository area. Homogeneous TH properties within each hydrostratigraphic unit are assumed for these calculations.

The LDTH-S submodel calculations are conducted in parallel with equivalent LDTH calculations, to develop a relation between the LDTH predicted liquid flux $3 \mathrm{~m}$ above the emplacement drift and the LDTH-S predicted flux. The LDTH-S inputs to the multi-scale approach are drift-seepage flux $q_{\text {seep }}($ LDTH-S) and liquid-phase flux contacting the WP $q_{\text {liq,wp }}($ LDTH-S).

\subsubsection{Procedure for Calculating EBS TH Conditions}

Complementary 1-D SDT submodels, 2-D LDTH submodels, and 3-D DDT submodels (Figure 3-46a, Figure 3-46b, and Figure 3-46c) are calculated for locations that are regularly spaced throughout the repository area, as described previously. The calculation procedure is described in detail in the following section.

Briefly, for each location, parallel SDT and LDTH calculations are conducted for AMLs of $85,56.67$, and $42.5 \mathrm{MTU} /$ acre (the lower two values represent cooler areas in the repository). This results in 6 model calculations per location, corresponding to 90 drift-scale model 
calculations for the $3 \times 5$ grid, or 210 calculations for the $5 \times 7$ grid. For TSPA-VA, the $5 \times 7$ grid provides finer resolution of the influence of spatially varying infiltration flux in the repository area (Figure 3-1c).

In addition, one DDT submodel calculation is required by the multi-scale approach; additional DDT calculations may be included to capture effects from the wide range of possible WP-type and emplacement-sequence scenarios.

This procedure captures the significant effects from mountain-scale heat flow as well as drift-scale TH behavior driven by heat output from individual WPs, each having distinctively different heat generation characteristics. To explicitly account for these effects in a single numerical TH model would require approximately 60 million grid blocks, which is beyond the computational capabilities of the current generation of $\mathrm{TH}$ simulators.

Altogether, using the $5 \times 7$ grid, a single TSPA-VA TH scenario requires 424 NUFT runs, including 2112 -D drift-scale calculations and one mountain-scale calculation. In a procedure that conditions the comparison of thermal conduction and TH model calculations, an additional 212 runs are used to initialize the models. Using the NUFT code, which is optimized for efficiency, a complete set of 424 runs requires 12 to $16 \mathrm{hr}$ on 9 Sun Ultra2 Sparc workstation processors.

The multi-scale model procedure is repeated to evaluate alternative TH parameter sets, such as the following:

- $\quad$ TSPA-VA (July 1997) base-case property set (Bodvarsson et al., 1997)

- TH (November 1997) property set, which is similar to the July 1997 property set but calibrated to SHT temperature data (Lawrence Berkeley National Laboratory memorandum to G.S. Bodvarsson from J. Birkholzer, November 19, 1997; Buscheck and Nitao, 1995)

- Modified-TH property set that is similar to the TH (November 1997) property set but calibrated to laboratory measurements of matrix imbibition diffusivity (Flint et al., 1996b)

The multi-scale procedure is also repeated to evaluate alternative repository models, including the reference point-load design, the line-load designs, and the use of engineered backfill in emplacement drifts.

The multi-scale TH modeling approach is outlined in Figure 3-54. The intermediate results of the calculation procedure are shown as the orange boxes in Figure 3-54. The interpolation/calculation steps are shown as the green boxes. This procedure involves seven major calculation steps, with several of the steps consisting of multiple parts.

\section{Figure 3-54 Flow chart for the multi-scale TH modeling approach}

\subsubsection{Step 1. Conduct Numerical Simulations Using NUFT}

The first step is to conduct numerical-model calculations with the use of the NUFT code (shown as blue boxes in Figure 3-54), including the following submodels:

- SMT model

- SDT model

- LDTH model using DKM

- DDT model

- LDTH-S model 


\subsubsection{Step 2. Construct Scanning Curves}

The second step is to construct the functional relations, called scanning curves, among the various model-output variables from complementary drift-scale models (shown as yellow boxes in Figure 3-54). The scanning curves include the following:

- Drift-wall TH temperature vs. drift-scale smeared-source conduction-only temperature

$T_{\mathrm{dw}}(\mathrm{LDTH})$ vs. $T_{\text {rep }}(\mathrm{SDT})$, which is the LDTH-predicted drift-wall temperature $T_{\mathrm{dw}}$ vs. the SDT predicted host rock temperature $T_{\text {rep }}$ (Figure 3-55a). This requires the parallel use of the LDTH and of SDT models. The variable $T_{\mathrm{dw}}$ is the average over the perimeter of the drift wall. This relation is developed for all 35 locations, with AMLs of 85, 56.67, and 42.5 MTU/acre. The three different AML "scanning" curves in Figure 3-55a guide the interpolation (in Step 3) of the local scanning curve that reflects the influence of local heating conditions and percolation flux on $T_{\mathrm{dw}}$ in a given region of the repository. At the center of the repository, it is likely that the 85-MTU/acre curve will apply; close to the repository edge, cooling may cause the local scanning curve to lie closer to the lower AML curves.

- Drift-wall TH $R H$ vs. drift-wall TH temperature $R H_{\mathrm{dw}}(\mathrm{LDTH})$ vs. $T_{\mathrm{dw}}(\mathrm{LDTH})$, which is the LDTH-predicted, drift-wall relative humidity $R H_{d w}$ vs. drift-wall temperature $T_{d w}$ (Figure 3-55b). The variables $T_{d w}$ and $R H_{\mathrm{dw}}$ are averages over the perimeter of the drift wall. This relation is developed for all 35 locations and AMLs of 85, 56.67, and $42.5 \mathrm{MTU} /$ acre. The three different AML scanning curves in Figure 3-55b guide the interpolation (in Step 3) of the local scanning curve that reflects the influence of local heating conditions and percolation flux on dryout behavior at a given location in the repository. During the heat-up phase, nonequilibrium fracture-matrix behavior causes a lag in rock dryout (and $\mathrm{RH}$ reduction), making it necessary for $T_{\mathrm{dw}}$ to rise well above the nominal boiling point before dryout begins. During cool-down, rewetting (and $R H$ rise) lag behind the decline in $T_{\mathrm{dw}}$.

- Drift-wall TH matrix saturation vs. drift-wall TH temperature $S_{\text {liq }}\left(\right.$ LDTH) vs. $T_{\text {dw }}($ LDTH), which is the LDTH-predicted, drift-wall liquid-phase saturation $S_{\text {liq }}$ vs. drift-wall temperature $T_{\mathrm{dw}}$ (Figure 3-55c) The variables $T_{\mathrm{dw}}$ and $S_{\text {liq }}$ are averages over the perimeter of the drift wall. This relation is developed for all 35 locations and AMLs of 85, 56.67, and 42.5 MTU/acre. The three different AML scanning curves in Figure 3-55c guide the interpolation (in Step 3) of the local scanning curve that reflects the local heating conditions and dryout conditions at a given location in the repository. During the heat-up phase, nonequilibrium fracturematrix behavior causes a lag in rock dryout (and $S_{\text {liq }}$ reduction), making it necessary for $T_{\mathrm{dw}}$ to rise well above the nominal boiling point before dryout begins. During cooldown, rewetting (and $S_{\text {liq }}$ rise) lag behind the decline in $T_{\mathrm{dw}}$.

- Host-rock TH liquid flux vs. drift-wall TH temperature $q_{\text {liq }}\left(\right.$ LDTH) vs. $T_{\text {dw }}\left(\right.$ LDTH), which is the LDTH-predicted, liquid-phase flux $q_{\text {liq }} 3 \mathrm{~m}$ above the drift crown, vs. drift-wall temperature $T_{\mathrm{dw}}$ (Figure 3-55d). This relation is developed for all 35 locations and AMLs of 85, 56.67, and 42.5 MTU/acre. The three different AML scanning curves in Figure 3-55d guide the interpolation (in Step 3) of the local scanning curve that reflects the influence of local heating conditions and percolation flux on liquid-phase flux at a given location in the repository. 
- WP TH air-mass fraction vs. drift-wall TH temperature

$X_{\text {air,gas }}\left(\right.$ LDTH) vs. $T_{\text {dw }}($ LDTH), which is the LDTH-predicted, gas-phase, air-mass

fraction in the drift (adjacent to the WP) vs. drift-wall temperature $T_{\mathrm{dw}}$ (Figure 3-55e).

This relation is developed for all 35 locations and AMLs of 85, 56.67, and

42.5 MTU/acre. The three different AML scanning curves in Figure 3-55e guide the interpolation (in Step 3) of the local scanning curve that reflects the influence of local heating conditions on gas-phase, air-mass fraction in a drift in a given region of the repository.

- Invert TH liquid saturation vs. lower drift-wall TH temperature $S_{\text {liq,inv }}(\mathrm{LDTH})$ vs. $T_{\text {ldw }}(\mathrm{LDTH})$, which is the LDTH-model-predicted liquid-phase saturation in the invert vs. the temperature $T_{\mathrm{ldw}}$ at the lower drift wall (Figure 3-55f). This relation is developed for AMLs of 85, 56.67, and $42.5 \mathrm{MTU} /$ acre and the $14 \mathrm{c} 3 \mathrm{drift}$ scale model location (Table 3-22). The three different AML scanning curves in Figure 3-55f guide the interpolation (in Step 3) of the local scanning curve that reflects the influence of local heating conditions on dryout behavior in the invert at a given location in the repository.

- Drift-wall conduction-only axial temperature variation $\Delta T_{\mathrm{dw}}$ (DDT; WP-type), which is the DDT-predicted temperature deviation $\Delta T_{\mathrm{dw}}$ from the average drift-wall temperature, axially along the drift, for a given WP type (Figure 3-56a). This step is required to modify the LDTH predicted $T_{\mathrm{dw}}$ to account for the influence of local heating conditions produced by the WP emplacement sequence. The value of $\Delta T_{\mathrm{dw}}$ will generally be positive for the point-load design because of the large gaps (in the axial direction) between WPs. Hot WP locations will have a large positive $\Delta T_{\mathrm{dw}}$, particularly at early time; cool WP locations will have a negative $\Delta T_{\mathrm{dw}}$. The value $\Delta T_{\mathrm{dw}}$ is determined for each WP location in the DDT model by subtracting the average $T_{\mathrm{dw}}$ along the entire drift in the DDT model from the local $T_{\mathrm{dw}}$ at a given WP location. This relation is developed for an AML of $85 \mathrm{MTU} /$ acre and the 14c3 drift-scale model location, which is at the geographic center of the repository layout.

- WP conduction-only axial temperature variation $\Delta T_{\text {wp }}$ (DDT; WP-type), which is the DDT-predicted temperature difference $\Delta T_{\text {wp }}$ between the WP and drift wall for a given WP type (Figure 3-56b). This calculation is required to predict the WP temperature. The value of $\Delta T_{\text {wp }}$ will always be positive for all WP locations. Hot WP locations will have a large $\Delta T_{\mathrm{wp}}$, particularly at early time; cool WP locations will have a smaller $\Delta T_{\mathrm{wp}}$. For backfill cases, $\Delta T_{\mathrm{wp}}$ will be extremely large, particularly immediately after backfill is emplaced. The value $\Delta T_{\mathrm{wp}}$ is determined for each WP in the DDT model by subtracting the average $T_{\mathrm{dw}}$ along the entire drift in the DDT model from the WP temperature $T_{\mathrm{wp}}$. This relation is developed for an AML of $85 \mathrm{MTU} /$ acre and the 14c3 drift-scale model location, which is at the geographic center of the repository layout.

Figure 3-55 Functional relations plotted for the process-model inputs to the multi-scale TH modeling approach

Figure 3-56 Functional relations plotted for the process-model inputs to the multi-scale TH modeling approach as functions of time 


\subsubsection{Step 3. Interpolate the Distribution of Average Drift-Wall Temperature}

The drift-wall temperature corresponding to a 3-D mountain-scale TH model with emplacement drifts modeled by line-average heat sources is approximated by interpolating scanning curves. The interpolant $T_{\mathrm{dw}}(\mathrm{LMTH} ; x, y)$ is calculated for average drift-wall conditions using the smeared repository heat-source temperature $T_{\text {rep }}(\mathrm{SMT} ; \mathrm{x}, \mathrm{y})$, and the $T_{\mathrm{dw}}(\mathrm{LDTH})$ vs. $T_{\text {rep }}(\mathrm{SDT})$ scanning curve, at the 35 locations and 3 AMLs (Figure 3-55a). This process involves spatial interpolation between the 35 locations and also involves interpolation between the 3 AML scanning curves in Figure 3-55a. The result of this step is the distribution of average drift-wall temperatures as a function of location in the repository. Because this process approximates a 3-D LMTH model prediction of the distribution of $T_{\mathrm{dw}}$ in the repository, the resulting distribution is called $T_{\mathrm{dw}}(\mathrm{LMTH} ; x, y)$, where $x$ and $y$ refer to the coordinate location in the repository.

\subsubsection{Step 4. Interpolate the Distribution of Drift-Wall Temperature for Each WP Type}

The drift-wall temperature distribution in the repository, as a function of location, is calculated for each WP type by adding $\Delta T_{\mathrm{dw}}$ (DDT; WP-type) to the $T_{\mathrm{dw}}(\mathrm{LMTH} ; x, y)$ distribution determined in the previous step. The net result of this step is comparable to having a 3-D discrete-heat source, mountain-scale thermohydrologic- (DMTH) model prediction of the $T_{\mathrm{dw}}$ distribution in the repository for each WP type; hence, the resulting distribution is called $T_{\mathrm{dw}}(\mathrm{DMTH} ; x, y, \mathrm{WP}$-type), where $x$ and $y$ refer to the coordinate location in the repository.

\subsubsection{Step 5. Interpolate the Distribution of Near-Field and In-Drift Hydrologic Conditions}

This step involves interpolating the distribution of near-field and in-drift TH conditions in the repository for each WP type using the temperature distribution from Step 4 and the scanning curves from Step 2. The $R H$ at the WP, the drift-wall matrix saturation, the liquidphase flux above the drift, the air-mass fraction at the WP, and the invert liquid-phase saturation are calculated for each WP type, as a function of location in the repository.

- Relative humidity at the drift wall $R H_{\mathrm{dw}}(\mathrm{DMTH} ; x, y$, WP-type), which is the distribution of $R H$ for each WP type, is interpolated using the abstracted drift-wall temperature $T_{\mathrm{dw}}(\mathrm{DMTH} ; x, y$, WP-type $)$ and the drift-wall relative humidity $R H_{\mathrm{dw}}(\mathrm{LDTH})$ vs. $T_{\mathrm{dw}}(\mathrm{LDTH})$ scanning curve at the 35 locations and 3 AMLs (Figure 3-55b). This process involves spatial interpolation between the 35 locations and interpolation between the 3 AML scanning curves in Figure 3-55b. The result of this step is comparable to having a DMTH-model prediction of the distribution of $R H_{\mathrm{dw}}$ in the repository for each WP type; hence, the resulting distribution is called $R H_{\mathrm{dw}}(\mathrm{DMTH} ; x, y, \mathrm{WP}$-type), where $x$ and $y$ refer to the coordinate location in the repository.

- Drift wall matrix saturation

$S_{\text {liq }}$ (DMTH; $x, y, \mathrm{WP}$-type), which is the distribution of saturation for each WP type, is interpolated using the abstracted drift-wall temperature $T_{\mathrm{dw}}(\mathrm{DMTH} ; x, y, \mathrm{WP}$-type $)$ and the drift-wall liquid-phase saturation $S_{\text {liq }}\left(\right.$ LDTH) vs. $T_{\mathrm{dw}}(\mathrm{LDTH})$ scanning curve at the 35 locations and 3 AMLs (Figure 3-55c). This process involves spatial interpolation between the 35 locations and interpolation between the 3 AML scanning curves in Figure 3-55c. The result of this step is comparable to having a DMTH-model prediction of the distribution of $S_{\operatorname{liq}}$ in the repository for each WP type; hence, the resulting distribution is called $S_{\text {liq }}$ (DMTH; $x, y$, WP-type), where $x$ and $y$ refer to the coordinate location in the repository. 
- Liquid flux above the drift

$q_{\text {liq }}$ (DMTH; $x, y$, WP-type), which is the distribution of liquid flux for each WP type, is interpolated using the abstracted drift-wall temperature $T_{\mathrm{dw}}(\mathrm{DMTH} ; x, y, \mathrm{WP}$-type) and the liquid-phase flux $3 \mathrm{~m}$ above the drift $q_{\text {liq }}(\mathrm{LDTH})$ vs. $T_{\mathrm{dw}}(\mathrm{LDTH})$ scanning curve at the 35 locations and 3 AMLs (Figure 3-55d). This process involves spatial interpolation between the 35 locations, and interpolation between the $3 \mathrm{AML}$ scanning curves in Figure 3-55d. The result of this step is comparable to having a DMTH-model prediction of the distribution of $q_{\mathrm{liq}}$ in the repository for each WP type; hence, the resulting distribution is called $q_{\text {liq }}$ (DMTH; $x, y$, WP-type), where $x$ and $y$ refer to the coordinate location in the repository.

- Air mass fraction at the WP

$X_{\text {airgas }}$ (DMTH; $x, y$, WP-type), which is the distribution of air-mass fraction for each WP type, is interpolated using the abstracted drift-wall temperature $T_{\mathrm{dw}}(\mathrm{DMTH} ; x, y, \mathrm{WP}-$ type) and the gas-phase air-mass fraction $X_{\text {air,gas }}(\mathrm{LDTH})$ vs. $T_{\mathrm{dw}}(\mathrm{LDTH})$ scanning curve at the 35 locations and 3 AMLs (Figure 3-55e). This process involves spatial interpolation between the 35 locations, and interpolation between the 3 AML scanning curves in Figure 3-55e. The result of this step is comparable to having a DMTH-model prediction of the distribution of $X_{\text {air,gas }}$ in the repository for each WP type; hence, the resulting distribution is called $X_{\text {air,gas }}(\mathrm{DMTH} ; x, y, \mathrm{WP}$-type), where $x$ and $y$ refer to the coordinate location in the repository.

- Invert liquid-phase saturation $S_{\text {liq,inv }}($ DMTH; $x, y$, WP-type), which is the invert liquid saturation for each WP type, is interpolated using the abstracted drift-wall temperature $T_{\mathrm{dw}}(\mathrm{DMTH} ; x, y, \mathrm{WP}$-type $)$ and the invert liquid phase saturation $S_{\text {liqinv }}(\mathrm{LDTH})$ vs. lower drift wall temperature $T_{\text {ldw }}($ LDTH) scanning curve at the 35 locations and 3 AMLs (Figure 3-55f). This process involves spatial interpolation between the 35 locations and interpolation between the 3 AML scanning curves in Figure 3-55f. The result of this step is comparable to having a DMTH-model prediction of the distribution of $S_{\text {liq,inv }}$ in the repository for each WP type; hence, the resulting distribution is called $S_{\text {liq,inv }}$ (DMTH; $x, y$, WP-type), where $x$ and $y$ refer to the coordinate location in the repository.

\subsubsection{Step 6. Determine the Distribution of WP Temperature for Each WP Type}

Step 6 is to use the drift-wall temperature distribution from Step 3 and scanning curves from Step 2 to determine the distribution of temperature on WPs throughout the repository area for each WP type. The result is the abstracted WP temperature distribution $\left(T_{\mathrm{wp}}\right)$ for each WP type, $T_{\mathrm{wp}}(\mathrm{DMTH} ; x, y$,WP-type). This is calculated by adding the temperature variation at the WP from Step 2, $\Delta T_{\text {wp }}$ (DDT; WP-type), to the abstracted temperature for an LMTH model, $T_{\mathrm{dw}}(\mathrm{LMTH} ; x, y)$ obtained in Step 3. This step determines the distribution of WP temperature as a function of WP type and location in the repository. The result of this step is comparable to having a DMTH-model prediction of the $T_{\text {wp }}$ distribution in the repository for each WP type; hence the resulting distribution is called $T_{\text {wp }}(\mathrm{DMTH} ; x, y, \mathrm{WP}$-type), where $x$ and $y$ refer to the coordinate location in the repository.

\subsubsection{Step 7. Determine the Distribution of Relative Humidity on WPs for Each WP Type}

The final step is to determine the distribution of $R H$ on WPs throughout the repository area for each WP type using the drift wall temperature $T_{\mathrm{dw}}$ distribution from Step 2, the WP temperature $T_{\mathrm{wp}}$ distribution from Step 6, and the relation $R H_{\mathrm{wp}}=R H_{\mathrm{dw}}\left[P_{\mathrm{sat}}\left(T_{\mathrm{dw}}\right) / P_{\mathrm{sat}}\left(T_{\mathrm{wp}}\right)\right]$, where $P_{\text {sat }}$ is the saturated vapor pressure. The result of this step is the DMTH-predicted WP $R H$ distribution, $R H_{\mathrm{wp}}(\mathrm{DMTH} ; x, y, \mathrm{WP}$-type), calculated using the abstracted drift-wall 
temperature $T_{\mathrm{dw}}\left(\mathrm{DMTH} ; x, y, \mathrm{WP}\right.$-type), the abstracted WP temperature $T_{\mathrm{wp}}(\mathrm{DMTH} ; x, y, \mathrm{WP}-$ type), and the relation $R H_{\mathrm{wp}}=R H_{\mathrm{dw}}\left[P_{\text {sat }}\left(T_{\mathrm{dw}}\right) / P_{\text {sat }}\left(T_{\mathrm{wp}}\right)\right]$, where $P_{\text {sat }}$ is the saturated vapor pressure. The result of this step is comparable to having a DMTH-model prediction of the $R H_{\text {wp }}$ distribution in the repository for each WP type; hence the resulting distribution is called $R H_{\mathrm{wp}}$ (DMTH; $x, y, \mathrm{WP}$-type), where $x$ and $y$ refer to the coordinate location in the repository.

\subsubsection{Constructing Probability Density Functions of EBS TH Conditions}

Predictions from the multi-scale TH modeling approach are used by several modeling and analysis groups that support TSPA-VA:

- WP corrosion

- NFE geochemistry

- Waste form dissolution

- Transport of radionuclides through the engineered barriers

It is possible to provide the full set of TSPA-VA performance measures (Table 3-20) for all seven major WP types (Table 3-24) at every WP location in the repository area. However, because it is unfeasible to perform the necessary calculations for all four of the listed groups, it is necessary to group WP environmental conditions into "bins."

For WP corrosion analysis, the process of "binning" the WP environments involves several steps. First, the repository area is subdivided into six major subdomains with respect to infiltration flux $q_{\text {inf }}$ using the infiltration map (Flint et al., 1996a). The boundaries between the subdomains (Figure 3-57) are chosen to minimize the variability of $q_{\text {inf }}$ within each subdomain. Thus, there are low- $q_{\text {inf }}$ subdomains and high- $q_{\text {inf }}$ subdomains. The rationale for grouping the WP environments into these subdomains is that $q_{\mathrm{inf}}$ is a major factor in determining how long and whether conditions during the thermal period are relatively hot and dry or cool and humid. For purposes of drift-seepage modeling, $q_{\text {inf }}$ is a major factor determining the tendency for water to seep into emplacement drifts. The six $q_{\text {inf }}$ subdomains correspond to northeast and northwest corners of the repository area, the central region, the south-central region, and the southwest and southeast corners of the repository area.

Figure 3-57 The six infiltration-flux subdomains and the infiltration-flux $q_{\text {inf }}$ distribution (Flint et al., 1996a) shown for the SMT-model representation of the repository area

The second step for binning WP environmental conditions is to group the five CSNF WP types into a single CSNF-WP category and to group the co-disposal DHLW WPs with the direct-disposal DOE SNF WPs into a single DHLW-WP category.

The third and fourth steps of binning involve developing probability density functions of temperature and $\mathrm{RH}$ conditions for the CSNF-WP category and for the DHLW-WP category in each of the six $q_{\text {inf }}$ subdomains. The probability density functions are expressed as $R H$ and temperature histograms. The environmental conditions on WPs are binned according to the time required by WPs to return to $R H=85 \%$ and to temperature on the WP surface at $5000 \mathrm{yr}$. An $R H$ of $85 \%$ was selected because it is a typical of the critical $R H$ for atmospheric corrosion $\left(R H_{\text {crit }}\right)$ (i.e., the value of $R H$ where atmospheric corrosion is initiated). Once $R H>R H_{\text {crit }}$, the corrosion rate for the outer corrosion-allowance material on the WP also depends on temperature at the WP surface. Because $R H$ generally exceeds $85 \%$ within $5000 \mathrm{yr}$ (unless an engineered backfill is used), temperature at $5000 \mathrm{yr}$ is a good indicator of whether the WP environment is relatively hot or relatively cool when humidity returns.

In each of the subdomains based on infiltration flux, the CSNF and DHLW-WP categories are each grouped into $10 \mathrm{RH}$ bins, and each $\mathrm{RH}$ bin is subdivided into three temperature bins, resulting in a total of 30 bins for CSNF WPs and 30 bins for DHLW WPs. For example, if all 
WPs attain $R H=85 \%$ within $3000 \mathrm{yr}$, the WPs could then be subdivided into groups where $R H=85 \%$ is attained within 0 to $300 \mathrm{yr}, 300$ to $600 \mathrm{yr}$, and so on, with the last $R H$ bin containing WPs that attain $R H=85 \%$ from 2700 to $3000 \mathrm{yr}$. Step 3 of the binning process is complete when all of the WPs have been placed in their respective $R H$ bins.

Step 4 of the WP environment binning process involves subdividing the WPs in a given $\mathrm{RH}$ bin group into three temperature bins, corresponding to temperature on the WP at 5000 yr. After completing Step 4, all CSNF WPs and all DHLW WPs will fall somewhere within their respective $30 \mathrm{RH}$ and temperature bins. The sum of the probabilities for the 30 bins will be $100 \%$, for both CSNF RH and temperature and for both DHLW RH and temperature. It is possible for many of the $\mathrm{RH}$ and temperature bins to contain no WPs.

Step 5 of the binning process involves selecting a typical WP location for each of the 30 bins that contain a non-zero probability. The mean rewetting time (to $\mathrm{RH}=85 \%$ ) and the mean temperature at $5000 \mathrm{yr}$ is calculated for all WPs in an $\mathrm{RH}$ and temperature bin. Leastsquares minimization is used to find the WP location that is closest to having the mean $R H=85 \%$ rewetting time and the mean temperature at $5000 \mathrm{yr}$. The temperature and $R H$ histories for the typical WP within each bin, along with the probabilities for each of the $R H$ and temperature bins, are provided to TSPA-VA for WP corrosion analysis. The other NFE conditions listed as performance measures in Table 3-20 are also provided to TSPA-VA for each of these typical WPs.

The procedure for binning near-field conditions for geochemistry analysis is similar to that for WP corrosion, but a smaller number of bins is generated. For each $q_{\text {inf }}$ subdomain, typical locations for CSNF and DHLW WPs are selected on the basis of the short-term, medium-term, and long-term temperature histories. The mean temperature on WPs is determined for 100, 1000, and $5000 \mathrm{yr}$ for all CSNF WPs. Least-squares minimization is used to find the CSNF-WP location with the smallest sum of the squared deviations in temperature (from the mean for all CSNF WPs) at 100, 1000, and $5000 \mathrm{yr}$, with equal weight given to all 3 times. This procedure is repeated for the DHLW WP group. A full set of NFE conditions, including the liquid-phase saturation in the invert $S_{\text {liq,inv }}$ is provided to TSPA-VA for the typical CSNF WP and the typical DHLW WP in each of the six $q_{\text {inf }}$ subdomains. This information is also used by the groups analyzing waste form dissolution and analyzing transport in the engineered barriers.

\subsubsection{Multi-Scale Model Results and Analysis}

The multi-scale TH modeling approach integrates results from complementary drift- and mountain-scale models to simultaneously account for the influence of mountain-scale heat flow and drift-scale TH behavior on TH conditions in the NFE (Table 3-20). Results from the drift- and mountain-scale models and the calculation procedure for the multi-scale approach are described in previous sections of this chapter.

This section describes the results of thermal-conduction-only models and then the results of the abstraction of NFE performance measures for TSPA-VA. The sensitivity of the abstracted results to uncertainty in TH properties, infiltration flux, repository edge effects, WP type, and repository design options is discussed in a series of subsections.

\subsubsection{Thermal-Conduction-Only, Smeared-Source, Mountain-Scale Submodel}

The evolution of temperature in the UZ, predicted by the SMT submodel, is shown in Figure 3-58a Figure 3-58d, and Figure 3-59. The areal temperature distribution is shown just for the heated footprint of the repository (Figure 3-58a, d). Figure 3-8a shows the distribution of hydrostratigraphic units at the repository horizon. The effects from edge cooling and thermal properties of the host rock units are evident at $100 \mathrm{yr}$ (Figure 3-58a). 


\section{Figure 3-58 Temperature distribution in the repository host rock predicted by the} SMT model

\section{Figure 3-59 Mountain-scale temperature distribution}

Temperature at a particular location within the repository is strongly affected by the thermal conductivity values of the local host-rock unit. At early time, when dryout occurs, the value of thermal conductivity at dry conditions $\left(K_{\mathrm{th}, \mathrm{dry}}\right)$ is particularly informative. The TSw34 unit, which is the predominant host-rock unit along the eastern edge of the repository (Figure 3-8a) has the highest value of $K_{\text {th, dry }}$ among the three host-rock units. The TSw35 unit, which is the host-rock unit throughout much of the repository layout, has the lowest value of $K_{\mathrm{th} \text {, dry }}$. Consequently, areas of the repository within the TSw35 have the largest increases in temperature, whereas areas within the TSw34 have smaller increases, and areas within the TSw36 unit are intermediate. Because the reference repository design lies within the TSw36 only at the western edge, edge-cooling effects tend to dominate thermal evolution in that unit.

The following general observations, calculated from the SMT submodel, can be made about the temperature distribution in the NFE:

- Dry thermal conductivity $K_{\mathrm{th} \text {, dry }}$ of the host rock has a strong influence during the dryout period, particularly during the early heat-up period.

- Edge cooling effects, which are initially confined to the outer edges of the repository, influence a progressively wider region at the edges with time.

- Topographic effects, which are negligible during the first 300 to $500 \mathrm{yr}$, become increasingly important with time. Topographic effects are relatively unimportant at the repository edges and are increasingly important toward the center. The greatest long-term temperature rise ( $t>500 \mathrm{yr}$ ) occurs where the repository depth is greatest. Consequently, the long-term temperature rise is greatest at locations approximately one-third of the way from the western repository boundary (Figure 3-58d).

In the altered zone, which extends outward from the NFE, the influence of topography on the temperature distribution is evident from Figure 3-59. At $100 \mathrm{yr}$, topography has no influence. At 1000 and $5000 \mathrm{yr}$, the elevations of the $40^{\circ}$ and $60^{\circ} \mathrm{C}$ isotherms are depressed below topographic lows (Figure 3-59b and Figure 3-59c), and raised below topographic highs (Figure 3-59e and Figure 3-59f).

The importance of including the SZ in mountain-scale models is also evident from Figure 3-59. The modern water table elevation is approximately $732 \mathrm{~m}$. At $1000 \mathrm{yr}$, temperature at the water table is projected by the SMT submodel to increase from $32^{\circ}$ to about $60^{\circ} \mathrm{C}$; at $5000 \mathrm{yr}$, it is projected to increase to about $80^{\circ} \mathrm{C}$. This thermal disturbance could penetrate far into the SZ; for example, at $400 \mathrm{~m}$ below the water table, the temperature is projected to increase from the modern value of about $45^{\circ}$, to about $60^{\circ} \mathrm{C}$ at $5000 \mathrm{yr}$.

The potential for buoyant convection in the SZ is also evident from Figure 3-59. Buoyant liquid-phase convection in the SZ has been modeled in the past (Buscheck and Nitao, 1993) using mountain-scale, 2-D, axisymmetric models. The effect is caused by transport of heat into the water that underlies the repository. If the bulk (fracture) permeability is sufficient and buoyant convection is not overwhelmed by regional SZ flow, the effect could significantly influence liquid-phase flow in the SZ. (As discussed in a previous section, the influence of regional groundwater flow in the SZ on heat flow in the SZ and UZ was not included in the SMT submodel calculations.) The thermal gradients that could drive such flow are steepest in the east-west direction (Figure 3-59e and Figure 3-59f). Consequently, lateral flow associated with thermally driven buoyant convection could be strongest in the east-west direction. 


\subsubsection{TH Multi-Scale Model Results and Analysis}

The results of the multi-scale TH modeling approach are comparable to using a 3-D, DMTH model. The ideal DMTH model would comprise finely gridded drift-scale models nested within a mountain-scale model. It is important to note that this ideal TH model would not utilize smeared-heat sources. This is crucial because adequate representation of condensate drainage between drifts and temperature variability in the NFE requires the use of discrete- or line-averaged heat sources.

As discussed in previous sections, the multi-scale TH modeling approach generates a set of corrections to the SMT-predicted repository host-rock temperature distribution $\left(T_{\text {rep }}\right)$ to account for the effects of convective heat transfer and drift-scale processes not incorporated in the SMT submodel. One of the more important corrections is for the influence of discrete heat sources. Because the SMT submodel uses a smeared-heat source, it does not represent either the drift-wall temperature $\left(T_{\mathrm{dw}}\right)$ or the WP temperature $\left(T_{\mathrm{wp}}\right)$ or the spatial variability of these temperatures. Another important aspect that the SMT model does not represent is the heat-pipe effect, which increases the efficiency of heat transfer (decreases the thermal gradient) such that the SMT model overpredicts $T_{\text {rep }}$.

The multi-scale procedure uses functional relations (scanning curves) between $T_{\mathrm{dw}}$ predicted by LDTH submodels and $T_{\text {rep }}$ predicted by SDT submodels. The LDTH submodel captures the heat-pipe effect and the influence of the local ambient percolation flux $\left(q_{\text {perc }}\right)$ on local heat flow in the UZ and on temperature in the NFE. Comparing Figure 3-58a and Figure 3-58d with Figure 3-58b and Figure 3-58e shows how the multi-scale-predicted $T_{\mathrm{dw}}$ is significantly lower than the SMT-predicted $T_{\text {rep }}$. The primary reason is the heat-pipe effect. Comparing Figure 3-58c and Figure 3-58f with Figure 3-58b and Figure 3-58e shows that the WP surface temperature $\left(T_{\text {wo }}\right)$ is higher than the temperature on the adjacent drift wall; however, $T_{\text {wp }}$ is still lower than the SMT-predicted $T_{\text {rep }}$.

There are two potentially important processes that the multi-scale $\mathrm{TH}$ modeling approach described in this report does not represent: (1) mountain-scale, buoyant, gas-phase convection; and (2) mountain-scale lateral diversion of condensate. The first process could be evaluated by replacing the SMT model with a SMTH model. The second process must be evaluated with a DMTH model. A computationally feasible DMTH model might consist of a 2-D east-west cross-sectional TH model oriented perpendicular to the structural dip of hydrostratigraphic units. A quasi-2-D version of the multi-scale TH modeling approach could then be applied to the east-west geometry, and the results could be compared with the eastwest DMTH model.

In the following five sections, the sensitivity of TH conditions in the NFE to the following factors is considered:

- TH properties significantly influence the magnitude of matrix-imbibition flux. Matrix imbibition in the host rock plays a key role in determining the spatial and temporal extent of rock dryout and $R H$ reduction.

- Ambient percolation flux $q_{\text {perc }}$ strongly influences the spatial and temporal extent of rock dryout and $R H$ reduction. Because $q_{\text {perc }}$ varies with location in the repository, the NFE is assessed at several locations.

- Edge cooling effects strongly influence the NFE, and conditions are assessed for TSPA-VA at "center" and "edge" locations.

- WP type is important because heat generation varies substantially among adjacent WPs, influencing local temperatures and rock dryout. Variation of heat output among WPs is more important for the point-load design because it accentuates the axial differences in NFE conditions 
- Repository design alternatives-including the point-load and line-load designs and engineered backfill- - have a strong influence of NFE conditions. Point-load and lineload designs differ with respect to (1) line-average thermal loading; (2) variability of thermal loading along the drifts; and (3) drift spacing, which can be greater for the line-load design and which greatly improves the effectiveness of condensate drainage between drifts. Engineered backfill increases the WP temperature and thereby reduces the $R H$ on WPs.

\subsubsection{Influence of TH Properties}

This section makes a qualitative comparison of multi-scale results obtained with three TH property sets, which differ chiefly with respect to representation of matrix imbibition diffusivity $D_{\text {imb }}$ in the tsw36 unit (the lowermost repository host-rock unit) and in the tsw37 unit (the basal vitrophyre). Matrix imbibition diffusivity is not an input parameter for NUFT, but can be calculated from the input parameters (Buscheck, 1997). The reported value for matrix imbibition is specific to a particular matrix saturation (chosen to be $90 \%$ by Buscheck, 1997) because the diffusivity decreases with increasing saturation. As such, the imbibition diffusivity can be used as an index of the tendency for rewetting by flow in the tuff matrix.

In the following discussion, the $\mathrm{TH}$ property sets considered are those that correspond to the nominal, TSPA-VA base-case infiltration map of Flint et al. (1996a). This proviso is needed because, in the development of TH property sets, the values obtained are specific to the magnitude of infiltration assumed (Bodvarsson et al., 1997). The three property sets are distinguished as follows:

- $\quad$ TSPA-VA base-case (July 1997) property set has large values of $D_{\text {imb }}$ in the TSw36 and TSw37 units, resulting in strong capillary-driven matrix flux. The July 1997 property set also has small values for the van Genuchten alpha parameter $\alpha_{\mathrm{f}}$ for fractures throughout the UZ, causing strong capillary-driven flux of water in fractures. Stronger capillary behavior reduces the spatial and temporal extent of dryout and $R H$ reduction in the NFE.

- "TH" (November 1997) property set was modified from the July 1997 set on the basis of temperature data from the SHT (Lawrence Berkeley National Laboratory memorandum to G.S. Bodvarsson from J. Birkholzer, November 19, 1997; Buscheck et al., 1993). The value of $D_{\text {imb }}$ in the TSw36 unit is reduced by a factor of 12 relative to the July 1997 set. This property set has larger values for $\alpha_{\mathrm{f}}$ and thus less capillarydriven flux in fractures than does the July 1997 set. It has the same value of $D_{\text {imb }}$ in the TSw37 unit.

- "Modified-TH" property set is a slightly modified version of the November 1997 set. The modification was based on experimental imbibition data (Flint et al., 1996b). The value for $D_{\text {imb }}$ in the TSw36 unit is reduced by a factor of 2.9 relative to the November 1997 property set, and the value for $D_{\mathrm{imb}}$ in the TSw37 unit is reduced by a factor of 28 relative to both the July 1997 and November 1997 sets.

The influence of matrix imbibition in the TSw36 unit, for the TSPA-VA base-case property set, is apparent from Figure 3-60. This is a contour map of WP RH for average 21-PWR WPs with medium heat output (see Table 3-24). On the western side of the repository, which lies within the TSw36 unit (Figure 3-8b), matrix imbibition greatly thwarts rock dryout and $R H$ reduction in the NFE (Figure 3-60a and Figure 3-60c).

Figure 3-60 Relative humidity $R H$ on the surface of "average" 21-PWR medium-heat CSNF WPs plotted for the 7/97 TSPA-VA I $\times 1$ hydrologic-parameter set and for the 12/97 modified-TH parameter set 
Reducing imbibition diffusivity for the TSw36 unit in the "TH" property set increases rock dryout and $R H$ reduction in the NFE, especially on the western side of the repository (compare Figure 3-60b and Figure 3-60e with Figure 3-60a and Figure 3-60d). Also, the smaller magnitude of capillary-driven flux in fractures (larger $\alpha_{\mathrm{f}}$ ) allows more rock dryout and $R H$ reduction throughout the repository area.

For the "modified-TH" property set, changes to matrix imbibition diffusivity in the TSw36 and TSw37 units resulted in more dryout in those units. However, the changes produced only a small reduction in $R H$ in the NFE, primarily in the southwest region of the repository (compare Figure 3-60b and Figure 3-60e with Figure 3-60c and Figure 3-60f).

\subsubsection{Influence of Ambient Percolation Flux Magnitude}

Three different infiltration flux $\left(q_{\text {inf }}\right)$ scenarios are considered in this section. (The assumption is made that infiltration flux $q_{\text {inf }}$ and percolation flux $q_{\text {perc }}$ are equal at each location in the repository because lateral diversion is apparently minor (comparing net infiltration and repository-level percolation maps from Bodvarsson et al., 1997). The "modified-TH" parameter set is used in this section. The three $q_{\text {inf }}$ scenarios are as follows:

- In the $\mathbf{I} \times \mathbf{1}$ infiltration flux scenario, I stands for the nominal TSPA-VA base-case flux from the map of Flint et al. (1996a). The mean value for the total repository area $\left(q_{\text {inf }}=\right.$ $7.80 \mathrm{~mm} / \mathrm{yr}$ ) is computed directly from the infiltration flux map and used as model input for the $\mathrm{I} \times 1$ calculations in Section 3.7. (The number of significant figures used for infiltration flux values does not reflect the uncertainty associated with the infiltration flux map, but rather the inherent precision with which values are represented in the numerical model.)

- The $\mathbf{I} / \mathbf{5}$ infiltration-flux scenario is the nominal infiltration-flux map divided by 5 . The corresponding value for the total repository area $\left(q_{\text {inf }}=1.56 \mathrm{~mm} / \mathrm{yr}\right)$ is used as model input for the I/ 5 calculations in Section 3.7.

- The $\mathbf{I} \times 5$ infiltration-flux scenario is the nominal infiltration-flux map multiplied by 5 . The corresponding value for the total repository area $\left(q_{\text {inf }}=39.0 \mathrm{~mm} / \mathrm{yr}\right)$ is used as model input for the $\mathrm{I} \times 5$ calculations in Section 3.7.

The influence of the $q_{\text {inf }}$ distribution in the infiltration map (Figure 3-1b and Figure 3-1d) is evident from Figure 3-60. The areas of greatest reduction of $R H$, and greatest duration of dryout, correspond to areas of lower $q_{\text {inf }}$. In general, $q_{\text {inf }}$ is lower in the eastern part of the proposed repository layout than in the western part. The region with the lowest $q_{\text {inf }}$ lies beneath Drill-Hole Wash in the northeast quadrant of the repository. The part of the repository lying beneath the summit of Yucca Mountain has the highest $q_{\text {inf. }}$. The southcentral area also experiences high $q_{\text {inf }}$. Accordingly, the magnitude and duration of $R H$ reduction in the NFE is less in those regions.

The influence of $q_{\text {inf }}$ on rock dryout and $R H$ reduction in the NFE is evident from Figure 3-61, which is a contour plot of WP RH for average 21-PWR WPs with medium heat output. The I $x 5$ scenario greatly thwarts rock dryout and $R H$ reduction in the NFE, causing WP RH to exceed $80 \%$ at $500 \mathrm{yr}$ and to exceed $90 \%$ at $1000 \mathrm{yr}$ over most of the repository area (Figure 3-61a and Figure 3-61d). For the $\mathrm{I} \times 1$ scenario, rock dryout and $\mathrm{RH}$ reduction in the NFE are greater, causing WP RH to be less than $60 \%$ at $500 \mathrm{yr}$ and less than $80 \%$ at $1000 \mathrm{yr}$ (Figure 3-61b and Figure 3-61e). For the I/ 5 scenario, rock dryout and $R H$ reduction in the NFE are much more extensive, causing WP RH to be less than $50 \%$ at $500 \mathrm{yr}$ and less than $70 \%$ at 1000 yr (Figure 3-61c and Figure 3-61f).

Figure 3-61 Relative humidity $R H$ on the surface of "average" 21-PWR medium-heat CSNF WPs plotted for three infiltration-flux $q_{\text {inf }}$ cases 


\subsubsection{Repository-Edge Effects}

For the multi-scale models used to evaluate repository-edge effects, the "modified-TH" property set is used, with nominal $(\mathrm{I} \times 1)$ infiltration. The supporting calculations compare four locations in the repository including the following (refer to Table 3-22 for location nomenclature):

- $15 \mathrm{c} 2$ location is located in the northwest quadrant of the repository layout, where the nominal infiltration flux is $8.5 \mathrm{~mm} / \mathrm{yr}$. The local host-rock unit is the TSw35 unit, and the repository depth is $384 \mathrm{~m}$.

- $15 \mathrm{c} 4$ location is located in the northeast quadrant of the repository layout, where the nominal infiltration flux is $2.0 \mathrm{~mm} / \mathrm{yr}$. The local host-rock unit is the TSw35 unit, and the repository depth is $303 \mathrm{~m}$.

- 15c5 location is located in the northeast quadrant of the repository layout, where the nominal infiltration flux is $0.67 \mathrm{~mm} / \mathrm{yr}$. The local host-rock unit is the TSw34 unit, and the repository depth is $282 \mathrm{~m}$.

- 12c5 location is located in the southeast quadrant of the repository layout, where the nominal infiltration flux is $8.5 \mathrm{~mm} / \mathrm{yr}$. The local host-rock unit is the TSw34 unit, and the repository depth is $231 \mathrm{~m}$.

The comparative calculations are summarized in following text.

For analysis of edge effects, two attributes of long-term temperature behavior in the NFE are considered: (1) duration of boiling conditions, and (2) whether boiling temperatures are superheated $\left(\mathrm{T}>96^{\circ} \mathrm{C}\right)$ or are indicative of long-term reflux/ heat-pipe conditions at the drift wall $\left(\mathrm{T} \cong 96^{\circ} \mathrm{C}\right)$. Conditions that are found to influence the duration of boiling temperatures in the NFE are as follows:

- Duration of boiling temperatures is not sensitive to the local percolation flux.

- Duration increases toward the repository center.

- Duration increases with repository depth below the ground surface, particularly for central locations. This correlation weakens with proximity to the repository edges. Conditions that are found to influence the tendency for long-term reflux/heat-pipe activity are as follows:

- The tendency for temperatures of $\sim 96^{\circ} \mathrm{C}$ to persist increases with greater dry thermal conductivity $K_{\text {th,dry }}$.

- The tendency for temperatures of $\sim 96^{\circ} \mathrm{C}$ to persist increases with greater local percolation flux.

The duration of decreased $R H$ in the NFE increases with smaller local percolation flux, and increases toward the center of the repository. The duration of reduced air-mass fraction depends primarily on the boiling period duration and is therefore less sensitive to the local percolation flux. The maximum decrease of $R H$ in the NFE during heat-up depends primarily on the peak temperature; peak temperature is achieved early in the thermal evolution and is relatively sensitive to dry thermal conductivity but relatively insensitive to topographic effects and repository-edge effects. The maximum liquid-phase flux $3 \mathrm{~m}$ above the drift crown occurs early in the thermal evolution and depends primarily on thermal loading. Flux above the drift declines throughout much of the thermal period until (at the end of the boiling period) it is approximately equal to the local percolation flux.

\subsubsection{Influence of Waste Package Type}

For the multi-scale models used to evaluate the influence of different WP types, the "modified-TH" property set is used, with nominal $(\mathrm{I} \times 1)$ infiltration. Comparative calculations for the 15c2 location are used to compare the effects of different WP types, while holding constant the reference point-load design and the areal thermal-loading of 
$85 \mathrm{MTU}$ / acre (refer to Table 3-22 for location information). The 15c2 location is in the northwest quadrant of the repository layout, where the nominal infiltration flux is $8.5 \mathrm{~mm} / \mathrm{yr}$. The local host-rock unit is the TSw35 unit, and the repository depth is $384 \mathrm{~m}$. It was selected because the local percolation flux is near the average for the repository and because the unit is near the center of the layout.

Three WP types are considered: a high-output 21-PWR CSNF WP, a medium-output 21PWR WP, and a low-output direct-disposal DHLW WP (see Table 3-24). The comparative calculations are summarized in $\mathrm{f}$ text.

Peak temperature at the drift wall ranges from $120^{\circ}$ to $170^{\circ} \mathrm{C}$ for these three WP types, while peak WP surface temperature ranges from $130^{\circ}$ to $205^{\circ} \mathrm{C}$ (Figure 3-62a and Figure 3-62b). These calculations were made without backfill, which would result in significantly higher WP temperatures. The duration of the boiling period is nearly the same for the three WP types; thus, the duration of decreased air-mass fraction at the WPs is also the same (Figure 3-62f). The value of the air-mass fraction approaches zero in $1 \mathrm{yr}$ for the spent-fuel WPs; it takes $10 \mathrm{yr}$ for the DHLW WP.

\section{Figure 3-62 Summary of TH conditions for three WP types}

The magnitude of $R H$ reduction during heat-up and the duration of decreased $R H$ are both directly related to the WP heat output (Figure 3-62c and Figure 3-62d). The magnitude of liquid-phase flux $3 \mathrm{~m}$ above the emplacement drift differs among the three WP types during the first $100 \mathrm{yr}$ (Figure 3-62e). The high-output WP has the earliest and greatest peak flux, whereas the low-output WP has the latest and smallest peak flux. After $100 \mathrm{yr}$, all three WP types have about the same liquid-flux history $3 \mathrm{~m}$ above the drift crown. During the first $800 \mathrm{yr}$, the flux at this location remains relatively large $\left(q_{\text {liq }}>40 \mathrm{~mm} / \mathrm{yr}\right)$. From $1000 \mathrm{yr}$ until the end of the boiling period at about $5000 \mathrm{yr}$, the flux gradually declines until it approaches the ambient percolation flux $\left(q_{\text {perc }} \rightarrow 8.5 \mathrm{~mm} / \mathrm{yr}\right)$.

\subsubsection{Influence of Repository Design Alternatives}

For the multi-scale models used to evaluate repository design alternatives, the "modified$\mathrm{TH}^{\prime \prime}$ property set is used, with nominal $(\mathrm{I} \times 1)$ infiltration. Comparison of the effects of lineload and point-load designs, and drift backfill, are made by computing the TSPA-VA performance measures over the entire repository footprint area and by computing drift-scale behavior at a representative location. Four repository design alternatives are compared:

- Point load with no backfill, the TSPA-VA base-case design (Figure 3-53)

- Line load with no backfill, in which the WPs are spaced $10 \mathrm{~cm}$ apart axially (end-toend) and the spacing between drifts is doubled to $56 \mathrm{~m}$ (Figure 3-53)

- Point load with backfill, in which backfill is placed between and around WPs and does not completely fill drift

- Line load with backfill, which assumes that measures are taken to prevent backfill from filling the 10-cm gaps between WPs

The backfill is assumed to be a quartz sand, with low imbibition diffusivity, that results in negligible capillary-driven moisture flux toward the WPs. It is assumed that the backfill is emplaced at $100 \mathrm{yr}$. The thermal conductivity of the backfill is $0.6 \mathrm{~W} / \mathrm{mK}$.

Differences between the line-load and point-load designs, with respect to rock matrix saturation and $R H$ reduction in the NFE, are evident from Figure 3-63. The line load causes much greater decrease of WP RH. For the line-load design with medium-output 21-PWR WPs, the WP RH is projected to be less than $60 \%$ at $1000 \mathrm{yr}$ for a large fraction of the 
repository area, compared to $80 \%$ for the point-load design (Figure 3-63a and Figure 3-63b). At $2000 \mathrm{yr}$, the area of the repository with WP RH less than $80 \%$ is projected to be about 10 times greater for the line load than for the point load (Figure 3-63d and Figure 3-63e).

\section{Figure 3-63 RH on the surface of "average" 21-PWR medium-heat CSNF WPs plotted for the point-load design}

For further analysis of differences in drift-scale TH effects, the 15c2 location was selected for the reasons previously discussed. Three WP types are considered, including the highoutput, medium-output, and low-output types described in earlier text. The influence of repository design alternatives on $R H$ in the NFE is evident from Figure 3-64. The line-load design produces more intense heating near the drifts and more efficient condensate drainage between the drifts. The result is greater decrease of $R H$ and more persistent $R H$ reduction on the drift wall and on WPs. The duration of decreased $R H$ is three times longer for the lineload design than it is for the point-load design. The most substantial improvement in $R H$ conditions is for the low-output DHLW WPs (Figure 3-64f) because radiative heat transfer between WPs helps to eliminate the cold trap effect.

Figure 3-64 Temperature and $R H$ on the drift wall and on the WP for the point-load and design-load designs with no backfill

There is a profound contrast in $R H$ reduction in the NFE between the line-load design without backfill and with backfill. For medium-output WPs in a backfilled line-load repository, the WP RH is projected to be less than 35\% at 1000 yr over the entire repository (Figure 3-63c). At 2000 yr, the WP RH is less than 55\% for the same design (Figure 3-63f); at $10,000 \mathrm{yr}$, the WP $R H$ is less than $75 \%$, over the entire repository area, for the same design (Figure 3-65d). With backfill, $R H$ reduction occurs mainly within the drift backfill and not within the host rock. Consequently, the projected performance of backfill is insensitive to edge-cooling effects.

The use of backfill in the point-load design causes the WPs to be thermally isolated. Consequently, for medium-output and high-output WPs, drift-wall temperature increases abruptly at $100 \mathrm{yr}$ when the backfill is emplaced, (Figure 3-65a and Figure 3-65c). Before backfill is emplaced, thermal radiation smoothes out the heat flux and temperature along the drift wall. After backfilling, heat flux from a WP is conducted locally, sharply increasing the temperature. The temperature increase with backfill in the point-load design is enormous for the hottest WPs, even compared with the line-load design. By contrast, low-output WPs produce a decrease in temperature at the drift wall after backfilling because radiative heat transfer from neighboring WPs is eliminated (Figure 3-65e).

Figure 3-65 Temperature and $R H$ on the drift wall and on the WP for the point-load and line-load designs with backfill at $100 \mathrm{yr}$

Radiative heat transfer between WPs in the backfilled line-load design enables "sharing" of the thermal load, which equalizes the distribution of temperature. The result is that the low-output WPs are much hotter and drier in line-load designs (with or without backfill) than they are in the point-load design (Figure 3-65f).

\subsection{Summary}

Thermohydrologic (TH) models provide estimates for the distributions of temperature and flow velocity in rock surrounding the repository, permitting the use of partially coupled models described in other chapters to determine whether or not more rigorous altered-zone 
(AZ) models are needed. TH models are also used to calculate the evolution of liquid water seepage flux, temperature, $R H$, and mass-fraction air within the drifts as functions of time after closure. These results are used directly to develop total system performance assessment (TSPA) abstractions for these NFE performance measures. They also provide input parameters for other process-level calculations, such as models for changes in water chemistry as water works its way into and through the different components of the engineered barrier system (EBS), which are documented elsewhere in this report.

\subsubsection{Conceptual Models and Codes}

The physical principles of heat and mass flow in fractured porous media are well understood and can easily be expressed as a set of coupled partial-differential equations. Using well-known and documented methods, these equations are approximated by finitedifference or similar formulations that can be solved by several computer codes (principally NUFT, VTOUGH, and TOUGH2) available to the project. These codes have been thoroughly tested in applications within and outside the Yucca Mountain Project and have been qualified under the project quality assurance (QA) program. They will produce essentially equivalent results given the same input data, so the choice of code is largely a matter of preference of the individual modeler, perhaps influenced by relative computational efficiency and ease of use for specific types of problems.

All these codes can perform calculations using any of several different ways of treating the coupling of heat and mass flow between matrix elements and their bounding fractures. All actual rock units underlying Yucca Mountain are fractured, with the highest frequency of fractures in the densely welded tuffs and the lowest in the nonwelded tuffs. A fully rigorous model would have to incorporate a 3-D variably connected network of fractures embedded within a 3-D matrix. Because hydrologic and thermal properties of both components may be highly variable spatially, many millions of grid blocks would be required in a model for rigorous calculation of a rock mass of even a few tens of meters on a side.

Because it is clearly impractical to model repository and mountain-scale processes in explicit detail, it has been necessary to develop approximations listed in the following text. Examples of all three of these approaches have been mentioned in this chapter; applications of the first two methods are described more fully because these have been used in the majority of TH studies for Yucca Mountain.

\section{Equivalent-Continuum Method}

The first approximation used in studies of Yucca Mountain unsaturated (UZ) hydrology and UZ thermohydrology is the ECM. By assuming that there are no local differences in flow or thermal potential between a matrix block and its bounding fractures, the potential equations can be written for the bulk rock, with properties given as volume-weighted averages of fracture and matrix properties. In effect, this assumes instantaneous heat and mass transfer between the two components of the bulk rock. ECM is the least computationally demanding method for treating fracture-matrix coupling, but it is not adequate if transient effects are important. Transient, episodic infiltration may be the predominant mechanism for local recharge at Yucca Mountain and will be represented more realistically by alternative methods.

\section{Dual-Permeability Method}

The next level of approximation is known as the DKM. In this approach, two sets of properties (one for fractures, the other for matrix) are associated with each geometric grid block. The coupling between them is assumed to be given by the product of a material constant times the local difference in potential between the fractures and the adjacent matrix. 
In effect, this assumes that a local steady-state condition exists everywhere. Adjustment of the coupling constant allows this model to represent more realistically the role of fractures in flow and transport calculations. However, the coupling constant is not directly measurable and must be inferred by comparison of model predictions of observable phenomena with actual field data.

\section{Discrete-Fracture Method}

The DFM is a more refined approach that requires explicit representation of both fractures and matrix components with grid blocks, including the interblock-transfer functions for heat and mass. Because of mathematical limitations on the numerical solution of the relevant equations, DFM can be applied only for the simplest geometry of fracture networks with a high degree of spatial symmetry so that the model domain can be spanned by a practical number of grid blocks. These problems on even a modest spatial scale quickly exceed the capabilities of professional workstations and even the current generation of supercomputers.

\subsubsection{Process Model Applications}

\subsubsection{Field tests}

Field tests in unsaturated tuffs provide the only means of building confidence in TH models. Although not discussed in detail in the present report, the single-heater G-Tunnel test was a key step in developing the project's current TH modeling capability. It clearly showed that a zone of dry rock develops around the heater as water vaporizes due to heating, and it led directly to the idea that heat could be used constructively to help prevent the contact of waste by water. Conduction was found to be the dominant heat-transfer mechanism throughout most of the heated volume, except within a zone of active boiling. Early modeling of this test, using ECM, produced reasonably good agreement between calculated and measured temperatures, but the calculated values of liquid saturation in the boiling zone were much higher than the neutron log measurements.

This test stimulated both conceptual and mathematical model development and provided some of the impetus for developing the NUFT code. The G-Tunnel test was recently reanalyzed using NUFT and the DFM. The results of these calculations agreed more closely with saturation values determined from the neutron logs than did the ECM results.

The SHT, currently in its final cool-down phase, closely resembles the G-Tunnel experiment in test geometry, instruments used, and duration. The SHT, however, is being conducted in actual repository host rock near the planned location of emplacement drifts, and pre-test characterization was more comprehensive for the SHT than for the G-Tunnel test.

Preliminary modeling results discussed in this report focus on the effects of parameters that determine the extent to which capillary forces control the distributions of temperature and liquid water around the heater borehole. Six property sets were considered, using different values for TH parameters. In Figure 3-7a, plots of temperature vs. time are shown at a radial distance of about $0.4 \mathrm{~m}(0.33 \mathrm{~m}$ above and $0.22 \mathrm{~m}$ to the left of the heater borehole axis) and about $1 \mathrm{~m}$ in from the end of the heater nearest the drift. The experimental measurements increase monotonically with time, except for a few, relatively short downward excursions associated with power outages. There is no indication of a plateau at the nominal boiling point, as would be expected if a boiling front of finite width passed through this measurement point. In contrast, all but one of the calculated temperature-vs.-time plots show a plateau at $96^{\circ} \mathrm{C}$. In Property Set 6, using the data set selected in July 1997 for TSPA-VA, the 
plateau lasts throughout the heating phase, and the calculated temperature never rises above boiling. This data set corresponds to the strongest capillary forces of any set considered. Property Set 4, with both matrix and fracture capillarity reduced, agrees well with the experimental data and did not exhibit a plateau. Although the saturation distributions were also calculated for each of the six sets of parameters, comparison of the model results with experimental data is still in progress.

The LBT is still in progress, using a partially excavated $3-\mathrm{m} \times 3-\mathrm{m} \times 4.5 \mathrm{~m}$ block in an outcrop of the repository host rock at Fran Ridge east of the crest of Yucca Mountain. Heat is supplied through an array of five parallel, horizontal boreholes placed approximately $1 \mathrm{~m}$ above the base of the block. The intent was to produce a heat source as nearly planar as possible, maximizing the likelihood of generating an observable condensate bank and heatpipe zone above the heater plane. Results are given in Section 3.4.3 for the first 300 days of the heating phase. Most of the calculations are in 3-D and based on the ECM, although some have used the DKM. Calculations were performed using the same six parameter sets that were used in modeling the SHT. Sensitivity of the temperature to capillary properties in the LBT models is qualitatively similar to that of the SHT cases, with the additional complication that moisture could escape from the model domain in the LBT. Detailed comparison of all measured temperatures and saturation values is in progress at this writing.

The actual data also show several episodes of instability, in which measured temperatures at points within the superheated region suddenly drop as much as $40^{\circ} \mathrm{C}$, reaching the nominal boiling point in less than an hour. These events are believed to have resulted from rapid fracture flow of condensate from above the superheated region, triggered by greater than normal heat loss at the top of the block. These anomalous heat losses were caused in one case by the loss of heating at the top of the block and in another by a storm. This phenomenon has yet to be modeled.

\subsubsection{Reference Calculations}

The NUFT code was used in a series of 2-D models to calculate drift-scale spatial distributions of temperature, liquid saturation, and liquid flux in the rock surrounding the emplacement drifts, and of the NFE variables of interest within the drifts, as functions of time. The results provide input for preliminary coupled-process investigations as well as for estimating waste package (WP) lifetime. To provide model parameters over the range of uncertainty consistent with experimental data, these calculations used two different sets of hydrologic properties, two different infiltration rates, and two different thermal-loading options. Five of the eight possible combinations of these parameters were used.

These reference calculations indicate that liquid flux near the emplacement drifts, $q_{\mathrm{liq}}$ is initially much greater than the ambient percolation flux and gradually declines, returning to ambient within 100 to $500 \mathrm{yr}$. The maximum flux is not sensitive to the ambient flux. In addition, $q_{\mathrm{liq}}$ is about the same for point-load and line-load designs, indicating that the total return flow of condensate does not depend on the details of moisture distribution within the heated zone. This implies that, in both designs, about the same fraction of total heat released supplies the latent heat for evaporating water somewhere in the heated zone. What differs is the effect of evaporation. A larger dryout zone is developed for the line load than for the point load because there is less refluxing and more efficient drainage of condensate through the pillars. 


\subsubsection{Drift-Scale Seepage}

The determination of temporal and spatial distribution of seepage into the emplacement drifts is the most important problem for both ambient and thermally driven UZ hydrology at Yucca Mountain. It is also the most difficult to treat rigorously because the location of seeps and their magnitude depend on the spatial heterogeneity of flow properties at scales much smaller than typical grid-block dimensions used in site-scale models. Ideally, a model should have grid-block dimensions somewhat less than the corresponding correlation lengths. The results documented in Section 3.6 should be viewed as a progress report on this topic, but they are probably good within an order of magnitude. Some of the tentative conclusions are summarized here, and will be tested to some extent, as data become available from the DST. Models have been developed, using the NUFT code, for estimating seepage into drifts under isothermal and nonisothermal conditions.

Isothermal models include 2-D and 3-D steady-state ECM and 2-D and 3-D transient DKM. Heterogeneity was introduced in the form of spatially correlated, random distributions of hydrologic parameters, and results were compared with cases with homogenous properties having the same mean values as in the heterogeneous cases. Grid spacing was on the order of one-third the correlation length. Nonisothermal calculations so far have been limited to 2-D ECM; accordingly, the results comparing isothermal with nonisothermal behavior should be used with caution.

The studies to date indicate that seepage increases with the degree of heterogeneity in hydrologic properties and is greater in 2-D than in 3-D models with similar properties and assumptions. Transient DKM simulations of episodic infiltration produce higher seepage rates than does the steady infiltration at the same average rate.

For models with heated drifts, some sets of hydrologic parameters lead to seepage into the drifts when the wall temperature is below boiling; others do not. This suggests that an immediate, short-term application of these models should be to investigate systematically the dependence of seepage on the capillary properties of the fracture-matrix system, particularly in consideration of the sensitivity of condensate drainage found in the analyses of the LBT and SHT results.

Perhaps the most important result to date of drift-scale seepage models is that the seepage flux is only a small fraction of total percolation flux in the rock near the repository. When combined with the possibility that much of the seepage will run down the inner surfaces of the drift and liner, rather than dripping onto the waste, a case could be made that very little water would contact waste even without such measures as drip shields and backfill. Making these speculations as credible as possible, by an appropriate combination of experiments and models, should receive very high priority as the project approaches license application.

\subsubsection{TSPA Multi-Scale Abstraction}

A multi-scale TH abstraction methodology has been developed and provides critical input to TSPA-VA. The abstraction tool calculates performance measures, including WP temperature and drift-air $R H$ at the WP surface. The need for a methodology that is abstracted from TH (NUFT) simulations, rather than using TH simulations directly, arises from the limited number of simulation runs that can reasonably be conducted. The abstraction methodology accurately estimates the TSPA performance measures-while accommodating the expected variability of TH properties-percolation flux and WP configurations in the repository. It does this while using the DKM problem-formulation with radiative thermal coupling inside the drift openings, but it substantially limits the required 
number of drift-scale TH simulations and completely avoids the need for detailed (drift-scale) 3-D TH simulation of the entire repository. The computational effort required for TSPA-VA is substantial, but it is within the capabilities of the network of professional workstations at LLNL.

The abstraction tool exploits several aspects of the repository TH problem to reduce computational effort. Scaling relations are used in which the repository is modeled as a smeared-heat source for calculating large-scale effects such as 3-D repository-edge effects. Another important aspect is that much of the heat transfer in certain parts of the repository TH problem occurs primarily by thermal conduction, which requires less effort to simulate than does heat transfer by coupled conduction and convection. For regions of the host rock that are dried out, or beyond the influence of water reflux activity, the influence on the TSPA performance measures can be accurately represented by conduction-only models. Assumptions used in the abstraction tool have been tested by comparing the results of approximate analysis with computationally intensive, full simulations.

The methodology has made it possible to compare the effects of different TH property sets and percolation flux maps and to compare the performance of point-load vs. line-load repository designs. Comparisons are facilitated by calculating the TSPA performance measures over the entire repository layout. Results show that, depending on the values selected for capillary properties and infiltration flux, rewetting behavior can significantly prolong or shorten the period of reduced $R H$ in the emplacement drifts. The most reliable results are obtained using TH property sets that have been calibrated against the results from thermal testing in the Exploratory Studies Facility (ESF) The magnitude of estimated infiltration is arguably the strongest influence on the calculated performance measures.

The abstraction methodology has been constructed so that refinements in the repository design can be readily analyzed. Backfill has been incorporated as an option in the methodology. Seepage models have been developed and show that spatial heterogeneity of fracture properties in the host rock can strongly influence the TSPA performance measures. Spatial heterogeneity can be readily accommodated in the present implementation of the abstraction methodology, although the computational effort will increase significantly.

\subsubsection{Improving TH Models to Reduce Uncertainty}

TH modeling has led the way in predicting NFE conditions for several years and will continue to do so until and beyond the repository license application. Improved accuracy and reliability of TH predictions will contribute directly to success in licensing and to potential cost-saving features of the repository design.

The abstraction methodology described in the previous subsection is one of the most significant accomplishments in TH modeling for near-field studies because it consolidates all the knowledge that has been gained about TH processes and optimizes the use of the available modeling tools. The full benefit of this methodology has not yet been realized by the Yucca Mountain Project. This methodology can be used to incorporate EBS features such as backfill and drip shields and can be extended to efficiently estimate the TH effects associated with alternative repository development sequences. For each major advance in the application of the abstraction methodology, additional work will be needed to verify the method against computationally intensive, full-scale simulations of the processes of interest. For the present, some additional verification against full-scale simulations is needed for TSPA-VA. In addition, the NUFT pre- and post-processors that implement the abstraction methodology will need to be qualified for use in licensing. 
The usefulness of TH simulations depends critically on the properties used to set up the models. Problems were identified with property sets proposed in 1997 for use in TSPA-VA and were corrected through ad hoc measures. In the future, a new approach to parameterestimation is needed-one that identifies which TSPA performance measures are most affected and then constructs the complete UZ hydrostratigraphy using heuristic principles that maximize confidence in the most sensitive (host-rock) properties. As a first step, nonisothermal TH data from field tests should be incorporated, with systematic weighting relative to data on the ambient system, in parameter-estimation to represent TSPA sensitivities. Uncertainty on the ambient percolation flux needs to be estimated and incorporated quantitatively into parameter estimation. Episodic flux boundary conditions should also be evaluated as means to improve confidence in TH property sets.

In the near future, more emphasis will be given to TH models for evaluating EBS design alternatives that will control in-drift flow processes. Modeling of drip shield and backfill options will involve very finely gridded models, application of existing software for radiative heat transfer in drift openings, conceptualization of the properties of failed concrete and rockfall, and representation of the partial failure of EBS components. In addition, the chemical and thermal conditions likely to be present in backfill will facilitate THC effects that may degrade or enhance EBS performance. Modification of TH simulators to investigate these effects is underway, as demonstrated by the drift-scale NUFT calculations presented in this report.

\section{Return to Table of Contents}

\subsection{References}

Bahney, R.H. (1997). “Preliminary Design Basis for WP Thermal Analysis.” (DI \#BBAA0000001717-0200-00019, Rev. 00). Las Vegas, NV: Civilian Radioactive Waste Management System, Management and Operating Contractor.

[MOL.19980127.0718]

Bear, J., and Y. Bachmat (1990). Introduction to Modeling of Transport Phenomena in Porous Media. Dordrecht, The Netherlands: Kluwar Acad. Publishers.

[NNA.19930507.0079]

Birkholzer, J. (1997). “T-H Modeling of the Single-Heater Test with Site-Scale Calibrated Properties." Lawrence Berkeley National Laboratory: memo to G.S. Bodvarsson, Lawrence Berkeley National Laboratory. November 19, 1997.

Birkholzer, J.T., C.F. Tsang, and J.S.Y. Wang (1996). “Drift Scale Modeling.” S.J. Altman et al. (primary authors). Multi-Scale Modeling to Evaluate Scaling Issues, Percolation Flux, and Other Processes for PA Recommendations. (WBS 1.2.5.4.6, WA-210 Rev. 00, Level 3 Milestone T6540) [MOL.19970512.0010]

Bodvarsson, G.S., and T.M. Bandurraga (1996). "Development and Calibration of the ThreeDimensional Site-Scale Unsaturated Zone Model of Yucca Mountain, Nevada." Berkeley, CA: Lawrence Berkeley National Laboratory, Earth Sciences Division. [MOL.19970211.0176]

Bodvarsson, G.S., T.M. Bandurraga, and Y.S. Wu (1997). “The Site-Scale Unsaturated Zone Model of Yucca Mountain, Nevada, for the Viability Assessment." June 1997. (LBNL-40376, UC-814). Berkeley, CA: Lawrence Berkeley National Laboratory, in collaboration with the U.S. Geological Survey. [MOL.19971014.0232] 
Buesch, D.C., R.W. Spengler, T.C. Moyer, and J.K. Greslin (1995). “Revised Stratigraphic Nomenclature and Macroscopic Identification of Lithostratigraphic Units of the Paintbrush Group Exposed at Yucca Mountain, Nevada." Open-File Report. (OFR 94-469). Denver, CO: U.S. Geological Survey. [MOL.19960702.0036]

Buscheck, T.A. (1996). "Hydrothermal Modeling." D.G. Wilder (ed.). Near-Field and AlteredZone Environment Report (Volume II). Livermore, CA: Lawrence Livermore National Laboratory. Section 1.0. (UCRL-JC-124998) [MOL.19961212.0122]

Buscheck, T.A. (1997). "Analysis of Thermal-Hydrological Behavior During the Heating Phase of the Single-Heater Test at Yucca Mountain." Milestone report for the CRWMS Management and Operating Contractor, U.S. Department of Energy. Livermore, CA: Lawrence Livermore National Laboratory. [MOL.19980109.0241]

Buscheck, T.A., J. Gansemer, T. Delorenzo, J.J. Nitao, R.J. Shaffer, M.J. Cordery, and K.H. Lee (1997a). "Thermal-Hydrological Models of the Distribution of Temperature, Relative Humidity, and Gas-Phase Air-Mass Fraction in Repository Drifts." Milestone report for the CRWMS Management and Operating Contractor, U.S. Department of Energy. (SPLD1M4 and SPLD2M4). Livermore, CA: Lawrence Livermore National Laboratory. [MOL.19980109.0248]

Buscheck, T.A., and J.J. Nitao (1988). "Estimates of the Width of the Wetting Zone Along a Fracture Subjected to an Episodic Infiltration Event in Variably Saturated, Densely Welded Tuff." (UCID-21579). Livermore, CA: Lawrence Livermore National Laboratory. [HQX.19890522.0064]

Buscheck, T.A., and J.J. Nitao (1993). “Repository-heat-driven-hydrothermal flow at Yucca Mountain, Part II: Large-scale in situ heater tests." Nucl. Tech. 104(3):449-471. (Also UCRL-JC-114132—P-2, Lawrence Livermore National Laboratory, Livermore, CA). [235095]

Buscheck, T.A., and J.J. Nitao (1995). “Thermal-Hydrological Analysis of Large-Scale Thermal Tests in the Exploratory Studies Facility at Yucca Mountain." (UCRL-ID121791). Livermore, CA: Lawrence Livermore National Laboratory. [MOL.19960501.0392; 223657]

Buscheck, T.A., J.J. Nitao, J. Birkholzer, and Y.W. Tsang (1997b). "Recommended Guidelines for Hydrological Parameters Used by TSPA-VA." Lawrence Livermore National Laboratory: memo to G.S. Bodvarsson, Lawrence Berkeley National Laboratory. September 8, 1997.

Buscheck, T.A., J.J. Nitao, and D.A. Chesnut (1991). “The impact of episodic nonequilibrium fracture-matrix flow on geological repository performance." In proceedings from A.N.S. FOCUS '91 Conference on Nuclear Waste Packaging. Las Vegas, NV: Sept. 29-Oct. 2, 1991. (Also UCRL-JC-106759-SUM, Lawrence Livermore National Laboratory, Livermore, CA) [NNA.911231.0023; 2032525]

Buscheck, T.A., J.J. Nitao, and D.A. Chesnut (1993). “Hydrothermal Modeling.” D.G. Wilder (ed.). Preliminary Near-Field Environment Report, Vol. II: Scientific Overview of Near-Field Environment and Phenomena. (Chapter 1) Livermore, CA: Lawrence Livermore National Laboratory. pp. 5-27. (UCRL-LR-107476, Vol. II) [226944] 
Buscheck, T.A., J.J. Nitao, and L.D. Ramspott (1996). “Near-field thermal-hydrological behavior for alternative repository designs at Yucca Mountain." In proceedings from XX International Symposium Science Basis Nuclear Waste Management. Boston, MA: Dec. 2, 1996. Mater. Res. Soc. (Also UCRL-JC-124629, Lawrence Livermore National Laboratory, Livermore, CA) [MOL.19961014.0031]

Buscheck, T.A., R.J. Shaffer, K.H. Lee, and J.J. Nitao (1997c). “1997 Analysis of ThermalHydrological Behavior During the Heating Phase of the Single-Heater Test at Yucca Mountain." Supplemental submission of milestone report for the CRWMS Management and Operating Contractor, U.S. Department of Energy. (SP9266M4). Livermore, CA: Lawrence Livermore National Laboratory. [MOL.19980109.0262]

Buscheck, T.A., R.J. Shaffer, and J.J. Nitao (1997d). "Pretest Thermal-Hydrological Analysis of the Drift-Scale Thermal Test at Yucca Mountain." (SP9318M4). Livermore, CA: Lawrence Livermore National Laboratory.

CRWMS M\&O (1996a). "Characterization of the ESF Thermal Test Area." (B00000000-017175705-00047, Rev. 1). Las Vegas, NV: Civilian Radioactive Waste Management System Management and Operation Contractor: TRW Environmental Safety Systems, Inc. [MOL.19970116.0187]

CRWMS M\&O (1996b). “Test Design, Plans, and Layout for the First ESF Thermal Test.” (BAB000000-01717-4600-00025, Rev. 1). Las Vegas, NV: Civilian Radioactive Waste Management System Management and Operation Contractor: TRW Environmental Safety Systems, Inc. [MOL.19970114.0166]

CRWMS M\&O (1997). “Waste Isolation Study.” May 13, 1997. (B00000000-01717-5705-00062 Rev. 00). Las Vegas, NV: Civilian Radioactive Waste Management System Management and Operating Contractor: TRW Environmental Safety Systems, Inc. [234282]

Dunlap, B., N.D. Francis, C.K. Ho, and M.L. Wilson (1997). “Provide Input to XPA07, PA204, PA208, PA210 Models." Las Vegas, NV: CRWMS M\&O/INTERA, Inc., and Sandia National Laboratories (Albuquerque, NM). [MOL.19980205.0250]

Edlefsen, N.E., and A.B.C. Anderson (1943). “Thermodynamics of Soil Moisture.” Hilgardia 15:31-298. [235679]

Flint, A., J.A. Hevesi, and L.E. Flint (1996a). “Conceptual and Numerical Model of Infiltration for the Yucca Mountain Area, Nevada." Water Resources Investigation Report. (3GUT623M). Denver, CO: U.S. Geological Survey. (U.S.G.S. Survey Milestone Report) [MOL.19970409.0087]

Flint, L.E., A.L. Flint, C.A. Rautman, and J.D. Istok (1996b). "Physical and Hydrologic Properties of Rock Outcrop Samples at Yucca Mountain, Nevada." Open File Report. (95-280). Denver, CO: U.S. Geological Survey. [225884]

Francis, N.D., C.K. Ho, and M.T. Itamura (1997). “UZ-Thermohydrology: Complete VA T/H Sensitivity Analysis." (SLX09F9). Albuquerque, NM: Sandia National Laboratories. [MOL.19980210.0914]

Ho, C.K. (1997). "Models of fracture-matrix interactions during multiphase heat and mass flow in unsaturated fractured porous media." In proceedings from 1997 ASME International Mechanical Engineering Congress and Exposition. Dallas, TX: ASME Fluids Engineering Division. [MOL.19971125.0236] 
Klavetter, E.A., and R.R. Peters (1986). "Estimation of Hydrologic Properties of an Unsaturated, Fractured Rock Mass." (SAND-84-2642). Albuquerque, NM: Sandia National Laboratories. [NNA.870317.0738; 202727]

Lee, K.H. (1995). “Progress Report on Pre-Test Calculations for the Large Block Test." (UCRLID-118699). Livermore, CA: Lawrence Livermore National Laboratory. [MOL.19950314.0178; 214965]

Lee, K.H. (1996). “Forecast Thermo-Hydrologic Results of the Single Heater Test." Milestone report for the CRWMS Management and Operating Contractor, U.S. Department of Energy. (SP911M4). Livermore, CA: Lawrence Livermore National Laboratory. [MOL.19970121.0144]

Lee, K.H., and T.-S. Ueng (1991). "Air-Injection Field Test to Determine the Effect of a Heat Cycle on the Permeability of Welded Tuff." (UCRL-ID-105163). Livermore, CA: Lawrence Livermore National Laboratory. [NNA.19910912.0001; 203447]

Lin, W., D. Wilder, S. Blair, T. Buscheck, W. Daily, G. Gdowski, W. Glassley, K. Lee, A. Meike, A. Ramirez, J. Roberts, D. Ruddle, J. Wagoner, D. Watwood, T. Williams, R. Carlson, and D. Neubauer (1998). "An overview of progress on the large-block test of the ESF thermal tests." In proceedings from 1998 International High-Level Radioactive Waste Management Conference. May 11-14, 1998. Am. Nucl. Soc. (Also UCRL-JC-128796 Ext. Abs., October 1997 preconference print by Lawrence Livermore National Laboratory, Livermore, CA) [MOL.19980120.0041]

Mualem, Y. (1976). “A new model for predicting the hydraulic conductivity of unsaturated porous media." Water Resour. Res. 12:513-522. [NNA.19881228.0005; 217339]

Nitao, J.J. (1988a). “Numerical Modeling of the Thermal and Hydrological Environment Around a Nuclear Waste Package Using the Equivalent Continuum Approximation: Horizontal Emplacement." (UCRL-ID-21444). Livermore, CA: Lawrence Livermore National Laboratory. [NNA.890317.0021; 201832]

Nitao, J.J. (1988b). "VTOUGH-An Enhanced Version of the TOUGH Code for Thermal and Hydrologic Simulation of Large-Scale Problems in Nuclear Waste Isolation." (UCID-21954). Livermore, CA: Lawrence Livermore National Laboratory.

Nitao, J.J. (1991). "Theory of matrix and fracture flow regimes in unsaturated, fractured porous media." In proceedings from Second International High-Level Radioactive Waste Management Conference. Las Vegas, NV. (Also UCRL-104933-SUM, Lawrence Livermore National Laboratory, Livermore, CA) [NNA.910523.0117; 234964]

Nitao, J.J. (1993). “The NUFT Code for modeling nonisothermal, multiphase, multicomponent flow and transport in porous media." In proceedings from EOS. Washington, DC.: American Geophysical Union. Supplement 73:31. (Also UCRL-JC-14769 Abs, Lawrence Livermore National Laboratory, Livermore, CA)

Nitao, J.J. (1995). Reference Manual for the NUFT Flow and Transport Code, Version 1.0. Livermore, CA: Lawrence Livermore National Laboratory. (UCRL-IC-113520)

Nitao, J.J. (1997a). “Distribution of Post-Emplacement Seepage into the Repository Drifts with Parametric Variation of Intrinsic Properties: Models and Bounds for PostEmplacement Seepage into the Repository." Milestone report for the CRWMS Management and Operating Contractor, U.S. Department of Energy. (SPLC2M4 and SPLC3M4). Livermore, CA: Lawrence Livermore National Laboratory. [MOL.19980107.0400] 
Nitao, J.J. (1997b). "Models for the Distribution of Percolation at the Repository Level and Seepage into Drifts under Pre-Emplacement Conditions." Milestone report for the CRWMS Management and Operating Contractor, U.S. Department of Energy. (SPLB2M4). Livermore, CA: Lawrence Livermore National Laboratory.

Nitao, J.J. (1997c). "Preliminary Bounds for the Drift-Scale Distribution of Percolation and Seepage at the Repository Level under Pre-Emplacement Conditions." Letter report to CRWMS Management and Operating Contractor, U.S. Department of Energy. Livermore, CA: Lawrence Livermore National Laboratory. [LLYMP 9703034; 234515]

Nitao, J.J., and J. Bear (1994). "Potentials and Their Role in Transport in Porous Media." (UCRL-JC-111741). Livermore, CA: Lawrence Livermore National Laboratory.

Nitao, J.J., and T.A. Buscheck (1995). “Discrete-fracture modeling of thermal-hydrological processes at Yucca Mountain and the LLNL G-Tunnel field test." In proceedings from Mater. Res. Soc. XIX Internat. Symp. Sci. Basis Nuclear Waste Management. Pittsburgh, PA: Nov. 27-Dec. 1, 1995. Mater. Res. Soc. [MOL.19970825.0089]

Nitao, J.J., T.A. Buscheck, and D.A. Chesnut (1993). "Implications of episodic nonequilibrium fracture-matrix flow on repository performance." Nucl. Technol. 104(3):385402. (Also UCRL-JC-109216, Lawrence Livermore National Laboratory, Livermore, CA). [NNA.19930727.0005; 208836]

Pruess, K. (1991). “TOUGH2: A General-Purpose Numerical Simulator for Multiphase Fluid and Heat Flow." (LBL-29400, EC-251). Berkeley, CA: Lawrence Berkeley National Laboratory. [213489]

Pruess, K., and Y. Tsang (1994). “Thermal Modeling for a Potential High-Level Nuclear Waste Repository at Yucca Mountain, Nevada." (LBL-35381). Berkeley, CA: Lawrence Berkeley National Laboratory. [NNA.19940427.0248; 212744]

Pruess, K., J.S.Y. Wang, and Y.W. Tsang (1990). “On thermohydrologic conditions near highlevel nuclear wastes emplaced in partially saturated fractured tuff, Part I: Simulation studies with explicit consideration of fracture effects." Water Resour. Res. 26(6):1235-1248. [NNA.19910328.0073]

TRW (1997). “Waste-Package Size Study Report.” Prepared for the U.S. Department of Energy by the CRWMS M\&O Contractor, TRW Environmental Safety Systems, Inc. (B00000000-01717-5705-00079 Rev 00). Las Vegas, NV: TRW Environmental Safety Systems, Inc. (Concurrence Draft B, October 1997)

Tsang, C.-F., J. Birkholzer, G. Li, and Y. Tsang (1997). “Drift Scale Modeling: Progress in Studies of Seepage into a Drift." Milestone report. (SP331CM4). Berkeley, CA: Lawrence Berkeley National Laboratory. [MOL.19971204.0420]

van Genuchten, M.T. (1980). “A closed form equation for predicting the hydraulic conductivity unsaturated soils." Soil Sci. Soc. Am. J. 44:892-898.

[NNA.19890522.0287; 217327]

Vanmarcke, E. (1988). Random Fields. Cambridge, MA: MIT Press.

Wang, J.S.Y. (1992). "Variations of hydrological parameters of tuff and soil." In proceedings from Third Annual International High-Level Radioactive Waste Management Conference. Las Vegas, NV: Am. Nucl. Soc., La Grange, IL. pp. 727-731. 
Wang, J.S.Y. (1996). "Evaluation of Moisture Evolution in the Exploratory Studies Facility." Berkeley, CA: Lawrence Berkeley National Laboratory. (Tracking no. TR31K6M) [MOL.19961231.0089]

Wilder, D.G., W. Lin, S.C. Blair, T.A. Buscheck, R.C. Carlson, K. Lee, A. Meike, A.L. Ramirez, J.L. Wagoner, and J.S.Y. Wang (1997). "Large Block Test Status Report." (UCRL-ID-128776). Livermore, CA: Lawrence Livermore, CA. [234859]

Zimmerman, R.W., and G.S. Bodvarsson (1989). "An approximate solution for onedimensional absorption in unsaturated porous media." Water Res. Res. 25(6):1422-1428. [221942] 


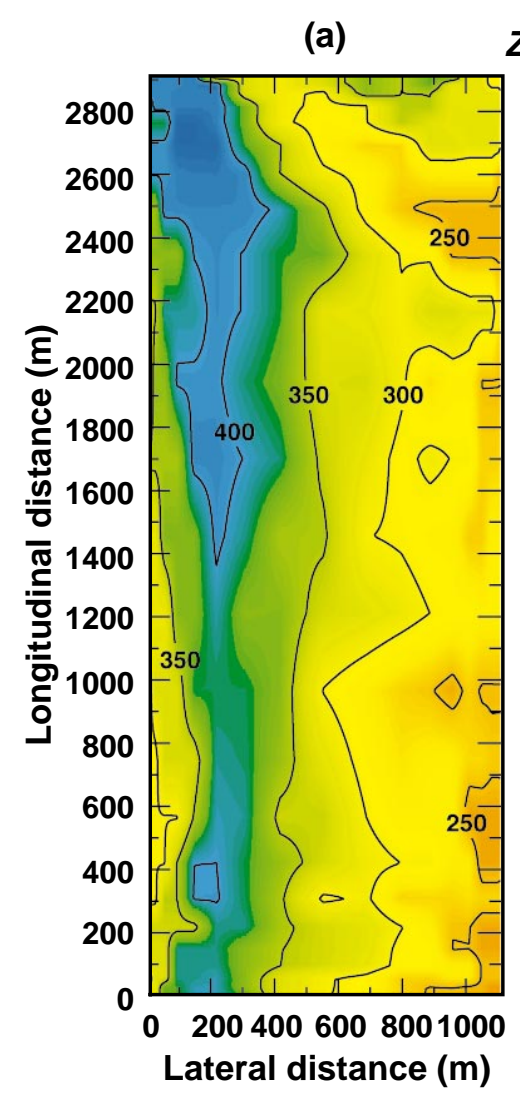

$Z_{\text {depth }}(\mathrm{m})$
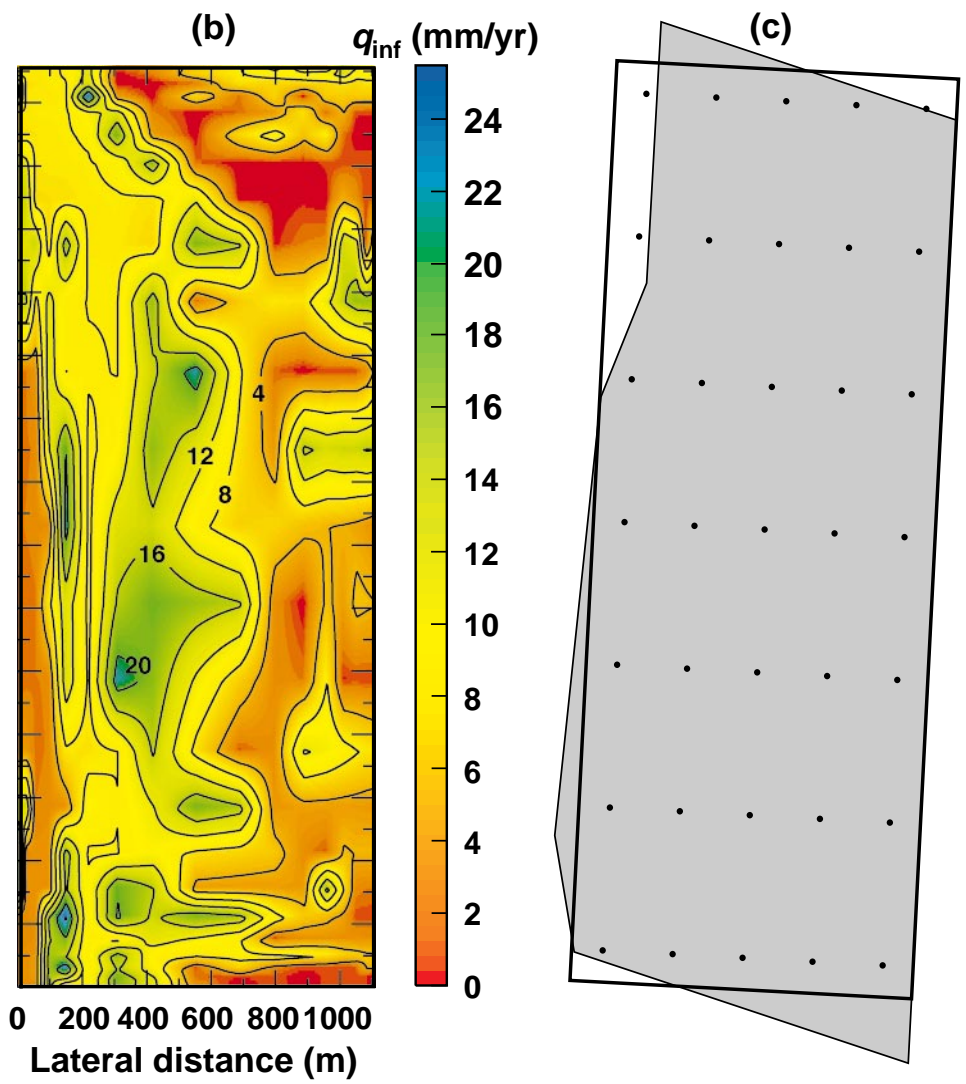

(d)

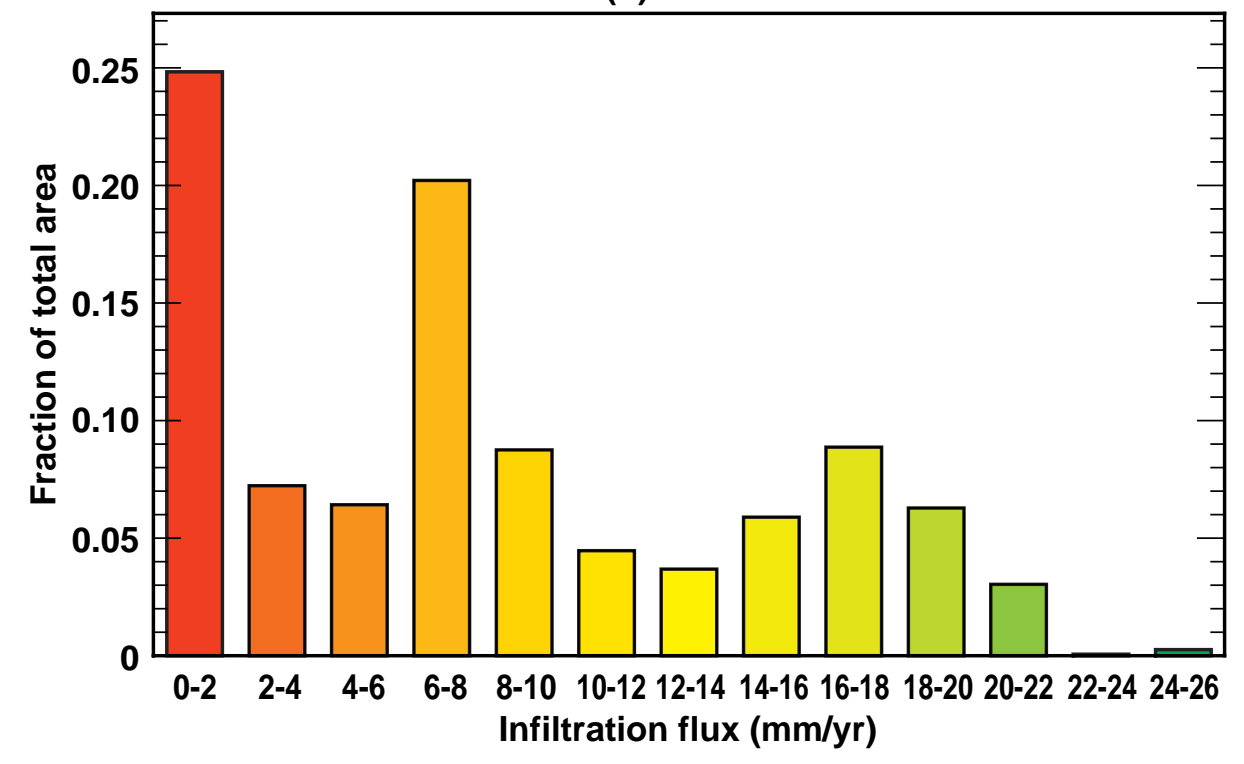

Figure 3-1. Surface topography, shallow infiltration, repository footprint, and model domain for site-scale thermal modeling at Yucca Mountain: (a) contour map showing repository depth $Z_{\text {rep }}$ below the ground surface; (b) contour map of infiltration-flux distribution [Flint, 1996] plotted over the SMT-model representation of the repository area; (c) SMT-model representation of the repository area compared with the actual repository area (depicted in gray); also shown - the 35 drift-scale-model locations in the $5 \times 7$ grid used in the multi-scale TH modeling approach; (d) frequency histogram for the shallow infiltration flux, corresponding to the contour map of $(b)$ 


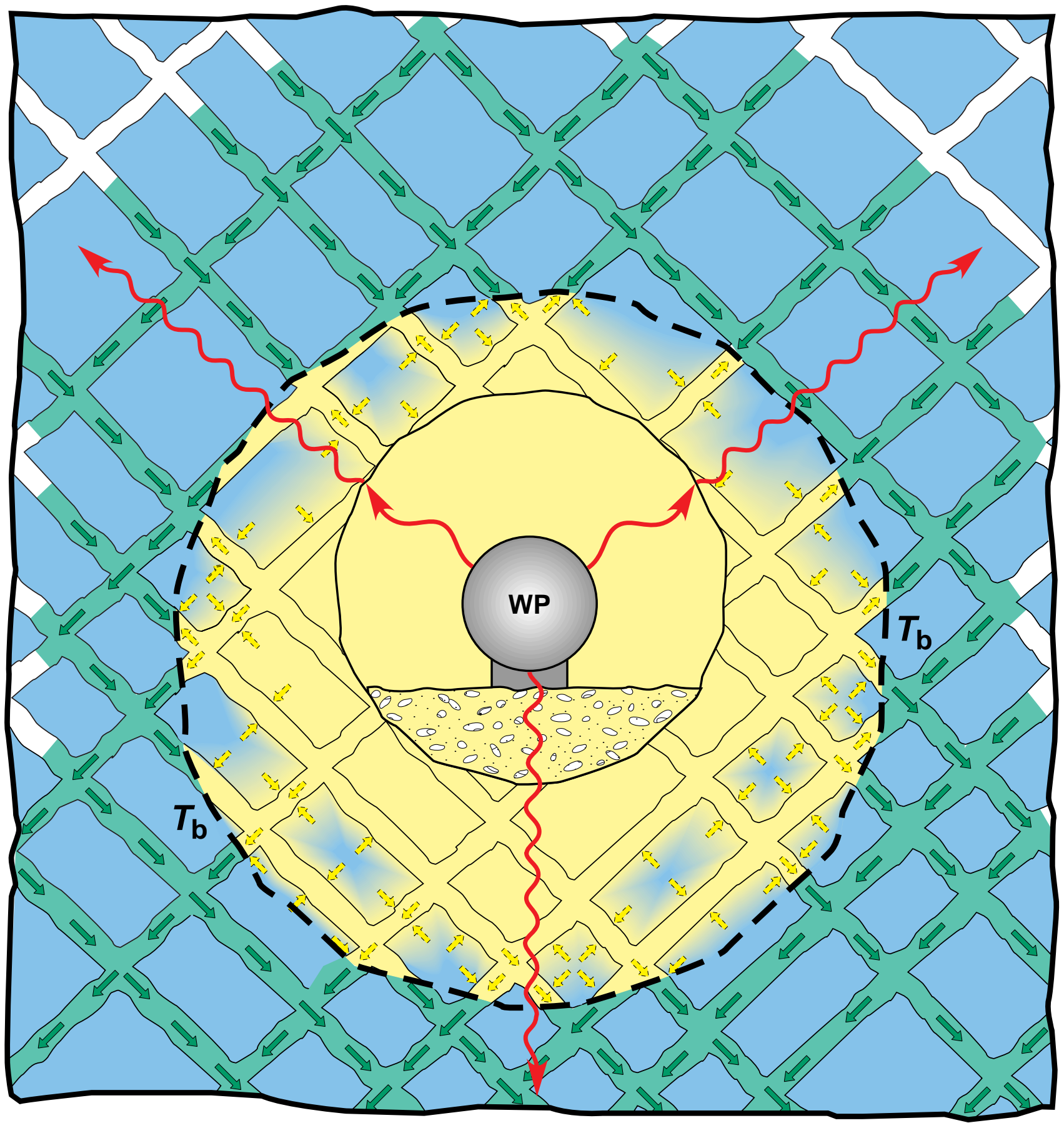

Figure 3-2. Drift-scale schematic showing decay-heat-driven TH flow and transport processes, including (1) radiative, conductive, and convective heat flow (red arrows), (2) boiling in the rock matrix and matrix-tofracture vapor transport (yellow arrows), (3) vapor flow in fractures (yellow arrows), and (4) condensation and condensate drainage (green arrows) 


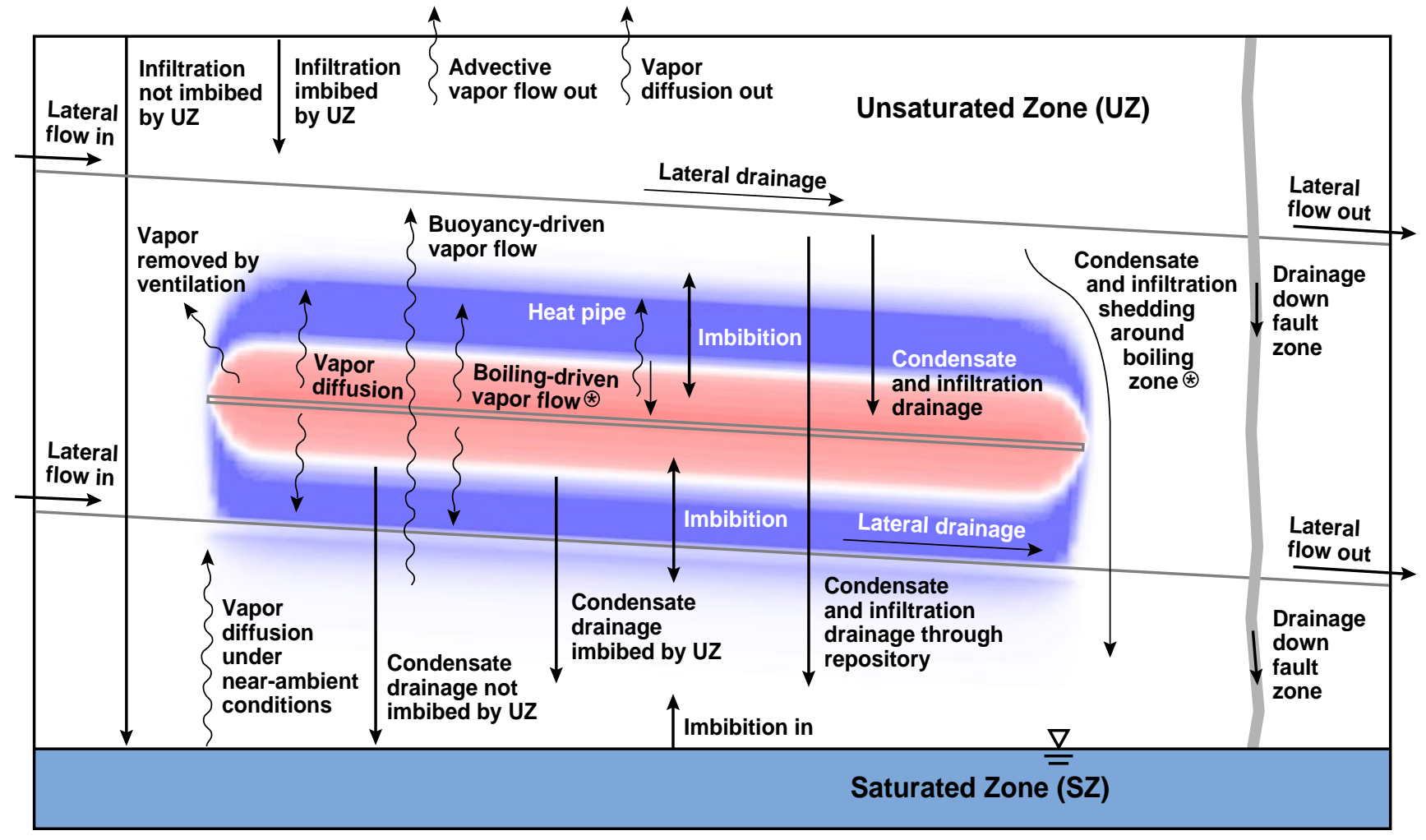

Figure 3-3. Mountain-scale schematic showing decay-heat-driven TH flow and transport processes that influence moisture redistribution and the moisture balance in the UZ 

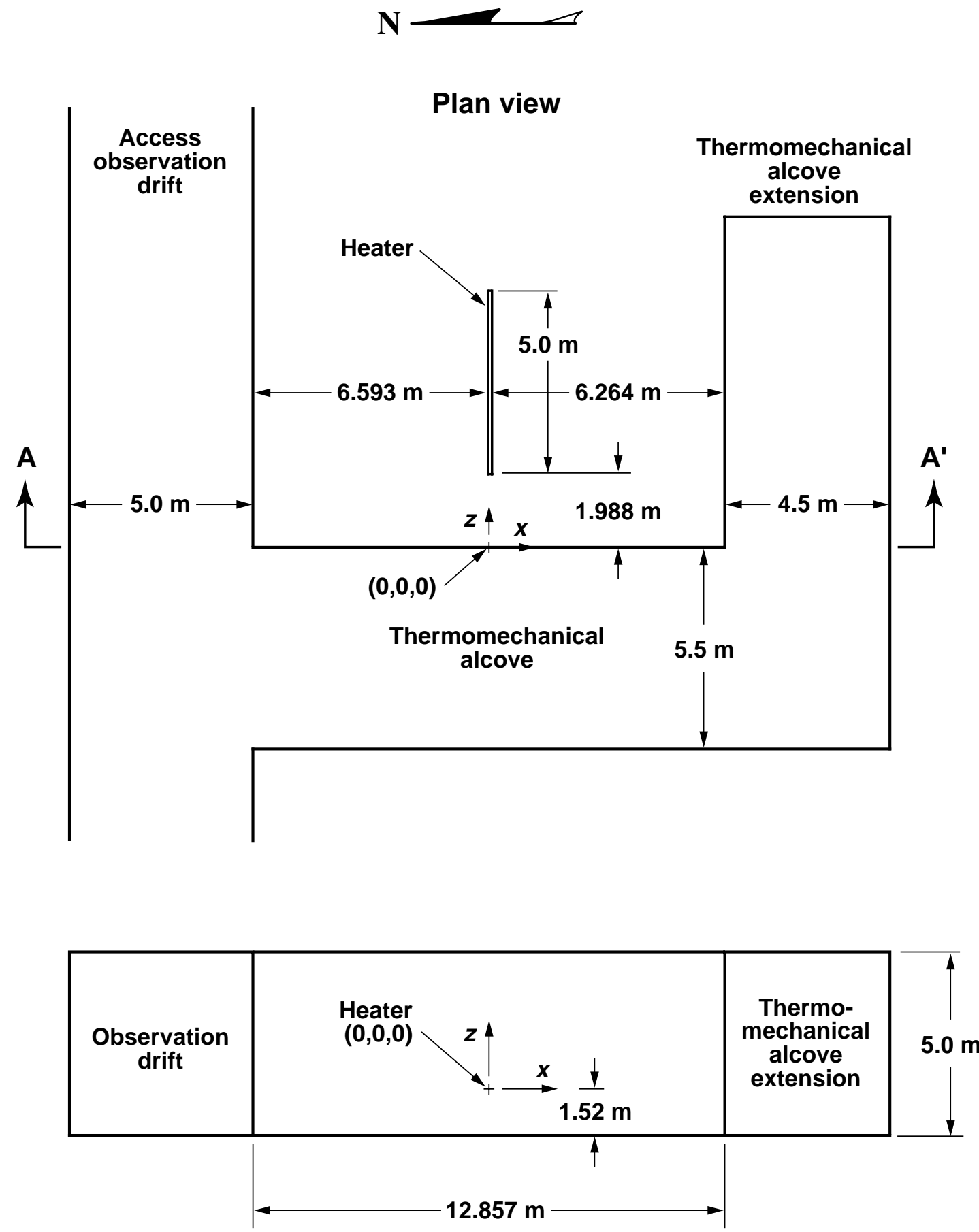

Vertical section A-A'

Figure 3-4. SHT layout, including dimensions and SHT-model coordinate system, in plan view and vertical Section A-A' 


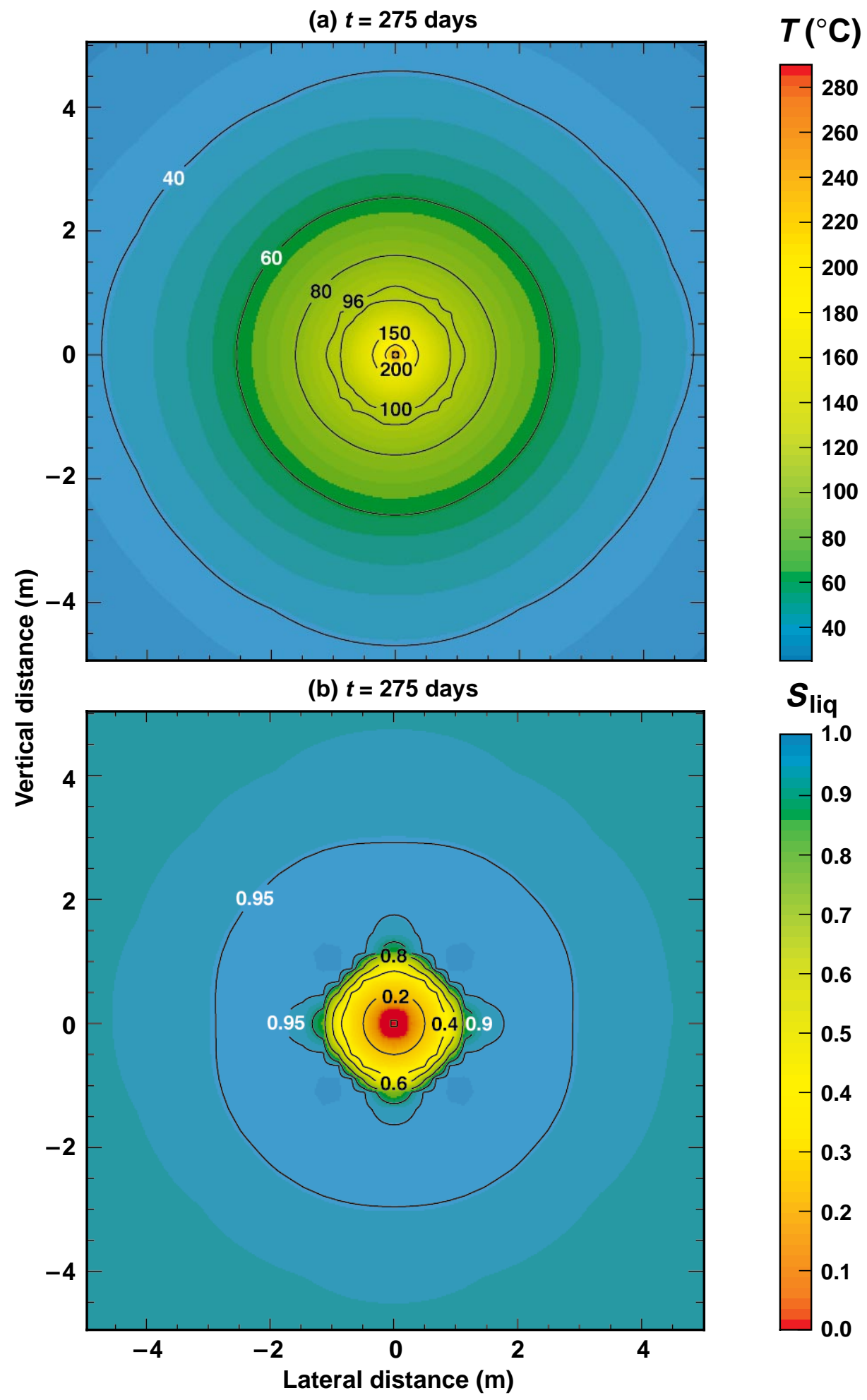

Figure 3-5. (a) Temperature $T$ distribution and (b) liquid-phase saturation $S_{\text {liq }}$ distribution at the end of the SHT heating phase $(t=275$ days $)$ in a vertical $(x-z)$ plane transverse to the midpoint of the heater for Case 2 


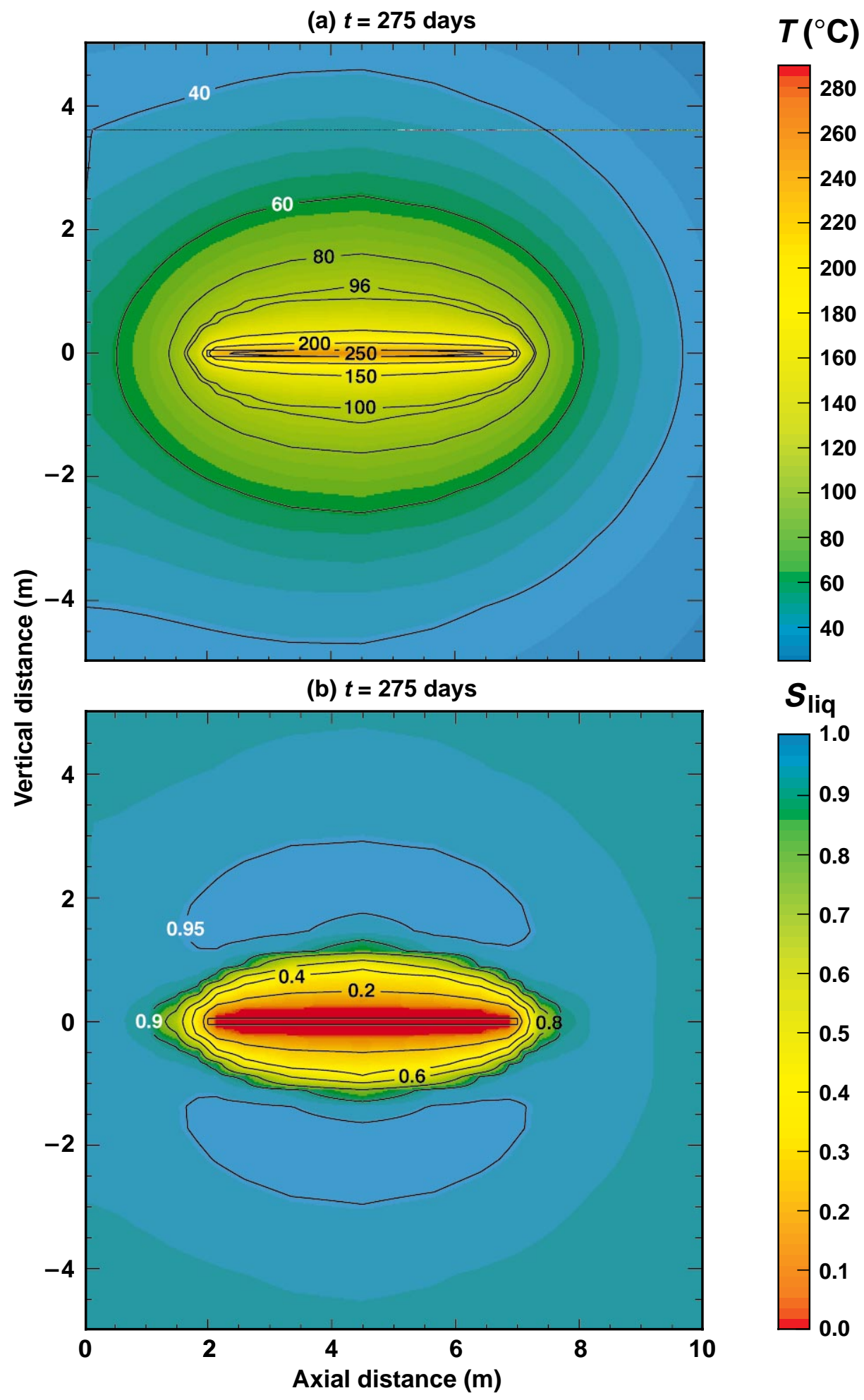

Figure 3-6. (a) Temperature $T$ distribution and (b) liquid-phase saturation $S_{\text {liq }}$ distribution at the end of the SHT heating phase $(t=275$ days) in a vertical $(y-z)$ plane along the axis of the heater for Case 2 (zero axial distance corresponds to the collar of the heater borehole) 
(a) thermocouple TMA-TC-1A-9

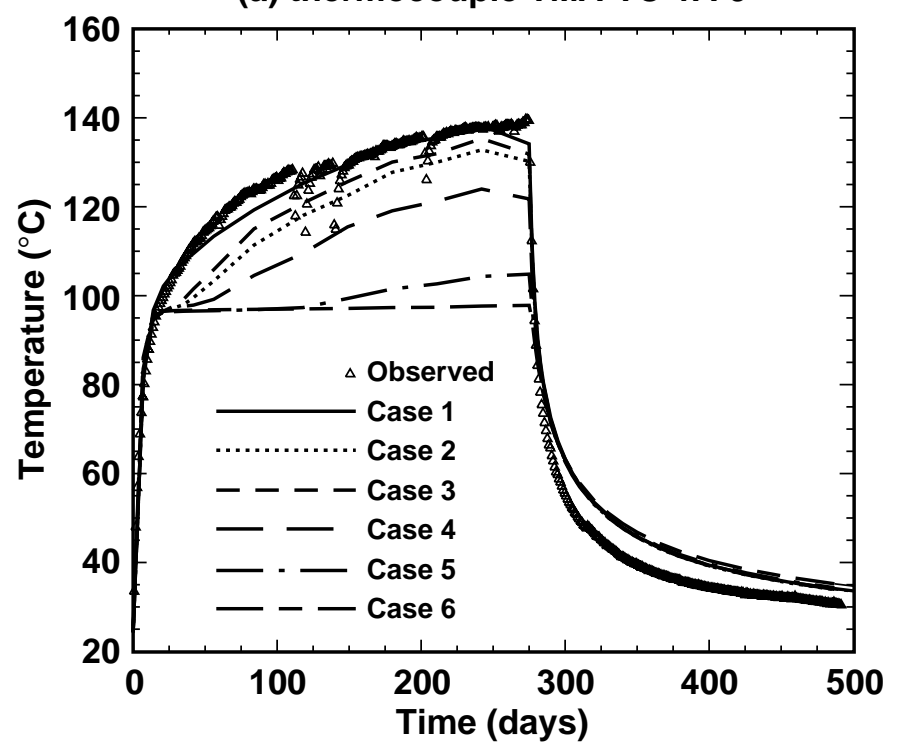

(b) borehole TMA-TC-5

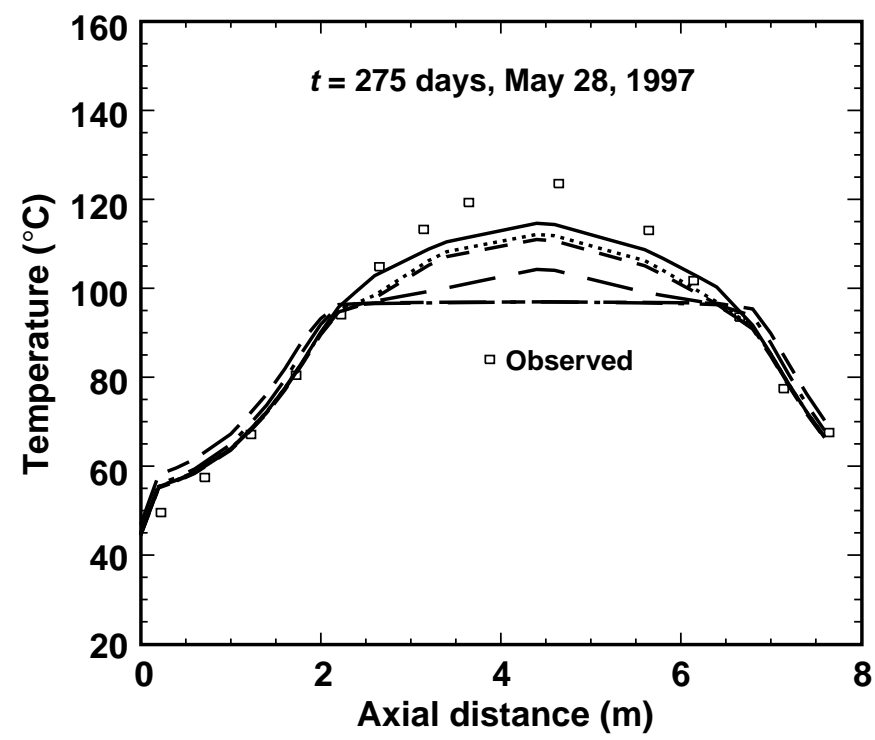

(c) borehole TMA-TC-5

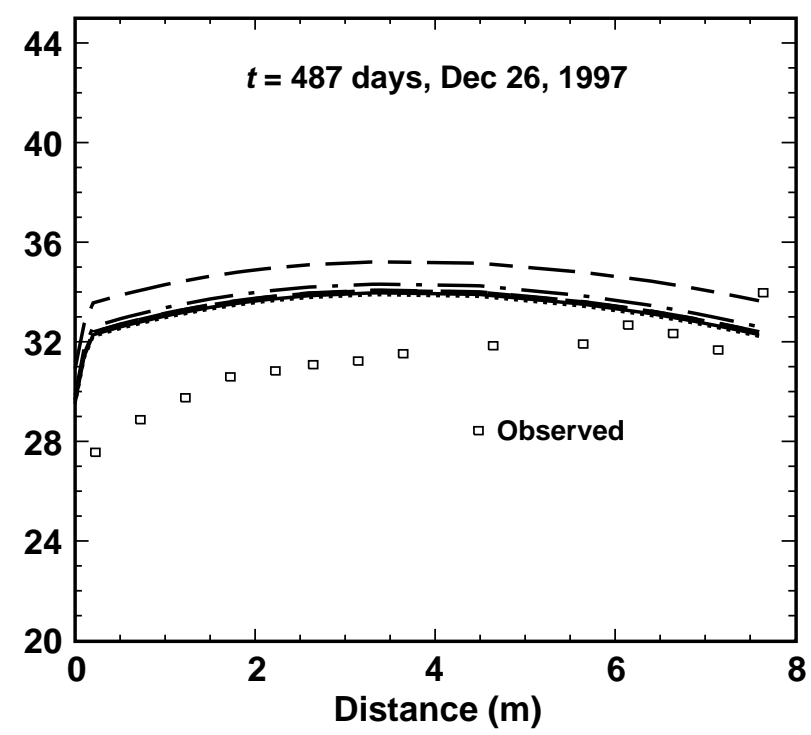

Figure 3-7. Calculated and measured temperature histories (a) compared at thermocouple TMA-TC-1A-9, located at $x=-0.221, y=2.978$, and $z=0.331$; (b) calculated and measured temperature distributions compared along borehole TMA-TC- 5 at the end of the SHT heating period ( $t=275$ days); (c) 213 days after the end of heating ( $t=487$ days) 
(a)

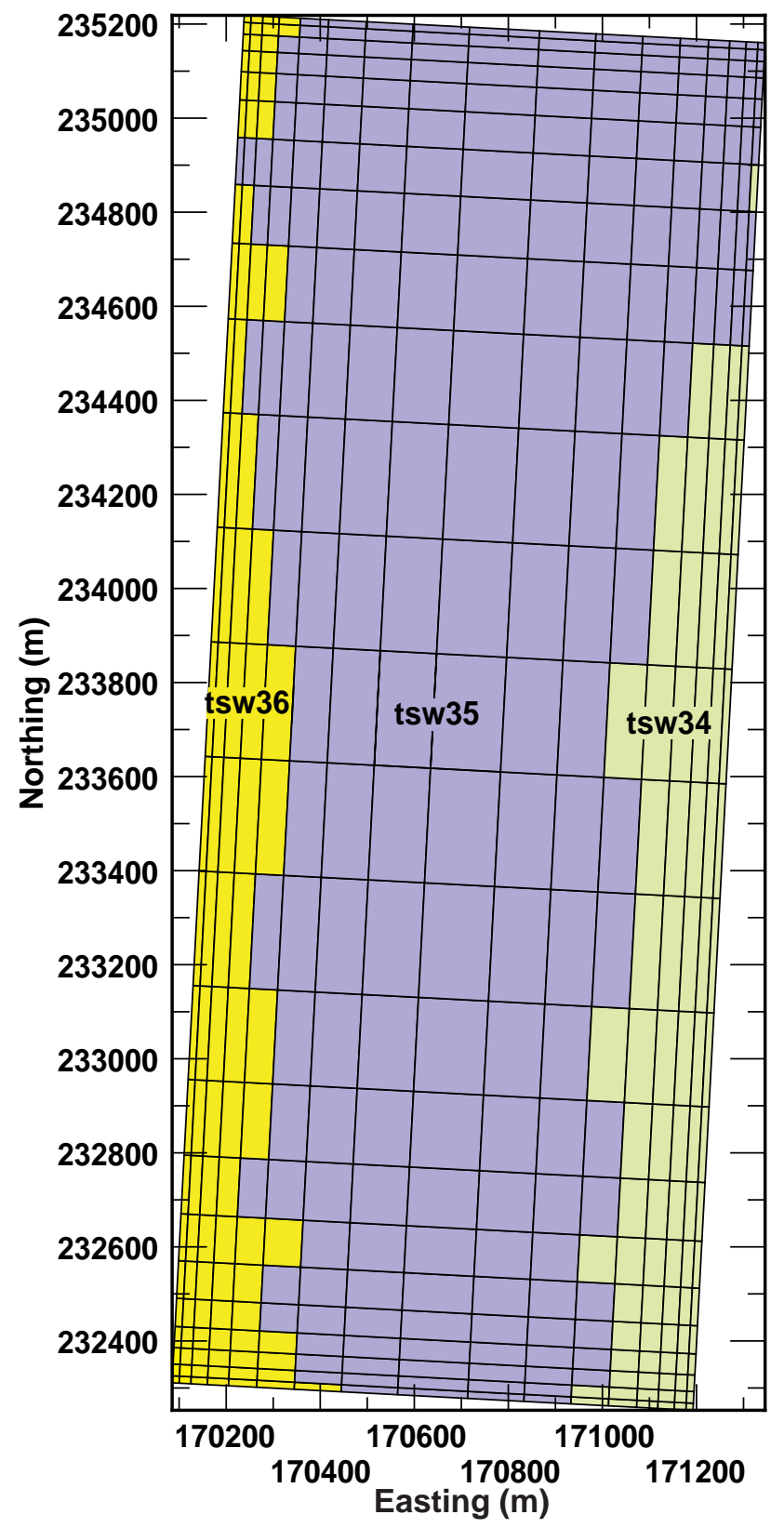

(b)

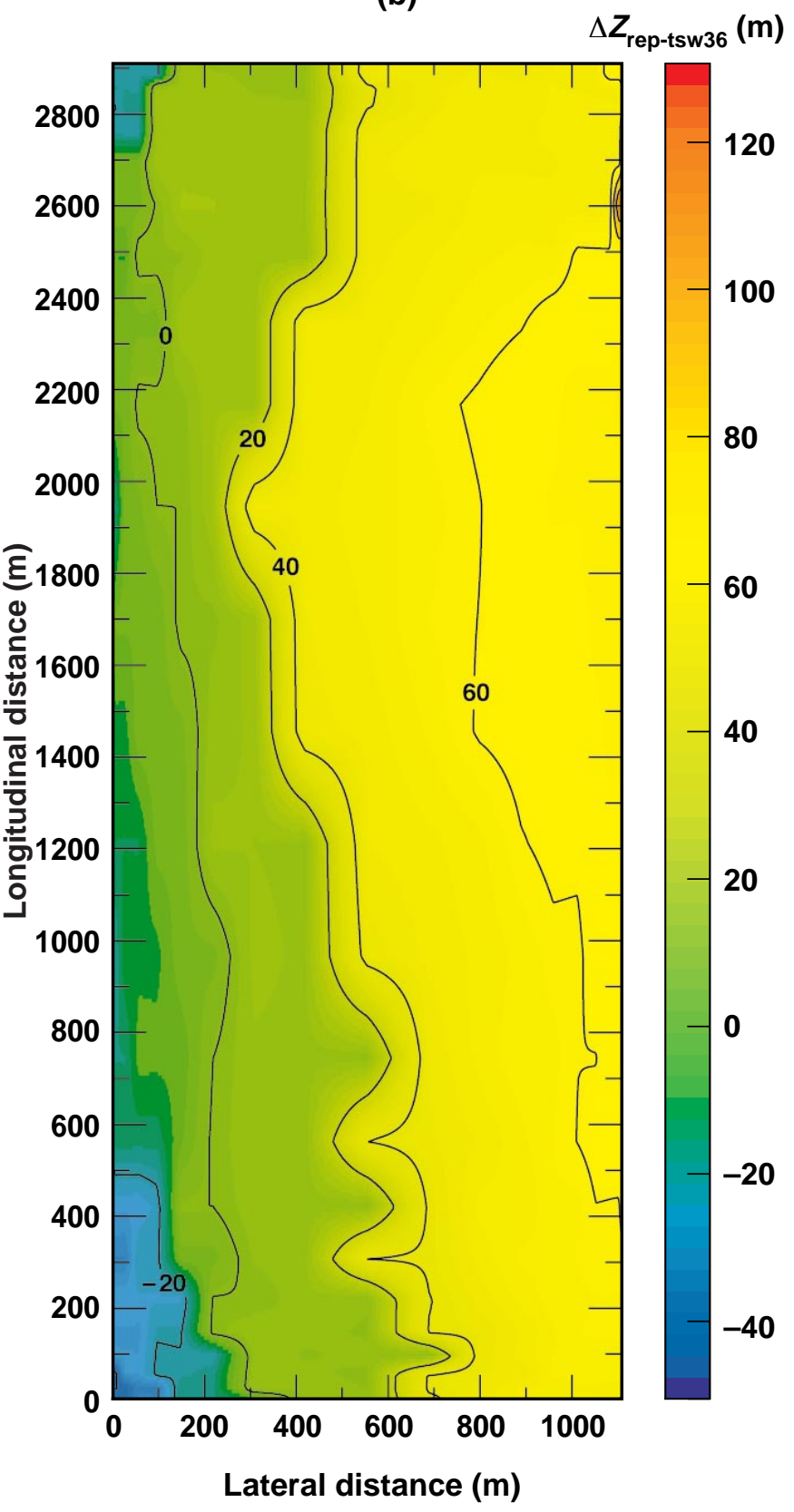

Figure 3-8. Plan view of the host-rock distribution (a) shown for the SMT-model grid of the repository area as a function of the Nevada-State coordinates; (b) contour map of the vertical distance $\Delta Z_{\text {rep-tsw36 }}$ between the repository horizon and the top of the tsw36 hydrostratigraphic model unit shown as a function of distance from the southwest corner of the repository area in the SMT model 


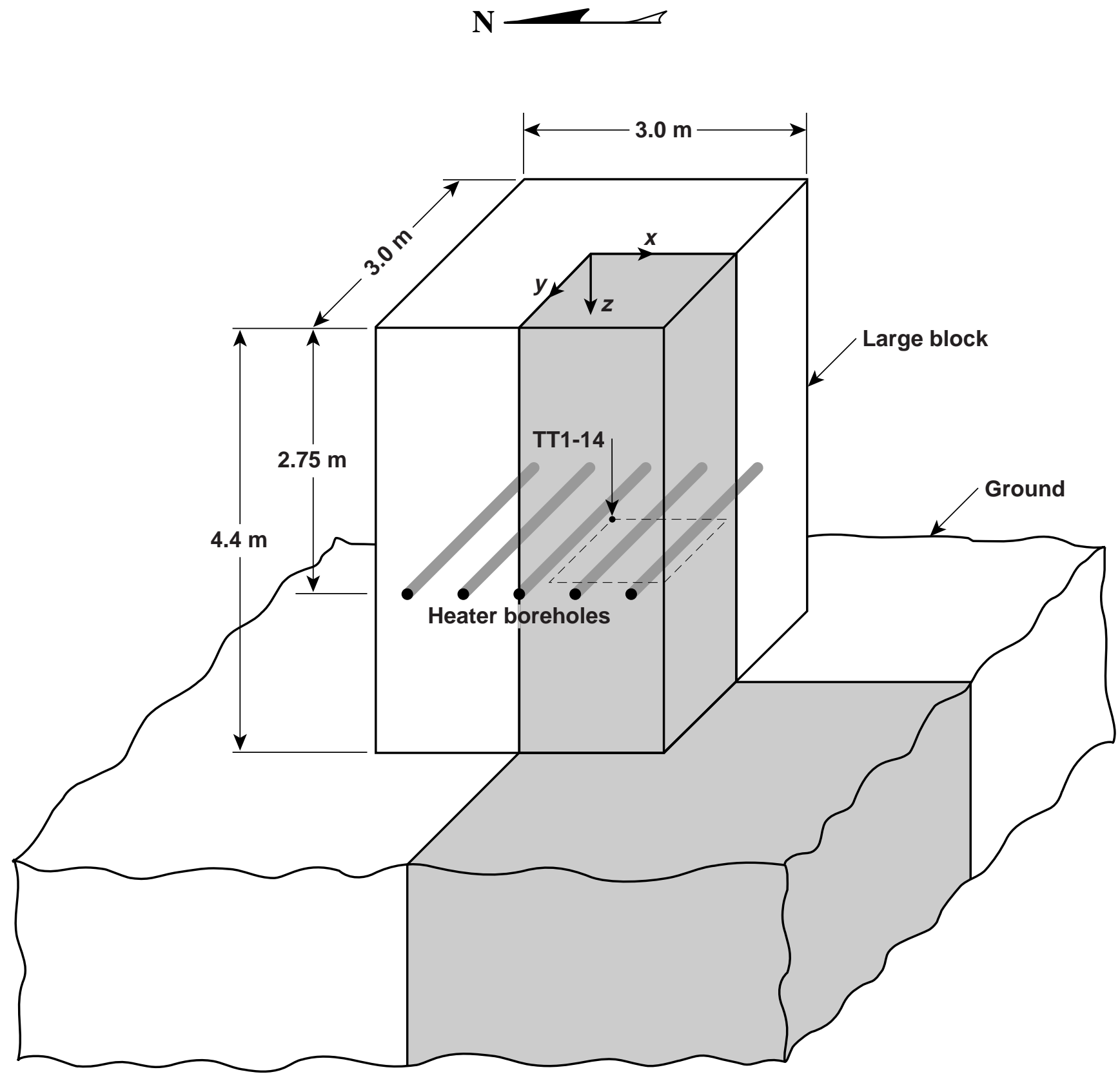

Figure 3-9. Schematic of the LBT showing the location of the five heater boreholes and the LBT-model coordinate system 
(a) $t=100$ days

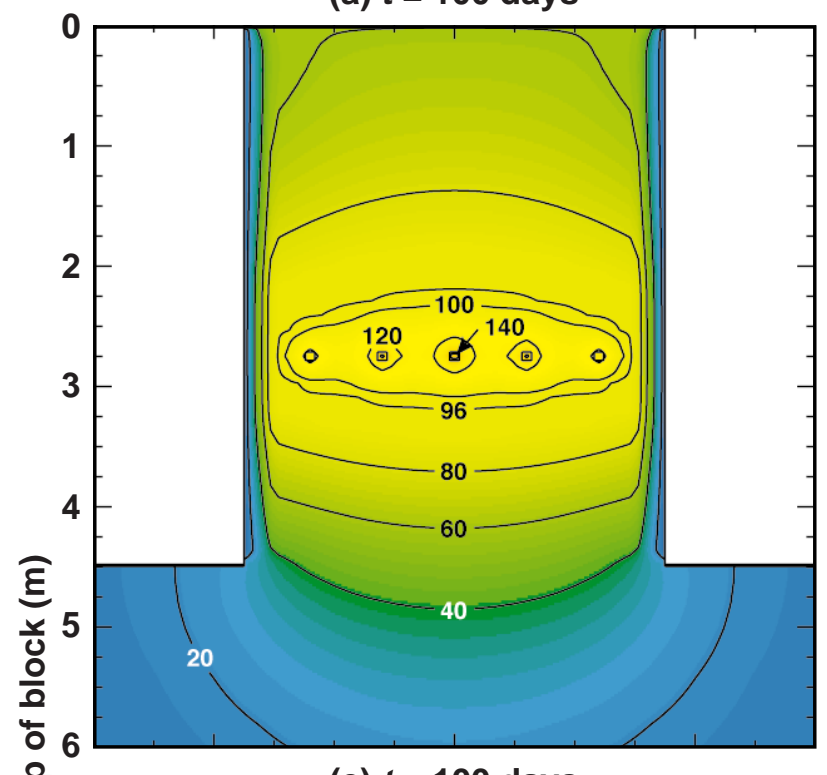

(c) $t=100$ days

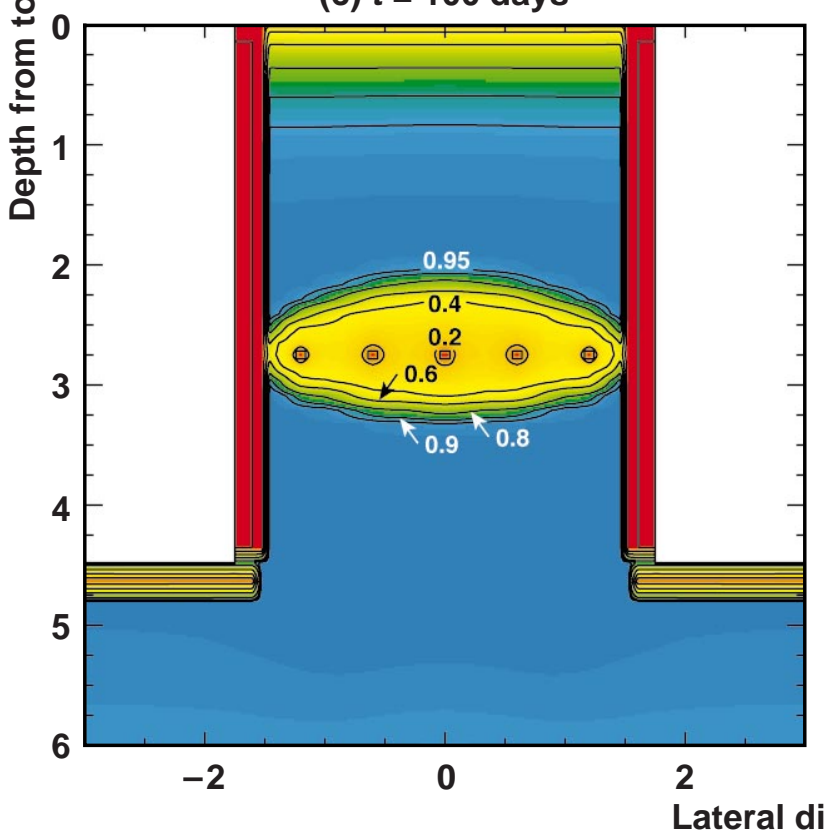

(b) $t=300$ days

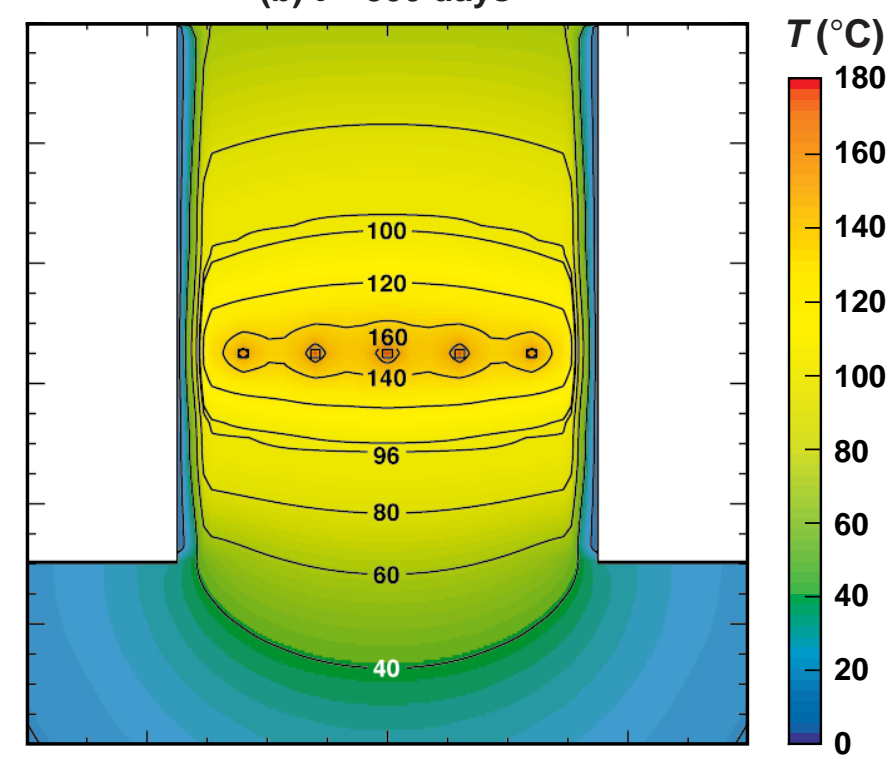

(d) $t=300$ days

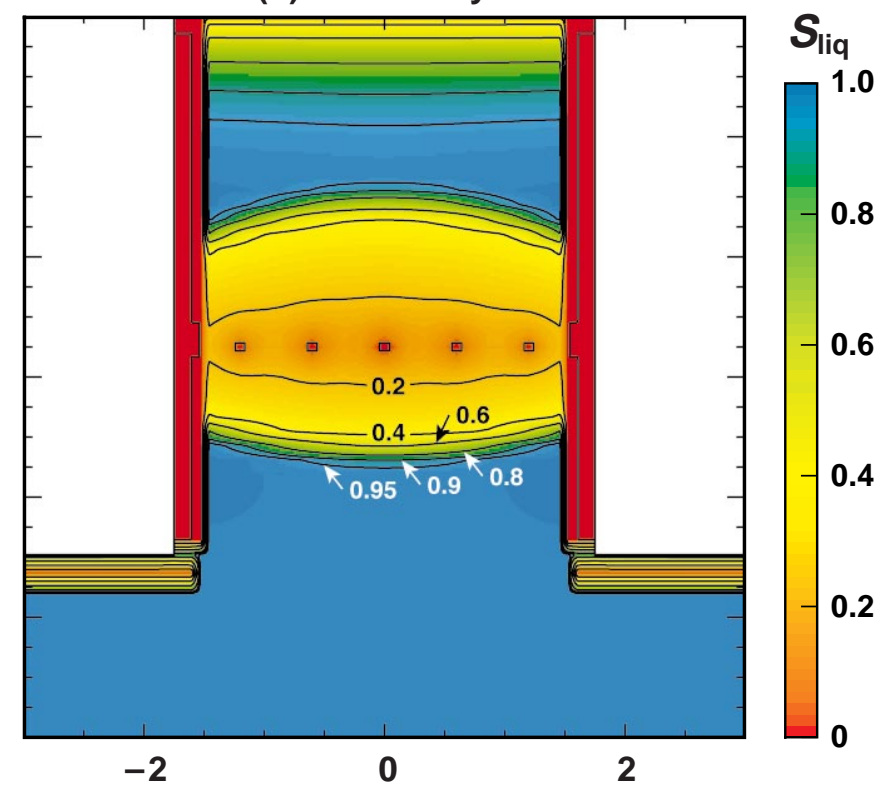

Figure 3-10. Temperature $T$ distributions (a) 100 days and (b) 300 days and liquid-phase saturation $S_{\text {liq }}$ distribution at (c) 100 days and (d) 300 days in a vertical $(x-z)$ plane transverse to the midpoint of the heaters in the LBT 
(e) $t=100$ days

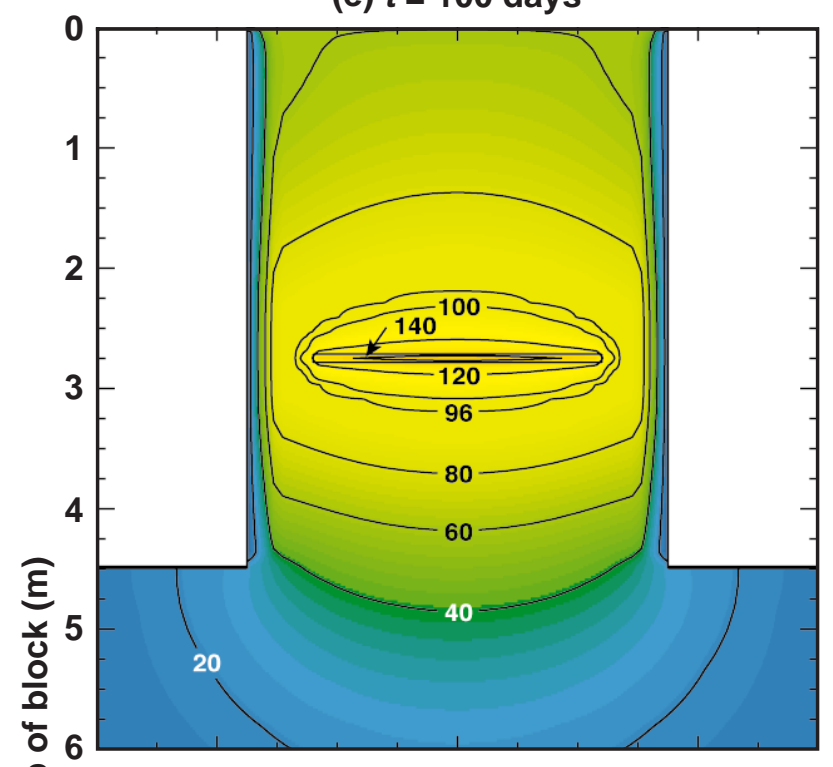

응

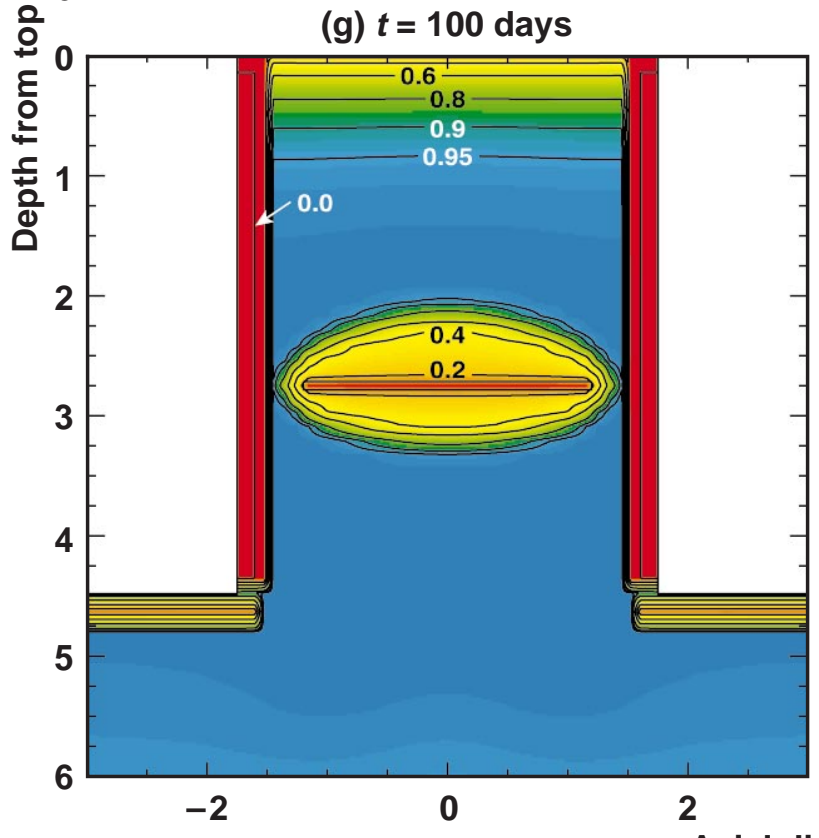

(f) $t=300$ days

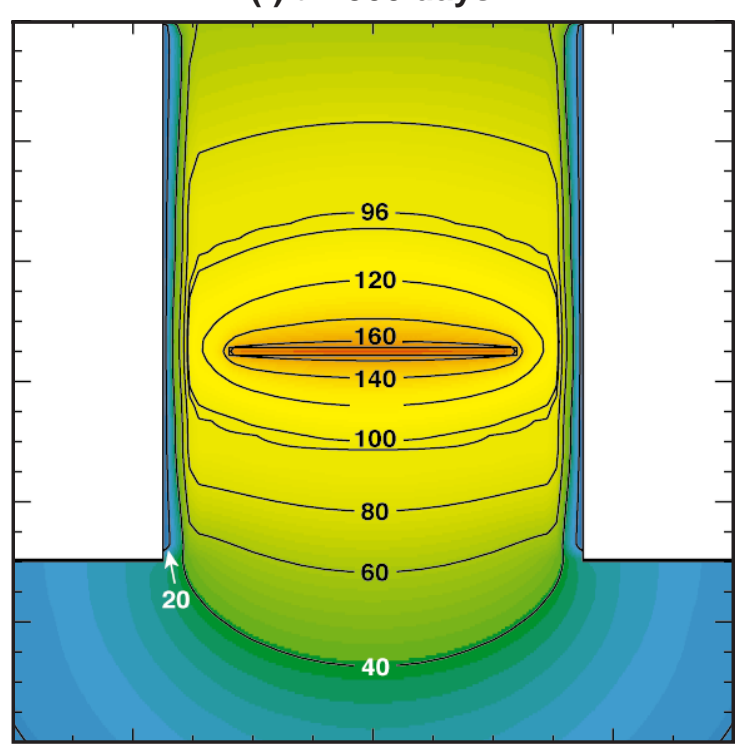

(h) $t=\mathbf{3 0 0}$ days

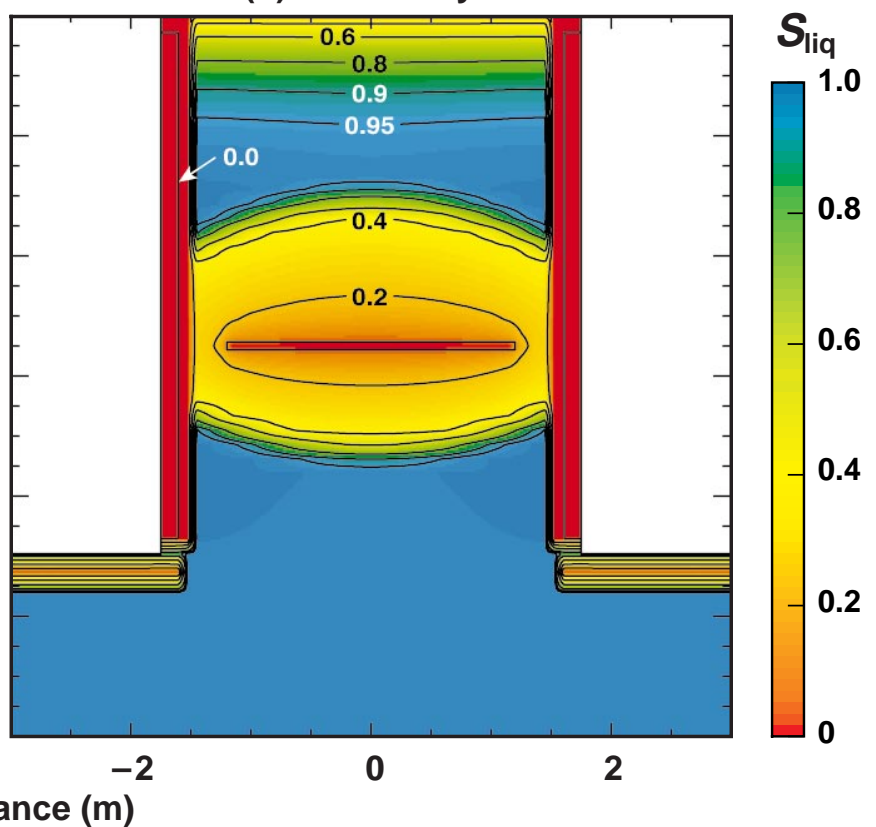

Figure 3-10 Temperature $T$ distribution at (e) 100 days and (f) 300 days and liquid-phase saturation $S_{\text {liq }}$ distribution at (g) 100 days and (h) 300 days in a vertical $(y-z)$ plane along the central heater axis in the LBT 
(a)

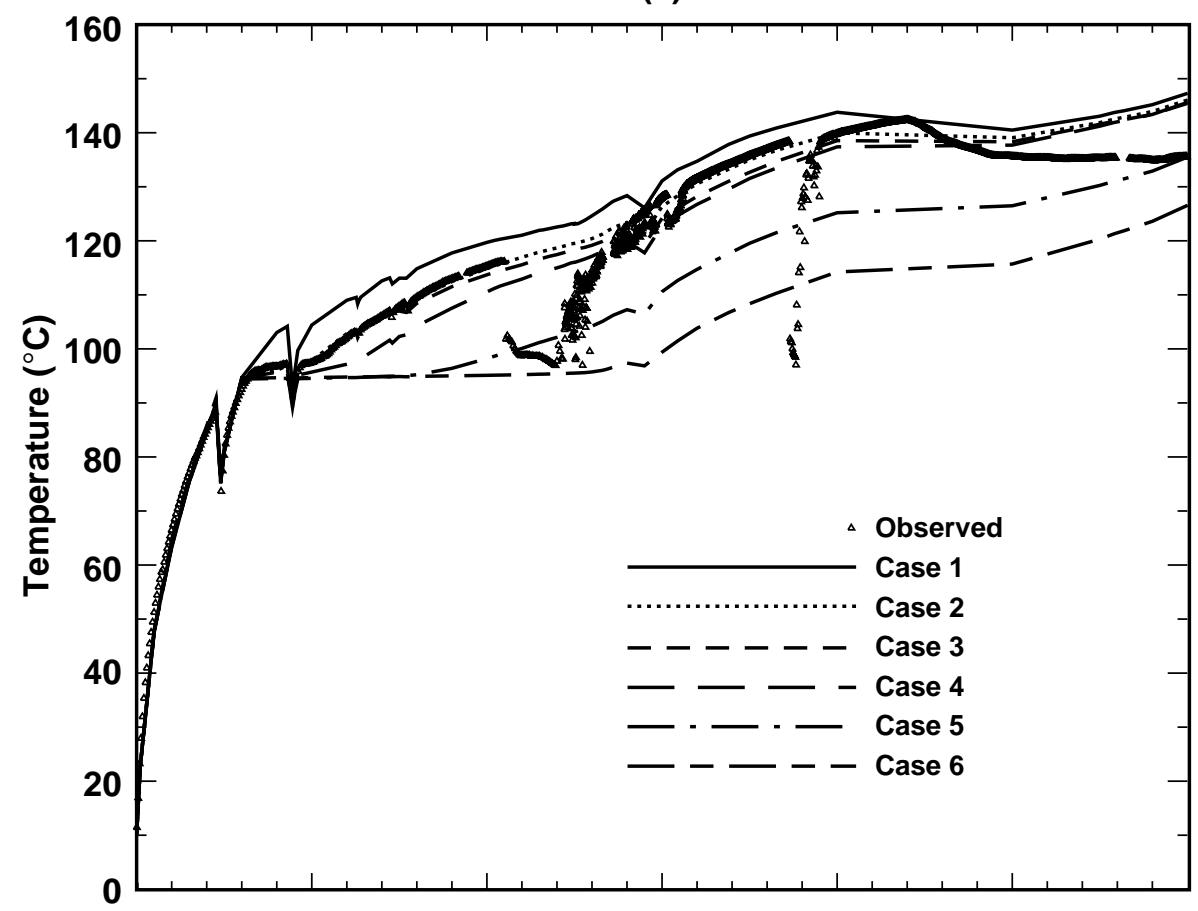

(b)

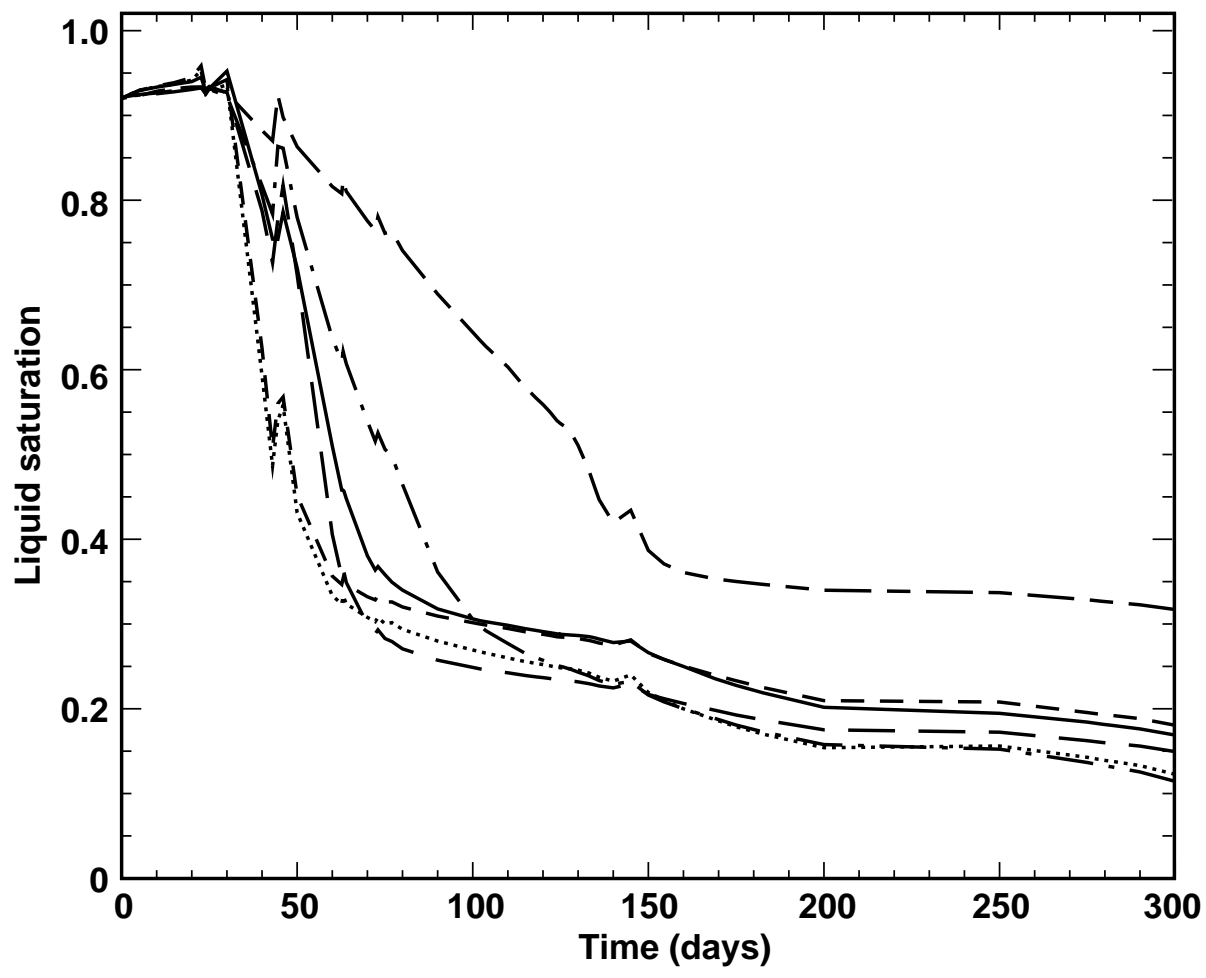

Figure 3-11. Calculated and measured temperature histories (a) compared at thermocouple TT-114; (b) calculated liquid-phase saturation $S_{\text {liq }}$ histories compared at the same point 
(a)

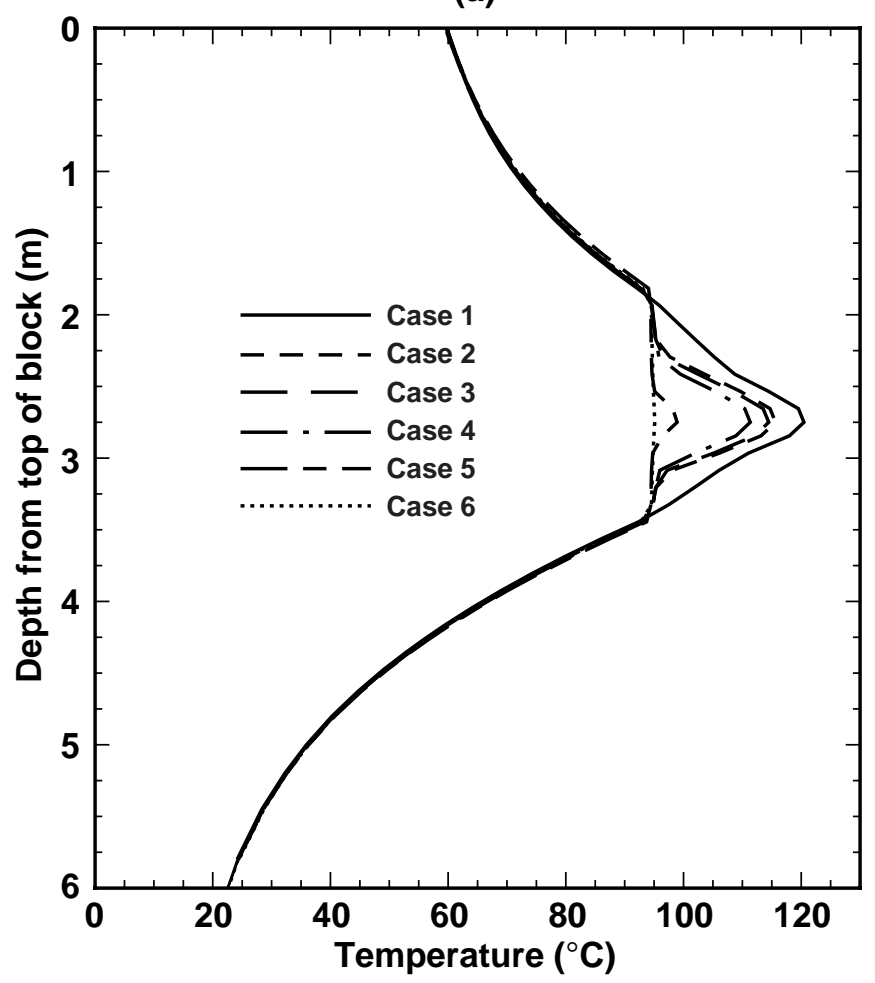

(b)

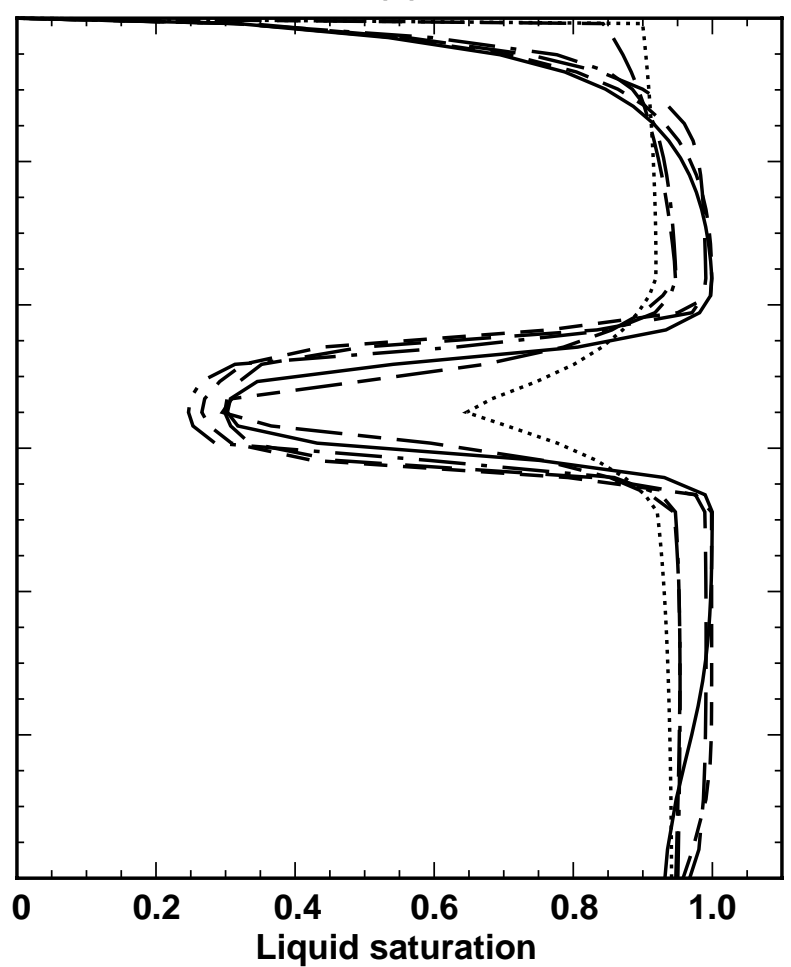

Figure 3-12. (a) Vertical temperature $T$ profile and (b) liquid-phase saturation profile $S_{\text {liq }}$ at the center of the LBT 


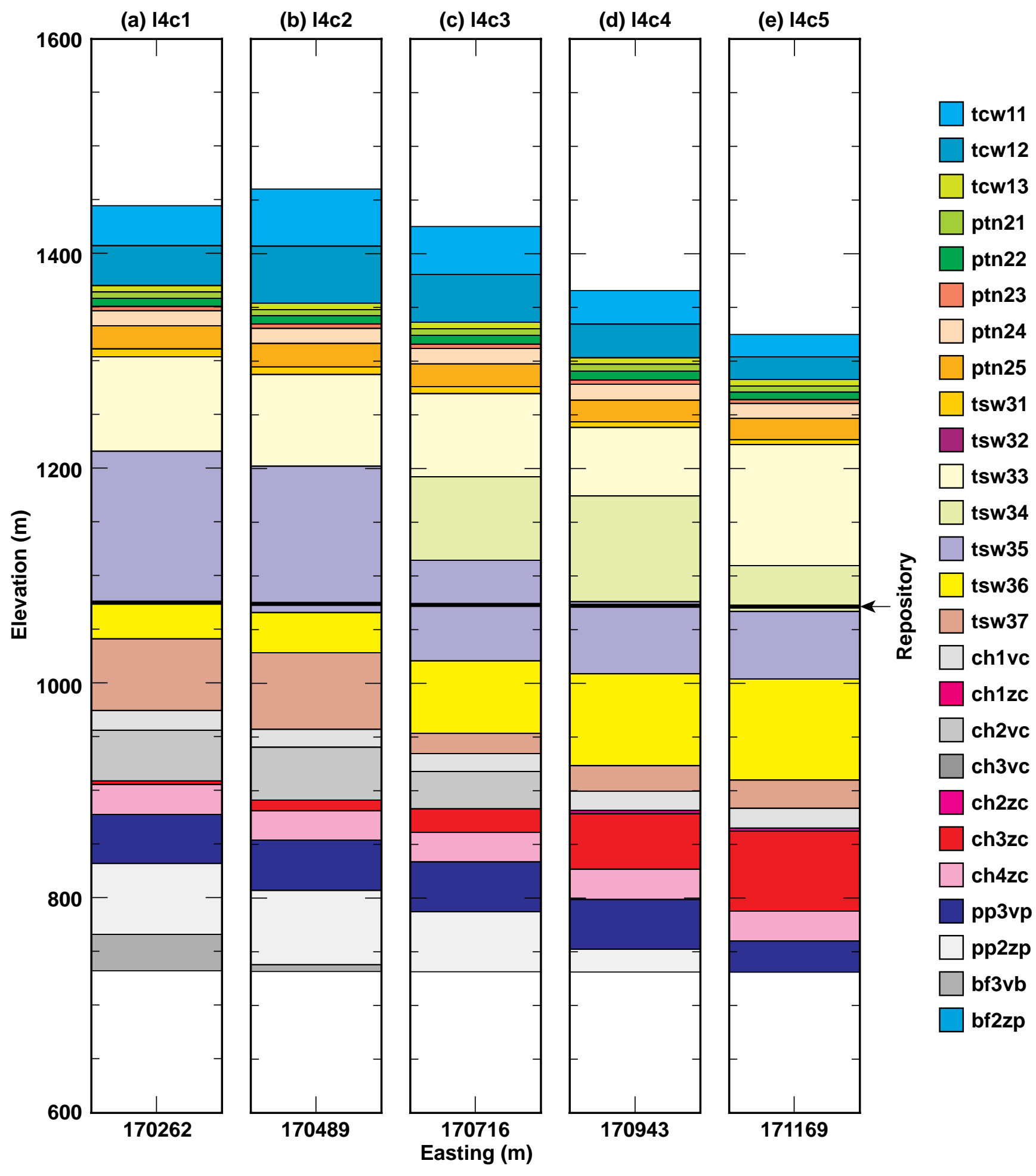

Figure 3-13. Vertical distribution of hydrostratigraphic model units shown for drift-scale model locations (in Nevada-State coordinates), including (a) 14c1 at Northing $=233760 \mathrm{~m}$, (b) $14 \mathrm{c} 2$ at Northing $=233748 \mathrm{~m}$, (c) 14c3 at Northing $=233736 \mathrm{~m},(\mathrm{~d}) 14 \mathrm{c} 4$ at Northing $=233724 \mathrm{~m}$, and (e) 14c5 at Northing $=233712 \mathrm{~m}$ 


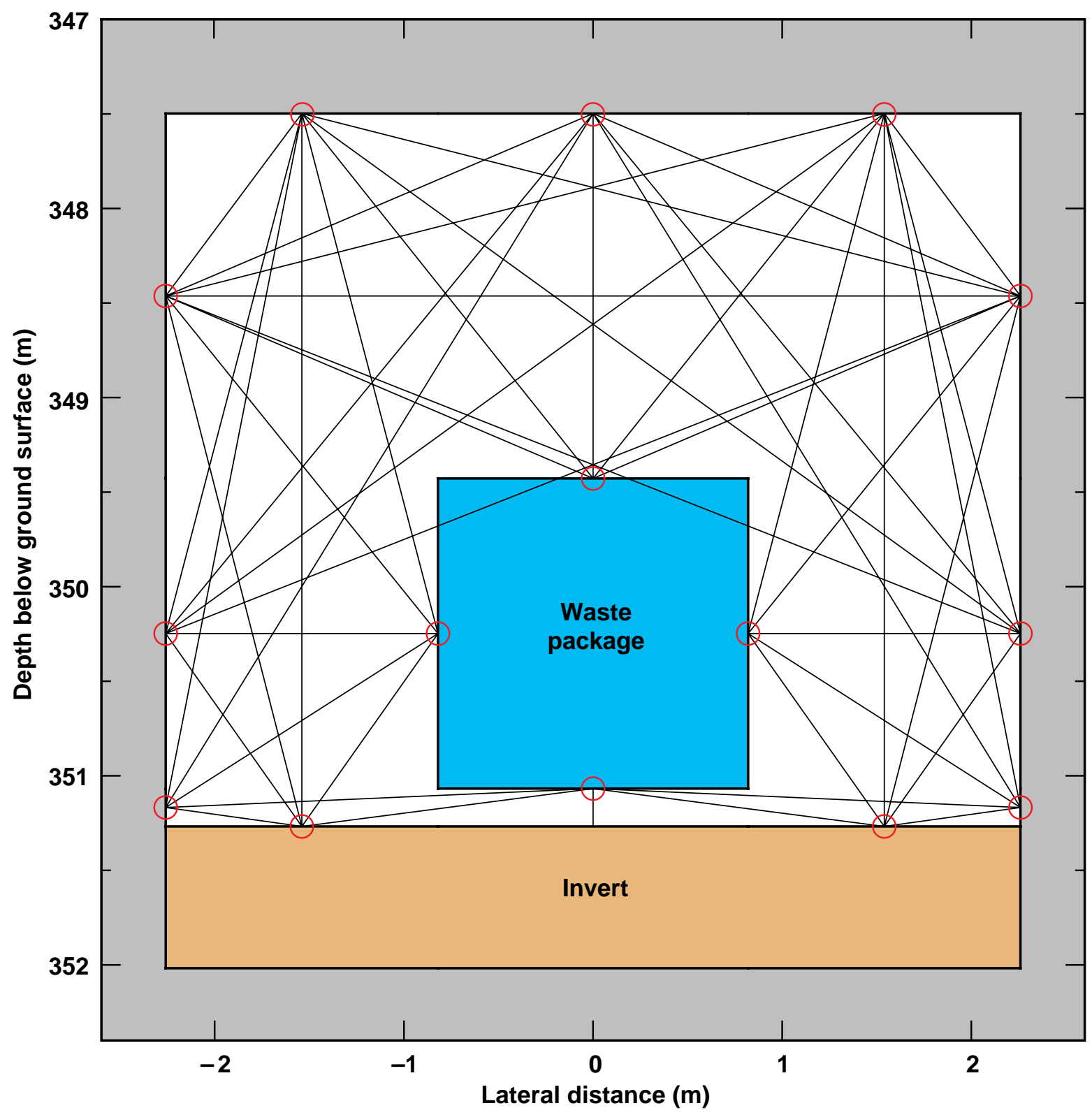

Figure 3-14. Vertical cross-section of the emplacement drift in the DDT and LDTH model showing the thermal radiation connections among the surfaces in the drift that are used in thermal simulations 


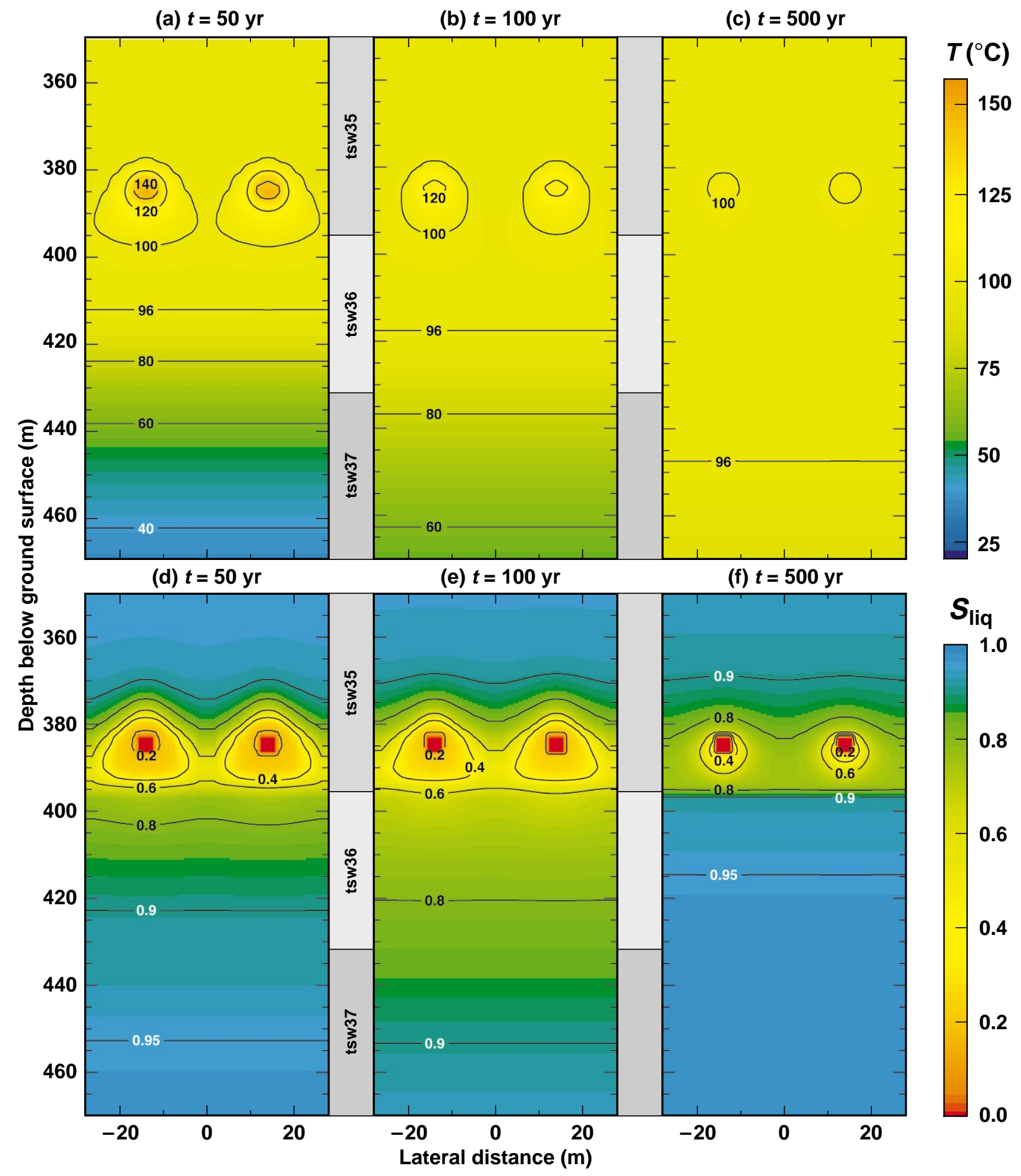

Figure 3-15. Temperature $T$ distributions at (a) $50 \mathrm{yr}$, (b) $100 \mathrm{yr}$, and (c) $500 \mathrm{yr}$, and liquid-phase saturation $S_{\text {liq }}$ distributions at (d) $50 \mathrm{yr}$, (e) $100 \mathrm{yr}$, and (f) $500 \mathrm{yr}$ plotted in a vertical $(x-z)$ plane transverse to the drift for Case 1, which assumes the point-load design, the $7 / 97$ TSPA-VA "I $\times 1$ " hydrologic-parameter set, and a percolation flux of $16.0 \mathrm{~mm} / \mathrm{yr}$, where I stands for nominal infiltration flux 


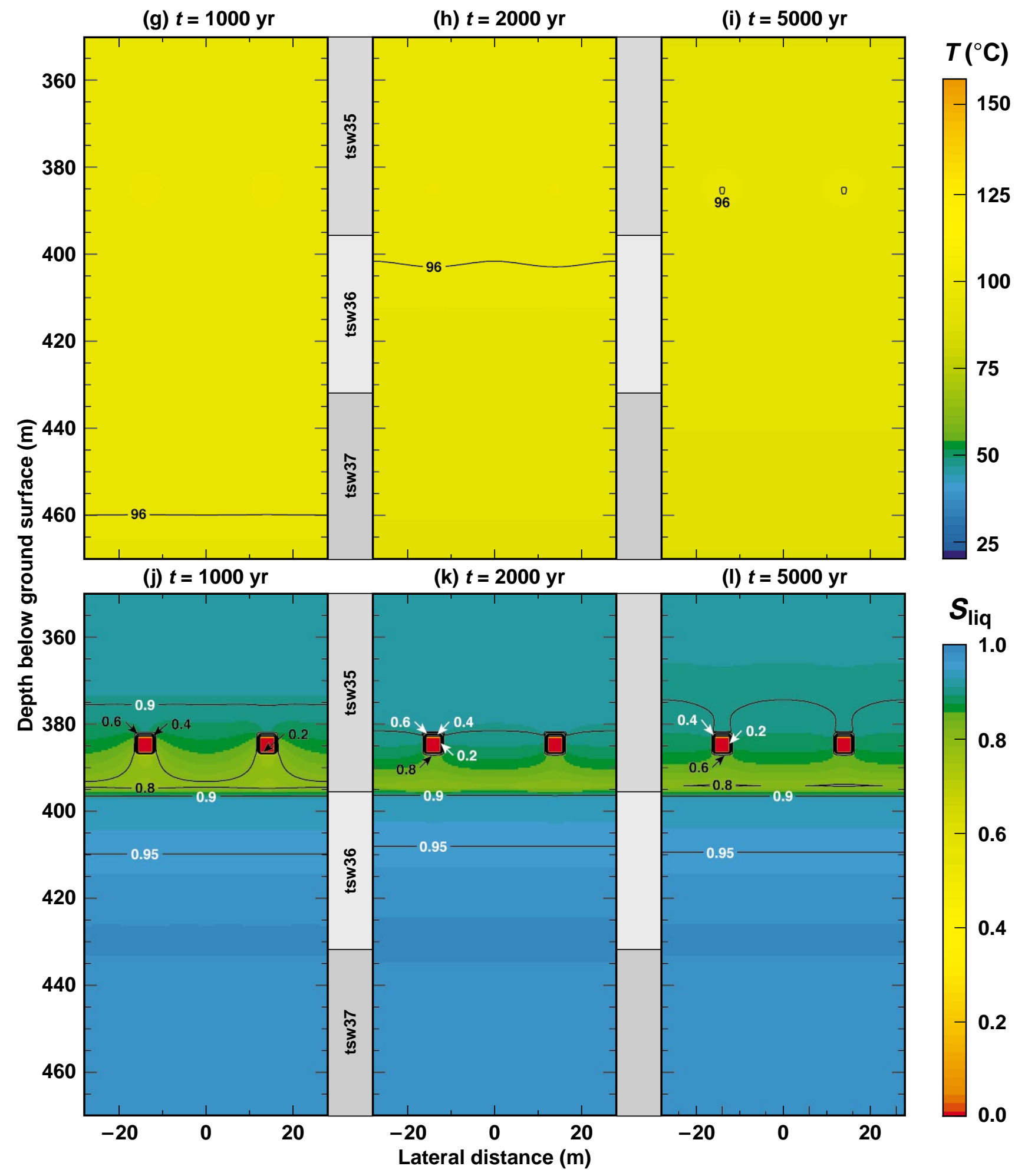

Figure 3-15. Temperature $T$ distributions at (g) $1000 \mathrm{yr}$, (h) $2000 \mathrm{yr}$, and (i) $5000 \mathrm{yr}$, and liquid-phase saturation $S_{\text {liq }}$ distributions at (j) $1000 \mathrm{yr},(\mathrm{k}) 2000 \mathrm{yr}$, and (l) $5000 \mathrm{yr}$ plotted in a vertical $(x-z)$ plane transverse to the drift for Case 1, which assumes the point-load design with no backfill, the 7/97 TSPA-VA "I $\times 1$ " hydrologicparameter set, and a percolation flux of $16.0 \mathrm{~mm} / \mathrm{yr}$, where I stands for nominal infiltration flux 


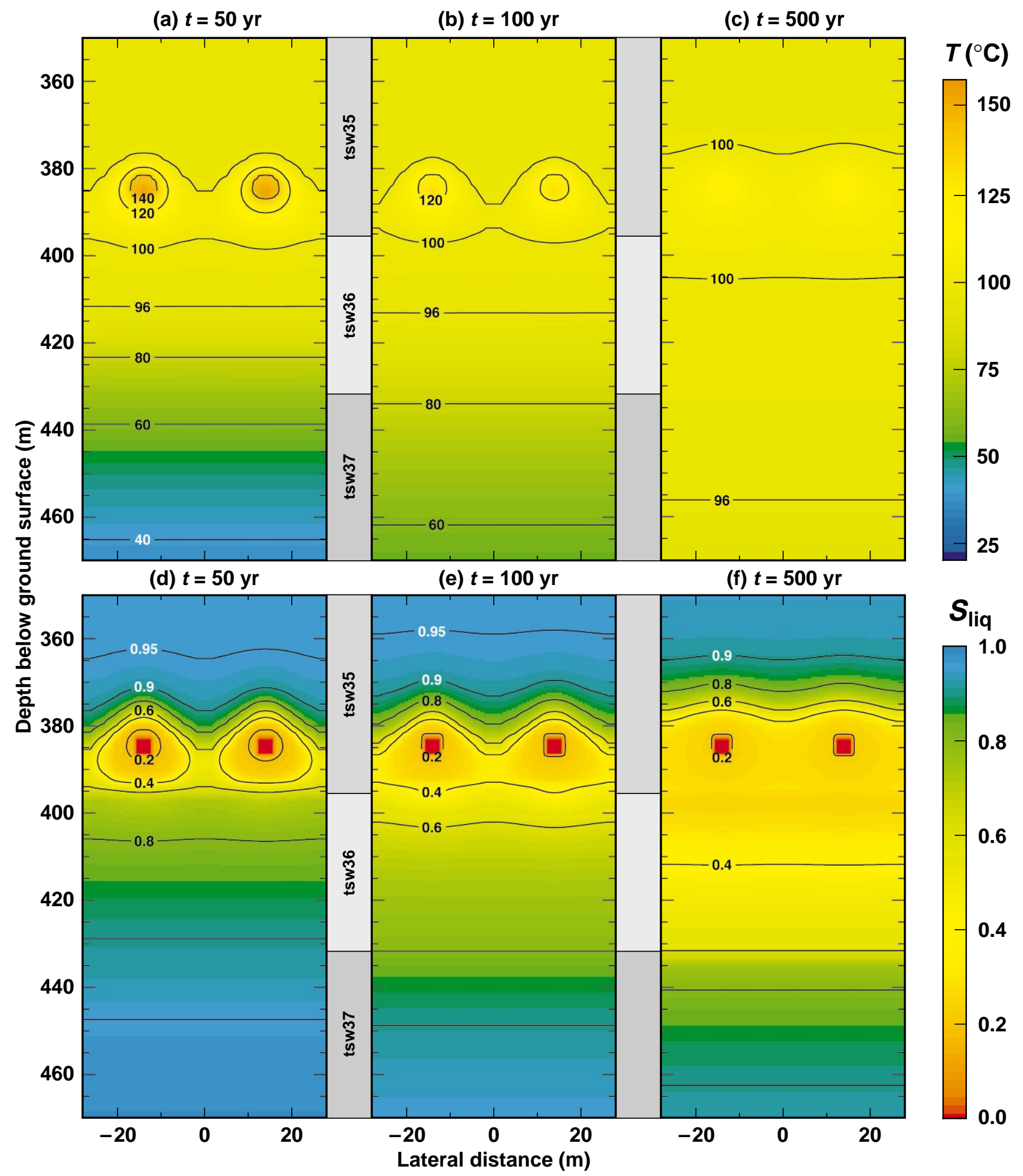

Figure 3-16. Temperature $T$ distributions at (a) $50 \mathrm{yr},(\mathrm{b}) 100 \mathrm{yr}$, and (c) $500 \mathrm{yr}$, and liquid-phase saturation $S_{\text {liq }}$ distributions at (d) $50 \mathrm{yr},(\mathrm{e}) 100 \mathrm{yr}$, and (f) $500 \mathrm{yr}$ plotted in a vertical $(x-z)$ plane transverse to the drift for Case 2, which assumes the point-load design, the 7/97 TSPA-VA “I/5" hydrologic-parameter set, and a percolation flux of $3.2 \mathrm{~mm} / \mathrm{yr}$, where I stands for nominal infiltration flux 


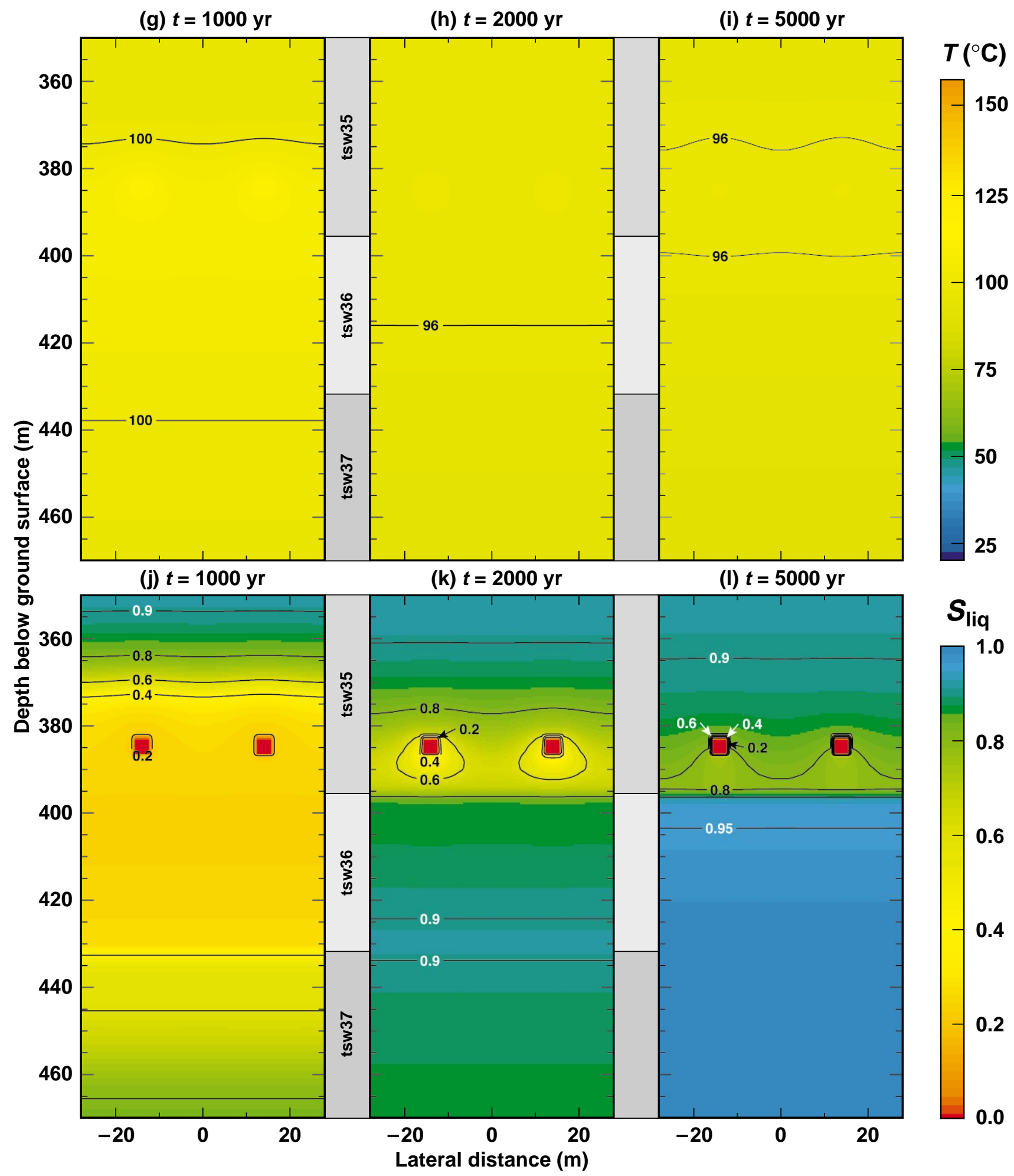

Figure 3-16. Temperature $T$ distributions at (g) 1000 yr, (h) $2000 \mathrm{yr}$, and (i) $5000 \mathrm{yr}$, and liquid-phase saturation $S_{\text {liq }}$ distributions at (j) $1000 \mathrm{yr},(\mathrm{k}) 2000 \mathrm{yr}$, and (l) $5000 \mathrm{yr}$ plotted in a vertical $(x-z)$ plane transverse to the drift for Case 2, which assumes the point-load design with no backfill, the 7/97 TSPA-VA "I/5" hydrologicparameter set, and a percolation flux of $3.2 \mathrm{~mm} / \mathrm{yr}$, where I stands for nominal infiltration flux 


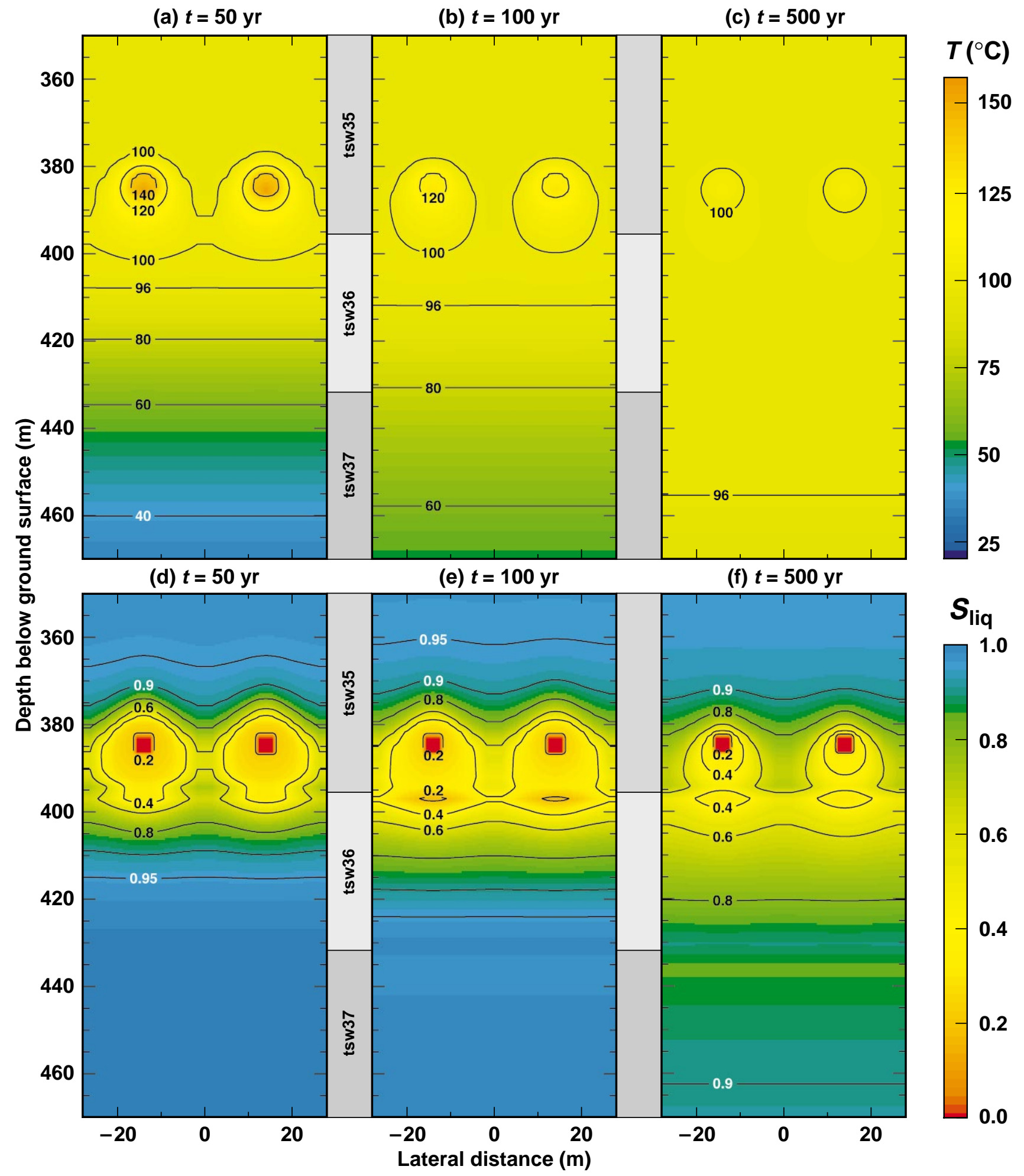

Figure 3-17. Temperature $T$ distributions at (a) $50 \mathrm{yr},(\mathrm{b}) 100 \mathrm{yr}$, and (c) $500 \mathrm{yr}$, and liquid-phase saturation $S_{\text {liq }}$ distributions at (d) $50 \mathrm{yr},(\mathrm{e}) 100 \mathrm{yr}$, and (f) $500 \mathrm{yr}$ plotted in a vertical $(x-z)$ plane transverse to the drift for

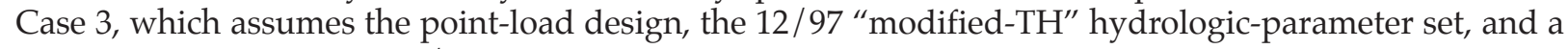
percolation flux of $16.0 \mathrm{~mm} / \mathrm{yr}$ 


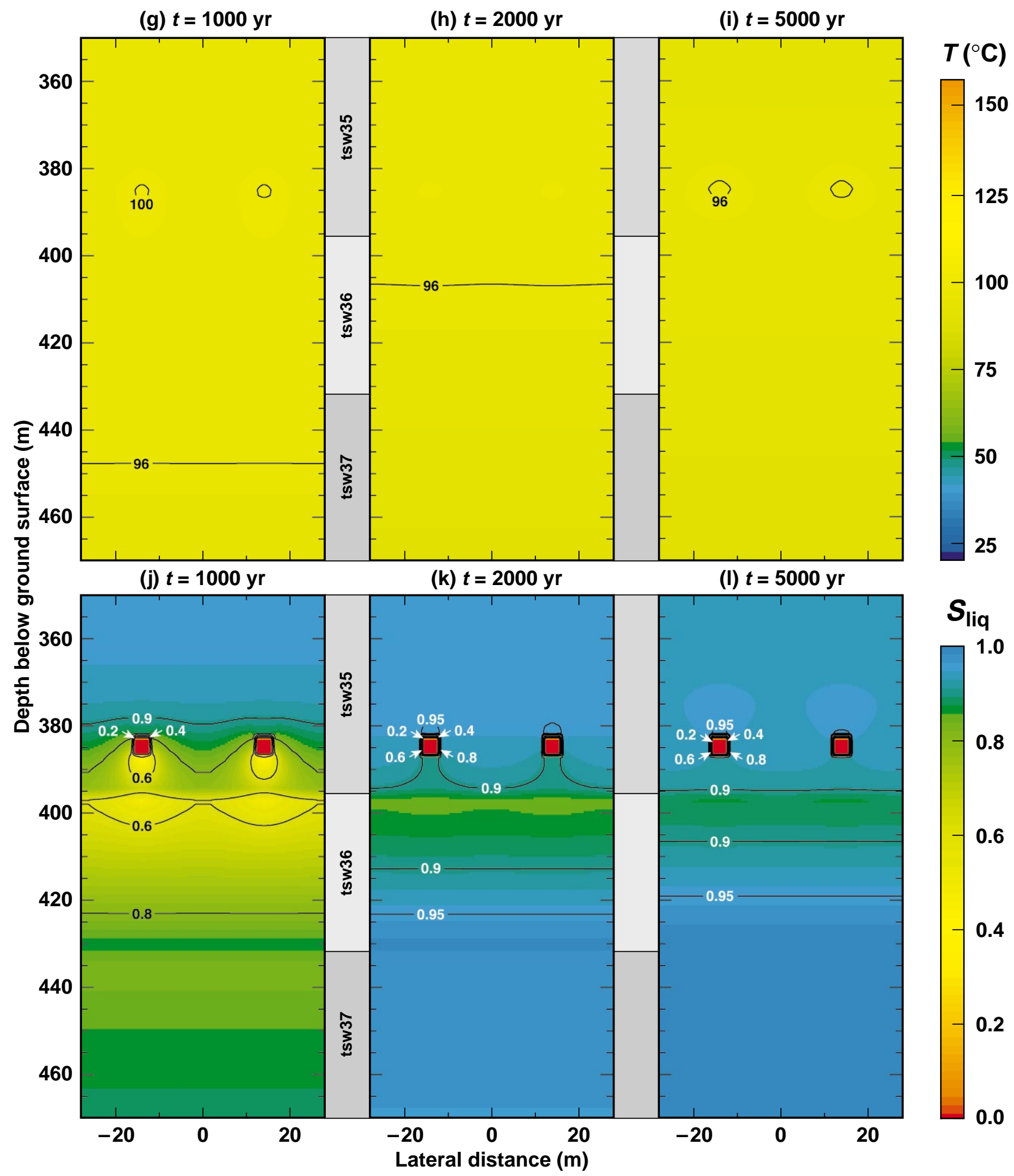

Figure 3-17. Temperature $T$ distributions at (g) 1000 yr, (h) $2000 \mathrm{yr}$, and (i) $5000 \mathrm{yr}$, and liquid-phase saturation $S_{\text {liq }}$ distributions at (j) $1000 \mathrm{yr},(\mathrm{k}) 2000 \mathrm{yr}$, and (l) $5000 \mathrm{yr}$ plotted in a vertical $(x-z)$ plane transverse to the drift for Case 3, which assumes the point-load design with no backfill, the 12/97 "modified-TH" hydrologicparameter set, and a percolation flux of $16.0 \mathrm{~mm} / \mathrm{yr}$ 


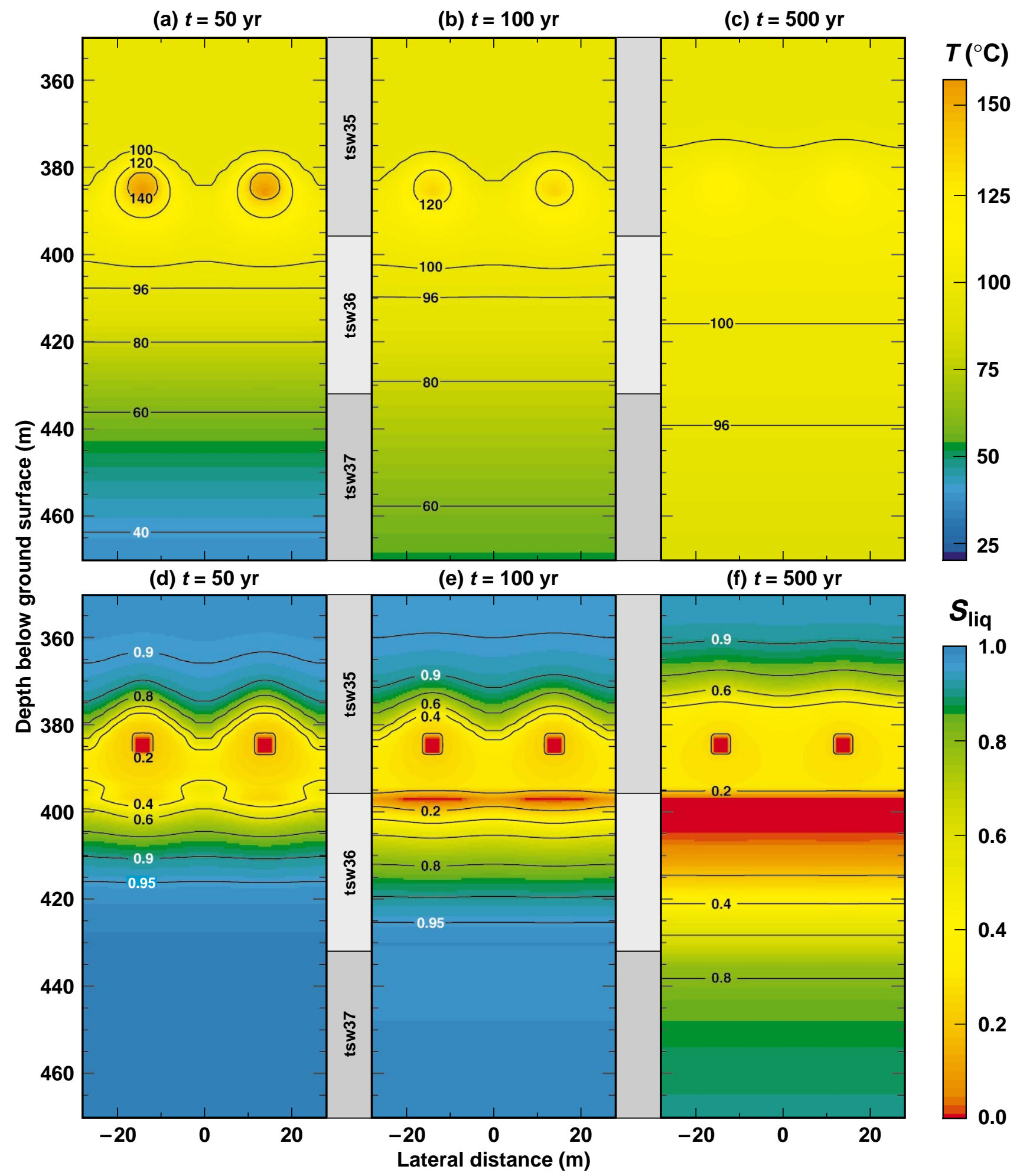

Figure 3-18. Temperature $T$ distributions at (a) $50 \mathrm{yr}$, (b) $100 \mathrm{yr}$, and (c) $500 \mathrm{yr}$, and liquid-phase saturation $S_{\text {liq }}$ distributions at (d) $50 \mathrm{yr},(\mathrm{e}) 100 \mathrm{yr}$, and (f) $500 \mathrm{yr}$ plotted in a vertical $(x-z)$ plane transverse to the drift for Case 4, which assumes the point-load design with no backfill, the 12/97 "modified-TH" hydrologicparameter set, and a percolation flux of $3.2 \mathrm{~mm} / \mathrm{yr}$ 


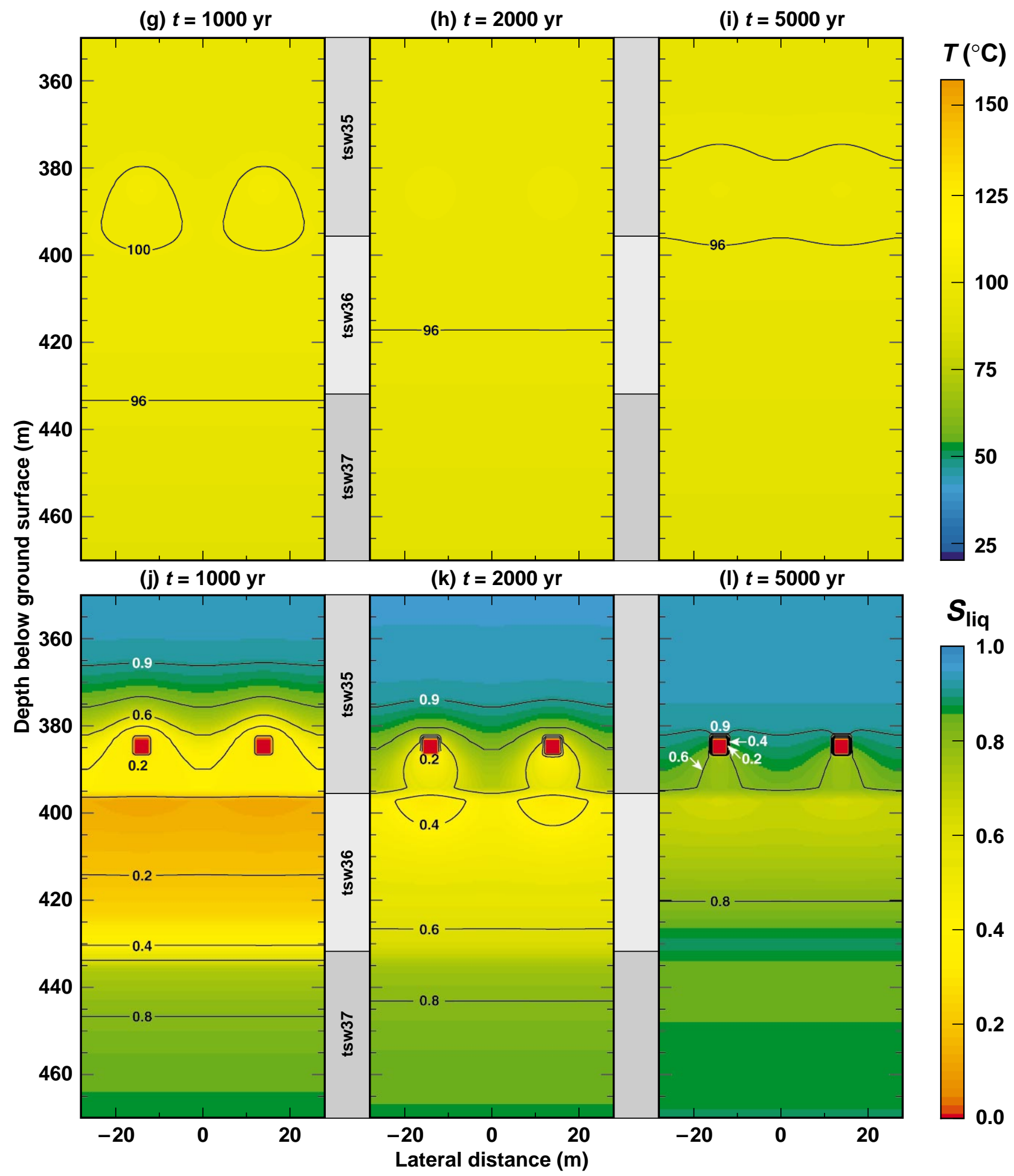

Figure 3-18. Temperature $T$ distributions at (g) 1000 yr, (h) $2000 \mathrm{yr}$, and (i) $5000 \mathrm{yr}$, and liquid-phase saturation $S_{\text {liq }}$ distributions at (j) $1000 \mathrm{yr},(\mathrm{k}) 2000 \mathrm{yr}$, and (l) $5000 \mathrm{yr}$ plotted in a vertical $(x-z)$ plane transverse to the drift for Case 4, which assumes the point-load design with no backfill, the 12/97 "modified-TH" hydrologicparameter set, and a percolation flux of $3.2 \mathrm{~mm} / \mathrm{yr}$ 


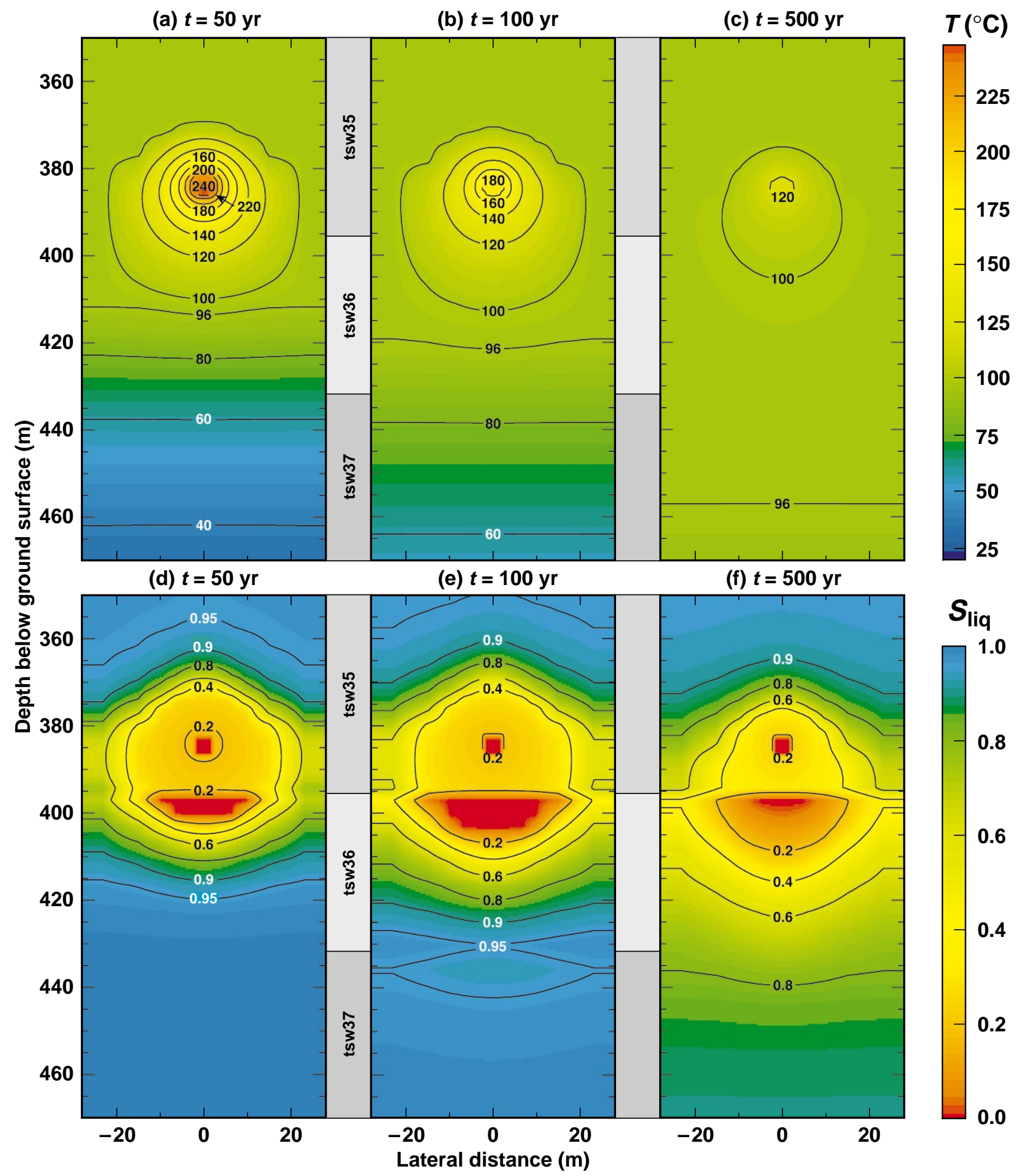

Figure 3-19. Temperature $T$ distributions at (a) $50 \mathrm{yr},(\mathrm{b}) 100 \mathrm{yr}$, and (c) $500 \mathrm{yr}$, and liquid-phase saturation $S_{\text {liq }}$ distributions at (d) $50 \mathrm{yr},(\mathrm{e}) 100 \mathrm{yr}$, and (f) $500 \mathrm{yr}$ plotted in a vertical $(x-z)$ plane transverse to the drift for Case 5, which assumes the line-load design with no backfill, the 12/97 "modified-TH" hydrologic-parameter set, and a percolation flux of $16.0 \mathrm{~mm} / \mathrm{yr}$ 


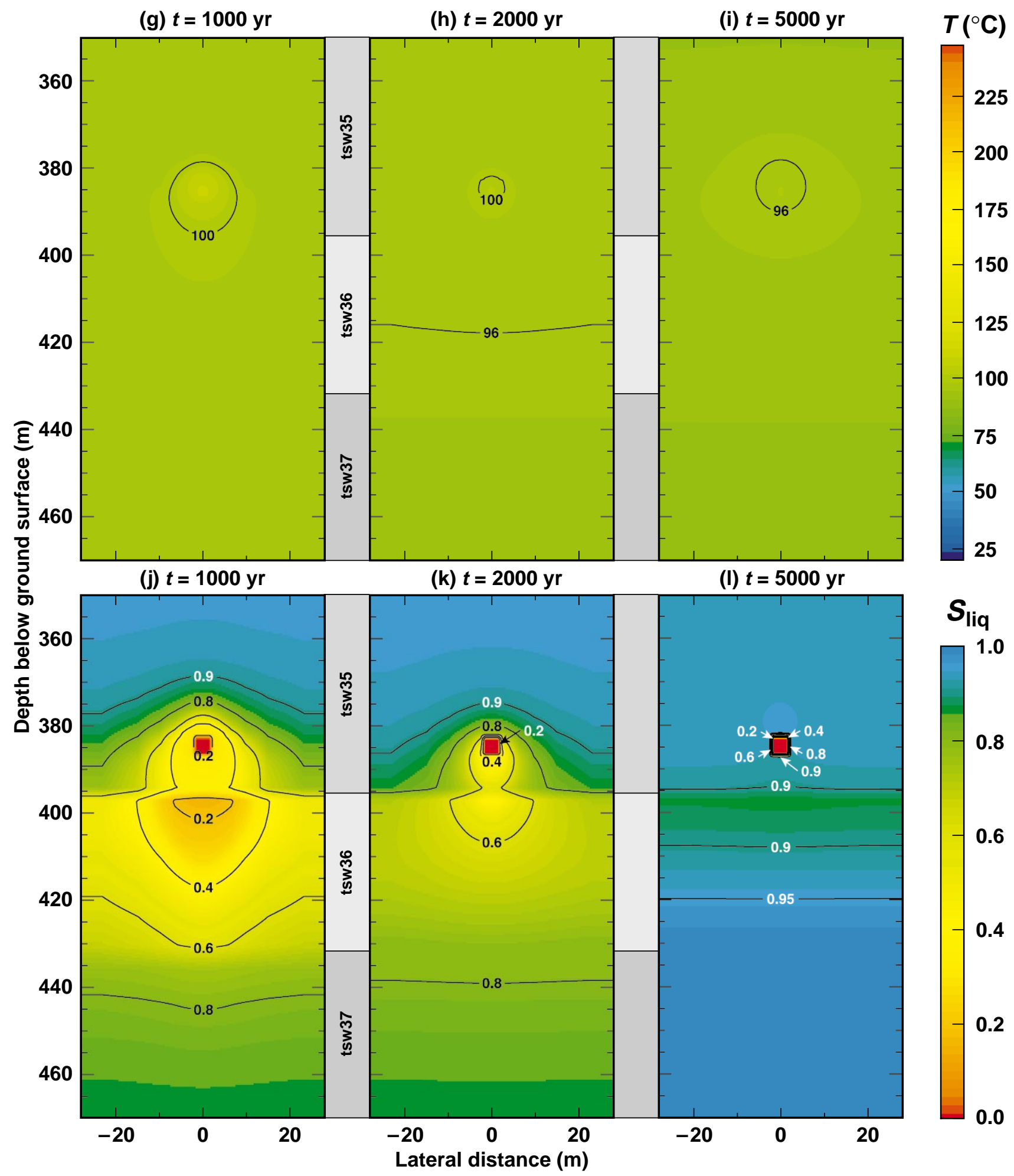

Figure 3-19. Temperature $T$ distributions at (g) 1000 yr, (h) $2000 \mathrm{yr}$, and (i) $5000 \mathrm{yr}$, and liquid-phase saturation $S_{\mathrm{liq}}$ distributions at (j) $1000 \mathrm{yr},(\mathrm{k}) 2000 \mathrm{yr}$, and (l) $5000 \mathrm{yr}$ plotted in a vertical $(x-z)$ plane transverse to the drift for Case 5, which assumes the line-load design, the 12/97 "modified-TH" hydrologic-parameter set, and a percolation flux of $16.0 \mathrm{~mm} / \mathrm{yr}$ 


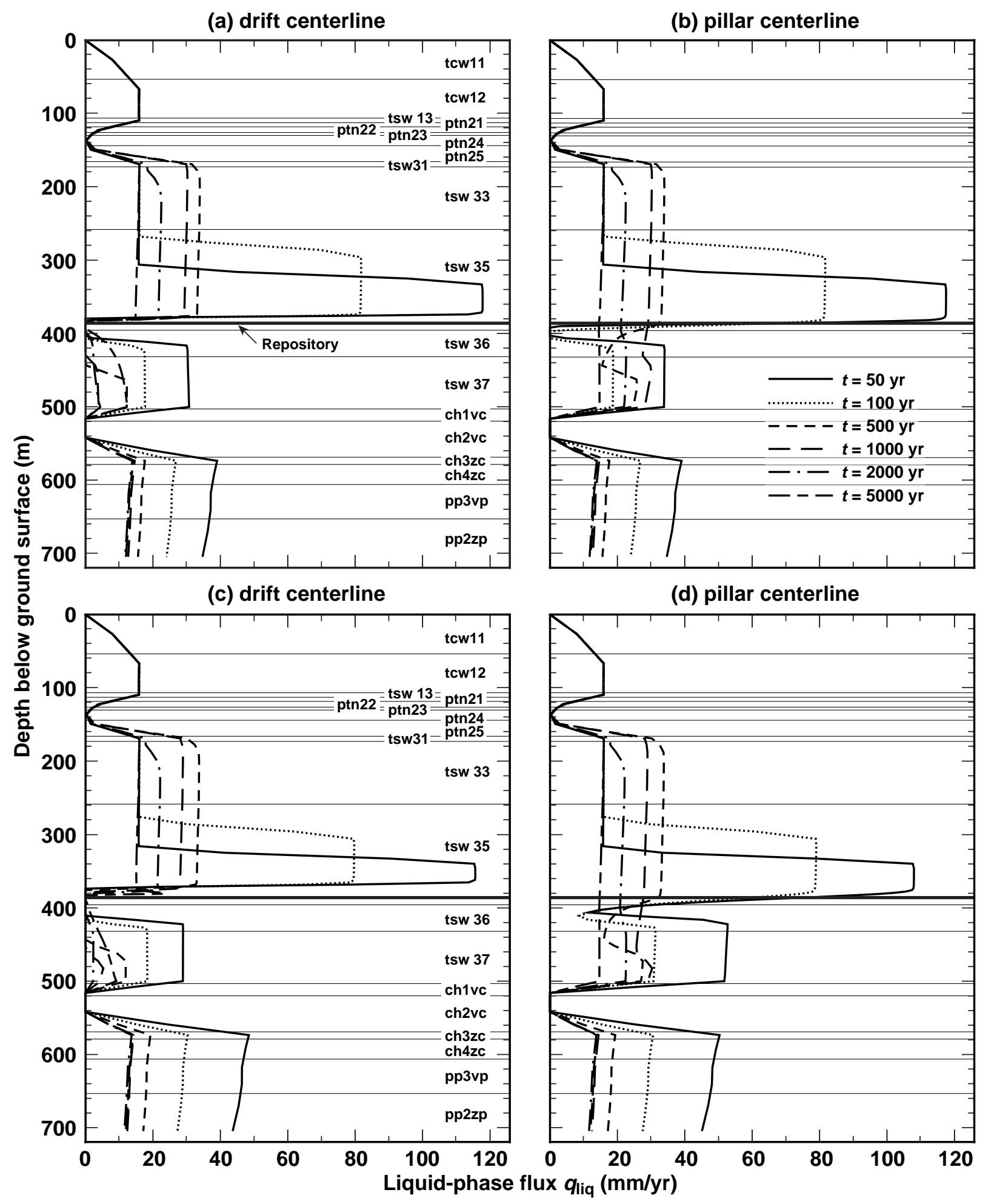

Figure 3-20. Vertical distribution of liquid-phase flux $q_{\text {liq }}$ plotted at (a) the drift centerline and (b) the pillar centerline at various times for Case 3 which assumes the point-load design with no backfill; vertical distribution of $q_{\text {liq }}$ also plotted at (c) the drift centerline and (d) the pillar centerline for Case 5, which assumes the line-load design with no backfill (all distributions assume the 12/97 "modified-TH" hydrologic-parameter set, and a percolation flux of $16.0 \mathrm{~mm} / \mathrm{yr}$ ) 
(a)

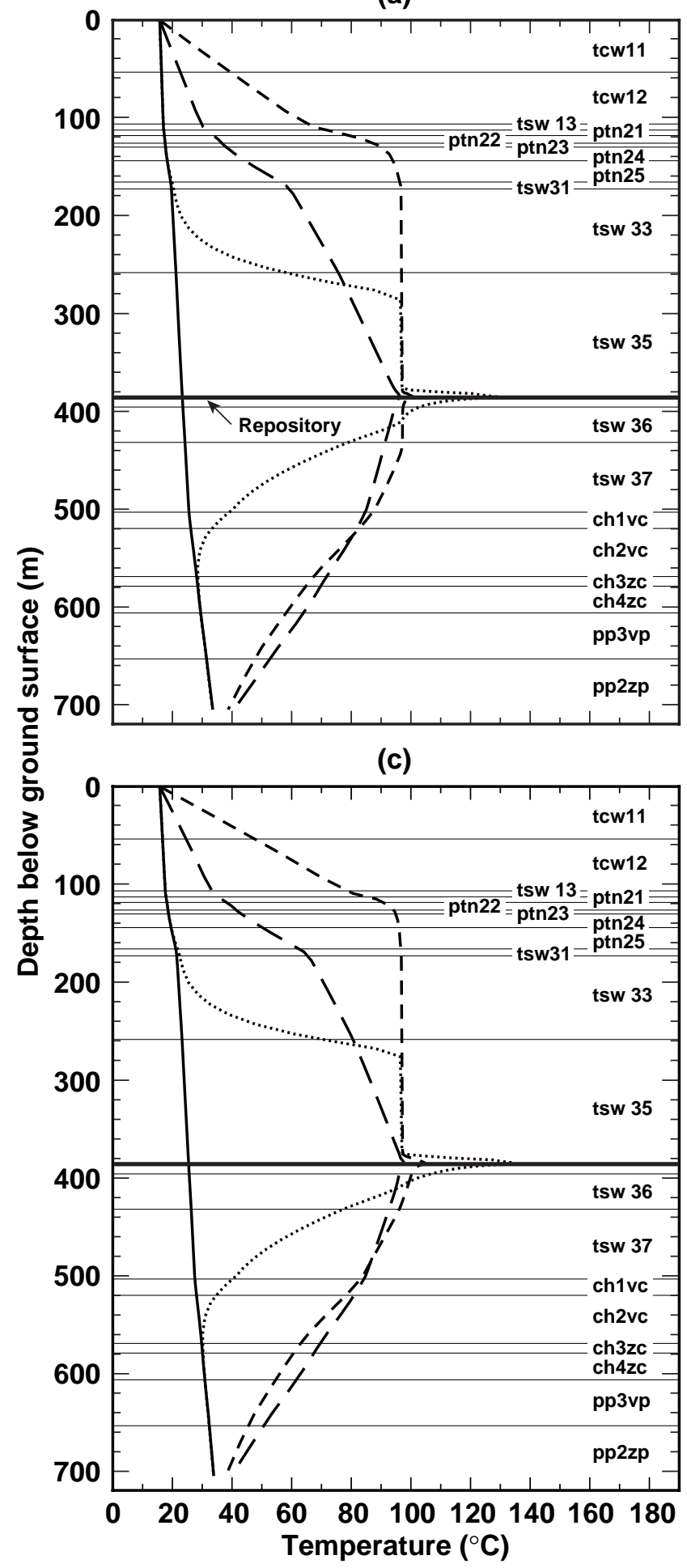

(b)

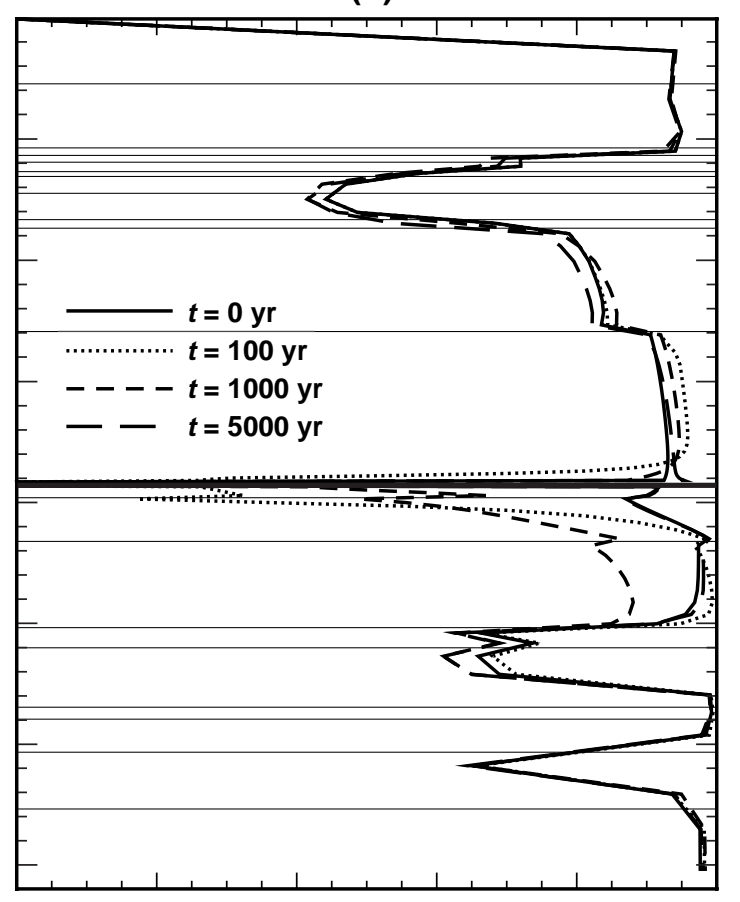

(d)

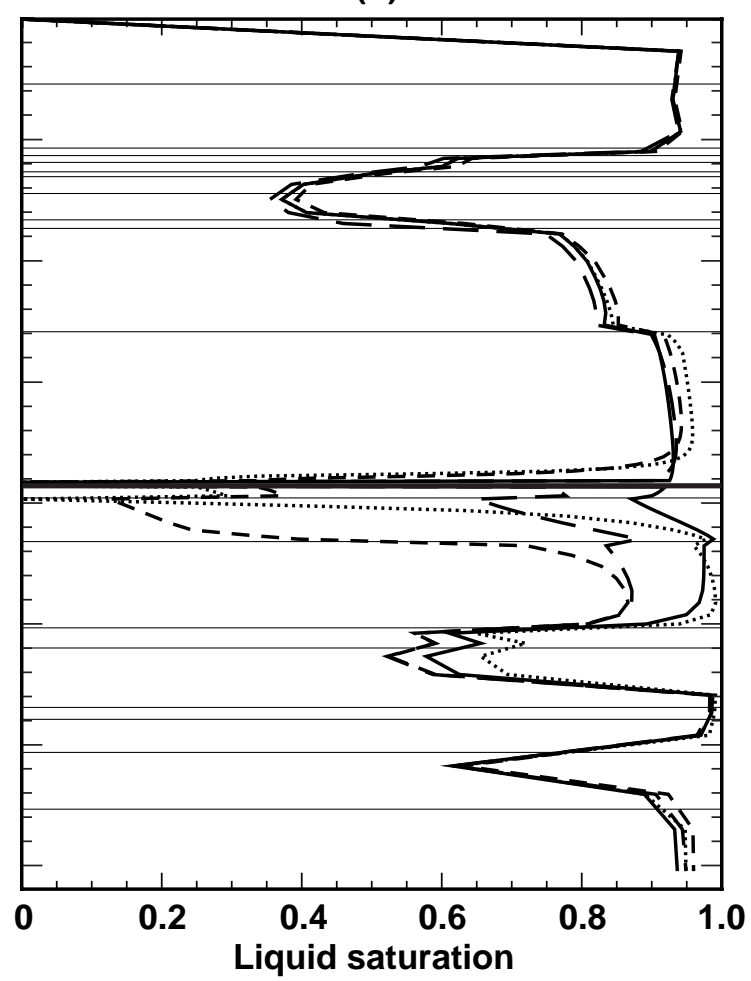

Figure 3-21. Vertical temperature $T$ distribution (a) and liquid-phase saturation $S_{\text {liq }}$ distribution (b) at the drift centerline are plotted at various times for Case 3, which assumes the point-load design with no backfill; vertical $T$ distribution (c) and $S_{\text {liq }}$ distribution (d) at the drift centerline also plotted for Case 5, which assumes the line-load design with no backfill (all distributions assume the 12/97 "modified-TH" hydrologic-parameter set, and a percolation flux of $16.0 \mathrm{~mm} / \mathrm{yr}$ ) 

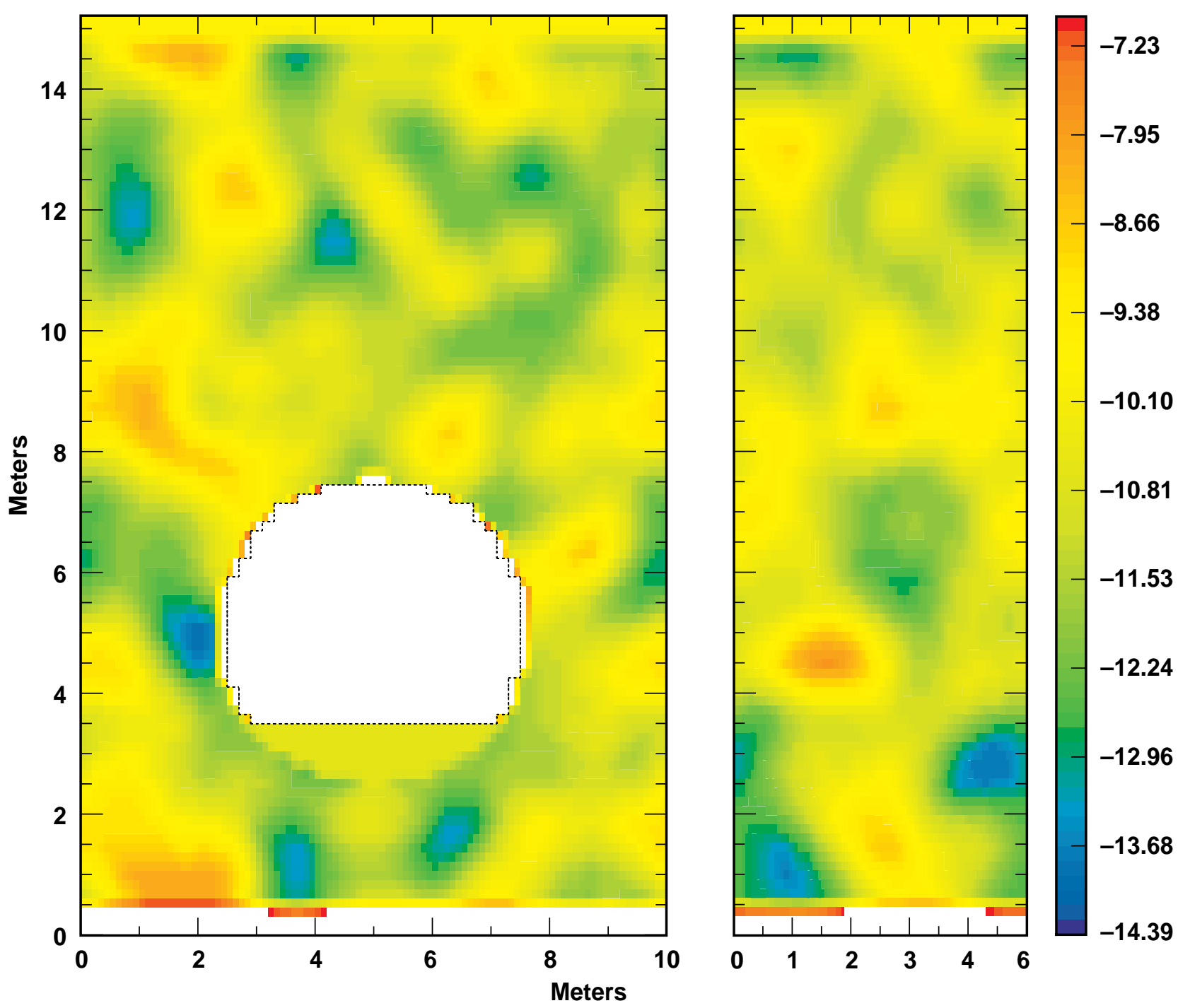

Figure 3-22. Front and side views of 3-D base-10 logarithm of hydraulic conductivity $(\mathrm{m} / \mathrm{s})$ field for the matrix continuum 

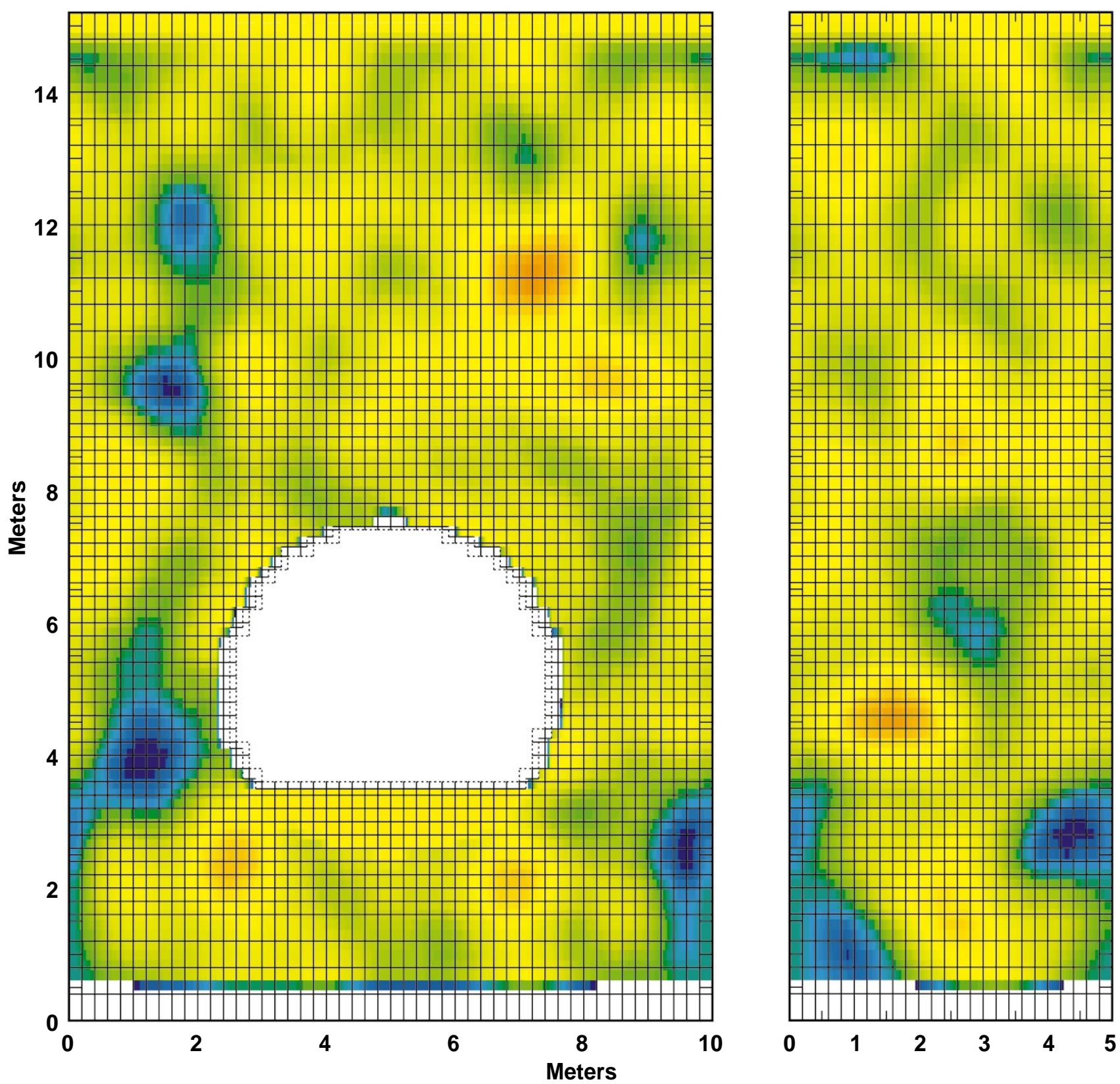

Figure 3-23. Front and side views of the 3-D grid. A random field of $\log 10$ of the fracture permeability is also shown. 


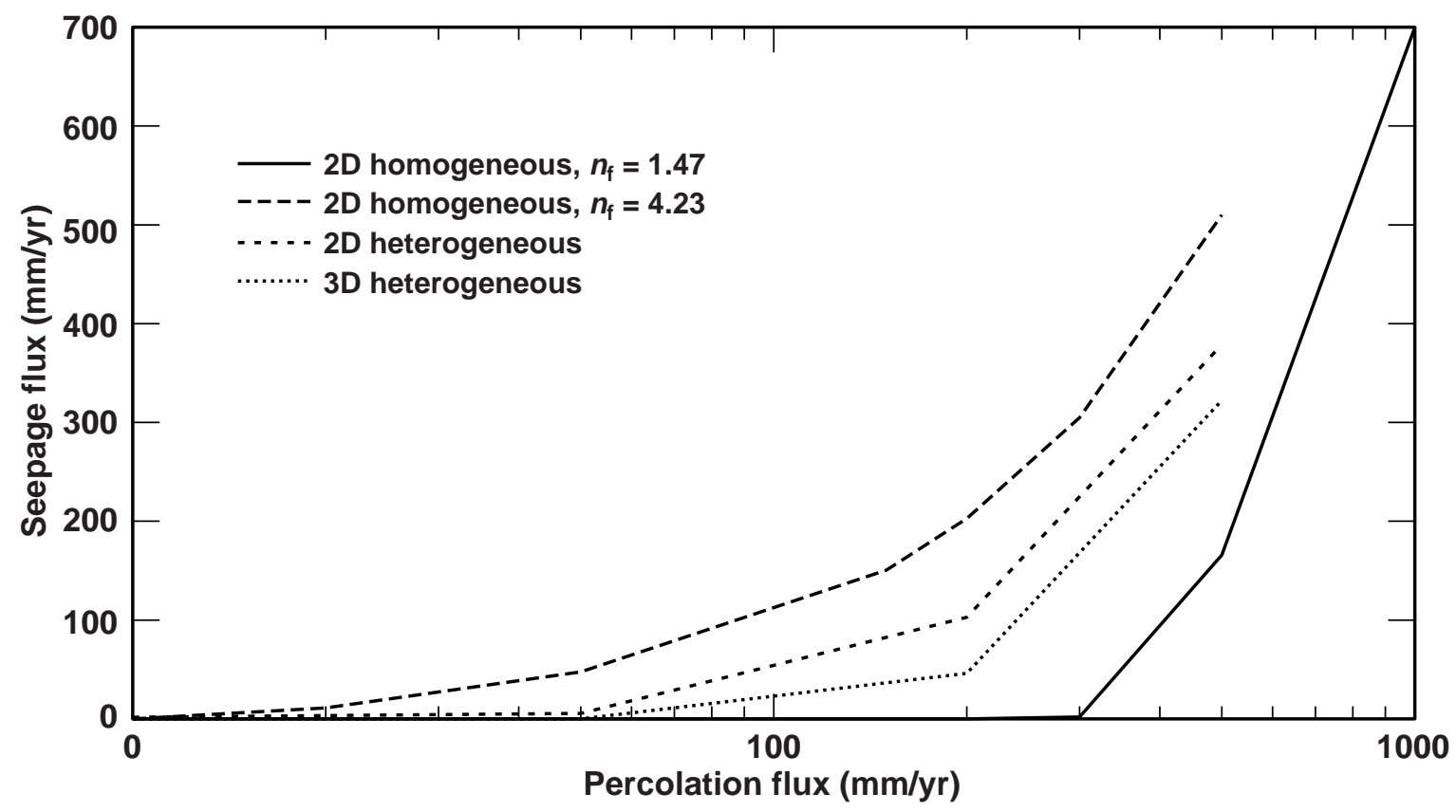

Figure 3-24. Seepage flux into drift vs. average percolation flux into top of model

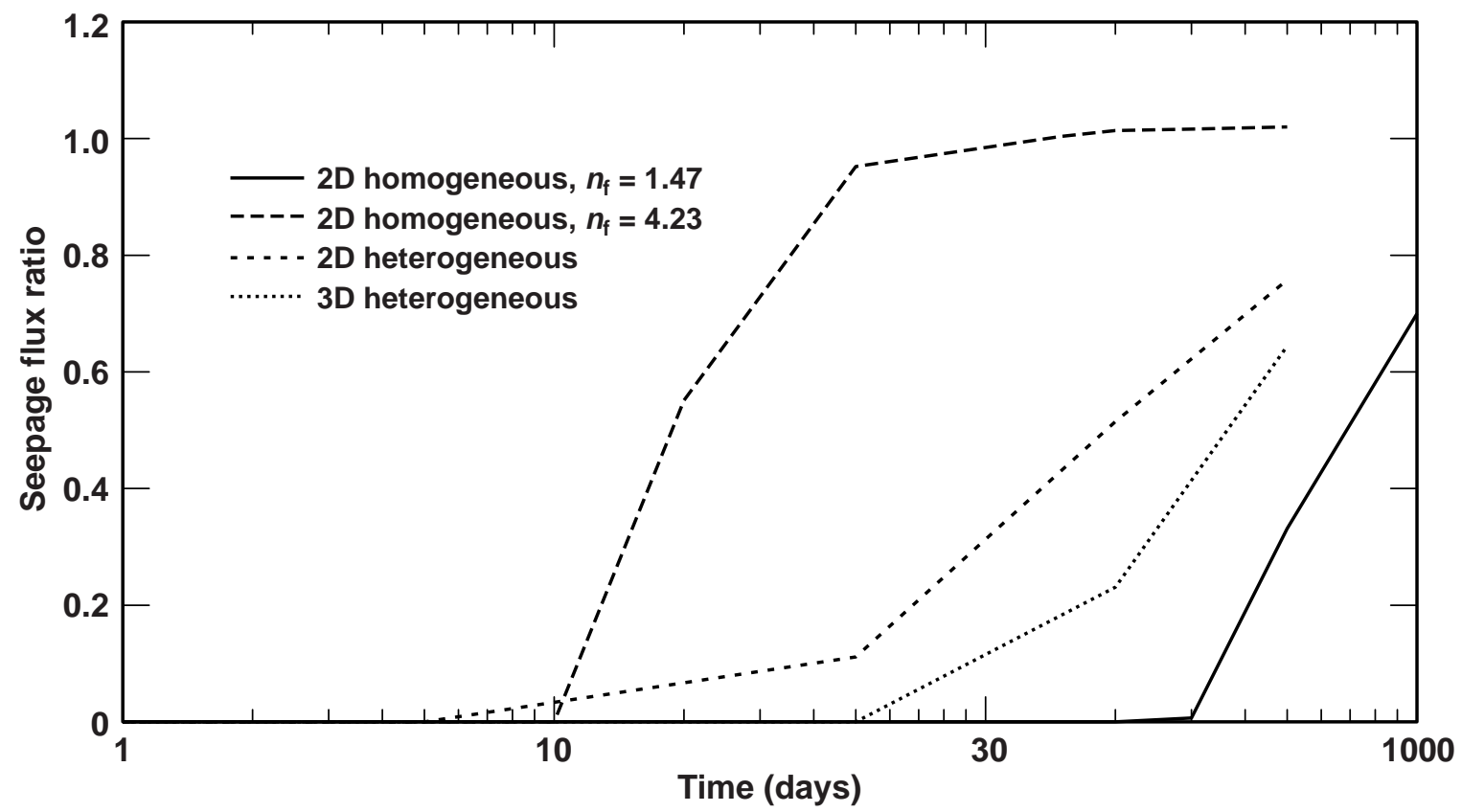

Figure 3-25. Ratio of seepage flux into the drift divided by ambient percolation flux vs. simulation time for the initial value problem. 


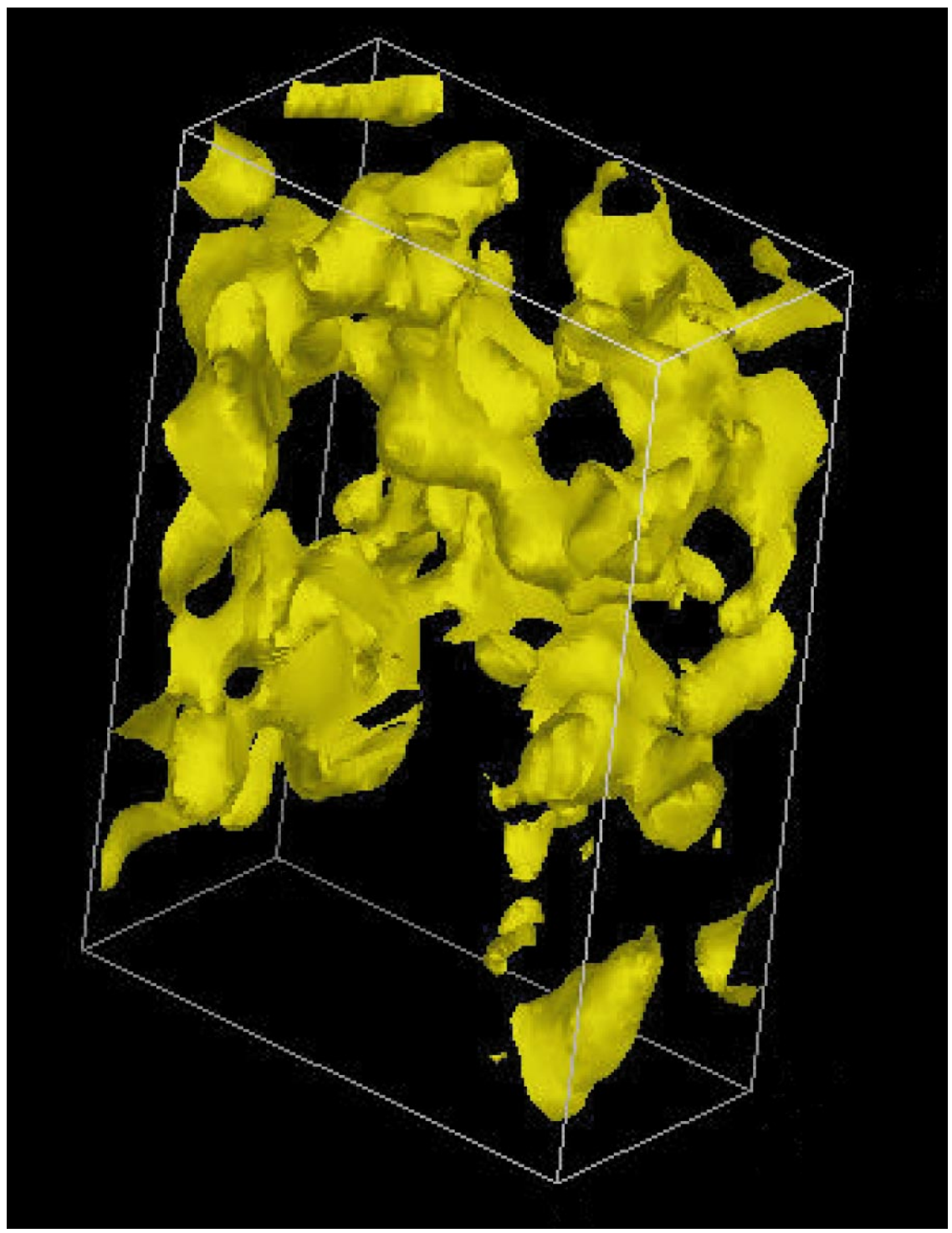

Figure 3-26. Steady-state isosurfaces of 50\% fracture saturation for a 3-D simulation 


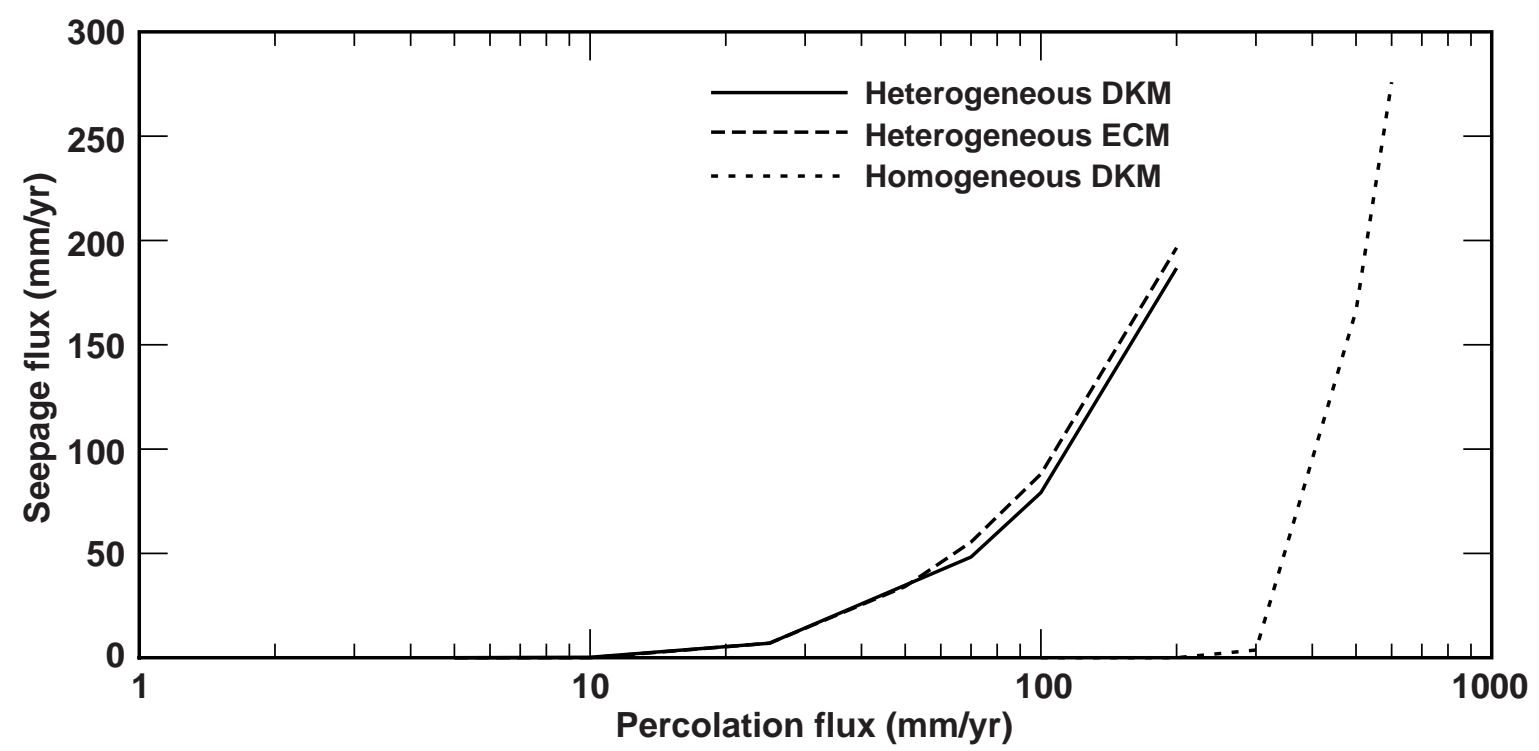

Figure 3-27. Seepage flux into the drift vs. percolation flux into the top of the model for the heterogeneous dual-permeability base case, heterogeneous equivalent continuum case, and homogeneous dual-permeability case

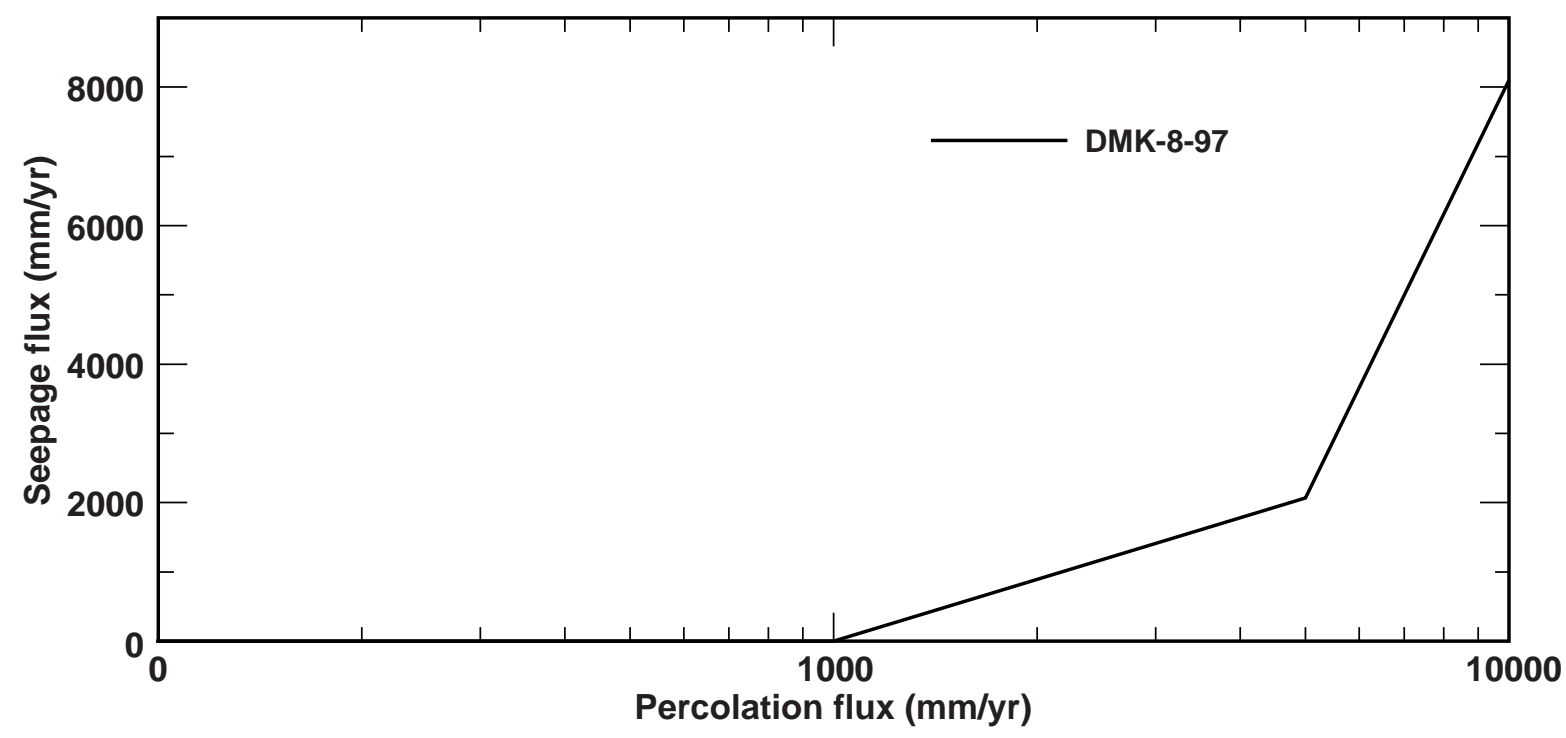

Figure 3-28. Seepage flux vs. percolation flux for the July 1997 TSPA-VA DKM case 


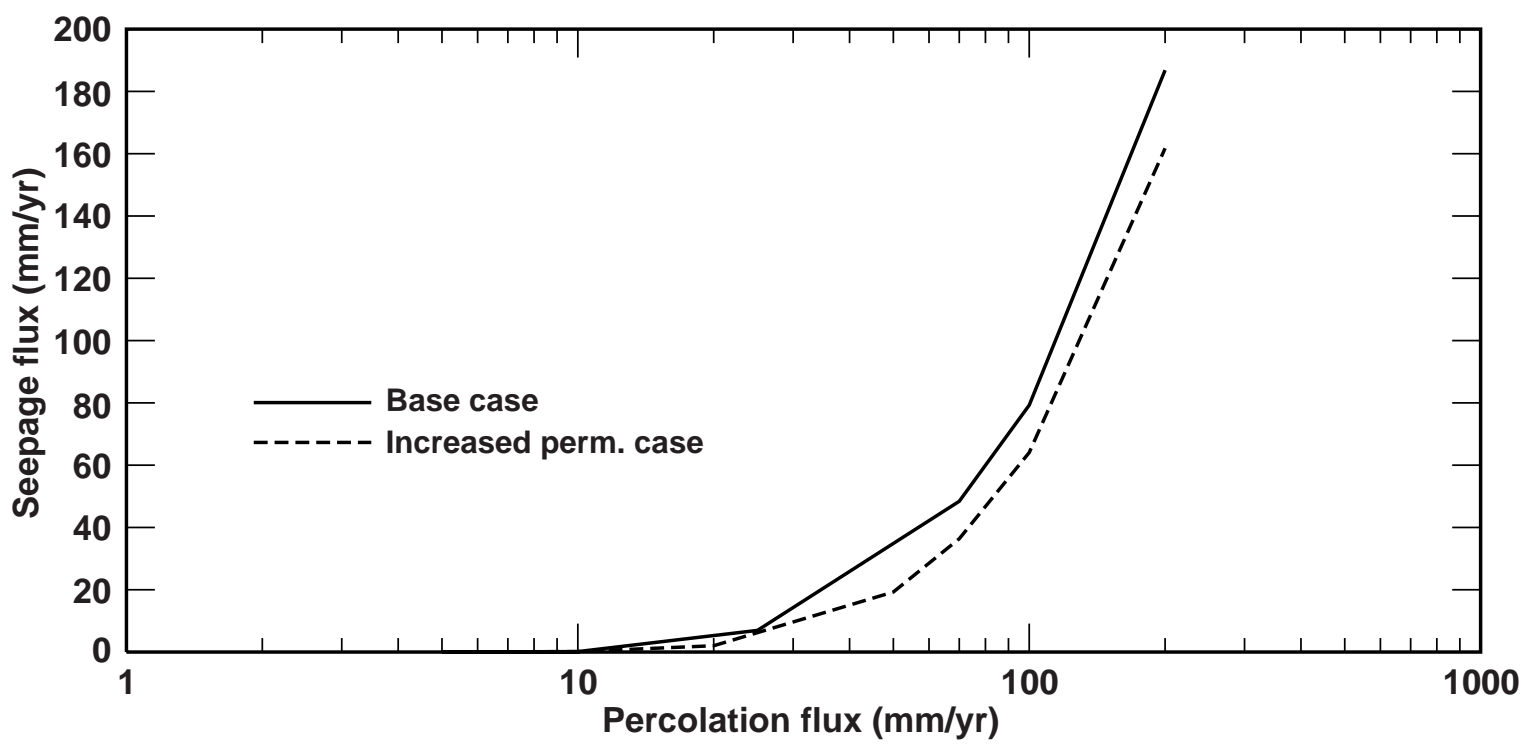

Figure 3-29. Seepage flux vs. percolation flux is $1.2 \mathrm{~m}$ around the drift for the base case and the increased fracture permeability case

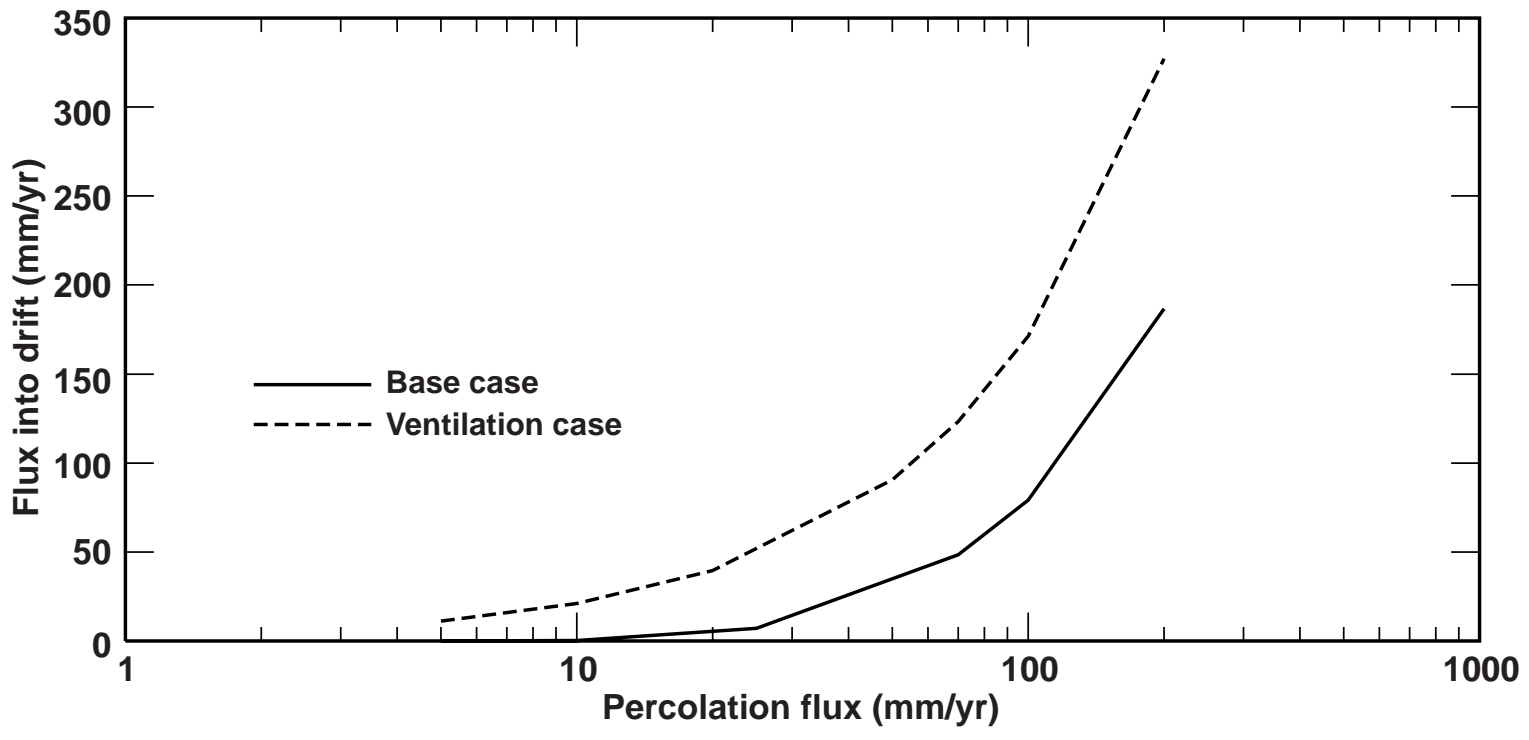

Figure 3-30. Total flux into drift vs. percolation flux for the base case and the case with $50 \% \mathrm{RH}$ in the drift to simulate ventilation 


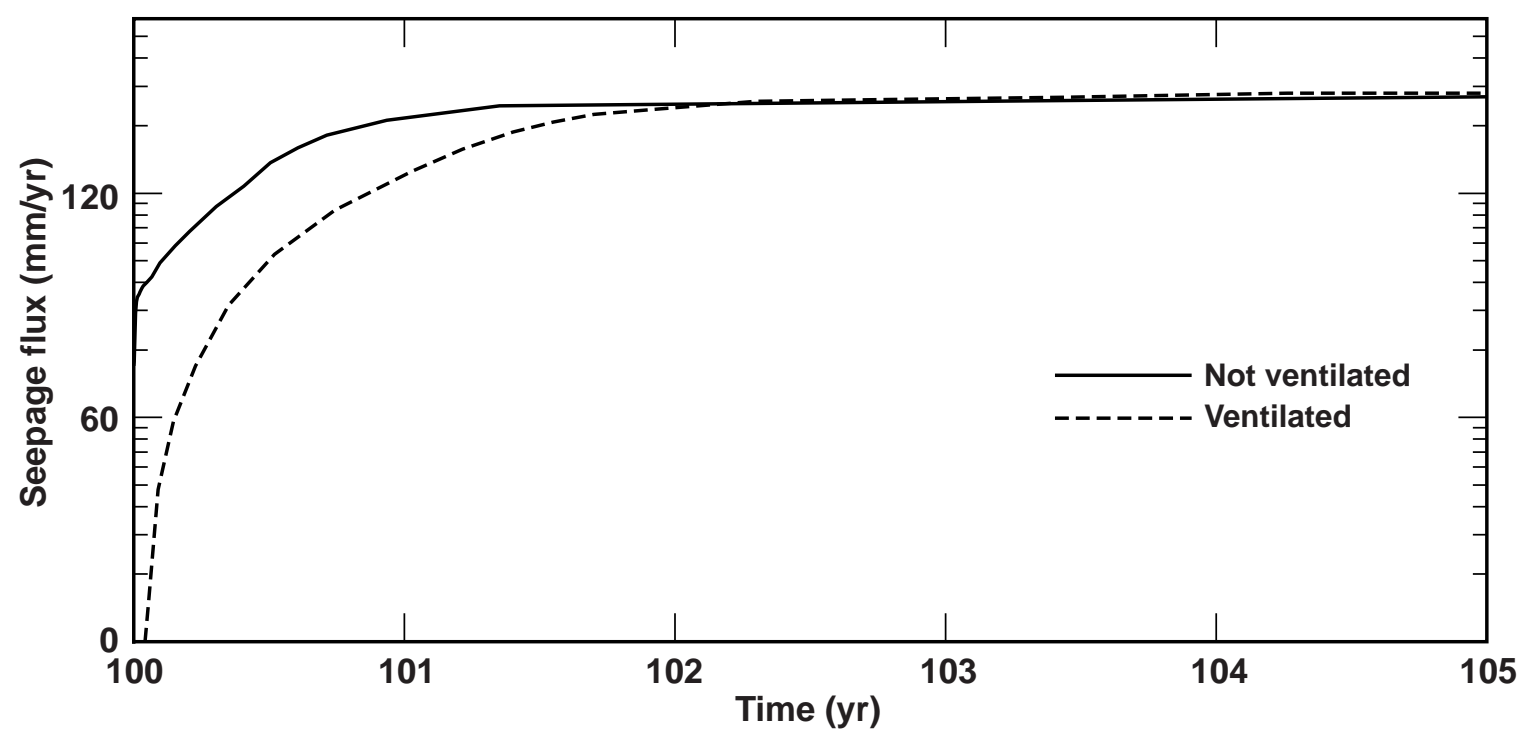

Figure 3-31. Seepage flux vs. time for cases with and without ventilation at $50 \%$ relative from time 0 to $100 \mathrm{yr}$ (Percolation flux is increased from $5 \mathrm{~mm} / \mathrm{yr}$ to $50 \mathrm{~mm} / \mathrm{yr}$ at $100 \mathrm{yr}$ )

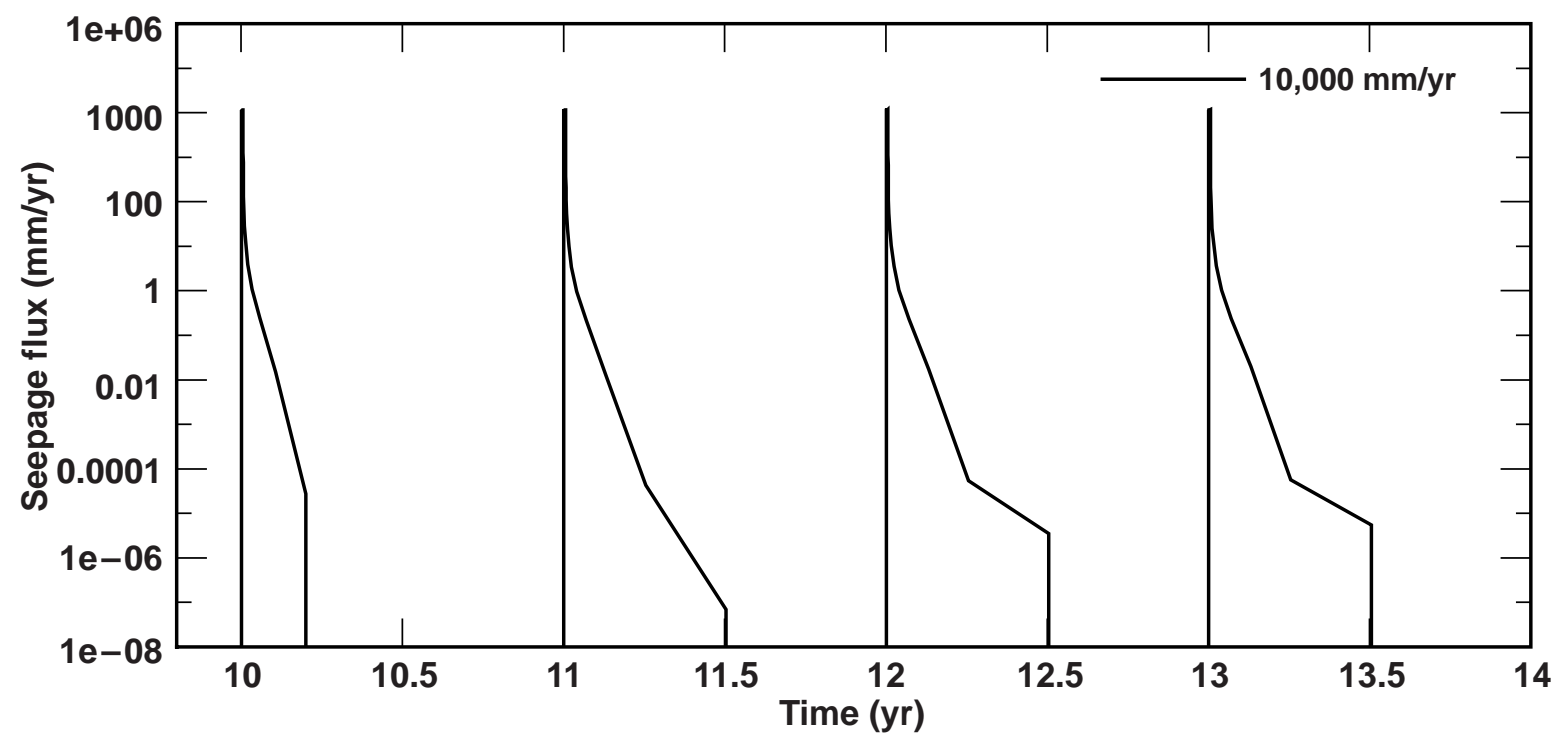

Figure 3-32. Seepage flux vs. time for the case with 10,000-mm/yr pulse every year, lasting over a 2-day period, imposed on a background flux of $5 \mathrm{~mm} / \mathrm{yr}$ 
(a) Start of infiltration pulse

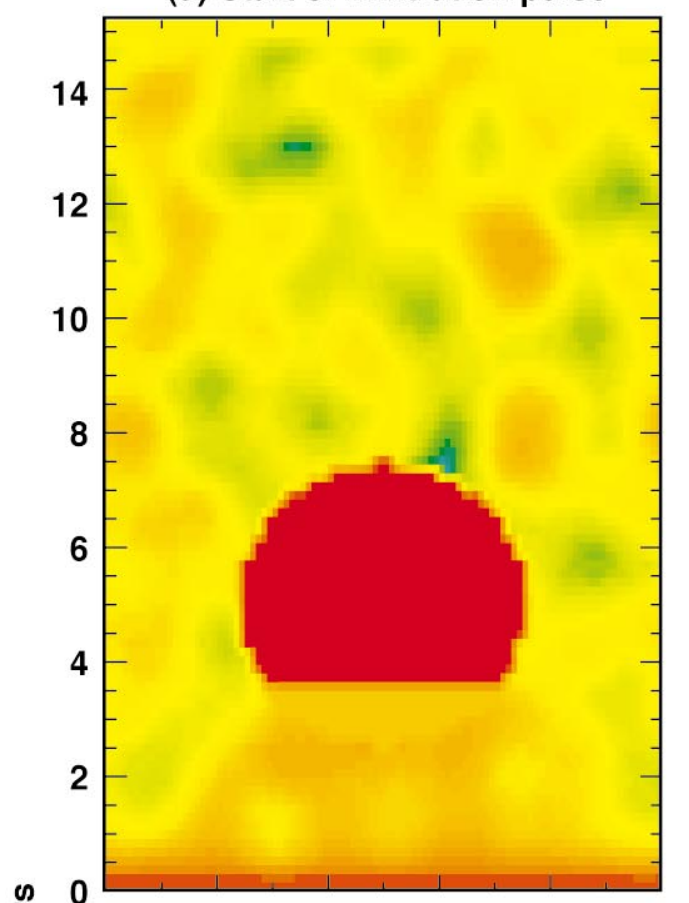

(c) 1.2 hours

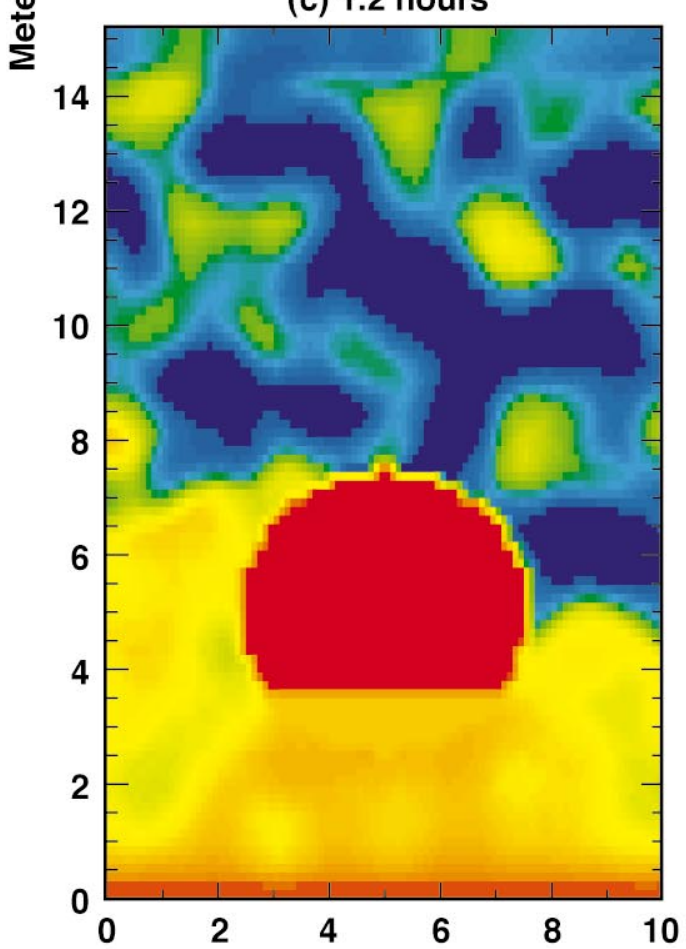

(b) 0.7 hours after start of pulse

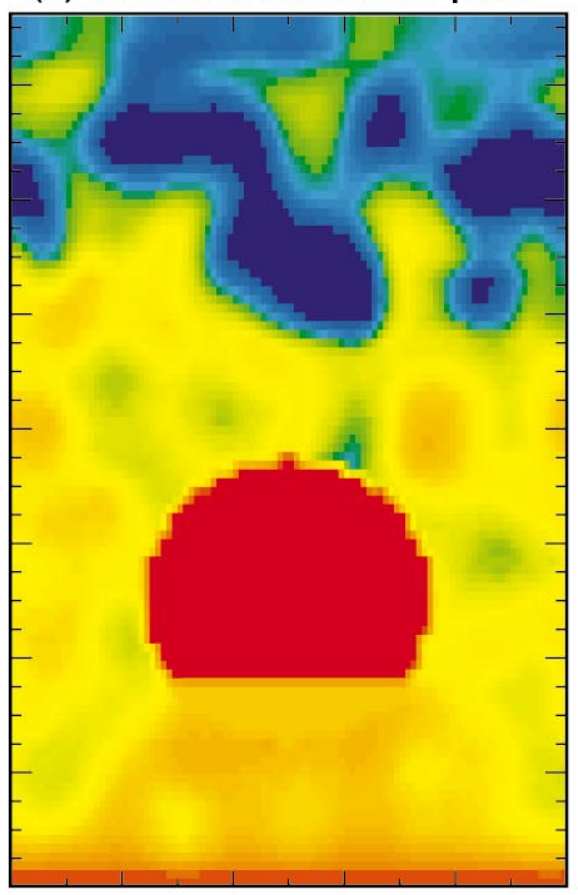

(d) 3.2 hours

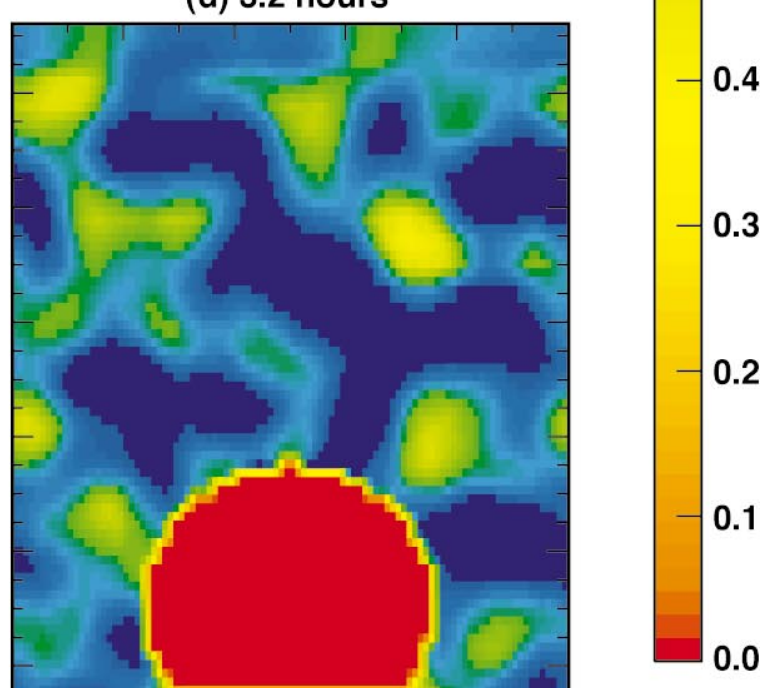

Figure 3-33. Liquid fracture saturation during the start of an infiltration event with a flux into the top of the model of 10,000 mm/yr over a 2-day period 


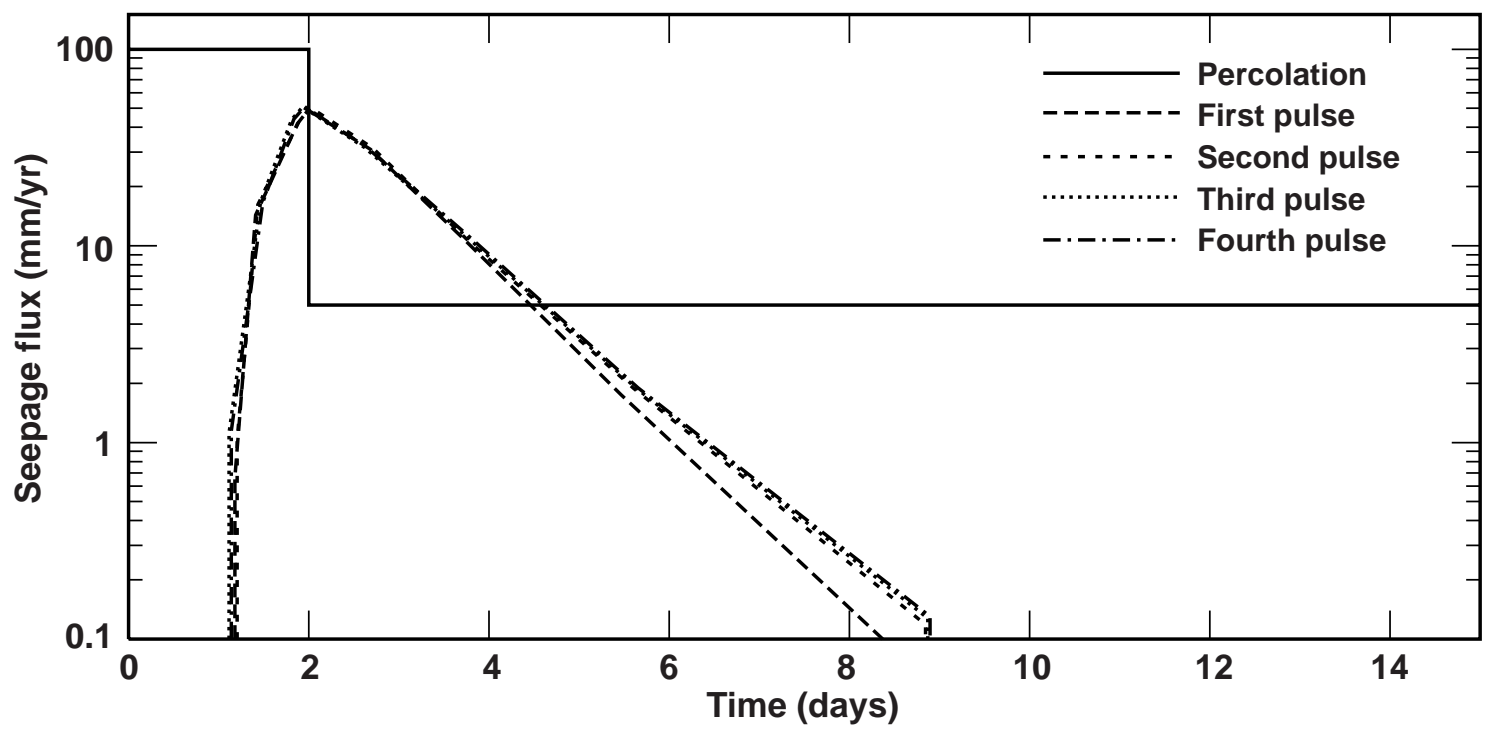

Figure 3-34. Seepage flux vs. time from beginning of each year for the case with $100-\mathrm{mm} / \mathrm{yr}$ pulse every year

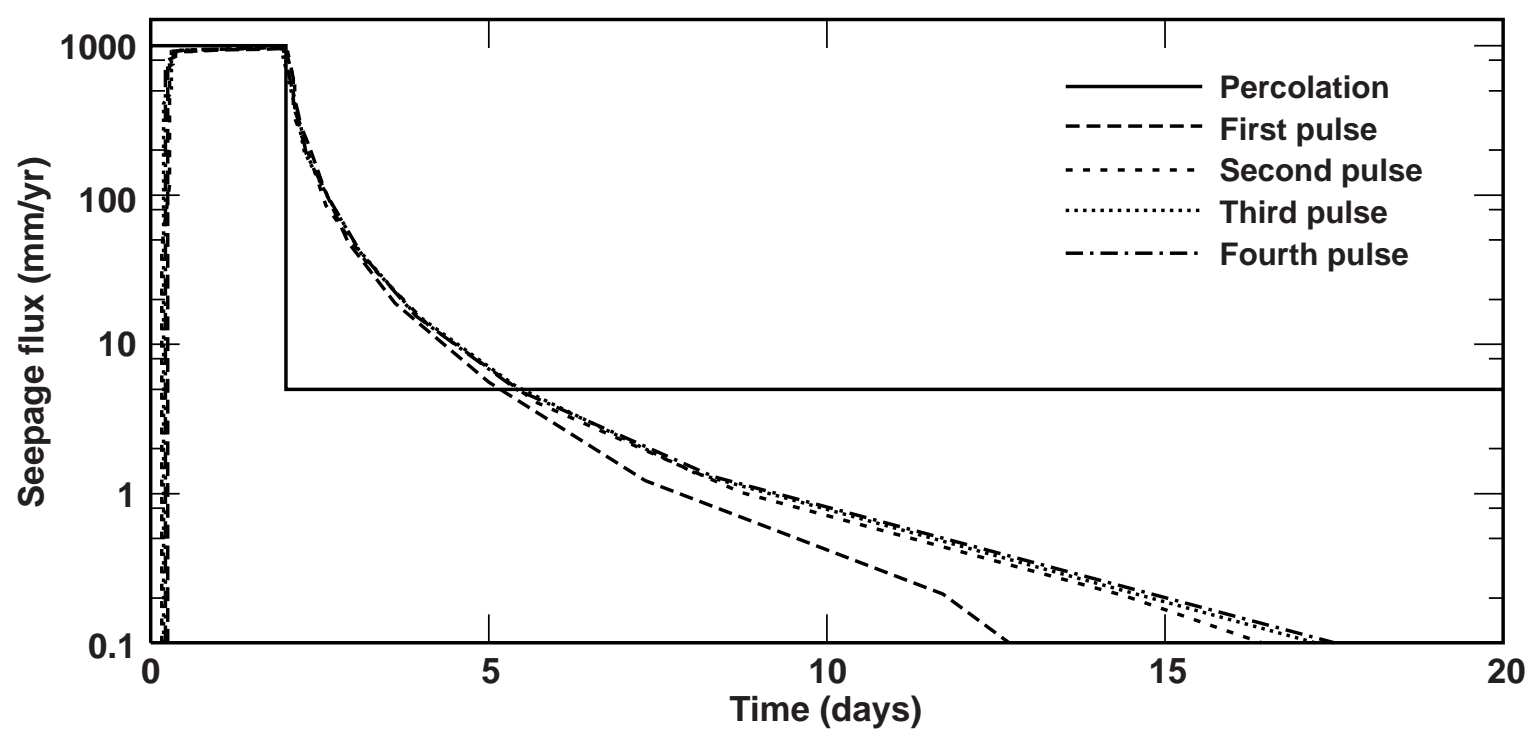

Figure 3-35. Seepage flux vs. time from beginning of each year for the case with $1000-\mathrm{mm} / \mathrm{yr}$ pulse every year 


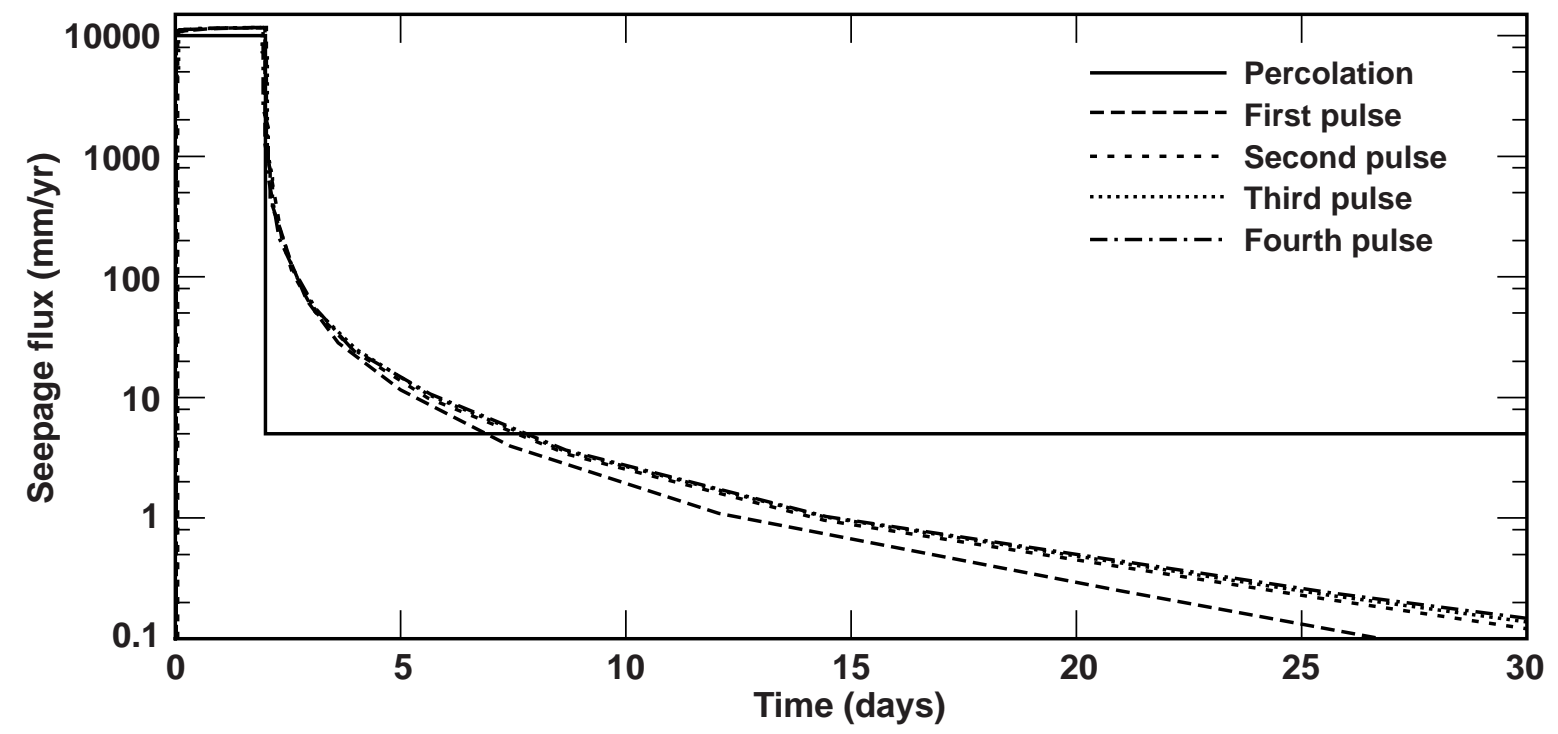

Figure 3-36. Seepage flux vs. time from beginning of each year for the case with 10,000-mm/yr pulse every year

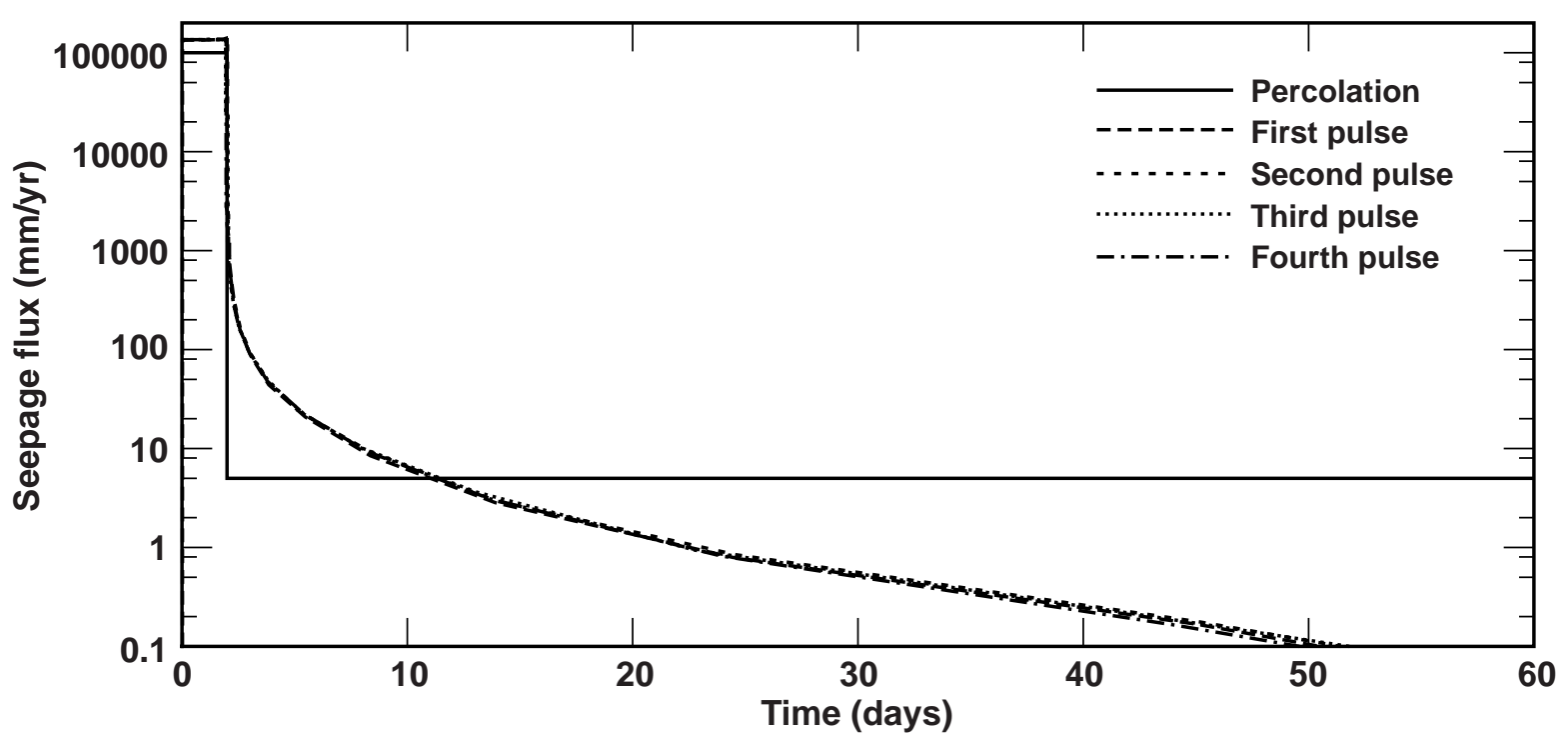

Figure 3-37. Seepage flux vs. time from beginning of each year for the case with 100,000-mm/yr pulse every year 


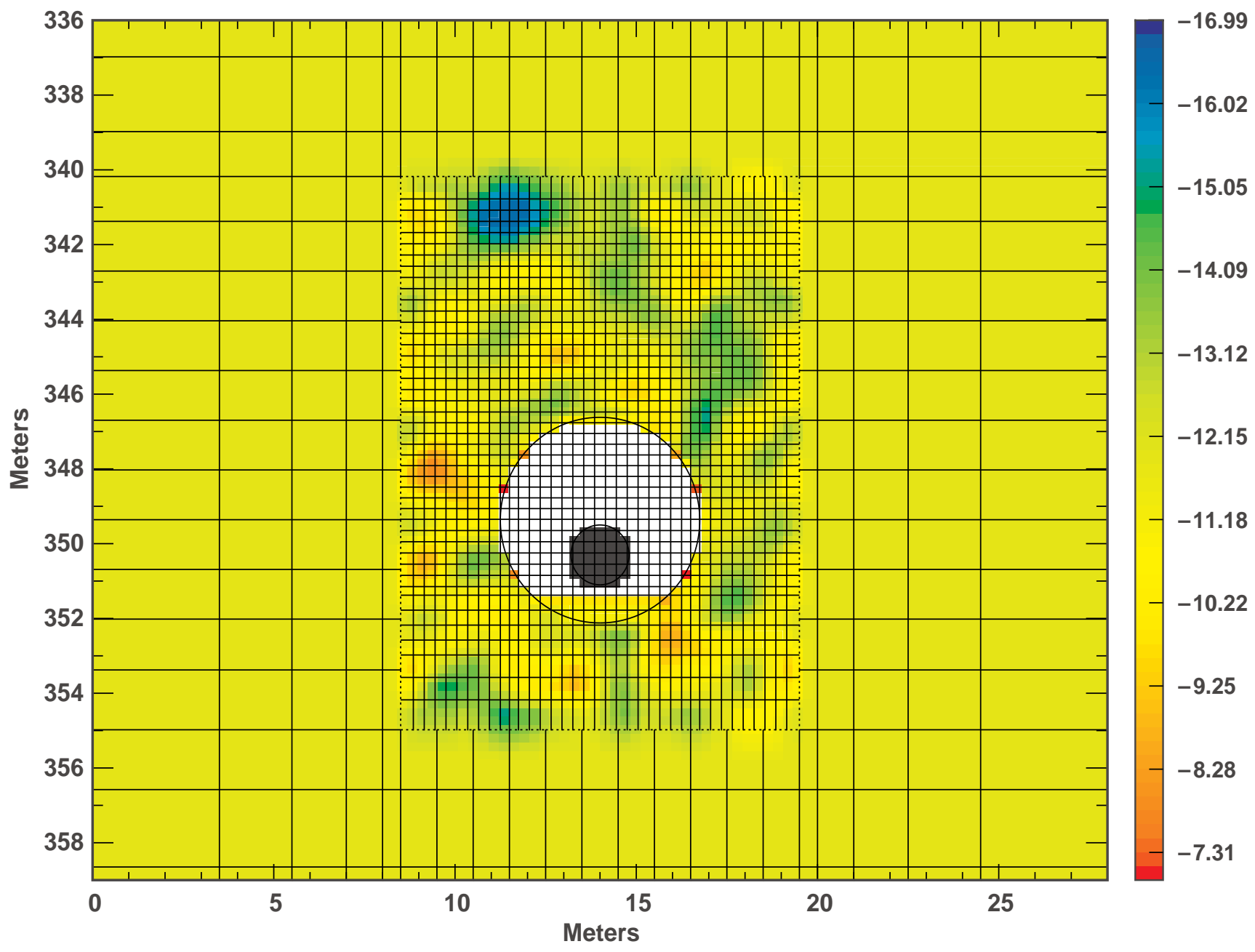

Figure 3-38. Closeup of the nested grid and the $\log _{10}$ bulk permeability $\left(\mathrm{m}^{2}\right)$ field around the drift 


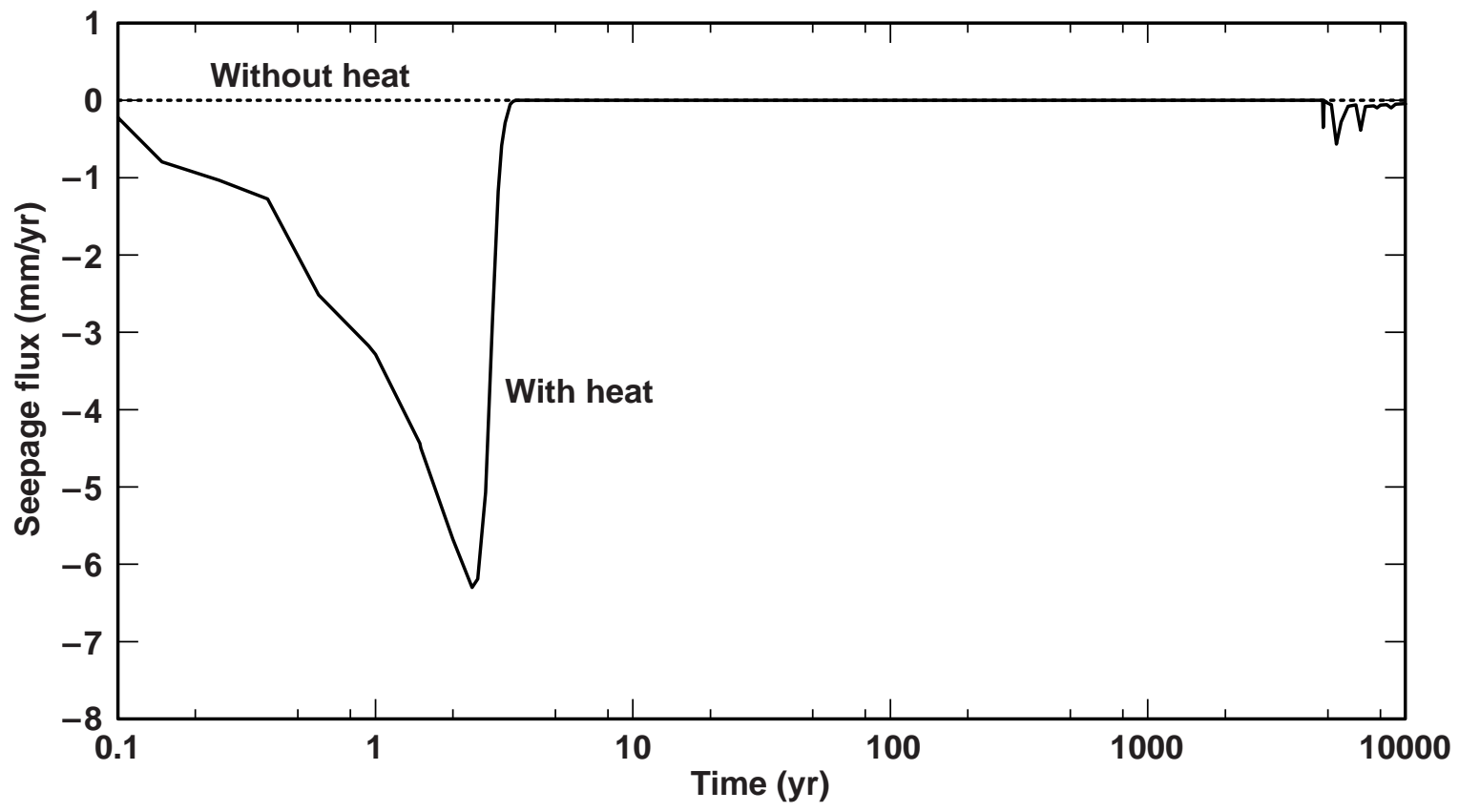

Figure 3-39. Seepage flux into the drift for the homogeneous, 5-mm/yr case under point loading with the TSPA-VA

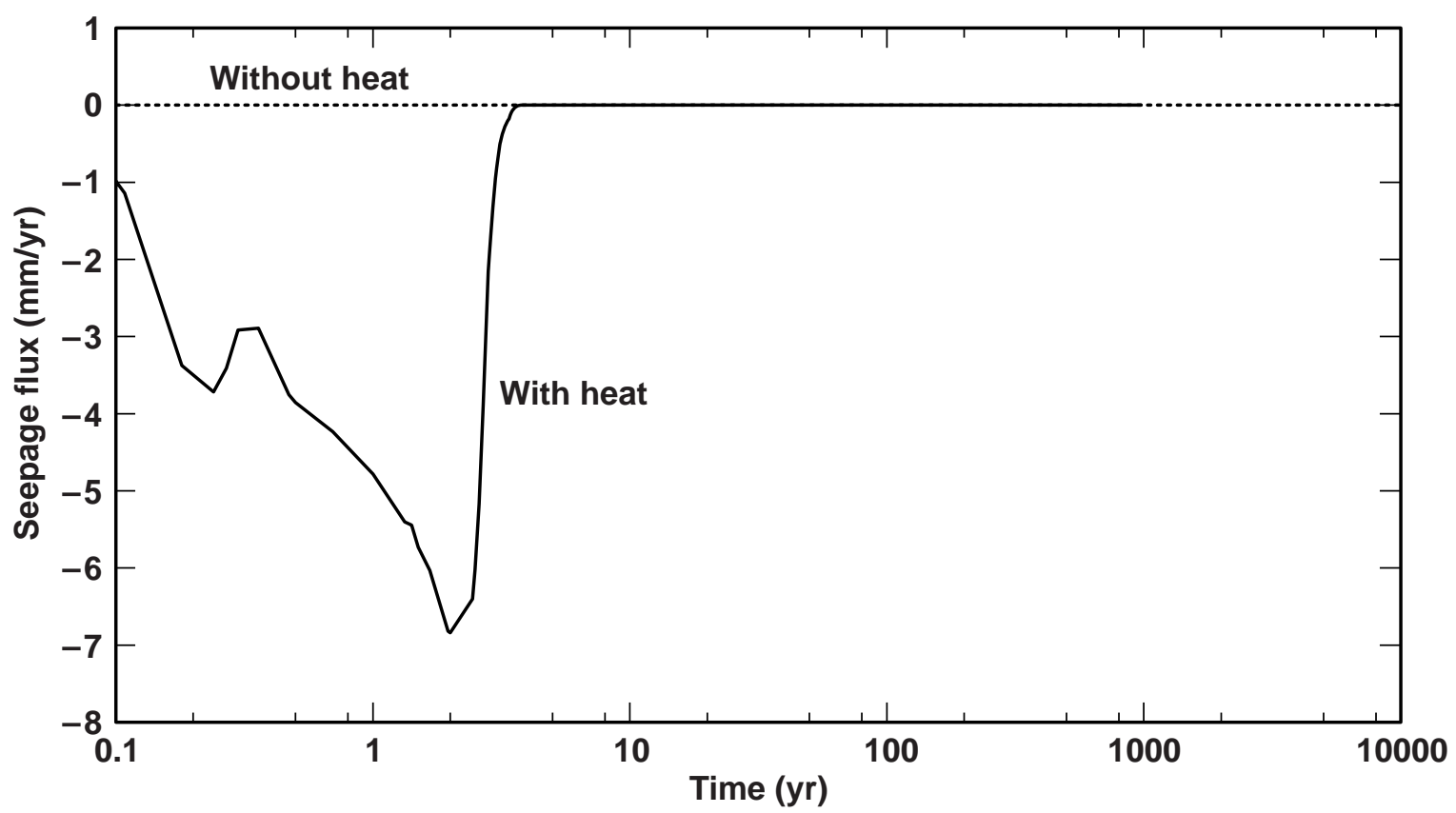

Figure 3-40. Seepage flux into the drift for heterogeneous, 5-mm/yr case under point loading with TSPA-VA property set 


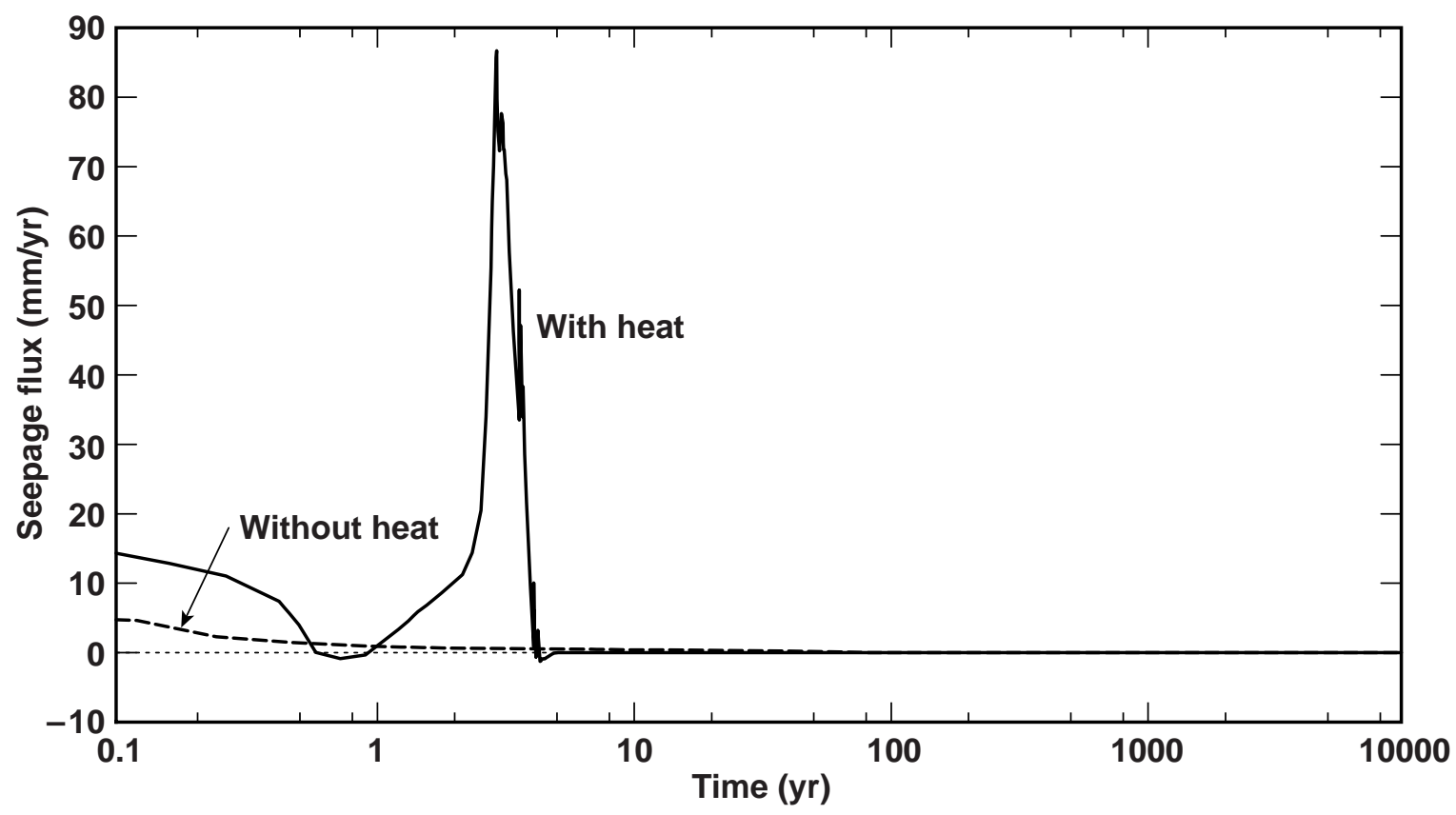

Figure 3-41. Seepage flux into the drift for the homogeneous, 5-mm/yr case under point loading with the Klavetter and Peters (1986) property set

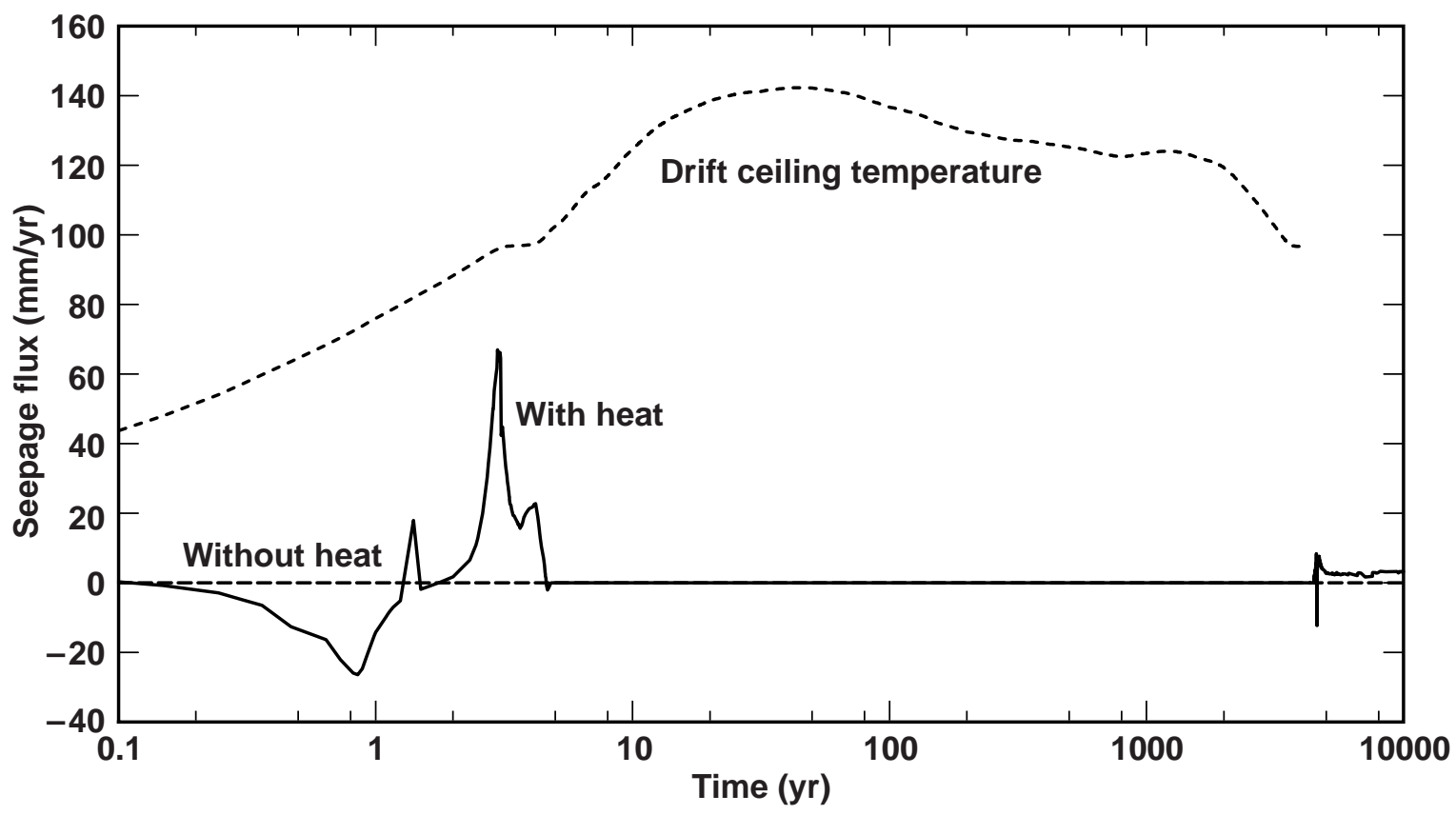

Figure 3-42. Seepage flux into the drift for the heterogeneous, 5- $\mathrm{mm} / \mathrm{yr}$ case under point loading with the Klavetter and Peters (1986) property set 


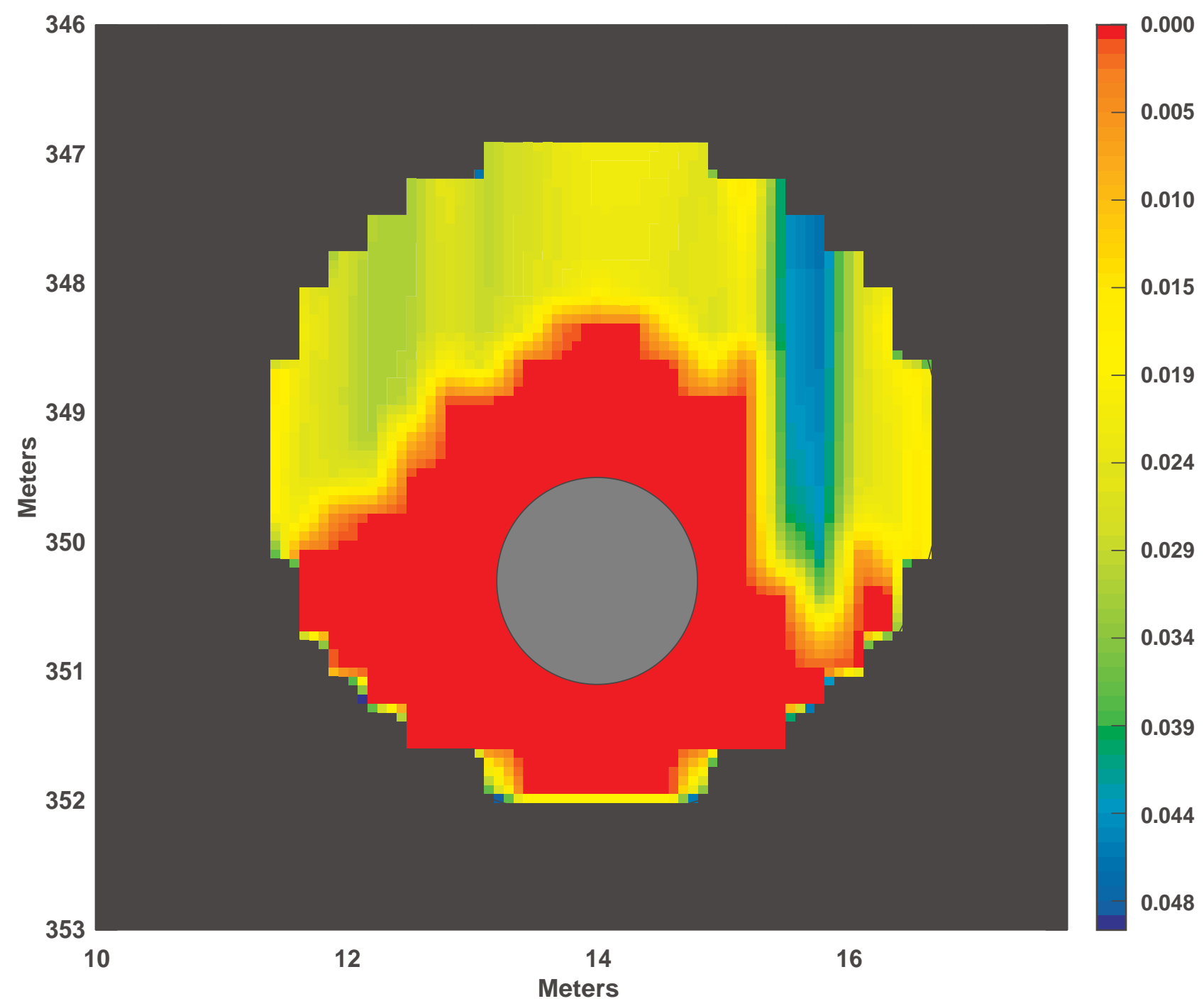

Figure 3-43. Liquid saturation in the drift at $3 \mathrm{yr}$ for $5-\mathrm{mm} / \mathrm{yr}$ percolation flux using the Klavetter and Peters (1986) property set with heterogeneous various (Seepage in the drift is caused by condensation of water vapor onto and within the cooler drift wall at early times. Seepage ceases after temperatures in the rock reach the boiling point. Evaporation of water is overestimated in the model because of artificially low relative permeability in the drift to facilitate numerical computation.) 


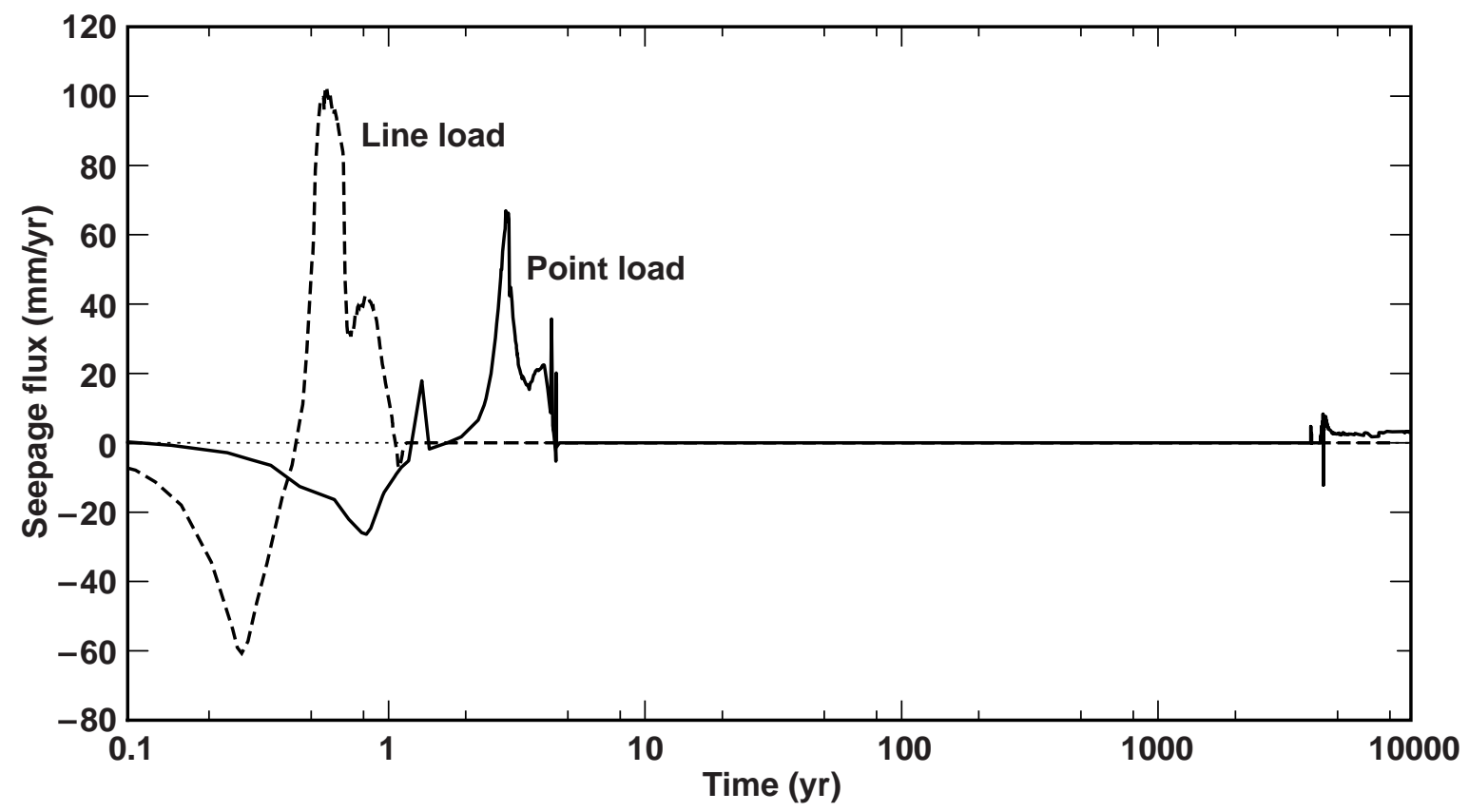

Figure 3-44. Seepage flux into the drift for the heterogeneous 5-mm/yr case with the Klavetter and Peters (1986) property set-comparison between line and point loading

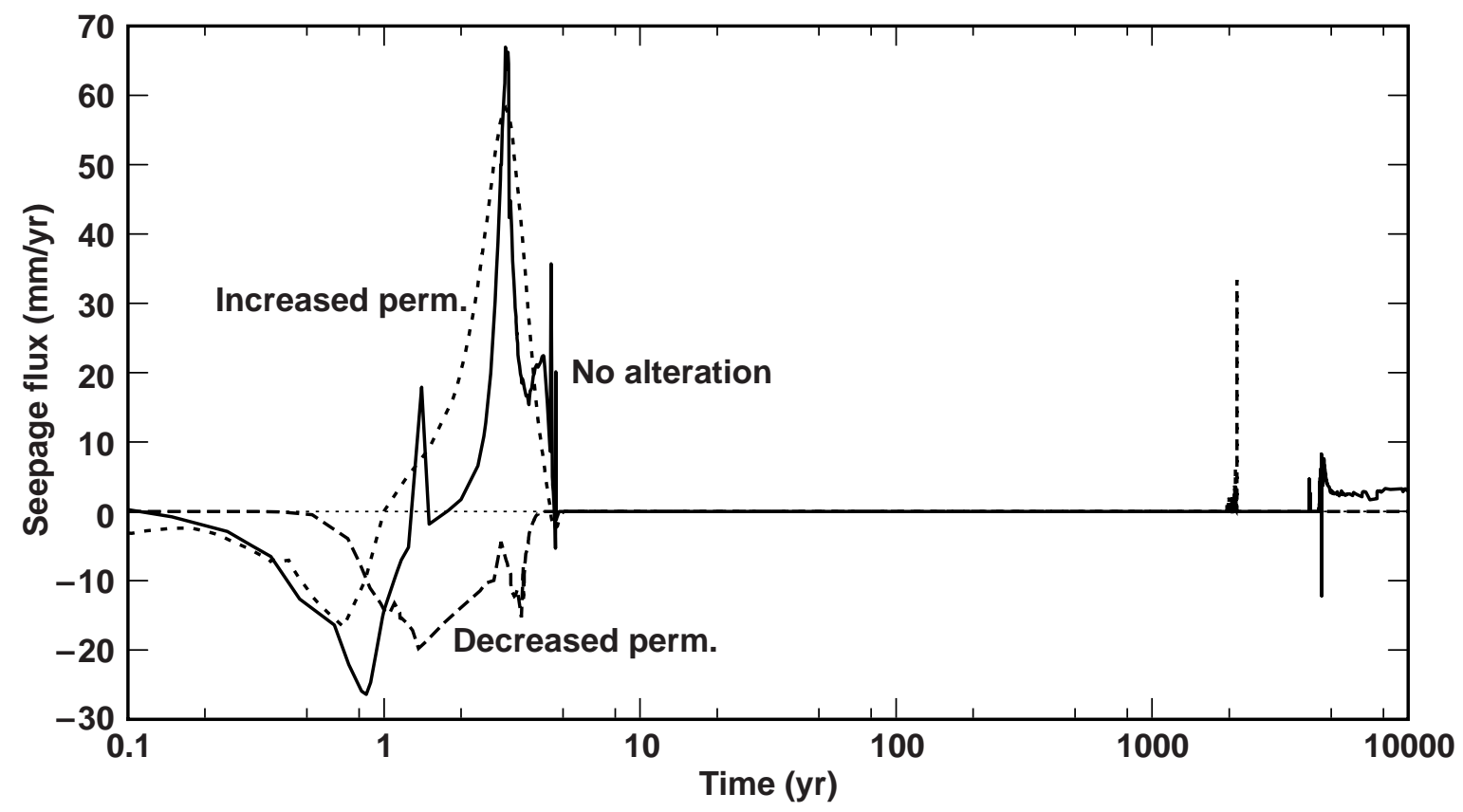

Figure 3-45. Seepage flux into the drift for the heterogeneous 5-mm/yr case under point loading with the Klavetter and Peters (1986) property set-effect of altering the degree of fracturing around the drift 
(a) SDT model

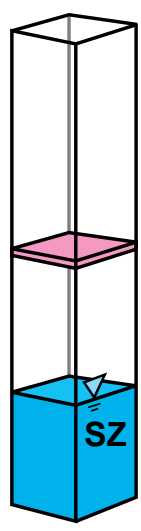

(b) LDTH model

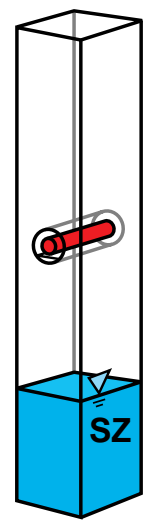

(c) DDT model

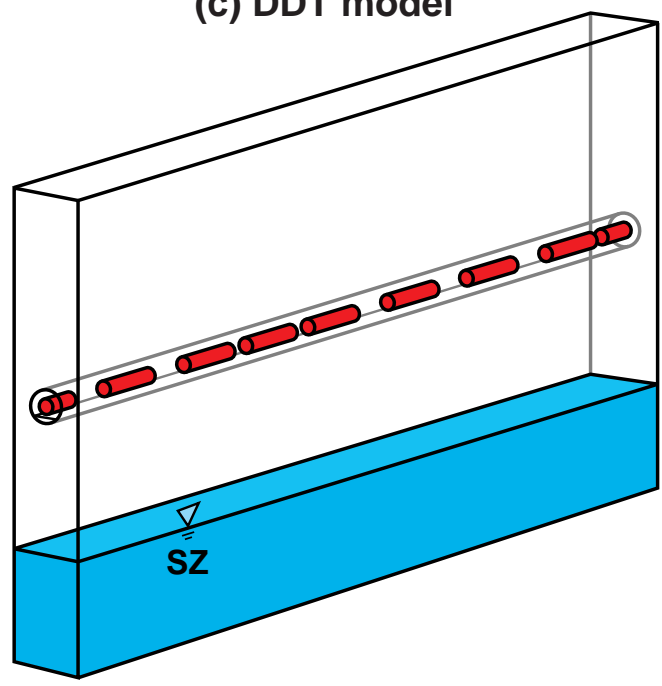

(d) SMT model

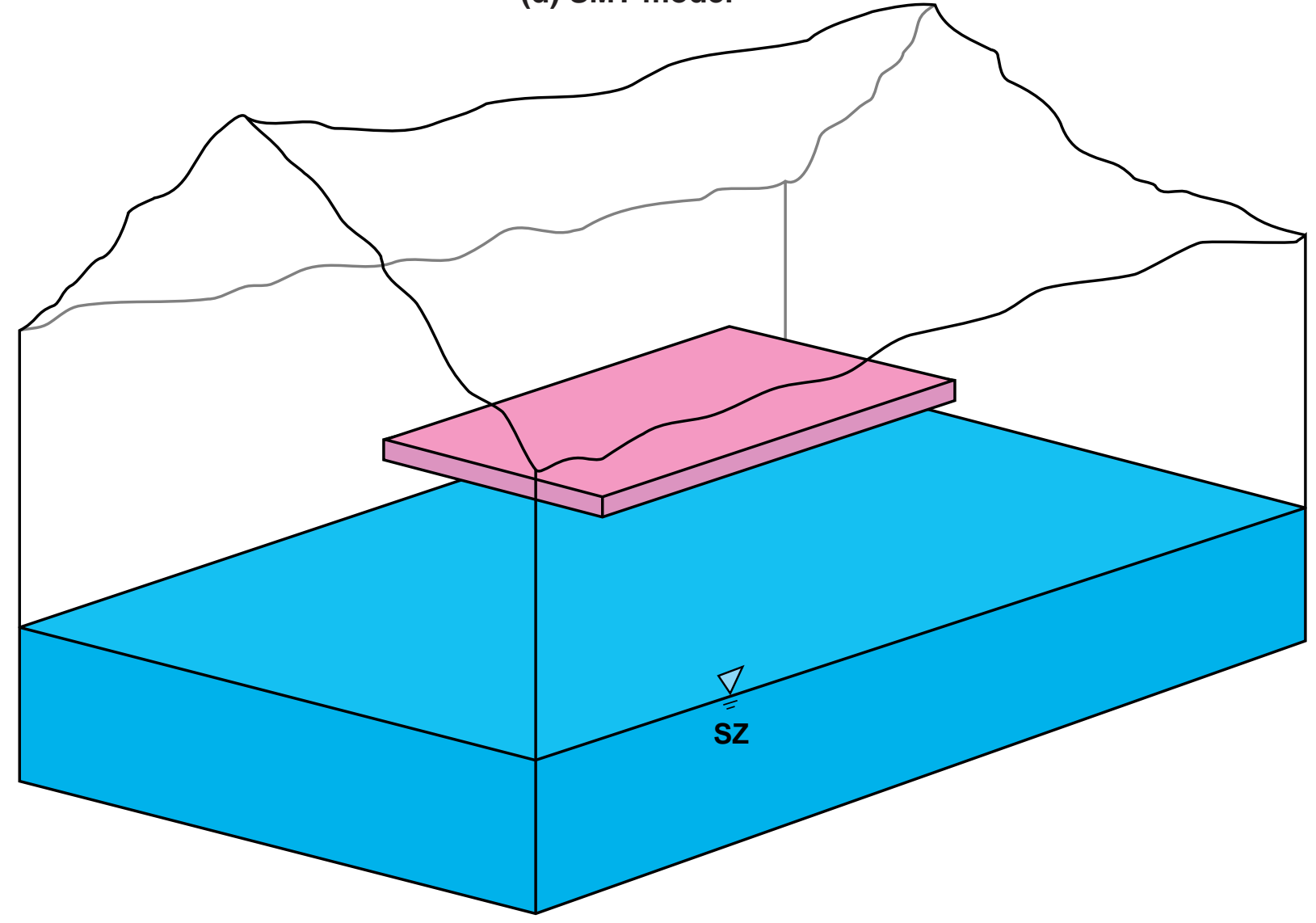

Figure 3-46. Schematic of conceptual models used by the multi-scale TH modeling approach, including (a) SDT model, (b) LDTH model, (c) DDT model, and (d) SMT model 


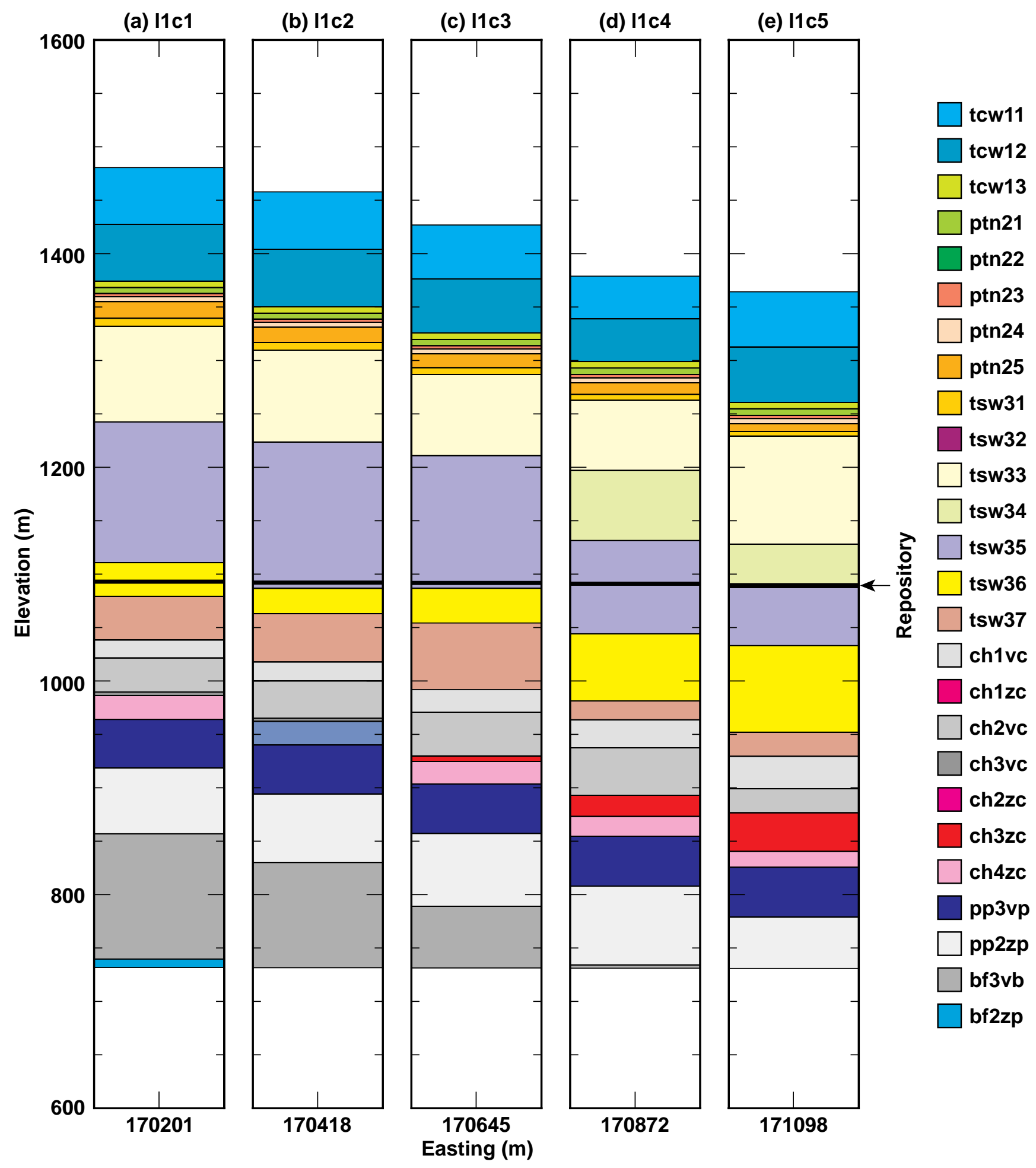

Figure 3-47. Vertical distribution of hydrostratigraphic model units shown for several drift-scale model locations (in Nevada-State coordinates), including (a) 11c1 at Northing $=232406 \mathrm{~m}$, (b) 11c2 at Northing $=232394 \mathrm{~m}$, (c) 11c3 at Northing $=232382 \mathrm{~m}$, (d) 11c4 at Northing $=232370 \mathrm{~m}$, and (e) $11 \mathrm{c} 5$ at Northing $=232358 \mathrm{~m}$ 


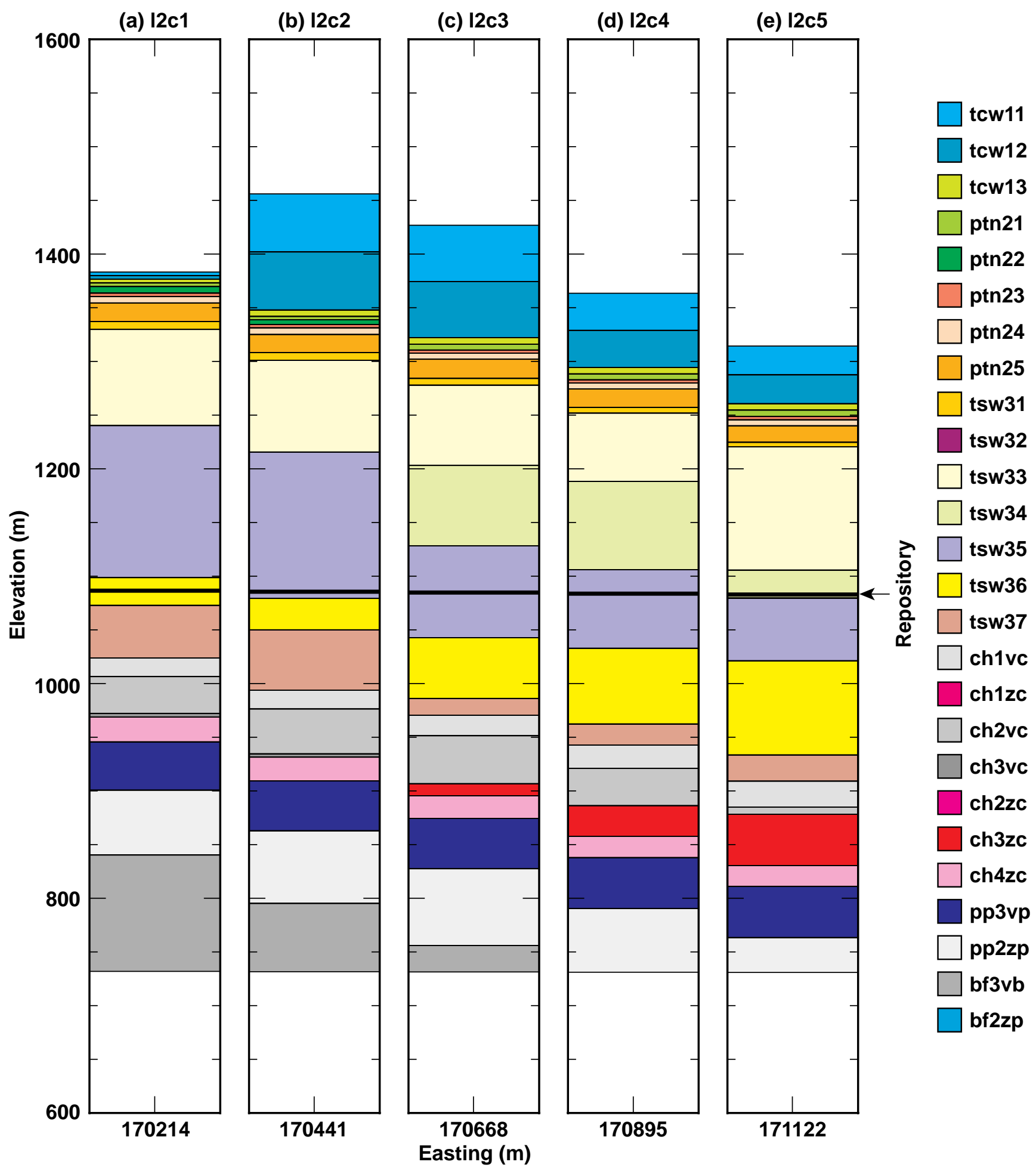

Figure 3-48. Vertical distribution of hydrostratigraphic model units shown for drift-scale model locations (in Nevada-State coordinates), including (a) 12c1 at Northing $=232857 \mathrm{~m},(\mathrm{~b}) 12 \mathrm{c} 2$ at Northing $=232845 \mathrm{~m}$, (c) 12c3 at Northing $=232833 \mathrm{~m},(\mathrm{~d}) 12 \mathrm{c} 4$ at Northing $=232821 \mathrm{~m}$, and (e) 12c5 at Northing $=232809 \mathrm{~m}$ 


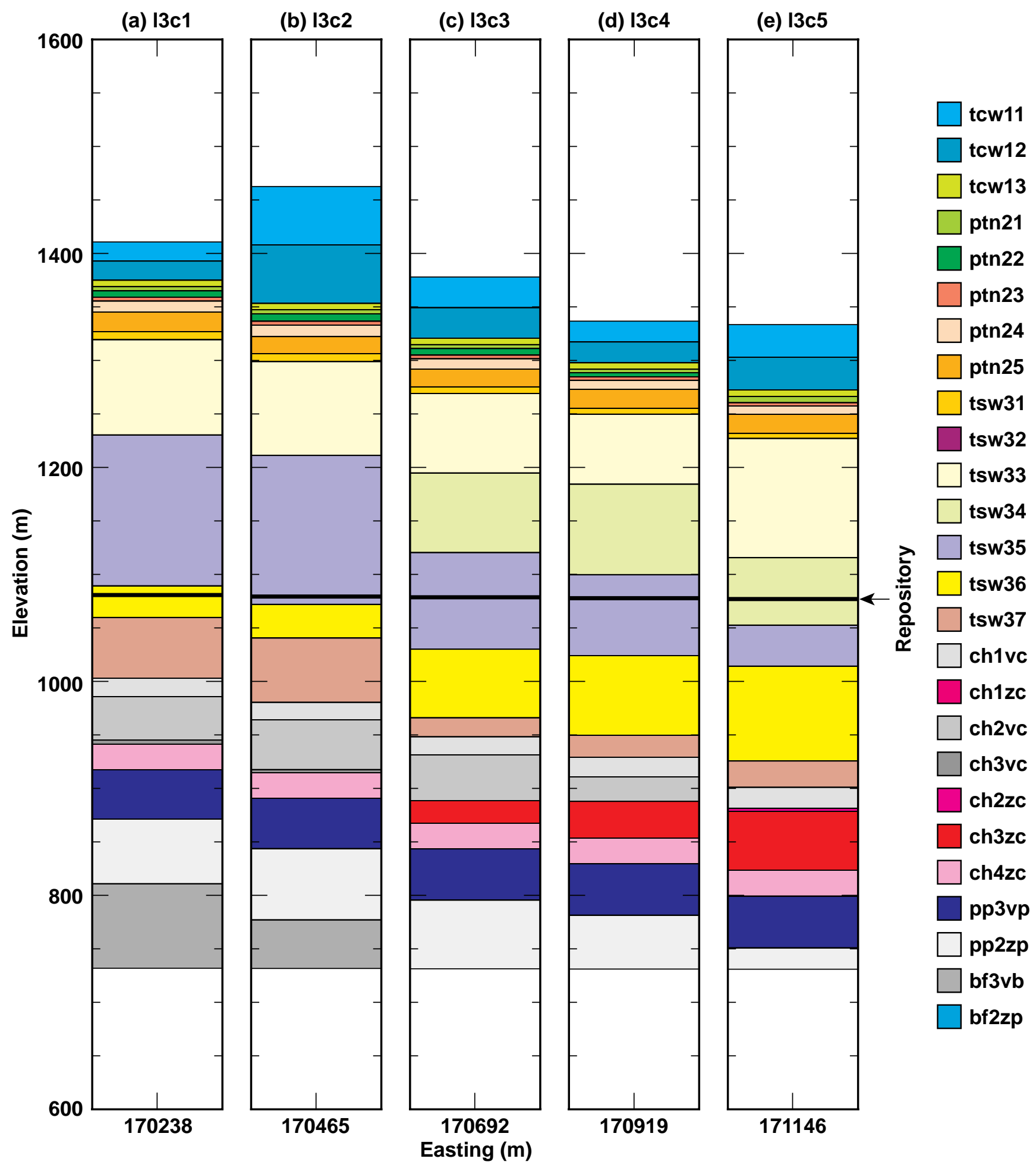

Figure 3-49. Vertical distribution of hydrostratigraphic model units shown for drift-scale model locations (in Nevada-State coordinates), including (a) 13c1 at Northing $=233308 \mathrm{~m},(\mathrm{~b}) 13 \mathrm{c} 2$ at Northing $=233296 \mathrm{~m}$, (c) 13c3 at Northing $=233285 \mathrm{~m}$, (d) $13 \mathrm{c} 4$ at Northing $=233273 \mathrm{~m}$, and (e) $13 \mathrm{c} 5$ at Northing $=233261 \mathrm{~m}$ 


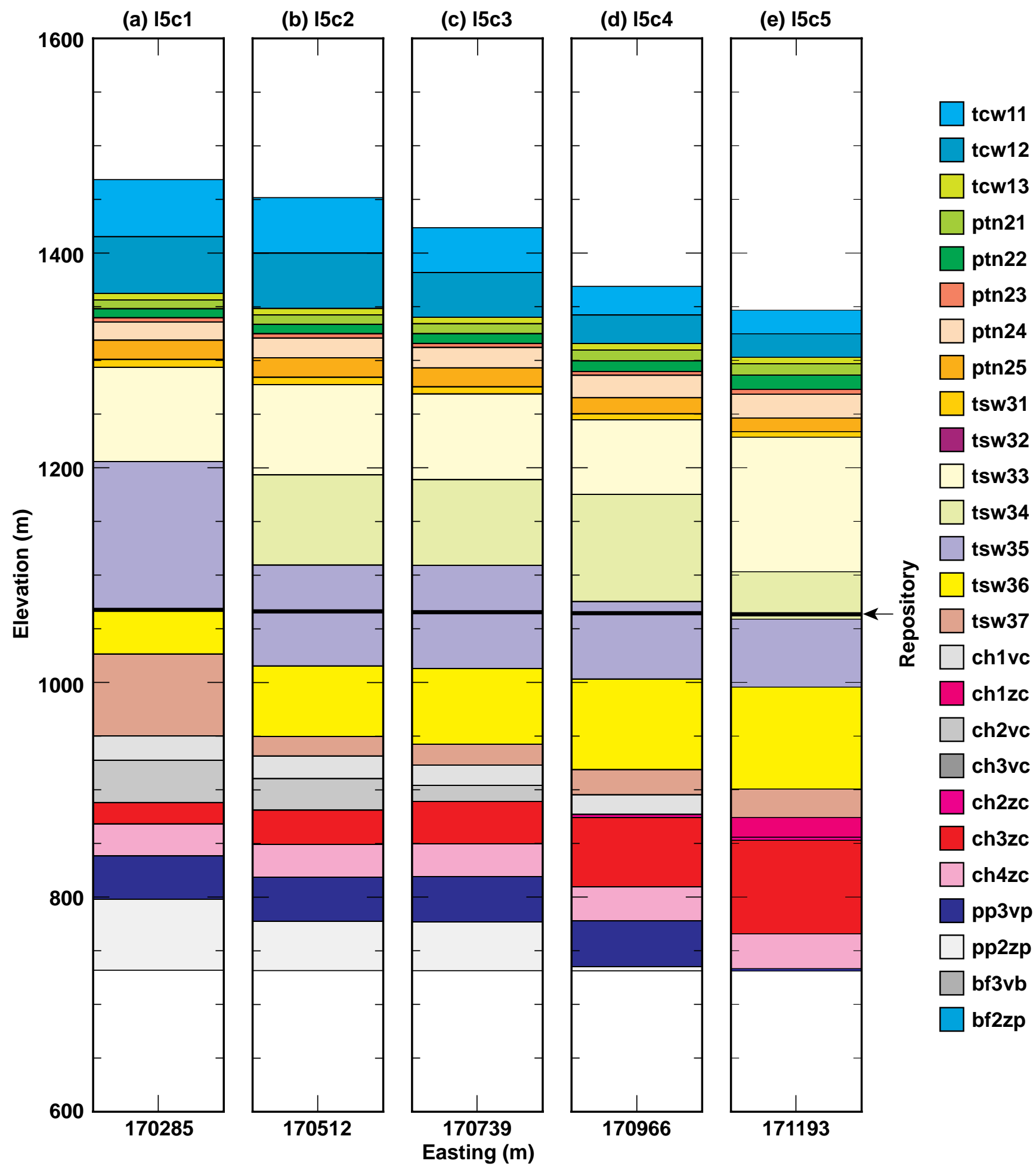

Figure 3-50. Vertical distribution of hydrostratigraphic model units shown for drift-scale model locations (in Nevada-State coordinates), including (a) 15c1 at Northing $=234211 \mathrm{~m}$, (b) 15c2 at Northing = 234199 m, (c) 15c3 at Northing $=234187 \mathrm{~m},(\mathrm{~d}) 15 \mathrm{c} 4$ at Northing $=234175 \mathrm{~m}$, and (e) $15 \mathrm{c} 5$ at Northing $=234164 \mathrm{~m}$ 


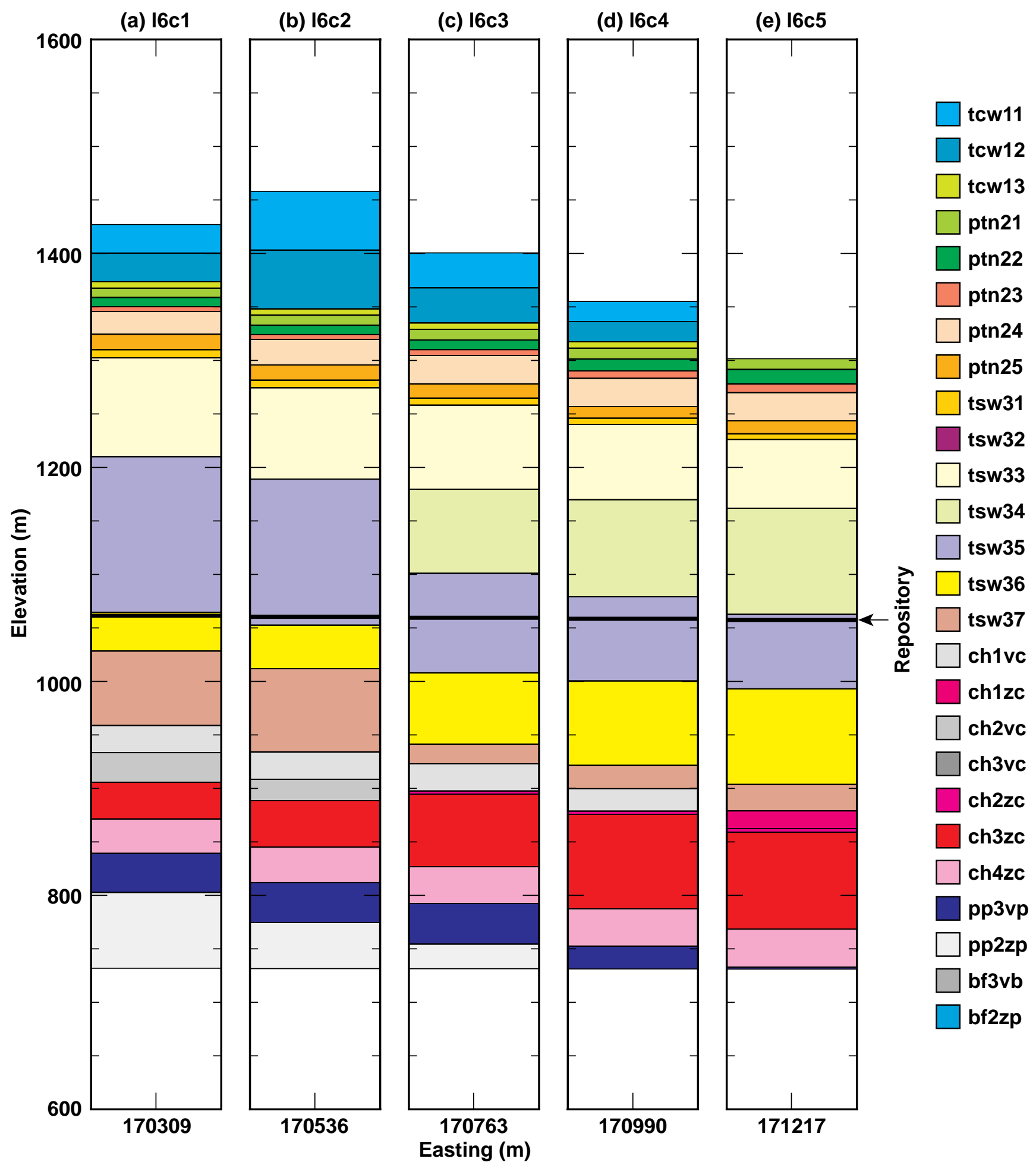

Figure 3-51. Vertical distribution of hydrostratigraphic model units shown for drift-scale model locations (in Nevada-State coordinates), including (a) 16c1 at Northing $=234663 \mathrm{~m},(\mathrm{~b}) 16 \mathrm{c} 2$ at Northing $=234651 \mathrm{~m}$, (c) 16c3 at Northing $=234639 \mathrm{~m}$, (d) $16 \mathrm{c} 4$ at Northing $=234627 \mathrm{~m}$, and (e) $16 \mathrm{c} 5$ at Northing $=234615 \mathrm{~m}$ 


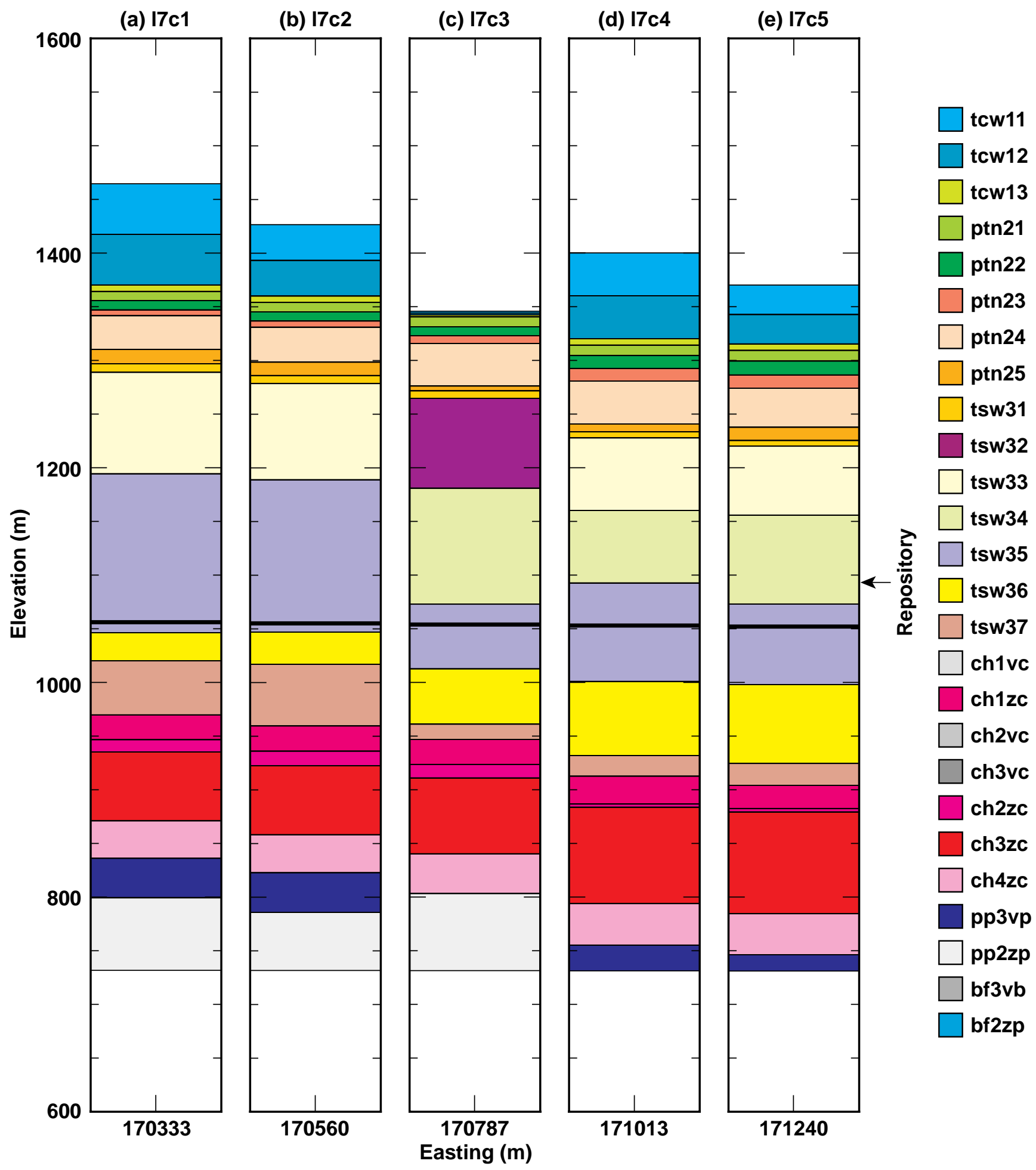

Figure 3-52. Vertical distribution of hydrostratigraphic model units shown for drift-scale model locations (in Nevada-State coordinates), including (a) $17 \mathrm{c} 1$ at Northing $=235114 \mathrm{~m},(\mathrm{~b}) 17 \mathrm{c} 2$ at Northing = $235102 \mathrm{~m}$, (c) 17c3 at Northing $=235090 \mathrm{~m},(\mathrm{~d}) 17 \mathrm{c} 4$ at Northing $=235078 \mathrm{~m}$, and (e) $17 \mathrm{c} 5$ at Northing $=235066 \mathrm{~m}$ 
(a) Point-load design, LML $=0.588 \mathrm{MTU} / \mathrm{m}$

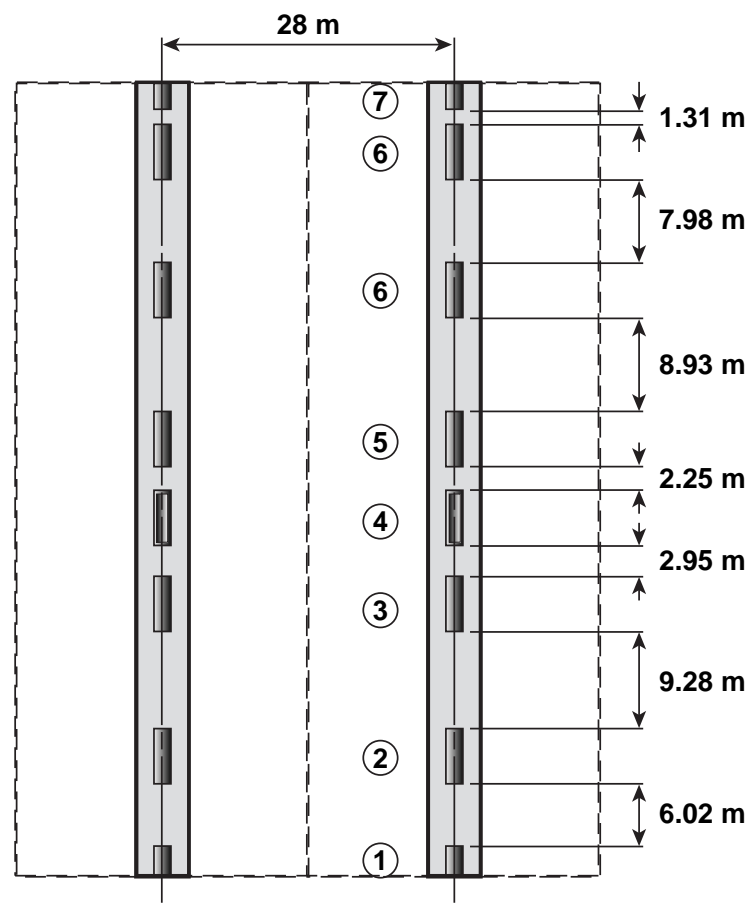

(b) Line-load design, LML $=1.189 \mathrm{MTU} / \mathrm{m}$

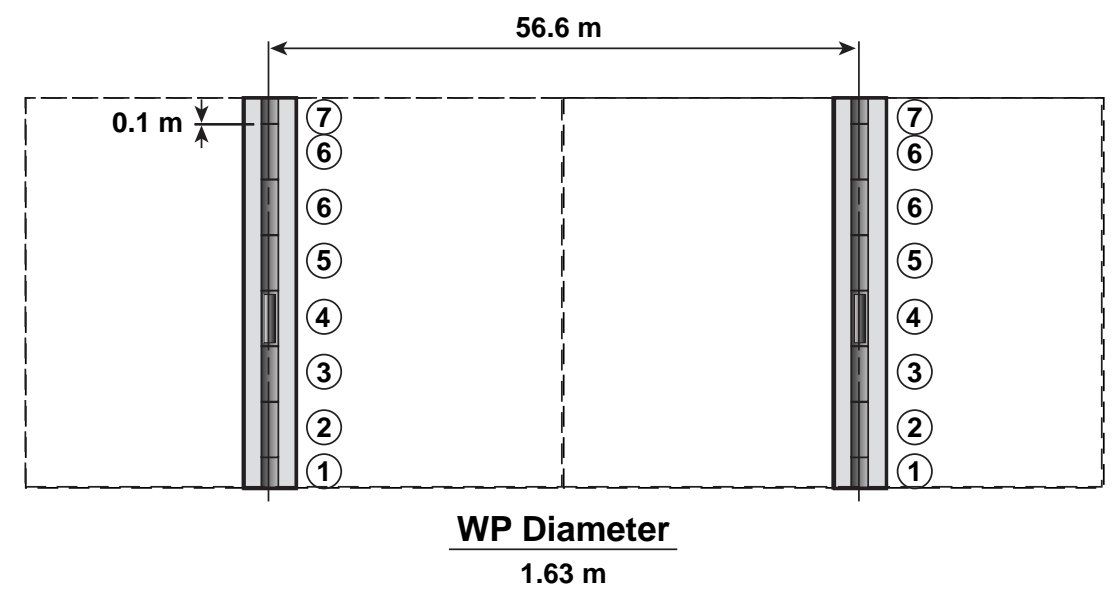

\begin{tabular}{|c|c|c|c|c|c|c|}
\hline \multicolumn{7}{|c|}{ Legend } \\
\hline (1) & (2) & (3) & (4) & (5) & (6) & (7) \\
\hline 12 PWR & 21 PWR & 21 PWR & 21 PWR & 2x44 PWR & Co-Disposal & Direct-Disposal \\
\hline (1/2) $6.25 \mathrm{MTU}$ & $7.4 \mathrm{MTU}$ & $9.4 \mathrm{MTU}$ & $8.93 \mathrm{MTU}$ & $7.81 \mathrm{MTU}$ & $4.06 \mathrm{~kW}$ & $(1 / 2) 0.793 \mathrm{~kW}$ \\
\hline (1/2) $10.48 \mathrm{~kW}$ & $2.91 \mathrm{~kW}$ & $17.85 \mathrm{~kW}$ & $9.34 \mathrm{~kW}$ & $6.44 \mathrm{~kW}$ & $5.37 \mathrm{~m}$ & (1/2) $5.37 \mathrm{~m}$ \\
\hline$(1 / 2) 5.87 \mathrm{~m}$ & $5.3 \mathrm{~m}$ & $5.3 \mathrm{~m}$ & $5.3 \mathrm{~m}$ & $5.3 \mathrm{~m}$ & & \\
\hline
\end{tabular}

Figure 3-53. Plan view of the WP layout represented in the DDT models for (a) the point-load design, which is the TSPA-VA base-case design, and (b) the line-load design, which is an alternative design for TSPA-VA 
3-D DDT model

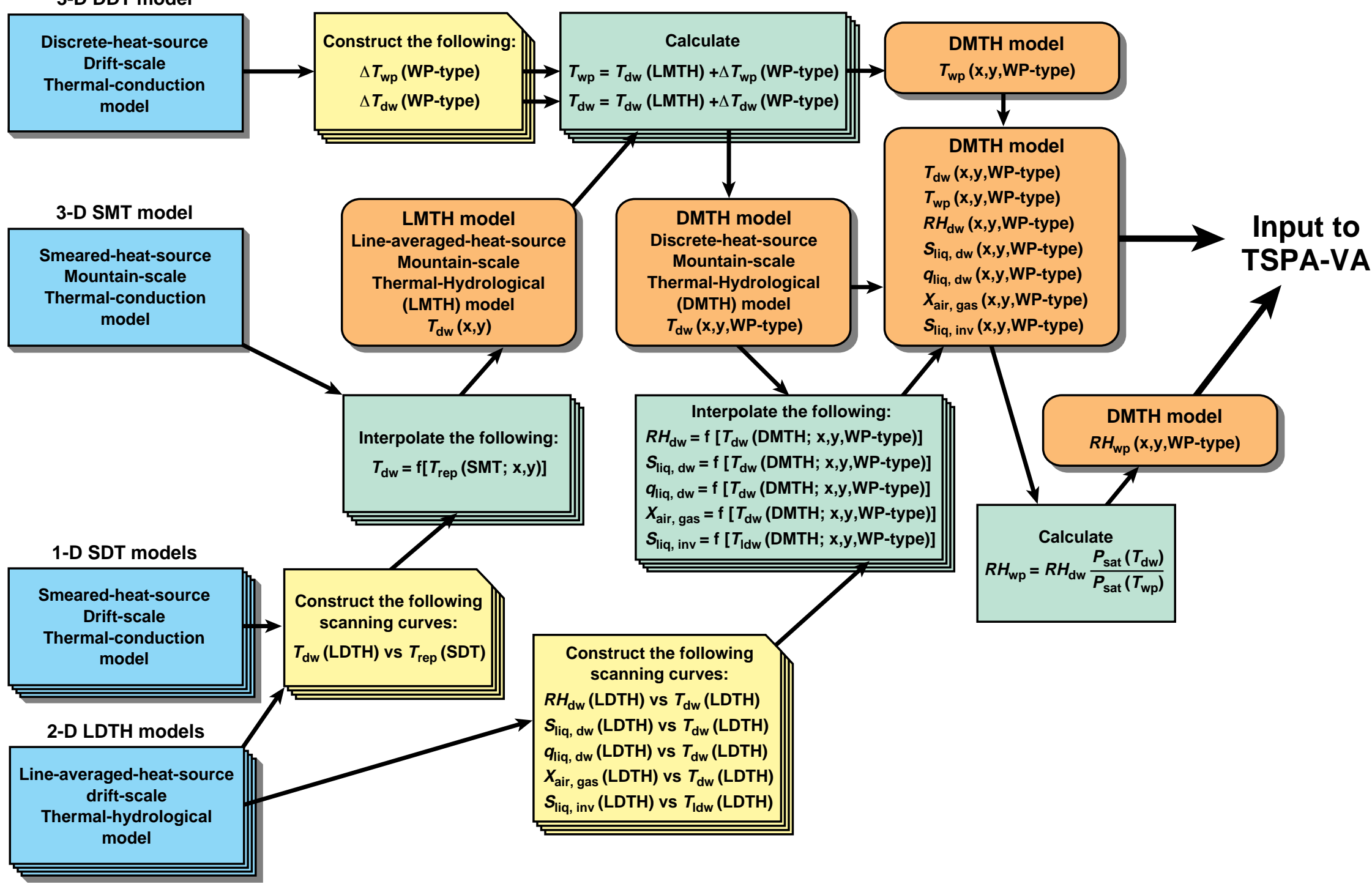

Figure 3-54. Flow chart for the multi-scale TH modeling approach with process models depicted in blue and abstracted models depicted in orange 

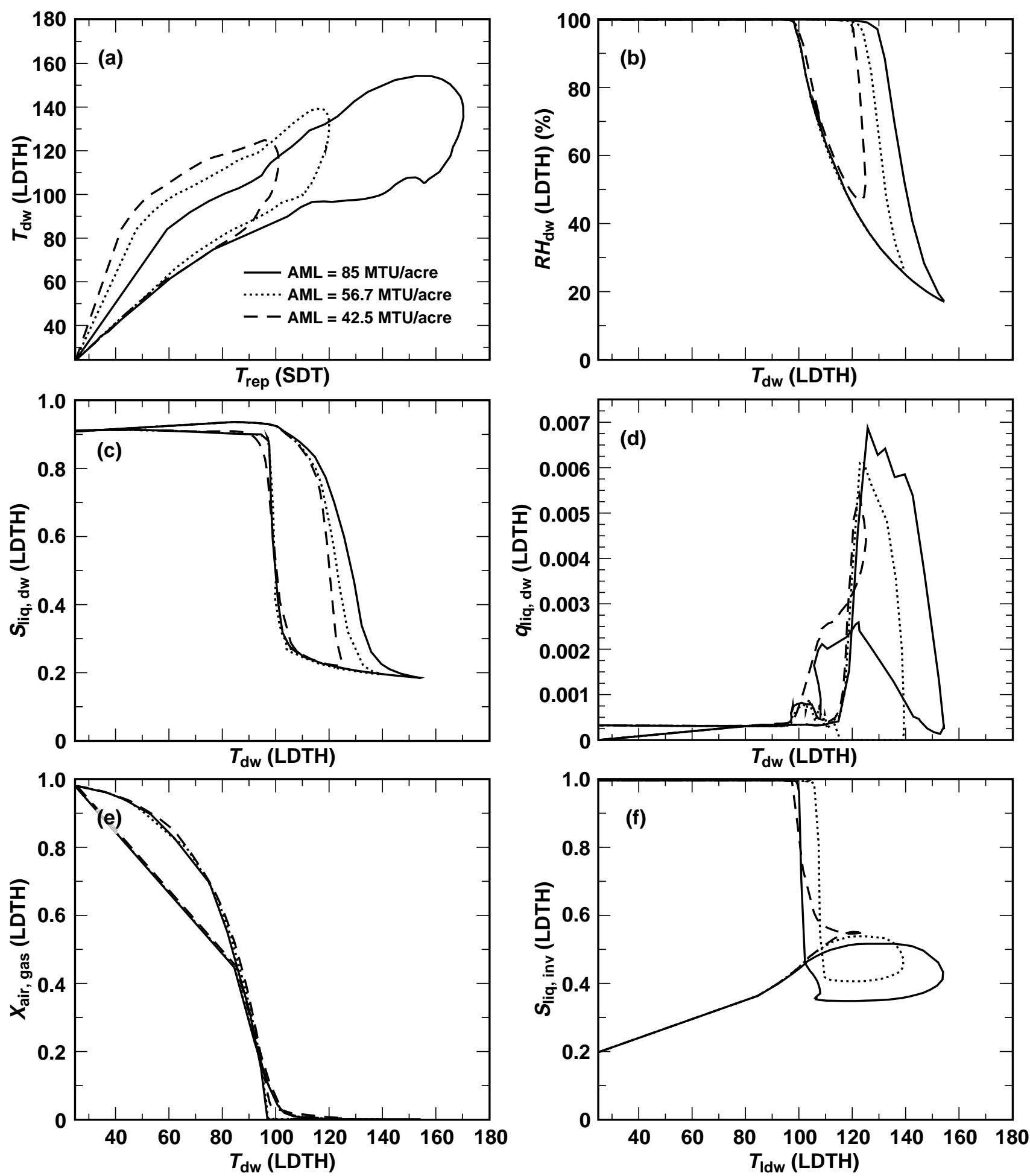

Figure 3-55. Functional relations plotted for the process-model inputs to the multi-scale TH modeling approach: (a) LDTH-model-predicted drift-wall temperature $T_{\mathrm{dw}}$ (LDTH) vs. SDT-model-predicted repository host-rock temperature $T_{\text {rep }}$ (SDT), (b) LDTH-model-predicted drift-wall relative humidity $R H_{\mathrm{dw}}(\mathrm{LDTH}) \mathrm{vs}$. $T_{\mathrm{dw}}$ (LDTH), (c) LDTH-model-predicted drift-wall liquid-phase saturation $S_{\text {liq,dw }}$ (LDTH) vs. $T_{\mathrm{dw}}(\mathrm{LDTH})$, (d) LDTH-model predicted liquid-phase flux $3 \mathrm{~m}$ above the drift $q_{\text {liq,dw }}(\mathrm{LDTH}) \mathrm{vs}$. $T_{\mathrm{dw}}$ (LDTH), (e) LDTHmodel-predicted gas-phase air-mass fraction in the drift $X_{\text {air,dr }}\left(\right.$ LDTH) vs. $T_{\mathrm{dw}}(\mathrm{LDTH})$, (f) LDTH-model-predicted liquid-phase saturation in the invert $S_{\text {liq,inv }}(\mathrm{LDTH})$ vs. $T_{\mathrm{dw}}(\mathrm{LDTH})$ 

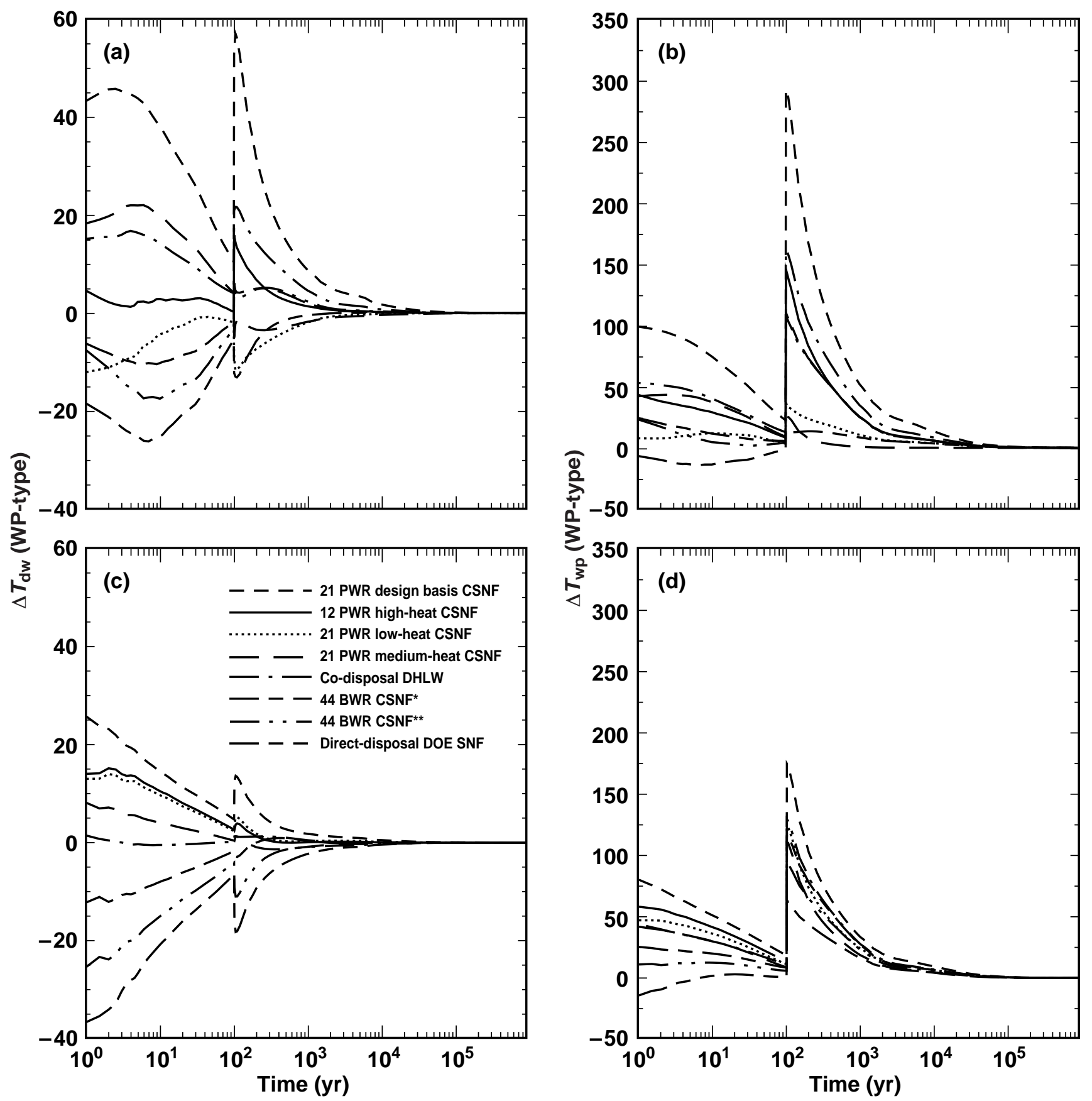

Figure 3-56. Functional relations plotted for the process-model inputs to the multi-scale TH modeling approach as functions of time: (a) WP-type/ location-specific temperature deviations $\Delta T_{\mathrm{dw}}$ (WP-type) from the average drift-wall temperature along the drift and (b) WP-type/location-specific temperature difference $\Delta T_{\text {wp }}$ (WP-type) between the WP surface and the average drift-wall temperature along the drift for the point-load design. The relations for $\Delta T_{\mathrm{dw}}$ (WP-type) (c) and $\Delta T_{\mathrm{wp}}$ (WP-type) (d) are also given for the line-load design. 


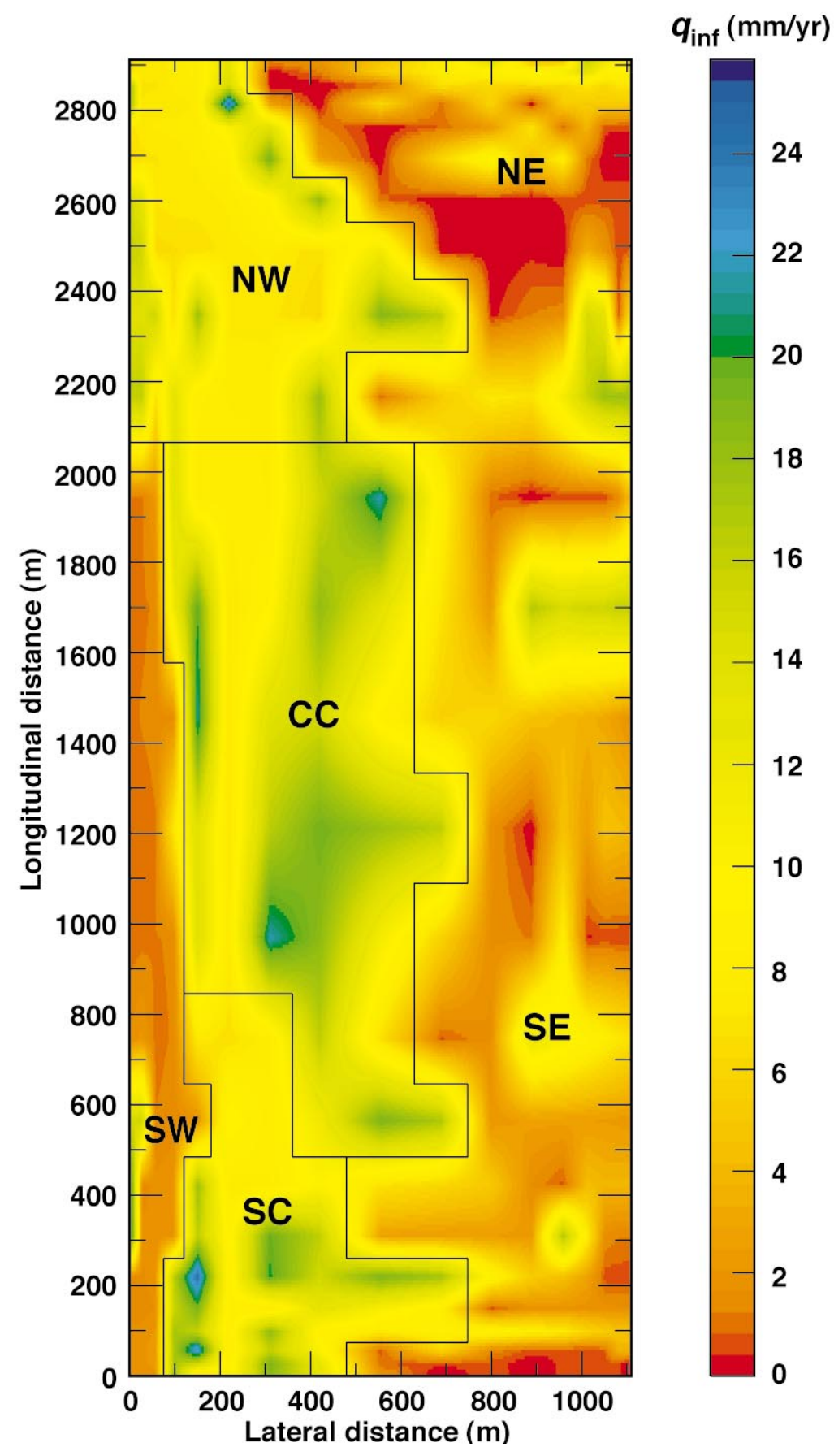

Figure 3-57. The six infiltration-flux subdomains and the infiltration-flux $q_{\text {inf }}$ distribution (Flint et al., 1996a) shown for the SMT-model representation of the repository area (infiltration-flux subdomains used to bin the WP environments for EBS subsystem analysis for TSPA-VA): increasing order of mean $q_{\text {inf }}$ the subdomains (1) southwest SW, (2) northeast NE, (3) southeast SE, (4) northwest NW, (5) south-central SC, and (6) centralcentral CC 
(a) SMT model

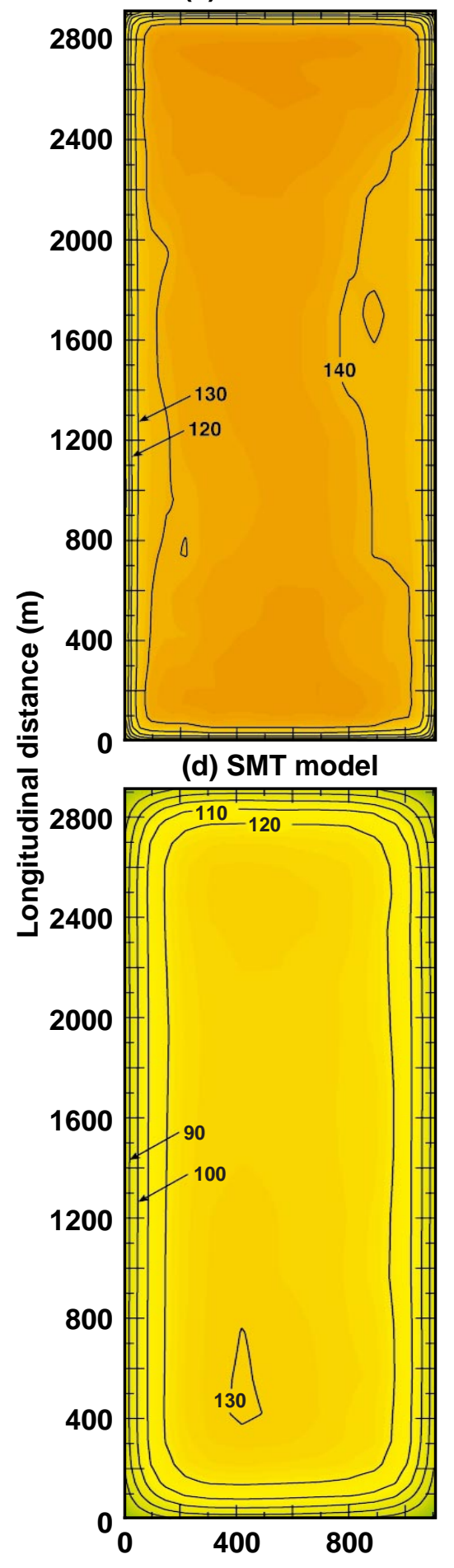

(b) Driftwall

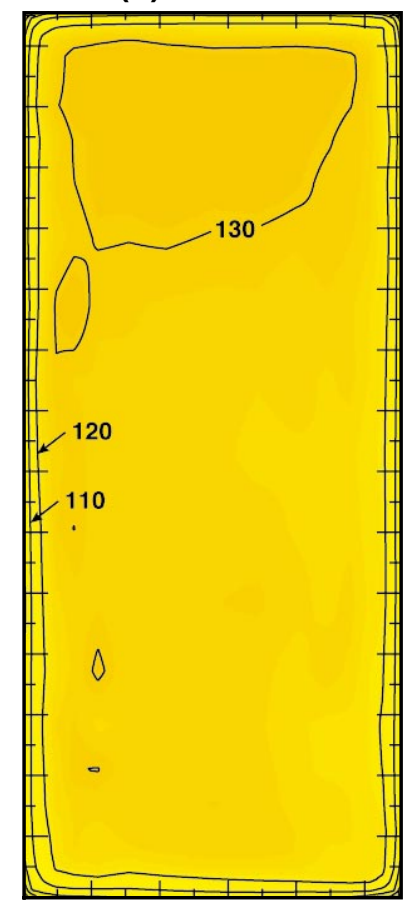

(e) Driftwall

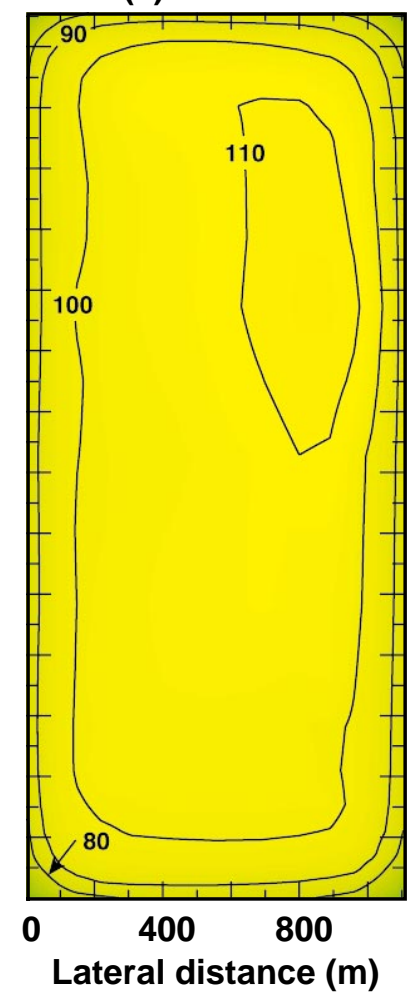

(c) WP surface

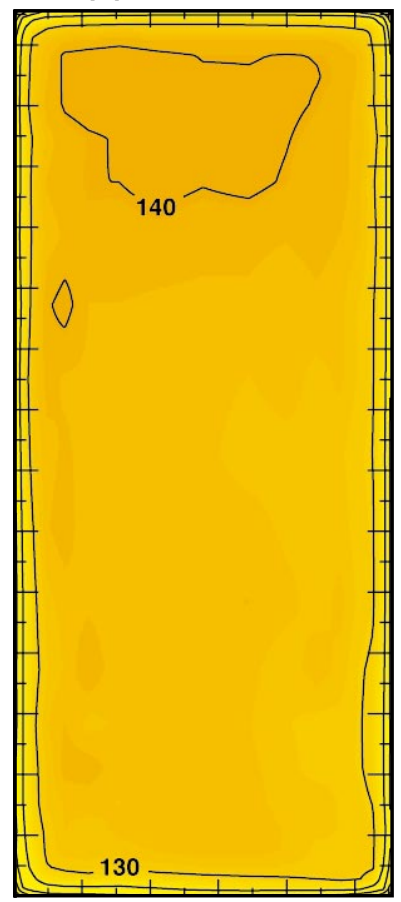

$T\left({ }^{\circ} \mathrm{C}\right)$

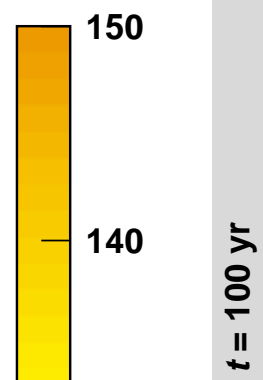

$-120$

100

80
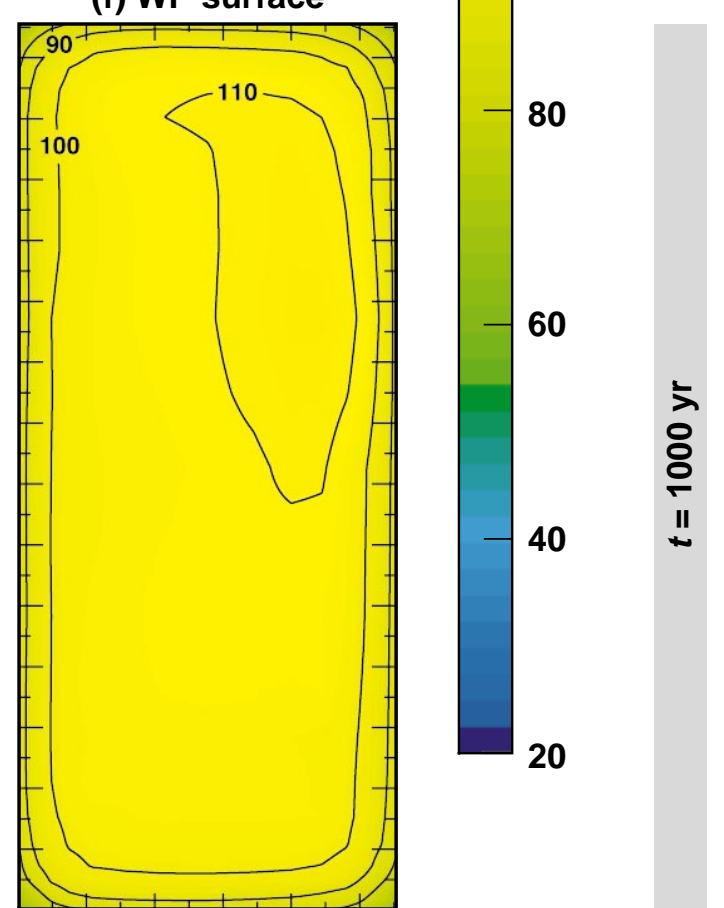

Figure 3-58. Temperature $T$ distribution in the repository host rock predicted by the SMT model shown at (a) $100 \mathrm{yr}$ and (d) $1000 \mathrm{yr}$; near-field temperatures predicted for "average" 21-PWR medium-heat CSNF WPs by the multi-scale TH modeling approach shown for the drift wall at (b) $100 \mathrm{yr}$ and (e) $1000 \mathrm{yr}$ and for the WP surface at (c) $100 \mathrm{yr}$ and (f) 1000 yr; distributions (b, c, e, and f) assume the 12/97 "modified-TH" hydrologicparameter set and the nominal "I $\times 1$ " infiltration-flux $q_{\text {inf }}$ map with an average $q_{\text {inf }}$ of $7.8 \mathrm{~mm} / \mathrm{yr}$ 


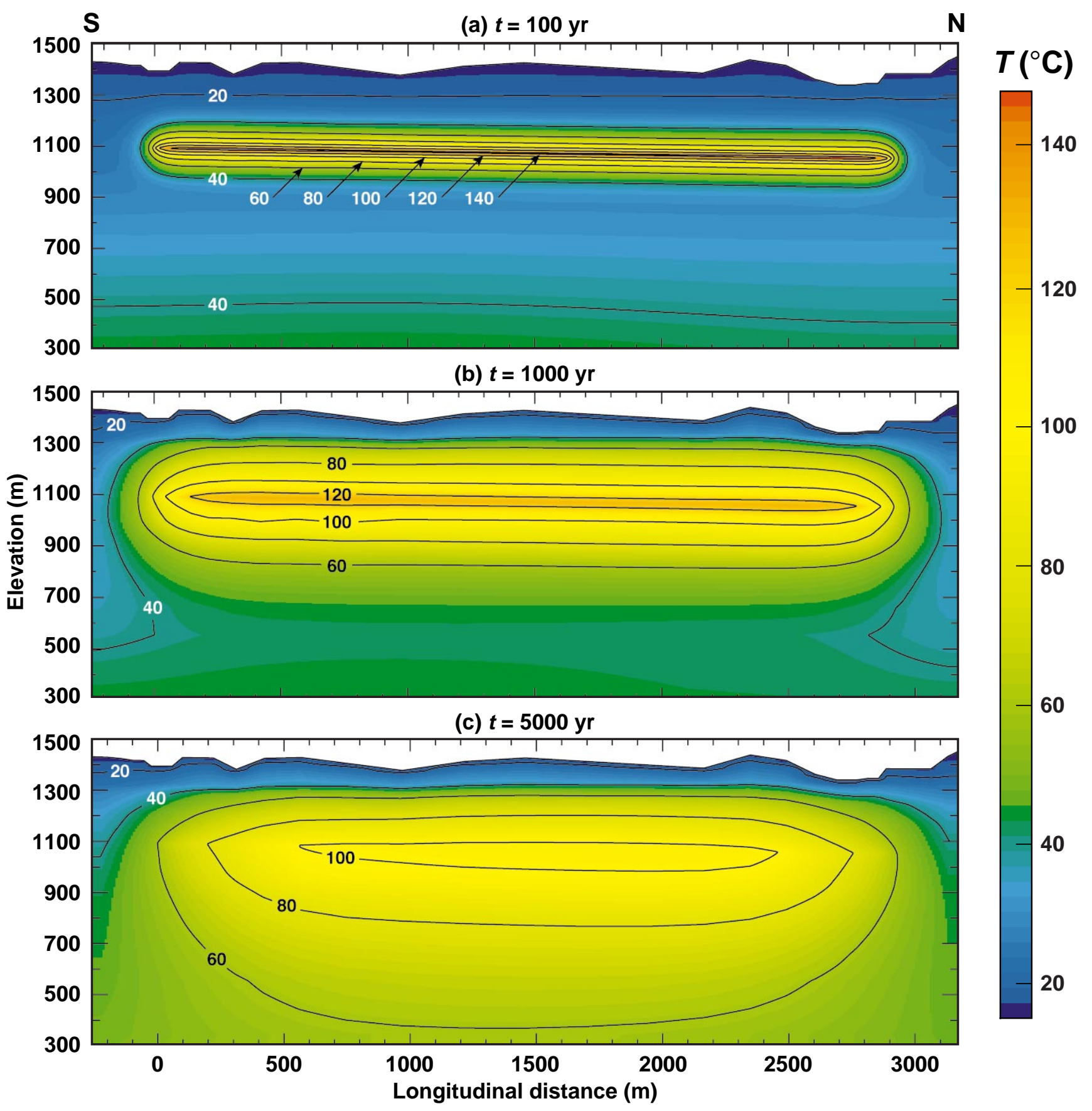

Figure 3-59. Mountain-scale temperature $T$ distribution in a vertical $(y-z)$ plane along the north-south centerline of the repository area predicted by the SMT model at (a) $100 \mathrm{yr}$, (b) $1000 \mathrm{yr}$, and (c) $5000 \mathrm{yr}$; zero longitudinal distance corresponding to southern edge of the repository area 


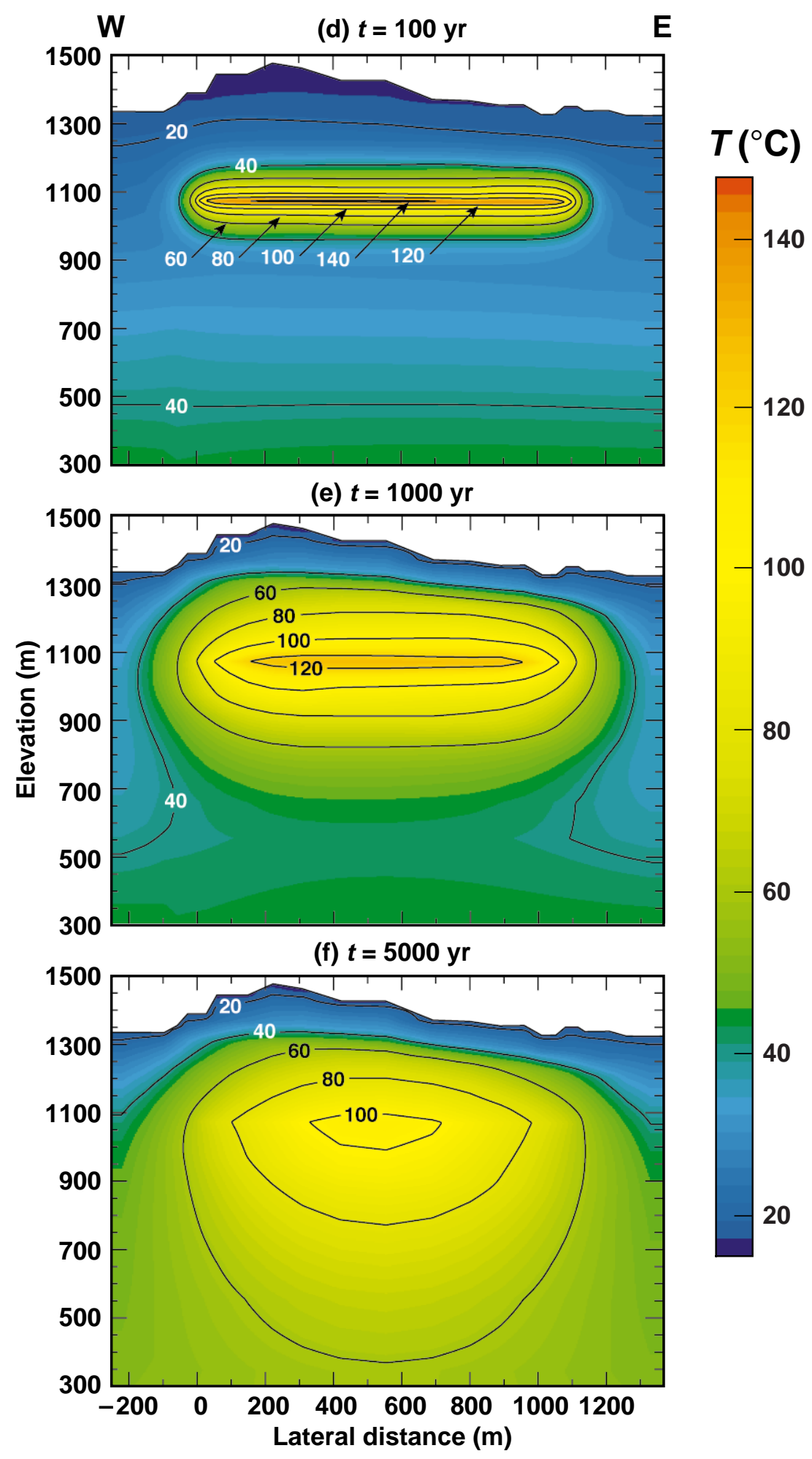

Figure 3-59. Mountain-scale temperature $T$ distribution in a vertical $(x-z)$ plane along the east-west centerline of the repository area predicted by the SMT model at (a) $100 \mathrm{yr}$, (b) $1000 \mathrm{yr}$, and (c) $5000 \mathrm{yr}$; zero lateral distance corresponding to western edge of the repository area 
(a) $7 / 97$ set

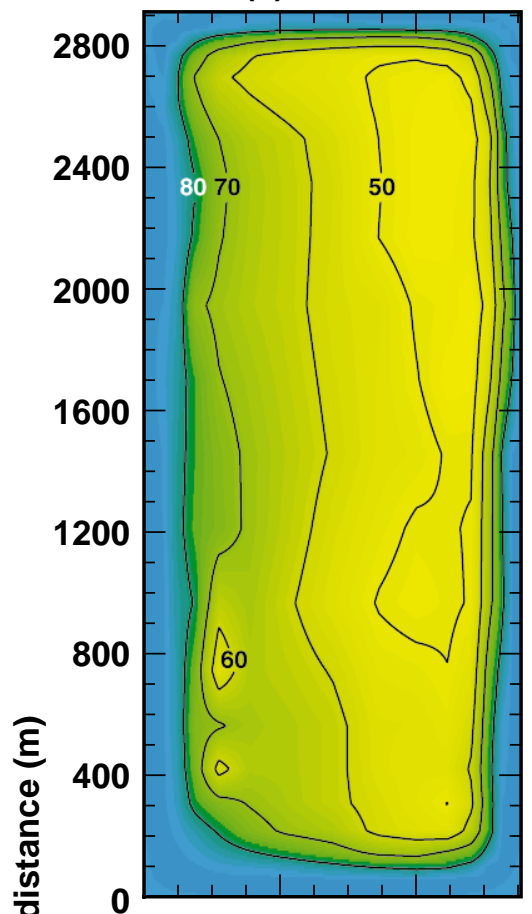

(d) $7 / 97$ set

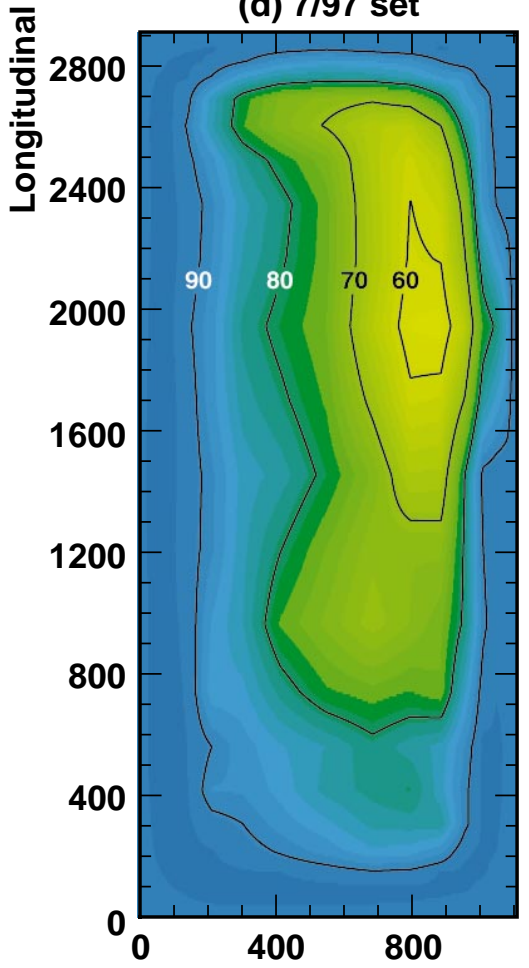

(b) $11 / 97$ set

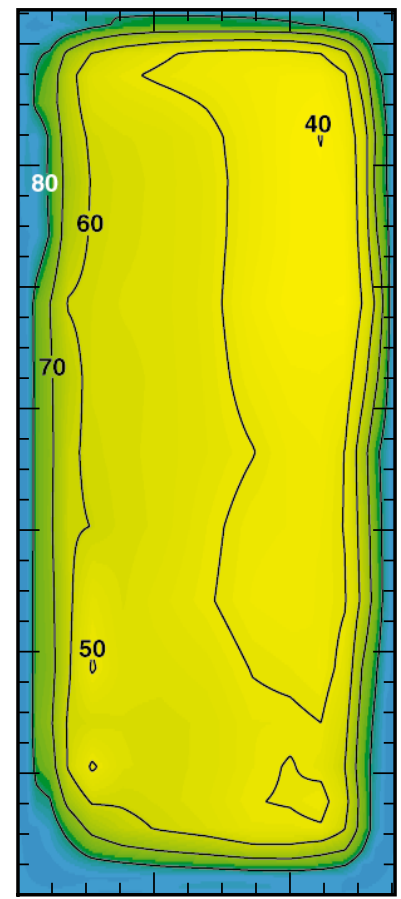

(e) $11 / 97$ set

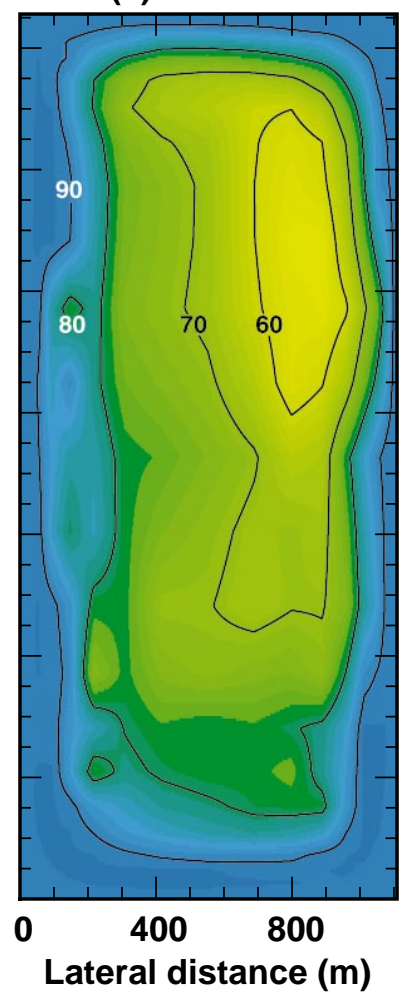

(c) $12 / 97$ set

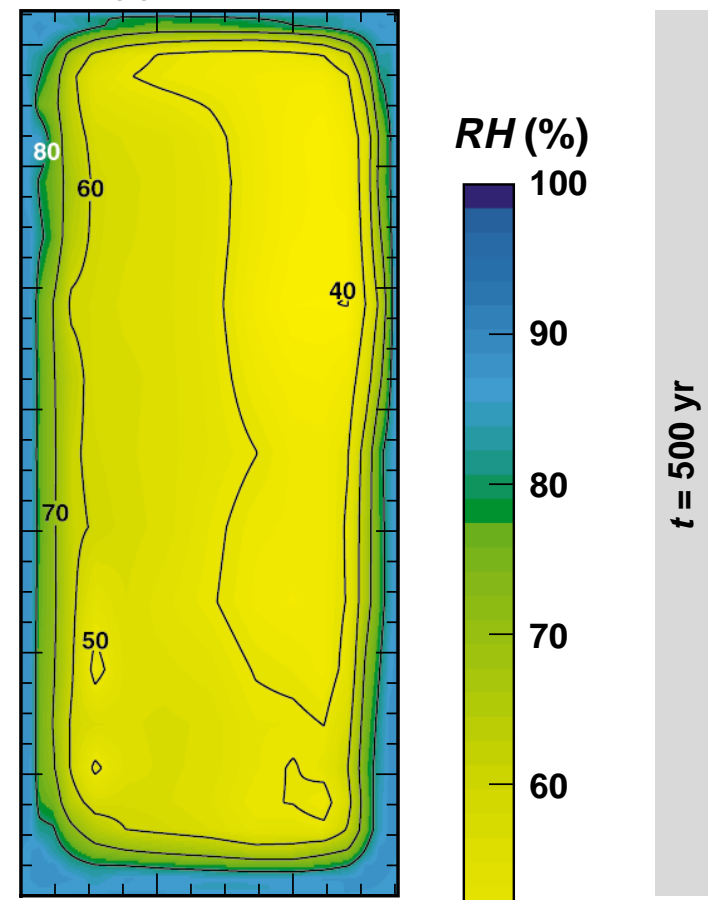

$-50$

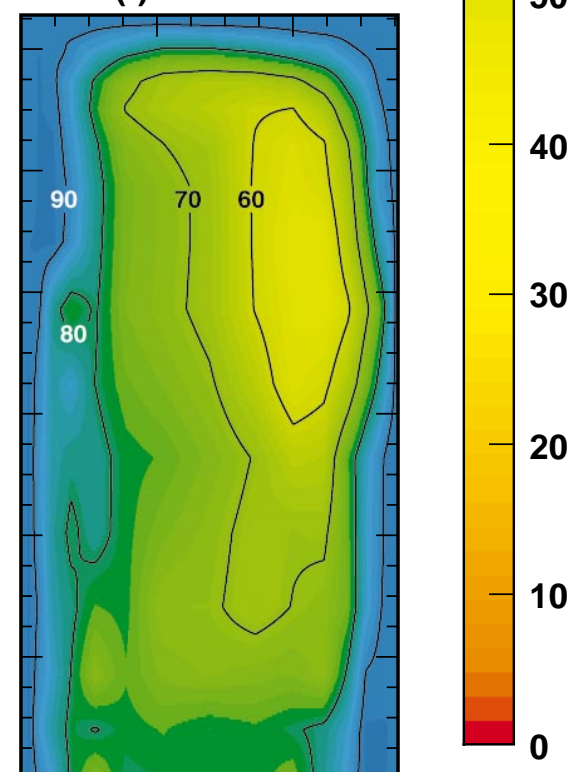

응

Figure 3-60. Relative humidity $R H$ on the surface of "average" 21-PWR medium-heat CSNF WPs plotted for the 7/97 TSPA-VA "I × 1" hydrologic-parameter set at (a) $500 \mathrm{yr}$ and (d) $1000 \mathrm{yr}$, for the 11/97 "TH" parameter set at (b) $500 \mathrm{yr}$ and (e) $1000 \mathrm{yr}$, and for the 12/97 "modified-TH" parameter set at (c) $500 \mathrm{yr}$ and (f) $1000 \mathrm{yr}$; all distributions assume the nominal infiltration-flux $q_{\text {inf }}$ map (average $q_{\text {inf }}=7.8 \mathrm{~mm} / \mathrm{yr}$ ) 
(a) high $q_{\text {inf }}$

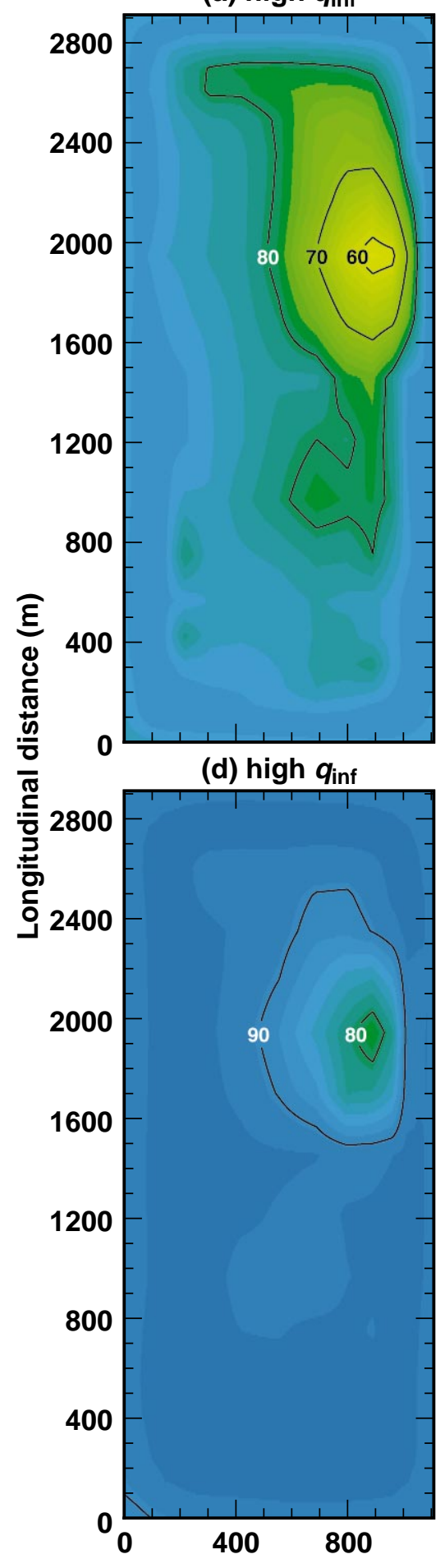

(b) medium $q_{\text {inf }}$

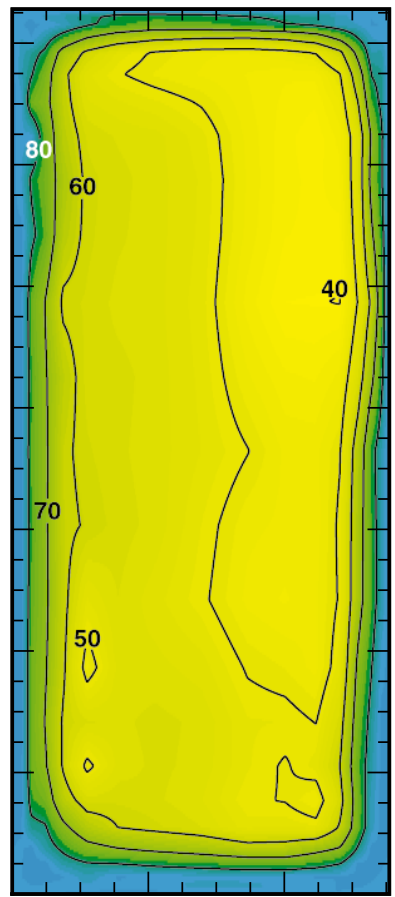

(e) medium $q_{\text {inf }}$

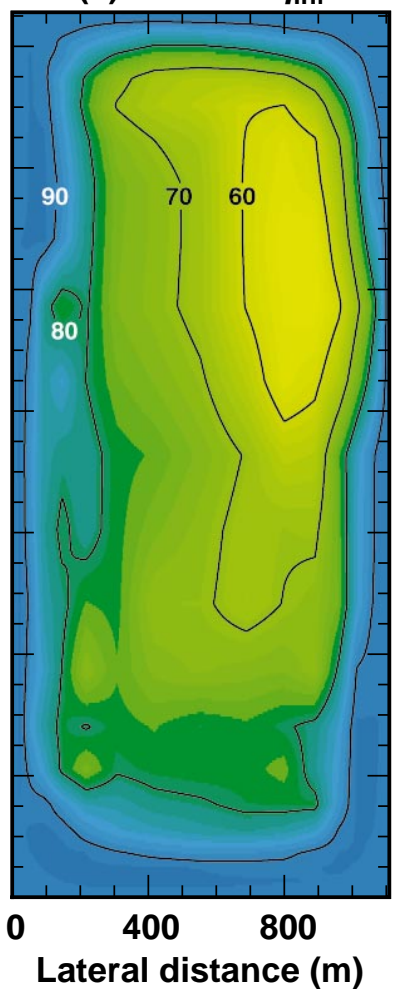

(c) low $q_{\text {inf }}$

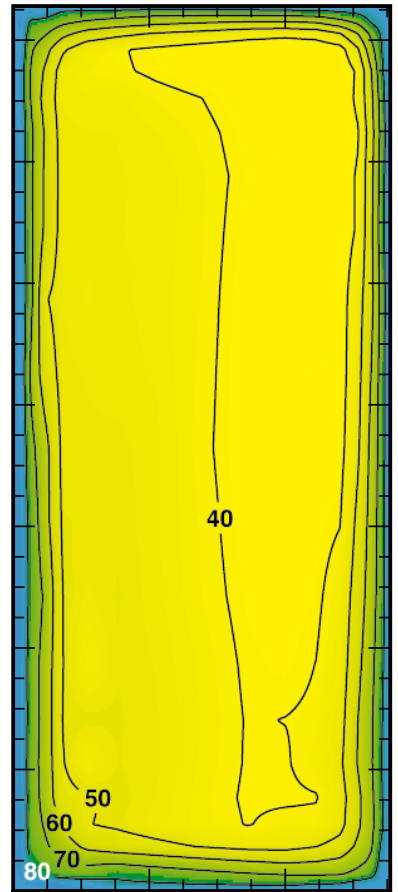

(f) low $q_{\text {inf }}$

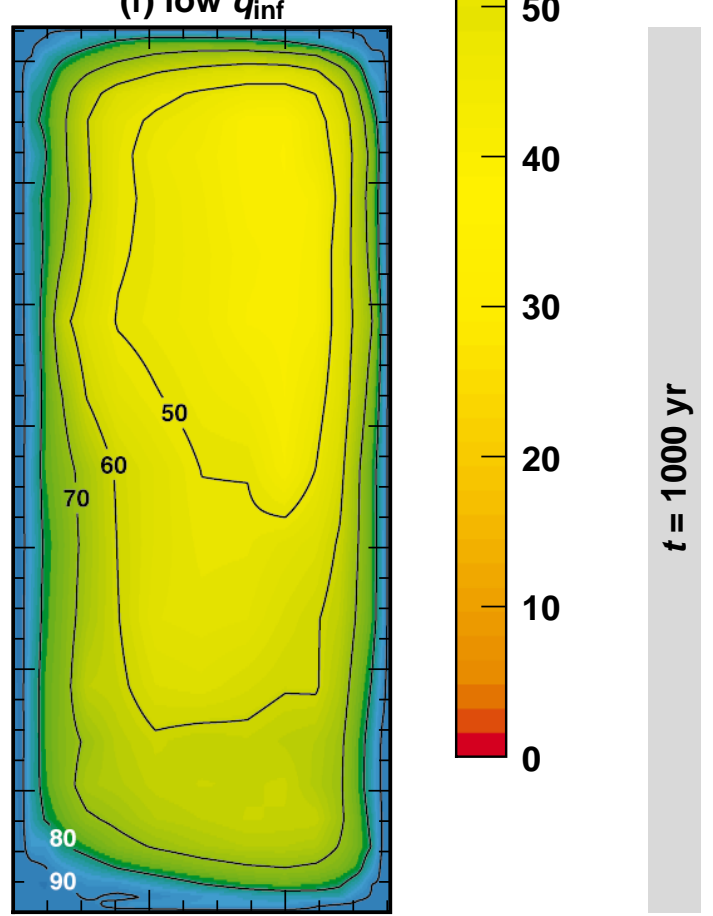

Figure 3-61. Relative humidity $R H$ on the surface of "average" 21-PWR medium-heat CSNF WPs plotted for three infiltration-flux $q_{\text {inf }}$ cases, including the high- $q_{\text {inf }} \mathrm{I} \times 5$ case (average $q_{\text {inf }}=39 \mathrm{~mm} / \mathrm{yr}$ ) at (a) $500 \mathrm{yr}$ and (d) $1000 \mathrm{yr}$, the medium- $q_{\text {inf }} \mathrm{I} \times 1$ case (average $q_{\text {inf }}=7.8 \mathrm{~mm} / \mathrm{yr}$ ) at (b) $500 \mathrm{yr}$ and (e) $1000 \mathrm{yr}$, and the low- $q_{\text {inf }}$ I $/ 5$ case (average $q_{\text {inf }}=1.56 \mathrm{~mm} / \mathrm{yr}$ ) at (c) $500 \mathrm{yr}$ and (f) $1000 \mathrm{yr}$; all distributions assume the 12/97 "modified-TH" parameter set 

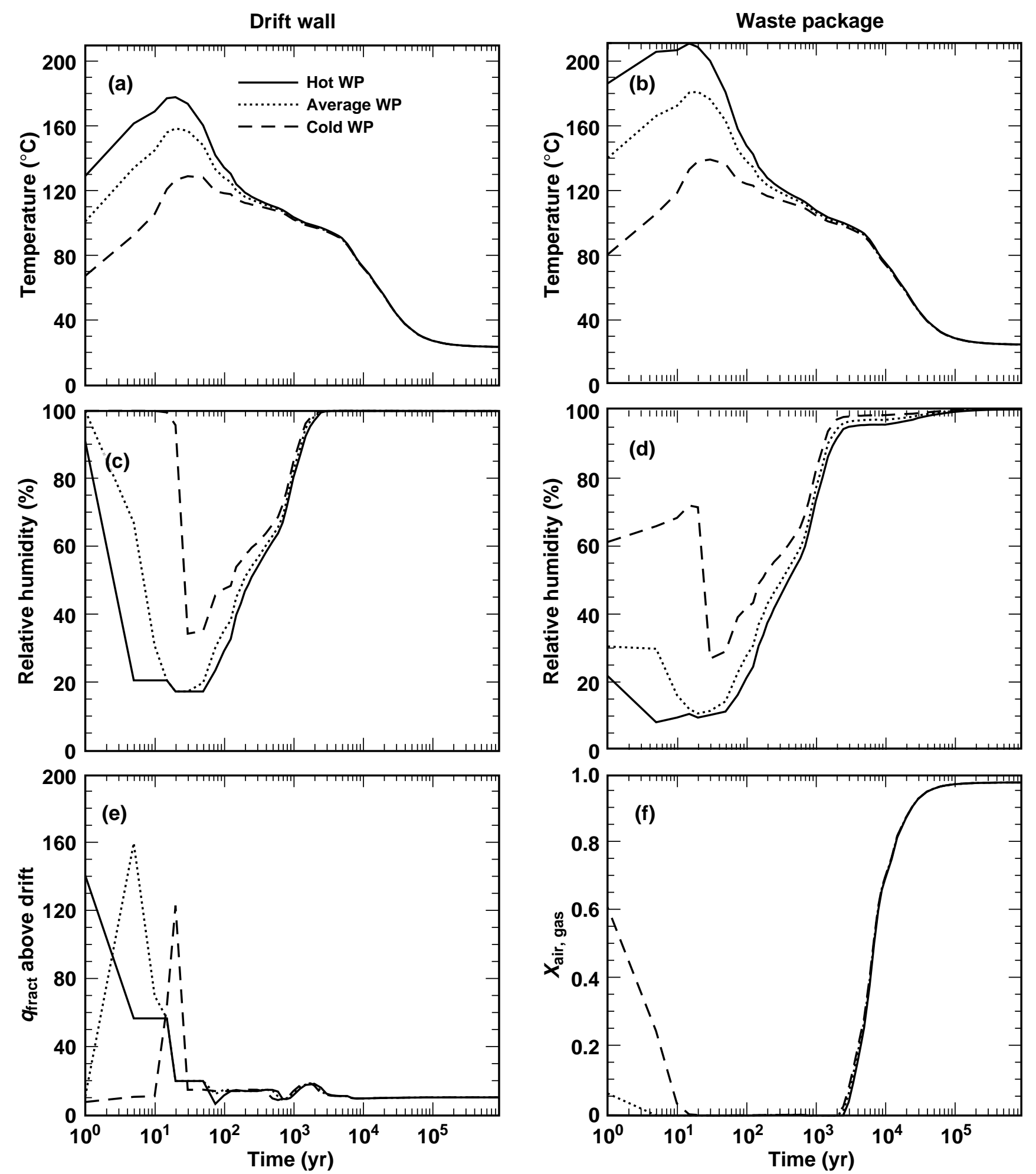

Figure 3-62. Summary of TH conditions for three WP types, including a "hot" 21-PWR design-basis CSNF WP, an "average" 21-PWR medium-heat CSNF WP, and a "cold" direct-disposal DHLW WP; conditions including temperature on the (a) drift wall and on the (b) WP, RH on the (c) drift wall and on the (d) WP, (e) liquid-phase flux $3 \mathrm{~m}$ above the drift, and (f) gas-phase air-mass fraction in the drift; all curves assume the 15c2 location, the 12/97 "modified-TH" parameter set, and local percolation flux of $8.53 \mathrm{~mm} / \mathrm{yr}$ 


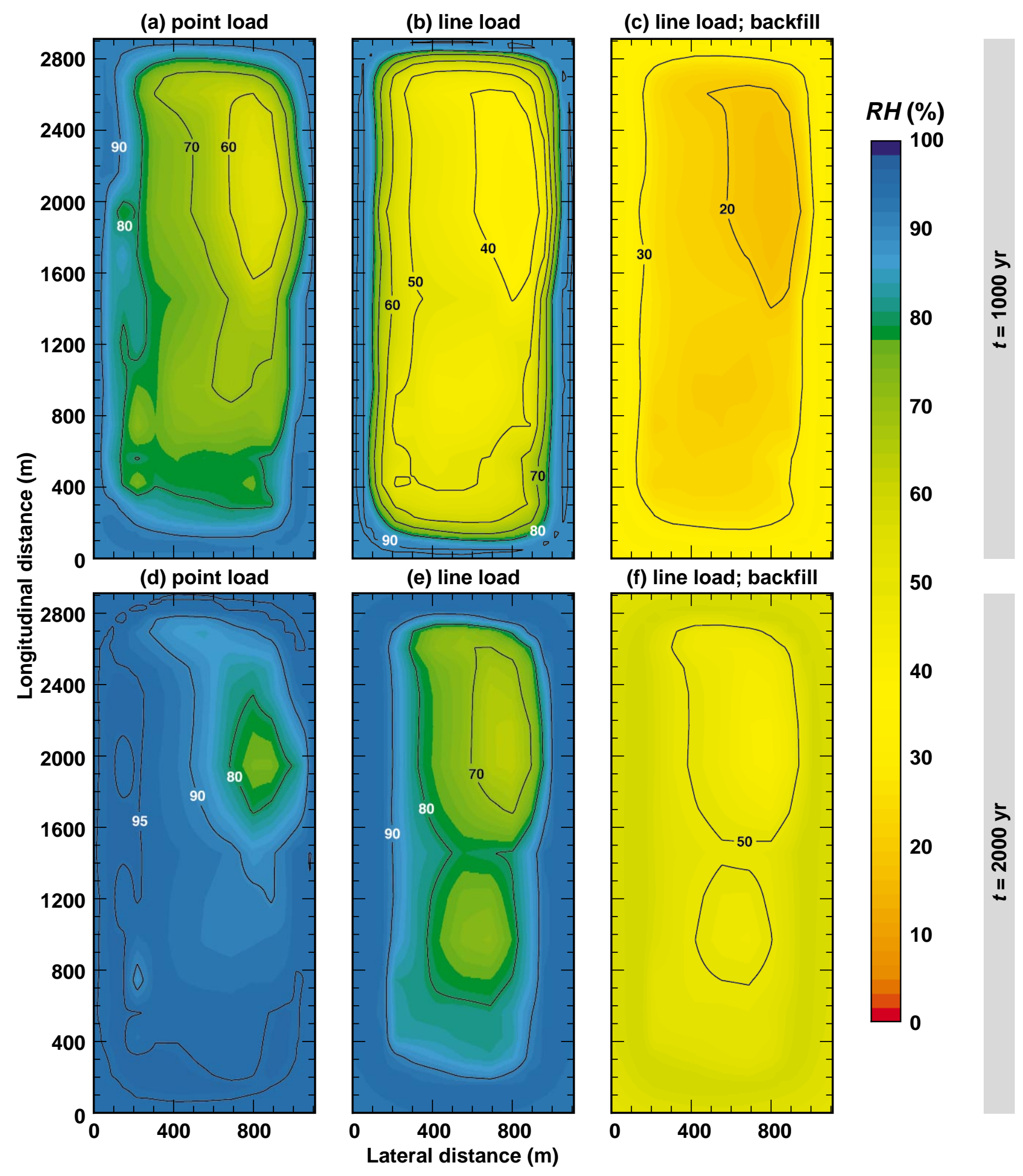

Figure 3-63. Relative humidity $R H$ on the surface of "average" 21-PWR medium-heat CSNF WPs plotted for the point-load design at (a) $1000 \mathrm{yr}$ and (d) $2000 \mathrm{yr}$, the line-load design at (b) $1000 \mathrm{yr}$ and (e) $2000 \mathrm{yr}$, and the backfilled line-load design at (c) $1000 \mathrm{yr}$ and (f) $2000 \mathrm{yr}$; all distributions assume the 12/97 "modified-TH" parameter set and the nominal infiltration-flux $q_{\text {inf }}$ map (average $q_{\text {inf }}=7.8 \mathrm{~mm} / \mathrm{yr}$ ) 

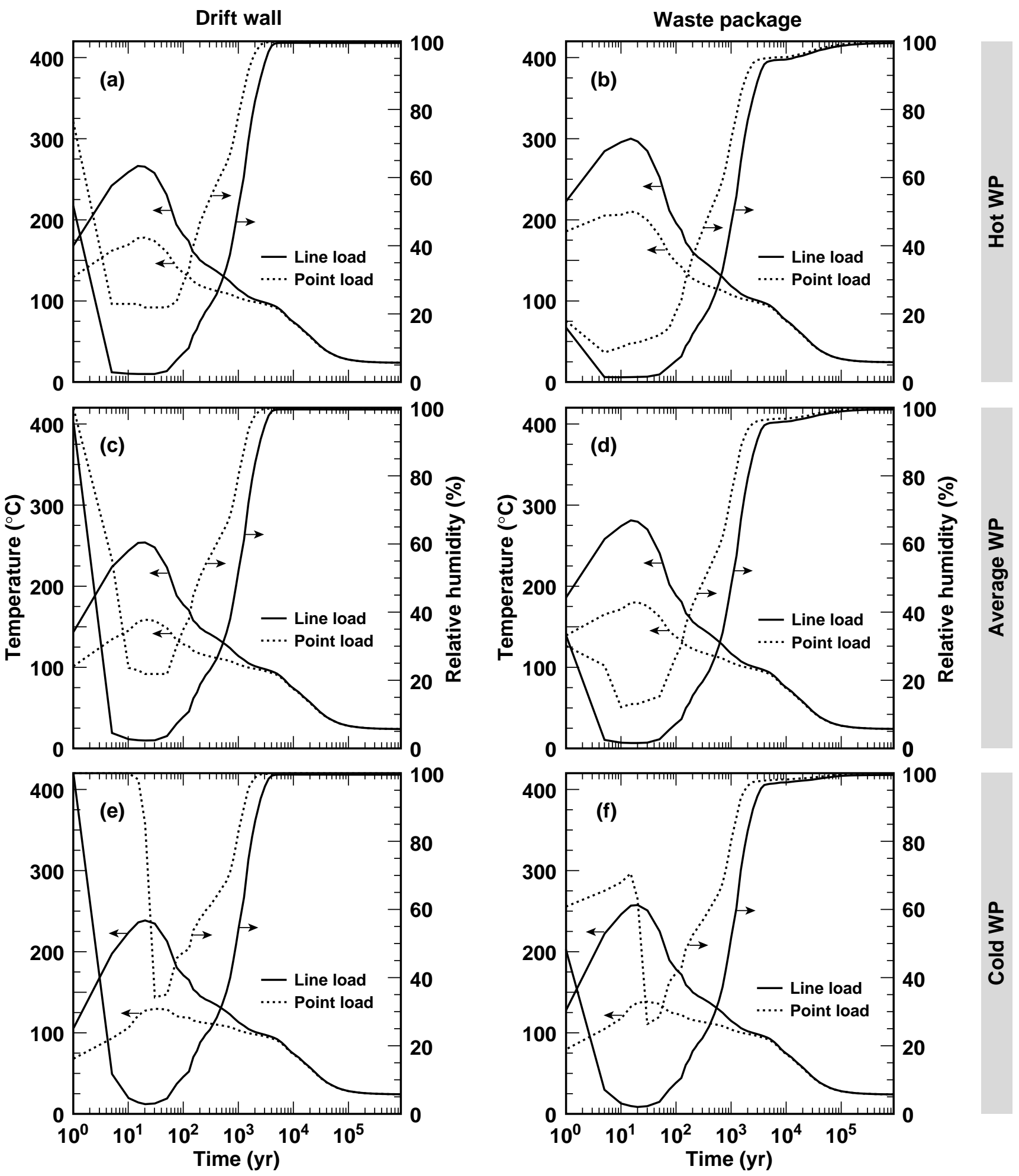

Figure 3-64. Temperature and relative humidity on the drift wall $(a, c, e)$ and on the WP $(b, d, f)$ for the pointload and line-load designs with no backfill; curves given for three WP types: a "hot" 21-PWR design-basis CSNF WP, an "average" 21-PWR medium-heat CSNF WP, and a "cold" direct-disposal DHLW WP; all curves

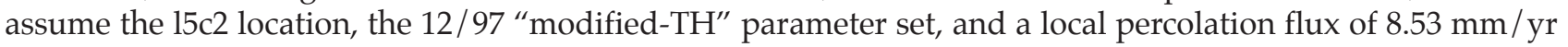



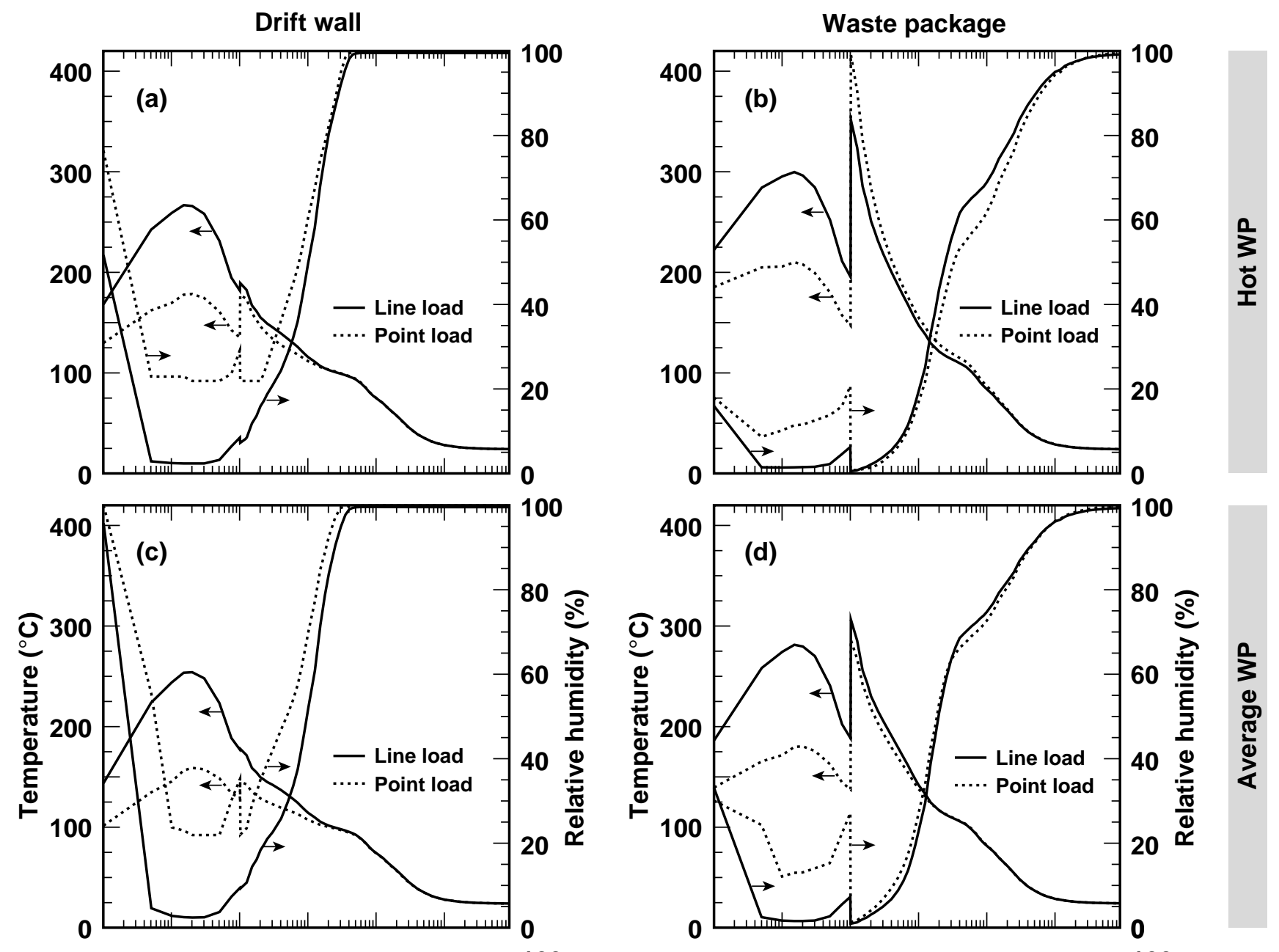

100
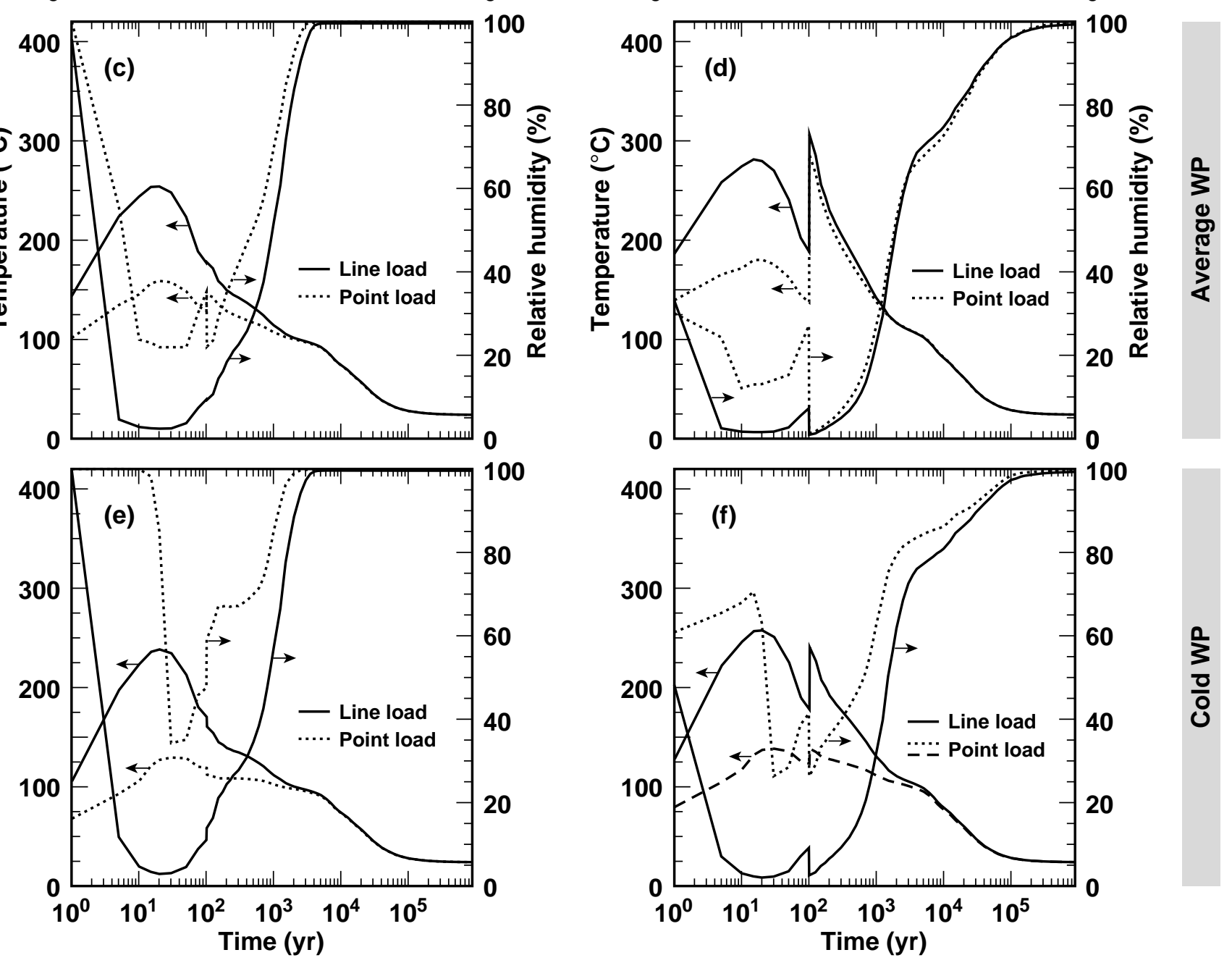

Figure 3-65. Temperature and relative humidity on the drift wall $(\mathrm{a}, \mathrm{c}, \mathrm{e})$ and on the WP $(\mathrm{b}, \mathrm{d}, \mathrm{f})$ for the pointload and design-load designs with backfill at $100 \mathrm{yr}$; curves given for three WP types: a "hot" 21-PWR designbasis CSNF WP, an "average" 21-PWR medium-heat CSNF WP, and a "cold" direct-disposal DHLW WP; all curves assume the 15c2 location, the 12/97 "modified-TH" parameter set, and a local percolation flux of $8.53 \mathrm{~mm} / \mathrm{yr}$ 



\title{
4. Thermomechanical and Thermohydromechanical Models
}

\author{
by Stephen C. Blair
}

\subsection{Introduction to Thermomechanical and Thermohydromechanical Models}

The hydrologic behavior of rock surrounding emplacement drifts in the proposed repository will depend on the mechanical response of the fractured rock mass to excavation and heating. This chapter describes the thermomechanical (TM) behavior of rock in the nearfield environment (NFE) and the coupling of this behavior with hydrologic processes.

The stress field in the rock mass surrounding emplacement drifts at Yucca Mountain will be altered by excavation of drifts and by heating associated with radioactive decay. The directions and magnitudes of the principal stresses will change significantly due to thermal loading and then will revert during cool-down. Elevated horizontal compressive stress will build up rapidly in the host rock, approaching maximum values within approximately 100 yr after waste emplacement. Such stress presents ground-support performance issues that are the subject of engineering analyses not discussed in this report.

Rock-mass bulk permeability is an important thermohydrologic $(\mathrm{TH})$ property that is controlled by fractures in the host rock, and the fractures will deform as stress conditions evolve. Two types of fracture deformation will contribute to thermohydromechanical (THM) coupling:

- Normal displacement (perpendicular to the fracture plane)

- Shear displacement (parallel)

Associated with these two types of deformation are important differences in the magnitude and the durability of fracture aperture changes:

Normal displacement is associated with changes in normal stress. Fractures stiffen readily as they close in response to normal compression, which limits the relative magnitude of the permeability change that can be produced. If the average normal stress is maintained well below the intact rock strength, and if creep is not significant, then fractures generally return to their previous aperture when unloaded (i.e., normal displacement is reversible). Fractures open in response to decreasing normal stress and eventually open at zero load. If fracture surfaces remain well aligned, and if shear displacement does not occur, fracture opening is also reversible.

Shear displacement is produced when the stress conditions in the fracture plane exceed the slip criteria for the fracture. The tendency for slip in response to shear stress is controlled by the frictional properties and geometry of the fracture surfaces, the hydraulic conditions within the fracture, the deformation history of the fracture, and the normal stress on the fracture. Frictional and geometrical properties are represented for all fractures in the host rock at Yucca Mountain by average property values. Fractures in the host rock may be wet, but there will be no significant positive pressure head in the unsaturated zone (UZ). The deformation history of individual fractures is unknown; as an approximation, deformability is assumed to be independent of past deformation. The normal stress is a major determinant of shear behavior and is considered extensively in this chapter.

Whereas this chapter discusses changes in fracture permeability, which may be equated to changes in bulk permeability of the repository host rock, the importance of these changes to repository performance is discussed in Chapter 8. Following are a few of the key questions that arise when considering performance issues: 
- What is the magnitude of permeability decrease associated with closure of fractures that are radially oriented and proximal to the emplacement drifts, and is this change reversible during cool-down?

- What is the magnitude of the decrease in vertical permeability of the host rock caused by closure of subvertical fractures in response to horizontal thermal stress, and is this change reversible?

- What are the magnitude and durability of local changes in horizontal permeability, associated with temperature gradients that occur during expansion and collapse of the thermal field, caused by opening of subhorizontal fractures in response to shear stress?

- What are the magnitude and durability of local changes in permeability caused by shear deformation associated with irregularity in the predicted temperature field and with stress concentration around drift openings?

This chapter reviews the conceptual and numerical models available for TM analysis of fractured rock and presents TM analyses of the Drift-Scale Test (DST) and the reference cases used for investigation of coupled processes in this report. The likely durable changes in bulk permeability associated with the calculated stresses are inferred using the calculated TM stresses. This chapter is structured as follows:

- Section 4.2 presents background information on the nature of TM behavior expected in the near field/altered zone and a discussion of the general types of numerical models and techniques available for TM calculations.

- Section 4.3 presents rock-mass constitutive models and supporting data, including recent results of fracture deformation and fracture flow experiments using $0.5-\mathrm{m}$ blocks of tuff.

- Section 4.4 describes simulation of the drift-scale TM response for the reference cases used to investigate coupled processes in this report. In this presentation, stress and displacement are calculated using two different modeling approaches. The temperature fields for these calculations were taken directly from $\mathrm{TH}$ simulations, or matched to TH simulations; thus, these calculations are weakly coupled. Predictive TM calculations for the DST and the Large-Block Test (LBT) are also discussed.

- Section 4.5 discusses conceptual models for changes in fracture permeability resulting from changes in stress conditions. A conceptual model is developed for THM analysis in this report.

- In Section 4.6, the conceptual model for THM coupling is applied to prediction of fracture permeability changes for the DST.

- In Section 4.7, the conceptual model is applied to the reference cases for investigation of drift-scale coupled processes in the repository.

- Section 4.8 is a summary of results.

\subsubsection{Identification of Users for Thermomechanical and Thermohydromechanical Models}

This chapter describes two types of mechanical models:

1. Drift-scale TM models

2. Models of THM coupling

These models are listed in Appendix A (Table A-9) along with the other models that make up this report.

Drift-scale TM models are used to predict the stress conditions that will exist in the repository host rock and ground support during the thermal period (Section 4.4). TM loading is also important for preclosure performance of repository subsystems and for analysis of 
ground-support longevity and rock fall after permanent closure. These models are used directly or indirectly by performance assessment (PA) and engineered barrier system (EBS) / Repository Design.

The conceptual model for THM coupling is based on laboratory and field experiments and on modeling of TM stresses in the host rock. The effects of fracture deformation on bulk permeability are of particular interest to PA because they influence seepage conditions during the post-thermal period.

\subsection{Conceptual Basis for Rock-Mass Thermomechanical Models}

\subsubsection{Geomechanical Aspects of the Near-Field Environment}

The proposed repository host rock is a fractured, densely welded, ash-flow tuff. The rock has low matrix permeability, and the bulk permeability is mainly associated with fractures. Any changes in rock mass permeability from TM effects can be attributed to the fractures because changes to matrix permeability in response to stress and temperature are small (Hardin and Chesnut, 1997).

Laboratory studies (Bandis et al., 1983; Barton et al., 1985) have shown that greater impact often results from shear deformation of fractures rather than from normal deformation. Fractures may be more deformable in shear under certain stress conditions, in which case the magnitude of changes in fracture aperture is greater than it is for perpendicular (normal) deformation. With greater deformation, there is increased likelihood of permanent displacement, which could produce durable changes in fracture permeability. This may be especially true for stress-unloading conditions during repository cool-down. Permanent changes in hydrologic properties or flow paths can result from normal deformation, but they tend to be smaller than those caused by shear displacements. This has been observed in field studies (e.g., Wilder, 1987). A “qualitative evaluation of the coupling complexity between mechanical behavior and void space evolution between the joint walls during shear displacement." has been suggested by Archambault et al. (1997) but has not yet been applied to prediction of NFE conditions.

\subsubsection{Available Model Approaches}

Several numerical codes have been developed for simulating rock-mass behavior. These codes employ finite-element, distinct-element, and boundary-element numerical techniques and can incorporate a wide variety of constitutive models. Some have been generalized to three dimensions. A wide range of codes is discussed in the following sections, and calculations, using a subset of these codes (FLAC and FRACROCK), are presented in this chapter. The quality assurance (QA) status for codes used for calculations is summarized in Appendix A.

To predict rock-mass response over long time periods and for changing temperature and hydrologic conditions, simulators should accommodate appropriate constitutive equations for elasto-plastic behavior, nonlinear properties of joints (natural fractures), and fracture propagation. The rock properties that are included in these equations are the intact rock compressive and tensile strengths, coefficient of friction, fracture stiffness, and fracture toughness. The codes use these parameters, along with boundary stresses and calculated thermal stresses, to obtain a stress distribution throughout the region of interest in the NFE.

Two different methods are typically used to estimate the stress in a fractured rock mass: the continuum method and the discrete-element method. The more common is the continuum approach, which uses the finite-element, the finite-difference, or the boundary element method for computing stress and displacement. The rock mass is assumed to be a 
continuum, and the network of cracks and fractures contained in the rock behave in a manner similar to that of an individual crack. Equations describing the response of an ensemble of cracks are assumed to be identical in form to those describing the response of a single crack. These models handle the creation of new cracks and fractures in various ways. One method is to derive a damage vector $\mathbf{D}$ that describes the number of cracks and fractures accumulated during the entire history of deformation (Costin, 1987).

\subsubsection{Finite-Difference and Finite-Element Methods}

In finite-difference and finite-element methods, the behavior of a structure is simulated by representing the material as elements or zones that form a grid. Each element behaves according to a prescribed constitutive law in response to applied forces or boundary conditions. Both finite-difference and finite-element methods translate a set of differential equations into matrix equations for each element relating forces at nodes to displacements at nodes. The Fast Lagrangian Analysis of Continua (FLAC) User's Manual (Itasca Consulting Group Inc., 1996) describes these methods as follows:

In the finite-difference method, every derivative in the set of governing equations is replaced directly by an algebraic expression written in terms of the field variables (e.g., stress or displacement) at discrete points in space; these variables are undefined anywhere else.

In contrast, the finite-element method has a central requirement that the field quantities (stress, displacement) vary throughout each element in a prescribed fashion, using specific functions controlled by parameters. The formulation consists in adjusting these parameters to minimize error terms or local or global energy.

Both methods produce a set of algebraic equations to solve. Even though these equations are derived in quite different ways, it is easy to show (in specific cases) that the resulting equations are identical for the two methods. Over the years, certain "traditional" ways of doing things have taken root: for example, finite element programs often combine the element matrices into a large global stiffness matrix, whereas this is not normally done with finite differences because it is relatively efficient to regenerate the finite difference equations at each step. [Then] an "explicit," time-marching method [can be used] to solve the algebraic equations. Implicit, matrix-oriented solution schemes are more common in finite elements.

\section{The Fast Lagrangian Analysis of Continua (FLAC) Code}

FLAC is a numerical code that uses a time-dependent finite-difference algorithm. The code is capable of treating both mechanical and thermally induced stresses and deformations. In FLAC, materials are represented by arbitrarily shaped, quadrilateral zones; several builtin, constitutive models are available for describing material behavior. FLAC is often used in two-dimensional (2-D) modeling, assuming plane-strain geometry and linear mechanical and thermal properties where mechanical properties (e.g., elastic moduli) and thermal properties (e.g., thermal expansion coefficient) are independent of stress and temperature. Constitutive relations often are either isotropic and elastic or make use of the ubiquitous joint model capability of the FLAC code (Itasca Consulting Group Inc., 1996)). In the code, heat transport is usually isotropic heat conduction. FLAC has recently become available for three- 
dimensional (3-D) modeling, and this new version is currently being configured to simulate the 3-D stress and displacement field in the NFE over time. FLAC capabilities include the following:

- The thermal module simulates transient heat flow and development of thermal stresses and can be coupled to the mechanical stress calculation.

- Failure criteria such as the Mohr-Coulomb criterion (Jaeger and Cook, 1976) or the Hoek-Brown criterion (1980) can be incorporated with equilibrium calculations.

- Interface elements are allowed to undergo tensile separation or Coulomb slip to simulate faults, joints, or frictional boundaries. These elements can have any spatial orientation in three dimensions, but are treated as impermeable boundaries for fluid flow; hence the FLAC 3-D code cannot directly solve the coupled THM problem.

- Ubiquitous fractures (see subsequent text) can be incorporated as part of the rockmass properties (e.g., Itasca Consulting Group Inc., 1996). This feature allows closely spaced joint sets to be modeled as part of a continuum, as long as the scale of the model is much larger than the spacing between individual joints. The ubiquitousfracture model assumes a series of parallel, weak planes embedded in a MohrCoulomb solid. Yield behavior may occur either in the solid or along the slip plane. A joint tensile-strength limit is also tested.

FLAC is used to simulate TM effects for the reference cases defined for analysis of coupled processes, as discussed in the following subsections.

\section{ABAQUS}

Another widely available code is ABAQUS, a 3-D finite-element code with capability for time-dependent analysis of thermal and mechanical behavior. The numerical model is weakly coupled in the sense that the temperature field produces thermal stresses, but these stresses do not, in turn, influence temperatures. The temperature field is calculated as a function of time from a thermal-diffusion model. The mechanical model uses the temperature field, which can vary with time, to apply thermal loading.

\subsubsection{Boundary-Element Method}

The boundary-element method (BEM) is a very different continuum method that can be used to simulate the NFE. With this method, the boundary of a region of interest is divided into elements, and the numerical solution of the problem of interest builds on the analytical solutions that have already been obtained for simple, singular problems. These mathematical singular solutions are combined using superposition to form the overall solution of the larger problem being studied. Because each of the singular solutions already satisfies the governing partial differential equations in the region of interest, there is no need to divide the region into a network of elements as in done in finite-element and finite-difference methods (Crouch and Starfield, 1990). Blair (1994) developed a numerical code called FRACROCK that calculates elastic deformation, as well as crack nucleation and propagation; FRACROCK is based on the BEM. FRACROCK has since been modified to incorporate thermal effects and is suited to study of fracturing and fracture behavior in the vicinity of drifts. FRACROCK is used to simulate TM effects for the reference cases defined for analysis of coupled processes, as discussed subsequently.

\subsubsection{Distinct-Element Method}

The distinct-element method represents a fundamentally different approach to analysis of the mechanical behavior of a fractured rock mass. In the distinct-element method, the rock mass is composed of an assembly of deformable blocks that are interfaced by discontinuities. 
In some of the distinct-element models, the blocks are discretized by means of triangular zones where the strains are assumed to be constant. In a distinct-element code, fractures in the rock mass are entered individually. One of the most commonly used distinct-element codes is called UDEC. Millard, et al., (1995) discusses the use of UDEC in a THM simulation. Zhang and Sanderson (1996) employed a distinct-element code to illustrate why rock masses with one or more systematic sets of oriented fractures should not be represented as an equivalent continuum. The distinct-element method has also been formulated in 3-D and is available in a code named 3-DEC.

Four distinguishing characteristics of the distinct-element method have been identified by Hart and Cundall (1992):

1. Discrete bodies within the system can undergo finite displacements and rotations.

2. New contacts develop between bodies as the calculation evolves.

3. Contacts are deformable.

4. The equations of motion are solved in an explicit time-stepping scheme.

A quasi-static solution scheme can be substituted for (4) in the preceding list. As with the continuum codes, Coulomb slip and joint dilation are incorporated into the model. An important application for distinct-element modeling, rather than for continuum modeling, is use with problems in which major failure occurs.

Distinct-element models were initially designed to model the interaction of independent, tumbling grains or bodies. Because of the need for generality, allowing particles to interact at any angle, these algorithms perform search-and-contact angle operations that are computationally slow. Attempts to extend distinct models to well-ordered, jointed rock masses (where displacements are small, with slippage on a few fractures) have resulted in distinct-element models that resemble continuum models. The boundaries must be represented in detail, but the search-and-contact angle operations are unnecessary.

\subsubsection{Conceptual Models for Incorporating the Effects of Fractures}

How best to incorporate fractures and fracture behavior is a fundamental question in geomechanical modeling. The simplest way is to assume that the fractured rock is a homogeneous, isotropic, and elastic medium having elastic properties that can be calculated from measured properties of intact rock and from ubiquitous fractures with known properties and abundance (e.g., Berryman, 1996). The simplest effective-medium approach requires a minimum of four parameters: density and two elastic moduli for the unfractured rock and a parameter describing the fracture abundance and deformability. More sophisticated effective-medium theories require an additional parameter that describes the shape or aperture of typical fractures. Although this approach is a good way to estimate mechanical properties of fractured rock, information about fracture orientation and connectivity is not used, and some of the effective-medium theories break down for high concentrations of thin fractures. The effective-isotropic-continuum approach is best for randomly oriented fractures, such as thermally induced microcracks, that are moderately small and sparse.

The preceding approach can be readily modified, by relaxing the assumption of isotropy, to incorporate information about fracture orientation. For an elastic but anisotropic constitutive model, additional elastic moduli are required (e.g., 3 more for the case of transverse anisotropy or as many as 21 independent parameters for general anisotropy) (Auld, 1973). There is some evidence from laboratory measurements that the Topopah Spring tuff matrix is slightly anisotropic in a transverse sense because of horizontal foliation (Blair and Berge, 1996a; Martin et al., 1992). The presence of a dominant set of subvertical fractures striking approximately to the north, as mapped in the LBT (Lin, 1994), suggests that the rock- 
mass deformability may be anisotropic with orthorhombic symmetry. Because the LBT also contains other fractures in different orientations, the anisotropy may be weak. Available laboratory or field measurements are not sufficient to quantify all the independent elastic parameters that would be required to describe anisotropy in the Topopah Spring tuff (Blair and Berge, 1996a). For the purposes of this report, the host rock is assumed to be mechanically isotropic.

An isotropic, elastic, but inhomogeneous model can be used by assuming the tuff is homogeneous except for a few fractures that can be represented in the computer model as interfaces where displacement is allowed. Several of the modeling codes described in a previous subsection (e.g., FLAC, ABAQUS) allow such interfaces. Parameters required for an explicit interface model include density and two elastic moduli for the tuff itself, plus fracture length, orientation, and strength information for each interface. Information about fracture orientation is used in this approach, but it is impractical to incorporate many interfaces in the model. Simulation of discrete interfaces (fractures) is best for representing a few major fractures that are considered most significant for fluid flow. This simulation is complementary to the effective-medium-theory approach described previously, and both methods are planned for future use in repository performance calculations.

If the assumption of purely elastic behavior is dropped and the ubiquitous-fracture model (e.g., Arulmoli, 1987; Blair et al., 1995) is used, the necessary parameters then include density, two elastic moduli, cohesion, dilation angle, tension limit, and internal friction angle for the tuff matrix, plus joint angle, joint cohesion, joint tension, and joint friction angle for the fracture set represented in the model. The main limitation of the ubiquitous-fracture approach is that it assumes a high abundance of parallel fractures, which controls the behavior of the rock mass. Note that the ubiquitous-fracture approach, the effective-medium theory, and the explicit-interface approaches are complementary; thus, all three have appropriate applications in modeling the repository host-rock response.

\subsubsection{Approach to Study of Coupled Behavior}

Coupling of TM and TH behavior is principally through the temperature field because the permeability is controlled by fractures, which may be modified significantly by thermal stress. Other, more fully coupled approaches have been developed. For example, work conducted in conjunction with the DECOVALEX program (see Section 4.2.4) includes development of an analytical solution for coupled THM behavior by Rehbinder (1995) and a study of THM behavior of sparsely fractured rock by Nguyen and Selvadurai (1995). In addition, Jiao and Hudson (1995) have presented a fully coupled model for engineered systems in rock.

At present, the available TH algorithms are more sophisticated than are the available fully coupled THM algorithms. Sophisticated simulators such as NUFT (see Chapter 3) are needed to capture the TH behavior, which controls the temperature field. Accordingly, for this report, temperature distributions are generated by TH simulators, thermal-stress distributions are calculated using weakly coupled TM calculations, and the results are analyzed using alternative conceptual models for fracture-permeability change in response to deformation. This approach represents the state-of-the-art in combining the information needed to assess THM coupled behavior for a Yucca Mountain repository.

\subsubsection{Model Validation Studies}

An international cooperative project was established for theoretical and experimental studies of coupled thermal, hydrologic, and mechanical processes in hard (crystalline) rock. This project is called DECOVALEX and is described by Jing, Tsang, and Stephansson (Jing et 
al., 1995). In this work, different mathematical models and computer codes were used to study problems of interest to geologic disposal of nuclear waste. In particular, 11 codes were evaluated; these included both 2-D and 3-D finite-element codes in which the rock was modeled as a porous / fracture media and a 2-D discrete-element code in which the rock was modeled as discrete deformable block assemblages. Much of the work done for DECOVALEX is documented in a special issue of the International Journal of Rock Mechanics (Stephansson, 1995).

As part of the DECOVALEX program, two intercode benchmark comparisons were carried out. The benchmark test BMT1 considered a large-scale (kilometer-scale) mass of rock as described by Millard et al. (1995). The benchmark problem BMT2 involved a system of nine blocks of intact, hard rock separated by two pairs of soft fractures, as described by Chan et al. (1995). For each of these exercises, both finite-element and distinct-element methods were used. Chan et al. found that, for the BMT2 test, heat convection significantly affects the distribution of temperature, thermal stresses, and displacements and that the predominant coupled effect is fracture closure caused by thermal expansion of the rock blocks. Results for DECOVALEX also indicate that, for tests BMT1 and BMT2, the different modeling techniques produced very similar results. This indicates that use of the continuum codes is adequate for study of host-rock behavior for a Yucca Mountain repository.

Another study used a physical model to simulate waste emplacement for evaluating a nuclear waste disposal concept in Japan. The large-scale laboratory test involved heating and flow testing, and was simulated with a version of ABAQUS (Hibbitt, 1994)) that includes vapor-flow processes (Borgesson and Hernelind, 1995). The test was performed using a partially saturated buffer material surrounding a simulated waste package (WP) in a hollow cylinder of rock. The calculations resulted in a prediction of void ratio, temperature, water saturation, pore pressure, and effective stress. The calculations were coupled THM; the hydrologic and thermal results compared well with the experiment, but the prediction of mechanical response did not. The 3-D finite-element model THAMES underestimated the experimental measurements of temperature in the buffer material (Ahola et al., 1994).

For another field-scale experiment, FLAC 2-D (Itasca Consulting Group Inc., 1996) was used to model heat and water flow through a 50-m $\times 50-\mathrm{m}$ block of fractured granite (Israelsson, 1995). Stress-induced permeability changes were limited to one order of magnitude, and fractures were assumed to be randomly oriented, thus allowing use of the continuum approach in FLAC.

\subsubsection{Testing Model Predictions for the Large-Block Test}

\subsubsection{Field Observations from the Large-Block Test}

Efforts to understand and characterize coupled processes in a fractured rock mass include the LBT currently underway at Fran Ridge, near Yucca Mountain, Nevada. The LBT is being conducted on a rectangular prism of rock that is $3 \mathrm{~m} \times 3 \mathrm{~m}$ in cross section and $4.5 \mathrm{~m}$ high. It is a fractured rock mass that was exposed from an outcrop by excavating the surrounding rock to leave the block in place. Two subvertical sets of fractures and one set of subhorizontal fractures intersect the block. The subvertical fracture sets are approximately orthogonal, with spacing of 0.25 to $1 \mathrm{~m}$, and are oriented generally in the NE-SW and NW-SE directions. The block also contains one major subhorizontal fracture located approximately $0.5 \mathrm{~m}$ below the top surface. 
Results from the LBT (Lin, 1998) show that the overall temperature profile is consistent with conduction-dominated heat flow, except for major temperature excursions at 2520 and $4475 \mathrm{hr}$. These excursions probably represent the formation of transient heat pipes, with episodic flow conditions.

Preliminary analysis of deformation from the LBT has been conducted using data from the multiple-point borehole extensometers (MPBX) and fracture-deformation monitoring systems. Results from these systems show that, within a few hours of the heater start-up, the block began to expand $0.1 \mathrm{in}$. The total deformation observed is approximately $0.1 \mathrm{in}$. Data from MPBX systems show similar expansion in the E-W and the N-S directions and indicate that horizontal expansion is directly dependent on height above the base. This is in disagreement with continuum calculations (Blair et al., 1995) that show a nonlinear profile of horizontal expansion associated with the thermal gradient imposed on the block. MPBX data and fracture-deformation data show that most of the deformation occurs in discrete, vertically oriented zones and is probably caused by opening of vertical fractures. In summary, the mechanical measurements indicate that the block expanded in the horizontal direction more than in the vertical direction and that macroscopic deformation was dominated by movement along fractures.

The hydrothermal response of the block is probably dominated by flow in vertical fractures. Interestingly, for the major thermal excursion at $2520 \mathrm{hr}$, the largest associated deformation was movement on a horizontal fracture.

\subsubsection{Thermomechanical Simulation of the LBT}

Blair, Berge, and Wang (Blair et al., 1995) analyzed the TM behavior of the LBT. This work was to provide analysis and interpretation of coupled THM behavior of the block as it was heated. Objectives of the modeling were to aid in experimental design of the test, to evaluate alternative thermal and constitutive models, and to evaluate different numerical methods. In this study, TM simulations of the heat-up phase of the LBT were conducted using two different commercially available numerical codes: FLAC and ABAQUS (see Section 4.2.2.1).

A 2-D, plane-strain, FLAC model was constructed in a plane orthogonal to the heater holes in the LBT. The thermal predictions made with FLAC showed a 2-D temperature field near the heaters at early time. At later time, the code predicted a nearly 1-D temperature field because the sides of the block are insulated. Temperature fields in the 3-D simulation were very similar to those predicted by the 2-D FLAC model.

Initial TM modeling of the LBT calculated temperatures, stresses, and displacements in 2-D and 3-D using a simplified continuum representation of the block. Although numerous fractures intersect the block, these simulations provided a general understanding of TM behavior. In these simulations, the gridding used in the models was similar to that used by Lee (1995) for hydrothermal modeling. Two additional simulations were conducted in which discrete fractures were represented and smaller stress values were predicted, demonstrating that the presence of compliant fractures tends to reduce the stress levels.

Blair et al. concluded that the TM FLAC and ABAQUS thermal-conduction modeling produced temperature fields similar to that of Lee (1995), who used a code containing a more sophisticated TH model (Blair et al., 1995). This suggests that the FLAC and ABAQUS codes could be used with a TH model to better investigate coupled processes. The 2-D modeling was useful for evaluating constitutive models, but was unable to simulate the outside face of the LBT where stresses are highest because the plane-strain assumption is equivalent to assuming that the model plane lies at the center of the block. The 3-D model required an order of magnitude more computer time, but it computed stresses and displacements everywhere in the block. 
Blair et al. compared 2-D and 3-D models using FLAC and ABAQUS, respectively (Blair et al., 1995; Itasca Consulting Group Inc., 1996). The plane-strain assumption in the 2-D model is overly restrictive for this application because the block is limited in the third dimension and is stress-free at those boundaries, whereas the plane-strain assumption corresponds to infinite extent in the third dimension. (The plane-strain assumption is less restrictive underground, where the extent of the medium in the third dimension can be much greater.) It was found that temperature predictions in the block were similar with both the 2-D and 3-D models, but differences in predicted stresses became more pronounced near the edges of the block.

\subsection{Initial Conditions and Rock-Mass Rheological Behavior at Ambient and Elevated Temperature}

Simulation of TM effects in the repository host rock requires specific input on the rockmass properties, initial conditions, and boundary conditions. The types of parameters required depend on the constitutive model used to represent stress-strain behavior. This section reviews the information assessed in the development of TM models for this report. The parameter values selected for analysis of the five reference cases and used in calculations in described in subsequent text are a subset of the information assessed. The QA status of the data sources used for this assessment is reports in Appendix A.

Three different types of stratigraphic nomenclature are used in this discussion. The TM stratigraphy of Ortiz et al. (1985) is the original system and is currently used most often for repository engineering analyses. In this stratigraphy, the repository horizon is located entirely within the TSw2 unit. The more detailed hydrostratigraphy used in the UZ site-scale model (Bodvarsson et al., 1997) is also used here, and there is a direct correspondence to the nomenclature of Flint (1998) (given in parentheses). In these systems, the repository horizon lies mostly within the tsw35 (Tptpmn), but in peripheral areas the horizon crosses into the overlying tsw34 (Tptpml) and the underlying tsw36 (Tptpll). The tsw37 (Tptpv) unit underlies the repository host rock and may also contribute to altered-zone mechanical response (this is the TSw3 unit of Ortiz et al., 1985).

\subsubsection{In Situ Stress}

In situ stress has been measured, by hydraulic fracturing, in drillholes USW G-1, USW G-2, and USW G-3 at Yucca Mountain (Stock et al., 1984, 1985). Stock et al. found that the vertical stress has an average value of approximately $7 \mathrm{MPa}$, with a range of values from 5 to $10 \mathrm{MPa}$. The minimum horizontal stress was found to have a bearing of about $\mathrm{N} 57^{\circ} \mathrm{W}\left( \pm 8^{\circ}\right)$; the bearing for the maximum horizontal stress was found to be about $\mathrm{N} 32^{\circ} \mathrm{E}\left( \pm 8^{\circ}\right)$. The ratio of horizontal stress to vertical stress had an average value of approximately 0.5 or 0.6 and a range of about 0.3 to 0.8 for the minimum horizontal stress or 0.3 to 1.0 for the maximum horizontal stress. Table 4-1 shows the mean value and ranges for vertical stress, which is the maximum principal stress and which is determined from the overburden thickness and density. The table also shows the ratios of minimum and maximum horizontal stresses to the vertical stress and the directions of the horizontal stresses. 
Table 4-1 Values and ranges of principal stresses in the potential repository horizon at Yucca Mountain (Stock et al., 1984, 1985)

\begin{tabular}{|l|c|c|}
\hline \multicolumn{1}{|c|}{ Parameter } & Average Value $^{\mathrm{a}}$ & Range \\
\hline \hline Maximum principal stress (vertical) & $7.0 \mathrm{MPa}(1015 \mathrm{psi})$ & 5.0 to 10.0 \\
\hline $\begin{array}{l}\text { Ratio of minimum horizontal stress } \\
\text { to vertical stress }\end{array}$ & 0.5 & 0.3 to 0.8 \\
\hline $\begin{array}{l}\text { Ratio of maximum horizontal stress } \\
\text { to vertical stress }\end{array}$ & 0.6 & 0.3 to 1.0 \\
\hline $\begin{array}{l}\text { Bearing of minimum horizontal } \\
\text { stress }\end{array}$ & $\mathrm{N} 57^{\circ} \mathrm{W}$ & $\mathrm{N} 50^{\circ} \mathrm{W}$ to $\mathrm{N} 65^{\circ} \mathrm{W}$ \\
\hline $\begin{array}{l}\text { Bearing of maximum horizontal } \\
\text { stress }\end{array}$ & $\mathrm{N} 32^{\circ} \mathrm{E}$ & $\mathrm{N} 25^{\circ} \mathrm{E}$ to $\mathrm{N} 40^{\circ} \mathrm{E}$ \\
\hline
\end{tabular}

Average value for a depth of about $0.3 \mathrm{~km}(1000 \mathrm{ft})$

A stress profile for the in situ stress near the Exploratory Studies Facility (ESF) was estimated using a 2-D finite-element analysis, similar to that presented in Hardy and Bauer (1991). The stress profile can be found in Wilder (1996) Sec. 4.0). Information about in situ stress is also discussed in Blair and Berge (1996c).

\subsubsection{Mechanical Properties of Topopah Spring Tuff}

Laboratory measurements (Table 4-2) of the mechanical strength of intact samples have been made on a substantial number of samples from the proposed repository horizon. These data indicate that the intact rock is strong relative to the stress magnitudes expected, having a uniaxial strength of $155 \mathrm{MPa}( \pm 59 \mathrm{MPa})$ and a Young's modulus of approximately 30-35 GPa. Upon loading, unfractured samples produce stress-strain curves that show nearly linear elastic behavior until failure. Fractured samples exhibit less brittle behavior, and the onset of yield typically occurs at $50 \%$ of the ultimate strength. Most of the tests for compressive strength have been conducted on samples that were saturated with water and tested under drained conditions. This represents a minimum value because rocks are moderately weaker when saturated with water. When uniaxial loading is used to measure elastic constants the results can be strongly influenced by inhomogeneities.

Price (1986) studied the effect of sample size on mechanical properties of Topopah Spring tuff and found that both the ultimate strength and the axial strain at failure are inversely related to sample diameter, whereas Young's modulus and Poisson's ratio are independent of sample diameter.

Table 4-2 Mechanical properties of Topopah Spring tuff and fractures

\begin{tabular}{|l|l|l|l|l|}
\hline \multicolumn{1}{|c|}{ Property } & \multicolumn{1}{|c|}{ Conditions } & \multicolumn{1}{c|}{ Value } & \multicolumn{1}{c|}{ Source } & \multicolumn{1}{c|}{ Comments } \\
\hline \hline porosity & $\begin{array}{l}\text { ambient temperature, } \\
\text { pressure }\end{array}$ & $9 \%-28 \%$ & Lin and Roberts, 1996 & $\begin{array}{l}\text { laboratory } \\
\text { measurements on } \\
\text { cores, small blocks }\end{array}$ \\
\hline bulk density & \multicolumn{1}{|c|}{$"$} & $\begin{array}{l}2280-2360 \\
\mathrm{~kg} / \mathrm{m}^{3}\end{array}$ & Blair and Berge, 1996c & $\begin{array}{l}\text { laboratory } \\
\text { measurements on } \\
\text { dry cores }\end{array}$ \\
\hline peak strength & $\begin{array}{l}25^{\circ} \mathrm{C}, \text { unconfined } \\
\text { uniaxial compression }\end{array}$ & $155 \pm 59 \mathrm{MPa}$ & Price, 1986 & $\begin{array}{l}\text { laboratory tests on } \\
\text { cores }\end{array}$ \\
\hline
\end{tabular}




\begin{tabular}{|c|c|c|c|c|}
\hline Property & Conditions & Value & Source & Comments \\
\hline$"$ & $"$ & $154 \pm 36 \mathrm{MPa}$ & Blair and Berge, 1996c & $"$ \\
\hline$"$ & $"$ & $139 \pm 73 \mathrm{MPa}$ & " & $\begin{array}{l}\text { laboratory tests on } \\
\text { irradiated cores }\end{array}$ \\
\hline$"$ & $\begin{array}{l}22^{\circ} \mathrm{C} \text {, unconfined } \\
\text { uniaxial compression }\end{array}$ & 68-102 MPa & Price et al., 1987 & $\begin{array}{l}\text { laboratory tests on } \\
\text { dry and saturated } \\
\text { cores }\end{array}$ \\
\hline$"$ & $\begin{array}{l}150^{\circ} \mathrm{C} \text {, unconfined } \\
\text { uniaxial compression }\end{array}$ & 89-136 MPa & $"$ & $\begin{array}{l}\text { laboratory tests on } \\
\text { saturated cores }\end{array}$ \\
\hline " & $\begin{array}{l}22^{\circ} \mathrm{C}, 5 \mathrm{MPa} \text { confirmed } \\
\text { pressure }\end{array}$ & $155-220 \mathrm{MPa}$ & $"$ & $"$ \\
\hline$"$ & $\begin{array}{l}150^{\circ} \mathrm{C}, 5 \mathrm{MPa} \text { conf. } \\
\text { press. }\end{array}$ & $48-155 \mathrm{MPa}$ & $"$ & $"$ \\
\hline$"$ & $\begin{array}{l}22^{\circ} \mathrm{C}, 10 \mathrm{MPa} \\
\text { confirmed pressure }\end{array}$ & $152-305 \mathrm{MPa}$ & $"$ & $"$ \\
\hline $\begin{array}{l}\text { indirect tensile } \\
\text { strength }\end{array}$ & ambient temperature & 4-16 MPa & $\begin{array}{l}\text { Martin et al., 1994, } \\
1995 b\end{array}$ & $\begin{array}{l}\text { Brazil tests on } \\
\text { saturated cores }\end{array}$ \\
\hline Young's modulus & $\begin{array}{l}25^{\circ} \mathrm{C} \text {, unconfined } \\
\text { uniaxial compression. }\end{array}$ & $33 \pm 5 \mathrm{GPa}$ & DOE, 1990 & $\begin{array}{l}\text { laboratory tests on } \\
\text { cores }\end{array}$ \\
\hline$"$ & $\begin{array}{l}25^{\circ} \mathrm{C} \text {, unconfined } \\
\text { uniaxial compression }\end{array}$ & $38-40 \mathrm{GPa}$ & Price, 1993 & $"$ \\
\hline$"$ & $\begin{array}{l}25^{\circ} \mathrm{C} \text {, unconfined } \\
\text { uniaxial compression }\end{array}$ & $25 \pm 3 \mathrm{GPa}$ & Blair and Berge, 1996c & $"$ \\
\hline$"$ & $\begin{array}{l}25^{\circ} \mathrm{C} \text {, unconfined } \\
\text { uniaxial compression }\end{array}$ & $23 \pm 5 \mathrm{GPa}$ & $"$ & $\begin{array}{l}\text { laboratory tests on } \\
\text { irradiated cores }\end{array}$ \\
\hline$"$ & $\begin{array}{l}22^{\circ} \mathrm{C} \text {, unconfined } \\
\text { uniaxial compression }\end{array}$ & $27-46 \mathrm{GPa}$ & Price et al., 1987 & $\begin{array}{l}\text { laboratory tests on } \\
\text { dry and saturated } \\
\text { cores }\end{array}$ \\
\hline$"$ & $\begin{array}{l}150^{\circ} \mathrm{C} \text {, unconfined } \\
\text { uniaxial compression }\end{array}$ & 28-33 GPa & $"$ & $\begin{array}{l}\text { laboratory tests on } \\
\text { saturated cores }\end{array}$ \\
\hline$"$ & $\begin{array}{l}22^{\circ} \mathrm{C}, 5 \mathrm{MPa} \text { confirmed } \\
\text { pressure }\end{array}$ & $36-41 \mathrm{GPa}$ & $"$ & $"$ \\
\hline$"$ & $\begin{array}{l}150^{\circ} \mathrm{C}, 5 \mathrm{MPa} \\
\text { confirmed pressure }\end{array}$ & $16-36 \mathrm{GPa}$ & $"$ & $"$ \\
\hline$"$ & $\begin{array}{l}22^{\circ} \mathrm{C}, 10 \mathrm{MPa} \\
\text { confirmed pressure }\end{array}$ & $33-41 \mathrm{GPa}$ & $"$ & $"$ \\
\hline$"$ & $\begin{array}{l}\text { ambient temperature, } \\
\text { unconfined, } 8.5 \mathrm{MPa} \\
\text { uniaxial compression }\end{array}$ & $3.6-31 \mathrm{GPa}$ & Blair and Berge, 1996b & $\begin{array}{l}\text { laboratory tests on } \\
0.5-\mathrm{m} \text { block, } \\
\text { measured across } \\
\text { matrix and } \\
\text { matrix+fracture }\end{array}$ \\
\hline$"$ & $"$ & $0.2-0.65 \mathrm{GPa}$ & $"$ & $\begin{array}{l}\text { measured across } \\
\text { fracture only }\end{array}$ \\
\hline
\end{tabular}




\section{Thermomechanical and Thermohydromechanical Models}

\begin{tabular}{|c|c|c|c|c|}
\hline Property & Conditions & Value & Source & Comments \\
\hline " & $\begin{array}{l}\text { ambient temperature, } \\
\text { unconfined, } 0.1-4 \mathrm{MPa} \\
\text { uniaxial compression }\end{array}$ & $\begin{array}{l}\text { approximately } \\
4 \mathrm{GPa}\end{array}$ & $"$ & $\begin{array}{l}\text { measured across } \\
\text { matrix+fracture }\end{array}$ \\
\hline$"$ & $\begin{array}{l}\text { ambient temperature, } \\
\text { unconfined, 4-8.5 MPa } \\
\text { uniaxial compression }\end{array}$ & $\begin{array}{l}\text { approximately } \\
6 \mathrm{GPa}\end{array}$ & $"$ & $"$ \\
\hline$"$ & $\begin{array}{l}\text { ambient temperature, } \\
\text { unconfined, 5-8. 5-5 } \\
\text { MPa stress cycle }\end{array}$ & 15-20 GPa & " & " \\
\hline " & $\begin{array}{l}\text { ambient temperature, } \\
\text { unconfined, 5-8. 5-5 } \\
\text { MPa stress cycle }\end{array}$ & $\begin{array}{l}\text { approximately } \\
54 \mathrm{GPa}\end{array}$ & $"$ & $\begin{array}{l}\text { measured across } \\
\text { matrix only }\end{array}$ \\
\hline " & $\begin{array}{l}85^{\circ} \mathrm{C} \text {, unconfined, } 5-8 . \\
\text { 5-5 MPa stress cycle }\end{array}$ & $\begin{array}{l}\text { approximately } \\
10 \mathrm{GPa}\end{array}$ & " & $\begin{array}{l}\text { meas. across } \\
\text { matrix+fracture }\end{array}$ \\
\hline$"$ & $\begin{array}{l}85^{\circ} \mathrm{C} \text {, unconfined, } 5-8 . \\
5-5 \mathrm{MPa} \text { stress cycle }\end{array}$ & $\begin{array}{l}\text { approximately } \\
32 \mathrm{GPa}\end{array}$ & $"$ & $\begin{array}{l}\text { measured across } \\
\text { matrix only }\end{array}$ \\
\hline " & $\begin{array}{l}\text { ambient temperature, } \\
\text { unloading from } 8.5 \mathrm{MPa}\end{array}$ & $\begin{array}{l}\text { approximately } \\
11 \mathrm{GPa}\end{array}$ & " & $\begin{array}{l}\text { measured across } \\
\text { matrix+fracture } \\
\text { (permanent strain } \\
\text { observed) }\end{array}$ \\
\hline Poisson's ratio & $\begin{array}{l}22^{\circ} \mathrm{C} \text {, unconfined } \\
\text { uniaxial compression }\end{array}$ & $0.09 \pm 0.07$ & Martin et al., 1993 & $\begin{array}{l}\text { laboratory tests on } \\
\text { cores }\end{array}$ \\
\hline$"$ & $"$ & $0.14-0.40$ & $\begin{array}{l}\text { Martin et al., } 1994, \\
\text { 1995b }\end{array}$ & $"$ \\
\hline$"$ & $"$ & $0.22-0.30$ & DOE, 1990 & $"$ \\
\hline$"$ & $"$ & $0.12-0.32$ & Price et al., 1987 & $"$ \\
\hline " & $\begin{array}{l}22^{\circ} \mathrm{C}, 5 \mathrm{MPa} \text { conf. } \\
\text { press. }\end{array}$ & $0.17-0.21$ & " & $"$ \\
\hline$"$ & $\begin{array}{l}22^{\circ} \mathrm{C}, 10 \mathrm{MPa} \\
\text { confirmed pressure }\end{array}$ & $0.18-0.24$ & $"$ & $"$ \\
\hline cohesion for tuff & & $18-38 \mathrm{MPa}$ & DOE, 1990 & \\
\hline $\begin{array}{l}\text { tuff angle of } \\
\text { intermediate } \\
\text { friction }\end{array}$ & & $20-37^{\circ}$ & $"$ & \\
\hline fracture cohesion & $\begin{array}{l}\text { ambient temperature, } \\
\text { unloading from } 8.5 \mathrm{MPa}\end{array}$ & $\begin{array}{l}\text { approximately } \\
1 \mathrm{MPa}\end{array}$ & Blair and Berge, 1996b & $\begin{array}{l}\text { laboratory tests on } \\
0.5 \text {-m block }\end{array}$ \\
\hline $\begin{array}{l}\text { friction angle for } \\
\text { fracture }\end{array}$ & & $28.4^{\circ}$ & MacDougall et al., 1987 & \\
\hline $\begin{array}{l}\text { coefficient of } \\
\text { friction for fracture }\end{array}$ & $\begin{array}{l}\text { ambient temperature, } \\
\text { 5-10 MPa normal stress }\end{array}$ & $0.8-1.1$ & Olsson and Brown, 1994 & $\begin{array}{l}\text { laboratory tests on } \\
\text { fractures in dry } \\
\text { cores }\end{array}$ \\
\hline $\begin{array}{l}\text { dilation angle for } \\
\text { fracture }\end{array}$ & $"$ & $5-11^{\circ}$ & $"$ & $"$ \\
\hline
\end{tabular}




\subsubsection{Thermal Properties of Topopah Spring Tuff}

Simulation of TM responses requires the following thermal properties for Topopah Spring welded tuff, for temperatures between about $25^{\circ}$ and $250^{\circ} \mathrm{C}$ : thermal conductivity, thermal capacitance, and thermal expansivity. These parameters are discussed in detail in Blair and Berge (1996b) and summarized in Table 4-3. The observations from which these properties are estimated are mainly from core samples at the scale of a few centimeters. New information from field-scale tests will be incorporated as it becomes available.

Table 4-3 Thermal properties of Topopah Spring tuff

\begin{tabular}{|c|c|c|c|c|}
\hline Property & Conditions & Value & Source & Comments \\
\hline $\begin{array}{l}\text { Thermal } \\
\text { conductivity }\end{array}$ & in situ & $2.1 \pm 0.2 \mathrm{~W} / \mathrm{m}-\mathrm{K}$ & Nimick, 1990 & in situ tests \\
\hline$"$ & $30-290^{\circ} \mathrm{C}$ & $1.67 \pm 0.15 \mathrm{~W} / \mathrm{m}-\mathrm{K}$ & TRW, 1996 & $\begin{array}{l}\text { laboratory tests on } \\
\text { rock from ESF }\end{array}$ \\
\hline Specific heat & & $840 \mathrm{~J} / \mathrm{kg}-\mathrm{K}$ & DOE, 1990 & \\
\hline$"$ & $25-300^{\circ} \mathrm{C}$ & $928 \mathrm{~J} / \mathrm{kg}-\mathrm{K}$ & TRW, 1996 & $\begin{array}{l}\text { laboratory tests on } \\
\text { rock from ESF }\end{array}$ \\
\hline $\begin{array}{l}\text { Thermal } \\
\text { capacitance }\end{array}$ & $\begin{array}{l}25-94 \text { and } \\
115-275^{\circ} \mathrm{C}\end{array}$ & $2.0-2.3 \mathrm{~J} / \mathrm{cm}^{3}-\mathrm{K}$ & DOE, 1990 & \\
\hline$"$ & $95-114^{\circ} \mathrm{C}$ & $11 \mathrm{~J} / \mathrm{cm}^{3}-\mathrm{K}$ & $"$ & (dehydration) \\
\hline $\begin{array}{l}\text { Thermal expansion } \\
\text { coefficient }\end{array}$ & $25-100^{\circ} \mathrm{C}$ & $7.7-10.8 \times 10^{-6} \mathrm{~K}^{-6}$ & Martin et al., 1996 & $\begin{array}{l}\text { laboratory tests on } \\
\text { cores }\end{array}$ \\
\hline$"$ & $"$ & $5.4-9.1 \times 10^{-6} \mathrm{~K}^{-1}$ & DOE, 1990 & $"$ \\
\hline$"$ & near $250^{\circ} \mathrm{C}$ & $14.2-20.6 \times 10^{-6} \mathrm{~K}^{-1}$ & Martin et al., 1996 & $"$ \\
\hline$"$ & $"$ & $25-35.6 \times 10^{-6} \mathrm{~K}^{-1}$ & DOE, 1990 & $"$ \\
\hline
\end{tabular}

Thermal conductivity and thermal capacitance have been measured in the laboratory as summarized by Hardin and Chesnut (1997), and values are found in the Reference Information Base (RIB; DOE, 1990). Measured values for these parameters vary by less than $10 \%$ over a wide temperature range. The thermal-expansion coefficient, however, increases with temperature (Martin et al., 1996). Data from the RIB show this parameter increases by a factor of 5 or more for measurements made at temperatures of approximately $250^{\circ} \mathrm{C}$ compared to measurements made at approximately $25^{\circ} \mathrm{C}$.

The thermal properties of rock samples from the proposed repository horizon have been the subject of several laboratory and field studies. Results reported in the RIB indicate that the dry matrix thermal conductivity is $1.49 \pm 0.44(\mathrm{~W} / \mathrm{mK})$. The in situ thermal conductivity has been measured dry and at a saturation of $65 \%$ with the same result $(2.1 \pm 0.2 \mathrm{~W} / \mathrm{mK})$. Brodsky et al. (1997) found that saturation had an effect. Using the stratigraphic nomenclature of Ortiz et al. (1985), for the TSw2 unit, the thermal conductivity for saturated samples is $2.29 \pm 0.42 \mathrm{~W} / \mathrm{mK}$, whereas that for dry samples is $1.50 \pm 0.44 \mathrm{~W} / \mathrm{mK}$.

The effect of temperature on strength of the intact host rock has not been intensively investigated. Rock strength appears to decline somewhat at elevated temperature, but the effect is probably not significant. Price et al. (1987) reported that, for samples from the repository horizon, Young's modulus decreased an average of $16 \%$ as temperature increased 
from $22^{\circ}$ to $150^{\circ} \mathrm{C}$. The average ultimate strength decreased $16 \%$ as temperature increased from $22^{\circ}$ to $150^{\circ} \mathrm{C}$ for both unconfined conditions and $5 \mathrm{MPa}$ confining pressure. Further discussion of temperature effects is provided by Hardin and Chesnut (1997).

Measurements of linear thermal expansion have been made on samples from unit TSw2 and are reported in the RIB. In general, the coefficient of linear thermal expansion increases with increasing temperature. At temperatures less than $100^{\circ} \mathrm{C}$, the coefficient of thermal expansion is reported to be in the range 7.7 to $10.8 \times 10^{-6}{ }^{\circ} \mathrm{C}^{-1}$. At temperatures near $250^{\circ} \mathrm{C}$, the coefficient increases to the range 14.2 to $20.6 \times 10^{-6} \mathrm{C}^{-1}$. Conclusions reached by Wilder (1993a, 1993b) regarding the use of these values to simulate processes in the near field, and for simulation of cooling, still apply. Recent measurements (Brodsky et al., 1997) indicate that thermal expansivity is strongly temperature-dependent and that there is a hysteresis between heating and cooling. Brodsky et al. reported some permanent elongations. Because the coefficients are strongly temperature-dependent, ranges of values must be specified at different temperatures (Table 4-4).

The increase with temperature is attributed to local distortions and formation of microcracks from differential expansion of constituent grains. Brodsky et al. (1997) attributed the hysteresis observed at temperatures near $200^{\circ} \mathrm{C}$ to phase changes in cristobalite and possibly in trydimite. Data on mineralogical phase changes in the host rock are summarized by Hardin and Chesnut (1997). Cristobalite is expected to represent about $30 \%$ of total volume, but a limited region of the rock mass is likely to get hot enough for the phase change to occur. If it does occur, the phase change is reversible, and a volume decrease may occur upon cooling well below $200^{\circ} \mathrm{C}$.

Table 4-4 Coefficient of thermal expansion in TSw2 unit at different temperatures, from Brodsky et al. (1997)

\begin{tabular}{|c|c|c|c|c|c|c|c|c|c|c|c|c|}
\hline \multicolumn{13}{|c|}{ Mean Coefficient of Thermal Expansion $\left(\times 10^{-6}\right.$ per $\left.{ }^{\circ} \mathrm{C}\right)$ During Heat-Up } \\
\hline $\begin{array}{c}\text { Saturation } \\
\text { State }\end{array}$ & Statistic & $\begin{array}{l}25- \\
50^{\circ}\end{array}$ & $\begin{array}{l}50- \\
75^{\circ}\end{array}$ & $\begin{array}{l}75- \\
100^{\circ}\end{array}$ & $\begin{array}{l}100- \\
125^{\circ}\end{array}$ & $\begin{array}{l}125- \\
175^{\circ}\end{array}$ & $\begin{array}{l}150- \\
175^{\circ}\end{array}$ & $\begin{array}{l}175- \\
200^{\circ}\end{array}$ & $\begin{array}{l}200- \\
225^{\circ}\end{array}$ & $\begin{array}{l}225- \\
250^{\circ}\end{array}$ & $\begin{array}{l}250- \\
275^{\circ}\end{array}$ & $\begin{array}{l}275- \\
300^{\circ}\end{array}$ \\
\hline Saturated & Mean & 7.14 & 7.47 & 7.46 & 9.07 & 9.98 & 11.74 & 13.09 & 15.47 & 19.03 & 25.28 & 37.13 \\
\hline Saturated & Std. dev. ${ }^{a}$ & 0.65 & 1.51 & 1.21 & 2.41 & 0.77 & 1.28 & 1.40 & 1.75 & 3.09 & 6.87 & 14.27 \\
\hline Saturated & Count & 19 & 19 & 19 & 19 & 19 & 19 & 19 & 16 & 16 & 16 & 16 \\
\hline Dry & Mean & 6.67 & 8.31 & 8.87 & 9.37 & 10.10 & 10.96 & 12.22 & 14.54 & 20.79 & 25.13 & 35.13 \\
\hline Dry & Std. dev. & 1.20 & 0.42 & 0.40 & 0.55 & 0.88 & 1.16 & 1.50 & 2.57 & 17.03 & 10.07 & 14.56 \\
\hline Dry & Count & 40 & 40 & 40 & 40 & 40 & 38 & 38 & 35 & 35 & 35 & 35 \\
\hline \multicolumn{13}{|c|}{ Mean Coefficient of Thermal Expansion $\left(\times 10^{-6}\right.$ per $\left.{ }^{\circ} \mathrm{C}\right)$ During Cool-down } \\
\hline Saturated & Mean & 21.89 & 27.83 & 26.55 & 21.38 & 17.31 & 14.06 & 12.49 & 11.52 & 10.27 & 9.48 & 8.81 \\
\hline Saturated & Std. dev. & 6.16 & 10.36 & 10.01 & 5.70 & 3.07 & 1.38 & 1.32 & 2.00 & 0.62 & 0.63 & 0.62 \\
\hline Saturated & Count & 16 & 16 & 16 & 16 & 19 & 19 & 19 & 19 & 19 & 19 & 19 \\
\hline Dry & Mean & 20.57 & 24.31 & 24.20 & 21.16 & 18.45 & 14.34 & 11.74 & 10.51 & 9.54 & 8.87 & 7.48 \\
\hline Dry & Std. dev. & 4.88 & 7.55 & 8.08 & 6.24 & 9.36 & 4.23 & 3.03 & 2.26 & 1.79 & 1.56 & 1.99 \\
\hline Dry & Count & 35 & 35 & 35 & 35 & 38 & 38 & 40 & 40 & 40 & 40 & 40 \\
\hline
\end{tabular}

Standard deviation 
Calculating stress changes due to mechanical or thermal loads requires information about the original in situ stresses, mechanical properties, and thermal properties of the rock being modeled. The second step-determining how stresses affect fractures-requires statistical information about the numbers and sizes of the fractures and data on rock strength and mechanical properties of the fractures. The third step-estimating permeability changesrequires either assumptions or additional information about the permeability of individual fractures under different stress and temperature conditions. Field measurements of in situ permeability for networks of fractures would also be useful. The following sections discuss these input parameters in terms of the input parameters that are required and of the observations that are available for different parameters.

\subsubsection{Fracture Mechanical Properties}

Calculating stress changes from mechanical or thermal loads requires data on the strength and other mechanical properties of fractures and on the distribution of fractures.

\subsubsection{Mechanical Properties of Fractures}

The available information on fracture mechanical properties includes laboratory measurements on cores at the scale of a few centimeters (e.g., Martin et al., 1994; Price et al., 1987; Blair and Berge, 1996c), laboratory measurements on small blocks of tuff at 0.5-m scale (Blair and Berge, 1996a), and field measurements at a scale of a few meters (e.g., Zimmerman et al., 1986). Observations include elastic moduli and strength data for the tuff under different temperature conditions (e.g., Blair and Berge, 1996b; Price et al., 1987) as well as mechanical properties of the fractures at laboratory scales of a few centimeters to a few tens of centimeters (e.g., Blair and Berge, 1996b; Olsson and Brown, 1994). Laboratory measurements may overestimate rock-mass strength and stiffness because the scale of measurement cannot readily incorporate multiple fractures or fractures that are longer or more widely spaced than the laboratory samples. On the other hand, laboratory tests can provide information that may not be available from field tests (e.g., Blair and Berge, 1996c).

\subsubsection{Fracture Statistics}

Required information on the distribution of fractures includes statistical estimates of fracture length, orientation of fracture sets, fracture frequency or abundance, and fracture apertures. This information is available at various scales, including descriptions of cracks and fractures in tuff core samples (e.g., Blair and Berge, 1996a; Lin et al., 1993), observations of cracks and vugs in small blocks of tuff at the 0.5-m scale (Blair and Berge, 1996a), detailed maps of fractures in the LBT (Lin, 1994; Lin et al., 1995), and information about fractures mapped in the ESF (Badan et al., 1991). Fracture statistics are discussed in detail by Lin (1994). In summary, fracture mapping at the LBT and the ESF found subvertical fractures striking predominantly N-S (Lin, 1994), with typical fracture concentrations of about 1.5 to 2.5 fractures / meter for the LBT and 3 to 4 fractures / meter for the ESF (Lin, 1994). The orientation data are consistent with earlier data from boreholes (Lin et al., 1993). Subhorizontal fractures were also mapped at the LBT and the ESF, but they are less abundant than vertical fractures.

Fracture apertures observed on the surface of the LBT varied from hairline $(<0.5 \mathrm{~mm})$ to approximately $2 \mathrm{~cm}$. Borehole camera images from vertical holes in the LBT showed large openings (apertures of several centimeters) for some vertical fractures in areas near the base of the block. Detailed information is not available about the fracture surface morphology that could be used to infer which fractures are tensile and which originated from shearing (Einstein and Dershowitz, 1990). 


\subsubsection{Fracture Permeability}

Several investigators have shown that increasing stress across fractures causes a reduction in fracture aperture, and, to a first approximation, flow in a fracture is related to the cube of the fracture aperture (Raven and Gale, 1985). Heating the rock generally increases stress and thereby reduces the fracture aperture. Studies have also shown that fracture shear displacement can significantly increase fracture permeability and that the magnitude of the change may readily exceed that due to normal displacement (Barton et al., 1985). Finally, it is widely accepted that the hydrologic behavior of a fractured rock mass is controlled by relatively few, well-connected fractures.

In other work Blair and Berge (1996a) linked several concepts to predict where permeability may be changed significantly in the DST:

- Flow along critically stressed fractures (Barton et al., 1985)

- Mapped fracture sets

- Evolution of the TM stress field

- Permeability increase due to initial shear deformation

This analysis predicted that significant TM effects on permeability may extend to a distance of several tens of meters from the drift; this is in contrast to previous work that suggested the effects will be limited to the immediate vicinity. Enhanced permeability is likely in regions of increased thermal stress gradients. This means that a region of increased permeability may accompany the thermal pulse as it travels outward from the heat source.

Estimation of permeability changes requires information on ambient conditions. Fieldscale borehole pneumatic tests provide the most reliable information on bulk permeability, which is attributed mainly to fracture permeability in this rock type. In addition, information is needed about the permeability of individual fractures and the connectivity of fracture networks.

Air-permeability data from pneumatic injection tests performed in the LBT and for tests at G-Tunnel show that measured permeability at the scale of a few meters can vary over about five orders of magnitude (Lin, 1994; Lin et al., 1995). For example, LeCain and Walker (1994) found that air permeability measured in a borehole at Yucca Mountain was several orders of magnitude greater than for tuff core samples. Measured permeability values for the Topopah Spring tuff are in the range 1.1 to $12.0 \times 10^{-13} \mathrm{~m}^{2}$ (0.11 to 1.2 D) (LeCain and Walker, 1994). For the LBT, measured air-permeability values range from $10^{-16} \mathrm{~m}^{2}$ to $7 \times 10^{-12} \mathrm{~m}^{2}$ $(0.1 \mathrm{mD}$ to $7 \mathrm{D})$, with the highest values measured where open, subvertical fractures were encountered (Lin, 1994). The G-Tunnel test data show that permeability changed where the tuff was strongly heated. Regions in which permeability was low before heating developed microcracks, and the permeabilities increased by approximately an order of magnitude (Lin, 1994).

\subsubsection{Laboratory Tests on 0.5-m-Scale Blocks}

Tests on small block samples of tuff have contributed to understanding of fracture deformability and permeability. Laboratory tests have the advantage that known boundary and environmental conditions can be imposed on samples that contain one or more fractures, whereas field tests have inherent limitations in this regard.

The laboratory samples were $0.5-\mathrm{m}$, artificially fractured blocks of Topopah Spring welded tuff containing an artificial, horizontal fracture (for data source, see Table A-4 in Appendix A). A uniaxial load was applied perpendicular to the fracture plan using a 300-ton press. The sample assembly was heated using heaters on copper plates at the top and bottom. Fluid flow was generated by an injection source at the center of the fracture plane (Figure 4-1). 
Figure 4-1 Illustration of small-block experiment for flow through horizontal fracture

The experiment first characterized flow in the fracture at ambient (unstressed) conditions, then cycled the uniaxial stress to values from 0.5 to $8 \mathrm{MPa}$, while measuring the injectivity and monitoring flow paths by detecting permeant seepage at the fracture edges. The fluid inlet pressure $\left(P_{\mathrm{f}}\right)$ and cumulative flow in and out of the sample are shown as a function of elapsed time in Figure 4-2. Uniaxial stress vs. elapsed time is shown in Figure 4-3. Fluid was injected into the fracture only for discrete time intervals.

Figure 4-2 History of fluid flow and axial stress on sample SB-3 during first $600 \mathrm{hr}$ of testing

\section{Figure 4-3 Axial load (uniaxial stress) vs. elapsed time for Test 7195}

Figure 4-2 shows cumulative flow vs. elapsed time for the entire test. Cumulative injected water volume is plotted along with the cumulative weight of fluid collected from the fracture. The close match between these two curves indicates that the flow occurred primarily in the fracture, with little or no diversion in the rock matrix. Total flow out of the fracture at all edges is shown as a function of time in Figure 4-4.

\section{Figure 4-4 Cumulative flow data for Test 7195}

Total flow out of each port is shown graphically in Figure 4-5. All but a small fraction of the water was collected in just a few of the ports. The dominant flow paths were in the center of each face and were oriented in the E-W and N-S directions. This is consistent with analysis of the surface topology.

Figure 4-5 Spatial representation of total flow in plane of fracture for sample SB-3 during Test 7195

Flow rate through the fracture at several fluid inlet pressures is plotted as a function of axial stress in Figure 4-6. This figure shows that the flow rates through this artificial fracture are quite high and that axial stress had a minor effect on the flow rate, even at low axial stresses for which fracture closure was observed. This indicates that, even for a relatively smooth, artificial fracture, most flow occurred in channels that were not affected by increased normal stress. Several channels were observed in the fracture profiles shown in Figure 4-4, and these do not close at the stress levels applied in this experiment.

Figure 4-6 Flow rate at four different fluid inlet pressures, plotted as a function of axial stress for Test 7195

The laboratory data provide approximate values to assign to individual fractures in modeling changes in rock-mass permeability and provide guidance for how permeability is likely to vary with environmental conditions. Field data provide information about bulk permeability for networks of fractures and indicate the magnitude of changes to be expected from heating. A new experiment is underway in which water flow through a single fracture in a block of tuff is being measured as a function of temperature and stress. The LBT and ongoing thermal tests in the ESF are expected to provide additional field observations of permeability changes due to heating. 


\subsubsection{Time-Dependent Joint/Rock Mass Properties}

Microcracking and subcritical crack growth in the host rock over long time periods are of interest because the formation, growth, and coalescence of microcracks could lead to rockfall. Little work has been done to predict rock fall at elevated temperatures over hundreds of years or longer. Kemeny and Cook (1990) used a probabilistic approach that included timedependent crack-growth measurements to examine borehole emplacement schemes for waste disposal. They estimated that, over the lifetime of the repository, spalling would be likely to occur in a significant portion of emplacement boreholes. (Emplacement boreholes are not included in the repository reference design for viability assessment.)

Martin et al. (1995a) performed laboratory experiments to investigate creep in Topopah Spring tuff samples at ambient and elevated temperatures. They found that observed creep deformation is consistent with a mechanism of time-dependent crack growth, particularly for stress magnitude greater than $90 \%$ of the uniaxial compressive strength. Temperature caused a reduction in strength, but further testing would be required to quantify the strength and creep deformation as a function of temperature, saturation, and applied load.

Blair and Berge (1996c) found that imposing low levels of compressive stress for periods of a few days on 0.5-m blocks of Topopah Spring tuff caused time-dependent, inelastic crack closure to occur for cracks oriented parallel to the applied stress.

\section{Deformation Results}

Fracture flow and uniaxial compression tests have been conducted with a small block of Topopah Spring tuff $(0.5-\mathrm{m}$ scale) that contains an artificial fracture oriented in the horizontal direction. Results show that ash-flow textures in the rock fabric are associated with significant anisotropy in both fluid flow and geomechanical properties. Observed flow in the plane of the fracture was anisotropic, with more flow along the direction of the rock fabric.

It was also found that, for this fracture, increasing normal stress across the fracture from 0.1 to $8 \mathrm{MPa}$ did not substantially reduce the flow rate, indicating that flow in the plane of the fracture was due primarily to flow in channels that were unaffected by the normal stress. Profiling of the fracture surface indicated the presence of several channels a few millimeters in width and depth. Similar features probably exist in natural fractures.

The rock sample was tested in compression in the direction parallel to the rock fabric, and it exhibited a Young's modulus of $40 \mathrm{GPa}$ for compressive stress in the range 4 to $8 \mathrm{MPa}$. This value is much higher than that observed in a similar sample of tuff that was tested in the direction perpendicular to the fabric (Blair and Berge, 1996c). This result indicates that the fracture mechanical properties may have significant anisotropy correlated with the rock fabric. Anisotropy in Young's modulus is at least 25\% and can be greater when vuggy zones occur. This amount of anisotropy is significant for simulations of TM behavior in the repository host rock.

Displacement measurements made on the block surface during uniaxial load cycling indicate that much of the cumulative deformation of the block was caused by fracture deformation, but that, when loaded, the artificial fracture became quite stiff in compression (Table 4-5). Significantly different results were obtained with two blocks that were prepared with the fracture parallel to the ash-flow fabric and with the fracture perpendicular to fabric. The fracture parallel to fabric (SB-3) was apparently stiffer than the fracture perpendicular to fabric (SB-1). 
Table 4-5 Young's modulus values for Experiment 7195 (SB-3 fracture parallel to rock fabric)

\begin{tabular}{|c|c|}
\hline Axial Stress (MPa) & Young's Modulus (GPa) \\
\hline \hline $0-1$ & 2.8 \\
\hline $1-4$ & 17 \\
\hline $4-8$ & 40 \\
\hline
\end{tabular}

Thus, for sample SB-1, values of Young's modulus were highly dependent on the location of the transducers, indicating that the rock is very heterogeneous. For the other sample, SB-3, all transducers produced similar values for the modulus. The Young's modulus of $40 \mathrm{GPa}$ for the stress range 4-8 $\mathrm{MPa}$ is significantly higher than any moduli observed for sample SB-1 (Table 4-5). For sample SB-3, the fracture closure data show that most closure occurred for axial stress in the range $0-0.5 \mathrm{MPa}$, with little closure for stress greater than $1 \mathrm{MPa}$. Thus, it is possible for an artificial fracture to be hydraulically conductive and stiff at the same time. The conductance is associated with aberrations of the surface, and the stiffness is probably associated with the high contact area for sawcuts. The stiffening behavior is consistent with the idea that increasing TM stresses normal to fractures may not significantly reduce permeability.

\subsection{Simulation of Thermomechanical Responses to Thermal Loading}

\subsubsection{Thermomechanical Simulation of Drift Scale Test}

The Yucca Mountain Site Characterization Project is currently conducting the DST in the ESF at Yucca Mountain, and analysis of the DST presents an opportunity to predict TM effects on permeability. This test is well suited for investigation of TM and THM effects for the following reasons:

- The geometry and heat load are well known.

- The access drift and heated drift were thoroughly mapped.

- The TH response to heating has been simulated (Buscheck and Nitao, 1995).

- Multiple data sets (e.g., rock-mass deformation, bulk permeability, and temperature) will be collected and jointly interpreted.

In this subsection, the nature of TM stress changes in response to heating will be evaluated. The DST is shown schematically in Figure 4-7. A 2-D TM analysis was made of the test along cross section $\mathrm{A}-\mathrm{A}^{\prime}$, which is perpendicular to the access and heated drifts.

Figure 4-7 Schematic of the Drift-Scale Test

\subsubsection{Thermomechanical Modeling}

\subsubsection{Model Description: 2-D FLAC Model of Drift-Scale Test}

The TM analysis is based on the 2-D version of the geomechanical FLAC code developed by the Itasca Consulting Group, Inc. (1996). FLAC is an uncoupled TM model: the stress field depends on the temperature field, but the temperature field is independent of the stress field. Therefore, the thermal-conduction problem can be solved independently of the mechanicalequilibrium problem. 
The DST TM model represents a 2-D, 87- $\mathrm{m} \times 75-\mathrm{m}$ cross-sectional region using 2170 grid elements, with the smallest grid spacing being approximately $0.1 \mathrm{~m}$ in the central part of the model (Figure 4-8). The rock making up the DST is modeled as a homogeneous elastic medium having the same mechanical properties as those found from laboratory measurements made on Topopah Spring tuff cores and small blocks (e.g., Blair and Berge, 1996c). Thermal properties were chosen to match those used in TH simulations (Buscheck and Nitao, 1995).

\section{Figure 4-8 Total FLAC grid}

Heater drift and canister are at the center of the left edge of the model. The access drift is located at about $x=30 \mathrm{~m}$ and $y=0$. For the canister heaters in the DST TM model, the FLAC model uses interior sources within grid elements. The DST TM model simulates a line heat source of $0.9-\mathrm{m}$ radius that is aligned perpendicular to the model in the center of a $5-\mathrm{m} \times 5-\mathrm{m}$ drift with linear power density of $0.8 \mathrm{~kW} / \mathrm{m}$ in the direction perpendicular to the model. The DST model also includes the wing heaters in a plane perpendicular to the model and extending about 4 to $14 \mathrm{~m}$ from the center of the drift Figure 4-9. These are the wing heaters that Buscheck and Nitao (1995) modeled using planar heat sources having aerial power densities of $105 \mathrm{~W} / \mathrm{m}^{2}$ and $157.5 \mathrm{~W} / \mathrm{m}^{2}$ in planar strip sections $5 \mathrm{~m}$ wide.

\section{Figure 4-9 Enlarged portion of grid between heated drift and access drift}

The mechanical initial conditions in the DST TM model are chosen to be

$$
\begin{aligned}
& \sigma_{\mathrm{xx}}=-5.0 \mathrm{MPa} \text { (compression negative) } \\
& \sigma_{\mathrm{yy}}=-10.0 \mathrm{MPa} \\
& \sigma_{\mathrm{zz}}=-5.0 \mathrm{MPa}
\end{aligned}
$$

The center of the heater drift is taken to be a symmetry boundary (zero horizontal displacement, in contrast to the stress boundary condition used in the reference cases discussed subsequently). The top, bottom, and far side boundaries are fixed in both the horizontal and vertical directions. These boundary conditions will create artifacts due to edge effects, but will not significantly affect results in the central part of the model within about $30 \mathrm{~m}$ of the heater drift. The edges of the model are far enough away to ensure accurate results for the region of interest between the heated drift and the access drift. The TM simulation for the DST is performed in several stages:

1. The mechanical model containing the heated drift and access drift excavations is run until force equilibrium is achieved.

2. The heated drift is backfilled between the heater and the drift wall as one method of providing a thermal connection to the repository rock. The mechanical model is again run to achieve force equilibrium.

3. The mechanical mode in FLAC is switched off, and the thermal option is switched on. The thermal-conduction solution is saved at $0.5 \mathrm{yr}$ (183 days), $1.0 \mathrm{yr}$ (365 days), $2.0 \mathrm{yr}$ (730 days), 3.0 yr (1090 days), and 4.0 yr (1460 days). The temperature, stress, and displacement fields for these five times are calculated. The temperatures are in general agreement with those in the TH model of Buscheck and Nitao (1995).

4. Finally, the thermal option is switched off, and the mechanical mode is switched on to calculate the thermally coupled stress field for each of the saved time steps. The TM solution is calculated at $0.5,1.0,2.0,3.0$, and $4.0 \mathrm{yr}$. 


\subsubsection{Modeling Results}

The temperature fields, stresses, and displacements are calculated for $0.5,1,2,3$, and $4 \mathrm{yr}$ of heating to compare with results from TH modeling (Buscheck and Nitao, 1995). After 4 yr of heating, the region in the DST TM model having temperatures greater than $100^{\circ} \mathrm{C}$ forms an ellipsoid extending approximately $10 \mathrm{~m}$ above and below the center of the heated drift and extending approximately $17 \mathrm{~m}$ out to the sides of the heated drift. This is roughly equivalent to the dryout zone in the TH model of Buscheck and Nitao (1995). For regions outside the heated drift, the calculated temperature field for the DST model agrees with the TH modeling results. Inside the drift, the temperatures in the FLAC model and peak temperature at the center of the heater are higher than those for the TH model because FLAC uses sources with finite cross-sectional areas to represent the heaters, whereas the TH code (NUFT) used line sources. The calculated temperatures, at the drift wall and beyond, match for the $\mathrm{TH}$ and FLAC models.

After 4 yr of heating, calculated horizontal stresses reach maximum values of approximately 30 to $70 \mathrm{MPa}$ compression in the region outside the drift. Corresponding vertical stresses range from 20 to $60 \mathrm{MPa}$ compression. The highest stresses are found at the drift wall, where temperatures are highest. Shear stresses are low: less than $20 \mathrm{MPa}$. Horizontal displacements are as great as $20 \mathrm{~mm}$ after $4 \mathrm{yr}$ of heating, and vertical displacements are as great as $12 \mathrm{~mm}$. At this time step, a stress gradient develops in the region between the drift wall and the end of the wing heater plane a few meters away. Although the FLAC modeling approach may tend to overestimate stresses and displacements because the effects of fractures are ignored, this stress gradient results from a large temperature gradient and is probably important to interpretation of test results.

The agreement between the TH and the FLAC temperature calculations indicates that stress and displacement results calculated using FLAC can be related to the TH modeling for the ESF tests. Coupled THM processes can also be modeled using temperature fields taken directly from the TH code as input for FLAC calculations of stress and displacement.

\subsubsection{FLAC Simulation of Reference Case 1-Total System Performance Assessment-Viability Assessment (TSPA-VA) Base Case}

In this subsection, a suite of TM simulations is presented for the reference Case 1. As indicated in Chapter 3, this case is based on the reference repository point-load design, with no backfill, and using the TSPA-VA base-case TH property set. The other case evaluated here is Case 5, which represents a line-load design with double drift spacing $(56 \mathrm{~m})$, and the "modified TH" property set. These calculations demonstrate the importance of coupling TM and TH models to capture behavior that is not predicted by either model. The other cases ( 2 , 3 , and 4) are not analyzed for TM response because the temperature fields are nearly identical to those of Case 1.

Temperature changes can substantially change the stress levels in a confined rock mass because rock has a relatively high deformation modulus. Temperature also has other influences on rock mechanical behavior and properties, including a general decrease in deformation modulus with increasing temperature and an increase in subcritical crackgrowth rate with increasing temperature. Other behaviors that are expected to contribute significantly only as temperatures rise above $200^{\circ} \mathrm{C}$ are rock creep and elasto-plastic behavior.

Even though these effects may become important over long time periods, or in the hottest parts of the repository, they are considered minor when compared to thermal expansion and the associated increase in stress that occurs as temperature in the host rock increases to as high as $200^{\circ} \mathrm{C}$. 
For the analyses discussed in this section, two different TM models are used: the FLAC model (Itasca Consulting Group Inc., 1996) and the FRACROCK model (Blair, 1994). For both simulations, a 5.1-m-diameter drift is located in the TSw2 unit. Simulations are conducted for time steps of $0,50,100$, and $1000 \mathrm{yr}$ after emplacement.

\subsubsection{FLAC Implementation}

The FLAC code (version 3.22) is used to calculate stresses and displacements for the temperature fields from the reference Case $1 \mathrm{TH}$ model calculated using TSPA-VA base-case properties, nominal infiltration, and a point-load repository design. (Reference cases for investigation of coupled processes in the NF/AZ Models Report are visually depicted and linked in the presentation on the CD-ROM that accompanies this report.) The NUFTgenerated temperature fields are used directly as input, and the grids are identical to the NUFT grids, except that a few additional grid lines have been added to maintain grid-block aspect ratios within acceptable limits. Block dimensions are all between 0.5 and $2.5 \mathrm{~m}$, except at the extreme edges of the model. The FLAC model contains about 5775 blocks.

The model is $170 \mathrm{~m} \times 98 \mathrm{~m}$ and represents depths from $300 \mathrm{~m}$ to $470 \mathrm{~m}$ in the vertical direction. The horizontal direction represents drifts with centers spaced $28 \mathrm{~m}$ apart. To ensure that the central portion is relatively free of artifacts caused by edge effects, several drifts are represented in the model grid. The drifts are circular in cross-section to avoid stress concentration at the corners. The drift diameter is $5.1 \mathrm{~m}$, and drifts are centered at a depth of $385 \mathrm{~m}$. A portion of the grid used for these simulations is shown in Figure 4-10.

\section{Figure 4-10 Portion of mesh used for FLAC simulations of reference Case 1}

The rock in the model is represented using properties appropriate for geologic unit Tptpmn (tsw35, within the TSw2 TM unit) for depths from $300 \mathrm{~m}$ to $395.6 \mathrm{~m}$; unit Tptpll (tsw36, TSw2) for depths from $395.6 \mathrm{~m}$ to $431.8 \mathrm{~m}$; and Tptpv (tsw37, TSw3) for depths from $431.8 \mathrm{~m}$ to $470 \mathrm{~m}$. Values for physical and thermal properties were obtained from the same source used for NUFT parameters, and are consistent with the UZ site-scale model (Bodvarsson et al., 1997).

The FLAC code requires specification of the properties shown in Table 4-6 for each zone in the model.

\section{Table 4-6 FLAC required properties and sources}

\begin{tabular}{|l|l|}
\hline \multicolumn{1}{|c|}{ Property } & \multicolumn{1}{c|}{ Source } \\
\hline \hline Bulk modulus & $\begin{array}{l}\text { Values selected for Near-Field/Altered-Zone Models } \\
\text { Report and Site Description Document }\end{array}$ \\
\hline Shear modulus & $\begin{array}{l}\text { Values selected for Near-Field/Altered-Zone Models } \\
\text { Report and Site Description Document }\end{array}$ \\
\hline Density & UZ Site-Scale Model \\
\hline Thermal conductivity & UZ Site-Scale Model \\
\hline Specific heat & UZ Site-Scale Model \\
\hline Coefficient of thermal expansion & Values selected for Near-Field/Altered-Zone Models \\
& Report and Site-Description Document \\
\hline
\end{tabular}

The bulk and shear moduli values and densities do not vary much for the different tuff layers, and small variations in these properties are not expected to affect the modeling results. The bulk modulus value used is $20 \mathrm{GPa}$, and the bulk shear modulus is $13 \mathrm{GPa}$ for the 
tuff. The bulk density is $2285 \mathrm{~kg} / \mathrm{m}^{3}$. Although thermal conductivity and specific heat values are supplied for the model, they are not used in the calculations because there are no heat sources in the model. Heating is instead accomplished by applying a temperature field. A thermal expansion coefficient value of $1.0 \times 10^{-5} \mathrm{~K}^{-1}$ is used because this is a representative value over the range $20^{\circ}$ to $200^{\circ} \mathrm{C}$. It is possible to use thermal properties that vary with temperature in FLAC, but these properties were not allowed to vary with temperature for this model. Future modeling will explore this issue. Physical property values for concrete and air are used for zones within the drifts, where appropriate. The WP is represented using two grid elements in each drift, and the model results are not sensitive to the physical properties selected to represent the WP.

The boundary conditions used in the FLAC modeling are (1) applied vertical stress of $7 \mathrm{MPa}$ compression at the top of the model (representing lithostatic stress) and (2) horizontal compressive stress of $5 \mathrm{MPa}$ acting at one side of the model domain. The other side of the domain is fixed in the horizontal direction, and the bottom of the model is fixed both horizontally and vertically; it is necessary to fix at least two corners to prevent the entire model from sliding or rotating. This configuration produces initial horizontal stresses of 4 to $6 \mathrm{MPa}$ and initial vertical stresses of 8 to $10 \mathrm{MPa}$ at the depths where the drifts are located. The vertical stress increases with depth due to gravitational loading. The variation in horizontal stress (approximately $2 \mathrm{MPa}$ ) across the model is a result of the side boundary conditions and gives an estimate of model uncertainty in the center of the model due to edge effects. Making the model large will not reduce the edge effects.

Initial temperatures are set to the same uniform vertical gradient used in the NUFT model, in which the surface temperature is $15.8^{\circ} \mathrm{C}$, and the water table temperature is $34.6^{\circ} \mathrm{C}$. Thus, the FLAC model initially has a temperature of approximately $23.5^{\circ} \mathrm{C}$ at $300 \mathrm{~m}$ depth and a temperature of approximately $27.9^{\circ} \mathrm{C}$ at $470 \mathrm{~m}$ depth, with a uniform vertical gradient between those depths.

FLAC calculations for each drift-scale problem, representing pre-waste emplacement conditions, were initialized using the following procedure:

1. Construct the grid.

2. Assign properties to all zones in the model.

3. Set the initial and boundary conditions as described.

4. Run FLAC in the mechanical mode to bring the model to initial mechanical equilibrium.

5. Run FLAC in the thermal mode to set the initial temperatures as described previously.

6. Run FLAC again in the mechanical mode to bring the model to mechanical equilibrium.

7. Define the initial displacements to be zero.

The result is a model with temperature and stress values appropriate for the unheated state and representing the effects of excavation. To compute thermal stresses and displacements that result from heating, two additional steps are followed:

8. Run FLAC in the thermal mode to apply the new temperature field.

9. Run FLAC in the mechanical mode to calculate stresses and displacements. This procedure was followed for temperature fields representing the 50-, 100-, and 1,000-yr time steps from the TH models for the reference cases considered. In each case, the temperature difference relative to the initial temperature distribution was used to produce the stress and displacement fields. In other words, thermal effects were calculated relative to pre-waste emplacement conditions. 
Although temperature fields were calculated by NUFT for time steps beyond $1000 \mathrm{yr}$, the maximum extent of thermal evolution generally does not change much for time steps beyond $100 \mathrm{yr}$; thus, time steps beyond $1000 \mathrm{yr}$ are not analyzed. This is consistent with the emphasis given in this chapter to permanent hydrologic effects from nonreversed shear deformation of fractures. In addition, only reference Cases 1 and 5 are analyzed for TM effects because the NUFT-calculated TH temperature fields for Cases 1 through 4 are similar.

\subsubsection{Effects from Drift Excavation}

The resulting vertical compressive stress near the drift spring line is calculated to be 12 to $20 \mathrm{MPa}$, and the horizontal compressive stress at the drift crown (and the invert) is 4 to $8 \mathrm{MPa}$. Initially the shear stresses are less than $2 \mathrm{MPa}$ everywhere in the model. The mechanical equilibration calculations that simulate excavation are the same for all the reference cases. Figure 4-11, Figure 4-12, Figure 4-13, and Figure 4-14 show the vertical and horizontal normal stress fields, and the vertical, horizontal, and shear stress fields. $\square$

Figure 4-11 Principal stresses computed for excavation only using FLAC for Case 1

Figure 4-12 Vertical stress field computed using FLAC for excavation only, Case 1

Figure 4-13 Horizontal stress field computed using FLAC for excavation only, Case 1

Figure 4-14 Shear stress field computed using FLAC for excavation only, Case 1

\subsubsection{Case 1: TM Results at 50 Years (FLAC)}

- Temperature Field-The NUFT-calculated temperature field shows temperatures at or above boiling within a few meters above the drifts, and extending more than $10 \mathrm{~m}$ below the drifts (Figure 4-15). The maximum temperature at the WPs is about $140^{\circ} \mathrm{C}$. It should be noted that the WP temperature varies with local repository conditions and design features, as discussed in Chapter 3.

- Stress Field-The maximum stress in the region near the drifts is increased by about $20 \mathrm{MPa}$ (Figure 4-16), and the principal stress directions are rotated.

- Vertical Stress and Displacement-After $50 \mathrm{yr}$ heating, the vertical stress increases slightly to approximately 10 to $15 \mathrm{MPa}$ compression near the drifts, with values of 15 to $30 \mathrm{MPa}$ within one diameter of the drift springline (see Figure 4-17). Lower vertical stress values of 5 to $10 \mathrm{MPa}$ compression are found in the pillars and above and below the drifts. Vertical displacements are 2 to $8 \mathrm{~cm}$ near the drifts (see Figure 4-18)

- Horizontal Stress and Displacement-Stress conditions differ above and below the drifts because of asymmetry of the temperature field. Elevated values of 15 to $30 \mathrm{MPa}$ compression are found within about one diameter above and below the drifts (Figure 4-19). Horizontal displacements are approximately 1 to $6 \mathrm{~cm}$ near the drifts, as shown in Figure 4-20.

- Shear Stress-Shear stress magnitudes (Figure 4-21) are less than $2 \mathrm{MPa}$ above the drifts, increasing to 4 to $6 \mathrm{MPa}$ below the drifts, and as much as $10 \mathrm{MPa}$ within one drift diameter.

Figure 4-15 Temperature field for Case 1 at $50 \mathrm{yr}$

Figure 4-16 Principal stress field computed using FLAC, Case 1 at $50 \mathrm{yr}$ 
Figure 4-17 Vertical stress field computed using FLAC, Case 1 at 50 yr

Figure 4-18 Vertical displacement field computed using FLAC, Case 1 at 50 yr

Figure 4-19 Horizontal stress field computed using FLAC, Case 1 at 50 yr

Figure 4-20 Horizontal displacement field computed using FLAC, Case 1 at 50 yr

Figure 4-21 Shear stress field computed using FLAC, Case 1 at $50 \mathrm{yr}$

\subsubsection{Case 1: TM Results at 100 Years (FLAC)}

- Temperature Field-The NUFT-calculated temperatures are at or above boiling, extending from $30 \mathrm{~m}$ above the drifts to nearly $30 \mathrm{~m}$ below. Maximum temperatures of about $120^{\circ} \mathrm{C}$ are calculated for the WPs (see Figure 4-22).

- Stress Field-The computed maximum stress in the region near the drifts is less than that for $50 \mathrm{yr}$ (Figure 4-23), but the stress field is still rotated between and below the drifts.

- Vertical Stress and Displacement-Vertical stress decreases slightly to approximately 5 to $15 \mathrm{MPa}$ compression near the drifts (Figure 4-24) and to 15 to $25 \mathrm{MPa}$ compression near the drift springline. Vertical displacements are about 4 to $9 \mathrm{~cm}$ near the drifts (Figure 4-25), which is 1 to $2 \mathrm{~cm}$ more than values at $50 \mathrm{yr}$. This result is due to the extension of the heated region above and below the drifts.

- Horizontal Stress and Displacement-Horizontal stress (Figure 4-26) is approximately 5 to $10 \mathrm{MPa}$ compression near the drifts and 8 to $13 \mathrm{MPa}$ between and below the drifts. Horizontal stresses of 10 to $20 \mathrm{MPa}$ compression are computed at about one diameter above and below the drifts. The horizontal displacements are similar to those at $50 \mathrm{yr}: 1$ to $6 \mathrm{~cm}$ near the drifts (Figure 4-27).

- Shear Stress-The shear stress magnitudes remain at about $2 \mathrm{MPa}$ near the drifts, but values as large as $10 \mathrm{MPa}$ are found within one diameter of the drifts (Figure 4-28).

Figure 4-22 Temperature field for Case 1 at $100 \mathrm{yr}$

Figure 4-23 Principal stress field computed using FLAC, Case 1 at $100 \mathrm{yr}$

Figure 4-24 Vertical stress field computed using FLAC, Case 1 at $100 \mathrm{yr}$

Figure 4-25 Vertical displacement field computed using FLAC, Case 1 at $100 \mathrm{yr}$

Figure 4-26 Horizontal stress field computed using FLAC, Case 1 at $100 \mathrm{yr}$

Figure 4-27 Horizontal displacement field computed using FLAC, Case 1 at $100 \mathrm{yr}$

Figure 4-28 Shear stress field computed using FLAC, Case 1 at $100 \mathrm{yr}$ 


\subsubsection{Case 1: TM Results at 1000 Years (FLAC)}

- Temperature Field-After 1000 yr of heating, NUFT calculations show temperature within a few degrees of boiling throughout the FLAC model domain (Figure 4-29).

- Stress Field-The maximum stress near the drifts is less than that after $100 \mathrm{yr}$, and the stress field is no longer rotated between the drifts (Figure 4-30).

- Vertical Stress and Displacement-The vertical stress remains at 5 to $15 \mathrm{MPa}$ compression near the drifts (Figure 4-31), with values of $20 \mathrm{MPa}$ compression near the drift springline. Vertical stress is approximately $5 \mathrm{MPa}$ compression within one diameter above and below the drifts. The pattern is basically the same as that for $100 \mathrm{yr}$, but stresses are slightly less near the drifts. Vertical displacements are shown in Figure 4-32. Values are 5 to $11 \mathrm{~cm}$ near the drifts, which is 1 to $2 \mathrm{~cm}$ more than after 100 yr.

- Horizontal Stress and Displacement-The horizontal stress is 5 to $10 \mathrm{MPa}$ compression near the drifts (Figure 4-33). Horizontal stresses of $10 \mathrm{MPa}$ compression are computed within one diameter above and below the drifts, and values as low as $2 \mathrm{MPa}$ are found within one diameter of the drift springline. Stresses are less than those at $100 \mathrm{yr}$ because thermal loading has decreased by an order of magnitude. Horizontal displacements (Figure 4-34) are about 1 to $6 \mathrm{~cm}$ near the drifts, the same as those for 50 and $100 \mathrm{yr}$.

- Shear Stress-Shear stress magnitude after $1000 \mathrm{yr}$ is still about $2 \mathrm{MPa}$ near the drifts (Figure 4-35), which is similar to conditions after $100 \mathrm{yr}$.

Figure 4-29 Temperature field for Case 1 at $1000 \mathrm{yr}$

Figure 4-30 Principal stress field for 1000 yr computed using FLAC

Figure 4-31 Vertical stress field for 1000 yr computed using FLAC

Figure 4-32 Vertical displacement field for 1000 yr computed using FLAC

Figure 4-33 Horizontal stress field for 1000 yr computed using FLAC

Figure 4-34 Horizontal displacement field for 1000 yr computed using FLAC

Figure 4-35 Shear stress field for 1000 yr computed using FLAC

\subsubsection{FRACROCK Simulation of Reference Cases 1 and 5}

The FRACROCK model consists of two main parts: a boundary-element module for computation of mechanical effects due to external boundary conditions, excavations, and fractures; and the HEAT (Hart, 1981) module for computing thermal stresses. (Reference cases for investigation of coupled processes in the NF/AZ Models Report are visually depicted and linked in the presentation on the CD-ROM that accompanies this report.) The FRACROCK code is based on the boundary-element method presented by Crouch and Starfield (1990). In particular, the displacement-discontinuity technique, as formulated in the TWODD computer program, is the form of the boundary-element method used in FRACROCK. 
The thermal stresses for TM analysis are computed using the HEAT code, which is a straightforward algorithm that computes thermally induced stresses in a continuum, without provision for excavations or multiple material types. HEAT does allow multiple heat sources, which can be placed anywhere and turned on and off independently. The HEAT routine is used to produce a temperature field that closely matches the NUFT-calculated TH temperature fields at selected time steps and that calculates the continuum stress field for each of these fields. The thermal stress fields are then superimposed on the mechanical stress fields computed using the boundary-element module.

The problem defined in the FRACROCK simulations is shown in Figure 4-36. This figure shows that the drift-pillar system is contained in a continuous region $400 \mathrm{~m}$ wide and extending from the free surface to a depth of $910 \mathrm{~m}$. The region is anchored at the bottom, and the right and left sides are essentially free surfaces, with minimal values of shear and normal stress applied to maintain stability of the solution. The vertical stress at each location is due to the overburden at that location, assuming a constant rock density of $2.3 \mathrm{~g} / \mathrm{cc}$. The horizontal stress at each location within the region is proportional to the vertical stress at the location, and a proportionality constant of 0.55 is used. Three drifts are simulated. These are located at a depth of $385 \mathrm{~m}$ with center-to-center spacing of $28 \mathrm{~m}$. Each of these drifts has a radius of $2.55 \mathrm{~m}$ (5.1-m diameter). A subregion 14-m wide and 120-m high is used for detailed calculation of temperature and stress fields in the vicinity of the drifts. Stress and temperature values are calculated at points distributed uniformly over this subregion on a 0.5-m square grid. Input values for this calculation are shown in Table 4-7. An interesting feature of the FRACROCK boundary-element approach is that drift geometries are circular and smooth, whereas discretized, rectilinear boundaries are developed for FLAC. The model used for Case 5 is similar, but has drift spacings of $56 \mathrm{~m}$.

Figure 4-36 Zone used in FRACROCK for Case 1 stress analysis

Table 4-7 Input parameters for FRACROCK and HEAT

\begin{tabular}{|l|c|}
\hline \multicolumn{1}{|c|}{ Parameter } & Value \\
\hline \hline Thermal Expansion Coefficient & $10 \times 10^{-6}(\mathrm{~K})^{-1}$ \\
\hline Young's Modulus & $15 \mathrm{GPa}$ \\
\hline Poisson's Ratio & 0.21 \\
\hline
\end{tabular}

The FRACROCK model is used to calculate stress fields corresponding to 50 and $100 \mathrm{yr}$ of heating, for reference Cases 1 and 5. Case 1 is the TSPA-VA base case for which FLAC calculations are discussed above. Case 5 corresponds to the line-load design, using the "modified TH" property set discussed in Chapter 3, with the same average areal thermal loading as that for Case 1, but double the drift spacing.

The first step in simulation of the TM response is to compute the stress field produced by drift excavation. The calculations (not shown) indicate vertical stress (sig1e) and horizontal stress (sig2e) decrease with distance from the drift, converging to the applied far-field stresses, in a manner consistent with the elastic response to a circular opening. This calculation was consistent with the FLAC calculation and suggests that excavation will not cause significant local movement along fractures. 


\subsubsection{Case 1: TM Results at 50 Years (FRACROCK)}

- Temperature Field-Matching contours generated using NUFT and HEAT are shown in Figure 4-37 and Figure 4-38. The $100^{\circ} \mathrm{C}$ isotherm extends approximately $10 \mathrm{~m}$ below the drift, but only about $3 \mathrm{~m}$ above the drift.

- Vertical Stress (sigyye)-Vertical stress near the drifts has increased moderately (Figure 4-39). The asymmetrical distribution of temperature has caused the stress contours around the drift to become butterfly shaped, with greater vertical stress near the springline and lower stress directly above and below the drifts. The computed peak stress near the drift wall is approximately $35 \mathrm{MPa}$, a bounding value, which is considerably less than the intact rock strength.

- Horizontal Stress (sigxxe) - The horizontal stress magnitude is in the range of 10 to $25 \mathrm{MPa}$ (Figure 4-40). Lobes of greater stress are shown around the drifts. Locally elevated horizontal stress in the pillars is associated with the temperature field.

- Shear Stress (sigxye) - The asymmetry of the temperature field is reflected in the diagonal configuration of shear-stress contours (Figure 4-41).Values of shear stress are low $(<1 \mathrm{MPa})$ at distances greater than one drift diameter.

Figure 4-37 Temperature field for Case 1 at 50 yr, as computed by NUFT

Figure 4-38 Simulation of 50-yr temperature field for Case 1 for input to FRACROCK

Figure 4-39 Vertical stress field for Case 1 at $50 \mathrm{yr}$, computed using FRACROCK

Figure 4-40 Horizontal stress field for Case 1 at $50 \mathrm{yr}$, computed using FRACROCK

Figure 4-41 Shear stress field for Case 1 at $50 \mathrm{yr}$, computed using FRACROCK

\subsubsection{Case 1: TM Results at 100 Years (FRACROCK)}

- Temperature Field-Matching contours generated using NUFT and HEAT are shown in Figure 4-42 and Figure 4-43. All the rock within about one diameter of the drift wall is at or above the boiling temperature, but the zone of superheated rock is smaller than it is at $50 \mathrm{yr}$. It should be noted that, whereas the drift appears square in the figures, the appearance is an artifact of the plotting algorithm because the drift wall is modeled as a smooth, circular boundary.

- Vertical Stress-The largest vertical stress magnitudes are somewhat lower than those computed for $50 \mathrm{yr}$ (Figure 4-44). The configuration of stress contours is similar to that at $50 \mathrm{yr}$, with somewhat more uniform conditions in the middle of the pillar. Normal stress magnitude near the drift wall is still approximately $30 \mathrm{MPa}$.

- Horizontal Stress-Horizontal stress magnitude is slightly lower than it is for $50 \mathrm{yr}$ (Figure 4-45). Stress concentrations persist immediately above and below the drift.

- Shear Stress-Shear stress contours continue to be aligned along diagonals (Figure 4-46). Overall, the distribution is similar to that for $50 \mathrm{yr}$, with greater shears above the centerline.

Figure 4-42 Temperature field for Case 1 at $100 \mathrm{yr}$, as computed by NUFT

Figure 4-43 Simulation of 100-yr temperature field for Case 1 for input to FRACROCK 
Figure 4-44 Vertical stress field for Case 1 at $100 \mathrm{yr}$, computed using FRACROCK

Figure 4-45 Horizontal stress field for Case 1 at $100 \mathrm{yr}$, computed using FRACROCK

Figure 4-46 Shear stress field for Case 1 at $100 \mathrm{yr}$, computed using FRACROCK

\subsubsection{Case 1: TM Results at 1000 Years (FRACROCK)}

- Temperature Field-The temperature distributions by NUFT and HEAT are in good agreement, as shown in Figure 4-47 and Figure 4-48.

- Vertical Stress-Stress magnitudes of 15 to $20 \mathrm{MPa}$ at the center of the pillar are similar to those for $100 \mathrm{yr}$ (Figure 4-49). Vertical stresses within a meter of the drift are lower than they are at $100 \mathrm{yr}$.

- Horizontal Stress-The horizontal stress magnitudes are very similar to those for 100 yr: e.g., 12-MPa compression at the center of the pillar (Figure 4-50).

- Shear Stress-The magnitudes of shear stresses are similar to those at $100 \mathrm{yr}$ (Figure 4-51), and, when compared with $100 \mathrm{yr}$, the peak shear stress at $1000 \mathrm{yr}$ is generally less near the drift. Shear stresses are low and do not indicate rock failure or instability.

Figure 4-47 Temperature field for Case 1 at $1000 \mathrm{yr}$, as computed by NUFT

Figure 4-48 Simulation of 1000-yr temperature field for Case 1 for input to FRACROCK

Figure 4-49 Vertical stress field for Case 1 at $1000 \mathrm{yr}$, computed using FRACROCK

Figure 4-50 Horizontal stress field for Case 1 at $1000 \mathrm{yr}$, computed using FRACROCK

Figure 4-51 Shear stress field for Case 1 at $1000 \mathrm{yr}$, computed using FRACROCK

\subsubsection{Case 5: TM Results at 50 Years (FRACROCK)}

- Temperature Field-Temperatures are much greater at the drift wall than for Case 1 $\left(240^{\circ}\right.$ vs. $\left.140^{\circ} \mathrm{C}\right)$, and thermal gradients of $10^{\circ} \mathrm{C} / \mathrm{m}$ extend as far as $10 \mathrm{~m}$ into the rock (Figure 4-52). The asymmetry about the horizontal centerline observed in Case 1 is enhanced, and a greater region is heated below the drift than above the drift. The HEAT temperature field captures many attributes of the NUFT temperature field, but the match is not as close as it is for Case 1 (Figure 4-53).

- Vertical stress-The vertical stress distribution is much different than that predicted for Case 1 at 50 yr (Figure 4-54). Stress magnitudes are greater, especially near the drift and 1 to 2 diameters below the drift.

- Horizontal Stress-Horizontal stress magnitudes are in the range 10 to $20 \mathrm{MPa}$, with stresses as great as $30 \mathrm{MPa}$ concentrated around the drift (Figure 4-55).

- Shear Stress-Shear-stress magnitudes are generally low (1 to $2 \mathrm{MPa}$ ), except near the drifts (Figure 4-56). Shear stresses of approximately $5 \mathrm{MPa}$ extend from the drift wall about one drift diameter into the rock.

Figure 4-52 Temperature field for Case 5 at $50 \mathrm{yr}$, as computed by NUFT 
Figure 4-53 Simulation of 50-yr temperature field for Case 5 for input to FRACROCK

Figure 4-54 Vertical stress field for Case 5 at $50 \mathrm{yr}$, computed using FRACROCK

Figure 4-55 Horizontal stress field for Case 5 at 50 yr, computed using FRACROCK

Figure 4-56 Shear stress field for Case 5 at $50 \mathrm{yr}$, computed using FRACROCK

\subsubsection{Case 5: TM Results at 100 Years (FRACROCK)}

- Temperature Field-The temperature field calculated with HEAT is comparable to that calculated using NUFT, but trends in the $100^{\circ} \mathrm{C}$ isotherm are not accurately expressed, and shear-stress magnitudes are likely to be underestimated (Figure 4-57 and Figure 4-58). Temperatures at the drift wall are decreasing $\left(180^{\circ} \mathrm{C}\right)$. The $100^{\circ} \mathrm{C}$ isotherm extends nearly $30 \mathrm{~m}$ below the drift and $20 \mathrm{~m}$ to the side.

- Vertical Stress-The overall stress level is 15 to $20 \mathrm{MPa}$ (Figure 4-59), with greater stresses concentrated near and below the drifts.

- Horizontal Stress-Horizontal stress contours form oblate regions centered below the drifts and extending laterally (Figure 4-60). Computed stresses reach magnitudes of about $23 \mathrm{MPa}$ very near the drift and $15 \mathrm{MPa}$ within 1 to 2 diameters of the drift wall.

- Shear Stress-Shear stress magnitudes are approximately $0.5 \mathrm{MPa}$, increasing to 7 to $9 \mathrm{MPa}$ near the drifts (Figure 4-61).

Figure 4-57 Temperature field for Case 5 at $100 \mathrm{yr}$, as computed by NUFT

Figure 4-58 Simulation of 100-yr temperature field for Case 5 for input to FRACROCK

Figure 4-59 Vertical stress field for Case 5 at $100 \mathrm{yr}$, computed using FRACROCK

Figure 4-60 Horizontal stress field for Case 5 at $100 \mathrm{yr}$, computed using FRACROCK

Figure 4-61 Shear stress field for Case 5 at $100 \mathrm{yr}$, computed using FRACROCK

\subsubsection{Discussion of TM Calculations}

FLAC calculations for Case 1 (TSPA-VA base case) are in reasonable agreement with previous predictions of thermal stress and displacement for the repository (e.g., Bauer and Costin, 1990). Direct comparisons with previous studies are difficult because the temperature fields are not readily available. FLAC simulations were run using a constant-stress boundary condition rather than a zero-displacement boundary condition at the sides of the model. Whereas the zero displacement condition represents rock-mass response near the center of the repository, the constant-stress boundary condition represents conditions closer to the edge. The constant-stress condition may actually represent a greater portion of the repository area and serves as a reasonable lower bound on horizontal stress magnitudes.

FRACROCK simulations produced much higher vertical stresses because an intact rock deformation modulus was used (from tests on core samples), and the vertical boundary condition was that of an infinite space rather than of a free surface. Horizontal stresses predicted using the FRACROCK model are similar to those predicted by other investigators 
(e.g., Bauer and Costin, 1990). The FRACROCK calculations show that implementation of the Case-5 line-load scheme significantly increases both the temperature and thermal stress magnitudes. More sophisticated calculations using temperature-dependent rock properties (e.g., thermal expansivity) are warranted for the line-load design.

It is important to note that these are idealized calculations of a simple cross-section, with heavy use of symmetry, that do not account for third-axis effects or sequential emplacement of waste. It is expected that, if the base-case point-load design is implemented, complex variation of the stress and displacement fields will arise along the drift axis.

\subsection{Conceptual Models of Fracture-Permeability Change in Response to Stress and Deformation}

The permeability of the host rock is an important parameter for characterizing the host rock's hydrologic performance. Knowledge and understanding of rock permeability and how it may change over the lifetime of a geologic repository is important to the prediction and evaluation of repository performance.

To assess the performance of the proposed repository over long times, it is necessary to estimate bounds on how the permeability of the rock mass forming the proposed repository may be altered by TM effects. TM effects will primarily include changes in stress and rock properties during heating and cooling.

Generally, as compressive stress across a fracture is increased, the aperture is reduced, which reduces the fluid flow. Thus, as the general level of stress in the potential repository horizon increases from TM effects, some fracture apertures may be reduced, decreasing the permeability. However, increasing the magnitude of stress in the rock may also increase shear stresses on favorably oriented fractures, causing shear displacement and increasing the fracture aperture and, thus, increasing the permeability.

TM coupling is generally thought to influence the permeability of fractured rock via two basic mechanisms. First, increasing temperature causes the rock to expand and normal stress magnitudes to increase. As this happens, fractures close and permeability decreases. In addition, as hot rock cools, it contracts, and TM stresses relax, causing some fractures to open and permeability to increase. Second, thermal expansion in the presence of drift openings or a thermal gradient may increase the shear stress, leading to fracture slip. This second mechanism has the greatest potential to cause significant change in permeability because small shear displacements can produce relatively large increases in fracture permeability. Fracture shear displacement is less likely to be reversed on cooling than is normal displacement.

\subsubsection{Shear-Slip Model}

\subsubsection{Stress Criterion for Shear Slip}

Barton et al. (1997) presented evidence that hydraulically conductive fractures are the fractures that are critically stressed. To estimate permeability changes due to heating, this concept is applied to the stress field calculated from TM considerations. The ratio of the shear stress to normal stress was calculated for potential slip planes in $30^{\circ}$ increments between $0^{\circ}$ and $180^{\circ}$. The planes are defined by the angle $\theta$ of their normals to the $x$ axis. Therefore, an angle of $0^{\circ}$ corresponds to a vertical plane, and an angle of $90^{\circ}$ corresponds to a horizontal plane. The rotation formulas for this definition of angle $\theta$ are taken from Turcotte and Schubert (1982): 


$$
\begin{aligned}
& -S_{\mathrm{n}}=\sigma_{x^{\prime} x^{\prime}}=\sigma_{x x} \cos ^{2} \theta+\sigma_{y y} \sin ^{2} \theta+\sigma_{x y} \sin 2 \theta \\
& \sigma_{y^{\prime} y^{\prime}}=\sigma_{x x} \sin ^{2} \theta+\sigma_{y y} \cos ^{2} \theta-\sigma_{x y} \sin 2 \theta
\end{aligned}
$$

and

$$
\tau=\sigma_{x^{\prime} y^{\prime}}=\frac{1}{2}\left(\sigma_{y y}-\sigma_{x x}\right)+\sigma_{x y} \cos 2 \theta
$$

where $\tau$ is the shear stress and $S_{\mathrm{n}}$ is the compressive normal stress. The Mohr-Coulomb criterion is

$$
|\tau| \geq c+f S_{\mathrm{n}}
$$

where $c$ is the cohesion and $f$ is the coefficient of friction. The maximum potential for frictional slip occurs for a cohesion value of zero (i.e., shear offset occurs when the ratio $\left.|\tau| /\left(f S_{n}\right) \geq 1\right)$. This ratio can be contoured for values greater than one for the six angles at the five times. Thus, contoured regions are those in which the slip criterion is satisfied and shear offset is expected.

\subsubsection{Relation of Slip to Permeability Change}

Knowing which fractures are hydraulically conductive is necessary for estimating how the permeability of a critically stressed fracture will change if slip actually occurs. Brown (1995) and Brown and Bruhn (1997) developed a methodology to estimate permeability from fracture-aperture distributions. Their basic result is summarized in the following equation:

$$
m_{0}=-C \frac{\pi s^{\alpha-1} \sec (\alpha \pi / 2)}{2^{\alpha-1}\left[\Gamma\left(\frac{\alpha+1}{2}\right)\right]^{2}}
$$

where $m_{0}$ is the variance of the fracture aperture, $s$ is the shear offset along the fracture, $\alpha$ is a scaling exponent, $C$ is a scale factor, and $\Gamma$ is the specialized mathematical gamma function. The aperture $b$ is related to variance by $b=3 \sqrt{m_{0}}$ for a Gaussian distribution (Brown, 1995). Finally, the permeability is assumed to satisfy the standard parallel-plate equation $k=\mathrm{b}^{2} / 12$. Combining these assumptions and taking logarithmic derivatives give the fractional change in $k$ as

$$
\frac{\Delta k}{k}=(\alpha-1) \frac{\Delta s}{s}
$$

The scaling exponent $\alpha$ is related to the fractal dimension $D$ of the fracture surface according to $\alpha=7-2$.D. Typical values of $D$ range between 2 , for smooth fractures, and 3 , for rough fractures (Brown, 1995). Therefore, maximum changes occur for $\alpha=3$. Assuming that the shear offset from thermal stress is the same as for a pre-existing slip (i.e., $\Delta s / s=1$ ) gives $\Delta k / k=2$. If $\alpha=1$, no change occurs in the permeability. In other words, the maximum change in permeability with this set of assumptions is that it doubles as a result of frictional slip. 
One limitation of the shear-slip model derived here is that stresses are not redistributed as a result of slip. Therefore, the possibility that multiple slip events could occur during heating has not been addressed. Under different assumptions from those used here, each occurrence of fracture slip could enhance the permeability.

\subsubsection{Discussion of Normal Deformation Models}

Models of normal stress effects on permeability fall into two general categories: explicitfracture models and porous-medium models. Porous-medium models typically conceptualize the medium as a packing of spherical grains. The stiffness of elastic sphere-tosphere contacts is well known in mechanics and is used to derive the macroscopic deformability. A rock mass with sufficient fracturing can be considered an effective porous medium for describing hydraulic response, and this assumption is made in TH simulations. However, the porous-medium model is not well suited for mechanistic description of THM coupling in fractured media because few, if any, of the model parameters are measurable.

Explicit-fracture models generally assume parallel-plate fracture geometry and use the "cubic law" representation of hydraulic transmissivity for a single fracture (Snow, 1965). Variations on this approach account for the hydraulic effects of aperture variability by using a tortuosity constant that reduces the parallel-plate transmissivity or by using statistical models that describe aperture as a spatially correlated random variable with corresponding average fracture-flow properties. Conceptual models for the deformation response of explicit fractures are based on abundant bench-scale normal and shear-deformation measurements reported in the literature. Shear displacement of a parallel-plate fracture does not change the transmissivity, but shear of a variable aperture fracture can produce large increases.

Extending an explicit model for fracture-normal displacement to estimate the effective properties for a rock mass, a set of parallel fractures is assigned values for the effective bulk permeability and deformation modulus. Fractures within a set are often assumed to have identical properties, to have uniform spacing, and to be hydraulically connected. The fracture spacing is required to describe rock-mass properties. Where there are multiple, hydraulically significant fracture sets, the directional flow properties from each can be superimposed. The deformability tensor for one or more sets of parallel fractures can be approximated using an approach such as that of Schoenberg (1980).

The concept of ubiquitous fractures is commonly used to model the deformability of a fractured medium. Whereas the hydraulic behavior of individual, randomly oriented fractures could be represented by the cubic law, the bulk permeability depends strongly on the connectivity between fractures. Connectivity is tedious to describe and difficult to measure, and it is not used in ubiquitous fracture models of medium deformability.

THM coupling in explicit-fracture models requires that the fractures be mechanically soft compared to the rock matrix, which is a reasonable representation for the repository host rock in which the ambient normal stress magnitudes are small. Medium deformability is partitioned between fractures and the intact matrix. The mechanical and hydraulic responses of fractures are inherently directional; thus, they determine directional properties of a fractured medium. THM coupling in partially saturated media differs from the general case for saturated media because the fluid pressure in fractures is negligible, and there is no effective stress correction.

For a 2-D medium with two perpendicular sets of fractures, a conceptual model for deformability and hydraulic conductivity of the medium is given by Ouyang and Elsworth (1993). Major points from their presentation are given below. The hydraulic conductivity of a set of parallel fractures is 


$$
K=\frac{g B^{3}}{12 \mu S}
$$

where $g$ is the acceleration of gravity, $\mu$ is the kinematic viscosity, $B$ is the fracture aperture, and $S$ is the fracture spacing. When fracture normal displacement $\Delta u$ occurs, the conductivity becomes

$$
K_{\Delta}=\frac{g}{12 \mu S}(B+\Delta u)^{3}
$$

Displacement $\Delta u$ can be defined in terms of the stress change $\Delta \sigma$ perpendicular to the fractures

$$
K_{\Delta}=\frac{g}{12 \mu S}\left(B+\frac{S \Delta \sigma}{E_{m}}\left[\frac{1}{R}-1\right]\right)^{3}
$$

where $R=E / E_{m}$ is the ratio of the rock-mass modulus $E$ to the intact matrix modulus $E_{m}$. Comparing the modified conductivity to the initial conductivity, the relative change is

$$
\frac{K_{\Delta}}{K}=\left(1+\frac{S \Delta \sigma}{B E_{m}}\left[\frac{1}{R}-1\right]\right)^{3}
$$

The notion of a constant fracture-normal stiffness is implicit in this formulation, thus its application is limited to displacements less than the initial aperture $(\Delta u \leq B)$. This is the simplest stiffness model and will tend to overestimate fracture closure, depending on the value selected for the rock-mass modulus reduction factor. The rock-mass modulus $(E)$ and modulus reduction factor $\left(R_{m}\right)$ describe deformability over the range of in situ loading conditions and are assumed not to vary with changes in stress.

It is of interest to apply this model in estimating the relative change in vertical conductivity of the repository host rock due to horizontal thermal compressive stress. Values for several rock-mass parameters are required. For representative values of fracture spacing $(S=0.3 \mathrm{~m})$ and bulk vertical permeability $\left(k=3.79 \times 10^{-12} \mathrm{~m}^{2}\right.$ is the fracture permeability in base-case TH simulations), the initial hydraulic fracture aperture can be calculated $(B=239 \mu \mathrm{m})$. From the FLAC calculations discussed previously, the horizontal stress increases during thermal loading from 5 to $15 \mathrm{MPa}(\Delta \sigma=-10 \mathrm{MPa})$. The matrix deformation modulus can be estimated from values in Table 4-2 ( $\left.E_{m}=35 \mathrm{GPa}\right)$. Using a value of 0.5 for the rock mass modulus reduction factor, the estimated relative change in permeability is about four-fold $\left(K_{\Delta} / K=0.26\right)$. For larger values of the modulus-reduction factor, smaller changes in conductivity are produced (e.g., for $R=0.75, K_{\Delta} / K=0.68$ ).

The permeability change suggested by the preceding calculation may be the product of elastic deformation and, therefore, reversible during unloading. The Topopah Spring tuff is competent, and the anticipated stress magnitudes are much less than the laboratorymeasured strength at elevated temperature. There are few data available upon which to judge the reversibility of permeability reduction in the repository host rock. Aquifer response to shallow underground mining sometimes exhibits reversal, but such observations transpire over days or months, not hundreds of years, and the exact mechanism is not generally known. Pneumatic-packer injectivity measurements for the SHT indicate a reduction in 
permeability at elevated temperature; again, the exact mechanism has not been determined. The duration of the repository effect will be on the order of $2000 \mathrm{yr}$, which is much longer than any modern observation and longer than any foreseeable field test at Yucca Mountain.

Assessment of the reversibility of fracture-normal displacement must be based on the few facts available. Fractures in tuff are loaded by in situ stress, and they exhibit elastic relaxation behavior when mechanically unloaded (e.g., Zimmerman and Finley, 1987; Zimmerman et al., 1986). The present condition of natural fractures is the result of equilibration, at ambient temperature, over geologic time. Based on a few laboratory tests, rheological behavior of the Topopah Spring tuff matrix is not observed to change much at $96^{\circ} \mathrm{C}$ relative to the behavior at ambient temperature (Hardin and Chesnut, 1997). It is therefore likely that some, if not all, of the fracture closure caused by TM stress will be recovered during cool-down. The residual effect after cool-down will be some fraction of the initial change, which can be estimated from the DST. The expected small magnitude of residual changes is the basis for selection of the shear-slip model to predict the measurable rock-mass response to heating in the DST.

\subsubsection{Discussion}

From the preceding discussion it is clear that, whereas understanding of rock-mass permeability changes in the repository host rock is important, no rigorous basis has yet been developed for estimating TM effects. Available models are based on concepts such as the cubic law, Mohr-Coulomb slip criteria, idealized fracture stiffness, hypothetical scaling relations, and statistical assumptions. The calculated stress changes also depend on repository design features that are not finalized. As described in Section 4.4.1, analysis of the DST presents a unique opportunity to experimentally test conceptual models for TM effects on permeability. Prediction of rock-mass permeability changes for the DST, using a method based on shear-slip criteria, is presented in Section 4.6.

\subsection{Thermohydromechanical Changes Predicted for the Drift-Scale Test}

\subsubsection{Predicted Changes}

In general, three fracture sets have been identified in the ESF (Albin et al., 1997):

- Set $\# 1$ is a steeply dipping set of fractures striking E-W.

- Set \#2 is a steeply dipping set of fractures striking N-S.

- Set \#3 is a subhorizontal set of fractures striking E-W.

The axis of the heated drift is oriented E-W; hence, set \#1 and set \#3 strike perpendicular to the plane of the 2-D FLAC model representing the DST. Thus, the calculations of shear slip for vertical and horizontal fractures correspond to set \#1 and set \#3, respectively.

To estimate regions of increased permeability for the DST, the conceptual model developed in the previous section is applied, with the assumption that permeability will double at any location where fracture slip is predicted to occur. It is also assumed that slip on one set of fractures does not interfere with slip on any other set and that changes in permeability predicted for one set of fractures can be added linearly to changes predicted for the other set. Thus, if a zone of enhanced permeability predicted for slip along a vertical set of fractures overlaps a zone of enhanced permeability predicted for a set of horizontal fractures, four-fold total (isotropic) permeability enhancement is predicted. This is comparable to an assumption that all connected pathways for fluid movement traverse fractures from both systems. In addition, small changes are assumed, and thus they are additive. 
Predicted zones of enhanced permeability due to excavation of the DST are shown in Figure 4-62. This figure indicates that drift excavation will increase permeability in a region extending up to half a drift diameter from the drift wall. This is typical for underground excavations.

\section{Figure 4-62 Zones of enhanced permeability predicted for the DST before heating}

Permeability changes predicted to occur after $0.5 \mathrm{yr}$ of heating are shown in Figure 4-63. Permeability may be enhanced in two, large, symmetric V-shaped regions, above and below the plane of the guard heaters. The scale of these regions is on the order of the separation of the drifts, and the width is on the order of half the drift separation.

\section{Figure 4-63 Zones of enhanced permeability predicted for the DST at 0.5 $\mathrm{yr}$}

As discussed previously, these regions are derived from the estimates of frictional slip for fracture sets oriented in both vertical and horizontal planes. The regions of frictional slip for planes of different orientation correlate with the principal stresses. Vertical fractures are expected to be favorably oriented for slip when they are approximately $30^{\circ}$ to the maximum principal stress direction; inspection of the stress field shows that $0.5 \mathrm{yr}$ after the start of heating, the ratio of maximum to minimum principal stresses $10 \mathrm{~m}$ above and below the heater plane becomes quite large and that the maximum stress direction rotates approximately $30^{\circ}$ relative to the vertical.

Horizontal fractures are favorably oriented for slip when the maximum principal stress lies approximately $30^{\circ}$ to the horizontal and the ratio of maximum to minimum principal stress becomes large. These conditions are met for horizontal fractures in regions between the wing heater and the access drift, centered at a distance of about $4 \mathrm{~m}$ above and below the plane of the wing heaters.

In Figure 4-63, two zones are shown where permeability is predicted to increase four-fold. These zones occur where both fracture sets are expected to slip, and they are also symmetric above and below the heater plane. They are to the side of the heater and constitute approximately $25 \%$ of the total area of permeability enhancement.

Figure 4-64, Figure 4-65, Figure 4-66, and Figure 4-67 show that the predicted zone of enhanced permeability will grow with time, while the same basic shape is maintained. Figure 4-66 and Figure 4-67 indicate that the zone of enhanced permeability may recede outward from the heaters after $2 \mathrm{yr}$ of heating. Comparison with stress plots shows that the permeability is enhanced in areas of high thermal gradients, as required by the formulation.

Figure 4-64 Zones of enhanced permeability predicted for the DST at 1.0 yr

Figure 4-65 Zones of enhanced permeability predicted for the DST at 2.0 $\mathrm{yr}$

Figure 4-66 Zones of enhanced permeability predicted for the DST at $3.0 \mathrm{yr}$

Figure 4-67 Zones of enhanced permeability predicted for the DST at $4.0 \mathrm{yr}$ 


\subsection{Thermohydromechanical Changes Predicted for Reference Drift-Scale Repository Cases}

The objective of this section is to evaluate the TM stress fields generated for reference Case 1 and Case 5 in terms of the potential to cause shear slip on fractures and consequent permanent alteration of TH properties. No effort is made to quantify the resulting permeability changes. (Reference cases for investigation of coupled processes in the NF/AZ Models Report are visually depicted and linked in the presentation on the CD-ROM that accompanies this report.)

The potential for permeability change is evaluated by first assuming that for slip to occur on a given fracture plane, the stress must exceed a Mohr-Coulomb slip criterion such as that given in Eq. 4-4. Values for friction parameters are given in Table 4-2. The repository host rock generally contains three sets of fractures: two subvertical and one subhorizontal. For this discussion, shear stress / strength ratios are calculated for vertical and horizontal fractures. Note that, due to the 2-D idealized geometry, these represent only a subset of the fractures and shear directions possible in the repository. For example, for vertical fractures, the fracture plane is assumed to be oriented parallel to the drift axis. Similarly, slip on horizontal fractures is considered only in the direction perpendicular to the drift axis.

Potential zones of slip are predicted using FLAC for vertical and horizontal fractures for Case 1 at 50 and 100 yr. These zones are shown in Figure 4-68, Figure 4-69, Figure 4-70, Figure 4-71, respectively, as areas where the shear ratio is greater than or equal to 1 . Slip on fractures due to induced shear stresses is expected to be limited to a few small zones that are within about one drift diameter of the drift wall. If equivalent calculations are performed using thermal-stress magnitude estimates from the FRACROCK simulations, similar zones of shear are obtained. This is in contrast to the relatively large zones of slip that are predicted for the DST using this same technique. Shear is predicted to develop in the DST because of the geometry of the wing heaters. The repository drift problem has more symmetry, and only line-heat sources perpendicular to the model are considered. Calculation of stress on a crosssection along the axis of the drift would produce a different result.

Figure 4-68 Ratio of shear stress to frictional strength for vertical fractures for Case 1 after 50 yr heating, computed using FLAC

Figure 4-69 Ratio of shear stress to frictional strength for horizontal fractures for Case 1 after 50 yr heating, computed using FLAC

Figure 4-70 Ratio of shear stress to frictional strength for vertical fractures for Case 1 after 100 yr heating, computed using FLAC

Figure 4-71 Ratio of shear stress to frictional strength for horizontal fractures for Case 1 after 100 yr heating, computed using FLAC

\subsection{Summary of Thermomechanical Models}

This chapter presents a method for estimating changes in permeability from TM effects. The method is similar to published approaches (Barton et al., 1985) and considers only effects from shear displacement, which has greater potential to produce permanent changes than does normal displacement. The method will provide the basis for estimating the magnitude 
of permanent changes in permeability associated with repository heating and will be tested by comparison to changes in permeability associated with the DST.

For the DST, TM effects may cause significant enhancement of permeability in a significant volume of the rock mass. Permeability is predicted to increase where temperature and thermal-stress gradients develop. A zone of enhanced permeability is likely to accompany the outwardly diffusing thermal "wave" as it emanates from the heaters. Permeability is predicted to increase by a factor of 2 to 4 , within a significant portion of the rock mass heated by the DST, within the first few months of heating. For the reference cases used to investigate coupled processes in this report, small changes in permeability are predicted using the FLAC-calculated stress fields for Case 1. Slip on fractures due to induced shear stresses is expected to be limited to a few small zones that are within about one drift diameter of the drift wall.

A critical link in the proposed method for THM coupling is the concept that permeability enhancement occurs as a result of shear offset due to Mohr-Coulomb slip on pre-existing fractures. This concept can be tested by comparing to predictions both displacement measurements and permeability measurements made during the DST. Other experiments, including the LBT and the SHT (Lin et al., 1997) provide similar opportunities for model testing.

As a final note, the guard heater geometry used in the DST introduces TM effects that may differ significantly from repository drift-scale effects. The DST heater arrays were designed primarily to achieve representative TH conditions (Wilder, 1996). Additional work is needed to improve predictions of permeability changes in response to repository heating.

\section{Return to Table of Contents}

\subsection{References}

Ahola, M.P., A.H. Chowdhury, S.M. Hsiung, and J. Philip (1994). “Comparison of Coupled Thermal-Mechanical-Hydrological Analyses of a Fractured Rock Mass." In proceedings from Fifth Annual International High-Level Radioactive Waste Management Conference. Las Vegas, NV: Am. Nucl. Soc. and Am. Soc. of Civ. Eng. 4:2492-2499. [235563]

Albin, A.L., W.L. Singleton, T.C. Moyer, A.C. Lee, R.C. Lung, G.L.W. Eatman, and D.L. Barr (1997). "Geology of the Main Drift-Station 28+00 to 55+00, Exploratory Studies Facility. Yucca Mountain Project, Yucca Mountain, Nevada." Denver, CO: Bureau of Reclamation and U.S. Geological Survey. [MOL.19970625.0096]

Archambault, G., S. Gentier, J. Riss, and R. Flamand (1997). “The evolution of void spaces (permeability) in relation with rock joint shear behavior." Int. J. Rock Mech. Min. Sci. 34:3-4.

Arulmoli (1987). “Analysis of Horizontal Waste Emplacement Boreholes of a Nuclear Waste Repository in Tuff." (SAND88-7133). Albuquerque, NM: Sandia National Laboratories. [202838]

Auld, B.A. (1973). Acoustic Fields and Waves in Solids, Vol. 1 and 2. New York, NY: John Wiley \& Sons.

Badan, B., M. Magrini, and E. Ramous (1991). “A study of the microbiological-corrosion products of steel and cast iron pipes in fresh water." J. Mater. Sci. 26:1951-1954.

Bandis, S., A.C. Lumsden, and N.R. Barton (1983). "Fundamentals of rock joint deformation." Int. J. Rock Mech. Min. Sci. and Geomech.(Abstr. 20):249-268. [224201] 
Barton, C.A., S. Hickman, R. Morin, M.D. Zoback, T. Finkbeiner, J. Sass, and D. Benoit (1997). "Fracture permeability and its relationship to in-situ stress in the Dixie Valley, Nevada, Geothermal Reservoir." In proceedings from Twenty-Second Workshop on Geothermal Reservoir Engineering. Stanford University, CA: Jan. 27-29, 1997.

Barton, N.R., S. Bandis, and K. Bakhtar (1985). "Strength, deformation, and conductivity coupling of rock joints." Int. J. Rock Mech. Min. Sci. and Geomech. 22(3):121-140. [217135]

Bauer, S.J., and L.S. Costin (1990). "Thermal and Mechanical Codes First Benchmark Exercise Part II: Elastic Analysis." (SNL-89-0757). Albuquerque, NM: Sandia National Laboratories. [200472]

Berryman, J.G. (1996). “Mixture Theories for Rock Properties.” T.J. Ahrens (ed.). Rock Physics $\mathcal{E}$ Phase Relations, A Handbook of Physical Constants. Washington, DC: American Geophysical Union. pp. 205-228. (AGU Reference Shelf 3)

Blair, S.C. (1994). "Analysis of Compressive Fracture in Rock Using Statistical Techniques." Berkeley, CA: University of California at Berkeley, Dept. of Materials Science and Mineral Engineering. Ph.D. Thesis.

Blair, S.C., and P.A. Berge (1996a). “Geomechanical Properties of Topopah Spring Tuff at the $0.5 \mathrm{~m}$ Scale: Preliminary Results of Compression Tests at Elevated Temperature." (UCRL-ID-125089). Livermore, CA: Lawrence Livermore National Laboratory. [MOL.19961107.0096; 226211]

Blair, S.C., and P.A. Berge (1996b). "Geomechanics.” Near-Field and Altered-Zone Environment Report, Vol. II. Livermore, CA: Lawrence Livermore National Laboratory. Chapter 4. (UCRL-LR-124998, Vol. II) [MOL.19961212.0122]

Blair, S.C., and P.A. Berge (1996c). “Uniaxial compression behavior of small blocks of welded tuff." In proceedings from Seventh International High-Level Radioactive Waste Management Conference. New York: Am. Nucl. Soc., La Grange Park, IL. 409411. (Also UCRL-JC-122733, Lawrence Livermore National Laboratory, Livermore, CA) [231905]

Blair, S.C., P.A. Berge, and H.F. Wang (1995). “Geomechanical Analysis of the Large Block Test." (UCRL-ID-122898). Livermore, CA: Lawrence Livermore National Laboratory. [MOL.19970506.0117]

Bodvarsson, G.S., T.M. Bandurraga, and Y.S. Wu (1997). “The Site-Scale Unsaturated Zone Model of Yucca Mountain, Nevada, for the Viability Assessment." June 1997. (LBNL-40376, UC-814). Berkeley, CA: Lawrence Berkeley National Laboratory, in collaboration with the U.S. Geological Survey. [MOL.19971014.0232]

Borgesson, L., and J. Hernelind (1995). “DECOVALEX I - Test Case 3: Calculation of the BIG BEN Experiment - Coupled Modelling of the Thermal, Mechanical and Hydraulic Behaviour of Water-Unsaturated Buffer Material in a Simulated Deposition Hole." Technical Report. (SKB 95-29). Stockholm, Sweden: Swedish Nuclear Fuel and Waste Management Co. [224376]

Brodsky, N.S., M. Riggins, and J. Connolly (1997). “Thermal Expansion, Thermal Conductivity, and Heat Capacity Measurements at Yucca Mountain, Nevada." Int. J. Rock Mech. Min. Sci. 34:3-4. (paper no. 40).

Brown, S.R. (1995). "Simple mathematical model of a rough fracture." J. Geophys. Res. 100:5941-5952. [222415]

Brown, S.R., and R.L. Bruhn (1997). "Fluid Permeability of Deformable Fracture Networks." (SAND97-0159). Albuquerque, NM: Sandia National Laboratories. 
Buscheck, T.A., and J.J. Nitao (1995). “Thermal-Hydrological Analysis of Large-Scale Thermal Tests in the Exploratory Studies Facility at Yucca Mountain." (UCRL-ID121791). Livermore, CA: Lawrence Livermore National Laboratory. [MOL.19960501.0392; 223657]

Chan, T., K. Khair, L. Jing, M. Ahola, J. Noorishad, and E. Vuillod (1995). “International comparison of coupled thermo-hydro-mechanical models of a multiplefracture bench mark problem: DECOVALEX Phase I, bench mark test 2 (in "Thermo-Hydro-Mechanical Couplings in Rock Mechanics")." Int. J. Rock Mech. Min. Sci. E Geomech. Abstr. 32(5).

Costin, L.S. (1987). “Time-Dependent Deformation and Failure.” B.K. Atkinson. Fracture Mechanics of Rock. London: Academic Press. (pp. 167-215). (ISBN: 0-12-0662655) [NNA.19890928.0110]

Crouch, S.L., and A.M. Starfield (1990). Boundary Element Methods in Solid Mechanics. Cambridge, MA: Unwin Hyman.

DOE (1990). “Yucca Mountain Project Reference Information Base, Version 4.” (YMP/CC0002). Las Vegas, NV: Yucca Mountain Site Characterization Project Office, U.S. Department of Energy. [221856]

Einstein, H.H., and W.S. Dershowitz (1990). "Tensile and shear fracturing in predominantly compressive stress fields-a review." Eng. Geol. 29:149-172.

Flint, L.E. (1998). “Characterization of Hydrogeological Units Using Matrix Properties, Yucca Mountain, Nevada." Water Resources Investigation Report. (WRIR 97-4243). U.S. Geological Survey.

Hardin, E.L., and D.A. Chesnut (1997). “Synthesis Report on Thermally Driven Coupled Processes." Milestone report for the CRWMS Management and Operating Contractor, U.S. Department of Energy. (SPL8BM3). Livermore, CA: Lawrence Livermore National Laboratory. (Also UCRL-ID-128495, Lawrence Livermore National Laboratory, Livermore, CA) [MOL.19980120.0035]

Hardy, M.P., and S.J. Bauer (1991). “Drift Design Methodology and Preliminary Application for the Yucca Mountain Site Characterization Project." (SAND89-0837). Albuquerque, NM: Sandia National Laboratories.

Hart, R.D. (1981). "Fully Coupled Thermal-Mechanical-Fluid Flow Model for Nonlinear Geologic Systems." Minneapolis, MN: University of Minnesota, Ph.D. Thesis. [NNA.900522.0114; 220092]

Hart, R.D., and P.A. Cundall (1992). “Microcomputer programs for explicit numerical analysis in geotechnical engineering." In proceedings from International Seminar on Numerical Methods in Geomechanics. Moscow, Russia.

Hibbitt, K.S., Inc., (1994). ABAQUS/Standard User's Manual (Version 5.4), Vols. I and II. Pawtucket, RI: Hibbitt, Karlsson \& Sorensen, Inc.

Hoek, E., and E.T. Brown (1980). Underground Excavation in Rock. London: The Institute of Mining and Metallurgy. [217420]

Israelsson, J. (1995). “DECOVALEX I — Bench-Mark Test 3: Thermo-Hydro-Mechanical Modelling." SKB Technical Report. (SKB 95-30). Stockholm, Sweden: Swedish Nuclear Fuel and Waste Management Company. [223944]

Itasca Consulting Group Inc. (1996). FLAC, Fast Lagrangian Analysis of Continua, Version 3.3, Vol. I-IV, User's Manuals. Minneapolis, MN: Itasca Consulting Group, Inc. 
Jaeger, J.C., and N.G.W. Cook (1976). Fundamentals of Rock Mechanics. London: Chapman and Hall. [209863]

Jiao, Y., and J.A. Hudson (1995). "The full-coupled model for rock engineering systems." Int. J. Rock Mech. Min. Sci. Geomech. Abst. 32(5):491-512.

Jing, L., C.-F. Tsang, and O. Stephansson (1995). “DECOVALEX—an international cooperative research project on mathematical models of coupled THM processes for safety analysis of radioactive waste repositories." Int. J. Rock Mech. Min. Sci. $\mathcal{E}$ Geomech. Abstr. 32(5).

Kemeny, J., and N. Cook (1990). "Rock Mechanics and Crustal Stress, Demonstration of a Risk-Based Approach to High-Level Waste Repository Evaluation." (EPRI NP7057). Palo Alto, CA: Electric Power Research Institute. (R.K. McGuire, ed.) [NNA.910813.0004; 216677]

LeCain, G.D., and J.N. Walker (1994). "Results of air-permeability testing in a vertical borehole at Yucca Mountain, Nevada." In proceedings from Fifth Annual International High-Level Radioactive Waste Management Conference. Las Vegas, NV: Am. Nucl. Soc., La Grange Park, IL. pp. 2782-2788. [MOL.19940829.0015]

Lee, K.H. (1995). “Progress Report on Pre-Test Calculations for the Large Block Test." (UCRLID-118699). Livermore, CA: Lawrence Livermore National Laboratory. [MOL.19950314.0178; 214965]

Lin, M., M.P. Hardy, and S.J. Bauer (1993). “Fracture Analysis and Rock Quality Designation Estimation for the Yucca Mountain Site Characterization Project." (SAND920449). Sandia National Laboratories. [NNA.19921204.0012; 205823]

Lin, W. (1994). “Preliminary characterization data for the Large Block Test obtained primarily to support construction decisions. Includes permeability, fracture density and initial moisture content." Scientific notebook. Livermore, CA: Lawrence Livermore National Laboratory. (Non-Q) [DTN LL940800804244.001]

Lin, W. (1998). “Update of the Large-Block Test.” March 6, 1998. Biweekly electronic report. (UCRL-MI-129071). Livermore, CA: Lawrence Livermore National Laboratory.

Lin, W., S. Blair, M. Buettner, T. Buscheck, W. Daily, G. Gdowski, G. Glassley, K. Lee, A. Ramirez, D. Ruddle, J. Roberts, S. Trettenero, D. Watwood, and R. Carlson (1997). "Thermal-mechanical-hydrological-chemical responses in the Single Heater Test at the ESF." In proceedings from International Society of Rock Mechanics and Geomechanics Abstract. New York, NY: June 29-May 2, 1997. International Symposium of the Int'1 Soc. Rock Mech. (UCRL-JC-125611) [MOL.19971210.0044]

Lin, W., and J.J. Roberts (1996). “Laboratory-Determined Hydrologic Properties and Processes." Near Field Environment Report. Livermore, CA: Lawrence Livermore National Laboratory. Chapter 2. (UCRL-124998, Rev. 1) [MOL.19961212.0122]

Lin, W., D. Wilder, J. Blink, P. Berge, S. Blair, V. Brugman, K. Lee, M. Owens, C. Radewan, A. Ramirez, N. Rector, J. Roberts, D. Ruddle, and J. Wagoner (1995). "A Progress Report on the Large Block Test." (UCRL-JC-119106-95). Livermore, CA: Lawrence Livermore National Laboratory. [MOL.19960208.0304]

MacDougall, H.R., L.W. Scully, and J.R. Tillerson (1987). “Site Characterization Plan Conceptual Design Report." (SAND84-2641). Albuquerque, NM: Sandia National Laboratories. [NNI.880902.0014; HQX.19871215.0048; 203922; 203538; 206486; 206487; 206488; 206489] 
Martin, R.J., J.S. Noel, P.J. Boyd, and R.H. Price (1996). “Thermal expansion as a function of confining pressure for welded tuff from Yucca Mountain." In proceedings from Second Annual North American Rock Mechanics Symposium. Aubertin, Hassani and Mitri (eds.). Montreal, Canada: June 19-21, 1996. Rotterdam: Balkema.

Martin, R.J., J.S. Noel, R.H. Price, and P.J. Boyd (1995a). “Creep Properties of the Paintbrush Tuff Recovered from Borehole NRG-7/7A." (SAND95-1759). Albuquerque, NM: Sandia National Laboratories. [MOL.19960419.0313]

Martin, R.J., R.H. Price, P.J. Boyd, and R.W. Haupt (1992). "Anisotropy of the Topopah Spring Member Tuff." (SAND91-0894). Albuquerque, NM: Sandia National Laboratories. [NNA.19920522.0041; 204665]

Martin, R.J., R.H. Price, P.J. Boyd, and J.S. Noel (1993). “Unconfined Compression Experiments on Topopah Spring Member Tuff at $22^{\circ} \mathrm{C}$ and a Strain Rate of $10^{-}$ 9 s ${ }^{-1}$." (SAND92-1810). Albuquerque, NM: Sandia National Laboratories. [NNA.19930728.0088; 208175]

Martin, R.J., R.H. Price, P.J. Boyd, and J.S. Noel (1994). “Bulk and Mechanical Properties of the Paintbrush Tuff Recovered from Borehole USW NRG-6." (SAND93-4020). Albuquerque, NM: Sandia National Laboratories. [MOL.19940811.0001; 213265]

Martin, R.J., R.H. Price, P.J. Boyd, and J.S. Noel (1995b). “Bulk and Mechanical Properties of the Paintbrush Tuff Recovered from Borehole USW NRG-7/7A." (SAND941996). Albuquerque, NM: Sandia National Laboratories. [MOL.19950316.0087; 215382]

Millard, A., N. Druin, A. Stietel, A. Thoraval, E. Vuillod, H. Baroudi, F. Plas, A. Bougnoux, G. Vouille, A. Kogayaski, K. Hara, T. Fujita, and Y. Ohnishi (1995). “Discrete and continuum approaches to simulate the thermo-hydro-mechanical couplings in a large, fractured rock mass." Int. J. Rock Mech. Min. Sci. E Geomech. Abstr. 32(5).

Nguyen, T.S., and A.P.S. Selvadurai (1995). “Coupled thermal-mechanical-hydrological behaviour of sparsely fractured rock: implications for nuclear fuel wasted disposal." Int. J. Rock Mech. Min. Sci. \& Geomech. Abstr. 32(5).

Nimick, F.B. (1990). "The Thermal Conductivity of the Topopah Spring Member at Yucca Mountain, Nevada." (SAND86-0090). Albuquerque, NM: Sandia National Laboratories. [NNA.19890516.0183; 202814]

Olsson, W.A., and S.R. Brown (1994). "Mechanical Properties of Seven Fractures from Drillholes NRG-4 and NRG-6 at Yucca Mountain, Nevada." (SAND94-1995).

Albuquerque, NM: Sandia National Laboratories. [MOL.19950208.0124; 213047]

Ortiz, T.S., R.L. Williams, F.B. Nimick, B.C. Whittet, and D.L. South (1985). “A ThreeDimensional Model of Reference Thermal/Mechanical and Hydrologic Stratigraphy at Yucca Mountain, Southern Nevada." (SAND84-1076). Albuquerque, NM: Sandia National Laboratories. [NNA.890315.0013; 201909]

Ouyang, Z., and D. Elsworth (1993). "Evaluation of groundwater flow into mined panels." Int. J. Rock Mech. Min. Sci. E Geomech. Abstr. 30(2).

Price, R. (1993). "Laboratory Determination of the Mechanical Properties of Fractures." (YMP Study Plan 8.3.1.15.1.4 RO). Washington, DC: U.S. Department of Energy.

[MOL.19960701.0275; 226121] 
Price, R.H. (1986). “Effects of Sample Size on the Mechanical Behavior of Topopah Spring Tuff." (SAND85-0709). Albuquerque, NM: Sandia National Laboratories. [NNA.891106.0125; 206259]

Price, R.H., J.R. Connolly, and K. Keil (1987). “Petrologic and Mechanical Properties of Outcrop Samples of the Welded, Devitrified Topopah Spring Member of the Paintbrush Tuff." (SAND86-1131). Albuquerque, NM: Sandia National Laboratories. [NNA.870601.0013; 202821]

Raven, K.G., and J.E. Gale (1985). "Water flow in a natural rock fracture as a function of stress and sample size." Int. J. Rock Mech. Min. Sci. E Geomech. Abstr. 22(4):251-261.

Rehbinder (1995). "Analytical solutions of stationary coupled thermo-hydro-geomechanical problems." Int. J. Rock Mech. Min. Sci. E Geomech. Abstr. 32(5).

Schoenberg, M. (1980). “Elastic wave behavior across linear slip interfaces." J. Acoustical Soc. Am. 68(5):1516.

Snow, D.T. (1965). “A Parallel Plate Model of Fractured Permeable Media.” Berkeley, CA: University of California at Berkeley, Ph.D. Thesis. [223751]

Stephansson, O. (1995). "Introduction to special issue on thermo-hydro-mechanical coupling in rock mechanics." Int. J. Rock Mech. Min. Sci. E Geomech. Abstr. 32(5).

Stock, J.M., J.H. Healy, and S.H. Hickman (1984). "Report on Televiewer Log and Stress Measurements in Core Hole USW G-2, Nevada Test Site." (OFR-84-172). Denver, CO: U.S. Geological Survey. [NNA.870406.0157; 208153]

Stock, J.M., J.H. Healy, S.H. Hickman, and M.D. Zoback (1985). “Hydraulic fracturing stress measurements at Yucca Mountain, Nevada, and relationship to regional stress field." J. Geophys. Res. 90(B10):8691-8706. [HQS.19880517.1509; 219009]

TRW (1996). “Test Design, Plans and Layout Report.” July 30, 1996. (BAB000000-01717-460000025). TRW Environmental Safety Systems, Inc. [MOL.19961122.0319]

Turcotte, D.L., and G. Schubert (1982). Geodynamics Applications of Continuum Physics to Geological Problems. New York, NY: John Wiley \& Sons. (450 pp.)

Wilder, D.G. (1987). "Influence of Stress-Induced Deformations on Observed Water Flow in Fractures at the Climax Granitic Stock." (UCRL-95539, Rev. 1). Livermore, CA: Lawrence Livermore National Laboratory. [NNA.19880425.0044; 203036]

Wilder, D.G. (1993a). "Preliminary Near-Field Environment Report, Volume I: Technical Bases for EBS Design." (UCRL-LR-107476, Vol. I). Livermore, CA: Lawrence Livermore National Laboratory. (NNA.19920501.0002)

Wilder, D.G. (1993b). "Preliminary Near-Field Environment Report, Volume II: Scientific Overview of the Near-Field Environment and Phenomenon." (UCRL-LR107476, Vol. II). Livermore, CA: Lawrence Livermore National Laboratory. [NNA.19920501.0002]

Wilder, D.G. (1996). “Near-Field and Altered-Zone Environment Report, Vol. II.” (UCRL-LR124998, Vol. II). Livermore, CA: Lawrence Livermore National Laboratory. [MOL.19961212.0121; MOL.19961212.0122]

Zhang, X., and D.J. Sanderson (1996). "Effects of stress on the two-dimensional permeability tensor of natural fracture networks." Geophys. J Intern 125(3).

Zimmerman, R.M., and R.E. Finley (1987). "Summary of Geomechanical Measurements Taken In and Around G-Tunnel Underground Facility, Nevada Test Site." (SAND86-1015). Albuquerque, NM: Sandia National Laboratories. [NNA.19870526.0015; 202819] 
Zimmerman, R.M., R.L. Schuch, D.S. Mason, M.L. Wilson, M.E. Hall, M.P. Board, R.P. Bellman, and M.L. Blanford (1986). "Final Report: G-Tunnel Heated Block Experiment." (SAND84-2620). Albuquerque, NM: Sandia National Laboratories. [MOL.19961217.0085; 202721] 



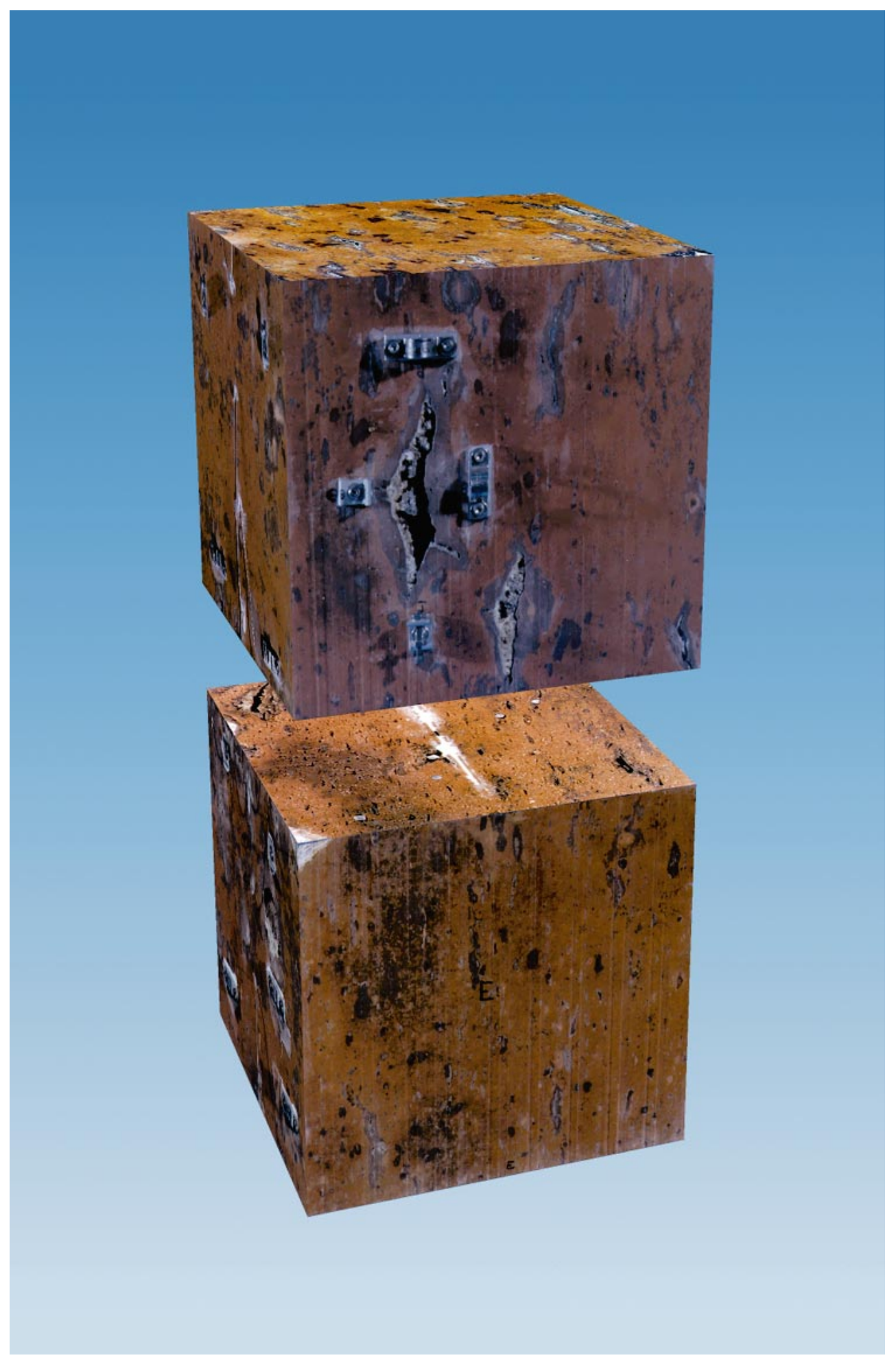

Figure 4-1. Illustration of small-block experiment for flow through horizontal fracture (fracture separation exaggerated) 
(a) history of fluid flow

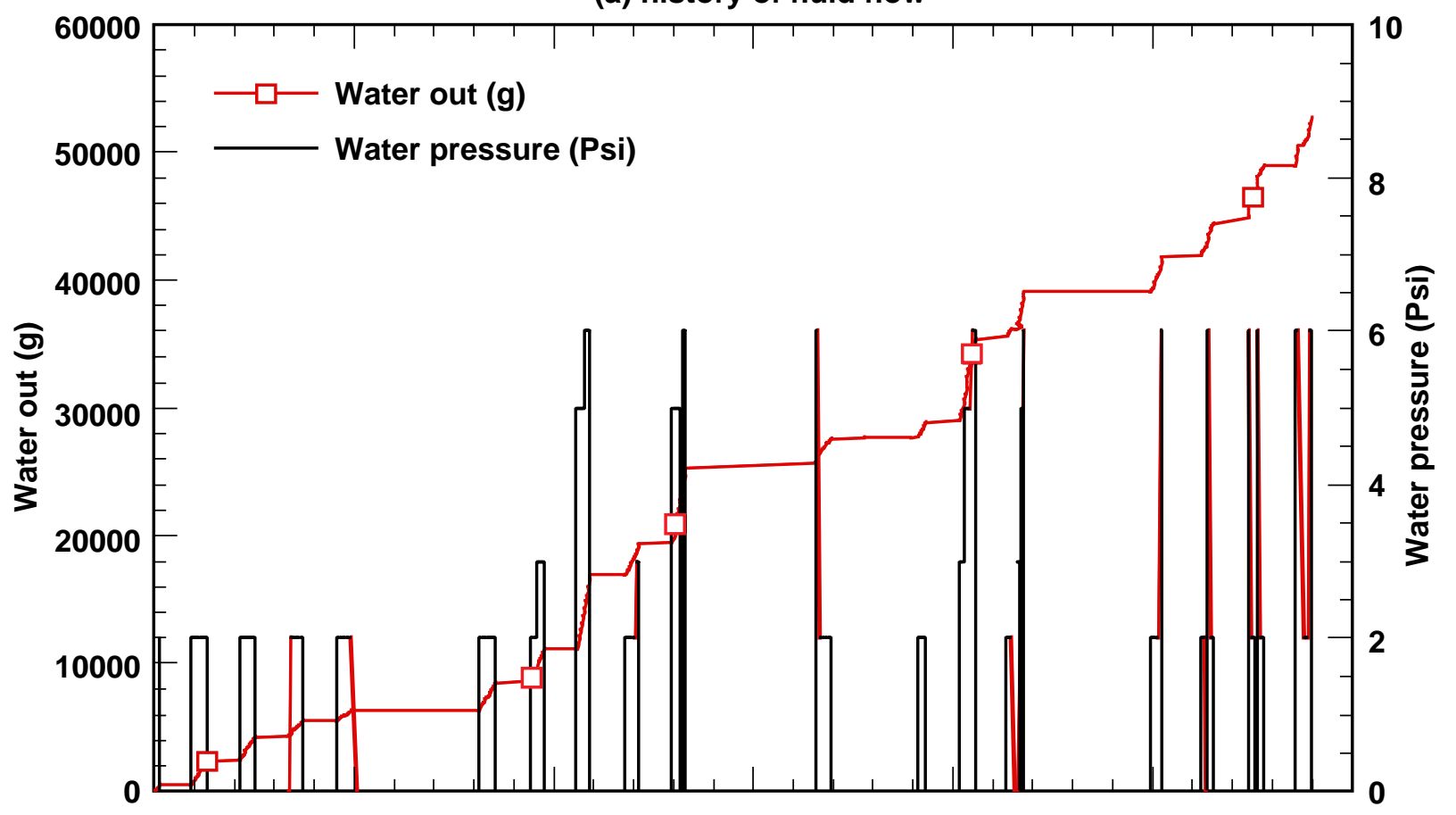

(b) history of axial stress

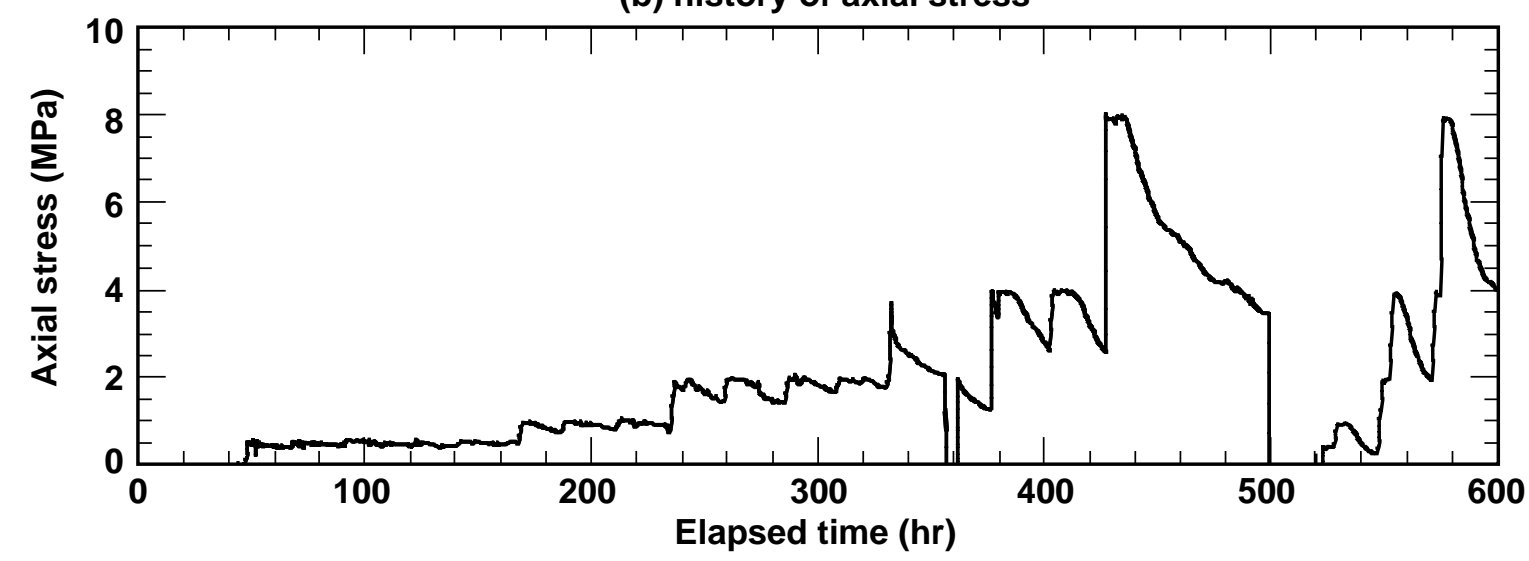

Figure 4-2. History of fluid flow and axial stress on sample SB-3 during first $600 \mathrm{hr}$ of testing: (a) history of fluid flow during first $600 \mathrm{hr}$ of testing of sample SB-3 during Test 7195 showing flow intervals and inlet pressures for the first portion of flow testing on this sample; (b) history of axial stress imposed on sample SB-3 during first $600 \mathrm{hr}$ of testing 


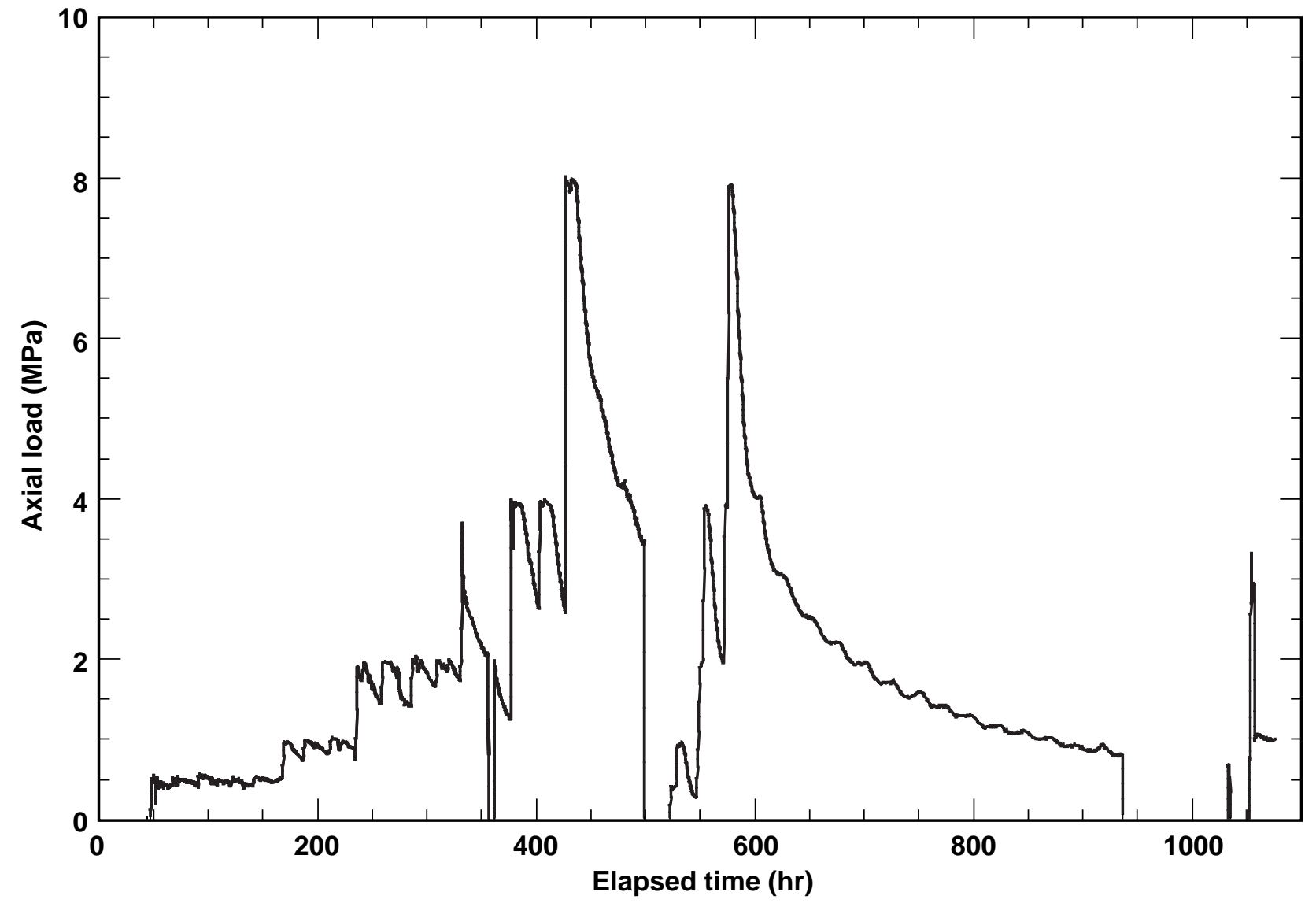

Figure 4-3. Axial load (uniaxial stress) vs. elapsed time for Test 7195 


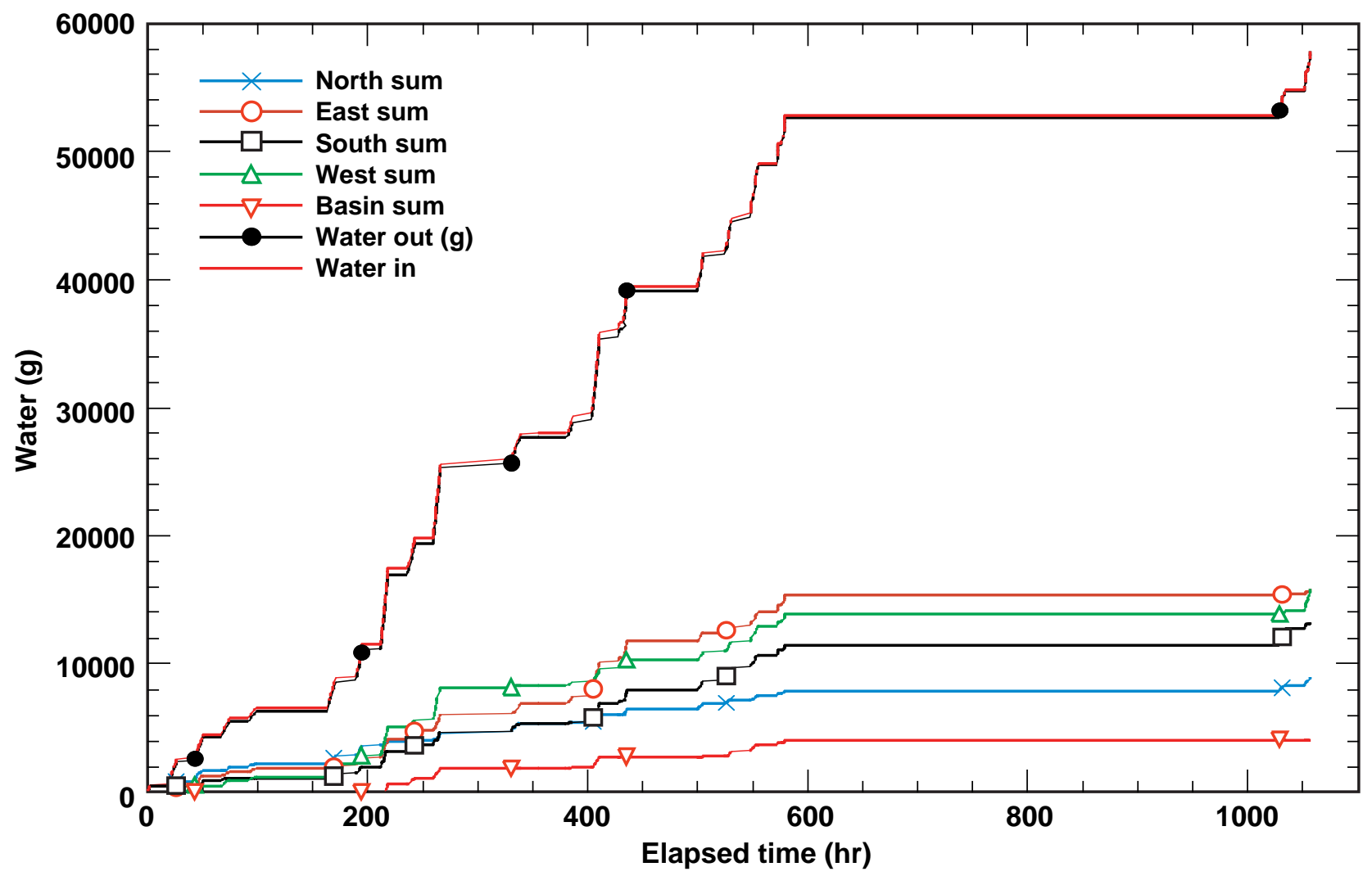

Figure 4-4. Cumulative flow data for Test 7195: shows overall cumulative flow into and out of the sample and cumulative flow out of the fracture along each face 


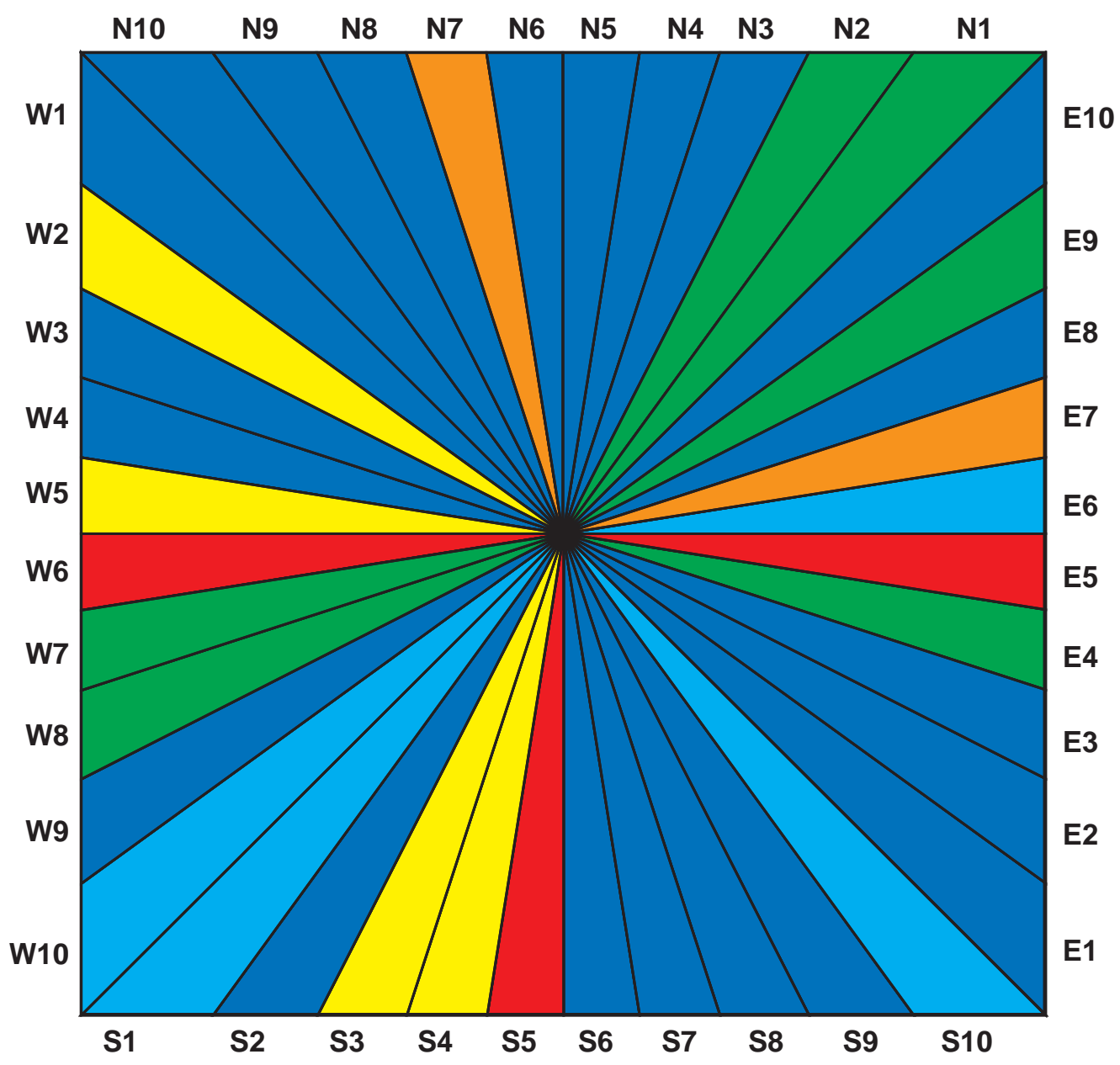

Figure 4-5. Spatial representation of total flow in plane of fracture for Sample SB-3 during Test 7195: assumes linear flow paths between the injection point at the center of the fracture plane and each port (note that the highest flows occur in the E-W direction, which is parallel to the textural anisotropy shown in Figure 4-3) 


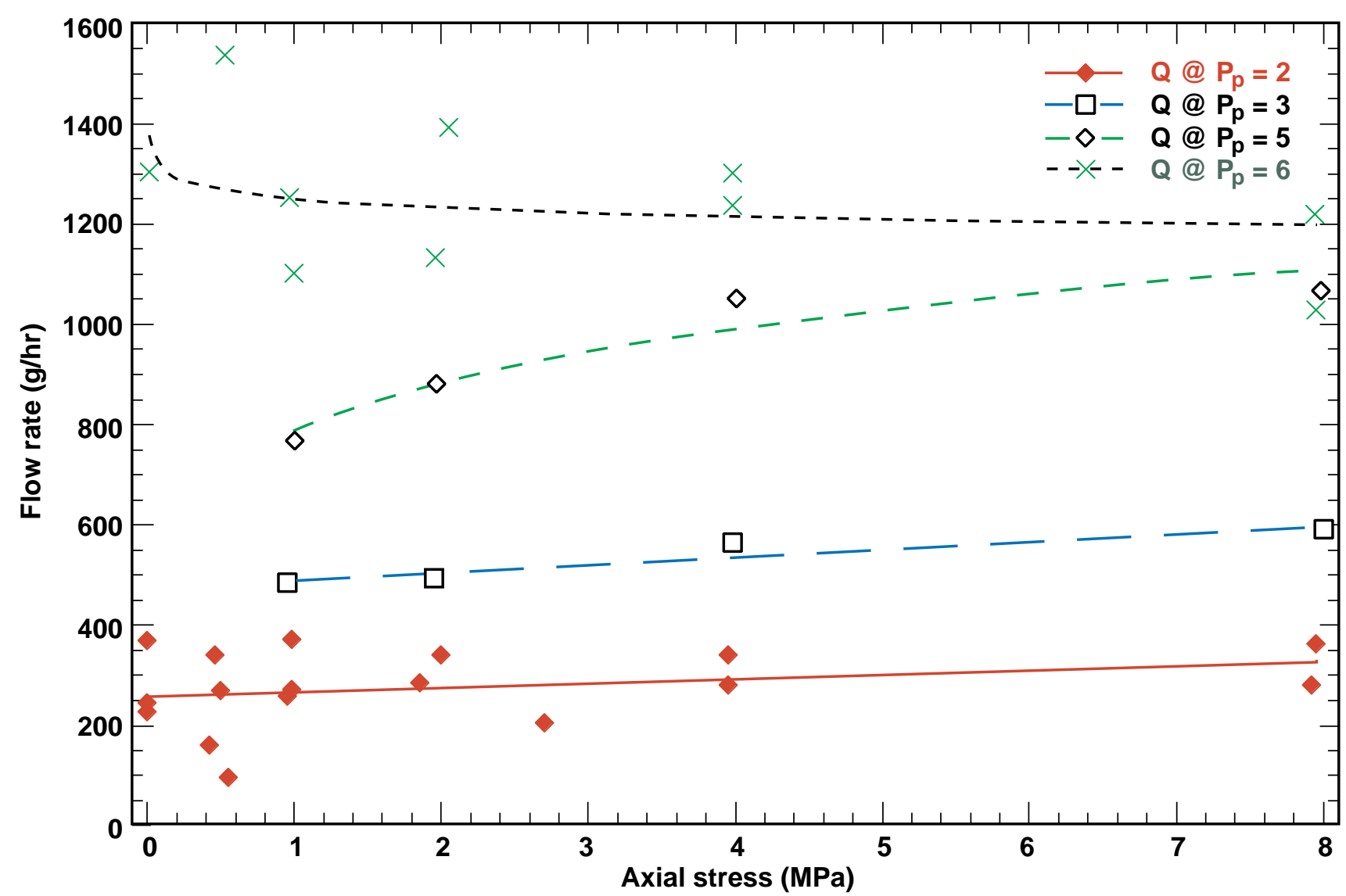

Figure 4-6. Flow rate at four different fluid inlet pressures, plotted as a function of axial stress for Test 7195 


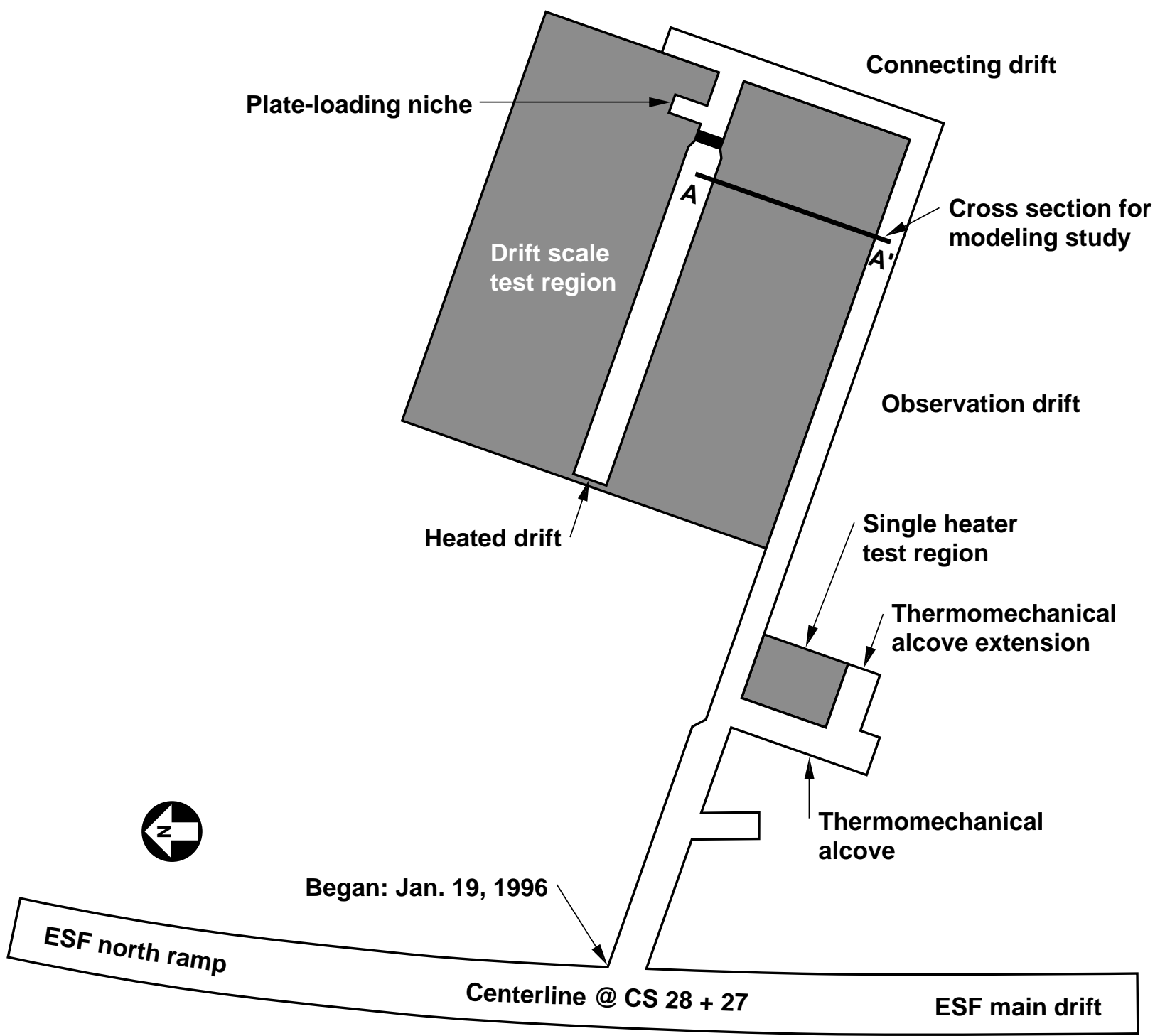

Reference only (scale approximate)

Figure 4-7. Schematic of the Drift-Scale Test 


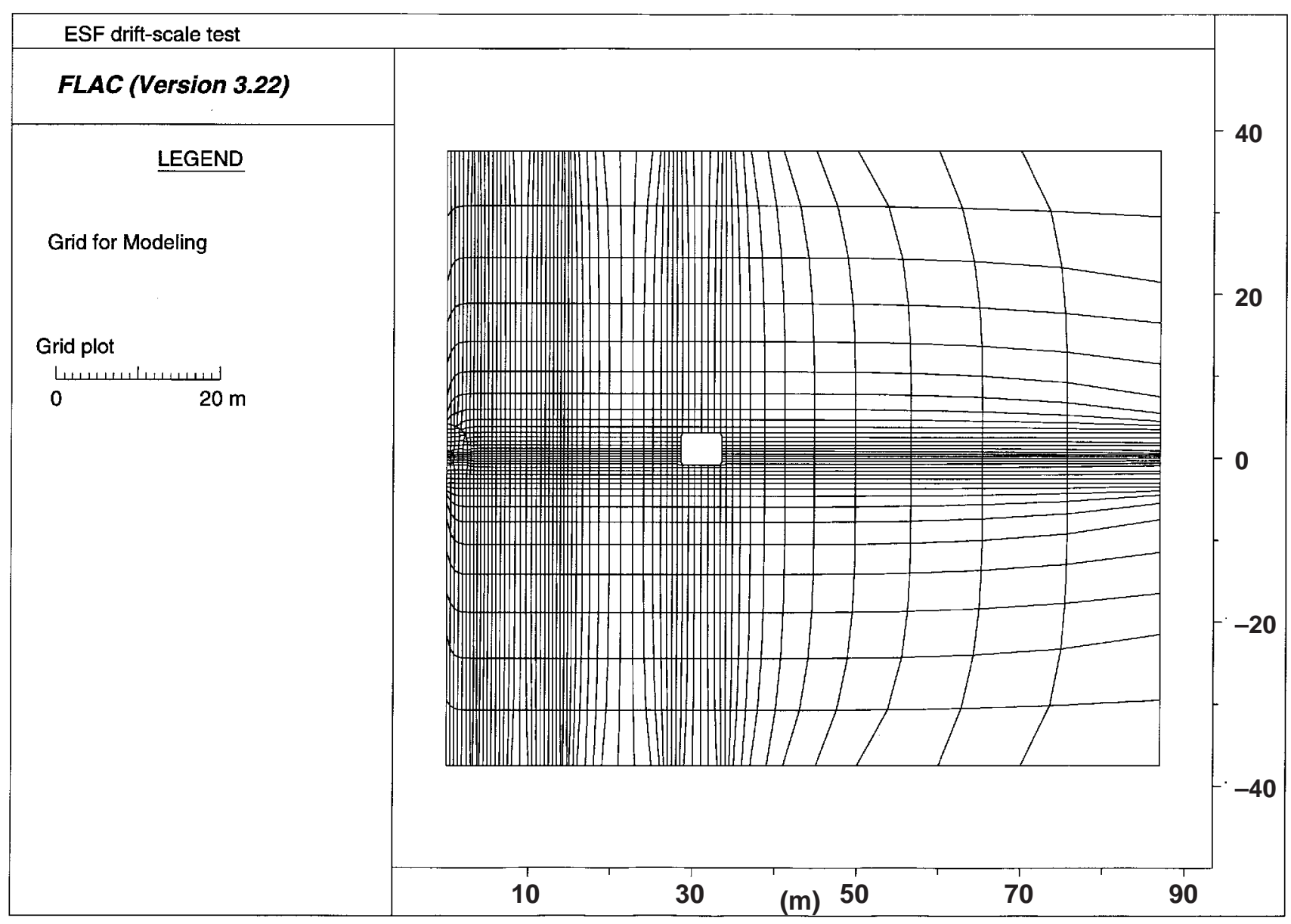

Figure 4-8. Total FLAC grid 


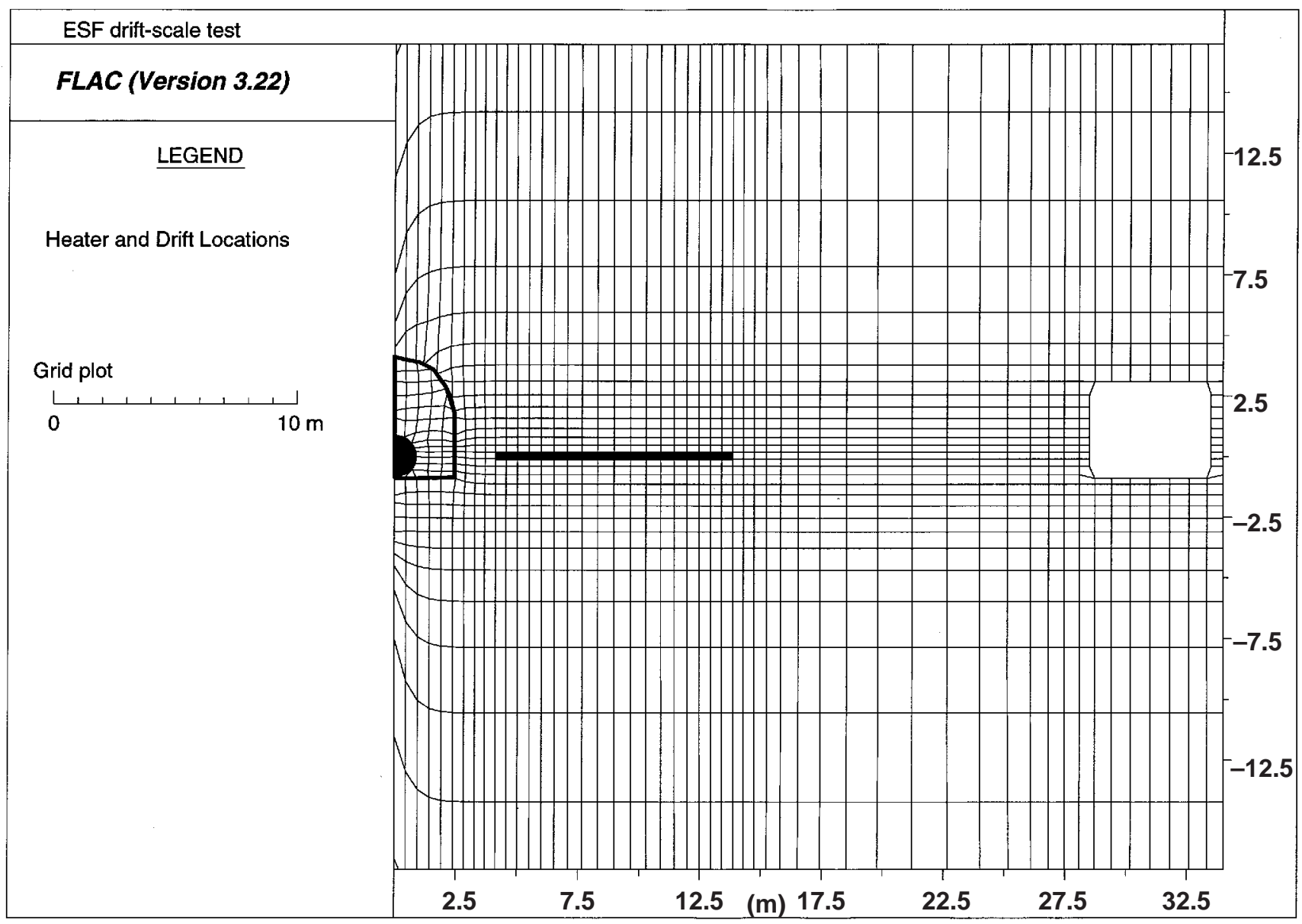

Figure 4-9. Enlarged portion of grid between heated drift and access drift 


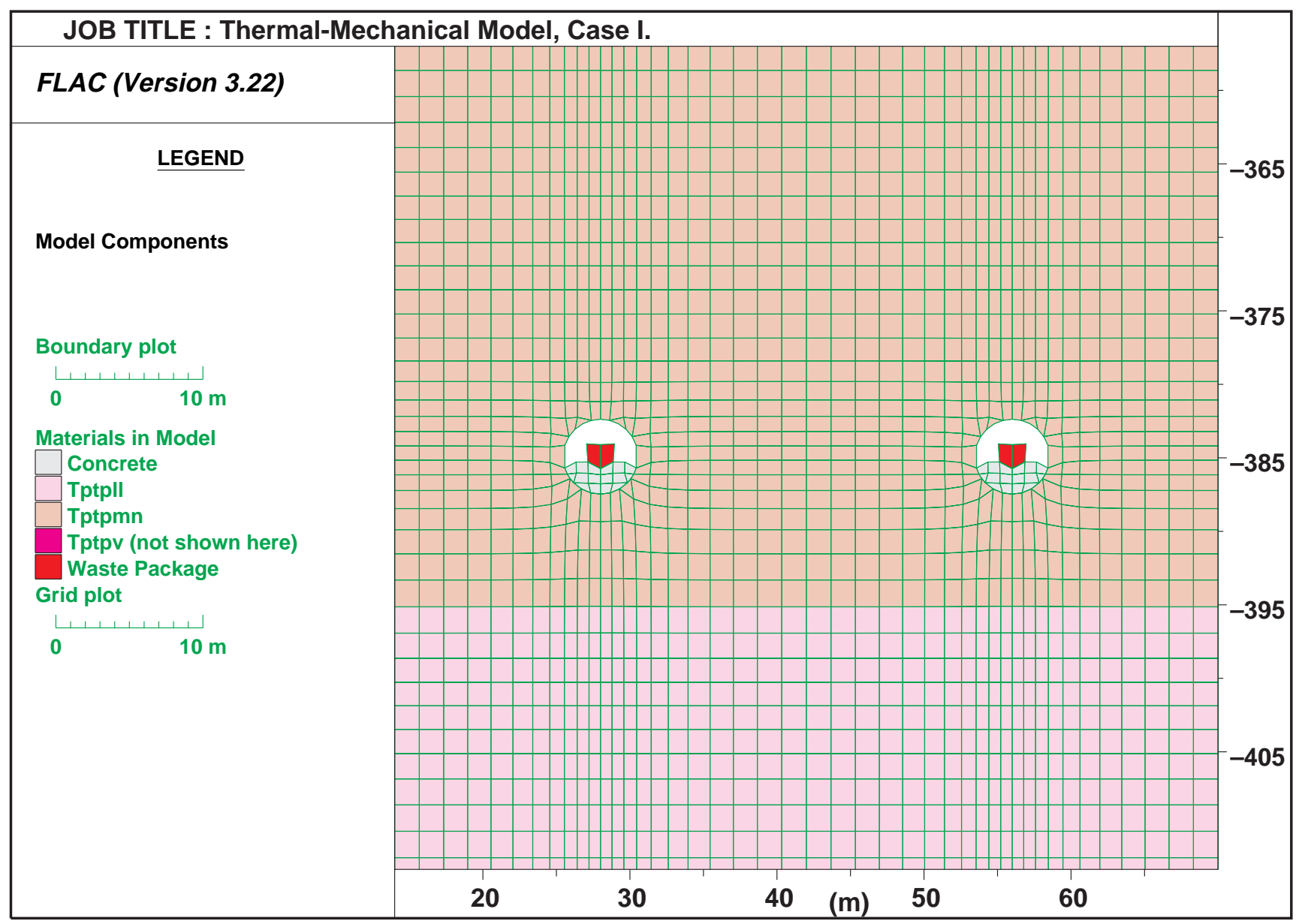

Figure 4-10. Portion of mesh used for FLAC simulations of reference Case 1 


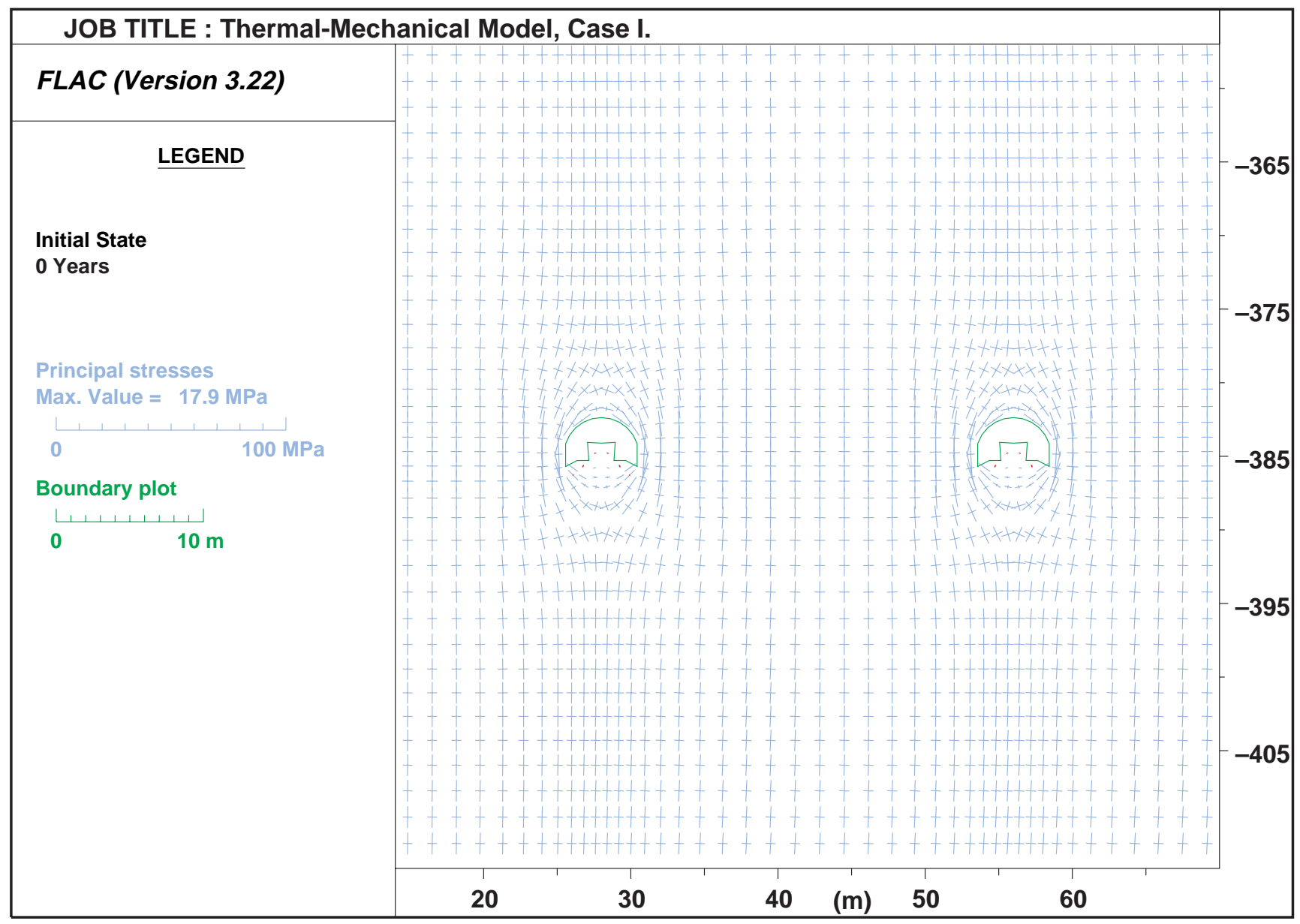

Figure 4-11. Principal stresses computed for excavation only using FLAC for Case 1 


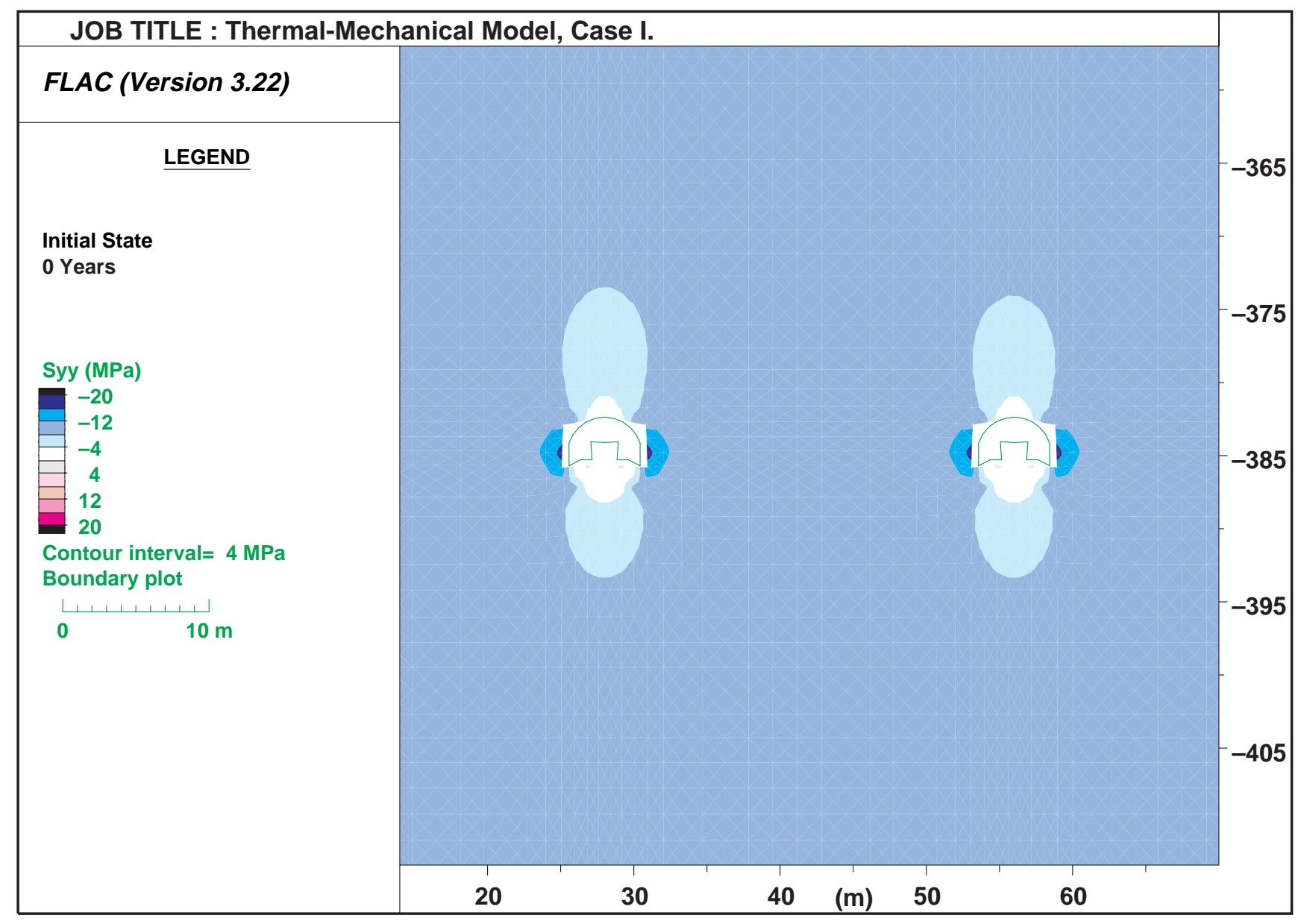

Figure 4-12. Vertical stress field computed using FLAC for excavation only, Case 1 


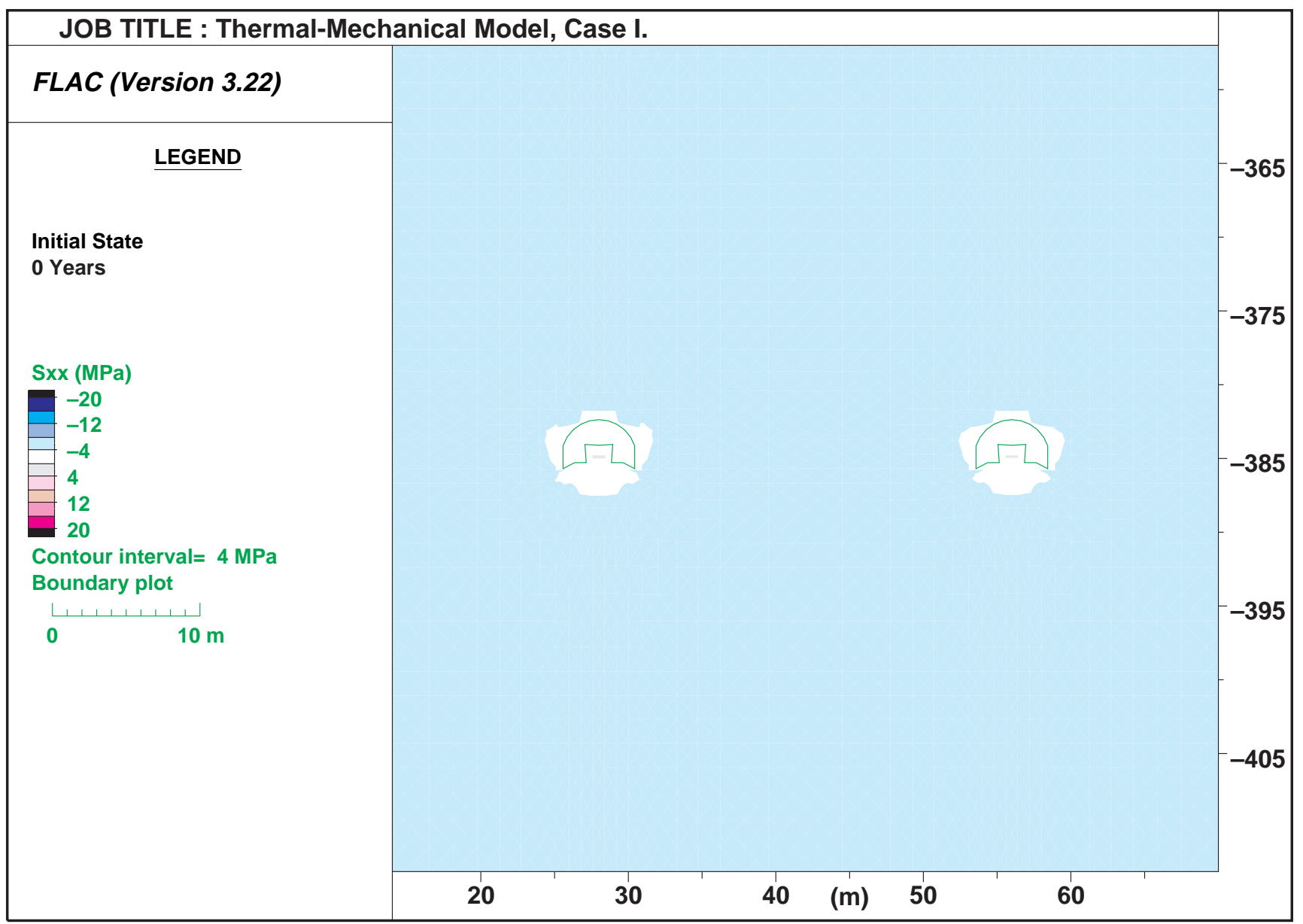

Figure 4-13. Horizontal stress field computed using FLAC for excavation only, Case 1 


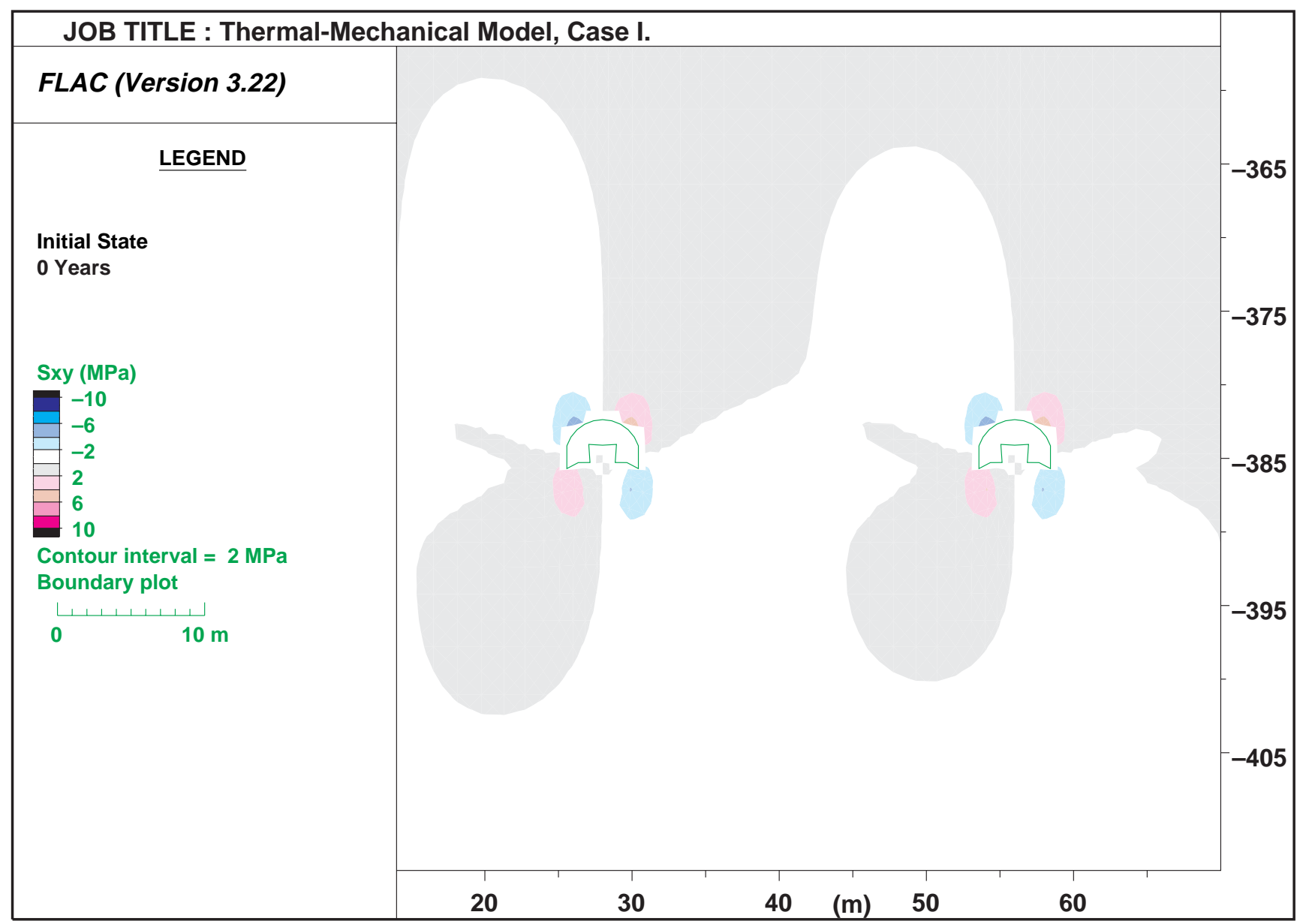

Figure 4-14. Shear stress field computed using FLAC for excavation only, Case 1 


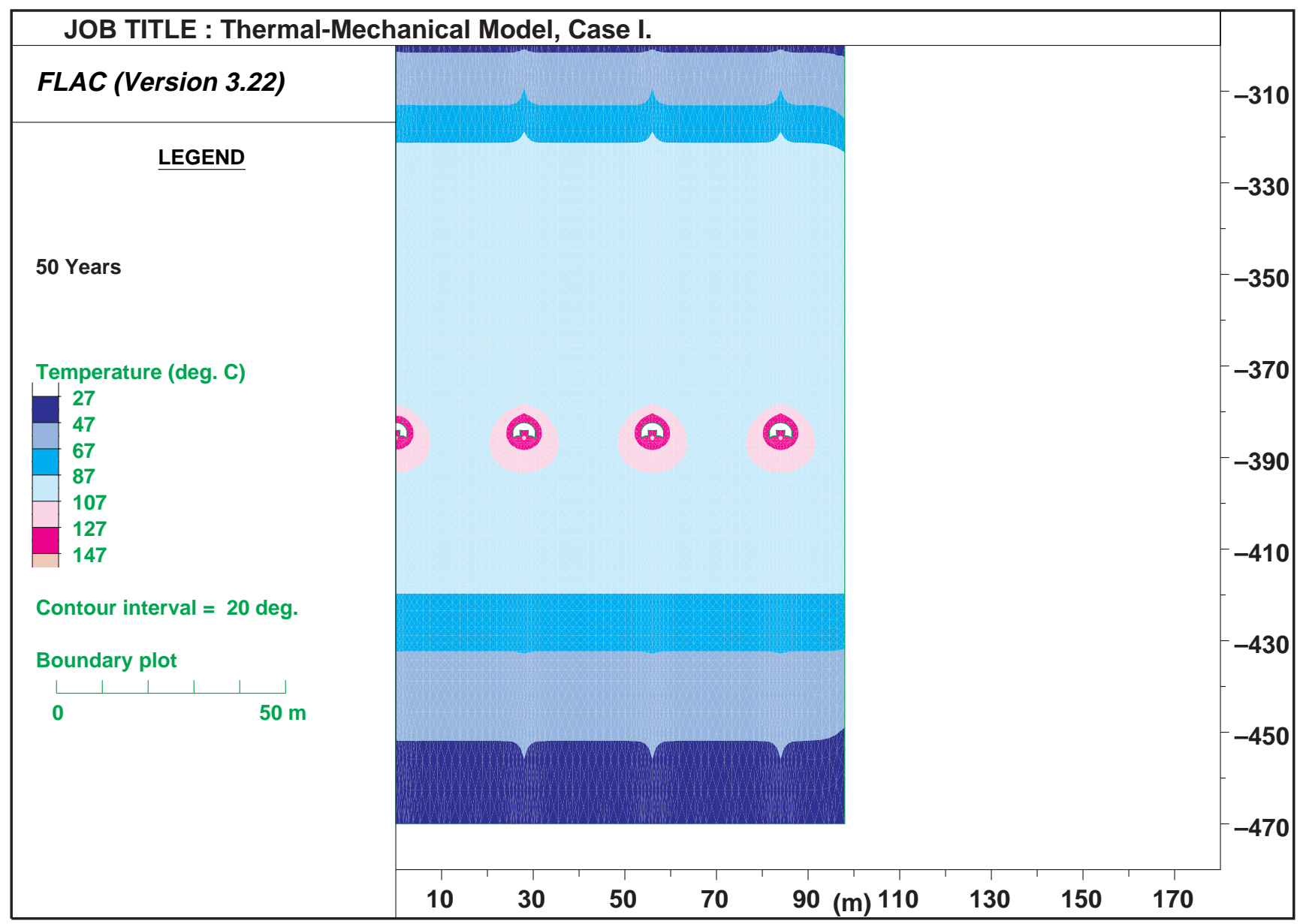

Figure 4-15. Temperature field for Case 1 at $50 \mathrm{yr}$ 


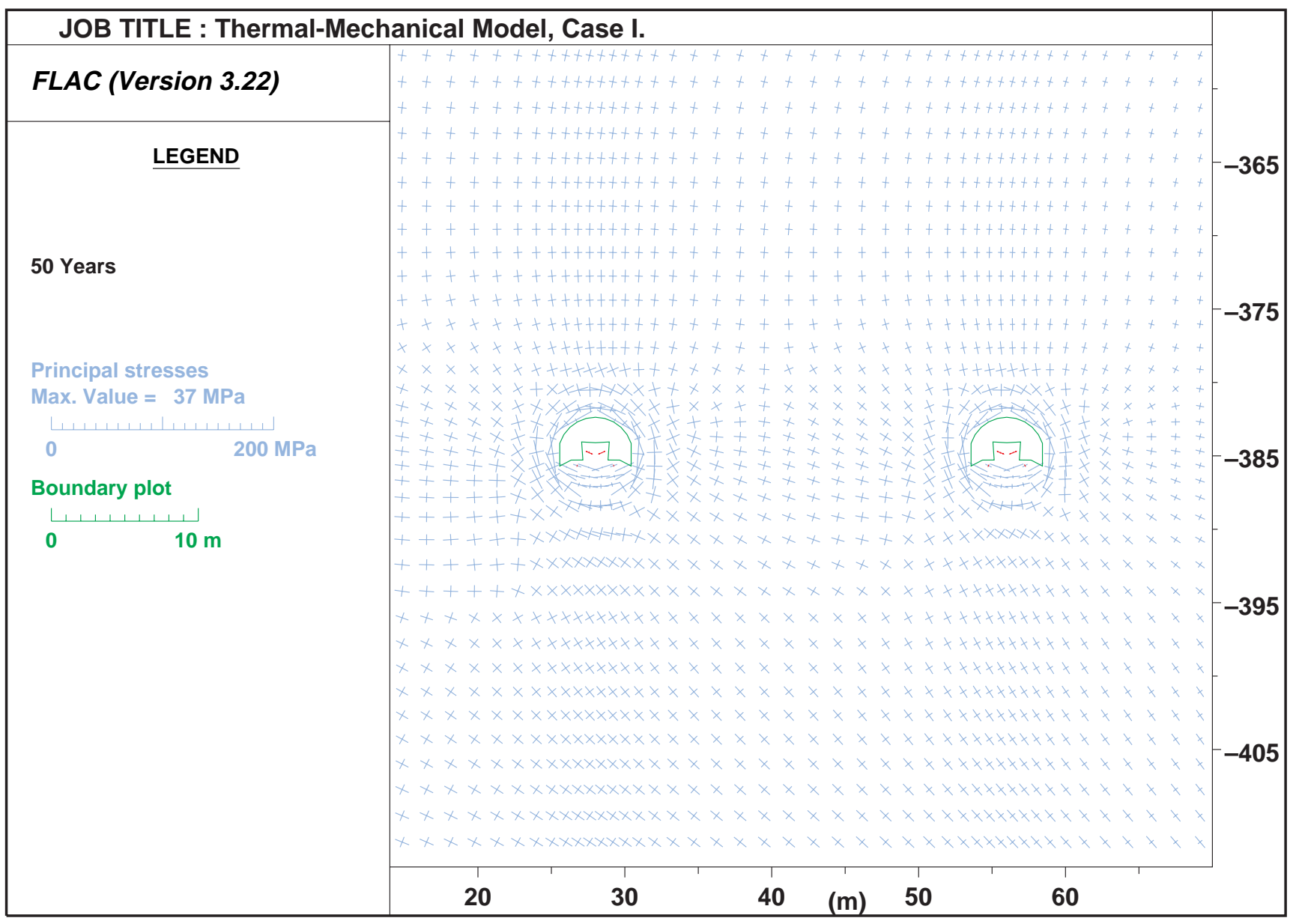

Figure 4-16. Principal stress field computed using FLAC, Case 1 at $50 \mathrm{yr}$ 


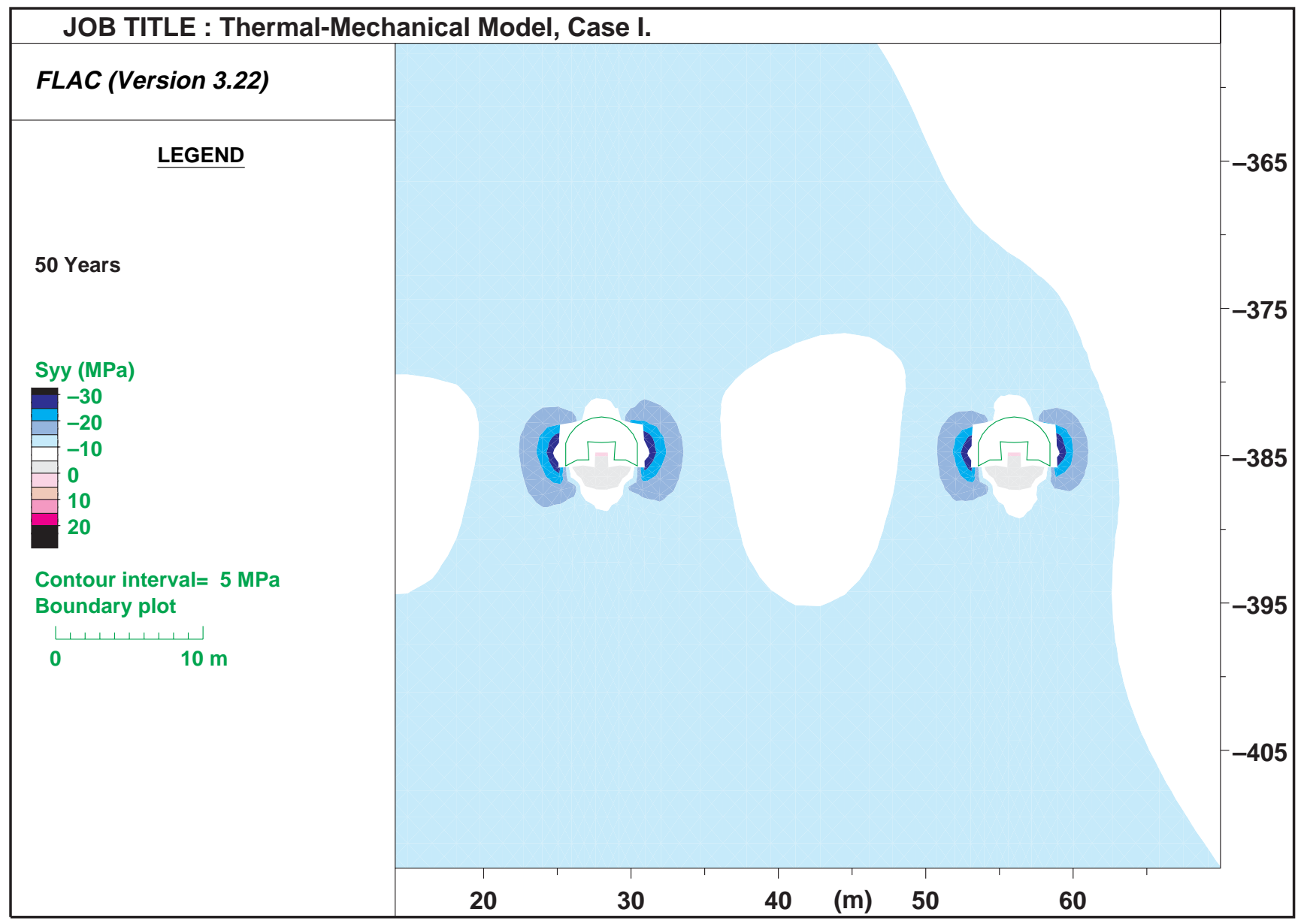

Figure 4-17. Vertical stress field computed using FLAC, Case 1 at 50 yr 


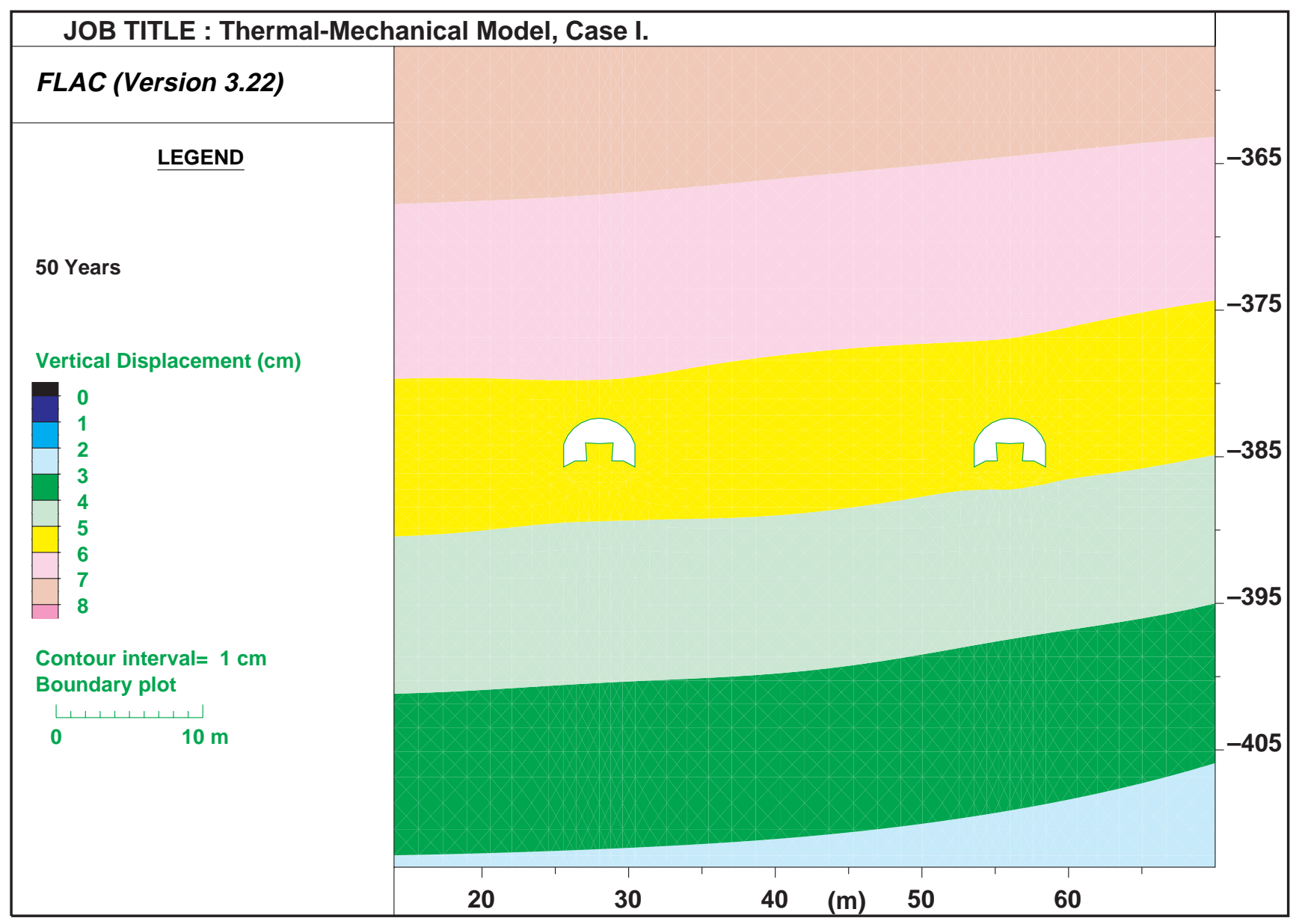

Figure 4-18. Vertical displacement field computed using FLAC, Case 1 at $50 \mathrm{yr}$ 


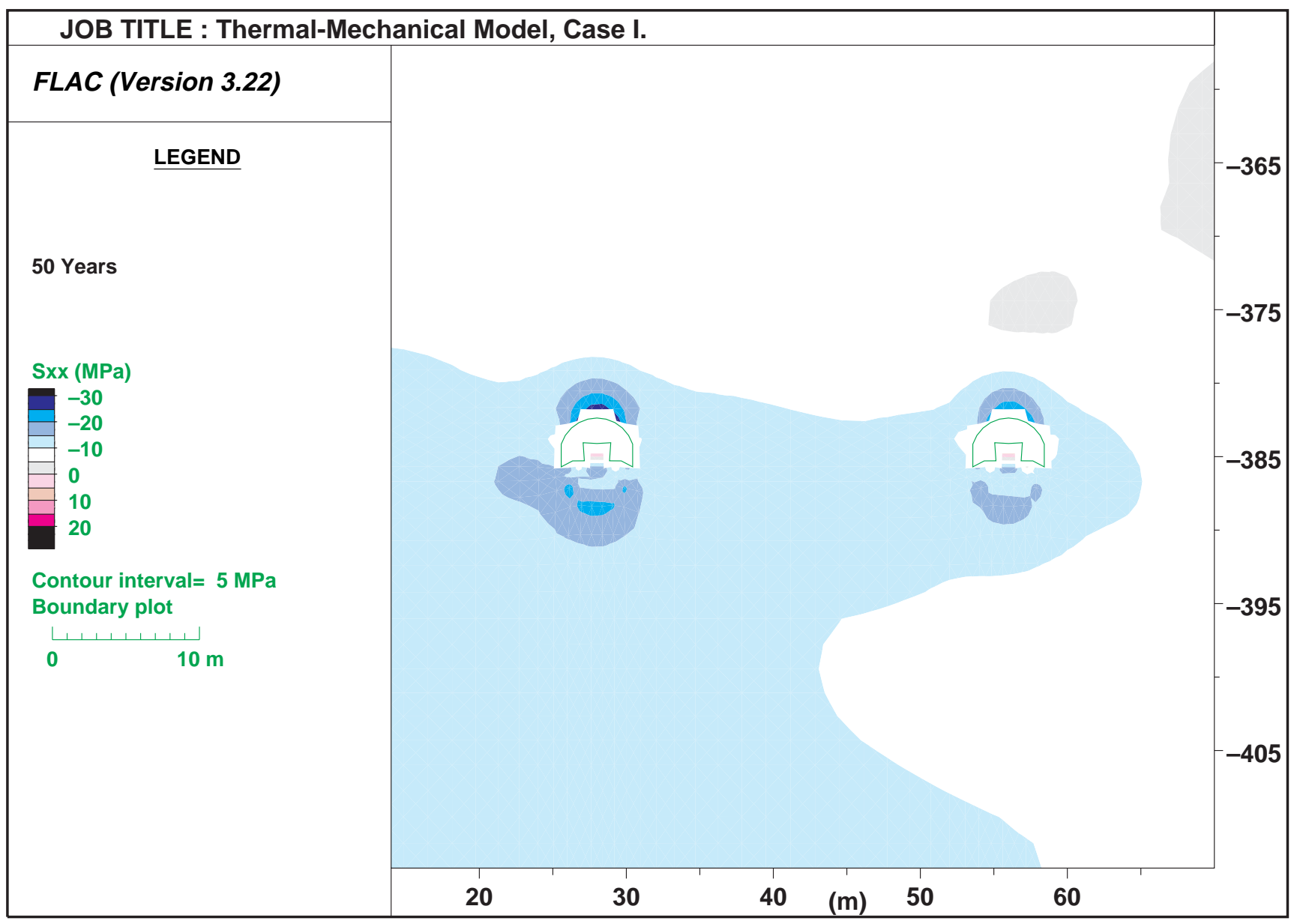

Figure 4-19. Horizontal stress field computed using FLAC, Case 1 at $50 \mathrm{yr}$ 


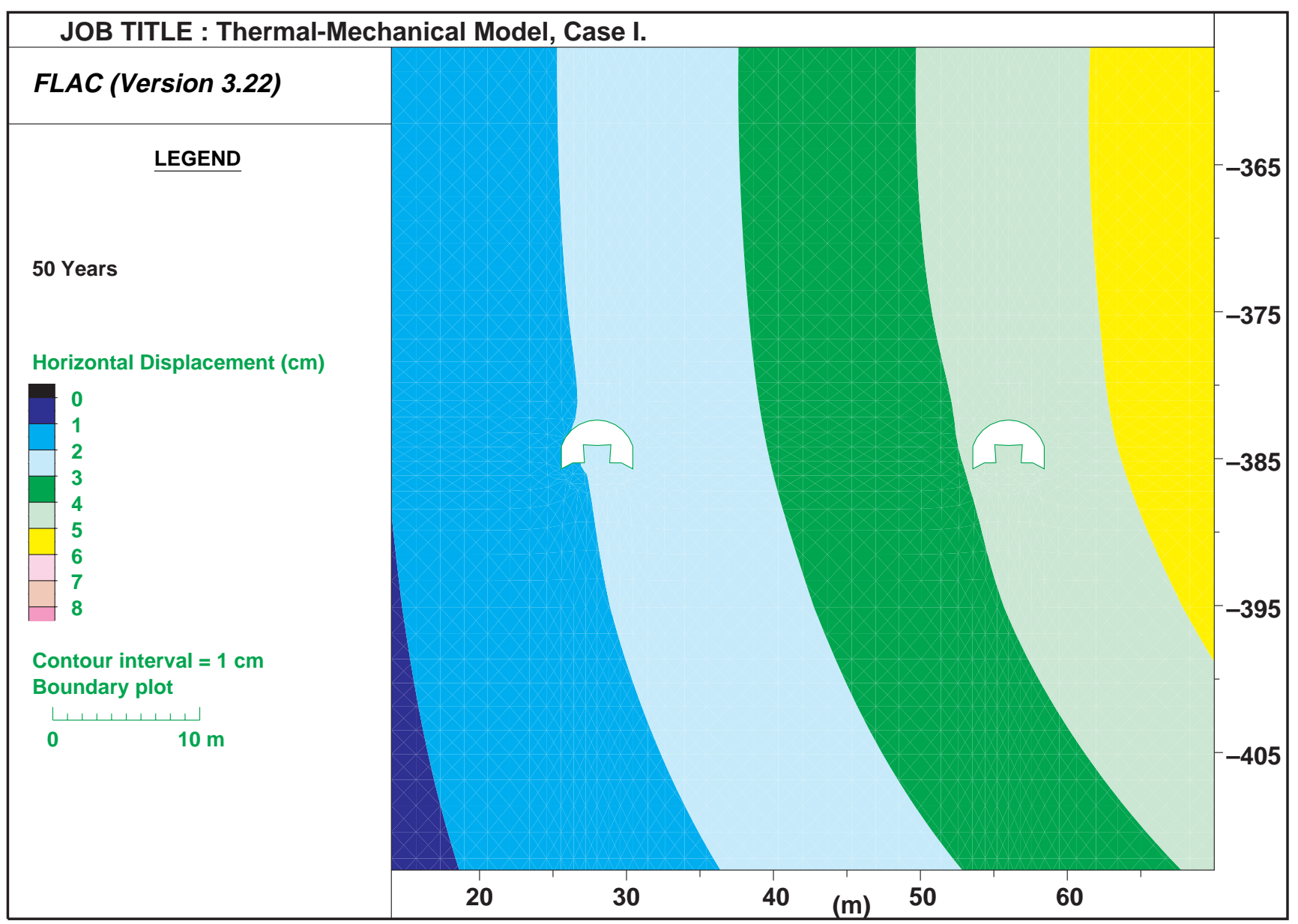

Figure 4-20. Horizontal displacement field computed using FLAC, Case 1 at 50 yr 


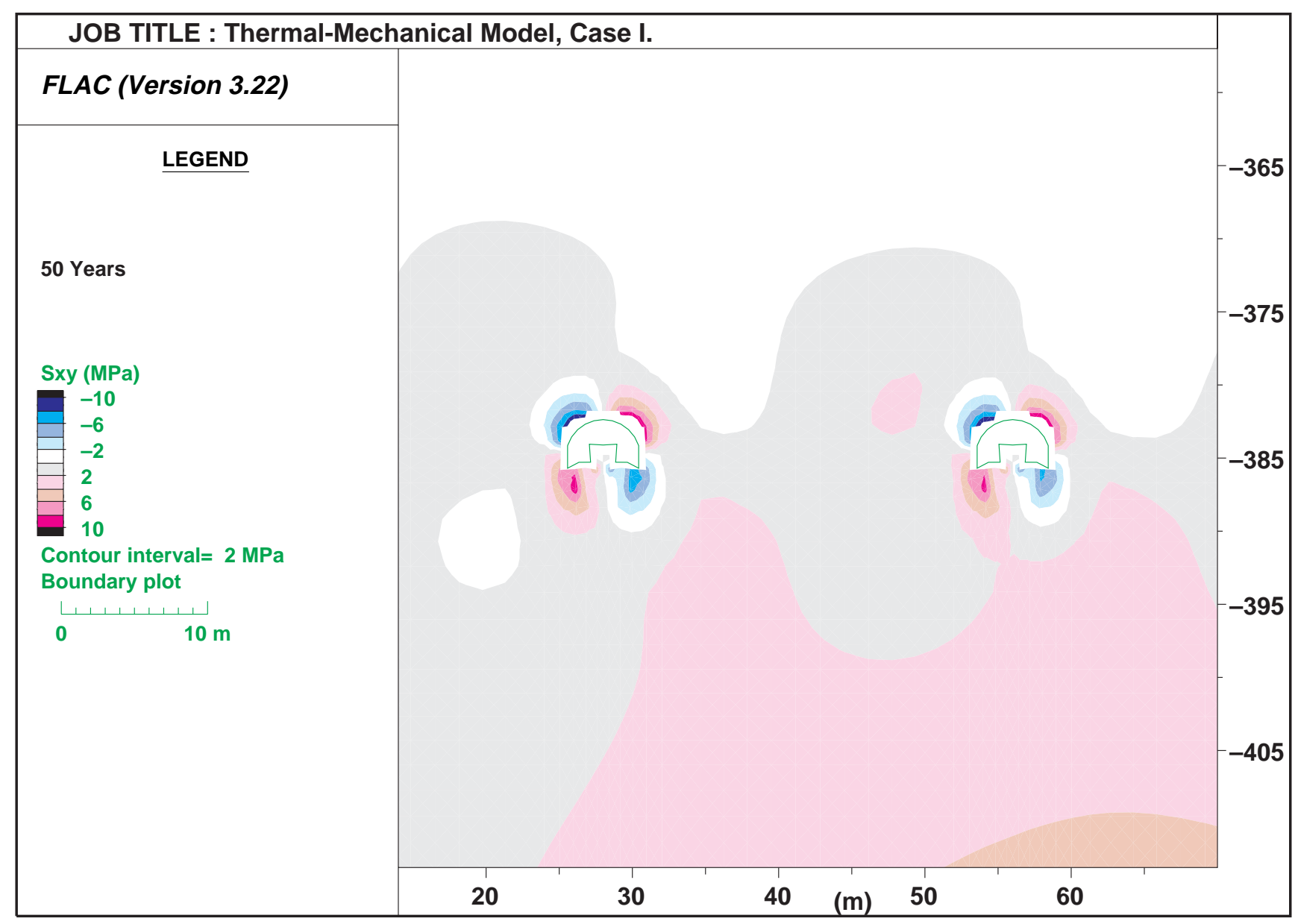

Figure 4-21. Shear stress field computed using FLAC, Case 1 at $50 \mathrm{yr}$ 


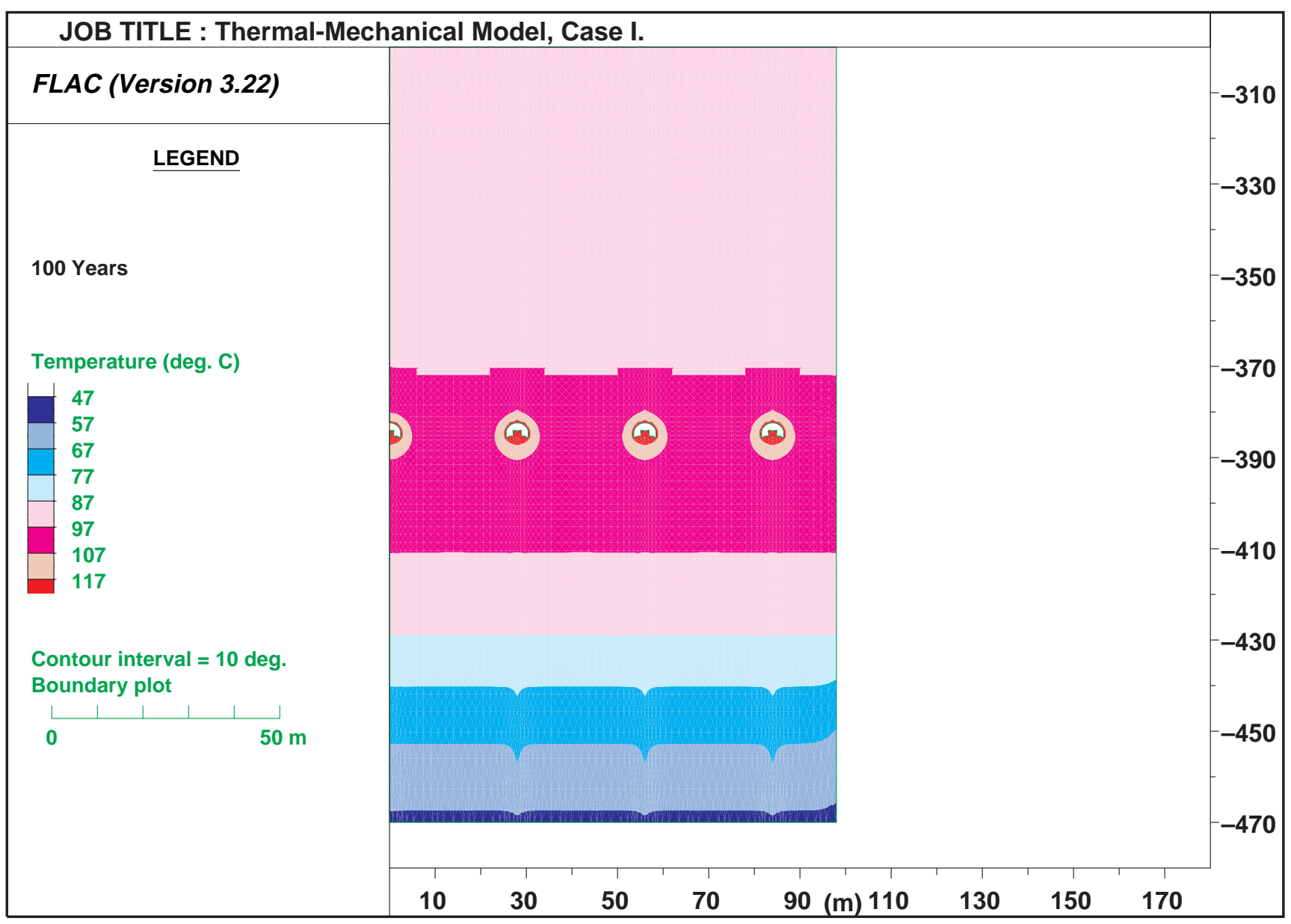

Figure 4-22. Temperature field for Case 1 at $100 \mathrm{yr}$ 


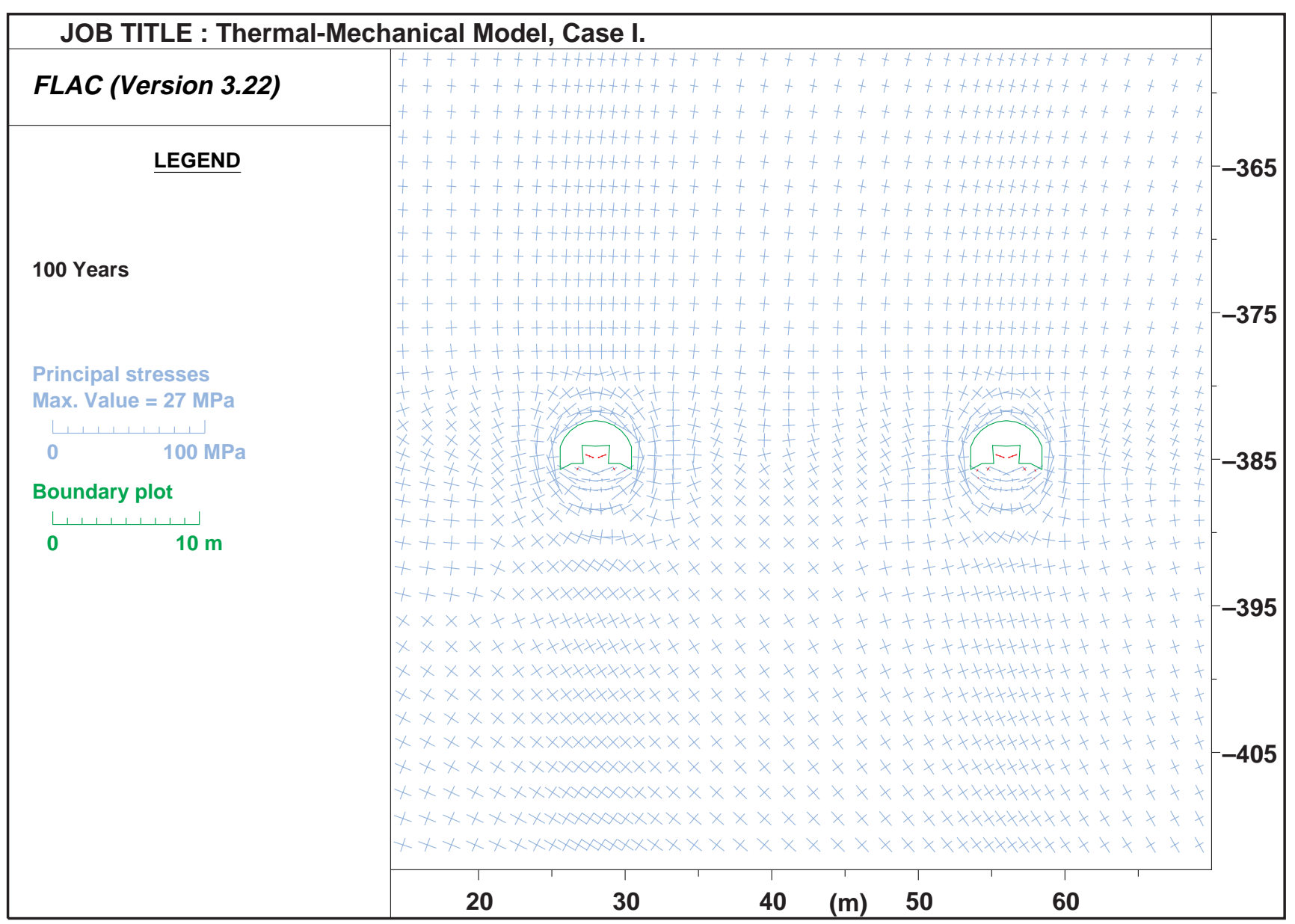

Figure 4-23. Principal stress field computed using FLAC, Case 1 at $100 \mathrm{yr}$ 


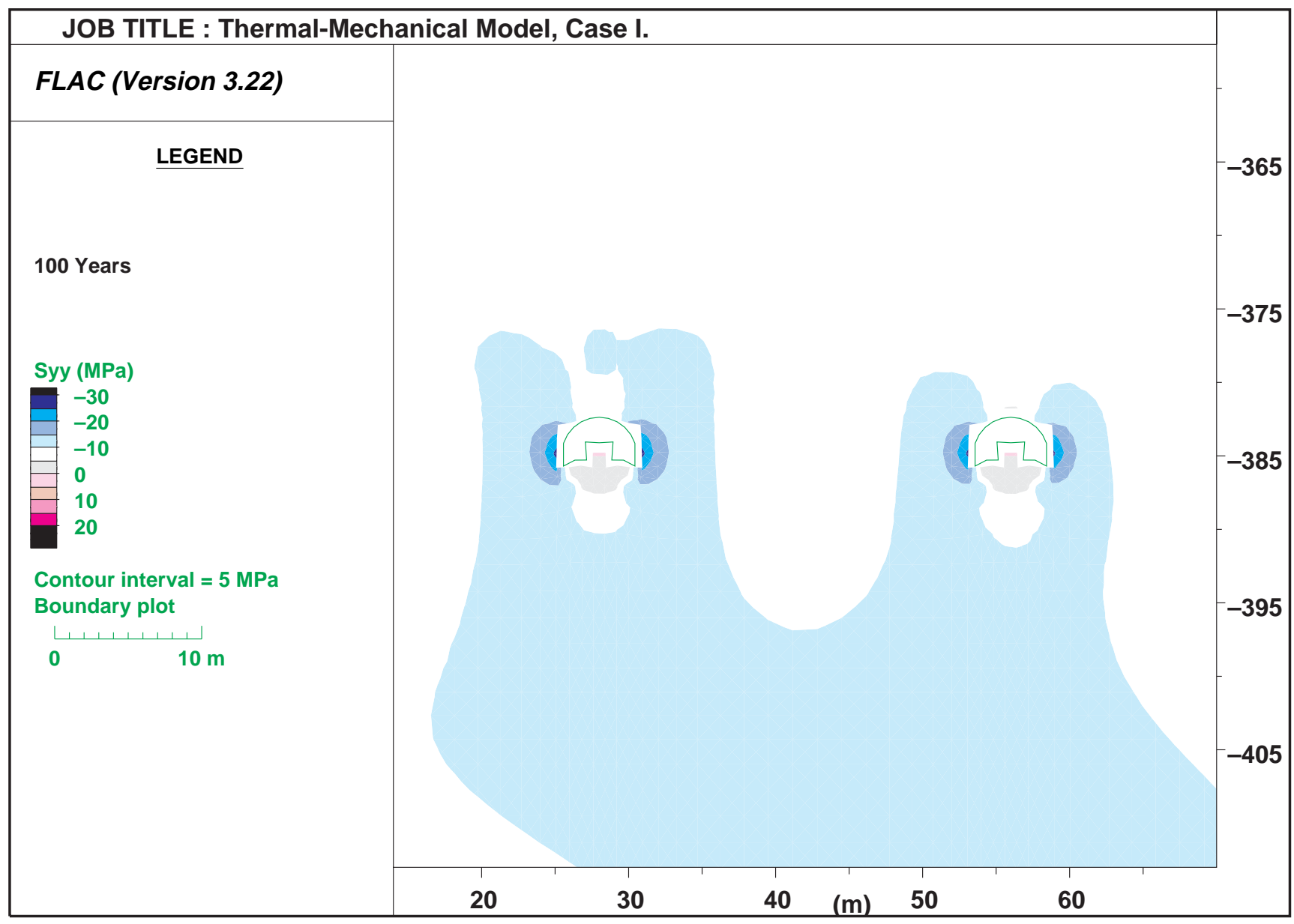

Figure 4-24. Vertical stress field computed using FLAC, Case 1 at $100 \mathrm{yr}$ 


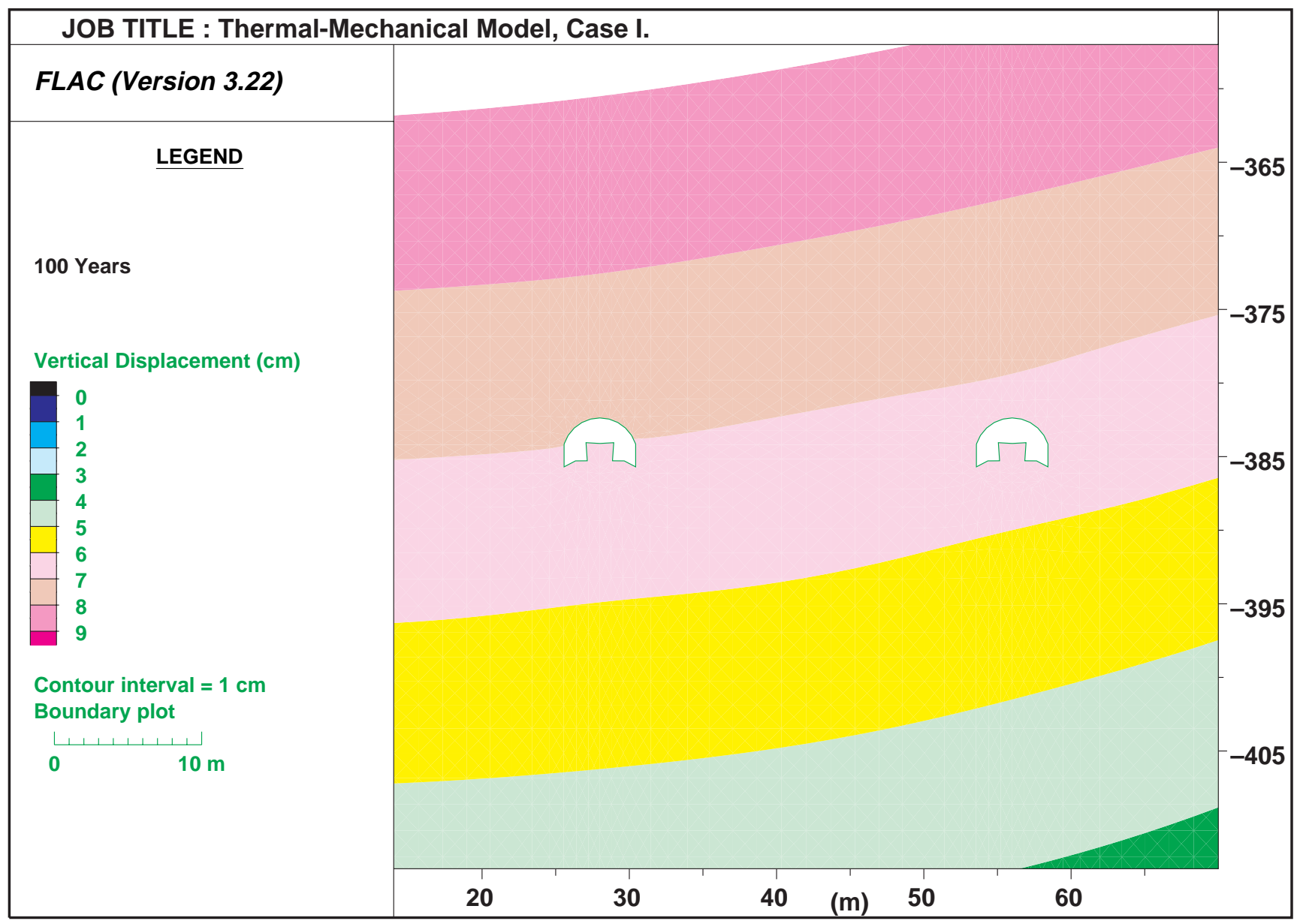

Figure 4-25. Vertical displacement field computed using FLAC, Case 1 at $100 \mathrm{yr}$ 


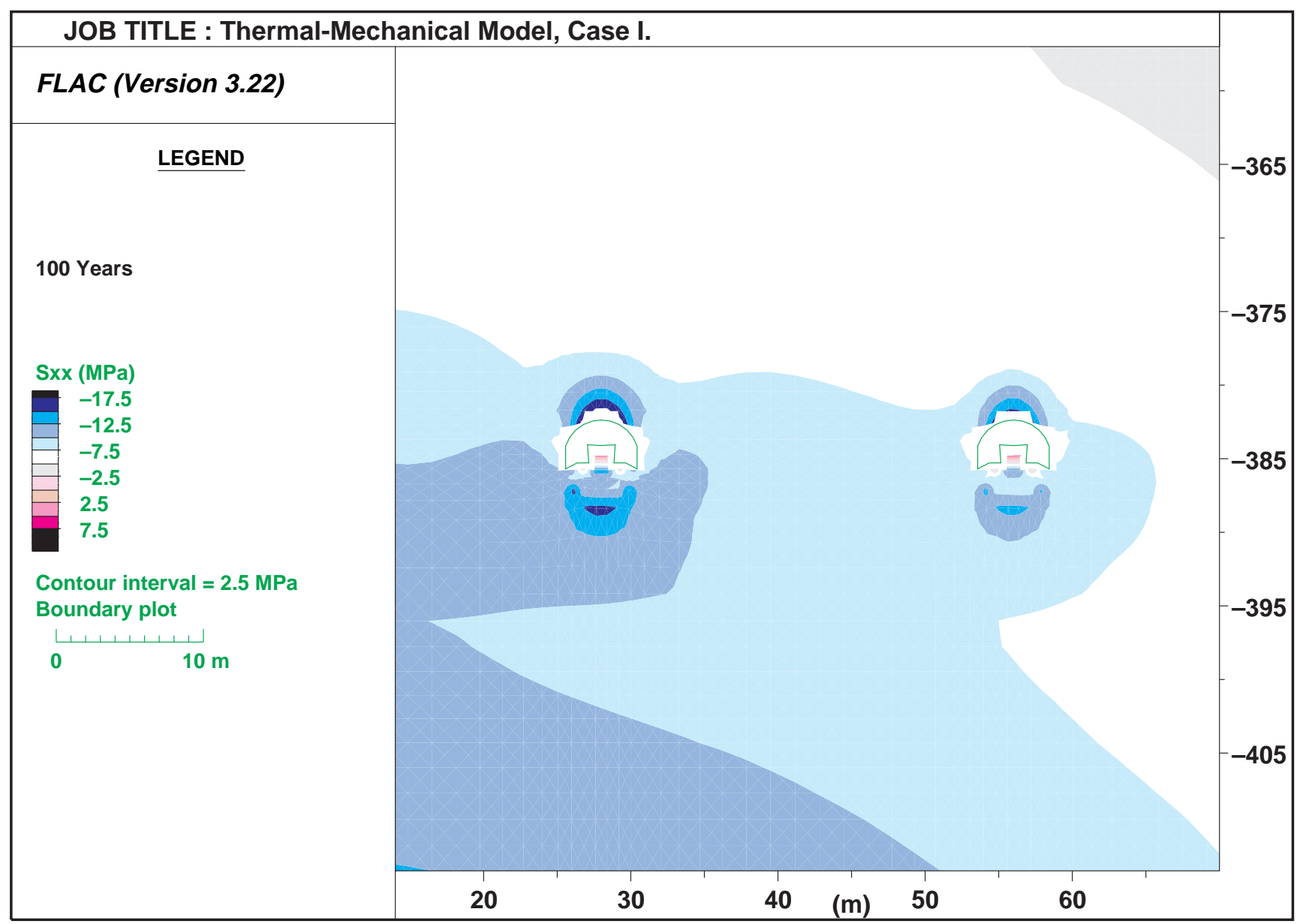

Figure 4-26. Horizontal stress field computed using FLAC, Case 1 at $100 \mathrm{yr}$ 


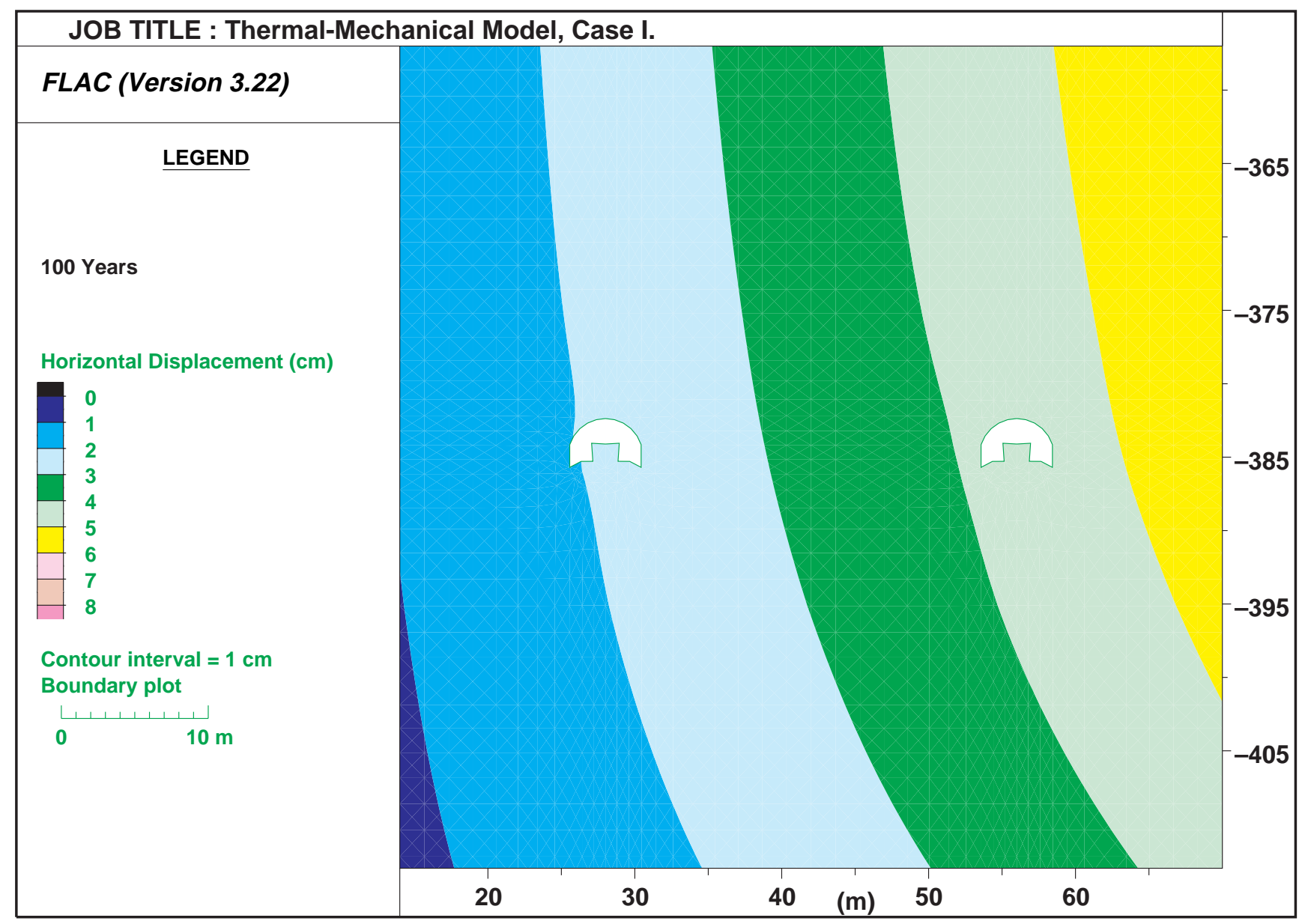

Figure 4-27. Horizontal displacement field computed using FLAC, Case 1 at $100 \mathrm{yr}$ 


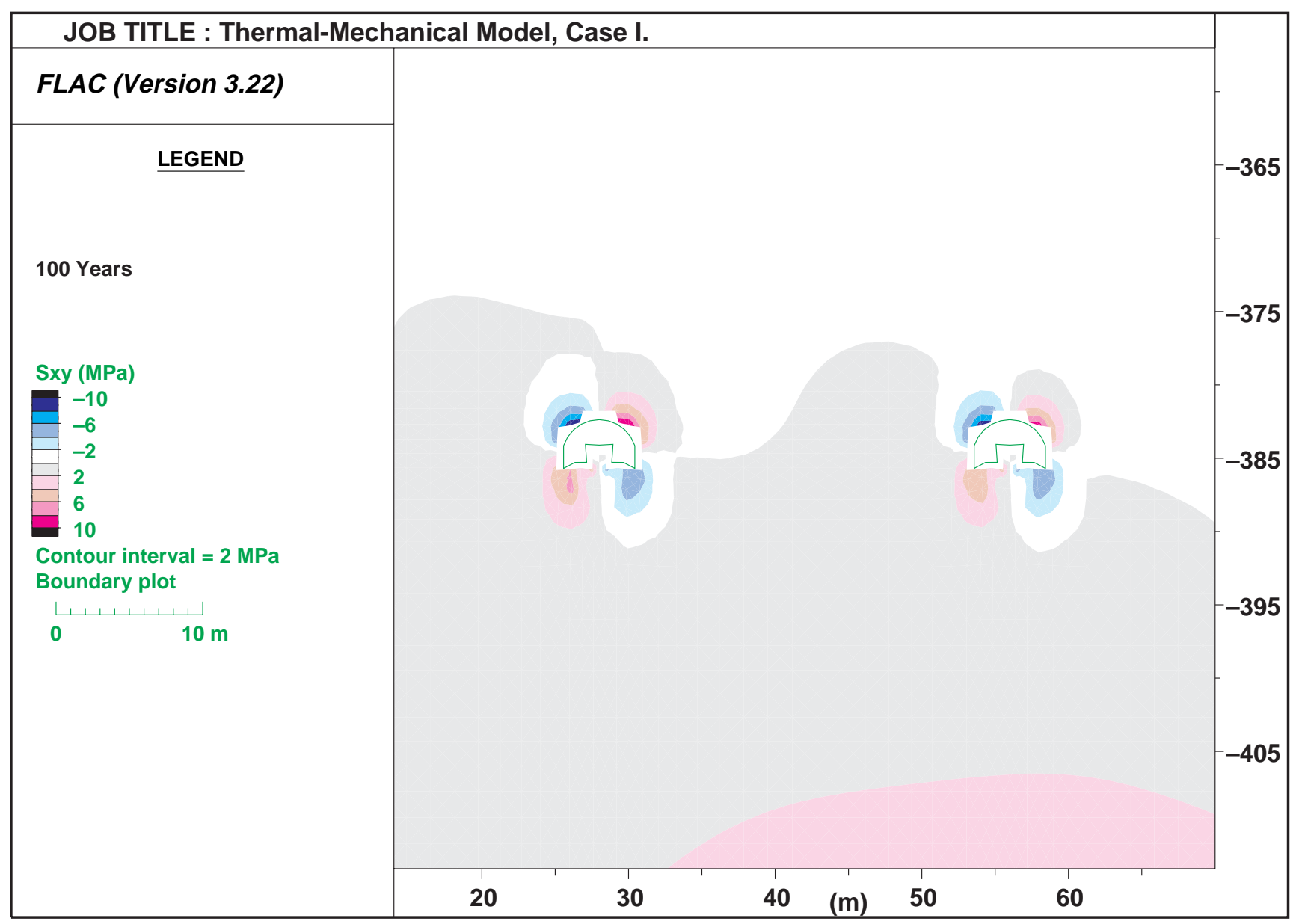

Figure 4-28. Shear stress field computed using FLAC, Case 1 at $100 \mathrm{yr}$ 


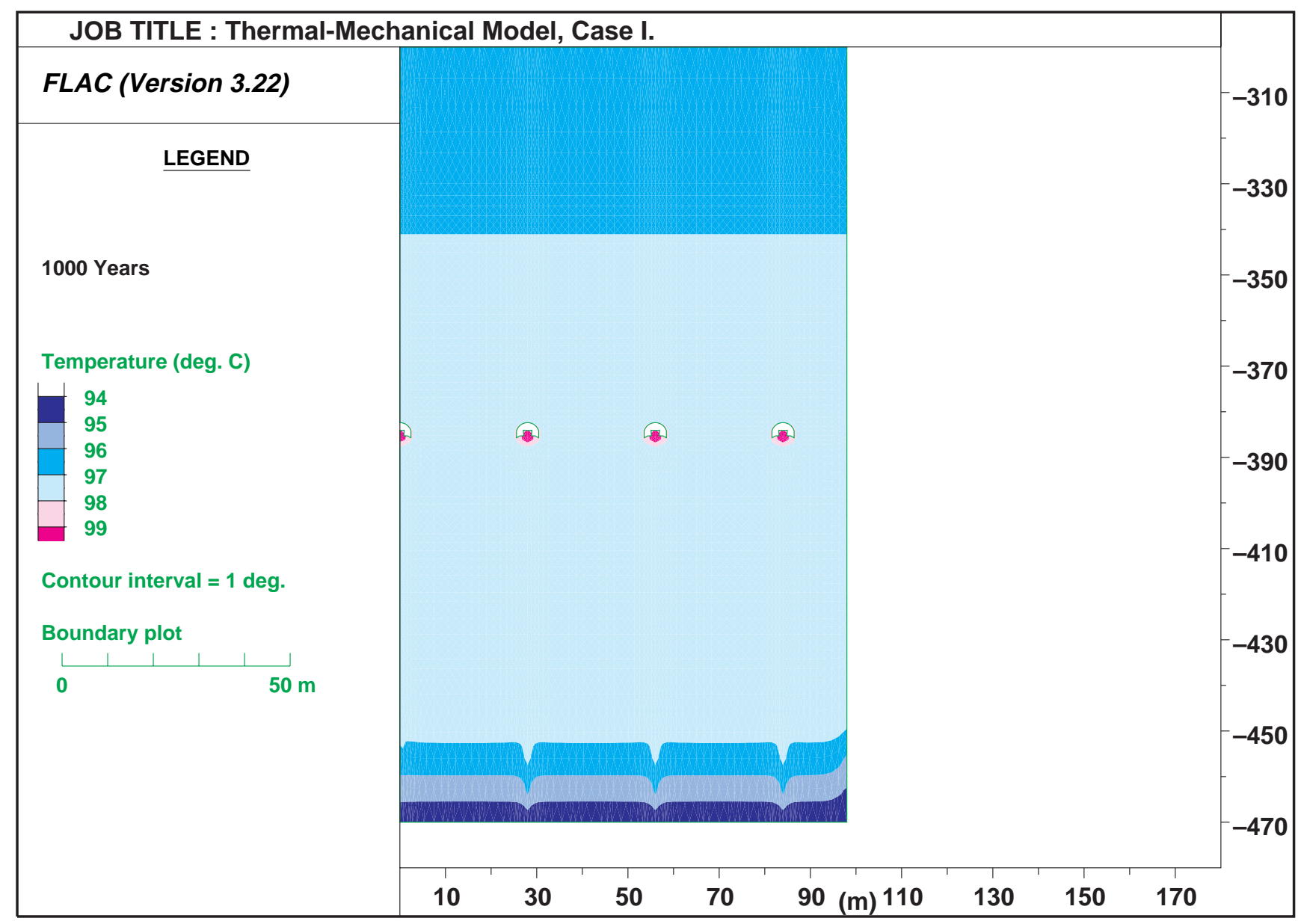

Figure 4-29. Temperature field for Case 1 at $1000 \mathrm{yr}$ 


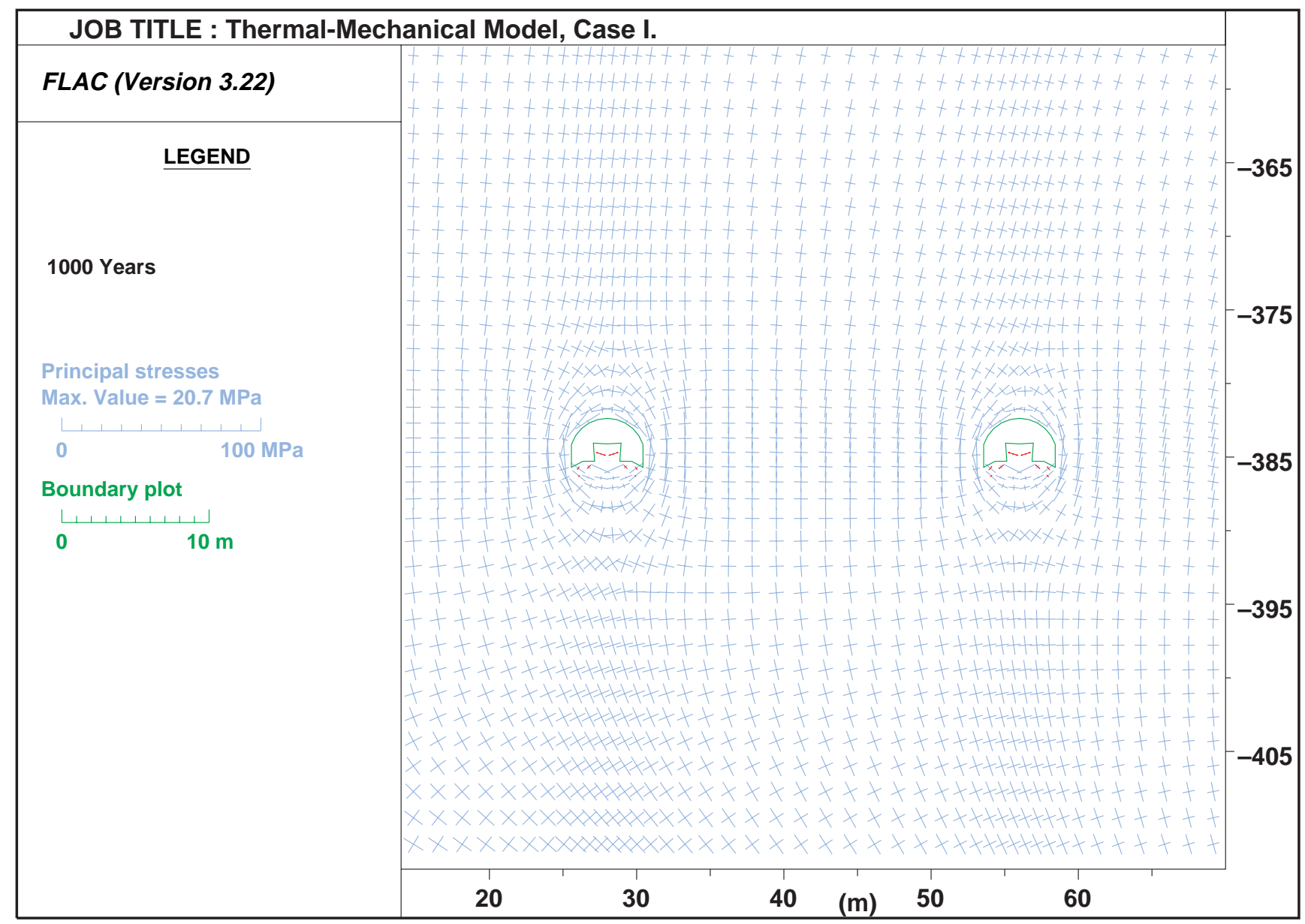

Figure 4-30. Principal stress field for $1000 \mathrm{yr}$ computed using FLAC 


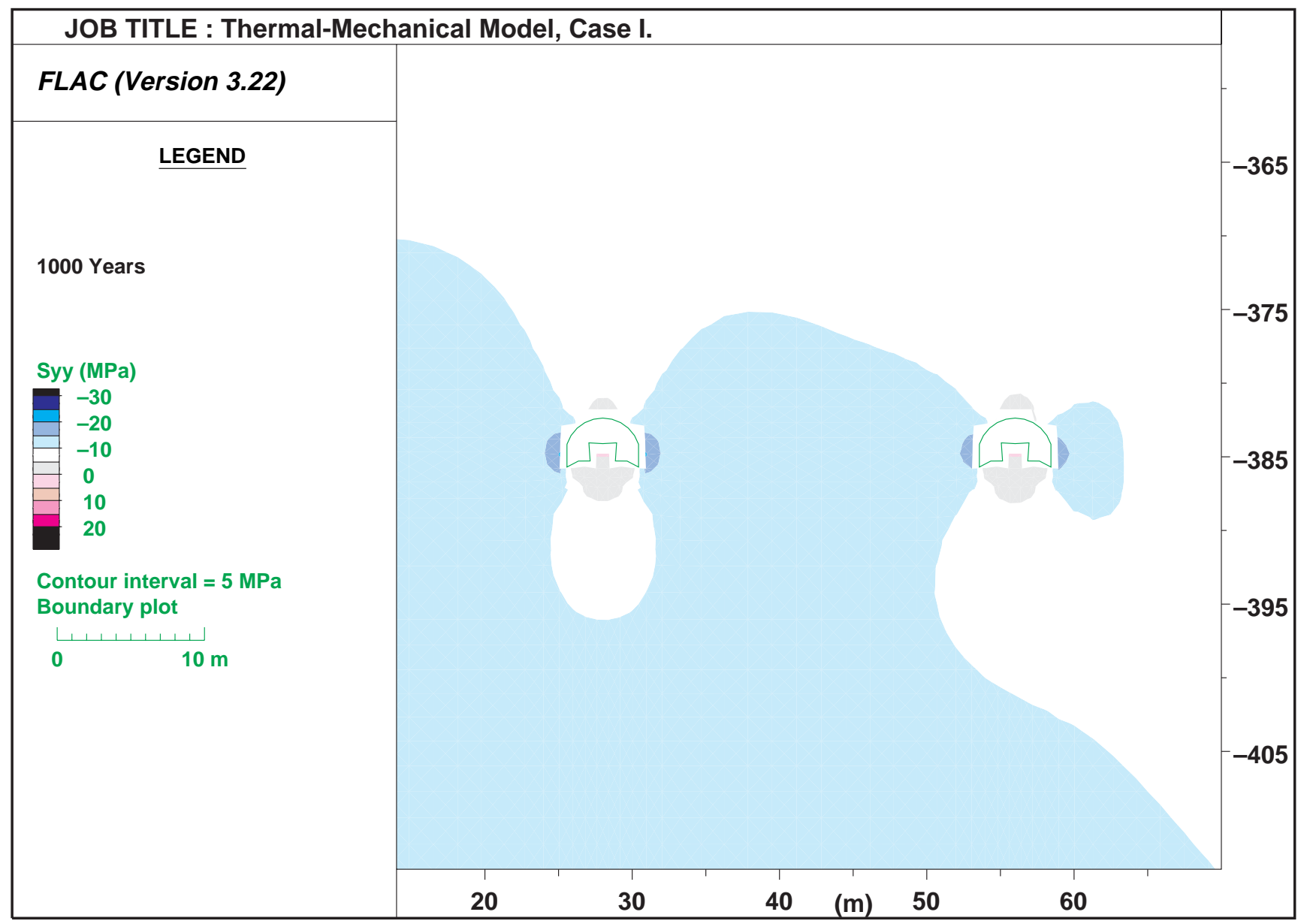

Figure 4-31. Vertical stress field for 1000 yr computed using FLAC 


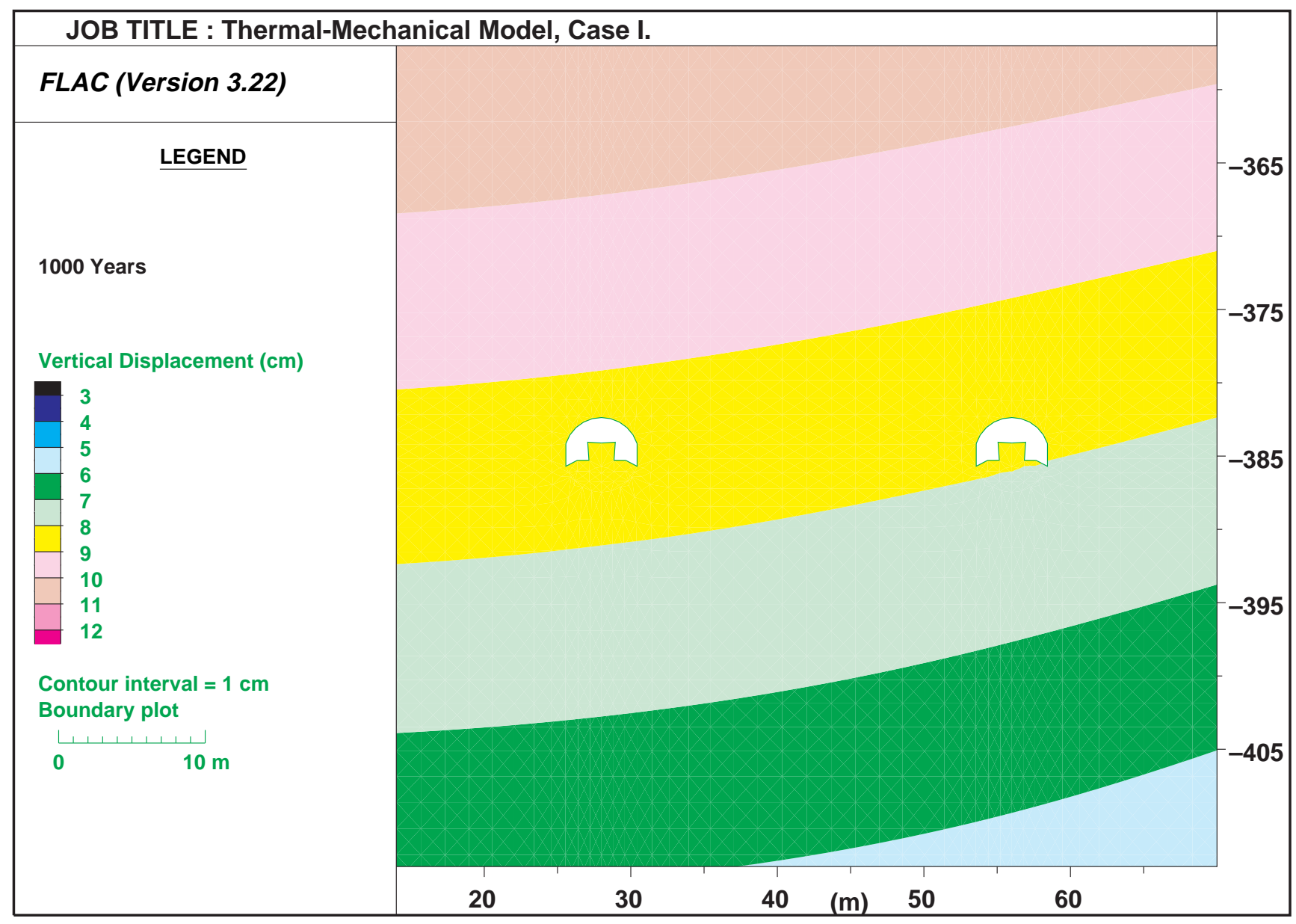

Figure 4-32. Vertical displacement field for $1000 \mathrm{yr}$ computed using FLAC 


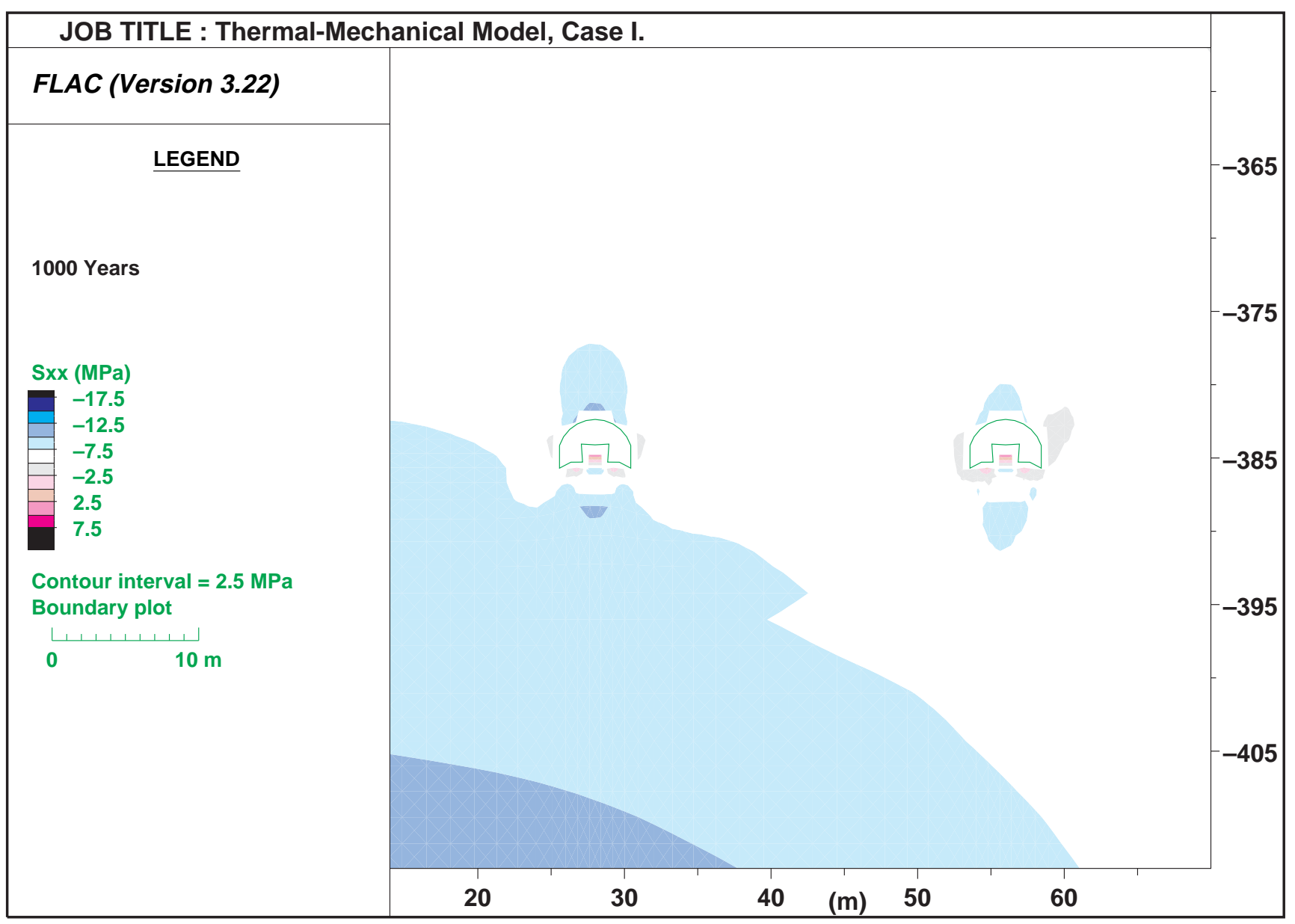

Figure 4-33. Horizontal stress field for $1000 \mathrm{yr}$ computed using FLAC 


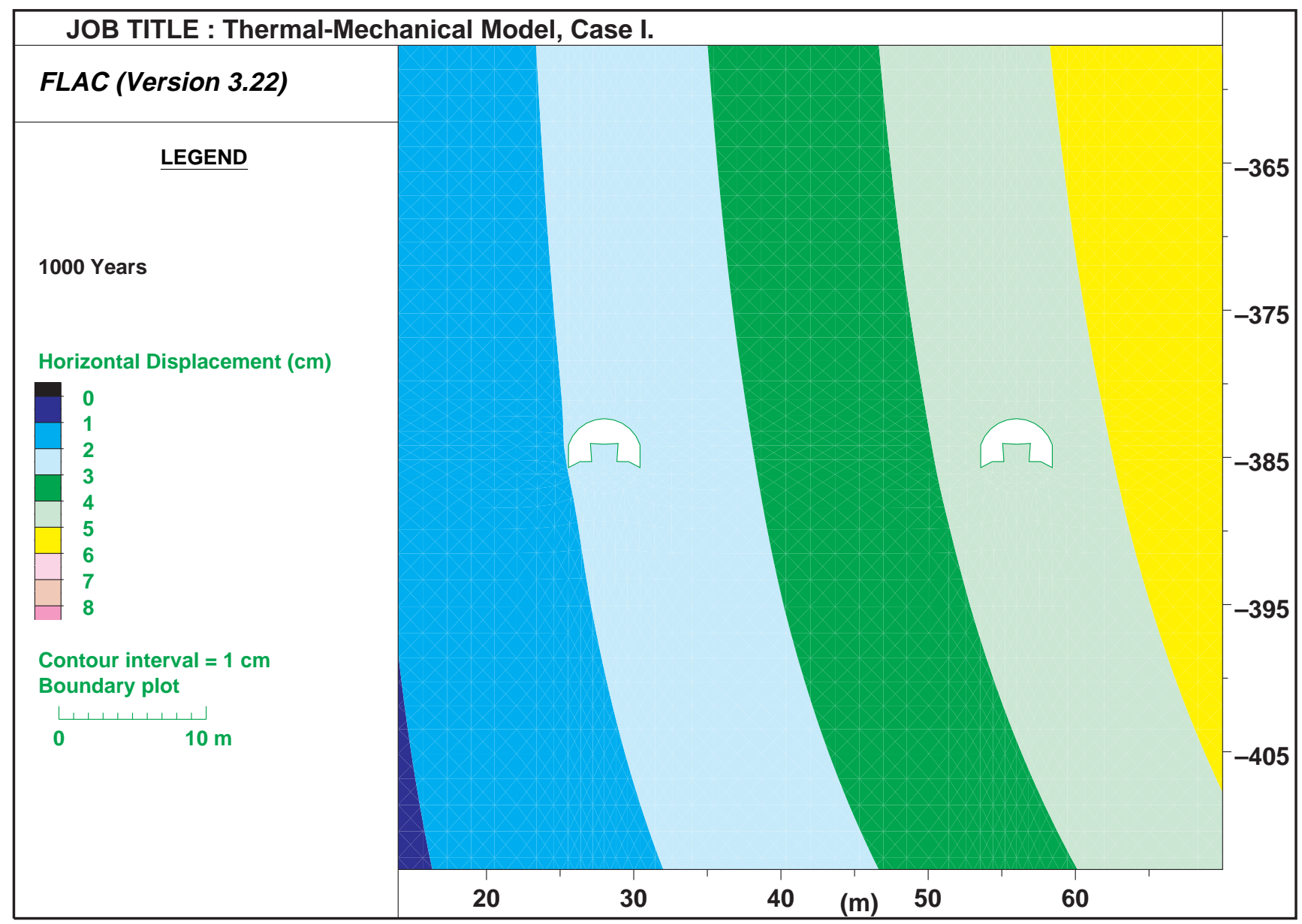

Figure 4-34. Horizontal displacement field for $1000 \mathrm{yr}$ computed using FLAC 


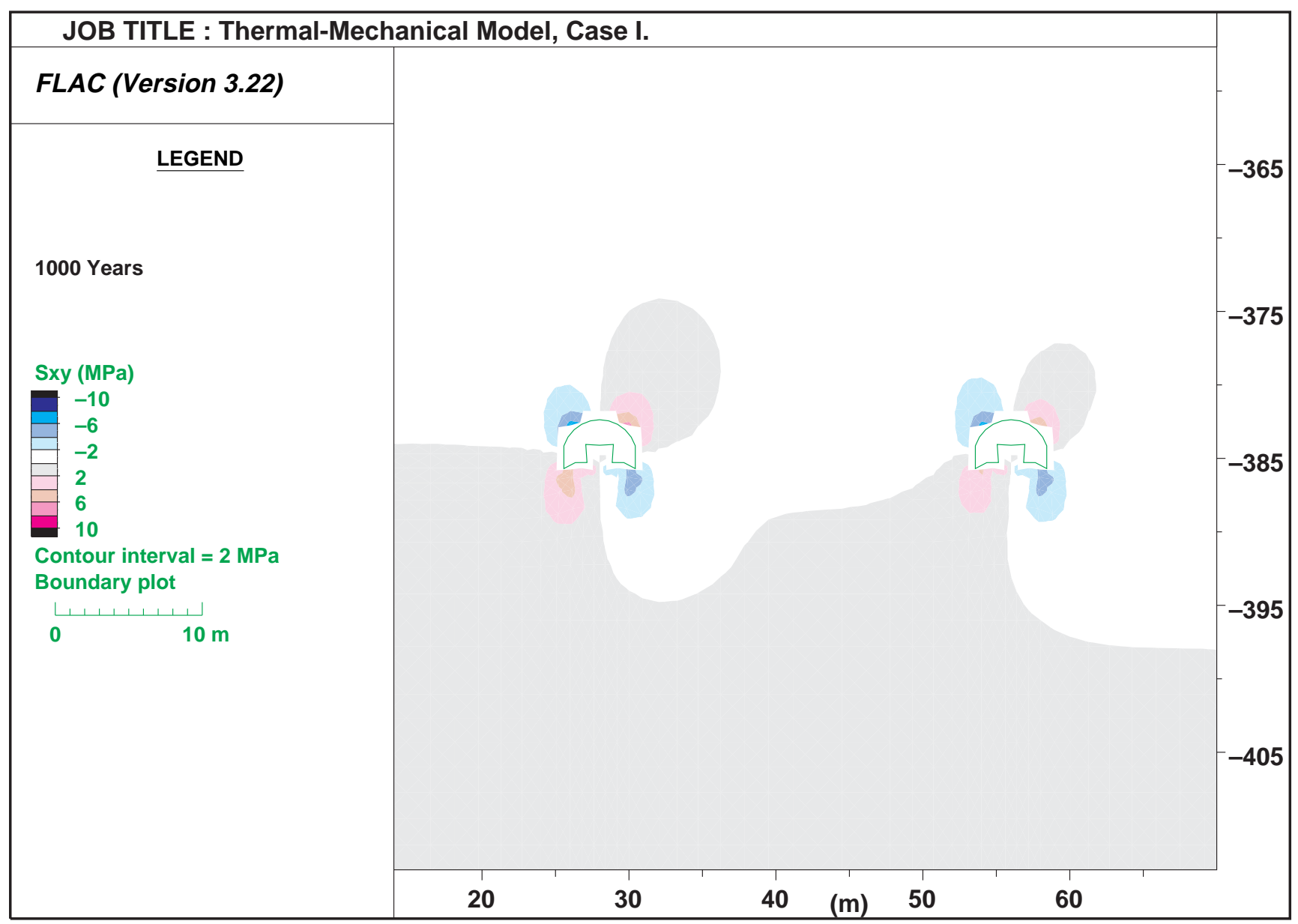

Figure 4-35. Shear stress field for 1000 yr computed using FLAC 


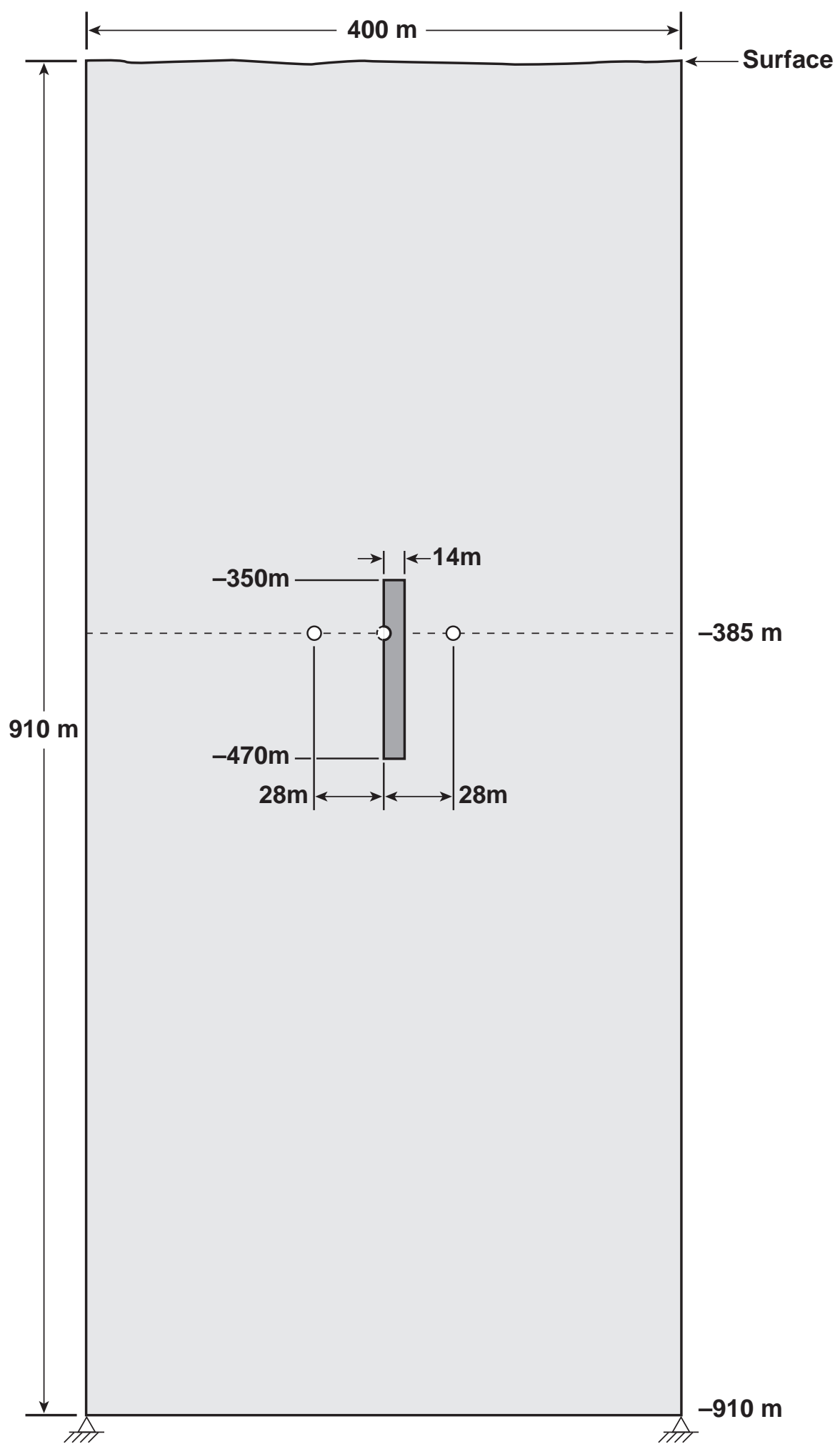

Figure 4-36. Zone around drift used in FRACROCK for Case 1 stress analysis 


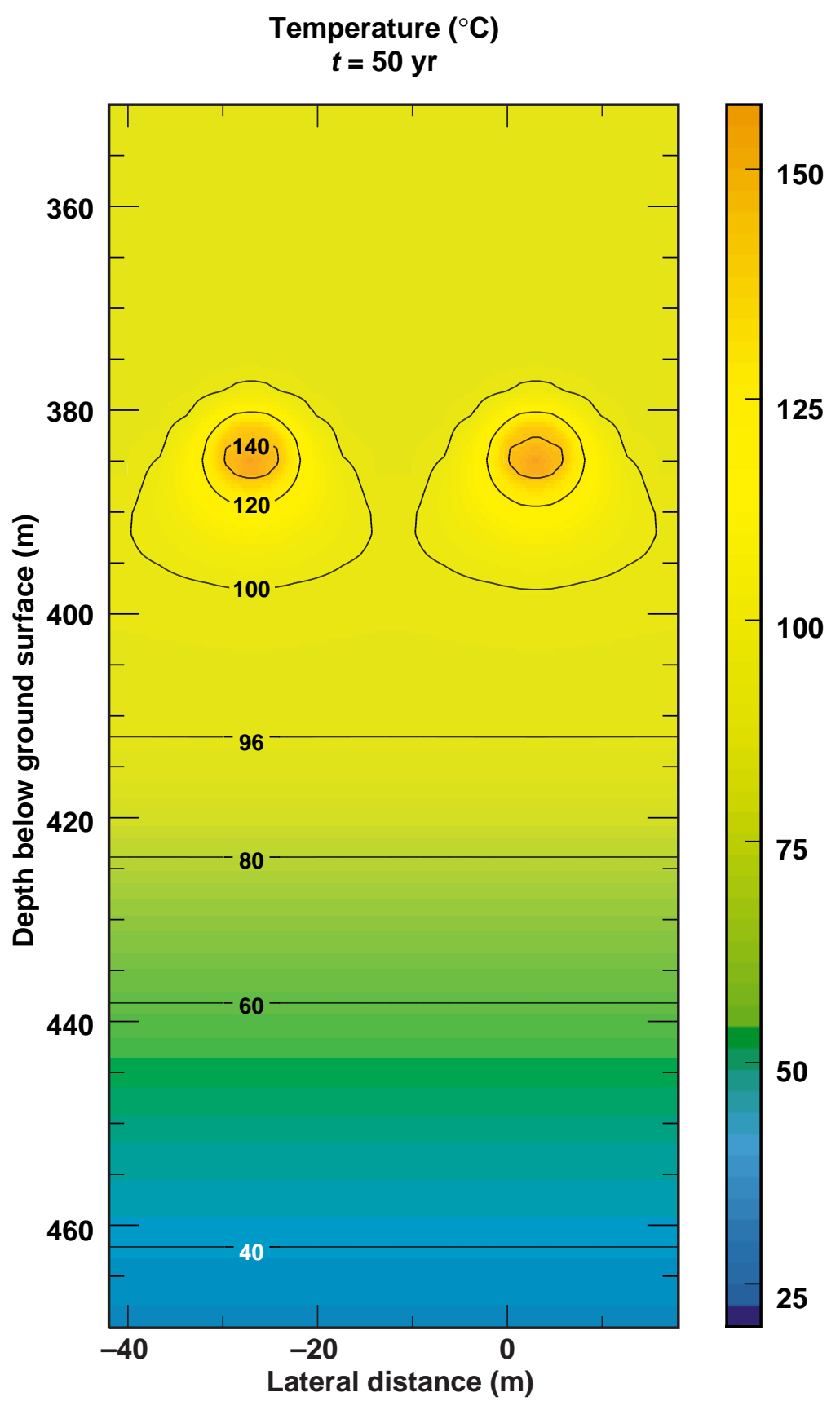

Figure 4-37. Temperature field for Case 1 at $50 \mathrm{yr}$, as computed by NUFT 


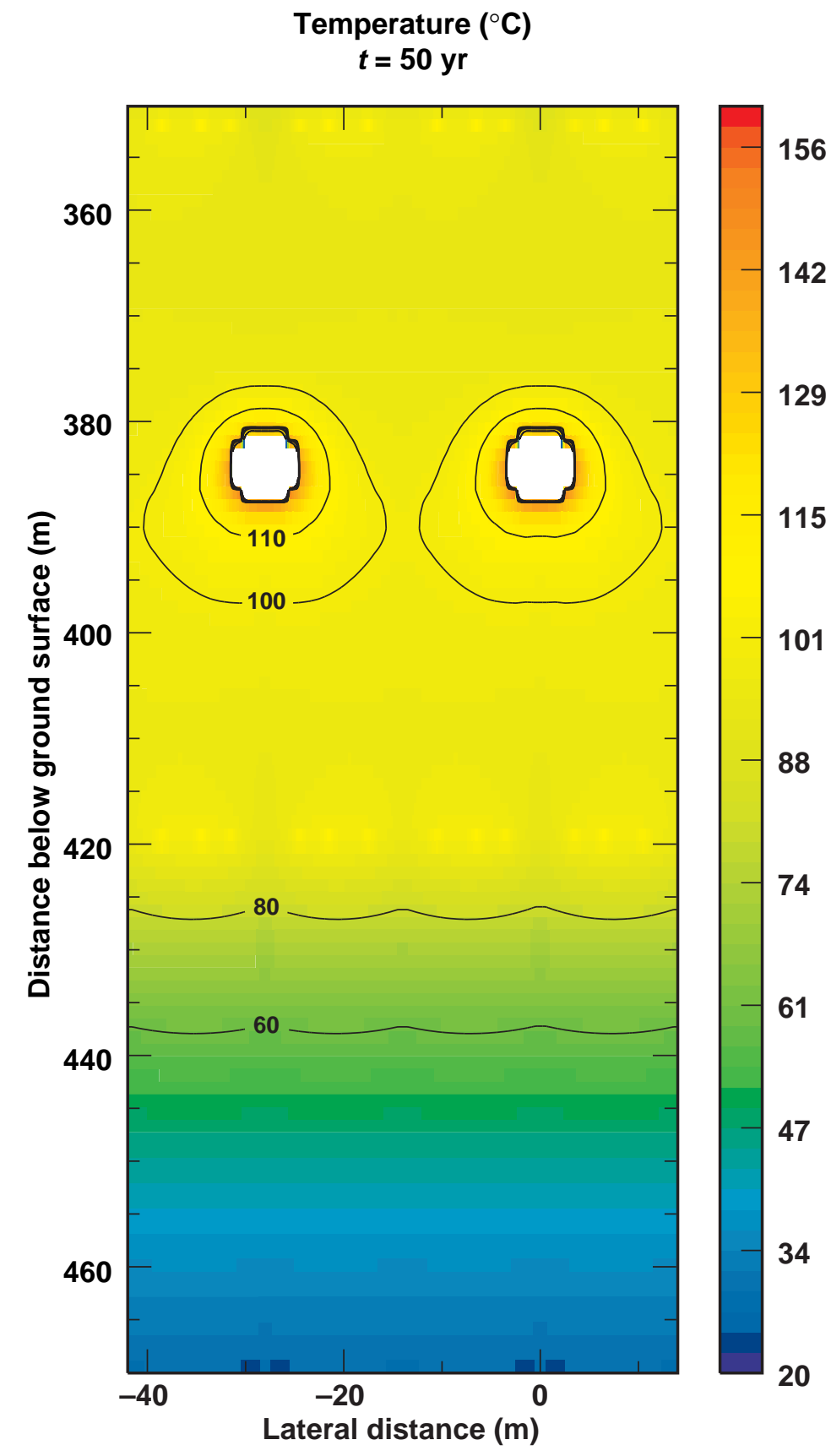

Figure 4-38. Simulation of 50-yr temperature field for Case 1 for input to FRACROCK 


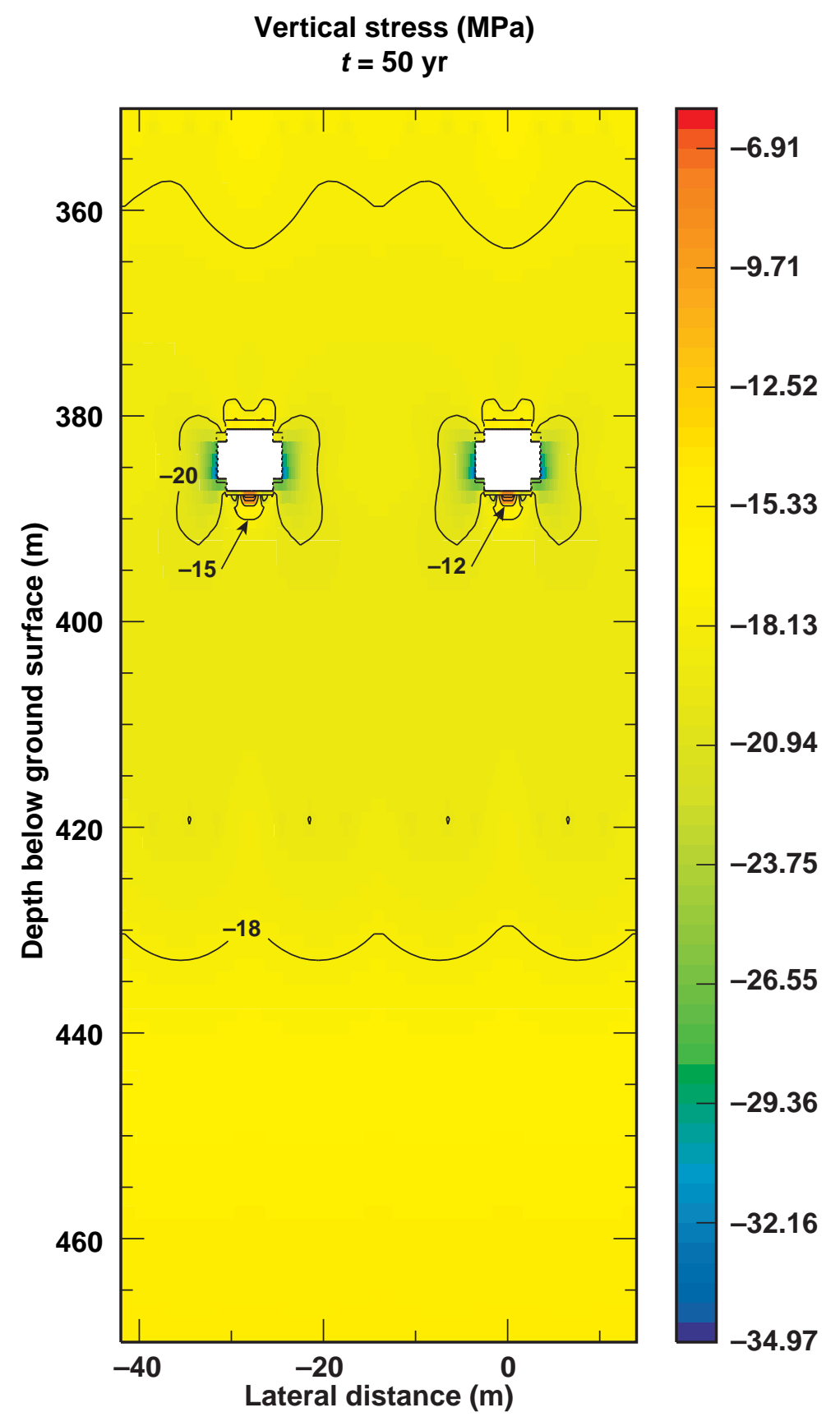

Figure 4-39. Vertical stress field for Case 1 at $50 \mathrm{yr}$, computed using FRACROCK 


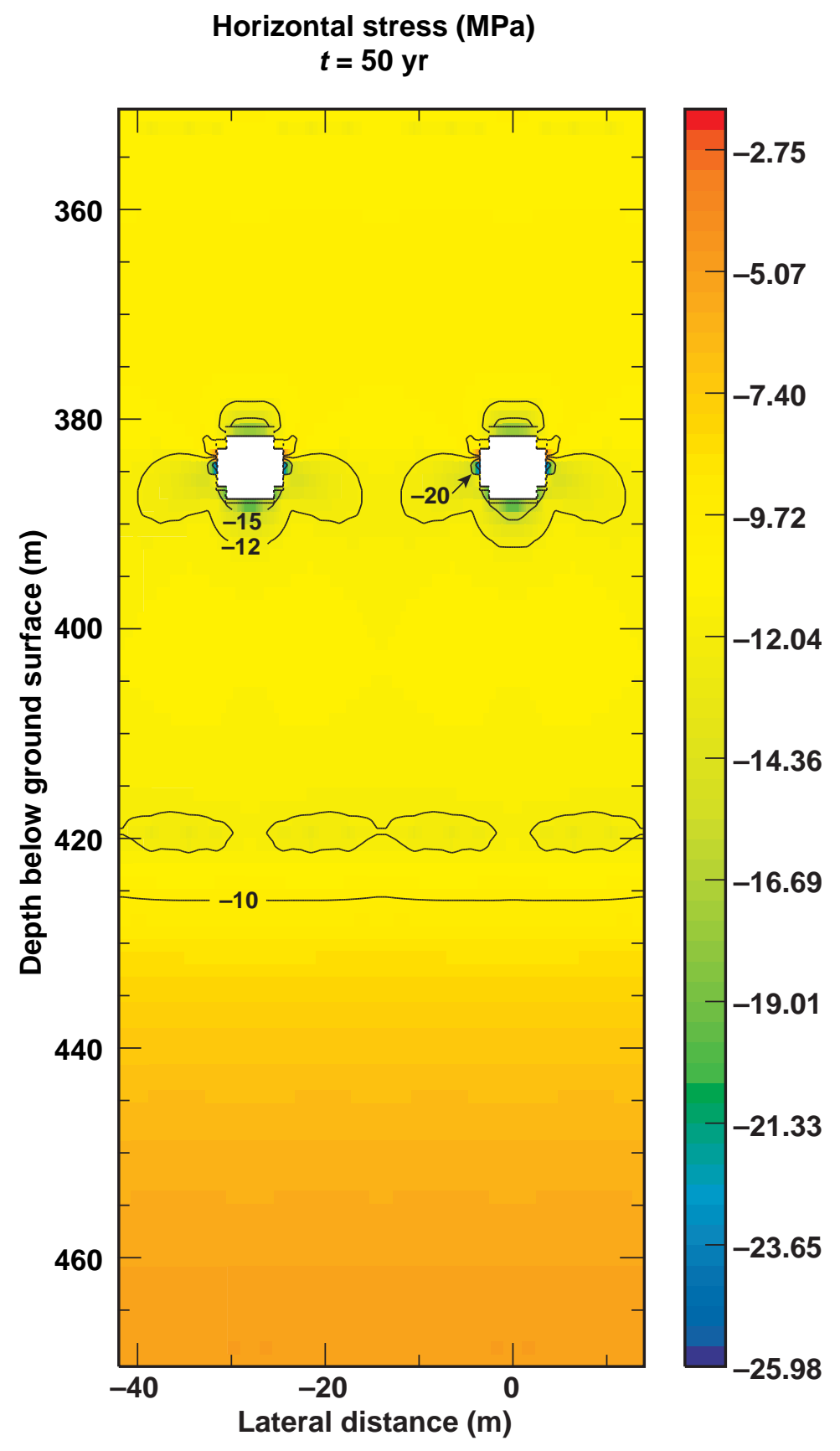

Figure 4-40. Horizontal stress field for Case 1 at $50 \mathrm{yr}$, computed using FRACROCK 


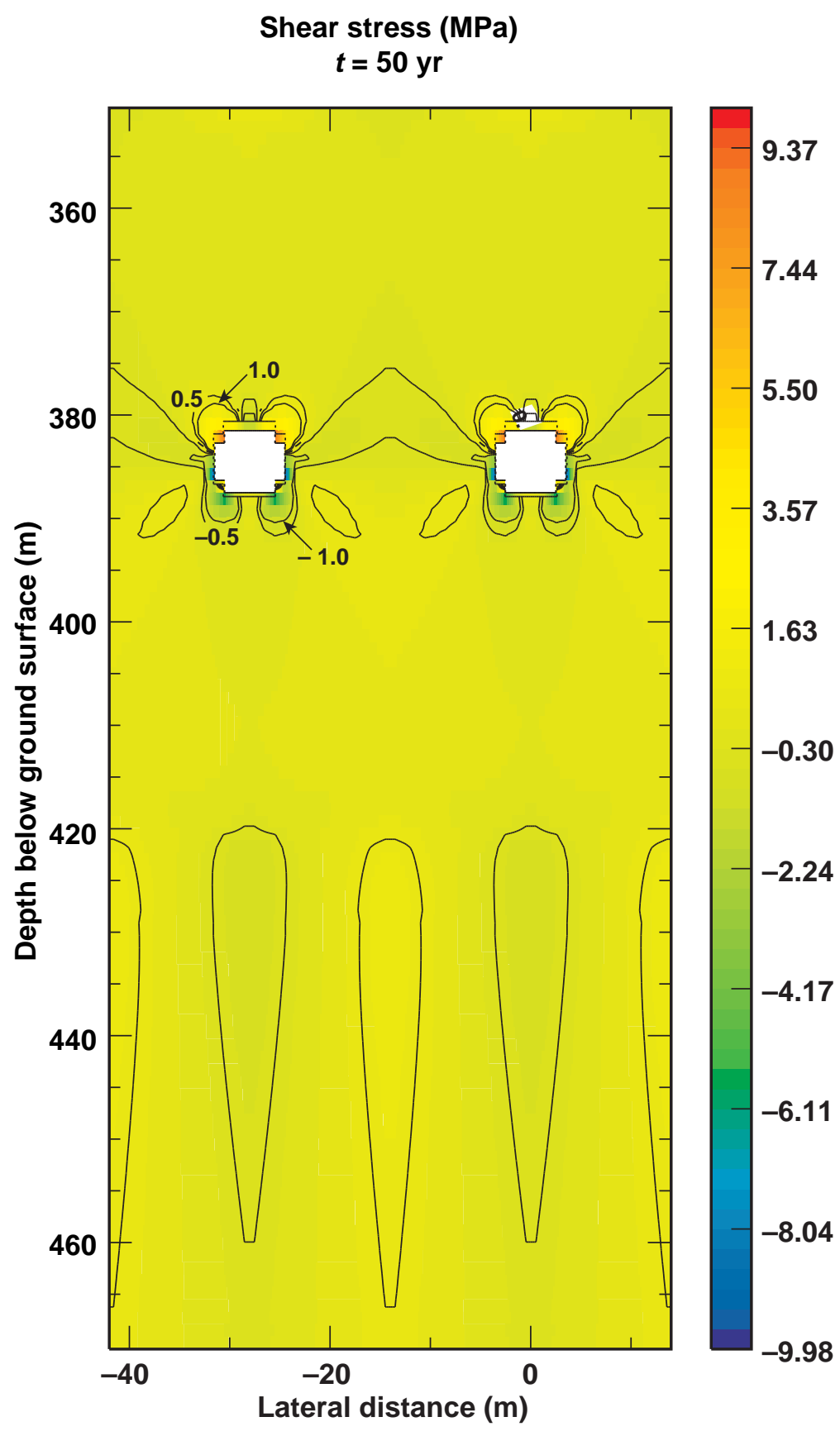

Figure 4-41. Shear stress field for Case 1 at $50 \mathrm{yr}$, computed using FRACROCK 


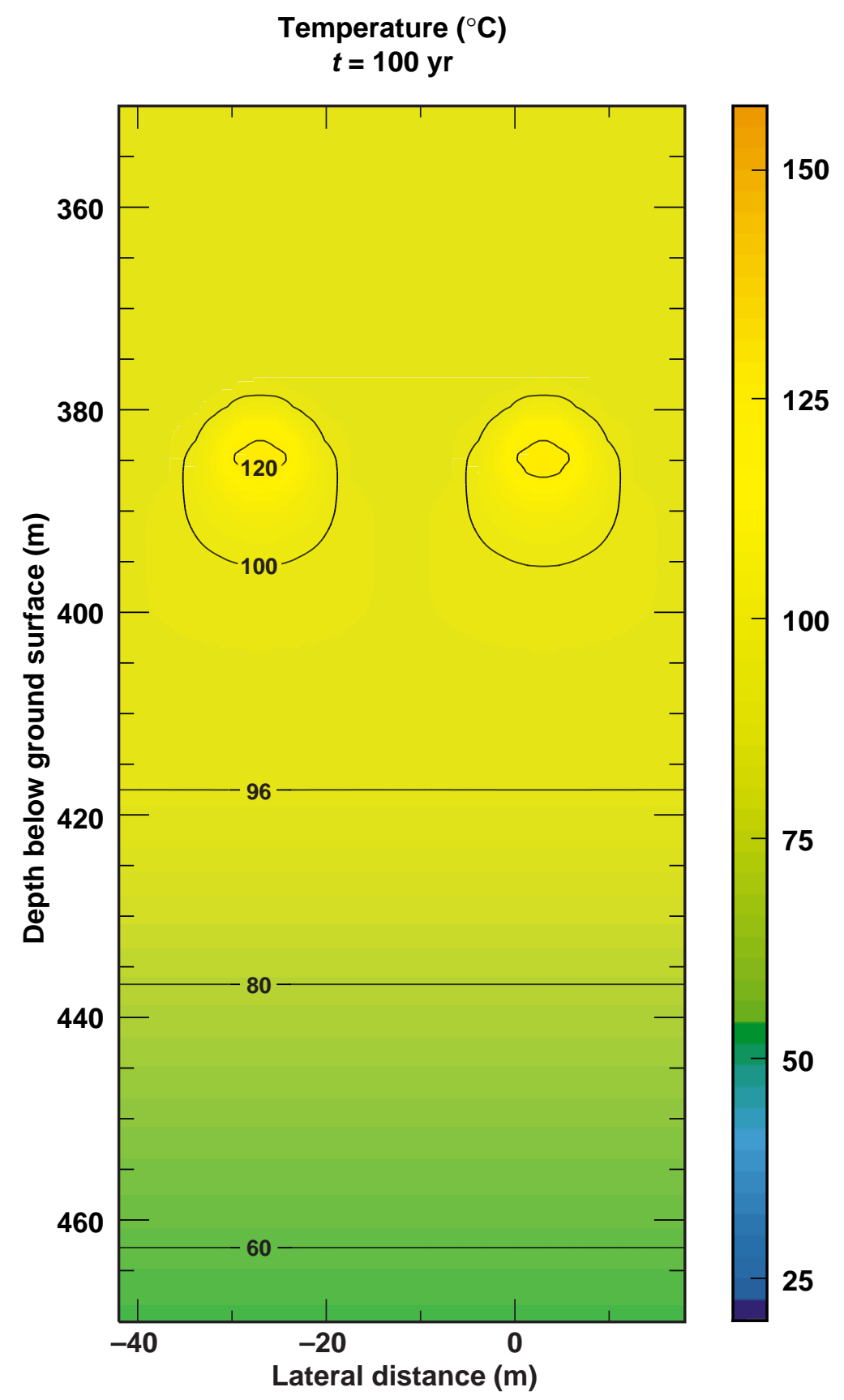

Figure 4-42. Temperature field for Case 1 at $100 \mathrm{yr}$, as computed by NUFT 


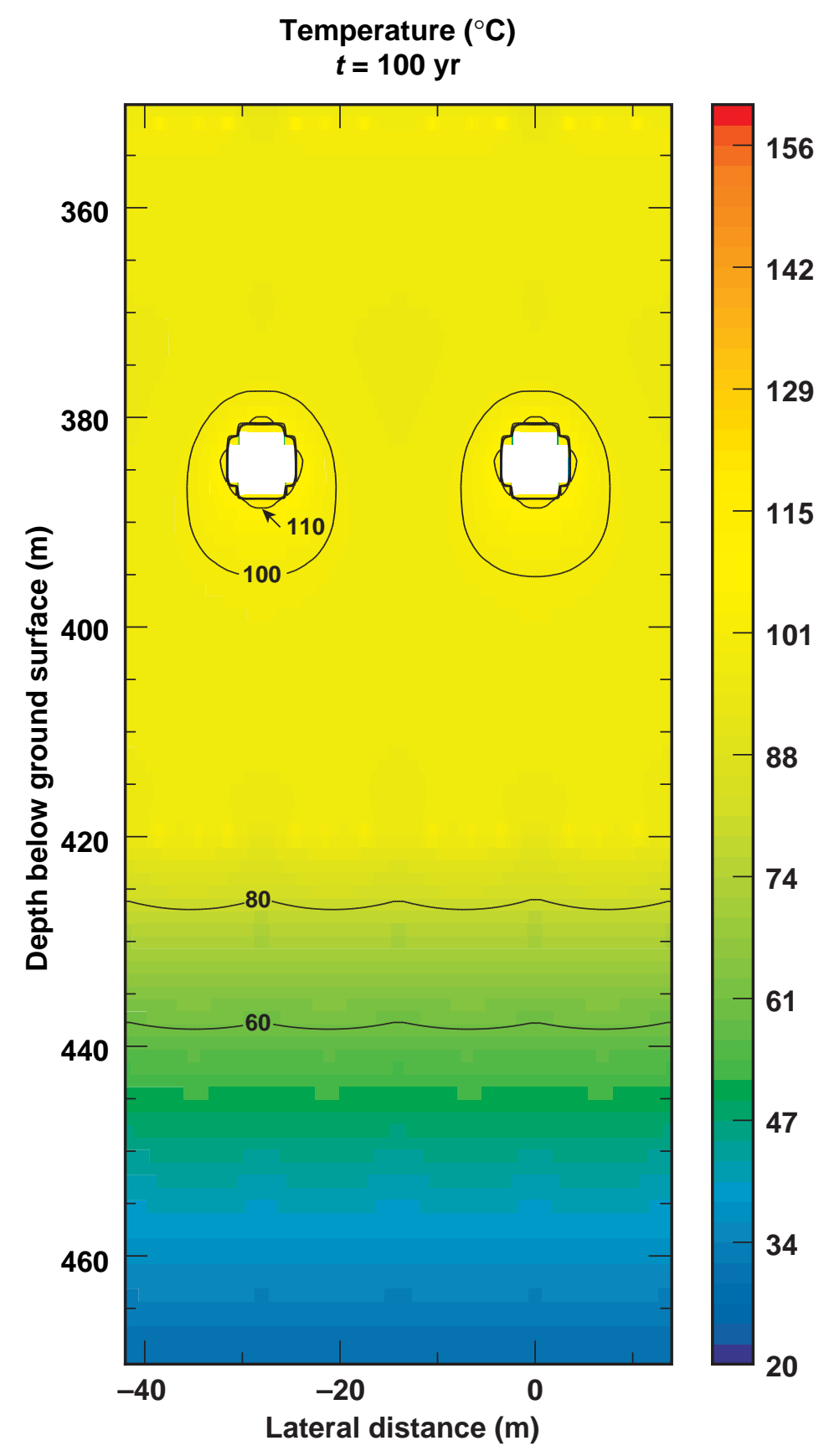

Figure 4-43. Simulation of $100 \mathrm{yr}$ temperature field for Case 1 for input to FRACROCK 


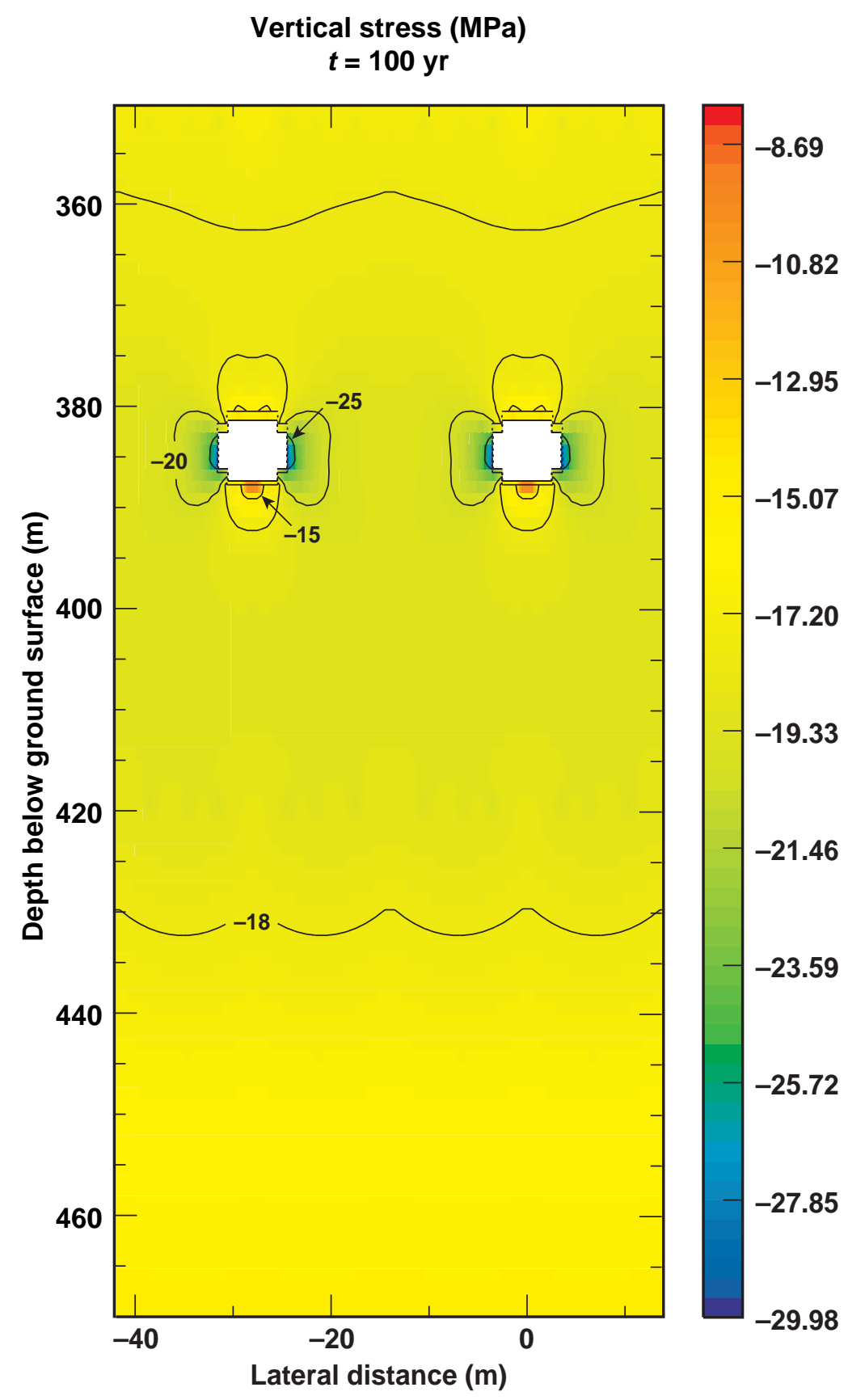

Figure 4-44. Vertical stress field for Case 1 at $100 \mathrm{yr}$, computed using FRACROCK 


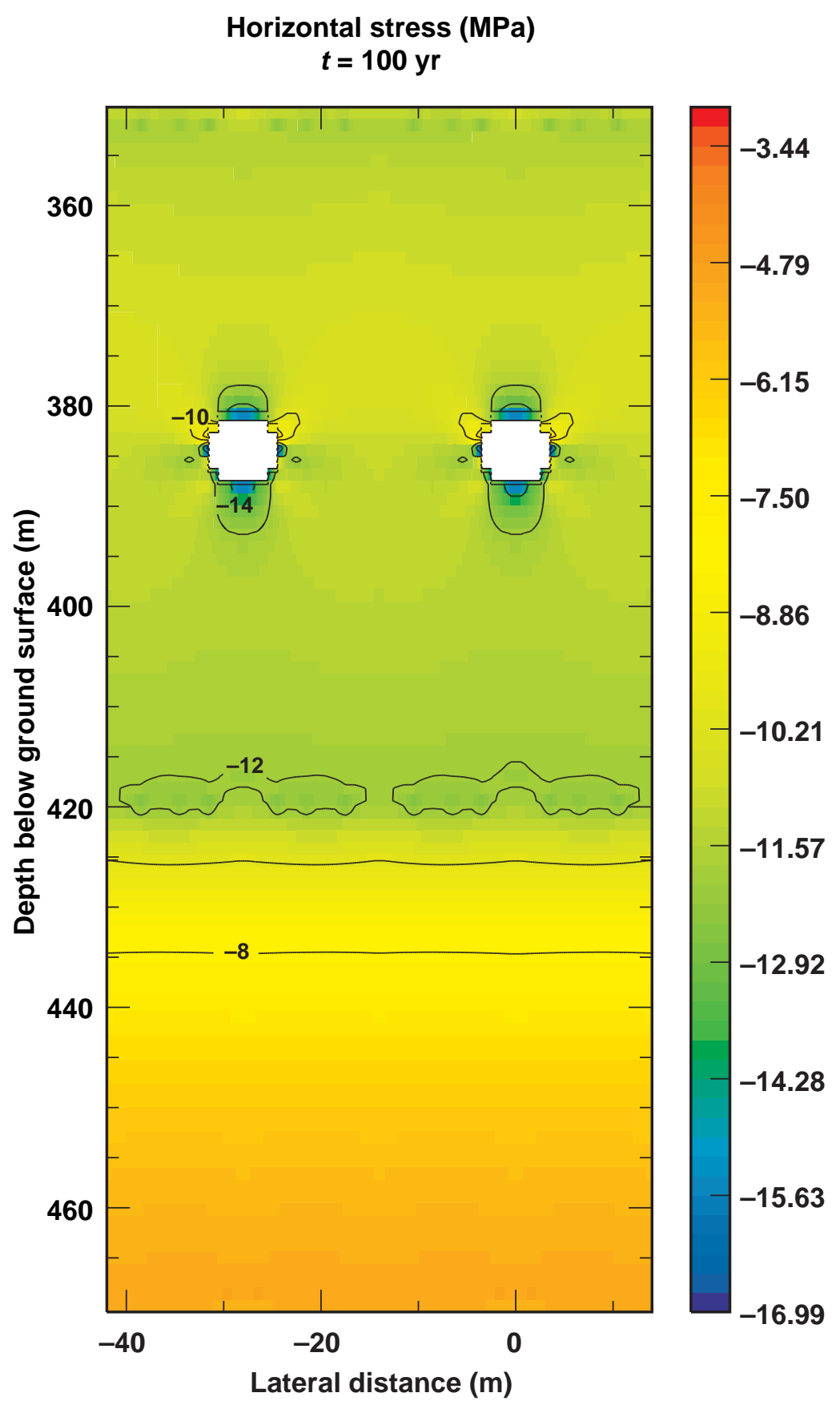

Figure 4-45. Horizontal stress field for Case 1 at $100 \mathrm{yr}$, computed using FRACROCK 


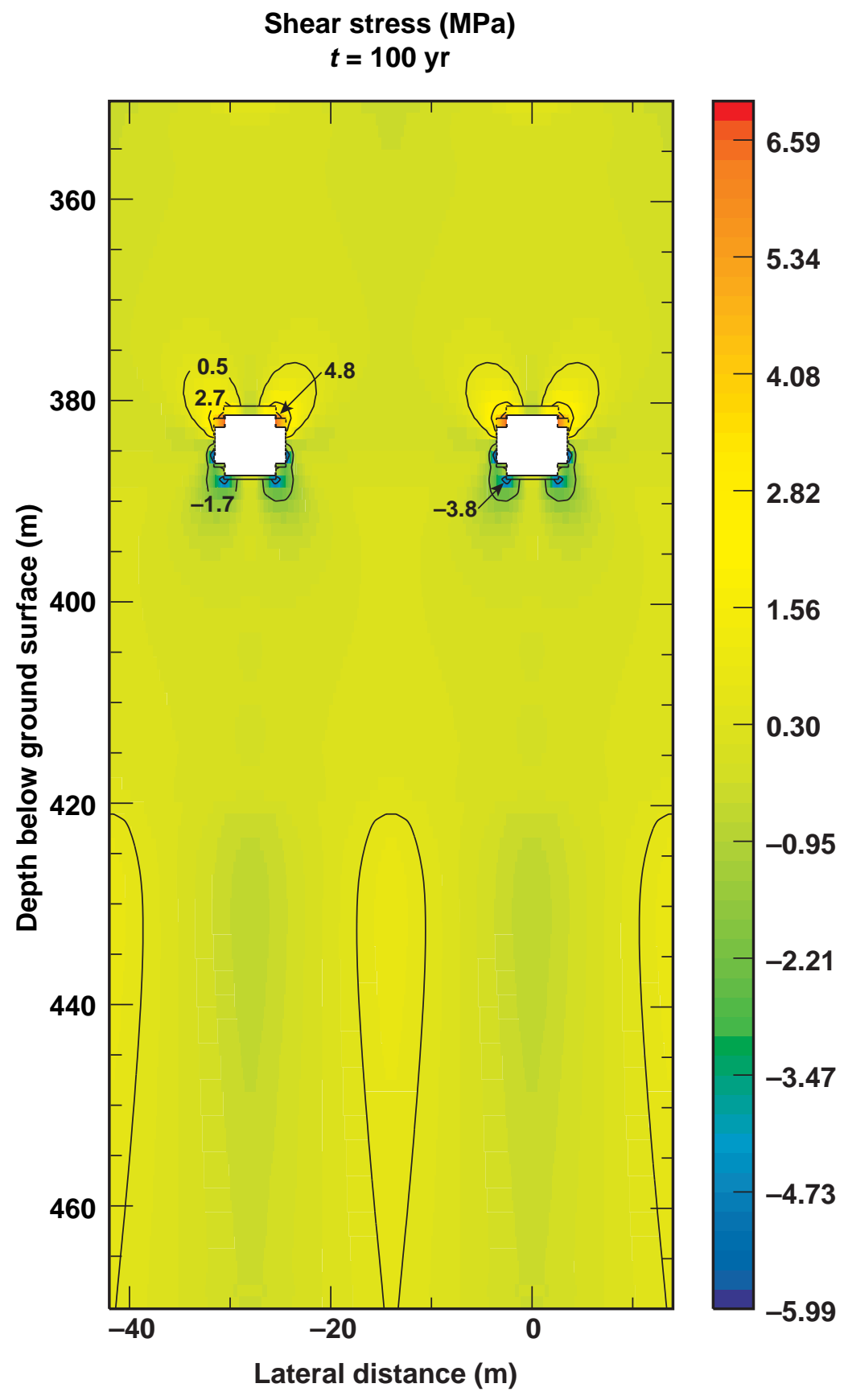

Figure 4-46. Shear stress field for Case 1 at $100 \mathrm{yr}$, computed using FRACROCK 


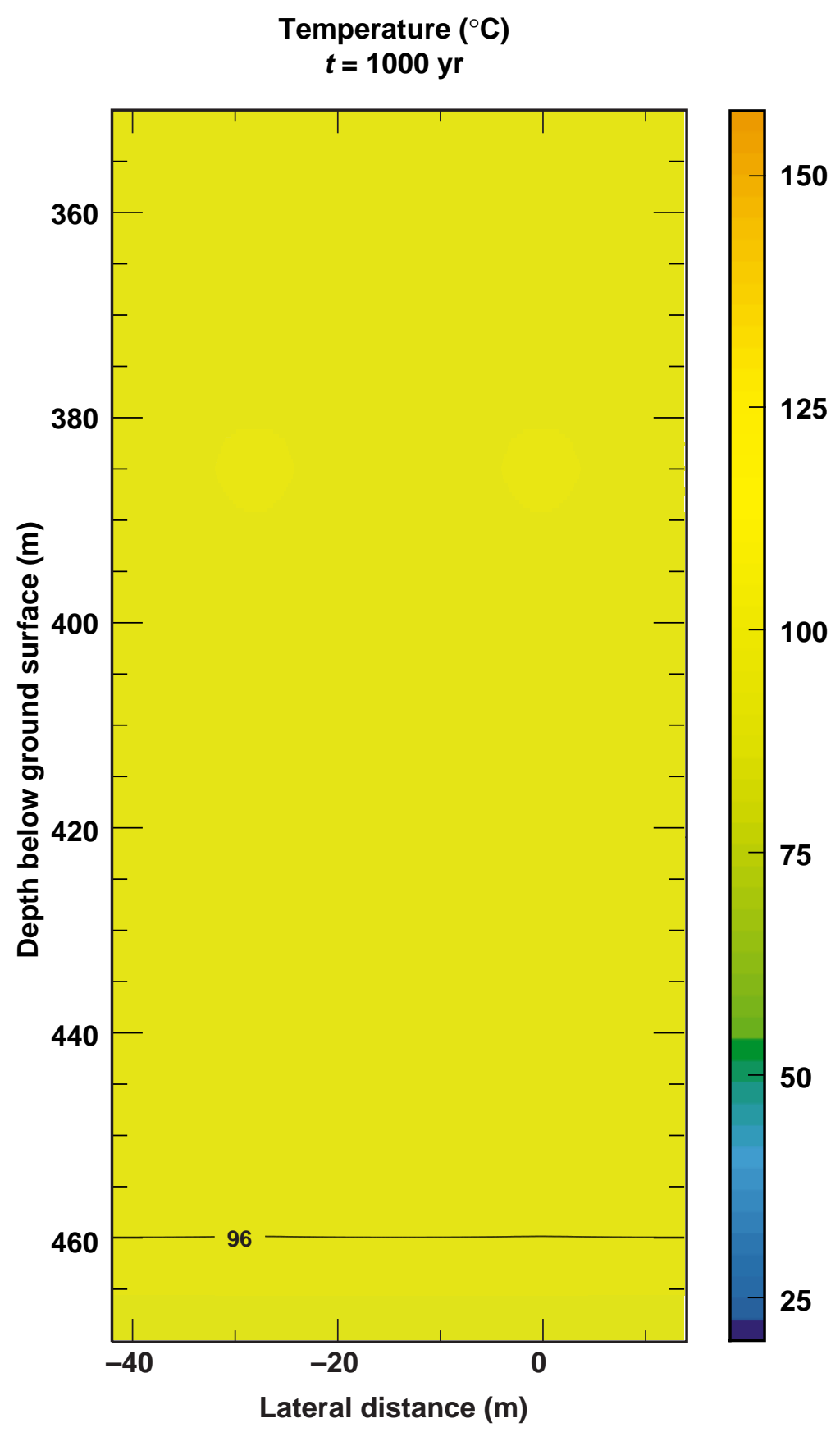

Figure 4-47. Temperature field for Case 1 at $1000 \mathrm{yr}$, as computed by NUFT 


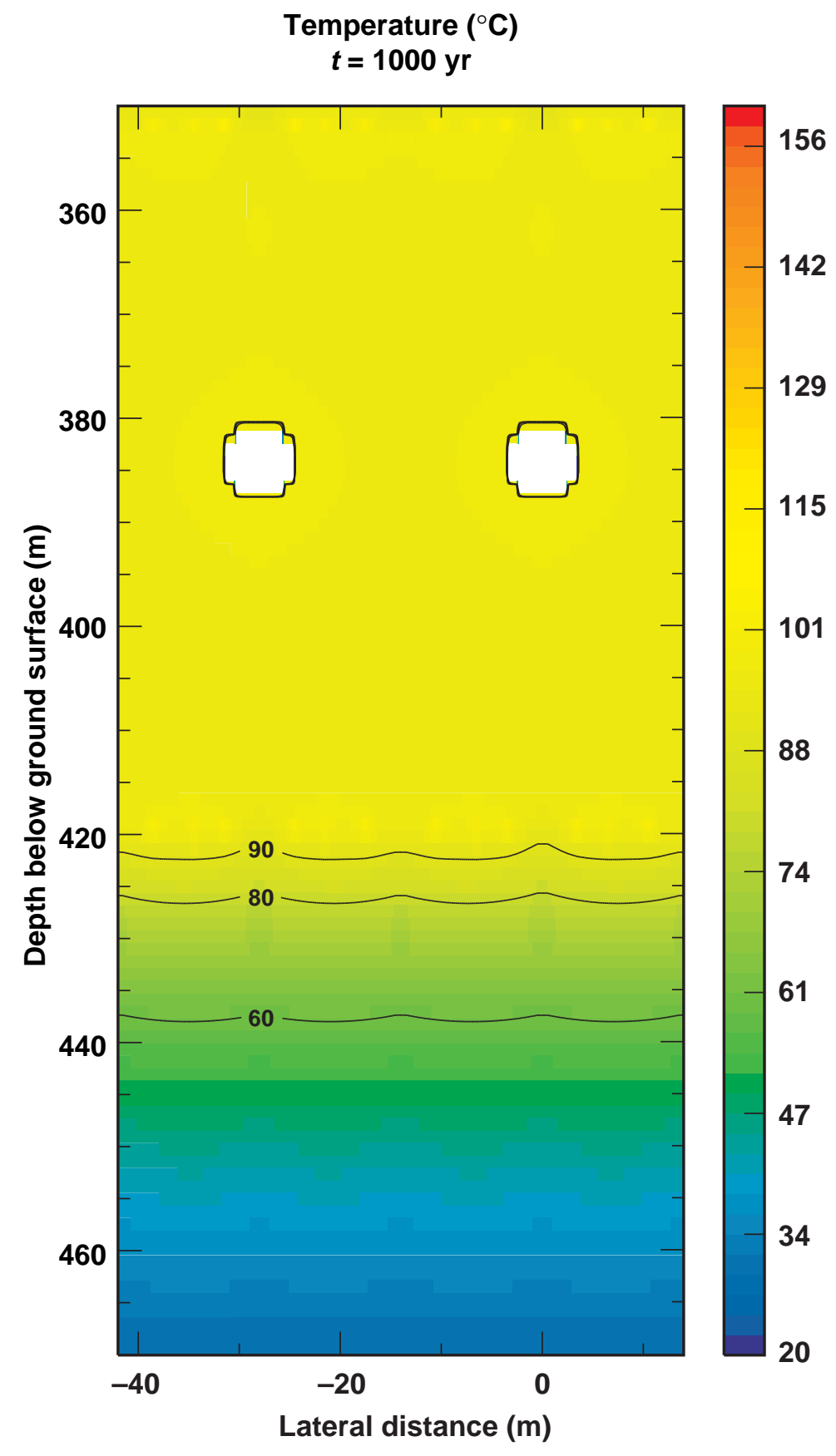

Figure 4-48. Simulation of $1000 \mathrm{yr}$ temperature field for Case 1 for input to FRACROCK 


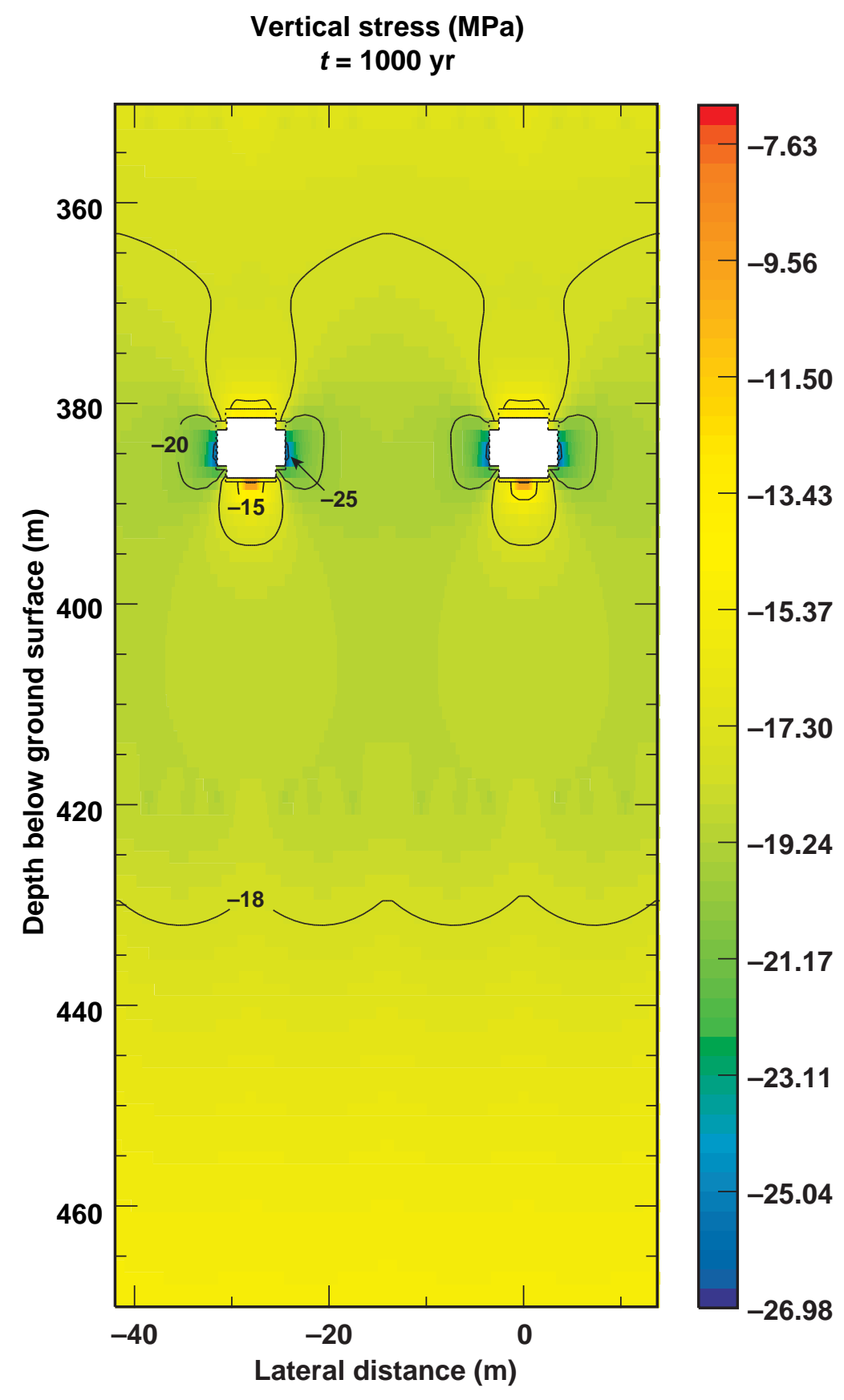

Figure 4-49. Vertical stress field for Case 1 at $1000 \mathrm{yr}$, computed using FRACROCK 


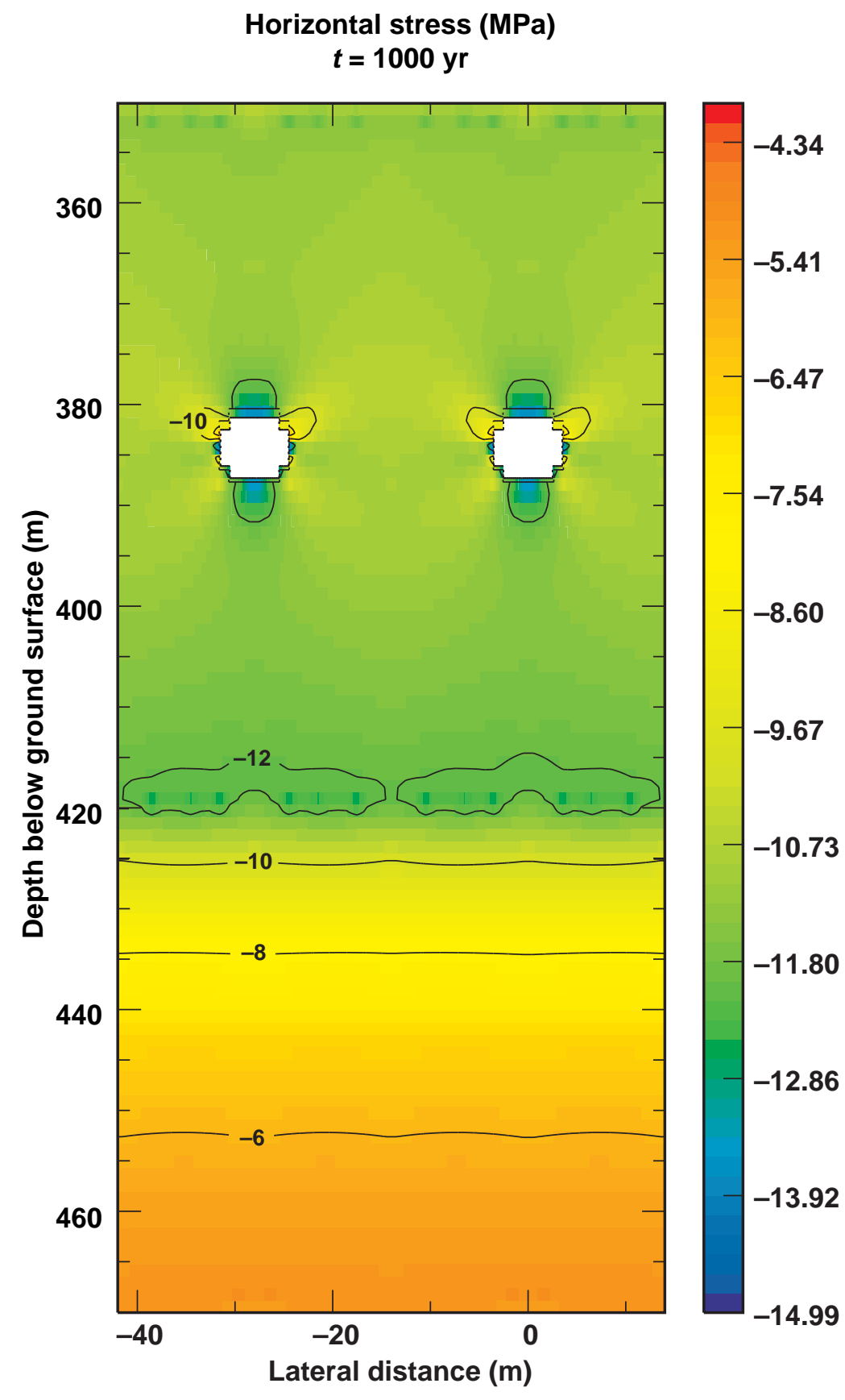

Figure 4-50. Horizontal stress field for Case 1 at $1000 \mathrm{yr}$, computed using FRACROCK 


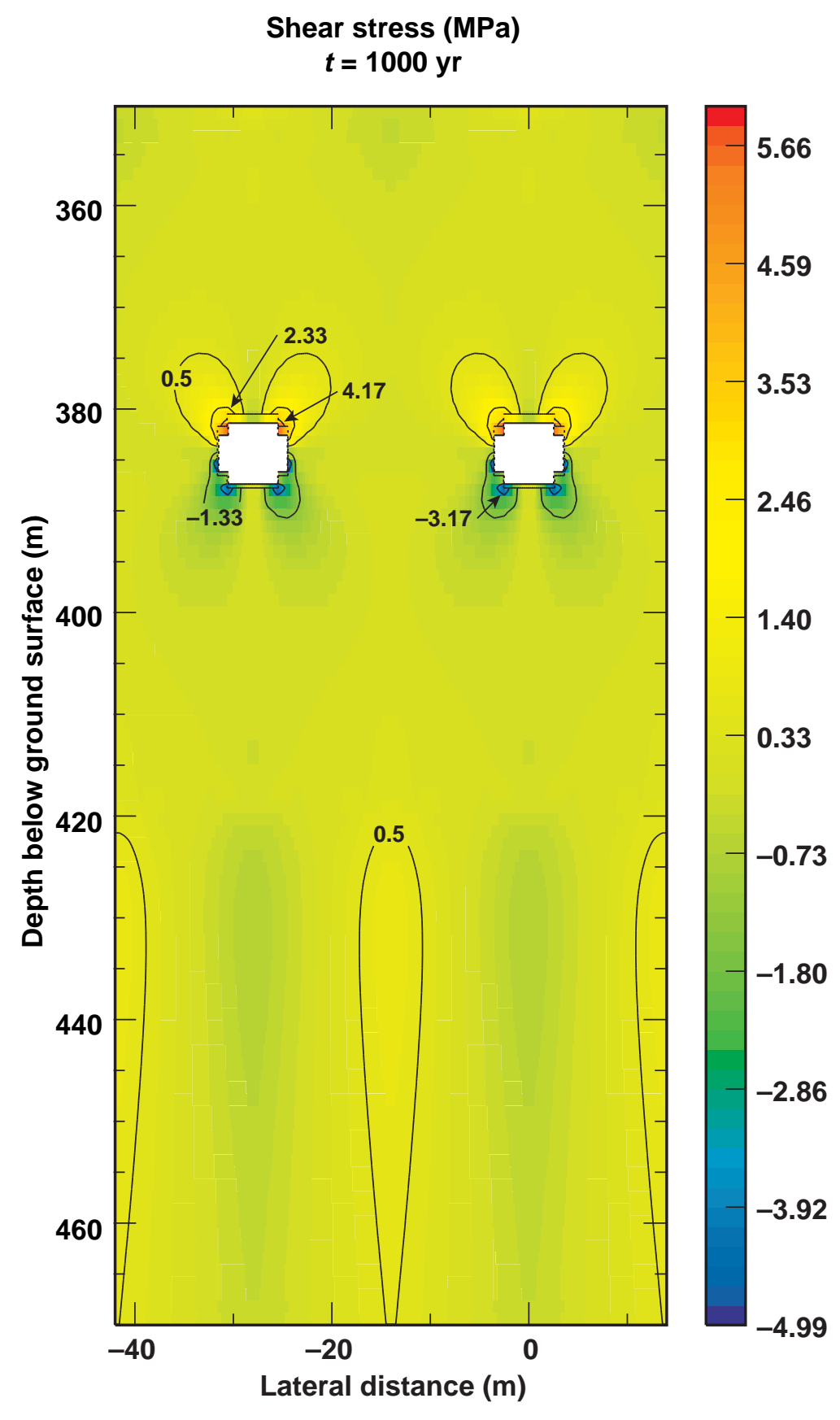

Figure 4-51. Shear stress field for Case 1 at $1000 \mathrm{yr}$, computed using FRACROCK 


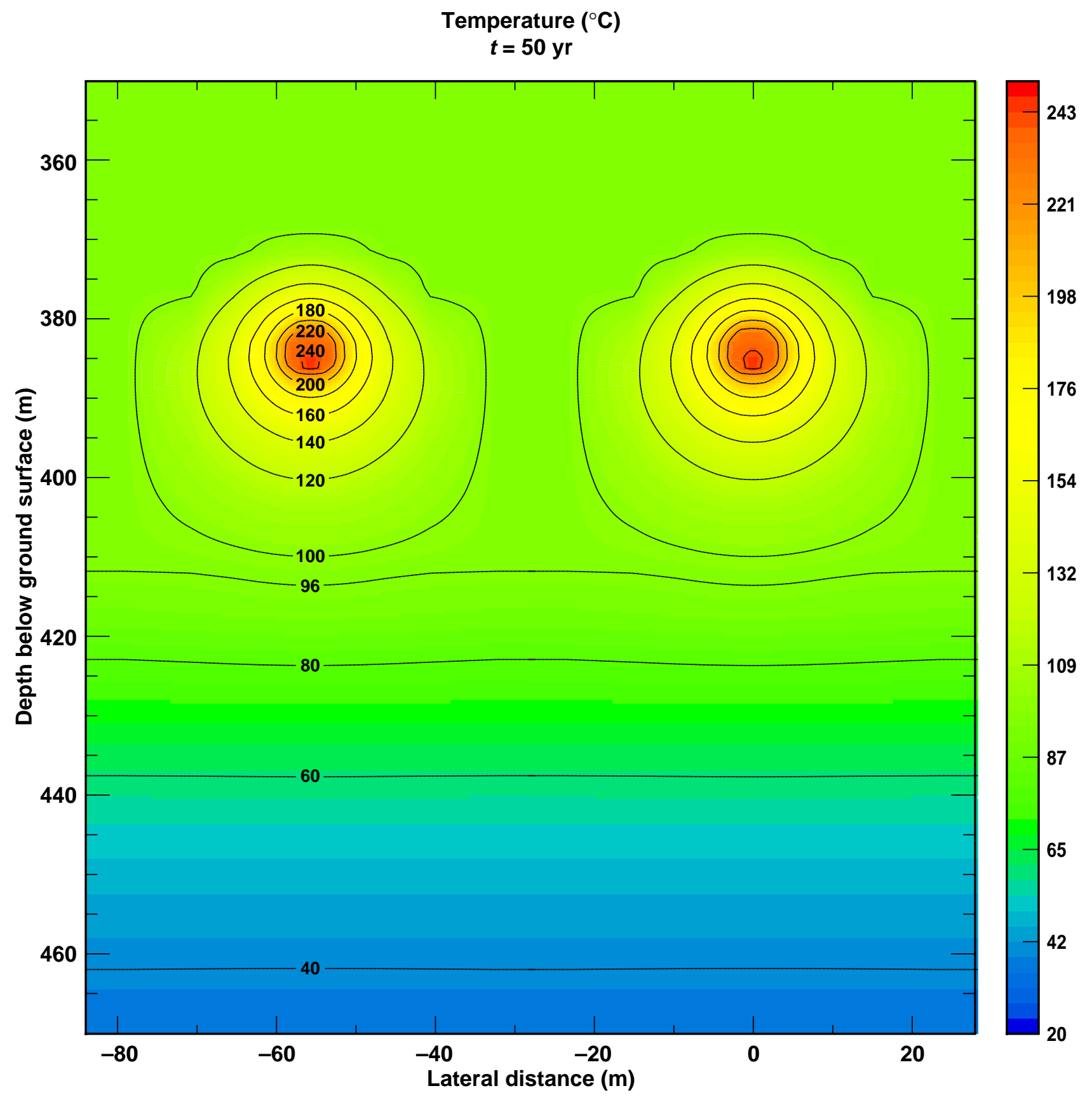

Figure 4-52. Temperature field for Case 5 at $50 \mathrm{yr}$, as computed by NUFT 


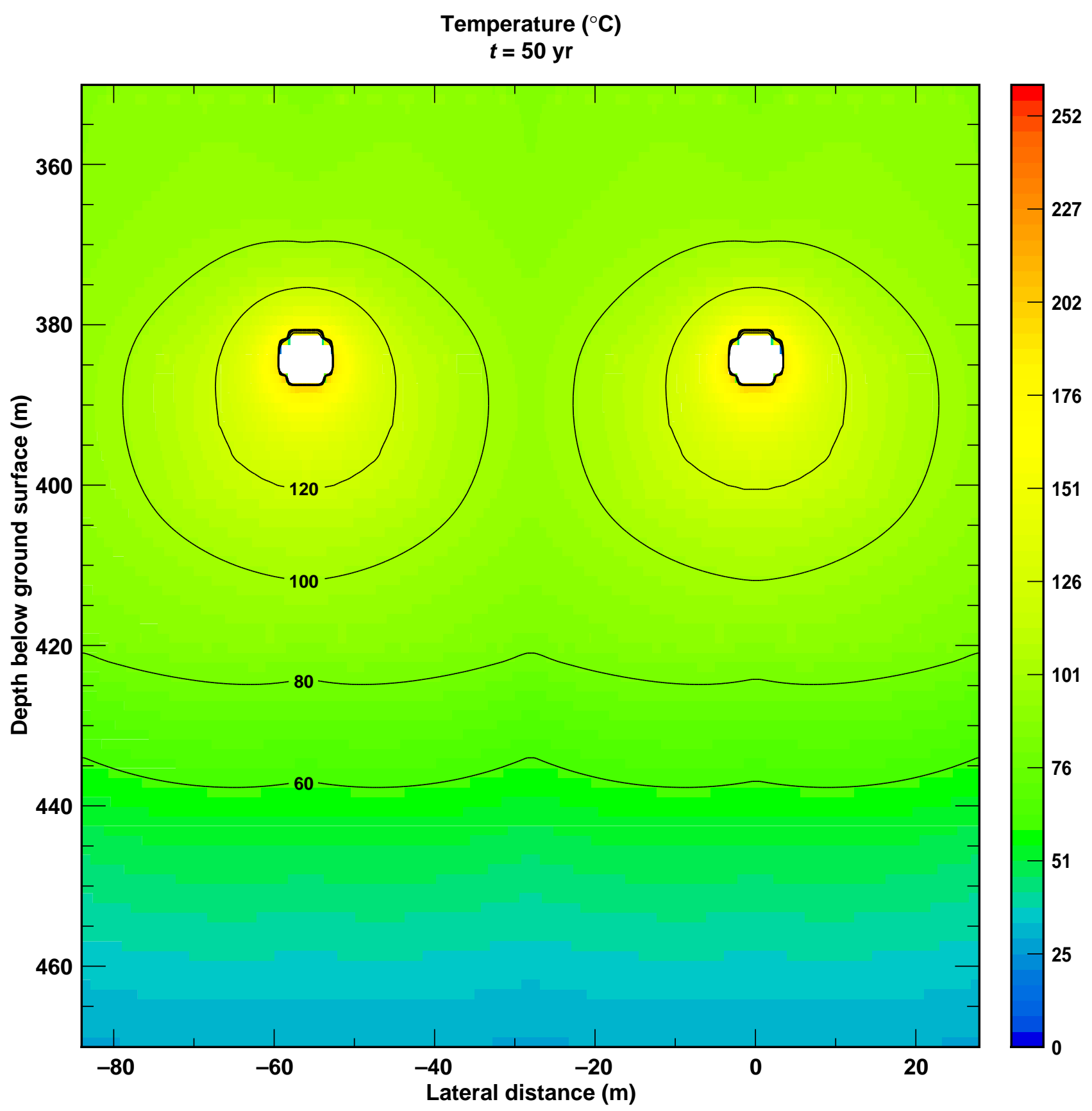

Figure 4-53. Simulation of 50-yr temperature field for Case 5 for input to FRACROCK 


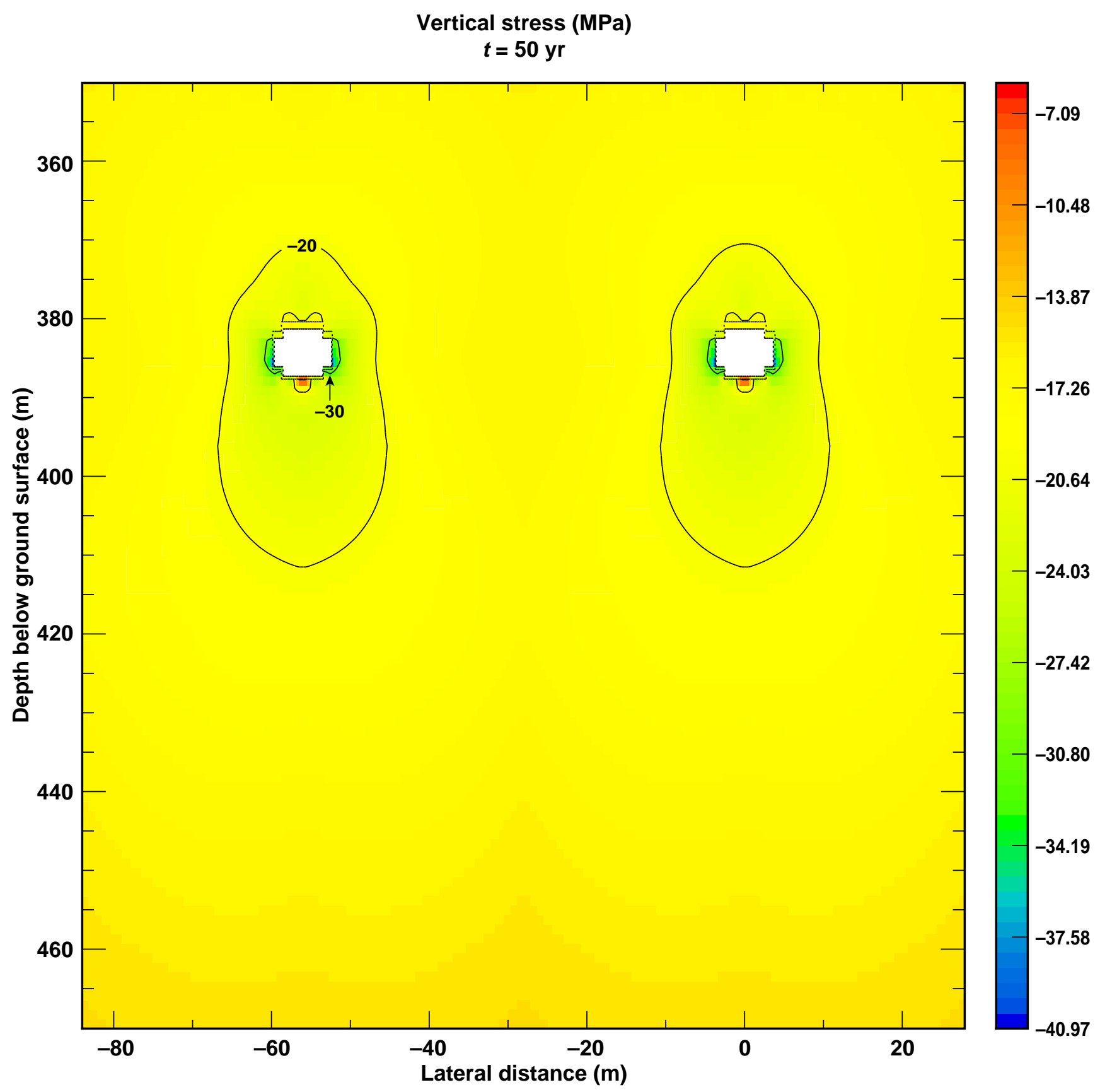

Figure 4-54. Vertical stress field for Case 5 at $50 \mathrm{yr}$, computed using FRACROCK 


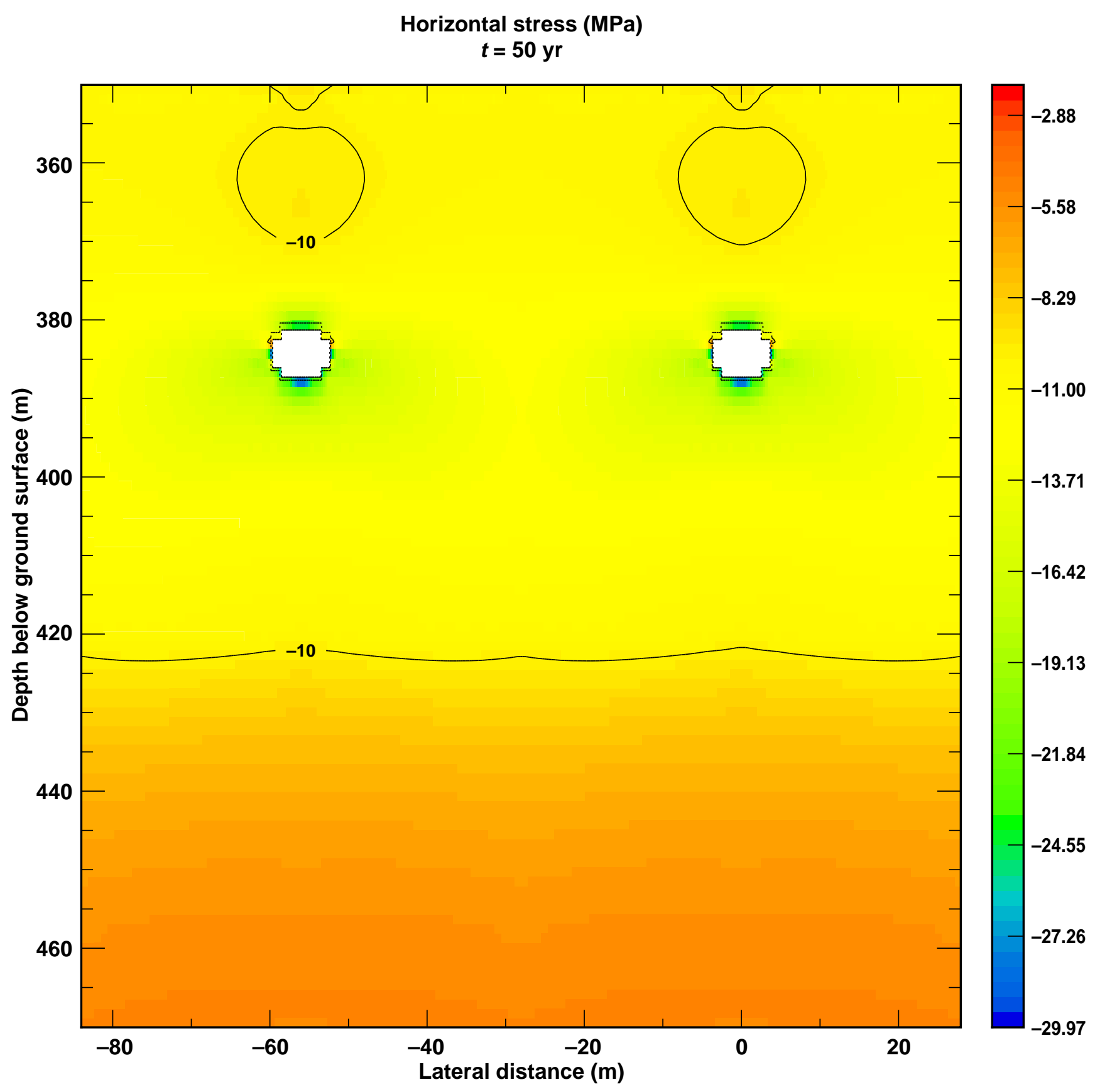

Figure 4-55 Horizontal stress field for Case 5 at $50 \mathrm{yr}$, computed using FRACROCK 


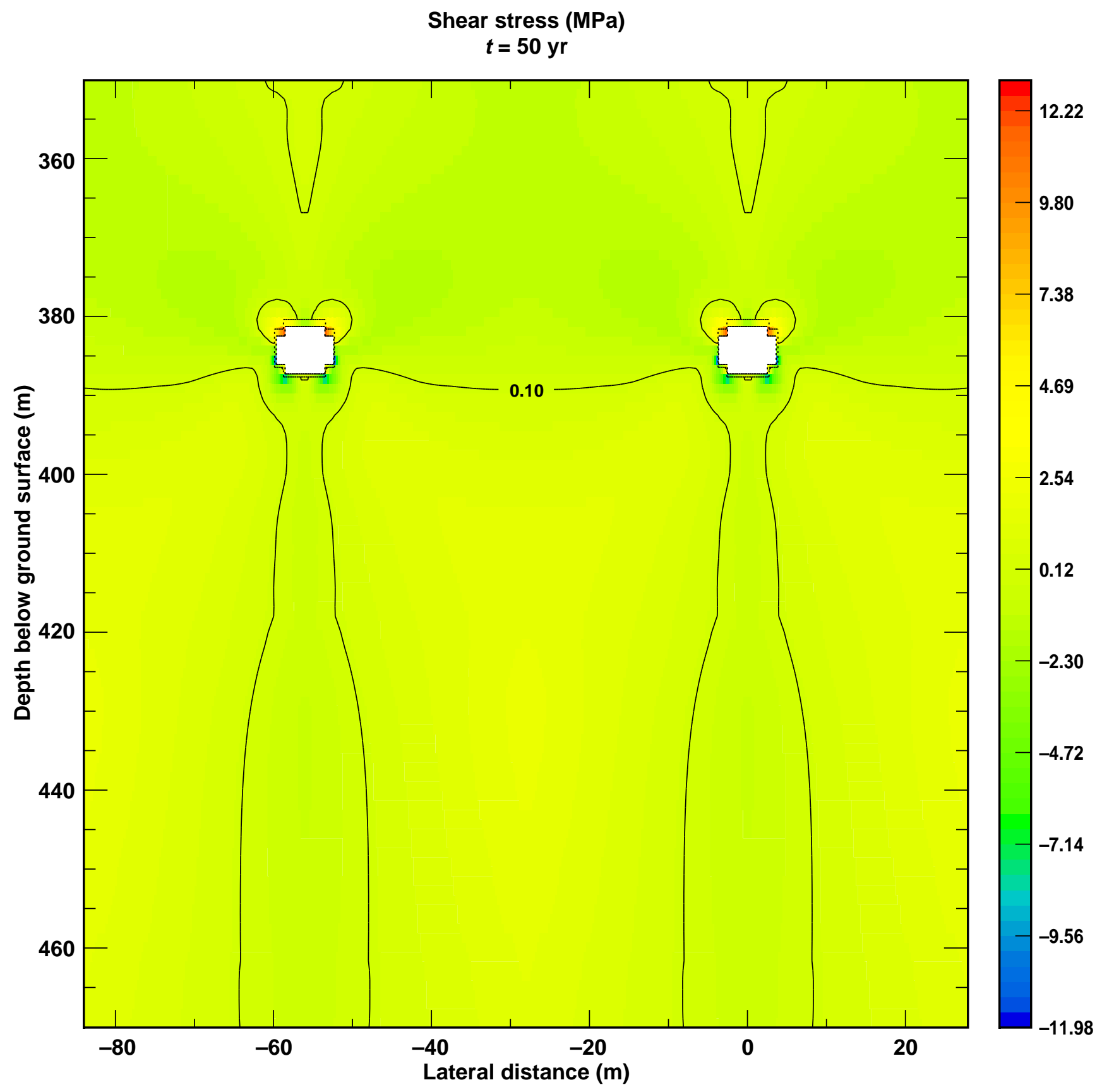

Figure 4-56. Shear stress field for Case 5 at $50 \mathrm{yr}$, computed using FRACROCK 


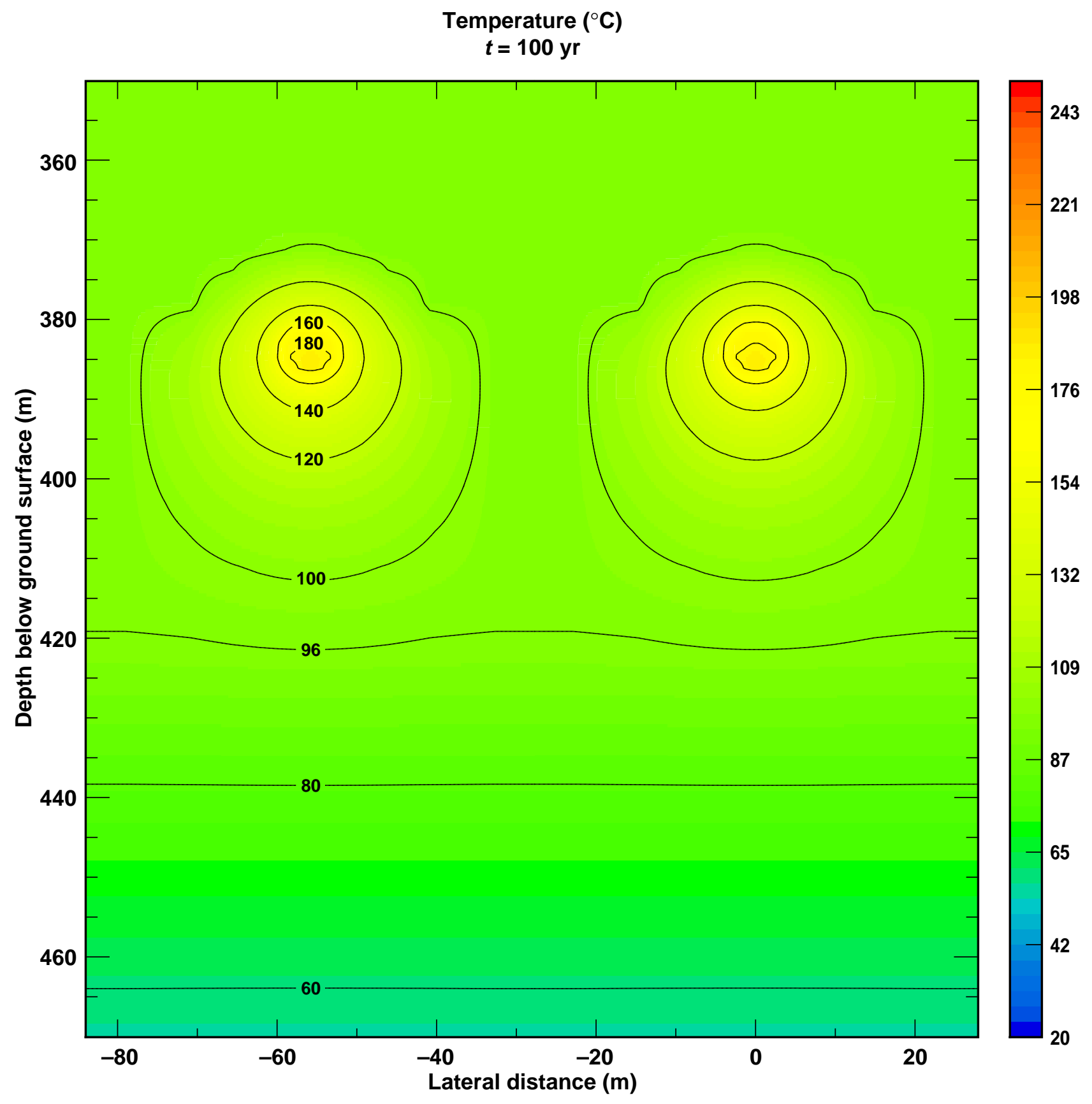

Figure 4-57 Temperature field for Case 5 at $100 \mathrm{yr}$, as computed by NUFT 


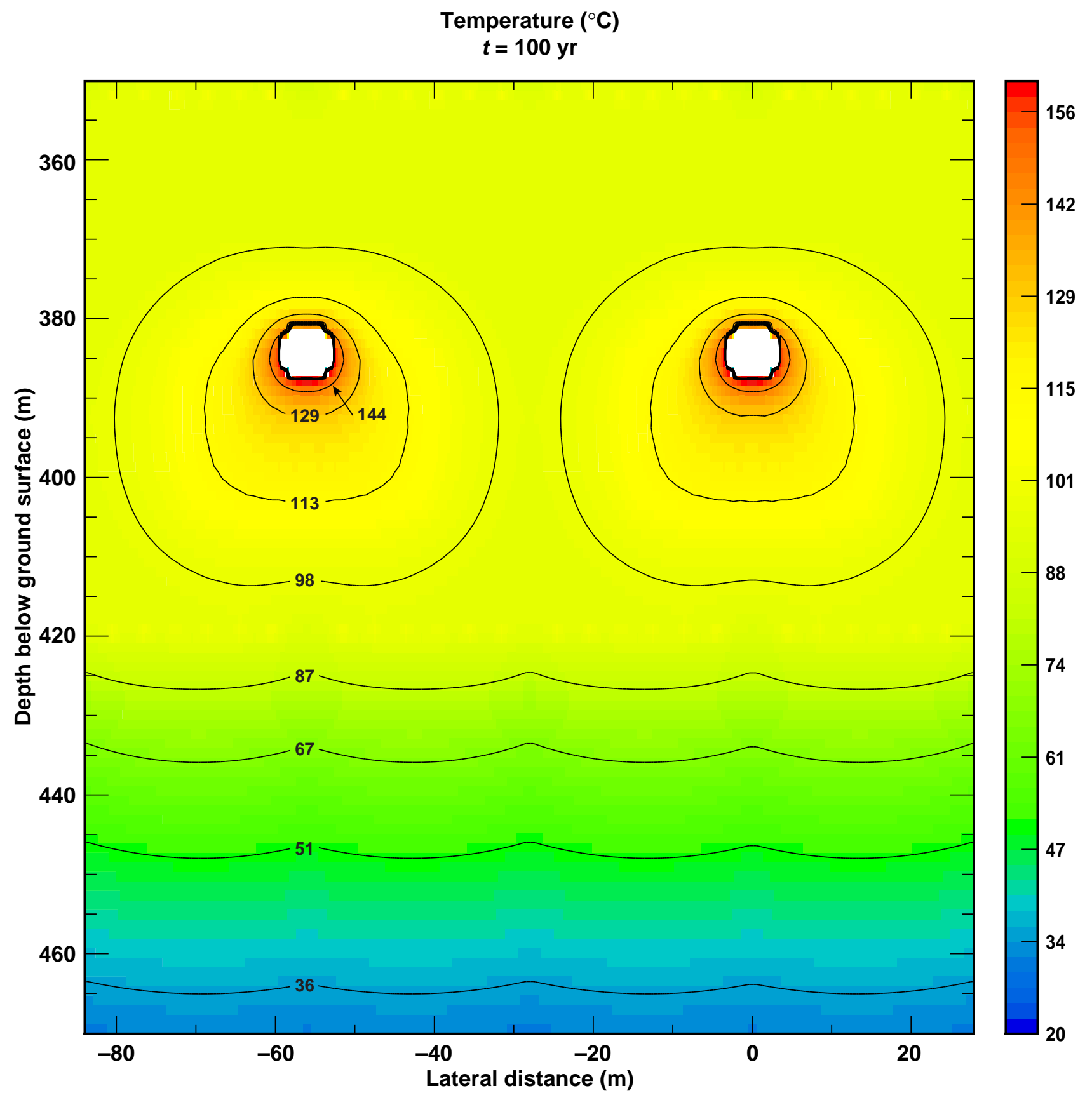

Figure 4-58. Simulation of 100-yr temperature field for Case 5 for input to FRACROCK 


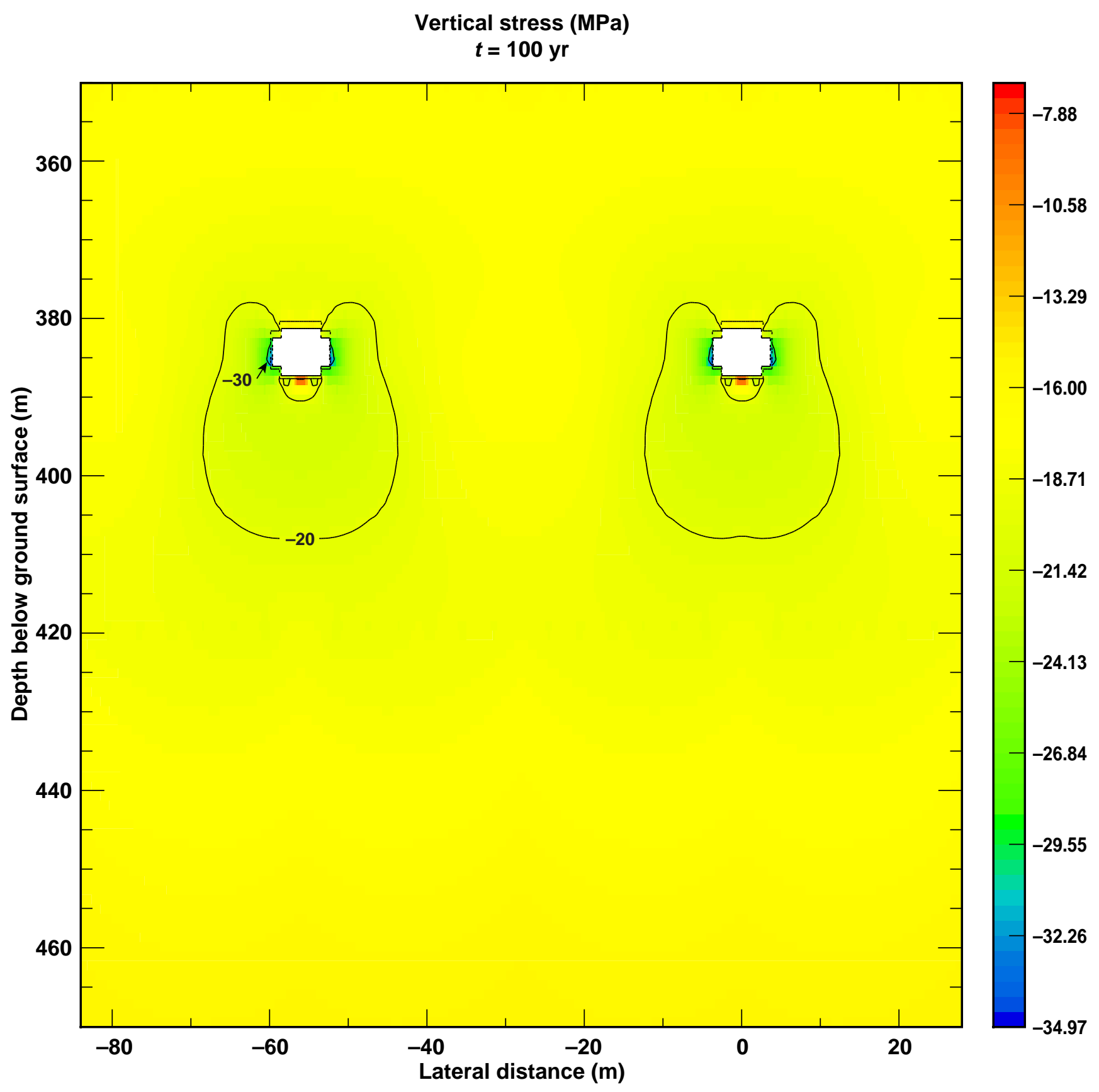

Figure 4-59. Vertical stress field for Case 5 at $100 \mathrm{yr}$, computed using FRACROCK 


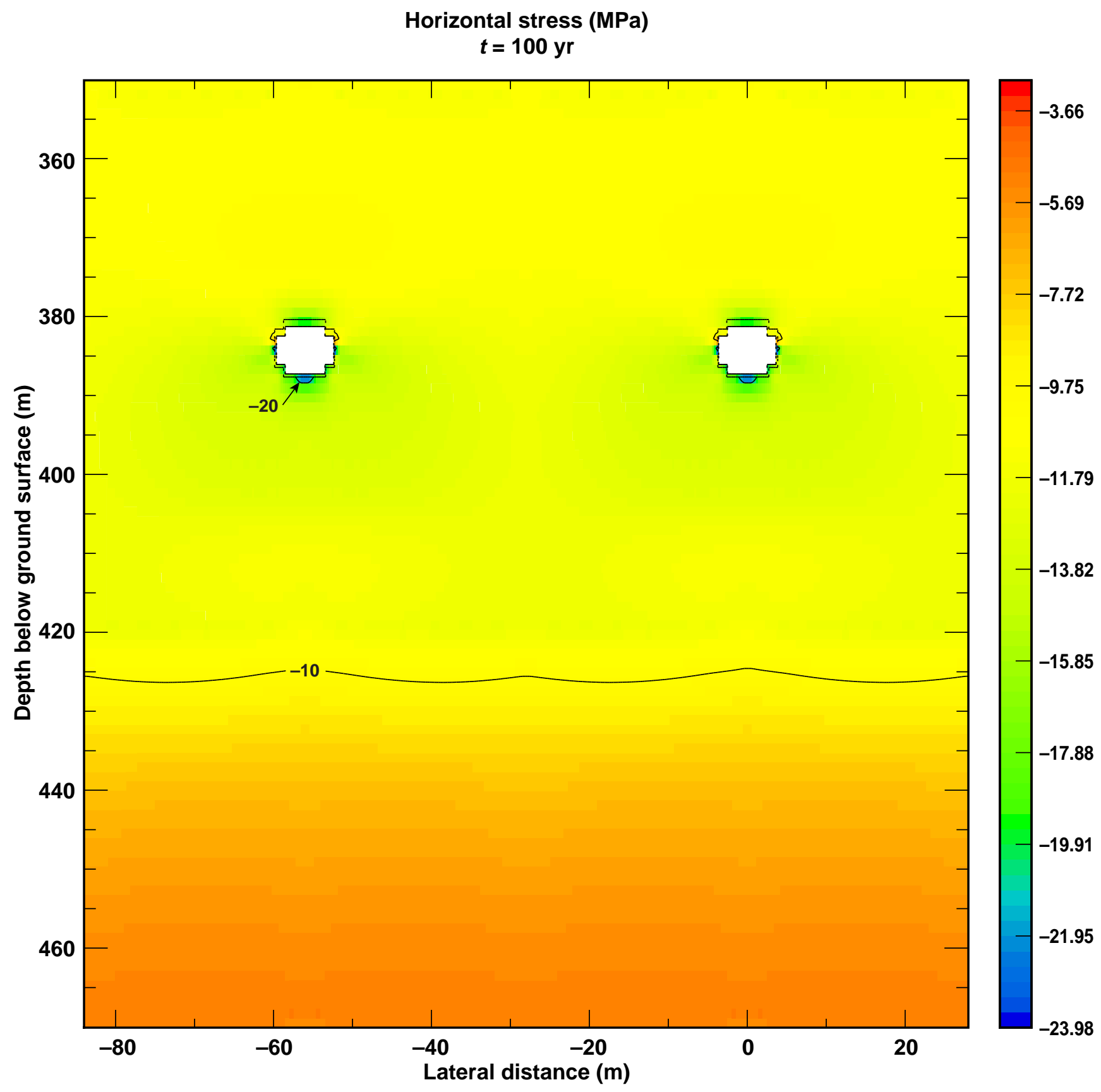

Figure 4-60. Horizontal stress field for Case 5 at $100 \mathrm{yr}$, computed using FRACROCK 


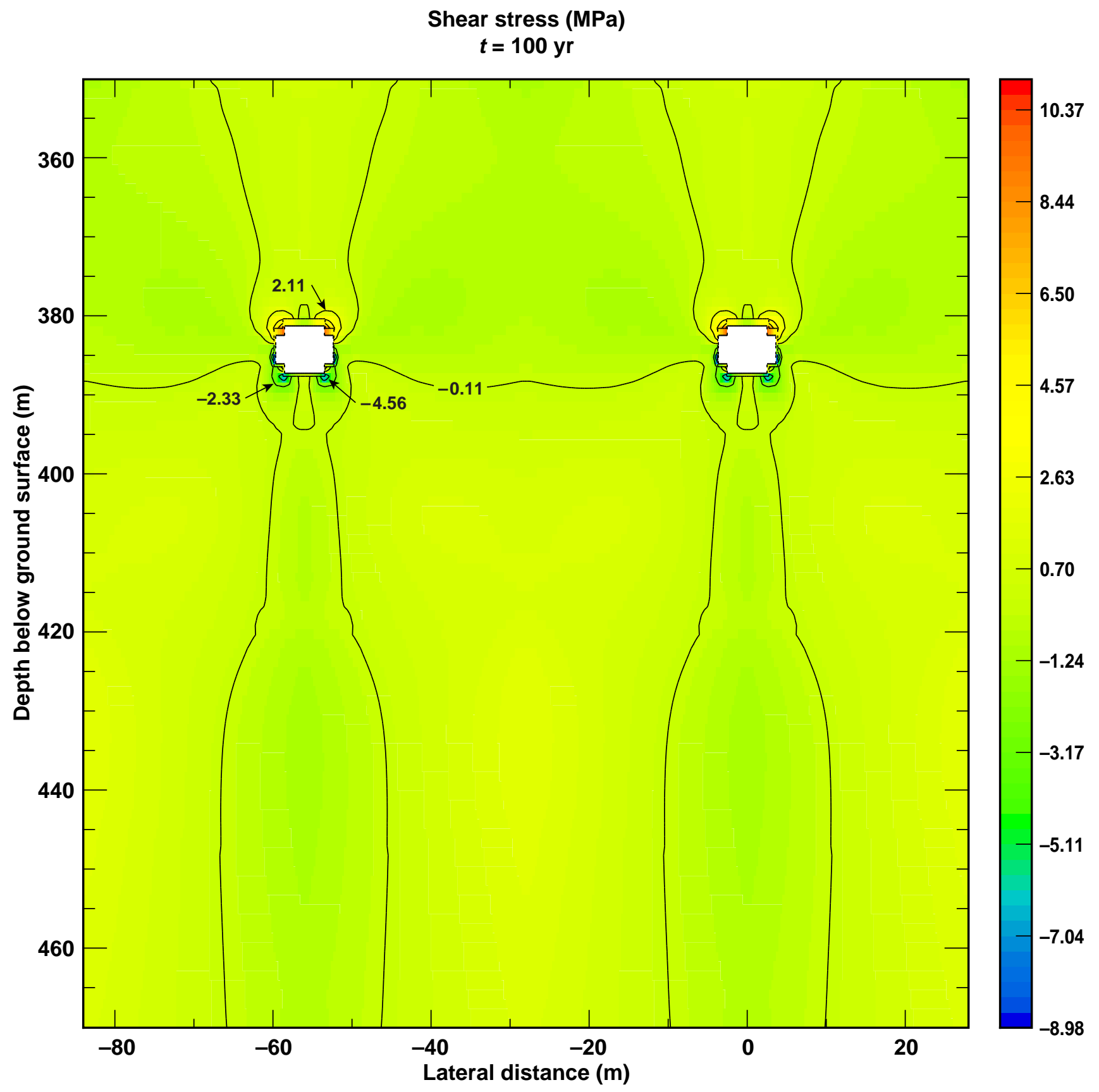

Figure 4-61. Shear stress field for Case 5 at $100 \mathrm{yr}$, computed using FRACROCK 


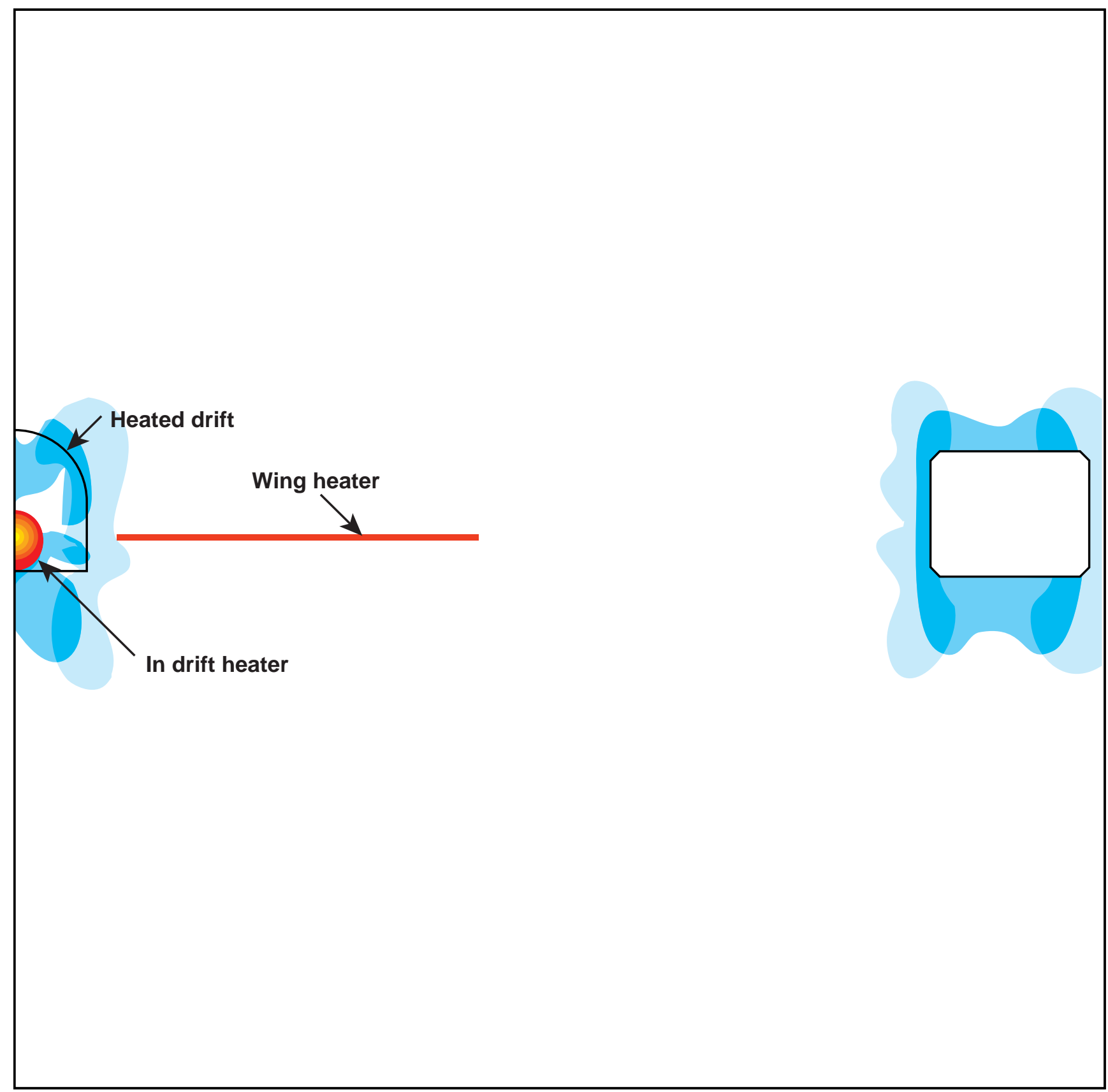

Figure 4-62. Zones of enhanced permeability predicted for the DST before heating: light shade = zone where permeability is enhanced due to slip on vertical fractures; medium shade = zone where permeability is enhanced due to slip on horizontal fractures; dark shade = zone where permeability is enhanced due to slip on both vertical and horizontal fractures 


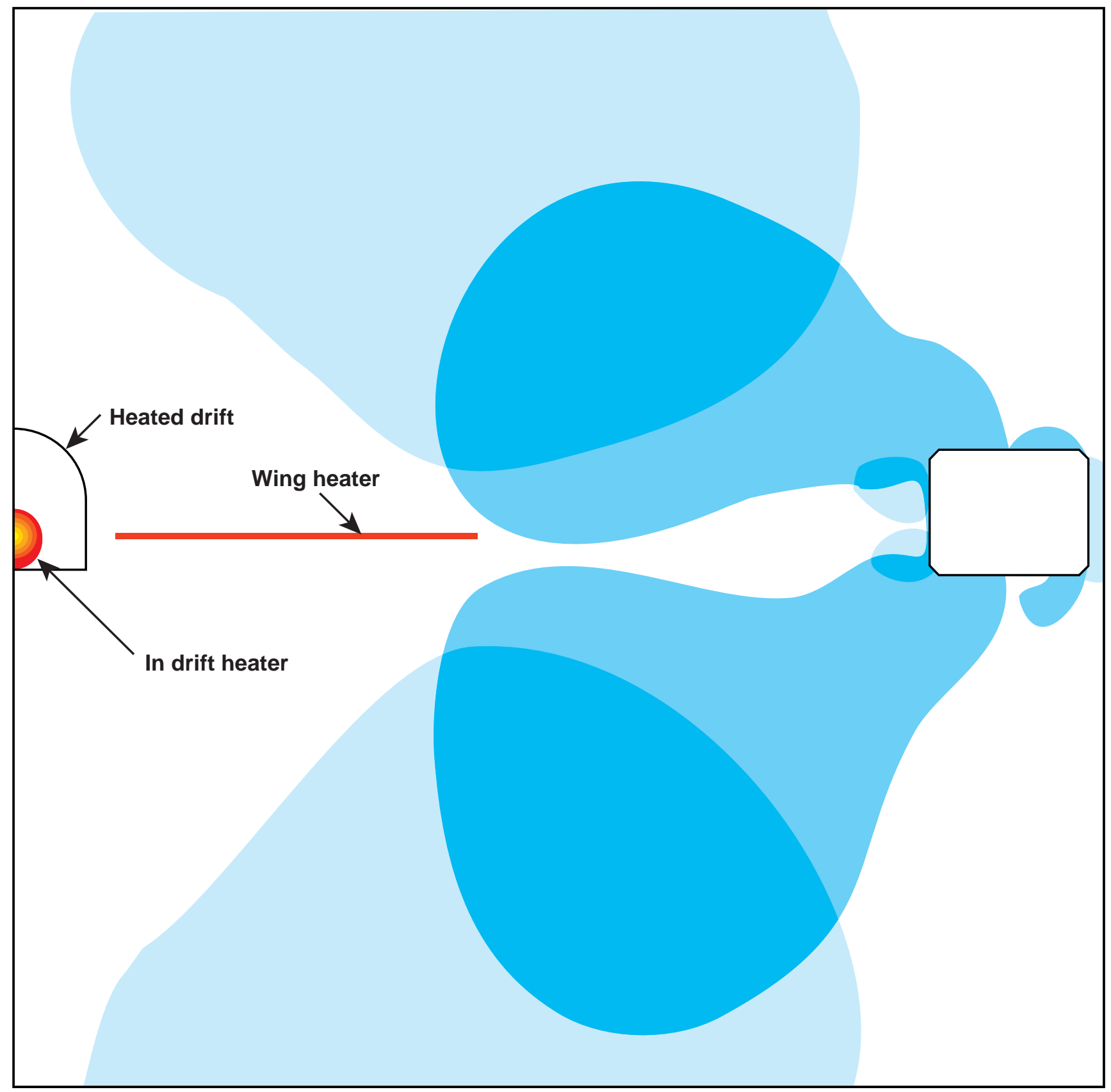

Figure 4-63. Zones of enhanced permeability predicted for the DST at $0.5 \mathrm{yr}$ : light shade $=$ zone where permeability is enhanced due to slip on vertical fractures; medium shade = zone where permeability is enhanced due to slip on horizontal fractures; dark shade = zone where permeability is enhanced due to slip on both vertical and horizontal fractures 


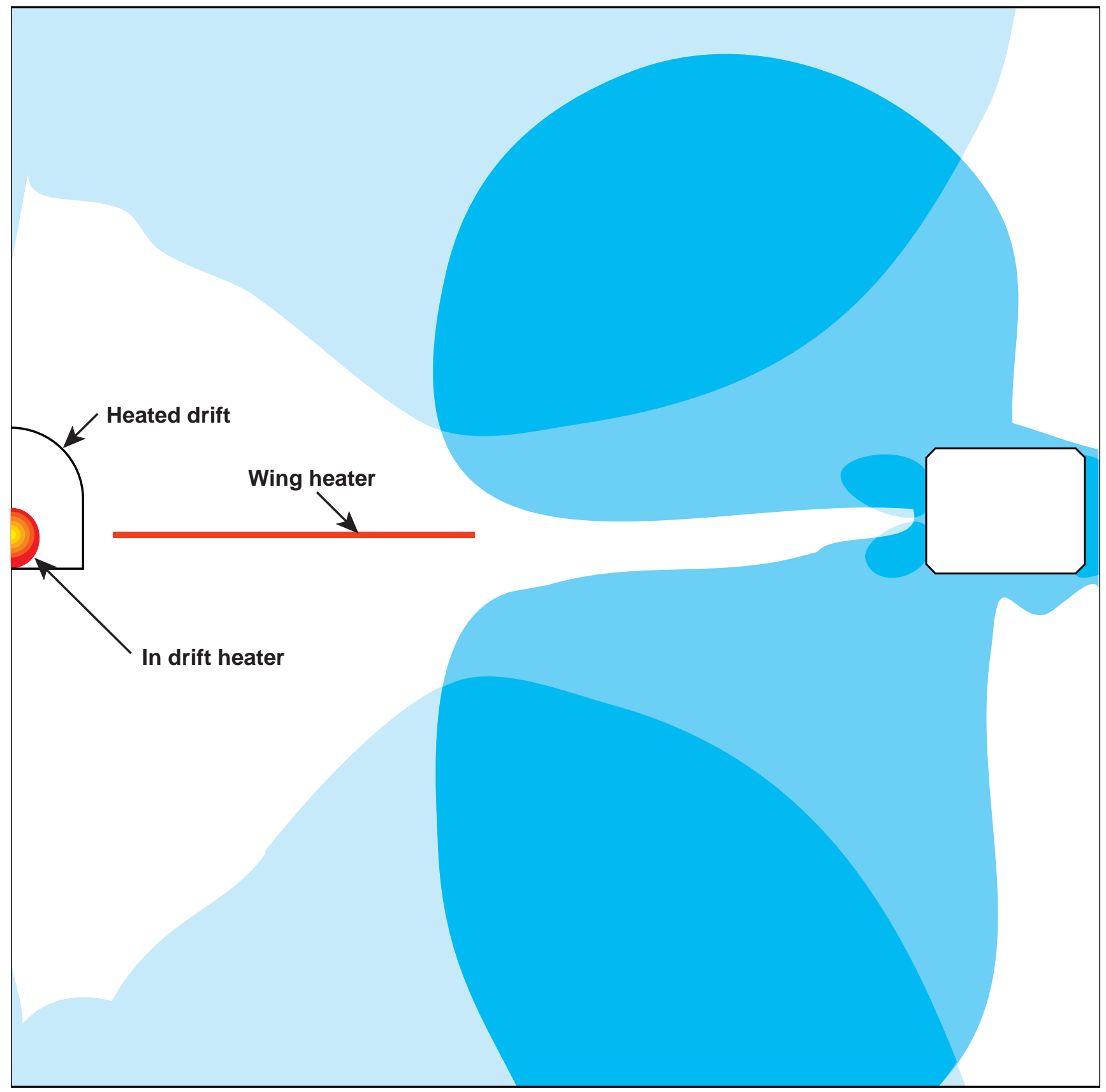

Figure 4-64. Zones of enhanced permeability predicted for the DST at $1.0 \mathrm{yr}$ : light shade = zone where permeability is enhanced due to slip on vertical fractures; medium shade = zone where permeability is enhanced due to slip on horizontal fractures; dark shade = zone where permeability is enhanced due to slip on both vertical and horizontal fractures 


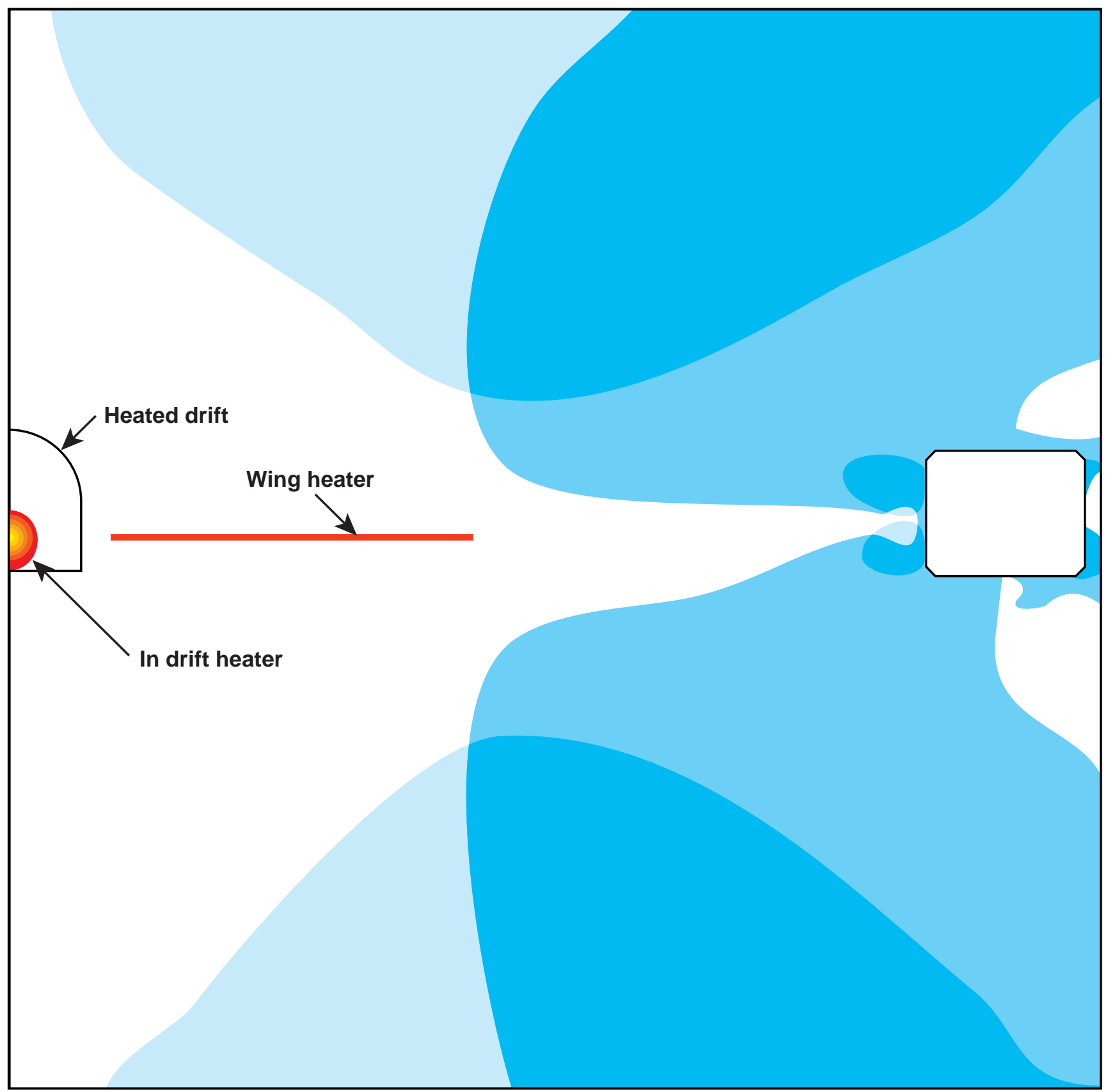

Figure 4-65. Zones of enhanced permeability predicted for the DST at $2.0 \mathrm{yr}$ : light shade = zone where permeability is enhanced due to slip on vertical fractures; medium shade = zone where permeability is enhanced due to slip on horizontal fractures; dark shade = zone where permeability is enhanced due to slip on both vertical and horizontal fractures 


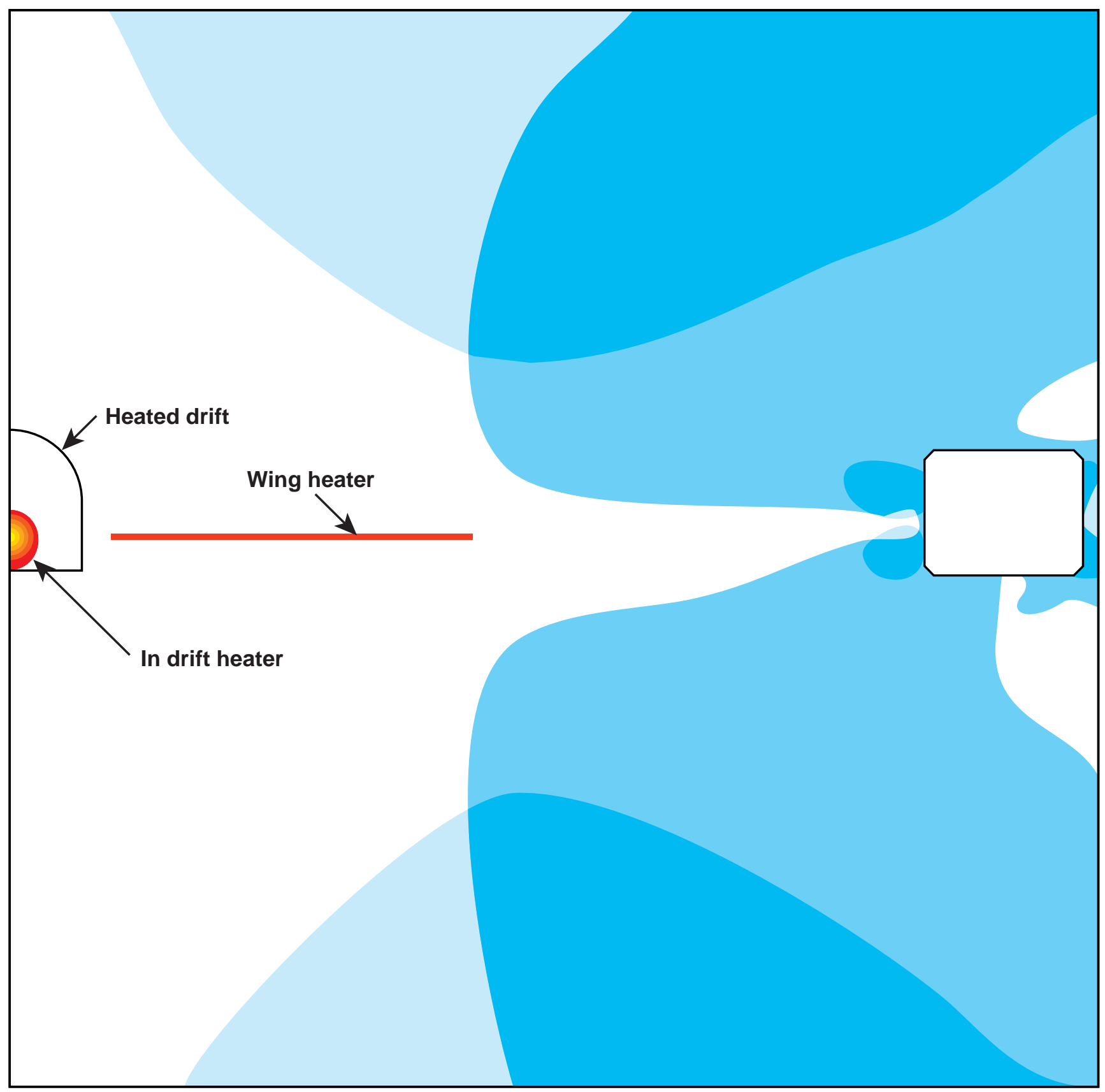

Figure 4-66. Zones of enhanced permeability predicted for the DST at $3.0 \mathrm{yr}$ : light shade = zone where permeability is enhanced due to slip on vertical fractures; medium shade = zone where permeability is enhanced due to slip on horizontal fractures; dark shade = zone where permeability is enhanced due to slip on both vertical and horizontal fractures 


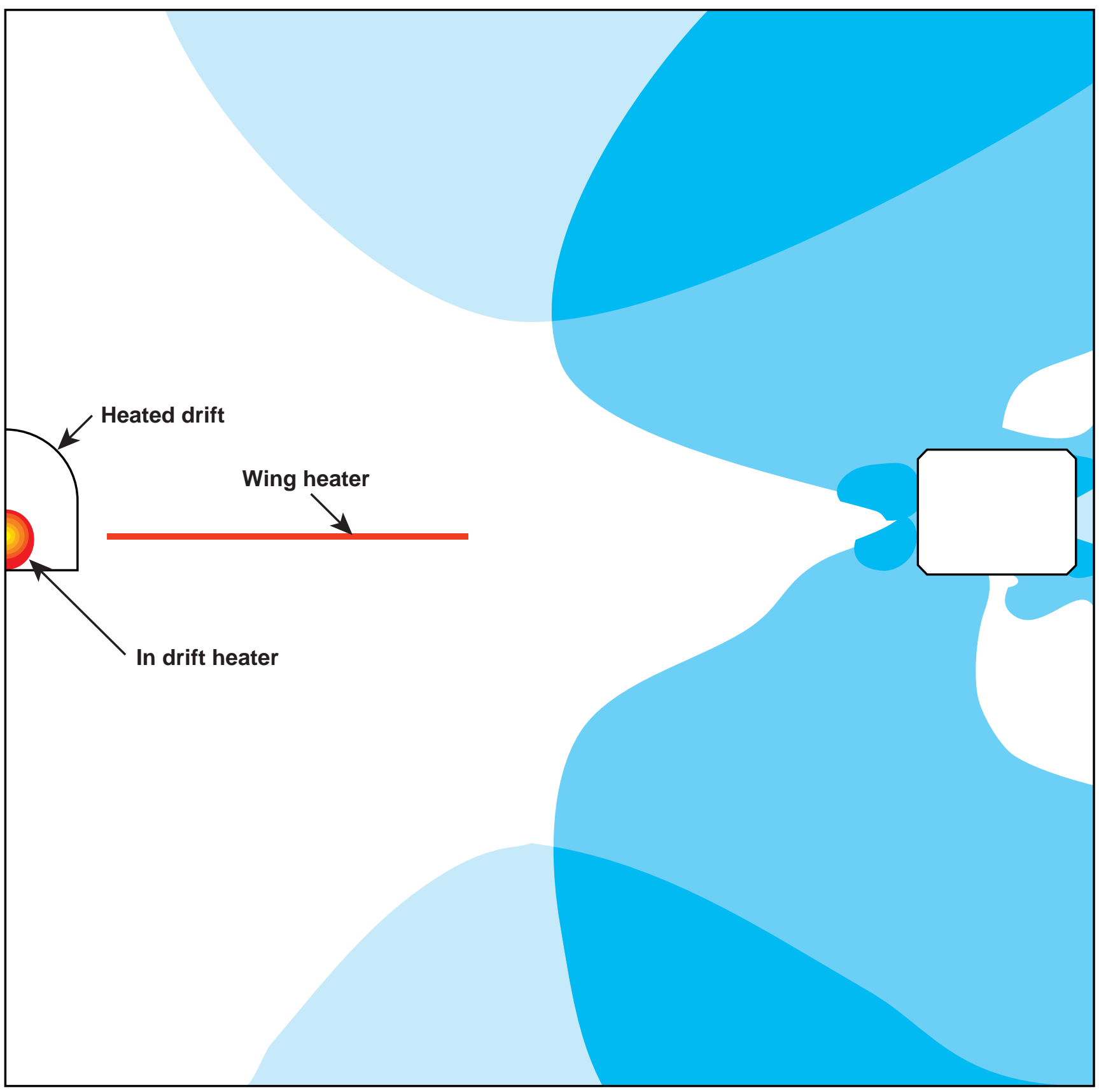

Figure 4-67. Zones of enhanced permeability predicted for the DST at $4.0 \mathrm{yr}$ : light shade = zone where permeability is increased due to slip on vertical fractures; medium shade $=$ zone where permeability is increased due to slip on horizontal fractures; dark shade = zone where permeability is increased due to slip on both vertical and horizontal fractures 


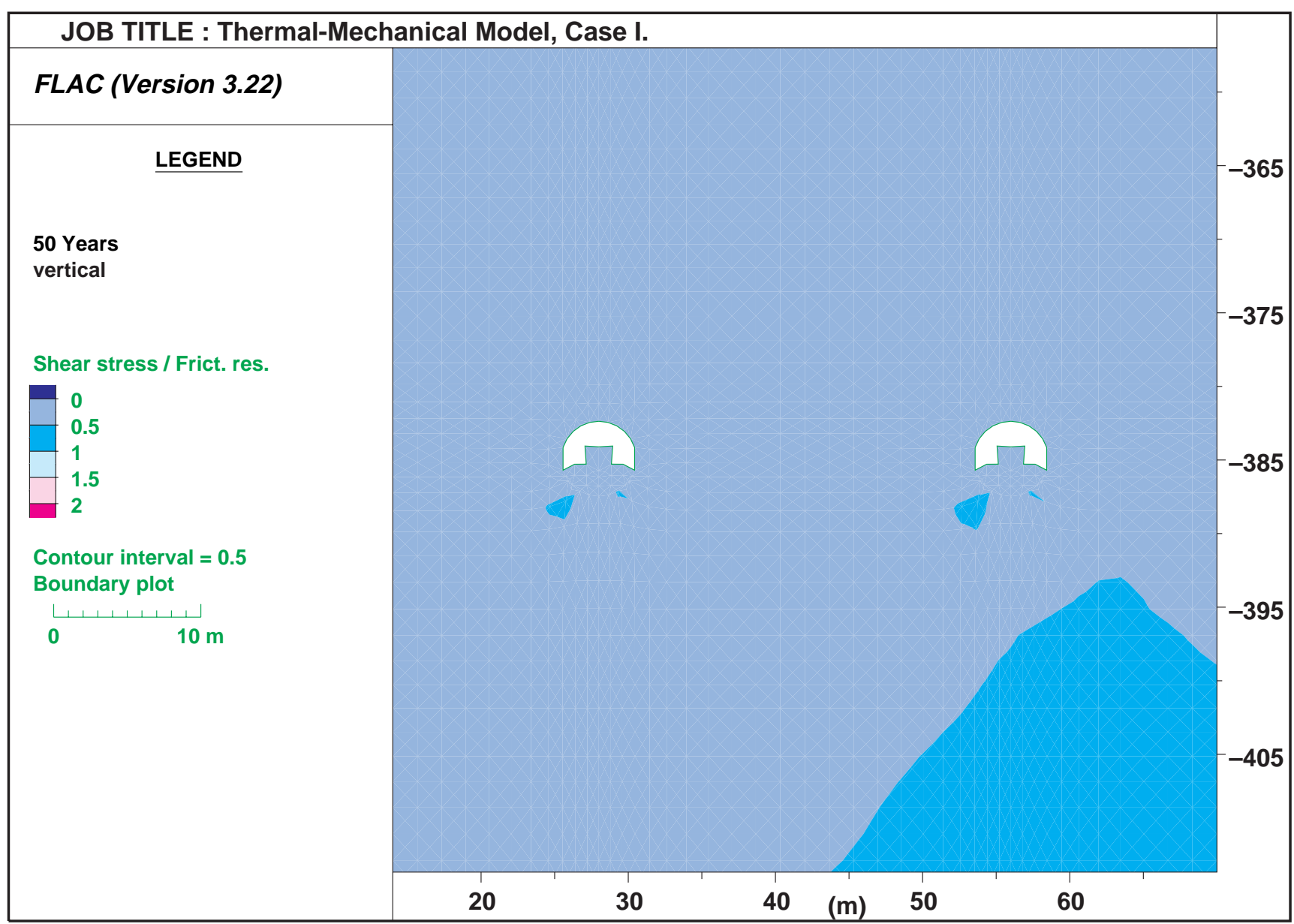

Figure 4-68. Ratio of shear stress to frictional strength for vertical fractures for Case 1 after $50 \mathrm{yr}$ heating, computed using FLAC 


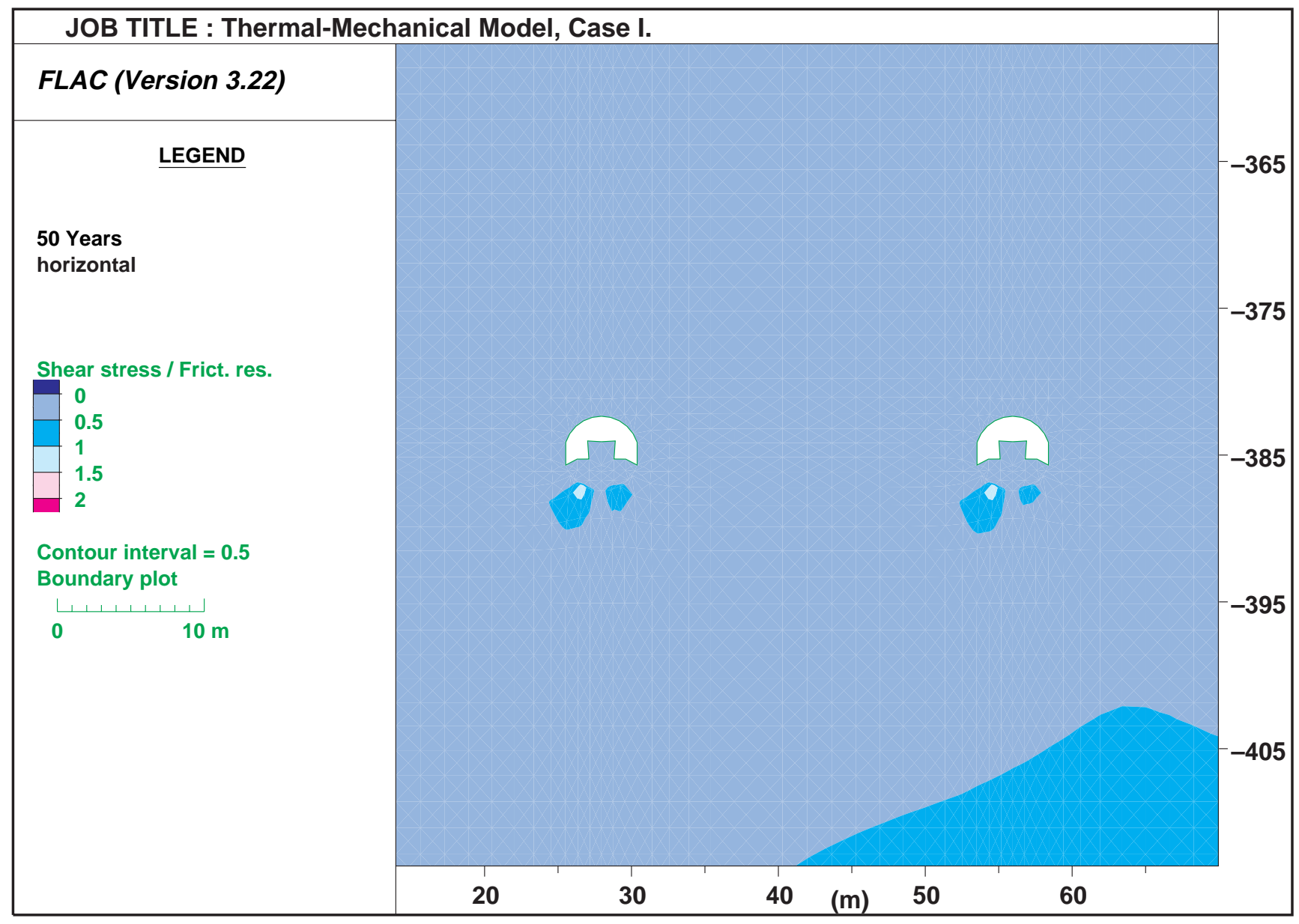

Figure 4-69. Ratio of shear stress to frictional strength for horizontal fractures for Case 1 after 50 yr heating, computed using FLAC 


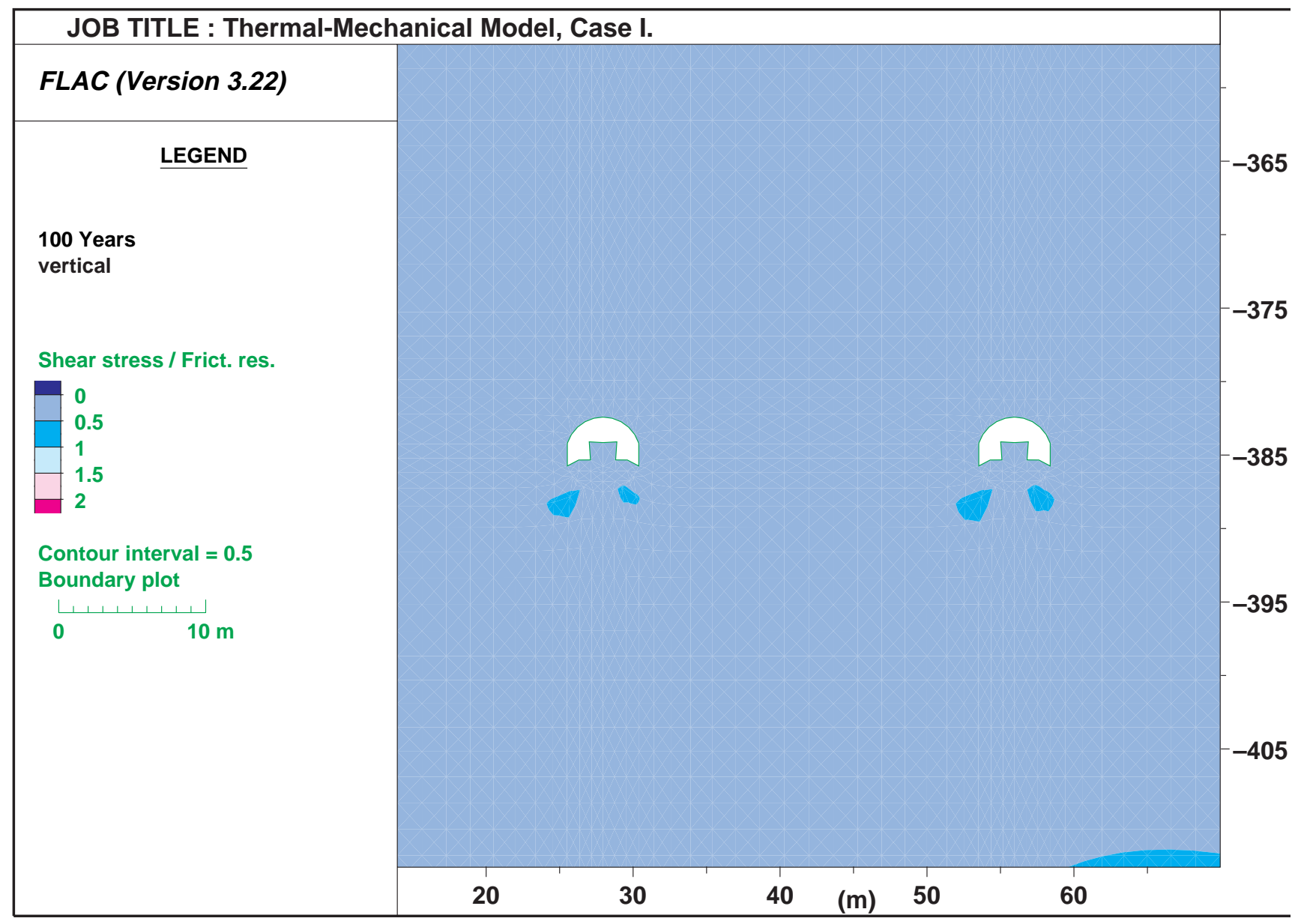

Figure 4-70. Ratio of shear stress to frictional strength for vertical fractures for Case 1 after $100 \mathrm{yr}$ heating, computed using FLAC 


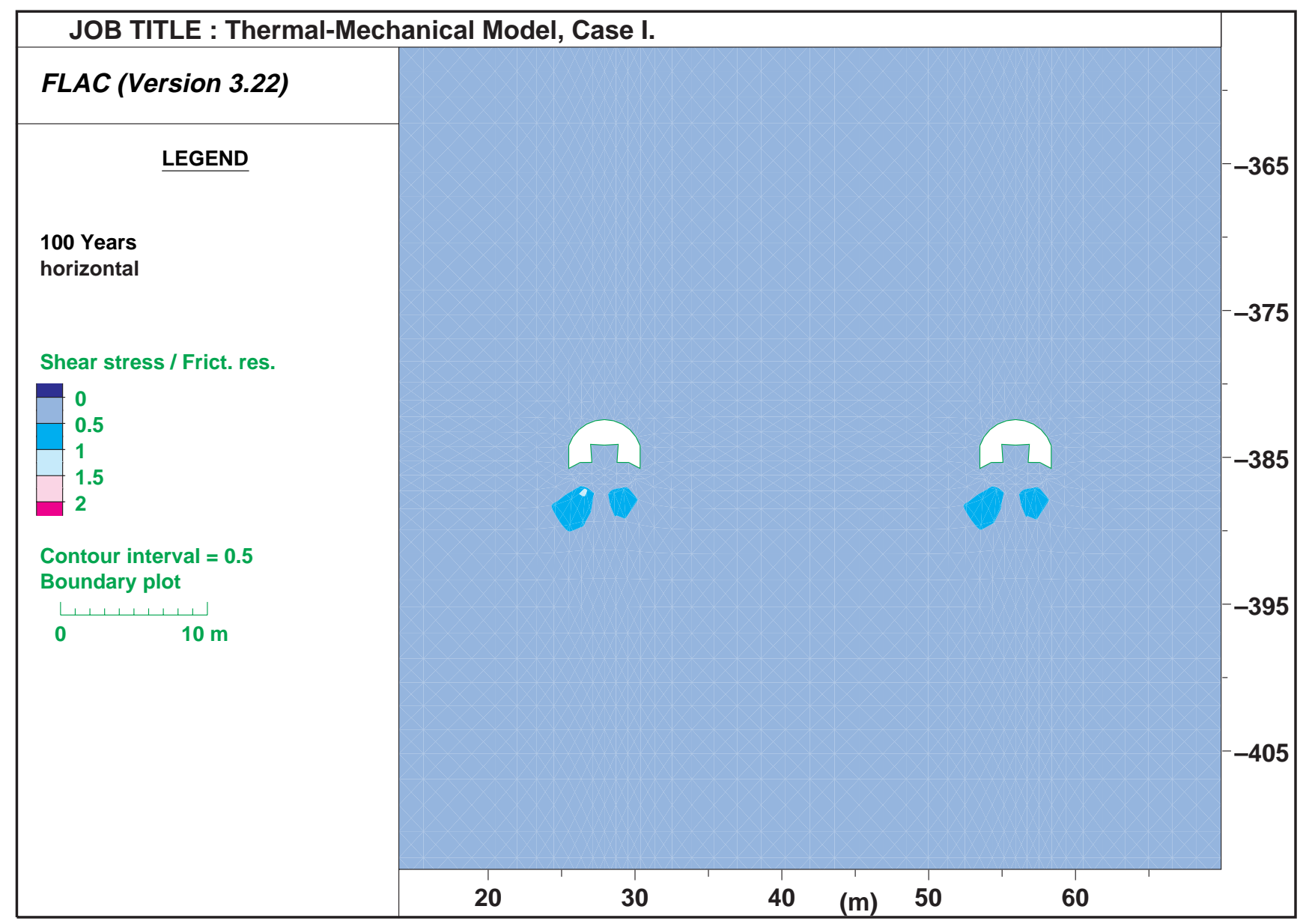

Figure 4-71. Ratio of shear stress to frictional strength for horizontal fractures for Case 1 after $100 \mathrm{yr}$ heating, computed using FLAC 



\section{Thermohydrochemical Models of the Altered Zone and the Near Field}

\subsection{Introduction to Thermohydrochemical Models}

The importance of thermohydrochemical (THC) processes in the near-field environment (NFE) arises from key aspects of repository performance including drift seepage flux, composition of water that will contact the engineered barriers, and transport of released radionuclides. The impacts of these factors on performance will depend generally on the effects of coupling between chemical and thermohydrologic $(\mathrm{TH})$ processes.

This chapter presents the basis for thermodynamic and reactive-transport models and applies them to three problems that emphasize the nature and effects of chemical reactions that will occur in the altered zone (AZ). A reaction-path model is presented first; it shows how water evolves during interaction with the host rock, as could be expected along a flow path for condensate drainage. Two fully coupled, THC models are presented next; these examine the movement of silica in the host rock. Finally presented is a reactive-transport model that can simulate many more reactions that are possible with multiple chemical components. The quality assurance (QA) status of these models is summarized in Appendix A.

Information is presented in this chapter in the following manner:

- Section 5.2 presents an overview of thermodynamic modeling and a detailed description of the conceptual, mathematical, and numerical basis for the EQ3/6 modeling package. The capabilities of EQ3/6 include modeling of rate processes and flow-through processes and reaction-path modeling.

- Section 5.3 defines reactive-transport modeling and how it is applied in studies of the near field and AZ. Various conceptual, mathematical, and numerical models for reactive transport have been proposed in the open literature, and a survey of these models is presented. The basis for the OS3D/GIMRT reactive-transport modeling code is included in this discussion.

- Section 5.4 presents a reaction path $(\mathrm{EQ3} / 6)$ model developed to describe the evolution of water composition in the $\mathrm{AZ}$, where water interacts with tuff at elevated temperature. The results show how water composition changes with progressive interaction.

- Section 5.5 describes a simulation study, in a repository-scale model, of THC coupling involving silica. This was one of the first simulations to examine the coupling, using realistic chemical reaction rate constants, of chemical effects to hydraulic permeability.

- Section 5.6 presents recent developments, for drift-scale problems, in fully coupled THC simulation involving silica. Silica accumulates where water vaporizes in these models, forming a "mineral cap." To compare coupled-process models, a series of simulations is presented for several of the reference cases used in this report. These simulations emphasize the dissolution and precipitation of silica. To include a more complete range of possible chemical reactions, a more general reactive-transport model, such as OS3D/GIMRT, is used. 
- Section 5.7 describes, for several of the same reference cases, a series of reactivetransport calculations using OS3D/GIMRT. These calculations show how dissolution and precipitation of calcite and silicates occur in fractures in the AZ. Dissolution occurs in the condensation zone and along pathways for condensate drainage. Precipitation occurs where the fracture-water residence time is greater, which allows for greater progress of the rate reactions that precipitate clays and zeolites.

- Section 5.8 presents a summary.

Models for the composition of water in the AZ, the composition of water interacting with cementitious materials, and transport of radionuclides in the NFE are presented in Chapter 6.

\subsubsection{Identification of Users for Thermohydrochemical Models}

This chapter describes three THC models: (1) a chemical model of AZ water composition, (2) a fully coupled simulation of THC effects involving silica only and (3) partially coupled simulations of reactive transport in the AZ involving a full set of aqueous chemical reactions. These models are listed in Appendix A (Table A-9) along with the other models that make up this report.

The AZ-water-composition model is used to estimate the concentrations of major chemical species in water that interacts with the host rock at ambient and elevated temperature along flow paths upgradient from the NFE. The composition of fracture water in the AZ is used for performance assessment (PA) and design as the influent composition for models of water composition in contact with introduced materials and engineered barriers in the NFE.

Fully coupled THC models have been demonstrated for repository-scale and drift-scale problems and integrate thermohydrology and reactive transport. Currently available models include dual-permeability TH processes, but can accommodate only a few chemical components (e.g., silica) and are limited with respect to the representation of complex processes such as boiling. These models are used to support sensitivity cases for PA and address the implications of coupled processes for repository performance. The predicted changes in fracture porosity and permeability are more reliable for the host rock above the emplacement drifts than they are for host rock below the drifts.

Partially coupled THC models can be used to examine the evolution of aqueous reactions driven by dissolution and precipitation rate reactions. These models are available for use in PA to assess changes in fracture porosity and permeability and changes in the abundance of sorbent minerals along pathways for radionuclide transport downgradient of the repository.

\subsection{Thermodynamic Models (EQ3/6)}

\section{by Thomas J. Wolery}

Thermodynamic model refers to models of geochemical processes (e.g., water-rock and other interactions) that generally take place in well-mixed systems. There is typically no consideration of spatial coordinates in the modeled systems. A time variable may be included in so-called reaction-path models. There is no time variable in static models as represented by speciation-solubility calculations. In contrast, reactive-transport models (see Section 5.2) always contain a time variable and have a full 1-D or greater spatial representation of a reacting system. Reactive-transport models are therefore always dynamic. A reactivetransport model generally involves an aqueous fluid and provision for both flow (including dispersion) and solute diffusion. Thermodynamic models, however, generally do not provide for fluid flow or solute diffusion, although certain extensions do allow a limited treatment of systems incorporating fluid flow without dispersion or diffusion. 
This section emphasizes the thermodynamic models that can be used in the context of the EQ3 / 6 software package (Daveler and Wolery, 1992; Wolery, 1992a, 1992b; Wolery and Daveler, 1992; Wolery et al., 1988; Wruck and Palmer, 1997). This software has been largely developed to meet the needs of the Yucca Mountain Site Characterization Project and has a history of usage on the project, as noted in examples discussed subsequently. Recent versions of this software (versions 7.2a, 7.2b) have been certified for use in quality-affecting work. Some examples of EQ3 / 6 application are discussed in this section. Other examples may be found elsewhere (e.g., Bruton, 1995; Delany, 1985; Glassley, 1996; Knauss et al., 1986; TRW, 1996; Viani and Bruton, 1992; Wolery et al., 1988). This section, however, discusses concepts that are generally applicable to all thermodynamic models.

\subsubsection{Conceptual Models}

Thermodynamic models predate reactive-transport models (Nordstrom et al., 1979; Wolery, 1992a). Consequently, thermodynamic modeling codes are presently in a more mature state than are reactive-transport codes. Thermodynamic codes tend to represent chemical processes and phenomena in greater breadth and detail than do existing reactivetransport codes. In many cases, thermodynamic codes have been the test beds for the development and testing of submodels for describing various individual processes or phenomena. Examples include the role of MINEQL (Westall et al., 1976) in the development of surface-complexation models and the role of EQ3 / 6 (Wolery, 1992a; Wolery and Daveler, 1992; Wolery et al., 1988) in the development of kinetics-based models for rock-water interaction (e.g., Delany, 1985). Even so, no thermodynamic modeling code in existence accounts for all the many phenomena that might occur in a geochemical system. Some of the phenomena often missing from such codes are solid-solution (coprecipitation), ion-exchange, surface-complexation, and gas-phase aqueous-solution interactions. EQ3/6 (see references cited previously) and PHREEQC (Parkhurst, 1995) are two of the most comprehensive thermodynamic modeling codes. Some other codes are noted in Section 5.2.3.3.

Computational constraints have limited the inclusion of detailed chemistry in reactivetransport modeling codes. Important phenomena such as solid solution have been ignored, and the incorporation of ion exchange and sorption have tended to be limited to relatively simple forms that do not greatly increase the computational burden. However, reactivetransport-model development (e.g., Johnson et al., 1997; Steefel and Yabusaki, 1995) is accelerating, and increasingly detailed chemical models are being included (see Section 5.2). This is partly the result of greater computing power and is also a response to practical needs in the earth sciences. Reactive-transport models have superior potential for dealing with many simulation problems. However, thermodynamic models will retain an important role in problems involving analysis and submodel development and will remain the most convenient tools for many simulation problems.

Both types of models generally have been developed for the study of natural systems (e.g., water-rock interactions). However, they may be applied equally to the study of engineered systems. Thus, rock may be replaced or augmented by substances such as waste forms (spent fuel, ceramics, and glass), metals, concretes and grouts, and other human-made materials of interest. The range of applicability for such models is quite extensive and cuts across scientific and engineering disciplines. These models are applicable to problems in the NFE, the AZ, and the far-field environment. One of the advantages of geochemical models is that entire chemical systems, including human-made materials, can be modeled in a fully integrated way that accounts for all important interactions. For example, one would jointly model the system containing tuff, water, and concrete to see the influence of the concrete on the tuff-groundwater interactions in addition to seeing the degradation of the concrete. 
The use of these models is critical in interpreting experimental and field data and in properly extrapolating the results to repository conditions over the long time scales associated with repository performance. Some important chemical processes may be so slow that laboratory experiments that run for months (or years) exhibit the relevant behavior to only a small degree, or the behavior of interest is not observable. One example is the precipitation of silica as quartz in tests such as the Single-Heater Test (SHT). Another example might be the precipitation of $\mathrm{NpO}_{2}$, which could result in negligible dissolved neptunium in Yucca Mountain groundwaters (Wolery et al., 1995). Accelerated conditions (e.g., high temperatures or $\mathrm{pH}$ conditions outside anticipated repository conditions) may be required to observe these reactions and to develop rate laws (as has been done for quartz; e.g., Rimstidt and Barnes, 1980) that can be extrapolated to repository conditions and time scales. A simple parametric approach to the application of short-term laboratory and field results would ignore these processes and might yield predictions at variance with reality. For example, such an approach might calculate high long-term dose rates associated with ${ }^{237} \mathrm{~Np}$ because it ignores the potential role of $\mathrm{NpO}_{2}$ as a sink for $\mathrm{Np}$ over very long time periods.

For speciation-solubility modeling (Wolery, 1992b), a static form of thermodynamic modeling, the only essential feature is the presence of an aqueous solution. However, reaction-path modeling (Wolery and Daveler, 1992) and reactive-transport modeling are inherently dynamic, and something is required to drive the reaction. The driving force is commonly the presence of another substance (e.g., rock, concrete, or waste form) with which the initial solution is not in equilibrium. It may also be a second aqueous solution, generally termed the "reactants." The driving force might equally well be a change in temperature (or pressure). It could even be an internal disequilibrium within the aqueous solution itself, possibly associated with the internal reduction-oxidation (redox) state of the solution. In the case of reaction-path calculations, the thermodynamic driving forces change (decrease overall) with increase in time or a reaction progress variable. In the case of reactive-transport calculations, the system changes as a function of time, and the driving forces typically vary temporally and spatially.

Both thermodynamic and reactive-transport models rely heavily on chemical thermodynamics and require supporting thermodynamic data in the form of equilibrium constants or, equivalently, Gibbs free energies. The usage of chemical thermodynamics naturally focuses on an array of simple reactions typified by

$$
\mathrm{NpO}_{2}^{+}+\mathrm{OH}^{-}=\mathrm{NpO}_{2} \mathrm{OH}_{(\mathrm{aq})}
$$

(an aqueous complexation reaction) and

$$
\mathrm{CaCO}_{3}(\text { Calcite })+\mathrm{H}^{+}=\mathrm{Ca}^{2+}+\mathrm{HCO}_{3}^{-}
$$

(a mineral dissolution reaction). The number of such reactions that must be treated in a calculation may be quite large (in the hundreds to thousands). Thus, large thermodynamic databases are often required to support such calculations. Equilibrium constants and Gibbs free energies depend on temperature and pressure; provision for dealing with the dependency is also necessary for many applications. This provision adds to the size and complexity of the required databases.

Although thermodynamic models have much to do with thermodynamic equilibrium and can be used to compute equilibrium states for systems of defined pressure, temperature, and chemical composition, most thermodynamic models only assume that some (usually most) reactions (as represented by Eq. 5-1 and Eq. 5-2) are individually in an equilibrium state. Usually this assumption is made for most aqueous ion-pairing and ion-complexing reactions. 
The remaining reactions are assumed to be more sluggish and may be out of equilibrium individually. Typically, this set of reactions includes many mineral-dissolution reactions, some mineral-precipitation reactions, and some redox reactions (especially involving the nonmetal elements such as $\mathrm{C}, \mathrm{H}, \mathrm{N}$, and S). Most silicate minerals dissolve slowly at $25^{\circ} \mathrm{C}$. It is commonly assumed in modeling that dissolution reactions are slow and that precipitation reactions are fast. Therefore, rate expressions are often assigned to dissolving minerals, whereas precipitating minerals are frequently assumed to follow equilibrium controls. Quartz, hematite, dolomite, $\mathrm{NpO}_{2}$, and $\mathrm{PuO}_{2}$ are examples of solids known to precipitate quite sluggishly under almost all conditions at low temperature. Some solids (e.g., calcite) may precipitate rapidly or sluggishly depending on the aqueous solution composition. Many redox reactions are very slow. Certain redox species (e.g., $\mathrm{SO}_{4}^{2-}, \mathrm{NO}_{3}$, and dissolved organics) may persist metastably (i.e., resisting thermodynamic driving forces) for long periods of time. Biologically mediated reactions, instead of inorganic processes, may control the rates involved. A system in which some individual reactions are at equilibrium and others are not is said to be in a state of partial equilibrium.

Thermodynamic models may be used in either an analytical mode or in a simulation mode. Reactive-transport models always operate in a simulation mode. The analytical mode of thermodynamic models is represented by speciation-solubility calculations.

\subsubsection{Speciation-Solubility Modeling}

One of the two principal objectives of speciation-solubility modeling is to determine the speciation of dissolved components such as $\mathrm{Np}$ (e.g., how much is $\mathrm{NpO}_{2}$, how much is $\mathrm{NpO}_{2} \mathrm{CO}_{3}^{-}$). (Chemical components are defined as the building blocks that can be combined to form all the constituents of a chemical system.) Common analytical techniques only give the total amount of the dissolved component (e.g., total dissolved Np). More specialized analytical techniques can distinguish between the oxidation states of a dissolved element [e.g., $\mathrm{Fe}(\mathrm{II})$ vs. $\mathrm{Fe}(\mathrm{III})$ ], but even this information is not commonly determined in groundwater analysis or in most experimental studies. Analytical techniques specific to an individual aqueous species such as $\mathrm{NpO}_{2} \mathrm{CO}_{3}^{-}$may exist, at least for solutions of limited composition, but are generally not applied to the analysis of complex waters such as groundwaters. Therefore, it is usually necessary to calculate the speciation in such waters using a thermodynamic model and using the appropriate equilibrium constants. Besides the component concentrations, such models generally also require as input the $\mathrm{pH}$ and some measure of the redox state of the solution (commonly the oxygen fugacity or the redox potential Eh).

The second principal goal of speciation-solubility calculations (the solubility part) is to calculate saturation indices (SI) for reactions that are not assumed to be at equilibrium. Most commonly, these indices are calculated for mineral dissolution reactions, and the objective is to determine whether the solution is undersaturated $(\mathrm{SI}<0)$, saturated $(\mathrm{SI}=0)$, or supersaturated $(\mathrm{SI}>0$ ) with respect to a given mineral. The SI (a thermodynamic driving force) is calculated according to

$$
\mathrm{SI}=\log Q / K
$$

where $Q$ is the ion activity product for the dissolution reaction (e.g., Eq. 5-2) and $K$ is the corresponding equilibrium constant. Here, $Q$ depends on the thermodynamic activities of the species appearing in the reaction (the activity of a pure solid is unity by definition). The thermodynamic activity of an aqueous solute species is the product of its molal concentration and its molal activity coefficient. For the case of calcite dissolution (Eq. 5-2), this takes the form 


$$
\log Q(\text { Calcite })=\log a\left(\mathrm{Ca}^{2+}\right)+\log a\left(\mathrm{HCO}_{3}^{-}\right)-\log a\left(\mathrm{H}^{+}\right)
$$

where $a$ is the thermodynamic activity of the indicated species. The thermodynamic activity of a pure phase such as calcite is unity; therefore, $\log a$ (Calcite) is zero, and a corresponding term need not appear in Eq. 5-4. The thermodynamic activity of water is the product of its mole fraction and its mole-fraction activity coefficient. Activity coefficients must be calculated from an activity coefficient model such as the Davies equation, the B-dot equation, or Pitzer's equations (see chapter 3 of Wolery, 1992b). A single model must be applied to all aqueous species.

Saturation indices can also be calculated for complete aqueous redox reactions exemplified by

$$
\mathrm{NO}_{3}^{-}+2 \mathrm{Fe}^{2+}+2 \mathrm{H}^{+}=\mathrm{NO}_{2}^{-}+2 \mathrm{Fe}^{3+}+\mathrm{H}_{2} \mathrm{O}
$$

where nitrate is reduced to nitrite and Fe(II) is oxidized to Fe(III). It is also possible to characterize the driving force, or degree of disequilibrium, of such a reaction by calculating a theoretical redox potential (Eh) for each redox couple (here, $\mathrm{NO}_{3}^{-} / \mathrm{NO}_{2}^{-}$and $\mathrm{Fe}^{2+} / \mathrm{Fe}^{3+}$ ) and determining the difference between them (Wolery, 1992b). To calculate the degree of disequilibrium or driving force for a reaction involving a redox couple, one must generally have two analytical measurements [e.g., one for each of $\mathrm{Fe}(\mathrm{II})$ and $\mathrm{Fe}(\mathrm{III})]$. In this example, a single measurement of total dissolved iron is insufficient.

EQ3NR (Wolery, 1992b) is the speciation-solubility code in the EQ3/ 6 package. Some partial results for J-13 well water (Harrar et al., 1990) are illustrated in Table 5-1, Table 5-2, and Table 5-3. These calculations were made using the j13wsf.3i input file (a part of the EQ3 / 6 test case library) and Revision 6 of the composite thermodynamic data file (discussed in Section 5.2.2). The water composition has been theoretically spiked with trace amounts (arbitrarily set at $1.0 \times 10^{-12}$ molal) of various radionuclides, including uranium, plutonium, and neptunium. The main purpose of this test case is to initialize a reaction-path simulation in which the groundwater reacts with spent fuel. However, it will be used here simply to illustrate some of the results that can be obtained from EQ3NR. The calculated speciation of dissolved uranium, neptunium, and plutonium is shown in Table 5-1. Uranium is entirely dominated by $\mathrm{U}(\mathrm{VI})$ species, plutonium by $\mathrm{Pu}(\mathrm{IV})$ species, and neptunium by $\mathrm{Np}(\mathrm{V})$ species. The code actually considered the IV, V, and VI (and other) oxidation states of these radionuclides. 
Table 5-1 The calculated speciation of uranium, neptunium, and plutonium present in trace amounts in spiked $\mathrm{J}-13$ well water $\left(\mathrm{Eh}=340\right.$ millivolts; $\left.T=298^{\circ} \mathrm{K}\right)$

Other species $\mathrm{U}, \mathrm{Np}$, and $\mathrm{Pu}$ are present, but those shown account for $>99 \%$ of the totals. The contribution of a species to the corresponding total is the product of its stoichiometric factor and its molality [e.g., for $\left(\mathrm{UO}_{2}\right)_{3}(\mathrm{OH})_{5}^{-}$, the factor is 3 because one mole of this species contains 3 moles of uranium].

\begin{tabular}{|c|c|c|c|}
\hline Species & Factor & Molality & Percentage \\
\hline \multicolumn{4}{|l|}{ Uranium } \\
\hline $\mathrm{UO}_{2}\left(\mathrm{CO}_{3}\right)_{2}{ }^{2-}$ & 1.0 & $7.664 \times 10^{-13}$ & 76.64 \\
\hline $\mathrm{UO}_{2}\left(\mathrm{CO}_{3}\right)_{3}{ }^{4-}$ & 1.0 & $1.745 \times 10^{-13}$ & 17.45 \\
\hline $\mathrm{UO}_{2}(\mathrm{OH})_{2}(\mathrm{aq})$ & 1.0 & $0.408 \times 10^{-13}$ & 4.08 \\
\hline $\mathrm{UO}_{2} \mathrm{CO}_{3}(\mathrm{aq})$ & 1.0 & $0.141 \times 10^{-13}$ & 1.41 \\
\hline Total & & $1.0 \times 10^{-12}$ & 99.58 \\
\hline \multicolumn{4}{|l|}{ Neptunium } \\
\hline $\mathrm{NpO}_{2}^{+}$ & 1.0 & $8.844 \times 10^{-13}$ & 88.44 \\
\hline $\mathrm{NpO}_{2} \mathrm{CO}_{3}^{-}$ & 1.0 & $0.840 \times 10^{-13}$ & 8.40 \\
\hline $\mathrm{NpO}_{2} \mathrm{OH}(\mathrm{aq})$ & 1.0 & $0.268 \times 10^{-13}$ & 2.68 \\
\hline Total & & $1.0 \times 10^{-12}$ & 99.53 \\
\hline \multicolumn{4}{|l|}{ Plutonium } \\
\hline $\mathrm{Pu}(\mathrm{OH})_{4}(\mathrm{aq})$ & 1.0 & $9.595 \times 10^{-13}$ & 95.95 \\
\hline $\mathrm{Pu}\left(\mathrm{HPO}_{4}\right)_{4}{ }^{4-}$ & 1.0 & $0.393 \times 10^{-13}$ & 3.93 \\
\hline Total & & $1.0 \times 10^{-12}$ & 99.88 \\
\hline
\end{tabular}

These results are sensitive to the inputs for $\mathrm{pH}(7.41)$ and redox $(\mathrm{Eh}=340 \mathrm{mV})$. The uranium and neptunium results are further sensitive to other constraints on the carbonate system, here a reported alkalinity of $128.9 \mathrm{mg} / \mathrm{L}$ (expressed as $\mathrm{HCO}_{3}^{-}$). It has recently been suggested that the $\mathrm{pH}$ of J-13 water is actually about 8.2 (Apps, 1997). The use of this value would, for example, lead to decreased domination of $\mathrm{NpO}_{2}^{+}$in the mass balance for neptunium. However, a greater uncertainty lies in the redox constraint. The dissolved oxygen content of J-13 water (5.6 mg/L) corresponds to an Eh of $778 \mathrm{mV}$ (as obtained via EQ3NR). This also corresponds to an $\mathrm{O}_{2}$ partial pressure of 0.138 bar, somewhat less than the atmospheric value of 0.20 bar. This is probably more realistic. Eh values are generally incapable of giving meaningful results in oxidizing systems (e.g., Hostetler, 1984; Lemire, 1984). Use of the higher Eh value makes almost no difference to the calculated speciation of uranium and neptunium. However, it leads to major differences for that of plutonium; as shown in Table 5-2, the plutonium mass balance then would be dominated by $\mathrm{Pu}(\mathrm{V})$ and $\mathrm{Pu}(\mathrm{VI})$ species (all the species shown are $\mathrm{Pu}(\mathrm{V})$ species, except for $\mathrm{PuO}_{2}\left(\mathrm{CO}_{3}\right)_{2}{ }^{2-}$ ). 
Table 5 - 2 The calculated speciation of plutonium present in trace amounts in theoretically spiked $\mathrm{J}-13$ well water $(\mathrm{Eh}=778 \mathrm{mV})$

Compare with Plutonium in Table 5- 1.

\begin{tabular}{|l|c|c|c|}
\hline \multicolumn{1}{|c|}{ Species } & Factor & Molality & Percent \\
\hline \hline $\mathrm{PuO}_{2} \mathrm{~F}_{3}{ }^{-}$ & 1.0 & $5.257 \times 10^{-13}$ & 52.57 \\
\hline $\mathrm{PuO}_{2}\left(\mathrm{CO}_{3}\right)_{2}{ }^{2-}$ & 1.0 & $2.939 \times 10^{-13}$ & 29.39 \\
\hline $\mathrm{PuO}_{2}{ }^{+}$ & 1.0 & $0.745 \times 10^{-13}$ & 7.45 \\
\hline $\mathrm{PuO}_{2} \mathrm{~F}_{2(\text { aq) }}$ & 1.0 & $0.517 \times 10^{-13}$ & 5.17 \\
\hline $\mathrm{PuO}_{2} \mathrm{~F}_{4}{ }^{2-}$ & 1.0 & $0.481 \times 10^{-13}$ & 4.81 \\
\hline Total & & $1.0 \times 10^{-12}$ & 99.88 \\
\hline
\end{tabular}

Table 5-3 illustrates some of the calculated saturation indices (all from the Eh $\quad 340 \mathrm{mV}$ calculation). Fundamentally, one should expect near-zero saturation indices to be obtained in the case of secondary minerals that are present in the real underground system an $\mathrm{d}$ that form under ambient conditions. An example is calcite. The large , negative saturation index of $\theta .75$ found here seems unrealistic. The accuracy of reported $\mathrm{f} 13$ water $\mathrm{pH}$ values has recently been questioned by Apps (1997), who has suggested that the $\mathrm{pH}$ be calculated instea d by assuming that the water was in equilibrium with calcite (a procedure that gives a $\mathrm{pH}$ near 8.2). Some of the other results shown in Table 5-3 also depend on the $\mathrm{pH}$. Many, however, also depend strongly on the input total concentrations of aluminum or iron. The data that were used here are probably close to, or less than, detection limits and are therefore not necessarily reliable. Thus, the actual groundwater is probably not supersaturate $d$ with respect to analcime or gibbsite (which contain $\mathrm{Al}$ ) or $\mathrm{Fe}\left(\mathrm{OH}_{3}\right)$ or goethite (which contain $\mathrm{Fe}$ ). On the other hand, the saturation indices for quartz, chalcedony, and the two forms of cristobalite depend essentially only on the input for total dissolved silica, which is well above the analytica l detection limit. The supersaturations in this case are undoubtedly real, in accord with the known kinetics of formation of these minerals. The groundwater should be undersaturated or, at mos $t$, about saturated with respect t o unstable primary feldspar, represented here by sanidine. The very positiv e saturation index for this phase is another reason to doubt the accuracy of the input for total dissolved aluminum. On the other hand, one should expect somewhat positive saturation indices for stable feldspars, such as albite and K-feldspar, becaus e they might form in response to the long-term dissolution of unstable primary feldspar.

The saturation indices calculated for $\mathrm{NpO}_{2}$ and $\mathrm{PuO}_{2}$ are both positive, indicating supersaturation for both phases, even in $\mathrm{Np}$ and $\mathrm{Pu}$ molalities of $1.0 \times 1$. This indicates the tremendous insolubility of these two solids, at least according to the presently known thermodynamic data (se eSection 5.2. 2). However, results were obtained for an Eh of $340 \mathrm{mV}$; for an $\mathrm{Eh}$ of $780 \mathrm{mV}$, the water is undersaturated with respect to both solids. 
Table 5-3 Calculated saturation indices $(\log Q / K)$ for selected solids in theoretically spiked J-13 well water $(\mathrm{Eh}=340 \mathrm{mV})$

\begin{tabular}{|c|c|}
\hline Solid & $\log Q / K$ \\
\hline Albite & +1.80 \\
\hline Analcime & +0.75 \\
\hline Calcite & -0.75 \\
\hline Ca-Clinoptilolite & +13.60 \\
\hline Cristobalite(alpha) & +0.45 \\
\hline Cristobalite(beta) & +0.01 \\
\hline $\mathrm{Fe}(\mathrm{OH})_{3}$ & +0.16 \\
\hline Gibbsite & +1.11 \\
\hline Goethite & +5.28 \\
\hline Hematite & +11.52 \\
\hline K-feldspar & +3.65 \\
\hline Kaolinite & +4.93 \\
\hline Ca-Montmorillonite & +5.68 \\
\hline Quartz & +0.88 \\
\hline Sanidine(high) & +2.45 \\
\hline $\mathrm{NpO}_{2}$ & +0.88 \\
\hline $\mathrm{PuO}_{2}$ & +4.86 \\
\hline
\end{tabular}

EQ3NR permits the user to define an aqueous solution using many possible input options. Other speciation-solubility codes may also offer some of these options. Normally, the input parameters include the usual components of a groundwater or experimental fluid analysis (i.e., the $\mathrm{pH}$, some measure of the redox state, and the total concentrations of the solute components). A useful output not noted previously is the calculated charge imbalance. If it exceeds $10 \%$ to $20 \%$ of the total charge, it may indicate that some of the input analytical data are incorrect or that the analytical dataset is incomplete. It is possible to replace some of the code inputs with alternative constraints, thus effectively switching some inputs and outputs. By applying a charge-balance constraint to one of the components of a $\mathrm{pH}$ buffer (e.g., $\mathrm{Na}^{+}$when $\mathrm{NaOH}$ is a buffer constituent), one can calculate the composition of a custom $\mathrm{pH}$ buffer solution for use in experimental studies. This has been done as part of studies of the $\mathrm{pH}$ dependence of the dissolution kinetics of silicate minerals (e.g., Knauss and Wolery, 1989). One can calculate, for example, how much $\mathrm{NaOH}$ is required to adjust a borate buffer to a certain $\mathrm{pH}$ value at elevated temperatures. Alternatively, by applying the charge-balance constraint to $\mathrm{H}^{+}$itself, one can calculate the $\mathrm{pH}$ of the buffer as a function of temperature.

The total concentration of a dissolved component can also be calculated by assuming equilibrium with either a solid or a component of a gas phase (the component must appear in the reaction involving the solid or gas species). For example, the concentration of dissolved aluminum can be estimated by assuming equilibrium with a mineral such as kaolinite $\left[\mathrm{Al}_{2} \mathrm{Si}_{2} \mathrm{O}_{5}(\mathrm{OH})_{4}\right]$. Calculations involving such assumptions are commonly made under three circumstances. One is when the analytical data fail to include useful results for certain components of interest. Some analyses may have been omitted or the reported concentrations 
may be less than or very close to the corresponding detection limits. This is often the case with certain components such as aluminum and iron that tend to be relatively insoluble. The second case is when some of the reported data are suspect for various reasons. Unless special procedures for sampling and analysis are employed, groundwater $\mathrm{pH}$ and alkalinity can fall in this category. For example, degassing $\left(\mathrm{CO}_{2}\right.$ loss $)$ can change the $\mathrm{pH}$ of a groundwater and may occur while the sample is being pumped out of the ground or in the time between collection and analysis. Alkalinity is commonly measured as a proxy for measurements of total dissolved $\mathrm{HCO}_{3}^{-}\left(\right.$or $\mathrm{CO}_{2}$ ). Such usage is subject to several assumptions (see APHA, 1976), not all of which may always hold. The third case is when one desires to calculate the solubility of a component in a fluid that does not normally contain that component (e.g., the solubility of $\mathrm{Np}$ in J-13 well water). This usage is usually subject to the restriction that the solubility must be sufficiently low not to disturb the properties of the water as a medium (e.g., its $\mathrm{pH}$ and ionic strength).

Speciation-solubility calculations have one other important use: they are required to initialize reaction-path and reactive-transport calculations.

\subsubsection{Reaction-Path Modeling}

In reaction-path modeling and reactive-transport modeling, the objective is not analysis but simulation. Reactions not assumed to be at equilibrium are assigned rate expressions. The saturation index or other measure of the degree of disequilibrium may appear in the rate law assigned to a reaction. Realistically, the dependence should be included such that the rate goes to zero as the driving force goes to zero and such that the direction of the reaction is consistent with the sign of the driving force. The most commonly used rate laws follow a form suggested by transition state theory (e.g., Helgeson et al., 1984; Wolery and Daveler, 1992). In some reaction-path calculations, all rates are specified as relative rates in terms of an overall reaction progress variable. Then, the modeled process is akin to a titration and has no explicit time frame.

The use of rate laws requires rate constants and usually some understanding of the dependence of rate constants on temperature (e.g., activation energies). There may be additional dependencies on solution composition parameters such as the $\mathrm{pH}$ and on parameters such as surface areas for reactions (e.g., mineral dissolution or precipitation) that occur across an interface (for a detailed discussion, see Wolery and Daveler, 1992, and references cited therein). Two types of uncertainties are involved: the first is represented by the uncertainties in the fundamental rate-law parameters, mainly rate constants and activation energies; the second applies to the nature of the rate laws themselves-if they are not completely general and fundamentally correct, their proper application may be scenariodependent.

In reaction-path modeling, the reactions assumed to be controlled by equilibrium do not have zero rates. Rather, they adjust dynamically to maintain their equilibrium states in response to changes driven by the other reactions or changes in temperature or pressure. For example, a reaction controlled by equilibrium and a reaction controlled by a rate expression may be linked by a common dependency on $\mathrm{H}^{+}$or some other aqueous species. If the equilibrium control on the former reaction were not applied, the progress of the latter reaction would throw the former out of equilibrium. The magnitude of the rate of an equilibrium-controlled reaction is unconstrained. It may achieve values that are unrealistic, in which case it should be treated instead by specifying a rate expression.

EQ6 (Wolery and Daveler, 1992) is the reaction-path code in the EQ3/6 package. A simple example of a reaction-path calculation is the reaction of microcline (a form of K-feldspar: $\mathrm{KAlSi}_{3} \mathrm{O}_{8}$ ) with a dilute ( $\left.\mathrm{pH} 4.0\right)$ solution of hydrochloric acid $(\mathrm{HCl})$. The progress of the 
reaction is measured in terms of the overall reaction progress variable, $\xi$. This value is equal to the number of moles of microcline that have reacted with a mass of solution initially containing $1 \mathrm{~kg}$ of solvent water. This problem is part of the EQ3/ 6 test case library (in the input file micro.6i). The phases quartz, chalcedony, and tridymite (three pure silica phases) are not permitted to precipitate (positive saturation indices for these phases are tolerated). Any other phases are to precipitate upon reaching saturation. The results discussed here are taken from Section 6.4 of the EQ6 user's manual (Wolery and Daveler, 1992), which discusses the problem in detail.

The dissolution reaction can be written as

$$
\mathrm{KAlSi}_{3} \mathrm{O}_{8(\mathrm{c})}+4 \mathrm{H}^{+}+\mathrm{Al}^{3+}+\mathrm{SiO}_{2(\mathrm{aq})}+2 \mathrm{H}_{2} \mathrm{O}
$$

As the microcline dissolves, and $\xi$ increases, the total dissolved molalities of $\mathrm{Al}, \mathrm{K}$, and $\mathrm{SiO}_{2}$ all increase, as shown in Figure 5-1. The $\mathrm{pH}$ (Figure 5-2) also changes as the reaction progresses. When $\log \xi$ achieves a value of approximately -5.45 , gibbsite $\left[\mathrm{Al}(\mathrm{OH})_{3}\right]$ starts to precipitate (Figure 5-3). This grows into the system until $\log \xi$ reaches approximately -5.75 . At this point, kaolinite $\left[\mathrm{Al}_{2} \mathrm{Si}_{2} \mathrm{O}_{5}(\mathrm{OH})_{4}\right]$ begins to precipitate. It competes with the gibbsite for aluminum, and the mass of gibbsite immediately starts to decrease. The gibbsite becomes completely redissolved very shortly thereafter (at $\log \xi$ equal to about -4.45 ). At $\log \xi$ near -4.0 , muscovite $\left[\mathrm{KAl}_{3} \mathrm{Si}_{3} \mathrm{O}_{10}(\mathrm{OH})_{2}\right]$ begins to precipitate, setting off a competition for $\mathrm{Al}$ with kaolinite. The mass of kaolinite then declines. However, before it can entirely redissolve, the solution becomes saturated with microcline, and the calculation ends. Throughout the simulation, the affinity (a measure of the thermodynamic driving force) decreases, reaching zero at the end (Figure 5-4). The affinity $A$ of a phase to dissolve is formally related to the saturation index $(\log Q / K)$ by

$$
A=-2.3026 R T \log Q / K
$$

where $R$ is the gas constant and $T$ the absolute temperature.

Figure 5-1 Changes in the total molalities of dissolved aluminum, potassium, and silica as a function of reaction progress $\xi$ in closed-system reaction of microcline with $\mathrm{pH} 4.0 \mathrm{HCl}$ solution

Figure 5-2 Changes in the $\mathrm{pH}$ as a function of reaction progress $\xi$ in closed-system reaction of microcline with $\mathrm{pH} 4.0 \mathrm{HCl}$ solution

Figure 5-3 The number of moles of secondary minerals $n_{\phi}$ as a function of reaction progress $\xi$ in closed-system reaction of microcline with $\mathrm{pH} 4.0 \mathrm{HCl}$ solution

Figure 5-4 The affinity of microcline to dissolve as a function of reaction progress $\xi$ in closed-system reaction of microcline with $\mathrm{pH} 4.0 \mathrm{HCl}$ solution

There is no defined concept of time in the preceding simulation. It is only known that time should increase as reaction progress increases. Time can be brought into the preceding simulation by supplying a kinetic rate law for the process of microcline dissolution.

An example of a reaction-path model with a time frame is taken from Section 6.8 of the EQ6 user's manual (Wolery and Daveler, 1992). This problem is also part of the EQ3/ 6 test case library (in the input file pquartz.6i). It simulates the precipitation of quartz at $105^{\circ} \mathrm{C}$ in an experiment reported by (Rimstidt and Barnes, 1980). This is a seeded-growth simulation, in 
which the rate law depends on a surface-area parameter. Because quartz is the stable form of $\mathrm{SiO}_{2}$, the less stable forms such as tridymite, chalcedony, and cristobalite are suppressed. In the actual experiment, kinetics provided the suppression, including the fact that only quartz seeds were present. Figure 5-5 shows the results of the calculation, plotted against time. The concentration of dissolved silica drops, reaction progress increases, and the affinity to precipitate, which has the opposite sign of the affinity to dissolve, decreases.

Figure 5-5 The precipitation kinetics of quartz in the system $\mathrm{SiO}_{2}-\mathrm{H}_{2} \mathrm{O}$ as a function of time

\subsubsection{Further Discussion}

Another important concept is local equilibrium. The assumption is that, at any given location in the system, a chemical reaction is always in a state of equilibrium. The assumption is more important in reactive-transport modeling, which deals more with spatial coordinates. Local equilibrium does not mean that a reaction so controlled has a zero rate. Rather, as in the case of partial equilibrium, it implies a dynamic adjustment at whatever rate is required (in whichever direction) to maintain the equilibrium condition.

Some extensions of thermodynamic models do allow limited provision for fluid flow, but they treat the reacting system in a less-than-full 1-D sense (Wolery and Daveler, 1992). An example is a fluid-centered, flow-through system, which follows the evolution of the first packet of water to flow through an initially uniform medium in an open system. The example is not fully 1-D because the model does not treat the evolution of any succeeding packets of water, which must now traverse a nonuniform reacting medium due to interactions associated with the traverse of the first packet. Another example is a solid-centered, flowthrough system (patterned after a leaching cell). This system follows the evolution of a small volume containing solids in which fluid flows in one end and flows out the other. It is also not fully 1-D because it does not follow the evolution of any solids in upstream or downstream cells. The fluid-centered, flow-through system is presently included in EQ6; the solid-centered, flow-through system is not.

A key point is to maximize confidence in thermodynamic models and reactive-transport models by testing the modeling methodology against experimental and field data under anticipated repository conditions and under accelerated conditions. For example, EQ3/6based models for tuff/groundwater interactions at elevated temperatures in well-mixed systems have been developed (e.g., Delany, 1985; Knauss et al., 1987, 1986; Knauss and Peifer, 1986) that correspond reasonably well with laboratory experiments. Figure 5-6 shows the aqueous solution chemistry results for the simulation (Delany, 1985) of an experiment (Knauss et al., 1985) in which Topopah Spring tuff was reacted with J-13 well water at $150^{\circ} \mathrm{C}$. These models account for the evolution of fluid chemistry and the formation of product minerals. An additional point is that such work links observations of a complex process with thermodynamic and kinetic data. The work cited shows that tuff/groundwater systems approach a near-steady-state condition on the time scales of the experiments (typically 60 to 90 days). Thermodynamic analysis shows that this is a metastable condition, not an equilibrium state. A parametric analysis would not be able to make the distinction.

Figure 5-6 Comparison of simulated (EQ3/6) and experimental aqueous solution compositions for the $150^{\circ} \mathrm{C}$ reaction between Topopah Spring tuff (Tpt) (core wafer) and J-13 well water 
A second key point is that meaningful results are only likely to be obtained when a thermodynamic modeling code or a reactive-transport code is used by an informed user because the results often depend heavily on the details of how the input describing the problem is constructed. A word problem such as, "What is the solubility of plutonium in J-13 water?" may be interpreted many ways. Asking six users of the same software to answer such a question may quite legitimately result in six different answers, varying widely in order of magnitude. The question needs to be more precise. One question that might have to be answered first is, "What exactly is J-13 water?" (questions regarding the $\mathrm{pH}$ and redox state have been noted previously). Another question might concern the time scale of interest and how the solubility equilibrium is to have been achieved (e.g., from supersaturation or from undersaturation and perhaps how these initial states were achieved).

A key action in answering solubility questions is to decide the appropriate solubilitycontrolling phase, giving consideration to the time scale and known kinetic factors. For geologically long time scales, the appropriate phase might simply be the most stable phase. For short periods of time, the answer might instead be a metastable phase. For the question, "What is the solubility of neptunium in J-13 water?" the appropriate phase might be $\mathrm{NpO}_{2}$ (stable; highly insoluble even under oxidizing conditions) or $\mathrm{Np}_{2} \mathrm{O}_{5}$ (metastable; highly soluble) (see Wolery et al., 1995, and references cited therein). Another interesting complication is that, when solubility is very high, one can no longer treat the original water chemistry as a fixed medium. For example, because so much $\mathrm{Np}$ must be put into J-13 water to achieve saturation with $\mathrm{Np}_{2} \mathrm{O}_{5}$, the basic character of the water itself (e.g., its $\mathrm{pH}$ and ionic strength) must change. The final solubility then depends on the details of how the Np was added to the groundwater. If a small amount of concentrated Np solution were added, the result may depend on whether the $\mathrm{Np}$ was present as $\mathrm{Np}(\mathrm{IV})$ or $\mathrm{Np}(\mathrm{V})$. It may also depend on what other solutes are present in this solution and on the solution $\mathrm{pH}$.

\subsubsection{Supporting Thermodynamic Data}

As noted in Section 5.2.1, a large body of supporting thermodynamic data is essential for broad application of both thermodynamic models and reactive-transport models. This section addresses the current status of thermodynamic data. Much of the discussion focuses on the adequacy of available data in regard to aqueous speciation, mineral solubilities, and radionuclide solubilities in the NFE. Although kinetic data are relevant to thermodynamic modeling, as noted in Section 5.1.1, the present section does not address the status of the available data of this kind. Such data are discussed in conjunction with reactive-transport modeling in the introduction to Chapter 3.

The first effort to develop a large thermodynamic database for natural-systems modeling over a wide range of temperature $\left(0^{\circ}\right.$ to $\left.300^{\circ} \mathrm{C}\right)$ was Helgeson (1969). The work was part of the development of a database to support calculations of the first reaction-path code, PATHI (Helgeson, 1968; Helgeson et al., 1970). The principal applications of this code were to model rock-water interactions in hydrothermal systems (e.g., of ore deposition, Helgeson, 1970). As the development of thermodynamic modeling codes has progressed, so has the development of supporting databases. Although some of the database development of the last nearly $30 \mathrm{yr}$ has naturally taken place in conjunction with modeling code development, much has also occurred independently of the development of any such code. A complete historical review of such development is beyond the scope of this report. The following discussion focuses mainly on developments associated with the Lawrence Livermore National Laboratory's (LLNL's) GEMBOCHS database, which is the main source of data used to support EQ3/6. 
GEMBOCHS is capable of producing several, distinct thermodynamic data files for EQ3 / 6 (Daveler and Wolery, 1992). The most commonly used data file is the COM, or composite, data file, which integrates data from a variety of principal sources. This file supports calculations over the broadest range of chemical components (more than half of the elements in the periodic table are represented, including the principal fission products and actinide elements). The COM data file supports calculations over the temperature range $0^{\circ}$ to $300^{\circ} \mathrm{C}$. It is intended to be used in conjunction with the B-dot or Davies equations (see Section 3 of Wolery, 1992b) and may therefore only be used in conjunction with the modeling of fairly dilute aqueous solutions (those with ionic strengths less than 1.0 molal). The COM data file is based on numerous sources.

It is heavily based on SUPCRT92 (Johnson et al., 1992), which is both a database and software for extrapolating the data over a wide range of temperatures and pressures. SUPCRT92 is the source of most of the data for the common rock-forming minerals, nearly all of the aqueous species commonly found in natural waters (e.g., groundwaters, hydrothermal solutions), and most of the common gas species.

Most of the data for the radionuclide elements (apart from Sr and Tc) are largely drawn from other sources. The uranium data, for example, are taken from the volume on uranium thermodynamics (Grenthe et al., 1992) sponsored by the Nuclear Energy Agency (NEA). The neptunium data are drawn from Linderberg and Runnells (1984); the plutonium data from Lemire and Tremaine (1980). These data will be revised when the NEA-sponsored datasets for neptunium and plutonium are published. The americium data are from a draft of Silva et al. (1993), which has since been published. The COM data file draws on too many sources to discuss here; all sources are documented internally in the file.

Modeling more concentrated solutions requires different and more elaborate models of the activity coefficients of aqueous species. At present, Pitzer's equations (see Wolery, 1992b, and references cited therein) form the only widely used basis for such modeling (and the only one presently usable in EQ3/6). In contrast to the minimal data requirements of the B-dot and Davies equations, Pitzer's equations impose a significant burden in the form of additional required data (a large array of interaction coefficients). These equations are the basis of a famous thermodynamic model of the $\mathrm{Na}-\mathrm{K}-\mathrm{Mg}-\mathrm{Ca}-\mathrm{H}-\mathrm{Cl}-\mathrm{SO}_{4}-\mathrm{OH}-\mathrm{HCO}_{3}$ $-\mathrm{CO}_{3}-\mathrm{H}_{2} \mathrm{O}$ (sea-salt) system at $25^{\circ} \mathrm{C}$ ). These data exist as a subset of the GEMBOCHS database and are used exclusively to create the HMW (Harvie-Moller-Weare) data file used by EQ3/6. Although this data file can be used to model aqueous solutions with very high ionic strength $(>12$ molal), its usage is limited by the fairly small component set and the restriction to $25^{\circ} \mathrm{C}$ calculations. These data files (COM and HMW) are, in a sense, the two prototypical EQ3/ 6 data files. Other data files (similar to one or the other of these) also are created as products of GEMBOCHS.

Table 5-4 summarizes the characteristics of some of the EQ3/ 6 data files that are part of the Revision 6 (December 3, 1996) set. The SUP data file is based purely on SUPCRT92 (Johnson et al., 1992) and subsequent updates cited in the references section of the data file itself. This data file is primarily usable only for rock-water interaction studies because the present version contains no representation of uranium and the actinide elements. Essentially all of the data in this file are present in the COM data file. The SKB data file is a variant of the COM file in which some of the data have been replaced by data from a database supplied by the Swedish Nuclear Fuel Co. (SKB). The purpose of this file is mainly to allow certain comparative calculations. The NEA data file is based almost exclusively on the thermodynamic datasets recommended by the NEA. In the present version, these data are all from Grenthe et al. (1992). 
There are several other EQ3/ 6 data files in the Revision 6 set. In Version 7 and earlier versions of EQ3 $/ 6$, temperatures are generally limited to the range $0^{\circ}$ to $300^{\circ} \mathrm{C}$, and the pressure is generally fixed at 1.013 bar up to $100^{\circ} \mathrm{C}$, and is thus equal to the pressure for liquid-vapor equilibrium for pure water (see Daveler and Wolery, 1992). Thermodynamic data (mostly equilibrium constants) are represented on a fixed temperature grid $\left(0^{\circ}, 25^{\circ}, 60^{\circ}\right.$, $100^{\circ}, 150^{\circ}, 200^{\circ}, 250^{\circ}$, and $300^{\circ} \mathrm{C}$ ). There is no provision for correcting the thermodynamic data for pressure effects; thus, pressure in the modeled system must correspond closely to the pressure represented on the data file. In the forthcoming EQ3/ 6 Version 8 (Wolery, 1994), these restrictions have been relaxed. Each data file may have a unique temperature grid, and the upper limit to the temperature range on some files is much greater than $300^{\circ} \mathrm{C}$. Each data file does have a reference pressure curve, but it need not be the old standard described previously. In some data files, the reference pressure curve is a constant value (e.g., 500 bars or $1 \mathrm{kbar}$ ). Some of these files (all of which are currently based on SUPCRT92 and updates thereto) contain additional thermodynamic data in the form of volumes of reaction and related thermodynamic functions, which allow pressure corrections off the data file reference pressure curve. Data files that contain additional data or that use a temperature grid other than the former standard are only usable with the Version 8 software.

Table 5-4 Some of the EQ3/6 data files in the Revision 6 set and their characteristics

\begin{tabular}{|l|l|l|c|c|c|c|c|c|}
\hline $\begin{array}{c}\text { File } \\
\text { Tag }\end{array}$ & Source & $\begin{array}{c}\text { Activity } \\
\text { Coefficient } \\
\text { Models }\end{array}$ & $\begin{array}{c}\text { Temp. } \\
\text { Range }\end{array}$ & $\begin{array}{c}\text { Chemical } \\
\text { Elements }\end{array}$ & $\begin{array}{c}\text { Aqueous } \\
\text { Species }\end{array}$ & $\begin{array}{c}\text { Pure } \\
\text { Minerals }\end{array}$ & $\begin{array}{c}\text { Solid } \\
\text { Solutions }\end{array}$ & $\begin{array}{c}\text { Gas } \\
\text { Species }\end{array}$ \\
\hline \hline COM & GEMBOCHS & $\begin{array}{l}\text { B-dot, } \\
\text { Davies }\end{array}$ & $\begin{array}{c}0^{\circ} \text { to } \\
300^{\circ} \mathrm{C}\end{array}$ & 81 & 1769 & 1120 & 12 & 93 \\
\hline SUP & SUPCRT92+ & $\begin{array}{l}\text { B-dot, } \\
\text { Davies }\end{array}$ & $\begin{array}{c}0^{\circ} \text { to } \\
300^{\circ} \mathrm{C}\end{array}$ & 69 & 1000 & 131 & 12 & 18 \\
\hline SKB & SKB & $\begin{array}{l}\text { B-dot, } \\
\text { Davies }\end{array}$ & $\begin{array}{c}0^{\circ} \text { to } \\
300^{\circ} \mathrm{C}\end{array}$ & 81 & 1279 & 1119 & 0 & 93 \\
\hline NEA & NEA92+ & $\begin{array}{l}\text { B-dot, } \\
\text { Davies }\end{array}$ & $\begin{array}{c}0^{\circ} \text { to } \\
300^{\circ} \mathrm{C}\end{array}$ & 33 & 182 & 216 & 0 & 82 \\
\hline HMW & HMW84 & $\begin{array}{l}\text { Pitzer's } \\
25^{\circ} \mathrm{C} \\
\text { only }\end{array}$ & 9 & 17 & 51 & 0 & 3 \\
\hline
\end{tabular}

SUPCRT92+ = Johnson et al., 1992, and subsequent updates cited in the SUP file itself

$\mathrm{SKB}=$ Swedish Nuclear Fuel Co., plus other sources; see the text

NEA92+ = Grenthe et al., 1992, and updates cited in the NEA data file itself

$\mathrm{HMW} 84=\mathrm{H} 05$

The thermodynamic data in GEMBOCHS and the EQ3/ 6 data files are continually updated and expanded. With regard to the actinide elements, updates are now or soon will be made to conform to the NEA recommendations for Am (Silva et al., 1993), Np (forthcoming), and $\mathrm{Pu}$ (forthcoming). Updates will also continue to be made as SUPCRT92 is expanded and revised.

This discussion would not be complete without some mention of problem areas. With regard to the actinides, there is a need for data on certain metastable forms of plutonium and neptunium, particularly $\mathrm{Np}(\mathrm{IV})$ polymer and $\mathrm{Pu}(\mathrm{IV})$ polymer. A need exists for a kinetic understanding of the aging and dehydration of these polymers. This is also true of the formation of certain stable solids, particularly $\mathrm{NpO}_{2}$ and $\mathrm{PuO}_{2}$ (which may be thought of as the ultimate dehydration products of the respective polymers). It does not appear these 
problems will be adequately addressed, if at all, in the forthcoming NEA datasets. Especially under reducing conditions (which may form temporarily in the repository horizon and which pertain at sufficient depth below the water table), other currently unknown actinide-bearing phases may form. One possibility is a plutonium coffinite $\left(\mathrm{PuSiO}_{4}\right)$, analogous to the known uranium mineral coffinite $\left(\mathrm{USiO}_{4}\right)$.

Another problem area is the formation of solid solutions (coprecipitation). Although considerable progress has occurred in this area in recent years for clays and zeolites (e.g., Viani and Bruton, 1992), which are natural products of rock-water interaction, more work may be required to model all necessary Yucca Mountain-related scenarios. Very little is known about how the precipitation of secondary uranium solids in and below the repository horizon might affect actinides such as plutonium by coprecipitation mechanisms. Another area of uncertainty is reaction of the actinides with poorly characterized organic substances that may be found in the NFE.

\subsubsection{Numerical Approach and Description of EQ3/6}

\subsubsection{General}

Section 5.2.1 presents the basic concepts behind modeling with EQ3/6. The modeling is generally divided into two parts: speciation-solubility modeling using the EQ3NR code, and reaction-path modeling with the EQ6 code. Speciation-solubility calculations are generally performed for three different purposes. The first of these is to analyze reported water chemistries in a thermodynamic context. The second is to synthesize special water compositions such as custom $\mathrm{pH}$ buffers. The third, which may overlap with the other two, is to calculate a model of the water to initialize a reaction-path calculation. A reaction-path calculation addresses the question (among others) of how the water chemistry changes during reaction with reactant substances or due to a change in temperature or pressure. One of these reactants may be another aqueous solution. A reaction-path calculation also addresses the formation of secondary minerals (which may be transient, as noted in the example in Section 5.2.1.2). Depending on the kind of reaction-path model, there may or may not be a time variable. In general, a reaction-path model takes place in a well-mixed system; thus there are no gradients in fluid composition and no spatial variables. This is the primary distinction of thermodynamic models from reactive-transport models. As noted in Section 5.2.1.3, there are some limited, pseudo-1-D extensions to reaction-path models. One of these-the fluid-centered, flow-through open system-is presently an option in EQ6. Otherwise, EQ6 essentially does calculations for closed systems. It can also model either closed or fluid-centered, flow-through systems for which the fugacities (partial pressures) of selected gases are fixed at specified values. These systems then behave as though they are open to a large external gas reservoir. A "closed" system to which this is applied is actually partially open.

A fixed fugacity capability works by creating a fictive mineral with the composition of the corresponding gas species. This mineral is placed in the equilibrium system. The corresponding equilibrium constant is defined that equilibrium imposes the desired fugacity. The user can specify a starting amount of each phase in addition to the corresponding fugacity. During the reaction-path calculation, the amount of a fugacity-fixing phase may increase or decrease. If the phase is entirely consumed, the fugacity is no longer fixed. This option is commonly applied to $\mathrm{CO}_{2}$ and $\mathrm{O}_{2}$ to fix their fugacities at the atmospheric values.

The EQ3/ 6 user's manuals (Daveler and Wolery, 1992; Wolery, 1992a, 1992b; Wolery and Daveler, 1992) describe the software and its usage in detail. Version 7 (of which the most recent release is version 7.2b) is written in Fortran 77. The forthcoming Version 8 is written in 
Fortran 90. The most recent version (7.2b) is certified for use in quality-affecting activities on the Yucca Mountain project. Maintenance and continuing development are conducted under a qualified QA program.

Version 8.0 (Wolery, 1994) will be released in 1998. Apart from representing a major rewrite of the software, it will contain new capabilities for making thermodynamic pressure corrections (noted in Section 5.2.2) and for modeling ion-exchange processes. The ionexchange modeling capability can deal with multiple exchange substrates and uses the Gapon and Vanselow exchange models and multisite extensions thereof. The new version will also have a capability for modeling redox disequilibrium in EQ6 (previously possible only in EQ3NR). The new version will greatly facilitate fluid-mixing calculations and will also have a new, improved user interface. Immediate future development will focus on improving the capability of the software for modeling boiling systems. EQ3/6 presently does not contain options for surface-complexation modeling (another possible future-development activity).

\subsubsection{Governing Equations and Methods}

From a numerical perspective, the function of EQ3/ 6 is to solve a large set of simultaneous equations, commonly numbering in the hundreds, sometimes in the thousands. Wolery (1992b) and Wolery and Daveler (1992) describe these equations in detail. There are two kinds of equations: the chemistry equations are algebraic in nature, and the rate-law equations are ordinary differential equations (ODE). The approach in EQ3/6 is always to solve the chemistry equations using algebraic methods and to solve the rate-law equations (when necessary) separately, using ODE integration methods. When these equations are coupled, as in a kinetic reaction-path calculation, the equations are solved using an operator splitting approach. An alternative approach would be to differentiate the chemistry equations, converting them to ODEs, and then to solve the whole set of equations using ODE methods. This was, in fact, the approach in the old PATHI program (Helgeson, 1968; Helgeson et al., 1970). PATHI exhibited problems with drift errors, however. For example, an initially charge-balanced aqueous solution could become significantly unbalanced because of cumulative integration errors. The method used in EQ3/ 6 avoids this problem.

Using the EQ3 / 6 approach, a reaction-path calculation can be treated as a sequence of equilibrium calculations, each of which involves solving the chemistry equations. In stepping along the reaction path, the total number of moles for each component can be changed because of mass transfer from the reactant system to the equilibrium system. Alternatively to mass transfer, or in addition to it, reaction might be driven by changes in temperature and/or pressure, which would change the equilibrium constants and activity coefficients.

\section{The Chemistry Equations and Equilibrium Calculations}

The chemistry equations comprise three basic types:

- Mass balance (generally one for each aqueous component)

- Mass action (one for each chemical reaction of whichever type)

- Thermodynamic activity coefficients (one equation per chemical species)

The aqueous components (or basis species) are typically species such as $\mathrm{H}_{2} \mathrm{O}, \mathrm{H}^{+}, \mathrm{Na}^{+}, \mathrm{K}^{+}$, $\mathrm{Ca}^{2+}, \mathrm{Mg}^{2+}, \mathrm{Fe}^{2+}, \mathrm{Al}^{3+}, \mathrm{SiO}_{2(\mathrm{aq})}, \mathrm{Cl}^{-}, \mathrm{SO}_{4}{ }^{2-}, \mathrm{HCO}_{3}{ }^{-}, \mathrm{UO}_{2}{ }^{2+}$, and $\mathrm{NpO}_{2}{ }^{+}$. The identities of the basis species are predefined on the supporting data file and may vary from one data file to another. EQ3 / 6 presently uses $\mathrm{O}_{2}$, a fictive aqueous species, as the redox species. Some codes use $\mathrm{e}^{-}$, the fictive aqueous electron, for this purpose, or a real species representing a chemical element in a second oxidation state (e.g., $\mathrm{Fe}^{3+}$ in addition to $\mathrm{Fe}^{2+}$ ). EQ3/ 6 may offer such choices in future versions. A minimum basis set contains one species matched one-to-one to a 
chemical element (of which it is composed) plus a redox species. In EQ3/6, $\mathrm{H}_{2} \mathrm{O}$ is always matched to $\mathrm{O}$, and $\mathrm{H}^{+}$to $\mathrm{H}$. Typically $\mathrm{Na}^{+}$is matched to $\mathrm{Na}, \mathrm{K}^{+}$to $\mathrm{K}, \mathrm{Ca}^{2+}$ to $\mathrm{Ca}$, and so forth. Some chemical elements do not have aqueous species of such simple composition. The rule then is to use a relatively simple species that may also be composed of oxygen or hydrogen or both. For example, $\mathrm{SiO}_{2(a))}$ is normally matched to $\mathrm{Si}$, and $\mathrm{HCO}_{3}^{-}$is matched to $\mathrm{C}$.

To treat redox disequilibrium (or analogous disequilibria not involving redox), it is necessary to employ a larger basis set. Some chemical elements are represented by more than one basis species. For example, $\mathrm{Fe}$ might be represented by $\mathrm{Fe}^{3+}$ and $\mathrm{Fe}^{2+}$. Note: this presumes that one of these is not being used as the redox species. Typically, such treatment is more likely to be necessary or desirable for the nonmetal elements such as $\mathrm{C}, \mathrm{N}$, or $\mathrm{S}$.

Disequilibrium is more common because reduction-oxidation of the forms in different oxidation states requires breaking covalent bonds as opposed to just transferring an electron. EQ3/6 allows for this by defining some basis species as an auxiliary set on the supporting data file. Thus, if $\mathrm{Fe}^{2+}$ is in the minimal or strict basis set and $\mathrm{Fe}^{3+}$ is in the auxiliary set, the latter can be treated (depending on what the user specifies on the input file) either as a dependent species of $\mathrm{Fe}^{2+}$ (falls under the mass balance for that species) or as an active basis species (has its own mass balance).

In EQ3NR, a mass balance equation has a form exemplified by

$$
m^{T}\left(\mathrm{Ca}^{2+}\right)=m\left(\mathrm{Ca}^{2+}\right)+m\left(\mathrm{CaHCO}_{3}^{+}\right)+m\left(\mathrm{CaCO}_{3(\mathrm{aq})}\right)+\ldots
$$

where $m^{T}$ is the total molality of the component species, and $m$ denotes the molality of the indicated species. Here $m\left(\mathrm{Ca}^{2+}\right)$ is the "true" molality of $\mathrm{Ca}^{2+}$. The measured total molality (by common analytical techniques) is generally always the total molality. The summation is over all aqueous species composed of the component ion, as indicated by the corresponding dissociation reactions as stored on the supporting data file. In EQ6, mass balances are written in terms of the absolute numbers of moles $(n)$, not molalities, and the balance extends to any phases other than the aqueous solution that are in equilibrium with that solution (the aqueous solution and any such phases are said to make the "equilibrium system"). Such a mass balance is exemplified by

$$
n^{T}\left(\mathrm{Ca}^{2+}\right)=n\left(\mathrm{Ca}^{2+}\right)+n\left(\mathrm{CaHCO}_{3}^{+}\right)+n\left(\mathrm{CaCO}_{3(\mathrm{aq})}\right)+\ldots+n(\text { Calcite })+\ldots
$$

where it is presumed that calcite $\left(\mathrm{CaCO}_{3}\right)$ is present in the equilibrium system. In actuality, the form of mass balance shown in Eq. 5-9 is that used in Version 8 of EQ6. The earlier versions of EQ6 treat mass balances in terms of chemical elements, and a charge-balance equation is substituted for a mass balance in the case of the redox species $\mathrm{O}_{2}$.

The molality of the $i$-th component or species $\left(m_{\mathrm{i}}\right)$ is related to the corresponding number of moles $\left(n_{\mathrm{i}}\right)$ by

$$
m_{\mathrm{i}}=\Omega n_{\mathrm{i}} / n_{\mathrm{W}}
$$

where $\Omega$ is the number of moles of water in a 1-kg mass $(\approx 55.51)$ and $n_{\mathrm{w}}$ is the number of moles of solvent water (the species $\mathrm{H}_{2} \mathrm{O}$, not the component $\mathrm{H}_{2} \mathrm{O}$ ). This equation can be applied to either total or true quantities. In both EQ3NR and EQ6, the total quantities on the left side are taken to be knowns, and the quantities on the right side are treated as unknowns. In EQ6, this treatment includes mass-balance equations for $\mathrm{H}_{2} \mathrm{O}, \mathrm{H}^{+}$, and $\mathrm{O}_{2}$ (note: a chargebalance equation substitutes for a mass balance on $\mathrm{O}_{2}$ in Version 7 and earlier versions of EQ3/6). However, EQ3NR does not actually use this treatment for these species. Instead, the 
$\mathrm{H}_{2} \mathrm{O}$ is treated by fixing the solvent water at $1 \mathrm{~kg}$, the $\mathrm{H}^{+}$is normally constrained by a $\mathrm{pH}$, and the $\mathrm{O}_{2}$ is normally constrained by an input Eh or oxygen fugacity value. Alternate equations then substitute for mass balance in the case of each of these species (see Sections 2.3.1-2.3.3 of Wolery, 1992b). Eq. 5-8 is then applied after the speciation-solubility calculation to obtain the $m^{T}$ (and thence the $n^{T}$ ) values for these components for input to EQ6.

For each dependent or nonbasis aqueous species, there is a corresponding reaction written in terms of the species itself and the basis species. This is exemplified by

$$
\mathrm{CaHCO}_{3}^{+}=\mathrm{Ca}^{2+}+\mathrm{HCO}_{3}^{-}
$$

There are no reactions for the basis species themselves; thus, they are sometimes referred to as "building blocks" (Wolery, 1992b). For each such reaction, there is a corresponding massaction equation that describes the equilibrium constraint. Corresponding to reaction 5-11 is

$$
\log K\left(\mathrm{CaHCO}_{3}^{+}\right)=\log a\left(\mathrm{Ca}^{2+}\right)+\log a\left(\mathrm{HCO}_{3}^{-}\right)-\log a\left(\mathrm{CaHCO}_{3}^{+}\right)
$$

where $K$ is the equilibrium constant for the reaction corresponding to the indicated species, and $a$ is the thermodynamic activity of the indicated species.

For the $i$-th aqueous solute species (any species other than solvent water), the thermodynamic activity $\left(a_{i}\right)$ is given by

$$
\log a_{\mathrm{i}}=\log m_{\mathrm{i}}+\log \gamma_{\mathrm{i}}
$$

where $m_{\mathrm{i}}$ is the molality of the species and $\gamma_{\mathrm{i}}$ is the corresponding (molal) activity coefficient. The thermodynamic activity of water $\left(\mathrm{a}_{\mathrm{w}}\right)$ is given by

$$
\log a_{\mathrm{w}}=\log x_{\mathrm{w}}+\log \lambda_{\mathrm{i}}
$$

where $x_{\mathrm{w}}$ is the mole fraction of solvent water, and $\lambda_{\mathrm{i}}$ is its mole fraction thermodynamic activity coefficient. Solvent water refers to the species $\mathrm{H}_{2} \mathrm{O}$, not to the component $\mathrm{H}_{2} \mathrm{O}$.

For each pure mineral, there is also an analogous corresponding reaction. This is here exemplified by

$$
\text { Calcite }+\mathrm{H}^{+}=\mathrm{Ca}^{2+}+\mathrm{HCO}_{3}^{-}
$$

(a repetition of Eq. 5-2). For each such reaction, when equilibrium is presumed, there is a corresponding mass-action equation. Corresponding to Eq. 5-15 is

$$
\log k(\text { Calcite })=\log a\left(\mathrm{Ca}^{2+}\right)+\log a\left(\mathrm{HCO}_{3}^{-}\right)-\log a\left(\mathrm{H}^{+}\right)
$$

A term containing $\log a$ (Calcite) does not appear on the right side because, as was noted in Section 5.2.1.2, the log activity of a pure phase is defined to be zero. The right side of Eq. 5-16 is the $\log Q$ (the $\log$ activity product, as may be noted by comparison with Eq. 5-4). Thus, Eq. 5-16 is equivalent to stating that $\log Q / K=0$ (i.e., that equilibrium prevails). EQ6 uses a mass-action equation such as Eq. 5-16 for each pure mineral present in the equilibrium system. Such a mass-action equation may be substituted for a mass-balance equation in EQ3NR (e.g., if calcite equilibrium were to be applied to $\mathrm{Ca}^{2+}$ instead of specifying a total concentration). Somewhat more complex forms of mass-action equations apply to components of solids solutions. For a discussion of these, see Wolery (1992b). 
The thermodynamic activity coefficients of aqueous species may be calculated in EQ3/ 6 according to one of three models. The simplest of these models is the Davies equation, which may be written for solute species as

$$
\log \gamma_{i}=-A_{\gamma} z_{i}^{2}[(\sqrt{I} /(1+\sqrt{I}))-0.2 I]
$$

where $A_{\gamma}$ is the Debye-Hückel. A constant for molal activity coefficients, $z_{i}$ is the electrical charge number of the species, and $I$ is the ionic strength, given by

$$
I=\sum_{i} m_{i} z_{i}^{2}
$$

where the summation is over all solute species. This model, as the similar B-dot equation that is also an option in EQ3/6, gives the impression that the activity coefficients depend on the solution composition in a simple way: only through the ionic strength. However, this is only a limiting approximation (in the limit of infinite dilution). As a practical matter, the Davies and B-dot equations are merely approximations that are not too bad in sufficiently dilute solutions. In general, they are not usable for $I>1.0$ molal. Loss of accuracy may be notable at even lower ionic strengths (generally for $I>0.2$ molal). In more concentrated solutions, complex equations that depend on the specific solution composition (and involve many more parameters) are required. EQ3/ 6 offers the option of Pitzer's equations. For a detailed description of the B-dot equation and Pitzer's equations, see Chapter 3 of Wolery (1992b). Certain EQ3/ 6 data files support using either the Davies equation or the B-dot equation. Other data files (containing the necessary additional data) support using Pitzer's equations. The model chosen on the input file must be consistent with the supporting data file.

Computationally, the approach taken by EQ3/ 6 to solve the chemistry equations is based on a hybrid Newton-Raphson approach (Wolery, 1992b; Wolery and Daveler, 1992). The mass-action equations for dependent aqueous species are substituted into the mass-balance equations. The thermodynamic activity coefficients are treated as constants in a NewtonRaphson iteration. They are updated between such iterations (this gives the method its hybrid flavor). Thus, in an EQ3NR calculation, during a Newton-Raphson step, only the substituted mass-balance equations remain, and the operational unknowns to iterate upon are the corresponding $\log$ (true) molalities of the basis species (the $\log \mathrm{fO}_{2}$ applies for $\mathrm{O}_{2}$ ). As noted previously, alternate equations always apply in EQ3NR to the basis species $\mathrm{H}_{2} \mathrm{O}, \mathrm{H}^{+}$, and $\mathrm{O}_{2}$. Alternative equations may also apply to other basis species, depending on the options chosen (see Wolery, 1992b). Otherwise, iteration is made by generating a correction vector $(\delta)$ by solving a matrix equation of the form

$$
J \delta=\alpha
$$

where $J$ is a Jacobian matrix, and $\alpha$ is a residual function vector (it measures the extent to which the mass-balance equations and mineral mass-action equations are not satisfied). Iteration continues until the max norms of both $\alpha$ and $\delta$ are within a specified tolerance. For details, see Wolery (1992b) and Wolery and Daveler (1992).

EQ6 includes a modification in that, when minerals are present in the equilibrium system, the corresponding mass-action equations are added to mass-balance equations, and the set of unknowns is composed of the $\log$ number of moles of the basis species (but $\log \mathrm{fO}_{2}$ for $\mathrm{O}_{2}$ ) and the log number of moles of such minerals. In Version 7 and earlier versions of EQ6, a charge-balance constraint is used instead of a mass balance for $\mathrm{O}_{2}$, as noted earlier. Equilibrium phase assemblages are found by adding and deleting mineral phases (see Section 7.9 of Wolery and Daveler, 1992). If convergence is achieved for a given phase 
assemblage (initially just the aqueous solution), the saturation indices are calculated for all mineral phases. If there are any supersaturations, the mineral with the highest SI (scaled to account for the magnitude of the mineral's molecular formula) is added to the phase assemblage, and the calculation is repeated until no supersaturations remain (excepting those associated with user-suppressed phases). If a mineral is present in one of these iterated assemblages but does not belong in the final assemblage, the code has means to delete it. This condition always results in a crash of the hybrid Newton-Raphson iteration. The code determines the mineral to delete by scanning the record of the crash. Typically, the log number of moles variable for the mineral that needs to be removed will be diverging toward minus infinity, equivalent to the number of moles variable converging toward zero.

A number of mechanisms in EQ3/ 6 assist hybrid Newton-Raphson iteration, among them various under-relaxation schemes. For a description of these, see Chapter 7 of Wolery and Daveler (1992).

As EQ6 steps from point to point of reaction progress, it builds finite-difference expressions (up to sixth order) to describe the changes in certain variables. Among the variables are the log number of moles variable for the aqueous basis species and for any minerals present in the equilibrium system. The finite-difference functions for these variables are used to predict starting values for the equilibrium calculations at succeeding points. Such finite-difference expressions are also used to predict the location (e.g., the reaction progress value) of certain events such as where a mineral in the equilibrium system will disappear or where a new one will appear.

\section{The Reaction-Rate Equations and Mass-Transfer Calculations}

As noted earlier, a reaction-path calculation can be treated as a sequence of equilibrium calculations. To accomplish this, one must account for how the total number of moles variable $\left(n^{T}\right)$ for the components changes due to mass transfer from the reactant system to the equilibrium system. Mass transfer is accomplished by integrating rate expressions for the reactions associated with the defined reactants. One may define reactants of various kinds, but the typical reactant is a pure mineral such as $\mathrm{K}$-feldspar $\left(\mathrm{KAlSi}_{3} \mathrm{O}_{8}\right)$, for which the dissolution reaction may be written as

$$
\mathrm{K} \text { - feldspar }+4 \mathrm{H}^{+}=\mathrm{K}^{+}+\mathrm{Al}^{3+}+\mathrm{SiO}_{2(\mathrm{aq})}+2 \mathrm{H}_{2} \mathrm{O}
$$

In general, a reaction such as this is always written so that the associated reactant has a coefficient of unity. For pure minerals and some other types of reactants, the reaction is taken from the supporting data file. For others such as the "special" reactants, the user must specify the reaction on the input file when constructing the reactant. Typical special reactants might be a second aqueous solution, a whole rock, a volcanic glass, a nuclear waste glass, or whole spent nuclear fuel.

Corresponding to the $j$-th reactant, such as K-feldspar in Eq. 5-20, is a reaction progress variable $\xi_{j}$. The notion is that, as $\xi_{j}$ progresses from zero to one, one mole of the corresponding reactant is destroyed. A stoichiometrically equivalent amount of each of the constituent components (here, the other species present in the reaction) appears in (or disappears from) the equilibrium system. The mass increments that so appear (or disappear) are added to (or subtracted from) the corresponding $n^{T}$ values for the equilibrium system. Using Eq. 5-20 as an example, when $\xi_{j}$ goes from zero to one, the $n^{T}$ value for $\mathrm{K}^{+}$is incremented by one mole (ditto for those of $\mathrm{Al}^{3+}$ and $\mathrm{SiO}_{2(\mathrm{aq})}$ ), that for $\mathrm{H}_{2} \mathrm{O}$ is incremented by two moles, and that for $\mathrm{H}^{+}$is decremented by four moles. The component species $\mathrm{H}_{2} \mathrm{O}, \mathrm{H}^{+}$, and $\mathrm{O}_{2}$ are frequently both consumed and produced in such reactions. Their $n^{T}$ values, in fact, 
may become zero or even negative. That is not a problem, because the mass balance totals do not have a physical meaning. Negative values may, in fact, be input to EQ6 from EQ3NR. For example, the $n^{T}$ value for $\mathrm{H}^{+}$is almost always a negative number for an alkaline solution.

In Version 7 of EQ6 and in earlier versions of EQ3/6, the situation is somewhat different than that described in the previous paragraph because the mass balances are handled in terms of chemical elements instead of component species, and a charge-balance equation is matched with the redox species $\mathrm{O}_{2}$. Here the coefficients used to compute the mass-transfer increments come not from the associated reactions, but from the defined chemical compositions. For pure minerals and related reactants, the compositions come from the supporting data file. For special reactants, the user specifies the chemical composition on the input file.

The overall reaction progress variable $(\xi)$ is defined as

$$
\xi=\sum_{j}\left|\xi_{j}\right|
$$

where the summation is normally over all reactants (an exception is noted later). This variable is the one used as the primary stepping variable in EQ6, even for calculations for which there is a time frame. Absolute values are used in Eq. 5-21 to account for the fact that the progress variable for an individual reaction formally may be negative (e.g., if a reactant mineral is precipitating according to a rate law instead of dissolving).

When a reaction-path calculation takes place with no time frame, all rates are specified in the form of so-called relative rates $\left(v_{j}^{\text {rel }}=d \xi_{j} / d \xi\right)$. These rates are almost always treated as constants, but can be simple functions of $\xi$. The values (or functional parameters) are specified by the user on the input file. For a given increment of overall reaction progress $(\Delta \xi)$, the corresponding increments for the individual progress variables $\left(\Delta \xi_{j}\right)$ are obtained by integration of the relative rates. This integration is based on the used of closed-form solutions. It is not a numerical integration. For example, in the case of a constant relative rate, the expression is simply

$$
\Delta \xi i=v_{j}^{\text {rel }} \Delta \xi
$$

From the individual progress increments, it is a simple matter to calculate the corresponding increments to the mass balance totals:

$$
\Delta n_{i}^{T}=\sum_{j} b_{i j} \Delta \xi_{j}
$$

Here the $b_{i j}$ are the appropriate stoichiometric coefficients.

Any change in temperature or pressure is normally specified as a comparably simple function of reaction progress (i.e., usually the rate is a constant). Such a specification is also made by the user on the input file. The treatment then involves simply computing the new temperature and pressure at a new point of reaction progress and recomputing the equilibrium constants and other thermodynamic data (such as Debye-Hückel constants) that depend on the temperature and pressure.

In reaction-path calculations having a time frame, EQ3/6 still uses the overall reaction progress as the primary variable. The time is then computed as a secondary variable. For a reaction-path calculation to use a time frame, there must usually be at least one reaction for which the rate is specified by a kinetic rate law (kinetic rate laws and relative rates may be mixed). A kinetic rate law specifies the reaction rate in terms of time; it describes the rate in the form $d \xi_{i} / d t$. An example is a simple form for mineral dissolution based on transition state theory: 


$$
d \xi_{j} / d t=s_{j} k_{+, j}\left[1-\exp \left(A_{+, j} / \sigma_{+j} R T\right)\right]
$$

where $s_{j}$ is the mineral's surface area, $k_{+, j}$ is the dissolution (forward) rate constant, $A_{+, j}$ is the affinity to dissolve, $\sigma_{+j}$ is a stoichiometric parameter (usually having a value of unity), $R$ is the gas constant, and $T$ is the absolute temperature. For further discussion of kinetic rate laws programmed into EQ6, see Section 3.3.3 of Wolery and Daveler (1992).

In calculations having a time frame, the overall reaction progress variable is defined in a slightly different way. The summation in Eq. 5-21 is then only over those reactants for which a kinetic rate law is specified. Differentiation of that equation with respect to time yields

$$
v=\sum_{j}\left|v_{j}\right|
$$

where $v=d \xi / d t$ and $v_{j}=d \xi_{j} / d t$. The $v_{j}$ can be obtained by evaluating the kinetic rate laws. Then $v$ can be obtained from Eq. 5-25. It is then possible to calculate the corresponding relative rates from

$$
v_{j}^{\mathrm{rel}}=v_{j} / v
$$

If there are any other reactants present whose rates are described directly by relative rates, these can now be evaluated directly from the specified expressions (usually constants as noted previously). With relative rates established for all reactants, individual progress increments and mass-balance total increments can now be obtained by integrating. However, obtaining the individual progress increments now requires a numerical integration (discussed in a later paragraph). Time is handled by defining a quantity call the inverse rate $\left(v_{1 / t}\right)$ :

$$
v_{1 / t}=1 / v
$$

Note that $v_{1 / t}=d t / d \xi$. This can be integrated (also numerically) to obtain the time increment $\Delta t$.

When time is involved, the key factor is that the required integration is numerical. EQ3/6 uses a type of predictor-corrector ODE method for performing this integration (see Section 8.4 of Wolery and Daveler, 1992). Stepping to a new point of reaction progress, the mass transfer and time increments are predicted using finite-difference expressions for the governing rates (these finite-difference functions are similar to those described previously). For the purposes of checking ODE integration accuracy, the relative rates and the inverse rate at the new point are also predicted using finite differences. An equilibrium calculation is then performed. Then the relative rates and the inverse rate are recalculated, and the values are compared with those that were predicted. If the differences are not all within a specified tolerance, the stepping is repeated with a smaller step size. The method is not a full predictorcorrector method. If it were, such a method correction would first be attempted using a corrector function, a finite-difference expression that would be based in part on the new calculated rate functions at the new point. The adequacy of the present ODE integration method is limited. For certain kinds of problems, it can result in the step size becoming stuck at a minimum value. A more sophisticated ODE integration method is planned to replace it in 1998. 


\subsubsection{Other Codes}

Although the number of thermodynamic modeling codes is legion (e.g., Nordstrom et al., 1979), due to space limitations, only a few relevant examples are discussed-and then only in broad terms. Unless otherwise noted, all software discussed here includes both speciationsolubility and reaction-path modeling capabilities.

\section{PHREEQC}

PHREEQC (Parkhurst, 1995) is a code with many interesting capabilities, mostly aimed at interactions involving low-temperature groundwaters. It includes ion-exchange and surfacecomplexation models. A unique capability of this code is its ability to do inverse modeling (e.g., deduce the processes that modified a groundwater while it flowed through a rock formation). It also has an advective transport capability. PHREEQC does not use Pitzer's equations. A related code, PHRQPITZ (Plummer et al., 1988), does use them, but lacks many of the other capabilities of PHREEQC.

\section{Geochemist's Workbench ${ }^{T M}$}

The Geochemist's Workbench, or GWB (Bethke, 1994), has an unrivaled strength in terms of graphics. Some of the graphics capabilities are highly useful for analyzing thermodynamic data and phase relations, apart from speciation-solubility or reaction-path modeling. The current version also includes a surface-complexation modeling capability (but, at least at the time of release, the substrate had to be hydrous Fe(III) oxide due to supporting data limitations). LLNL uses GEMBOCHS to produce data files for GWB in the same manner as it does for EQ3/6.

\section{SOLVEQ and CHILLER}

Another interesting package is composed of the codes SOLVEQ (Spycher and Reed, 1989b) and CHILLER (Spycher and Reed, 1989a). SOLVEQ is a speciation-solubility code much like EQ3NR. CHILLER is a reaction-path code with specific adaptations for modeling boiling systems. This software does not use Pitzer's equations.

\section{INSIGHT}

A code with a strong bent toward Pitzer's equations is INSIGHT (Sterner et al., 1997). It is aimed at modeling the thermodynamics of systems containing concentrated aqueous solutions using the formalism of Pitzer's equations. It does not perform reaction-path calculations per se, only equilibrium calculations. Its unique strength lies in its ability to analyze all kinds of thermodynamic measurements to obtain the necessary Pitzer interaction parameters. At present, it appears to be the premier tool for this kind of work.

\subsection{Reactive-Transport Models}

Reactive-transport models always contain a time variable and a full 1-D, or greater, spatial representation of a reacting system. A reactive-transport model generally involves an aqueous fluid and provision for transport by advection and dispersion (and possibly diffusion). Chemical reactions are treated as rate processes with specific reaction constants.

Reactive-transport models are dynamic and require a source of energy to drive reactions. This is typically the presence of a substance such as rock, concrete, or the waste form with which the aqueous phase is not in equilibrium. Energy may also be derived from differences in chemical potential compared to another aqueous phase or from differences in temperature or pressure. It could also be an internal disequilibrium such as differences in the redox states 
of solution components. In reactive-transport simulations, the system changes with time, and the driving forces may vary both temporally and spatially. The main difference between thermodynamic models, such as EQ3/6, and reactive-transport models is that transport of mass and/or energy can perturb the system from its equilibrium state.

For near-field and AZ studies, reactive transport models are used to simulate chemical reactions that are associated with the temperature and flow regimes predicted by TH models. They are used to identify significant reactions and to determine where in the AZ the reactions will occur. Chemical dissolution and precipitation are evaluated with respect to the likely feedback effects on porosity, permeability, and other TH properties.

The use of reactive-transport models is critical in interpreting experimental and field data and in extrapolating the results to repository conditions. Some important chemical processes may be so slow that they are not observable in controlled experiments, yet they are important to repository performance; other processes are relatively fast. If rate-dependent conditions or slow reactions are ignored, direct application of results from short-term tests could lead to erroneous predictions of long-term performance. Reactive transport models are used throughout the geochemical community for research, waste-disposal siting, and system evaluation by regulatory agencies.

Reactive-transport models may be applied not only to the study of water-rock interaction, but also to the interaction of water with waste forms, metals, cemetitious materials, and other human-made materials of interest. They are closely related to thermodynamic models, which include static, flow-through, and reaction-path models as discussed in Section 5.2. Reactive-transport models rely heavily on chemical thermodynamics, requiring supporting thermodynamic data in the form of equilibrium constants or free-energy data, which are commonly used as parameters in reaction-rate-law equations (see Section 5.3.2)

\subsubsection{Mathematical and Numerical Approaches to Reactive-Transport Modeling}

\section{by William E. Glassley, William L. Bourcier, and James W. Johnson}

This section outlines the fundamental chemical and transport processes that must be accounted for in reactive-transport models and reviews the existing software that encodes these processes in explicitly coupled form. Any reactive-transport simulator must provide explicit mathematical coupling of the processes and be encoded within a workable software package. Several such modeling packages currently are available in the public domain. However, the rigor with which they address each of the coupled processes relevant to the repository $\mathrm{AZ}$ varies depending on the applications envisioned by the developer. In other words, there are significant differences in the conceptual and mathematical models, the numerical implementations, and the solution methods used.

The limitations of numerical processors (when the codes were developed), the absence of well-constrained models and theoretical constructs for certain flow and reactive-transport problems, and the general lack of experimental data against which codes can be tested make it difficult to construct a code that embodies all of the capabilities that may be appropriate for repository application. The following review of existing codes provides a snapshot of current capabilities.

\subsubsection{Descriptions of Selected Reactive-Transport Software}

The list of codes discussed here is not exhaustive; it is intended to represent the range of modeling approaches among software that is widely available. The software is described in alphabetical order. The list contains few proprietary codes. Note that the equations used to solve for heat flow, fluid flow, and solute transport in all of the codes are quite similar. 
Hence, in the following discussions, a detailed presentation of them is only given in the first description (1DREACT). In the other descriptions, functions are presented only if they significantly differ from those in the synopsis of 1DREACT.

\section{DREACT (OS3D/GIMRT)}

This program is a comprehensive, 1-D and 2-D reactive-transport simulator that accounts for single-phase advective, diffusive, and dispersive mass flux, conductive and convective heat flow, kinetically controlled fluid rock-mass transfer along fracture surfaces, and permeability variations that arise from porosity changes caused by mineral precipitation/dissolution. 1DREACT necessarily incorporates a variety of simplifying assumptions; nevertheless, the program provides a versatile and convenient numerical laboratory for modeling reactive transport in hydrothermal systems with imposed thermal gradients associated with the emplacement of a nuclear waste repository. The following discussion also applies to the OS3D/GIMRT code (Steefel and Yabusaki, 1995) developed by the authors of 1DREACT as its successor.

Heat transport and fluid flow are modeled by simultaneous account of the conservation of thermal energy, which can be expressed as (Steefel and Lasaga, 1994)

$$
\rho_{m} C_{P, m} \frac{\partial T}{\partial t}=\nabla \cdot\left(\lambda_{m} \nabla T-\rho_{f} C_{P, f} \mathbf{u}_{f} T\right)
$$

and the fluid-continuity equation, which is defined by Steefel and Lasaga (1994)

$$
\frac{\partial\left(\phi_{m} \rho_{f}\right)}{\partial t}=-\nabla \cdot\left(\rho_{f} \mathbf{u}_{f} T\right)
$$

where $\rho$ refers to density, $C_{P}$ denotes isobaric heat capacity, $T$ stands for temperature, $t$ signifies time, $\lambda$ represents thermal conductivity, $\phi$ defines porosity, the subscripts $\mathrm{f}$ and $\mathrm{m}$ refer to the fluid phase and bulk medium, respectively, and the fluid flux, $\mathbf{u}_{\mathrm{f}}$ is obtained from Darcy's law, which can be written as (Steefel and Lasaga, 1994)

$$
\mathbf{u}_{f}=-\frac{k}{\mu_{f}}\left(\nabla P-\rho_{f} \mathbf{g}\right)
$$

where $k$ denotes permeability, $\mu$ stands for dynamic viscosity, $P$ represents pressure, and $\mathbf{g}$ is the gravity vector. As is common practice, these conservation and continuity equations reasonably neglect, as second-order effects, the potential thermal energy contributions from radioactive decay or chemical reactions and fluid-mass contributions from hydration or dehydration reactions. The continuity equation is further simplified by presuming that both the fluid and rock matrix are essentially incompressible, which leads to $\nabla \cdot \mathbf{u}_{\mathrm{f}}=0$. Moreover, in the context of these equations, the fluid phase is presumed to be pure $\mathrm{H}_{2} \mathrm{O}$, and its properties $\left(p_{\mathrm{f}}, \mu_{\mathrm{f}}\right.$ and $\left.C_{p, t}\right)$ are evaluated using several approximations to more rigorous formulations. Again, these simplifications are typical (in fact, they are incorporated in most of the programs evaluated in this review) and are not unreasonable.

1DREACT permits definition of as many as six zones of distinct initial porosity (and permeability) in the model system. However, in its present configuration, the code cannot model any aspects of two-phase flow or variations in the stress field. 
The reaction module of 1DREACT explicitly accounts for multicomponent chemical systems, complexation reactions in the fluid phase, and surface-controlled mineral dissolution/precipitation kinetics. The conservation of solute mass is described by Steefel and Lasaga (1994)

$$
\frac{\partial\left(\phi_{\mathrm{m}} U_{j}\right)}{\partial t}+\nabla \cdot\left(\mathbf{u}_{\mathrm{f}} U_{j}-\mathrm{D} \nabla U_{j}\right)=R_{j}^{\min }
$$

where $U_{j}$ refers to the total solution concentration of the $j$ th chemical component (accounting for a primary $j$ th solute and the $N_{i} j$-bearing complexes), $\mathbf{u}_{\mathrm{f}} U_{j}$ and $\mathrm{D} \nabla U_{j}$ represent the advective and diffusive/dispersive fluxes, D stands for the dispersion/diffusion coefficient, and $R_{j}^{m i n}$ denotes the reaction-rate term, which is the product $v_{j, m} r_{m}$ summed over the $N_{m}$ minerals, where $v_{j, m}$ refers to the stoichiometric coefficient of the $j$ th primary solute in the $m$ th mineral, and $r_{m}$ is the precipitation/dissolution rate for that mineral. The surface-controlled kinetic rate is given by Steefel and Lasaga (1994)

$$
r_{m}=\operatorname{sgn}\left[\log \left(\frac{Q_{m}}{K_{m}}\right)\right] A_{m} k_{m}\left|K^{-1} \prod_{j=1}^{N_{j}}\left(\gamma_{j} C_{j}\right)^{v_{j, m}}\right|
$$

where sgn denotes the sign of the expression, $K_{m}$ refers to the aqueous dissolution constant for the $m$ th mineral, $Q_{m}$ stands for the associated ion activity product (defined by the numerator of the product term), $r_{j}$ signifies the activity coefficient of the subscripted primary solute, $A_{m}$ denotes the reactive surface area, and $k_{m}$ is the reaction-rate constant, whose temperature dependence is accounted for by an Arrhenius equation (Steefel and Lasaga, 1994):

$$
r_{m}=k_{25} \exp \left[-\frac{E_{\mathrm{a}}}{\mathrm{R}}\left(\frac{1}{T}-\frac{1}{298.15}\right)\right]
$$

where $k_{25}$ is the rate constant at $25^{\circ} \mathrm{C}, E_{\mathrm{a}}$ represents the activation energy, $R$ stands for the gas constant, and $T$ denotes temperature (in Kelvin).

Similarly to the transport equations, these specific statements of solute mass conservation and the kinetic rate law are obtained following several simplifying approximations: all complexation (homogeneous) reactions are considered reversible (i.e., local equilibrium is imposed on the fluid phase; hence, there is no provision for treating redox disequilibria), the dispersion/ diffusion coefficient of each aqueous solute is considered identical, and the rate laws are strictly linear and do not account for the inhibiting or catalyzing effect of specific aqueous solutes. With the possible exception of uniformly reversible complexation reactions, these approximations are not overly restrictive for simulating the repository AZ. Moreover, the kinetic description avoids imposition of the local equilibrium constraint on fluid-rock (heterogeneous) reactions. There is no theoretical limit to the number of chemical components and species that can be considered, and the nucleation process associated with mineral precipitation is explicitly accounted for (supersaturation catalyzes "instantaneous" growth of a 10-micron radius crystal). In addition, it is possible to run 1DREACT using both standard and customized thermodynamic databases.

This model does not explicitly account for the effects of sorption, colloids, ion exchange, or solid solutions. Because the program calculates activity coefficients from an extended Debye-Hückel formulation, its range of applicability is limited to systems characterized by relatively dilute aqueous solutions. 
Temporal variations in the volume fraction of specific minerals as a function of irreversible dissolution/precipitation are explicitly accounted for with

$$
\frac{\partial \phi_{m}}{\partial t}=V_{m} r_{m}
$$

where $V_{m}$ refers to the standard molal volume of the subscripted mineral, and $\phi_{m}$ is the mineral volume fraction used to calculate the dependent change in porosity

$$
\phi=1-\sum_{m=1}^{N_{m}} \phi_{m}
$$

By assuming an idealized permeability structure composed of three mutually orthogonal sets of smooth, parallel fractures having uniform aperture $\delta$ and spacing $d$ (isotropic permeability), the functional relation between flow porosity $\phi_{\mathrm{F}}$ and permeability $k$ can be defined as (Phillips, 1991)

$$
k=\frac{\phi_{\mathrm{F}} \delta^{2}}{36}
$$

where $\phi_{\mathrm{F}}=\delta / d$, and variations in $\phi_{\mathrm{F}}$ as a function of $\phi$ are restricted to the those affecting fracture aperture.

This relation among permeability, porosity, and dissolution/precipitation incorporates another key simplifying assumption beyond those already noted for the fracture network; namely, all mineral dissolution/precipitation is restricted to the fracture surfaces themselves (i.e., reaction-controlled porosity changes within the rock matrix are not accounted for; hence, the matrix is effectively impermeable from a chemical standpoint). This approximation leads to the constraint that the sum of mineral surface areas must equal the total surface area of rock in contact with the fluid. In sum, these permeability-structure and reaction assumptions create a permeability-porosity-reaction model that is significantly reduced in complexity relative to that which holds in natural systems. However, as noted by Steefel and Lasaga (1994), such simplification is needed at the current stage of theoretical development.

Using an integrated finite-difference scheme, 1DREACT discretizes the partial differential equations that represent the transport and reaction processes (deMarsily, 1986; Patankar, 1980; Steefel, 1993; Steefel and Lasaga, 1994), and employs a one-step or "global implicit" method to solve the coupled nonlinear transport and reaction equations simultaneously (Kee et al., 1985). As discussed by Steefel and Lasaga (1994), there are pros and cons to these choices as well as several alternatives. The principal advantage of the integrated finitedifference method is that variable grid-spacing (which permits dynamic adaptive gridding) and fluid velocities are readily incorporated. Moreover, in 1DREACT, this method is implemented using Patankar's (1980) "power-law" scheme for the transport terms, which ensures that the appropriate (upwind-weighted or fully centered) difference formulation is used depending on the relative importance of advective and dispersive/ diffusive transport. The one-step-solution method has two important potential advantages relative to the alternative two-step strategies: (1) the global convergence properties of this method are at times superior, and (2) in the typical case in which a wide range of reaction rates must be accounted for, it is often possible to take larger time steps for a given numerical stability tolerance. 
The numerical methods used in 1DREACT permit incorporation of many useful features that facilitate improved numerical stability, run-time performance, and modeling flexibility, including the following:

- Dynamically calculated time steps

- Adaptive gridding

- Basis (i.e., primary solute) species switching

- The option to run the program in either transient or stationary-state mode

In transient mode, mineral volume fractions and surface areas are held constant for the duration of each time step; in stationary-state mode, these mineral properties are held constant until the solute concentrations achieve a quasi-stationary state (Lichtner, 1988, 1992). The latter option is an efficient method for modeling systems for which the rate in mineral property variation is much slower than is the rate at which solute concentrations achieve a stationary state (Steefel, 1993).

1DREACT can be used to conduct both 1-D and 2-D simulations (Steefel and Yabusaki, 1995). There are no restrictions to the physical scales that can be represented. In this regard, note that, in situations for which the relative reaction rates of minerals can be considered constant, it is possible to perform scale transformations on the simulation results (Lichtner, 1993).

\section{Basin2}

Basin2 is a numerical model developed to trace through time the evolution of groundwater regimes within sedimentary basins (Bethke, 1985; Bethke et al., 1993). It is therefore unique with respect to the other codes described in this document in that it is meant to be applied over a larger spatial area and to evolve over longer time periods. However, Basin2 uniquely combines some features of fluid flow and chemistry that could be useful for modeling some key aspects of repository processes.

Basin2 uses a finite-difference technique to solve the fluid- and heat-flow and transport equations. It is formulated in Lagrangian coordinates and considers 2-D flow in heterogeneous, anisotropic, and accreting domains. The user supplies a description of the finite-difference grid and boundary conditions over which the calculation is performed. Basin2 can simulate flow through an unlimited number of strata, which can have different hydrologic and lithologic properties. Faults can also be included in the grid.

The code takes into account density and heat capacity variations of the rock and fluid as a function of pressure-temperature conditions. It also accounts for density and heat capacity changes in the fluid with salinity variations. Fluid viscosity values are generated internally using a look-up table based on data in Phillips et al. (1981). The stress field generated during compaction is also calculated and used to predict porosity changes on the basis of the simple empirical relation:

$$
\phi=\phi_{0} e^{-\beta \sigma_{\mathrm{e}}}+\phi_{1}
$$

where $\phi_{0}$ is the initial reducible porosity, $\phi_{1}$ is the irreducible (final) porosity, $\beta$ is the compressibility of the reducible pore volume, and $\sigma_{\mathrm{e}}$ is the effective stress (Bethke et al., 1993). Permeability is then calculated from porosity using the relation:

$$
\log k_{x}=A \phi+B
$$

where $k_{x}$ is the permeability in the $x$ direction, and $A$ and $B$ are empirical fitting parameters. The user defines the permeability in the $z$ direction as a simple function of $k_{x}$. The user also defines the heat flow into or out of the basin along all the boundaries. 
Two simple methods for coupling fluid chemistry with flow are used in Basin2. Built into the modeling code are the solubilities of four silica polymorphs (quartz, chalcedony, cristobalite, and amorphous silica) and calcium sulfate (anhydrite). The solubilities of these phases are assumed to be a function of temperature only (for silica) and temperature and pressure (for anhydrite). The code computes the saturation state for these phases in each finite-difference volume and adjusts the amount of phase present to ensure saturation. Equilibrium is assumed at all locations. The code then updates the porosity and permeabilities on the basis of the previously mentioned relations for the subsequent fluidflow calculation.

A more general method for coupling mineral precipitation/dissolution to fluid flow makes use of the chemical modeling code React (Bethke, 1994). A React simulation for the appropriate fluid composition is carried out over the temperature range of the basin. React calculates the amount of mineral phases present at each temperature using a rigorous speciation calculation. Basin2 is then linked to React using interface tables generated by React. In each finite-difference element, Basin2 assumes the equilibrium of the secondary mineral phases with the host fluid on the basis of the masses listed in the React output file. The rock porosity and permeability are adjusted appropriately.

Although each of these approaches is based on reasonable assumptions regarding local equilibrium in basins, no reaction kinetics are considered. Neither approach is consistent with a rigorous mass balance for chemical elements. If a mineral is calculated to be supersaturated, it is allowed to be present regardless of whether sufficient mass is present to form it. For example, all the mass may have been used up in precipitating minerals in adjacent elemental volumes.

Basin2 solves the equations describing flow through a deforming medium using a finitedifference technique (Bethke, 1985). It works with a curvilinear coordinate system that follows basin stratigraphy. This approach maintains an axis of the coordinate system that is parallel to the direction of greatest permeability. This approach introduces a small error into the calculation. Basin2 automatically adjusts the time step of the flow calculation based on criteria involving the amount of sediment produced, the size of the last time step, and limiting maximum values for changes in temperature, pressure, and salinity during the step.

To apply Basin2 to hydrologic problems at Yucca Mountain, the user must turn off some of the features of the code that are irrelevant, including features such as erosion and uplift rates, rock compaction, sedimentation rates, and a few others. This can be done by choosing appropriate input parameters.

Basin2 contains three algorithms for calculating the extent of the reaction of organic materials. Although Basin2 was developed to predict petroleum generation, its kinetic relations could probably be modified so it will be useful in modeling the alteration of organic matter (such as diesel fuel) present in the vicinity of the waste package (WP).

Basin2 also has an excellent graphics postprocessor that can be used to visualize the numerical results. This code is documented in a 225-page user's manual (Bethke et al., 1993) and in several publications from the literature (Bethke, 1985, 1986; Bethke et al., 1988). There have been no explicit verification/validation studies performed with the code, but there is a test library of simulations that could be used for that purpose. The code is commercial and cannot be modified at will without the consent of the owner.

\section{FEHM}

FEHM (Finite Element simulation of coupled Heat and Mass transport) is a finite-element code used in a variety of applications (Zyvoloski, 1983, 1986; Zyvoloski and Dash, 1991; Zyvoloski et al., 1988). This code was designed to describe heat and mass transfer in the 
porous media that may be encountered in hydrothermal systems and nuclear waste repositories. It has primarily been applied to the evaluation of multiphase transport in the Los Alamos hot-dry rock project and to PA for the Yucca Mountain repository project.

Heat and mass transfer are explicitly considered in the code. Heat budgets during boiling are accounted for through thermodynamic functions that describe the properties of water. A temperature-dependent Henry's law treatment of dissolved species accounts for partitioning between liquid and vapor phases. Saturation conditions are explicitly calculated at each time step. The user determines how often output for these parameters is retrieved. Humidities are not part of the current output file, but are calculated internally and can be readily included. Boiling, unsaturated conditions, and two-phase flow are incorporated. The stress state is updated at appropriate time steps, depending on the rate of stress change. The code accounts for changes in heat transfer, physical properties, and hydrologic properties as stress conditions change.

Reaction kinetics are incorporated via a forward- and reverse-rate law for each reversible reaction describing the behavior of each species. The effective rate law for each reaction is then represented by

$$
\partial\left[S_{j}\right] / \partial t= \pm a_{j}\left\{k_{\text {for }} \prod_{i=1}^{m}\left[S_{i}\right]^{b(i)}-k_{\mathrm{rev}} \prod_{i=m+1}^{n}\left[S_{i}\right]^{b(i)}\right\}
$$

where $S_{j}$ is the concentration of solute species $j, S_{i}$ is the concentration of $j$ in reactant species 1 through $m$ and in product species, $m+1$ through $n, b(i)$ is the appropriate exponential for the forward or reverse reaction, $a_{j}$ is the stoichiometric coefficient, and the $k$ terms are the forward (for) and reverse (rev) rate constants for the appropriate reactions. Temperature dependence of the rate constants is derived from the Arrhenius expression

$$
k=A \exp (-E / R T)
$$

where $A$ is the pre-exponential factor, $E$ is the activation energy, $R$ is the gas constant, and $T$ is temperature in degrees Kelvin. Similar laws are used for the kinetics applied to sorbed species (discussed subsequently), but concentrations are defined in terms of bulk rock density, fluid density, porosity, and specific isotherm parameters.

The code relies on user-defined reactions to describe the rates of reactions and reaction stoichiometry. The net reaction, defining the speciation state for each species, is determined from the sum of the forward and reverse reactions. Temperature dependence of the reactions is accounted for via the rate expressions described previously. The code can currently handle as many as 10 species, and more may be added. Activity coefficient models are not employed in the code; i.e., activity is assumed to be equivalent to concentration. Sorption isotherms can be computed using Langmuir or modified Freundlich approaches. The kinetics of sorption can be included, in which case forward and reverse rates are user specified.

The FEHM code is not currently designed to change porosity or permeability as dissolution or precipitation occur. However, a version of the code has been used in which user-defined relations are employed to make changes in porosity and permeability as dissolution or precipitation occur (see Section 5.5). The code is not currently designed to deal with the effects of permeability and porosity on mass transfer although, as with the effect of dissolution and precipitation on porosity and permeability, user-defined modules may be added. 
FEHM is fully 2-D or 3-D. An automatic, finite-element mesh generator is available for use with the code. Nonorthogonal meshes can be used. The cell dimensions for each lithologic unit are independent of those from other units. The user defines the region to be considered in terms of blocks. A given block then uses the same type of element, although element size may vary within the block, depending on the calculational needs. For 2-D simulations, four-noded quadrilaterals or three-noded triangles can be used as elements; 3-D simulations can use eight-noded hexahedrons or six-noded triangular prisms. Properties of individual lithologic units can be included and permeability anisotropy accounted for. The standard model employed for the Yucca Mountain simulations is on the scale of kilometers. However, simulations of mineralogical systems on the scale of millimeters or microns seems possible, provided the chemical heterogeneity is not too complex. This needs to be evaluated because of potential numerical problems at locations where changes in chemistry occur as a result of contrasting mineralogy.

A user's manual, in draft form, is available, and documents describing the application of the code to different problems can be obtained. The code is undergoing verification and certification and is not proprietary.

\section{LEHGC}

The LEHGC code (Lagrangian-Eulerian-HydroGeochemical Code) is an enhanced version of the HYDROGEOCHEM code (Yeh and Tripathi, 1992) LEHGC's major improvement over HYDROGEOCHEM is that the numerical solution procedure has been modified to implement aspects of the Lagrangian approach for the advective component of transport. This hybrid Lagrangian-Eulerian scheme allows larger time steps to be used in advection-dominated calculations and causes less numerical dispersion than do traditional Eulerian schemes. LEHGC retains most of the features of HYDROGEOCHEM, which are described in the manual for that code (Yeh and Tripathi, 1992).

LEHGC is a 2-D finite-element code that provides for fluid advection and dispersion/diffusion. The elemental grid can be a homogeneous porous media or can account for discrete fractures. It can also include heterogeneous and anisotropic media. In all cases, it provides for saturated/ unsaturated flow conditions. Because there is no heat transport considered in this code, it cannot account for boiling or any type of two-phase behavior for water. The relation between moisture content and pressure head must therefore be supplied by the user. There is no provision for stress fields generated during reactive transport.

LEHGC incorporates a comprehensive chemical model. It currently includes aqueous complexation, adsorption/desorption, ion exchange, precipitation/dissolution, redox reactions, and acid-base reactions. Redox reactions and acid-base reactions are essentially a special case of aqueous complexation reactions.

The speciation algorithm is a conventional one that uses the total analytical concentration of each element as the primary dependent variable. The total amount of element is divided among those fractions in solution as free or complexed species, adsorbed species, ionexchanged species, and precipitated solids. This calculation is constrained by the mass-action equations that describe these equilibria. Local equilibrium is assumed at all points along the reaction; i.e., all these reactions are assumed to be at equilibrium throughout the system. No mineral precipitation/dissolution or other types of chemical kinetics are considered.

Equilibrium constants rather than free energies of formation are used to define thermodynamic equilibrium. Standard Debye-Hückel activity coefficients are used to calculate species activities from species concentrations.

The treatment of adsorption is based on the fundamental relation: 


$$
B_{i}^{y}=K_{i}^{y}\left(\prod_{k=1}^{N_{A}} X_{k}^{a_{i k}^{y}}\right)\left(\prod_{k=1}^{N_{s}} Y_{k}^{b_{i k}^{y}}\right), i=1,2, \ldots, M_{y}
$$

where $B_{i}^{y}$ is the activity of the $i$ th adsorbed surface species, $K_{i}^{y}$ is the equilibrium constant of the $i$ th adsorbed species, $X_{k}^{a_{i k}^{y}}$ is the activity of the $k$ th aqueous component species, $a_{i k}^{y}$ is the stoichiometric coefficient of the $k$ th aqueous component in the $i$ th adsorbed species, $Y_{k}^{b_{i k}^{y}}$ is the activity of the $k$ th adsorbed species, and $b_{i k}{ }^{y}$ is the stoichiometric coefficient of the $k$ th aqueous component in the $i$ th adsorbed species. The treatment uses the double layer theory to calculate activities of surface species (Davis and Leckie, 1978).

Ion exchange is defined by the mass action expression:

$$
K_{i j}=\left(\frac{B_{i}}{A_{i}}\right)^{v_{j}}\left(\frac{A_{j}}{B_{j}}\right)^{v_{i}}, i=1,2, \ldots, M_{z}
$$

where $A_{j}$ is the activity of the $i$ th aqueous species, $B_{j}$ is the activity of the $i$ th ion-exchanged species, $v_{i}$ is the charge of the $i$ th species, and $M_{z}$ is the number of ion-exchanged species. It is difficult to derive a simple analytical expression for anything but homovalent exchange. LEHGC can rigorously compute the Jacobian matrix for hetereovalent ion-exchange reactions.

It should be noted that the treatments of sorption and ion exchange are linked to a fictive sorbing compound or ion exchanger and not to real minerals actively precipitating and dissolving during the reaction. The amount of sorbing compound or ion exchanger must be provided as input by the user. The code is not capable of linking the precipitation/ dissolution process to sorption and ion exchange. For example, if during the reactive transport simulation, a zeolite phase were to precipitate, the code would not apply appropriate ion-exchange properties to that mineral precipitate for the corresponding mass of precipitate. This type of scenario could not be rigorously modeled in one step with the LEHGC code. However, reasonable approximations could be generated using a carefully chosen sequence of simulations.

The most recent version of LEHGC provides for multiple-surface complexation and ionexchange sites. It also provides for colloid transport, although there is not yet any documentation to describe how it is implemented.

The hydrologic and chemical constraints are coupled using the direct substitution approach, in which the chemical equilibrium equations are substituted into the hydrologic transport equations to result in a set of nonlinear, partial differential equations. Different sets of dependent variables can be chosen for this approach (Yeh and Tripathi, 1989). In LEHGC, the total analytical concentrations of aqueous components are used as the dependent variables. LEHGC does not provide for changes in matrix porosity/permeability with mineral precipitation/dissolution.

The algorithm for solving the fluid-flow problem is the finite-element Galerkin model in two dimensions for an incompressible fluid. LEHGC solves the standard equations that relate pressure head, moisture content, and hydrologic conductivity. The solutions to these equations provide the temporal-spatial distribution of pressure head, moisture content, and the Darcy's velocity. No adaptive gridding is included in the code. 
Because LEHGC is an updated version of HYDROGEOCHEM, much of the documentation for HYDROGEOCHEM and its hydrologic modeling code predecessors (FEMWATER and FEMWASTE) apply to LEHGC. However, no comprehensive manual exists for LEHGC, and it is difficult to determine exactly what changes have been made in developing LEHGC from these earlier codes.

Some verification and validation work has been performed using HYDROGEOCHEM (Yeh and Tripathi, 1992) and LEHGC (Siegel, 1993; Siegel et al., 1993). This work has demonstrated that the ion-exchange and sorption modules can correctly simulate some simple ion-exchange and sorption experiments. The development plan for LEHGC includes developing capabilities to more rigorously model unsaturated conditions, heat transport, and full 3-D capabilities.

\section{PRECIP}

The PRECIP code was originally developed for modeling dissolution and precipitation reactions accompanying fluid flow in hot, dry rock geothermal systems (Noy, 1991). It was later employed for modeling mineral evolution in hyperalkaline systems (Clark et al., 1992; Khoury and Milodowski, 1992) and for modeling cement pore-fluid chemistry (Savage et al., 1992; Savage and Rochelle, 1993). Its emphasis is on reaction relations and phase equilibria rather than on hydrologic properties.

PRECIP does not compute heat transfer, although calculations can be done in either an isothermal system or one in which a temperature gradient exists along the flow path. The code does deal with mass transfer. The user defines a hydraulic head gradient for the system, which will control the computed Darcy velocities, given the other system properties. Only single-phase systems are considered by this code. The transport and reaction equations are fully coupled and solved simultaneously. These effects of changes in hydrologic properties on mass transport can be accounted for by employing a user-defined porosity-permeability relation. The code considers a porous medium, the properties of which may evolve through time as reaction proceeds, but it does not have the capability to deal with dual-porosity systems. The assumption is made that the system under consideration is a single-phase, fully saturated system. Humidities are not a relevant part of the calculations, and the stress state is not considered in the calculations.

Reaction kinetics are incorporated as a function, whose rate depends on the saturation ratio. The general form of the equation considered in the code is

$$
\text { Rate }=a k\left\{W^{m}-1\right\}^{n}
$$

where $a$ is the effective surface area of the mineral, $k$ is the rate constant, and $W$ is the saturation ratio, which is defined as the activity product $Q$ divided by the equilibrium constant $K$ for the reaction. The exponents $m$ and $n$ are reaction-specific constants. Although specific values for these exponents have been incorporated in the code, user-defined values can be added, as can other user-defined rate functions. Temperature dependence of the rate is incorporated into the rate constant and in the function that describes $W$.

The reactions to be considered are hydrolysis reactions for the solids of interest, and these are directly incorporated into the code. The total mass of each component is accounted for in mass-conservation equations that include the kinetic expressions for each phase as well as longitudinal dispersivity and pore-water diffusion terms. Incorporation of terms for porosity in these conservation equations then allows the solution of the hydrologic and chemical conditions simultaneously as a function of kinetics. Sorption and ion exchange are not considered. 
The code relies on user-defined reactions to describe the rates of reactions and reaction stoichiometry. The net reaction, defining the speciation state for each species, is determined from the sum of the hydrolysis reactions. Temperature dependence of the reactions is accounted for via the rate expressions described previously. Activity coefficient models are not employed in the code; thus, activity-composition relations are not defined. Either activity can be assumed to be equivalent to concentration, or user-defined routines can be used to compute these. As the code currently exists, computational results are presented as concentrations.

PRECIP computes changes in porosity on the basis of volume changes resulting from precipitation or dissolution of solids. To translate porosity change into a change in permeability, a user-defined function must be added to the code; such a function is not explicitly incorporated into the supplied code. Nevertheless, the chemical transport and the dissolution and precipitation effects are solved simultaneously, thus avoiding the common difficulty of sequential calculations for transport and chemistry.

The PRECIP code is solely 1-D. Simulations of mineralogical systems are done on the scale of millimeters or microns to meters. The actual scale is determined by the flow velocity and reaction rates of the system under examination.

Documentation describing the application of PRECIP to different problems has been published. Code development is continuing. Verification and validation activities have taken the form of published applications of the code (described previously). Some experiments are planned to test the simulations.

\section{STELE}

The STELE code was constructed by merging the transport code METIS (Goblet, 1989) with the chemical transport code CHIMERE (Coudrain-Ribstein and Jamet, 1989). STELE (or its component parts) has been applied to model the evolution of sedimentary basins (Coudrain-Ribstein and deMarsily, 1989), evolution of aquifers in which hydrothermal fluids have been injected (Coudrain-Ribstein et al., 1989a, 1989b; Coudrain-Ribstein and Gouze, 1993), uranium transport in soils (Jamet et al., 1993), and ore formation in Mississippi Valley type ore deposits (Schmitt et al., 1991). It has also been employed in the code comparison exercises of the Chemval project (Madé and Jamet, 1993).

STELE has no capability to account for boiling, condensation, or two-phase flow. Heat and mass transfer are computed under conditions assuming complete saturation. Heat transfer is treated via the temperature dependence of the dynamic fluid viscosity (described by the Bingham formula [Madé and Jamet, [1993]) and by taking into account dispersion and convection during fluid flow. Darcy velocities are computed using

$$
V=\left(k / \mu_{\mathrm{d}}\right)(\operatorname{grad} P+\rho g \operatorname{grad} z)
$$

where $k$ is the intrinsic permeability, $\mu_{\mathrm{d}}$ is the fluid dynamic viscosity (Pa-sec), $P$ is the pressure, $\rho$ is the mass per unit volume of fluid, $g$ is the gravitational constant, and $z$ is the vertical distance. Because boiling is not considered, only liquid velocities are considered. Dual-porosity conditions are considered, but stress is not considered.

Reaction kinetics are treated using the rate law

$$
\Omega=(1 / d l)\left[\operatorname{TOT}_{1}\left(B_{j}\right)-\operatorname{TOT}_{0}\left(B_{j}\right)\right]
$$


where $\Omega$ is the geochemical flux (mol/Ls), $d l$ represents one or more kinetic terms, $T_{O T}\left(B_{j}\right)$ is the concentration of component $B_{j}$ at equilibrium with some mineral phase, and $T O T_{0}\left(B_{j}\right)$ is the total aqueous concentration of $B_{j}$. This expression reduces to the classic relation for reaction kinetics when considering only single phases

$$
\Omega=k_{\mathrm{c}}[1-(Q / K)]
$$

where $k_{\mathrm{c}}$ is the rate constant, $Q$ is the activity product, and $K$ is the equilibrium constant. To take into account the current state of the art, further development of this portion of the code would be useful.

Sorption can be treated using a partition-coefficient approach defined by distribution coefficient values. Speciation calculations are done employing a user-selected thermodynamic data file. Either of two activity-coefficient models (Davies or Bromley [Madé and Jamet, 1993]) can be used. Saturation and precipitation or dissolution conditions are determined using standard mass-action laws, employing the selected activity coefficient models. There is no limit to the number of species considered.

Hydrologic properties are assumed to remain constant throughout a simulation. In the currently available versions of the code, there is no capability for updating porosity, permeability, or mechanical properties as dissolution or precipitation occurs. Mass transfer is assumed to occur under constant volume conditions. Hence, there is no coupling between mass transfer and changes in hydrologic properties. Deposition and dissolution of material is treated as a bulk process in which moles of material are deposited or removed per cubic meter of porous media.

All computations are 2-D, using an orthogonal finite-element gridding scheme. Adaptive gridding is not employed, although nonuniform gridding is possible. The code is designed to conduct simulations on the scale of meters to kilometers. The primary application has been in the study of basin and ore deposit development, evaluating flow regimes in hydrothermal systems.

Adequate STELE documentation exists as user's manuals (in French) and numerous publications describing the application of the code to different problems. These applications constitute a verification effort. It has also been extensively employed in the Chemval project, which represents an international verification and validation activity.

\subsubsection{Summary}

The codes described in the foregoing text can be used to simulate a wide range of problems relevant to study of the NF/ AZ, but it is clear that no single code will satisfy all current modeling needs. The OS3D/GIMRT code has been used for reactive-transport simulations of the AZ (see Section 5.7), but does not incorporate the THC effects of boiling. To address this gap, the TH simulator NUFT (see Chapter 3 ) has been modified to perform fully coupled THC calculations that include boiling reactions (see Section 5.6); to date it has been applied to simulation problems that are limited with respect to the number of chemical components and mineral phases. 


\title{
5.3.2 Supporting Kinetic Data
}

\author{
by James W. Johnson, Kevin G. Knauss, William E. Glassley, and Laura D. DeLoach
}

\subsubsection{Geochemical Kinetics in Reaction-Path and Reactive-Transport Modeling}

In reaction-path modeling and in reactive-transport modeling, reactions that are not assumed to be at equilibrium are assigned rate expressions. The SI or other measure of the degree of disequilibrium may appear in the rate law assigned to a reaction, but this is not always the case. Realistically, this dependence should be included in a manner such that the rate goes to zero as the driving force goes to zero and such that the direction of the reaction is consistent with the sign of the driving force. The most commonly used rate laws are based on a form suggested by transition-state theory. In some reaction-path calculations, all rates are specified as relative rates in terms of an overall reaction-progress variable. In this case, the modeled process is akin to a titration and has no explicit time frame.

The use of rate laws requires rate constants and usually some understanding of the dependence of rate constants on temperature (e.g., an Arrhenius-type activation energy). There may be additional dependencies on solution composition parameters (e.g., $\mathrm{pH}$ or a catalyzing or inhibiting aqueous species) as well as on parameters such as surface areas for reactions that occur across an interface (e.g., mineral dissolution or precipitation). There are two kinds of uncertainties involved:

- The first is represented by the uncertainties in the fundamental rate-law parameters, mainly rate constants and activation energies (parameter uncertainty).

- The second applies to the nature of the rate laws themselves. If they are not completely general and fundamentally correct, their proper application may be scenario-dependent (conceptual uncertainty).

In reaction-path modeling (see Section 5.4) using a code such as EQ3/6 (Wolery, 1992a), it is important to note that the reactions assumed to be controlled by equilibrium do not have zero rates. Rather, they adjust dynamically to maintain their equilibrium states in response to changes driven by the other reactions or to changes in temperature or pressure. For example, a reaction controlled by equilibrium and a reaction controlled by a rate expression may be linked by a common dependency on $\mathrm{H}^{+}$or some other aqueous species. If the equilibrium control on the former reaction were not applied, the progress of the latter reaction would throw the former reaction out of equilibrium. The magnitude of the rate of an equilibriumcontrolled reaction is unconstrained. It may achieve values that are unrealistic, in which case it should be treated instead by specifying a rate expression and by providing the requisite kinetic parameters.

\subsubsection{Reaction Rate Equations}

The mathematical representation of mineral dissolution/precipitation kinetics encoded in a reactive-transport simulator (e.g., GIMRT [Steefel and Yabusaki, 1995]) or in a reaction-path code (e.g., EQ3/6 [Wolery, 1992a]) effectively controls the compositional evolution of fluid and mineral phases in the model system. The accuracy of this representation depends on which processes are accounted for in the adopted rate law, its functional form, and the values selected for reference-state rate constants, activation energies, reactive-specific surface areas, and nucleation threshold saturations. Hence, one cannot assess the relevance of a given set of simulations without a clear understanding of the underpinning kinetic treatment.

In reaction-path calculations having an absolute (i.e., real) time frame, equilibrium-based codes such as EQ3/ 6 and React (Bethke, 1994) use the overall reaction progress as the primary variable. The time is then computed as a secondary variable. For a reaction-path 
calculation to use a time frame, there must usually be at least one reaction for which the rate is specified by a kinetic rate law (kinetic rate laws and relative rates may be mixed in EQ3/6). A kinetic rate law specifies the reaction rate in terms of time; it describes the rate in the form $d \xi_{\mathrm{i}} / d t$. An example is a simple form for mineral dissolution loosely based on transition state theory:

$$
\frac{d \xi_{j}}{d t}=s_{j} k_{+j}\left[1-\exp \left(\frac{A_{+j}}{\sigma_{+j} R T}\right)\right]
$$

where $\xi_{j}$ is the reaction progress variable, $s_{j}$ is the mineral surface area, $k_{+, j}$ is the dissolution (forward) rate constant, $A_{+, j}$ is the affinity to dissolve, $\sigma_{+j}$ is a stoichiometric parameter (usually having a value of unity), $R$ is the gas constant, and $T$ is the absolute temperature. The temperature dependence of the rate constant is accounted for by specifying an Arrhenius activation energy $\left(E_{\mathrm{a}}\right)$.

Although functional relations between measured and actual reactive specific surface areas of a given mineral of specified size fraction are of critical importance, these are not presently quantified (Mogollon et al., 1996; White and Peterson, 1990). One typically makes the assumption that the specific surface areas available from gas adsorption BET measurements (Brunauer-Emmett-Teller method) are in fact equivalent to the reactive specific surface areas.

In reactive-transport calculations using a code such as OS3D/GIMRT, the rate at which a mineral $\mathrm{m}$ dissolves or precipitates with respect to a given aqueous composition, $r_{\mathrm{m}}\left(\mathrm{mol} \mathrm{m}^{-3} \mathrm{~s}^{-1}\right)$, can be expressed in general form as (Steefel and Lasaga, 1994; Steefel and Yabusaki, 1996)

$$
r_{\mathrm{m}}=A_{\mathrm{m}, \mathrm{tot}} k_{\mathrm{m}} f\left(a_{i}\right) f\left(\Delta G_{r}\right)
$$

where $A_{\mathrm{m}, \text { tot }}$ is the total surface area $\mathrm{m}_{\mathrm{m}}{ }^{2} \mathrm{~m}_{\text {rock }}{ }^{-3}$, and $k_{\mathrm{m}}$ stands for the temperature-dependent dissolution/precipitation rate constant $\left(\mathrm{mol} \mathrm{m}^{-2} \mathrm{~s}^{-1}\right)$ dependence of $r_{\mathrm{m}}$ on the concentrations of specific aqueous solutes $i=1$. $\mathrm{N}$, expressed as activities, $a_{i}$, is given by

$$
f\left(a_{i}\right)=\prod_{i=1}^{N} a_{i}^{p}
$$

where the exponents $p$ are determined empirically, and the dependence on Gibbs free energy of the mineral's specified hydrolysis reaction $\Delta G_{r}$ is given by

$$
f\left(\Delta G_{\mathrm{r}}\right)=\operatorname{sgn}\left(\Delta G_{\mathrm{r}}\right)\left|\left(\frac{Q}{K}\right)^{M}-1\right|^{n}
$$

where $K$ and $Q$ refer to the standard-state equilibrium constant of the hydrolysis reaction and the analogous ion activity product of the solution, respectively, $\operatorname{sgn}\left(\Delta G_{\mathrm{r}}\right)$ denotes the sign of $\Delta G_{\mathrm{r}}$ (positive for supersaturation; negative for undersaturation), and the exponents $n$ and $M$ are positive numbers determined empirically by experiment (given the dearth of experimental data, both are most commonly taken to be unity).

The temperature dependence of the rate constant is given by an Arrhenius-type expression (Steefel and Lasaga, 1994): 


$$
k_{\mathrm{m}}=k_{\mathrm{m}, P_{\mathrm{r}}, T_{\mathrm{r}}} \exp \left[\frac{-E_{\mathrm{a}}}{R}\left(\frac{1}{T}-\frac{1}{T_{r}}\right)\right]
$$

where $k_{\mathrm{m}, P_{\mathrm{r}}, T_{\mathrm{r}}}$ stands for the reference-state $T_{r}=298.15^{\circ} \mathrm{C}, P_{r}=1$ bar rate constant, $E_{\mathrm{a}}$ represents the apparent activation energy $\left(\mathrm{cal} \mathrm{mol}^{-1)}\right), R$ denotes the gas constant (1.9872 $\mathrm{cal} \mathrm{mol}^{-1} \mathrm{~K}^{-1}$ ) and $T$ stands for the temperature $(\mathrm{K})$. In practice, $k_{\mathrm{m}}$ can be represented either by $k_{\mathrm{m}, P_{\mathrm{r}}, T_{\mathrm{r}}}$ and $E_{\mathrm{a}}$ or by substituting a known $k_{\mathrm{m}}(T)$ for $k_{\mathrm{m}, P_{\mathrm{r}}, T_{\mathrm{r}}}$ and setting $E_{\mathrm{a}}$ equal to zero.

As can be seen from the form of the reaction-rate laws described previously, the required kinetic parameters for near-field calculations are standard state $\left(25^{\circ} \mathrm{C}, 1\right.$-bar $)$ rate constants and an activation energy to extrapolate to rates at temperatures other than $25^{\circ} \mathrm{C}$.

\subsubsection{Laboratory Experiments to Quantify Kinetic Constants}

Extension of reactive-transport modeling to predict the coupled thermal, hydrologic, and chemical evolution of geologic systems is predicated on successful application of the approach to simulate well-constrained physical experiments. In an important laboratory study, steady-state effluent concentrations and dissolution-precipitation features associated with crushed quartz, amorphous silica, and tuff dissolution were determined experimentally using a plug-flow reactor and subsequently were modeled with the reactive-transport simulator.

Three plug-flow-reactor experiments were conducted and modeled using the reactivetransport software package OS3D/GIMRT (Steefel and Yabusaki, 1995, 1996). In each experiment, deionized water was uniformly forced, for a fixed period of time, through a confined plug of pulverized solid material under isothermal-isobaric conditions. The three experiments were distinguished primarily by the plug composition; quartz, amorphous silica, and Topopah Spring welded tuff were used. The fundamental data obtained in each experiment were the steady-state effluent concentrations and the time required for development of steady-state conditions, both of which provide important constraints on subsequent reactive-transport simulations. OS3D/GIMRT was used iteratively to model mineral-specific surface areas, dissolution-rate constants, nucleation thresholds, and the extent of secondary precipitation, based mainly on comparison of modeled effluent concentrations with observed data. Additional data regarding dissolution-precipitation characteristics were obtained from postmortem analysis of the plugs. More complete description of the experiments, observed data, and the reactive transport simulations, is provided by Johnson et al. (Johnson et al., 1997, 1998).

OS3D/GIMRT simulation of the effluent concentrations observed during tuff dissolution provided estimates of porosity changes, mineral-volume fractions, nucleation thresholds, and relative reaction rates for certain secondary precipitates. This information is typical of what can be obtained by iterative reactive-transport modeling of multicomponent, multiphase systems such as Topopah Spring tuff. In the plug-flow reactor containing pulverized tuff, the observed effluent composition was accurately simulated; however, minor discrepancies remained between the observed and predicted absolute concentrations, as well as between observed and predicted durations until attainment of steady-state effluent conditions. These discrepancies were largely attributable to subtle variations in the relative magnitude of nucleation thresholds and dissolution-precipitation rates for certain primary and secondary minerals and to limitations in the adopted kinetic rate law. 
It is important to acknowledge the approximations, simplifications, and uncertainties that are inherent to reactive-transport models at their present state of development. In this study, the constant flow field was not explicitly coupled to reaction-dependent changes in porosity and permeability, reactive and total mineral specific surface areas were approximated as equal and constant, the implicit nucleation-crystal growth algorithm was much simplified, and a linearized version of the OS3D/GIMRT kinetic rate law was adopted. For modeling of tuff, feldspar solid solution compositions were approximated as the corresponding endmember components. In general, the uncertain nature of many thermodynamic, especially kinetic data, is well documented. Despite these qualifications, close agreement was obtained between OS3D/GIMRT-projected and independently measured specific surface areas and dissolution rate constants and also between projected and measured effluent concentrations from tuff dissolution. The reported agreement between experimental and simulation results provides a measure of confidence for extending the modeling approach to more complex systems

\subsubsection{Adoption of Kinetic Data}

The reaction-rate equations coded into both reaction-progress and reactive-transport equations involve kinetic and thermodynamic terms. In most cases, the thermodynamic quantities are known with a much greater degree of confidence than are the kinetic quantities. This means that the thermodynamic terms dealing with activities of catalyzing or inhibiting species and the chemical affinity of the dissolution or precipitation reaction are probably calculated with a fair degree of accuracy. Given the stated ambiguity concerning reactive vs. total (gas adsorption) surface area, the specific surface-area term is less well known. The kinetic rate constants and activation energies, unfortunately, are much less well known than either of the preceding.

The critical importance of the chosen rate constants and activation energies to the validity of any reaction progress or reactive-transport calculation cannot be overemphasized. For example, given a $25^{\circ} \mathrm{C}$ rate constant and activation energies that differ by a factor of 2 (e.g., 16 vs. $8 \mathrm{kcal} / \mathrm{mol}$ ), the reaction rates calculated at $240^{\circ} \mathrm{C}$ would differ by more than a factor of 300. Experimental data that would constrain rate constants and activation energies are sparse, and many of the values used for chemical calculations are estimates for which there is considerable uncertainty.

Despite the uncertainty in critical parameters, best guesses can be made from uncritical review of the existing kinetic data. These guesses are highly uncertain for some mineral phases. When one considers the potential complicating effects of as-yet-unknown catalyzing or inhibitory impacts for certain minerals (e.g., the effect of $\mathrm{Al}$ on albite dissolution (Oelkers and Schott, 1995) or the impact of Na on quartz dissolution (Dove, 1994; Dove and Crerar, 1990), the uncertainty is increased.

Ignoring these complications, it can be said that the rate constants and activation energies for the silica polymorphs are probably known with a reasonable degree of certainty, and the equivalent data for some of the feldspars are less well known but probably adequate for firstorder estimates. On the other hand, rate constants and activation energies for many of the potential secondary phases, such as the zeolites, are poorly known. As a minimum, what is required for model calculations are the $25^{\circ} \mathrm{C}$ rate constants and activation energies, and an attempt is made here to provide some estimated values. 
In the Table 5-5 are the best guesses for the $25^{\circ} \mathrm{C}$ rate constants and activation energies for the important primary minerals in the Topopah Spring member of the Paintbrush tuff (Tpt). Several simplifying assumptions are involved in this compilation. First, it is assumed that the immediate need is for reactive-transport calculations; i.e., the code OS3D/GIMRT will be used. The type of kinetic data provided will be limited by the capabilities of that particular code. For example, OS3D/GIMRT does not handle solid solution explicitly, and this necessitates assuming that the reacting feldspar can be approximated as a mixture of the appropriate proportions of the pure end members of the series. Second, given the current state of kinetic data development, it seems inappropriate to attempt to provide the ability to account for catalyzing or inhibiting aqueous species, meaning that the exponent $p$ in Eq. 5-48 will be equal to 0 for all such species and that the exponents $M$ and $n$ in Eq. 5-34 are equal to 1 in the rate equations for all primary and secondary minerals.

Table 5-5 Provisional dissolution rate constants and activation energies for important minerals in the Topopah Spring member of the Paintbrush Tuff

\begin{tabular}{|c|c|c|c|}
\hline Mineral & $\begin{array}{l}\text { Rate Constant } \\
\left(\log \mathrm{k}^{+} \mathrm{mol} / \mathrm{m}^{2} \cdot \mathrm{s}\right)\end{array}$ & $\begin{array}{c}\text { Activation Energy } \\
\text { (kcal/mol) }\end{array}$ & Source \\
\hline Quartz & -13.9 & 20.9 & Tester et al., 1994 \\
\hline$\alpha$-cristobalite & -12.5 & 16.5 & Renders et al., 1995 \\
\hline Amorph. silica & -12.1 & 15.0 & Rimstidt and Barnes, 1980 \\
\hline Albite & -12.0 & 16.2 & Blum and Stillings, 1995 \\
\hline K-feldspar & $-12.0^{*}$ & 13.8 & Blum and Stillings, 1995 \\
\hline Anorthite & -8.8 & 8.4 & Blum and Stillings, 1995 \\
\hline Muscovite & -14.0 & 14.0 & Knauss and Wolery, 1989 \\
\hline Paragonite & $-14.0^{* *}$ & $14.0^{* *}$ & Assume = muscovite \\
\hline Kaolinite & -13.0 & 15.0 & Nagy, 1995 \\
\hline Pyrophyllite & $-13.0^{* *}$ & $15.0^{* *}$ & Assume = kaolinite \\
\hline Gibbsite & -12.5 & 15.0 & Nagy, 1995 \\
\hline Diaspore & $-12.5^{\star *}$ & $15.0^{* *}$ & Assume = gibbsite \\
\hline Boehmite & $-12.5^{\star *}$ & $15.0^{\star *}$ & Assume = gibbsite \\
\hline Heulandite & -11.7 & $15.0^{* *}$ & Ragnarsdottir, 1993 \\
\hline Clinoptilolite & -13.2 & $15.0^{* *}$ & Murphy et al., 1996 \\
\hline Analcime & -11 & $15.0^{* *}$ & Murphy et al., 1996 \\
\hline
\end{tabular}

In the case of minerals for which literature kinetic rate constants are available as a function of $\mathrm{pH}$, only the $\mathrm{pH}$ independent (i.e., $p=0$ ) constants are used. For most of the primary minerals in the Tpt unit, the standard-state rate constants are probably accurate to within a factor of 2 to 5, while the activation energies are probably known to within $25 \%$. For a few of the potential secondary minerals (e.g., kaolinite), the rate constants and activation energies are probably as well known as they are for the feldspars. However, for many of the 
secondary minerals (e.g., zeolites), the kinetic-rate constants and activation energies are either poorly known or unknown. In these latter cases, the kinetic parameters are simply assumed to be identical to some other, similar, mineral.

\subsubsection{Conceptual Model for Reactive Transport in the Altered Zone}

\section{by William E. Glassley}

This section qualitatively describes many processes that are expected to occur within the $\mathrm{AZ}$ and the nature of the linkages or couplings between them. Many experimental studies have coupled water-rock interaction and transport through geologic media and have demonstrated the interdependence of chemical reaction and fluid flow (Hardin and Chesnut, 1997). Examples of coupled processes observed in the laboratory include the following:

- Complete healing of natural and artificial fractures (Lin and Daily, 1989)

- Large decreases in permeability $(>90 \%)$ as a function of small reductions (a few percent) in the porosity of nonfractured rocks having initially low permeability and porosity (Brace, 1977; Camp, 1964; Keith et al., 1983; Lin and Daily, 1989; McClure et al., 1979; Moore et al., 1986; Morrow et al., 1981; Pearson, 1976; Pyrak-Nolte et al., 1987; Scheidegger, 1974; Vaughan, 1985; Verma and Pruess, 1988).

- Substantial sealing of flow pathways in crushed material, with strong dependence on reactive surface area (Rimstidt et al., 1989)

In simulations of natural systems, complete sealing of fractures has been projected to occur in rock overlying heat sources (e.g., Krupp and Seward, 1987); this is consistent with the observation of caprock sealing in geothermal systems. All of these results indicate that THC coupled processes are important and that TH simulations should account for the effects on rock-mass properties.

Numerous simulations of the TH evolution of the repository block have been conducted using different codes and assumptions (see Chapter 3). Common to all of these studies is the conclusion that changes in temperature and saturation will occur through time. Discussion of coupled processes is simplified by identifying four hydrologic regimes that will occur in response to the heating associated with the reference repository design (after Buscheck and Nitao, 1992, 1993a).

\section{Regime A: $\mathrm{T}<\mathrm{T}_{\text {boiling }}$}

This regime encompasses areas in which the temperature is between ambient and boiling. During heating, pore water vaporizes and moves down gas-phase pressure gradients. Water vapor moving in fractures to lower-temperature regions will condense on fracture surfaces. The condensate will be imbibed into the matrix or will flow along fracture surfaces, depending on local conditions. The liquid and vapor flow rates will depend on the rate of heating. Within a few tens of meters of the emplacement drifts, Regime A will be brief (less than $10 \mathrm{yr}$ ); but hundreds of meters away, Regime A will persist for hundreds to thousands of years.

Regime B: $T=T_{\text {boiling }}$

Within approximately $10 \mathrm{~m}$ of the emplacement drifts, the temperature will eventually reach boiling; a quasi-steady heat pipe region will then form as the outward heat flux is matched by the latent heat flux caused by liquid-vapor counterflow. Water will cycle from the boiling zone to the condensation zone (Regime A) as vapor and back again as liquid. Region B is the intervening zone of counterflow where the temperature is relatively constant. 
This thickness of this regime will grow as the thermal envelope around the repository expands. The thickness to which it develops will depend on the thermal output of the repository, the ambient percolation flux, the thermal conductivity, distance to the ground surface, and conditions that control drainage of condensate through the repository horizon (e.g. drift spacing). To the extent that a quasi-steady heat pipe forms, Regime B will be chemically active because temperature and water saturation will be elevated for a long time and will contribute to reaction progress.

Within Regime B, condensate will dissolve fracture lining minerals; the rate at which this occurs will be a function of temperature, reactive surface area, flow velocity, mineralogy, and evolving water chemistry. The balance between rate of water flow and rate of dissolution will determine whether the water achieves equilibrium with the fracture minerals (Glassley, 1993). The diversity of fracture-lining minerals (Carlos, 1987; Carlos et al., 1990, 1993) suggests that partial equilibrium (i.e., some of the reactions in a system achieve equilibrium while others do not) may persist for some time. The water may eventually reach the boiling front, where the dissolved solids will be deposited on the fracture walls. The minerals precipitated in the boiling zone generally will not be the same as those dissolved.

Alternatively, the condensate may be partially evaporated and drain through the repository horizon into cooler rock below. Depending on local conditions, tuff dissolution may continue along drainage pathways. As the solution cools, and where the water-residence time increases, precipitation will produce assemblages that contain minerals that are generally slower to form than precipitates in the boiling zone.

The total volume of the solids precipitated, for any aliquot of water, will be small. However, significant amounts of secondary phases are likely to accumulate at the boiling front, and significant amounts of material will be dissolved in Regime B. Also, the minerals that precipitate under aqueous conditions along condensate drainage pathways will have molar volumes that are greater than those for the original minerals dissolved.

\section{Regime $\mathrm{C}: \mathrm{T}>\mathrm{T}_{\text {boiling }}$}

Regime $\mathrm{C}$ is bounded by the boiling isotherm and the emplacement drifts. In general, the rock will be dry, and the humidity will be less than $90 \%$. Local persistence of an aqueous phase to temperatures as high as $150^{\circ}$ to $210^{\circ} \mathrm{C}$ may result from salinity of residual water in restricted pores or from throttling of the gas phase pressure within matrix blocks. In general, however, this regime will be dominated by rock dryout, and its chemical reactivity will be a function of relative humidity.

\section{Regime $D: T<T_{\text {local max }}$}

This regime develops after the local thermal maximum has been achieved. It encompasses rock that has been physically modified during the heat-up period, with effects evident as changes in bulk and fracture porosity and permeability and as changes in reactivity of exposed mineral surfaces. Eventually the heat-pipe zone and dryout zone will collapse because of decreasing thermal output, possibly combined with increased ambient percolation due to climate change. Thus, the conditions that prevail in Regimes A and B may eventually rework the precipitates deposited at the base of the heat-pipe zone.

The effect of evolving TH regimes at a particular location in the AZ will be to progressively modify the mechanical and hydrologic properties of fractures (Table 5-6). In regions of net dissolution, fracture apertures will increase, causing increased permeability and changing the rock mass deformability. In regions where precipitation occurs, complementary effects will occur. As temperature decreases in Regime $\mathrm{D}$, reaction rates also decrease; thus, the cumulative effects of previous TH regimes may persist. 
Table 5-6 Distribution of selected mineralogical, chemical, and mechanical processes that are likely to develop within Regimes A through D and to affect TH conditions

\begin{tabular}{|l|c|c|c|c|}
\hline \multicolumn{1}{|c|}{ Process } & A & B & C & D \\
\hline \hline Dissolution & Major & Major & Minor & Major \\
\hline Precipitation & Major & Major & Minor & Major \\
\hline lon exchange and sorption & Nil & Nil & Nil & Nil \\
\hline Dehydration of hydrous phases & Minor & Major & Major & Nil \\
\hline Rehydration of hydrous phases & Nil & Minor & Minor & Major \\
\hline Rock-mass thermal expansion & Minor & Major & Major & Minor \\
\hline Matrix deformation & Nil & Major & Major & Minor \\
\hline Fracture deformation & Nil & Major & Major & Minor \\
\hline
\end{tabular}

Major = process could have a significant role in determining hydrothermal properties

Minor $=$ process could have a second-order role in determining hydrothermal properties

$\mathrm{Nil}$ = process will probably be of trivial significance in determining hydrothermal properties

For the tuff matrix, water-rock interaction will occur as the temperature and saturation change. The result will be mineral dissolution and precipitation, leading to modification of the permeability and capillary properties. Molar volumes of the secondary phases that form may be significantly different from the original minerals. The impact of temperature and pore-water salinity on $\mathrm{H}_{2} \mathrm{O}$ properties will also affect the apparent hydrologic properties, an effect that will modify hydraulic conductivity and moisture-retention characteristics.

The overall result of these interacting processes will be to affect the $\mathrm{TH}$, mineralogical, chemical, and mechanical evolution of the repository block. Coupled-process models for the $\mathrm{AZ}$ provide boundary conditions for near-field processes that directly affect engineered barriers. This includes models for the range of possible water compositions entering the NFE.

\title{
5.4 Reaction-Path Model for Water Chemistry and Mineral Evolution in the Altered Zone
}

\author{
by Michael Whitbeck and William E. Glassley
}

\subsubsection{Introduction}

The proposed repository site is in the unsaturated zone (UZ), where ambient matrix saturation is $70 \%$ to $95 \%$ (Flint, 1998). Heat-driven evaporation, boiling, and condensation processes will cause water vapor to move away from the repository, resulting in the increased saturation of some rocks in some parts of the repository block (Buscheck and Nitao, 1992, 1993b; Nitao, 1988). In some operation scenarios, the saturation may approach $100 \%$ after waste is emplaced. Thus, in some parts of the NFE and in the rock units bounding the repository horizon, the potential for rock-water interaction may increase initially owing to the greater abundance of liquid water and the presence of elevated temperature. Eventually, as sufficiently high temperatures develop, all liquid water will be driven out, and rock-water interaction will effectively cease. During the subsequent cool-down, liquid water will probably return, especially by fracture flow.

Most of the minerals currently present in the rock that makes up the potential repository horizon are not in thermodynamic equilibrium with water at elevated temperatures, nor is the glassy material preserved within the Paintbrush tuff nonwelded unit (PTn) or the 
Topopah Spring welded 3 unit (TSw3). As a result, water will interact with existing minerals and glass and cause new minerals to form and some existing minerals to change composition or dissolve, thus modifying the water chemistry. To place bounds on these effects, a study was undertaken to model these rock-water-interaction processes. This effort focused on identifying the likely minerals that would form, determining the effect on water chemistry, and estimating the rates at which the changes would occur. A preliminary effort was also made to estimate the uncertainty in the calculations, based on the known uncertainty in the rate constants for dissolution and precipitation reactions.

\subsubsection{Approach}

The EQ3 / 6 code, Version 7.2a, (Daveler and Wolery, 1992; Wolery, 1992a, 1992b; Wolery and Daveler, 1992; Wolery et al., 1988) was used for the tuff/groundwater simulations reported here. The database used was the composite or COM data file, Revision 22a of the Version 7 series. All calculations were done using the B-dot activity coefficient model (see (Wolery, 1992b) for a description of this model). This model is adequate for treating relatively dilute aqueous solutions.

The rock units considered in the simulations were those of the Topopah Spring welded 2 (TSw2; devitrified, the repository horizon) and Topopah Spring welded 3 (TSw3; the underlying vitrophyre) units of the Topopah Spring tuff. The mineral abundances for the TSw2 unit, the dissolution rates of the mineral phases, and the mineral phases' respective surface areas were taken from Delany (1985), who reported the first kinetic reaction-path simulations of tuff-groundwater interactions. Her simulations were designed to model experiments at $150^{\circ}$ and $250^{\circ} \mathrm{C}$. The work for TSw2 presented here is aimed at lower temperatures $\left(40^{\circ}-90^{\circ} \mathrm{C}\right)$ and generally much longer times. The TSw3 tuff is nearly all glass. Its composition was taken to be that of Sample 2A from Table II of Broxton et al. (1989). Any crystalline components of TSw3 were ignored.

The initial water composition reacting with the rock materials was assumed to be that of J-13 water. The composition used was the average composition reported by Harrar et al. (1990). There are some problems with this composition that are not addressed in the work reported here (see 5.2.1.2). For example, the reported average $\mathrm{pH}$ is 7.41, whereas the actual $\mathrm{pH}$ is probably around 8.2 (Apps, 1997). The reported alkalinity is probably not an accurate measure of dissolved bicarbonate. The reported concentrations for some chemical components, such as $\mathrm{Al}$ and $\mathrm{Fe}$, are probably unreliable because the values are close to or below detection limits and because filtration of the water may have been insufficient to remove small particles of solids. However, the consequences of these problems in the present work are minor. The principal effect is an initial, instantaneous precipitation of small quantities of some phases, such as clay minerals or iron oxide (or oxyhydroxide), with which the actual initial fluids would probably begin in equilibrium. This effect, however, would be quickly overwhelmed by subsequent reaction with large amounts of rock.

The purpose of these simulations was to consider the effect of reaction progress on water chemistry and mineral assemblages. It was necessary to develop a conceptual model in which sufficient water was available to allow reactions to proceed until a very long-term steady state (not necessarily a final equilibrium state) was achieved. However, the extent to which most of the rock will actually be altered in the NFE and AZ is likely to be limited by the availability of water. Thus, from the point of view of the rock (most of it, anyway), the natural systems will only progress part way along the reaction paths suggested by the modeling described here. Small amounts of water, however, might be much more extensively altered by reaction with the rock. The actual extent of water reaction in the real system should probably be thought of as a statistical quantity described by some kind of bell curve. 
It was assumed in these calculations that reaction would take place in a system open to the atmosphere. Hence, the coexisting gas phase had constant $\mathrm{O}_{2}$ and $\mathrm{CO}_{2}$ partial pressures equivalent to those of present-day atmosphere ( 0.2 and 0.0003 bar, respectively). A somewhat higher $\mathrm{CO}_{2}$ partial pressure of 0.001 bar would be more appropriate (see Apps, 1997). This information was obtained after these calculations were done; the use of the higher value would somewhat alter the results.

Simulations were conducted for both rock types at $40^{\circ}, 50^{\circ}, 60^{\circ}, 75^{\circ}$, and $90^{\circ} \mathrm{C}$. These temperatures were selected because they cover the range between ambient conditions and boiling. Preliminary results of the simulations are presented in Section 10.4.4 of Volume II of the Near-Field and Altered-Zone Environment Report (Wilder, 1996). Reaction of rock and water at higher temperatures will be conducted at a later time when the EQ3 / 6 code has been modified to simulate reactions off the liquid-vapor saturation curve of water. Detailed simulations involving flow pathways with restricted flux also will be conducted at a later time.

Quartz, talc, and tridymite were suppressed from precipitation in all of the runs because these phases, although less soluble than other silica polymorphs or hydrous $\mathrm{Mg}$ silicates, do not readily precipitate because of kinetic barriers. It may be, however, that, after sufficient time has passed in natural systems, these phases would appear as the kinetic barriers are overcome. In that case, inclusion of these phases would be appropriate at some point in the reaction progress. When sufficient information is available to reasonably simulate this process, the type of simulations reported here will be redone.

To evaluate the effect of uncertainty in the dissolution rate, two methods were used. One method evaluated the propagation of error in a generalized reaction sequence:

$$
\mathrm{A} \Leftrightarrow \mathrm{B} \Leftrightarrow \mathrm{C}
$$

This simple system had an exact analytical solution yet was general enough to represent many of the subsets of more complex geochemical systems that are not strongly coupled with other parts of the system. The results of this evaluation will not be treated in detail here, but are described in Whitbeck and Glassley (1996).

The second method used was simulation, using different rate constants, of the reactions of water with the volcanic rocks. This method allowed representation of the specific effects on the simulations described in this study. To illustrate these effects, dissolution of sanidine (a potassic feldspar: $\mathrm{KAlSi}_{3} \mathrm{O}_{8}$; in natural sanidine some $\mathrm{K}$ is replaced by $\mathrm{Na}$ ) was considered. Sanidine has a $25^{\circ} \mathrm{C}$ dissolution rate constant of $\sim 5 \times 10^{-15} \mathrm{~mol} / \mathrm{cm}^{2}$-s (Holdren and Speyer, 1985). The activation energy is likely to be on the order of $9.5 \mathrm{kcal} / \mathrm{mol}$ (based on the $150^{\circ}$ and $250^{\circ} \mathrm{C}$ values used by Delany (1985). In the temperature range of interest, the rate increases by nearly an order of magnitude per $100^{\circ} \mathrm{C}$ increase in temperature. The uncertainty in the $25^{\circ} \mathrm{C}$ rate constant has not been formally assessed, but it is at least an order of magnitude and likely about two orders of magnitude. This uncertainty is thus greater than the magnitude of the temperature corrections from $25^{\circ} \mathrm{C}$ to the run temperatures of $40^{\circ}-90^{\circ} \mathrm{C}$. The reaction rate for a given mineral is proportional to the mineral's surface area. Thus, the overall reaction rate is a function of the total surface area and can vary over many orders of magnitude depending on, for example, whether water flows only along a preferred flow pathway in a fracture (small available surface area) or permeates a rock (large available surface area). The effect of different rates of glass dissolution was examined by using a slow relative rate $(8.66 \times$ $\left.10^{-14} \mathrm{~mol} / \mathrm{unit} \xi\right)$ and a very fast relative rate $\left(4.0 \times 10^{-4} \mathrm{~mol} / \mathrm{unit} \xi\right)$ in different suites of runs. The rate model used here has no explicit dependence on surface area. For the same amount of reacted glass, the same results are obtained in all runs. Here, $\xi$ is essentially a proxy for (and is proportional to) the product of the surface area and the time. To evaluate these effects, the 
runs with a slow reaction-rate constant had a total mass of $0.1735 \mathrm{~g}(0.08029 \mathrm{~mol})$ of glass per $\mathrm{kg}$ of water, and the runs with the fast reaction-rate constants had $17.29 \mathrm{~g}(8.0 \mathrm{~mol})$ of glass. The glass molecular weight was arbitrarily chosen to be $2.21454 \mathrm{~g} / \mathrm{mol}$ ).

\subsubsection{Results}

For the TSw2 tuff (composed of minerals but no glass), the water composition and secondary mineralogy obtained at steady-state conditions are primarily constrained by the rate-law expressions for the dissolution of the starting minerals and by the solubility of the secondary minerals. They are also constrained in part by the solubility of the starting minerals because the dissolution rates of these phases slow down as the corresponding equilibrium solubility is approached (e.g., in the case of cristobalite). Because the rate of dissolution varies from phase to phase, the dissolution of this tuff is highly incongruent. However, for the Tsw3 tuff, here taken to be entirely composed of glass, dissolution is treated as congruent. Hence, the amount of material dissolved (which is proportional to the extent of reaction progress; the proportionality constant is the specified relative rate of dissolution) will determine the secondary mineralogy and water chemistry. In this case, a near steadystate composition will not be achieved, except under extreme conditions in which a very large volume of glass is dissolved in a relatively small amount of water. Although this might occur for a small proportion of the overall water in the NFE and AZ, it could not occur for a large proportion because the dissolution of an unrealistically large volume of glass would be required. The mineralogical and chemical results obtained for the two rock types are described in the following text.

As noted in, for example, Section 3.4.2 of the Near-Field and Altered-Zone Environment Report, Vol. II (Wilder, 1996), the accuracy of predictions of mineral occurrences at any given time in a numerical simulation is limited by a variety of constraints. Inaccuracies in, or a complete absence of, thermodynamic data or kinetic data for specific minerals are examples of such constraints. Hence, the mineral suites described subsequently must be considered to be representative of the mineral assemblages that will actually form rather than exact predictions of those assemblages. For example, the occurrence of a zeolites such as clinoptilolite should only be taken to indicate that some similar zeolite is expected to form. The specific composition of that phase may differ because of factors such as current limitations on the available thermodynamic data and on the ability to model complex solid solutions. Similarly, the occurrence of muscovite (K-mica) should be taken to indicate only that some form of sheet silicate (likely a clay mineral) would actually form.

\subsubsection{Pre-Equilibration of the Water}

The initial water composition reflected a pre-equilibration of J-13 water at the temperature of the simulation. In part, this accounts for the effect of temperature on the water chemistry. However, the results also reflect, in part, some of the minor problems with the reported water chemistry noted previously. At each temperature, small amounts of saponite (an Mg-rich clay), "carbonate" (calcite, $\mathrm{CaCO}_{3}$, with about $10 \mathrm{~mol} \%$ substitution of $\mathrm{MgCO}_{3}$ ), and fluorapatite $\left[\mathrm{Ca}_{5}\left(\mathrm{PO}_{4}\right)_{3} \mathrm{~F}\right]$ formed in the initial equilibration step. More significant amounts of cristobalite (a primary mineral in the TSw2 tuff) also formed, reducing the dissolved silica. The pre-equilibration conducted here did not consider the possible presence of minerals that might dissolve in the pre-equilibration step as a consequence of the increase in temperature from $25^{\circ} \mathrm{C}$. At all temperatures, the initial equilibrated composition was moderately alkaline $\left(\mathrm{pH} \sim 8.5\right.$, up from the pre-equilibrated $25^{\circ} \mathrm{C}$ value of 7.41 and closer to 
the probable ambient value of 8.2 proposed by Apps [1997]), was oxidizing (calculated Eh between 580 and $680 \mathrm{mV})$, and had a low ionic strength $(\approx 0.0028$ molal). Concentrations of the dissolved elements were low. In decreasing order of abundance, they were:

- $\mathrm{Na}(\approx 40 \mathrm{mg} / \mathrm{L})$

- $\mathrm{Si}(\approx 30 \mathrm{mg} / \mathrm{L})$

- $\mathrm{Cl}(\approx 10 \mathrm{mg} / \mathrm{L})$

- $S(\approx 6.0 \mathrm{mg} / \mathrm{L})$

- $\mathrm{Ca}(\approx 5 \mathrm{mg} / \mathrm{L})$

- $\mathrm{F}(\approx 2.0 \mathrm{mg} / \mathrm{L})$

- $\operatorname{Mg}(\approx 0.1 \mathrm{mg} / \mathrm{L})$

- $\mathrm{Li}(\approx 0.05 \mathrm{mg} / \mathrm{L})$

- $\mathrm{Al}\left(<1.0 \times 10^{-4} \mathrm{mg} / \mathrm{L}\right)$

- $\quad \mathrm{P}\left(2.0 \times 10^{-5} \mathrm{mg} / \mathrm{L}\right)$

Note that the $\mathrm{Ca}$ and $\mathrm{Mg}$ are largely removed from solution. The Ca drops from 13 to $5 \mathrm{mg} / \mathrm{L}$, the $\mathrm{Mg}$ from 2.01 to $0.1 \mathrm{mg} / \mathrm{L}$.

As suggested in previous text, there are several ways in which the pre-equilibrated water composition model might be improved in future work. However, the compositions obtained here are still considered likely to give a reasonably good representation of the starting fluids. At even moderately low extent of reaction with rock, the errors and uncertainties in the starting compositions tend to be overwhelmed by the effect of rock-water interaction.

Although J-13 water is commonly taken as the likely starting water composition for fluids evolving in the NFE and AZ, it may be less than perfect for this purpose. At least some ambient groundwater in the repository may not exactly match it (see Apps, 1997) and references cited therein). However, a larger factor is that evaporative, boiling, and condensing processes will also come into play. Thus, in some parts of the system, the "starting" fluid may be condensate, not ambient groundwater or even ambient groundwater altered by rock-water interaction. Temperature will change along flow paths and add further complexity. Salts left behind by evaporation or boiling will be redissolved. In the NFE, human-made materials, such as concrete and metals, will lead to further alteration of water chemistry. Therefore, the models described here represent only a part of the whole-system processes, but they are representative of the kind of thermodynamic modeling that can be applied to additional parts of the system. Thus, one can extend the models presented in this section to ask what would happen if the waters produced by the processes considered here entered another part of the system and were exposed to a different set of conditions or materials.

The term "pre-equilibration" may be somewhat misleading because it is used here in a loose sense. The water produced by this process is not in a state of complete equilibrium. For example, if it were, the dissolved silica would be controlled by equilibrium with quartz, and its concentration would be substantially lower. Rather, the pre-equilibrated water is only adjusted to maintain equilibrium with a limited set of reactions (e.g., aqueous acid-base reactions, aqueous complexing reactions, and some reactions involving minerals).

\subsubsection{Secondary Mineralogy Produced by Reaction of the Devitrified TSw2 Tuff}

Reaction of the devitrified TSw2 tuff produced secondary mineral assemblages that varied with extent of reaction and temperature. Plots of the major members of the secondary mineralogy for both the TSw2 and TSw3 tuffs at all five temperatures are shown in Figure 5-7. The TSw2 results are shown with solid lines. The relatively small amounts of saponite, "carbonate" (calcite), and fluorapatite that formed in the pre-equilibration step persisted at low abundances to the maximum extent of reaction at all temperatures. 
Relatively early in each simulation, other clays (celadonite and smectite), authigenic feldspar (microcline), and zeolites joined those phases and persisted to long-term, near-steady-state conditions. Various zeolites were involved. Clinoptilolite formed at $40^{\circ} \mathrm{C}$, stilbite at $50^{\circ}$ and $75^{\circ} \mathrm{C}$, and mesolite throughout the $40^{\circ}-90^{\circ} \mathrm{C}$ range. The actual time of appearance of specific phases depended on the temperature. At $40^{\circ} \mathrm{C}$, important secondary aluminosilicate phases (i.e., those not present initially, but which later formed in significant abundance) did not appear until after $\sim 100$ days of reaction. At temperatures greater than $50^{\circ} \mathrm{C}$, important aluminosilicates appeared within a few days.

In all cases, near-steady-state conditions with relatively high abundances of secondary minerals were not approached until thousands of days had elapsed. In all cases, one of the last phases to appear was the silica polymorph chalcedony, but it quickly became the most abundant secondary phase. At $90^{\circ} \mathrm{C}$, garnet and tremolite appeared in the secondary mineral assemblage. Given evidence from metamorphic petrology in low-temperature systems, these phases probably would not be the stable phases that would form under these conditions (see Section 3.4.2 of the Near-Field and Altered-Zone Environment Report, Vol. II). Their presence in the simulations is likely due to limitations of the current thermodynamic data or to incompletely adequate solid-solution models, especially for clay minerals and zeolites.

Figure 5-7 Major secondary mineralogy as a function of time for the reaction of devitrified TSw2 tuff and glassy TSw3 tuff with J-13 well water

\subsubsection{Chemistry of the Water Reacted with the Devitrified TSw2 Tuff}

The composition of the water reacted with the TSw2 tuff remained relatively constant, at any temperature studied, until the reaction time reached $\sim 100$ days. At that point, the composition changed significantly owing to the formation of additional secondary mineral phases and to the dissolution of significant quantities of the rock components. Selected results are plotted in Figure 5-8 through Figure 5-11. Results for $\mathrm{Ca}$ and $\mathrm{Mg}$ were both largely precipitated from solution in the pre-equilibration step. The already low concentrations of these components merely became lower. Attention here will therefore focus on other components.

Figure 5-8 Changes in the $\mathrm{pH}$ of $\mathrm{J}-13$ well water during reaction with devitrified TSw2 tuff

Figure 5-9 Changes in the chemistry (Si) of J-13 well water during reaction with devitrified TSw2 tuff

Figure 5-10 Changes in the chemistry (Na) of J-13 well water during reaction with devitrified TSw2 tuff

Figure 5-11 Changes in the chemistry (Al) of J-13 well water during reaction with devitrified TSw2 tuff

Even though they were incorporated in the secondary minerals, the elements $\mathrm{Na}$, $\mathrm{Si}$, and $\mathrm{Al}$ increased significantly because the amounts added to solution by the dissolution of the primary solids were much greater than the amounts removed by precipitation in secondary phases. Nevertheless, the final concentration that these elements reached was controlled by 
the solubility of the major secondary phases. For example, at steady-state conditions, Si was buffered at chalcedony saturation. Other elements were controlled by a combination of secondary phases (many of these phases were complex, multicomponent, solid solutions).

The variation in $\mathrm{pH}$ was strongly controlled by the fact that $\mathrm{CO}_{2}$ fugacity was fixed in the simulations. This parameter, in turn, influenced carbonic acid equilibria in such a way that $\mathrm{pH}$ remained slightly alkaline throughout calculations. However, the final steady-state value differed by approximately one log unit between the $40^{\circ}$ and $90^{\circ} \mathrm{C}$ simulations. The redox state was controlled by fixing the $\mathrm{O}_{2}$ fugacity at 0.2 bar. The Eh changed, but only because the $\mathrm{pH}$ changed.

The conservative elements $\mathrm{Cl}, \mathrm{F}$, Li, and $\mathrm{P}$ showed no significant variation during reaction. They were not incorporated into any secondary phases other than apatite (formed in the pre-equilibration step), which maintained a constant abundance throughout the calculations.

\subsubsection{Secondary Mineralogy Produced by Reaction of the Vitric TSw3 Tuff}

Plots of the major members of the secondary mineralogy for the TSw2 and TSw3 tuffs at all five temperatures are shown in Figure 5-7. The TSw3 results are shown with broken lines.

For a relatively small extent of reaction of the glassy TSw3 tuff, the secondary phases that formed during reaction were pyrolusite $\left(\mathrm{MnO}_{2}\right)$, clays, and zeolites. This result is very similar to the assemblage formed in the case of the devitrified TSw 2 tuff. The principal differences were in the amounts of secondary phases and in the specific zeolites that formed. The results were qualitatively consistent with the description of natural glass alteration (Levy, 1984a, 1984b) in the vicinity of Yucca Mountain. At the end of the calculations, very similar end points were achieved for all temperatures considered. The main exception involved "carbonate" (calcite), which did not persist to the end point at $40^{\circ} \mathrm{C}$.

For large extent of reaction at any temperature, the only secondary phases present (apart from ones formed in the pre-equilibration step) were pyrolusite, chalcedony, and hematite. Finally, none of the simulations (at any temperature) with vitric material showed any formation of the reaction products muscovite or microcline. This situation contrasted with the TSw2 simulations in which these two phases were present throughout the calculations.

\subsubsection{Chemistry of the Water Reacted with the TSw3 (Vitric) Tuff}

Water composition in simulations for small extent of reaction with TSw3 (glassy) tuff reached end points that were nearly identical to those for the simulations involving TSw2 tuff. They also tended to follow very similar trends during reaction. Selected results are plotted in Figure 5-12 through Figure 5-17. For large extent of reaction, the simulations followed the same trends as early on, but ultimately achieved much higher total concentrations of all dissolved components except Si. Near the end of these simulations, large amounts of $\mathrm{Ca}$ and $\mathrm{Mg}$ were put into solution. The concentration of this component dropped to very low values buffered by equilibrium with chalcedony, which was precipitated in relatively large volumes. Also at high extent of reaction, the $\mathrm{pH}$ dropped to acidic values $(\approx 3.8)$ in the lower-temperature $\left(<90^{\circ} \mathrm{C}\right)$ simulations. The $\mathrm{pH}$ remained slightly alkaline $(\approx 7.8)$ in the $90^{\circ} \mathrm{C}$ simulation. In these simulations, total dissolved elemental carbon decreased by three orders of magnitude during reaction. The decrease profoundly influenced alkalinity and $\mathrm{pH}$ buffer capacity. The concentration of solutes increased from $\approx 0.02 \%$ (on a weight basis) at the beginning of a simulation to $\approx 0.6 \%$ at the end.

Figure 5-12 Changes in the $\mathrm{pH}$ of J-13 well water during reaction with glassy TSw3 tuff at $40^{\circ}, 50^{\circ}, 60^{\circ}, 75^{\circ}$, and $90^{\circ} \mathrm{C}$ 
Figure 5-13 Changes in the chemistry (Si) of J-13 well water during reaction with glassy TSw3 tuff

Figure 5-14 Changes in the chemistry (Na) of J-13 well water during reaction with glassy TSw3 tuff

Figure 5-15 Changes in the chemistry (Ca) of J-13 well water during reaction with glassy TSw3 tuff

Figure 5-16 Changes in the chemistry (Mg) of J-13 well water during reaction with glassy TSw3 tuff

Figure 5-17 Changes in the chemistry (Al) of J-13 well water during reaction with glassy TSw3 tuff

The development of low-pH solutions at the high extent of reaction below $90^{\circ} \mathrm{C}$ deserves some comment. Such a high extent of reaction is likely to be achieved, if at all, by only very small quantities of water. The factors leading to the development of this acidity were not fully analyzed, but are undoubtedly due to the oxidation of some minor or trace component in the glass coupled with the high extent of reaction. A possible reaction that might produce acidity is $\mathrm{S}^{2-}$ (in glass) $+\mathrm{O}_{2}+2 \mathrm{H}_{2} \mathrm{O}=4 \mathrm{H}^{+}+\mathrm{SO}_{4}{ }^{2-}$ (analogous to the reactions that form acid mine waters). However, this particular reaction would also have produced acidity at $90^{\circ} \mathrm{C}$. The glass component involved in the production of acidity is more likely to be a transition metal such as Cr or Mn.

The conservative elements $\mathrm{Cl}, \mathrm{F}, \mathrm{Li}$, and $\mathrm{P}$ were not included in the simulations of glassy tuff because no analyses were available for them.

\subsubsection{Propagation of Time Errors}

The effects of differences in rates on specific simulations were examined by varying the sanidine dissolution rate (actually the associated rate constant). To determine the propagated uncertainty in the amount of sanidine formed at a given time, the results shown in Figure 5-18 were used. Calculating the differences between these runs generated approximate error bars (shown in Figure 5-19). These results demonstrate that the propagated error increased with time until sanidine vanished. The "error," or uncertainty, can be characterized in one of two ways: The first addresses the uncertainty regarding the amount of the mineral present at any given time; the second focuses on the uncertainty in the time at which a specific mineral disappears from the system (is completely dissolved). The uncertainty in the predicted time when a phase appears or disappears can be substantially greater than the uncertainty in the predicted amount made of a phase. For example, the maximum uncertainty in the amount of sanidine produced is about $50 \%$. The corresponding uncertainty in the time of its consumption is about $-60 \%$ on the low side and about $2100 \%$ on the high side.

Figure 5-18 Changes in sanidine abundance resulting from a plus or minor one order of magnitude change in the dissolution rate of this mineral

Figure 5-19 Estimates of sanidine abundance, assuming the dissolution rates used to construct Figure 5-18 


\subsubsection{Discussion}

The simulations conducted were designed to be the preliminary simulations for bounding water compositions that could evolve in the AZ and migrate into the NFE. They were also designed to bound the mineralogy that could evolve in the AZ. Therefore, emphasis was placed on establishing a baseline on which refinements and extensions would be made at a future time. The following are the key constraints on which the simulations were based:

- Constant gas fugacities for $\mathrm{O}_{2}$ and $\mathrm{CO}_{2}$, equivalent to atmospheric values

- Suppression of precipitation of certain phases to account for long-term metastabilities

- Selection of rate constants and surface areas

- Both small and large extents of reaction considered, corresponding to a range of effective rock-water ratios

\subsubsection{Baseline Secondary Mineralogy}

Under all conditions, clays and zeolites occurred within the secondary mineral assemblages. The specific time in the simulations when these phases appeared and the compositions and structures that occurred varied, depending on the evolving chemical conditions and on the temperature. The respective volumes that developed at steady-state conditions were generally less than the volumes of other coexisting aluminosilicates. These phases, nevertheless, formed an important part of the secondary assemblages, owing to their potentially important role as materials in which radionuclides might be sorbed.

Silica polymorphs developed early in the simulations. This is an important observation because, under all conditions, silica polymorphs are the most abundant secondary phases to form. This suggests that silica may play an important role in coupling hydrology, geochemistry, and mineralogy via precipitation- or dissolution-induced changes in porosity and permeability (see Section 10.4.4 of the Near-Field and Altered-Zone Environment Report, Vol. II).

In all the simulations, carbonates played an important role in the secondary mineral assemblage, but they were not always present when steady state was reached. Because carbonates may be important in both influencing water composition and in sequestering ${ }^{14} \mathrm{C}$ through isotope exchange, the ubiquitous presence of these phases at various stages of reaction may be significant.

\subsubsection{Baseline Water Composition}

The composition of water that may enter the NFE is restricted to a relatively narrow range, except for the condition in which large volumes of glassy tuff are dissolved. For those conditions not involving large volumes of reacted glass, the bounds suggest that the solution will be approximately neutral to slightly basic, will be relatively oxidizing, will be saturated with respect to one silica polymorph or another, and will have low to moderate concentrations of dissolved species. Total ionic strength will remain below 0.1 molal. For the case in which large volumes of glass are dissolved, the total solute concentration can be quite high. The $\mathrm{pH}$ may also become strongly acidic.

Even for the more general case (apart from large extent of reaction with glass), the water chemistry varied significantly. During the course of reaction, large changes occur in the concentrations of the nonconservative dissolved components. These changes are correlated with the dissolution or precipitation of various mineral phases. 


\subsubsection{Uncertainty}

The uncertainty in the abundance of a dissolving or precipitating mineral at a given time is shown to be problem-specific and potentially significant with respect to long-term predictions of geochemical properties in slowly reacting systems. The results from the study in which sanidine dissolution was considered demonstrate that, for a relatively small initial uncertainty in the controlling rate parameter, there is a comparable uncertainty in the amount of the mineral present at a given time. However, there is a much larger uncertainty in the time at which a mineral may appear in or disappear from the system. The results presented here shows that an order-of-magnitude variation in the rate results in the disappearance of sanidine sometime between a few hundred days and nearly a hundred years of reaction.

\subsubsection{Conclusions}

The results presented here provide, for a very specific set of conditions and simulation constraints, initial bounds on secondary mineralogy and water chemistry. These constraints, along with similar efforts to bound pore-water chemistry during evaporation (Glassley [1995] and Section 3.4.1 of the Near-Field and Altered-Zone Environment Report, Vol. II), provide baseline information from which to refine simulation strategies and then to obtain improved bounds on water chemistry and secondary mineralogy.

These results also document the magnitude of the uncertainties that exist in establishing the nature of secondary minerals that may form, the resulting water chemistry, and the absolute times at which specific changes might be expected to develop during reaction. Much work is still needed before quantitative bounds can be confidently placed on the evolution of water chemistry entering the NFE and on the resulting secondary mineral assemblages that may influence radionuclide migration.

These results clearly document that both the times at which mineral phases may be present or not during reaction and their respective amounts when they are present may be uncertain by orders of magnitude. Because these parameters are also important for describing water chemistry as a function of time, it is clear that, unless more measurements of dissolution and precipitation rates are obtained, only very gross bounds can be placed on either the mineralogical or water-chemistry evolution.

This point is also important for radionuclide retardation in the NFE and the AZ. The magnitude of retardation depends on the mineral phases that are present and on their respective amounts. However, these results demonstrate that there is substantial uncertainty about when any specific phase may form or be consumed in reactions and reinforce the need for more precise values of dissolution and precipitation kinetic-rate constants.

These water chemistries are not the ones that a WP is likely to encounter. Materials introduced into the repository during construction and waste emplacement will probably be primary controls on the chemistry of the water that reaches the packages.

\subsection{Repository-Scale Thermohydrochemical Scoping Calculations Involving Silica}

\section{by William E. Glassley}

This section describes early scoping calculations done to bound the magnitude of coupled effects that may occur in a TH system with silica dissolution and precipitation. The study was done prior to issuance of the most recent infiltration model for Yucca Mountain (Flint et al., 1996), thus the values assumed for average percolation flux were probably low by a factor of ten. The effective continuum model (ECM) was used (see Chapter 3), and the TH properties were different from current total system performance assessment-viability assessment 
(TSPA-VA) property sets; therefore, there are inherent limitations on comparison to the reference cases computed for this report. Nevertheless, the study is of interest as an important attempt to directly couple THC processes in a repository simulation. (Reference cases for investigation of coupled processes in the NF/AZ Models Report are visually depicted and linked in the presentation on the CD-ROM that accompanies this report.)

The FEHM code was used with special modules to explicitly account for changes in permeability related to temperature-dependent silica dissolution and precipitation (Zyvoloski et al., 1988). The simulations were performed at repository scale to evaluate coupled processes throughout the AZ; the near field was not modeled in detail.

In the calculations described subsequently, steady-state THC conditions were achieved in the time period simulated ( 0 to $10,000 \mathrm{yr}$ ). The results show that the potential for diversion of percolation flux around the repository is enhanced, relative to noncoupled simulations, when silica redistribution is included. Significant differences in the quantity of silica deposition at various locations were projected to occur.

For properties and conditions considered in this study, temperatures greater than the boiling point of water were predicted to occur throughout an extensive dryout region (Buscheck and Nitao, 1992; Nitao, 1988). Large volumes of water vapor will be produced, migrate, and condense, causing changes in local saturation. This will lead to changes in the chemical environment at virtually all locations affected by heat. Mineral dissolution, transport of dissolved constituents, and precipitation of secondary minerals will occur. The extent to which they occur will vary with time and location. If thermodynamic equilibrium between water and tuff minerals is approached and sufficient volume of water is present, significant changes in porosity and permeability are possible.

In contrast with this view, Braithwaite and Nimick (1984) concluded from single-pass, fully saturated, flow-through experiments that the impact of silica redistribution on hydrologic properties would be minimal and would have no influence on evolution of thermal or hydrologic properties. Verma and Pruess (1988) reached a similar conclusion. Neither of these studies involved directly coupled simulation. The calculations described in this section include direct coupling and also compare models in which permeability was controlled in different ways. This study was not exhaustive, but was intended to support further refinement in THC simulation techniques and strategies.

\subsubsection{Processes Of Interest}

This discussion of THC coupled processes takes place around a conceptual model in which four TH regimes are represented (Buscheck and Nitao, 1992) (Figure 5-20). These four regimes are described in Section 5.3.3. Chapter 3 provides a more complete discussion of thermohydrology. When considering solute transport in a dynamic TH environment, it is useful to distinguish between boiling and sub-boiling effects and between the effects of increasing and decreasing temperature.

In this study, the areal power density ranged from 20 to $114 \mathrm{~kW} /$ acre (roughly comparable to areal mass loading [AML] from 20 to $100 \mathrm{MTU} /$ acre), and ambient percolation flux ranged from zero to $0.13 \mathrm{~mm} / \mathrm{yr}$.

\section{Figure 5-20 Hydrothermal Regimes A-C at 100 and 1000 yr after repository closure}

\subsubsection{Code Selection}

Several codes exist with the capability to couple hydrologic and geochemical processes (Bäverman, 1993; Knapp, 1989; Lichtner, 1988, 1993; Madé and Jamet, 1993; Noy, 1990; Steefel and Lasaga, 1992; Zyvoloski, 1983, 1986; Zyvoloski and Dash, 1991; Zyvoloski et al., 1988). A 
review of these codes (Bourcier et al., 1995) demonstrated that each has useful characteristics, but none couples the processes of concern in a way that is well suited to objectives of this study. The available codes essentially fall into two groups:

- Codes that rigorously compute chemical processes but do not deal explicitly with heat transfer and are typically designed to examine processes on the scale of meters in single-pass, flow-through systems

- Codes that deal with large-scale systems but that typically do not couple changes in porosity resulting from mineral reactions with changes in permeability

A review concluded that reconnaissance studies of simple chemical systems could be most readily be accomplished by using the FEHM code (Zyvoloski, 1983, 1986; Zyvoloski and Dash, 1991; Zyvoloski et al., 1988) and incorporating minor code changes to allow updating the porosity and permeability. The modified code is fully 3-D, although only 2-D simulations were conducted for this effort. It has dual-porosity and dual-permeability capabilities, but these simulations used an ECM approach. The simulations described subsequently were conducted in collaboration with Bruce Robinson at Los Alamos National Laboratory.

\subsubsection{Modeling Strategy}

The vertical cross-section model used in the simulations incorporated a $36 \times 34$-gridpoint, 2-D, finite-element grid. Distances were scaled to simulate the depth to the repository and to the saturated zone. The lower boundary of the grid was taken to be the saturated zone and was assumed to remain at a nominal, constant temperature of $20^{\circ} \mathrm{C}$. The sides of the grid were treated as no-flow boundaries for heat and mass. The top was also assumed to remain at a constant temperature of $20^{\circ} \mathrm{C}$. To simplify the simulations, the entire model domain was assumed to have fracture and matrix properties equivalent to the ECM properties for the Topopah Springs welded tuff reported by Klavetter and Peters (1986).

The initial state of the system was generated by allowing a uniform infiltration rate of $0.0365 \mathrm{~mm} / \mathrm{yr}$ to achieve a steady-state saturation profile. At steady state, the matrix saturation was approximately $75 \%$ at the top of the grid and nearly $100 \%$ at the water table. To model the effects of repository operation, the grid points representing the repository were assigned a temperature of $150^{\circ} \mathrm{C}$ after reaching steady state. This temperature was maintained for 10,000 yr, after which the temperature source was turned off, and the system was allowed to relax for an additional 90,000 yr.

The geochemistry was modeled using simplified, temperature-dependent forward and reverse reaction rates for the reaction:

$$
\mathrm{SiO}_{2} \text { (solid) } \Leftrightarrow \mathrm{SiO}_{2} \text { (aqueous) }
$$

Dissolution of the solid was represented by a zero-order reaction, and precipitation was represented by a first-order reaction. Temperature dependence of the reaction rates was represented by the Arrhenius relation:

$$
k=A \exp \left(-E_{\mathrm{A}} / R T\right)
$$

where $A$ is the pre-exponential factor for the rate expression, $k$ is the rate constant, $E_{\mathrm{A}}$ is the activation energy, $R$ is the gas constant $(8.314 \mathrm{~kJ} /$ mole-K$)$, and $T$ is the absolute temperature. This approach is a simplified model of reaction kinetics that satisfies the principal dependence of reaction rates on temperature (see, for example, Lasaga, 1981, 1984; Lasaga and Blum, 1986; Rimstidt and Barnes, 1980), although the sensitivity to solution composition (e.g., $\mathrm{pH}$, ionic strength) is not considered. 
Changes in porosity were linearly related to changes in the number of moles of $\mathrm{SiO}_{2}$ in the rock (initial $\mathrm{SiO}_{2}$ abundance was assumed to be 5 moles $/ \mathrm{kg}$-rock).

The relation between porosity and permeability for fractured rock is not well established (see, for example, Brace, 1977; Camp, 1964; McClure et al., 1979; Pearson, 1976; Pyrak-Nolte et al., 1987; Scheidegger, 1974; Vaughan, 1985; Verma and Pruess, 1988). To bound the breadth of changes observed in various laboratory studies (e.g., Keith et al., 1983; Lin and Daily, 1989; Moore et al., 1986; Morrow et al., 1981), simulations were conducted using different permeability-porosity relations. In one case, the classical Kozeny-Carman relation was assumed; in other simulations, a different relation was used to represent more sensitive coupling between porosity and permeability:

$$
\log _{10}\left(k / k_{0}\right)=a \cdot \text { porosity }+b
$$

where $k$ and $k_{0}$ are the altered and initial permeabilities, and $a$ and $b$ are constants used to define the relation. For the simulations presented here, one case was considered in which there was no coupling of permeability with porosity, and one case was considered in which strong coupling between porosity and permeability existed such that a change in porosity from 0.11 to 0.09 (i.e., $20 \%$ decrease) resulted in a permeability decrease of three orders of magnitude. Although no data are available for direct evaluation of the likelihood for strong coupling, the assumption was used as a bound on coupling these parameters in ECM simulations.

\subsubsection{Results}

Figure 5-21 shows temperature contours calculated for 10,000 yr. The thermal perturbation encompasses the entire repository block except at the upper and lower constant temperature boundaries of the model domain. The model grid, properties, boundary conditions, and representation of heat sources were sufficient to produce the $\mathrm{TH}$ processes that are observed in other simulations (see Chapter 3).

\section{Figure 5-21 Temperature contours at 10,000 yr after waste emplacement}

The ECM saturation conditions projected to occur at 10,000 $\mathrm{yr}$ for both the noncoupled and coupled cases are shown in Figure 5-22 and Figure 5-23, respectively. For both cases, a dryout zone at temperatures greater than boiling develops immediately around the repository (Regime C). Outside this zone, at temperatures at or near boiling, heat-pipe conditions develop above the repository, and saturation approaches 100\% (Regime B). The zone of elevated saturation appears to extend around the edge repository, apparently from condensate shedding due to gravity-driven drainage.

\section{Figure 5-22 Saturation contours at 10,000 yr for the case with no coupling between silica transport and permeability change}

Figure 5-23 Saturation contours at 10,000 yr for the case with coupling between silica transport and permeability change

The principal differences between the noncoupled and coupled simulations are the location of saturation contours and maximum degree of saturation. For the coupled case (Figure 5-23), the region of Regime $\mathrm{C}$ is projected to be wider, and the maximum saturation above the repository is greater. These differences result directly from redistribution of silica. 
Figure 5-24and Figure 5-25 show the overall redistribution of silica for the noncoupled and coupled cases. Steady-state silica distribution occurs in the 10,000-yr time frame because reaction rates are relatively fast. This result is consistent with arguments suggesting that, for most mineralogical reactions involving aqueous fluids, steady-state mass distribution occurs in a few thousand years. In the noncoupled and coupled cases, deposition of silica is projected to occur near the repository, while dissolution and removal of silica occur farther away (Figure 5-26, Figure 5-27, Figure 5-28, and Figure 5-29). For both cases, dissolution below the repository is restricted to an envelope less than $100 \mathrm{~m}$ thick, whereas above the repository the zone of dissolution is projected to be several hundred meters thick. The narrow envelope below the repository may have resulted, at least in part, from the constanttemperature condition at the bottom of the model domain. Similarities between the noncoupled and coupled simulations demonstrate that the processes responsible for silica redistribution are essentially the same. In both cases, dissolution of silica occurs where water is heated to boiling.

Figure 5-24 Distribution of silica at 10,000 yr with no coupling between silica transport and permeability change

Figure 5-25 Distribution of silica at 10,000 yr with coupling between silica transport and permeability change

Figure 5-26 Regions of net precipitation of silica at 10,000 yr with no coupling between silica transport and permeability change

Figure 5-27 Regions of net precipitation of silica at 10,000 yr with coupling between silica transport and permeability change

Figure 5-28 Regions of net dissolution of silica at 10,000 yr with no coupling between silica transport and permeability change

Figure 5-29 Regions of net dissolution of silica at 10,000 yr with coupling between silica transport and permeability change

Differences in the magnitude and location of silica concentration between the noncoupled and coupled cases reflect reduction in permeability caused by silica precipitation. For the coupled case, the range from minimum to maximum silica concentration (4.69 to

5.24 moles $/ \mathrm{kg}$-rock) is significantly less than for the noncoupled case (3.0 to 7.24 moles $/ \mathrm{kg}$ rock). Regions of the model with the greatest contrast in silica distribution are compressed in the noncoupled case, relative to the coupled case, but the most extreme values in both cases occur at the repository edges.

\subsubsection{Discussion}

Despite the sensitivity of permeability to silica distribution in the coupled case, the difference in liquid saturation contours between the noncoupled and coupled cases is relatively small. The most significant effect on liquid saturation is that the region of lowest saturation above and below the repository is expanded by several tens of meters in both directions in the coupled case, thus indicating that dryout is enhanced by coupling. In 
addition and consistent with the dramatic reduction in permeability from silica precipitation, the thickness of the region of increased saturation above the repository is significantly increased in the coupled case.

The most significant impact of coupling appears to be the distribution of solids. In the coupled case, boiling leads to silica precipitation, and the consequent reduction of permeability inhibits gravity-driven water reflux, which causes an increase of liquid saturation. As the system develops, the amount of precipitation in the vicinity of the repository is reduced, relative to the noncoupled case, because of the decrease in fluid flux. The net result is that permeability reduction changes the heat-pipe characteristics, which limits the amount of permeability reduction that will occur at steady state. This is numerical verification of the dimensionless analysis of heat-pipe characteristics published by Stubos et al. (1993a, 1993b). See Chapter 8 for more discussion of steady-state heat-pipe effects on bulk permeability.

The simulations projected the greatest effect of dissolution/precipitation on the distribution of silica to occur at the repository edges. In both the noncoupled and coupled cases, the regions of maximum dissolution occur in lobes at the edges of the dissolution haloes above and below the repository. Between these lobes is the region of maximum precipitation of silica. This relation appears to reflect the interplay between gravity-driven drainage and flow of water across temperature contours.

\subsubsection{Conclusions}

The principal objective for the simulations presented in this section was to evaluate the use of the FEHM code to simulate coupled THC processes on the repository scale for simple chemical systems. The results show that such simulations are possible, and there is no reason beyond computational effort they could not be performed at different scales or with higher dimensionality.

The results of the simulations place bounds on repository evolution for two extreme cases: the case in which TH and thermochemical (TC) processes are not coupled, and the case for which TH and TC processes are strongly coupled using a log-linear relation. These conditions are simplistic representations of the processes expected to occur during $\mathrm{TH}$ evolution of the proposed repository.

The simple, one-component chemical system does not completely represent the chemistry of Yucca Mountain tuffs, and more complex water compositions and mineralogical relations are needed for additional realism. Considerably more computational effort will be required when additional components and reactants are incorporated.

The simulations conducted here show that, during thermal evolution of the repository, if no competing reactions occur (which is unknown), fluid and solute movement will result in precipitation of silica near the repository. This will tend to isolate the repository from ambient percolation, but might also lead to "bathtub-ring" effects if water enters the emplacement drifts.

\subsection{Thermohydrochemical Alteration of Flow Pathways Above and Below the Repository}

\section{by John J. Nitao}

The interaction between $\mathrm{TH}$ and geochemical processes is an important facet of near-field behavior that has only recently been modeled. In earlier work, Robinson and Glassley (1995) used the ECM to simulate silica dissolution and precipitation around an idealized diskshaped repository model. Otherwise, there has been relatively little investigation of $\mathrm{TH}$ and 
geochemical coupling around a UZ repository. Robinson and Glassley's (1995) calculations predicted little silica precipitation around the repository. However, the ECM cannot distinguish between precipitation in the fractures and that in the matrix. In the ECM, it is relatively difficult for precipitated minerals to significantly plug the pore space because of the relatively large void volume of the bulk single continuum. Fractures, on the other hand, can be plugged more easily because of their small volume; yet, fractures play a key role in the movement of gas and liquid phases around the repository. The DKM used in this modeling effort alleviates some of the limitations of the ECM by considering the matrix and fractures as two connected, yet distinct, porous-medium effective continua.

In this section, calculations are presented for a coupled $\mathrm{TH}$ and geochemical reactivetransport system around a single drift. (Reference cases for investigation of coupled processes in the NF/AZ Models Report are visually depicted and linked in the presentation on the CDROM that accompanies this report.) Most of the fractures in the UZ at Yucca Mountain can be grouped according to whether they are of predominantly calcite mineralogy or of silica mineralogy; this work focuses on the silica system.

\subsubsection{Introduction}

Silica minerals at the repository horizon consist primarily of cristobalite, with smaller amounts of other minerals such as quartz. Therefore, dissolution of cristobalite and quartz to aqueous silica is expected to be a key reaction mechanism. Laboratory data are available for dissolution of these minerals, but the modeling of silica precipitation is problematic. Silica may precipitate as a variety of minerals, including chalcedony and amorphous silica. Precipitation to quartz is not observed in nature except at high temperatures (which significantly exceed the ambient boiling point of water at Yucca Mountain) despite the fact that the solubility of silica, with regard to quartz, is significantly lower. The much higher surface energy of quartz compared to, say, amorphous silica is thought to act as a kinetic barrier (Steefel and Van Cappellen, 1990) to nucleation.

The work reported here is only a first step in understanding the reactive-transport mechanisms of the complex mineralogy found in nature. To reduce the complexity of the problem, cristobalite and quartz are first considered as the mineral phases modeled. Then a cristobalite, quartz, and amorphous silica system is considered. In the future, other mineral phases will be considered. In the cristobalite-quartz model, precipitated silica is primarily quartz because of its lower solubility. To account for the often-observed abundance of amorphous silica, calculations are also performed with amorphous silica where precipitation of quartz and cristobalite are turned out.

The following reactions are considered in this simple silica-cristobalite-quartz system

$$
\begin{aligned}
& \mathrm{SiO}_{2 \text { (cristobalite) }} \Leftrightarrow \mathrm{SiO}_{2 \text { (aqueous) }} \\
& \mathrm{SiO}_{2 \text { (quartz) }} \Leftrightarrow \mathrm{SiO}_{2 \text { (aqueous) }} \\
& \mathrm{SiO}_{2 \text { (amorphous silica) }} \Leftrightarrow \mathrm{SiO}_{2 \text { (aqueous) }}
\end{aligned}
$$

The temperature-dependence and kinetic nature of these reactions and their effect on permeability are important issues that need to be considered in modeling altered conditions around the repository. 
Some important features of the silica-cristobalite-quartz system are listed here to help interpret the results of the current model. However, because several important silica minerals were not considered for this report, some of the following observations may not be applicable to the actual system at Yucca Mountain.

- Precipitation rates are, in general, much faster than dissolution rates. There is a significant lag in the dissolution of silica due to the flow of silica-undersaturated fluid.

- The solubility of silica as it goes to either cristobalite, quartz, or amorphous silica increases with temperature. Therefore, silica-saturated fluids will precipitate silica as it is transported from regions of hotter temperatures to regions of cooler temperatures.

- The solubility of silica-quartz is lower than that for the silica-cristobalite reaction. Therefore, in a closed cristobalite-quartz system, the cristobalite will dissolve, and aqueous silica will eventually precipitate to quartz.

- The dissolution rate constants for both reactions increase significantly with temperature. The rate constant for cristobalite increases by a factor of about 270 from $25^{\circ}$ to $100^{\circ} \mathrm{C}$, whereas the rate constant for quartz increases by a factor of about 1200 . Therefore, a significant increase in dissolution rates is expected in regions that have aqueous fluid present and that are heated by the repository.

\subsubsection{Description of Coupled-Process Phenomena}

The phenomenology of TH processes has been described in Chapter 3. One of the most notable features is the likely existence of gravity-driven heat pipes above the repository. In a heat pipe, water vapor driven outward through fracture pathways by gas pressure, either from steam generation or buoyant gas convection, will condense and travel downward by gravity, only to reboil as it approaches the superheated repository zone. The length of heat pipes can be many tens to hundreds of meters, depending on thermal design parameters of the repository.

From the perspective of the aqueous phase, condensation can be viewed as a source term of liquid water with no dissolved silica. When mixed with water already present, the condensed liquid water can lead to an undersaturated solution.

As the condensate liquid flows down the heat pipe through fractures, silica polymorphs will be dissolved along fracture walls until the fluid becomes silica-saturated. If the fluid happens to flow around the sides of the drift, it will eventually reach cooler regions, causing some of the silica to precipitate. If, instead, the fluid reaches the upper edge of the superheated repository zone, it will evaporate, acting as a sink term for liquid water. Silica concentrations will increase, resulting in silica saturation and precipitation.

From the preceding conceptual model, increased fracture apertures would be expected where the condensate zone has swept past. However, the greatest region of aperture increase is expected to occur where the condensate zone is almost stationary during the quasi-steady heating period characterized by a more-or-less stable heat pipe.

The approximate degree to which silica can precipitate in fractures can be demonstrated by the following "back-of-the-envelope" calculation. Consider the approximately tabular zone of refluxing over a 57-kW/acre repository. The specific mass flux $\left(\mathrm{kg} / \mathrm{s}-\mathrm{m}^{2}\right)$ of condensate in the heat-pipe region above the repository is approximately equal to $q_{l}=q_{H} / 2 h_{\text {vap }}$, where $q_{H}=14 \mathrm{~W} / \mathrm{m}^{2}$ is the areal heat flux over a $57 \mathrm{~kW} /$ acre repository, and $h_{v a p}=2.3 \times 10^{6} \mathrm{~J} / \mathrm{kg}$ is the specific enthalpy of vaporization of water at the nominal boiling point at the repository elevation. Therefore, we have $q_{l}=3.0 \times 10^{-6} \mathrm{~kg} / \mathrm{s}-\mathrm{m}^{2}$. If it is assumed that the condensate is saturated with silica, the flux of silica entering the boiling zone is equal to $q_{\text {silica }}=\omega q_{1}$, where $\omega=7.8 \times 10^{-3}$ is the mass fraction of silica in the liquid phase saturated 
with respect to cristobalite at the boiling point of water. Hence, $q_{\text {silica }}=2.34 \times 10^{-8} \mathrm{~kg} / \mathrm{s}-\mathrm{m}^{2}$. Because the mass density of the various silica polymorphs is around $\rho_{\mathrm{m}}=2$ to $2.6 \times 10^{3} \mathrm{~kg} / \mathrm{m}^{3}$, the rate of volume increase in silica precipitate, per unit area, is $d V / d t=q_{\text {silica }} / \rho_{\mathrm{m}}=9.0 \times 10^{-12}$ $\mathrm{m} / \mathrm{s}=2.8 \times 10^{-4} \mathrm{~m} / \mathrm{yr}$. For sake of argument, suppose there are approximately 3 parallel, 100 -micron aperture fractures per meter, giving a fracture porosity of $\phi_{\mathrm{f}}=3 \times 10^{-4}$. Now, if the precipitation occurs mostly within fractures, the region of the rock mass potentially filled by precipitate increases at a significant rate of $d z / d t=1 / \phi_{\mathrm{f}} d V / d t=1.0 \mathrm{~m} / \mathrm{yr}$. As the heat decays, the rate of increase will decrease proportionately from this value. Significant zones of precipitation can, therefore, occur over the several hundred- to several thousand-year span of the refluxing period.

The preceding analysis assumes that the condensate entering the boiling zone is silicasaturated. Further analysis has found that the dissolution of silica from the fracture walls does not occur fast enough to justify this assumption. In fact, it can be shown that, if only dissolution in the fractures is assumed, the rate of growth of the precipitation zone is equal to $d z / d t=k a_{\mathrm{s}} L / \rho_{\mathrm{m}} b$, where $k$ is the dissolution rate $\left(\mathrm{kg} / \mathrm{s}-\mathrm{m}^{2}\right)$ of the silica polymorph, $a_{\mathrm{s}}$ is the relative surface area of silica on the fracture walls, $L$ is the length $(\mathrm{m})$ of the refluxing zone, and $\mathrm{b}$ is the fracture half-aperture $(\mathrm{m})$. Substituting values of $k=4 \times 10^{-14} \mathrm{~kg} / \mathrm{s}-\mathrm{m}^{2}$ for cristobalite dissolution at boiling temperatures, taking $a_{\mathrm{s}}=0.30, L=100 \mathrm{~m}$, and $b=50 \mu \mathrm{m}$, one obtains $d z / d t=3.4 \times 10^{-4} \mathrm{~m} / \mathrm{yr}$, which amounts to approximately $3.4 \mathrm{~m}$ in 10,000 yr, slower by several orders of magnitude than observed in the simulations. However, this particular estimate does not include matrix-to-fracture diffusion. Molecular diffusion of aqueous silica from the pores of the matrix to the fracture is significant because the fracture has such a small volume compared to the pore volume of the matrix. The matrix becomes a source of aqueous silica, thereby increasing the effective surface area of the silica exposed to the condensate as it travels down the heat pipe and raises the silica concentration to saturated levels. Thus, if matrix diffusion is included, the assumption of saturated silica concentrations in the analysis of the previous paragraph is a good one. (Of course, the computer simulations presented later do not make this assumption; they calculate silica concentrations taking into account all of the relevant processes.)

\subsubsection{Mathematical Model for Reactive Transport}

The mathematical model for TH processes is presented in Chapter 3. The mathematical model for reactive transport used in performing this simulation is given here. A first-order reaction rate $\left(\mathrm{mol} / \mathrm{s}-\mathrm{m}^{3}\right)$ formula was used for dissolution and precipitation:

$$
r_{\gamma}=-a k_{\gamma}\left(1-n / K_{\gamma}\right), \gamma=\text { cristobalite, quartz }
$$

where the rate constant is given by the Arrhenius relation

$$
k_{\gamma}=k_{\gamma 0} \exp \{-E / R T\} / \exp \left\{-E / R T_{0}\right\}
$$

and the symbols are defined as follows:

$$
\begin{aligned}
& n=\text { mole fraction of silica } \\
& T=\text { absolute temperature }(\mathrm{K}) \\
& k_{\gamma 0}=\text { reaction rate constant }\left(\mathrm{mol} / \mathrm{s}-\mathrm{m}^{2}\right) \text { at } T=T_{0} \\
& E=\text { activation energy }(\mathrm{J} / \mathrm{mol}) \\
& R=\text { the gas constant }(\mathrm{J} / \mathrm{K}-\mathrm{mol}) \\
& a=\text { mineral-specific surface area }\left(\mathrm{m}^{2} / \mathrm{m}^{3}\right) \\
& K_{\gamma}=\text { equilibrium constant }
\end{aligned}
$$


The constant $k_{\gamma 0}$ depends on whether dissolution is occurring (if $n<K_{\gamma}$ ) or precipitation is occurring (if $n>K_{\gamma}$ ). It was found that, in regions of high evaporation (e.g., the refluxing of the heat pipe), the solution becomes extremely supersaturated, and the first-order law does not precipitate minerals fast enough; instead, it predicts unrealistically high concentrations. In the study of the quartz-cristobalite system, a precipitation constant that is three orders of magnitude times the dissolution constant was used to remedy this problem. For the system with amorphous silica, an improved approach was used; in this approach, the precipitation rate was multiplied by a nonlinear factor equal to the cube of the ratio $n / K \gamma$. This ratio is a measure of supersaturation that is equal to unity during equilibrium, but that increases with the degree of supersaturation.

The specific mineral surface area was assumed to be of the form

$$
a=a_{0}\left(\theta / \theta_{0}\right)^{2 / 3}, \text { if } r_{\gamma}>0
$$

where $\theta$ is the volume fraction, and $\theta_{0}$ is the initial volume fraction. The functional dependence of the equilibrium constant on temperature was of the form

$$
K_{\gamma}=B_{\gamma} T_{C}^{\sigma_{\gamma}}
$$

where $T_{C}$ is temperature in degrees centigrade. The transport equation of aqueous silica is

$$
\frac{\partial \phi \rho_{1} S_{1} \omega_{\gamma}}{\partial t}+\nabla \cdot\left[\omega_{\gamma} \mathbf{q}_{1}-\phi S_{1} \rho_{1} \tau_{1} D_{\gamma} \nabla \omega_{\gamma}\right]=-M r_{\gamma}
$$

where the following symbols are used:

$$
\begin{aligned}
& t=\text { time }(\mathrm{s}) \\
& \phi \quad=\text { porosity } \\
& \omega=\text { mass fraction of aqueous silica } \\
& S_{1}=\text { liquid phase saturation } \\
& \rho_{1}=\text { liquid phase mass density }\left(\mathrm{kg} / \mathrm{m}^{3}\right) \\
& q_{1}=\text { specific liquid phase mass flux }\left(\mathrm{kg} / \mathrm{m}^{2}\right) \\
& \tau_{1}=\text { liquid phase tortuosity factor } \\
& D \gamma=\text { diffusion coefficient }\left(\mathrm{m}^{2} / \mathrm{s}\right) \\
& M=\text { molecular mass of silica }(\mathrm{kg} / \mathrm{mol})
\end{aligned}
$$

The mineral dissolution-precipitation equation is

$$
\frac{\partial \rho_{\mathrm{m}} \theta_{\gamma}}{\partial t}=M r_{\gamma}
$$

where $\rho_{\gamma}$ is the mass density of the mineral, and $\theta_{\gamma}$ is its volumetric fraction. The resulting porosity change is given by

$$
\frac{\partial \phi}{\partial t}=-\sum_{\gamma} \frac{\partial \theta_{\gamma}}{\partial t}
$$


The capillary pressure in the fracture and matrix continua were assumed to scale inversely with porosity,

$$
p_{c} / p_{c 0}=\phi_{0} / \phi \text {. }
$$

The intrinsic permeabilities in the fracture and matrix continua were assumed to scale as the cube of the porosity

$$
k / k_{0}=\left(\phi / \phi_{0}\right)^{3}
$$

In the case of fractures, Eq. 5-65 is equivalent to cubic law if the fracture aperture is assumed to vary linearly with the porosity of the fracture continua.

The van Genuchten formula was used for the capillary pressure and relative permeability curves of the fracture and matrix. The van Genuchten $m$ parameters for capillary pressure and relative permeability curves, as well as the residual saturation, are kept fixed with respect to changes in porosity. The hydrologic parameter values used are primarily from the July 1997 TSPA-VA Base Case as given in Table 3-5 and Table 3-6 of this report. One of the simulation cases that will be described is from the December 1997 "Modified TH" property set given by Table 3-9, Table 3-10, and Table 3-11 in this report.

The parameters used for modeling the cristobalite reaction are shown in Table 5-7, and those for quartz are shown in Table 5-8.

Table 5-7 Cristobalite dissolution base-case parameter values

\begin{tabular}{|c|c|c|}
\hline Parameter & Value & Unit \\
\hline \hline$\rho_{\gamma}$ & $2.33 \times 10^{3}$ & $\mathrm{~kg} / \mathrm{m}^{3}$ \\
\hline$k_{0}$ & $3.16 \times 10^{-13}$ & $\mathrm{~mol} / \mathrm{m}^{2}-\mathrm{s}$ \\
\hline$B_{\gamma}$ & $1.73 \times 10^{-6}$ & \\
\hline$\sigma_{\gamma}$ & 1.584 & \\
\hline$T_{0}$ & 298.15 & $\mathrm{~K}$ \\
\hline$E$ & $6.91 \times 10^{4}$ & $\mathrm{~J} / \mathrm{mol}$ \\
\hline$a_{0}$ & $9.42 \times 10^{4}$ & $\mathrm{~m} / \mathrm{m}^{3}$ \\
\hline$\theta_{0}$ & 0.30 & \\
\hline
\end{tabular}

The initial specific surface area $a_{0}$ was that for spheres with a radius of 10 microns multiplied by the relative composition of the mineral, which is assumed to be equal to its initial relative volume $\theta_{0}$. 
Table 5-8 Quartz dissolution base-case parameter values

\begin{tabular}{|c|c|c|}
\hline Parameter & Value & Unit \\
\hline \hline$\rho_{\gamma}$ & $2.65 \times 10^{3}$ & $\mathrm{~kg} / \mathrm{m}^{3}$ \\
\hline$k_{0}$ & $1.26 \times 10^{-14}$ & $\mathrm{~mol} / \mathrm{m}^{2}-\mathrm{s}$ \\
\hline$B_{\gamma}$ & $2.03 \times 10^{-7}$ & \\
\hline$\sigma_{\gamma}$ & 1.853 & \\
\hline$T_{0}$ & 298.15 & $\mathrm{~K}$ \\
\hline$E$ & $8.75 \times 10^{4}$ & $\mathrm{~J} / \mathrm{mol}$ \\
\hline$a_{0}$ & $1.57 \times 10^{4}$ & $\mathrm{~m}^{2} / \mathrm{m}^{3}$ \\
\hline$\theta_{0}$ & 0.05 & \\
\hline
\end{tabular}

\subsubsection{Numerical Solution of Coupled Equations}

In this application, the coupled $\mathrm{TH}$ and reactive-transport equations are solved by the NUFT code in a sequential manner for computational efficiency. Each solution cycle proceeds as follows. First, the TH equations are solved over a single time step. Next, the reactivetransport equations are solved using time steps that are equal to or smaller than the TH time step. The use of smaller time steps reflects the fact that time constants for reactive transport are often smaller than those for thermohydrology. Time-linear interpolated values based on the beginning and the end of the TH step are used for the flux $q_{1}$, mass density $\rho_{v}$, saturation $S_{1}$, and temperature $T$ fields in the reactive-transport model. The reactive-transport model is solved in this manner until it reaches the end time of the TH step. The simulation then passes the calculated porosity field to the TH model, which begins its next time step, as shown in Figure 5-30. The cycle is repeated until the ending simulation time is reached.

Both the TH and reactive-transport models have automatic time-stepping for controlling the changes in the solution variables. For this simulation, the transport time steps were about an order of magnitude smaller than the TH time steps. A complete simulation run out to 900,000 yr requires about 1 to $1.5 \mathrm{hr}$ of CPU time on a Sun UltraSparc II workstation with a $300-\mathrm{MHz}$ processor.

Figure 5-30 Passing of parameter fields during the sequential solution of the TH and reactive-transport models

\subsubsection{Drift-Scale Simulation}

\subsubsection{Model Description}

The model domain contains a single drift within a 2-D columnar section of Yucca Mountain extending vertically from the water table to the ground surface. The geometry, gridding, and boundary conditions are identical to those of the 14c2 model described in Section 3.5 of this report. The lithology of the column is shown in Figure 3-13(b). The WP depth is approximately $387 \mathrm{~m}$ from the ground surface. The heat output is from the TSPA-VA base-case design shown in Table 3-24. The DKM was used to model the 2-D fracture-matrix system. 


\subsubsection{Point-Load Base Case}

The simulation referred to as the base case here is the point-load TSPA-VA base case, which has $16 \mathrm{~mm} / \mathrm{yr}$ of infiltration for the $14 \mathrm{c} 2$ column. The drift spacing is $28 \mathrm{~m}$.

Figure 5-31 and Figure 5-32 show the relative porosity change (defined as the ratio of the porosity to the initial porosity) at $1000 \mathrm{yr}$ and the sensitivity to the reacting mineral surface area. Figure 5-31 shows results for the fractures, and Figure 5-32 shows results for the matrix. Three different surface areas are considered: (a) 0.01 times the base-case surface area $\left(0.01 \times A_{\mathrm{s}}\right),(\mathrm{b}) 1$ times the base-case surface area $\left(1 \times A_{\mathrm{s}}\right)$, and (c) 100 times the base-case surface area $\left(100 \times A_{\mathrm{s}}\right)$.

Figure 5-31 shows significant precipitation of silica above and to the sides of the drift in all cases; it is caused by the drying of silica-laden condensate from the heat pipe. The fracture aperture has been reduced by approximately two orders of magnitude; therefore, assuming the cubic law, the permeability has been reduced by six orders of magnitude. The location of the precipitation zone, or cap, is essentially swept by the superheated region as it passes through on its outward and inward movement. Because no significant refluxing occurs below the repository in the absence of a gravity-driven heat pipe, there is no precipitation zoning there. A capillary-driven heat pipe exists, but, because of the low permeability of the matrix, any precipitation that arises appears to be very small.

Figure 5-31 Relative change in fracture porosity (ratio of the porosity to the initial porosity) at $\mathbf{1 0 0 0} \mathrm{yr}$ for base case properties and point-load reference design (corresponding to reference Case 1) and incorporating dissolution and precipitation of quartz and cristobalite

Figure 5-32 Relative change in matrix porosity (ratio of the porosity to the initial porosity) at $\mathbf{1 0 0 0} \mathrm{yr}$ for base case properties and point-load reference design (corresponding to reference Case 1) and incorporating dissolution and precipitation of quartz and cristobalite

Although the $0.01 \times A_{\mathrm{s}}$ and $1 \times A_{\mathrm{s}}$ cases are quite similar, significantly more precipitation nearly encompasses the drift. Also note the increased porosity above the precipitation cap from mineral dissolution caused by the condensation of liquid water. The precipitation cap protruding downwards in the pillars midway between adjacent drifts is caused by gravity drainage of condensate.

For the $0.01 \times A_{\mathrm{s}}$ case in Figure 5-31(d), there is very little vertical porosity change in the fractures, except around the repository. As shown in Figure 5-31(e), noticeable mineral dissolution in the fractures occurs at the upper condensation zone for the $1 \times A_{\mathrm{s}}$ case. In the $100 \times A_{\mathrm{s}}$ case shown in Figure 5-31(f), there is significant dissolution in the fractures over the entire UZ with very large porosity increases in upper condensate zone. Significant porosity changes are also observed below the repository.

As shown in Figure 5-32(a) and Figure 5-32(d), changes to the matrix porosity are negligible for the $0.01 \times A_{\mathrm{s}}$ case, except for slight porosity increases occurring below the repository around the area of the basal vitrophyre.

In Figure 5-32(b), (c), (e), and (f), the $1 \times A_{\mathrm{s}}$ and $100 \times A_{\mathrm{s}}$ cases have qualitatively similar porosity changes, with increase in porosity occurring primarily in the basal vitrophyre areas and above the repository where there is a gravity-driven heat pipe.

As discussed earlier, the precipitation of silica to quartz is not observed in nature except at temperatures much higher than that of the ambient boiling point of water at Yucca Mountain. In a sense, this system substitutes the precipitation to other minerals (e.g., amorphous silica) with the precipitation to quartz. Any differences resulting from the 
difference in volume between the two minerals has been neglected. To interpret the simulation, it is important to understand the reaction kinetics of this idealized system. Because the solubility of quartz is less than that of cristobalite, cristobalite has a tendency to transform to quartz resulting from the dissolution of cristobalite and precipitation of quartz. At room temperature, this process increases significantly as temperature increases.

Figure 5-33(a) shows the volumetric fraction of quartz in the fracture-porous-medium continua. Most of the precipitate that forms above the drift is quartz. In reality, the precipitate will most likely be in the form of other types of silica-based minerals. Figure 5-33(b) shows the same plot for the volumetric fraction of cristobalite. Most cristobalite precipitation occurs at the pillars, possibly because precipitation there is not so much from drying as from the movement of condensate downwards to cooler rock regions. Not shown is the eventual transformation of cristobalite over most of the UZ to quartz, which is significantly accelerated by repository heat.

\section{Figure 5-33 Mineral volumetric fractions of quartz and cristobalite in the fracture plane at $\mathbf{1 0 0 0} \mathrm{yr}$ for the base case (corresponding to reference Case 1)}

Figure 5-34(a) shows the temperature at $1000 \mathrm{yr}$ for the $1 \times A_{\mathrm{s}}$ case. The boiling point of water under ambient conditions at the repository is approximately $96^{\circ} \mathrm{C}$. However, the slight increase in pressure caused by the boiling of water elevates the boiling point slightly. Above the repository, the temperature is flat over some distance, as expected in a heat pipe. The location of the upper boundary of the superheated zone is the end of the heat pipe, and temperatures there start to climb toward the repository. This point is approximately the upper boundary of the precipitation zone. The temperature is flat below the drift as well. The vertical extent of this lower heat pipe is only $30 \mathrm{~m}$ tall, as opposed to the upper heat pipe, which is $200 \mathrm{~m}$ tall. This lower heat pipe is not present with silica reactive transport. With reactive transport, the upper heat pipe is shorter because of the decreased fracture permeability in the region just above the drift. Heat can only flow there by conduction; therefore, a temperature gradient must be present. To compensate for the loss in heat-transfer efficiency above the drift, more heat must flow downward toward the water table. Evidently, this additional heat creates the observed lower heat pipe. Figure 5-34(b) shows the log base 10 of the fracture permeability $\left(\mathrm{m}^{2}\right)$. The initial fracture permeability at the repository unit was $9.1 \times 10^{-10} \mathrm{~m}^{2}$. In the precipitation cap, the permeability has decreased to about $10^{-14} \mathrm{~m}^{2}$.

\section{Figure 5-34 Base-case (corresponding to reference Case 1) conditions at $1000 \mathrm{yr}$}

Figure 5-34(c) shows the liquid saturation in the fracture continuum at $1000 \mathrm{yr}$. At this time, the boiling region has come back to the drift, along with a rewetting saturation front in the matrix and fracture. The high saturation in the precipitation cap is caused by the increased capillary in the fracture due to the inverse scaling with aperture, as applied by the model. Figure 5-34(d), representing the liquid saturation in the matrix at $1000 \mathrm{yr}$, clearly shows the return of the wetting front to the drift. Although the region around the drift is not dry, a heat pipe of approximately $200 \mathrm{~m}$ remains above the drift, and a much shorter one remains below the drift. Figure 5-34(e) is contour plot of gas pressure at $1000 \mathrm{yr}$. There is a slight, but noticeable, gas-phase pressurization below the precipitation cap.

Figure 5-35 compares the WP temperature history for the case with and without silica reactive transport. Before the peak, the temperatures for the two cases are close together; after the peak, the reactive-transport case is slightly higher because the precipitation cap retards the action of the heat pipe during the time when the rewetting front reaches the drift. The relative humidity time history for the WP is shown for the two cases in Figure 5-35(b). Once again, the two curves are quite similar. Figure 5-35(c) is the liquid saturation time history in 
the rock just above the crown of the drift. During the drying period, which takes place from $0 \mathrm{yr}$ to approximately $250 \mathrm{yr}$ in this case, the saturation is slightly lower for the reactivetransport case. After $250 \mathrm{yr}$, the rewetting front returns noticeably earlier in the reactivetransport case.

Figure 5-35 (a) Temperature history of the WP with and without silica reactive transport, (b) relative humidity on the WP, and (c) liquid saturation in the rock above the drift crown

\subsubsection{Time Evolution of the Precipitation Cap}

To better understand the time evolution of the formation of the precipitation cap, Figure 5-36 through Figure 5-40 show fracture porosity change, temperature, matrix liquid saturation, and fracture liquid saturation at 100,500,1000,5000, and $9 \times 10^{5} \mathrm{yr}$ for the $1 \times A_{\mathrm{s}}$ base case.

Figure 5-36 Base-case (corresponding to reference Case 1) conditions at $100 \mathrm{yr}$

Figure 5-37 Base-case (corresponding to reference Case 1) conditions at $500 \mathrm{yr}$

Figure 5-38 Base-case (corresponding to reference Case 1) conditions at $1000 \mathrm{yr}$

Figure 5-39 Base-case (corresponding to reference Case 1) conditions at $5000 \mathrm{yr}$

Figure 5-40 Base-case (corresponding to reference Case 1) conditions at 900,000 yr

As shown by the matrix saturation in Figure 5-36(c), the dryout zone at 100 yr extends to only a few meters around the drift. Depending on the heat load and infiltration rate, this zone can, in some cases, be much larger. The zone where most of the water boils is in the steepsaturation-gradient region around the drift. In Figure 5-36(a), the precipitation cap occurs within that region.

As shown in Figure 5-37(a), the precipitation cap has increased at 500 yr. In Figure 5-37(c), the water has come back toward the drift, especially in the region above the drift. From Figure 5-37(b), most of the region around the drift is above boiling, and ongoing two-phase refluxing brings silica-rich condensate toward the drift area where it precipitates. In Figure 5-37(d), the liquid saturation within the precipitate cap region is very large because of the large capillary there, but the absolute amount of water is very small because of the small porosity of the fracture in the cap.

Figure 5-38(a) shows that at 1000 yr the precipitate cap has grown even further. Figure 5-38(c) shows almost complete rewetting above the drift.

At 5000 yr, the precipitation cap shown in Figure 5-39(a) has about reached its maximum size. Figure 5-39(c), representing the matrix saturation, shows that the wetting front has arrived at the drift walls. However, as shown in Figure 5-39(b), the temperatures around the drift are above the boiling point so that some two-phase refluxing occurs.

At 900,000 yr, Figure 5-40(a) shows the result of the slow dissolution of the precipitation cap as infiltration flows down fractures. Because of the geothermal gradient included in the model, the temperature increases slightly with depth. The solubility of quartz and cristobalite increases with temperature so that the infiltration reaching the repository level can be slightly undersaturated in silica. Most of the dissolution appears to occur in the rock along the sides of the drift. 


\subsubsection{Effect of Infiltration}

Figure 5-41 shows the relative porosity change in the fractures at $1000 \mathrm{yr}$ for the $1 \times A_{\mathrm{S}}$ base case, but with the infiltration rate reduced by a factor of one-fifth from $16 \mathrm{~mm} / \mathrm{yr}$ to $3.2 \mathrm{~mm} / \mathrm{yr}$. The lower infiltration rate allows for a larger boiling region so the boiling regime is more horizontal and higher above the repository. The impact is a precipitation cap that is less arched and less formation of precipitate below the drift elevation. The overall volume of the precipitation cap appears to be nearly the same, however.

Figure 5-41 Relative change to fracture porosity at $1000 \mathrm{yr}$ for the base case with infiltration reduced by a factor of $1 / 5$ (corresponding to reference Case 2)

\subsubsection{Effect of Hydrological Parameters}

Figure 5-42(a) shows the relative porosity change in the fractures for the $1 \times A_{\mathrm{S}}$ base case at 1000 yr, but the December 1997 "Modified TH" property set given in Tables 3-9 through 3-11 of this report is used instead. Notice that the precipitation cap entirely surrounds the drifts. This property set has, among other differences, a higher fracture capillarity. Thus, the gravity-driven fingers of precipitate between drifts seen in the base case are now pulled in the horizontal direction. In Figure 5-42(b), the precipitation cap at 10,000 yr has grown to a substantial thickness.

Figure 5-42 Relative change to fracture porosity for the base case using hydrologic properties from the December 1997 "Modified TH" property set (corresponding to reference Case 3)

\subsubsection{Line-Load Case}

Also considered was the 57-MTU/acre, line-load case with 85-m drift spacing. The heat load is twice as linearly concentrated, compared to the base case. Figure 5-43(a) shows the relative fracture porosity change at $1000 \mathrm{yr}$ for $3.2-\mathrm{mm} / \mathrm{yr}$ infiltration with $1 \times A_{\mathrm{S}}$ mineral surface area. The combination of line-load and lower infiltration flux allows for the boiling zone to extend away from the drift at $1000 \mathrm{yr}$. The result is a distinctly semicircular precipitation cap that is different from the previous one for the base case. Note that the cap does not coalesce between drifts, but can allow for drainage between drifts.

Figure 5-43 Relative change to fracture porosity at $1000 \mathrm{yr}$ for the line-load case (corresponding to reference Case 5)

Figure 5-43(b) shows the same case, except that infiltration flux is increased to $16 \mathrm{~mm} / \mathrm{yr}$. The higher infiltration flux reduces the radial distance of the boiling zone around the drift. Therefore, the radius of the precipitation cap is correspondingly diminished. Additional numerical studies will be conducted to determine whether the thick, bulbous segment of the precipitate cap above the drift is real or is an artifact of low grid resolution. In any case, the general trend of the lateral extent of the cap on infiltration flux is clearly evident.

Now described are simulations that include amorphous silica with cristobalite and quartz. The precipitation of cristobalite and quartz is turned off to allow for amorphous silica precipitation, which is often observed in the field. Parameters for amorphous silica were from Rimstidt and Barnes (1980). Parameters for cristobalite and quartz are the same as in the previous runs and were found to be numerically close those in Rimstidt and Barnes, given the experimental errors involved, especially for measurements at low temperatures. 
Figure 5-44(a) shows the relative porosity change in the fractures at $100 \mathrm{yr}$ for the basecase point load (16 mm/y infiltration rate). The precipitation of amorphous silica occurs over two regions: as the formation of a relatively thin arch above the drift and as rectangular zones at the drift pillars below the depth of the drifts. The latter zones arise from precipitation of silica as condensate drains to the cooler regions rather than from evaporation of water, as is the case for the precipitation at the arch. Figure 5-44(b) shows the relative porosity change in the fractures at $1000 \mathrm{yr}$. The arch has increased because of refluxing above the drift. However, there are now "dissolution channels" where infiltration and condensate flux can flow down along each side of the drift. The location of the channels is determined by the fact that there is a relatively thin region of precipitate between the rectangular pillar zones and the drift. Above the drift, dissolution cannot not occur because precipitation is continuing to form by refluxing of the gravity-driven heat pipe. Figure 5-44(c) shows the relative porosity change in the fractures at 10,000 yr. The dissolution channels become partially plugged as temperatures cool and silica is transported from above the arch to the channels.

Figure 5-44 Relative change to fracture porosity for the base case (corresponding to reference Case 1) only, allowing precipitation of amorphous silica

The 57-MTU / acre line-load case (16 mm/y infiltration rate) with $85-\mathrm{m}$ drift spacing is considered next. Figure 5-45(a) shows the relative porosity change in the fractures at $100 \mathrm{yr}$. There is a considerable difference compared to results for the point-load case. The initial refluxing zone is established further from the drift, leading to the formation of a wide precipitate arch, or cap. Figure 5-45(b) shows, at $1000 \mathrm{yr}$, the same arch but with two vertical arms hanging down at its ends. These arms are from silica precipitation as condensate drains down the tips of the arch to cooler regions of the rock. The precipitation zone above the drift has thickened as refluxing in the boiling zone moves closer to the drift. Figure 5-45(c) illustrates the relative porosity change in the fractures at 10,000 $\mathrm{yr}$ and shows that the arch has further thickened above and to the sides of the drift. Note that the fractures in the pillar region remain open, allowing for drainage of any increases in infiltration.

Figure 5-45 Relative change to fracture porosity for the line-load (corresponding to reference Case 5) only, allowing precipitation of amorphous silica

\subsubsection{Conclusions and Potential Ramifications}

A new and unique capability has been developed for numerically modeling coupled TH and geochemical phenomena in a fractured rock mass driven by repository heat. Although the geochemistry is idealized, this model can be used with other geochemical modeling methods to analyze more detailed processes. In particular, the flow field from this model will be used, but with more sophisticated geochemistry, as input to other models that do not have TH flow capabilities. Future studies will include modeling of the calcite system.

The preliminary modeling study presented here illustrates how geochemical phenomena can potentially alter the multiphase fluid flow field and vice versa. The consequences to repository performance may be substantial. Reduction in fracture aperture around the drifts may lead to reduced seepage into the drifts. However, at high heat loads with close drift spacing, the region in which there is precipitation in the fractures will probably be of significant lateral extent in the repository horizon, possibly leading to perched condensate in some locations. One of the advantages of the line-load thermal design is that the regions of precipitation are less likely to coalesce between drifts, thereby allowing condensate to drain between drifts. Moreover, precipitation in the fractures will tend to occur over regions of high refluxing such as over hotter WPs rather than over regions of low refluxing such as over 
cooler WPs. Thus, spatial flux conditions caused by heating heterogeneity is potentially further increased by geochemical changes. These considerations emphasize the importance of the role of engineering design in increasing the likelihood that geochemical changes will enhance rather than detract from repository performance.

Another potential impact is the change in the transport and flow properties below the repository. According to this preliminary modeling study, most of the alteration within fractures may be expected to occur above and to the sides of the drifts. However, if the capillary pressure in the fractures is sufficiently strong, as demonstrated in one of the property sets that was modeled, an alteration can also be present below the drifts. In many of the modeled cases, significant porosity changes in the matrix were, indeed, present below the repository.

\subsection{Drift-Scale THC Scoping Calculations and Alteration of Flow Pathways Above and Below Repository Drifts}

\section{by William Glassley}

The purpose of the work presented in this section is to document the sensitivity of mineral development to differences in temperature and thermally influenced liquid flux. This study provides preliminary bounds on the nature and extent of mineralogical change within the $\mathrm{AZ}$ and the extent to which these changes are sensitive to TH rock properties, configuration of heat sources within emplacement drifts, and coexisting gas composition.

\subsubsection{Introduction}

The region considered is a vertical cross-section through the mountain from the ground surface to the top of the saturated zone (approximately $725 \mathrm{~m}$ ). The horizontal distance extends 15 to $30 \mathrm{~m}$ from a hypothetical emplacement drift center, depending on the case considered (see Chapter 3 for descriptions of the reference cases). This volume of rock was chosen for consideration to establish the extent of mineral alteration during heating of the repository block.

For each case considered, arrays of values for the horizontal and vertical flux vectors for the liquid fracture flux, fracture saturation, and the temperature were extracted at time steps of 10, 100, 1000, and 10,000 yr. These data were extracted from NUFT line-averaged-heatsource, drift-scale thermohydrologic (LDTH) simulations for the reference cases (Chapter 3). The flux values at each time step were multiplied by the saturation values to estimate mass flux in an equivalent, saturated fracture continuum such that proportional effects of chemical processes on hydrologic properties would be comparable to partially saturated conditions predicted by NUFT.

Reactive-transport simulations were conducted using the code GIMRT (Steefel and Yabusaki, 1996) to investigate key processes at each of the time steps-10, 100, 1000, and $10,000 \mathrm{yr}$-assuming that the temperature and flow fields at each of these time steps would remain the same for a 10-yr period. Mineral development during each 10-yr period was examined after 1,5, and 10 yr of reaction. The simulations were carried out using a rectangular mesh with uniform cell dimensions of $0.5 \mathrm{~m}$ horizontally and $16 \mathrm{~m}$ vertically. These dimensions were selected to allow evaluation of the large-scale features that will affect porosity-permeability structure and chemical-mineralogical evolution within the AZ. Finer meshes will be used in future simulations to evaluate local characteristics of AZ evolution.

The approach just outlined permitted evaluation of the sensitivity of secondary mineral development to differences in flow fields resulting from different $\mathrm{TH}$ properties and heating scenarios used in four of the reference cases. The consequences of permeability modification by dissolution and/or precipitation effects are not incorporated by this approach. 
Also considered was the effect of varying the $\mathrm{CO}_{2}$ content of the gas phase coexisting with condensate. Analyses of in situ pore gas from the UZ at Yucca Mountain (Yang et al., 1988) indicate that the pore gas has $\mathrm{CO}_{2}$ contents that are elevated, relative to atmospheric values, by a factor of 1.5 to 3 . Measurements of gases taken to establish the pore-gas baseline for the Drift-Scale Test (DST) also indicate the level of $\mathrm{CO}_{2}$ is elevated relative to atmospheric values.

In addition, the host rock at Yucca Mountain contains a minor amount of $\mathrm{CO}_{2}$ in the gas phase and about $10^{3}$ times as much in matrix pore water as dissolved inorganic carbon (DIC). $\mathrm{CO}_{2}$ has been extracted, using published methods, from preserved core samples by distillation at temperatures as high as $200^{\circ} \mathrm{C}$ (Davidson et al., 1995). The extraction results have shown that $\mathrm{CO}_{2}$ is more abundant than might be expected. One set of extractions is summarized in Table 5-9, which shows that if the total carbon yield is equated to DIC, then the equivalent pore water bicarbonate concentration is 2 to 10 times that obtained using Henry's Law with a pore gas $\mathrm{CO}_{2}$ partial pressure of $10^{-3} \mathrm{~atm}$, and assuming near-neutral $\mathrm{pH}$. Similar observations were reported by Yang et al. (1996) and Davidson et al. (1995). The carbon samples described in Table 5-9 were analyzed for carbon isotopes and found to contain $45 \%$ to $105 \%$ modern carbon, which is comparable to the UZ pore gas at Yucca Mountain. There are several candidate explanations for the abundance of $\mathrm{CO}_{2}$, including $\mathrm{pH}>8.5$ in the matrix pore water and sorption of bicarbonate at the water-rock interface. For this discussion, it suffices that distillation of the tuff matrix produces abundant $\mathrm{CO}_{2}$, and that, because of carbonate precipitation during the extraction, the laboratory extractions may underestimate the $\mathrm{CO}_{2}$ actually present.

Table 5-9 Carbon dioxide extraction yield from distillation of condensable gases from preserved Yucca Mountain core samples

\begin{tabular}{|l|c|c|c|c|c|c|}
\hline $\begin{array}{c}\text { Sample ID } \\
\text { Borehole/Depth (ft) }\end{array}$ & $\begin{array}{c}\text { Dry } \\
\text { Mass }\end{array}$ & $\begin{array}{c}\mathbf{H}_{2} \mathbf{O} \\
\text { Yield } \\
(\mathbf{g})\end{array}$ & $\begin{array}{c}\text { Water/ } \\
\mathbf{R o c k} \\
(\mathbf{g} / \mathbf{k g})\end{array}$ & $\begin{array}{c}\text { Carbon } \\
\text { Yield } \\
(\mathbf{m g})\end{array}$ & $\begin{array}{c}\text { Carbon/ } \\
\left.\mathbf{H}_{\mathbf{2}} \mathbf{O} / \mathbf{m g}\right)\end{array}$ & $\begin{array}{c}\text { As } \\
\mathbf{H C O}_{3} \\
(\mathbf{m g} / \mathbf{k g})\end{array}$ \\
\hline \hline SD-12/691.3-693.3 & 3.04 & 125 & 41.0 & 9.6 & 77 & 393 \\
\hline SD-7/1810.2-1814.0 & 2.63 & 133 & 50.5 & 14.9 & 112 & 571 \\
\hline SD-9/1360.1-1363.8 & 3.07 & 92 & 29.9 & 5.4 & 59 & 299 \\
\hline SD-7/1131.7-1133.3 & 2.54 & 87 & 34.3 & 6.2 & 71 & 360 \\
\hline NRG-7/7A/960.2-961.5 & 3.17 & 126 & 39.8 & 4.3 & 34 & 172 \\
\hline NRG-7/7A/386.7-388.0 & 2.99 & 81 & 27.1 & 2.4 & 29 & 148 \\
\hline SD-7/409.7-412.6 & 2.56 & 81 & 31.6 & 4.0 & 49 & 249 \\
\hline SD-12/1262.1-1263.8 & 1.92 & 58 & 30.3 & 2.7 & 47 & 239 \\
\hline
\end{tabular}

Finally, modeling of water compositions obtained from the SHT suggest that condensate formation occurs in equilibrium with elevated $\mathrm{CO}_{2}$ partial pressures (Glassley and DeLoach, 1997). This suite of data suggests that the effects of different $\mathrm{CO}_{2}$ partial pressures need to be considered when doing reactive-transport simulations. Described in this report are preliminary simulations that examine this effect.

\subsubsection{Conceptual Model}

Studies in the Exploratory Studies Facility (ESF) have documented that fractures showing evidence of water flow in the past consistently are lined by calcite and are accompanied by minor quantities of silica polymorphs, alkali feldspars, and clay (Paces et al., 1996; Vaniman et al., 1997). The proportions of these mineral phases are not well constrained by field 
observations, but calcite consistently dominates the observed mineral assemblages. On the basis of these reported characteristics, the relative proportions of the mineral phases shown Table 5-10 were selected to represent water-mineral interaction in fractures.

Table 5-10 Minerals considered in the simulations, their initial abundances and surfaces along the fracture surfaces, and the controlling rate constants and respective activation energies for the applicable dissolution reactions

\begin{tabular}{|l|c|c|c|c|}
\hline \multicolumn{1}{|c|}{ Minerals } & $\begin{array}{c}\text { Initial Volume } \\
\text { Fraction }\end{array}$ & $\begin{array}{c}\text { Rate } \\
\text { Constant }^{\star}\end{array}$ & $\begin{array}{c}\text { Activation } \\
\text { Energy }^{\star \star}\end{array}$ & $\begin{array}{c}\text { Surface } \\
\text { Area }^{\star \star *}\end{array}$ \\
\hline \hline Calcite & $9.5 \mathrm{e}-3$ & $1.000 \mathrm{E}-11$ & 10.0 & 1000.0 \\
\hline Quartz & $1.0 \mathrm{e}-4$ & $1.259 \mathrm{E}-14$ & 20.9 & 10.0 \\
\hline alpha Cristobalite & $1.0 \mathrm{e}-4$ & $3.467 \mathrm{E}-13$ & 16.47 & 100.0 \\
\hline Kaolinite & $1.0 \mathrm{e}-5$ & $1.000 \mathrm{E}-13$ & 15.0 & 100.0 \\
\hline K-Feldspar & $1.0 \mathrm{e}-5$ & $1.000 \mathrm{E}-12$ & 13.8 & 100.0 \\
\hline Albite & $5.0 \mathrm{e}-6$ & $1.000 \mathrm{E}-12$ & 16.2 & 100.0 \\
\hline Diaspore & 0.0 & $3.000 \mathrm{E}-13$ & 15.0 & 0.0 \\
\hline Mesolite & 0.0 & $3.000 \mathrm{E}-13$ & 15.0 & 0.0 \\
\hline Stilbite & 0.0 & $3.000 \mathrm{E}-13$ & 15.0 & 0.0 \\
\hline Porosity & 0.990275 & n.a. & n.a. & n.a. \\
\hline
\end{tabular}

${ }^{*}$ moles $/ \mathrm{m}^{2}$-sec at $25^{\circ} \mathrm{C}$

** $\mathrm{kcal} / \mathrm{mole}$

*** $\mathrm{m}^{2} / \mathrm{m}^{3}$ of rock

n.a. not applicable

For all cases, three characteristic regions develop during the TH evolution of the system considered here. Directly below the heated region, water vapor will condense at approximately the location of the boiling-point isotherm and will drain under the influence of gravity toward the saturated zone. This lower zone characteristically has a simple thermal gradient going from high temperature near the heated region toward low temperature near the saturated zone and has fluid flux vectors that are predominately directed downward.

A second region accounts for the rock mass between heated areas (i.e., the pillar region). The pillar region is assumed to extend tens of meters vertically above and below the repository horizon. The thermal conditions in this region will be complex, with highest temperatures occurring adjacent to the heated areas in the central portion of the pillar region. Fluid flow here may include both water draining from the reflux zone above the heated region and condensate that forms in this region. Fluid flow through the pillar region eventually enters the lower zone, in which water flows under the influence of gravity to the saturated zone and down the local temperature gradient.

Above the heated area, water vapor generated during heating will migrate upward and condense in cooler regions. Within this third region, the condensed water will flow, under the influence of gravity, back toward the heated region, eventually reaching the boiling zone where it will vaporize and deposit the solute load it contains. The coupled THC phenomenology of that reflux zone cannot be fully simulated with GMIRT code because boiling processes are not incorporated in the code. However, with the exception of mineral deposition at the boiling front, evolution of this zone can be modeled using the same approach as that used for the pillar region. In summary, the regions considered in this report 
are the lower zone of condensate formation and flow below the heated region, the pillar region (which also encompasses the lower zone), and the region above the heated drift. This approach permits consideration of the effects of condensate formation and drainage in the regions most likely to be affected.

Different strategies are followed for modeling these areas. For the lower zone, it is assumed that reactive transport was constrained to occur only within the region that extends from the lower boiling-point isotherm down to the saturated zone. This provides a realistic evaluation of mineral evolution in those regions that will not be affected by condensate drainage from above (e.g., the area immediately below the heated drifts).

A second strategy was implemented to account for condensate formation within the pillar region and to evaluate the consequence on mineral development in the lower zone if liquid flow traverses a significant path length through the pillar region before entering the lower zone. In this second strategy, condensate formation and associated reactive transport are allowed to commence above the upper boiling-point isotherm, in the region where temperatures are approximately $90^{\circ} \mathrm{C}$, and at a distance of $7.5 \mathrm{~m}$ from the heated drift. This "stand-off" distance was chosen to avoid any influence of lateral drainage on mineral development so that the observed evolution reflects only that of drainage within the pillars.

The third strategy allows reactive transport throughout the area and assumes only that condensate formation occurred at the $90^{\circ} \mathrm{C}$ isotherm and that no reactive transport occurred in the vicinity of the heated drift where conditions will generally be at or higher than boiling. This last scenario allows evaluation of the interplay between fluid movement in the various regions.

\subsubsection{Model Input}

Table 5-10 shows the parameters specified as the initial conditions for all reactivetransport simulations. Reaction-rate constants are based on published results or on values derived from plug-flow reactor experiments documented previously (Johnson et al., 1997, 1998) or are estimated.

The mineral-reactive surface areas are selected to approximate the relative reactive surface areas along a fracture surface. The surface-area parameter is highly uncertain and probably varies along the surface of fractures both between and within specific lithologic units.

The initial abundances of the mineral phases used in the simulations reflect the approximate relative proportions of these minerals reported in studies of the ESF fracture mineralogy (Paces et al., 1996; Vaniman et al., 1997). As with the surface areas, the relative mineral abundances actually vary from location to location within a fracture. Sensitivity studies will be conducted to evaluate the extent to which modification of the results of this study will result with variation in the surface-area parameters and within absolute and relative abundances of the initial mineral phases.

Because the flux conditions used in the simulations are those within the fracture system, the absolute abundances of the initial mineral phases are selected such that porosity modification has little influence on the flow field. The intent is to evaluate the effects on mineral development. By constraining the system to have initial high porosity and initial low mineral abundances, the flux vectors are ensured to remain essentially constant throughout the time period for which simulations are conducted. This approach leads to the minimum possible mineral development and provides a lower limit to the extent of porosity change during reactive transport and a lower limit on the magnitude of mineral growth. The effects on the locations of mineral change are probably minimal. 
The initial water composition is assumed to be that of condensate (i.e., essentially, distilled water) in equilibrium with a $\mathrm{CO}_{2}$ partial pressure of 0.1 bars. This elevated $\mathrm{CO}_{2}$ partial pressure was selected to account for the effects of exsolution of dissolved gases during heating and boiling of pore water (Arthur and Murphy, 1989; Glassley and DeLoach, 1997; Criscenti and Arthur, 1989) and to take into account the apparent elevation of $\mathrm{CO}_{2}$ partial pressure in the ambient site pore gases.

The secondary minerals selected for inclusion in these simulations are those that consistently appear in simulations of rock-water interaction involving the fracture mineralogy and high-silica rhyolitic rocks (Glassley, 1997; Glassley and DeLoach, 1997). The system considered in this study comprises the components $\mathrm{CaO}-\mathrm{Na}_{2} \mathrm{O}-\mathrm{K}_{2} \mathrm{O}-\mathrm{SiO}_{2}-\mathrm{Al}_{2} \mathrm{O}_{3}-$ $\mathrm{H}_{2} \mathrm{O}-\mathrm{CO}_{2}$. Not considered in this suite of simulations are minerals that contain Fe or $\mathrm{Mg}$. Although these components account for a small mass fraction of the rock system, they may be important in the development of the redox conditions $(\mathrm{Fe})$ and in clay and zeolite formation (Fe and $\mathrm{Mg}$ ). In general, however, it has been found that exclusion of these components, and their respective phases, has little impact on the distribution and abundance of the secondary mineral phases considered in this study. The principle differences to be expected are that no Fe oxide or hydroxide phases will develop in these simulations, and the specific zeolite and clay mineral species occurring here (stilbite and mesolite, and kaolinite, respectively) may be partially or completely superseded by such species as clinoptilolite, heulandite, mordenite, or smectite clays. These differences are not significant for the nature and extent of mineral alteration and effects on rock TH properties. However, for considerations regarding radionuclide transport, these differences in mineralogy may be significant.

\subsubsection{Results}

Although a large number $(>100)$ of simulations has been conducted to determine the sensitivity of this system to the variables of interest, description of the entire suite of results is beyond the scope of this report. Instead, succinct presentation of the results is attempted by comparing results for a selected time interval. Because it was apparent that the largest magnitude changes are in the amount of calcite dissolved, the calcite dissolution behavior was compared for all cases at all time intervals, and a "standard" time was selected for all other comparisons.

To evaluate the nature of the changes and the rates at which they occur, the results are compared for the 1,5, and 10-yr calcite abundances, Cases 1 and 3, for all time intervals (Figure 5-46, Figure 5-47, Figure 5-48, Figure 5-49, Figure 5-50, Figure 5-51, Figure 5-52, and Figure 5-53), within the lower zone. In these figures, the abundance of calcite, as a percentage of the total solids, is shown. Only the $93 \%$ to $95 \%$ abundance envelope is shown because it is the one that encompasses most of the spatial distribution of the change. Similar results are obtained for simulation suites examining the pillar region and the region above heated drifts.

Figure 5-46 Calcite abundance as a function of location for Case 1 after $10 \mathrm{yr}$ heating

Figure 5-47 Calcite abundance as a function of location for Case 1 after 100 yr heating

Figure 5-48 Calcite abundance as a function of location for Case 1 after 1000 yr heating

Figure 5-49 Calcite abundance as a function of location for Case 1 after 10,000 yr heating

Figure 5-50 Calcite abundance as a function of location for Case 3 after $10 \mathrm{yr}$ heating 
Figure 5-51 Calcite abundance as a function of location for Case 3 after $100 \mathrm{yr}$ heating

Figure 5-52 Calcite abundance as a function of location for Case 3 after $1000 \mathrm{yr}$ heating

Figure 5-53 Calcite abundance as a function of location for Case 3 after 10,000 yr heating

For both cases, the results show that the spatial distribution of calcite dissolution is rapidly evolving between years 1 and 5 . However, the differences between 5 and $10 \mathrm{yr}$ are small. This pattern directly reflects the rates of dissolution reactions in this system: calcite dissolution in mildly acidic solutions is much faster than that for the silicate phases. As a result, calcite dissolution will rapidly proceed in regions where condensate forms. Relatively rapid chemical evolution of the condensate will then occur along the flow path. Within about $5 \mathrm{yr}$, the main features of the mineralogical system are established. The principal changes that occur are dissolution of the initial phases (calcite, feldspars, and clay) and formation of zeolites and hydrated aluminous phases. It was concluded that comparison of mineralogical changes after $5 \mathrm{yr}$ of reaction indicates the representative changes for comparison among cases.

Figure 5-54 shows behavior typical of all of the primary and secondary minerals. For all cases, the extent of mineralogical change is relatively minor during the first $10 \mathrm{yr}$ heating. For all other time periods (i.e., for times $\geq 100 \mathrm{yr}$ ), the magnitude of mineralogical change is much larger. After the initial buildup, the time rate of change is approximately constant for these longer time intervals. The observed change in porosity (Figure 5-55) shows the same relatively constant rate of change for longer time periods; for the early period considered, however, rate of change is higher, for reasons discussed subsequently. The results from all simulations are similar for time steps at $1000 \mathrm{yr}$ or more. Hence, all the remaining discussion describes changes observed for the 1000-yr interval, based on a 5-yr reaction.

Figure 5-54 Production rate of stilbite (a zeolite) for each case examined as a function of time

Figure 5-55 Porosity rate of change, for each case examined as a function of time

It is important to emphasize that the absolute abundance of specific mineral phases and the locations at which they will dissolve or precipitate depend on the initial condition and the specific TH characteristics of the relevant flow field. No single time period is exactly representative of all time periods or reaction duration, and the 5-yr reaction at the 1000-yr time step is used only as a means of comparing cases. This will be termed the "comparison period" in the following discussion.

For the sake of completeness, Figure 5-56 shows the 93\% to 95\% calcite abundance distribution for Cases 2 and 5 for the comparison period.

\section{Figure 5-56 Calcite abundance as a function of location for Cases 2 and 5 after 1000 yr heating}

Figure 5-57 through Figure 5-60 and Figure 5-61 through Figure 5-64 show, respectively, the temperature distribution and liquid fracture flux vectors for the comparison period for each case. It is evident that the differences in temperature distribution are very small, but the flow distributions are significantly different, even though all cases show a predominance of downward-directed, gravity-driven flux.

Figure 5-57 Temperature distribution for Case 1 after 1000 yr heating 
Figure 5-58 Temperature distribution for Case 2 after 1000 yr heating

Figure 5-59 Temperature distribution for Case 3 after 1000 yr heating

Figure 5-60 Temperature distribution for Case 5 after 1000 yr heating

Figure 5-61 Flux vector distribution for Case 1 after $1000 \mathrm{yr}$ heating

Figure 5-62 Flux vector distribution for Case 2 after 1000 yr heating

Figure 5-63 Flux vector distribution for Case 3 after $1000 \mathrm{yr}$ heating

Figure 5-64 Flux vector distribution for Case 5 after $1000 \mathrm{yr}$ heating

In all cases, the location where condensate first interacts with rock is a region in which dissolution of calcite occurs and porosity increases. The region of dissolution tends to be relatively narrow vertically, but extends laterally throughout the region where significant condensate flux occurs because the solution is far from equilibrium with the minerals with which it is in contact, thus resulting in dissolution of the initial mineralogy. After a period of time, the length of which is determined by the dissolution kinetics of the initial mineral phases, the solution tends to achieve concentration levels of dissolved species that are close to saturation values for secondary minerals to form and, hence, for calcite dissolution to diminish. Because the system is dynamic, the time required for significant progress of rate reactions is sufficient for the liquid phase to flow a significant distance along the flow path. Hence, for all scenarios, porosity will increase over distances of as many as several meters to tens of meters in the regions where condensate forms.

It is also observed in all cases that zeolite formation consistently occurs in the low-flux region between elevations of $\sim 145 \mathrm{~m}$ to $\sim 245 \mathrm{~m}$ above the saturated zone (the ch2 $\mathrm{vc}$ unit; see Chapter 3). This phenomenon reflects the fact that saturation in the zeolite phases is only achieved after there has been sufficient contact time between the evolving condensate solution and the fracture mineralogy. The time needed to achieve this state is consistently satisfied within this zone where fracture fluxes drop to very low values (between 0 and $250 \mathrm{~m} / \mathrm{yr}$ ) and thus allow long contact times. (Fracture flux is lower because the matrix flux is greater.) Because the total mass flux through this region is small, the absolute abundance of secondary mineral phases formed in this region is also small. The conditions needed to result in saturation in a zeolite phase are also satisfied along some of the faster flow pathways, reflecting unique features of the individual cases considered, as discussed in subsequent text.

\subsubsection{Contrasts Between Cases: No Drainage Considered In Pillar Region}

The pattern of mineral development considered here (Figure 5-65, Figure 5-66, Figure 5-67, Figure 5-68, and Figure 5-69) reflects the conditions for the lower zone simulations. Note that, for this comparison, reactive transport was not permitted in the regions higher than $\sim 325 \mathrm{~m}$ above the saturated zone. Mineralogical evolution shown in the accompanying figures in this higher-elevation area results solely from the equilibration of the condensate with the fracture mineralogy at elevated temperatures. The results representing the reactive-transport simulations for this lower-zone instance are shown in the following five figures. 
Figure 5-65 Porosity, calcite, stilbite, and diaspore distributions for Case 1 after 1000 yr thermal evolution

Figure 5-66 Porosity, calcite, stilbite, and diaspore distributions for Case 2 after 1000 yr thermal evolution

Figure 5-67 Porosity, calcite, stilbite, and diaspore distributions for Case 3 after 1000 yr thermal evolution

Figure 5-68 Porosity, calcite, stilbite, and diaspore distributions for Case 5 after 1000 yr thermal evolution

Figure 5-69 Mesolite distributions for the cases represented in the preceding four figures

The impact of different infiltration rates can be evaluated by comparing the results for Cases 1 and 2 where the initial TH properties are the same but the infiltration rates differ by a factor of 5 (Figure 5-65 and Figure 5-66). In general, the lower flux values (Case 2) lead to smaller volumes of secondary minerals forming along the flow path. This reflects the fact that the extent of secondary mineral development is, in part, a function of the total mass flux. In addition, for lower flux values, the distribution of flux is more uniform in nonequilibrium TH models. Hence, the chemical alteration effects are, for a given volume of water, distributed through a larger volume of rock.

The impact of modifying the TH rock properties, at a constant infiltration rate, can be assessed by considering the differences between Cases 1 and 3 . In this instance, the properties used in Case 3 lead to flux distributions that are less affected by fracture capillarity, which causes dissolution to be restricted to smaller areas than those for Case 1 . The only exception is the region immediately below the heated area where zeolite development extends to shallower levels. This latter condition reflects the effect of decreasing the flux in the area immediately below the drifts, which results in greater contact time.

The most significant differences in mineral distribution are between Cases 1 and 5, which respectively consider the point load, using base-case TSPA-VA TH rock properties, and the line load, using the "modified $\mathrm{TH}^{\prime}$ " property set. For both cases, the nominal infiltration flux is used. Unlike any of the other scenarios, calcite dissolution in Case 5 is concentrated in the area immediately below and adjacent to the heated area. Very little dissolution occurs within the pillar region laterally adjacent to the heated region, although fluxes are such that dissolution is also observed at the edge of the modeled region. Furthermore, zeolite development occurs in a more complex distribution, extending within the pillar region up to the elevation of the heated area.

\subsubsection{Contrasts Between Cases: Drainage Permitted in Pillar Region}

In this case, the simulations were run allowing water and mineral evolution to proceed in that region more than $7.5 \mathrm{~m}$ laterally away from the heated area and at depths deeper than the shallowest $90^{\circ} \mathrm{C}$ isotherm. Hence, drainage through the pillar region was allowed to affect mineral evolution in the pillar and to modify mineral development in the lower zone. In this instance, the differences between Cases 2 and 5 (Figure 5-70, Figure 5-71, Figure 5-72, Figure 5-73, Figure 5-74, and Figure 5-75) were considered because they have the greatest contrast in initial conditions (point load vs. line load, TSPA-VA base-case DKM properties vs. enhanced properties, reduced infiltration vs. nominal infiltration). 
Figure 5-70 Porosity distribution for Case 2 after 1000 yr thermal evolution, under the pillar flow constraints

Figure 5-71 Stilbite distribution for Case 2 after 1000 yr thermal evolution, under the pillar flow constraints

Figure 5-72 Mesolite distribution for Case 2 after $1000 \mathrm{yr}$ thermal evolution, under the pillar flow constraints

Figure 5-73 Porosity distribution for Case 5 after 1000 yr thermal evolution, under the pillar flow constraints

Figure 5-74 Stilbite distribution for Case 5 after 1000 yr thermal evolution, under the pillar flow constraints

Figure 5-75 Mesolite distribution for Case 5 after $1000 \mathrm{yr}$ thermal evolution, under the pillar flow constraints

In both cases, mesolite (a zeolite) distributions extend into the pillar region and above the level of the heated area. Stilbite distributions remain virtually unchanged. In addition, the absolute abundance of zeolites increased. Both of these effects are due to increasing the flow path length and thus enhancing the contact time between solution and fracture mineralogy. In the regions between the saturated zone and the heated area, zeolite distributions are similar to those seen for the case in which no reaction was permitted in the pillar area.

Porosity increase in the pillar region, in both cases, is concentrated in that area where condensate is modeled to first contact the fracture mineralogy. There is no noticeable change in the calcite dissolution below the heated area compared to the case in which no pillar flow was considered.

\subsubsection{Contrasts Between Cases: Drainage Permitted in Pillar Region and Over Drifts}

In this instance, the only limitation placed on reactive transport was that it was not allowed in the immediate vicinity of the heated area. The volume of nonreaction extended $7.5 \mathrm{~m}$ toward the pillar region and from $5 \mathrm{~m}$ below to $27 \mathrm{~m}$ above the heated area.

Comparison of the results of these simulations (Case 1, Figure 5-76; Case 5, Figure 5-77) shows that porosity increase and calcite dissolution occur in approximately the same areas and to the same extent for both cases. The most significant result with respect to these properties is that dissolution of calcite and porosity increase occur within the main region of condensate formation and extend continuously from the region over the heated area into the pillar region.

Figure 5-76 Porosity, calcite, stilbite, and mesolite distributions for Case 1 after 1000 yr thermal evolution

Figure 5-77 Porosity, calcite, stilbite, and mesolite distributions for Case 5 after 1000 yr thermal evolution

The principal differences are in secondary mineral development. The most extensive mineralogical contrast is in the distribution of mesolite formation. In Case 5, mesolite forms throughout the region of condensate flow and at small but significant abundances, in contrast 
to its development in Case 1 where it is less extensively developed. In Case 5, development of this zeolite completely encompasses the heated area. Stilbite formation, in both cases, is slightly more extensive than for the case in which condensate flow in the pillar is considered.

In this case, the difference that would develop in mineral assemblages if the gas phase composition were an order of magnitude less than atmospheric values was also examined. The results are shown in Figure 5-78, for Case 1. Comparison of these results with those in Figure 5-76 (also Case 1) show that the lower gas $\mathrm{CO}_{2}$ partial pressure leads to more extensive development of secondary zeolites. For both stilbite and mesolite, total abundance increases, and the distribution expands. Furthermore, the area of dissolution of calcite is more extensive. Porosity, however, is more extensively reduced. These differences are caused by the interactions in solution of the carbonate and bicarbonate complexes with other metal ions. The result is that a drop in $\mathrm{CO}_{2}$ partial pressure, relative to in situ or atmospheric values, modifies solution chemistry sufficiently to lead to increased saturations in the solid phases.

Figure 5-78 Porosity, calcite, stilbite, and mesolite distributions for Case 1 after 1000 yr thermal evolution

\subsubsection{Discussion}

\subsubsection{Implications for Porosity Modification by Mineralogical Change}

The simulations conducted here are designed to allow evolution of fracture mineralogy without leading to significant absolute changes in porosity. This is done to allow evaluation of the effects that initial flow-field conditions and thermal-field conditions would have on mineralogical evolution without complications arising from large changes in the porositypermeability structure of the system. To accomplish this, large fracture porosities and small mineral volumes are used. As a result, the absolute changes seen in mineral volumes are very small. These values will not reflect the actual changes expected in the repository. To represent such changes, further simulations will be conducted in which significant porosity evolution is allowed to proceed as mineralogical changes occur. In essence, future simulations will be done for conditions having much lower fracture porosities and higher initial mineral volumes and reactive surface areas. Nevertheless, regardless of the porosity structure, the locations of the mineralogical changes are expected to be similar for similar flux conditions.

One of the predominate effects observed in this suite of simulations is the consistent increase of porosity in regions where condensate first forms. Because condensate is far from equilibrium with the mineralogical assemblage, it will dissolve the ambient mineral assemblage at the site of formation. This process will take place relatively rapidly. The magnitude of porosity increase at a particular location will depend on the duration of condensate formation there and on its $\mathrm{pH}$. The latter, in turn, will be a reflection of the local $\mathrm{CO}_{2}$ partial pressure. Hence, some variability in the extent of porosity evolution should be expected.

The distribution of the condensate zones requires that dissolution be concentrated in two key areas. One area is that above the heated areas, which will be approximately where the upper boiling-point isotherm will be located. However, this area is also the locus of boiling behavior, which is not incorporated in GIMRT simulations and which is expected to cause reduction of fracture porosity and permeability. The other area will be the region below drifts, just below the boiling isotherm. This area is also the location of mineral precipitation in some scenarios. In all scenarios considered, regions of porosity increase will develop above and below the heated region. 


\subsubsection{Sensitivity of Mineral Development to Ambient Site Conditions}

These results show that, the lower the fluid velocity conditions at any particular location, the more concentrated the effects of reactions involving dissolution and precipitation. Low effective velocities can be achieved through low infiltration rate, increased matrix flux relative to fracture flux, $\mathrm{TH}$ conditions that lead to low flow rates, and local increases in porosity. These conditions increase contact time, thus providing more complete reaction extent within a given distance. For all the cases considered, there are certain locations that generally satisfy these conditions and lead to secondary mineral development.

Under all conditions, condensate interaction with fracture mineralogy leads to zeolite development in that region of consistently low fluid velocities at $\sim 150 \mathrm{~m}$ above the saturated zone, which is within the ch2vc unit. The volume of zeolite development here will be a function of the total mass flux in this region. As indicated in Chapter 3, liquid saturations in this area may be extremely low, which would result in only a very small extent of mineral growth. However, the persistence of this condition for the 10,000-yr period of the repository suggests that cumulative mineral development may be significant. Further work to conduct simulations at lower fracture porosities will evaluate the long-term implications of this condition for modification of transport pathways.

The impact of infiltration flux on mineral development is an example of a site parameter that may strongly influence the location of secondary mineral growth. For example, the differences between Cases 1 and 2 in distribution of stilbite directly demonstrate this effect. Lower overall infiltration flux ultimately diminishes the liquid fracture flux in the area below the heated zone in Case 2, thus resulting in longer residence times and more extensive condensate-fracture interaction.

\subsubsection{Differences between Loading Scenarios}

Different point-load and line-load scenarios lead to different patterns of secondary mineral development because each scenario generates different thermal fields and liquid flux distributions. However, for the specific cases considered here, the differences in mineral distribution are relatively minor. In both cases, although there are differences in specific mineral abundances and the details of the mineral distribution patterns, secondary mineral development occurs within the pillar regions. As noted previously, the patterns of fluid movement do not dramatically differ in these two cases within the pillar region; hence, the differences in mineralogical development are small.

What remains significantly different in these two cases are the areas where dissolution is focused. The distinctions suggest that the line-load scenario may lead to more significant dissolution of fracture calcite below the heated drifts than for the conditions modeled in the TSPA-VA base case. The rate of porosity change and its extent are large enough to quickly modify fluid-flow pathways. Finer-scale simulations are underway to evaluate the details of the spatial distribution of this effect and its sensitivity to site parameters.

\subsubsection{Differences Resulting from $\mathrm{CO}_{2}$ Pressure Variation}

The effect of different $\mathrm{CO}_{2}$ partial pressures is to modify the aqueous speciation. This, in turn, influences ion concentrations that determine the saturation state of mineral phases. The consequences seen in these simulations of varying $\mathrm{CO}_{2}$ partial pressure by several orders of magnitude are that lower pressures increase the amount and distribution of the secondary minerals considered. This effect needs further evaluation, however, because not all mineral saturation states will be changed the same way. Work to examine a wider range of minerals is underway. 


\subsubsection{Conclusions}

Flow pathways in the repository block will probably be modified by mineralogical changes during rock-water interaction, especially with TH processes. Most of the changes that impact flow will be concentrated within fracture systems because of the localization of flow there. A suite of simulations has been carried out to examine the sensitivity of dissolution and precipitation in fracture systems to ambient site properties and heating scenarios.

The results demonstrate that, regardless of ambient site conditions or heating strategy, fracture porosity will be enhanced in those areas where condensate forms. This effect occurs because condensate is far from chemical equilibrium with the fracture mineralogy and will tend to dissolve minerals that are present along fracture surfaces until the solution reaches saturation in some secondary phase or phases.

The suite of simulations also demonstrates that the locations where secondary mineral saturations are achieved depend on the ambient site conditions and the specific heating strategy considered. The principal system property that determines whether saturation is achieved is the integrated residence time of the solution on the fracture surface. In general, regardless of the heating scenario or the ambient site properties, low liquid-fluid velocities are consistently observed in the region approximately $150 \mathrm{~m}$ above the saturated zone. Hence, it is expected that, within a few decades of the initiation of repository heating, secondary minerals (primarily zeolites) will form in that region, in those locations where fluid flow has been maintained.

Secondary minerals are also expected to form, after several decades of fluid flow, within the pillars between emplacement drifts. Although the precise distributions of this mineral development are affected by the particular heating strategy and site properties, the occurrence of these minerals in the pillar region is not scenario-dependent.

\subsection{Summary of Thermohydrochemical Models}

Two broad categories of chemical models are described in this chapter: thermodynamic (including reaction-path) models and reactive-transport models. Thermodynamic models calculate the state of a reaction cell based on equilibrium behavior and may also include nonequilibrium behavior expressed as relative reaction rates and controls on reactant availability. The conceptual and mathematical basis for thermodynamic and reaction-path modeling of water-rock interaction is described in detail. Features of the EQ3/6 modeling software package are described, with references to support documentation, and the current status and future developments for the software are discussed.

Reactive-transport models involve rate reactions and a time variable, and they represent a spatial domain. Described are several reactive-transport simulators that incorporate a wide range of chemical species and that represent the past few years of model development reported in the open literature. The 1DREACT simulator is typical; its successor, the OS3D/GIMRT code, is used to evaluate reference cases in this report. The conceptual and mathematical models that form the basis for these codes are presented in detail. Whereas these simulators handle chemical problems with multiple components, strong coupling between components, and heterogeneous phases, they model only aqueous reactions and do not include a vapor phase in mass or energy balances. Hence, they cannot readily be used to simulate processes driven by evaporation or boiling.

Another category of reactive-transport simulators is also presented in this chapter. TH codes such as FEHM and NUFT have been modified to incorporate the chemical behavior of a small number of components. This approach has been useful for studying the effects of boiling processes on mineral precipitation, using silica as a single component. 
A series of four modeling studies was presented in this chapter:

- A reaction-path study of water evolution in contact with the host rock (EQ3/6)

- A reactive-transport simulation of silica movement at the repository scale (FEHM)

- Fully coupled calculations involving silica movement at the drift scale (NUFT)

- Reactive-transport simulations of mineral dissolution and alteration in the $\mathrm{AZ}$ (OS3D/GIMRT)

\section{Reaction-Path Study}

This study analyzed the impact of uncertainty in relative reaction rates used in the EQ3/6 model on the capability to simulate observed water composition in the host rock (J-13 water). Results show that evolution of recharge water to the J-13 composition depends on several rate reactions and requires substantial reaction progress. Uncertainty analysis of relativereaction rate constants shows that the mineral phases chosen, and the reaction constants used, are consistent with evolution of the J-13 composition.

\section{Coupled Repository-Scale Calculation with Silica}

Fully coupled THC calculations were performed using the FEHM finite-element code, which was modified to incorporate the behavior of a single chemical component and to couple changes in fracture porosity with changes in permeability. The TH aspects of these calculations were done using the ECM model (see Chapter 3). The entire repository block, from the water table to the ground surface, was modeled using a coarse mesh and simple constant-temperature or no-flow boundary conditions. Ambient percolation flux was introduced to the top of the model at a very low rate.

The calculations show that sufficient silica will be mobilized by repository $\mathrm{TH}$ processes to cause significant local changes in the fracture porosity. Boiling occurs predominantly at the bottom of the isothermal (heat-pipe) zone, and the residual solute deposits from boiling accumulate there. This accumulation was not inhibited by coupling of the fracture porosity and permeability (relative permeability and moisture-characteristic relations were not coupled). Repository edge effects were reflected in the manner of silica deposition, and similar effects can be expected to result from repository-loading scenarios.

\section{Drift-Scale TH Simulations with Silica}

Fully coupled THC calculations were performed using the NUFT code, modified to incorporate the behavior of silica. Model input included relations between porosity and permeability, and between changes in permeability and changes in the water characteristic and relative permeability curves. The DKM conceptual model was used for these calculations (see Chapter 3), and chemical dissolution/ precipitation reactions were modeled in both the fracture and matrix continua.

Formation of a mineral cap above the emplacement drifts was predicted by this model for a broad range of conditions such as infiltration flux and specific surface area available for silica dissolution. During repository heating, simulations show that dissolution and precipitation occur in both the fractures and matrix, depending on location.

Calcite dissolution leading to deposition of additional species in the boiling zone (e.g. calcite, calcium silicate hydrate, or calcium chloride) is not incorporated in this model. When included, this effect is likely to increase the initial rate of precipitate formation because calcite dissolves one to two orders of magnitude faster than do silicates, such as cristobalite.

In these simulations, the precipitate forming the mineral cap is assigned the chemical properties of quartz, which dissolves slowly. This choice may be consistent with aging of the precipitate for thousands of years at elevated temperatures. In the simulations shown, the 
mineral cap does not dissolve when the heat pipe and dryout zone collapse, nor does it dissolve when contacted by ambient percolation long after the thermal period. This may be related to the choice of quartz, but is also caused by decreased reaction rates at lower temperatures, restricted access of fluids to the mineral cap, and previous equilibration of ambient percolation with exposed minerals in the UZ.

A lower bound on permeability reduction in the boiling zone is incorporated in the model, to reflect the relation between permeability and heat-pipe formation, one that is known from years of TH simulations. When the permeability decreases to a threshold value ( 10 millidarcy), throttling occurs and resists liquid reflux penetration. The lower end of the heat-pipe zone then migrates away from the heat source. Thus, although precipitation is concentrated at the bottom of the heat-pipe zone where boiling occurs, the zone of permeability reduction migrates upward with time.

\section{Reactive-Transport Calculations}

Coupled reactive-transport simulations were performed, using the OS3D/GIMRT code, for the reference cases developed for this report (Chapter 3). The temperature and flow fields for reactive-transport simulations were taken directly from the output for specific time steps, from NUFT simulations of thermohydrology. The GIMRT simulations were thus 2-D, were based only on fracture-flow conditions extracted from DKM simulations, and ignored matrix processes. This conceptualization applies to steady-state hydraulic conditions in which the fracture flux is large compared to the matrix flux and in which fracture-matrix interaction is limited. Reactive processes were simulated for a few years at each of these time steps, assuming steady-state temperature and flow fields. Using this approach, the chemical activity at each time step was characterized with respect to the affected mineral species and to the time to achieve steady state for a given set of initial conditions.

The GIMRT simulations show that dissolution of calcite will occur rapidly, particularly during a transient period of increased $\mathrm{CO}_{2}$ fugacity caused by evolution of $\mathrm{CO}_{2}$ from the rock during the first few years after the start of heating. Aqueous dissolution and precipitation reactions involving silicates are likely to produce significant changes in fracture porosity, over longer time periods, on the order of tens of years.

There are areas in the $\mathrm{AZ}$ where $\mathrm{TH}$ conditions will lead to longer water-rock contact time and, thus, to more complete reaction. It is in these areas that there will be more extensive precipitation of silicates, such as stilbite, and other zeolites.

Later in the repository thermal evolution, $\mathrm{CO}_{2}$ will be displaced from the gas phase by water vapor as the air-mass fraction approaches zero (see Chapter 3). GIMRT simulations show that decreased $\mathrm{CO}_{2}$ fugacity will have little impact on dissolution processes at nearneutral $\mathrm{pH}$. The presence of condensate that is less chemically aggressive to calcite will result in physical spreading of zones of precipitation and dissolution.

Comparison of GIMRT simulations with the silica distributions calculated using NUFT shows that aqueous dissolution and boiling processes may be incompatible above the emplacement drifts. Whereas the lower end of the heat-pipe zone is at relatively high saturation and elevated temperature, condensation may not occur because the water composition is evaporatively concentrated. An integrated model that accommodates a full set of chemical reactions-with evaporation, boiling, and gas-phase transport-is the next step for improved realism of THC simulations. This topic receives ample attention in the open literature, and development of this capability is underway at LLNL and elsewhere.

Return to Table of Contents 


\subsection{References}

APHA (1976). Standard Methods for the Examination of Water and Wastewater, 15th Edition. Washington, DC: American Public Health Association, American Water Works Association, Water Pollution Control Federation.

Apps, J.A. (1997). “Chapter 14: Hydrogeochemical Analysis.” The Site-Scale Unsaturated Zone Model of Yucca Mountain, Nevada, for the Viability Assessment (Chapter 14). Las Vegas, NV: Civilian Radioactive Waste Management System, Management and Operating Contractor.

Arthur, R.C., and W.M. Murphy (1989). "An analysis of gas-water-rock interactions during boiling in partially saturated tuff." Sci. Geol. Bull. 42(4):313-327.

Bäverman, C. (1993). “Development of 'CHEMFRONTS,' a Coupled Transport and Geochemical Program to Handle Reaction Fronts." AB Technical Report. (93-21). Svensk Kärnbränslehantering AB. (235 pp.)

Bethke, C.M. (1985). “A numerical model of compaction-driven groundwater flow and heat transfer and its application to the paleohydrology of intracratonic sedimentary basins." J. Geophy. Res. 90:6817-6828.

Bethke, C.M. (1986). "Hydrologic constraints on genesis of the Upper Mississippi Valley Mineral District from Illinois Basin brines." Econ. Geol. 81(81):233-249.

Bethke, C.M. (1994). The Geochemist's Workbench ${ }^{\mathrm{TM}}$, Version 2.0. A Users Guide to Rxn, Act2, Tact, React, and Gtplot. Champaign, IL: Hydrology Program, University of Illinois.

Bethke, C.M., W.J. Harrison, C. Upson, and S.P. Altaner (1988). "Supercomputer analysis of sedimentary basins." Science 239:261-267.

Bethke, C.M., M.-K. Lee, H.A.M. Quinodoz, and W. Kreiling (1993). “Basin Modeling with Basin 2." Urbana-Champaign, IL: University of Illinois, Urbana-Champaign. (225 pp.)

Blum, A.E., and L.L. Stillings (1995). “Feldspar dissolution kinetics.” A.F. White and S.L. Brantley (eds.). Chemical Weathering Rates of Silicate Minerals. Washington, DC: Min. Soc. Am. 31:291-351.

Bourcier, W.L., W.E. Glassley, and J. Johnson (1995). “A Review of Existing Coupled Reactive Transport-Hydrothermal Computer Codes." Livermore, CA: Lawrence Livermore National Laboratory. [MOL.19950726.0110]

Brace, W.F. (1977). "Permeability From Resistivity and Pore Shape." J. Geoph. Res. 82(3343-3349).

Braithwaite, J.W., and F.B. Nimick (1984). "Effect of Host-Rock Dissolution and Precipitation on Permeability in a Nuclear Waste Repository in Tuff." (SAND84-0192). Albuquerque, NM: Sandia National Laboratory. [HQZ.19870131.6415]

Broxton, D.E., W.G. Warren, R.C. Hagen, and G. Luedemann (1989). “Chemistry of Diagenetically Altered Tuffs at a Potential Nuclear Waste Repository at Yucca Mountain, Nevada." (LANL-10802-MS). Los Alamos, NM: Los Alamos National Laboratory.

Bruton, C.J. (1995). “Testing EQ3/ 6 and GEMBOCHS using fluid-mineral equilibria in the Wairakei Geothermal System." Chemical and Mineralogical Properties of the Waste Package Environment: Natural System Simulations. (Yucca Mountain Project Letter Report, Milestone MOL206; also UCRL-ID-129280, Lawrence Livermore National Laboratory, Livermore, CA.) [MOL.19960409.0131] 
Buscheck, T.A., and J.J. Nitao (1992). “The impact of thermal loading on repository performance at Yucca Mountain." In proceedings from Third International High-Level Radioactive Waste Management Conference. Las Vegas, NV: April 12-16, 1992. Am. Nuc. Soc., La Grange, IL. pp. 1003-1017. (Also UCRL-JC-109232, Lawrence Livermore National Laboratory, Livermore, CA) [NNA.19920408.0008]

Buscheck, T.A., and J.J. Nitao (1993a). “The analysis of repository-heat-driven hydrothermal flow at Yucca Mountain." In proceedings from Fourth International High-Level Radioactive Waste Management Conference. Las Vegas, NV: April 26-30, 1993. Am. Nuc. Soc., La Grange, IL. (Also UCRL-JC-112444, Lawrence Livermore National Laboratory, Livermore, CA) [NNA.19930315.0010]

Buscheck, T.A., and J.J. Nitao (1993b). “The impact of repository heat on thermo-hydrological performance at Yucca Mountain." In proceedings from Am. Nuc. Soc. Topical Meeting on Site Characterization and Model Validation (Focus 93). Las Vegas, NV: September 26-30, 1993. (UCRL-JC-114791) [NNA.19940121.0144; 114791]

Camp, T.R. (1964). “Theory of water infiltration.” J. Am. Soc. Civ. Eng. 90:1-3.

Carlos, B. (1987). "Minerals in Fractures of the Saturated Zone from Drill Core USW-G4, Yucca Mountain, Nye County, Nevada." (LA-10827-MS). Los Alamos, NM: Los Alamos National Laboratory. (32 pp.) [HQS.19880517;1112]

Carlos, B.A., D.L. Bish, and S.J. Chipera (1990). "Manganese-Oxide Minerals in Fractures of the Crater Flat Tuff from Drill Core USW G-4, Yucca Mountain, Nevada." (LA-11787-MS). Los Alamos, NM: Los Alamos National Laboratory. [NNA.19900206.0163]

Carlos, B.A., S.J. Chipera, D.L. Bish, and S.J. Craven (1993). “Fracture-lining manganeseoxideminerals in silicic tuff, Yucca Mountain, Nevada." Chem. Geol. 107(47-69). [NNA.19931028.0002]

Clark, I.D., H.N. Khoury, E. Salameh, P. Fritz, H.K. Seidlitz, and T.E. Milodowski (1992). "Origin of the Maqarin, Jordan, Hyperalkaline Groundwaters: Isotopic and Geochemical Evidence for In-Situ Combustion, Calcination, and Recarbonation of Bituminous Marls." Y.K. Kharaka and A.S. Maest (eds.). Water-Rock Interaction. Rotterdam: Balkema. pp. 1485-1489.

Coudrain-Ribstein, A., and G. deMarsily (1989). "Modeling of mass transfer arising from buoyancy flow in sedimentary basins." Sci. Geol. Bull. 42:299-312.

Coudrain-Ribstein, A., P. Goblet, and G. deMarsily (1989a). "Coupled Modeling of Flow, Mass Transport, Heat Transport, and Chemistry: An Example." H.E. Kobus and W. Kinzelbach (eds.). Contaminant Transport in Groundwater. Rotterdam: Balkema. pp. 173-177.

Coudrain-Ribstein, A., and P. Gouze (1993). "Quantitative study of geochemical processes in the Dogger Aquifer, Paris Basin, France." Appl. Geochem. 8:495-506.

Coudrain-Ribstein, A., and P. Jamet (1989). “Choix des composantes et spéciation d'une solution." C.R. Acad. Sci. Paris t. 308(série II):239-244.

Coudrain-Ribstein, A., A. Vinsot, and P. Iris (1989b). "Space and time evolution of the geochemical processes arising from geothermal injection in an aquifer." Geothermics 18:57-64.

Criscenti, L.J., and R.C. Arthur (1989). "The calculated effects of isothermal boiling on tuff-water interactions." Radiochim. Acta 52/53:513-517. [HQX.19891005.0043] 
Daveler, S.A., and T.J. Wolery (1992). “EQPT, A Data File Preprocessor for the EQ3/ 6 Software Package: User's Guide and Related Documentation (Version 7.0)." (UCRL-MA-110662). Livermore, CA: Lawrence Livermore National Laboratory. (part 2) [NNA.19930129.0101]

Davidson, G.R., E.L. Hardin, and R.L. Bassett (1995). “Extraction of ${ }^{14}$ C from pore water in unsaturated rock using vacuum distillation." Radiocarbon 37:861-874.

Davis, J.A., and J.C. Leckie (1978). "Surface ionization and complexation at oxide water interface, part 2." J. Colloid Interface Sci. 67:90-107. [NNA.19891016.0123]

Delany, J.M. (1985). “Reaction of Topopah Spring Tuff with J-13 Water: A Geochemical Modeling Approach Using the EQ3/ 6 Reaction Path Modeling Code." (UCRL-53631). Livermore, CA: Lawrence Livermore National Laboratory. (48 pp.) [NNA.871111.0114; HQS.19880517.2419]

deMarsily, G. (1986). Quantitative Hydrogeology. London: Academic Press, Inc. [222592]

Dove, P.M. (1994). “The dissolution kinetics of quartz in sodium chloride solutions at $25^{\circ} \mathrm{C}$ to 300․" Am. J. Sci. 294:665-712.

Dove, P.M., and D.A. Crerar (1990). "Kinetics of quartz dissolution in electrolyte solutions using a hydrothermal mixed flow reactor." Geochim. Cosmochim. Acta 54:955969.

Flint, A., J.A. Hevesi, and L.E. Flint (1996). "Conceptual and Numerical Model of Infiltration for the Yucca Mountain Area, Nevada." Water Resources Investigation Report. (3GUT623M). Denver, CO: U.S. Geological Survey. (U.S.G.S. Survey Milestone Report) [MOL.19970409.0087]

Flint, L.E. (1998). “Characterization of Hydrogeological Units Using Matrix Properties, Yucca Mountain, Nevada." Water Resources Investigation Report. (WRIR 97-4243). U.S. Geological Survey.

Glassley, W.E. (1993). “Coupled hydro-geochemical processes and their significance for Yucca Mountain site characterization." In proceedings from ANS Topical Mtg. on Nuclear Waste Isolation in an Unsaturated Zone, Focus '93. Am. Nuc. Soc., La Grange Park, IL. pp. (Also ICRL-JC-114783, Lawrence Livermore National Laboratory, Livermore, CA) [MOL.19971031.0400]

Glassley, W.E. (1995). “Report on Near-Field Geochemistry: Water Composition Changes Due to Evaporation." Milestone report for the CRWMS Management and Operating Contractor, U.S. Department of Energy. (M0L206). (UCRL-ID-129280, Lawrence Livermore National Laboratory, Livermore, CA) [MOL.19950406.0153]

Glassley, W.E. (1996). “Equilibrium Bounds on Water Chemistry and Mineralogical Changes Produced by Near-Field Relative Humidity Changes." D.G. Wilder (ed.). Near-Field and Altered-Zone Environment Report, Vol. II. Livermore, CA: Lawrence Livermore National Laboratory. pp. 3-11-3-17. (UCRL-124998, Vol. II) [MOL.19961212.0122]

Glassley, W.E. (1997). “Thermo-Chemical Analysis of the Drift-Scale Heater Test: Mineralogical and Geochemical Characteristics." Milestone report for the CRWMS Management and Operating Contractor, U.S. Department of Energy. (SP9320M4). Livermore, CA: Lawrence Livermore National Laboratory. [MOL.19980105.0566] 
Glassley, W.E., and L. DeLoach (1997). “Second Quarter Results of Chemical Measurements in the Single Heater Test." Milestone report for the CRWMS Management and Operating Contractor, U.S. Department of Energy. (SP9240M4). Livermore, CA: Lawrence Livermore National Laboratory. [MOL.19971218.0917]

Goblet, P. (1989). “Programme METIS, Notice de Conception.” ENSMP Technical Report. $(89 / 23)$.

Grenthe, I., J. Fuger, R.J.M. Konings, R.J. Lemire, A.B. Muller, C. Nguyen-Trung, and H. Wanner (1992). Chemical Thermodynamics, Vol. 1: Chemical Thermodynamics of Uranium. Amsterdam: North Holland. [NNA.19940415.0009]

Hardin, E.L., and D.A. Chesnut (1997). "Synthesis Report on Thermally Driven Coupled Processes." Milestone report for the CRWMS Management and Operating Contractor, U.S. Department of Energy. (SPL8BM3). Livermore, CA: Lawrence Livermore National Laboratory. (Also UCRL-ID-128495, Lawrence Livermore National Laboratory, Livermore, CA) [MOL.19980120.0035]

Harrar, J., J.F. Carley, W.F. Isherwood, and E. Raber (1990). “Report of the Committee to Review the Use of J-13 Well Water in Nevada Nuclear Waste Storage Investigations." (UCRL-ID-21867). Livermore, CA: Lawrence Livermore National Laboratory. [NNA.910131.0274]

Helgeson, H.C. (1968). “Evaluation of irreversible reactions in geochemical processes involving minerals and aqueous solutions-I. Thermodynamic relations." Geochim. Cosmochim. Acta 32:853-877. [HQS.19880517.2454]

Helgeson, H.C. (1969). "Thermodynamics of hydrothermal systems at elevated temperatures and pressures." Am. J. Sci. 267:729-804. [NNA.19900816.0012]

Helgeson, H.C. (1970). “A Chemical and Thermodynamic Model of Ore Deposition in Hydrothermal Systems at Elevated Temperatures and Pressures." Mineralogical Society of American Special Paper 3. Washington, DC: Mineralogical Society of America. pp. 155-186.

Helgeson, H.C., T.H. Brown, A. Nigrini, and T.A. Jones (1970). “Calculation of mass transfer in geochemical processes involving aqueous solutions." Geochim. Cosmochim. Acta 34:569-592. [225570]

Helgeson, H.C., W.M. Murphy, and P. Aagard (1984). “Thermodynamic and kinetic constraints on reaction rates among minerals and aqueous solutions, II. Rate constants, effective surface area, and the hydrolysis of feldspar." Geochim. Cosmochim. Acta 48:2405-2432. [220177; 220013]

Holdren Jr., G.R., and P.M. Speyer (1985). “Reaction-rate-surface-area relationships during the early stages of weathering: II. Data on eight additional feldspars." Geochim. Cosmochim. Acta 51:2311-2318. [MOL.19940517.0029]

Hostetler, J.D. (1984). “Electrode electrons, aqueous electrons, and redox potentials in natural waters." Am. Journ. Sci. 284:734-759.

Jamet, P., P.J. Hooker, J.M. Schmitt, E. Ledoux, and P. Escalier des Orres (1993).

"Hydrogeochemical modeling of an active system of uranium fixation by organic soils and sediments (Needle's Eye, Scotland)." Mineral. Deposita 28:66-76. 
Johnson, J.W., K.G. Knauss, W.E. Glassley, and L.D. DeLoach (1997). “Reactive Transport through Topopah Spring Tuff: Summary of Initial PFR Experiments and Associated Computer Simulations." Milestone report for the CRWMS Management and Operating Contractor, U.S. Department of Energy. (SPL1BM4). Livermore, CA: Lawrence Livermore National Laboratory. [MOL.19970507.0178]

Johnson, J.W., K.G. Knauss, W.E. Glassley, and L.D. DeLoach (1998). “Reactive transport modeling of plug-flow-reactor experiments: significance, initial results, and future applications." J. Hydrol. (in press)

Johnson, J.W., E.H. Oelkers, and H.C. Helgeson (1992). “SUPCRT92: A software package for calculating the standard molal thermodynamic properties of minerals, gases, aqueous species, and reactions from 1 to 5000 bars and $0^{\circ} \mathrm{C}$ to $1000^{\circ} \mathrm{C}$." Comput. Geosci. 18:899-947. (UCRL-JC-107907). [234273]

Kee, R.J., L.R. Petzold, M.D. Smooke, and J.F. Grcar (1985). “Implicit methods in combustion and chemical kinetics modeling." J.U. Brackbill and B.I. Cohen (eds.). Multiple Times Scales. New York, NY: Academic Press. pp. 113-144.

Keith, L.A., P.T. Delaney, and D.E. Moore (1983). "Permeability reduction due to precipitation of quartz under nonisothermal conditions." In proceedings from Ninth Workshop on Geothermal Reservoir Engineering. Stanford, CA: Stanford University.

Khoury, H.N., and T.E. Milodowski (1992). "High Temperature Metamorphism and Low Temperature Retrograde Alteration of Spontaneously Combusted Marls: The Maqarin Cement Analogue, Jordan." Y.K. Kharaka and A.S. Maest (eds.). Water-Rock Interaction. Rotterdam: Balkema. pp. 1515-1518.

Klavetter, E.A., and R.R. Peters (1986). “Estimation of Hydrologic Properties of an Unsaturated, Fractured Rock Mass." (SAND-84-2642). Albuquerque, NM: Sandia National Laboratories. [NNA.870317.0738; 202727]

Knapp, R.B. (1989). “A Lagrangian Reactive Transport Simulator with Successive Paths and Stationary States: Concepts, Implementation, and Verification." (UCRL-100952). Livermore, CA: Lawrence Livermore National Laboratory. [NNA.19900419.0143]

Knauss, K.G., W.J. Beiriger, and D.W. Peifer (1987). “Hydrothermal Interaction of Solid Wafers of Topopah Spring Tuff with J-13 Water and Distilled Water at 90 and $150^{\circ} \mathrm{C}$ Using Dickson-Type, Gold-Bag Rocking Autoclaves: Long-Term Experiments." (UCRL-53722). Livermore, CA: Lawrence Livermore National Laboratory. [NNA.19870713.0081; 203013]

Knauss, K.G., W.J. Beiriger, D.W. Peifer, and A.J. Piwinskii (1985). “Hydrothermal Interaction of Solid Wafers of Topopah Spring Tuff with J-13 Water and Distilled Water at 90, 150, and $250^{\circ} \mathrm{C}$, Using Dickson-Type, Gold-Bag Rocking Autoclaves." (UCRL-53645). Livermore, CA: Lawrence Livermore National Laboratory. [NNA.19900207.0282; HQS.19980517.2482]

Knauss, K.G., J.M. Delany, W.J. Beiriger, and D.W. Peifer (1986). “Hydrothermal interaction of Topopah Spring tuff with J-13 water as a function of temperature." In proceedings from Mater. Res. Soc. Symp. Proc. 44:539-546. (Also UCRL-90853 Abs, Lawrence Livermore National Laboratory, Livermore, CA) 
Knauss, K.G., and D.W. Peifer (1986). “Reaction of Vitric Topopah Spring Tuff and J-13 Ground Water Under Hydrothermal Conditions, Using Dickson-Type, Gold-Bag Rocking Autoclaves." (UCRL-53795). Livermore, CA: Lawrence Livermore National Laboratory. [NAA.891102.0117]

Knauss, K.G., and T.J. Wolery (1989). "Muscovite dissolution kinetics as a function of $\mathrm{pH}$ and time at 70 C." Geochim. Cosmochim. Acta 53:1493-1501.

Krupp, R.E., and T.M. Seward (1987). “The Rotokawa Geothermal System, New Zealand: An active epithermal gold-depositing environment." Econ. Geol. 82:1109-1129.

Lasaga, A.C. (1981). “Rate Laws of Chemical Reactions.” A.C. Lasaga and R.J. Kirkpatrick (eds.). Reviews in Mineralogy. Washington, DC: Mineral Soc. Am. 9:1-67. (in Kinetics of Chemical Reactions) [NNA.19921103.0017]

Lasaga, A.C. (1984). “Chemical kinetics of water-rock interaction.” J. Geophys. Res. B9:4009-4025. [NNA.19900216.0060]

Lasaga, A.C., and A.E. Blum (1986). "Surface chemistry, etch pits, and mineral-water reactions." Geochim. Cosmochim. Acta 50:2363-2379. [NNA.19940517.0032]

Lemire, R.J. (1984). “An Assessment of the Thermodynamic Behavior of Neptunium in Water and Model Groundwater for 25 and $150^{\circ} \mathrm{C} . "$ (AECL-7817). Pinawa, Manitoba, Canada: Atomic Energy of Canada, Ltd. (53 pp.)

Lemire, R.J., and P.R. Tremaine (1980). “Uranium and plutonium equilibria in aqueous solutions to $200^{\circ} \mathrm{C} . "$ J. Chem. Eng. Data 25:361-370.

Levy, S.S. (1984a). "Petrology of Samples from Drill Holes USW H-3, H-4, and H-5, Yucca Mountain, Nevada." (LA-9706-MS). Los Alamos, NM: Los Alamos National Laboratory. (77 pp.) [NNA.870519.0048; 200558]

Levy, S.S. (1984b). "Studies of Altered Vitrophyre for the Prediction of Nuclear Waste Repository Induced Thermal Alteration at Yucca Mountain, Nevada." G.L. McVay (ed.). Scientific Basis for Nuclear Waste Management VII. New York, NY: Elsevier. pp. 959-966. [210392]

Lichtner, P.C. (1988). “The quasi-stationary state approximation to coupled mass transport and fluid-rock interaction in a porous medium." Geochim. Cosmochim. Acta 52:143-165.

Lichtner, P.C. (1992). “Time-space continuum description of fluid/ rock interaction in permeable media." Water Resour. Res. 28:3134-3155.

Lichtner, P.C. (1993). "Scaling properties of time-space kinetic mass transport equations and the local equilibrium limit." Am. Jour. Sci 293:257-296.

Lin, W., and W.D. Daily (1989). "Laboratory Study of Fracture Healing in Topopah Spring tuff-implications for near field hydrology." In proceedings from Topical Meeting on Nuclear Waste Isolation in the Unsaturated Zone, Focus '89. Las Vegas, NV: September 17-21, 1989. Am. Nuc. Soc., La Grange Park, IL. (Also UCRL-100624, Lawrence Livermore National Laboratory, Livermore, CA) [NNA.19900711.0241; 224040]

Linderberg, R.D., and D.D. Runnells (1984). "Ground water redox reactions: An analysis of equilibrium state applied to Eh measurements and geochemical modeling." Science 225:925-927. 
Madé, B., and P. Jamet (1993). "Modeling of hydrothermal systems with coupled chemical reactions transport code." In proceedings from Fourth International Symposium on Hydrothermal Reactions. M. Cuney and M. Cathelineau (eds.). Nancy, France:. pp. 139-142.

McClure, L.C., H.S. Fogler, and W.E. Kline (1979). “An experimental technique for obtaining permeability-porosity relationships in acidizing porous media." Ind. Chem. Eng. Fundam. 18:188-191.

Mogollon, J.L., J. Ganor, J.M. Soler, and A.C. Lasaga (1996). “Column experiments and the full dissolution rate law of gibbsite." Am. J. Sci. 296(7):729-765.

Moore, D.E., C.A. Morrow, and J.D. Byerlee (1986). "High-temperature permeability and groundwater chemistry of some Nevada test site tuffs." J. Geophys. Res. 91(B2):2163-2171.

Morrow, C.A., D. Lockner, D.E. Moore, and J.D. Byerlee (1981). “Permeability of granite in a temperature gradient." J. Geophys. Res. 86:3002-3008. [HQS.19980517.2501]

Murphy, W.M., R.T. Pabalan, J.D. Prikryl, and C.J. Goulet (1996). “Reaction kinetics and thermodynamics of aqueous dissolution and growth of analcime and Ma-clinoptilolite at 25ㄷ." Am. J. Sci. 296:128-186.

Nagy, K.L. (1995). “Dissolution and precipitation kinetics of sheet silicates.” A.F. White and S.L. Brantley (eds.). Chemical Weathering Rates of Silicate Minerals. Washington, DC: Min. Soc. Am. 31:173-233.

Nitao, J.J. (1988). “Numerical Modeling of the Thermal and Hydrological Environment Around a Nuclear Waste Package Using the Equivalent Continuum Approximation: Horizontal Emplacement." (UCRL-ID-21444). Livermore, CA: Lawrence Livermore National Laboratory. [NNA.890317.0021; 201832]

Nordstrom, D.K., L.N. Plummer, T.M.L. Wigley, T.J. Wolery, J.W. Ball, E.A. Jenne, R.L. Bassett, D.A. Crerar, T.M. Florence, B. Fritz, M. Hoffman, G.R. Holdren Jr., G.M. Lafon, S.V. Mattigod, R.E. McDuff, F. Morel, M.M. Reddy, G. Sposito, and J. Thrailkill (ed.). (1979). "Chemical Modeling of Aqueous Systems." ACS Symposium Series 93. E.A. Jenne (ed.). Washington, DC: Am. Chem. Soc.

Noy, D.J. (1990). “PRECIP: A Program for Coupled Groundwater Flow and Precipitation/Dissolution Reactions." Technical Report. (WE/90/38C). British Geological Survey.

Noy, D.J. (1991). “PRECIP: A Program for Coupled Groundwater Flow and Precipitation/Dissolution Reactions." Technical Report. (WE/90/38C). British Geological Survey. (37 pp.)

Oelkers, E.H., and J. Schott (1995). “Experimental study of anorthite dissolution and the relative mechanism of feldspar hydrolysis." Geochim. Cosmochim. Acta 59(24):5039-5053.

Paces, J.B., L.A. Neymark, B.D. Marshall, J.F. Whelan, and Z.E. Peterman (1996). “Ages and Origins of Subsurface Secondary Minerals in the Exploratory Studies Facility (ESF)." Milestone report for the CRWMS Management and Operating Contractor, U.S. Department of Energy. (3GQH450M). U.S. Geological Survey. [MOL.19970324.0052]

Parkhurst, D.L. (1995). "User's Guide to PHREEQC—A Computer Program for Speciation, Reaction-Path, Advective-Transport, and Inverse Geochemical Calculations." Water-Resources Investigations Report. (95-4227). Lakewood, CO: U.S. Geological Survey. (143 pp.) 
Patankar, S.V. (1980). Numerical Heat Transfer and Fluid Flow. New York, NY: Hemisphere Publications. (197 pp.)

Pearson, R.O. (1976). “Planning and Design of Additional East Mesa Geothermal Test Facilities (Phase IB), I. Final Report." (SAN/1140-1/1). Albuquerque, NM: Sandia National Laboratories.

Phillips, O.M. (1991). Flow and Reactions in Permeable Rocks. Cambridge, UK: Cambridge University Press. (277 pp.) [NNA.19930414.0024]

Phillips, S.L., A. Igbene, J.A. Fair, and H. Ozbec (1981). “A Technical Databook for Geothermal Energy Utilization.” (LBL-12810). Berkeley, CA: Lawrence Berkeley National Laboratory.

Plummer, L.N., D.L. Parkhurst, G.W. Fleming, and S.A. Dunkle (1988). “A Computer Program Incorporating Pitzer's Equations for Calculation of Geochemical Reactions in Brines." Water-Resources Investigations Report. (88-4153). Washington, DC: U.S. Geological Survey. (310 pp.)

Pyrak-Nolte, L.J., L.R. Myer, N.G.W. Cook, and P.A. Witherspoon (1987). “Hydraulic and mechanical properties of natural fractures in low permeability rock." In proceedings from Sixth International Congress on Rock Mechanics. Montreal, Canada:. [NNA.19900727.0306]

Ragnarsdottir, K.V. (1993). "Dissolution kinetics of heulandite at pH 2-12 and $25^{\circ} \mathrm{C}$. " Geochim. Cosmochim. Acta 57:2439-2449. (Also report LLYMP9212011B, University of Bristol). [NNA.19921218.0007]

Renders, P.J.N., C.H. Gammons, and H.L. Barnes (1995). “Precipitation and dissolution rate constants for cristobalite from 150 to $300^{\circ} \mathrm{C}$." Geochim. Cosmochim. Acta 49(1):77-85.

Rimstidt, J.D., and H.L. Barnes (1980). “The kinetics of silica-water reactions.” Geochim. Cosmochim. Acta 44:1683-1699. [NNA.19891006.0185]

Rimstidt, J.D., W.D. Newcomb, and J.L. Shettel Jr. (1989). “A vertical thermal gradient experiment to simulate conditions in vapor dominated geothermal systems, epithermal gold deposits, and high level radioactive repositories in unsaturated media." In proceedings from Water-Rock Interaction. D.L. Miles (ed.). Rotterdam: A.A. Balkema. 6:585-588. [NNA.19920319.0007]

Robinson, B., and W.E. Glassley (1995). "Coupled Flow and Reactive Transport at Yucca Mountain, Simulated Using the FEHMN Code." Los Alamos, NM: Los Alamos National Laboratory.

Savage, D., K. Bateman, P. Hill, C. Hughes, A. Milodowski, J. Pearce, E. Rae, and C. Rochelle (1992). "Rate and mechanism of the reaction of silicates with cement pore fluids." Appl. Clay Sci. 7:33-45.

Savage, D., and C.A. Rochelle (1993). "Modeling reactions between cement pore fluids and rock: implications for porosity change." J. Contam. Hydrol. 13:365-378.

Scheidegger, A. (1974). The Physics of Flow Through Porous Media. New York, NY: Macmillan.

Schmitt, J.-M., S. Makhoukhi, and P. Goblet (1991). “Modelling of Structure-Induced Hydrothermal Circulations in a Mississippi Valley Type Deposit." M. Pagel and J.L. Leroy (eds.). Source, Transport, and Deposition of Metals. Rotterdam: A.A. Balkema. pp. 489-492. 
Siegel, M.D. (1993). “Towards a realistic approach to validation of reactive transport models for performance assessment." In proceedings from FOCUS 93, Site Characterization and Model Validation. Las Vegas, NV: Am. Nuc. Soc. (SAND 93-2507C) [NNA.19940217.0082]

Siegel, M.D., D.B. Ward, C.R. Bryan, and P. Hopkins (1993). “Development and Validation of Reactive Transport Models for Performance Assessment." (WBS 1.2.5.4.6). Sandia National Laboratory.

Silva, R.J., G. Bidoglio, M.H. Rand, P.B. Robouch, H. Wanner, and I. Puigdomenech (1993). Chemical Thermodynamics of Americium. Amsterdam: North Holland. [NNA.19931022.0023]

Spycher, N.F., and M.H. Reed (1989a). CHILLER: A Computer Program for Computing Water-Rock Reactions, Boiling, Mixing and Other Reacting Processes in Aqueous-Mineral-Gas Systems. Eugene, OR: Department of Geological Sciences, University of Oregon. (Revised Preliminary Edition)

Spycher, N.F., and M.H. Reed (1989b). SOLVEQ: A Computer Program for Computing Aqueous-Mineral-Gas Equilibria. Eugene, OR: Department of Geological Sciences, University of Oregon. (Revised Preliminary Edition)

Steefel, C.I. (1993). “1DREACT: One-Dimensional Reaction-Transport Model: User Manual and Programmer's Guide." (Unpublished manuscript). Richland, WA: Pacific Northwest Laboratory, Battelle Memorial Institute. (42 pp.)

Steefel, C.I., and A.C. Lasaga (1992). "Putting Transport into Water-Rock Interaction Models." Geology 20:680-684. [NNA.19930217.0106]

Steefel, C.I., and A.C. Lasaga (1994). “A coupled model for transport of multiple chemical species and kinetic precipitation/dissolution reactions with application to reactive flow in single-phase hydrothermal systems." Am. J. Sci. 294:529-592. [235372]

Steefel, C.I., and P. Van Cappellen (1990). "A new kinetic approach to modeling water-rock interaction: The role of nucleation, precursors, and Ostwald ripening." Geochim. Cosmochim. Acta 54:2657-2677.

Steefel, C.I., and S.B. Yabusaki (1995). “OS3D/GIMRT: Software for Modeling Multicomponent-Multidimensional Reactive Transport." Richland, WA: Pacific Northwest Laboratory, Battelle Memorial Institute.

Steefel, C.I., and S.B. Yabusaki (1996). “User's Manual and Programmer's Guide, Version 1.0, for Software for Modeling Multicomponent, Multidimensional Reactive Transport." Richland, WA: Pacific Northwest National Laboratory. (Internal Report, 58 pp.)

Sterner, S.M., A.R. Felmy, J.R. Rustad, and K.S. Pitzer (1997). “Thermodynamic Analysis of Aqueous Solutions Using INSIGHT." (PNWD-SA-4436). Richland, WA: Pacific Northwest National Laboratory. (107 pp.)

Stubos, A.K., C. Satik, and Y.C. Yortsos (1993a). "Critical heat-flux hysteresis in vapor liquid counterflow in porous-media." Intl. J. Heat E Transf. 36(1):227-231.

Stubos, A.K., C. Satik, and Y.C. Yortsos (1993b). "Effects of capillary heterogeneity on vapor liquid counterflow in porous-media." Intl. J. Heat E Transf. 36(4):967-976.

Tester, J.W., W.G. Worley, B.A. Robinson, C.O. Grigsby, and J.R. Feerer (1994). “Correlating quartz dissolution kinetics in pure water from 25 to $625^{\circ} \mathrm{C}$." Geochim. Cosmochim. Acta 58(11):2407-2420. 
TRW (1996). “Degraded Mode Criticality Analysis of Immobilized Plutonium Waste Forms in a Geologic Repository." November 15, 1996. (A00000000-01717-5705-00014 Rev 00). Las Vegas, NV: TRW Environmental Safety Systems, Inc. [MOL.19970723.0016]

Vaniman, D.T., D.L. Bish, S.J. Chipera, B.A. Carlos, and S. Guthrie, Jr. (1997). “Summary and synthesis report on mineralogy and petrology studies for the Yucca Mountain Site Characterization Project." Milestone report for the CRWMS Management and Operating Contractor, U.S. Department of Energy. (3665). Los Alamos, NM: Los Alamos National Laboratory.

Vaughan, P.J. (1985). “Analysis of permeability reduction during flow of heated, aqueous fluid through westerly granite." In proceedings from International Symp. on Coupled Processes Affecting the Performance of a Nuclear Waste Repository. September 1985. Berkeley, CA, Lawrence Berkeley National Laboratory. (LBL-21850)

Verma, A., and K. Pruess (1988). "Thermohydrological conditions and silica redistribution near high-level nuclear waste emplaced in saturated geological formations." J. Geoph. Res. 93:1150-1173. [NNA.19891222.0004]

Viani, B.E., and C.J. Bruton (1992). "Modeling ion exchange in clinoptilolite using the EQ3/ 6 geochemical modeling code." In proceedings from Seventh International Symposium on Water-Rock Interaction. Y. Kharaka and A.S. Maest (eds.). Brookfield, VT:: A.A. Balkema. pp. 73-77. (Also UCRL-JC-109952, Lawrence Livermore National Laboratory, Livermore, CA) [MOL.19960607.0056; 230491]

Westall, J.C., J.L. Zachary, and F.M.M. Morel (1976). MINEQL, A Computer Program for the Calculation of Chemical Equilibrium Composition of Aqueous Systems. Cambridge, MA: Ralph M. Parsons Laboratory for Water Resources and Environmental Engineering, Department of Civil Engineering, Massachusetts Institute of Technology. (Technical Note 18) [NNA.19891016.0142]

Whitbeck, M., and W.E. Glassley (1996). "Preliminary Bounds on the Water Composition and Secondary Mineral Development that May Influence the Near-Field Environment." Milestone report for the CRWMS Management and Operating Contractor, U.S. Department of Energy. (MOL205). Livermore, CA: Lawrence Livermore National Laboratory. [MOL.19960726.0040]

White, A.F., and M.L. Peterson (1990). "Role of Reactive-Surface-Area Characterization in Geochemical Kinetic Models." D.C. Melchior and R.L. Bassett (eds.). Chemical Modeling of Aqueous Systems II. Washington, DC:. pp. 461-475.

Wilder, D.G. (1996). “Near-Field and Altered-Zone Environment Report, Vol. II.”

(UCRL-LR-124998, Vol. II). Livermore, CA: Lawrence Livermore National Laboratory. [MOL.19961212.0121; MOL.19961212.0122]

Wolery, T.J. (1992a). "EQ3/6, A Software Package for Geochemical Modeling of Aqueous Systems: Package Overview and Installation Guide." (UCRL-MA-110662). Livermore, CA: Lawrence Livermore National Laboratory. (part 1) [NNA.19921023.0028]

Wolery, T.J. (1992b). “EQ3NR, A Computer Program For Geochemical Aqueous SpeciationSolubility Calculations: Theoretical Manual, User's Guide, and Related Documentation." (UCRL-MA-110662). Livermore, CA: Lawrence Livermore National Laboratory. (Version 7.0) [NNA.19921218.0010] 
Wolery, T.J. (1994). “EQ3/6 Version 8: Differences From Version 7.” Letter report for the CRWMS Management and Operating Contractor, U.S. Department of Energy. Livermore, CA: Lawrence Livermore National Laboratory. (26 pp.)

Wolery, T.J., and S.A. Daveler (1992). “EQ6, A Computer Program for Reaction Path Modeling of Aqueous Geochemical Systems: Theoretical Manual, User's Guide, and Related Documentation (Version 7.0)." (UCRL-MA-110662). Livermore, CA: Lawrence Livermore National Laboratory. (part 4) [MOL.19980218.0570]

Wolery, T.J., K.J. Jackson, W.L. Bourcier, C.J. Bruton, B.E. Viani, K.G. Knauss, and J.M. Delany (1988). Current Status of the EQ3/6 Software Package for Geochemical Modeling. Washington, DC: Am. Chem. Soc. (Symposium Series 416. Also UCRL-98729, Lawrence Livermore National Laboratory, Livermore, CA) [NNA.19900117.0112]

Wolery, T.J., C.E. Palmer, and K.G. Knauss (1995). “The Neptunium Solubility Problem in Repository Performance Assessment: A White Paper." July 24, 1995. White Paper. Livermore, CA: Lawrence Livermore National Laboratory, Yucca Mountain Site Characterization Project.

Wruck, D.A., and C.E.A. Palmer (1997). "Analysis of Elevated Temperature Data for Thermodynamic Properties of Selected Radionuclides." Milestone report for the CRWMS Management and Operating Contractor, U.S. Department of Energy. (SPL4B1M4). Livermore, CA: Lawrence Livermore National Laboratory. (UCRL-ID-128955) [MOL.19971210.0037]

Yang, I.C., G.W. Rattray, and P. Yu (1996). “Interpretations of Chemical and Isotopic Data from Boreholes in the Unsaturated Zone at Yucca Mountain, Nevada." Water Resources Investigations Report. (WRIR 96-4058). U.S. Geological Survey. [MOL.19970715.0401]

Yang, I.C., A.K. Turner, T.M. Sayre, and P. Montazar (1988). “Triaxial-Compression Extraction of Pore Water from Unsaturated Tuff, Yucca Mountain, Nevada." Water Resources Investigations Report. (88-4189). U.S. Geological Survey. [NNA.19890309.0161]

Yeh, G.T., and V.S. Tripathi (1989). “A critical evaluation of recent developments in hydrogeochemical transport models of reactive multichemical components." Water Resour. Res. 25(1):93-108. [NNA.19931012.0014]

Yeh, G.T., and V.S. Tripathi (1992). "HYDROGEOCHEM: A Coupled Model of HDROlogic Transport and GEOCHEMical Equilibria in Reactive Multicomponent Systems." (ORNL-6371). Oak Ridge, TN: Oak Ridge National Laboratory. [NNA.19920207.0005]

Zyvoloski, G. (1983). "Finite element methods for geothermal reservoir simulation." Int. J. Numer. Anal. Methods Geomech. 7:75-86. [NNA.910806.0017]

Zyvoloski, G. (1986). "Incomplete factorization for finite element." Int. J. Numer. Methods in Eng. 23:1101-1109. [NNA.890523.0138]

Zyvoloski, G., and Z. Dash (1991). "Software Verification Report FEHM, Version 1.0." (LA-UR-91-609). Los Alamos, NM: Los Alamos National Laboratory. [NNA.910806.0018]

Zyvoloski, G., Z. Dash, and S. Kelbar (1988). “FEHM 1.0: Finite Element Heat and Mass Transfer Code." (LA-11224-MS). Los Alamos, NM: Los Alamos National Laboratory. [NNA.900918.0013] 


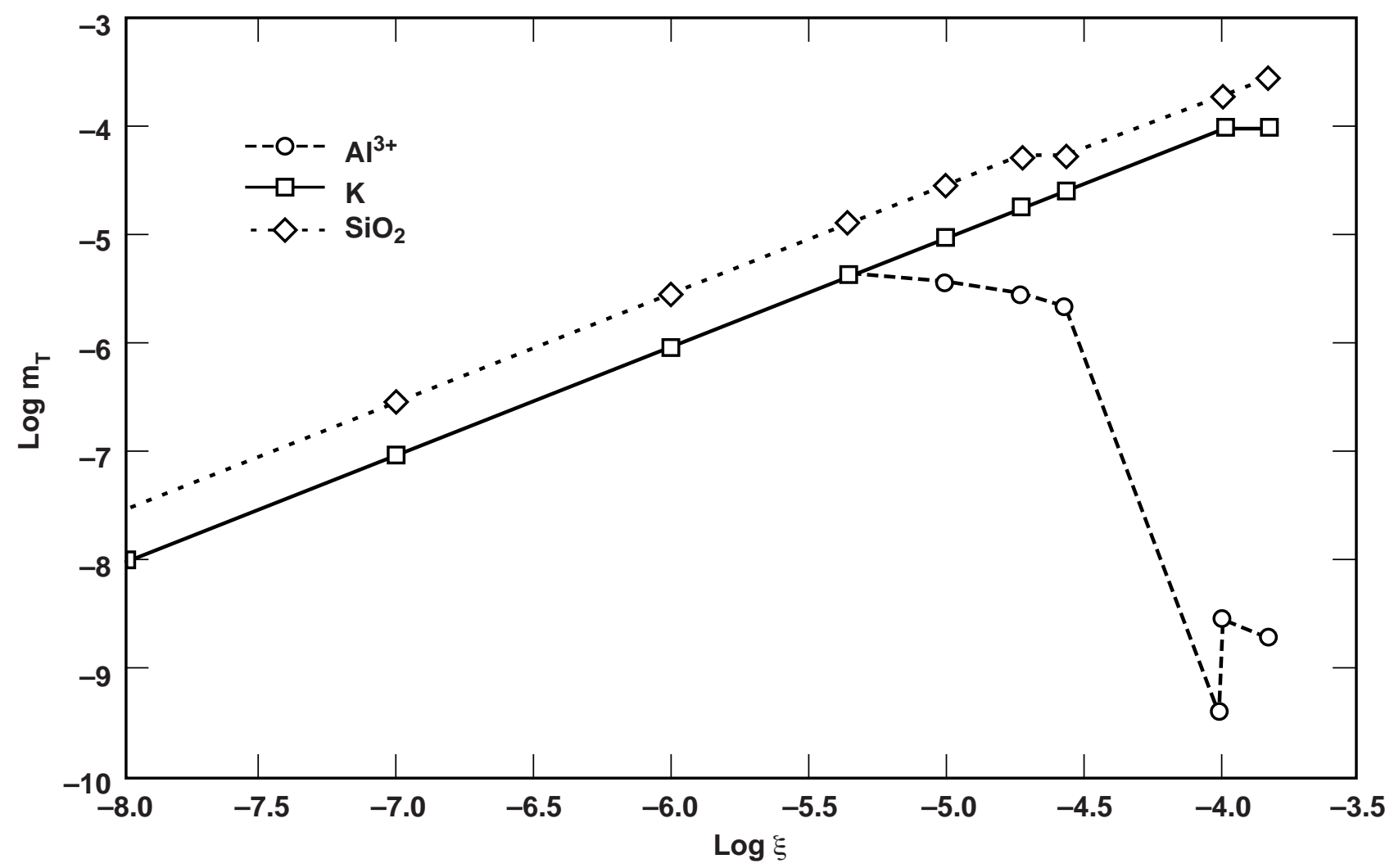

Figure 5-1. Changes in the total molalities of dissolved aluminum $\mathrm{Al}^{3+}$, potassium $\mathrm{K}$, and silica $\mathrm{SiO}_{2}$ as a function of reaction progress $\xi$ in a closed-system reaction of microcline with $\mathrm{pH} 4.0 \mathrm{HCl}$ solution. 


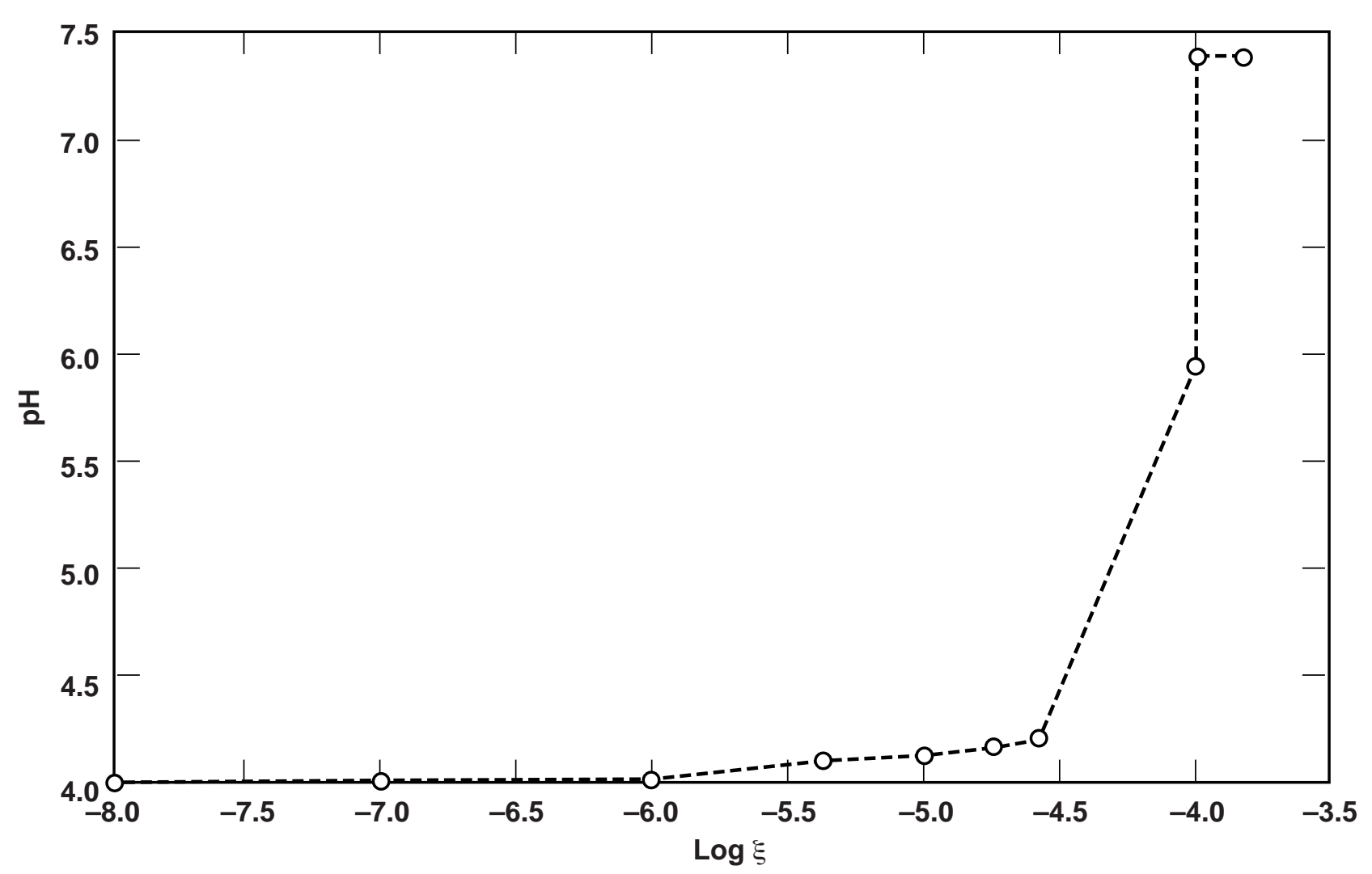

Figure 5-2. Changes in the $\mathrm{pH}$ as a function of reaction progress $\xi$ in a closed-system reaction of microcline with $\mathrm{pH} 4.0 \mathrm{HCl}$ solution. 


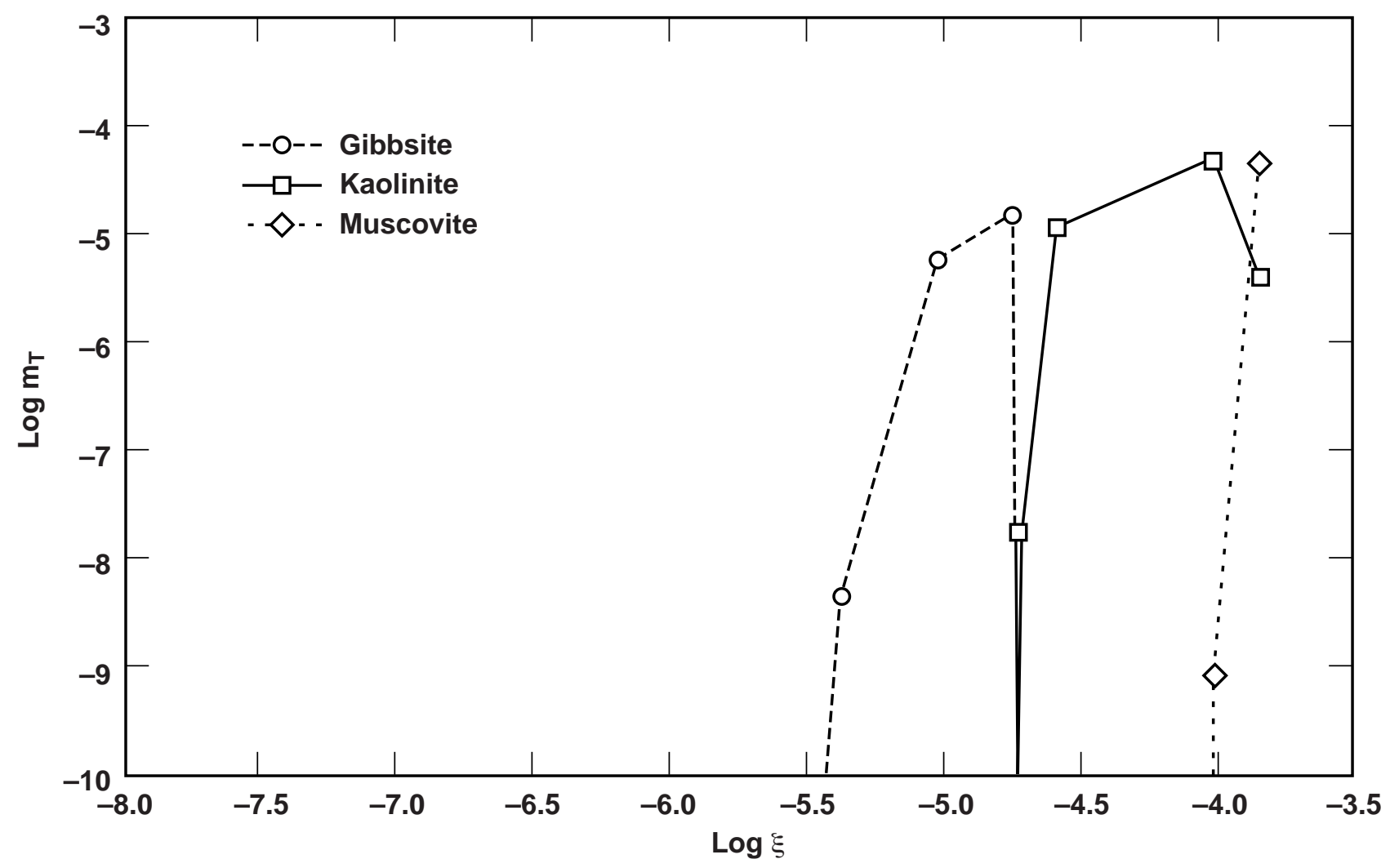

Figure 5-3. The number of moles of secondary minerals $n_{\phi}$ as a function of reaction progress $\xi$ in a closedsystem reaction of microcline with $\mathrm{pH} 4.0 \mathrm{HCl}$ solution. The reacting system originally contains $1 \mathrm{~kg}$ of solvent water. 


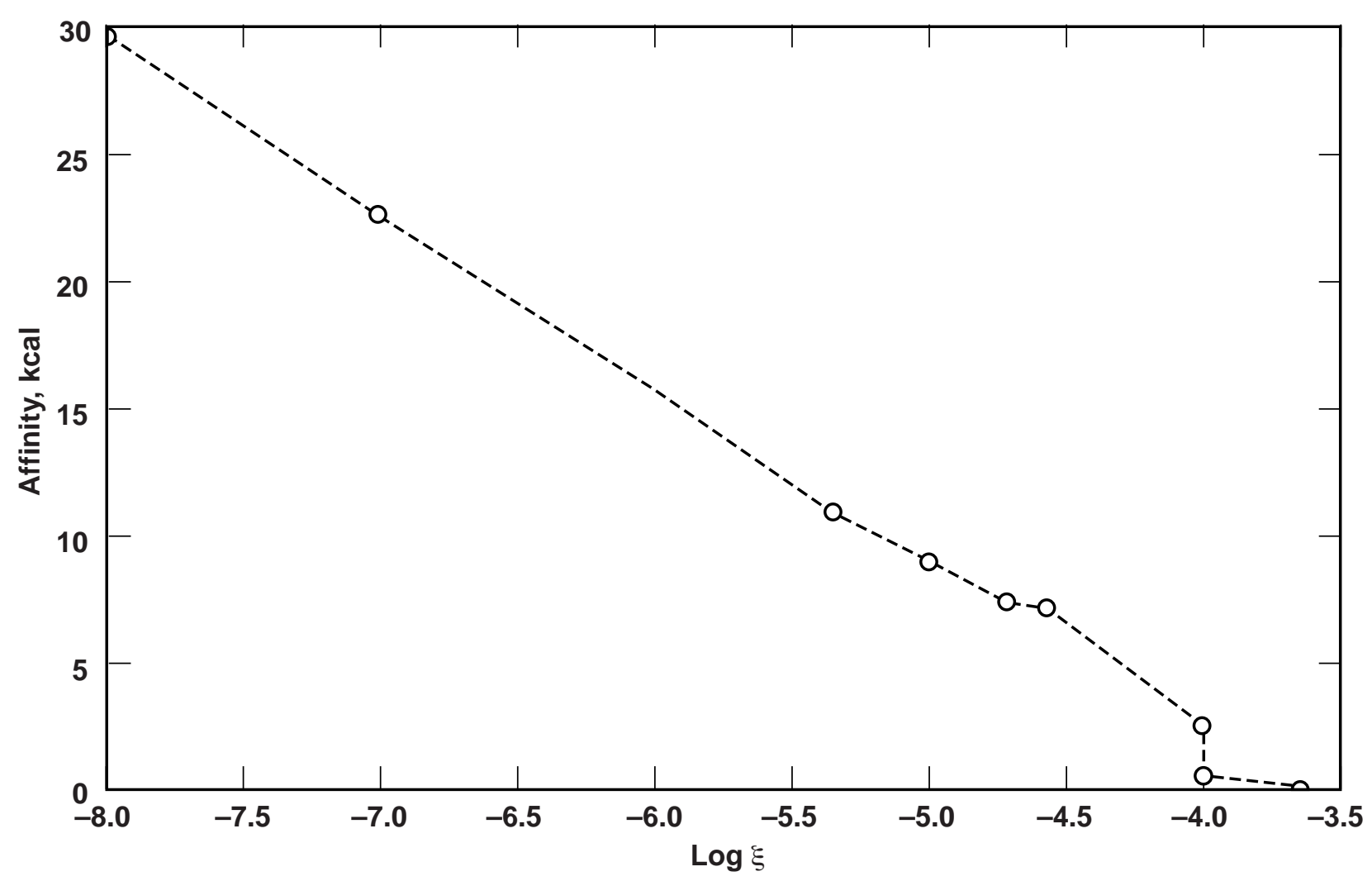

Figure 5-4. The affinity of microcline to dissolve as a function of reaction progress $\xi$ in a closed-system reaction of microcline with $\mathrm{pH} 4.0 \mathrm{HCl}$ solution. 
(a)

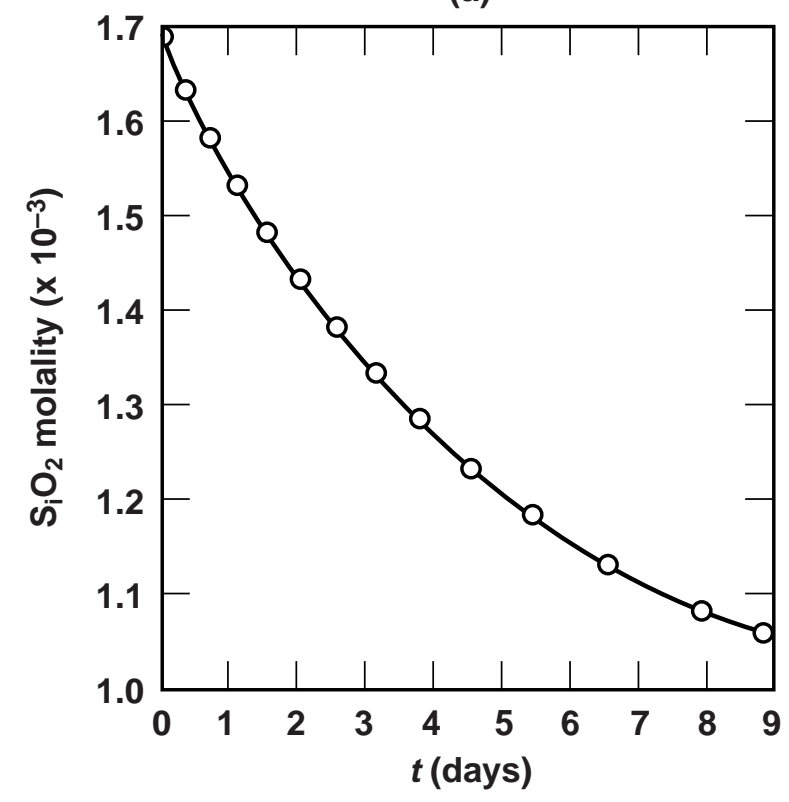

(c)

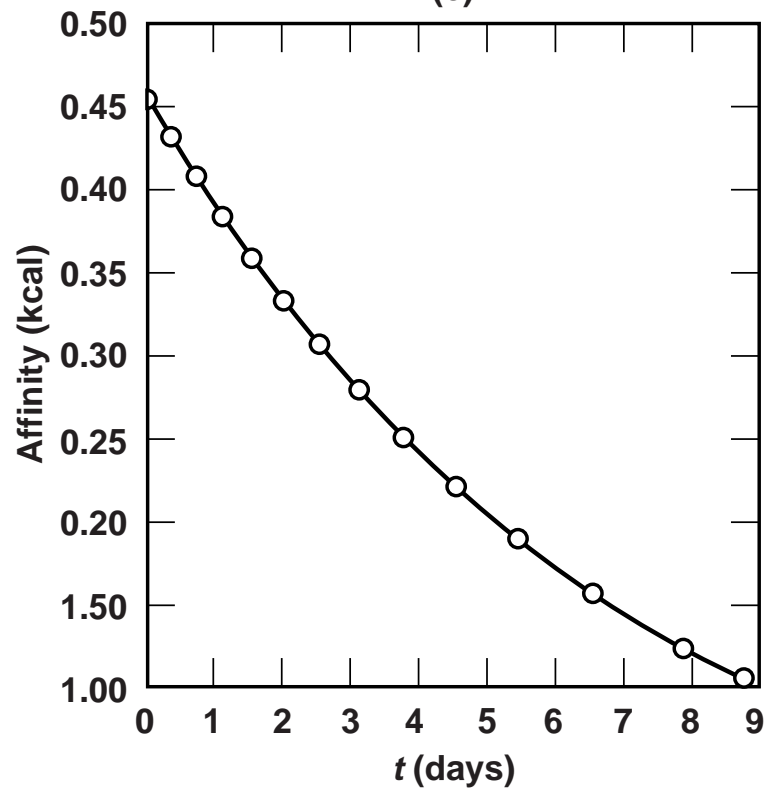

(b)

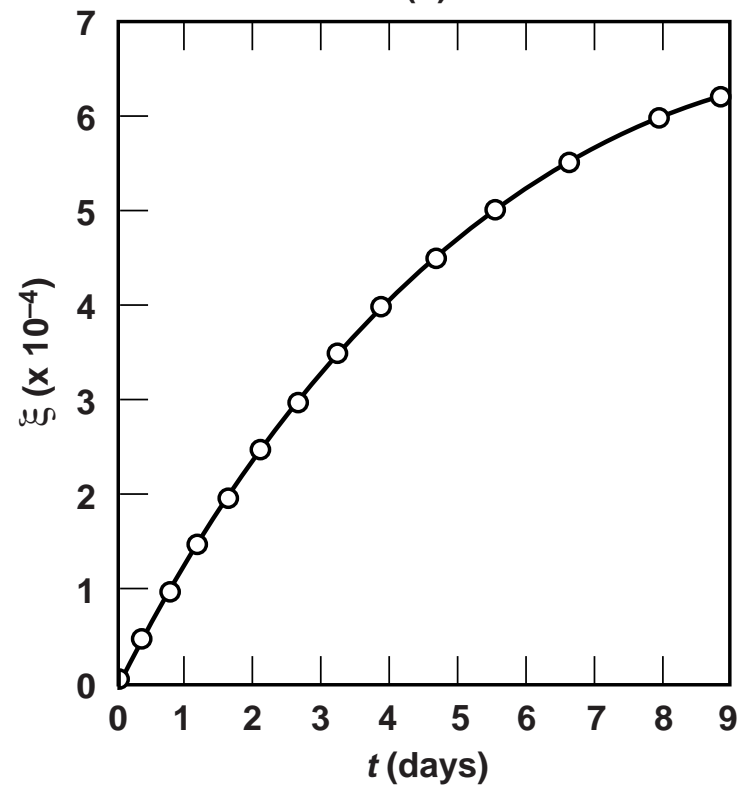

Figure 5-5. The precipitation kinetics of quartz in the system $\mathrm{SiO}_{2}-\mathrm{H}_{2} \mathrm{O}$ as a function of time $t$ : (a) molality of aqueous silica, (b) value of reaction progress $\xi$ (here equivalent to the number of moles of new quartz relative to an initial $1 \mathrm{~kg}$ of solvent water), and (c) affinity of quartz to precipitate. 


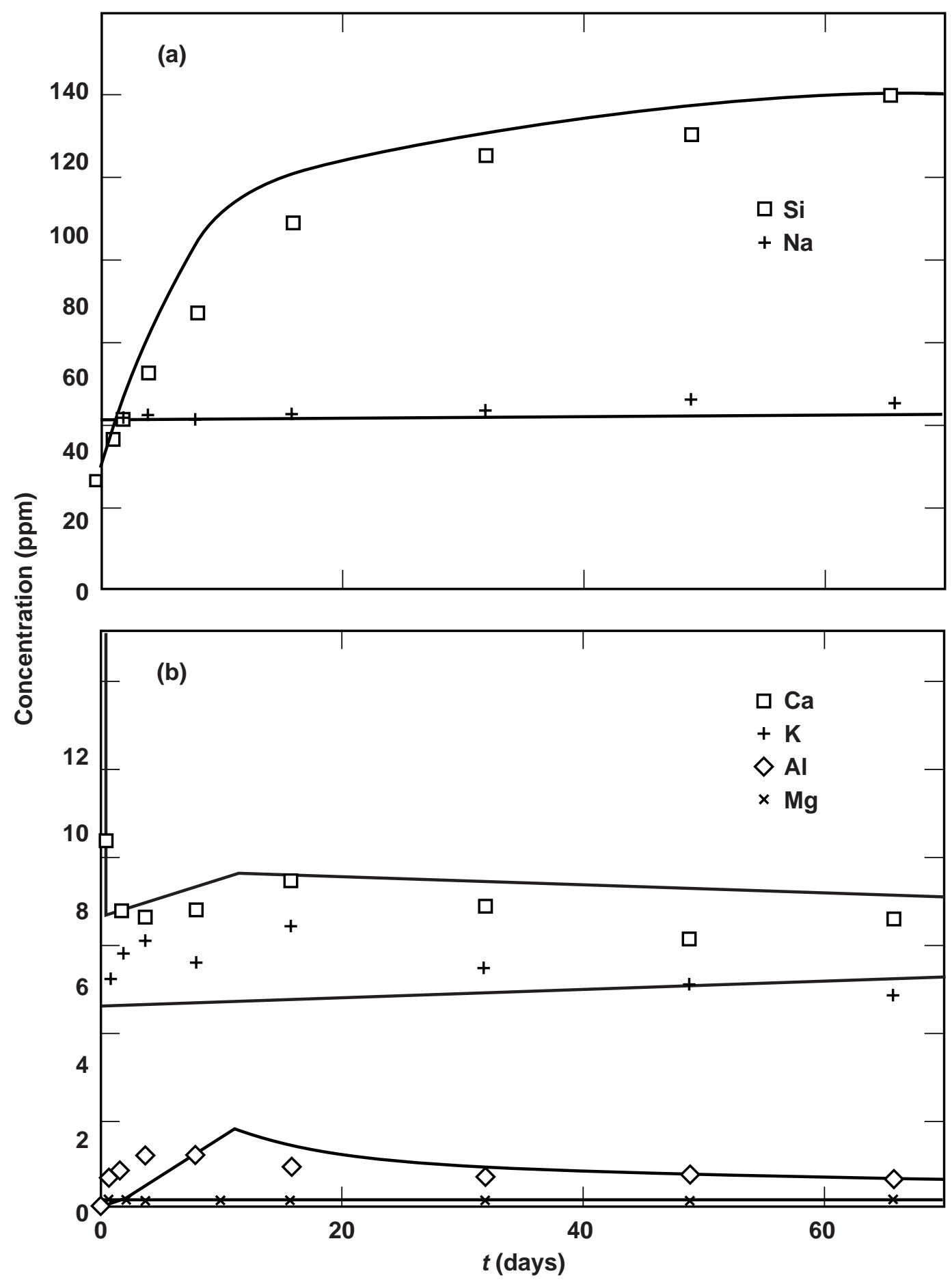

Figure 5-6. Comparison of simulated (EQ3/6) and experimental aqueous solution compositions for the $150^{\circ} \mathrm{C}$ reaction between Topopah Spring tuff (Tpt) (core wafer) and J-13 well water. 

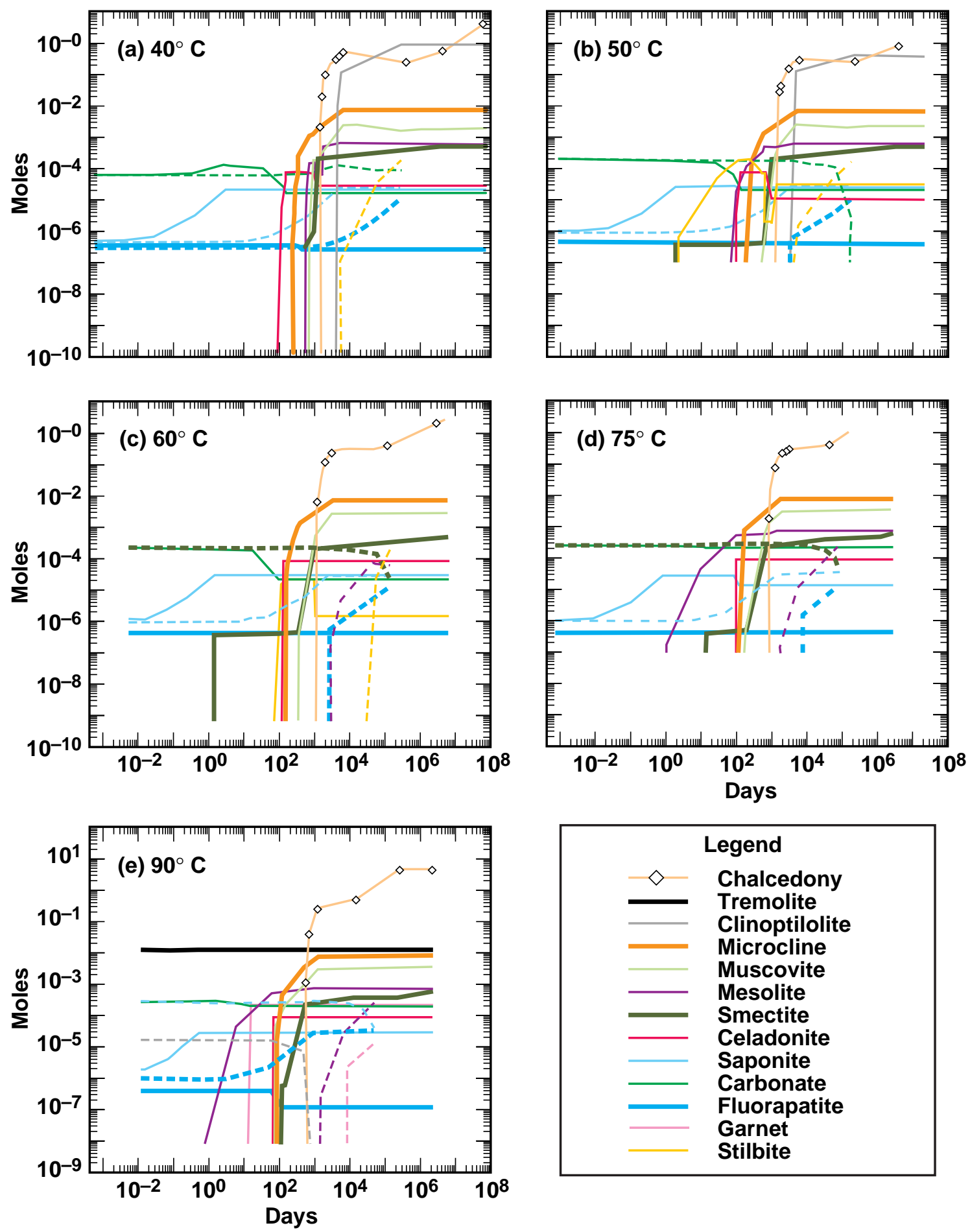

Figure 5-7. Major secondary mineralogy as a function of time for the reaction of devitrified TSw2 tuff (solid lines) and glassy TSw3 tuff (broken lines) with J-13 well water: (a) $40^{\circ} \mathrm{C}$, (b) $50^{\circ} \mathrm{C}$, (c) $60^{\circ} \mathrm{C}$, (d) $75^{\circ} \mathrm{C}$, and (e) $90^{\circ} \mathrm{C}$. Minor transition metal oxides such as hematite and pyrolusite are not shown. In the case of the devitrified tuff (TSw2), the time frame of these results actually depends on the surface areas presumed, which may be highly scenario-dependent. Increasing the total surface area would displace the curves to the left. In the case of the vitrophyre (TSw3), the rate of reaction is subject to similar considerations and was adjusted so that the timing of appearance of the secondary phases was similar to that in the case of the devitrified tuff. 


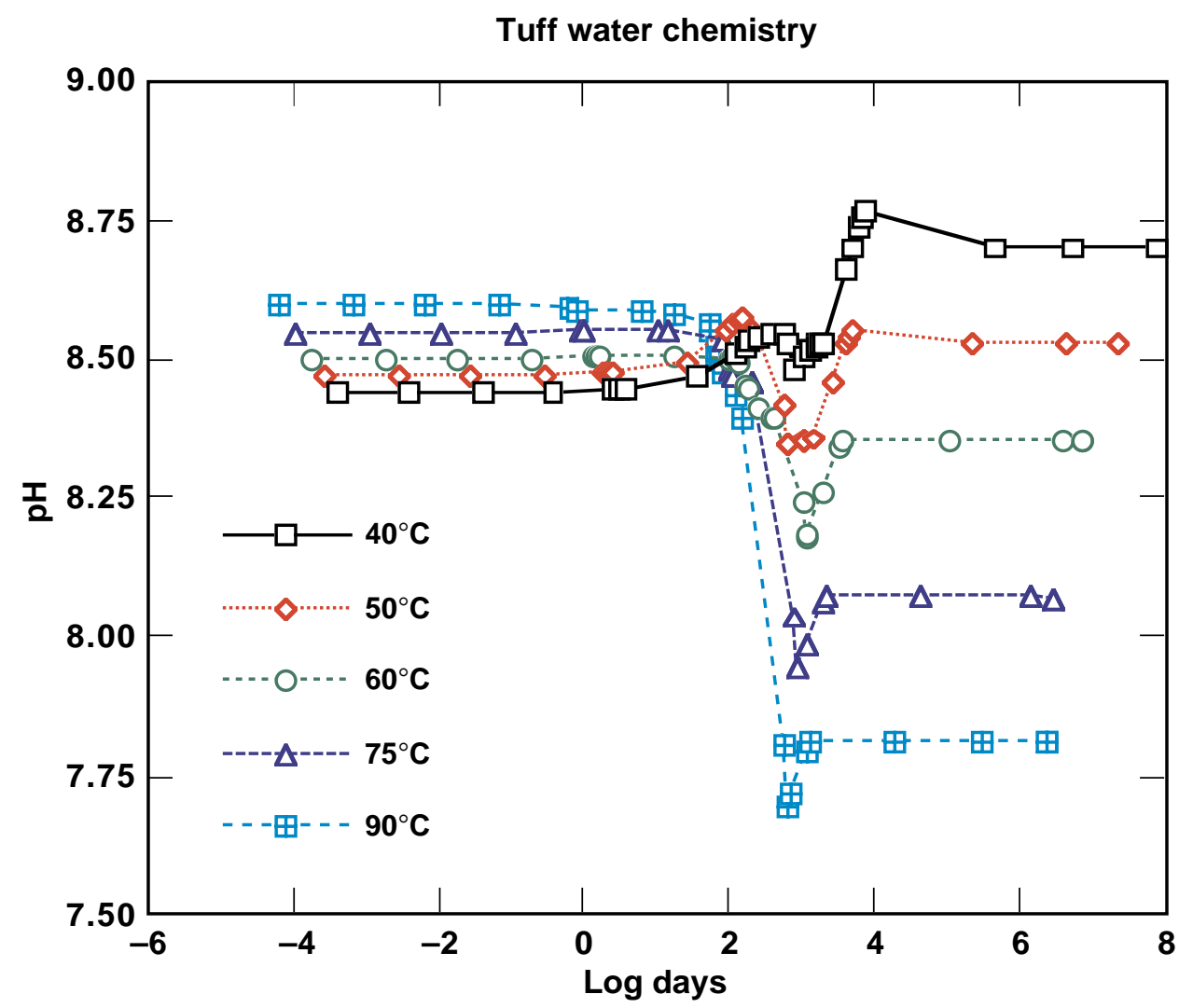

Figure 5-8. Changes in the chemistry of J-13 well water during reaction with devitrified TSw2 tuff at $40^{\circ}, 50^{\circ}$, $60^{\circ}, 75^{\circ}$, and $90^{\circ} \mathrm{C}(\mathrm{pH})$. 


\section{Tuff water chemistry}

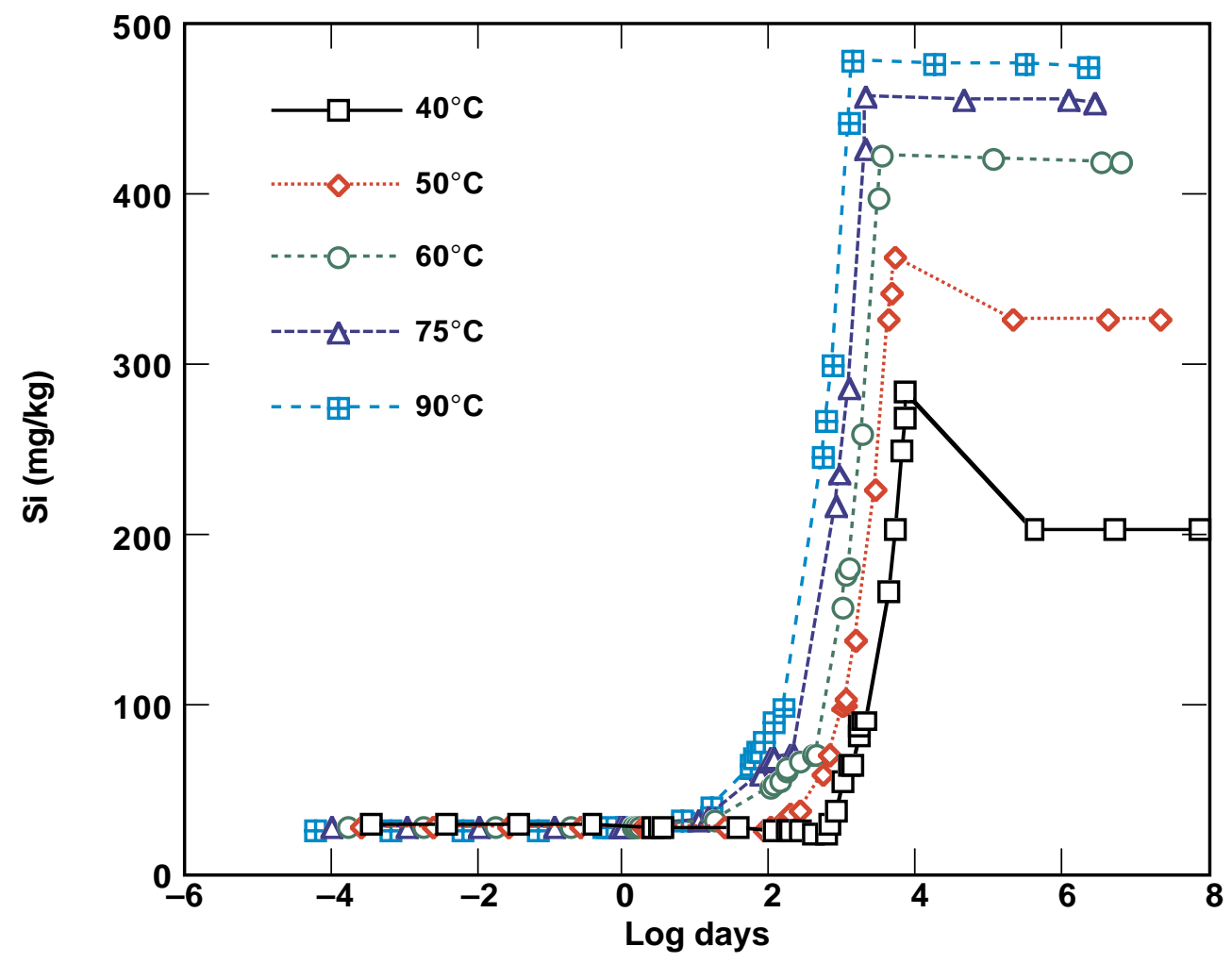

Figure 5-9. Changes in the chemistry of J-13 well water during reaction with devitrified TSw2 tuff at $40^{\circ}, 50^{\circ}$, $60^{\circ}, 75^{\circ}$, and $90^{\circ} \mathrm{C}(\mathrm{Si})$. 


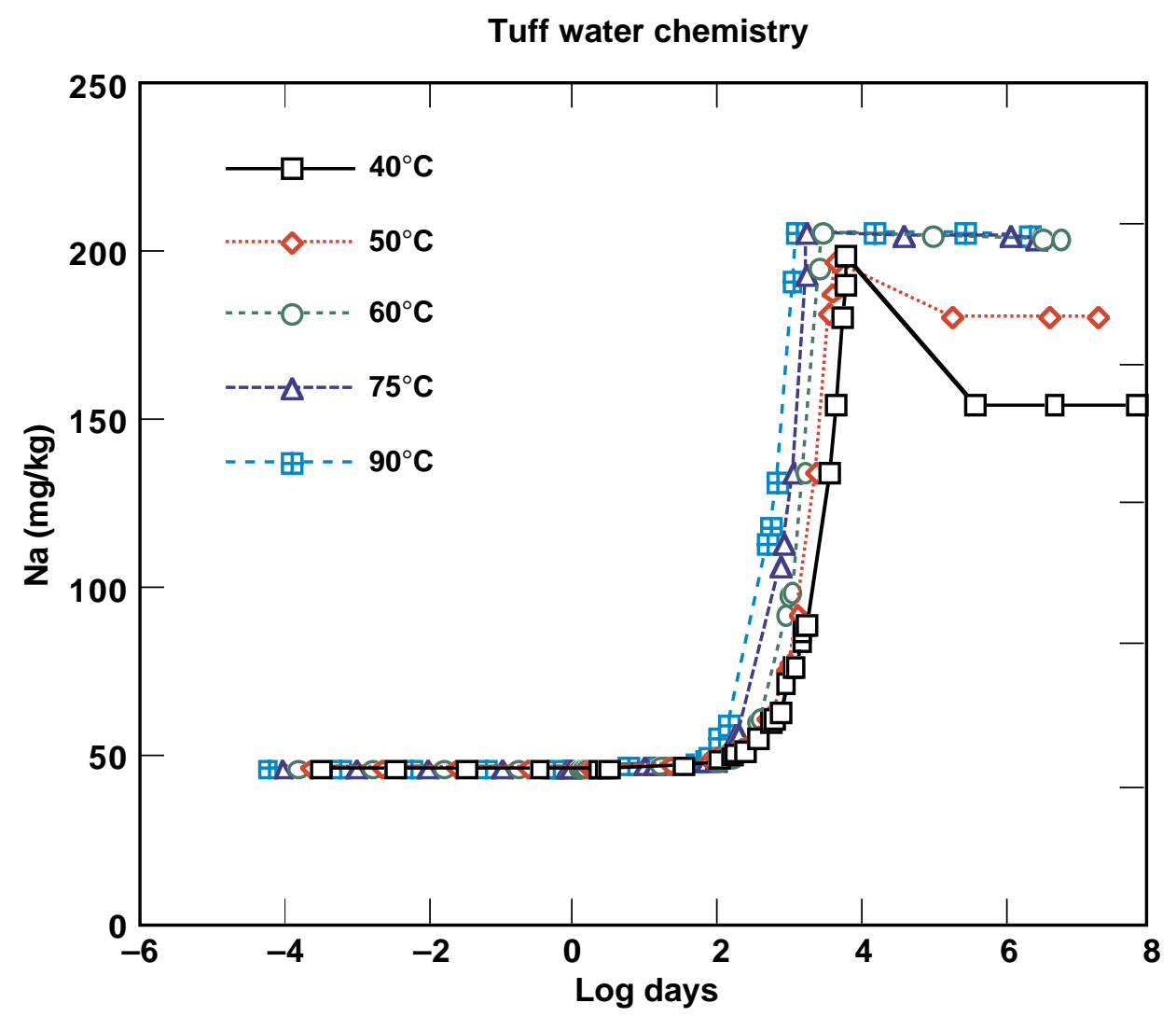

Figure 5-10. Changes in the chemistry of J-13 well water during reaction with devitrified TSw2 tuff at $40^{\circ}$, $50^{\circ}, 60^{\circ}, 75^{\circ}$, and $90^{\circ} \mathrm{C}(\mathrm{Na})$. 


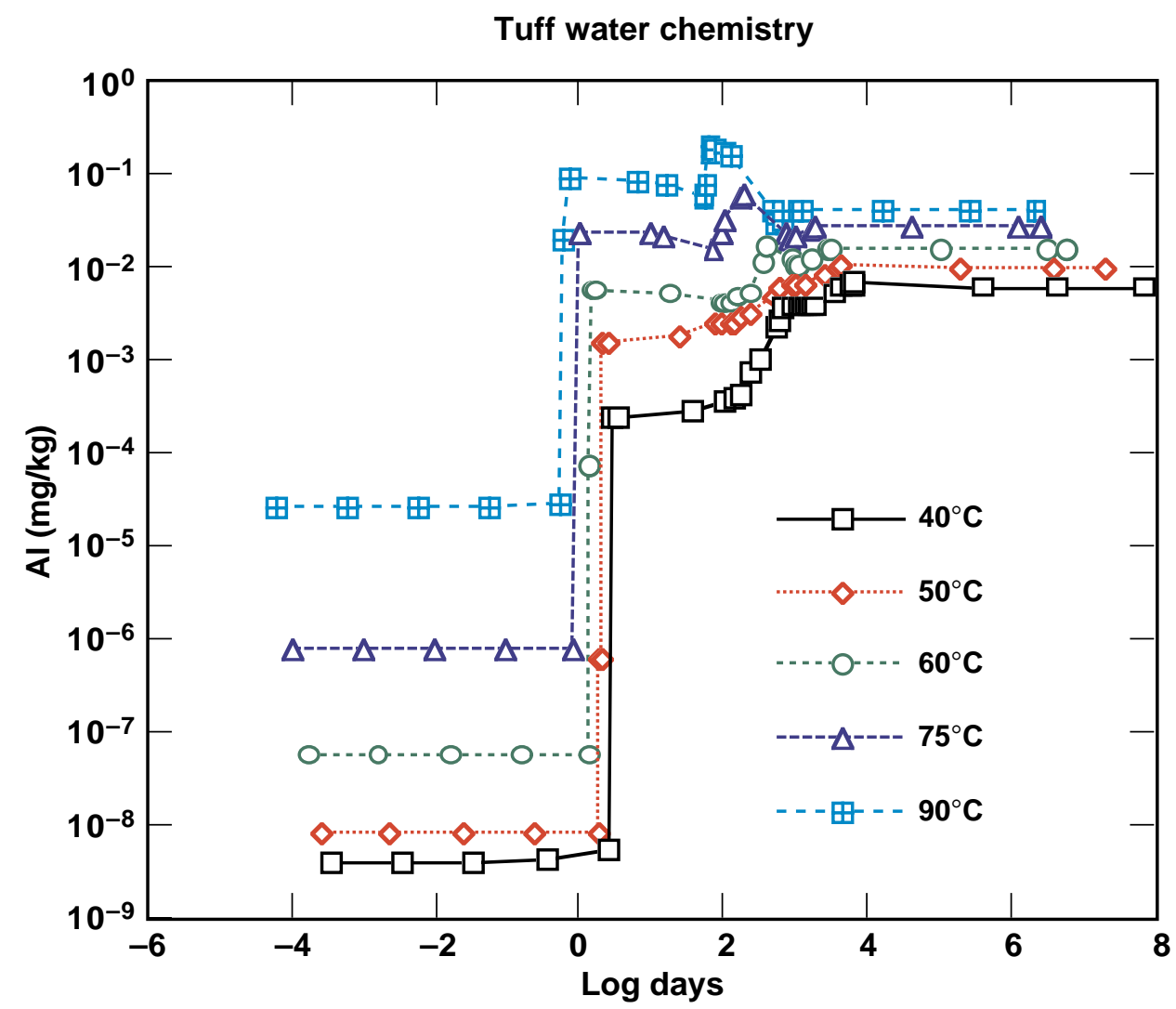

Figure 5-11. Changes in the chemistry of J-13 well water during reaction with devitrified TSw2 tuff at $40^{\circ}$, $50^{\circ}, 60^{\circ}, 75^{\circ}$, and $90^{\circ} \mathrm{C}(\mathrm{Al})$. 


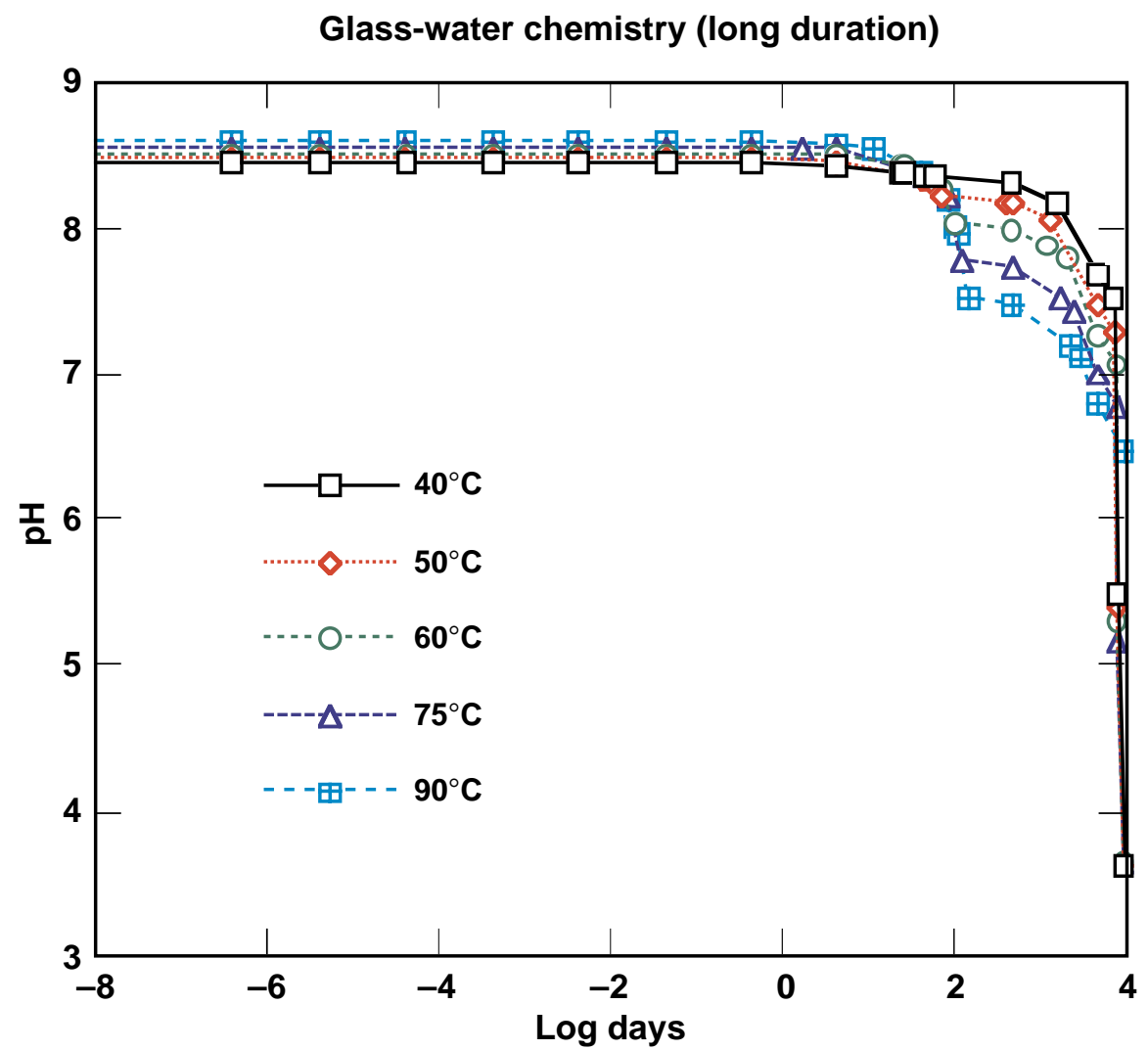

Figure 5-12. Changes in the $\mathrm{pH}$ of J-13 well water during reaction with glassy TSw3 tuff at $40^{\circ}, 50^{\circ}, 60^{\circ}, 75^{\circ}$, and $90^{\circ} \mathrm{C}$. 


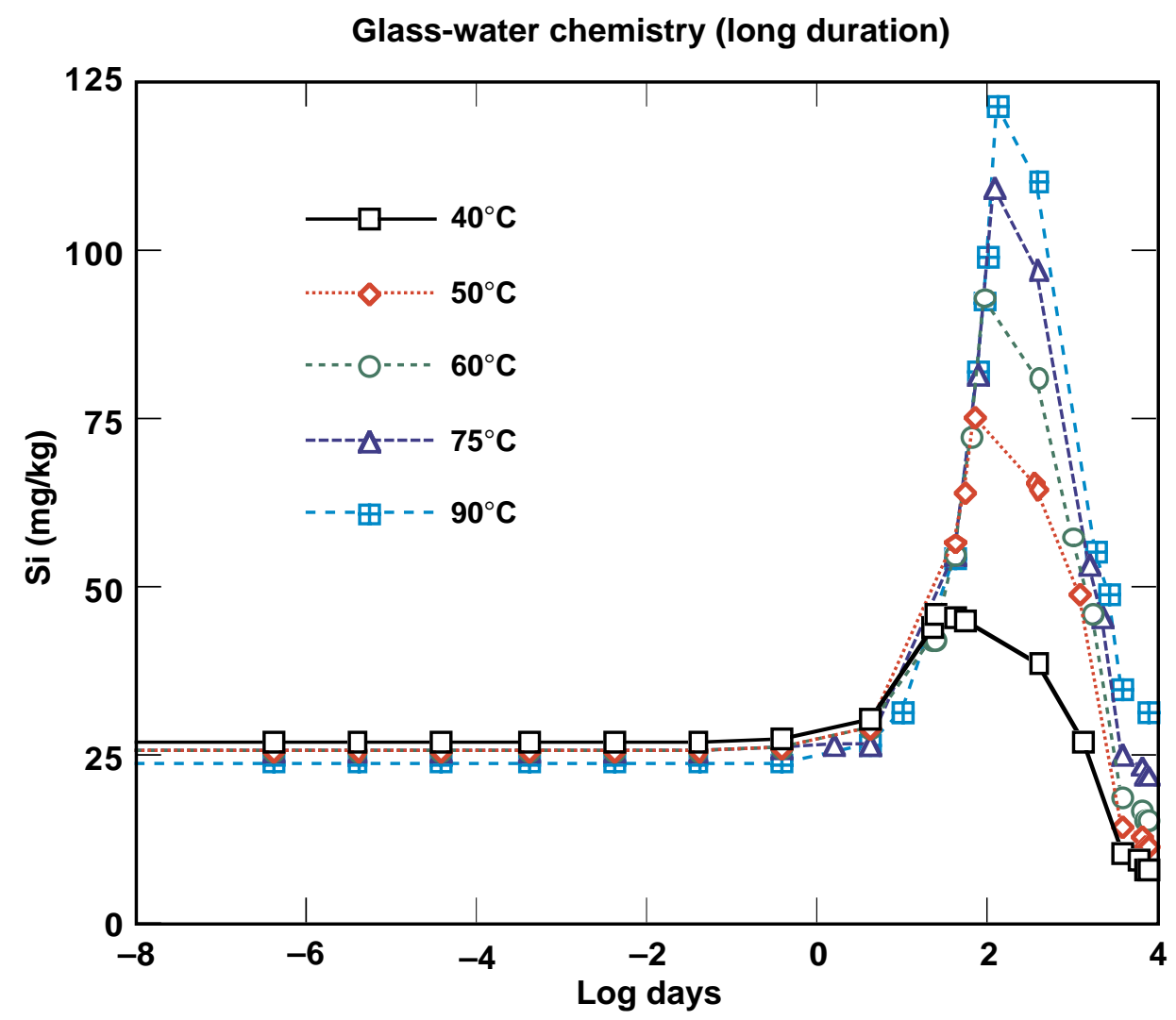

Figure 5-13. Changes in the chemistry of J-13 well water during reaction with glassy TSw3 tuff at $40^{\circ}, 50^{\circ}$, $60^{\circ}, 75^{\circ}$, and $90^{\circ} \mathrm{C}(\mathrm{Si})$. 


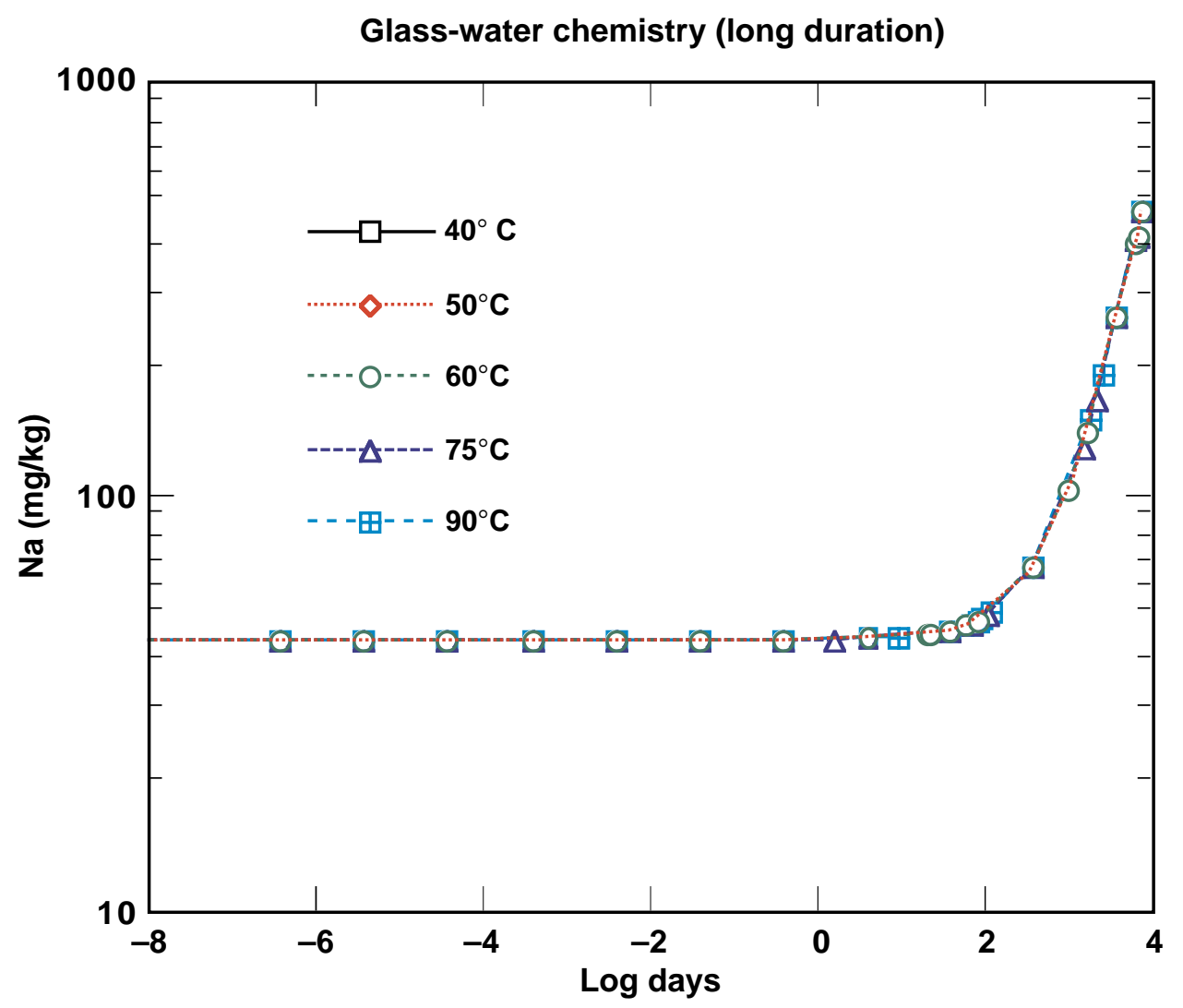

Figure 5-14. Changes in the chemistry of J-13 well water during reaction with glassy TSw3 tuff at $40^{\circ}, 50^{\circ}$, $60^{\circ}, 75^{\circ}$, and $90^{\circ} \mathrm{C}(\mathrm{Na})$. 


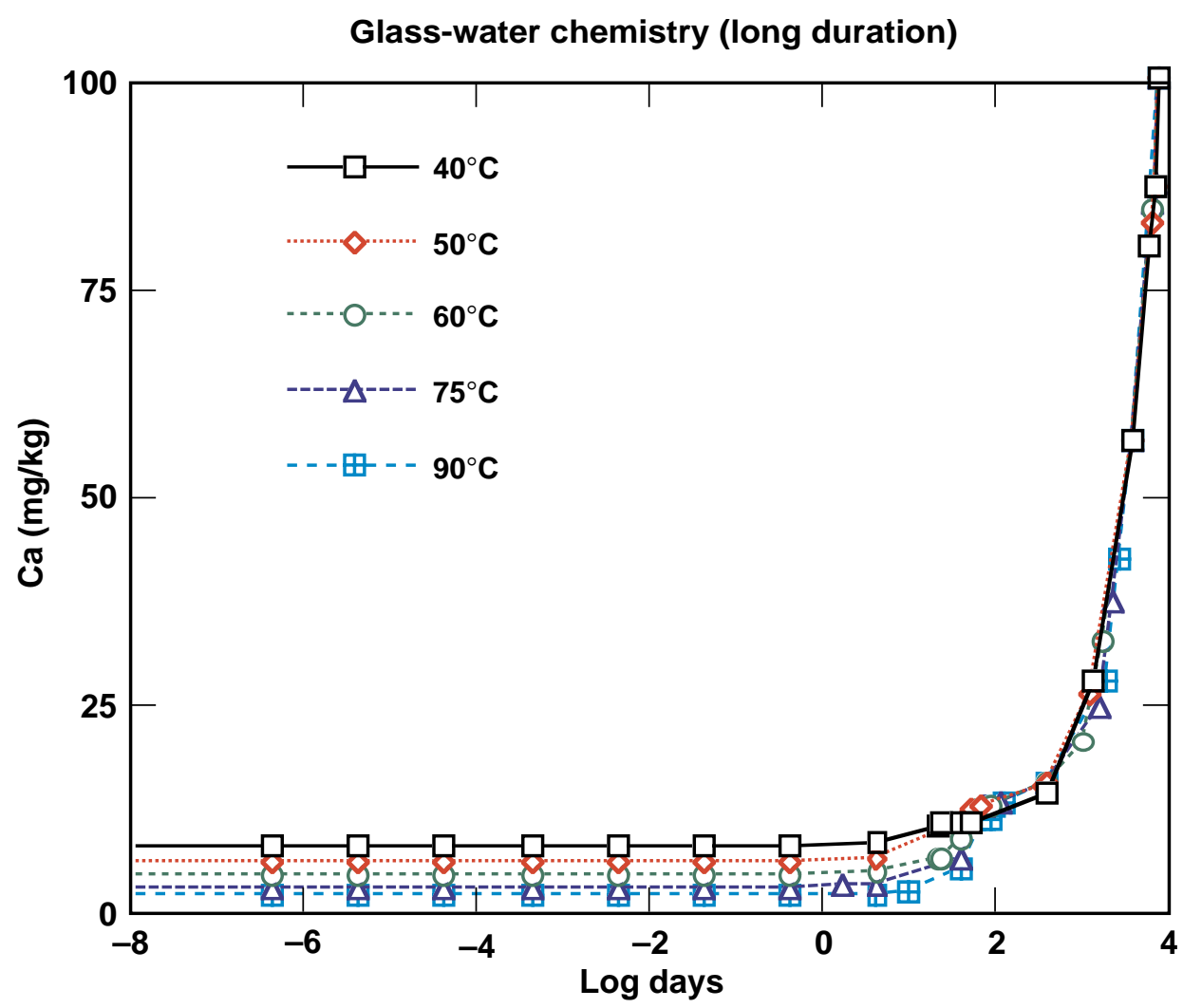

Figure 5-15. Changes in the chemistry of J-13 well water during reaction with glassy TSw3 tuff at $40^{\circ}, 50^{\circ}$, $60^{\circ}, 75^{\circ}$, and $90^{\circ} \mathrm{C}(\mathrm{Ca})$. 


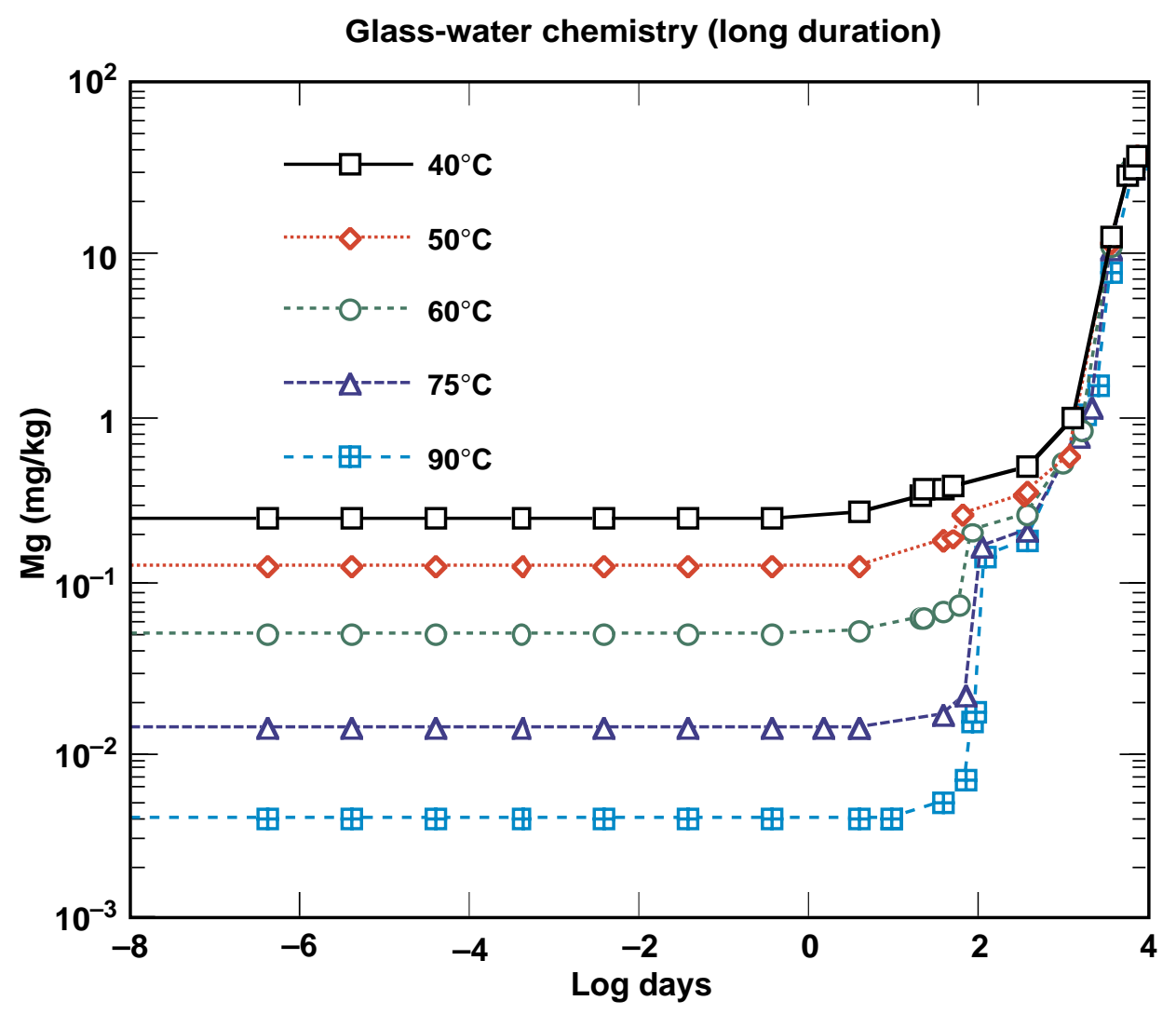

Figure 5-16. Changes in the chemistry of J-13 well water during reaction with glassy TSw3 tuff at $40^{\circ}, 50^{\circ}$, $60^{\circ}, 75^{\circ}$, and $90^{\circ} \mathrm{C}(\mathrm{Mg})$. 


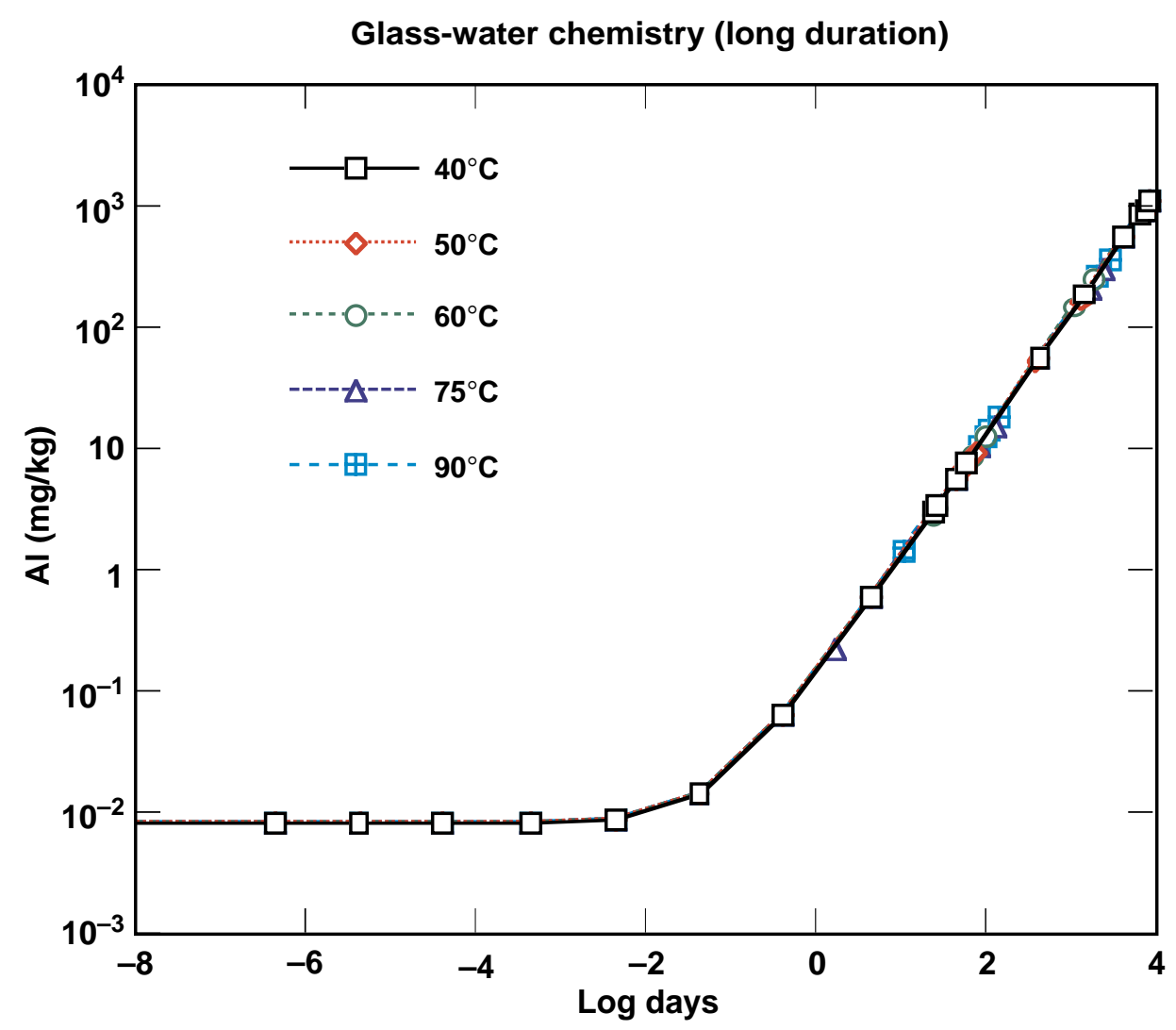

Figure 5-17. Changes in the chemistry of J-13 well water during reaction with glassy TSw3 tuff at $40^{\circ}, 50^{\circ}$, $60^{\circ}, 75^{\circ}$, and $90^{\circ} \mathrm{C}(\mathrm{Al})$. 


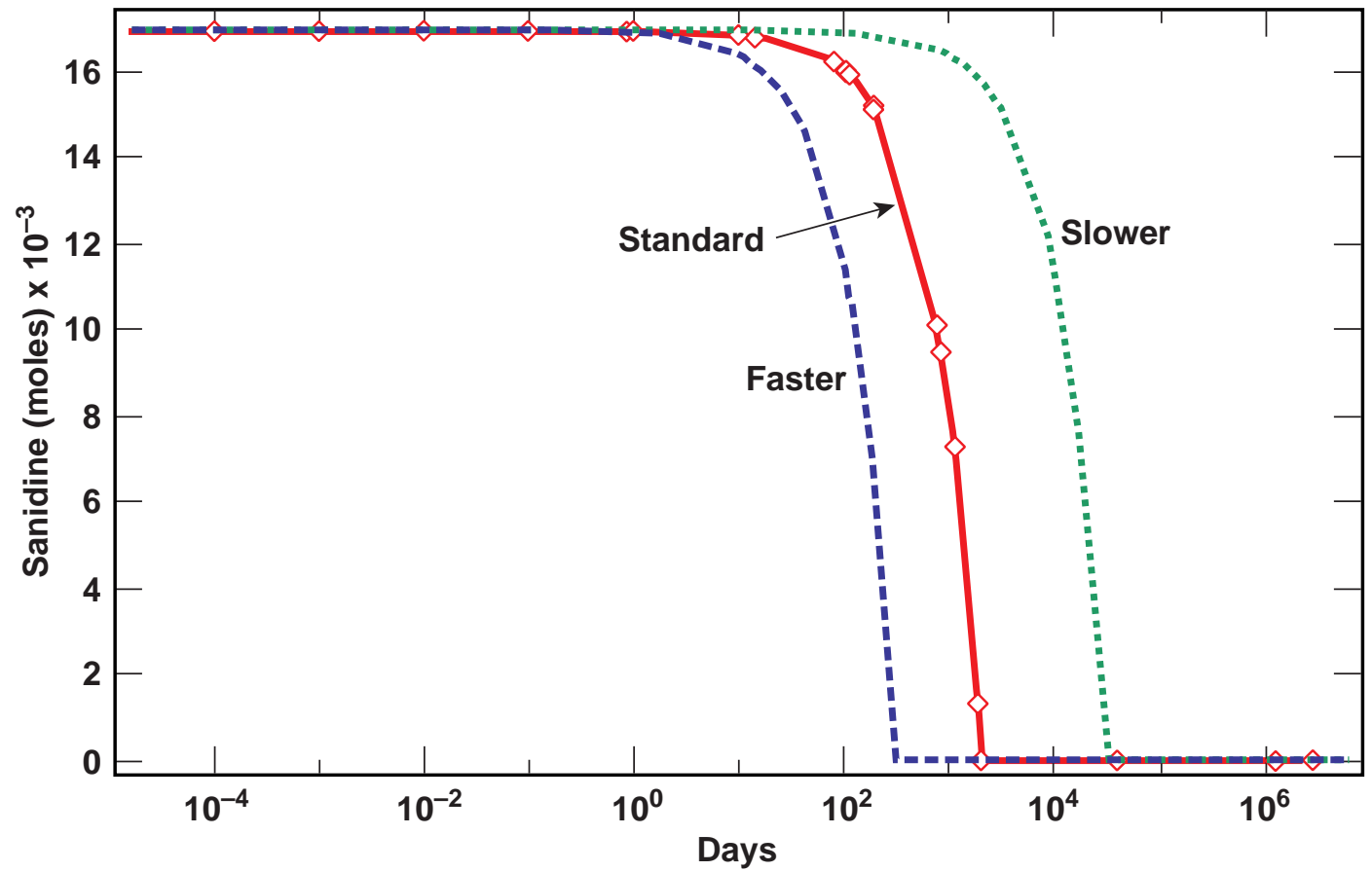

Figure 5-18. Changes in sanidine abundance resulting from a plus or minor 1 order of magnitude change in the dissolution rate of this mineral. 


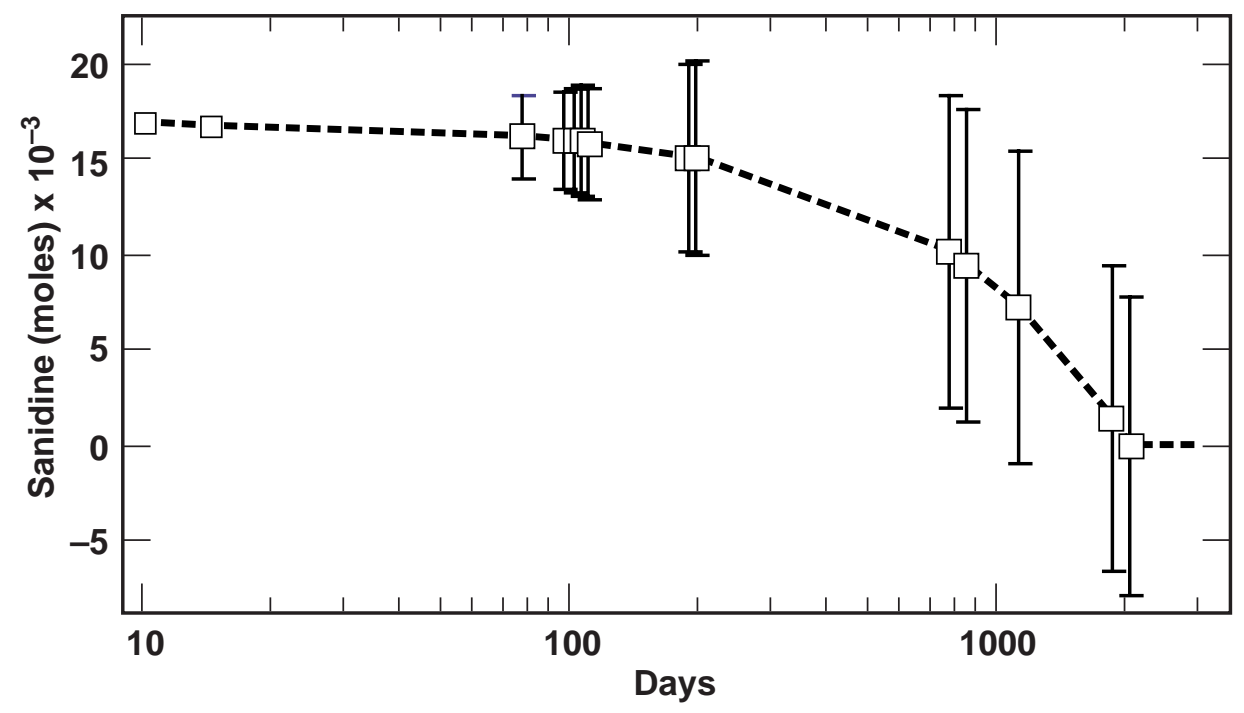

Figure 5-19. Estimates of sanidine abundance, assuming the dissolution rates used to construct Figure 5-18. The error bars represent the abundances computed from the curves in Figure 5-18. The negative abundance range is not physical. It is an artifact of calculating the uncertainty as a symmetric quantity. 
Repository heating can result in a single-phase above-boiling zone, a two-phase boiling zone, and a condensate zone

Dimensionless liquid saturation contours for 30 -yr-old SNF, an APD of $114 \mathrm{~kW} / \mathrm{acre}$, and a AML of 154.7 MTU/acre

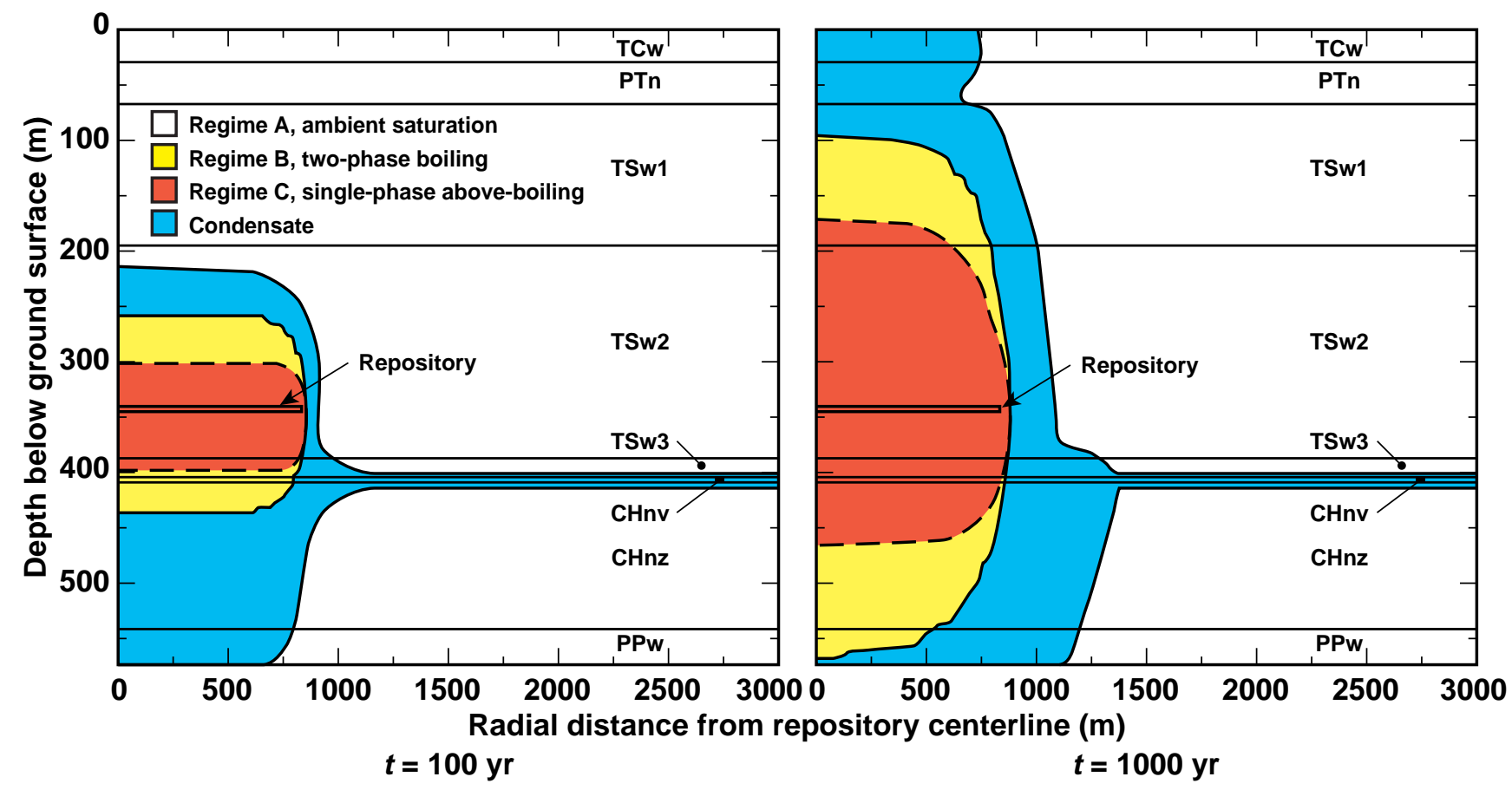

Figure 5-20. Hydrothermal regimes A-C at 100 and $1000 \mathrm{yr}$ after repository closure. 


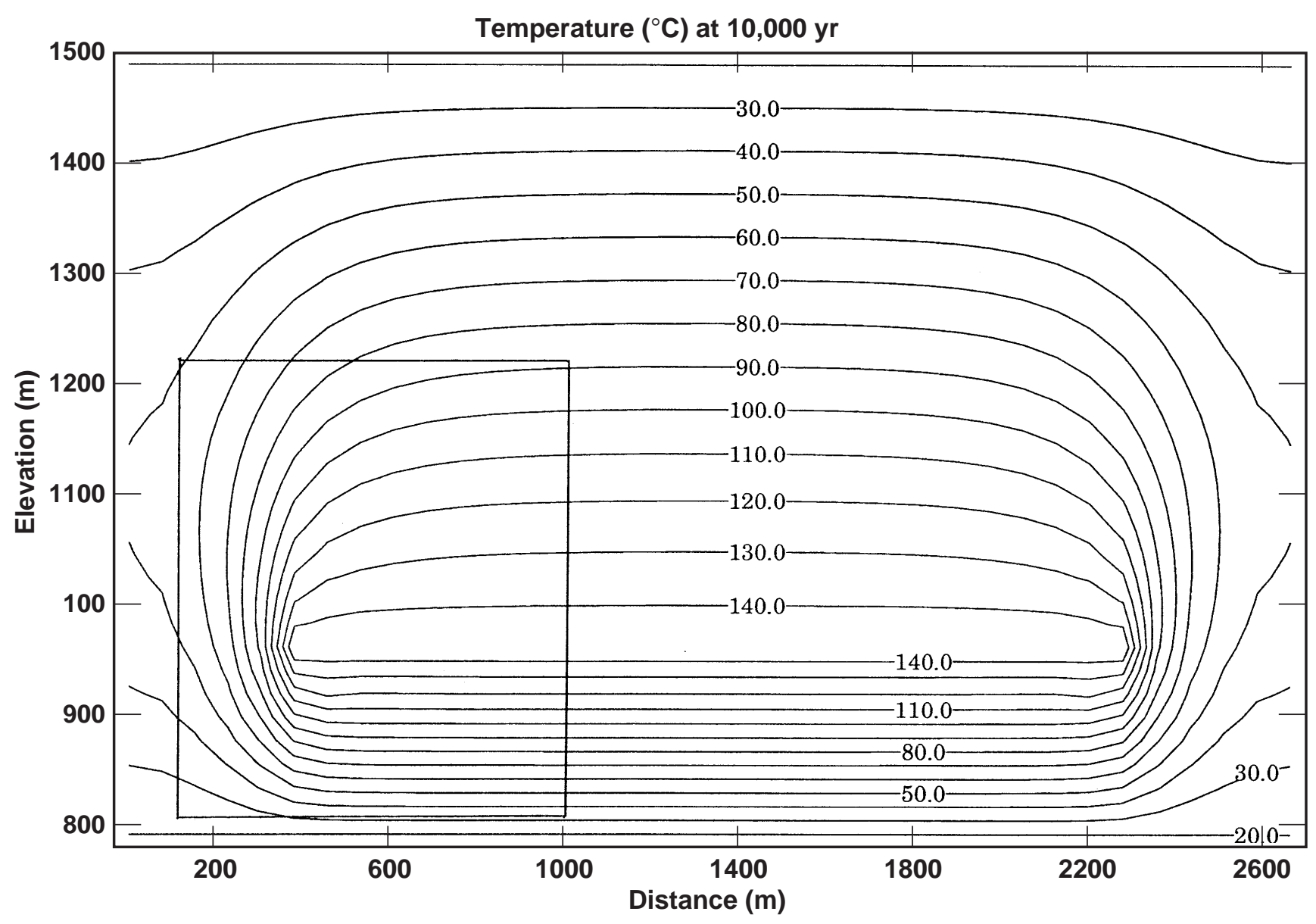

Figure 5-21. Temperature contours at 10,000 yr after waste emplacement. The repository was located at an elevation of $962 \mathrm{~m}$ and extends approximately 400 to $2250 \mathrm{~m}$. The large, boxed area indicates the location contoured in Figures 5-22 through 5-29. 


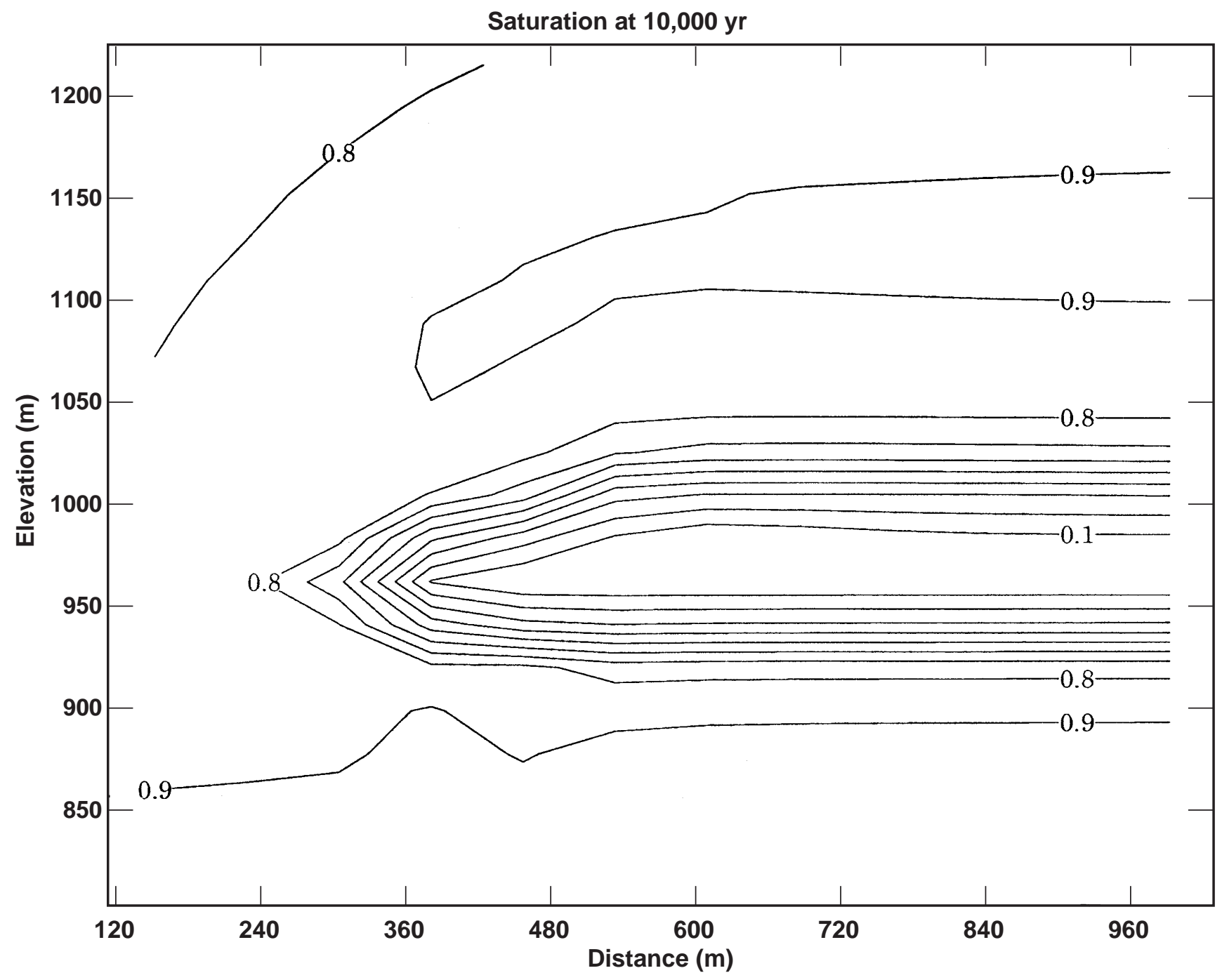

Figure 5-22. Saturation contours at 10,000 yr for the case with no coupling between silica transport and permeability change. 


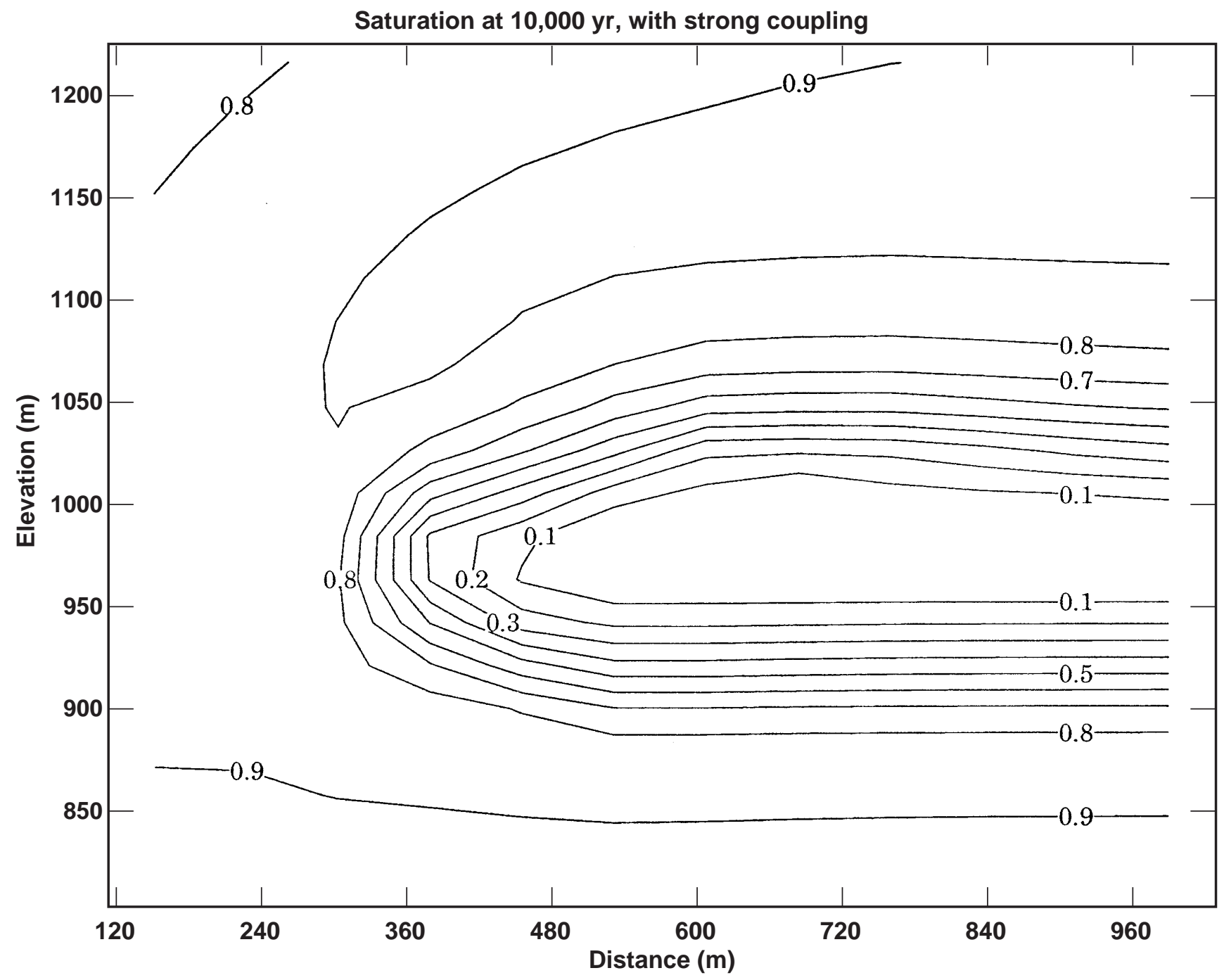

Figure 5-23. Saturation contours at 10,000 yr for the case with coupling between silica transport and permeability change. 


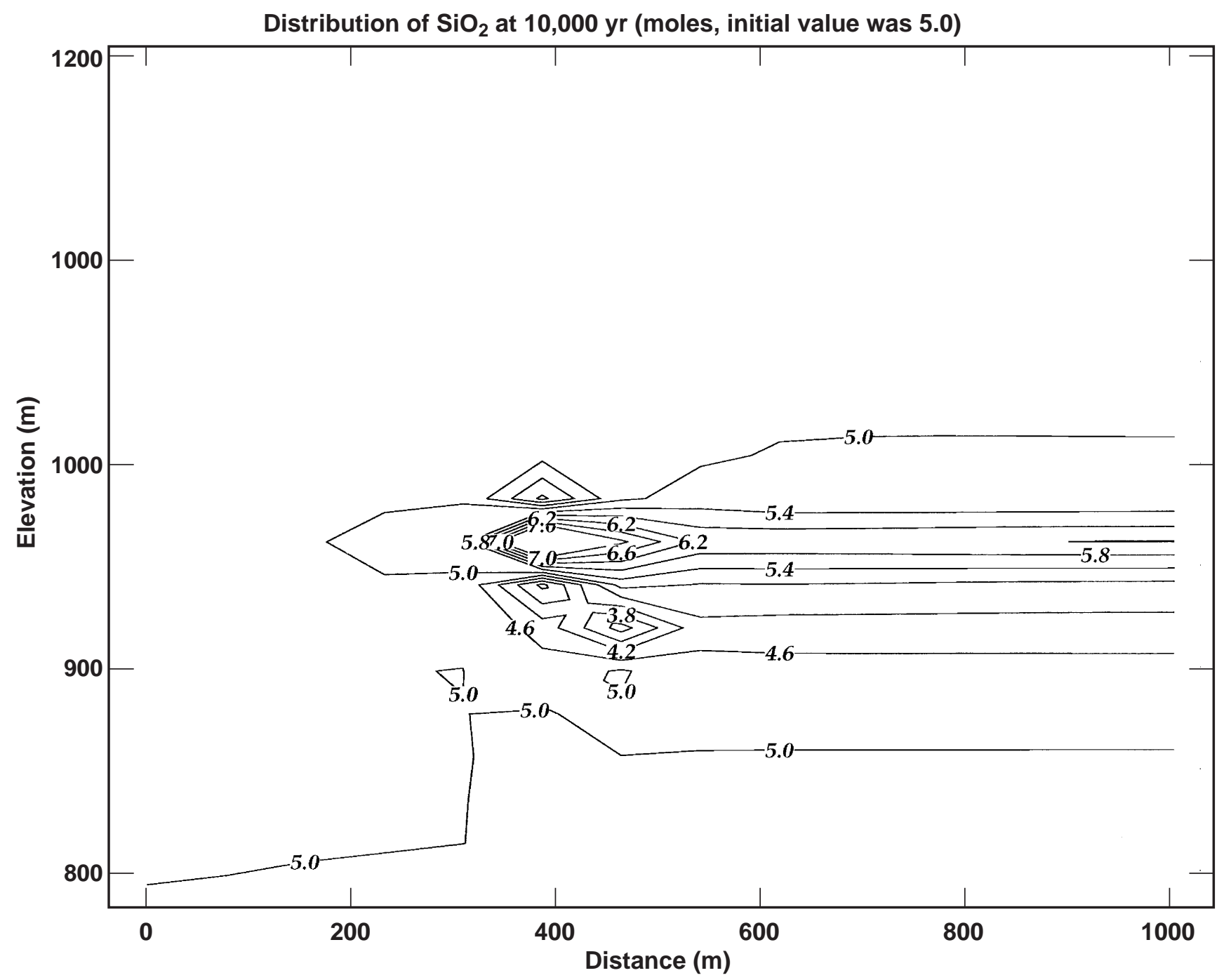

Figure 5-24. Distribution of silica at 10,000 yr with no coupling between silica transport and permeability change. The contours are in moles of $\mathrm{SiO}_{2} / \mathrm{kg}$ of rock. 


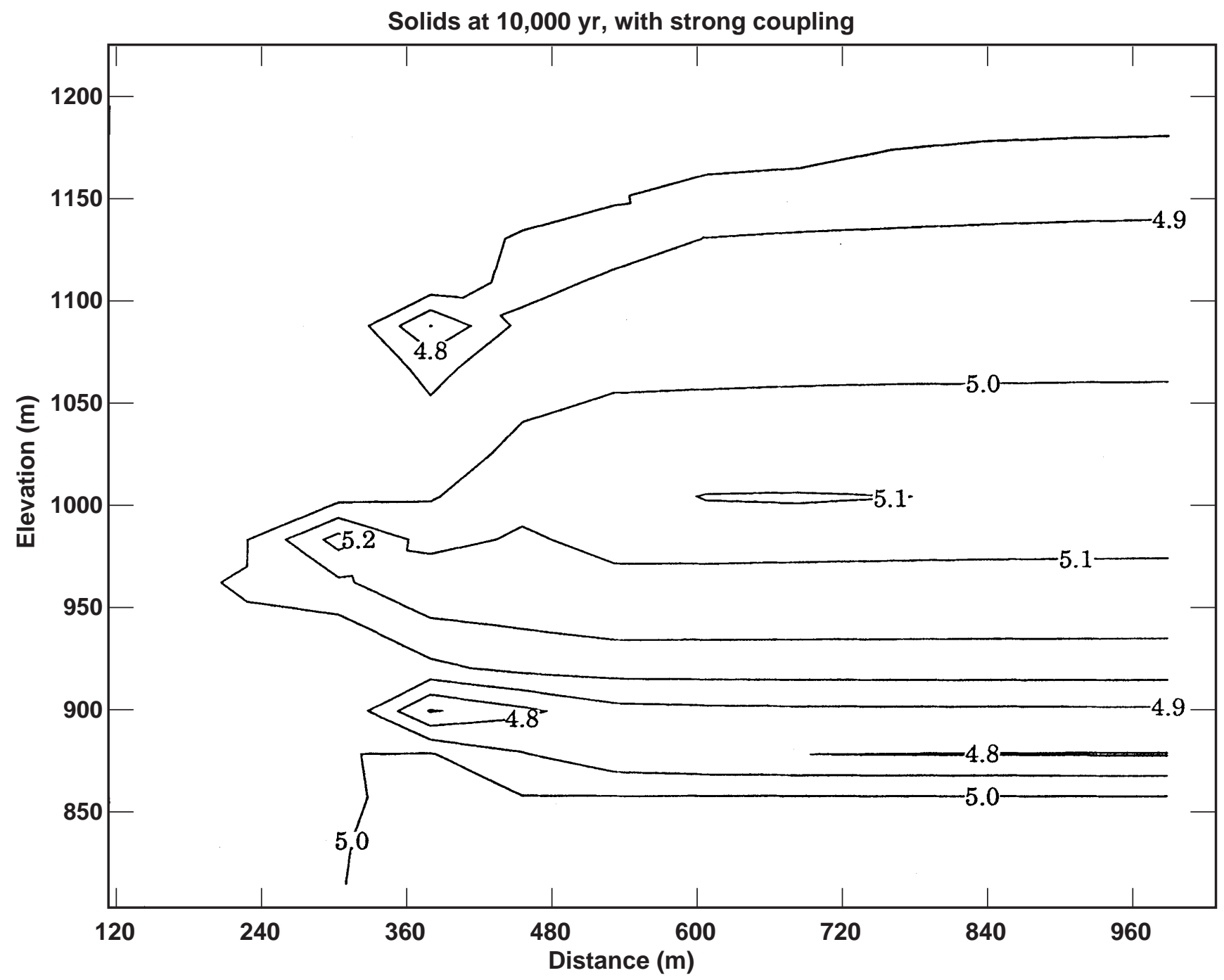

Figure 5-25. Distribution of silica at 10,000 yr with coupling between silica transport and permeability change. The contours are in moles of $\mathrm{SiO}_{2} / \mathrm{kg}$ of rock. 


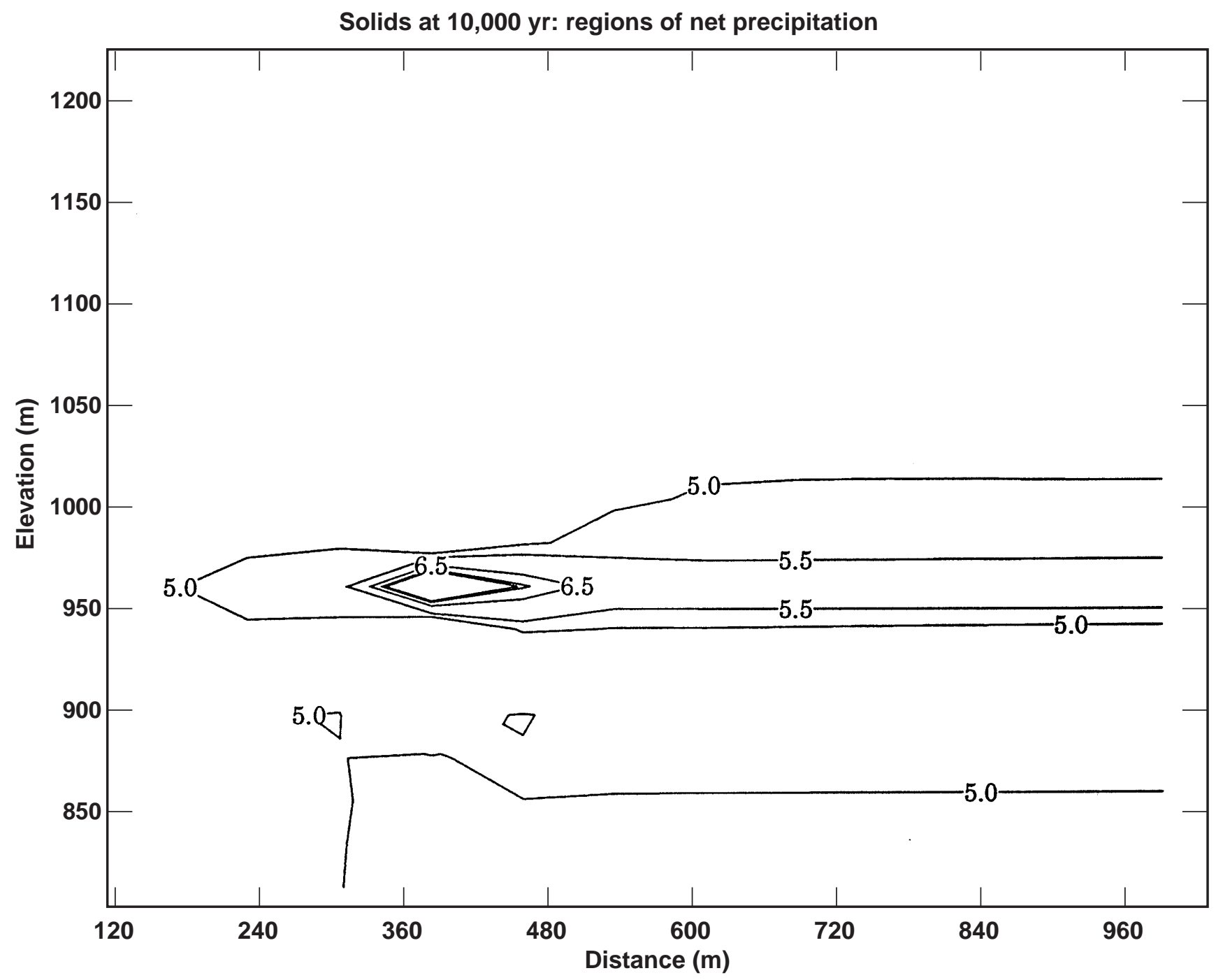

Figure 5-26. Regions of net precipitation of silica at 10,000 yr with no coupling between silica transport and permeability change. The contours are in moles of $\mathrm{SiO}_{2} / \mathrm{kg}$ of rock. The minimum silica concentration is $5.0 \mathrm{moles} / \mathrm{kg}$ of rock (the initial concentration), and the maximum is 7.3 moles $/ \mathrm{kg}$ of rock. 
Solids at $10,000 \mathrm{yr}$, with strong coupling: regions of net precipitation

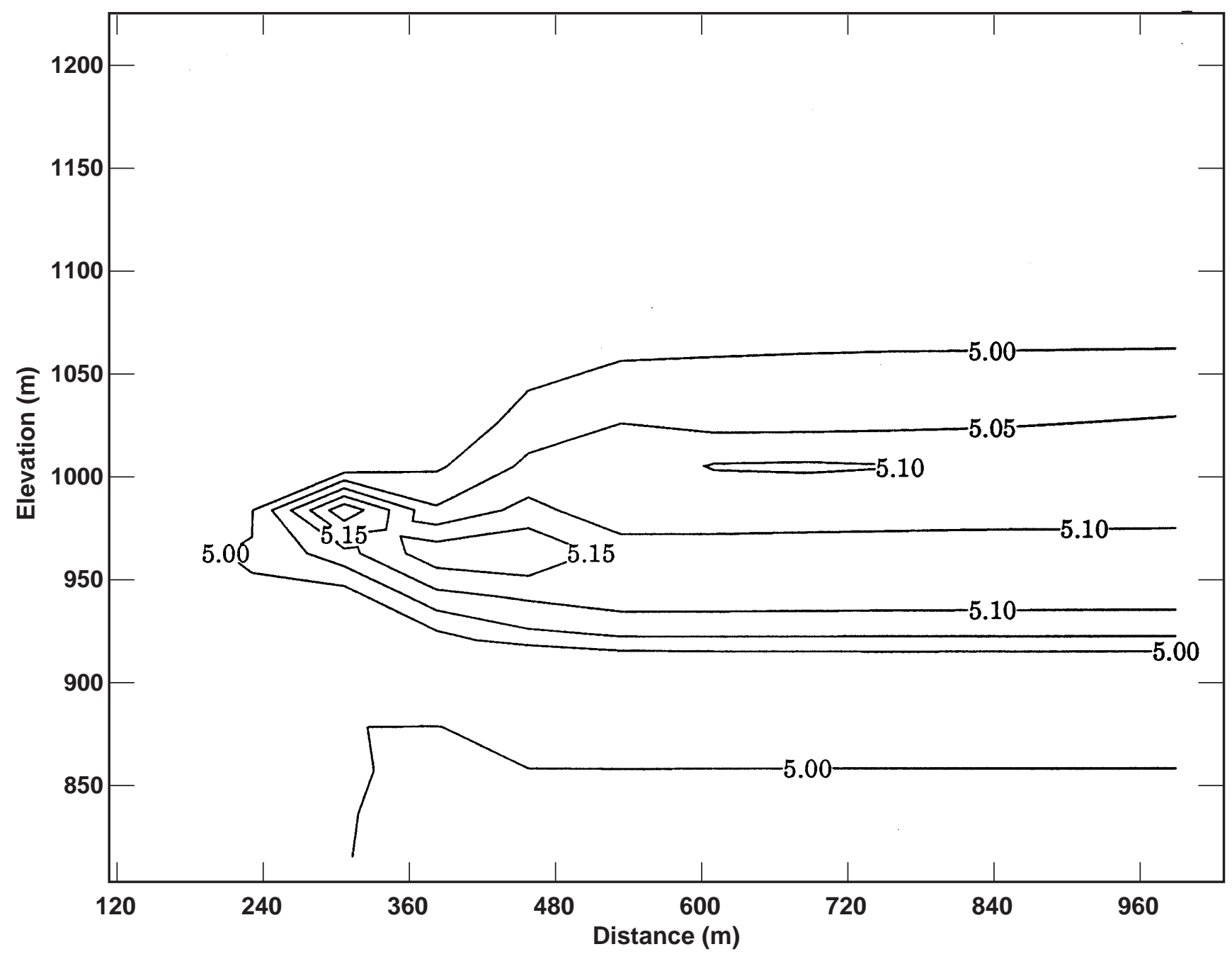

Figure 5-27. Regions of net precipitation of silica at 10,000 yr with coupling between silica transport and permeability change. The contours are in moles of $\mathrm{SiO}_{2} / \mathrm{kg}$ of rock. The minimum silica concentration is 5.0 moles $/ \mathrm{kg}$ of rock (the initial concentration), and the maximum is 5.24 moles $/ \mathrm{kg}$ of rock. 


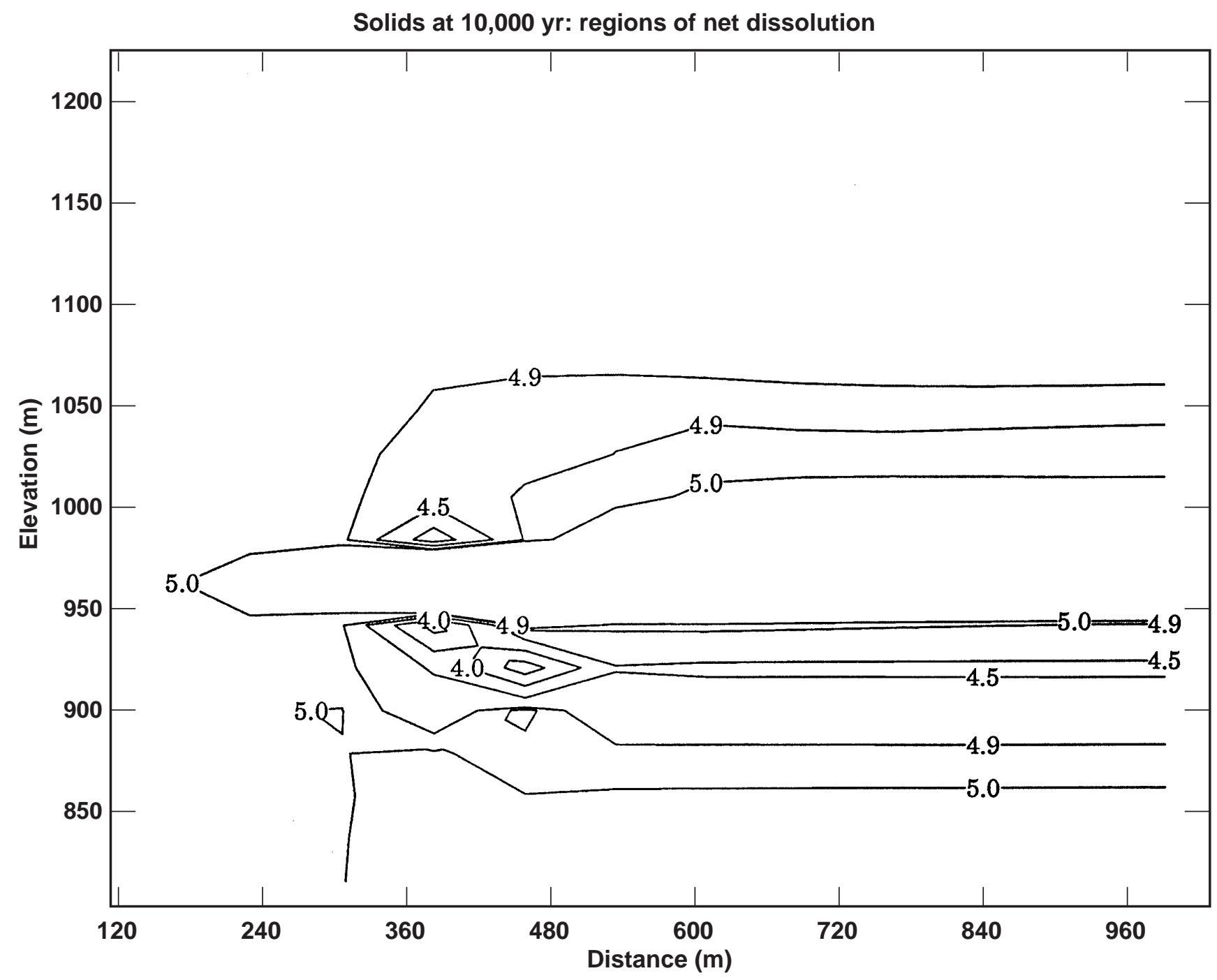

Figure 5-28. Regions of net dissolution of silica at 10,000 yr with no coupling between silica transport and permeability change. The contours are in moles of $\mathrm{SiO}_{2} / \mathrm{kg}$ of rock. The minimum silica concentration is 3.0 moles $/ \mathrm{kg}$ of rock, and the maximum is 5.0 moles $/ \mathrm{kg}$ of rock (the initial concentration). 


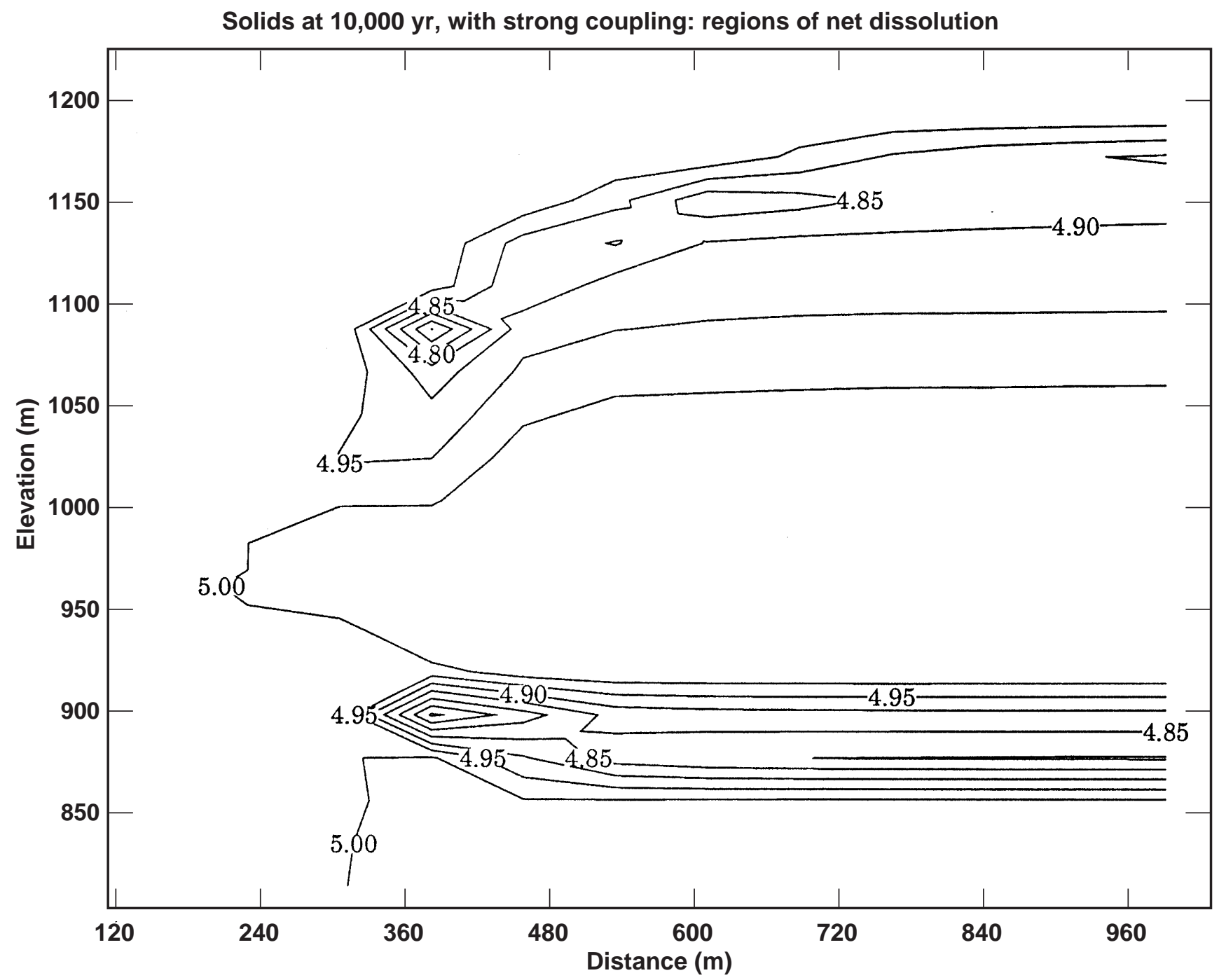

Figure 5-29. Regions of net dissolution of silica at 10,000 yr with coupling between silica transport and permeability change. The contours are in moles of $\mathrm{SiO}_{2} / \mathrm{kg}$ of rock. The minimum silica concentration is 4.69 moles $/ \mathrm{kg}$ of rock, and the maximum is 5.00 moles $/ \mathrm{kg}$ of rock (the initial concentration). 


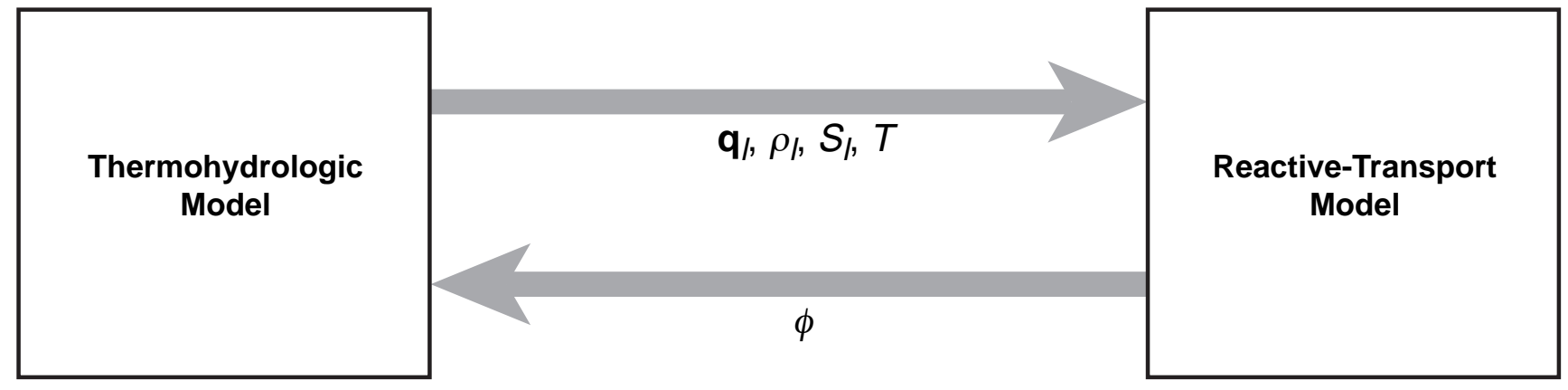

Figure 5-30. Passing of parameter fields during the sequential solution of the thermohydrologic and reactivetransport models. 
(a)

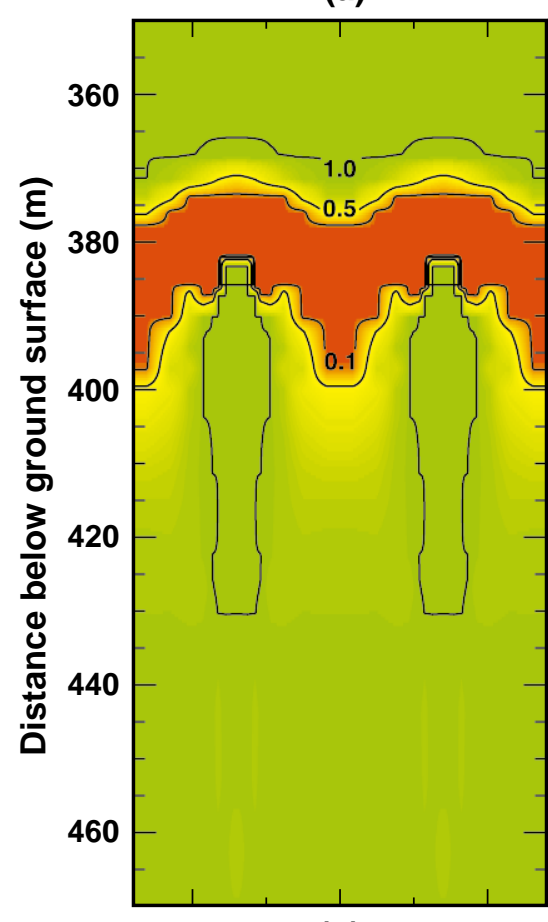

(d)

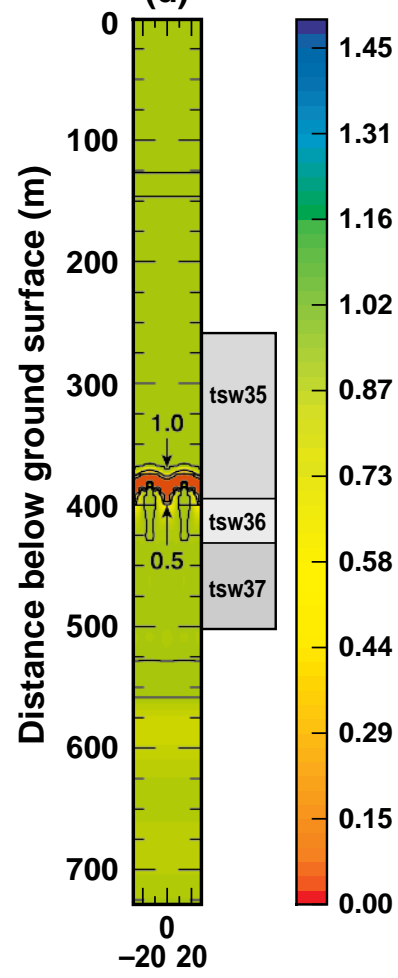

(b)

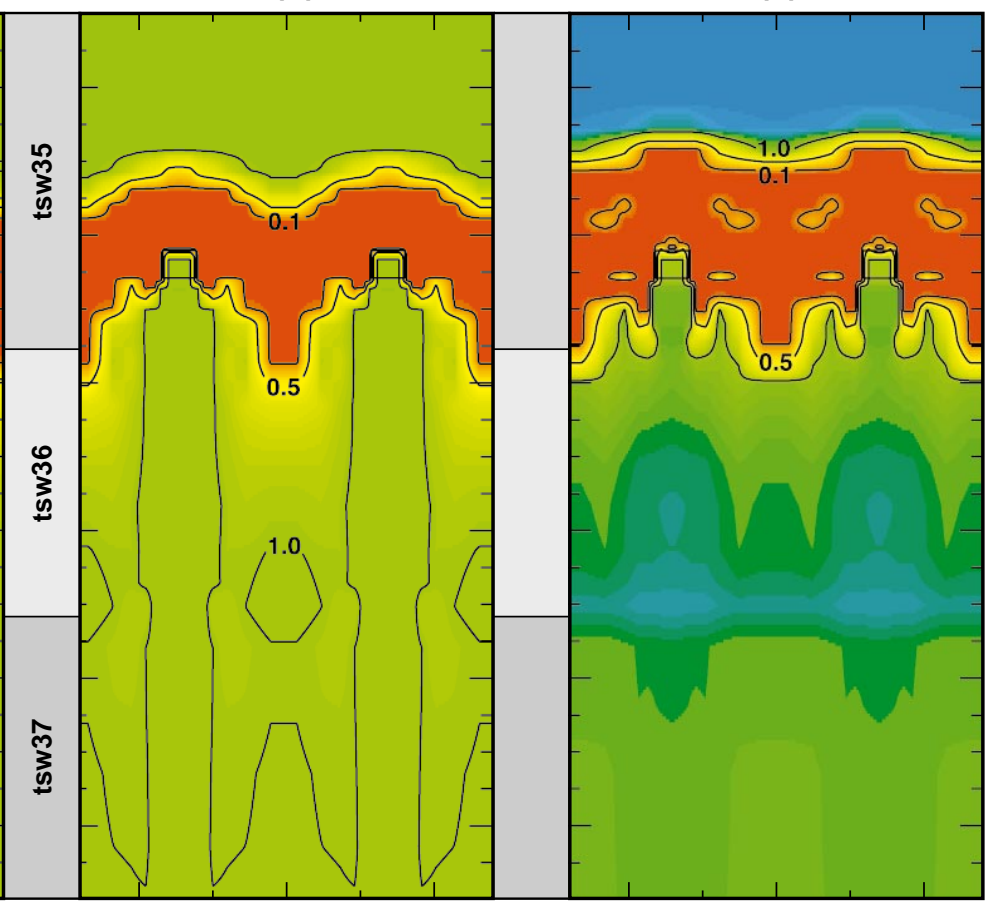

(e)

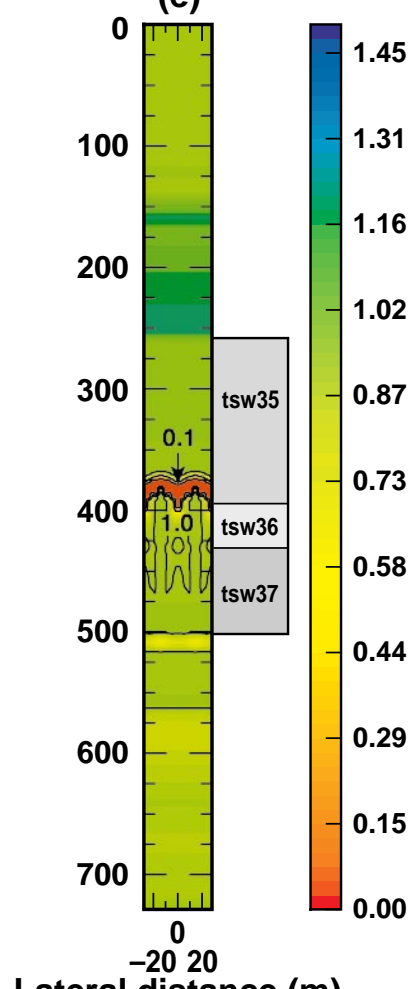

(f)

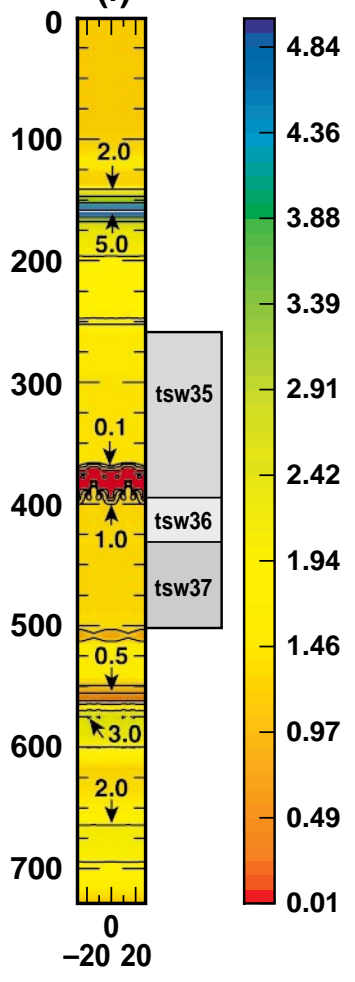

1.45

1.31

1.16

1.02

0.87

0.73

0.58

0.44

0.29

0.15

0.00

\section{Lateral distance (m)}

Figure 5-31. Relative change in fracture porosity (porosity: initial porosity) at $1000 \mathrm{yr}$ for base-case properties and point-load reference design (corresponding to reference Case 1), and incorporating dissolution and precipitation of quartz and crisotobalite. For (a) and (d), the reactive surface area is $0.01 \times$ the base-case value; for (b) and (e), the reactive surface is $1.0 \times$ the base-case value; for $(\mathrm{c})$ and $(\mathrm{f})$, the reactive surface area is $100 \times$ the base-case value. Porosity changes are fully coupled to permeability changes for all results shown. For (d) - (f), the entire model domain, ground surface to the water table, is shown mirrored horizontally across boundary symmetry planes. For (a) - (c), an inset region domain is expanded for greater detail. 
(a)

(b)

(c)

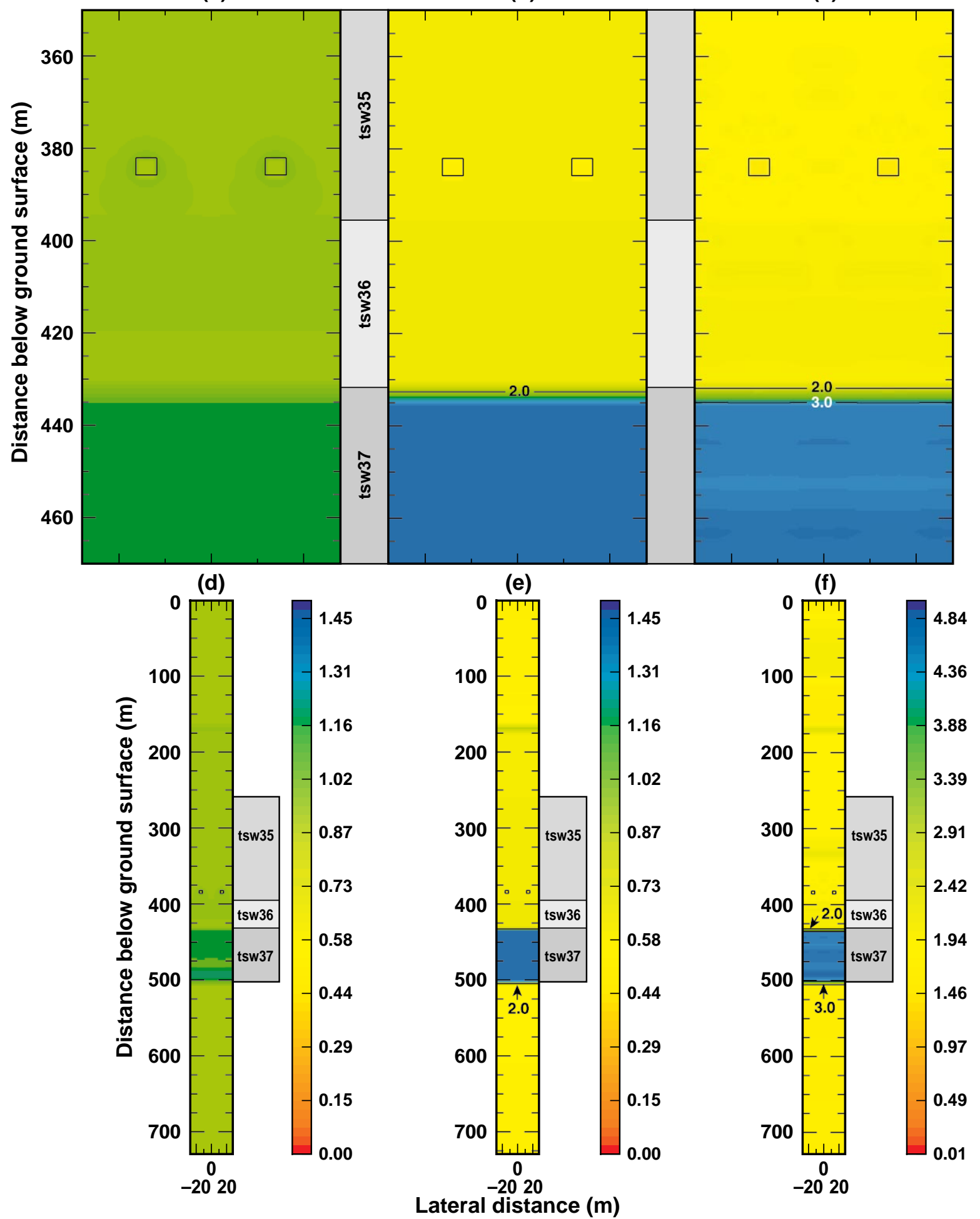

Figure 5-32. Relative change in matrix porosity (porosity: initial porosity) at $1000 \mathrm{yr}$ for base-case properties and point-load reference design (corresponding to reference Case 1), and incorporating dissolution and precipitation of quartz and crisotobalite. For (a) and (d), the reactive surface area is $0.01 \times$ the base-case value; for (b) and (e), the reactive surface is $1.0 \times$ the base-case value; for $(\mathrm{c})$ and $(\mathrm{f})$, the reactive surface area is $100 \times$ the base-case value. Porosity changes are fully coupled to permeability changes for all results shown. For (d) - (f), the entire model domain, ground surface to the water table, is shown mirrored horizontally across boundary symmetry planes. For (a) - (c), an inset region domain is expanded for greater detail. 
(a) quartz

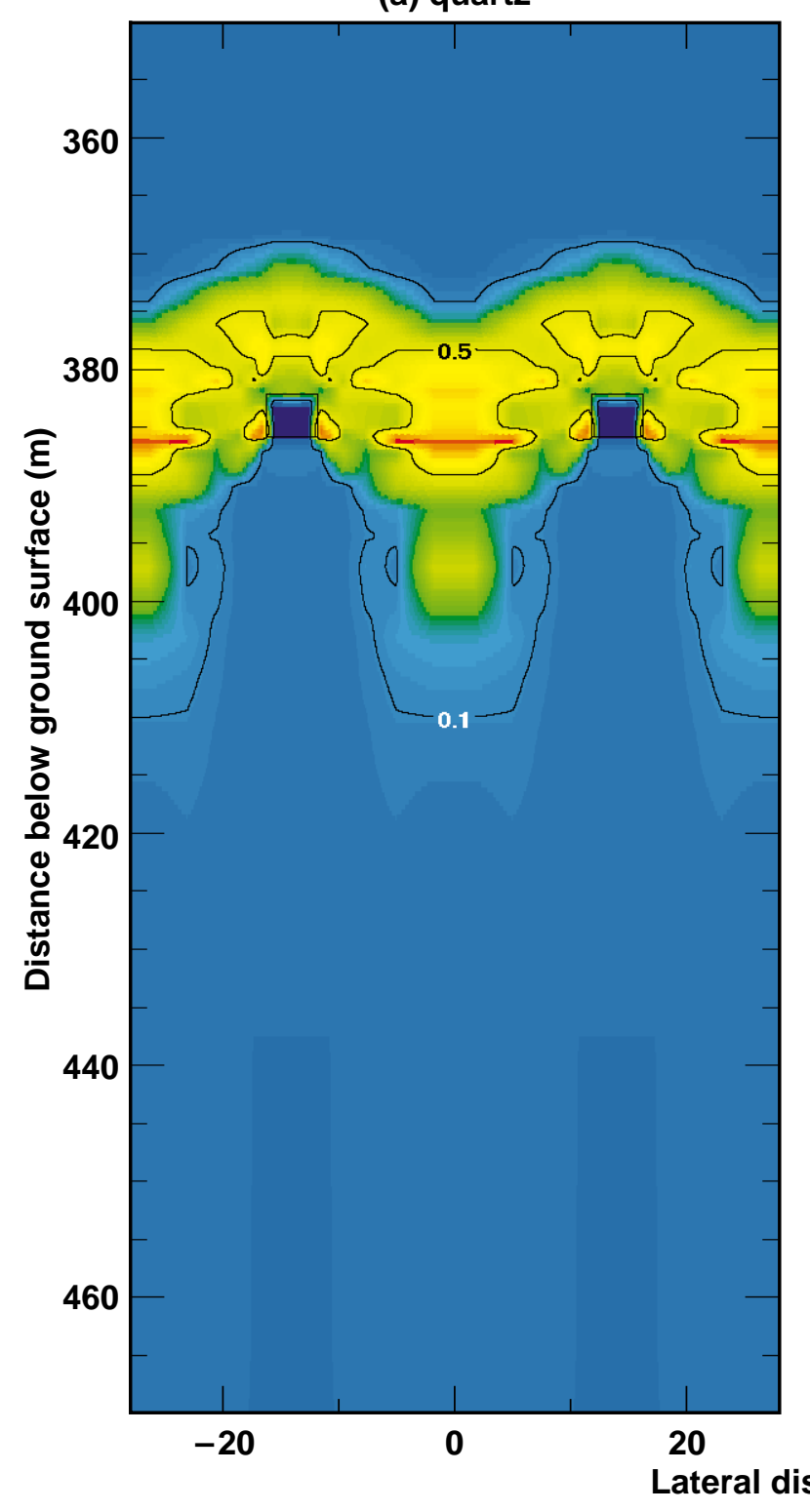

(b) cristobalite

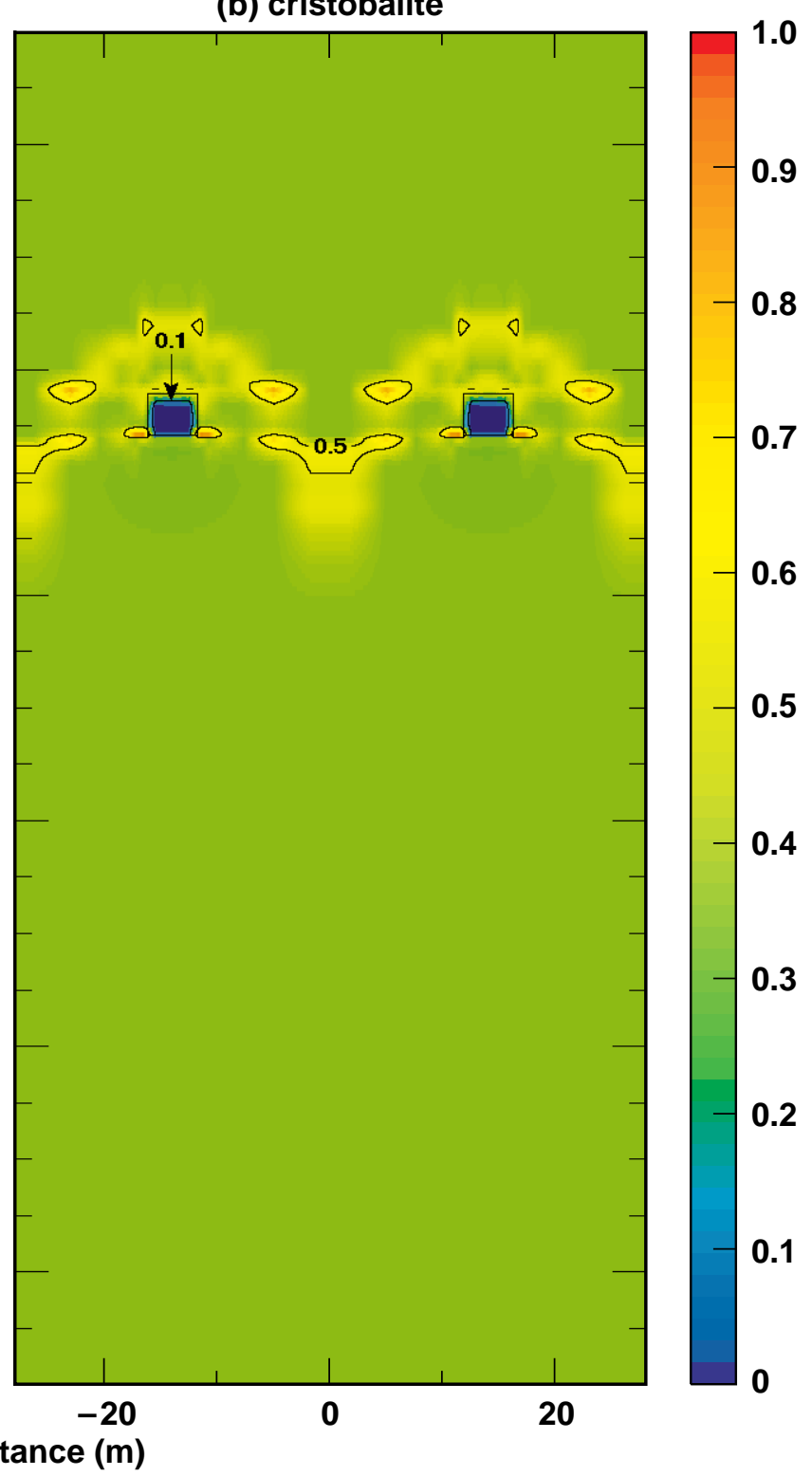

Figure 5-33. Mineral volumetric fractions in the fracture plane at $1000 \mathrm{yr}$ for the base case (corresponding to reference Case 1) : (a) quartz and (b) cristobalite. 
(a) temperature $\left({ }^{\circ} \mathrm{C}\right)$

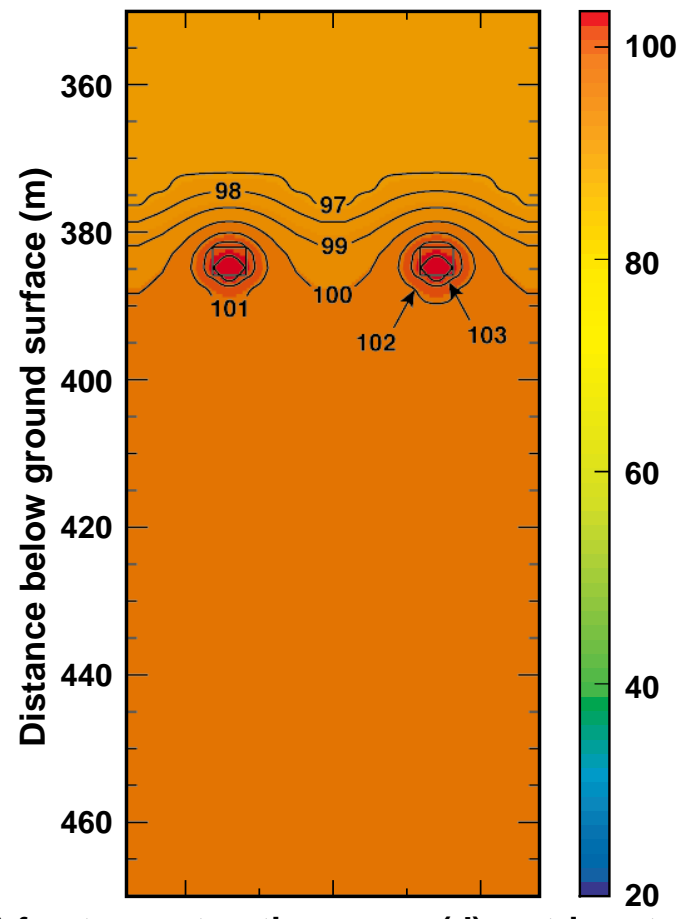

(c) fracture saturation

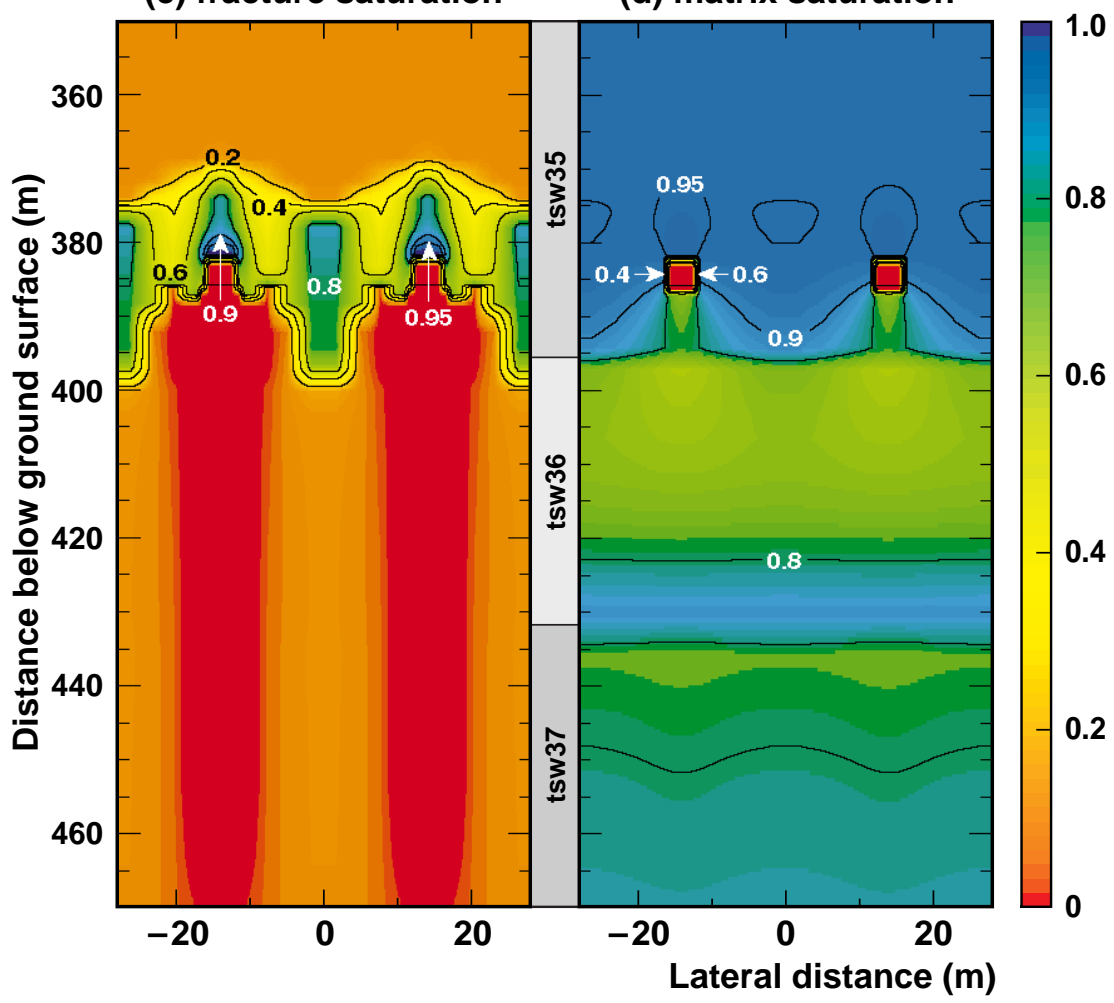

(b) fracture permeability

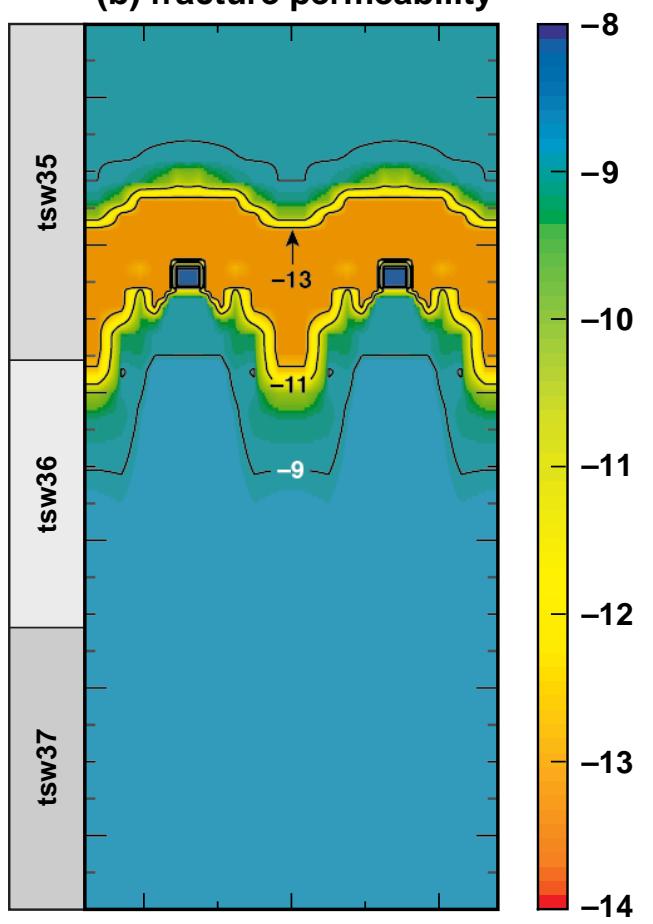

(e) gas pressure

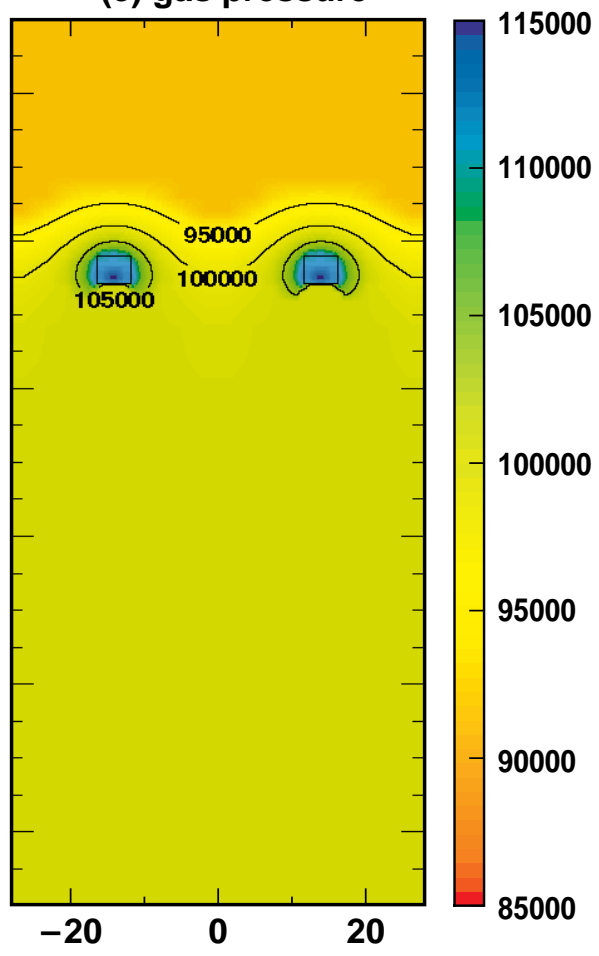

Figure 5-34. Base-case (corresponding to reference Case 1) conditions at $1000 \mathrm{yr}$ : (a) temperature in the fractures, (b) $\log$ base 10 of fracture permeability $\left(\mathrm{m}^{2}\right)$, (c) liquid saturation of the fractures, (d) liquid saturation of the matrix, and (e) gas pressure of the fractures. 

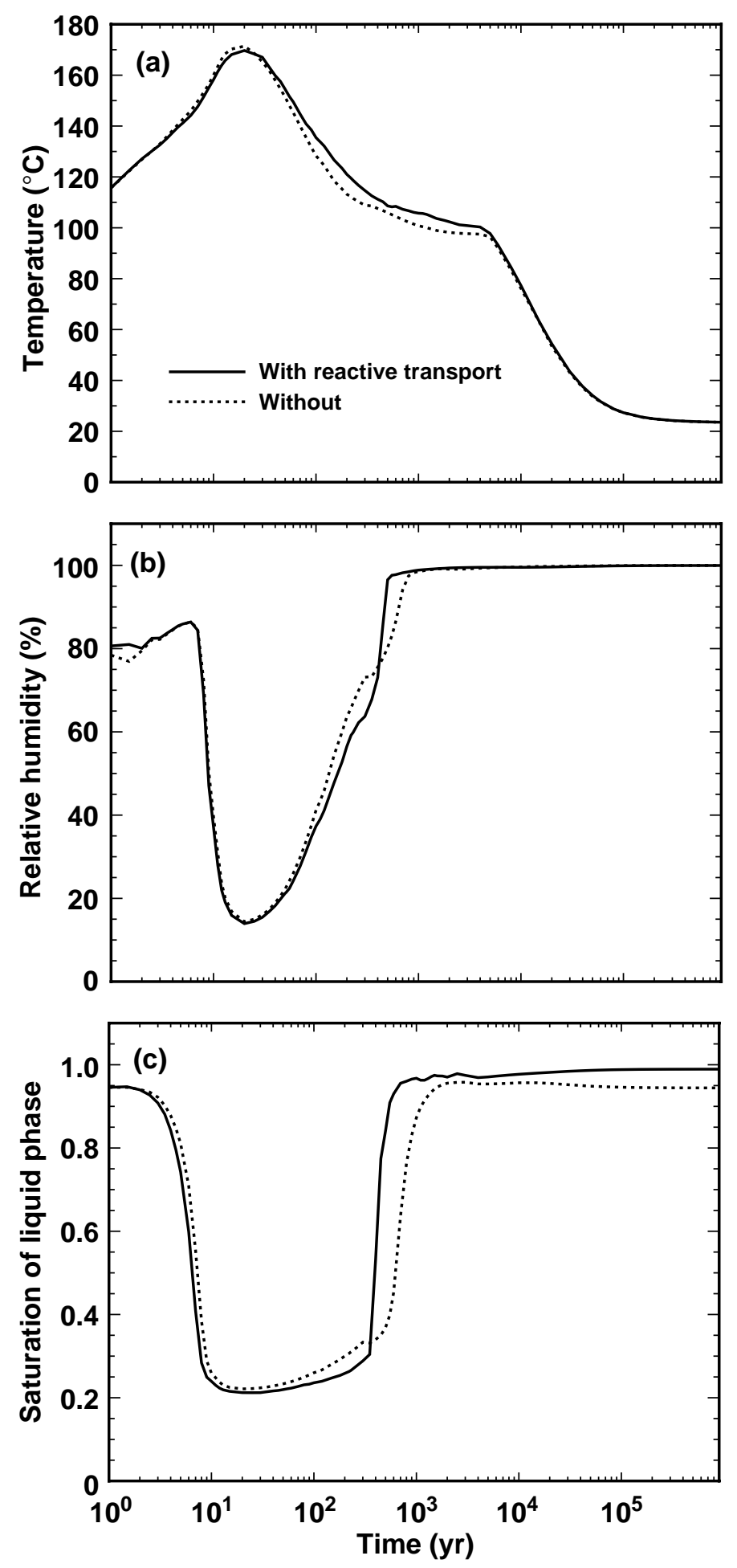

Figure 5-35. (a) Temperature history of the WP with and without silica reactive transport, (b) relative humidity on the WP, and (c) liquid saturation in the rock above the drift crown. 
(a) fracture porosity ratio change

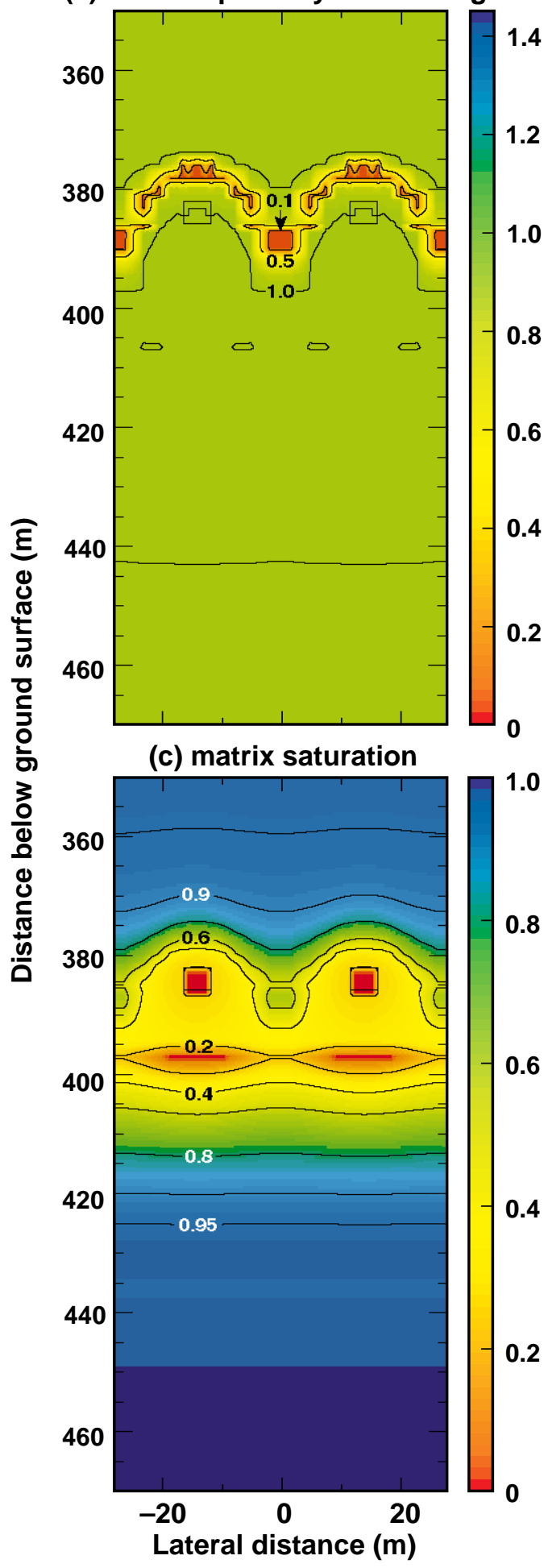

(b) temperature $\left({ }^{\circ} \mathrm{C}\right)$

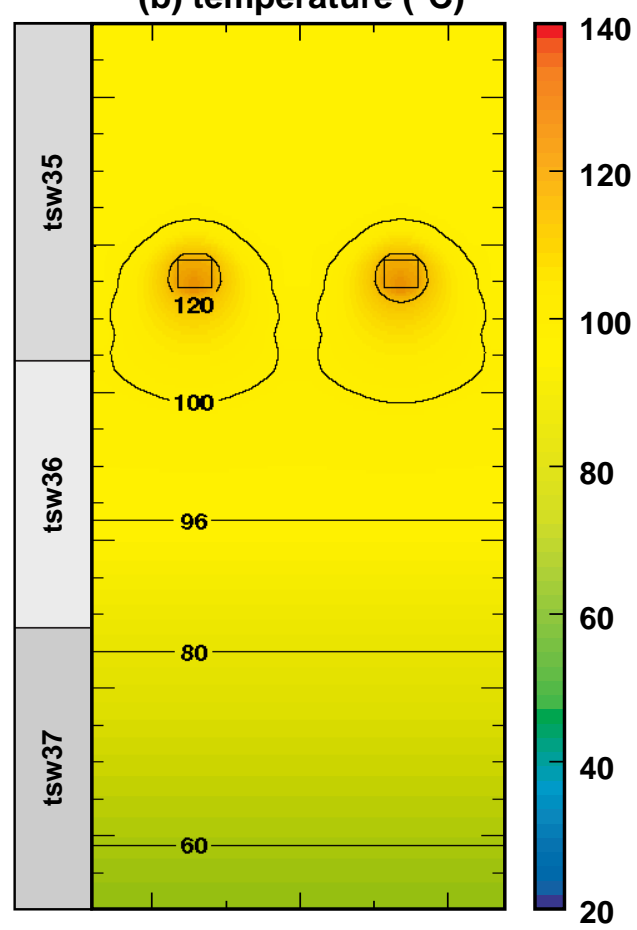

(d) fracture saturation

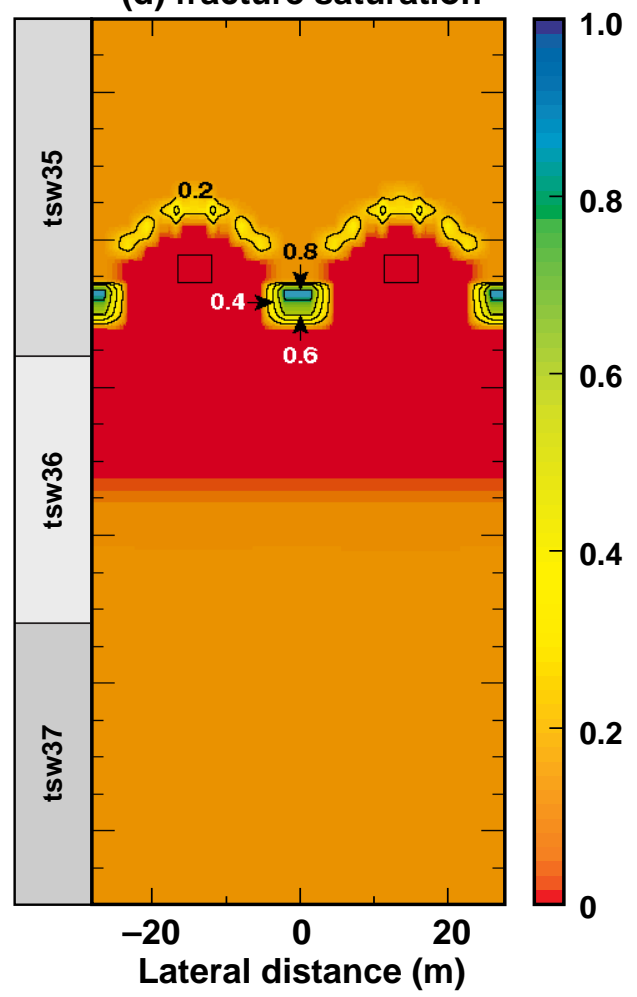

Figure 5-36. Base-case (corresponding to reference Case 1) conditions at $100 \mathrm{yr}$ : (a) fracture porosity ratio change, $(b)$ temperature, $(c)$ matrix saturation, and (d) fracture saturation. 
(a) fracture porosity ratio change

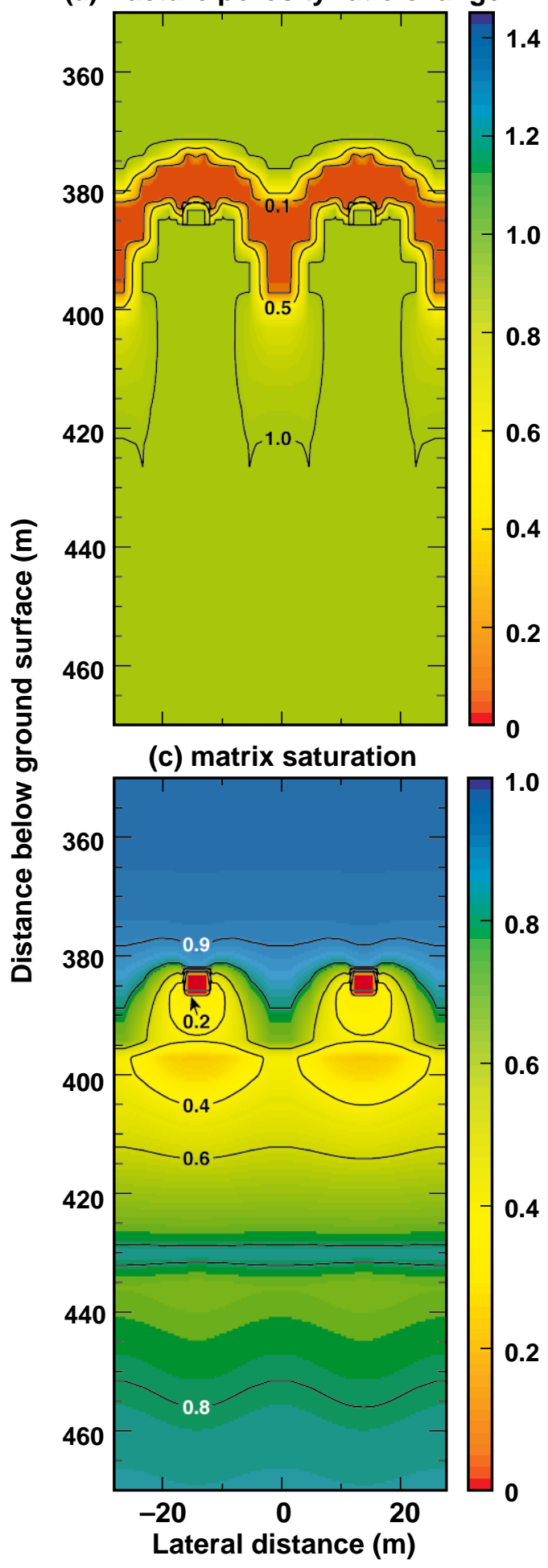

(b) temperature $\left({ }^{\circ} \mathrm{C}\right)$

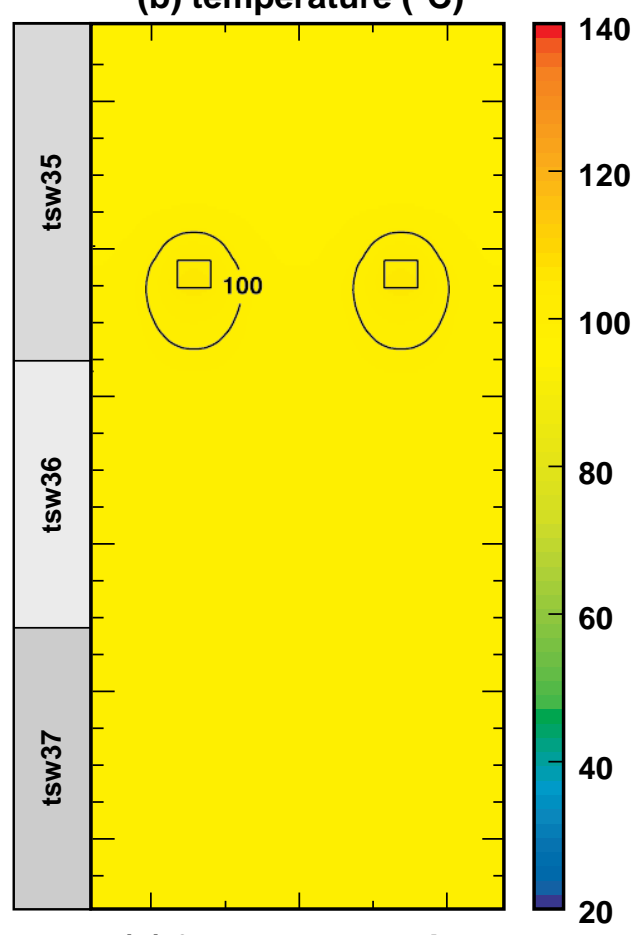

(d) fracture saturation

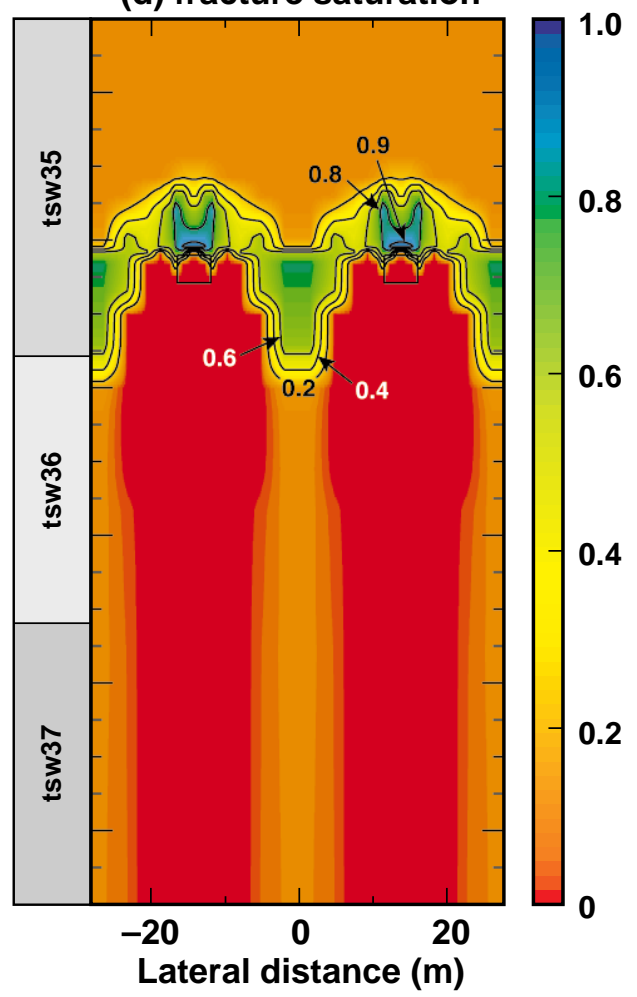

Figure 5-37. Base-case (corresponding to reference Case 1) conditions at $500 \mathrm{yr}$ : (a) fracture porosity ratio change, (b) temperature, (c) matrix saturation, and (d) fracture saturation. 
(a) fracture porosity ratio change

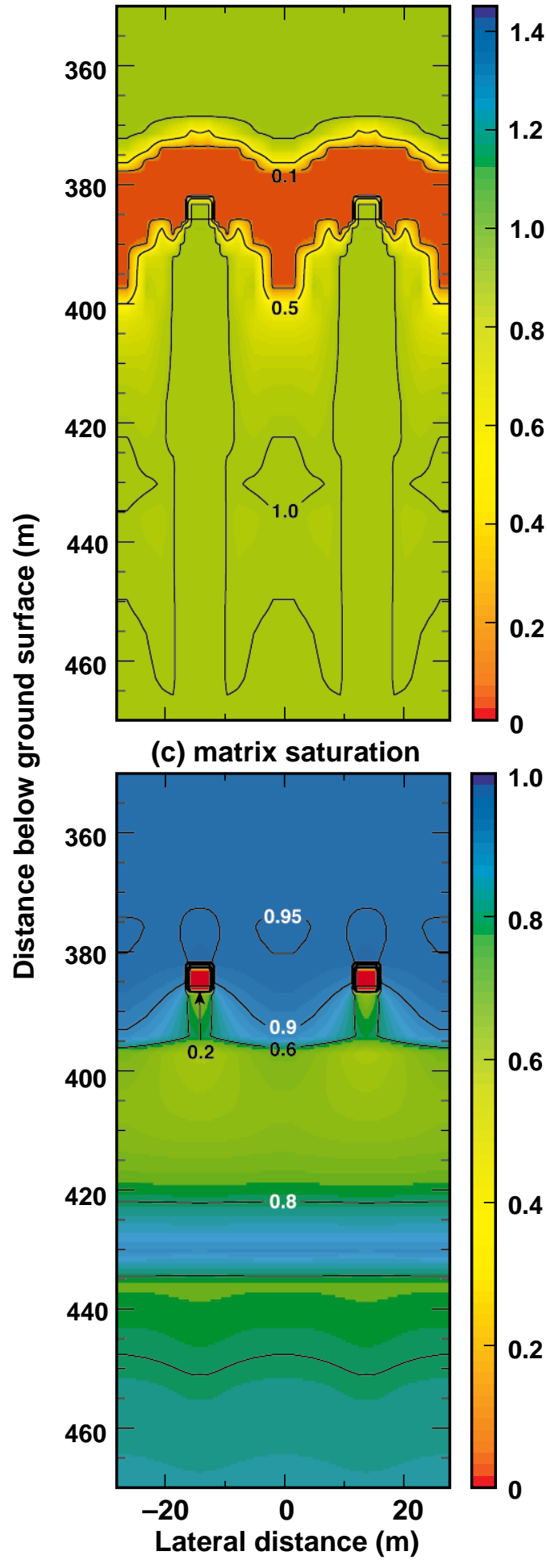

(b) temperature $\left({ }^{\circ} \mathrm{C}\right)$

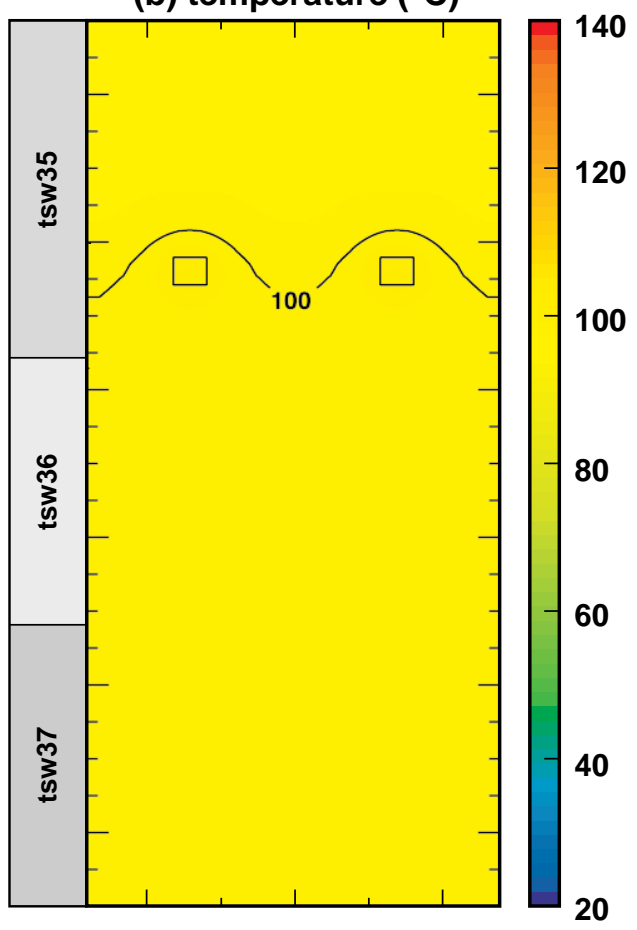

(d) fracture saturation

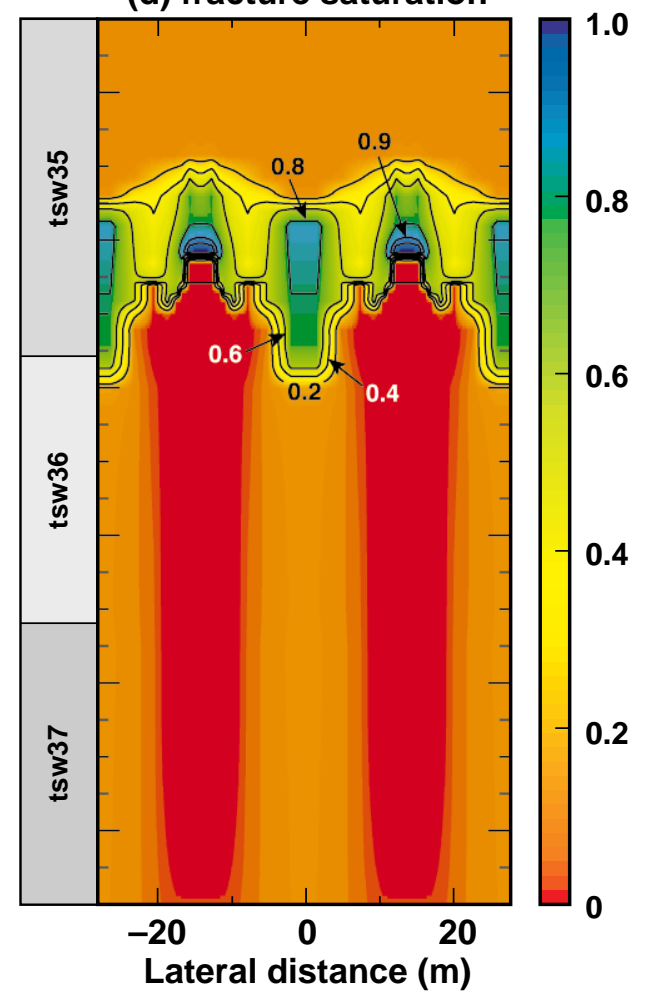

Figure 5-38. Base-case (corresponding to reference Case 1) conditions at $1000 \mathrm{yr}$ : (a) fracture porosity ratio change, $(b)$ temperature, $(c)$ matrix saturation, and $(d)$ fracture saturation. 
(a) fracture porosity ratio change

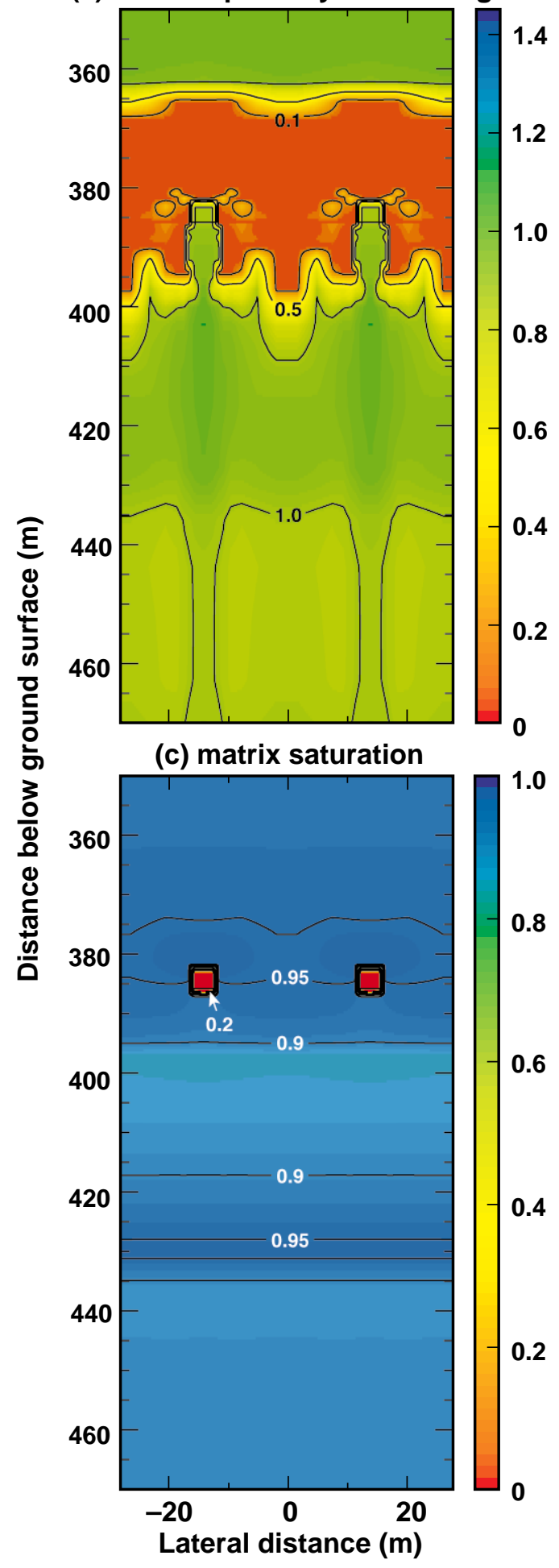

(b) temperature $\left({ }^{\circ} \mathrm{C}\right)$

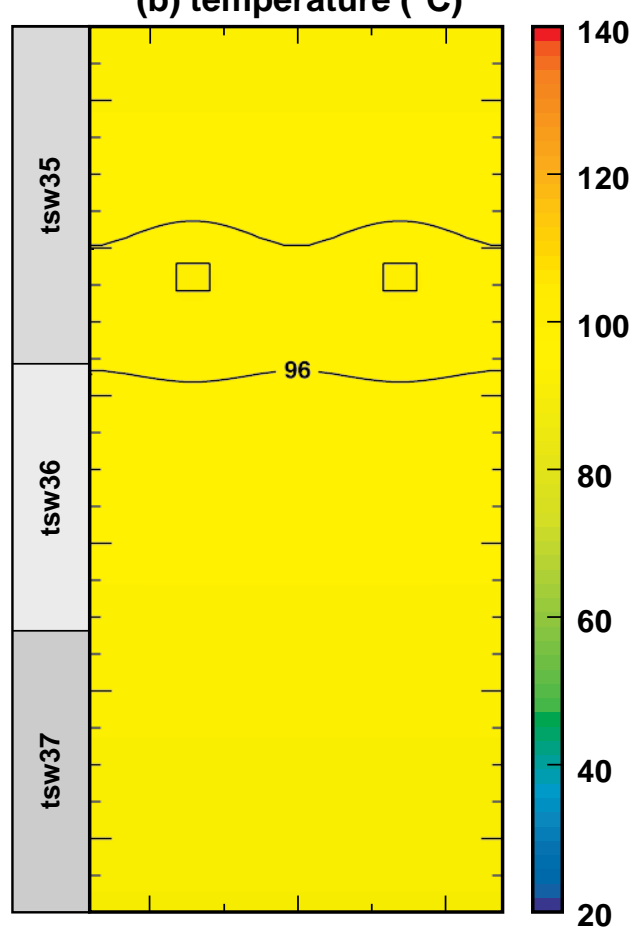

(d) fracture saturation

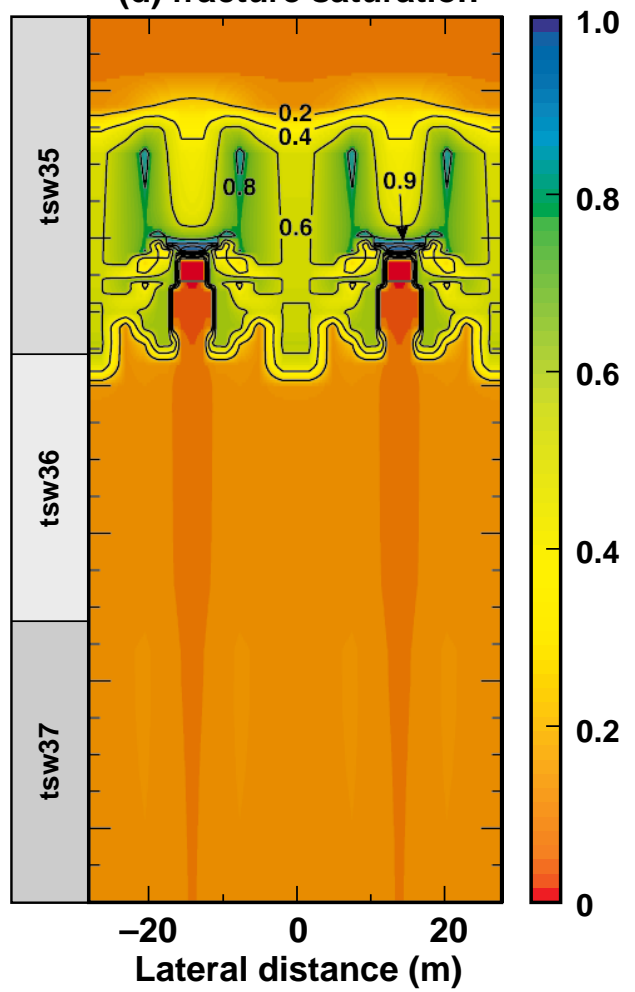

Figure 5-39. Base-case (corresponding to reference Case 1) conditions at $5000 \mathrm{yr}$ : (a) fracture porosity ratio change, (b) temperature, (c) matrix saturation, and (d) fracture saturation. 

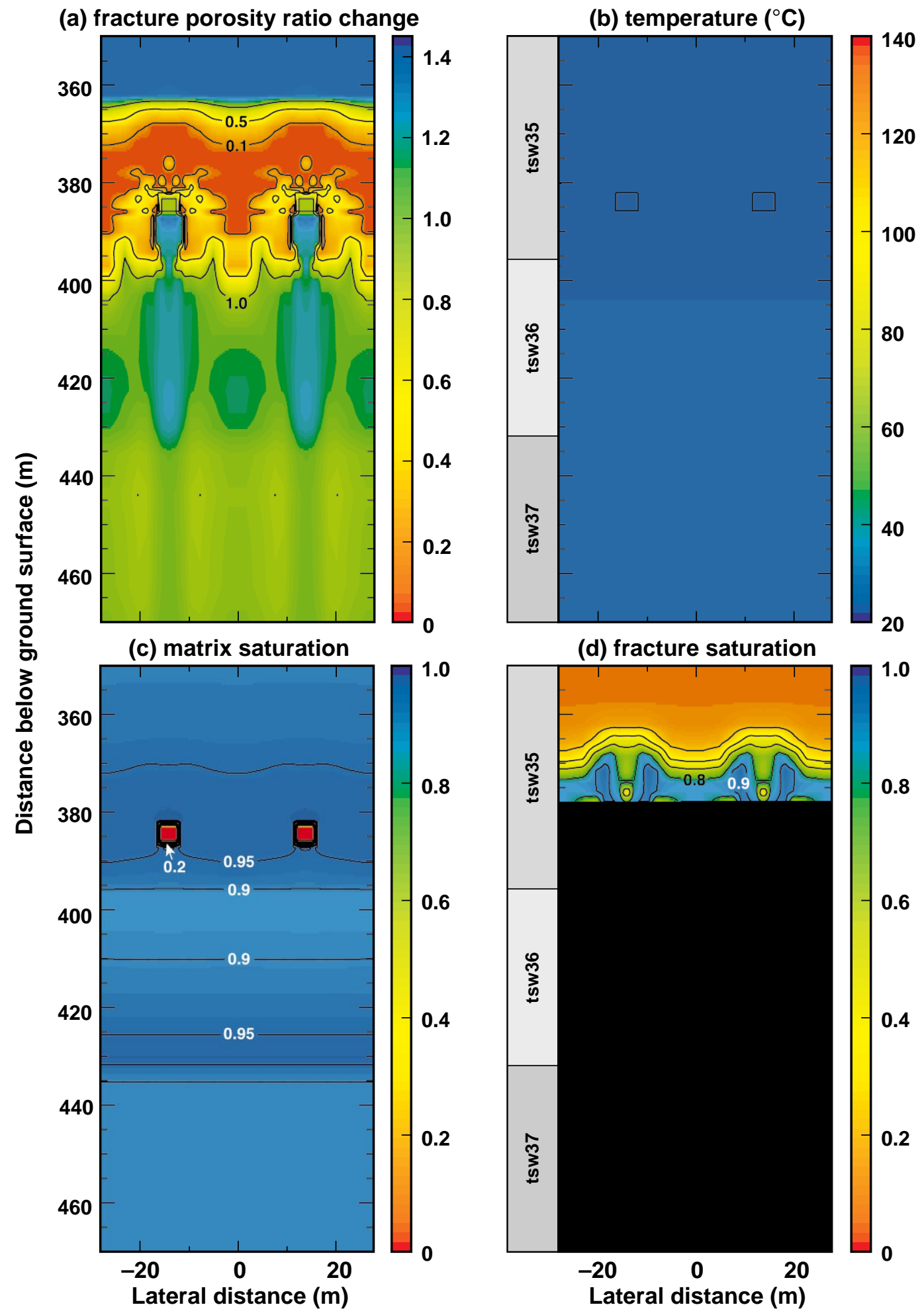

Figure 5-40. Base-case (corresponding to reference Case 1) conditions at 900,000 yr: (a) fracture porosity ratio change, $(b)$ temperature, $(c)$ matrix saturation, and (d) fracture saturation. 


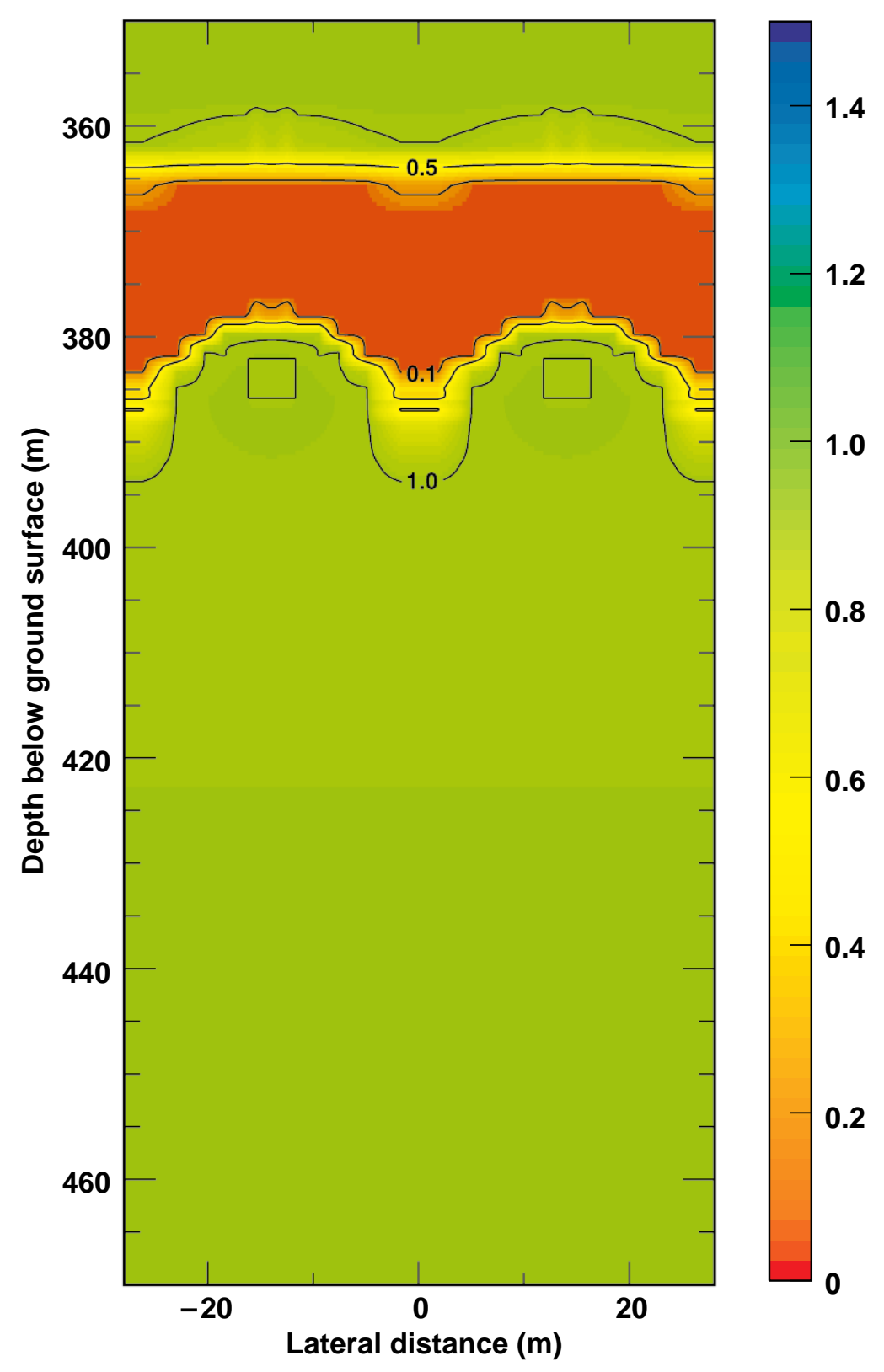

Figure 5-41. Relative change to fracture porosity at $1000 \mathrm{yr}$ for the base case (corresponding to reference Case 2) with infiltration reduced by a factor of $1 / 5$. 
(a) $t=1000 \mathrm{yr}$

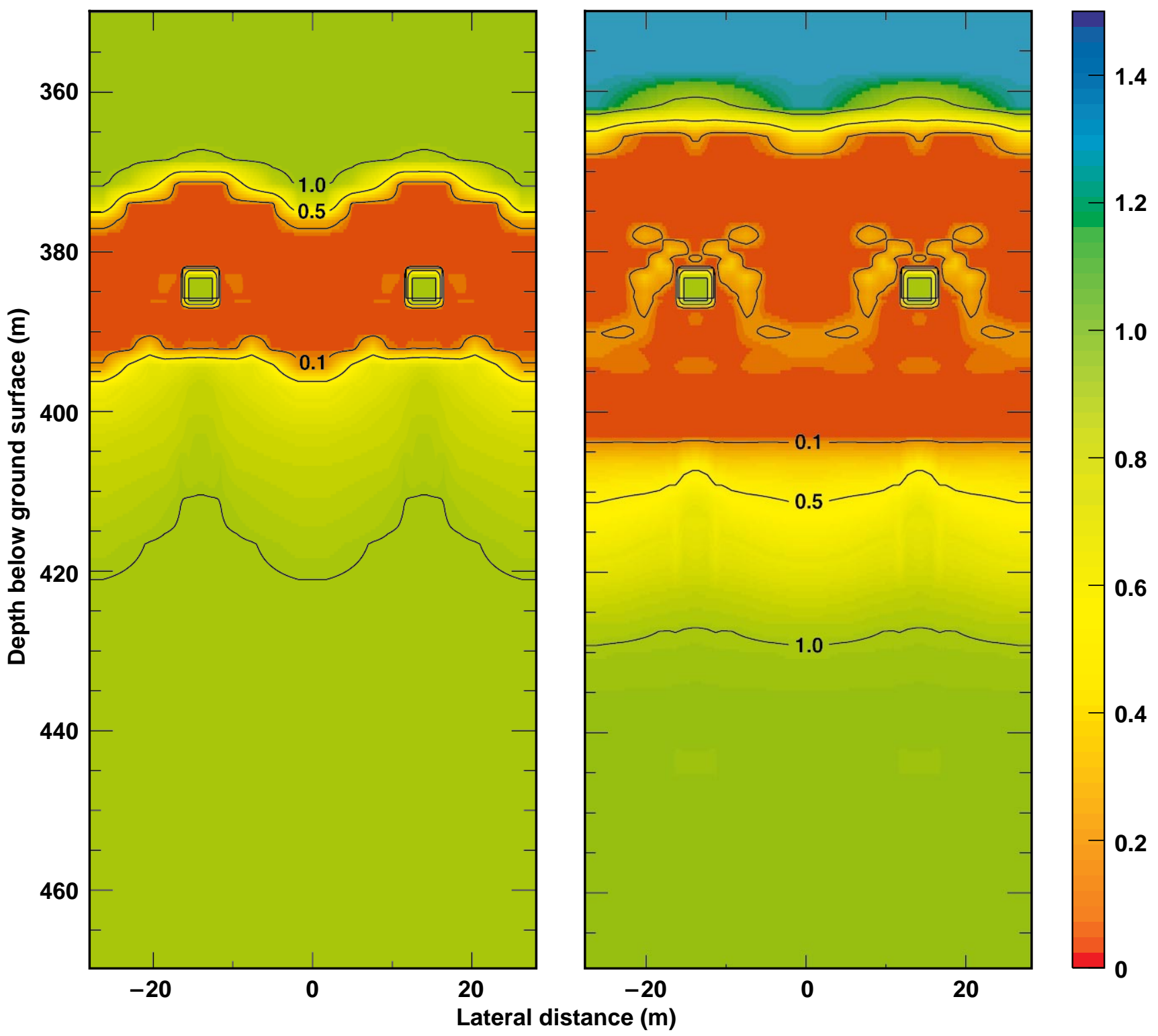

Figure 5-42. Relative change to fracture porosity for the base case (corresponding to reference Case 3) using hydrologic properties from the December 1997 "Modified TH" property set: (a) $1000 \mathrm{yr}$ and (b) 10,000 yr. 
(a) $3.2 \mathrm{~mm} / \mathrm{y}$ infiltration rate

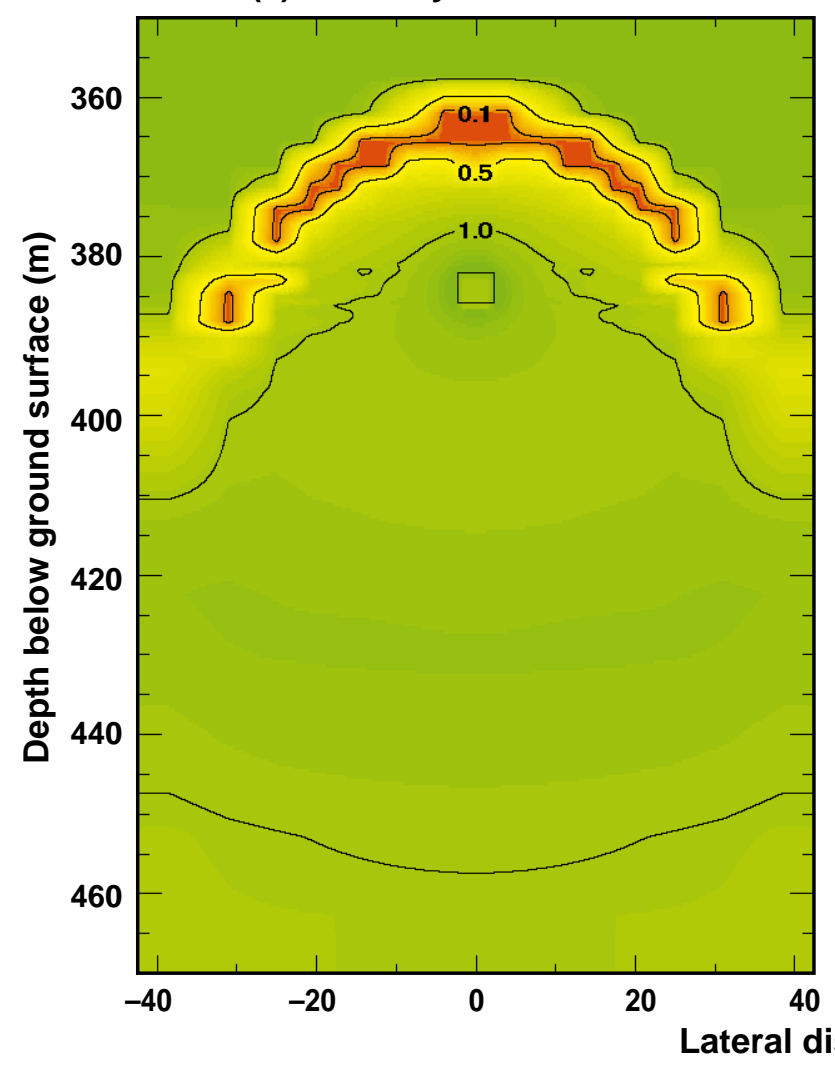

(b) $16 \mathrm{~mm} / \mathrm{y}$ infiltration rate

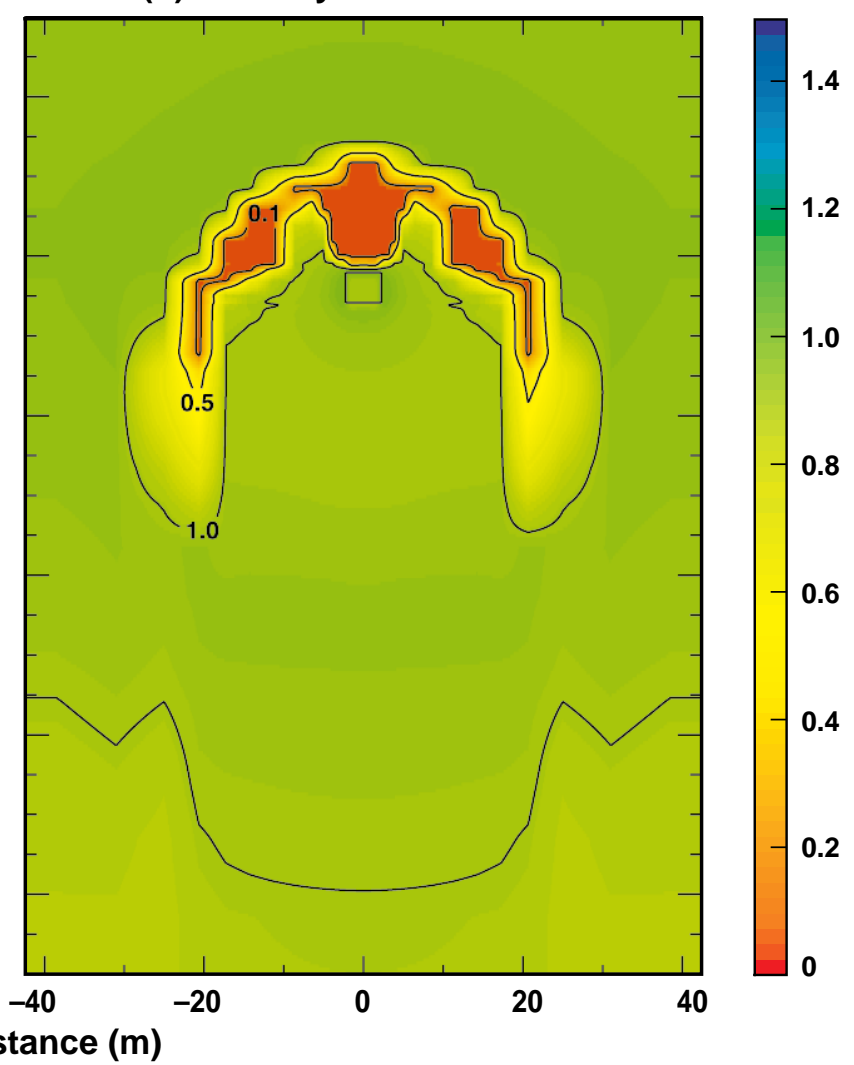

Figure 5-43. Relative change to fracture porosity at $1000 \mathrm{yr}$ for the line-load case: (a) $3.2 \mathrm{~mm} / \mathrm{y}$ infiltration rate and (b) $16 \mathrm{~mm} / \mathrm{y}$ infiltration rate (corresponding to reference Case 5). 


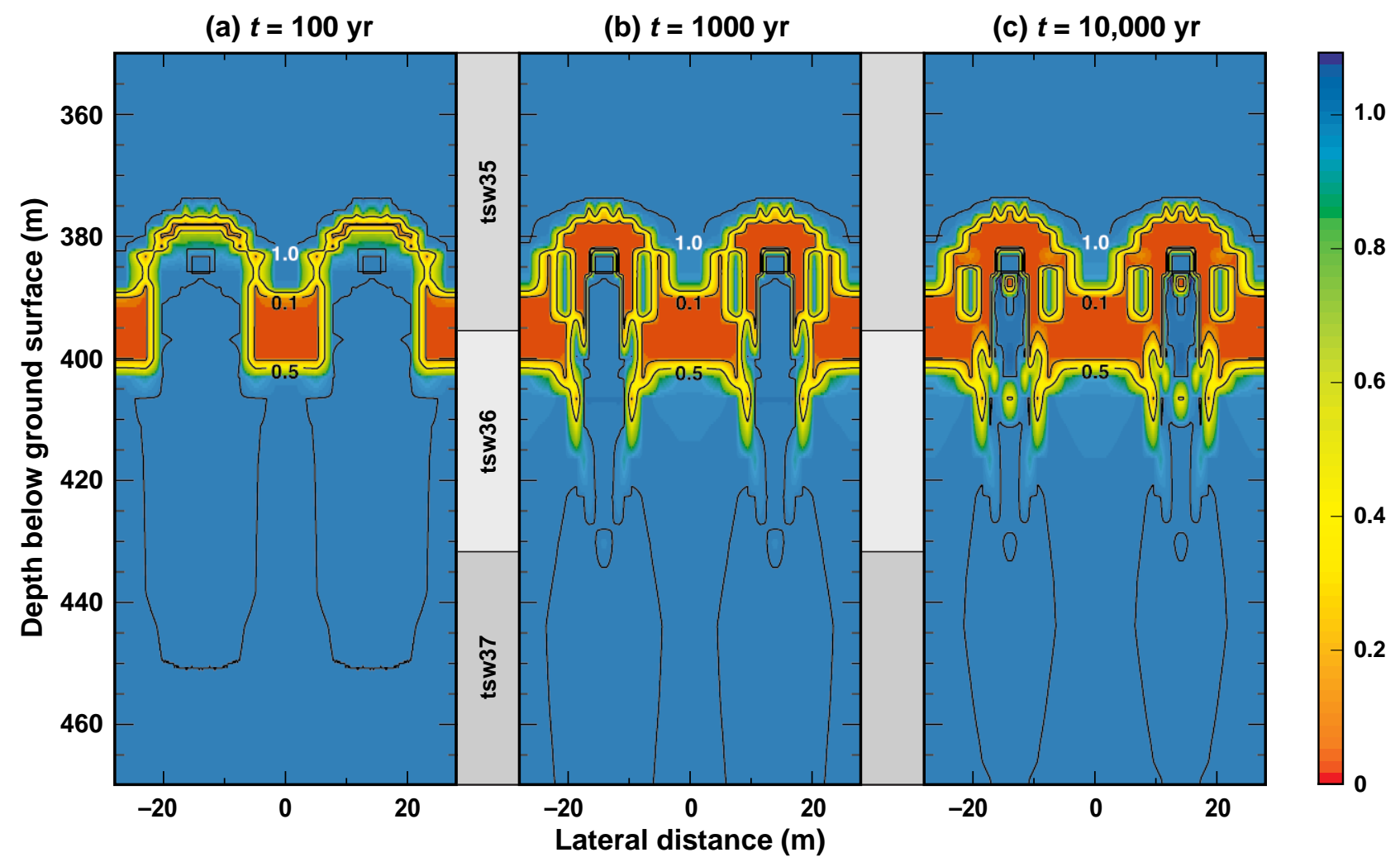

Figure 5-44. Relative change to fracture porosity for the base case (corresponding to reference Case 1) only allowing precipitation of amorphous silica, (a) $100 \mathrm{yr}$, (b) $1000 \mathrm{yr}$, and (c) 10,000 yr. 

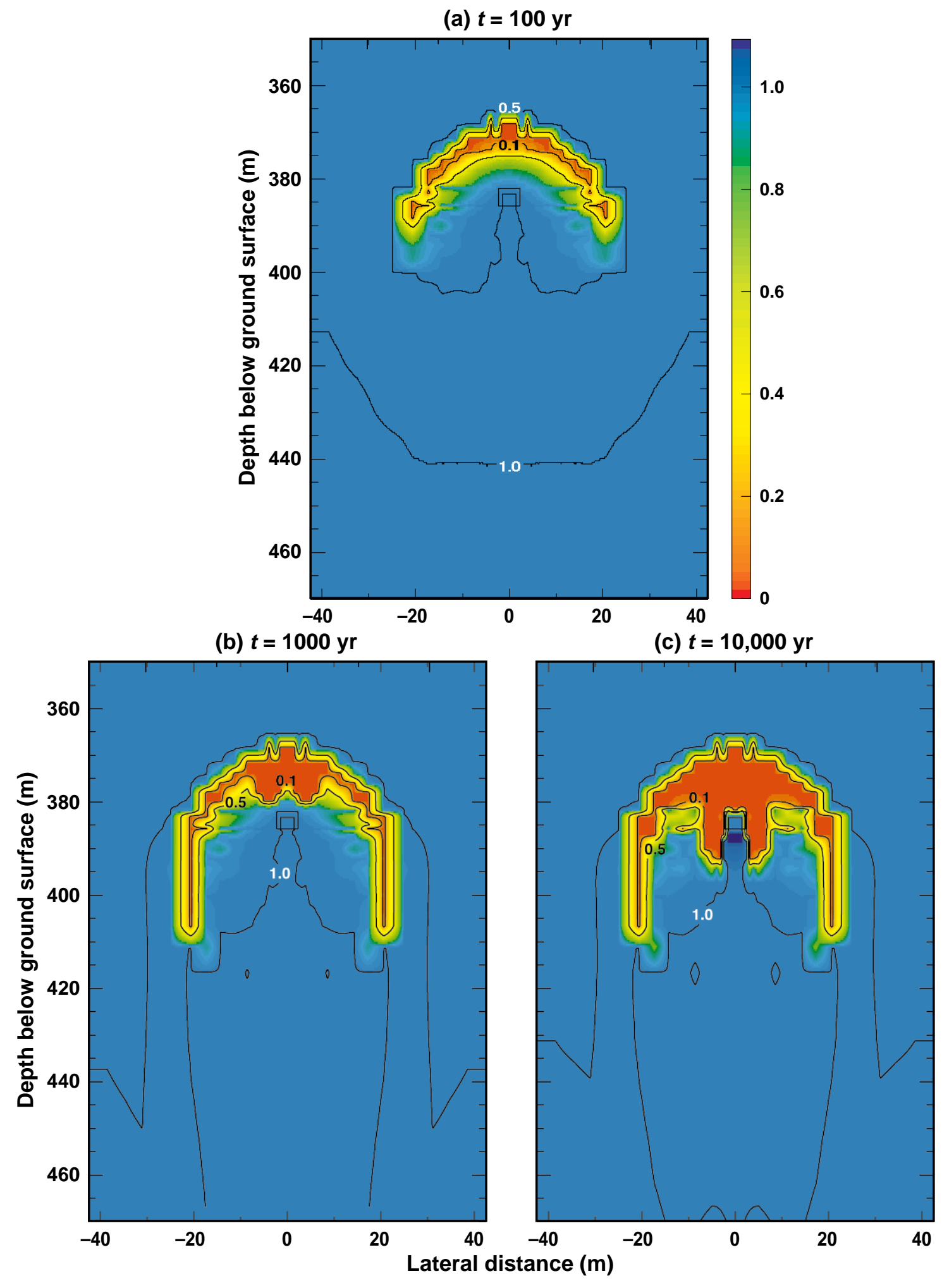

Figure 5-45. Relative change to fracture porosity for the line-load (corresponding to reference Case 5) only allowing precipitation of amorphous silica, (a) $100 \mathrm{yr}$, (b) $1000 \mathrm{yr}$, and (c) 10,000 yr. 


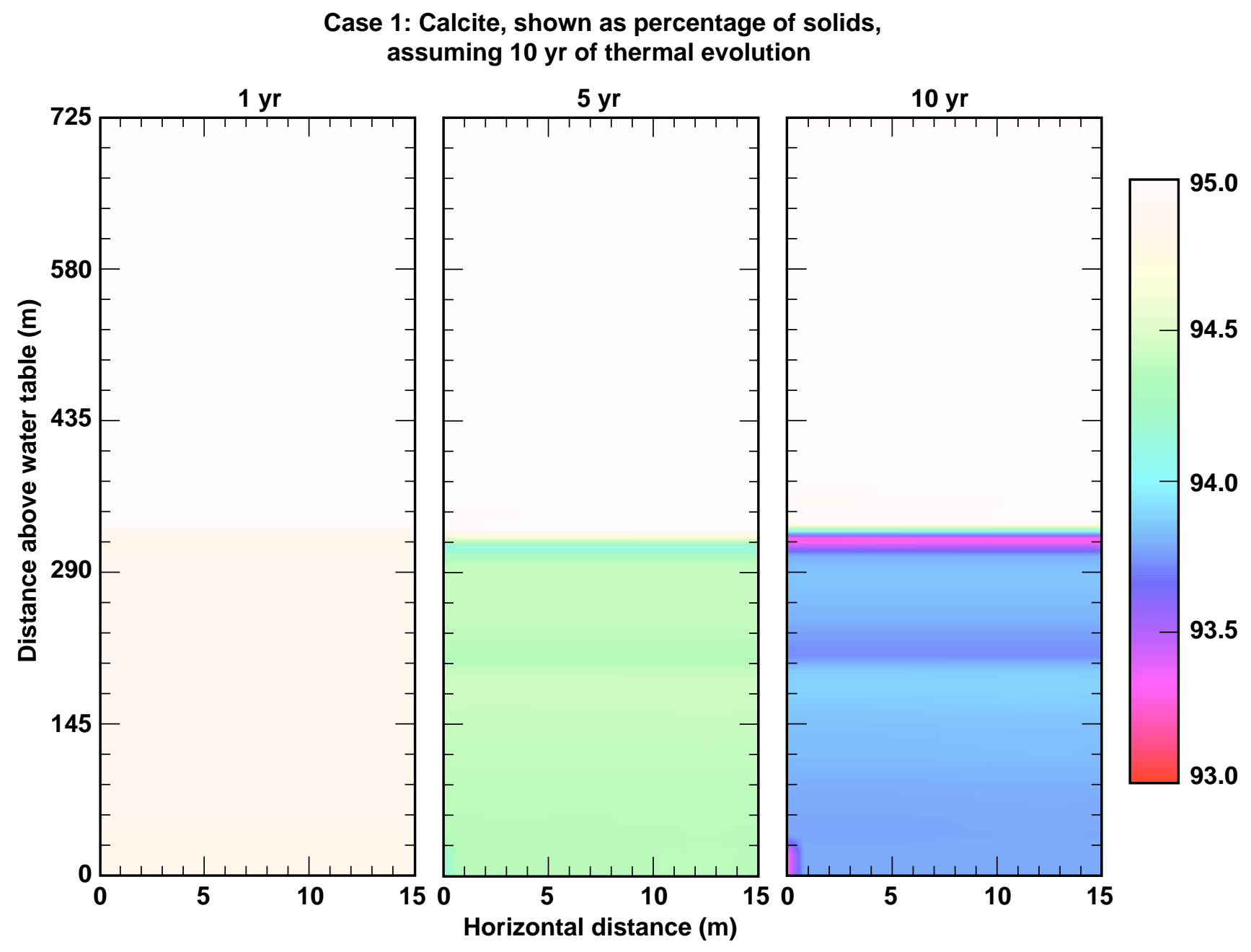

Figure 5-46. Calcite abundance as a function of location for Case 1 after $10 \mathrm{yr}$ heating. The vertical distance is referenced to the top of the saturated zone. The horizontal distance is referenced to the center of the heated drift. The calcite abundance is measured as the volume percentage of calcite composing the mineral assemblage in the 2-D slice. The reactive transport was conducted using the thermal and flow regimes resulting after $10 \mathrm{yr}$ heat output by WPs in the heated drift region. These properties were computed for the Case 1 set of conditions (see Chapter 3 for a definition of Case 1 conditions). The three vertical panels show the results of reactive-transport simulations, assuming that the computed 10-yr thermal and flow regimes persisted unchanged for 1,5 , and $10 \mathrm{yr}$. 


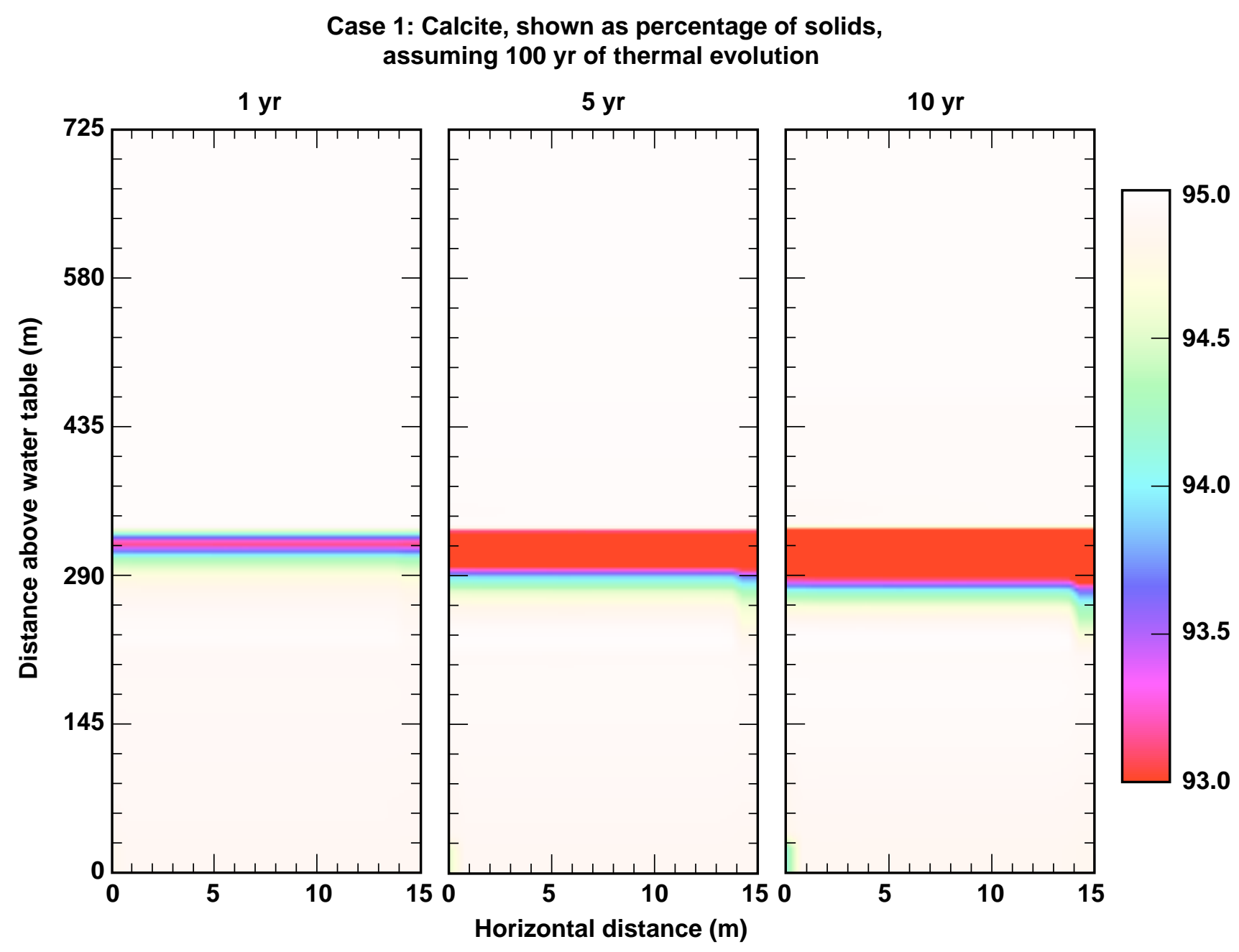

Figure 5-47. Calcite abundance as a function of location for Case 1 after $100 \mathrm{yr}$ heating. The vertical and horizontal scales and the calcite representation are the same as in Figure 5-46. The reactive transport was conducted using the thermal and flow regimes resulting after $100 \mathrm{yr}$ heat output by WPs in the heated drift region. The three vertical panels show the results of reactive-transport simulations, assuming that the computed $100 \mathrm{yr}$ thermal and flow regimes persisted unchanged for 1, 5, and $10 \mathrm{yr}$. 


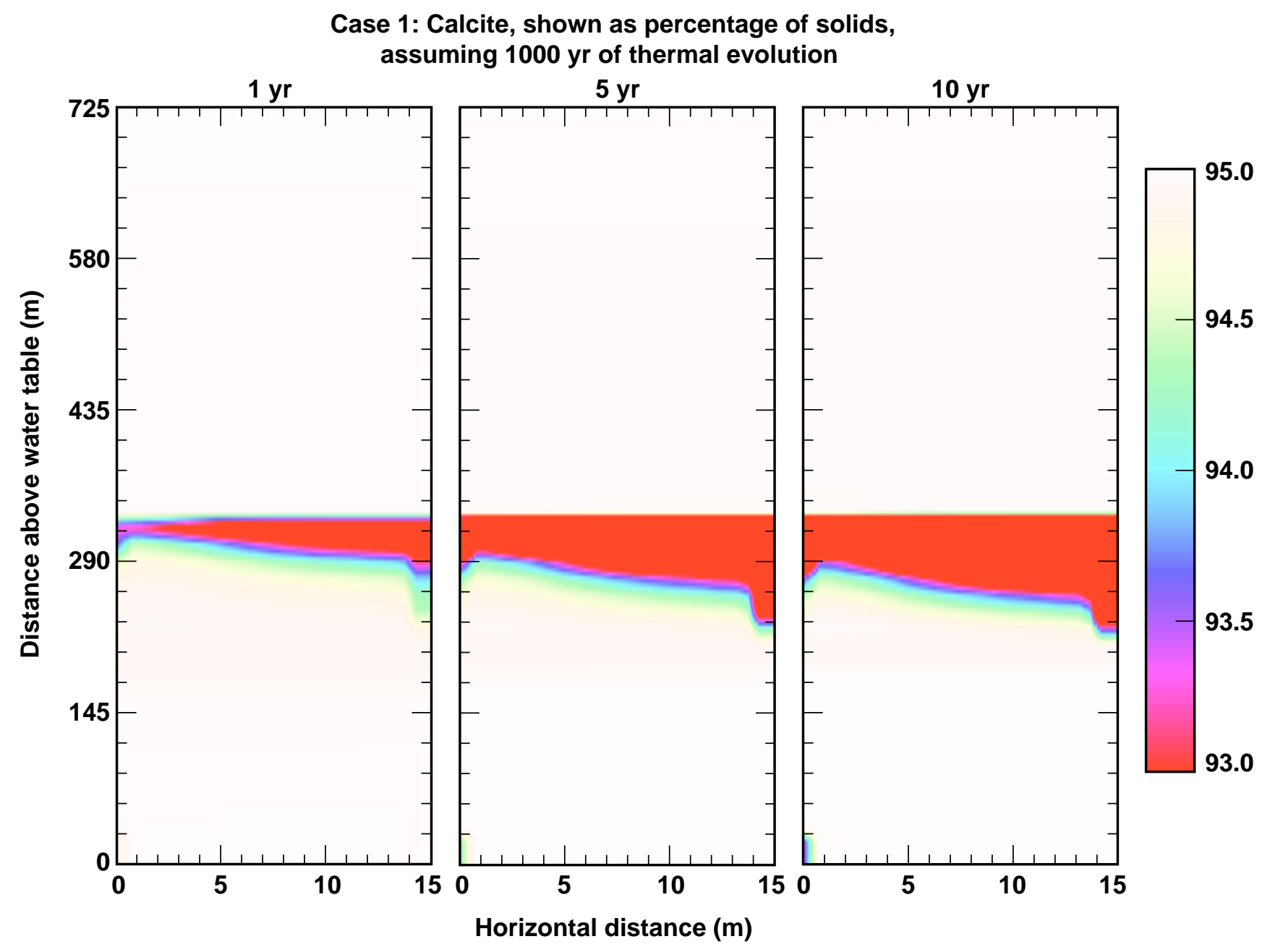

Figure 5-48. Calcite abundance as a function of location for Case 1 after $1000 \mathrm{yr}$ heating. The vertical and horizontal scales and the calcite representation are the same as in Figure 5-46. The reactive transport was conducted using the thermal and flow regimes resulting after $1000 \mathrm{yr}$ heat output by WPs in the heated drift region. The three vertical panels show the results of reactive-transport simulations, assuming that the computed 1000-yr thermal and flow regimes persisted unchanged for 1, 5, and $10 \mathrm{yr}$. 


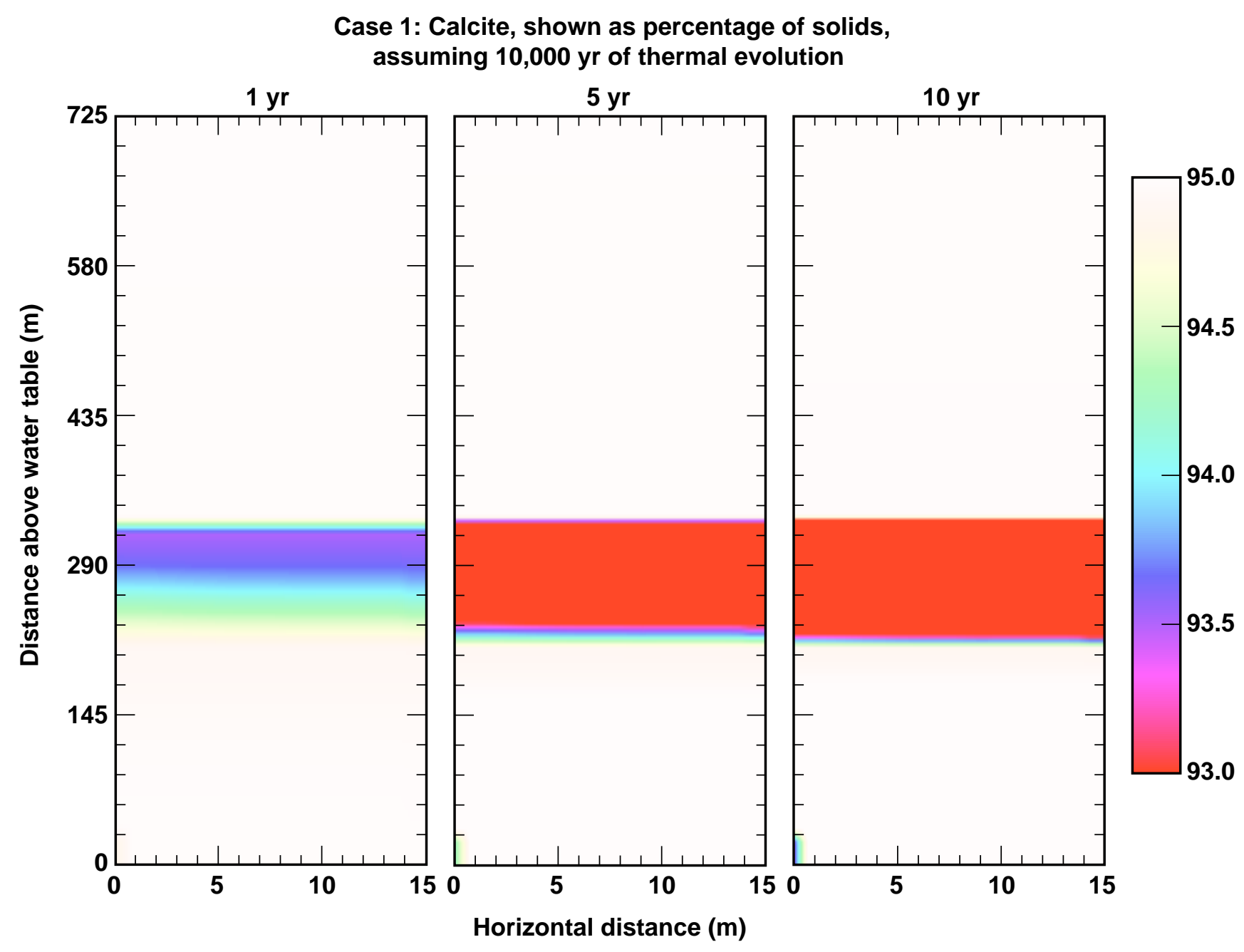

Figure 5-49. Calcite abundance as a function of location for Case 1 after 10,000 yr heating. The vertical and horizontal scales and the calcite representation are the same as in Figure 5-46. The reactive transport was conducted using the thermal and flow regimes resulting after 10,000 yr heat output by WPs in the heated drift region. The three vertical panels show the results of reactive-transport simulations, assuming that the computed 10,000-yr thermal and flow regimes persisted unchanged for 1, 5, and $10 \mathrm{yr}$. 


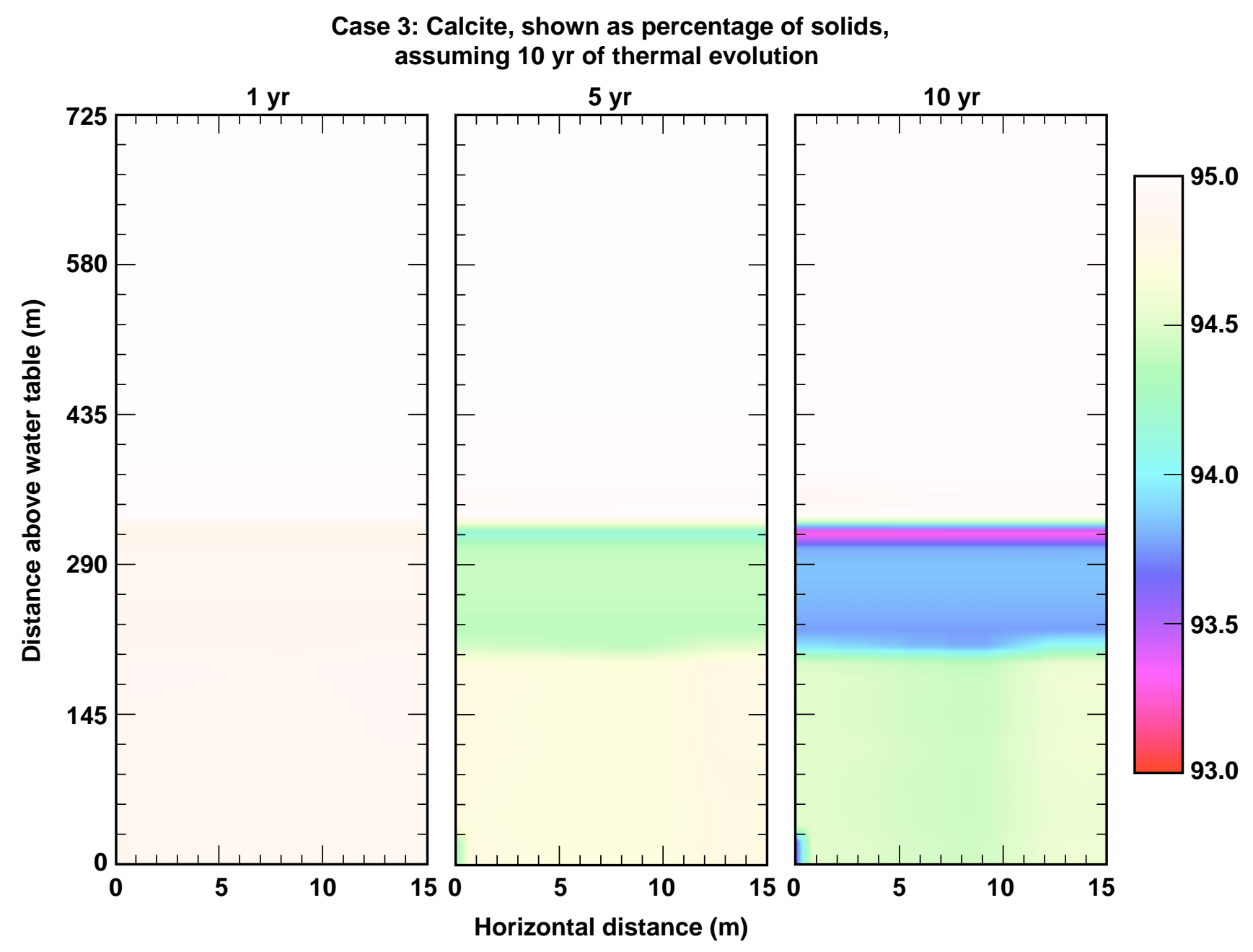

Figure 5-50. Calcite abundance as a function of location for Case 3 after $10 \mathrm{yr}$ heating. The vertical and horizontal scales and the calcite representation are the same as in Figure 5-46. The reactive transport was conducted using the thermal and flow regimes resulting after $10 \mathrm{yr}$ heat output by WPs in the heated drift region. These properties were computed for the Case 3 set of conditions (see Chapter 3 for a definition of Case 3 conditions). The three vertical panels show the results of reactive-transport simulations, assuming that the computed 10-yr thermal and flow regimes persisted unchanged for 1, 5, and $10 \mathrm{yr}$. 


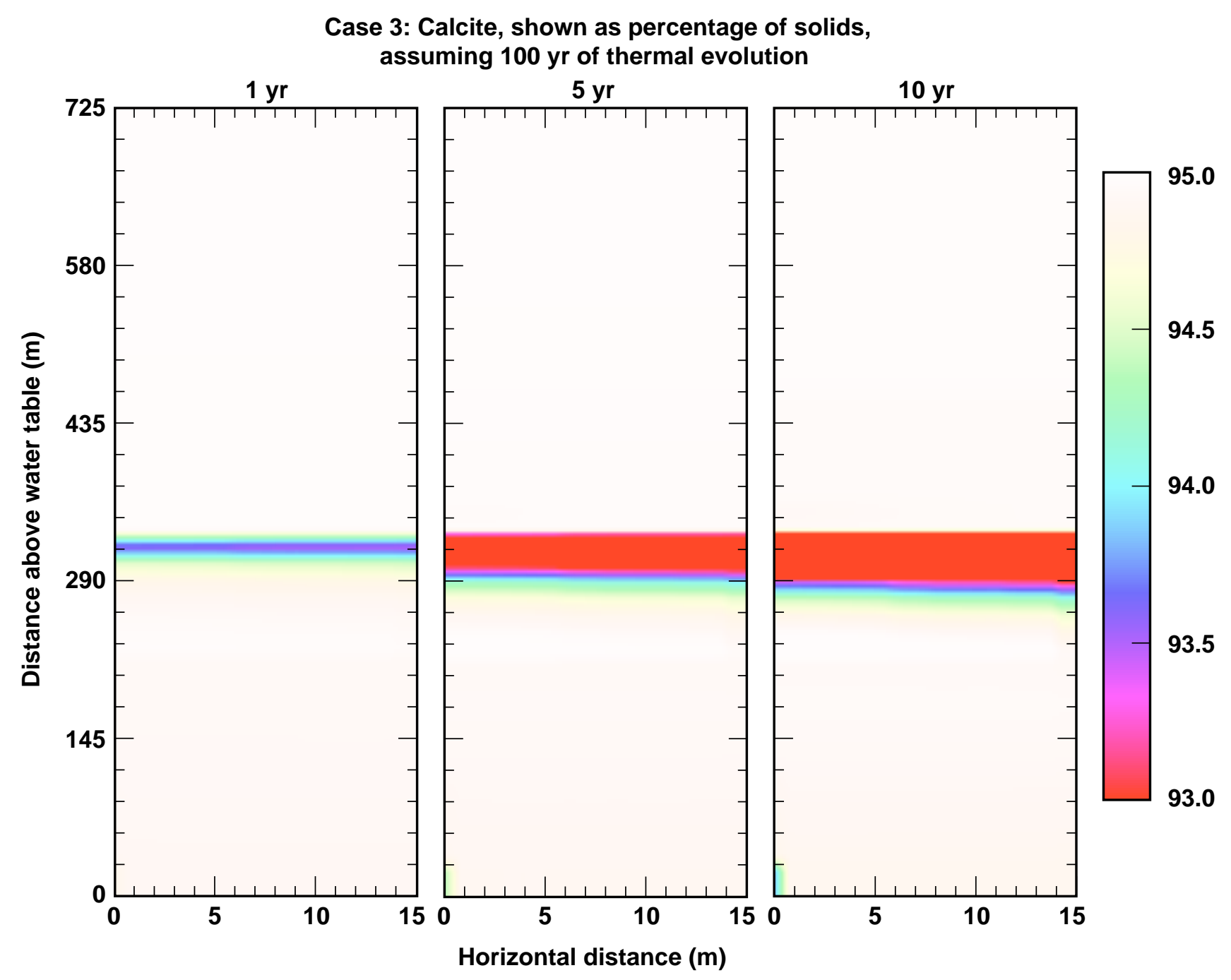

Figure 5-51. Calcite abundance as a function of location for Case 3 after $100 \mathrm{yr}$ heating. The vertical and horizontal scales and the calcite representation are the same as in Figure 5-46. The reactive transport was conducted using the thermal and flow regimes resulting after $100 \mathrm{yr}$ heat output by WPs in the heated drift region. The three vertical panels show the results of reactive-transport simulations, assuming that the computed 100-yr thermal and flow regimes persisted unchanged for $1 \mathrm{yr}, 5 \mathrm{yr}$, and $10 \mathrm{yr}$. 


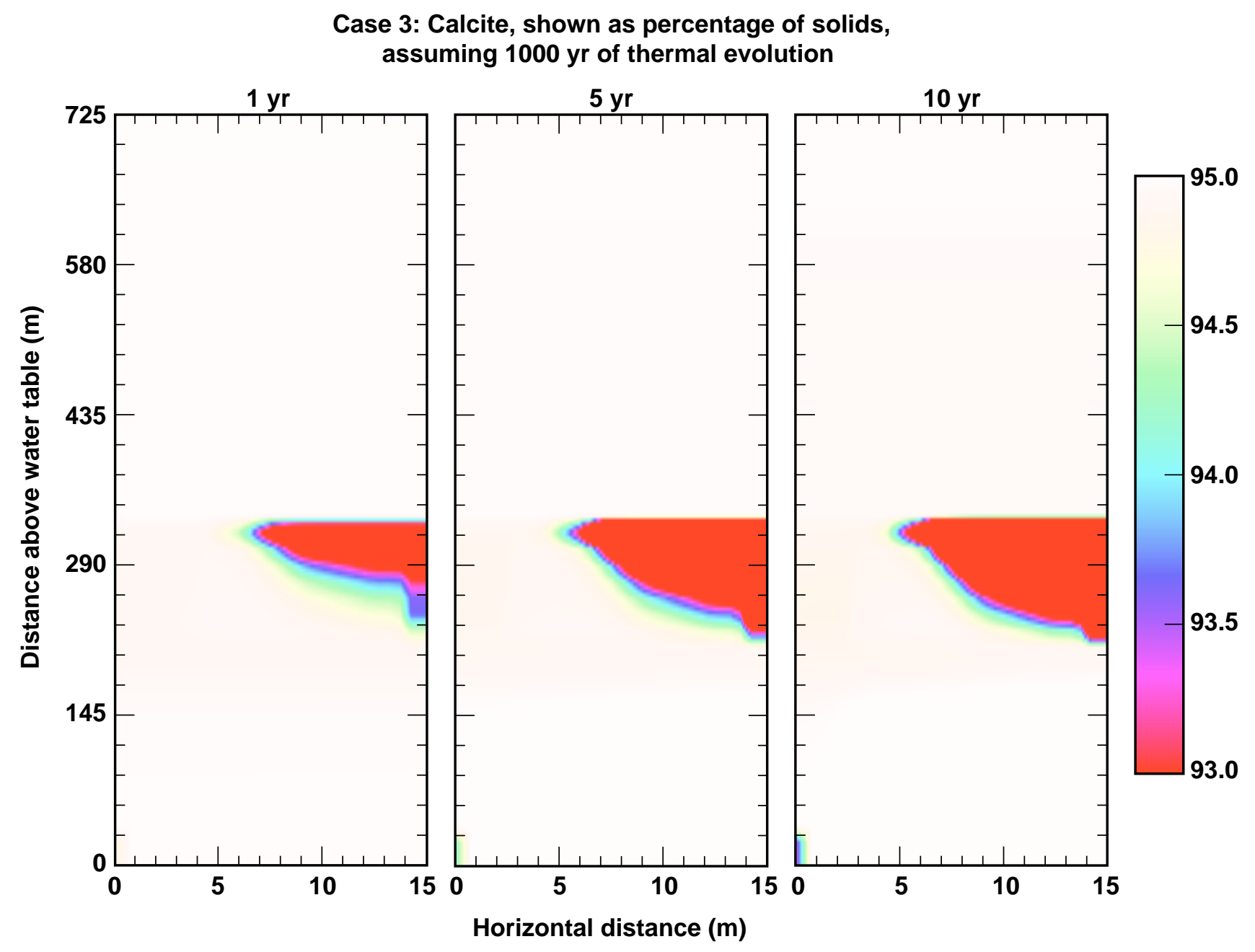

Figure 5-52. Calcite abundance as a function of location for Case 3 after $1000 \mathrm{yr}$ heating. The vertical and horizontal scales and the calcite representation are the same as in Figure 5-46. The reactive transport was conducted using the thermal and flow regimes resulting after $1000 \mathrm{yr}$ heat output by WPs in the heated drift region. The three vertical panels show the results of reactive-transport simulations, assuming that the computed 1000-yr thermal and flow regimes persisted unchanged for 1, 5, and $10 \mathrm{yr}$. 


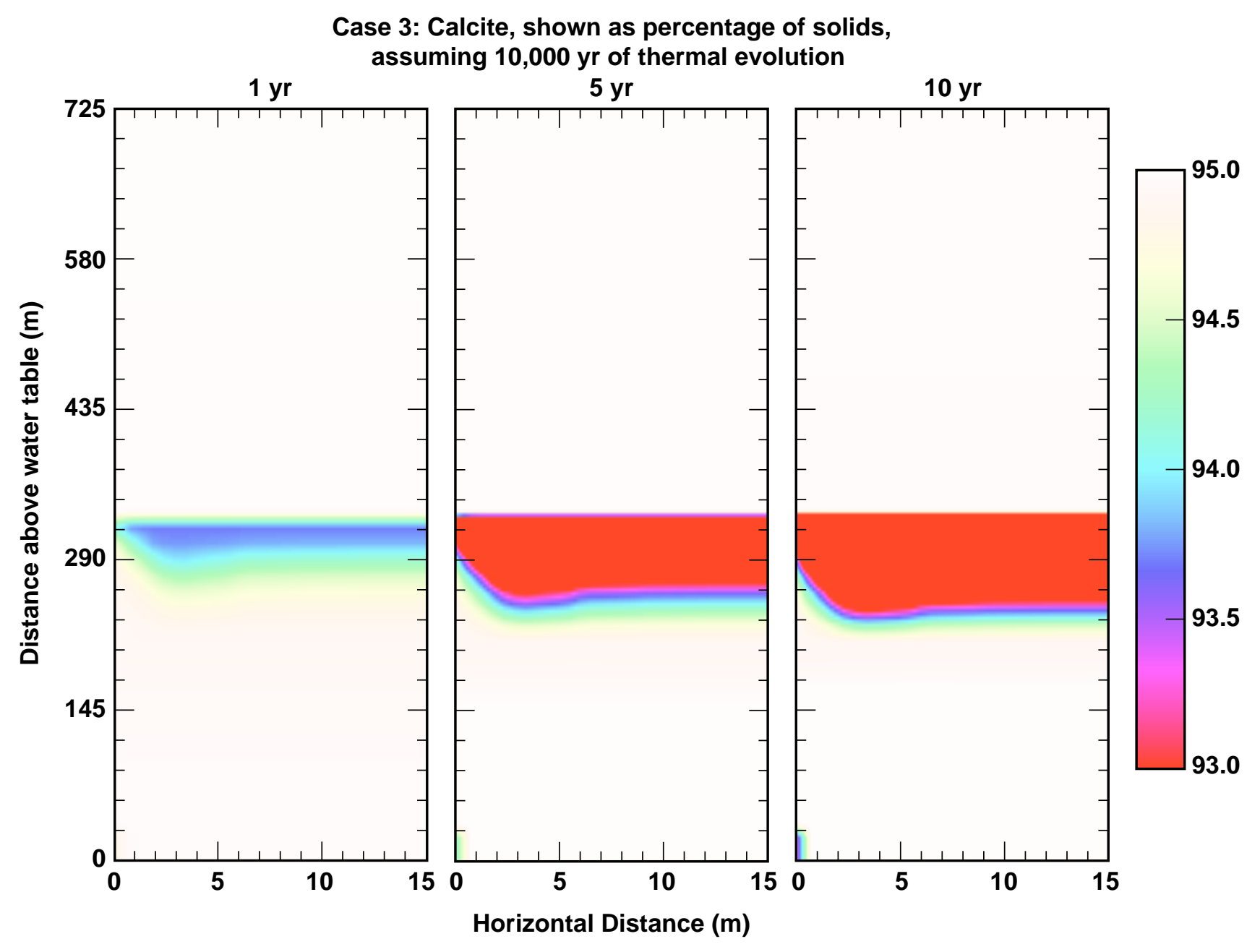

Figure 5-53. Calcite abundance as a function of location for Case 3 after 10,000 yr heating. The vertical and horizontal scales and the calcite representation are the same as in Figure 5-46. The reactive transport was conducted using the thermal and flow regimes resulting after $1000 \mathrm{yr}$ heat output by WPs in the heated drift region. The three vertical panels show the results of reactive-transport simulations, assuming that the computed 10,000-yr thermal and flow regimes persisted unchanged for 1, 5, and $10 \mathrm{yr}$. 


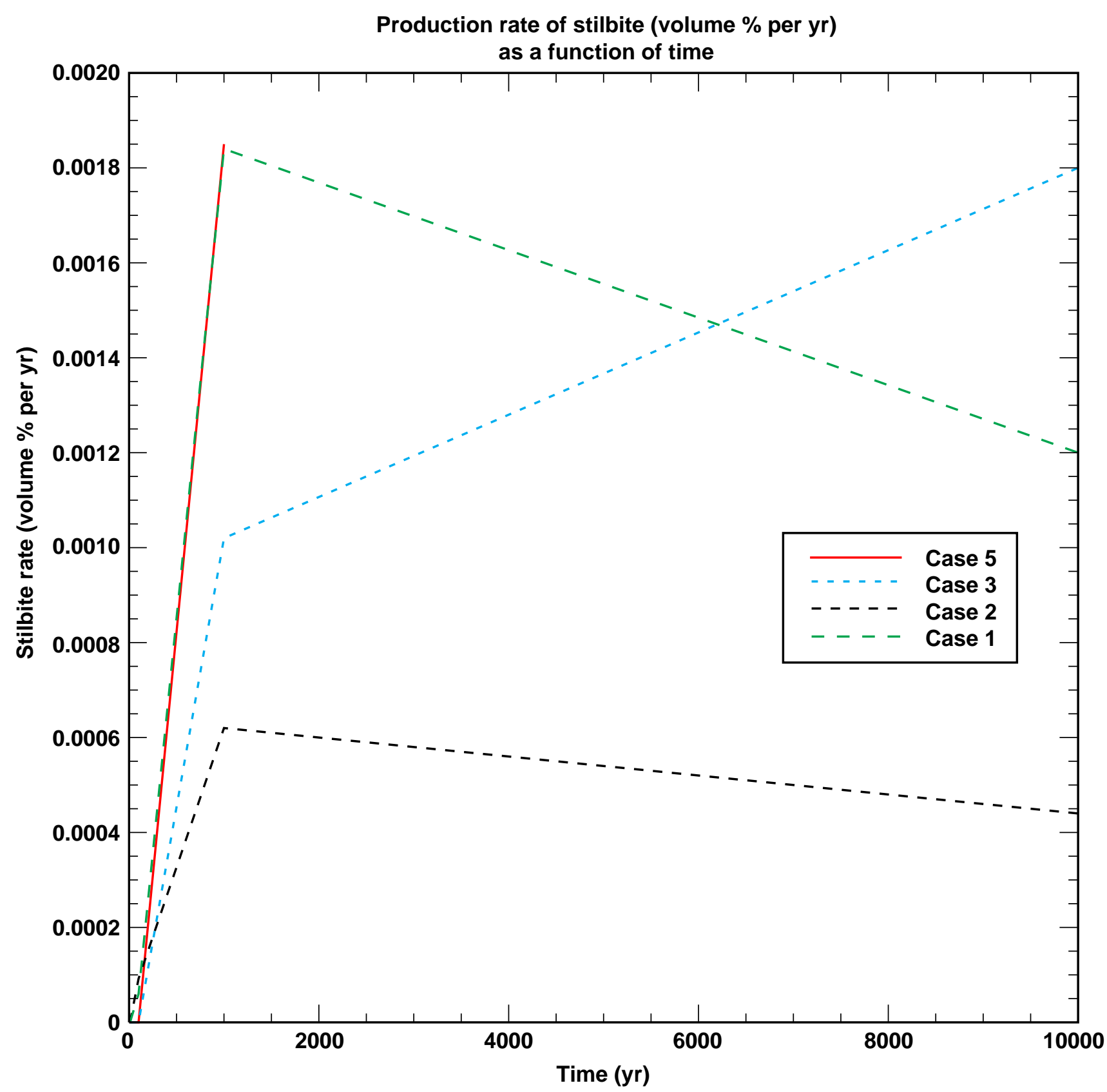

Figure 5-54. Production rate of stilbite (a zeolite) for each case examined as a function of time. Note the small rate of change for the period between 1000 and 10,000 yr compared with that of the first $1000 \mathrm{yr}$. 


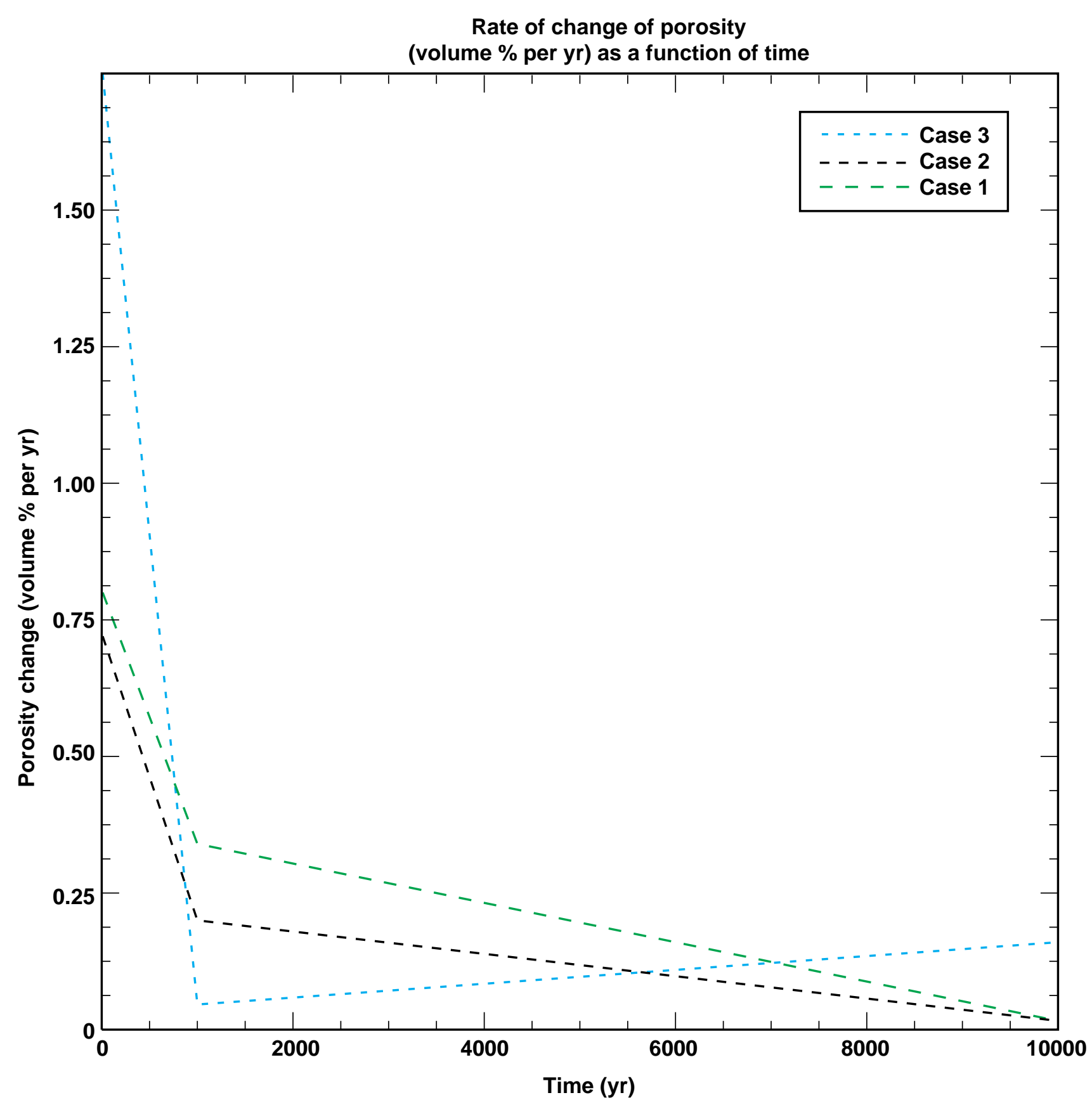

Figure 5-55. Porosity rate of change, for each case examined as a function of time. Note the small rate of change for the period between 1000 and 10,000 yr compared with that of the first $1000 \mathrm{yr}$. 


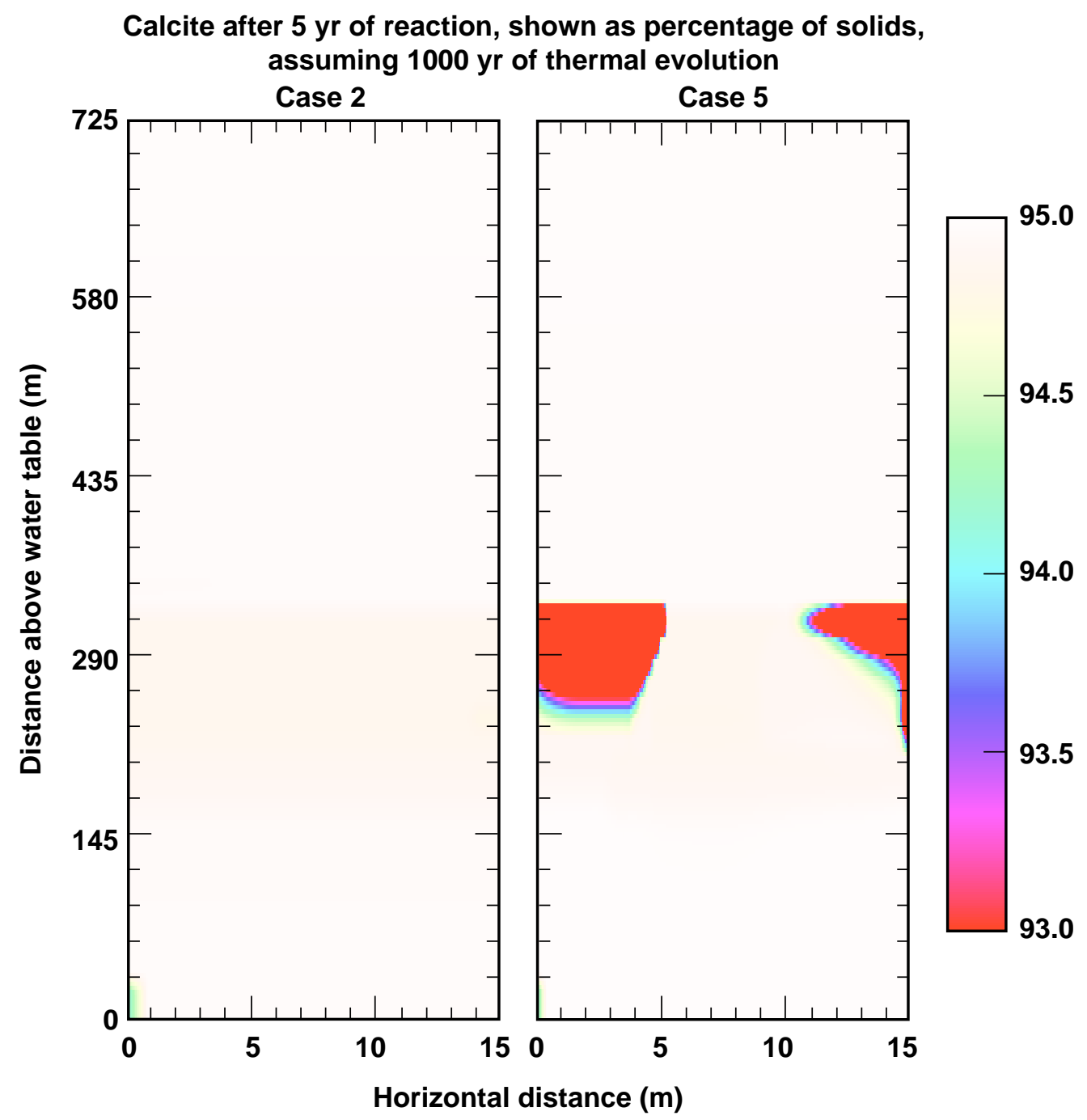

Figure 5-56. Calcite abundance as a function of location for Cases 2 and 5 after $1000 \mathrm{yr}$ heating. The vertical and horizontal scales and the calcite representation are the same as in Figure 5-46, except for Case 5, in which the horizontal scale is twice the distance as that in Figure 5-46. The reactive transport was conducted using the thermal and flow regimes resulting after $1000 \mathrm{yr}$ heat output by WPs in the heated drift region. The two vertical panels show the results of reactive-transport simulations, assuming that the computed 1000-yr thermal and flow regimes persisted unchanged for $5 \mathrm{yr}$. 

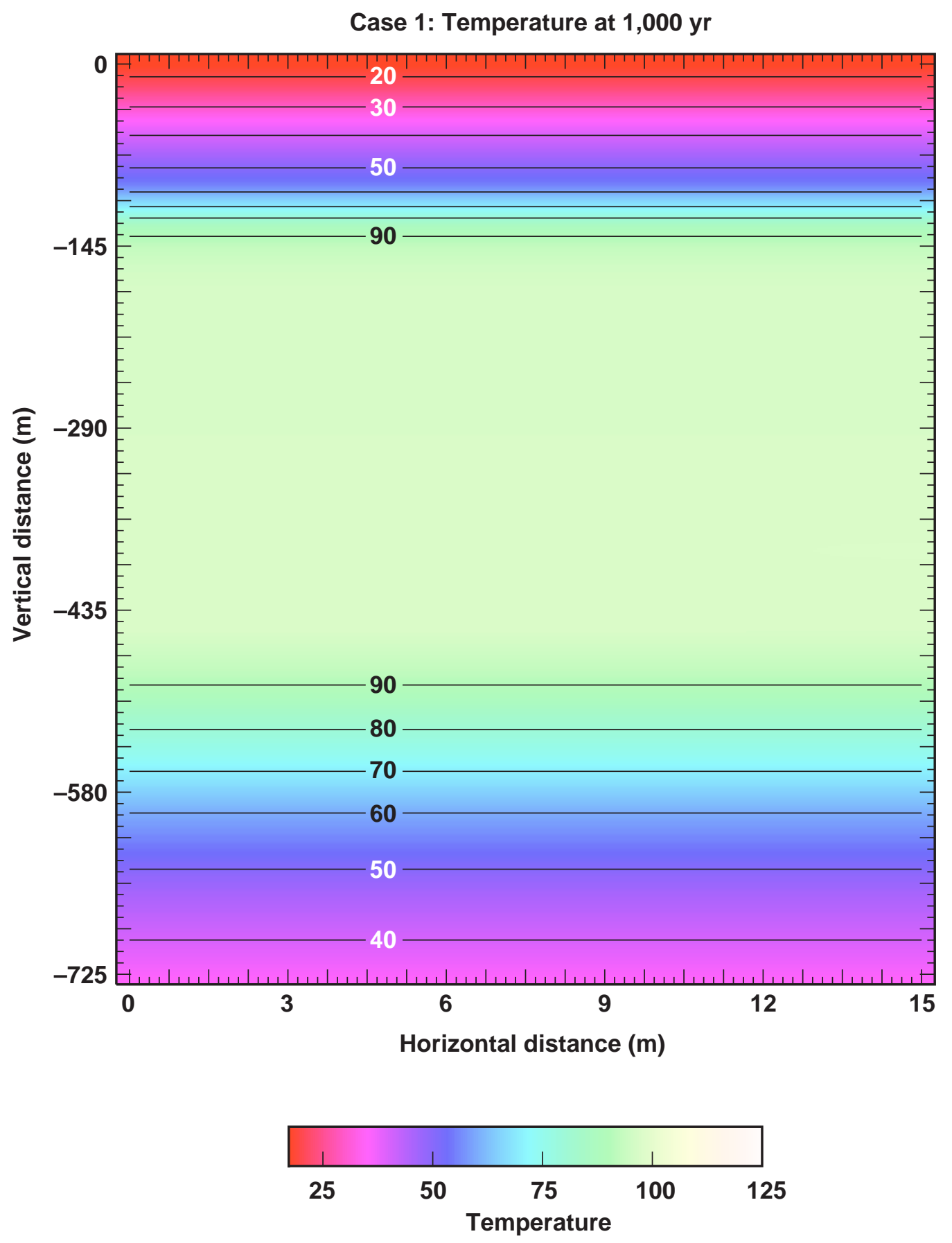

Figure 5-57. Temperature $\left({ }^{\circ} \mathrm{C}\right)$ distribution for Case 1 after $1000 \mathrm{yr}$ heating. The vertical and horizontal scales are the same as in Figure 5-46. 

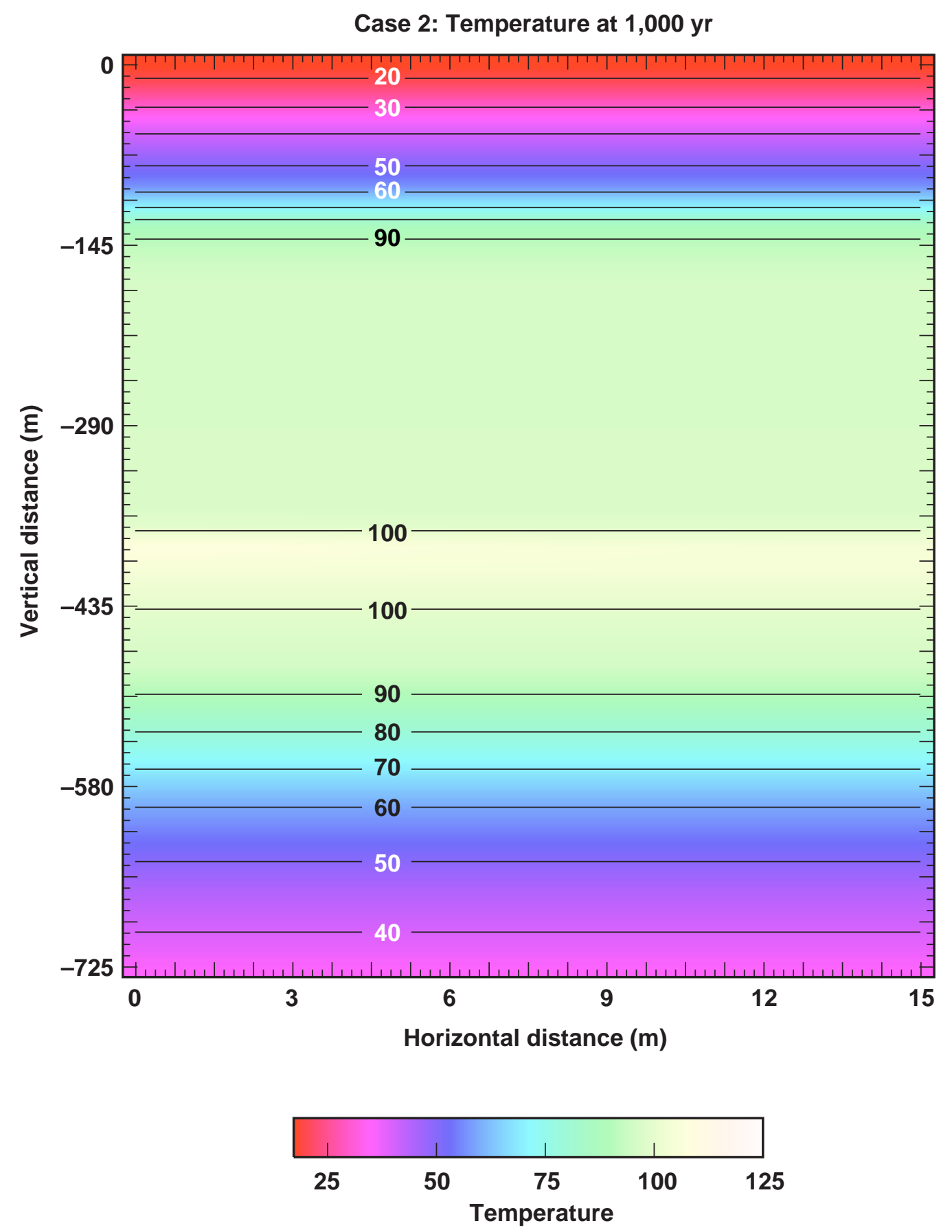

Figure 5-58. Temperature $\left({ }^{\circ} \mathrm{C}\right)$ distribution for Case 2 after $1000 \mathrm{yr}$ heating. The vertical and horizontal scales are the same as in Figure 5-46. 

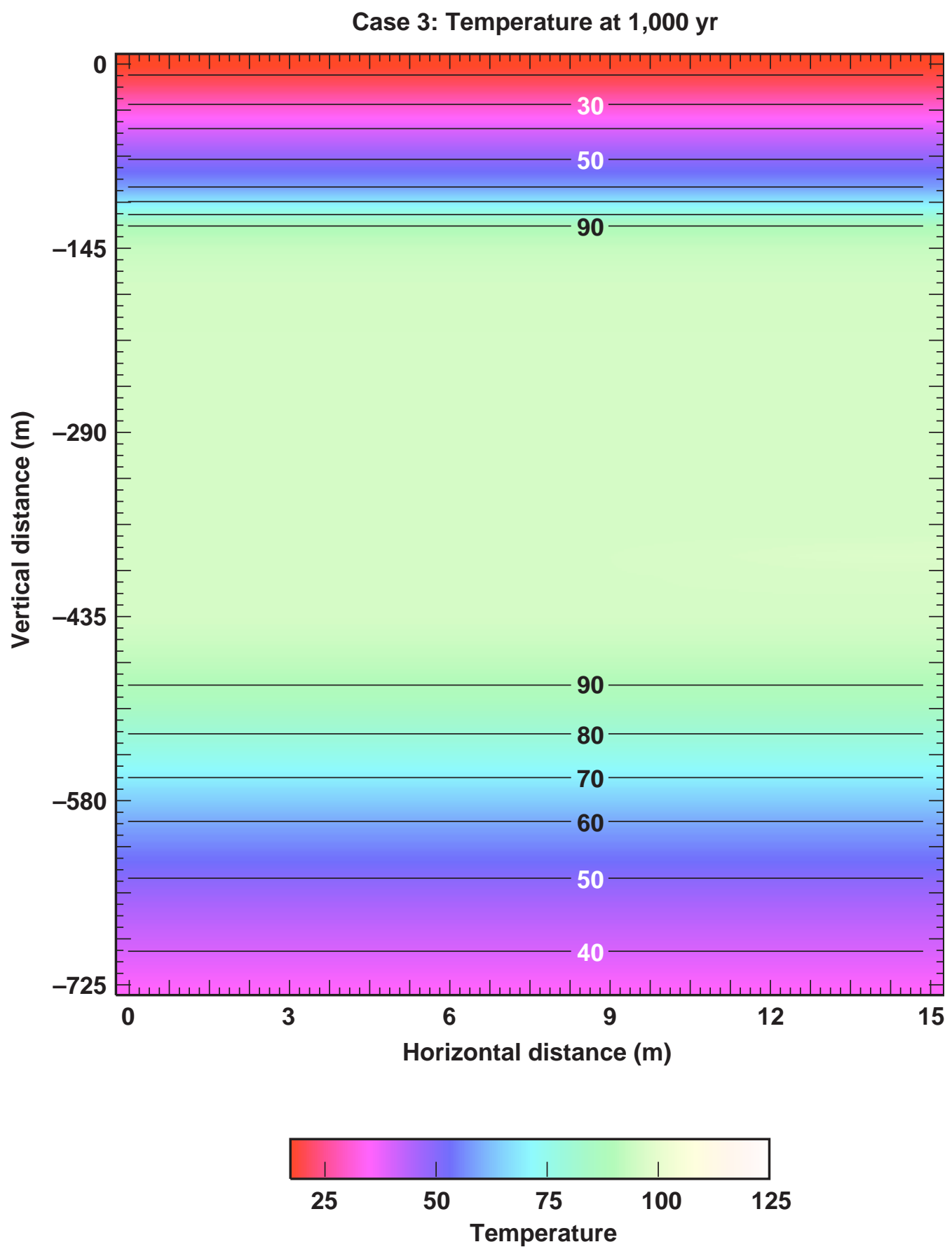

Figure 5-59. Temperature $\left({ }^{\circ} \mathrm{C}\right)$ distribution for Case 3 after $1000 \mathrm{yr}$ heating. The vertical and horizontal scales are the same as in Figure 5-46. 

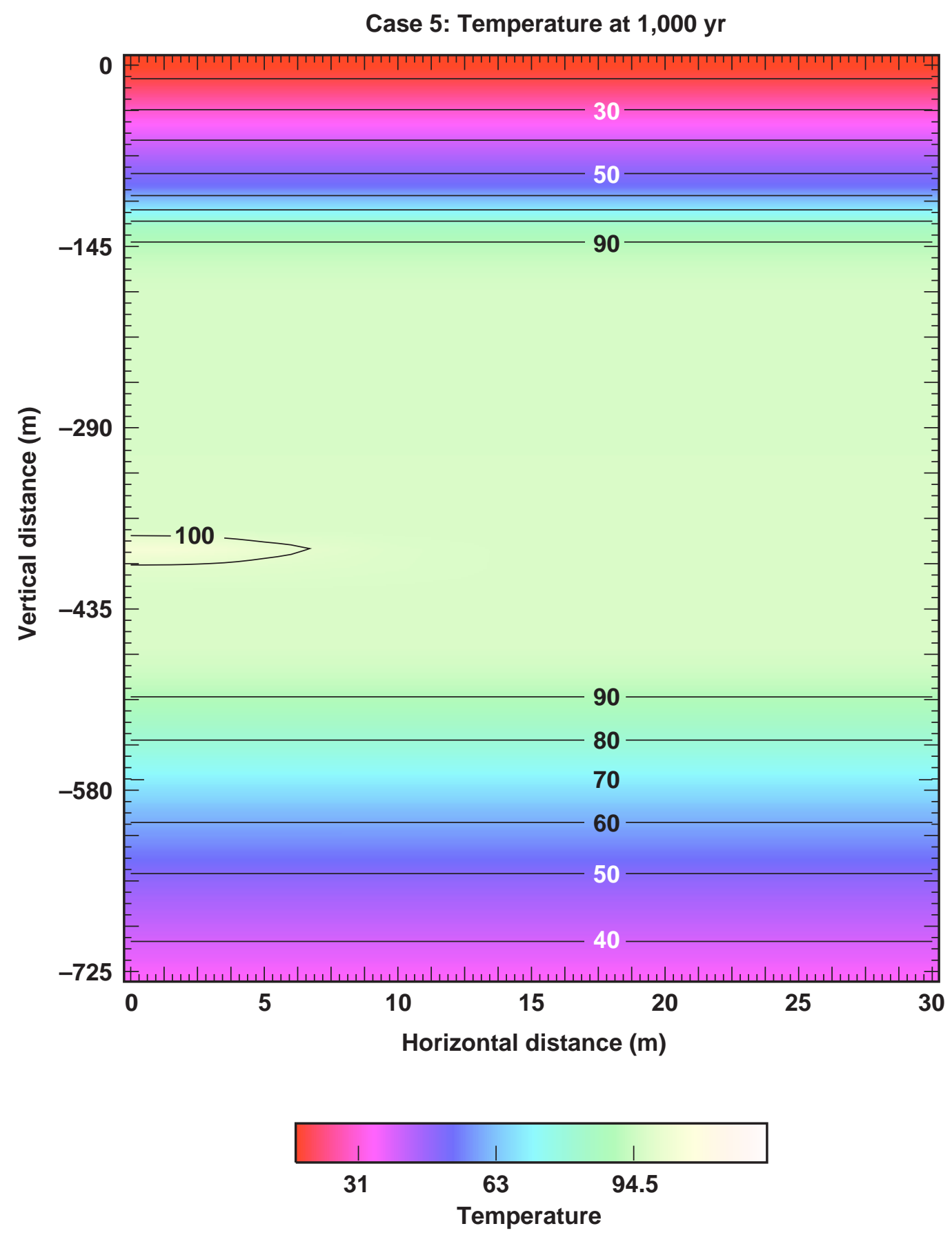

Figure 5-60. Temperature $\left({ }^{\circ} \mathrm{C}\right)$ distribution for Case 5 after $1000 \mathrm{yr}$ heating. The vertical and horizontal scales are the same as in Figure 5-46, while the horizontal scale is twice that in Figure 5-46. 
Case 1: $1,000 \mathrm{yr}$

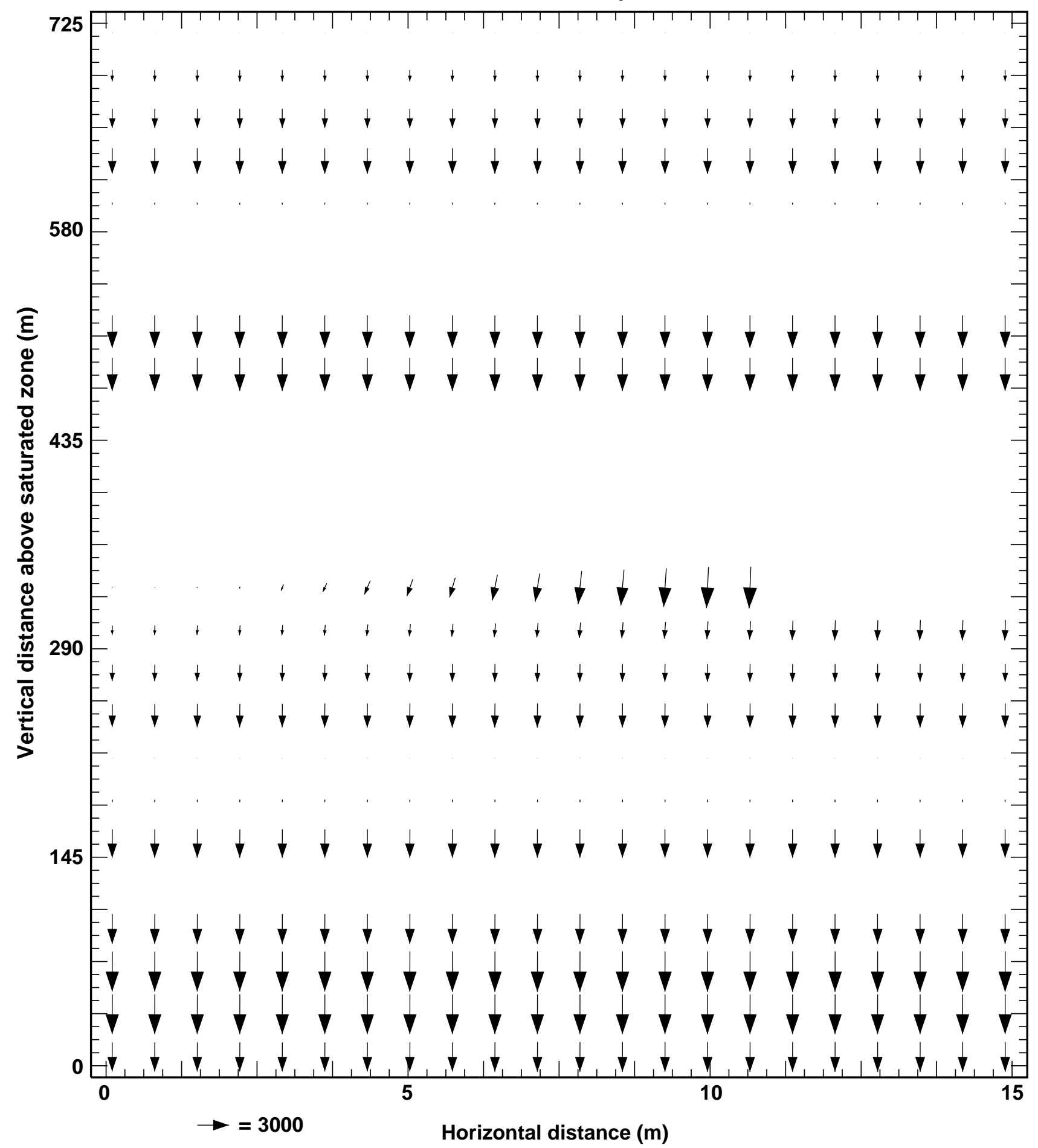

Figure 5-61. Flux vector distribution (m/yr) for Case 1 after $1000 \mathrm{yr}$ heating. The vertical and horizontal scales are the same as in Figure 5-46. 


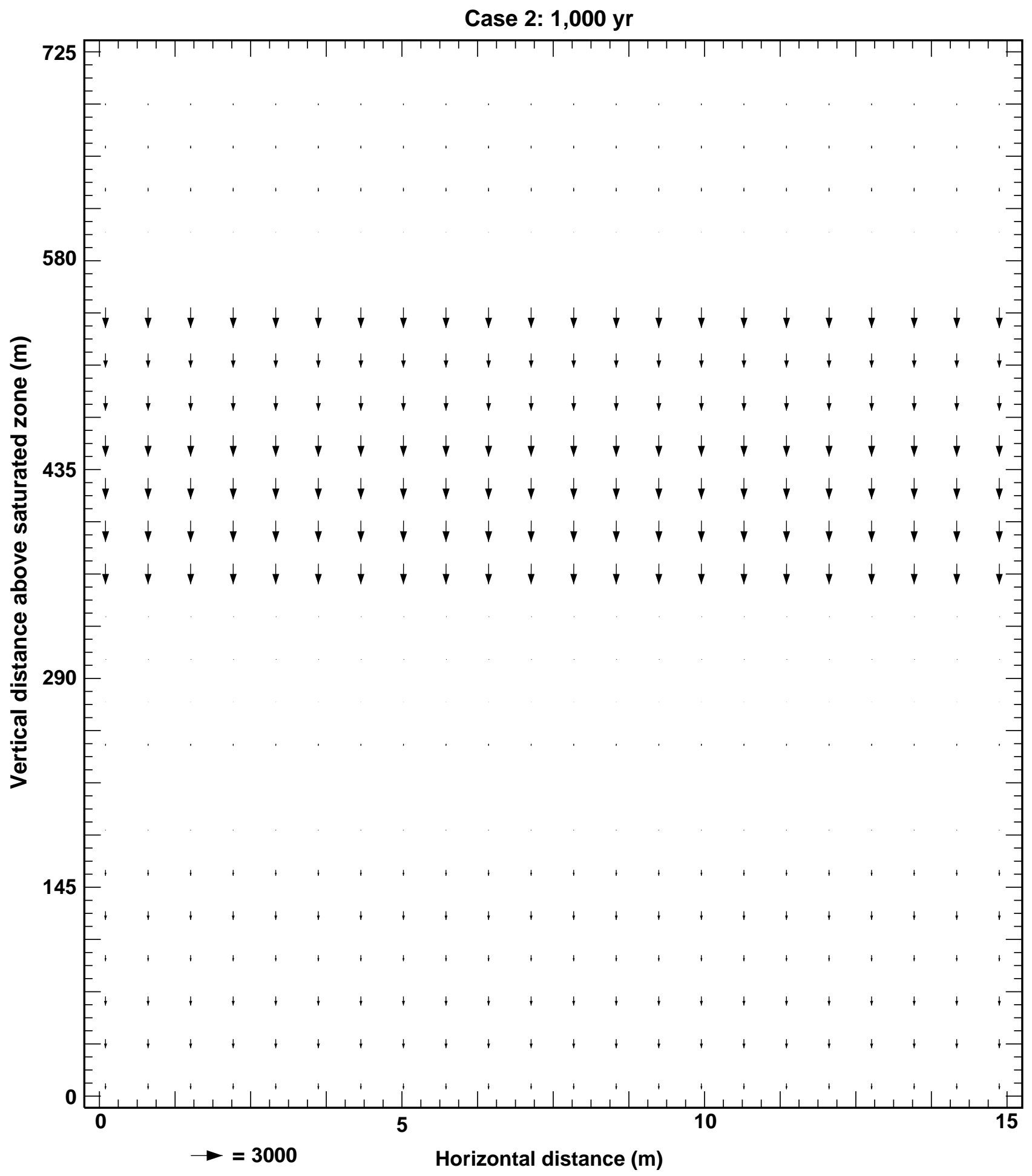

Figure 5-62. Flux vector distribution (m/yr) for Case 2 after $1000 \mathrm{yr}$ heating. The vertical and horizontal scales are the same as in Figure 5-46. 


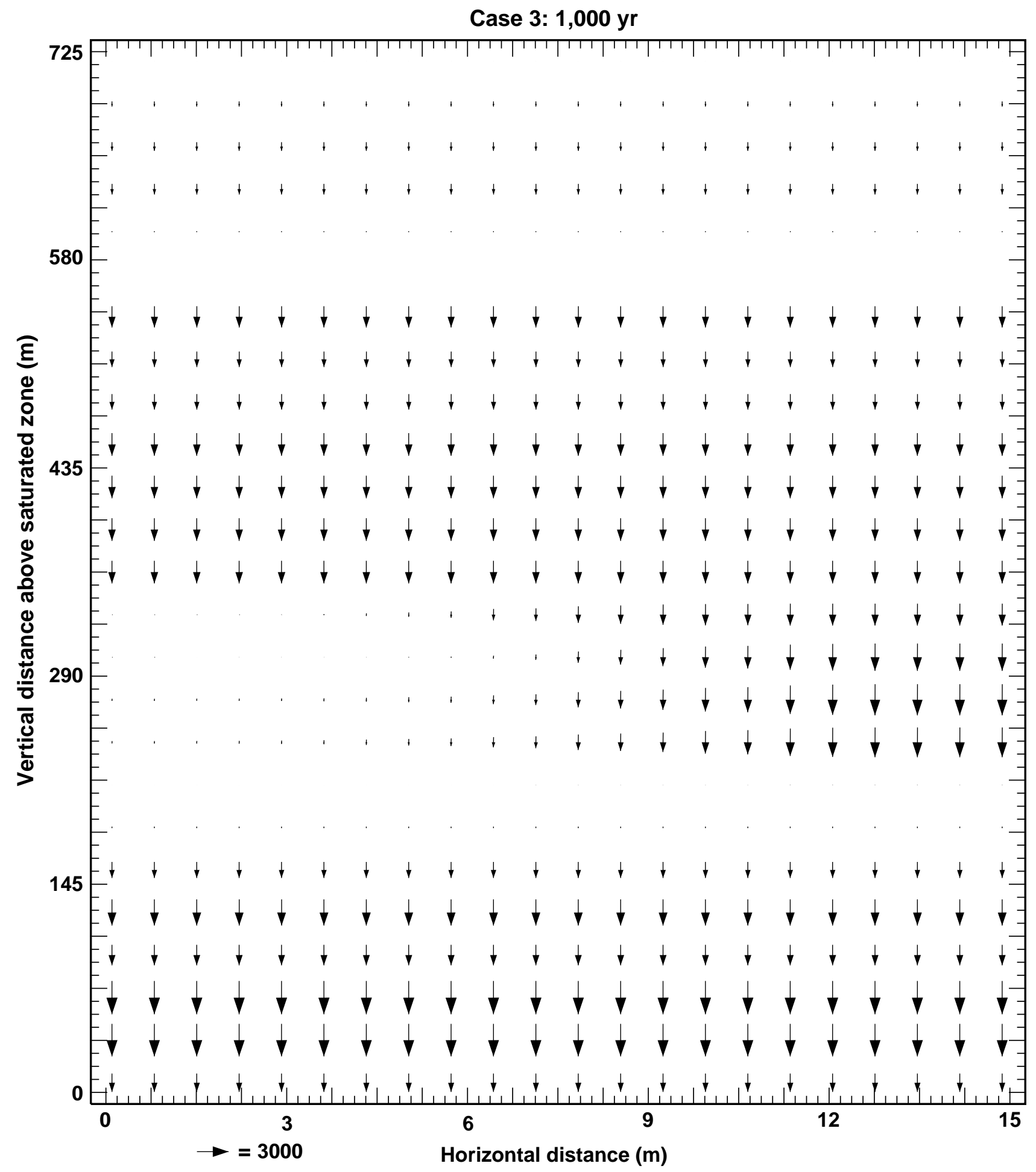

Figure 5-63. Flux vector distribution (m/yr) for Case 3 after $1000 \mathrm{yr}$ heating. The vertical and horizontal scales are the same as in Figure 5-46. 
Case 5: 1,000 yr

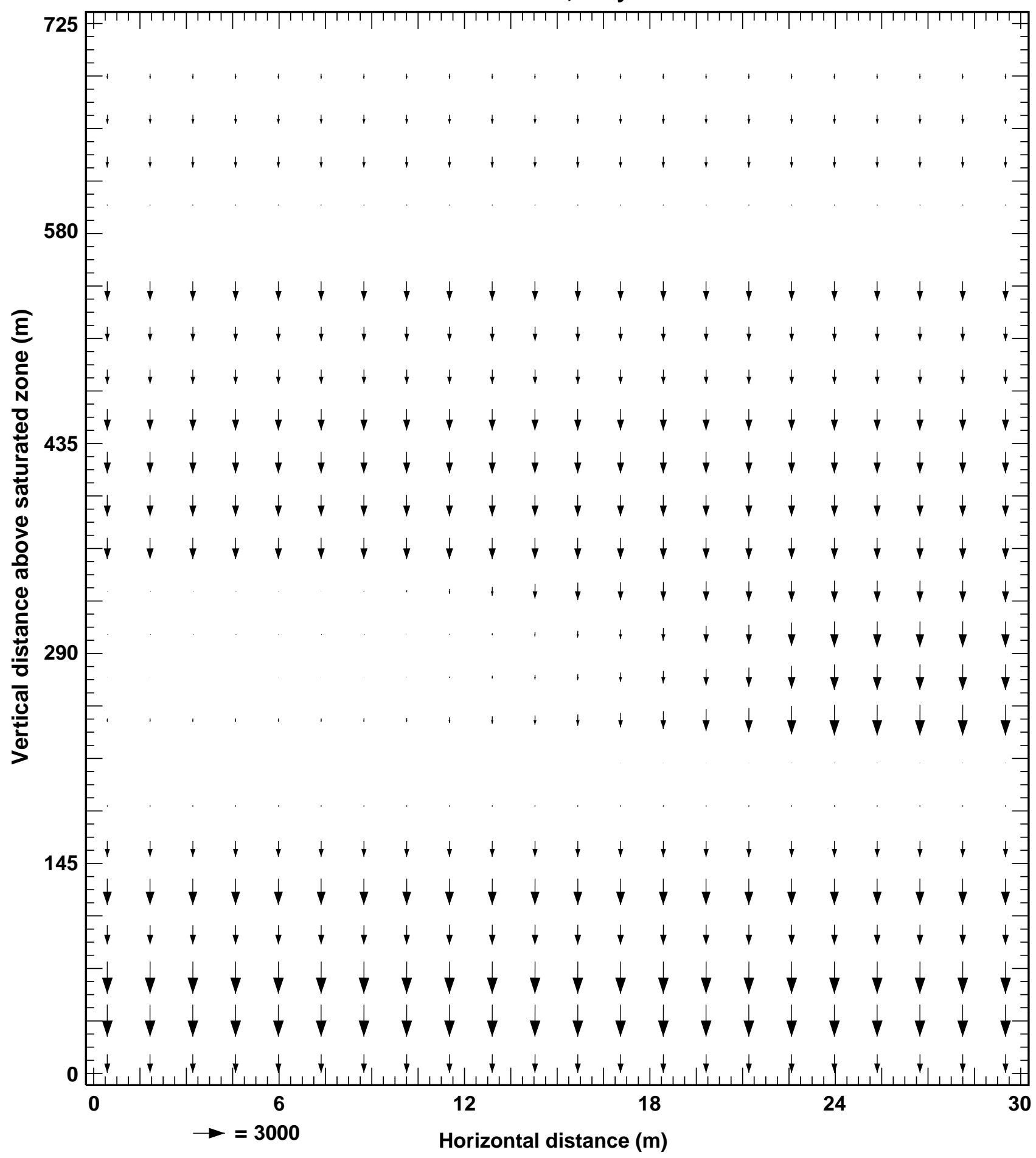

Figure 5-64. Flux vector distribution (m/yr) for Case 5 after $1000 \mathrm{yr}$ heating. The vertical and horizontal scales are the same as in Figure 5-46, while the horizontal scale is twice that in Figure 5-46. 
Case 1: Mineralogy and porosity after $5 \mathrm{yr}$, assuming 1,000 yr of thermal evolution

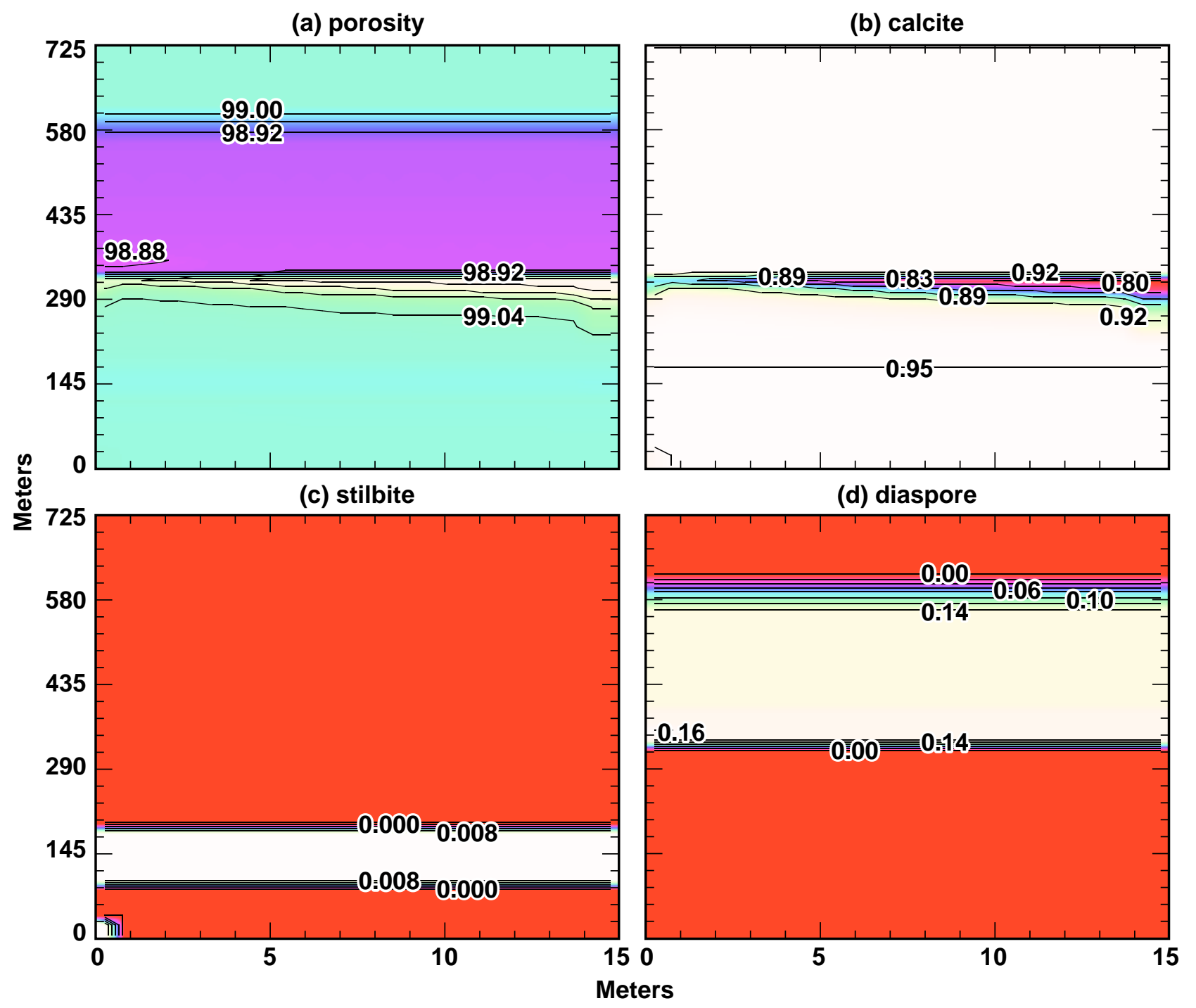

Figure 5-65. (a) Porosity, (b) calcite, (c) stilbite, and (d) diaspore distributions for Case 1 after 1000 yr of thermal evolution. The 2-D cross-section dimensions are the same as in Figure 5-46. All contour values are in volume $\%$. These calculations are conducted for a 5-yr period, assuming the flow and temperature regimes remained constant during that time. In these simulations, reactive transport is only permitted in the region at depths below $325 \mathrm{~m}$. At shallower depths, only thermal equilibration between fluid and rock is allowed. 
Case 2: Mineralogy and porosity after $5 \mathrm{yr}$, assuming 1,000 yr of thermal evolution

(a) porosity
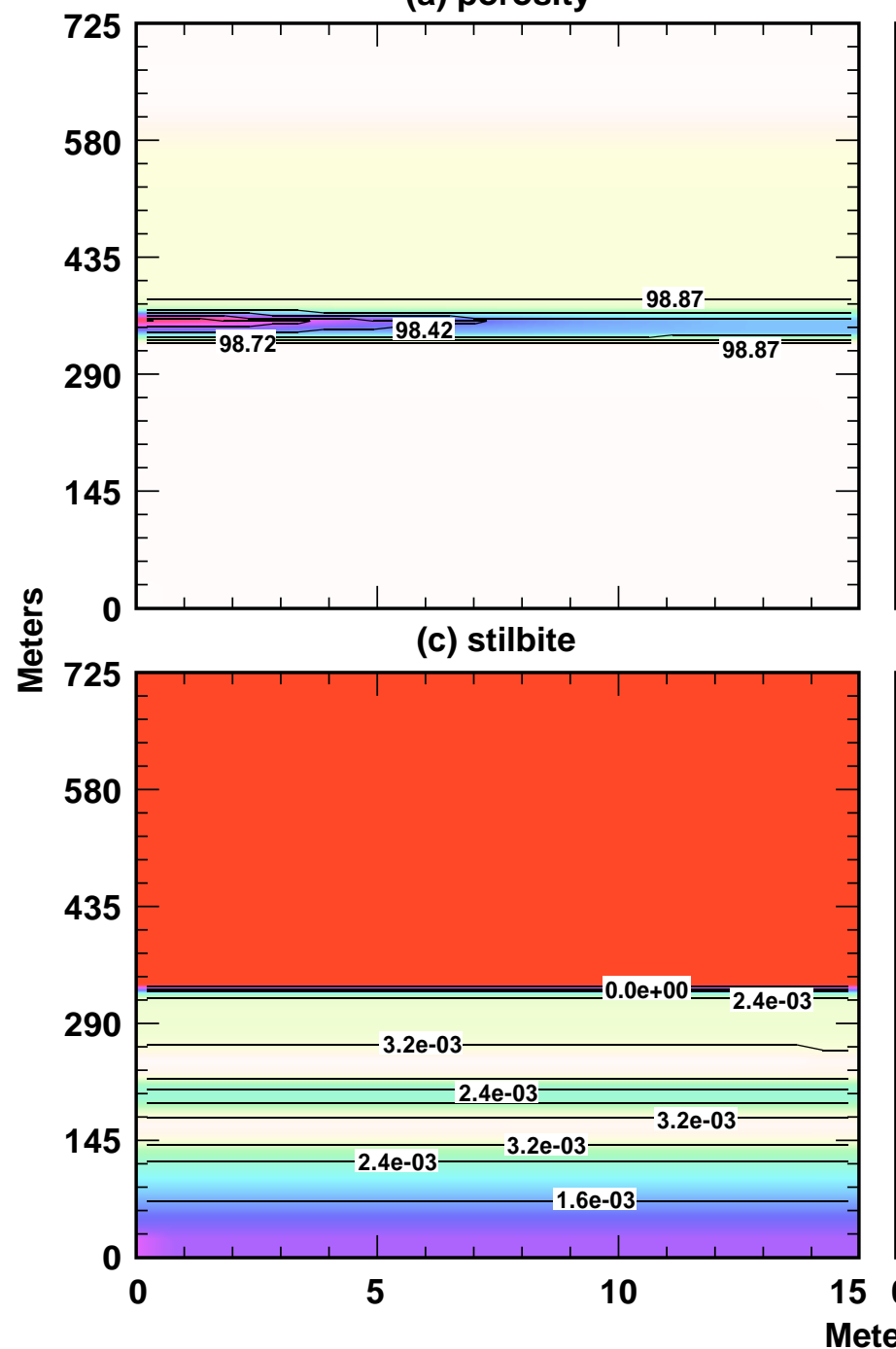

(b) calcite

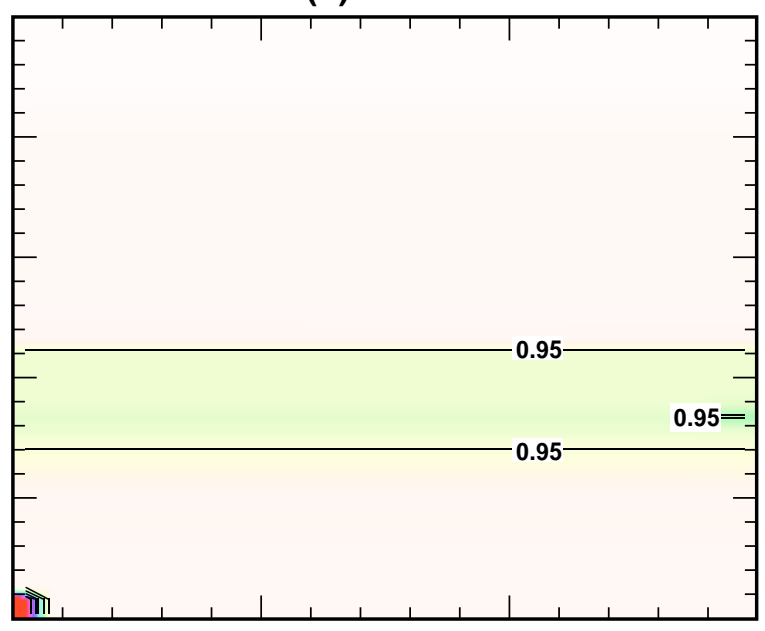

(d) diaspore

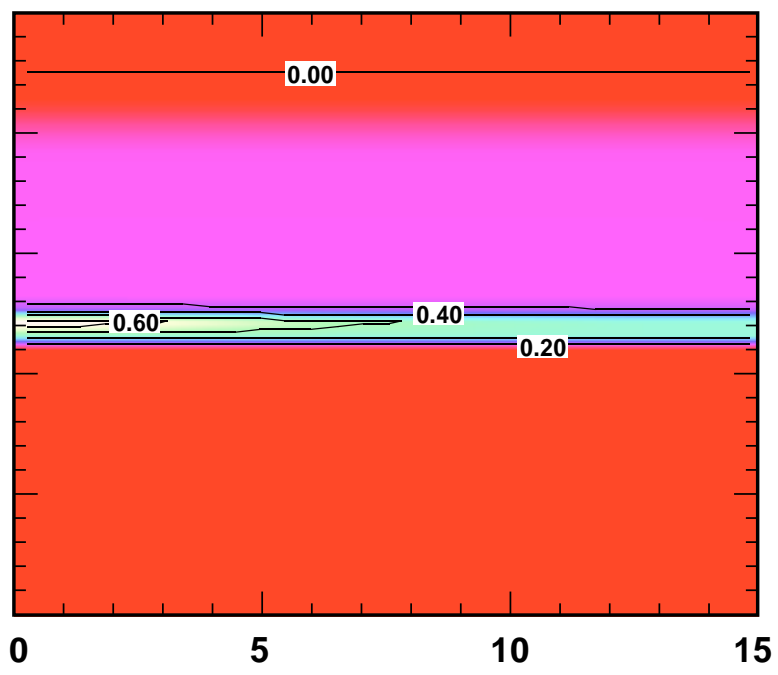

Figure 5-66. (a) Porosity, (b) calcite, (c) stilbite, and (d) diaspore distributions for Case 2 after 1000 yr of thermal evolution. The 2-D cross-section dimensions are the same as in Figure 5-46. All contour values are in volume $\%$. These calculations are conducted for a 5-yr period, assuming the flow and temperature regimes remained constant during that time. In these simulations, reactive transport is only permitted in the region at depths below $325 \mathrm{~m}$. At shallower depths, only thermal equilibration between fluid and rock is allowed. 


\section{Case 3: Mineralogy and porosity after $5 \mathrm{yr}$, assuming 1,000 yr of thermal evolution}

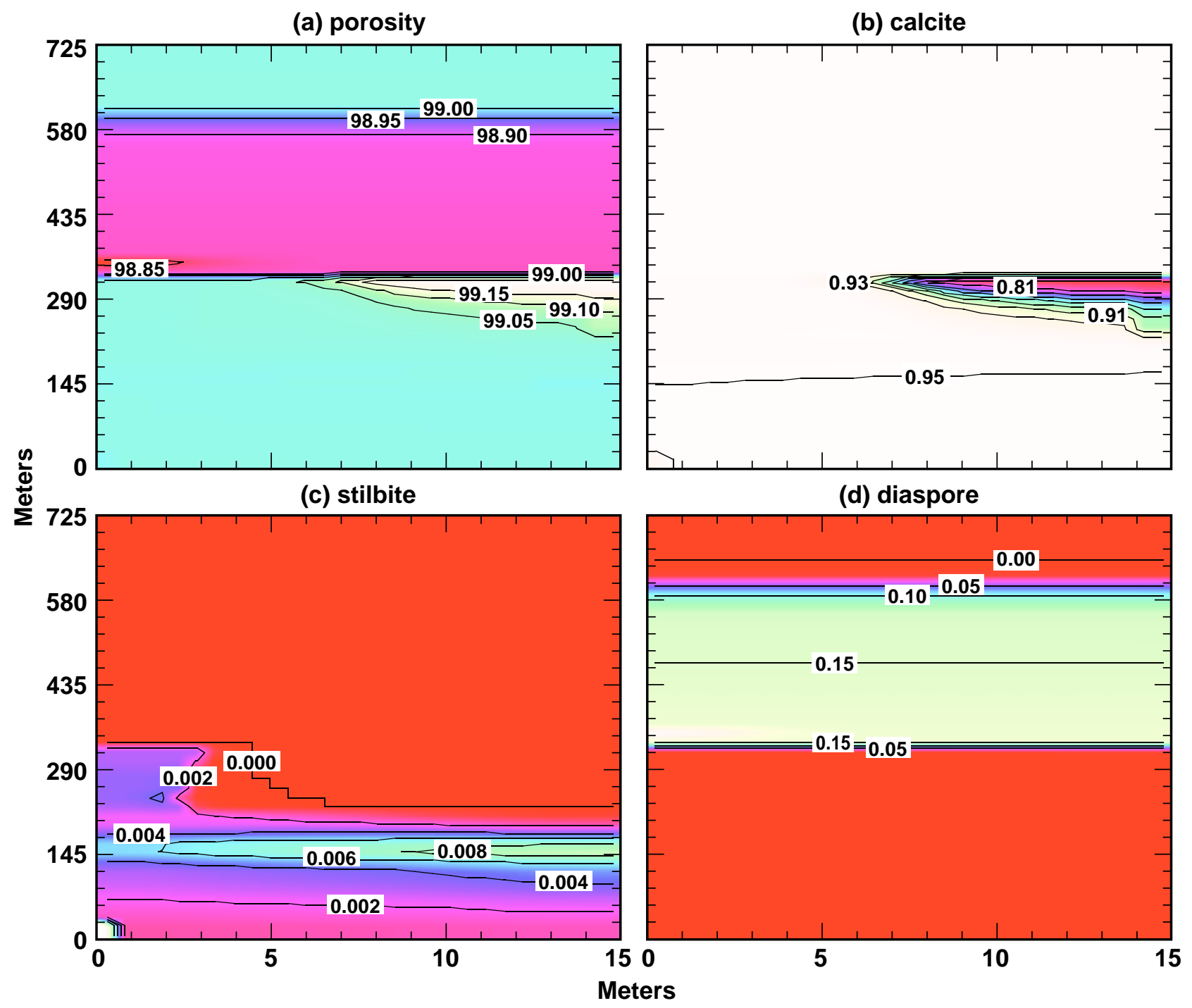

Figure 5-67. (a) Porosity, (b) calcite, (c) stilbite, and (d) diaspore distributions for Case 3 after 1000 yr of thermal evolution. The 2-D cross-section dimensions are the same as in Figure 5-46. All contour values are in volume $\%$. These calculations are conducted for a 5-yr period, assuming the flow and temperature regimes remained constant during that time. In these simulations, reactive transport is only permitted in the region at depths below $325 \mathrm{~m}$. At shallower depths, only thermal equilibration between fluid and rock is allowed. 


\section{Case 5: Mineralogy and porosity after $5 \mathrm{yr}$, assuming 1,000 yr of thermal evolution}

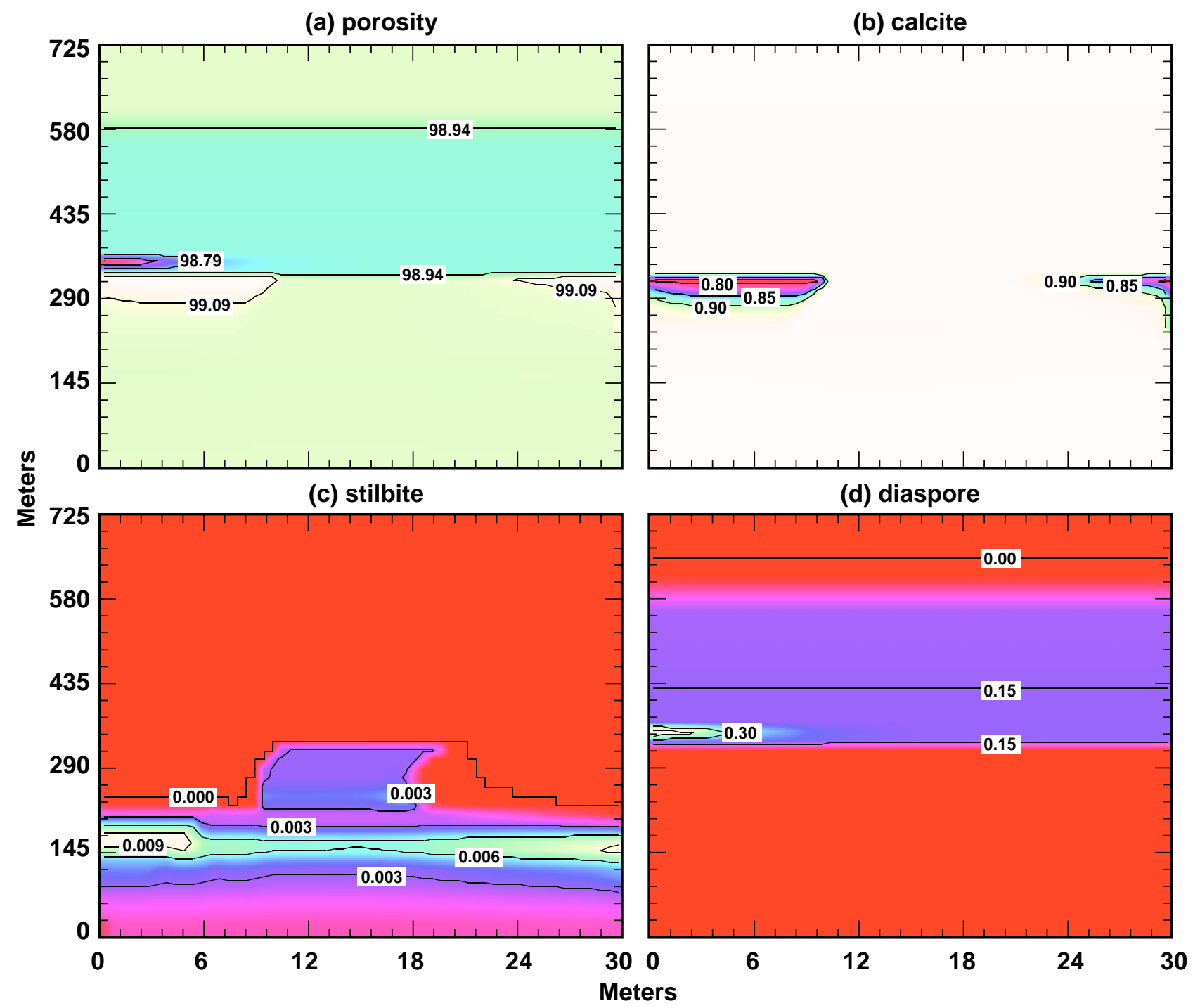

Figure 5-68. (a) Porosity, (b) calcite, (c) stilbite, and (d) diaspore distributions for Case 5 after $1000 \mathrm{yr}$ of thermal evolution. The 2-D cross-section dimensions are the same as in Figure 5-46. All contour values are in volume $\%$. These calculations are conducted for a 5-yr period, assuming the flow and temperature regimes remained constant during that time. In these simulations, reactive transport is only permitted in the region at depths below $325 \mathrm{~m}$. At shallower depths, only thermal equilibration between fluid and rock is allowed. Note that the horizontal axis is twice that in the preceding three figures. 


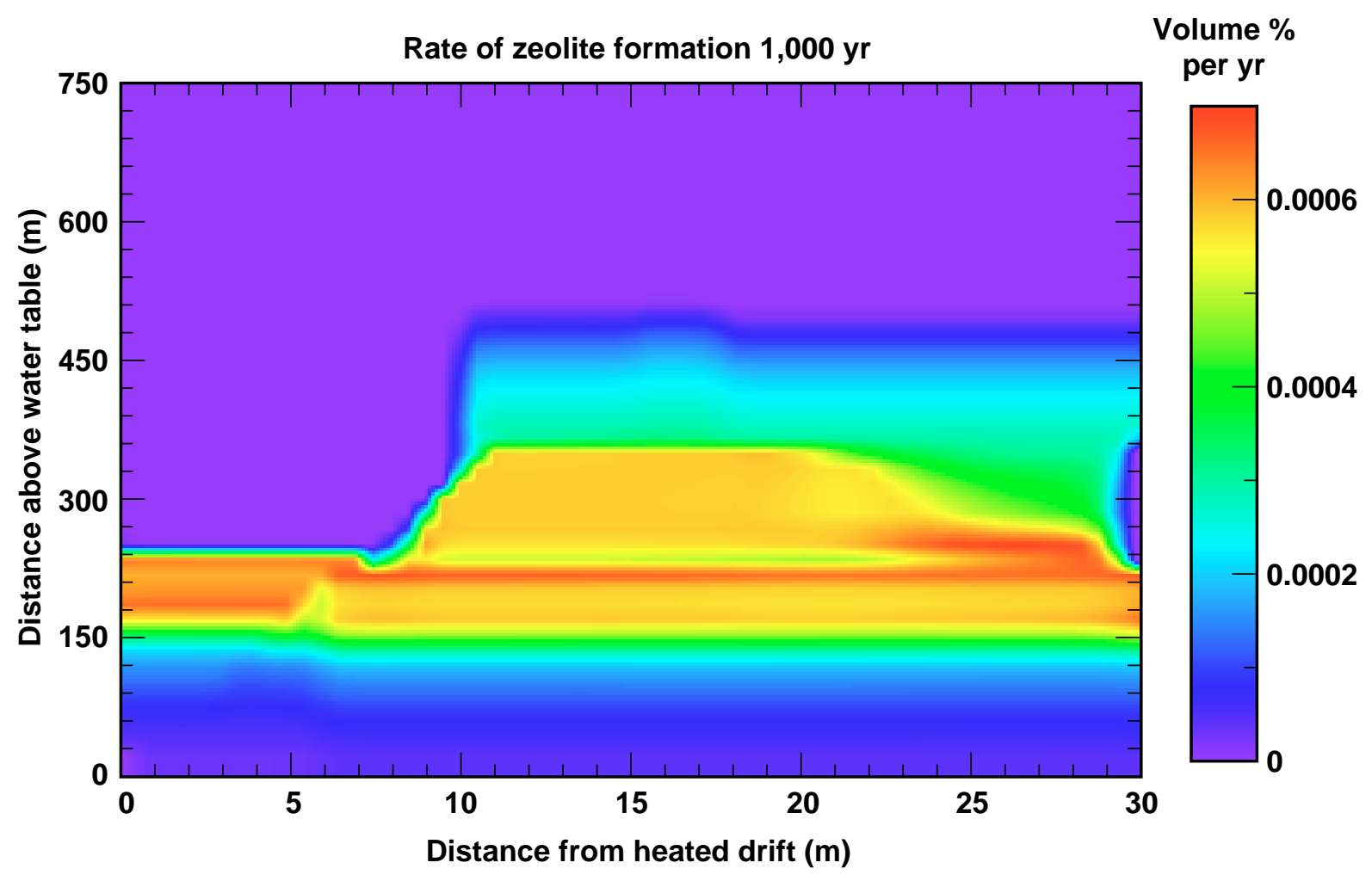

Figure 5-69. Mesolite distributions for the cases represented in Figure 5-65 through Figure 5-68. 
Case 2: Porosity, at $5 \mathrm{yr}$, after 1,000 yr of thermal evolution
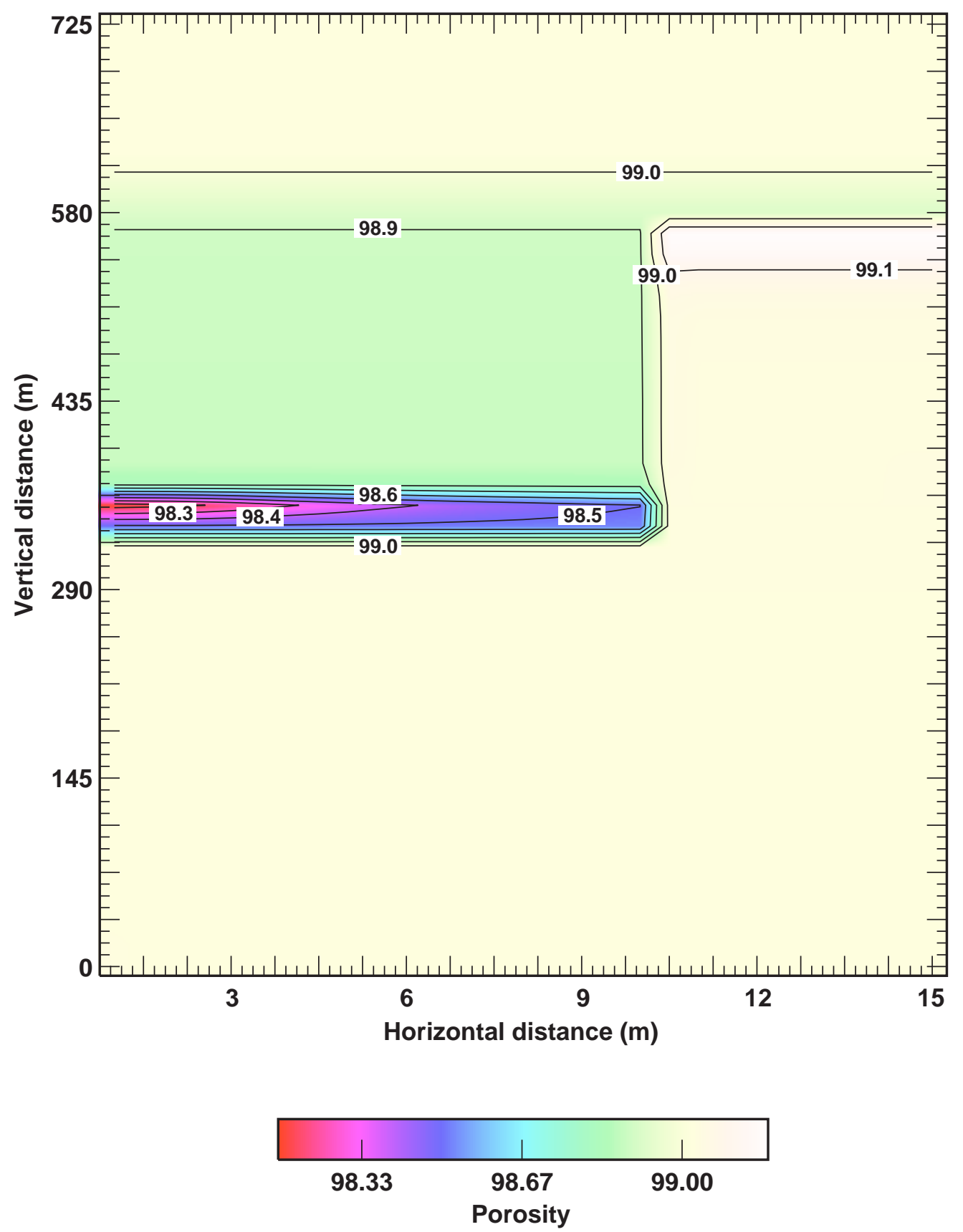

Figure 5-70. Porosity distribution for Case 2 after $1000 \mathrm{yr}$ of thermal evolution, under the pillar flow constraints. These calculations are conducted for a 5-yr period, assuming the flow and temperature regimes remained constant during that time. In these simulations, reactive transport is permitted in the pillar region and at depths below $325 \mathrm{~m}$. At shallower depths above the heated drift region, only thermal equilibration between fluid and rock is allowed. 

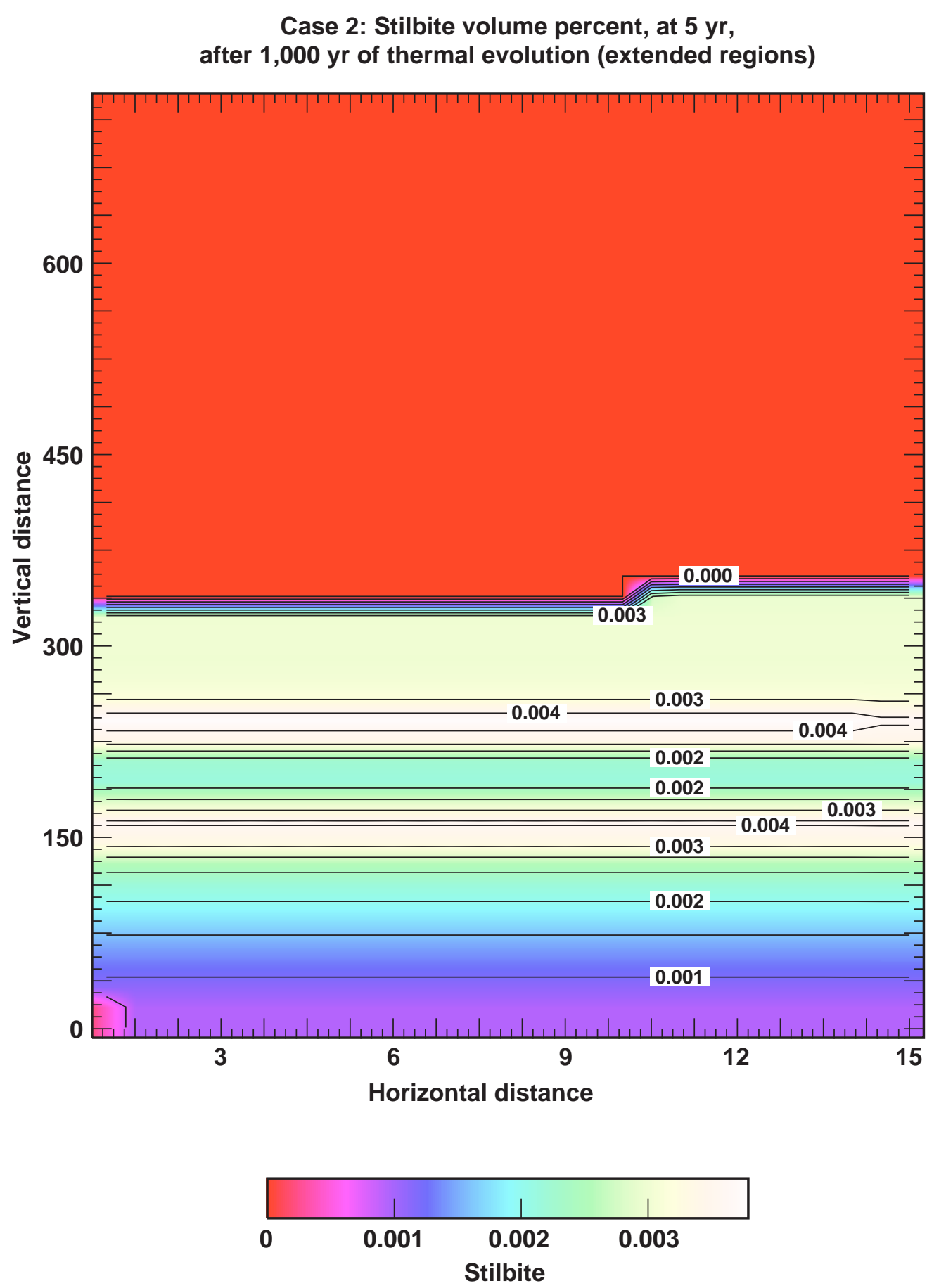

Figure 5-71. Stilbite distribution for Case 2 after $1000 \mathrm{yr}$ of thermal evolution, under the pillar flow constraints. These calculations are conducted for a 5-yr period, assuming the flow and temperature regimes remained constant during that time. In these simulations, reactive transport is permitted in the pillar region and at depths below $325 \mathrm{~m}$. At shallower depths above the heated drift region, only thermal equilibration between fluid and rock is allowed. 


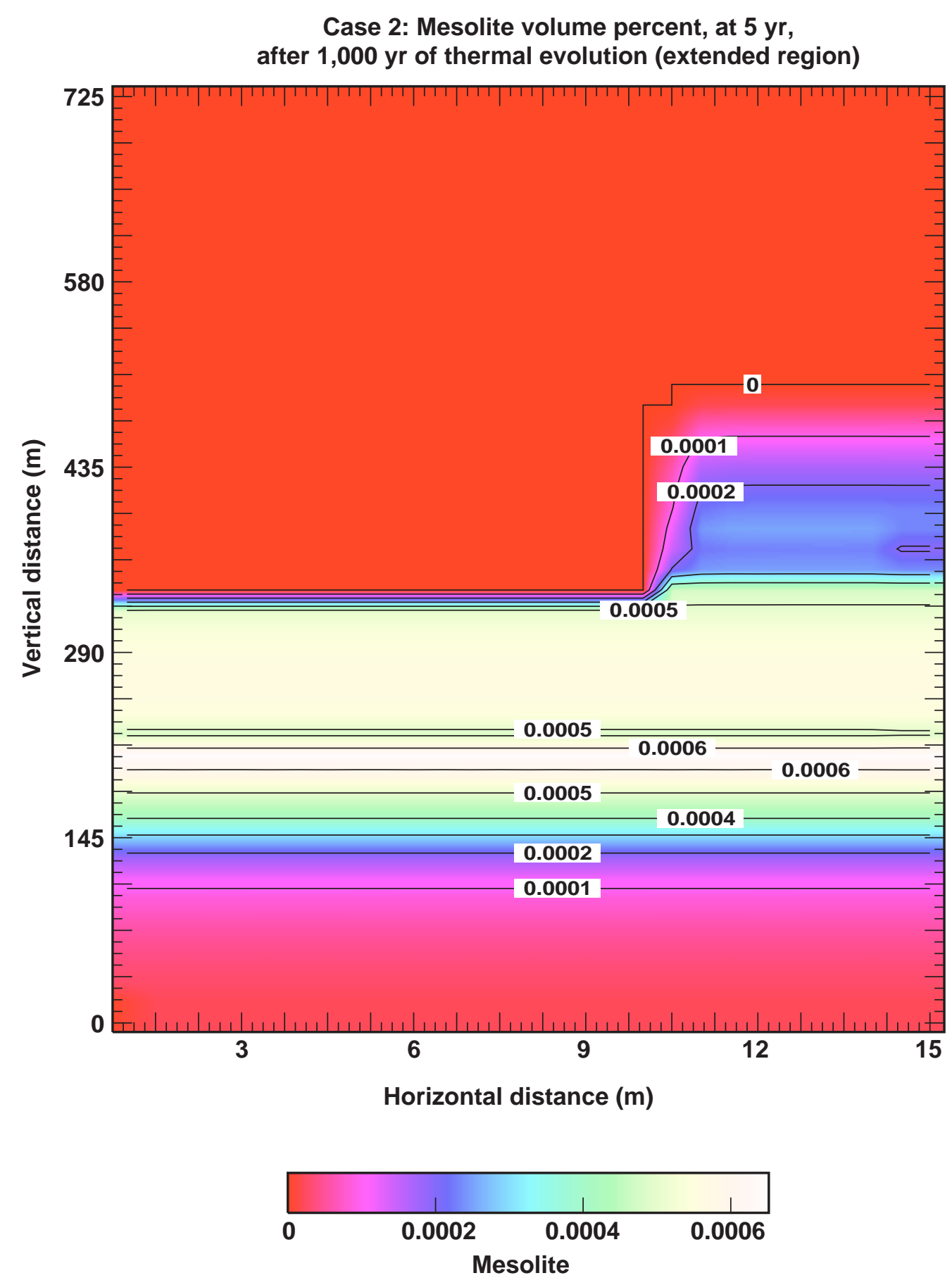

Figure 5-72. Mesolite distribution for Case 2 after $1000 \mathrm{yr}$ thermal evolution, under the pillar flow constraints. These calculations are conducted for a 5-yr period, assuming the flow and temperature regimes remained constant during that time. In these simulations, reactive transport is permitted in the pillar region and at depths below $325 \mathrm{~m}$. At shallower depths above the heated drift region, only thermal equilibration between fluid and rock is allowed. 
Case 5: Porosity, at $5 \mathrm{yr}$, after $1,000 \mathrm{yr}$ of thermal evolution
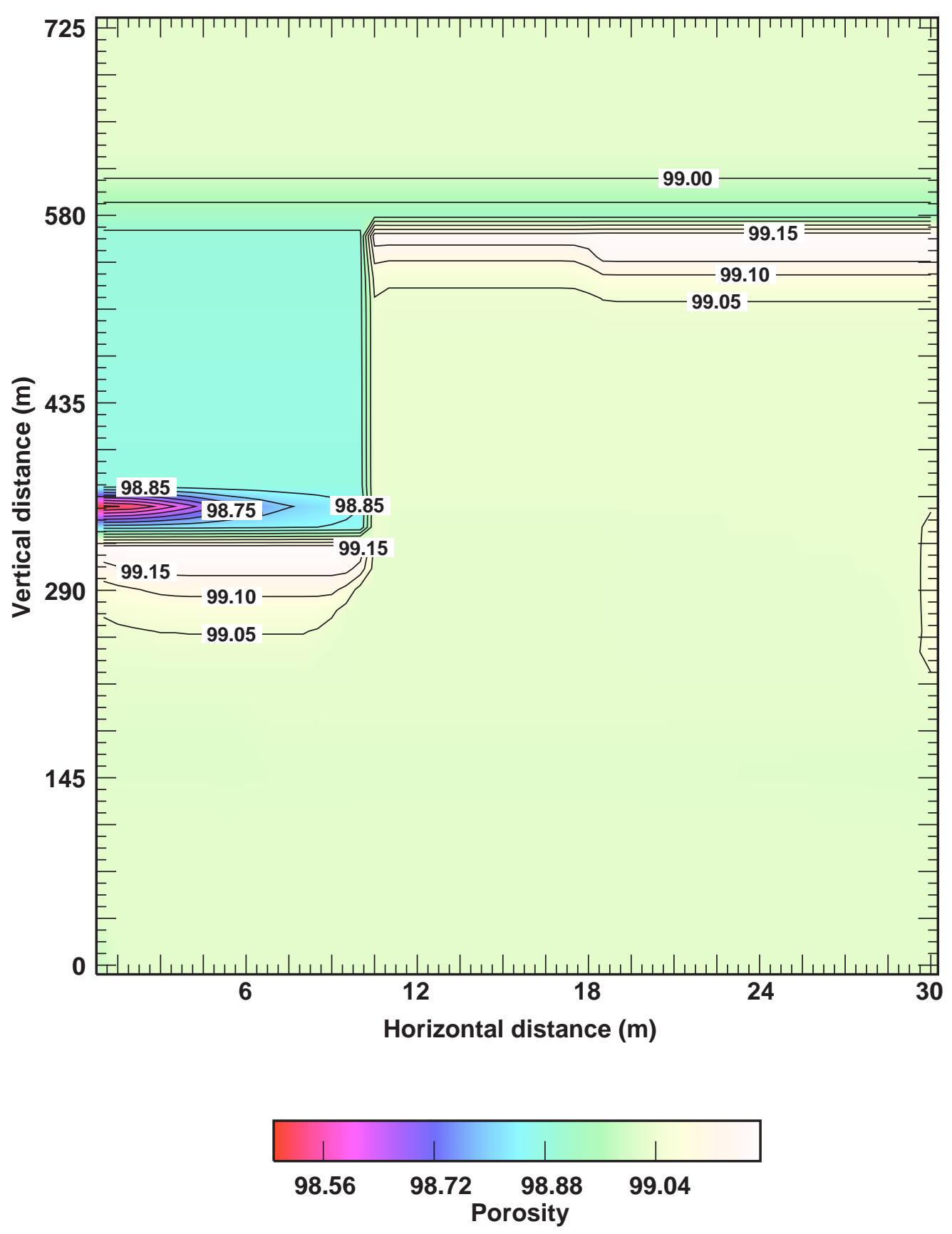

Figure 5-73. Porosity distribution for Case 5 after $1000 \mathrm{yr}$ of thermal evolution, under the pillar flow constraints. These calculations are conducted for a 5-yr period, assuming the flow and temperature regimes remained constant during that time. In these simulations, reactive transport is permitted in the pillar region and at depths below $325 \mathrm{~m}$. At shallower depths above the heated drift region, only thermal equilibration between fluid and rock is allowed. 


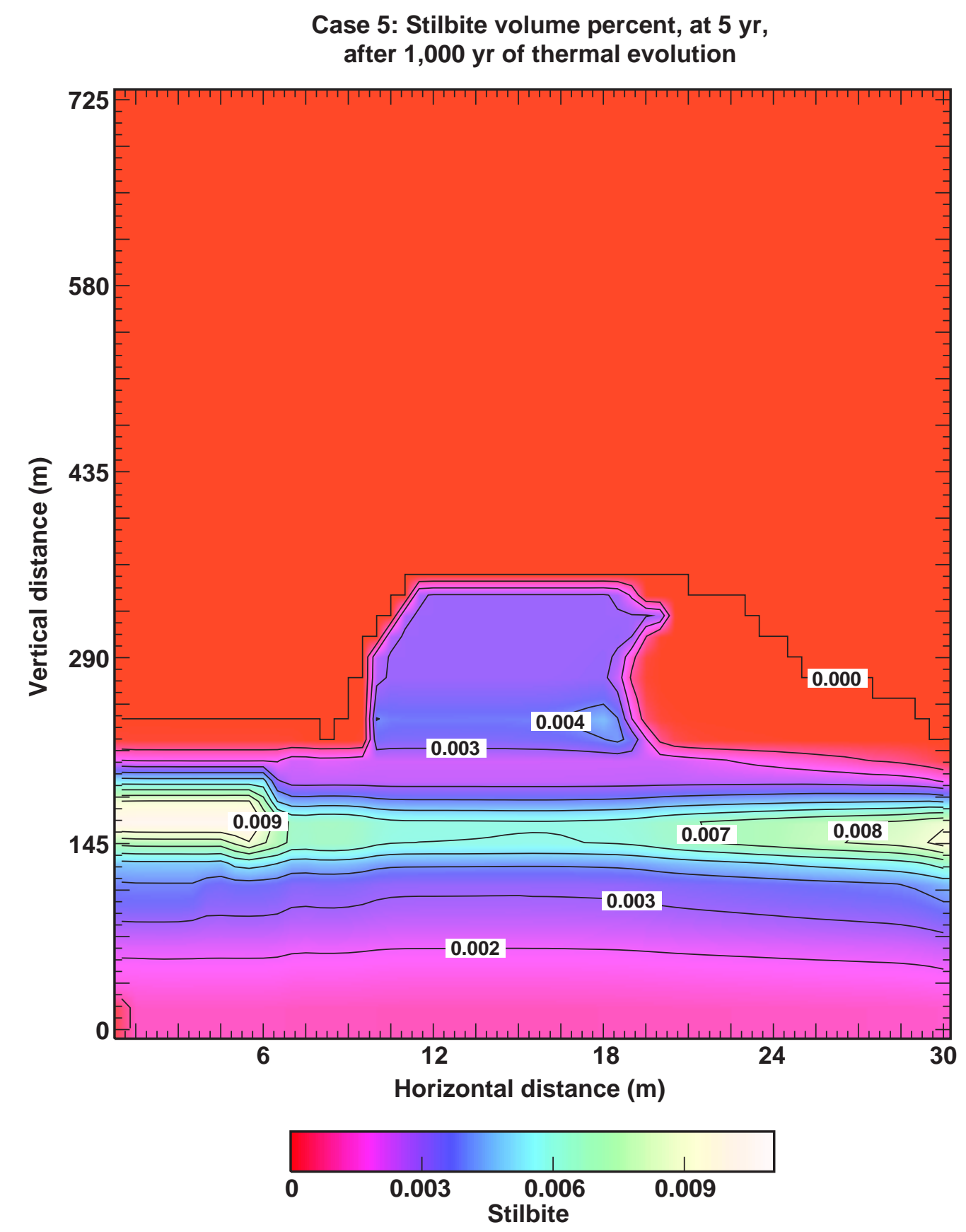

Figure 5-74. Stilbite distribution for Case 5 after $1000 \mathrm{yr}$ of thermal evolution, under the pillar flow constraints. These calculations are conducted for a 5-yr period, assuming the flow and temperature regimes remained constant during that time. In these simulations, reactive transport is permitted in the pillar region and at depths below $325 \mathrm{~m}$. At shallower depths above the heated drift region, only thermal equilibration between fluid and rock is allowed. 


\section{Case 5: Mesolite volume percent, at $5 \mathrm{yr}$, after $1,000 \mathrm{yr}$ of thermal evolution}

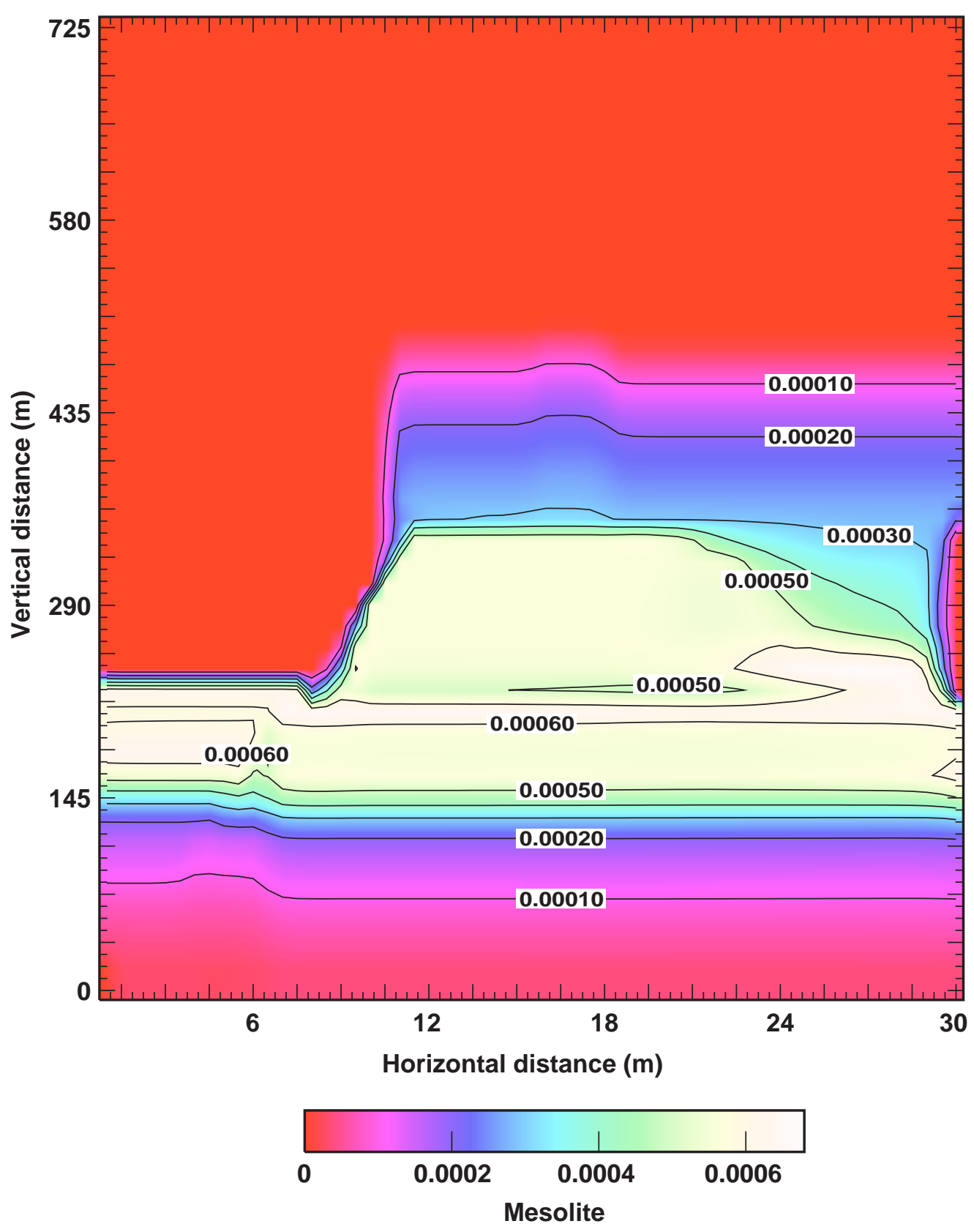

Figure 5-75. Mesolite distribution for Case 5 after $1000 \mathrm{yr}$ of thermal evolution, under the pillar flow constraints. These calculations are conducted for a 5-yr period, assuming the flow and temperature regimes remained constant during that time. In these simulations, reactive transport is permitted in the pillar region and at depths below $325 \mathrm{~m}$. At shallower depths above the heated drift region, only thermal equilibration between fluid and rock is allowed. 
Case 1: Mineralogy and porosity after $5 \mathrm{yr}$, assuming $1,000 \mathrm{yr}$ of thermal evolution, including over drift area

(a) porosity

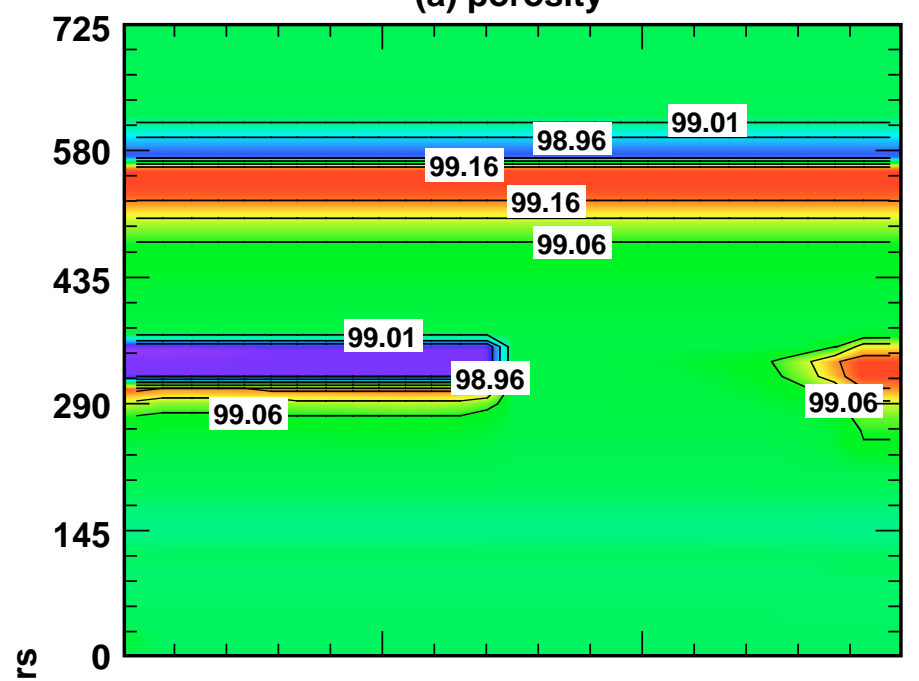

(c) stilbite

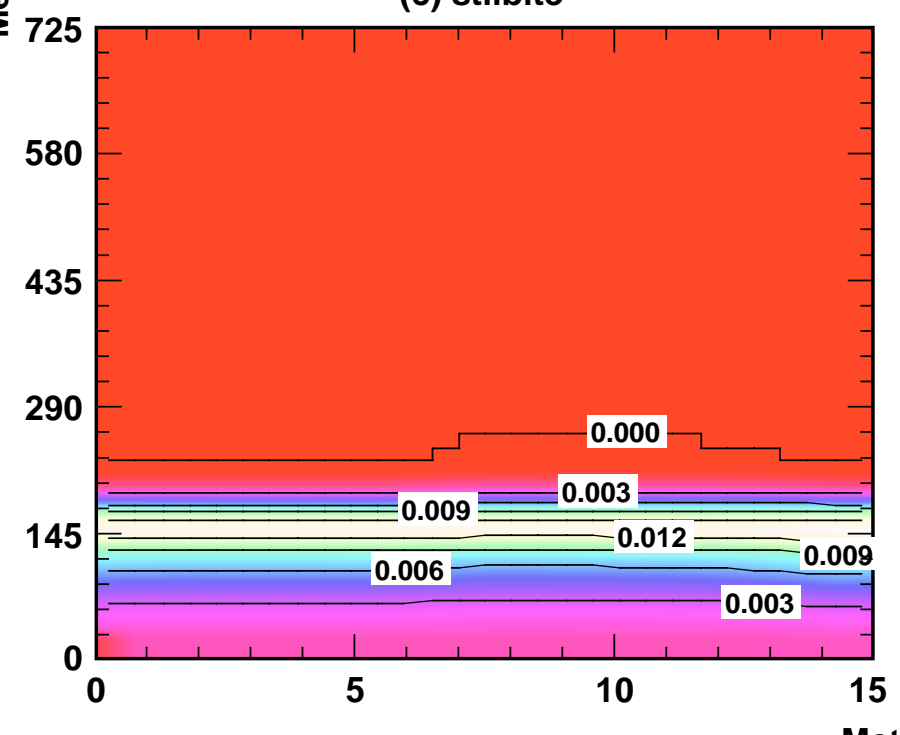

(b) calcite

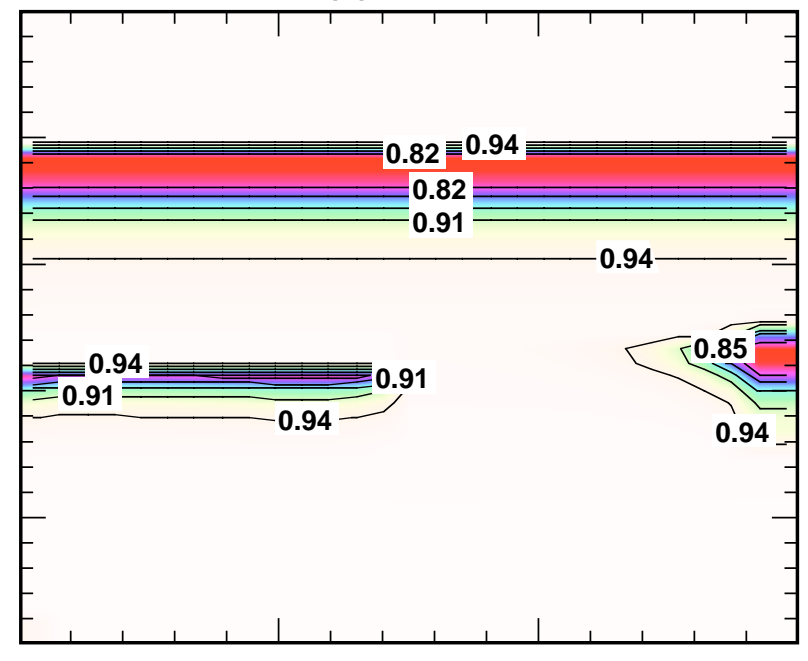

(d) mesolite

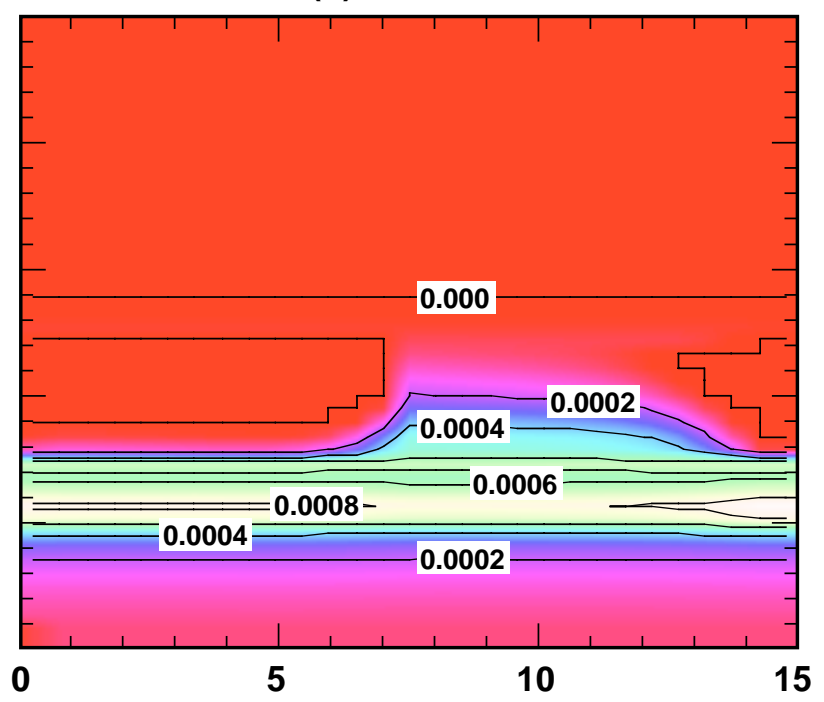

Meters

Figure 5-76. (a) Porosity, (b) calcite, (c) stilbite, and (d) mesolite distributions for Case 1 after 1000 yr of thermal evolution. The 2-D cross-section dimensions are the same as in Figure 5-46. All contour values are in volume $\%$. These calculations are conducted for a 5-yr period, assuming the flow and temperature regimes remained constant during that time. In these simulations, reactive transport is permitted throughout the region, except for a few meters immediately around the area of the heated drift. 
Case 5: Mineralogy and porosity after $5 \mathrm{yr}$, assuming $1,000 \mathrm{yr}$ of thermal evolution, including over drift area

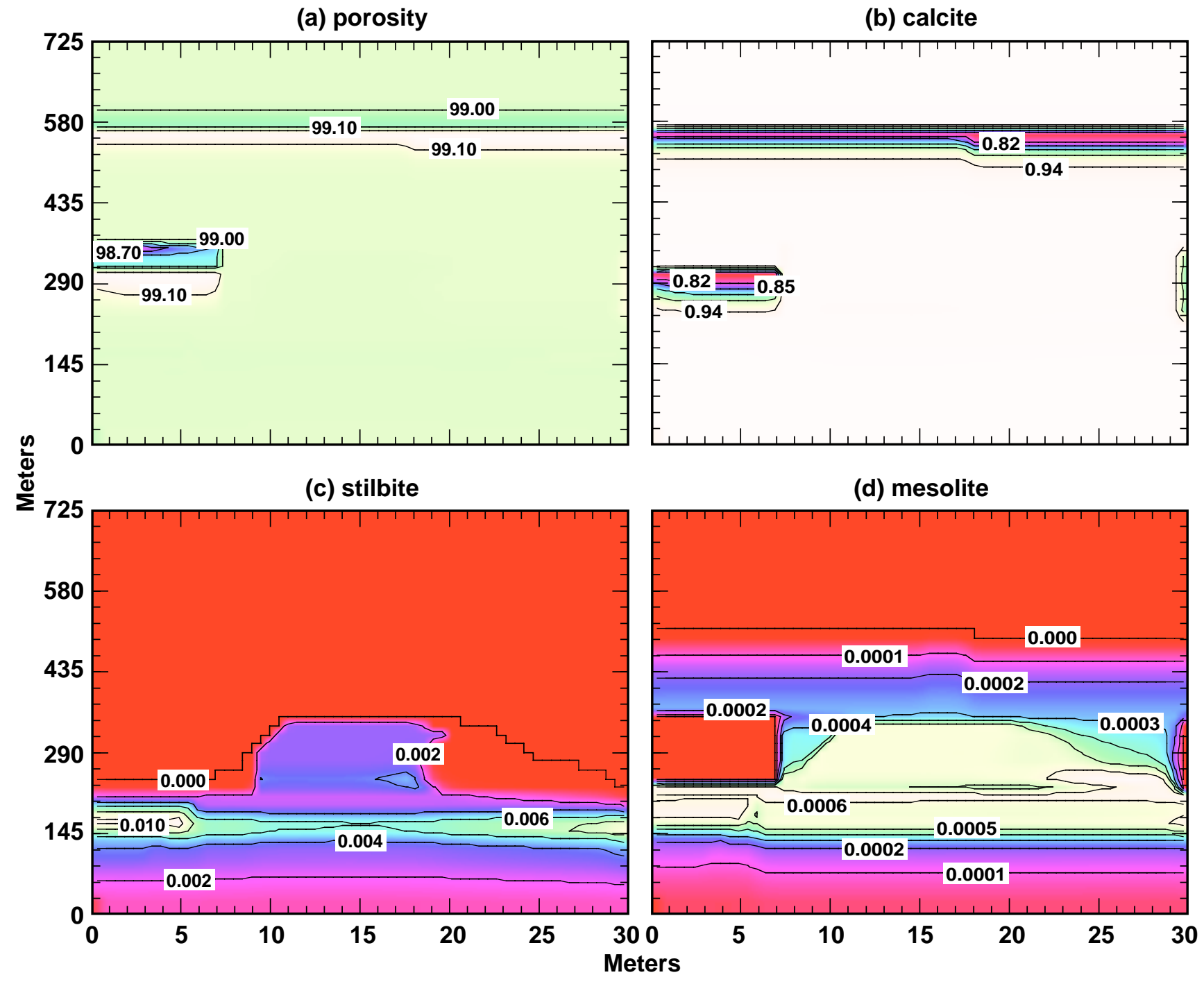

Figure 5-77. (a) Porosity, (b) calcite, (c) stilbite, and (d) mesolite distributions for Case 5 after $1000 \mathrm{yr}$ of thermal evolution. The 2-D cross-section dimensions are the same as in Figure 5-48. All contour values are in volume $\%$. These calculations are conducted for the same conditions as in Figure 5-76. 
Case 1: Mineralogy and porosity after $5 \mathrm{yr}$, assuming $1,000 \mathrm{yr}$ of thermal evolution, including over drift area and atmospheric $\mathrm{CO}_{2}$

(a) porosity

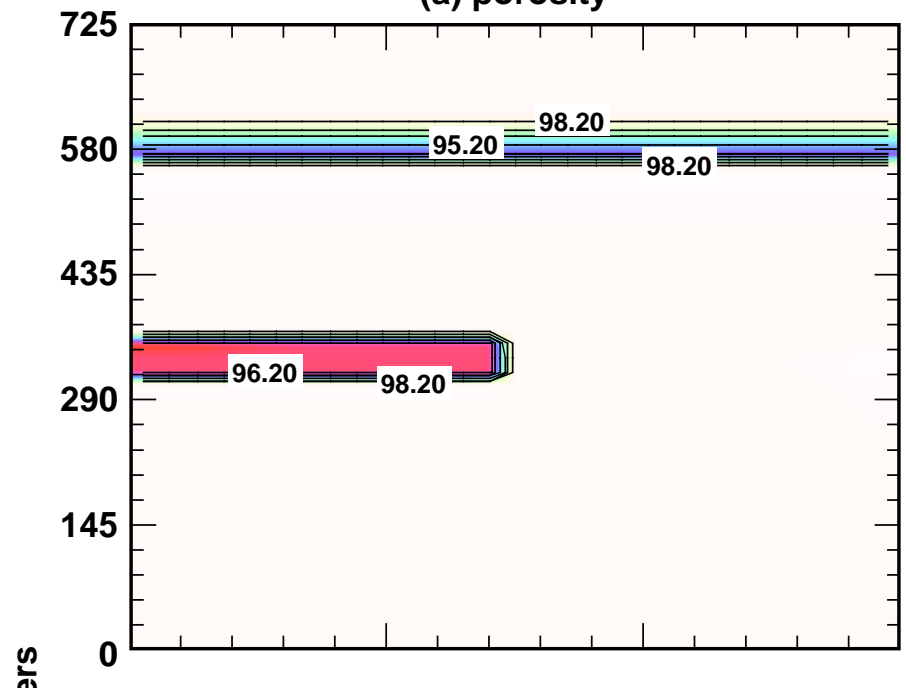

(c) stilbite

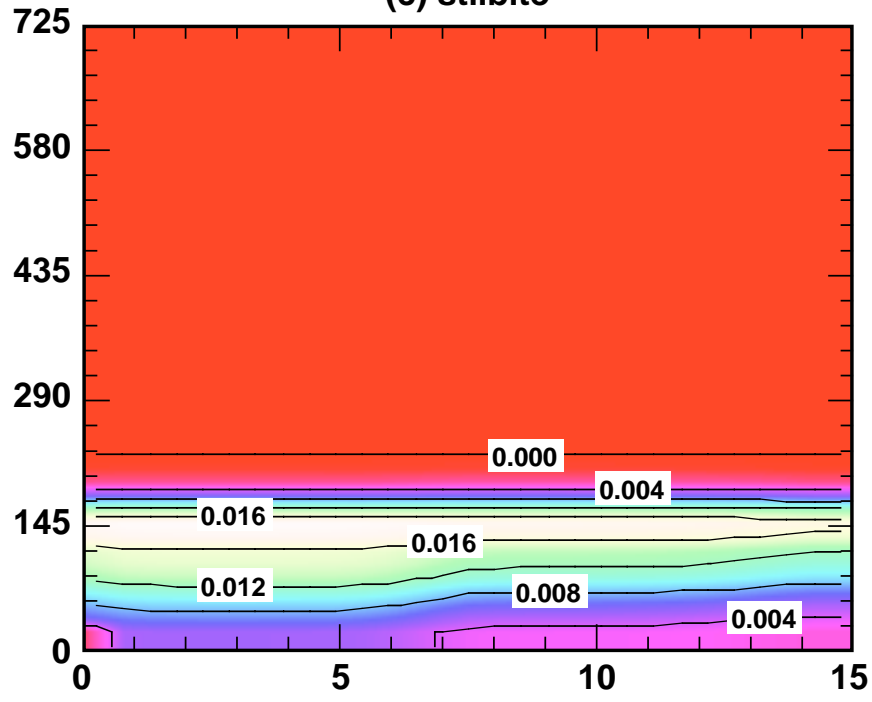

(b) calcite

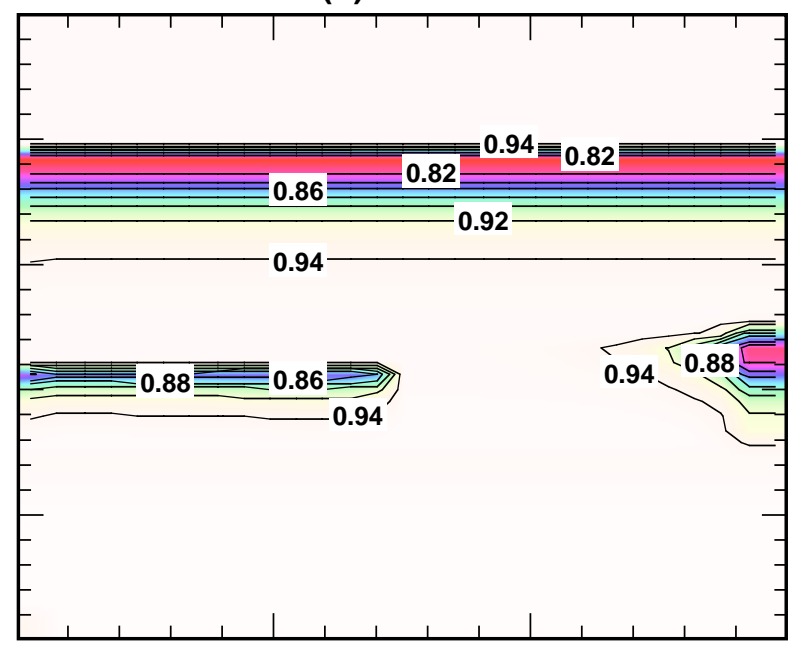

(d) mesolite

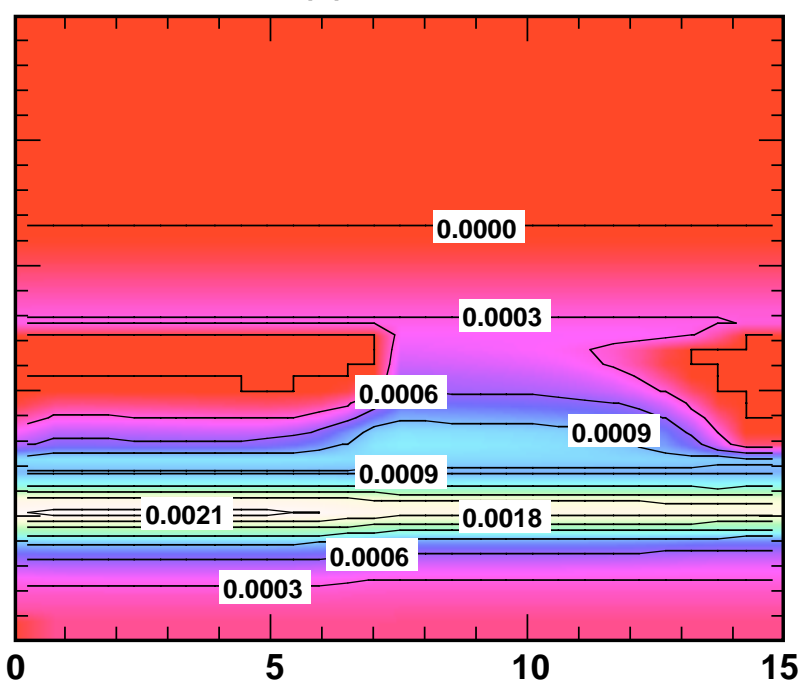

Meters

Figure 5-78. (a) Porosity, (b) calcite, (c) stilbite, and (d) mesolite distributions for Case 1 after 1000 yr of thermal evolution. The 2-D cross section dimensions are the same as in Figure 5-46. All contour values are in volume $\%$. These calculations are conducted for a 5-yr period, assuming the same conditions as presented in Figure 5-76, except that the initial condensate composition is assumed to be an order of magnitude less than that of atmospheric $\mathrm{CO}_{2}$. 


\section{Near-Field Geochemical Environment}

\subsection{Introduction to Models of the Near-Field Geochemical Environment}

The ambient geochemical environment of the host rock has been very steady for millions of years of geologic history, and external boundary conditions have been reasonably steady for the thousands of years of the present interglacial period. Repository heating, however, will strongly perturb geochemical processes in the near field and altered zone (NF/AZ). Moreover, the near-field environment (NFE) will contain abundant, chemically reactive, introduced materials such as concrete, steel, and the waste form itself. The waste package (WP) lifetime and the future release rates for key radionuclides will be particularly sensitive to the near-field geochemical environment.

This chapter describes modeling approaches for predicting conditions in the NFE. It emphasizes prediction of the water composition in the NFE, which is needed to assess the WP lifetime, and evaluation of the mobility of radionuclides, that may be released from the WPs.

The discussion of models begins with the interaction of condensate water and the host rock, at elevated temperature, in the altered zone (AZ) above the repository. A predictive model is presented for the chemical evolution of water, at temperatures from ambient to boiling, in the host rock. Under certain conditions (see Chapter 3), water could drain into the repository drifts where it would interact with introduced materials. The reference repository design calls for extensive use of concrete, and interaction of water with cement can produce alkaline fluids depending on the type of cement, the extent of cement maturation, and other factors. A second predictive model is presented for estimating the range of chemical compositions for water in contact with cementitious materials. This is the water composition that is assumed to contact the WPs.

If radionuclides are released to the NFE, interaction with introduced materials will cause retardation of some species. Presented in this chapter is a series of laboratory experiments that help to define the retardation behavior of $U$ and $\mathrm{Np}$ in contact with iron corrosion products and altered concrete. Also presented is a third chemical model that can be used to describe radionuclide speciation and precipitation, at conditions ranging from an alkaline environment influenced by cement to the host-rock environment at ambient temperature. The mobility of radionuclides in the NFE is addressed from the perspective of solution chemistry and from experimentally observed interactions with introduced materials.

Interaction of radionuclides with introduced materials may cause colloidal transport, which could be significant in the NF/ AZ, depending on chemical and hydraulic factors that include the magnitude and distribution of advective flow. True radiocolloids, pseudocolloids, and biocolloids are discussed as alternative modes of radionuclide transport. This chapter presents conceptual models that describe possible colloid source materials, processes that will generate colloids, and influences on colloidal sorption of radionuclides. Described are experiments that have addressed some aspects of colloid transport.

In summary, this chapter comprises the following sections:

Section 6.1 includes background information on the Near-Field and Altered-Zone Environment Report, Vol. II (Wilder, 1996), which contains more extensive discussion of projected conditions.

Section 6.2 describes two approaches to modeling, at ambient and elevated temperature, the composition of water in contact with the host rock in the AZ. 
Section 6.3 presents recent modeling of the water composition that is likely to result from contact with cementitious materials in the emplacement drifts.

Section 6.4 describes and analyzes several sets of batch and transport experiments to investigate the retardation of $\mathrm{U}$ and $\mathrm{Np}$ by iron corrosion products and cementitious materials.

Section 6.5 summarizes the scientific issues and current approach to modeling of radionuclide transport from the repository, through the AZ.

This chapter summarizes models for geochemical conditions in the NF/AZ that are available for use in repository design and performance analysis. Some calculations are provided as examples or to demonstrate model behavior over ranges of conditions. It is intended that these models be further developed and exercised extensively in support of the viability assessment and repository licensing. The models are currently limited by the availability of laboratory experimental data, the completeness of chemical databases, and limitations on the chemical processes that are incorporated (e.g., boiling and coprecipitation). Predictive capability is much less advanced for colloid transport than for other chemical models presented and, therefore, is much more dependent on experimental data.

\subsubsection{Previous Description of Conditions in the Near Field/Altered Zone}

Whereas this report is about conceptual and numerical models in the NF/ AZ, another set of reports has been prepared for the purpose of describing the environmental conditions. The Preliminary Near-Field Environment Report, Volume I: Technical Bases for EBS Design was published in 1993 to summarize, in a form that could be readily used by repository designers and analysts, the state of knowledge of expected near field conditions (Wilder, 1993). This volume was revised in 1997 and renamed the Near-Field and Altered-Zone Environment Report, Volume I: Technical Bases for EBS Design to emphasize the importance of thermally driven coupled processes in the host rock (Wilder, 1997). The revision addresses progress in site characterization, such as revised estimates of net infiltration, and progress in repository design. Volume II of the Near-Field and Altered-Zone Environment Report was published in 1996 as a collection of more detailed technical information developed to support assessment of environmental conditions (Wilder, 1996). The current schedule calls for revision of this detailed report (Near-Field and Altered-Zone Environment Report, Vol. II, Rev. 1) in late 1999.

\subsubsection{Identification of Users for Models of the Near-Field Geochemical Environment}

This chapter describes four models for the near-field geochemical environment:

1. A chemical model for predicting water composition in contact with introduced materials

2. A model for $\mathrm{U}$ and $\mathrm{Np}$ retardation on iron corrosion products and cementitious materials

3. A chemical model for assessing bounds on aqueous radionuclide concentrations based on solubility limits

4. A conceptual framework for colloidal and microbial processes that may affect radionuclide transport

These models are listed in Appendix A (Table A-9) along with the other models that comprise this report.

Chemical modeling of water composition in contact with introduced materials, particularly concrete, is important for assessing the lifetime of the WP and other engineered barriers for performance assessment (PA) and engineered barrier (EBS) and WP design. The model consists of a database developed for use with the EQ3/ 6 code. 
Retardation of $\mathrm{U}$ and $\mathrm{Np}$ on iron corrosion products and cementitious materials is important to PA. This model consists of experimental results in the form of sorption isotherms and surface complexation constants that are used to simulate transport. The potential radionuclide retardation from these materials in the NFE may be comparable to that associated with tens or hundreds of meters of the fractured host rock. Radionuclide mobility in the NFE, and along flowpaths from the NFE to the AZ, is also important to PA and is bounded by solubility calculations using a thermochemical database developed for use with the EQ3 / 6 code. Current models for possible colloidal and microbial effects on radionuclide transport are more conceptual, and less predictive in nature, than are other transport models discussed in this report. Information on colloidal processes is relevant primarily to PA, whereas microbial processes are important to both PA and EBS/WP design.

\subsection{Chemical Composition of Water in the Altered Zone Before Entering the Near-Field Environment}

\section{by William E. Glassley}

Placing high-level radioactive WPs in a geologic repository is expected to result in elevated temperatures around emplacement drifts. This thermal effect will heat in situ pore water and fracture water, resulting in local chemical interaction between the host rock and the ambient water. If temperatures are high enough to evaporate or boil the pore and fracture waters, the water vapor is expected to migrate to cooler regions, where it will condense.

These processes may result in water movement that will cause water to contact emplaced materials. The predominant mechanism for water movement is expected to be fracture flow, although some component of flow may result from water migration through the rock matrix. Because the mineralogical compositions of fracture linings and of the rock matrix are significantly different, it is expected that there will be two categories of water types, each reflecting interaction of liquid water with a different mineralogy.

The purpose of this section is to summarize the work completed to date that places constraints on the composition of the water expected to enter regions where emplaced materials are present. Because most water penetrating the NFE is expected to enter via fracture flow, this section focuses on the composition of this water. Extensive previous studies (Bruton, 1996; Bruton et al., 1993; Bruton and Viani, 1992; Carroll et al., 1996; Johnson and Glassley, 1996; Knauss, 1987; Knauss et al., 1987, 1986; Knauss and Copenhaver, 1995; Knauss and Peifer, 1986; Viani and Bruton, 1991 and see Section 5.3 of this report) have evaluated the likely interactions that may result through reaction primarily with matrix material. The results of these reports are not described here because the effect of such waters on repository materials is expected to be minimal. However, it should be noted that some water from the matrix might subsequently drain into fractures, through which it might rapidly penetrate the NFE. More work is needed to assess this possibility.

Although the overall composition of the fracture water will likely be dominated by interaction between condensate and fracture-lining minerals, the water may also be affected along the flow pathway by the dissolution of residues of previous evaporation. Section 6.2.1 describes water compositions influenced solely by rock-water interaction (see Section 5.3), and Section 6.2.2 describes the effects of evaporation on water chemistry and the deposition of mineral residues. Section 6.2.3 discusses the implications of these results for predicting bounding water compositions.

The compositions of NFE-penetrating waters predicted by thermodynamic modeling are generally more dilute than those of the ambient groundwaters. They evolve in concert with the formation of secondary zeolites, clays, and hematite $\left(\mathrm{Fe}_{2} \mathrm{O}_{3}\right)$. The limited experimental 
data appear to place upper limits on the dissolved species in NFE-penetrating waters. This is based on persistent supersaturations with carbonate and silicate phases and on the fact that experimentally obtained concentrations are generally higher than the corresponding values obtained from model simulations.

An integrated approach that combines thermodynamic modeling (including kinetics modeling) with short-term experimental data is the best means for predicting the long-term behavior of the thermohydrochemical (THC) system. This imposes the need to evaluate a number of the system variables, some of which remain uncertain. Additional experimental work is needed to reduce the uncertainties associated with predicting long-term reaction rates (mostly for certain mineral dissolution and precipitation reactions). Further simulations are required to better account for the effect of these uncertainties on the calculated long-term water compositions.

\subsubsection{Fracture-Water Composition in the Absence of Evaporation Effects}

A wide range of minerals has been observed in fractures sampled from boreholes and the perimeter drift at the potential repository site (Carlos, 1987, 1989, 1994; Glassley and Boyd, 1995; Lin and Daily, 1984). Models are being developed of the evolution of water flowing through the dominant suite of fracture minerals as defined by Carlos (1987). The simulations reported here are those to date, which have concentrated on a fracture-lining assemblage of calcite $\left(\mathrm{CaCO}_{3}\right)$ and a silica $\left(\mathrm{SiO}_{2}\right)$ polymorph (opal-CT or quartz), accompanied by minor amounts of clays and oxides. This assemblage is common in samples from the Large Block Test (LBT; Glassley and Boyd, 1995) and in samples of high-porosity, cemented breccia in drill core recovered from the Single-Heater Test (SHT)and the Drift-Scale Test (DST), in the Exploratory Studies Facility (ESF).

The composition of water that may flow in fractures lined with this mineral suite can be bounded in several ways:

- Consider the results of experimental studies of water compositions in fractured-tuff flow-through systems

- Examine water obtained from thermally perturbed fractured TSw2 tuff (nomenclature of Ortiz et al. [1985]); host-rock unit at or near the repository horizon

- Use thermodynamic models (see Section 5.1) and reactive-transport models (see Section 5.2) to calculate the appropriate water compositions, assuming appropriate reaction scenarios and using various thermodynamic and kinetic data

The following subsections address these three approaches.

\subsubsection{Flow-Through Experiments}

Several experiments have been conducted in which water flows through devitrified, welded Topopah Spring tuff under controlled temperature and pressure conditions (Daily et al., 1987; Lin and Daily, 1984, 1991; Lin et al., 1995). The most thorough characterization of water composition in these studies was that reported by Lin et al. (1995). In their study, water flowed through a natural calcite-opal fracture while the temperature was cycled between $25^{\circ} \mathrm{C}$ and $150^{\circ} \mathrm{C}$. Four complete temperature cycles, each lasting between $1200 \mathrm{hr}$ and $2000 \mathrm{hr}$, were carried out, for a total experiment duration of 267 days. The core length was $6.335 \mathrm{~cm}$. The water used in the experiment was J-13 well water (Harrar et al., 1990) doped with sodium azide to prevent the growth of bacteria. Water samples were periodically collected at the outlet of the flow chamber and were analyzed for total $\mathrm{Al}, \mathrm{B}, \mathrm{Ca}, \mathrm{Cl}, \mathrm{F}, \mathrm{Fe}, \mathrm{K}, \mathrm{Na}, \mathrm{Si}$, $\mathrm{SO}_{4}$, and $\mathrm{pH}$. From these analyses, the saturation indices of a variety of possible solid phases were evaluated using EQ3/6 (see Section 5.1). The solution compositions are presented in Figure 6-1. 
Figure 6-1 Bar graphs of water compositions from various fracture studies

Most elemental concentrations exhibited a clear positive correlation with temperature. Calcium was an exception, correlating negatively with temperature. This behavior is consistent with the well-known retrograde solubility of calcite and the normal, prograde solubility behavior of most silicate minerals. However, the calculations showed that the water was consistently supersaturated with respect to calcite and nontronite (a ferric-iron-rich) smectite clay: $(\mathrm{Na}, \mathrm{K})_{0.33-2 x}(\mathrm{Ca}, \mathrm{Mg})_{x} \mathrm{Fe}_{2} \mathrm{Al}_{0.33} \mathrm{Si}_{3.67} \mathrm{O}_{10}(\mathrm{OH})_{2}$. It is likely that the supersaturation in the case of calcite involves kinetic limitations on the rate of approach to equilibrium. This might also be the explanation in the case of the nontronite. However, this calculated supersaturation might equally well derive from analytical errors such as in the determination of dissolved $\mathrm{Fe}$ and $\mathrm{Al}$, for which the concentrations were quite low.

During the initial heating cycle, dissolved Si rose to approximately $75 \mathrm{mg} / \mathrm{L}$, even during the cool-down portion of that cycle (Figure 6-2). During the third and fourth cycles, the Si concentration followed the temperature changes, approximately maintaining a compositional trend expected for cristobalite saturation; for temperatures less than approximately $70^{\circ} \mathrm{C}$, it was consistently above the equilibrium Si concentrations for both cristobalite and amorphous silica (Figure 6-3). This behavior implies that precipitation of these silica polymorphs (and of other, more stable silica polymorphs) was inhibited at lower temperatures.

Figure 6-2 Temperature $\left({ }^{\circ} \mathrm{C}\right)$ and outlet $\mathrm{Si}$ concentration $(\mathrm{mg} / \mathrm{L})$ and temperaturedependent theoretical saturation Si concentration values for amorphous silica and cristobalite as a function of time for the fracture flow-through experiment (Lin et al., 1995)

Figure 6-3 Temperature $\left({ }^{\circ} \mathrm{C}\right)$, outlet Si concentration $(\mathrm{mg} / \mathrm{L})$, and temperaturedependent theoretical saturation Si concentration values for amorphous silica and cristobalite as a function of time for the fracture flow-through experiment (Lin et al., 1995)

\subsubsection{Field Observations}

Water samples have been collected from fractures at Rainier Mesa (Harrar et al., 1990) and from Borehole 16 in the SHT (Glassley and DeLoach, 1997). The compositions of these waters are shown in Table 6-1. They are quite similar with respect to the major cations. They are generally more dilute than J-13 well water, described by Harrar et al. (1990). These waters are supersaturated with respect to calcite, quartz, and cristobalite if it is assumed that they are in equilibrium with atmospheric $\mathrm{CO}_{2}$ (partial pressure of $0.0003 \mathrm{~atm}$ ). However, the waters are undersaturated with respect to other silicates. 
Table 6-1 Fracture-water compositions from field samples, experiments, and models

\begin{tabular}{|c|c|c|c|c|c|c|c|c|}
\hline $\begin{array}{l}\text { Temp. } \\
\left({ }^{\circ} \mathrm{C}\right)\end{array}$ & $\begin{array}{l}\text { Water } \\
\text { Source }\end{array}$ & $\begin{array}{c}\log \mathbf{H}^{+} \\
\text {(Molality) }\end{array}$ & $\begin{array}{c}\mathrm{Log} \mathrm{Mg}^{+2} \\
\text { (Molality) }\end{array}$ & $\begin{array}{c}\text { Log } \mathrm{K}^{+} \\
\text {(Molality) }\end{array}$ & $\begin{array}{c}\log \mathrm{Na}^{+} \\
\text {(Molality) }\end{array}$ & $\begin{array}{l}\mathrm{Log} \mathrm{Ca}^{+2} \\
\text { (Molality) }\end{array}$ & $\begin{array}{c}\text { Log } \\
\mathrm{SiO}_{2}(\mathrm{aq}) \\
\text { (Molality) }\end{array}$ & $\begin{array}{c}\mathrm{Log} \\
\mathrm{HCO}_{3}^{-} \\
\text {(Molality) }\end{array}$ \\
\hline 25 & $\mathrm{~J}-13^{1}$ & -7.40 & -4.08 & -3.90 & -2.70 & -3.49 & -3.00 & -4.14 \\
\hline 25 & $\begin{array}{l}\text { Rainier } \\
\text { Mesa }^{2}\end{array}$ & -7.50 & -4.21 & -3.92 & -2.82 & -3.68 & -3.05 & -2.79 \\
\hline 25 & $\begin{array}{l}\text { Single } \\
\text { heater test } \\
\text { hole } \# 16^{3}\end{array}$ & ND & -4.17 & -4.19 & -3.16 & -3.49 & -3.22 & ND \\
\hline 25 & $\begin{array}{l}\text { Model, } \\
15 \mathrm{~m}, 45 \mathrm{yr}\end{array}$ & -10.1 & -3.14 & -7.19 & -4.15 & -4.92 & -3.38 & -3.21 \\
\hline 95 & $\begin{array}{l}\text { Model, } \\
1.5 \mathrm{~m}, 5 \mathrm{mo}\end{array}$ & -8.52 & -7.98 & -6.27 & -4.14 & -3.88 & -2.69 & -3.55 \\
\hline 95 & $\begin{array}{l}\text { Model, } \\
15 \mathrm{~m}, 45 \mathrm{yr}\end{array}$ & -8.53 & -3.38 & -5.26 & -4.16 & -4.55 & -2.69 & -3.05 \\
\hline 104 & $\begin{array}{l}\text { Fracture } \\
\text { flow } \\
\text { experiment }^{4}\end{array}$ & -8.35 & ND & -3.54 & ND & -3.21 & -2.37 & ND \\
\hline \multicolumn{9}{|c|}{$\begin{array}{ll}1,2 & \text { see Harrar et al., } 1990 \\
3 & \text { see Glassley and DeLoach, } 1997 \\
4 & \text { see Lin et al., } 1995\end{array}$} \\
\hline
\end{tabular}

\subsubsection{Modeling Studies}

Hundreds of simulations have been conducted over the years in the attempt to establish bounds for water chemistry that may develop within the NFE. These studies have shown that, under repository-relevant conditions, waters interacting with rocks similar to TSw2 tuff become saturated with calcite, quartz, and cristobalite in periods of less than one year. Two primary variables control the degree of saturation with respect to various other silicate phases (primarily clays and zeolites):

- The first is whether or not equilibrium with atmospheric $\mathrm{CO}_{2}$ is assumed. The $\mathrm{CO}_{2}$ fugacity in the NFE during repository thermal evolution will probably vary between the present ambient value of about $10^{-3}$ atm and a much lower value controlled by distillation of pore water and displacement of the gas phase by water vapor. Abundant $\mathrm{CO}_{2}$ is present in the host rock, as demonstrated by extraction from rock core (see Chapter 5). When this $\mathrm{CO}_{2}$ inventory in the host rock is mobilized during heat-up, there will be a transient occurrence of elevated $\mathrm{CO}_{2}$ pressure that will be succeeded by depletion relative to present conditions because of displacement by water vapor. Eventually, over thousands of years as natural circulation recharges the host rock and the associated waters, the fugacity will return to present values. Atmospheric $\mathrm{CO}_{2}$ pressure is a representative value that lies between the projected extremes.

- The second variable controlling saturation with respect to silicates is the level of supersaturation that is allowed to occur before corresponding silica polymorph is permitted to precipitate. 
The simulations described here were conducted using the reactive-transport code GIMRT (Steefel and Yabusaki, 1995). It was assumed that quartz precipitation would not occur until the corresponding saturation index $(S I=\log Q / K$; see Section 5.1) reached a value of 0.5 . This results in reaching supersaturation with respect to cristobalite, which then precipitates before quartz can precipitate. All other phases were assumed to precipitate when (or if) the corresponding saturation indices reached 1.0. The flow-field length and flow duration were each varied ( $1.5 \mathrm{~m}$ to $15 \mathrm{~m}$ and $5 \mathrm{mo}$ to $45 \mathrm{yr}$, respectively). To consider the possible consequences of developing reflux regimes in which recirculating water interacts with fracture surfaces for long periods, the flux was assumed to be very high $(2.2 \mathrm{~m} / \mathrm{yr})$. It was assumed that the initial fracture mineralogy was calcite $(90 \%)$, opal $(5 \%)$, hematite $(0.5 \%)$, and smectite $(0.1 \%)$.

Representative results of the water chemistry from the simulations in which equilibrium with atmospheric $\mathrm{CO}_{2}$ was assumed are included in Table 6.1. The water composition obtained by reaction at $25^{\circ} \mathrm{C}$ can be compared with the compositions of water collected in the field. The water composition obtained by reaction at $95^{\circ} \mathrm{C}$ can be compared with water compositions obtained from experimental studies of reaction during flow through fractures. For both temperatures, the model solutions have higher $\mathrm{pH}$ values. They are also significantly more dilute. This reflects the effect of precipitation of clays, silica polymorphs, and zeolites on the solution chemistry and also reflects the relatively low solubility of calcite at near-neutral to high $\mathrm{pH}$ values at the selected flow rates.

The outlet solution composition for the $95^{\circ} \mathrm{C}, 15-\mathrm{m}$ outlet model is shown in Figure $6-4$ as a function of time. Figure 6-5 shows the evolution of porosity and mineralogy at the inlet of the fracture as a function of time. Figure 6-6 shows the corresponding evolution at the outlet.

Clearly, the solution composition reaches a steady-state condition within a few years after flow and reaction are initiated. However, this steady-state condition is not an equilibrium state because of the continued dissolution of at least some of the original fracture-lining minerals. The opal phase (modeled as amorphous silica) is removed within a few years along the entire flow path and is replaced by cristobalite. At the inlet, cristobalite also begins to dissolve once all of the opal has been converted to cristobalite. Dissolution of the calcite and smectite proceeds over the entire duration of even the longer flow period. On the other hand, hematite, which is one of the original fracture-lining minerals, precipitates throughout the modeling period. The dissolved species contribute to the deposition of potassium feldspar $\left(\mathrm{KAlSi}_{3} \mathrm{O}_{3}\right)$ and Ca-clinoptilolite $\left(\mathrm{Ca}_{1.7335} \mathrm{Al}_{3.45} \mathrm{Fe}_{0.017} \mathrm{Si}_{14.533} \mathrm{O}_{36}: 10.922 \mathrm{H}_{2} \mathrm{O}\right)$ along the flow path for the duration of the simulations. Kaolinite $\left[\mathrm{Al}_{2} \mathrm{Si}_{2} \mathrm{O}_{5}(\mathrm{OH})_{4}\right]$ is also initially deposited throughout the flow pathway; as flow progresses, however, this phase slowly dissolves in the outlet region. These mineralogical changes contribute to porosity changes along the flow path. At the inlet, porosity changes from $3.4 \%$ to $4.6 \%$; at the outlet, it increases to $4.04 \%$.

Figure 6-4 The log molality of selected dissolved species in the outlet solution of the $95^{\circ} \mathrm{C}, 15-\mathrm{m}$ fracture flow model, as a function of time

Figure 6-5 Porosity and mineralogy (all in volume percent) at the inlet position of the $95^{\circ} \mathrm{C}, 15-\mathrm{m}$ fracture-flow model, as a function of time

Figure 6-6 Porosity and mineralogy (all in volume percent) at the outlet position of the $95^{\circ} \mathrm{C}, 15-\mathrm{m}$ fracture flow model, as a function of time

The results presented here place bounds on the most likely compositions of the waters that will enter the NFE (ignoring the possible dissolution of evaporation residues). These bounds are defined by two different approaches for projecting the evolution of water 
composition. The first is the modeling approach (see Section 5.1), in which the water composition is calculated from thermodynamic models, including kinetic descriptions for the relatively slow reactions, and coupled equilibrium descriptions for the relatively faster ones. The calculations presented here are reactive-transport models (see Section 5.2), which include the effect of fluid flow. (For examples of batch system models of tuff-water interaction, see Section 5.3.) The relatively slow reactions are typically those for mineral dissolution and precipitation, though not all reactions of this kind are relatively slow. The rate-law expressions typically have forms suggested by transition-state theory. The rate laws are generally inferred from and calibrated by experiments in which a single mineral dissolves in or precipitates from an aqueous solution of simple composition. This approach has the advantage that the time frame is not limited. The potential disadvantage is that the model may be incomplete in some way (e.g., some important reactions are not considered) or that parameter values are poorly constrained or incorrect.

The other approach to defining likely bounds on the water chemistry is through experimental studies. If the studies are properly designed, all the relevant factors determining the overall process should be present. However, the results are only directly applicable for the time frames of the experiments. Unless a complete thermodynamic equilibrium is achieved (a condition that can be demonstrated by showing reversibility), the studied systems would continue to evolve. Potentially, depending on the time scales intrinsic to the actual processes, the studied systems could change considerably. Rock-water interaction studies typically run no longer than a few months. Current knowledge of the kinetics of some relevant reactions suggests that even multiyear experiments would fail to show near-complete evolution.

The compositions of NFE-penetrating waters predicted by the modeling approach are generally more dilute than those of ambient groundwaters. These compositions evolve in concert with secondary mineral development characterized by the formation of zeolites, clays, and hematite. The limited experimental data appear to place upper limits on the concentrations of dissolved species in NFE-penetrating waters. This conclusion is based on the persistent supersaturation of the waters with respect to carbonate and silicate phases (as shown by modeling calculations) and on the fact that the concentrations of the measured species are consistently higher for the flow-through experiments discussed here, relative to the results of model simulations.

The approaches are not mutually exclusive. The goal of modeling is to bridge the gap between what relatively short-term experimental studies can tell us and what will happen over experimentally inaccessible very long time scales. Thus, the models must be capable of explaining the experimental results and, at the same time, must contain the necessary thermodynamic and kinetic understanding of the processes involved to extrapolate the reaction processes over the very long times required for PA.

Such an integrated approach is the best means for considering the long-term behavior of the THC system, but it imposes the need to evaluate the many system variables, some of which remain uncertain. The principal uncertainties in this approach are in the kinetics of dissolution and precipitation; the kinetics used in these simulations are based on laboratory rates measured in simple systems. Recent work has documented that laboratory-measured rates may not always accurately reflect dissolution or precipitation kinetics in complex, natural systems (Carroll et al., 1996, 1995). Such cases might be the result of several factors, but the use of oversimplified rate models is one likely cause. Further experimental work is needed to resolve these differences and reduce the current uncertainties. Such work might account for additional factors that determine the reaction rates (e.g., resulting in rate laws that are not as simple in form as the ones presently employed). Regardless, further 
simulations are necessary to estimate the effect of uncertainty in rate law models on calculated water compositions over a wide range of reaction scenarios. One fact to consider is that the initial mineralogy used in the simulations discussed here is simple and did not take into account the effects of minor phases that have been reported in many fractures.

\subsubsection{Water Composition Affected by Evaporative Concentration}

Evaporation of water may deposit secondary precipitates along fracture surfaces or in rock pores during the heating of the repository. The specific precipitates that may develop will reflect the initial water composition. J-13 well water was used in this study because it has the approximate composition of the most dilute water that is close to equilibrium with highsilica rhyolite similar to that which makes up the repository horizon at Yucca Mountain. The effects of boiling per se are not considered because they have been addressed elsewhere (Arthur and Murphy, 1989; Criscenti and Arthur, 1989). Consideration is here given only to evaporation of water; no rock-water interaction is assumed. This may give a likely picture of the most concentrated solutions that will develop. Later work will consider the effects of the interaction between tuff and water concentrated by evaporation.

\subsubsection{Approach}

With the use of the EQ3 / 6 code package (Daveler and Wolery, 1992; Wolery, 1992a, 1992b; Wolery and Daveler, 1992), evaporation of water can be simulated by removing water at a specified rate in an EQ6 simulation (Wolery and Daveler, 1992). The evaporation was done at constant temperatures of $31^{\circ}$ and $90^{\circ} \mathrm{C}$, as well as in scenarios where the amount of water evaporated was correlated with increasing temperature (a linear water loss over the range $31^{\circ}$ to $95^{\circ} \mathrm{C}$ plus other nonlinear trends over the same range). Other factors being equal, the simulations at the two fixed temperatures, as well as the nonisothermal ones, gave virtually the same results (within $1 \%$ ).

The maximum amount of water removed in any of these simulations was in the range of $95 \%$ to $96 \%$. Thus a conservative solute would be concentrated by a factor of 20 to 25 . The ionic strength of the resulting solutions is therefore kept below roughly 0.2 molal, within the range of applicability of the B-dot activity coefficient equation (see Section 5.2) used in these calculations. For more concentrated solutions (e.g., those with ionic strength greater than about 1 molal), the B-dot equation is no longer applicable, and Pitzer's equations or something similar would have to be used instead (for a discussion of activity coefficient models, see Chapter 3 of Wolery (1992b).

Because only $95 \%$ to $96 \%$ of the water was removed in these calculations, the simulations are not sufficient to identify all of the residue minerals that would be precipitated by a nearcomplete to complete dryout. Some of the mineral phases that can only be precipitated by higher degrees of water loss and that would probably form in the real system include halite $(\mathrm{NaCl}), \mathrm{NaNO}_{3}$, and gypsum $\left(\mathrm{CaSO}_{4}: 2 \mathrm{H}_{2} \mathrm{O}\right)$. Further work is needed to identify the complete assemblage of residue minerals and to assess the effect of these on waters that will subsequently reenter the NFE and AZ (after the repository system has begun to cool).

These simulations were conducted both with and without controls on the composition of the co-existing gas phase (atmospheric values: $\mathrm{O}_{2}$ partial pressure $=0.2$ bar; $\mathrm{CO}_{2}$ partial pressure $=0.0004$ bar).

Some simulations conducted allowed the precipitation of quartz $\left(\mathrm{SiO}_{2}\right)$, tridymite (less stable $\left.\mathrm{SiO}_{2}\right)$, and talc $\left[\mathrm{Mg}_{3} \mathrm{Si}_{4} \mathrm{O}_{10}(\mathrm{OH})_{2}\right]$. In others, the precipitation of these phases was suppressed to simulate scenarios in which the metastable precipitation of other, more soluble phases is allowed to occur instead. Experimental studies of tuff-water interaction (e.g., Delany, 1985, and Knauss et al., 1986) indicate that one of the less-stable silica polymorphs, 
such as cristobalite, present in the rock usually controls aqueous silica. At low temperatures similar to those considered here, the minerals that form most readily in real systems (i.e., on relatively short time scales) are often metastable rather than the thermodynamically stable ones (see summary in Bruton et al., 1993). These contrasting approaches allow consideration of the relative importance of the precipitation of metastable phases on the aqueous chemistry. (Note: Talc bears certain chemical and mineralogical similarities to saponite [Mg-rich smectite clay]; saponite generally appears in simulations such as these when talc is suppressed.)

\subsubsection{Results and Discussion}

For some aqueous species and solids, the effect of a controlling gas-phase composition can be dramatic. For the calculations presented here (Figure 6-7 through Figure 6-17), squares indicate cases where gas-phase controls were implemented; circles indicate values computed with no gas-phase controls. Solid symbols indicate simulations in which quartz, tridymite, and talc precipitation was not suppressed, and open symbols indicate cases where it was suppressed.

Evaporation produced $\mathrm{pH}$ trends (Figure 6-7) that were markedly different, depending on the presence or absence of controls imposed by coexisting gases. With such controls, the $\mathrm{pH}$ drifted upward to relatively high values during evaporation due to the loss of $\mathrm{CO}_{2}$ resulting from the imposed, relatively low $\mathrm{CO}_{2}$ partial pressure. Without such controls, the $\mathrm{pH}$ drifted slightly downward, remaining close to neutral. Here, $\mathrm{CO}_{2}$ is not permitted to escape to a gas phase (which would raise the $\mathrm{pH}$ ). In the absence of gas-phase controls, the oxygen fugacity (or partial pressure) remains at about the atmospheric value because there is nothing in the evaporation process to induce a change. The Eh (Figure 6-8) shows a break into separate trends depending on whether or not gas-phase controls are imposed. This essentially correlates with the separate trends in the $\mathrm{pH}$ (Eh is related to oxygen fugacity and $\mathrm{pH}$; see Section 2.3.6.3 of Wolery, 1992b). Both systems remain oxidizing (equally so from the standpoint of $\mathrm{O}_{2}$ partial pressures).

Figure 6-7 The $\mathrm{pH}$ as a function of moles of water evaporated

\section{Figure 6-8 Eh, in volts, as a function of moles of water evaporated}

The Na (Figure 6-9) and $\mathrm{Cl}$ (Figure 6-10) concentrations increased linearly as the remaining amount of $\mathrm{H}_{2} \mathrm{O}$ decreased (reaching about 23 times the starting concentrations). This was also true for $\mathrm{SO}_{4}{ }^{2-}$ and $\mathrm{NO}_{3}^{-}$(neither shown here). Because of the insufficient removal of water in the present calculations, no solids were precipitated that incorporated these components. F also increased linearly (Figure 6-11), but with a smaller slope. It was incorporated into fluorapatite $\left[\mathrm{Ca}_{5}\left(\mathrm{PO}_{4}\right)_{3} \mathrm{~F}\right]$ and fluorite $\left(\mathrm{CaF}_{2}\right)$ during evaporation. Fluorapatite precipitated during the very early stages of evaporation and remained constant in abundance. Fluorite only occurred in small concentrations toward the end of the evaporation. Hence, the relative behavior of $\mathrm{F}$ in solution mimicked that of $\mathrm{Na}$ and $\mathrm{Cl}$, but only achieved about 15 times the starting concentration, vs. about 23 times in a case of $\mathrm{Na}$ and $\mathrm{Cl}$.

Figure 6-9 $\mathrm{Na}$, in $\mathrm{mg} / \mathrm{kg}$, as a function of moles of water evaporated

Figure 6-10 $\mathrm{Cl}$, in $\mathrm{mg} / \mathrm{kg}$, as a function of moles of water evaporated

Figure 6-11 F, in $\mathrm{mg} / \mathrm{kg}$, as a function of moles of water evaporated 
In contrast to the $\mathrm{Na}, \mathrm{Cl}$, and $\mathrm{F}$ concentrations, the concentrations of Si (Figure 6-12), $\mathrm{Ca}$ (Figure 6-13), and $\mathrm{P}$ (present as $\mathrm{PO}_{4}^{3-}$; not shown here) were affected, as evaporation proceeded, by precipitation of silicates, carbonates, and phosphates. The dissolved Si variation was controlled by the solubility limit of either quartz and chalcedony, which was the most abundant secondary precipitate (quartz, except where quartz was suppressed). Changes in the Ca concentration mainly correlated with the precipitation of calcite (Figure 6-14), the next-most-abundant precipitate. Dolomite precipitation (Figure 6-15) also influenced Ca concentration, but to a lesser degree. $\mathrm{P}\left(\mathrm{PO}_{4}{ }^{3-}\right)$ variation reflected the effects of precipitation of fluorapatite.

Figure 6-12 Si, in $\mathrm{mg} / \mathrm{kg}$, as a function of moles of water evaporated

\section{Figure 6-13 Ca, in $\mathrm{mg} / \mathrm{kg}$, as a function of moles of water evaporated}

Figure 6-14 Log of the number of moles of calcite precipitated, as a function of moles of water evaporated

Figure 6-15 Log of the number of moles of dolomite precipitated, as a function of moles of water evaporated

Clearly, in these simulations, the specific silica polymorph allowed to precipitate had only a second-order effect on the solution composition, apart from on the Si concentration itself. The Ca concentration was impacted by the Si concentration via the precipitation of Ca-bearing aluminosilicates. These include saponite clay (Figure 6-16) and the zeolite stilbite (Figure 6-17). Although the amounts of these phases formed are relatively small, they do include ion-exchange sites that could be significant in reactions with returning waters after cool-down, and they might also be significant for subsequent radionuclide migration.

Figure 6-16 Log of the number of moles of saponite precipitated, as a function of moles of water evaporated

Figure 6-17 Log of the number of moles of stilbite precipitated, as a function of moles of water evaporated

In all these simulations, regardless of the specific conditions imposed on the system and regardless of the degree of evaporation, fluorapatite, nontronite (ferric iron-rich clay), and pyrolusite $\left(\mathrm{MnO}_{2}\right)$ were continually present in small abundances. This reflects the low solubilities of these solids.

\subsubsection{Conclusions}

Water that will interact with repository materials will be a mixture of ambient waters and condensate, which have interacted with rock and fracture mineralogy, and of the residues of evaporative processes. The largest volume of water is expected to travel via fracture flow. The range of compositions that may be expected will fall within the following bounding conditions:

- During heat-up-The water will have compositions dominated by evaporative processes, in which case the composition will depend on the degree of evaporation. Most of this water will have originally been ambient groundwater. Some may be 
refluxed water-condensate from water evaporated or boiled off in other regions in or near the repository. The results presented in Section 6.2.2 bound the composition of most of this water. A very small portion, however, will be more concentrated.

- During cool-down-The water will be dominated by condensate, in which case the actual composition will depend on the extent of interaction of the water with minerals along the flow pathway. This, in turn, is a function of flow-path length and fluid velocity. The results presented in Section 6.2.2 summarize the range of expected compositions for these waters. It is expected that this water will be a significant, if not dominant, component of water interacting with repository materials.

- At the edges of the thermal pulse and in the late stages of cool-down-The water will be dominated by fracture flow of ambient fluids percolating through the repository block. These waters would be expected to fall within the range of waters previously reported and are generally considered to be similar to those of J-13 well water (see Harrar et al., 1990).

One can evaluate how reasonable these bounds are only by comparing the experimental and modeling results with each other and with observed water compositions in appropriate natural systems. Although there is a paucity of data that can be used for this purpose, the available data consistently suggest that the fracture waters will be dilute bicarbonate waters. However, significant discrepancies exist for some components, which suggests that the modeling strategies need to be refined. It also must be kept in mind that the actual water interacting with repository materials at various locations and times may well be a mixture of the three types of water noted previously.

To more accurately reproduce water compositions from field and laboratory studies, the following modifications to the modeling approach are indicated:

- Develop better estimates for the relation between precipitation and extent of supersaturation

- Establish the probable ranges of dissolution rate constants

- Characterize the abundance of minor phases along fracture surfaces to account for sources and sinks of minor species in solution (e.g., $\mathrm{Cl}, \mathrm{NO}_{3}, \mathrm{Mg}, \mathrm{K}$ )

- Improve estimates for effective surface areas for reactant phases

- Improve estimates for the gas-phase composition coexisting with the fluids

Once these modifications are achieved, the resulting models will provide a clearer description of the mechanisms responsible for the water compositions observed for field samples (e.g., the Rainier Mesa fracture water and the water from the SHT; Glassley and DeLoach, 1997; Harrar et al., 1990). This will allow tighter bounds to be placed on water compositions entering the NFE.

\subsection{Chemistry of Water in the Near-Field Environment}

\section{by Annemarie Meike}

This section applies a chemical database and mineral assemblages developed for watercement interaction studies (see Chapter 7) to calculate water composition in contact with cementitious materials, for various conditions. The ranges of water compositions presented here can be used to assess the performance of the WP and other engineered barriers, if cementitious materials are assumed to have a dominant influence on the near-field geochemical environment. This chapter presents a modeling approach and example calculations for several values of the system temperature. It is anticipated that additional calculations will be performed and sensitivity analyses conducted when this approach is used for total system performance assessment (TSPA) or repository design. 
Concrete is a major source of calcium and silicate chemical species. Depending on the formulation, sulfate and chloride may also be contributed. Organic components, used to condition the wet concrete paste before it is cured, are usually present in very small quantities. At low temperatures, such organic components may be a minor source of nutrients for microbes. During the repository thermal period, this organic component may volatilize and redistribute or even diffuse away from the emplacement drift.

In the reference design, concrete will be used for the drift liner and for the invert under the WPs, which also supports the transportation system. Iron corrosion could have a temporary effect on the redox conditions in the near-field geochemical environment, as investigated in a modeling study by Meike and Glassley (1997). In that study, hematite was assumed to be the final product of iron oxidation. Significant chemical impact was calculated only for a closed environment. In open system calculations, the iron was readily oxidized, after which there was little impact on water composition.

Because of the large volume of concrete in the reference design and because of the tendency to produce alkaline $\mathrm{pH}$ (depending on formulation and age), concrete could be the dominant influence on water composition in the near field. It is a major source of uncertainty in the water composition because of sensitivity to the initial formulation and to environmental conditions. The capability to predict the water composition in contact with concrete has progressed through the creation of a more precise conceptual model and expansion of the available thermodynamic database to include cement phases found in concrete at different stages of maturation.

The chemical environment within the emplacement drifts is expected to be extremely heterogeneous and to differ significantly from that in the surrounding rock, as discussed in Chapter 7. Prediction of the composition of water in contact with introduced materials is affected by uncertainties that also affect prediction of the water composition in contact with rock (see Section 6.2). In particular, chemical evolution depends on gas-phase composition, which will vary during repository thermal evolution and which is difficult to simulate. Use of the water composition ranges developed here should be undertaken with appreciation for the conceptual and parametric uncertainties associated with the available modeling approaches.

These calculations do not include the effects of microbially mediated chemical reactions on bulk composition of materials in the NFE, for which reaction models have not been developed. These results do provide a broad set of bounding conditions for the environment in which microbial activity could occur. There are known examples of microbial activity neutralizing or even acidifying the $\mathrm{pH}$ of water in contact with concrete. This report presents only abiotic simulations, while recognizing that microbial activity will extend an envelope of uncertainty toward more acidic conditions. It is unlikely that the effects of microbial activity will increase the $\mathrm{pH}$, so in this sense abiotic simulations provide an upper bound on $\mathrm{pH}$.

\subsubsection{Database}

Chapter 7 describes the process by which thermodynamic data available from the literature have been incorporated into a database that can be used by the geochemical modeling code EQ3/6. Three databases were constructed:

- The cem.R27 database contains sufficient data to simulate cement-water interaction at $25^{\circ} \mathrm{C}$ for a range of possible cement mineral phases.

- The cem.R28 database contains additional phases selected from the COM database (for EQ3 / 6; see Chapter 5) that belong to the cement-mineral system. This database has been used for calculations at elevated temperature.

- The cemcomp.28 database integrates the cem.R27 database with the COM database. 
The cem.R27 and cem.R28 databases are used to test the internal robustness of the more limited $\mathrm{Ca}-\mathrm{Si}-\mathrm{Al}-\mathrm{SO}_{3}-\mathrm{H}_{2} \mathrm{O}$ chemical system that represents the most significant phases found in Portland cement. The database used in the calculations below is cemcomp.28, which incorporates the cem.R27 database with the COM database to facilitate modeling a larger chemical system. Because it is new, the cemcomp. 28 database has not undergone the rigorous testing that has been applied to the other, standard EQ3/ 6 databases (see Section 5.1). It is intended that this database will be tested against experimental and analog data to understand its usefulness. The simulations presented in this section are examples of the application of the cemcomp.R28 database.

\subsubsection{EQ3 Calculations: Speciation of Incoming Water and Additional Constraints}

The influent water chosen for these simulations is J-13 well water (Harrar et al., 1990) that has been used historically as the point of departure for simulations of rock-water interaction at Yucca Mountain. Whereas the actual $\mathrm{pH}$ of J-13 water could differ from the value originally reported, for the purpose of these simulations, the interest is mainly in the $\mathrm{pH}$ conditions that result from steady interaction of water with concrete. J-13 water is relatively dilute and nearly neutral, compared with water that has interacted with cement, thus other choices in the formulation of this problem are more critical.

For these simulations, the influent water is preconditioned at the temperature of the EQ6 simulation to maintain a consistent methodology throughout the set of simulations that spans a temperature range from $25^{\circ}$ to $150^{\circ} \mathrm{C}$. All elements that will appear in the simulation must be represented in the influent J-13 chemistry in at least trace quantities. For this study, addition of trace species was not necessary. For reaction with other phases, such as the waste form that contains different elements, the J-13 water would be preconditioned to contain trace quantities of these elements. The J-13 input file was the same for every run, with the exception of the selected temperature, in this set of simulations.

\subsubsection{Suppressed Phases}

The EQ3 part of the simulation allows the modeler to suppress precipitation of minerals that would not be expected to form under the temperature conditions of the simulation. For example, it is common to suppress the precipitation of quartz at low temperatures. The simulations performed for this report were design to evaluate the usefulness of the cemcomp.R28 database. As a preliminary assessment, mineral phases that are not among typical cement minerals were successively suppressed until some of the newly introduced cement mineral phases began to precipitate. The intention here was to test the relations between newly added phases and the previous phases in the database, while varying the quantity of $\mathrm{CO}_{2}$ (gas) available for reaction. This test is not simply a function of the thermodynamic input data. Using this approach, the following phases were suppressed: maximum microcline, low-albite, k-feldspar, ordered dolomite, grossular garnet, prehnite, muscovite, paragonite, dolomite, talc, epidote, ordered epidote, high albite, andradite, tremolite, diopside, and wollastonite. Quartz was not suppressed because it is a reactant phase in the EQ6 run; instead, the precipitation rate was set to a very low value.

\subsubsection{EQ6: Interaction of Water with Solid Phases}

\subsubsection{Solid Inputs: Evolving Grout Plus Tuff}

The repository temperature and chemical conditions will be similar to those that have been characterized in geology as low-grade metamorphism. During low-grade metamorphism, metastable phases evolve by processes such as phase transformation 
(a solid-state process in which one mineral transforms into another: e.g., graphite into diamond), dissolution and precipitation, and the addition and subtraction of water from crystal structures (contributing to or subtracting from the overall liquid and vapor water budget). Those solid phases that are metastable with respect to each other evolve into a more stable assemblage, as shown in Figure 6-18. With this concept in mind, five mineral assemblages have been developed to represent cement in different stages of metamorphism under different conditions (Table 6-2). A discussion of the basis for these assemblages, including experimental observations and information from the open literature, is provided in Chapter 7.

Figure 6-18 Schematic diagram of conditions under which mineral assemblages described in Table 6-2 might develop from grout of an original Type $\mathrm{V}$ composition

Table 6-2 Solid reactants used in EQ6 simulations at $25^{\circ} \mathrm{C}$; Identical simulations have been conducted at $60^{\circ}$ and $150^{\circ} \mathrm{C}$.

\begin{tabular}{|c|c|c|c|c|c|c|}
\hline Run Name & & 25_Young & $\begin{array}{c}\text { 25_Young } \\
\text { AFm }\end{array}$ & $\begin{array}{c}\text { 25_Therma } \\
\mathrm{O}_{2}\end{array}$ & $\begin{array}{l}\text { 25_Therma } \\
\mathrm{O}_{2} / \mathrm{CO}_{2} \\
\end{array}$ & $\begin{array}{c}\text { 25_Therma } \\
\mathrm{CO}_{2}\end{array}$ \\
\hline \multirow[t]{2}{*}{ Temperature } & & $25^{\circ} \mathrm{C}$ & $25^{\circ} \mathrm{C}$ & $25^{\circ} \mathrm{C}$ & $25^{\circ} \mathrm{C}$ & $25^{\circ} \mathrm{C}$ \\
\hline & Formula & Moles/l.m & Moles/l.m & Moles/I.m & Moles/l.m & Moles/l.m \\
\hline \multicolumn{7}{|l|}{ Grout } \\
\hline C-S-H1.7gel & $1.7 \mathrm{Ca}-\mathrm{Si}-2 \mathrm{H}_{2} \mathrm{O}$ & $5.97 \times 10^{3}$ & $6.02 \times 10^{3}$ & & & \\
\hline Tobermorite & $\begin{array}{c}{\left[\mathrm{Ca}_{5}\left(\mathrm{Si}_{2} \mathrm{H}_{2} \mathrm{O}\right] \mathrm{Ca}-\right.} \\
4 \mathrm{H}_{2} \mathrm{O}\end{array}$ & & & $1.99 \times 10^{3}$ & $1.97 \times 10^{3}$ & \\
\hline Ettringite & $\begin{array}{c}{\left[\mathrm{Ca}_{3} \mathrm{Al}(\mathrm{OH})_{6} \mathrm{O}_{16}\right.} \\
\left.12 \mathrm{H}_{2} \mathrm{O}\right]_{3}\left(\mathrm{SO}_{4}\right)_{3} \cdot 2 \mathrm{H}_{2} \mathrm{O}\end{array}$ & $1.02 \times 10^{2}$ & & $1.02 \times 10^{2}$ & $1.02 \times 10^{2}$ & $1.02 \times 10^{2}$ \\
\hline Monosulfate & $\begin{array}{c}\mathrm{Ca}_{3} \mathrm{Al}(\mathrm{OH})_{6} \mathrm{Ca}\left(\mathrm{SO}_{4}\right) \\
-12 \mathrm{H}_{2} \mathrm{O}\end{array}$ & & $3.06 \times 10^{2}$ & & & \\
\hline $\begin{array}{l}\text { Gehlenite } \\
\text { Hydrate }\end{array}$ & $\mathrm{Ca}_{2} \mathrm{Al}_{2} \mathrm{SiO}_{7}: 8 \mathrm{H}_{2} \mathrm{O}$ & $4.09 \times 10^{2}$ & $3.58 \times 10^{2}$ & & & \\
\hline Hydrogarnet & $\mathrm{Ca}_{3} \mathrm{Al}_{2} \mathrm{O}_{6}: 6 \mathrm{H}_{2} \mathrm{O}$ & & & $4.09 \times 10^{2}$ & & \\
\hline Hematite & $\mathrm{Fe}_{2} \mathrm{O}_{3}$ & $2.69 \times 10^{2}$ & $2.69 \times 10^{2}$ & $2.69 \times 10^{2}$ & & \\
\hline Portlandite & $\mathrm{Ca}(\mathrm{OH})_{2}$ & $3.99 \times 10^{3}$ & $3.40 \times 10^{3}$ & $3.81 \times 10^{3}$ & $1.68 \times 10^{3}$ & \\
\hline Brucite & $\mathrm{Mg}(\mathrm{OH})_{2}$ & $7.23 \times 10^{2}$ & $7.23 \times 10^{2}$ & $7.23 \times 10^{2}$ & & \\
\hline Calcite & $\mathrm{CaCO}_{3}$ & & & & $1.68 \times 10^{3}$ & $1.42 \times 10^{4}$ \\
\hline $\begin{array}{l}\text { Hemicarbo- } \\
\text { aluminate }\end{array}$ & $\mathrm{Ca}_{3.5} \mathrm{Al}_{2} \mathrm{C}_{.5}\left(\mathrm{H}_{2} \mathrm{O}\right)\left(\mathrm{O}_{18}\right)$ & & & & $5.11 \times 10^{2}$ & \\
\hline Run Name & & 25_Young & $\begin{array}{c}\text { 25_Young } \\
\text { AFm }\end{array}$ & $\begin{array}{c}\text { 25_Therma } \\
\mathrm{O}_{2} \\
\end{array}$ & $\begin{array}{l}\text { 25_Therma } \\
\mathrm{O}_{2} / \mathrm{CO}_{2} \\
\end{array}$ & $\begin{array}{c}\text { 25_Therma } \\
\mathrm{CO}_{2}\end{array}$ \\
\hline \multirow[t]{2}{*}{ Temperature } & & $25^{\circ} \mathrm{C}$ & $25^{\circ} \mathrm{C}$ & $25^{\circ} \mathrm{C}$ & $25^{\circ} \mathrm{C}$ & $25^{\circ} \mathrm{C}$ \\
\hline & Formula & Moles/l.m & Moles/l.m & Moles/l.m & Moles/l.m & Moles/l.m \\
\hline $\begin{array}{l}\text { Tri carbo } \\
\text { aluminate }\end{array}$ & $\mathrm{Ca}_{6} \mathrm{Al}_{2} \mathrm{C}_{3} \mathrm{H}_{60} \mathrm{O}_{45}$ & & & & & $5.11 \times 10^{2}$ \\
\hline
\end{tabular}




\section{Near-Field Geochemical Environment}

\begin{tabular}{|c|c|c|c|c|c|c|}
\hline Grout & & & & & & \\
\hline $\begin{array}{l}\text { Hydromag- } \\
\text { nesite }\end{array}$ & $\begin{array}{c}\mathrm{Mg}_{5}\left(\mathrm{CO}_{3}\right) 4(\mathrm{OH})_{2}: \\
4 \mathrm{H}_{2} \mathrm{O}\end{array}$ & & & & $1.45 \times 10^{2}$ & \\
\hline Artinite & $\mathrm{Mg}_{2} \mathrm{CO}_{3}: 3 \mathrm{H}_{2} \mathrm{O}$ & & & & & $3.61 \times 10^{2}$ \\
\hline Hematite & $\mathrm{Fe}_{2} \mathrm{O}_{3}$ & & & & $1.34 \times 10^{2}$ & \\
\hline Siderite & $\mathrm{FeCO}_{3}$ & & & & $2.69 \times 10^{2}$ & $5.38 \times 10^{2}$ \\
\hline Chalcedony & $\begin{array}{c}\mathrm{SiO}_{2} \text { or micro- } \\
\text { xtalline qtz }\end{array}$ & & & & & $9.86 \times 10^{3}$ \\
\hline Silica Fume & & $1 / 3$ reacted & $1 / 3$ reacted & $\begin{array}{l}100 \% \\
\text { reacted }\end{array}$ & $100 \%$ reacted & $100 \%$ reacted \\
\hline Silica fume & $\mathrm{SiO}_{2}$ & $1.79 \times 10^{3}$ & $1.79 \times 10^{3}$ & 0 & 0 & 0 \\
\hline \multicolumn{7}{|l|}{$\begin{array}{l}\text { Aggregate, } \\
\text { Fine } \\
\end{array}$} \\
\hline Annite & & $1.70 \times 10^{1}$ & $1.70 \times 10^{1}$ & $1.70 \times 10^{1}$ & $1.70 \times 10^{1}$ & $1.70 \times 10^{1}$ \\
\hline Phlogopite & & 7.87 & 7.87 & 7.87 & 7.87 & 7.87 \\
\hline $\begin{array}{l}\text { Sanidine- } \\
\text { high }\end{array}$ & & $4.12 \times 10^{3}$ & $4.12 \times 10^{3}$ & $4.12 \times 10^{3}$ & $4.12 \times 10^{3}$ & $4.12 \times 10^{3}$ \\
\hline Albite & & $7.74 \times 10^{2}$ & $7.74 \times 10^{2}$ & $7.74 \times 10^{2}$ & $7.74 \times 10^{2}$ & $7.74 \times 10^{2}$ \\
\hline Anorthite & & $3.34 \times 10^{2}$ & $3.34 \times 10^{2}$ & $3.34 \times 10^{2}$ & $3.34 \times 10^{2}$ & $3.34 \times 10^{2}$ \\
\hline Pyrophyllite & & $1.05 \times 10^{2}$ & $1.05 \times 10^{2}$ & $1.05 \times 10^{2}$ & $1.05 \times 10^{2}$ & $1.05 \times 10^{2}$ \\
\hline Quartz & & $7.29 \times 10^{3}$ & $7.29 \times 10^{3}$ & $7.29 \times 10^{3}$ & $7.29 \times 10^{3}$ & $7.29 \times 10^{3}$ \\
\hline Cristobalite & & $1.62 \times 10^{4}$ & $1.62 \times 10^{4}$ & $1.62 \times 10^{4}$ & $1.62 \times 10^{4}$ & $1.62 \times 10^{4}$ \\
\hline \multicolumn{7}{|l|}{$\begin{array}{l}\text { Aggregate, } \\
\text { Coarse }\end{array}$} \\
\hline Annite & & $2.22 \times 10^{1}$ & $2.22 \times 10^{1}$ & $2.22 \times 10^{1}$ & $2.22 \times 10^{1}$ & $2.22 \times 10^{1}$ \\
\hline Phlogopite & & $1.03 \times 10^{1}$ & $1.03 \times 10^{1}$ & $1.03 \times 10^{1}$ & $1.03 \times 10^{1}$ & $1.03 \times 10^{1}$ \\
\hline $\begin{array}{l}\text { Sanidine- } \\
\text { high }\end{array}$ & & $5.38 \times 10^{3}$ & $5.38 \times 10^{3}$ & $5.38 \times 10^{3}$ & $5.38 \times 10^{3}$ & $5.38 \times 10^{3}$ \\
\hline Albite & & $1.01 \times 10^{3}$ & $1.01 \times 10^{3}$ & $1.01 \times 10^{3}$ & $1.01 \times 10^{3}$ & $1.01 \times 10^{3}$ \\
\hline Anorthite & & $4.36 \times 10^{2}$ & $4.36 \times 10^{2}$ & $4.36 \times 10^{2}$ & $4.36 \times 10^{2}$ & $4.36 \times 10^{2}$ \\
\hline Pyrophyllite & & $1.37 \times 10^{2}$ & $1.37 \times 10^{2}$ & $1.37 \times 10^{2}$ & $1.37 \times 10^{2}$ & $1.37 \times 10^{2}$ \\
\hline Quartz & & $9.53 \times 10^{3}$ & $9.53 \times 10^{3}$ & $9.53 \times 10^{3}$ & $9.53 \times 10^{3}$ & $9.53 \times 10^{3}$ \\
\hline Cristobalite & & $2.11 \times 10^{4}$ & $2.11 \times 10^{4}$ & $2.11 \times 10^{4}$ & $2.11 \times 10^{4}$ & $2.11 \times 10^{4}$ \\
\hline
\end{tabular}

The difference between the two ("young" in Table 6-2) concretes before the thermal pulse is in the representation of the sulfate-bearing phase. Young concrete is represented as ettringite, whereas the young -AFm concrete is represented as monosulphate. For these simulations, the impact of this distinction is small because relative rates are being used, as 
discussed subsequently. However, as the input data are improved and it becomes possible to distinguish the solubilities, this factor may govern the concentration of sulfur species in solution.

As understanding of thermally treated concrete improves through the use of experimental data and historical analogs, representation of these mineral assemblages will become more accurate. However, given the present state of knowledge of the minerals involved, more accurate representation of the assemblages will not significantly improve predictions of water chemistry. The dissolution rates of major cement minerals are not known and must be estimated.

These simulations are conducted in closed mode, and the mineralogical input can be viewed as a vehicle for getting the solid components to dissolve. The real effort associated with these simulations is to determine which minerals should precipitate because they are supersaturated, and to evaluate the resulting solution chemistry. The mineral assemblages presented in Chapter 7 were modified slightly to emphasize cement phases that are represented in the database, which simplifies this discussion. However, it should be recognized that the mineral assemblages presented in Chapter 7 can be used as input. Those minerals that are not represented in the database can be incorporated (and dissolved) as special reactants. The more fundamental problem is that phases that are not represented in the database cannot be precipitated. Thus, it is required that all solid phases that are expected to be a significant part of the chemical process be represented in the database.

Concrete differs from grout by the addition of aggregate, usually in two sizes: fine and coarse. For these simulations, a tuff formulation was assumed as the aggregate. The formulation used deviates somewhat from the standard tuff formulation in the EQ3/ 6 library, but incorporates the mineralogy given by Tang (1997). Comparison of the two tuff formulations shows some differences, but they are probably insignificant for these simulations. Coarse and fine aggregate are not distinguished in these simulations by composition, but by grain size. The difference in grain size translates to a difference in surface area, but instead of two separate aggregate sources, only one is assumed for these simulations. The intention is to represent the contribution of both mineral sizes to the water chemistry by an average relative dissolution rate. It would be preferable to simulate both size ranges, but this has little impact because the relative reaction rates estimated for the aggregate and grout are approximate. When the grout mineralogy is more accurately characterized, and the reactive properties of the phases are better known, it will be appropriate to represent the two aggregate grain sizes distinctly.

\subsubsection{Other Additives to the Original Concrete Formulation}

Of the possible additives to the original concrete formulations, only silica fume is represented. It is assumed for these simulations that only part of the silica fume is consumed in curing of the young concrete and AFm concrete. The silica is thus incorporated in other minerals in that assemblage, and the remaining silica fume is represented as amorphous silica. It is assumed that all of the silica fume is reacted in the thermally treated mineral assemblages, thus all of the silica is incorporated into other minerals. Superplasticizers and other additives are not represented in this simulation; they may be incorporated in future simulations.

\subsubsection{Dissolution Rates}

These simulations are conducted as reaction-path models (see Section 5.1) varying reaction progress instead of real time. Rate constants for some of the reactions that occur in these systems are constrained by experimental data, particularly dissolution reactions. It is theoretically possible to assign relative rate constants to the other reactions, and to show 
reaction progress as real time. However, given that many of the reaction rates are estimated, relative rates, the use of real time could give an inaccurate impression that time is constrained. For these simulations, all dissolution reaction rates are relative, and a simple approximation is made that grout components dissolve twice as fast as aggregate components.

\subsubsection{Gas Constraints: Atmospheric, Low $\mathrm{CO}_{2}$, Unfixed}

Three cases are examined for each mineral assemblage. In the first case, $\mathrm{O}_{2}$ and $\mathrm{CO}_{2}$ fugacities are fixed at atmospheric values. In the second case, $\mathrm{CO}_{2}$ and $\mathrm{O}_{2}$ values are fixed, but $\mathrm{CO}_{2}$ is set at a value far below atmospheric (0.01 times atmospheric). In the third case, the gas composition is not fixed. Because it is necessary to set a value for the maximum volume of gas in the simulation (the gas reservoir) and because reaction rates are not well constrained, $\mathrm{CO}_{2}$ is represented by the presence of calcite in this case (Table 6-2). In all cases, $\mathrm{CO}_{2}$ is eventually depleted by reaction with cement phases.

\subsubsection{Redox Conditions}

These simulations were conducted without control on the redox state, except for the atmospheric conditions (pyrite forms in the unfixed gas case). Because the contribution of Fe to the composition of the water is small (less than $10^{-9}$ molal in most of the simulations), it is unnecessary as a component in the simulations. In these simulations the amount of product hematite is constant throughout.

\subsubsection{Temperature Ranges}

Simulations were conducted at $25^{\circ}, 60^{\circ}$, and $150^{\circ} \mathrm{C}$. The $60^{\circ}$ and $150^{\circ} \mathrm{C}$ simulations require additional interpretation and examination because, in some cases, the new data are only applicable at $25^{\circ} \mathrm{C}$. The results for evolution of the young concrete mineral assemblages in Table 6-2, at a temperature of $60^{\circ} \mathrm{C}$ are presented here for discussion.

\subsubsection{Results and Discussion}

This section presents a conceptual model for the prediction of water composition in contact with concrete during the repository thermal evolution. The interpretation approach calls for selecting values of reaction progress for which the mineral assemblages can be readily described. The water-concrete evolution can then be described as a sequence of stages characterized by relatively stable assemblages.

The simulations presented here are intended as points of departure from which improvements will be made as understanding of these complex systems increases. In particular, it is anticipated that thermohydrochemical simulations can be used to reduce the uncertainty as to the evolution of $\mathrm{CO}_{2}$ fugacity in the NFE. Three of the simulation results at $60^{\circ} \mathrm{C}$ are presented for discussion in Figure 6-19, Figure 6-20, Figure 6-21, Figure 6-22, Figure 6-23, and Figure 6-24. The following discussion emphasizes the young concrete assemblages, for which features of the new database are especially important.

Figure 6-19 Plot of concentration of dissolved elements as a function of reaction progress for a simulation conducted at $60^{\circ} \mathrm{C}$, using a young concrete mineral assemblage and tuff aggregate composition and fixed $\mathrm{O} 2$ and $\mathrm{CO} 2$ compositions at atmospheric values

Figure 6-20 Plot of precipitated mineral phases as a function of reaction progress for the simulation described in Figure 6-19 
Figure 6-21 Plot of concentration of dissolved elements as a function of reaction progress for a simulation conducted at $60^{\circ} \mathrm{C}$, using a young concrete mineral assemblage and tuff aggregate composition and fixed $\mathrm{O}_{2}$ and $\mathrm{CO}_{2}$ compositions where $\mathrm{CO}_{2}$ is set at a low value (somewhat arbitrarily chosen)

Figure 6-22 Plot of precipitated mineral phases as a function of reaction progress for the simulation described in Figure 6-21

Figure 6-23 Plot of concentration of dissolved elements as a function of reaction progress for a simulation conducted at $60^{\circ} \mathrm{C}$, using a young concrete mineral assemblage and tuff aggregate composition and unfixed $\mathrm{O}_{2}$ and $\mathrm{CO}_{2}$ compositions

Figure 6-24 Plot of precipitated mineral phases as a function of reaction progress for the simulation described in Figure 6-23

Two plots are presented for each simulation. The first is a plot of total elemental concentration in the aqueous phase vs. reaction progress. The second shows the precipitation of solid phases as a function of reaction progress (within the precipitation constraints defined by the model). The precipitated minerals are presented to indicate which reactions control the water chemistry.

With respect to the fixed-gas composition cases, increase in $\mathrm{pH}$ represents the point at which the gas reservoir of the EQ3/6 model is depleted. Physical significance could be assigned to the depletion of the gas reservoir, but this is not the object of these simulations. The gas reservoir and titration parameters can be set to any scheme that can be justified by thermohydrochemical simulation of $\mathrm{CO}_{2}$ evolution in the host rock. It is important to note that a significant shift occurs in the precipitated phases, $\mathrm{pH}$, and some of the aqueous species when depletion occurs. For bounding the concentrations of aqueous species, in simulations with fixed gas fugacity, it is appropriate to consider the chemical evolution prior to depletion of $\mathrm{CO}_{2}$ gas.

Each of the three simulations plotted (fixed atmospheric fugacity, fixed at low fugacity, and unfixed) can be related to a repository performance scenario. The fixed cases represent open drifts in which the evolution of solids has no impact on gas composition. The unfixed case represents a NFE with highly restricted gas permeability.

Certain general observations can be made. The aqueous chemistry depends on gas composition, which is directly linked to $\mathrm{pH}$ in all simulations. The concentration of aluminum species in solution is greater at higher $\mathrm{pH}$. Other species such as $\mathrm{Mg}$ are less sensitive to $\mathrm{pH}$, and it is possible to develop first-order estimates for the concentrations of these species in solution. The concentrations of the more sensitive species can be bounded within an envelope that encompasses the range of values represented in the simulation. The concentration of a particular species in solution can be directly related to the solid phases that exist.

Further reaction progress is required to develop upper-bound concentrations for elements such as nitrogen, sulfur, and potassium, because they are still increasing when the simulations end. For these elements, there is no phase that reaches supersaturation in these models. Phases from the new database are precipitated in the fixed-fugacity simulations at $60^{\circ} \mathrm{C}$, and even more of them are apparent at $25^{\circ} \mathrm{C}$.

For the case in which gas composition is fixed at close to atmospheric values (Figure 6-19 and Figure 6-20), depletion of the $\mathrm{CO}_{2}$ gas reservoir does not occur until near the end of the simulation when the $\mathrm{pH}$ and the gas fugacity values change sharply. Elemental 
concentrations in the aqueous phase before that point depend to a large extent on the precipitation of calcite, but also on kaolinite, diaspore, and anhydrite. The relation between the formation of calcite and the fugacity of $\mathrm{CO}_{2}$ is more clear at $25^{\circ} \mathrm{C}$.

For the case in which gas composition is fixed, but for which the $\mathrm{CO}_{2}$ fugacity is fixed at a low value (Figure 6-21 and Figure 6-22) $\mathrm{CO}_{2}$ gas depletion also does not occur until near the end of the simulation, and the $\mathrm{pH}$ and the gas fugacity values are similarly affected. The range of concentrations of the various species contrasts with the first simulation. For example, the concentration of Si species in solution varies over a range from $10^{-3}$ to $10^{-6}$ molal, whereas in the atmospheric case it ranges from $10^{-3}$ to $10^{-4.5}$ molal.

The unfixed case (Figure 6-23 and Figure 6-24) does not seem physically realistic in the repository, except when the air-mass fraction approaches zero during the thermal pulse, because gas exchange will most likely occur along fractures. However, it may help to establish bounding concentrations for some chemical species. For example, a lower bound can be suggested for $\mathrm{Mg}$ at roughly $10^{-9}$ molal. In the unfixed case, even portlandite becomes supersaturated and precipitates. In all cases, $\mathrm{K}$ and $\mathrm{S}$ concentrations increase over the course of the simulation, although sulfur-bearing phases such as ettringite and anhydrite begin to form later in the simulation.

\subsection{Concentrations of Radionuclides in Water Leaving the Near Field}

\section{by Brian E. Viani}

Iron corrosion products and altered concrete will be present in large quantities in the reference repository design. Experimental data indicate that these materials have partition coefficients for $\mathrm{Np}$ and $\mathrm{U}$ that are at least several orders of magnitude larger than the coefficients that have been obtained for rock samples from Yucca Mountain. These materials, collectively, have the potential to retard $\mathrm{Np}$ and $\mathrm{U}$ in a manner equivalent to thousands of meters of rock. Concrete, in particular, appears to provide a secondary solubility constraint for radionuclides leaving the EBS, although the chemical mechanism has not yet been established.

Because of the conjunctive effects of sorption and solubility limits, iron-oxide corrosion products and concrete should be considered when establishing bounds on radionuclide concentrations in water leaving the NFE. The sorption capacity of iron oxides and cementitious materials is significant. To the extent that $\mathrm{Np}$ and $\mathrm{U}$ are solubility-limited in the NFE, interaction with concrete may have a long-lasting effect on the advective mode of radionuclide release. This section presents experimental data and interpretive analytical and numerical models, which support a conceptual model for significant retardation of these radioelements in the NFE. Also identified in this section are additional experiments and modeling that can reduce the uncertainty associated with these findings.

\subsubsection{Introduction}

Based on measured sorption properties for whole-rock samples of Yucca Mountain tuffs (Meijer, 1990) PA calculations suggest that the tuffs may not contribute significantly to retardation of key radionuclides (e.g., Np, Tc, U, Ni, Se). Demonstration of greater retardation performance would help ensure compliance with regulatory performance objectives. It is desirable to evaluate whether the NFE will provide additional retardation, and thereby decrease the source term relative to the projected releases from the waste form. It is also desirable to assess whether introduced materials in the NFE, or their degradation products, will promote radionuclide transport. 
A variety of introduced materials are needed to construct the repository and engineered barriers. Some of these materials and/or their alteration products will line the path that radionuclides must traverse in leaving the repository. The properties of these components, with regard to radionuclide transport, will differ significantly from those of the ambient tuffs.

Because of the limited seepage flux expected to invade the NFE during cool-down and the post-thermal period and except for brief episodes of increased fracture flow when water residence time will be decreased, groundwater chemistry will be strongly influenced by alteration products that will line flow pathways from the repository.

\subsubsection{Phases that Will Occur along Transport Pathways}

The path for radionuclide transport from the waste form through the NFE to the host rock will intersect one or more of the following materials:

- WP filler and packing materials and the degradation products

- WP corrosion-resistant material (CRM)

- WP corrosion-allowance material (CAM) and its corrosion products

- Other WP barrier materials and the alteration products

- Backfill and packing (e.g., crushed tuff) and their alteration products

- Invert materials (e.g., concrete and crushed tuff) and their degradation products

- Topopah Spring tuff (host rock) that has been altered by hydrothermal interaction with ambient fluids and/or interaction with introduced materials in the NFE

To derive a source term that reflects retardation processes in the NFE, transport of radionuclides through two plentiful and chemically important materials must be assessed. These materials are the corrosion products derived from the CAM and the cementitious materials (concrete, grout, and their alteration products) used in the repository construction. They will interact strongly with radionuclides, causing sorption, precipitation, and colloid transport (Viani, 1995, 1996a).

\subsubsection{Iron Corrosion Products}

It is expected that the CAM will alter mainly to iron oxide during the repository thermal evolution (Viani, 1996b). The most abundant corrosion products resulting from degradation of the waste container are expected to be iron oxides derived from a relatively thick overpack of low-carbon steel surrounding a corrosion-resistant inner barrier (Ahn and Soo, 1995; Van Luik et al., 1992). Based on proposed designs, approximately 1.6 to $2.0 \times 10^{4} \mathrm{~kg}$ of steel will surround each WP. This steel is expected to undergo generalized corrosion as a result of interaction with hot, humid atmosphere and contact by liquid groundwater during the cooldown and post-thermal periods. Depending on the specific oxide formed, the maximum mass of oxide that could form from each canister is in the range 2.2 to $3.9 \times 10^{4} \mathrm{~kg}$. The end product is expected to be a porous accumulation of oxides of iron surrounding the waste form and inner barrier. This medium will probably line the transport pathways taken by radionuclides that leave the waste canister via breaches in the corrosion-resistant inner barrier. Because the corrosion products are expected to be finely divided, there may be a potential for addition of iron oxide colloids to the groundwater. There will also be a potential for filtration of wasteform-derived colloids.

The waste container and steel overpack are expected to be subjected to three distinct thermohydrologic (TH) regimes during the repository lifetime. Following waste emplacement, the WPs will be subjected to elevated temperature and humidity in the absence of liquid water, during the period in which water is driven away from the repository. This regime will be followed by a period of contact by hot and relatively dry atmosphere during the thermal and cool-down periods of the repository thermal evolution (see Chapter 2). After 
temperatures in the drift drop below boiling, hot, humid conditions will return. Finally, rehydration of the repository horizon could result in liquid water contacting the container. It is assumed that oxic conditions will prevail for the life of the waste canister and that oxygen will not be limited.

\subsubsection{Expected Corrosion Products Formed from CAM}

Depending on redox and moisture conditions, low-carbon steel will alter more or less uniformly to oxides that can include $\mathrm{FeO}$ (wüstite), $\mathrm{Fe}(\mathrm{OH})_{2}, \mathrm{Fe}_{3} \mathrm{O}_{4}$ (magnetite), $\gamma-\mathrm{Fe}_{2} \mathrm{O}_{3}$ (maghemite), $\alpha-\mathrm{Fe}_{2} \mathrm{O}_{3}$ (hematite), $\mathrm{Fe}(\mathrm{OH})_{3}$ (amorphous iron hydroxide), $\gamma$-FeOOH (lepidocrocite), and $\alpha-\mathrm{FeOOH}$ (goethite) (Evans, 1960).

\section{Hot, Dry Atmosphere}

Under dry conditions in the presence of oxygen, carbon steel corrodes to form a film of anhydrous oxides dominated by $\alpha-\mathrm{Fe}_{2} \mathrm{O}_{3}$ and $\gamma-\mathrm{Fe}_{2} \mathrm{O}_{3}$ at relatively low temperatures $\left(<200^{\circ} \mathrm{C}\right)$, and dominated by $\mathrm{Fe}_{3} \mathrm{O}_{4}$ at higher temperatures (Evans, 1960). During the dryout period of the repository, if oxygen is available, anhydrous Fe(III)-oxides are expected to dominate the corrosion film formed on the steel CAM.

\section{Hot, Humid Atmosphere}

Based on observed corrosion of steel in humid atmospheres at ambient temperature, the corrosion products are expected to be $\mathrm{Fe}(\mathrm{OH})_{3}$, and $\alpha$ - and $\gamma$-FeOOH (Booker, 1976; Furet et al., 1990; Kucera and Mattsson, 1987). Acceleration of corrosion by water vapor at ambient temperatures is well known (Evans, 1960; Fyfe, 1976) but the effect of water vapor on the types of corrosion products formed at relatively low elevated temperatures $\left(<200^{\circ} \mathrm{C}\right)$ is not well documented.

\section{Liquid Groundwater Contact}

Assuming the availability of oxygen, low-carbon steel will likely corrode in the presence of groundwater to form products similar to those observed under humid unsaturated conditions (i.e., $\alpha$ - and $\gamma$-FeOOH, $\mathrm{Fe}(\mathrm{OH})_{3}$ ) (Booker, 1976).

\section{Microbially Induced Corrosion}

During periods of elevated humidity and/or groundwater contact, there is a potential for microbially induced corrosion (MIC; Hughes, 1976). Although sulfides could potentially form as a result of MIC even under aerobic conditions (Borenstein, 1994), the ultimate products of such corrosion will likely be dominated by the hydrated oxides of Fe(III) under long-term oxidizing conditions (e.g., Badan et al., 1991).

\section{Expected Oxide Phases}

Of the initial corrosion products that form under hot, dry or under humid, warm conditions, $\gamma$-FeOOH (goethite) and $\gamma-\mathrm{Fe}_{2} \mathrm{O}_{3}$ (hematite) are the most likely phases that will persist in the relatively low-temperature, oxygenated, humid, post-thermal repository environment. Based on the observed genesis and persistence of iron oxide phases in soils, other corrosion product phases such as $\gamma-\mathrm{Fe}_{2} \mathrm{O}_{3}, \mathrm{Fe}_{3} \mathrm{O}_{4}$, and $\gamma$-FeOOH are likely to be transitory (Schwertmann and Cornell, 1991). Although hydrated oxides of Fe(III) appear to be the most likely initial constituents of the corrosion layers formed on low-carbon steel in the presence of water, in the long term, transformation of these phases to anhydrous $\alpha-\mathrm{Fe}_{2} \mathrm{O}_{3}$ (hematite) may occur. 
The relative thermodynamic stability of goethite and hematite is not known with certainty, although tabulated data suggest hematite is slightly more stable than goethite (Johnson and Lundeen, 1994; Schwertmann and Cornell, 1991). Both goethite and hematite are widely distributed in soils, and each appears to persist as the most stable phase for specific ranges in earth surface conditions (Schwertmann and Cornell, 1991). Repository regimes that maintain lowered water activity and elevated temperatures would favor the transformation of goethite to hematite, although the kinetics of this transformation may be slow enough to allow goethite to persist for thousands of years (Schwertmann and Cornell, 1991).

\subsubsection{Interaction of Radionuclides with Corrosion Products}

Interaction of radionuclide-bearing fluids with WP corrosion products is expected to be dominated by surface chemical effects. Iron oxides are effective sorbents for a wide range of metal cations (e.g., transition metals, actinides) and many metal oxyanions (e.g., chromate, selenate, phosphate, carbonate, silicate) (Benjamin et al., 1996; Dzombak and Morel, 1990). In systems with relatively low concentrations of complexing ligands (e.g., carbonate), partition coefficients $\left(K_{\mathrm{d}}\right)$ for radionuclides such as $\mathrm{U}$ and $\mathrm{Np}$ can be as high as $10^{4}-10^{6} \mathrm{~mL} / \mathrm{g}$ (Ho and Miller, 1986; Kohler et al., 1992; Tochiyama et al., 1995; Triay et al., 1993; Turner, 1995). However, the presence of dissolved carbonate can significantly reduce sorption to iron oxides for metals such as $\mathrm{U}$ and $\mathrm{Np}$ that form solution carbonate complexes (Hsi and Langmuir, 1985; Turner, 1995; Van Geen et al., 1994).

It is clear that sorption coefficients for sorption of elements such as $\mathrm{U}, \mathrm{Np}$, and $\mathrm{C}$ on iron oxides are several orders of magnitude larger than for sorption on the repository host rock (Viani, 1996a). Because the products of corrosion are likely to be finely divided, a potential for introduction of colloids to the waste stream also exists. The potential for iron oxide colloid movement will depend on many factors, including fluid composition, oxide particle size, morphology and structure of the corrosion product layer, temperature, and the mode and flux of groundwater flow through the layer.

\subsubsection{Surface Complexation Model}

Partitioning of a radionuclide (sorbate) between a solid phase (sorbent) and solution is generally determined using a batch technique in which the sorbate concentration in solution is maintained below the saturation level of all potential radionuclide-bearing solids.

Depending on the purpose for measurement, partitioning is generally measured as a function of total concentration of added sorbate at constant $\mathrm{pH}$ and temperature $\mathrm{T}$ (sorption isotherm) or as a function of $\mathrm{pH}$ at constant total sorbate and $\mathrm{T}$ (sorption edge or envelope). The composition of the fluid in which the sorbate is dissolved can range from simple noninteracting supporting electrolytes to complex fluids that approximate natural groundwater (e.g., synthetic J-13 water). The data obtained from batch sorption experiments have been used to generate models ranging in simplicity from a constant sorption coefficient $\left(K_{\mathrm{d}}\right)$ model to multisite surface complexation models that incorporate sorption of multiple species (Fuentes et al., 1987; Turner, 1995).

Sorption isotherms are empirical models based on numerical fits to sorption isotherm data. They range from linear (constant $K_{\mathrm{d}}$ ) to nonlinear Freundlich and Langmuir isotherms. In general, these models can be used to predict sorption of a given sorbate only for the same conditions used in the experiment (i.e., fluid composition, $\mathrm{pH}$, sorbate/ sorbent ratio, temperature, and sorbent properties). To use the simplest models to assess radionuclide transport, the variation in $K_{\mathrm{d}}$ or other fit parameters with fluid composition, $\mathrm{pH}$, sorbate/sorbent ratio, surface area of sorbent, etc., must be known or estimated. In principle, 
because the simple isotherm or sorption coefficient models are empirical, no thermodynamic data (e.g., solution speciation) or solid-phase characterization is necessary for application over the specific property ranges encompassed by the experiments.

A simple $K_{\mathrm{d}}$ model cannot account for either the solution or surface chemical effects that have been shown to strongly influence sorption onto iron oxides (Dzombak and Morel, 1990). Solution composition, particularly $\mathrm{pH}$ and dissolved $\mathrm{CO}_{2}$, can significantly alter the partitioning of radionuclides between solution and surface. Mineral surface heterogeneity, especially the presence of a small proportion of sites with a high affinity for the sorbate (strong sites), can result in $K_{\mathrm{d}}$ that varies with the ratio of the mass of sorbent to mass of sorbate. Simple isotherms cannot account for competitive sorption or for prior "passivation" of the sorbent (i.e., saturation of sorption sites by other sorbates). Fugacity of $\mathrm{CO}_{2}, \mathrm{pH}$, concentrations of competing cations, and sorbate/ sorbent ratio are likely to be the most important variables controlling sorption of $\mathrm{U}, \mathrm{Np}, \mathrm{Pu}$, and other hydrolyzable cations under the oxidizing conditions expected in the repository. To account for these effects, a surfacecomplexation model that considers specific solution and mineral-surface-radionuclide interaction is necessary to predict the effect of a corrosion layer on radionuclide transport. The following discussion describes a modeling that is similar to models developed for other repository performance studies (Triay et al., 1997)

Surface-complexation models can, in principle, be used to model sorption of a given sorbate over a range in $\mathrm{pH}$, fluid composition, sorbent properties, sorbate concentrations, and sorbate/sorbent ratios. To use these models, both sorbent property parameters (specific surface area, site density, site heterogeneity) and surface-complexation parameters (acidity constants, binding constants) must be experimentally determined. In addition, thermodynamic data for solution species is necessary before surface-complexation binding parameters can be retrieved from the experimental sorption data.

\section{Diffuse, Double-Layer, Surface-Complexation Model}

The diffuse, double-layer, surface-complexation model was used to retrieve model parameters from batch and transport experiments involving $U$ adsorption on hematite and quartz (Viani and Torretto, 1997). The model was then used to simulate the effects of sorption on radionuclide transport through an iron oxide corrosion layer (Viani et al., 1997). Dzombak and Morel (1990) and Turner (1995) showed that the diffuse, double-layer model (DLM), the simplest of the surface-complexation models, is capable of effectively modeling a wide variety of metal-surface interactions. For each type of sorption site, equilibrium constants for two protonation reactions (acidity constants), the site density, and equilibrium binding constants for each sorbing species are required.

In the model discussed here, published acidity constants and site density estimates are used; these have been shown to be effective in modeling adsorption of radionuclides on iron oxides and other phases (Turner, 1995). Sorption of $U$ is modeled by assuming a single monodentate surface species (Turner, 1995). Turner showed that this assumption was sufficient for modeling $\mathrm{U}$ sorption onto iron oxide and other minerals over a relatively wide range of experimental values. 
The DLM model requires equilibrium constants for the following reactions:

$$
\begin{array}{rll}
>\mathrm{FeOH}^{0} & =>\mathrm{FeO}^{-}+\mathrm{H}^{+} & K_{-} \\
>\mathrm{FeOH}^{0}+\mathrm{H}^{+} & =>\mathrm{FeOH}_{2}{ }^{+} & K_{+} \\
>\mathrm{FeOH}^{0}+2 \mathrm{H}_{2} \mathrm{O}+\mathrm{UO}_{2}^{++} & =>\mathrm{FeOUO}_{2}(\mathrm{OH})_{2}{ }^{-}+3 \mathrm{H}^{+} & K_{\mathrm{B}}
\end{array}
$$

where $>\mathrm{FeOH}^{0}$ signifies the surface site, and $K_{-}, K_{+}$, and $K_{\mathrm{B}}$ are the deprotonation, protonation, and binding constant, respectively. The effects of electrostatic interactions are explicitly provided for in the formulation of the surface binding equilibrium constants (Dzombak and Morel, 1990).

The REACT geochemical modeling code (Bethke, 1994), which includes the DLM model, was used with the acidity constants and site densities shown in Table 6-3 to model the batch sorption data. The binding constant for uranium $\left(K_{\mathrm{B}}\right)$ was varied until the simulation visually fit the data. REACT makes use of the Lawrence Livermore National Laboratory (LLNL) thermodynamic database (GEMBOCHS) for solution and solid phases (Johnson and Lundeen, 1994). A 1-D reactive-transport code, X1t (Bethke, 1996), that simulates advective, diffusive, and dispersive transport while accounting for chemical changes due to precipitation/dissolution, surface complexation (DLM), and solution speciation was used to model U-transport data and to simulate the transport of $U$ through a corrosion layer composed of hematite.

Table 6-3 Parameters used for modeling sorption (DLM) and transport of uranium using REACT and X1t codes

\begin{tabular}{|c|c|c|c|c|c|c|c|}
\hline Phase & Vol. \%* & $\begin{array}{c}\text { Specific } \\
\text { Surface } \\
\mathbf{m}^{2} / \mathbf{g}\end{array}$ & $\begin{array}{c}\text { Site } \\
\text { Density } \\
\text { Sites/nm }\end{array}$ & $\begin{array}{c}\text { Fraction } \\
\text { Strong } \\
\text { Sites }\end{array}$ & $\begin{array}{c}\text { log } \boldsymbol{K}_{+}^{* *} \\
\text { Weak } \\
\text { Strong }\end{array}$ & $\begin{array}{c}\text { log } \boldsymbol{K}_{-} \\
\text {Weak } \\
\text { Strong }\end{array}$ & $\begin{array}{c}\text { log } \boldsymbol{K}_{\mathrm{B}} \\
\text { Weak } \\
\text { Strong }\end{array}$ \\
\hline \hline Hematite & 1.9 & 2.32 & 2.31 & 0.0325 & 7.35 & -9.17 & -11.7 \\
& & & & & 7.35 & -9.17 & -7.7 \\
\hline Quartz & 58.1 & 0.10 & 2.31 & 0.0 & $\mathrm{NA}^{* \star *}$ & -7.20 & -8.45 \\
\hline
\end{tabular}

Volume of mineral in column expressed as percent of total volume.

** $\quad \log K_{B}$ for weak sites was estimated by varying $\log K_{B}$ until the predicted fraction sorbed (REACT) visually matched the experimental batch data; $\log K_{\mathrm{B}}$ for the strong sites was set at $4 \log$ units more positive than $K_{\mathrm{B}}$ for weak sites; the fraction of strong sites was then estimated by varying the strong site density until the predicted U breakthrough curve (X1t) matched the experimental data.

*** Because the point of zero charge is so low for quartz $(\mathrm{pH} \sim 2)$, the protonation constant has not been defined nor is it needed to model this system.

\subsubsection{Batch Experiments with Iron-Corrosion Products}

A series of batch experiments was undertaken to obtain modeling parameters for sorption of $U$ on hematite (Viani and Torretto, 1997). Initial batch sorption experiments were also completed for $\mathrm{Np}$, but modeling parameters will not be retrieved until a complete set of experimental results has been obtained.

\section{Materials}

Hematite was chosen to simulate the dominant corrosion product expected to form from a ferrous corrosion-allowance layer on a WP. Although less-crystalline, more-hydrous and/or more-reduced iron-oxide phases (e.g.; ferrihydrite, goethite, magnetite) would almost certainly form during the corrosion process, these phases are likely to be transitory and would likely alter to hematite. Hematite is thought to have a lower binding affinity for 
radionuclides than do other Fe(III) oxides (185), hence sorption and retardation data obtained with this mineral are expected to be conservative relative to data for other oxide phases of equivalent specific surface.

Synthetic hematite (Aldrich Chemical, $\mathrm{Fe}_{2} \mathrm{O}_{3},<5 \mu \mathrm{m}, 2.32 \mathrm{~m}^{2} / \mathrm{g}$ ) was used for batchsorption and transport experiments (Viani and Torretto, 1997) and verified by X-ray diffraction to be crystalline and free of contaminants. Because the capacity for sorption is linearly related to specific surface area, use of this well-crystallized, low-surface-area material is likely to provide a conservative estimate of the sorption capacity of a real hematite corrosion layer. Passivation of hematite surfaces by aqueous interaction was not investigated in this study.

\section{Evolution of Crystallinity and Surface Area}

Surface area probably plays the major role in determining the efficacy of sorption by an iron oxide. However, there are measurable differences in the affinity of different iron oxides for metals (Hsi and Langmuir, 1985; Tochiyama et al., 1995). The less hydrous and / or more crystalline oxides, such as hematite, adsorb less than do amorphous $\mathrm{Fe}(\mathrm{OH})_{3}$ or ferrihydrite. The sorptive capacity of a given mass of corrosion products might be expected to decrease with time, as a result of increased crystallinity or particle size, or to transform to a less sorbing phase such as hematite. If radionuclides are sorbed to a material that subsequently alters to a form with less specific surface area or higher crystallinity, there is the potential for desorption that would be independent of changes in groundwater composition (Smith et al., 1994).

\section{Uranium and Neptunium Analysis}

A stock solution of natural uranium spiked with $1 \%{ }^{233} \mathrm{U}$ was used to prepare the experimental uranium solutions for both the sorption and transport experiments. Quantification of uranium in all experimental samples was done via alpha liquid scintillation spectroscopy using a Packard 2500TR/ AB liquid scintillation counter. Samples were prepared by placing $2-3 \mathrm{~mL}$ assays into $18-20 \mathrm{~mL}$ of Ecolite scintillation cocktail. The samples were then counted for $30 \mathrm{~min}$ or until a $1 \% 2 \sigma$ uncertainty was achieved. Experimental neptunium solutions for sorption and transport experiments were prepared from a ${ }^{237} \mathrm{NpO}_{2}^{+}$stock solution. Because the ${ }^{237} \mathrm{~Np}$ is in radioactive secular equilibrium with its $\beta^{-}$emitting daughter ${ }^{233} \mathrm{~Pa}$, the $\alpha / \beta$ discrimination was used to ensure that only the $\mathrm{Np}$ alpha radiation was being counted. It was possible to completely discriminate the ${ }^{233} \mathrm{~Pa}-\beta^{-}$while maintaining a $98 \%$ efficiency for the ${ }^{237} \mathrm{~Np}-\alpha$. The lower limit of detection at the $95 \%$ confidence level was calculated to be $8.084 \times 10^{-9} \mathrm{M}$ and $1.327 \times 10^{-8} \mathrm{M}$ for $\mathrm{Np}$ and $\mathrm{U}$, respectively.

\section{Batch sorption experiments}

Hematite/ solution mixtures, having a mass/volume $(\mathrm{M} / \mathrm{V})$ ratio of $1 \mathrm{~g} / \mathrm{L}$, were equilibrated for 4 days at $26^{\circ}, 38^{\circ}, 50^{\circ}$, and $76^{\circ} \mathrm{C}$. The solutions were 0.01 molar in $\mathrm{NaCl}$ and $2 \times 10^{-6}$ molar in $\mathrm{U}(\mathrm{VI})$, with $\mathrm{pH}$ varying between 4 and 10 . All solutions were prepared $\mathrm{CO}_{2}$-free, and the experiments were carried out in an argon atmosphere. Following equilibration in a constant temperature chamber, the final $\mathrm{pH}$ was measured on an aliquot of the supernatant. An aliquot of the supernatant was filtered through a $4.1 \mathrm{~nm}$ centrifugal filter and placed into a scintillation vial for uranium analysis. The concentration in the supernatant was used to compute the percent of the initial uranium sorbed by the hematite. Sorption of $\mathrm{Np}$ by hematite was measured using the same protocol at $26^{\circ} \mathrm{C}$ for an initial $\mathrm{Np}$ concentration of $1 \times 10^{-6} \mathrm{M}$. 


\section{Results}

Figure 6-25 shows the results of the batch sorption experiments at four temperatures. The data in Figure 6-25 indicate that, up to $50^{\circ} \mathrm{C}$, there is a slight increase in the binding of uranium at lower to neutral $\mathrm{pH}$, but there appears to be little effect on binding at neutral and higher $\mathrm{pH}$. Increasing temperature has been shown to increase the binding of metals to mineral surfaces (Brady, 1994).

Figure 6-25 Variation in percent sorbed and concentration of U(VI) in solution vs. final $\mathrm{pH}$ in four-day batch experiments

Figure 6-26 shows the results of simulating sorption using REACT and a single-site DLM (weak site) using the parameters in Table 6-3. The log $K_{\mathrm{B}}$ of the binding constant that best fit the data, -11.7 , is about $1.5 \log$ units smaller than that reported for goethite for the same adsorbed uranium species (Turner, 1995). Considering that site density and acidity constants are the same as used by Turner (1995), and assuming the measured specific surface is accurate and that the aqueous phase thermodynamic data used by Turner is similar to that in GEMBOCHS, the difference in $\log K_{\mathrm{B}}$ can be ascribed to a lower affinity of the hematite surface for uranium compared to goethite. This is consistent with the conclusions of Hsi and Langmuir (1985). It should be noted that to use this binding constant in a simulation would require using the same acidity constants and aqueous thermodynamic data used in the simulations.

\section{Figure 6-26 Comparison of DLM model (single-site) prediction}

It has been observed that, for many metals, a second surface site having a high affinity for the metal ("strong" site type) is necessary to describe sorption at low loading concentration or at low pH (Dzombak and Morel, 1990). Typically, the presence of a second site type is identified by measuring sorption isotherms over a wide range in sorbate concentrations and noting changes in slope due to saturation of strong sites. The strong site density necessary to describe the isotherm is commonly only a fraction $(<5 \%)$ of the total adsorption sites. For this analysis, the effect of surface heterogeneity was simulated by including a small fraction (0.0325) of strong sites. The fraction of strong sites was inferred from the breakthrough data for the $\mathrm{pH} 4$ transport experiment, as shown subsequently. Figure 6-27 shows a REACT / DLM simulation for which 3.25\% of the total sites are high affinity (strong sites) for which the $\log K_{\mathrm{B}}$ is -7.7 . It is important to note that the inclusion of the second site does not significantly change the visual fit of the simulation to the bulk of the data and that the effect of the second site on the fit is most evident at the lowest $\mathrm{pH}$ values.

\section{Figure 6-27 Comparison of DLM model (two-site) prediction}

The fraction of $\mathrm{Np}$ sorbed on hematite is not as large as $\mathrm{U}$ at the same $\mathrm{pH}$ (Figure 6-28). This observation is consistent with Turner's (1995) compilation of surface complexation parameters for sorption of $\mathrm{Np}$ and $\mathrm{U}$ on goethite and other iron oxides.

Figure 6-28 Percent $\mathrm{U}(\mathrm{VI})$ and $\mathrm{Np}(\mathrm{V})$ sorbed vs. final $\mathrm{pH}$ in four-day batch experiments

\subsubsection{Transport Experiments with Iron Corrosion Products}

Solutions containing 2 to $7.6 \times 10^{-6} \mathrm{~mol} / \mathrm{L}$ of U(VI), $1.33 \times 10^{-4} \mathrm{~mol} / \mathrm{L}$ of NaI, and $0.01 \mathrm{~mol} / \mathrm{L}$ of $\mathrm{NaCl}$ having $\mathrm{pH}$ values of 4.0, 5.0, 6.0, and 8.0 were pumped through columns $(15-\mathrm{cm} \times 1$-cm diameter) containing a mixture of quartz and hematite in the proportion of 94:6 by weight. Fine-grained quartz (Sigma Chemical, $\mathrm{SiO}_{2}, 2$ to $10 \mu \mathrm{m}$ ) was used in the transport experiments as a dilutant so that breakthrough of the uranium tracer would be 
observed in a reasonable time and because surface-complexation data for uranium adsorption on quartz are available (Turner, 1995). The quartz was treated with 6-M HCl to remove trace metals and/or impurity phases. X-ray diffraction analysis showed the treated quartz to be crystalline and free of impurity phases. The fractional porosity of the packed column was estimated to be $\sim 0.4(\sim 4.7 \mathrm{~mL})$. Flow rates were relatively constant within columns, but varied between columns from 2.2 to $3.3 \mathrm{~mL} / \mathrm{h}$.

Iodide was added to provide a conservative tracer against which to compare the relative retardation of $U$. Effluent samples were treated in the same manner as were the sorption samples, except that a specific ion electrode was used to measure the concentration of iodide. Because breakthrough of uranium at $\mathrm{pH} 6$ and 8 was not observed, it was decided to flush the $\mathrm{pH} 8$ column with untraced $0.01-\mathrm{M} \mathrm{NaCl}$ at $\mathrm{pH} 4$ and collect enough samples to observe elution of the sorbed $U$.

Results

Figure 6-29 shows the concentrations of iodide and uranium measured in the effluent for the four columns. Breakthrough of iodide was rapid (in approximately 1 pore-volume), and breakthrough of $U$ was significantly delayed relative to iodide. The shape of the $U$ breakthrough for the $\mathrm{pH} 4$ and $\mathrm{pH} 5$ experiments was quite sharp. After approximately $416 \mathrm{~mL}$ of the uranium-bearing, $\mathrm{pH} 8$ influent had been passed through the column, the influent was changed to a tracer-free solution at $\mathrm{pH}$ 4. The concentrations of iodide and $\mathrm{U}$ subsequent to the change in influent are shown in Figure 6-30. Breakthrough was observed at almost the same effluent volume as that observed for the $\mathrm{pH} 4$ column. Approximately $5 \%$ of the $\mathrm{U}$ input into the column was desorbed by the time the experiment was stopped.

Figure 6-29 Variation in concentration of $\mathrm{I}^{-}$and $\mathrm{U}(\mathrm{VI})$ vs. volume of effluent at $26^{\circ} \mathrm{C}$ for hematite:quartz columns at four $\mathrm{pH}$ values

Figure 6-30 Variation in concentration of $\mathrm{I}^{-}$and $\mathrm{U}(\mathrm{VI})$ vs. volume of effluent in $\mathrm{pH} 8$ column subsequent to the input of $\mathrm{pH} 4$ tracer-free solution

Simulating uranium breakthrough using the single-site DLM parameters derived from the sorption experiments resulted in a predicted breakthrough earlier than observed (Figure 6-31). If the binding constant for $U$ were made larger, the breakthrough point could be matched; but the breakthrough was much less sharp, occurring over 40-60 mL rather than $\sim 15$.

Figure 6-31 Comparison of X1t transport model (single- and two-site DLM) and observed $\mathrm{I}^{-}$and $U(\mathrm{VI})$ concentrations in effluent from $\mathrm{pH} 4$ column

To test the effect of a high-affinity site, the simulation was rerun including a site with a binding constant four orders of magnitude greater than the binding site deduced from the sorption results. This binding constant is large, but is in line with strong-site binding constants reported for transition metals on iron oxides, which vary between three and four orders of magnitude larger than the corresponding weak-site binding constants (Dzombak and Morel, 1990). The simulated breakthrough was very sensitive to the fraction of strong sites included in the model; thus, the fraction of strong sites could be estimated with precision. Including the strong binding site was the only means to match the breakthrough point and the shape of the experimental breakthrough curve (Figure 6-31). Although this match do not prove the presence of such sites, it is apparent that batch sorption data that does not address the presence of high-affinity sites may not provide appropriate parameters for modeling transport. The transport experiments apparently may provide a quite sensitive 
test for the presence of a small quantity of high-affinity sites. These results may mean that a simplified adsorption parameter set (Turner, 1995) may not be sufficient to accurately model transport.

In Figure 6-32, the experimental results for the entire $\mathrm{pH} 8$ column experiment (both initial loading at $\mathrm{pH} 8$ and desorption at $\mathrm{pH} 4$ ) are compared to the simulation prediction using the two-site model for hematite (described previously). As expected, the simulation correctly predicts the breakthrough of the iodide, but it predicts earlier breakthrough of the uranium and also predicts the uranium peak concentration to be much larger. The simulation predicts almost all of the uranium to be desorbed within the first $50 \mathrm{~mL}$ following breakthrough; this is in contrast to the approximately $5 \%$ observed in the experiment.

\section{Figure 6-32 Comparison of X1t transport model (two-site DLM) and observed $\mathrm{I}^{-}$and $\mathrm{U}(\mathrm{VI})$ concentrations in effluent from $\mathrm{pH} 8$ column}

For the X1t simulations shown, precipitation of any supersaturated phases was not allowed. If it is assumed that quartz solubility controls the level of silica in solution, the model predicts that the solution would become supersaturated with respect to the uranium silicate phase, soddyite. It is possible that precipitation of a uranium silicate occurred in the column during the passage of the $\mathrm{pH} 8$ solution. If so, a precipitate might not necessarily dissolve rapidly enough to be eluted by the subsequent $\mathrm{pH} 4$ fluid.

\subsubsection{Simulating Transport Through a Layer of Iron Corrosion Products}

Can the iron oxides derived from the CAM serve as a significant barrier to radionuclide transport? To bound the potential for radionuclide retardation, an upper limit to the radionuclide sorbed by a given mass of corrosion product can be estimated based on the maximum sorption site density and the surface area of the sorbent.

The maximum site densities estimated for iron oxides from measurements of adsorption maxima and estimates of proton donor/acceptor sites range between $\sim 2$ and 7 sites $/ \mathrm{nm}^{2}$ for cationic sorbates (Davis and Kent, 1990; Dzombak and Morel, 1990) Surface areas for natural and synthesized iron oxides vary widely. In general, natural and poorly ordered synthetic oxides have relatively large specific surface area (on the order of tens to hundreds $\mathrm{of}^{2} / \mathrm{g}$ ) (Davis and Kent, 1990; Dzombak and Morel, 1990). Assuming a maximum sorption site density of 2.31 sites $/ \mathrm{nm}^{2}$ (Dzombak and Morel, 1990), and specific surface of $20 \mathrm{~m}^{2} / \mathrm{g}$ (Davis and Kent, 1990) and considering $U$ as the sorbate, the limit imposed by sorption site density would be $\sim 0.02 \mathrm{~g} \mathrm{U} / \mathrm{g}$ oxide ( $\sim 550 \mathrm{~kg} \mathrm{U}$ per container). Depending on actual site densities and surface areas, this value could vary by an order of magnitude in either direction.

Sorption may be also be limited to a value less than the maximum site density when the solid/liquid partition coefficient $\left(K_{\mathrm{d}}\right)$ is less than some critical value. When the $K_{\mathrm{d}}$ is below this value, a radionuclide-bearing groundwater will "break through" the sorbing layer (i.e., exit at its inflow concentration) even though all the potential sorption sites not have been filled.

A simple calculation of the years for a radionuclide-bearing fluid to exceed the sorption capacity (either site density or $K_{\mathrm{d}}$-limited) of a sorbing layer of iron corrosion products was made based on the following assumptions:

- The maximum sorption density is 2.31 sites $/ \mathrm{nm}^{2}$.

- The specific surface of the oxides in the corrosion layer is $20 \mathrm{~m}^{2} / \mathrm{g}$.

- Sorption is linear and defined by a constant $K_{d}$.

- Six liters per year of groundwater contact the waste from one package.

- The concentration of radionuclides (e.g., U) in the groundwater after contacting the waste is $1 \mathrm{mg} / \mathrm{L}$. 
- The radionuclide-bearing groundwater contacts a fraction of the $3 \times 10^{4} \mathrm{~kg}$ of iron oxide corrosion product that surrounds the waste.

Figure 6-33 shows the time it would take for a radionuclide to breach the corrosion product layer for sorption $K_{\mathrm{d}}$ values ranging from $10^{2}$ to $10^{5} \mathrm{~mL} / \mathrm{g}$ and fractional contact volumes between 0.01 and 0.20 . For $K_{d}$ below about $10^{4} \mathrm{~mL} / \mathrm{g}$, capacity is limited by the $K_{\mathrm{d}}$. Above that value, capacity is limited by the sorption site density. Partition coefficients on the order of $10^{3}$ to $10^{6} \mathrm{~mL} / \mathrm{g}$ for $\mathrm{U}$ on iron oxides have been experimentally measured at low sorbent-to-solution ratios ( 1g/L) for $\mathrm{pH}$ between 5 and 9 (Ticknor, 1994; Turner, 1995). For $K_{\mathrm{d}}$ values greater than about $10^{3} \mathrm{~mL} / \mathrm{g}$ and volume fractions greater than 0.01 , the calculation suggests that retardation performance will be equivalent to the radionuclides released over hundreds of thousands of years. These retardation times imply that the corrosion layer can have a dramatic effect on the performance of the repository; especially for long-lived radionuclides such as $\mathrm{Np}$.

Figure 6-33 Calculated years to breakthrough of radionuclide-bearing groundwater through corrosion layer as a function of $K_{d}$ and volume fraction of the corrosion product from one waste canister $(\sim 30,000 \mathrm{~kg})$ contacted by the groundwater

\section{Transport Simulations}

Using the surface complexation parameters for $U$ sorption to hematite determined previously and a simulated flow path through a corrosion layer, a 1-D transport code X1t (Bethke, 1996) was employed to simulate $U$ transport for various fluid compositions. X1t simulations use the method of operator-splitting by which the calculation of mass transfer is performed separately from the chemical aspects of the computation. The physical and chemical parameters used in the simulations are shown in Table 6-4.

Table 6-4 Summary of parameters used to simulate transport of $U$ through hematite corrosion layer

\begin{tabular}{|l|l|c|}
\hline \multicolumn{2}{|l|}{ Properties of Flow System } \\
\hline \hline & Medium & Hematite \\
\cline { 2 - 3 } & Fractional porosity & 0.40 \\
\cline { 2 - 3 } & Specific discharge, $\mathrm{cm} / \mathrm{yr}$ & 2.1 \\
\cline { 2 - 3 } & Flow path length, $\mathrm{m}$ & 1 \\
\cline { 2 - 3 } & Dispersivity, $\mathrm{m}$ & 0.01 \\
\cline { 2 - 3 } & Diffusion coefficient, $\mathrm{cm}^{2} / \mathrm{s}$ & $1 \times 10^{-6}$ \\
\cline { 2 - 3 } & Simulation time, $\mathrm{yr}$ & $3 \times 10^{4}$ \\
\cline { 2 - 3 } & Number of nodes & 5 \\
\cline { 2 - 3 } & Limiting Courant number & 1 \\
\hline
\end{tabular}




\begin{tabular}{|l|l|c|}
\hline \multicolumn{2}{|c|}{ Properties of Hematite Sorbent } \\
\hline \hline \multirow{4}{*}{ Weak Sites } & Specific surface, $\mathrm{m}^{2} / \mathrm{g}$ & 20 \\
\hline & Site density, sites $/ \mathrm{nm}^{2}$ & 2.235 \\
\cline { 2 - 3 } & Log $K_{+}$ & 7.35 \\
\cline { 2 - 3 } & Log $K_{-}$ & -9.17 \\
\cline { 2 - 3 } & Log $K_{\mathrm{B}}$ & -11.7 \\
\hline \multirow{4}{*}{ Strong Sites } & Site density, sites $/ \mathrm{nm}^{2}$ & 0.075 \\
\cline { 2 - 3 } & Log $K_{+}$ & 7.35 \\
\cline { 2 - 3 } & Log $K_{-}$ & -9.17 \\
\cline { 2 - 3 } & Log $K_{\mathrm{B}}$ & -7.7 \\
\hline
\end{tabular}

The simulations are intended to evaluate the importance of the phenomena rather than to bound all possible groundwater compositions. Five different simulated fluids were used to assess the sensitivity of $U$ transport to $\mathrm{pH}$ and dissolved $\mathrm{CO}_{2}$ (Table 6-5). The range in $\mathrm{pH}$ and ionic strength considered largely spans the range displayed by Yucca Mountain groundwaters, but does not encompass $\mathrm{pH}$ extremes that might arise because of interaction of fluids with fresh concretes or microbiologically induced acidity. However, the $\mathrm{pH}$ values considered do span the range over which dissolved $\mathrm{CO}_{2}$ varies from being less important (low $\mathrm{pH}$ ) to very significant (high $\mathrm{pH}$ ) with respect to $\mathrm{U}$ transport. The fugacity of $\mathrm{CO}_{2}$ was varied between 0 and approximately 10-fold greater than atmospheric ("10× atm" fugacity $=0.003 \mathrm{~atm}$ ). Ionic strength was varied as necessary to maintain electrical balance at the given $\mathrm{pH}$.

Table 6-5 Composition of five hypothetical U-bearing groundwaters used to simulate transport of $U$ through hematite corrosion products

\begin{tabular}{|l|l|l|l|l|l|}
\hline $\mathrm{pH}$ & 6 & 6 & 8 & 8 & 8 \\
\hline $\mathrm{CO}_{2}$, fugacity & 0 & 0.003 & 0 & 0.0003 & 0.003 \\
\hline $\mathbf{U}, \mathbf{M}$ & $4 \times 10^{-6}$ & $4 \times 10^{-6}$ & $4 \times 10^{-6}$ & $4 \times 10^{-6}$ & $4 \times 10^{-6}$ \\
\hline $\mathbf{O}_{2}$, fugacity & 0.20 & 0.20 & 0.20 & 0.20 & 0.20 \\
\hline lonic strength, $\mathbf{M}$ & 0.11 & 0.11 & 0.01 & 0.01 & 0.01 \\
\hline
\end{tabular}

The concentration of $U$ in solution was set to $\sim 1 \mathrm{mg} / \mathrm{L}\left(4 \times 10^{-6} \mathrm{M}\right)$ to allow comparison with the results based on the simple mass balance calculation presented previously. Ongoing experiments using $\mathrm{U}$ and $\mathrm{Np}$ tracers will provide surface-complexation parameters and assess the role of site heterogeneity to be used in future models of this type involving $\mathrm{Np}$.

The ratio of sorbent to sorbate in a porous material, such as the detritus that is produced from engineered barrier degradation (for any given volume element), is much larger than the ratio of sorbent to sorbate in a batch experiment. The sorbent-to-sorbate ratio in a rock is controlled by the porosity and fluid saturation state of the medium. For these simulations, the ratio of sorbent to solution is $\sim 7875 \mathrm{~g} / \mathrm{L}$, almost 4 orders of magnitude larger than most laboratory experiments. Under conditions of unsaturated flow (for a given specific discharge and porosity), this ratio would be even larger. This ratio implies that sorption could be stronger than observed in vitro, depending on contact dynamics. 


\section{Simulation Results}

In Figure 6-34, the concentration of dissolved $U(C)$ relative to its influx concentration $\left(C_{0}\right)$ is shown at $10 \mathrm{~cm}$ into the corrosion layer for the 5 different influent solutions. Only one solution, $\mathrm{pH} 8,10 \times \mathrm{atm} \mathrm{CO}_{2}$, is close to breaking through after $3 \times 10^{4}$ years $\left(\mathrm{C} / \mathrm{C}_{0} \sim 0.8\right)$. Under these flow conditions, a conservative (nonretarded) species would be expected to break through in about $20 \mathrm{yr}$. As expected, $\mathrm{CO}_{2}$ concentrations have little effect on $\mathrm{U}$ transport at $\mathrm{pH} 6$, but significantly affect transport at $\mathrm{pH} 8$.

\section{Figure 6-34 Variation in relative concentration of $U\left(C / C_{0}\right)$ with time at node $1,10 \mathrm{~cm}$ into hematite corrosion layer}

At the other downstream nodes (not shown; see Viani et al., 1997) U concentrations are very low, and $\mathrm{C} / \mathrm{C}_{0}$ ratios are less than $10^{-5}$, except for the result at $\mathrm{pH} 8$, with $10 \times$ atm $\mathrm{CO}_{2}$, at $30 \mathrm{~cm}\left(\mathrm{C} / \mathrm{C}_{0} \sim 0.01\right)$. Under the given conditions, $\mathrm{U}$ breakthrough for corrosion layers thicker than $30 \mathrm{~cm}$ will not occur until well beyond $3 \times 10^{4} \mathrm{yr}$.

Figure 6-35(a) shows the variation in $K_{d}$ as calculated from the simulated solution and sorbate compositions at the $10-\mathrm{cm}$ node for 4 simulations. The calculated $K_{\mathrm{d}}$ values (especially at early times) are very large, and they decrease by two to three orders of magnitude with time. This decrease is directly related to surface site occupancy (Figure 6-35b). At $10 \mathrm{~cm}$, the strong sites are projected to completely fill in 11,000 yr. The weak sites are $\sim 5 \%$ filled after $30,000 \mathrm{yr}$ for the $\mathrm{pH} 6$ and $\mathrm{pH} 8\left(\right.$ no $\mathrm{CO}_{2}$ and atmospheric $\left.\mathrm{CO}_{2}\right)$ cases. After the strong sites are filled, the $K_{d}$ drops by more than an order of magnitude.

\section{Figure 6-35 Variation in $K_{d}$ vs. time at node $1(10-\mathrm{cm})$; concentration of $U$ vs. time on strong and weak sites at node $1,10 \mathrm{~cm}$ into hematite corrosion layer}

The calculated $K_{\mathrm{d}}$ values are much larger than those measured experimentally using batch techniques. At the earliest simulation times, the simulated concentrations of $U$ in the fluid are very low $\left(<<10^{-12} \mathrm{M}\right)$, and the ratio of strong sites to dissolved $U$ is very large. The large $K_{d}$ values at early times are not inconsistent with what is known about surface complexation on iron oxides. However, it is unlikely that a batch experiment can be used to verify the values predicted by these simulations, because both the $U$ concentration of $U\left(<<10^{-12} \mathrm{M}\right)$ and the solid/solution ratio $(8000 \mathrm{~g} / \mathrm{L})$ are not accessible experimentally. This fact has little bearing on the conclusion that iron corrosion products can significantly retard $\mathrm{U}$ and, by analogy, Np as well.

\subsubsection{Effect of Cementitious Materials on U Transport}

A preliminary assessment of the effect of cementitious material on $U$ transport through a corrosion product layer was made using model simulations. Model parameters were the same as described previously, but input solution compositions were adjusted to simulate the effect of concrete on fluid chemistry (Table 6-6). For the simulation using "cement water," two Ca surface species were added to the model to assess the effect of competitive adsorption. The Ca surface species and the $\log K_{\mathrm{B}}$ values for the following surface complexation reactions at weak and strong sites were taken from Dzombak and Morel (1990):

$$
\begin{array}{llll}
>(\mathrm{w}) \mathrm{FeOH}^{0}+\mathrm{Ca}^{++} & = & >(\mathrm{w}) \mathrm{FeOCa}^{+}+\mathrm{H}^{+} & \log K_{\mathrm{B}}=-5.85 \\
>(\mathrm{s}) \mathrm{FeOH}^{0}+\mathrm{Ca}^{++} & = & >(\mathrm{s}) \mathrm{FeOHCa}^{++} & \log K_{\mathrm{B}}=10.45
\end{array}
$$

where $>(w)$ and $>(s)$ refer to weak and strong sites, respectively. 
Table 6-6 Composition of fluids used for assessing the effect of a high $\mathrm{pH}$ cementitious material on transport of $U$ through a hematite corrosion product layer

\begin{tabular}{|c|c|c|c|c|}
\hline & \multicolumn{4}{|c|}{ Simulation Conditions } \\
\hline $\begin{array}{c}\text { Inlet Fluid } \\
\text { Composition }\end{array}$ & $\begin{array}{l}\mathrm{pH} \mathrm{10}, \\
\text { no } \mathrm{CO}_{2}\end{array}$ & $\begin{array}{r}\mathrm{pH} \mathrm{10,} \\
\mathrm{atm} \mathrm{CO}_{2} \\
\end{array}$ & $\begin{array}{c}\mathrm{pH} \mathrm{10} \\
10 \times \mathrm{CO}_{2} \\
\end{array}$ & $\begin{array}{l}\text { "Cement } \\
\text { Water"* }\end{array}$ \\
\hline pH & 10 & 10 & 10 & 12.17 \\
\hline $\mathrm{CO}_{2}$, fugacity & 0.0 & 0.0003 & 0.003 & $5.04 \times 10^{-13}$ \\
\hline $\mathrm{O}_{2}$, fugacity & 0.20 & 0.20 & 0.20 & 0.20 \\
\hline lonic strength, M & 0.01 & 0.357 & 12.3 & 0.232 \\
\hline $\mathrm{Na}(\mathrm{M})$ & 0.01 & 0.298 & 22.4 & 0.200 \\
\hline $\mathrm{Ca}(\mathrm{M})$ & $\mathrm{nc}^{\star \star}$ & $\mathrm{nc}$ & $\mathrm{nc}$ & 0.0139 \\
\hline $\mathrm{Al}(\mathrm{M})$ & $\mathrm{nc}$ & $\mathrm{nc}$ & $\mathrm{nc}$ & $1.78 \times 10^{-5}$ \\
\hline $\mathrm{U}(\mathrm{M})$ & $4.00 \times 10^{-6}$ & $4.00 \times 10^{-6}$ & $4.00 \times 10^{-6}$ & $4.00 \times 10^{-6}$ \\
\hline $\mathrm{SiO}_{2}(\mathrm{M})$ & $\mathrm{nc}$ & $\mathrm{nc}$ & $\mathrm{nc}$ & $3.13 \times 10^{-6}$ \\
\hline $\mathrm{Cl}(\mathrm{M})$ & 0.0099 & 0.01 & 0.01 & 0.200 \\
\hline $\mathrm{SO}_{4}(\mathrm{M})$ & $\mathrm{nc}$ & $\mathrm{nc}$ & $\mathrm{nc}$ & $1.85 \times 10^{-3}$ \\
\hline
\end{tabular}

* The composition of this fluid is based on the $\mathrm{Ca}, \mathrm{Al}, \mathrm{SiO}_{2}$, and $\mathrm{SO}_{4}$ predicted for a solution in equilibrium with typical cement phases: calcite, hillebrandite, katoite, ettringite, portlandite and hematite. The $\mathrm{NaCl}$ concentration was set to $0.2 \mathrm{M}$ (similar to that observed by Atkins et al. [1990] for an ordinary Portland cement). The $\mathrm{pH}$ and fugacity of $\mathrm{CO}_{2}$ were calculated using the REACT geochemical modeling code (data: thermo.com.V8.R6.230) by adjusting $\mathrm{OH}^{-}$to maintain electrical neutrality and by constraining the fugacity of $\mathrm{CO} 2$ by equilibrium with calcite.

** $\mathrm{nc}=$ component not considered in simulation

$\mathrm{pH}$ and $\mathrm{CO}_{2}$ Effects

A pH of 10 was used to simulate groundwater affected by interaction with cement, but not by interaction with fresh concrete $(\mathrm{pH}>12)$. These simulations can be compared directly with those described previously for lower $\mathrm{pH}$. Laboratory experiments described subsequently have shown that the $\mathrm{pH}$ of groundwater in contact with a hydrothermally altered concrete is between 10 and 11. It is clear that $\mathrm{pH}$ of 10 and $\mathrm{CO}_{2}$ fugacity equal to 10 times atmospheric are not compatible conditions (the ionic strength required would be on the order of 12 molal). The simulation under this scenario is not realistic, but it is included for comparison. At $\mathrm{pH} 10$, the simulated transport of $U$ through a hematite corrosion layer is the same for the atmospheric and 10× atmospheric $\mathrm{CO}_{2}$ levels (Figure 6-36), and U breakthrough is immediate. At $\mathrm{pH} 10$, in the absence of $\mathrm{CO}_{2}, \mathrm{U}$ is significantly retarded, and breakthrough would be more delayed than at lower $\mathrm{pH}$ in the absence of $\mathrm{CO}_{2}$ (compare with Figure 6-34). These results emphasize the importance of $\mathrm{CO}_{2}$ in estimating the $\mathrm{pH}$ of water in contact with the engineered barriers.

Figure 6-36 Variation in relative concentration of $U(\mathrm{C} / \mathrm{Co})$ with time at node 1 , which is $10 \mathrm{~cm}$ into hematite corrosion layer, for $\mathrm{pH} 10$ and no $\mathrm{CO}_{2}$

Transport of $U$ through the corrosion layer for a groundwater having the composition shown in the last column of Table 6-6 (cement water) is similar to that at $\mathrm{pH} 10$ in the absence of $\mathrm{CO}_{2}$. The simulation of $\mathrm{U}$ transport in water derived from interaction of groundwater with cement was modeled, assuming that $\mathrm{CO}_{2}$ levels were controlled by equilibrium with calcite. 
Under these conditions, the $\mathrm{pH}$ is fixed by the concrete chemistry, and the equilibrium $P_{\mathrm{CO}_{2}}$ is quite low. This composition might be expected to describe a fluid in the pore space of the concrete or at the point the fluid exits the concrete. The rock properties, rather than the fugacity of $\mathrm{CO}_{2}$ in an external gas phase, control the fluid composition. In contrast, for a fluid having extensive contact with a reservoir of $\mathrm{CO}_{2}$, the $\mathrm{pH}$ would be expected to be significantly lower: again probably controlled by calcite equilibrium. Under this scenario, for the same fluid composition shown in Table 6-6, the $\mathrm{pH}$ would be about 8.15 for equilibrium with atmospheric $\mathrm{CO}_{2}$ and calcite. Transport of $\mathrm{U}$ under those conditions would be expected to be similar to the $\mathrm{pH} 8 \mathrm{~atm} \mathrm{CO}_{2}$ simulation shown in Figure 6-34; that is, retardation of $\mathrm{U}$ by hematite would still be quite significant.

\section{Effect of Ca Sorption}

The effect of Ca sorption on $\mathrm{U}$ sorption appears to be minimal in these simulations as judged by comparison of the concentration of surface $\mathrm{U}$ species for $\mathrm{pH} 10$ (no $\mathrm{CO}_{2}$, no $\mathrm{Ca}$ ) and "cement water" results (Figure 6-37). Note that the concentrations of surface species in Figure 6-35 are plotted logarithmically to show both the Ca surface species concentrations and the $\mathrm{U}$ surface species on the same plot. Even in the presence of a large amount of sorbed $\mathrm{Ca}$, sorption of $\mathrm{U}$ onto strong and weak sites is almost identical to that predicted in the $\mathrm{pH} 10$ simulation. The concentrations of surface $U$ species shown in Figure 6-37 are essentially identical to those for the $\mathrm{pH} 6$ and $\mathrm{pH} 8\left(\right.$ no $\mathrm{CO}_{2}$, and atm $\mathrm{CO}_{2}$ ) simulations shown in Figure 6-35(b).

\section{Figure 6-37 Variation in concentration of $U$ and Ca surface species with time at Node 1, which is $10-\mathrm{cm}$ in hematite corrosion layer, for $\mathrm{pH} 10\left(\right.$ no $\left.\mathrm{CO}_{2}\right)$ and cement- derived groundwaters}

\subsubsection{Discussion}

The results of these simulations, and the conclusions drawn from them, are preliminary because a simplified chemical system is considered, and the range of chemical and hydraulic conditions considered is limited. It is important to recognize the sensitivity of radionuclide transport to the near-field geochemical environment.

The $\mathrm{pH}, P_{\mathrm{CO}_{2}}$, ionic strength and concentrations of competing cations and ligands are all important aqueous-phase compositional variables that could be better constrained. The specific sorptive oxide phase, its abundance and specific surface area, and the mode of groundwater movement should also be constrained to predict retardation in the NFE with confidence. The following text provides further discussion of ways to improve the veracity of this analysis.

\section{Effect of Specific Discharge}

If $6 \mathrm{~L}$ of groundwater contacts the waste from one WP each year, the specific discharge used in the simulations requires a cross-section equivalent to a circle with a diameter of approximately $65 \mathrm{~cm}$. A larger flux of groundwater or a smaller cross-sectional area for flow would necessitate a larger specific discharge for the simulations. A larger specific discharge would yield a similar result, but the time axis would be scaled downward accordingly (i.e., twice the discharge would result in breakthrough at half the time). 


\section{Mode of Groundwater Flux}

Groundwater movement was assumed to occur as a homogeneous, saturated flow. Retardation will depend on the volume of corrosion product contacted by a given volume of radionuclide-bearing groundwater. For unsaturated flow, the ratio of sorbent to sorbate will be larger than has been assumed here, and retardation would be enhanced. Although homogeneous unsaturated flow would be expected to yield qualitatively similar results (normalized to specific discharge), episodic saturated flow that occurs via fast paths would not, and the same specific discharge occurring episodically would likely yield different results (i.e., less retardation).

\section{Competitive Sorption and Solution Complexation}

These experiments and model analyses do not address the effect of competition for sorption sites or the effect of solution ligands other than calcium and carbonate. Competition and solution complexation will reduce retardation. Although the specific effects of dissolved carbonate on metal transport are well known, the $P_{\mathrm{CO}_{2}}$ values expected in the repository are not well constrained. Organic ligands derived from microbial processes could, for some radionuclides, potentially reduce the sorptive capacity of the corrosion layer significantly. The type, quantity, and affinity of these ligands for radionuclides are also poorly constrained.

\section{Precipitation/Dissolution}

The effect of precipitation of radionuclide-bearing phases has not been accounted for either in the transport experiments or in the simulations. These results may therefore be considered conservative, if, as suggested by the results of the quartz/hematite experiment, Usilicates can precipitate. Depending on when radionuclide-bearing groundwater contacts the corrosion layer, coprecipitation of the radionuclide with iron oxide may occur (Bruno et al., 1995). It is also appropriate to assess whether iron silicate formation can occur and thereby reduce the quantity of iron oxide available for sorption.

\subsubsection{Gaps in Experimental Data and Modeling}

\section{Data Needs}

Additional experimental data are needed to increase confidence in simulations of chemical retardation in the NFE. The experiments that can provide these data include the following:

- Independent assessment of the presence of strong sites for radionuclide sorption

- Transport experiments that include dissolved carbonate to assess the potential for rapid uranium breakthrough at near-neutral $\mathrm{pH}$

- Additional sorption and transport experiments for $\mathrm{Np}$ and other radionuclides

- Sorption and transport experiments with mixed radionuclides to assess competition

- Sorption and transport of radionuclides in more complex systems such as J-13 groundwater, "cement waters," multimedia transport columns, and mixed radionuclide solutions

\section{Simulation/Modeling Needs}

Dissolution/ precipitation of radionuclide-bearing phases needs to be addressed in NFE transport simulations. A wider range of hydraulic, chemical, and temperature conditions needs to be simulated. The effect of fast-flow pathways needs to be addressed by coupling a 
surface-complexation model to a hydrologic model that can simulate fracture flow and heterogeneous media. Finally, literature data, experimental data, and simulation results need to be integrated and simplified (e.g., in the form of response surfaces) for performance assessment calculations.

\section{Colloids}

The effect of colloids on radionuclide transport through oxide corrosion products has not been considered in this analysis. Whereas it is likely that colloidal particles will exist and be capable of transporting radionuclides through the corrosion layer, the magnitude of radionuclide transport via these mechanisms has not been determined. If corrosion products serve as a source for pseudo-colloids, breakthrough at the boundary of the corrosion layer may occur sooner and at greater total radionuclide concentrations than discussed here. If radionuclides are sorbed rather than coprecipitated onto colloidal iron oxides, desorption kinetics becomes important for assessing transport from the NFE into less-altered surrounding rock. The production and mobility of iron oxide colloids needs to be bounded for a range of hydrologic and chemical conditions.

\subsubsection{Conclusions for Transport Through Iron Corrosion Products}

Iron oxides derived from the WP CAM can potentially provide a sorptive barrier to radionuclide transport that could be equivalent to many meters of surrounding rock. There is the potential for retardation of radionuclides for thousands to tens of thousands of years following release from the WP. However, retardation will be strongly affected by fluid chemistry, particularly $\mathrm{pH}$ and $\mathrm{CO}_{2}$ fugacity, and by the nature of the flow path through the layer of corrosion products.

Model results indicate that, at high $\mathrm{pH}$, the presence of significant levels of $\mathrm{CO}_{2}$ can inhibit the sorption of $\mathrm{U}$ and, by analogy, $\mathrm{Np}$. The $\mathrm{CO}_{2}$ level expected in the repository and in the groundwater at the time of radionuclide transport is not well constrained. If the cement is not completely carbonated, the presence of large amounts of cementitious material will limit $\mathrm{CO}_{2}$ levels in the NFE to less than ambient (prerepository) levels. However, simulations suggest that retardation will nevertheless be significant for two scenarios that are likely to control fluid chemistry: (1) high-pH fluids in which $\mathrm{CO}_{2}$ fugacity is controlled by calcite equilibrium (e.g., fluids within cementitious material) and (2) initially high-pH fluids in which $\mathrm{pH}$ is decreased by reaction with ambient $\mathrm{CO}_{2}$ (e.g., fluids that exit cementitious material).

It is expected that the adsorption of $\mathrm{Np}$ onto iron oxides will generally be weaker than for adsorption of $U$ onto iron oxides; thus, simulations using $U$ provide an upper limit to the retardation expected for $\mathrm{Np}$. When the crystalinity of the iron corrosion products increases, as the materials age in the NFE, $\mathrm{U}$ and $\mathrm{Np}$ may desorb or be incorporated in the iron oxides. The fate of sorbed $U$ and $\mathrm{Np}$ under these conditions has not been investigated.

The emphasis in these studies is on the sorptive behavior of near-field materials, but simulations that include precipitation and dissolution will give a more realistic assessment of radionuclide migration through corrosion products.

Although gaps in data and modeling need to be filled, based on these results the presence of iron corrosion products in the flow field downgradient from the WPs will significantly enhance repository performance. 


\subsubsection{Cementitious Materials}

Cementitious materials in the form of concrete ground support and invert components, shotcrete, and grout for liner stabilization or rock bolt anchoring will be present in the repository in significant quantities (Meike, 1996). The current design calls for $3.85 \mathrm{~m}^{3}$ of concrete per lineal meter of emplacement drifts, which includes the concrete invert and the concrete drift liner but does not include the piers on which the WPs will rest. Concrete that underlies the WPs is the cementitious material that is most likely to interact with radionuclide-bearing fluids.

The chemical and mineralogical properties of concretes are dramatically different from those of the repository host rock. Pore fluids in relatively young concretes are typically alkaline, with $\mathrm{pH}>12$ and ionic strength that is significantly greater than that of Yucca Mountain groundwater (Atkinson et al., 1989; Glasser et al., 1985). Colloidal silica-rich particles are abundant in fluids derived from fresh concrete (Ramsay et al., 1988); this is in contrast to the much smaller concentrations of colloids observed in groundwater from Yucca Mountain (Triay et al., 1993; Viani, 1996a).

Fresh concrete and cement phases have been shown to strongly retard and/or immobilize certain actinides and, in some cases, alkali radionuclides (Albinsson et al., 1993; Atkins et al., 1988; Ma et al., 1996). Concrete interaction will alter the chemistry of water contacting the WP and waste form and will affect the interaction between radionuclide-bearing water and other downstream flow-path components such as iron oxides and the host rock. As discussed in the previous subsection, if the presence of concrete results in decreased $\mathrm{CO}_{2}$ fugacity and increased $\mathrm{pH}$, radionuclide retardation by corrosion products could be enhanced.

It is expected that, by the time radionuclides are released from the WPs, cementitious materials will have been altered by exposure to hot, dry conditions and by hot, humid conditions for hundreds or thousands of years. The extent to which cementitious materials will remain viable as chemical barriers over thousands of years will depend on the nature of alteration that occurs during the thermal period. Because of the chemical and mineralogical complexity of cementitious materials and because of the scarcity of relevant thermodynamic and kinetic data, an experimental approach should be taken to evaluating potential retardation behavior of altered concrete (Bruton and Viani, 1996). This was the motivation for the series of experiments described in the following text.

\subsubsection{Expected Concrete Alteration Products}

Fresh concrete is composed of a mixture of minerals and amorphous phases, including the aggregate and the predominantly calcium-silicate-hydrate phases composing the cement. Under hydrothermal repository conditions, the cement is expected to undergo significant mineralogical and chemical alteration. Alteration of cementitious materials is likely to be characterized by several processes, including conversion of poorly crystalline or amorphous $\mathrm{Ca}, \mathrm{Si}, \mathrm{Al}$, Fe-hydrated gels to more crystalline phases; leaching of alkalis; and formation of calcite by carbonation. The most important results of chemical alteration are the decrease in $\mathrm{pH}$ and ionic strength (relative to young concrete) and the increase in dissolved carbonate in fluids that have reacted with these materials.

\section{Alteration of Concrete Under Hot, Dry Conditions}

In contrast to the data related to alteration of metals, few empirical or thermodynamic data are available to predict mineralogical changes in cements that occur as a result of longterm exposure to dry heat (Meike, 1996). Although loss of capillary and adsorbed water is expected, the extent of dehydration of hydrated calcium silicate gels that make up the bulk of 
cement phases is difficult to predict. The rate and extent to which these gels recrystallize to more stable and less hydrated phases are also uncertain. For bounding predictions regarding the effects of dry heat on concrete, thermodynamic data and long-term thermal experiments are needed. Depending on the extent of dehydration and recrystallization during hot, dry conditions, rehydration to form more hydrated phases is expected during the longer, subsequent hot and humid period.

\section{Alteration of Concrete Under Hot, Wet Conditions}

Hydrothermal alteration of cements and concretes has been studied more thoroughly than has hot, dry heating. However, predicting the final assemblage of phases expected when radionuclide-bearing fluids are released from the WPs is complicated by large gaps in available thermodynamic and empirical data. Relatively short-term $(<1 \mathrm{yr})$ hydrothermal treatment of cements and concretes in the presence of liquid water, and with tuff in some experiments, has shown that the hydrated calcium silicate gels in fresh concrete partly transform to crystalline phases such as tobermorite and xonotlite (Bensted, 1989). Whether these same phases would form in the absence of liquid water during the unsaturated, hot and humid conditions expected in the repository is not known. In addition, hydrated calcium silicate phases, such as tobermorite, that have been observed in hydrothermally altered concretes may be metastable, and their persistence under repository NFE conditions is uncertain.

\section{Carbonation of Concrete}

In addition to the alteration of calcium-silicate-hydrate gels to more crystalline phases, interaction of $\mathrm{CO}_{2}$ with these alkaline materials can result in the formation of $\mathrm{CaCO}_{3}$ (Kobayashi and Uno, 1994; Taylor, 1990). The degree and extent to which the cementitious materials in the repository will alter to calcite will depend on a number of factors, including the atmospheric concentration of $\mathrm{CO}_{2}$ and $\mathrm{H}_{2} \mathrm{O}$, temperature, and the rate of diffusion of $\mathrm{CO}_{2}$ into the cement (Saetta et al., 1993). When the atmospheric $\mathrm{CO}_{2}$ concentration is high and diffusion is not a limiting factor, the conversion of finely divided calcium-silicate-hydrate minerals such as tobermorite or xonotlite to calcite and $\mathrm{SiO}_{2}$ can be rapid (Goto et al., 1995; Martin, 1994). Extensive carbonation of concrete would dramatically change the pore-fluid chemistry. In particular, highly alkaline pore fluids would no longer be expected, and pore fluid $\mathrm{pH}$ would decrease by more than $3 \mathrm{pH}$ units. This change in chemistry could significantly affect the concrete's potential for radionuclide retardation.

\subsubsection{Hydrothermally Altered Concrete Sample Preparation}

To examine the role of concrete in limiting radionuclide transport in the NFE, experiments are underway to measure $\mathrm{U}, \mathrm{Np}$, and iodide transport through samples of concrete from the invert material used in construction of the ESF. Transport is being investigated for both fresh and heat-treated concrete samples.

\section{Heat Treatment Applied to ESF Concrete Samples}

Concrete samples were heated (dry, and in the presence water) to alter the concrete to forms more likely to exist at the end of the dryout and rewetting periods. Although these laboratory treatments are not intended to closely reproduce actual NFE conditions, the treated samples are a more valid representation of concrete evolution in the repository than is fresh, untreated concrete. Both intact concrete (with artificial fractures) and crushed concrete were heat-treated according to the steps shown in Figure 6-38. 
Figure 6-38 Flow chart showing the treatments applied to ESF concrete samples

\section{Characterization of Fresh and Hydrothermally Altered Concrete Samples}

Samples of untreated and hydrothermally treated concrete were analyzed by X-ray diffraction and scanning electron microscopy. Results from these analyses (Table 6-7) indicate that the major mineralogical changes caused by the hydrothermal treatment are disappearance of portlandite $\left[\mathrm{Ca}(\mathrm{OH})_{2}\right]$ and the appearance of calcium-silicate-hydrate phases scawtite $\left[\mathrm{Ca}_{7} \mathrm{Si}_{6}\left(\mathrm{CO}_{3}\right) \mathrm{O}_{18}-2 \mathrm{H}_{2} \mathrm{O}\right]$, tobermorite $\left[\mathrm{Ca}_{5} \mathrm{Si}_{6}(\mathrm{OH})_{2}-4 \mathrm{H}_{2} \mathrm{O}\right]$, and xonotlite $\left[\mathrm{Ca}_{6} \mathrm{Si}_{6} \mathrm{O}_{17}(\mathrm{OH})_{2}\right]$, plus clay mineral phases with the 2:1 layer structure (smectite) and 1:1 layer structure (serpentine).

Table 6-7 Phases identified by X-ray diffraction of untreated and hydrothermally altered concrete

\begin{tabular}{|c|c|c|}
\hline Phase & Untreated Concrete & $\begin{array}{c}\text { Hydrothermally Treated } \\
\text { Concrete }\end{array}$ \\
\hline \hline Dolomite & major* & major \\
\hline Calcite & Major & major \\
\hline Quartz & Minor & mot observed \\
\hline Portlandite & Major & minor \\
\hline Scawtite & not observed & minor \\
\hline Tobermorite & not observed & minor \\
\hline Xonotlite & not observed & minor \\
\hline Smectite & not observed & not observed \\
\hline \multicolumn{2}{|c|}{ Serpentine } & Major, minor-qualitative assessment of the relative abundance of the phase based on X-ray peak heights
\end{tabular}

It should be noted that, because the aggregate used for the ESF invert is magnesium- and carbonate-rich (dolomitic limestone was used for the aggregate), the scawtite, serpentine, and smectite phases might not form if the aggregate is crushed tuff, as indicated in the reference repository design. The formation of clays, especially smectite, is expected to significantly alter the sorptive capacity of concrete for cationic radionuclides.

\subsubsection{Batch Sorption Experiments with Hydrothermally Altered Concrete}

Batch experiments were used for initial measurements of $U$ and Np sorption on hydrothermally altered concrete. A subsample of the crushed, altered concrete was pulverized to $45-53 \mu \mathrm{m}$. Sorption measurements were conducted in an argon glove box at room temperature $\left(23 \pm 2^{\circ} \mathrm{C}\right)$ with a $\mathrm{M} / \mathrm{V}$ ratio of $4 \mathrm{~g} / \mathrm{L}$. Initial concentrations of $\mathrm{U}$ and $\mathrm{Np}$ ranged from $10^{-7}$ to $10^{-5} \mathrm{M}$ in $0.01-\mathrm{M} \mathrm{NaCl}$. Concentrations of $\mathrm{U}$ and $\mathrm{Np}$ in the aqueous phase were determined using a liquid scintillation counter $\left({ }^{233} \mathrm{U}\right.$ and ${ }^{237} \mathrm{~Np}$ tracers).

\section{Determination of Ambient $\mathrm{pH}$}

A 0.1 -g aliquot of concrete was mixed with $25 \mathrm{~mL}$ of $0.01-\mathrm{M} \mathrm{NaCl}$ solution, and the $\mathrm{pH}$ in the aqueous phase was monitored at $24 \mathrm{hr}$, at $48 \mathrm{hr}$, and after a week. The $\mathrm{pH}$ stabilized at $10.8 \pm 0.1$ after $48 \mathrm{hr}$. 


\section{Sorption Isotherms}

Twenty-five $\mathrm{mL}$ of $0.01-\mathrm{M} \mathrm{NaCl}$ containing $\mathrm{U}(\mathrm{VI})$ and $\mathrm{Np}(\mathrm{V})$ at concentrations ranging from $10^{-7}$ to $10^{-5} \mathrm{M}$ were mixed with $0.1 \mathrm{~g}$ of the concrete in a reaction vessel. Two samples were taken from each reaction vessel for analysis of $U$ and $\mathrm{Np}$ after 5 days of equilibrium. One sample was an aliquot of $2 \mathrm{~mL}$ of supernatant withdrawn from near the liquid surface. The other sample was an aliquot of $1.9 \mathrm{~mL}$ filtered through a syringe filter with a pore size of $20 \mathrm{~nm}$. The $\mathrm{U}$ and $\mathrm{Np}$ concentrations were compared to the spiked starting solution, and the distribution ratios of $\mathrm{U}(\mathrm{VI})$ and $\mathrm{Np}(\mathrm{V})$ were calculated using

$$
K_{\mathrm{d}}=\left(\frac{A_{\mathrm{i}}-A}{m}\right)\left(\frac{V}{A}\right)
$$

where $A_{\mathrm{i}}$ and $A$ are activities $(\mathrm{cpm} / \mathrm{mL})$ in an initial and supernatant sample respectively, $m$ is the mass of concrete $(\mathrm{g})$, and $V$ is the volume of liquid used $(\mathrm{mL})$.

\section{Measurements of Partition Coefficient vs. $\mathrm{pH}$}

Twenty-five $\mathrm{mL}$ of $0.01-\mathrm{M} \mathrm{NaCl}$ containing $10^{-5} \mathrm{M} \mathrm{U}(\mathrm{VI})$ or $\mathrm{Np}(\mathrm{V})$ were introduced into reaction vessels with $0.1 \mathrm{~g}$ of concrete. The $\mathrm{pH}$ was adjusted to 9.8, 10.3, 11.3, and 11.8 using $6-\mathrm{M} \mathrm{HCl}$ or 1-M NaOH. Filtered and unfiltered samples were taken from each reaction vessel and counted for analysis of $\mathrm{U}$ and $\mathrm{Np}$ after four days of reaction time.

\section{Sorption Isotherms at Different Values of $\mathrm{pH}$}

The isotherms based on filtered and unfiltered samples (Figure 6-39) indicate that $U$ and Np strongly partition to the solid phase. For three $U$ and for all the $\mathrm{Np}$ samples, partitioning to the solid was so strong that concentrations in the filtered samples were at or below detection limits. The isotherms are nonlinear, but the reconnaissance nature of these measurements precludes fitting the data to a specific isotherm model. A linear fit, forced through the origin, is used to estimate the average $K_{\mathrm{d}}$ which varies from $12 \times 10^{3} \mathrm{~mL} / \mathrm{g}$ for the unfiltered samples to $90 \times 10^{3} \mathrm{~mL} / \mathrm{g}$ for the filtered samples. The large $K_{\mathrm{d}}$, even for the unfiltered samples, suggest that altered concrete is an effective sorbent. Conservative $K_{d}$ for $U$ and $\mathrm{Np}$ on this material would be on the order of $10^{4} \mathrm{~mL} / \mathrm{g}$ at this $\mathrm{pH}$.

Figure 6-39 Batch sorption isotherms $\left(26^{\circ} \mathrm{C} ; \mathrm{pH} 10.8\right)$ based on analysis of filtered and unfiltered supernatants for $\mathrm{U}$ and $\mathrm{Np}$ on hydrothermally altered crushed concrete

$K_{\mathrm{d}}$ vs. pH

As shown in Figure 6-40, partitioning between solution and solid is $\mathrm{pH}$-dependent. The measured $K_{\mathrm{d}}$ varies in the $\mathrm{pH}$ range 9.8 to 11.8 by more than 1 order of magnitude for $U$ and as much as 4 orders of magnitude for $\mathrm{Np}$. Both filtered and unfiltered samples exhibit the same dependence on $\mathrm{pH}$. Uranium exhibits a sorption maximum between $\mathrm{pH} 10.5$ and 11, and $\mathrm{Np}$ sorption increases monotonically with $\mathrm{pH}$.

Figure 6-40 Partition coefficient $K_{\mathrm{d}}$ vs. $\mathrm{pH}$ for $\mathrm{U}$ and $\mathrm{Np}$ on hydrothermally altered concrete 


\section{Solubility Constraints}

Preliminary solubility calculations suggest that, for a system in which $P_{\mathrm{CO}_{2}}$ is controlled by equilibrium with calcite, $\mathrm{Ca}$ is controlled by equilibrium with calcium silicate hydrate (e.g., gyrolite), Si is controlled by equilibrium with a silica polymorph (e.g., quartz or amorphous silica), and the predicted $\mathrm{Np}$ and $\mathrm{U}$ concentrations are at or below the measured (filtered) concentrations. The predicted solubility-controlling phases are $\mathrm{CaUO}_{4}$ and haiweeite (for $\mathrm{U}$ ) and $\mathrm{NpO}_{2}$ (for $\mathrm{Np}$ ). For these equilibrium solubility limits, the predicted $\mathrm{CO}_{2}$ fugacity is quite low: on the order of $10^{-8} \mathrm{~atm}$. Although $P_{\mathrm{CO}_{2}}$ was not explicitly controlled, the experiments were done in an argon glove box, thus it is expected that $P_{\mathrm{CO}_{2}}$ was very small. Solubility calculations show that the concentrations of $U$ and $N p$ controlled by equilibrium with these phases should increase monotonically with $\mathrm{pH}$, which is contrary to the observed behavior; thus, sorption is inferred instead of precipitation (Figure 6-40).

Calcium-uranium-oxide phases have been suggested as potential solubility-controlling phases for $U$ in fresh concretes and a natural alkaline spring water. Serne et al. (1996) suggest the $\mathrm{U}$ concentration in Portland cement pastes is controlled by a phase with the stoichiometry of $\mathrm{CaUO}_{4}$, but with greater solubility (an amorphous and/or hydrous form). Atkins et al. (1988) suggest that a poorly crystalline phase with the stoichiometry of $\mathrm{Ca}_{2} \mathrm{UO}_{5}\left(\mathrm{H}_{2} \mathrm{O}\right)_{1.3-1.7}$ may control $U$ concentration in fresh cements. Both studies suggest that $U$ concentration is controlled at a greater concentration than is predicted for solubility equilibrium with crystalline $\mathrm{CaUO}_{4}$. Analysis of alkaline spring waters ( $\mathrm{pH} 12.6$ to 12.9) derived from calciumsilicate-hydrate-containing rocks of the Maqarin area of Jordan (a natural analog for lowtemperature alteration of fresh concrete) shows that $U$ concentrations are greater than predicted based on $\mathrm{CaUO}_{4}$ equilibrium, but less than that predicted for equilibrium with Uoxide and hydroxide phases (Linklater et al., 1996). Linklater et al. (1996) suggest that amorphous Ca-U oxides and/or carbonates are the likely solubility-controlling phases in these groundwaters.

Relatively small increases in the $P_{\mathrm{CO}_{2}}$ (e.g., to a fugacity of $10^{-6}$ ) are predicted to increase the concentrations of $\mathrm{U}$ and $\mathrm{Np}$ in equilibrium with $\mathrm{CaUO}_{4}$ and $\mathrm{NpO}_{2}$ to values at or above the experimentally measured concentrations of these elements. To ascertain the role of precipitation in controlling the observed concentrations of $U$ and $\mathrm{Np}$ in these experiments, lower detection limits are needed, dissolved carbonate should be measured at experimental conditions, and the effect of $\mathrm{pH}$ should be examined intensively.

\section{Surface Complexation}

Because the observed variation of $U$ and $\mathrm{Np}$ partitioning with $\mathrm{pH}$ was contrary to that expected for solubility control by simple oxide phases, $\mathrm{U}$ and $\mathrm{Np}$ may be adsorbed onto the concrete through a surface-complexation mechanism. It has been shown that sorption of iodide and $\mathrm{Cs}^{+}$onto fresh Portland cement is dependent on $\mathrm{pH}$ and on the cement $\mathrm{Ca} / \mathrm{Si}$ ratio and that the variation in sorption of these elements could qualitatively be described by a diffuse-layer, surface-complexation model (Heath et al., 1996). Preliminary DLM binding constants for $\mathrm{U}, \mathrm{Np}, \mathrm{Pu}, \mathrm{Am}$, and $\mathrm{Sn}$ on high-ratio $\mathrm{Ca} / \mathrm{Si}$ cements were also reported by these authors, but confirmation of the corresponding $\mathrm{pH}$ dependence was not presented.

\section{Effect of Colloids}

The experiments presented here showed that filtered samples for both $\mathrm{U}$ and $\mathrm{Np}$ had significantly lower activities than did unfiltered samples and that, for $\mathrm{Np}$, all the detectable activity was associated with the particulate fraction except for the $10^{-5} \mathrm{M} \mathrm{Np}$ batches. Because the experiments used crushed and ground concrete that was shaken with the reaction 
solution, the quantity of particulate matter in the supernatants and the particle sizes are probably much larger than would be expected in groundwater passing through an altered, but generally intact concrete. The particles retained by filtration were not directly examined, but they probably were composed primarily of calcium silicate hydrate minerals, clays, and unreacted Ca-Si-hydrate gels. Importantly, even with the significant particulate load in the aqueous phase, the measured value of $K_{d}$ for unfiltered samples were quite large, thus retardation can be estimated conservatively.

\subsubsection{Transport Experiment with Hydrothermally Altered Concrete}

Experiments were conducted at ambient room temperature $\left(21^{\circ} \mathrm{C}\right)$ to study the transport of iodide, $\mathrm{NpO}_{2}{ }^{+}$, and $\mathrm{UO}_{2}{ }^{2+}$ through a fractured core of hydrothermally altered concrete.

A core sample containing one major longitudinal fracture was hydrothermally treated as described previously, machined to $5 \mathrm{~cm}$ in length, and installed in a permeameter device. The apparatus permitted tracer introduction upstream from the core at either constant-flow or at constant-pressure conditions (Viani and Martin, 1994) (Figure 6-41). The jacketed core was placed under confining pressure ( 35 bar) to prevent flow along the sides.

Filtered $\mathrm{NaCl}(0.01 \mathrm{M})$ was used as the permeant for all tracer experiments. Flow rate was constant at $1 \mathrm{~mL} / \mathrm{hr}$, and an automatic fraction collector was used to collect $0.25-$ to $5-\mathrm{mL}$ samples of the effluent for chemical analysis. Separate pulses of iodide, Np, and U tracers were injected (Table 6-8). Effluent samples were analyzed for iodide using a specific ion electrode and were analyzed for $\mathrm{U}$ and $\mathrm{Np}$ using alpha liquid-scintillation spectroscopy. The apparent permeability for the different experiments varied between 0.4 and 1.0 millidarcy, but the variation within each experiment was less than $10 \%$.

Figure 6-41 Schematic drawing of apparatus employed for experiments using intact, fractured, hydrothermally altered concrete core samples

Table 6-8 Tracer experiments with fractured, altered concrete core ${ }^{*}$

\begin{tabular}{|l|c|c|c|c|c|}
\hline Tracer & Run Date & $\begin{array}{c}\text { Pulse } \\
\text { Concentration } \\
(\mathbf{M})\end{array}$ & $\begin{array}{c}\text { Pulse } \\
\text { Volume } \\
(\mathbf{m L})\end{array}$ & $\begin{array}{c}\text { Effluent } \\
\text { Analyzed } \\
(\mathbf{m L})\end{array}$ & $\begin{array}{c}\text { Average } \\
\text { Permeability } \\
\text { (millidarcy) }\end{array}$ \\
\hline \hline lodide & $11 / 13 / 97$ & $7.5 \times 10^{-4}$ & 0.56 & 40 & $0.92 \pm 0.08$ \\
& $12 / 19 / 97$ & & 85 & $0.37 \pm 0.02$ \\
\hline $\mathrm{NpO}_{2}^{+}$ & $12 / 31 / 97$ & $2.599 \times 10^{-6}$ & 5.06 & 120 & $0.47 \pm 0.04$ \\
\hline $\mathrm{UO}_{2}^{2+}$ & $01 / 09 / 98$ & $2.10 \times 10^{-6}$ & 5.06 & 90 & $0.68 \pm 0.05$ \\
\hline
\end{tabular}

lodide Tracer

Although iodide can be used as a conservative tracer for tuff and most sedimentary rocks (Viani and Carman, 1996), batch sorption data for cementitious materials (not hydrothermally treated) indicate that iodide may not be conservative ( $K_{\mathrm{d}}$ values between 10 and $600 \mathrm{~mL} / \mathrm{g}$ ) (Atkins and Glasser, 1990; Baker et al., 1994; Heath et al., 1996). In future experiments, sorption of iodide will be independently assessed by batch studies using hydrothermally altered and untreated ESF concrete.

Pulses of NaI solution were injected upstream (Figure 6-42), and breakthrough occurred within $3 \mathrm{~mL}$ following injection. The breakthrough was similar to that previously observed for a sawcut sample of Topopah Spring tuff (Viani and Carman, 1996), except that dispersion and/or matrix diffusion resulted in a broader peak and longer "tailing" (Figure 6-42). 
$\begin{array}{ll}\text { Figure 6-42 } & \begin{array}{l}\text { Relative concentration of iodide }\left(\mathrm{C} / \mathrm{C}_{0}\right) \text { in } 0.01-\mathrm{M} \mathrm{NaCl} \text { effluent from } \\ \text { fractured, hydrothermally altered concrete core for two pulses of } \mathrm{NaI}\end{array}\end{array}$

Two iodide pulses were injected five weeks apart, over which time several hundred $\mathrm{mL}$ of $\mathrm{NaCl}$ had passed through the core under discontinuous flow conditions. Breakthrough for the second iodide pulse was significantly broader than for the first, possibly representing physical alteration of the sample. Lower apparent permeability also implied physical alteration.

Recovery of the iodide eluted from the sample was estimated by integration, after subtracting the small but measurable background level $\left(\sim 10^{-6} \mathrm{M}\right)$ derived from the concrete. Approximately $93 \%$ of the injected I was recovered after $40 \mathrm{~mL}$ of carrier fluid was eluted from the first iodide pulse and 109\% was recovered after $85 \mathrm{~mL}$ was eluted following the second pulse.

\section{Np and U Tracers}

Neptunium was not observed above the detection limit in the $210 \mathrm{~mL}$ eluted from the sample after injection of the pulse. A U pulse was injected when $120 \mathrm{~mL}$ was eluted after $\mathrm{Np}$ injection. Uranium was not detected in the first $90 \mathrm{~mL}$ eluted after injection. If $\mathrm{U}$ and $\mathrm{Np}$ precipitated in the sample, and their solubility is lower than analytical detection limits, continued elution would never produce observable breakthrough. If sorption controls the eluted $\mathrm{U}$ and $\mathrm{Np}$ concentrations and is reversible, breakthrough is possible. However, based on the relatively large $K_{d}$ values measured in batch studies, this could require elution of many hundreds of pore volumes.

It is apparent that the particulate-associated activity measured in the batch-sorption experiments is not significant for $\mathrm{Np}$ and $\mathrm{U}$ transport through the fractured core sample. Colloids, if present, did not carry $\mathrm{U}$ and $\mathrm{Np}$ at bulk concentrations above the detection limit, as was the case in the batch studies. This observation tends to confirm the hypothesis that particles suspended in the supernatant of the batch studies were larger and more concentrated than would be expected to occur without artificial comminution.

\section{Effluent $\mathrm{pH}$}

Before injection of the $\mathrm{Np}$ and $\mathrm{U}$ pulses, the effluent $\mathrm{pH}$ was about 10.5; after injection, the $\mathrm{pH}$ stabilized at about 8.5. Acidity contained in the tracer solutions $(5 \mathrm{~mL}$ at $\mathrm{pH} 3.89$ and 4.45 for $\mathrm{Np}$ and $\mathrm{U}$, respectively) neutralized the alkalinity in the pore fluid. The $\mathrm{pH}$ may be controlled by calcite in equilibrium with atmospheric $\mathrm{CO}_{2}$. Alternatively, if irreversible dissolution of calcium silicate hydrates and/ or unreacted Ca-Si-hydrate gel is significant, the fluid $\mathrm{pH}$ may increase during periods of reduced flow or no flow. The processes controlling $\mathrm{pH}$ in laboratory experiments with altered concrete will be investigated in future experiments.

\subsubsection{Gaps in Experimental Data and Modeling}

Published experimental investigations of the interaction of radionuclides with cementitious materials have been focused mostly on fresh, unaltered materials that are probably not appropriate analogs for concrete in the NFE at the time of future radionuclide release. Additional experimental work is needed to identify the radionuclide solubilitycontrolling phases and/or sorption mechanisms for altered concrete. This would be made easier if the range of expected phases in concrete were better constrained.

Although preliminary modeling of surface complexation has been used to explain adsorption of radionuclides on fresh cements (Heath et al., 1996), the applicability of such models to fresh or altered concrete has not been tested. Investigation of surface complexation 
will require additional experiments with lower detection limits. Experiments using simpler systems (i.e., single cement phases) are needed to discern chemically mechanistic sorption parameters (such as the parameters for iron oxides) for surface-complexation models.

In the absence of a mechanistic model for retardation (e.g., solubility or sorption models), $K_{d}$ measurements for a range of solution compositions and concrete compositions could be undertaken. In particular, the role of $\mathrm{CO}_{2}$ in radionuclide transport at high $\mathrm{pH}$ has not been addressed. Transport and sorption experiments using carbonate-bearing solutions are needed.

Additional experiments should be conducted using concrete having the composition proposed for the reference repository design (e.g., tuff aggregate instead of limestone).

\subsubsection{Conclusions for Transport Through Cementitious Materials}

Hydrothermal alteration of concrete decreases the pore fluid $\mathrm{pH}$ (relative to fresh concrete) and increases the relative abundance of crystalline Ca-Si-hydrates and clay minerals. The altered concrete examined in these experiments strongly retards $\mathrm{U}$ and $\mathrm{Np}$, either by sorption or solubility limits. Iodide was not retarded. Neptunium and U were not detected in the effluent from a transport experiment conducted with an altered, fracture core of concrete from the ESF. Measurable fractions of the $\mathrm{U}$ and $\mathrm{Np}$ in batch experiments were associated with particulate matter, but both the dissolved and particulate fractions were below detection in transport experiments.

\subsection{Radionuclide Transport in the Altered Zone}

Transport from the boundary of the NFE through the AZ is addressed in this report because AZ transport characteristics will be changed by hydrothermal alteration and by the downgradient effects from leaching of introduced materials in the near field. A comprehensive model describing transport in the unsaturated zone (UZ) for ambienttemperature, unaltered conditions has been published (TRW, 1997). An analysis that minimized the repository performance implications of actinide transport at elevated temperature was included with that model, but the effects of introduced materials and coupled processes on the transport-related properties of the host rock were not addressed.

Current understanding of thermally driven coupled processes, and the effects of introduced materials on the near-field geochemical environment, lead to the identification of several aspects of near-field performance that could significantly change the application of the UZ transport model (TRW, 1997) to the AZ downgradient from the NFE:

- $\quad$ THC processes during the thermal period will change the hydrologic and chemical characteristics of fracture flow paths downgradient from the repository. Silica polymorphs and other mineral species will form in fractures where boiling occurs during the thermal period. Dissolution and precipitation of secondary silicates will occur along pathways for condensate drainage and may increase or decrease fracture apertures and reactive mineral surface area. Thermally driven liquid reflux will very likely inhibit fracture-matrix hydrologic interaction in the host rock.

- Thermomechanical (TM) conditions during heating and cool-down of the host rock will affect hydrologic conditions and may also affect chemical conditions by exposing fresh mineral surfaces.

- According to the reference repository design, large amounts of concrete will be used in the repository emplacement drifts, and leachate from cementitious materials will affect transport pathways because of elevated $\mathrm{pH}$ and the associated dissolution and precipitation reactions. 
- Colloids will be generated from introduced materials, especially from iron corrosion products, which have high affinity for actinides. Whereas it is likely that colloidal particles will exist and be capable of transporting radionuclides through the corrosion layer, the magnitude of radionuclide transport via these mechanisms has not been determined. If corrosion products serve as a source for pseudocolloids, breakthrough at the boundary of the corrosion layer may occur sooner, and at greater total radionuclide concentrations, than discussed here.

In view of these perturbations on the UZ site-scale transport model, additional information is needed to estimate radionuclide mobility. The basic approach is to bound the aqueous concentrations of radionuclides in the AZ using solubility limits and to bound the contributions from alternative transport modes, including various types of colloids. There is significant uncertainty with respect to the importance of colloidal transport. This approach ignores sorptive activity along transport pathways in the $\mathrm{AZ}$, and this activity may increase relative to prerepository conditions because of secondary minerals formed by hydrothermal alteration.

The following sections describe the status of thermodynamic data used to calculate radionuclide solubility and also describe the status of investigations to assess the importance of alternative modes of transport.

\subsubsection{Thermodynamic Constraints on Water-Borne Radionuclide Concentrations}

\section{by Cynthia E.A. Palmer and David A. Wruck}

Aqueous transport is the most likely scenario for migration of nonvolatile radionuclides from a repository to the accessible environment (Silva and Nitsche, 1995). Solubility limits are important controls on radionuclide concentrations in water leaving the NF/ AZ and represent defensible upper bounds for noncolloidal concentrations. Solubility refers to the equilibrium total dissolved concentration of a radioelement (e.g., actinide or fission product) in a solvent (groundwater) at a specified set of conditions such as temperature, pressure, $\mathrm{pH}, \mathrm{CO}_{2}$ fugacity, and ionic strength.

Solubility calculations are performed using a thermodynamic model such as EQ3/6 (see Section 5.1) with an appropriate database. A thermodynamic database is a summary of measured thermodynamic constants for aqueous species, solids, and gases. Ideally, solubility measurements should be carried out from both oversaturation and undersaturation to demonstrate that equilibrium, rather than steady state, was attained. For unknown chemical systems, oversaturation experiments can reveal the solubility-controlling solid under steadystate conditions. Experiments are possible from undersaturation if the solid phase can be identified and synthesized.

A thermodynamic database is evaluated by testing predictions of solution species, secondary phases, and total concentrations against controlled laboratory experiments. To provide relevant input, experiments should address ranges of temperature, ionic strength, redox conditions, and solution composition.

In 1984, the Nuclear Energy Agency (NEA) initiated the preparation and publication of a series of critical reviews of the chemical thermodynamics of those elements that are of particular importance in radioactive waste disposal. The United States has participated through the Yucca Mountain Site Characterization Project and Lawrence Livermore National Laboratory. The NEA assembles teams of chemists internationally recognized in actinide and physical chemistry. Team members evaluate reported experiments and decide whether each measurement warrants inclusion in a final volume. Written comments are included in the review. A second panel is convened to verify the conclusions of the first. 
Interpretation of chemical thermodynamic data for the actinides and fission products requires the use of auxiliary data such as acid dissociation constants. These data must be internally consistent, and it is therefore necessary to always use the same auxiliary data with the NEA databases. The NEA project selected the database from the Committee on Data for Science and Technology as the principal source of auxiliary data. These data were evaluated as part of the NEA uranium review.

In 1992, the NEA published the volume, Chemical Thermodynamics of Uranium (NEA, 1992). In 1995, the NEA published the volume, Chemical Thermodynamics of Americium (NEA, 1995). At present, two additional volumes are in the review, namely the Chemical Thermodynamics of Neptunium and Plutonium and the Chemical Thermodynamics of Technetium. A new phase of the NEA review process is being initiated. At this time, it is anticipated that the initial volumes will be updated as needed and that additional reviews will be undertaken for zirconium and selenium or nickel.

Efforts to develop thermodynamic data for Yucca Mountain repository safety analysis have focused on providing the GEMBOCHS database (see Chapter 5) with data for the radionuclide solubility-limiting solid phases for water compositions similar to J-13 water. However, water composition in the NFE may depart significantly from J-13 water, and releases may occur when the temperature in the NFE (or inside the WP) is significantly elevated. Accordingly, there are gaps in the available thermodynamic data. A review of the status of thermodynamic data available for repository performance assessment and discussion of issues related to elevated temperature and alkaline $\mathrm{pH}$ are presented in the following sections.

\subsubsection{Status of Available Solubility Data}

\section{Reference Groundwater}

The chemical composition of groundwater samples from the Yucca Mountain region has been discussed in Palmer et al. (1996). The water from well J-13 is thought to be representative of interstitial and fracture waters in the Yucca Mountain region and is used as a reference water in this report. The effect of temperature on the composition of J-13 water in contact with Yucca Mountain tuff has been investigated in several studies (Knauss and Beiriger, 1984; Knauss et al., 1985a, 1987, 1985b; Knauss and Peifer, 1986; Oversby, 1985). There are only minor changes in solution composition over the temperature range of $25^{\circ}$ to $150^{\circ} \mathrm{C}$. As the temperature is raised, there is an increase in the dissolved Si concentration and slight decreases in the dissolved $\mathrm{Mg}, \mathrm{Ca}$, and carbonate concentrations. The J-13 water composition at $25^{\circ} \mathrm{C}$, and suggested maximum concentrations in interstitial and fracture waters at elevated temperatures (Glassley, 1986), are summarized in Table 6-9 and Table 6-10.

Table 6-9 Cation concentrations (mM) in the reference water

\begin{tabular}{|l|c|c|c|c|c|c|c|c|c|c|}
\hline & $\mathbf{L i}$ & $\mathbf{~ N a}$ & $\mathbf{K}$ & $\mathbf{M g}$ & $\mathbf{C a}$ & $\mathbf{A l}$ & $\mathbf{S i}$ & $\mathbf{M n}$ & $\mathbf{F e}$ & $\mathbf{p H}$ \\
\hline \hline $\mathrm{J}-13$ & 0.009 & 1.96 & 0.136 & 0.072 & 0.29 & 0.001 & 0.92 & 0.00002 & 0.0008 & 6.9 \\
\hline High T & - & $<2.8$ & $<0.38$ & $<0.21$ & $<0.37$ & $<0.19$ & $<5.7$ & - & - & $6.9-7.6$ \\
\hline
\end{tabular}


Table 6-10 Anion concentrations (mM) in the reference water

\begin{tabular}{|l|l|l|l|l|l|c|}
\hline & \multicolumn{1}{|c|}{$\mathbf{F}^{-}$} & \multicolumn{1}{|c|}{$\mathbf{C l}^{-}$} & \multicolumn{1}{|c|}{$\mathbf{N O}_{3}{ }^{-}$} & $\mathbf{H C O}_{3}{ }^{-}$ & $\mathbf{S O}_{4}{ }^{2-}$ & $\mathbf{O}_{2}$ \\
\hline \hline $\mathrm{J}-13$ & 0.11 & 0.18 & 0.16 & 2.34 & 0.19 & 0.18 \\
\hline High T & $<0.26$ & $<0.28$ & $<0.24$ & $<2.30$ & $<0.26$ & - \\
\hline
\end{tabular}

\section{Temperature Extrapolations}

Knowledge of the solubility-controlling solid phases is an important step in understanding the geochemical behavior of an element. Initially, relevant solids may be identified through solubility experiments conducted from oversaturation. This approach will not always be successful; for example, it may be difficult to identify the solid due to the presence of amorphous or mixed phases, or the time to reach equilibrium may be very long. Even when solubility-controlling solids can be identified through oversaturation experiments, one generally has data at only one or a few fixed temperatures. To model equilibrium reactions at an arbitrary temperature, the equilibrium constants must be extrapolated to the temperature of interest.

Temperature extrapolations of chemical equilibrium data, including solubility products, are described in detail in Langmuir (1997) and Puigdomenech et al. (1997). It is rare to have complete information on the temperature dependence of thermodynamic functions for the reactions of interest; thus, approximation methods must be used to predict the equilibrium constants, such as solubility products and complex stability constants, over the temperature intervals of interest. The integrated van't Hoff equation is useful over small temperature ranges (about $10 \mathrm{~K}$ or less):

$$
\log K^{0}(T)=\log K^{0}\left(T_{0}\right)+\frac{\Delta H^{0}\left(T_{0}\right)}{2.303 R}\left(\frac{1}{T_{0}}-\frac{1}{T}\right)
$$

Here, $R$ is the ideal gas constant $8.3145 \mathrm{~J} \mathrm{~mol}^{-1} \mathrm{~K}^{-1}$. Included in Eq. 6-2 is the assumption that the enthalpy of reaction $\Delta H_{0}$ is constant over the temperature range, and there is no change in heat capacity. Eq. 6-2 is applicable over a larger temperature range, approximately $20^{\circ}$ to $200^{\circ} \mathrm{C}$, when the reaction is isoelectric; that is, the sum of positive charges among the reactants equals the sum of positive charges among the products, and the sum of negative charges among the reactants equals the sum of negative charges among the products (Puigdomenech et al., 1997).

If the change in heat capacity $\Delta C_{\mathrm{v}}{ }^{0}$ is assumed to be a nonzero constant over the temperature range $T_{0}$ to $T$, the equation for the equilibrium constant becomes

$$
\log K^{0}(T)=\log K^{0}\left(T_{0}\right)+\frac{\Delta H^{0}\left(T_{0}\right)}{2.303 R}\left(\frac{1}{T_{0}}-\frac{1}{T}\right)+\frac{\Delta C_{p}^{0}}{2.303 R}\left(\frac{T_{0}}{T}-1+\ln \frac{T}{T_{0}}\right)
$$

In the next level of approximation, an empirical expression is used to describe the temperature dependence of $\Delta C_{\mathrm{v}}{ }^{0}$. However, such expressions are rarely available for the reactions of interest. Values of $\Delta H_{0}$ and $\Delta C_{\mathrm{v}}{ }^{0}$ can be determined by calorimetric measurements at two or more temperatures or by direct measurements of the equilibrium constant at several temperatures. 


\section{Uranium}

The estimated $\mathrm{U}$ solubility in $\mathrm{J}-13$ water at $25^{\circ} \mathrm{C}$ is about $10^{-4} \mathrm{M}$ (Kerrisk, 1984), and the principal aqueous species are $\mathrm{UO}_{2}\left(\mathrm{CO}_{3}\right)_{2}{ }^{2-}, \mathrm{UO}_{2}\left(\mathrm{CO}_{3}\right)_{3}{ }^{4-}$, and $\mathrm{UO}_{2} \mathrm{CO}_{3}{ }^{0}$ (Palmer et al., 1992) Potential solubility-controlling solids include schoepite $\left(\mathrm{UO}_{3} \cdot 2 \mathrm{H}_{2} \mathrm{O}\right.$ or $\left.\mathrm{UO}_{2}(\mathrm{OH})_{2} \cdot \mathrm{H}_{2} \mathrm{O}\right)$, rutherfordine $\left(\mathrm{UO}_{2} \mathrm{CO}_{3}\right)$, the sodium uranates, and $\mathrm{Na}_{4} \mathrm{UO}_{2}\left(\mathrm{CO}_{3}\right)_{3}$. Uranyl silicate $\left(\left(\mathrm{UO}_{2}\right)_{2} \mathrm{SiO}_{4} \cdot 2 \mathrm{H}_{2} \mathrm{O}\right)$ and mixed uranyl silicate phases $\left[\mathrm{Na}\left(\mathrm{H}_{3} \mathrm{O}\right)\left(\mathrm{UO}_{2}\right) \mathrm{SiO}_{4} \cdot \mathrm{H}_{2} \mathrm{O}\right.$, $\left.\mathrm{Na}_{2}\left(\mathrm{UO}_{2}\right)_{2}\left(\mathrm{Si}_{2} \mathrm{O}_{5}\right)_{3} \cdot 7 \mathrm{H}_{2} \mathrm{O}\right]$ may also be significant because of the high $\mathrm{Si}$ concentrations present in J-13 and other waters of the Yucca Mountain region. Under reducing conditions, important solid phases may include uraninite $\left(\mathrm{UO}_{2}\right)$, oxide phases $\mathrm{UO}_{x}$ with $2<x<3$, and the crystalline and amorphous forms of $\mathrm{USiO}_{4}$.

The OECD Nuclear Energy Agency Thermochemical Data Base (NEA-TDB) project has completed a critical review of thermodynamic data for $U$ compounds and species (Grenthe et al., 1992, 1995). Solubility reactions for schoepite, the uranium oxides, and $\mathrm{UO}_{2} \mathrm{CO}_{3}$ are relatively well understood over the temperature range of interest. A detailed discussion of original publications and data selection for these phases is available in the NEA-TDB review and is not be repeated here.

In a review of experimental data up to 1980, thermodynamic functions were calculated for several $\mathrm{U}$ species and compounds over the temperature range $25^{\circ}$ to $200^{\circ} \mathrm{C}$ (Lemire and Tremaine, 1980). The Atomic Energy of Canada Limited (AECL) thermodynamic database used for geochemical modeling of $U$ is derived from Lemire and Tremaine (1980) and subsequent revisions (Lemire and Garisto, 1989, 1992). A comparison of the NEA-TDB and AECL databases indicates that only minor differences exist in predicted U solubilities, based on the two databases (McMurry, 1997).

The dependence of $U$ solubility on ligand concentrations in groundwater has been discussed (Miuahara, 1993). The NEA-TDB data (Grenthe et al., 1992) were used for the evaluation. The $U$ concentration in solution increases very sensitively with increasing carbonate concentration. The temperature dependence was not considered.

Solubility data for uranyl orthosilicate and mixed uranyl silicate solids are available at $25^{\circ} \mathrm{C}$ (Casas et al., 1994; Moll et al., 1996; Nguyen et al., 1991). In a review of thermodynamic data for uranyl silicate minerals to 1995, a plan was outlined for the study of uranophane $\left[\mathrm{Ca}\left(\mathrm{UO}_{2}\right)_{2}\left[\mathrm{SiO}_{3}(\mathrm{OH})\right]_{2} \cdot 5 \mathrm{H}_{2} \mathrm{O}\right]$, soddyite $\left[\left(\mathrm{UO}_{2}\right)_{2} \mathrm{SiO}_{4} \cdot 2 \mathrm{H}_{2} \mathrm{O}\right]$, and schoepite $\left[\mathrm{UO}_{3} \cdot 2 \mathrm{H}_{2} \mathrm{O}\right.$ or $\left.\mathrm{UO}_{2}(\mathrm{OH})_{2} \cdot \mathrm{H}_{2} \mathrm{O}\right]$ solubilities at $25^{\circ}, 60^{\circ}$, and $90^{\circ} \mathrm{C}$ (Murphy and Pabalan, 1995). However, no data were found for the elevated-temperature solubility behavior of uranium silicates and mixed silicates.

Although phosphate concentrations are low in J-13, uranyl phosphate solids have low solubilities and may be significant (Grenthe et al., 1992; Sandino and Bruno, 1992). No data were found for the elevated-temperature solubility behavior of uranium phosphates.

Solubility of the $\mathrm{UO}_{2}$ matrix is an important parameter for predicting the stability of spent nuclear fuel under disposal conditions. The dissolution process depends on the redox conditions in the repository. Solubility experiments have been conducted using unirradiated $\mathrm{UO}_{2}$ pellets under anoxic conditions (Ollila et al., 1996). Steady-state results obtained at $\mathrm{pH}$ 9.0 and temperatures of $27^{\circ}$ to $30^{\circ} \mathrm{C}$ are listed in Table 6-11. Based on the NEA-TDB data (Grenthe et al., 1992), it was determined that $\mathrm{U}_{4} \mathrm{O}_{9}\left(\mathrm{UO}_{2.25}\right)$ was the solubility-controlling solid phase. 
Table 6-11 Steady-state results from Ollila et al. (1996)

\begin{tabular}{|l|l|}
\hline \multicolumn{1}{|c|}{ [U], $\mathbf{M}$} & \multicolumn{1}{c|}{ Medium } \\
\hline \hline $6-9 \times 10^{-9}$ & Deionized water \\
\hline $2 \times 10^{-8}$ & {$\left[\mathrm{CO}_{3}{ }^{2-}\right]: 60-275 \mathrm{ppm}$} \\
\hline $4 \times 10^{-8}$ & {$\left[\mathrm{CO}_{3}{ }^{2-}\right]: 600 \mathrm{ppm}$} \\
\hline $2.0-2.5 \times 10^{-8}$ & Synthetic groundwater \\
\hline
\end{tabular}

The dissolution mechanism of spent $\mathrm{UO}_{2}$ fuel under oxic conditions has also been reported (Grambow et al., 1990). A three-phase model was proposed:

1. The $\mathrm{UO}_{2}$ matrix

2. An oxidized surface such as $\mathrm{U}_{3} \mathrm{O}_{7}$

3. A U(VI)-containing solid alteration product (schoepite in deionized water)

The solubility of amorphous $\mathrm{UO}_{2} \cdot x \mathrm{H}_{2} \mathrm{O}$ has been studied at room temperature in the $\mathrm{pH}$ range 2 to 12 (Rai et al., 1990). The amorphous solid is expected to be metastable with respect to crystalline $\mathrm{UO}_{2}$, but the transformation kinetics may be slow.

The solubility of schoepite, uranium oxides, and $\mathrm{UO}_{2} \mathrm{CO}_{3}$ are relatively well understood under expected repository conditions. There are little or no data on the high-temperature solubility behavior of uranium silicates, uranium phosphates, sodium uranates, and mixed sodium uranium carbonates. Schoepite is generally assumed to be the solubility-controlling phase in systems in equilibrium with atmospheric $\mathrm{CO}_{2}$ at $25^{\circ} \mathrm{C}$ and $\mathrm{pH} 7$, but the carbonate and silicate phases also have low solubilities and may become solubility-controlling at elevated temperatures. Cristobalite in the tuff could be a major source of $\mathrm{SiO}_{2}$ for the formation of uranium silicates. $U$ solubility experiments could be performed from oversaturation in reference waters in contact with tuff at elevated temperatures. Such experiments may help bound the maximum $U$ concentrations at elevated temperatures, and solubility-controlling solid phases may also be identified.

\section{Neptunium}

The solubility and speciation of Np in J-13 and UE-25p1 waters have been investigated in a series of experiments (Nitsche, 1991; Nitsche et al., 1993a, 1995, 1993b). The experiments were conducted from oversaturation, and some of them were also conducted from undersaturation at $\mathrm{pH} 5.9$ to 8.5 and temperatures in the range $25^{\circ}$ to $90^{\circ} \mathrm{C}$. Np was introduced as $\mathrm{NpO}_{2}^{+}$. At steady-state, $\mathrm{NpO}_{2}{ }^{+}$and $\mathrm{NpO}_{2} \mathrm{CO}_{3}{ }^{-}$were the principal aqueous $\mathrm{Np}$ species. At all temperatures investigated, $\mathrm{NpO}_{2}^{+}$was the main species near $\mathrm{pH} 6$, and $\mathrm{NpO}_{2} \mathrm{CO}_{3}^{-}$was the dominant species near $\mathrm{pH} 8.5$.

Steady-state $\mathrm{Np}$ concentrations were in the range of $10^{-5}$ to $10^{-3} \mathrm{M}$, and the concentrations decreased as the $\mathrm{pH}$ increased. Reported solid phases are listed in Table 6-12. The solubilitycontrolling solid changed from a mixed sodium $\mathrm{Np}(\mathrm{V})$ carbonate to $\mathrm{Np}_{2} \mathrm{O}_{5}$ at hightemperature and low-pH values, but the steady-state $\mathrm{Np}$ concentrations showed little temperature-dependence. 
Table 6-12 Solubility-controlling solids from Nitsche, 1991; Nitsche et al., 1993a, $1995,1993 b$

\begin{tabular}{|c|l|l|l|}
\hline pH & \multicolumn{1}{|c|}{$\mathbf{2 5}^{\circ} \mathbf{C}$} & \multicolumn{1}{c|}{$\mathbf{6 0} \mathbf{0}^{\circ} \mathbf{C}$} & \multicolumn{1}{c|}{$\mathbf{9 0}^{\circ} \mathbf{C}$} \\
\hline \hline 5.9 & $\mathrm{Na}_{0.6} \mathrm{NpO}_{2}\left(\mathrm{CO}_{3}\right)_{0.8} \cdot \mathrm{H}_{2} \mathrm{O}$ & $\mathrm{Na}_{2 n-1} \mathrm{NpO}_{2}\left(\mathrm{CO}_{3}\right)_{n} \cdot \mathrm{xH}_{2} \mathrm{O}$ & $\mathrm{Np}_{2} \mathrm{O}_{5}$ \\
\hline 7.1 & $\mathrm{Na}_{0.6} \mathrm{NpO}_{2}\left(\mathrm{CO}_{3}\right)_{0.8} \cdot 2.5 \mathrm{H}_{2} \mathrm{O}$ & $\mathrm{Na}_{2 n-1} \mathrm{NpO}_{2}\left(\mathrm{CO}_{3}\right)_{n} \cdot \mathrm{xH}_{2} \mathrm{O}$ & $\mathrm{Np}_{2} \mathrm{O}_{5}$ \\
& & & $\mathrm{Na}_{2 n-1} \mathrm{NpO}_{2}\left(\mathrm{CO}_{3}\right)_{n} \cdot \mathrm{xH}_{2} \mathrm{O}$ \\
\hline 8.5 & $\mathrm{Na}_{0.6} \mathrm{NpO}_{2}\left(\mathrm{CO}_{3}\right)_{0.8} \cdot 2.5 \mathrm{H}_{2} \mathrm{O}$ & $\mathrm{Na}_{2 n-1} \mathrm{NpO}_{2}\left(\mathrm{CO}_{3}\right)_{n} \cdot \mathrm{xH}_{2} \mathrm{O}$ & $\mathrm{Na}_{2 n-1} \mathrm{NpO}_{2}\left(\mathrm{CO}_{3}\right)_{n} \cdot \times \mathrm{H}_{2} \mathrm{O}$ \\
\hline
\end{tabular}

The relatively high solubility of $\mathrm{Np}(\mathrm{V})$ solids is an important issue for geologic disposal of spent nuclear fuel. If risk calculations are made using the most conservative assumption [i.e., that the $\mathrm{Np}(\mathrm{V})$ solids control the long-term $\mathrm{Np}$ concentrations], calculated dose values exceed proposed regulatory levels (Wolery et al., 1995). Under reducing conditions, the $\mathrm{Np}$ solubility is several orders of magnitude smaller and is controlled by $\mathrm{NpO}_{2} \cdot x \mathrm{H}_{2} \mathrm{O}(x<2)$ or $\mathrm{Np}(\mathrm{OH})_{4}$, as shown in waste-form leaching experiments (Rai et al., 1982; Wilson and Bruton, 1990). It is thought that the $\mathrm{Np}(\mathrm{V})$ phases observed in the oversaturation experiments under oxidizing conditions are metastable with respect to $\mathrm{NpO}_{2}$, a $\mathrm{Np}(\mathrm{IV})$ phase. A better understanding is needed of the $\mathrm{Np}(\mathrm{V}) / \mathrm{Np}(\mathrm{IV})$ transformation kinetics involving the solid phases (Wolery et al., 1995). Continuing work on Np solubility-limiting phases is reported by Runde et. al. (1998a, 1998b).

The solubility of mixed sodium $\mathrm{Np}(\mathrm{V})$ carbonates has been investigated at $30^{\circ}, 50^{\circ}$, and $75^{\circ} \mathrm{C}$ (Lemire et al., 1993). Solid-state conversions, such as $\mathrm{NaNpO}_{2} \mathrm{CO}_{3} \rightarrow \mathrm{Na}_{3} \mathrm{NpO}_{2}\left(\mathrm{CO}_{3}\right)_{2}$ occurred very slowly, were difficult to detect, and complicated the data analysis. The results were analyzed using two models. In one model, it was assumed that $\mathrm{NaNpO}_{2} \mathrm{CO}_{3} \cdot x \mathrm{H}_{2} \mathrm{O}$ was the only equilibrium solid. In the other model, an equilibrium was assumed to occur between $\mathrm{NaNpO}_{2} \mathrm{CO}_{3} \cdot x \mathrm{H}_{2} \mathrm{O}$ and $\mathrm{Na}_{3} \mathrm{NpO}_{2}\left(\mathrm{CO}_{3}\right)_{2} \cdot y \mathrm{H}_{2} \mathrm{O}$. The results suggested the product $\left[\mathrm{NpO}_{2}{ }^{+}\right]\left[\mathrm{CO}_{3}{ }^{2-}\right]$ decreases as the temperature is raised at a fixed $\mathrm{Na}$ concentration.

Experimental and predicted $\mathrm{Np}(\mathrm{V})$ solubilities have been reported for concentrated $\mathrm{NaCl}$ solutions at $25^{\circ} \mathrm{C}$ in Runde et al. (1996) and in salt solutions at ionic strength usually less than 1 (Neck et al., 1992, 1994). The solubility-controlling solids were identified as $\mathrm{NaNpO}_{2} \mathrm{CO}_{3} \cdot x \mathrm{H}_{2} \mathrm{O}$ for $\left[\mathrm{CO}_{3}{ }^{2-}\right]<10^{-3} \mathrm{M}$ and $\mathrm{Na}_{3} \mathrm{NpO}_{2}\left(\mathrm{CO}_{3}\right)_{2} \cdot y \mathrm{H}_{2} \mathrm{O}$ for $\left[\mathrm{CO}_{3}{ }^{2-}\right]>10^{-3} \mathrm{M}$.

The solubility of amorphous $\mathrm{NpO}_{2} \mathrm{OH}$ has been investigated at room temperature (Itagaki et al., 1992; Robert et al., 1996). The results of the two studies are in agreement. The solubility product constant $\log \mathrm{K}^{0}\left(\left[\mathrm{NpO}_{2}^{+}\right]\left[\mathrm{OH}^{-}\right]\right)$was determined to be $-8.68 \pm 0.26$ and $-8.79 \pm 0.12$ in the two studies.

The solubility and speciation of $\mathrm{Np}(\mathrm{V})$ and $\mathrm{Np}(\mathrm{IV})$ in brine solutions has been investigated in long-term experiments (>2000 days) (Silber et al., 1994). The steady-state Np concentrations were $10^{-5}$ to $10^{-6} \mathrm{M}$ in the $\mathrm{pH}$ range 8 to 13 . Radiolysis effects caused the oxidation of $\mathrm{Np}(\mathrm{IV})$ to $\mathrm{Np}(\mathrm{V})$ and increased the apparent $\mathrm{Np}$ solubility with time.

The dissolution of $\mathrm{NpO}_{2}$ has been investigated over a range of $\mathrm{pH}$ values (2 to 6), ionic strengths $(0.001$ and $0.1 \mathrm{~m})$, and temperatures $\left(30^{\circ}\right.$ to $\left.90^{\circ} \mathrm{C}\right)$ in $\mathrm{NaClO}_{4}$ solutions $($ Nakayama and Nagano, 1991). Measured dissolution rates decreased as the $\mathrm{pH}$ increased, and they increased as the temperature increased. Solubility equilibria were not reached in all runs.

Solubility studies of $\mathrm{Np}(\mathrm{IV})$ hydrous oxide in water and $0.1-\mathrm{M} \mathrm{NaClO}_{4}$ have been reported (Nakayama et al., 1996). Measurements were carried out from oversaturation and undersaturation directions at $25^{\circ} \mathrm{C}$ and a $\mathrm{pH}$ range of 5.3 to 13.7 in the presence of reducing 
agents $\left(\mathrm{Na}_{2} \mathrm{~S}_{2} \mathrm{O}_{4}, \mathrm{Fe}\right.$, and $\left.\mathrm{Cu}\right)$. Steady-state $\mathrm{Np}$ concentrations were in the range $10^{-7}$ to $10^{-8} \mathrm{M}$. Very little information was given about the precipitates, but based on the analogous $\mathrm{Pu}(\mathrm{IV})$ system, the results are consistent with formation of $\mathrm{Np}(\mathrm{IV})$ hydrous oxides.

The NEA-TDB review of chemical thermodynamics of $\mathrm{Np}$ and $\mathrm{Pu}$ is in preparation.

The solubility of $\mathrm{Np}$ under expected repository conditions is reasonably well understood for reducing conditions and over short time scales (a few months to one year). As discussed previously, a better understanding of the $\mathrm{Np}(\mathrm{V}) / \mathrm{Np}(\mathrm{IV})$ solids is critically needed. Some experimental work is in progress (Palmer, C.E.A., and T.J. Wolery: Experimental work funded by Laboratory Directed Research and Development Office, Lawrence Livermore National Laboratory, Livermore, CA. However, considering the importance of the $\mathrm{Np}(\mathrm{V}) / \mathrm{Np}(\mathrm{IV})$ issue, more work should be done in this area. Additional experiments of lower priority could include investigations of long-term radiolysis effects and calorimetric measurements of the heats of dissolution of the $\mathrm{Np}(\mathrm{V})$ solids.

\section{Plutonium}

The solubility and speciation of $\mathrm{Pu}$ in J-13 and UE-25p1 waters have been investigated in a series of experiments (Nitsche, 1991; Nitsche et al., 1993a, 1995, 1993b). Experiments were conducted from oversaturation and undersaturation at several $\mathrm{pH}$ values and temperatures. Plutonium was added as $\mathrm{Pu}^{4+}$. At steady-state, the major oxidation state in solution was $\mathrm{Pu}(\mathrm{V})$. The $\mathrm{Pu}$ concentration decreased from about $2 \times 10^{-7} \mathrm{M}$ at $25^{\circ} \mathrm{C}$ to about $8 \times 10^{-9} \mathrm{M}$ at $90^{\circ} \mathrm{C}$. No significant influence of $\mathrm{pH}$ was observed. The precipitates were identified as mixtures of $\mathrm{Pu}(\mathrm{IV})$ hydrous oxide and $\mathrm{Pu}$ carbonates. It was not possible to further identify the solid phases; therefore, solubility product constants were not determined.

It is likely that slow transformations of the Pu solids were significant in the experiments. The observed decrease in $\mathrm{Pu}$ concentrations with increasing temperature may have resulted from the temperature dependence of the reaction rates rather than the temperature dependence of solubility products. Because of the kinetics problem, it may be impossible to uniquely identify the solubility-controlling solid phase. However, such identification is probably not necessary for repository PA, because maximum Pu concentrations will differ by many orders of magnitude, depending on whether $\mathrm{Pu}(\mathrm{V})$ or $\mathrm{Pu}(\mathrm{IV})$ solids are solubilitycontrolling. The measured $\mathrm{Pu}$ concentrations were inconsistent with $\mathrm{Pu}(\mathrm{V})$ solids, such as $\mathrm{NaPuO}_{2} \mathrm{CO}_{3}$ or $\mathrm{PuO}_{2} \mathrm{OH}$.

The solubility and speciation of $\mathrm{Pu}$ introduced as $\mathrm{Pu}^{4+}, \mathrm{PuO}_{2}{ }^{+}$, and $\mathrm{PuO}_{2}{ }^{2+}$ has been investigated in $\mathrm{J}-13$ water at $25^{\circ} \mathrm{C}$ (Nitsche and Edelstein, 1985) and dilute carbonic acid solutions at $\mathrm{pH}=6,0.057$-atm $\mathrm{CO}_{2}$ partial pressure, and $30^{\circ} \mathrm{C}(\mathrm{Neu}, 1993)$. The experiments were monitored for 25 to 150 days. When $\mathrm{Pu}$ was introduced as $\mathrm{Pu}^{4+}$, the $\mathrm{Pu}$ concentrations rapidly decreased to about $10^{-6} \mathrm{M}$. When $\mathrm{Pu}$ was introduced as $\mathrm{PuO}_{2}{ }^{+}$or $\mathrm{PuO}_{2}{ }^{2+}$, steady-state $\mathrm{Pu}$ concentrations on the order of $10^{-5} \mathrm{M}$ were reported. However, the oxidation state distribution of dissolved $\mathrm{Pu}$ was dependent on the initial $\mathrm{Pu}$ oxidation state, so it is likely that the results do not reflect true equilibrium conditions. When $\mathrm{Pu}$ is introduced as $\mathrm{PuO}_{2}^{+}$or $\mathrm{PuO}_{2}{ }^{2+}$, kinetic considerations may cause the initial precipitation of metastable $\mathrm{NaPuO}_{2} \mathrm{CO}_{3}$ hydrate followed by slow conversion to a less-soluble $\mathrm{Pu}(\mathrm{IV})$ hydrous oxide.

Disproportionation of $\mathrm{Pu}(\mathrm{V})$ is very slow under the conditions investigated in Nitsche and Edelstein (1985) and Neu (1993), but studies of $\mathrm{Pu}(\mathrm{V})$ in near-neutral solutions at $75^{\circ} \mathrm{C}$ indicate that the $\mathrm{Pu}$ concentration decreases to $10^{-6} \mathrm{M}$ in less than $18 \mathrm{hr}$ in the presence of carbonate (Wruck and Palmer, 1996).

Solubility studies have been reported for $\mathrm{Pu}$ introduced as $\mathrm{Pu}^{3+}, \mathrm{Pu}^{4+}, \mathrm{Pu}(\mathrm{IV})$ polymer, $\mathrm{PuO}_{2}{ }^{+}$, and $\mathrm{PuO}_{2}{ }^{2+}$ in a synthetic brine solution under oxic conditions (Nitsche et al., 1994). The experiments were performed at room temperature. Steady-state $\mathrm{Pu}$ concentrations were 
in the range $3 \times 10^{-8}$ to $8 \times 10^{-7} \mathrm{M}$. At steady-state, the major Pu oxidation state in solution was $\mathrm{Pu}(\mathrm{VI})$. The following solid phases were observed: for $\mathrm{Pu}(\mathrm{IV})$-polymer, $\mathrm{Pu}(\mathrm{IV})$ hydrous oxide; for $\mathrm{Pu}^{3+}$ and $\mathrm{Pu}^{4+}$, crystalline unidentified compounds; for $\mathrm{PuO}_{2}^{+}$and $\mathrm{PuO}_{2}^{2+}, \mathrm{NaPuO}_{2} \mathrm{CO}_{3}$.

$\mathrm{Pu}(\mathrm{IV})$ polymer [colloidal $\mathrm{Pu}(\mathrm{IV})$ hydrous oxide] is formed during the neutralization of acidic $\mathrm{Pu}(\mathrm{IV})$ solutions. The solubility behavior of $\mathrm{Pu}(\mathrm{IV})$ polymer has been described as intermediate between that of amorphous $\mathrm{Pu}(\mathrm{OH})_{4}$ and crystalline $\mathrm{PuO}_{2}$ (Rai and Swanson, 1981)]. The solubility products of amorphous $\mathrm{Pu}(\mathrm{OH})_{2}$ and crystalline $\mathrm{PuO}_{2}$ at $20^{\circ} \mathrm{C}$ have been determined by Kim and Kanellakopulos (1989), as follows:

$$
\mathrm{Pu}(\mathrm{OH})_{4}(\mathrm{am}) \Leftrightarrow \mathrm{Pu}^{4+}+4 \mathrm{OH}^{-} \log \mathrm{K}=-57.85 \pm 0.05
$$

and

$$
\mathrm{PuO}_{2}(\mathrm{cr})+2 \mathrm{H}_{2} \mathrm{O} \Leftrightarrow \mathrm{Pu}^{4+}+4 \mathrm{OH}^{-} \log \mathrm{K}=-60.20 \pm 0.17
$$

The solubility of amorphous $\mathrm{PuO}_{2} \cdot x \mathrm{H}_{2} \mathrm{O}$, with $x$ near 2 , has been investigated at room temperature (Rai, 1984). The analysis included the Pu redox equilibria in solution. The solubility product results were:

$$
\begin{aligned}
& \mathrm{PuO}_{2} \cdot x \mathrm{H}_{2} \mathrm{O}(\mathrm{am}) \Leftrightarrow \mathrm{Pu}^{4+}+4 \mathrm{OH}^{-}+(x-2) \mathrm{H}_{2} \mathrm{O} \log \mathrm{K}=-56.85 \pm 0.36 \\
& \mathrm{PuO}_{2} \cdot x \mathrm{H}_{2} \mathrm{O}(\mathrm{am}) \Leftrightarrow \mathrm{PuO}_{2}^{+}+\mathrm{e}^{-}+x \mathrm{H}_{2} \mathrm{O} \log \mathrm{K}=-19.45 \pm 0.23
\end{aligned}
$$

and

$$
\mathrm{PuO}_{2} \cdot x \mathrm{H}_{2} \mathrm{O}(\mathrm{am}) \Leftrightarrow \mathrm{PuO}_{2}^{2+}+2 \mathrm{e}^{-}+x \mathrm{H}_{2} \mathrm{O} \log \mathrm{K}=-35.61 \pm 0.39
$$

There is a large spread (3 to 4 orders of magnitude) in the solubility of amorphous $\mathrm{PuO}_{2} \cdot x \mathrm{H}_{2} \mathrm{O}$, colloidal $\mathrm{Pu}(\mathrm{IV})$ hydrous oxide, and crystalline $\mathrm{PuO}_{2}$ at room temperature. The differences in solubility may be related to differences in particle size and degree of hydration, similar to the situation for various forms of silica (Iler, 1979). Experiments using carefully prepared and characterized $\mathrm{Pu}(\mathrm{IV})$ colloid could investigate particle-size effects. In addition, experiments at various temperatures could be performed to see if increasing the temperature destabilizes the more hydrated, higher-solubility forms of $\mathrm{Pu}(\mathrm{IV})$ polymer.

The effect of complexation on the solubility of $\mathrm{Pu}(\mathrm{IV})$ in the aqueous carbonate system has been investigated at room temperature (Yamaguchi et al., 1994). The solubilitycontrolling solid was identified as $\mathrm{PuO}_{2} \cdot x \mathrm{H}_{2} \mathrm{O}$.

Ground and surface waters that contain Fe powder can maintain $\mathrm{Pu}$ in the $\mathrm{Pu}(\mathrm{III})$ oxidation state. The solubility of $\mathrm{Pu}(\mathrm{OH})_{3}$ has been studied from both oversaturation and undersaturation at $23^{\circ} \mathrm{C}$ (Felmy et al., 1989). The solubility product constant was determined to be $\log K_{\mathrm{sp}}^{0}\left[\mathrm{Pu}^{3+}\right]\left[\mathrm{OH}^{-}\right]^{-3}=-26.2 \pm 0.8$.

In a review of experimental data to 1980, equilibrium constants were estimated for the dissolution of several Pu oxide, fluoride, and phosphate compounds over the temperature range $25^{\circ}$ to $200^{\circ} \mathrm{C}$ (Lemire and Tremaine, 1980).

The NEA-TDB review of chemical thermodynamics of $\mathrm{Np}$ and $\mathrm{Pu}$ is in preparation.

The solubility behavior of Pu under expected conditions is reasonably well understood. Solid phases were not precisely determined in the oversaturation experiments; thus, model assumptions concerning the solid phases are required for geochemical modeling. The $\mathrm{Pu}(\mathrm{V})$ 
solids do not appear to be significant under expected repository conditions. Solubility models based on $\mathrm{Pu}(\mathrm{OH})_{4}$ or $\mathrm{PuO}_{2} \cdot x \mathrm{H}_{2} \mathrm{O}$ should include the $\mathrm{Pu}$ redox equilibrium in the solution phase under expected repository conditions.

\section{Americium}

The solubility of Am in J-13 and UE-25p1 waters was investigated in a series of experiments (Nitsche, 1991; Nitsche et al., 1993a, 1995, 1993b). Experiments were conducted from oversaturation and undersaturation with $\mathrm{Am}$ added as $\mathrm{Am}^{3+}$. No clear solubility trend was found with temperature $\left(25^{\circ}, 60^{\circ}\right.$, and $\left.90^{\circ} \mathrm{C}\right)$ and $\mathrm{pH}(6,7$, and 8.4). Steady-state Am concentrations ranged from roughly $10^{-10}$ to $10^{-6} \mathrm{M}$. The solubility-controlling solid phase was orthorhombic $\mathrm{AmCO}_{3} \mathrm{OH}$ under J-13 conditions and a mixture of $\mathrm{Am}_{2}\left(\mathrm{CO}_{3}\right)_{3} \cdot 2 \mathrm{H}_{2} \mathrm{O}$ and orthorhombic $\mathrm{AmCO}_{3} \mathrm{OH}$ under UE-25p1 conditions. Different solids were observed because the carbonate concentration is higher by about a factor of 6 in UE-25p1 water.

The NEA-TDB project has completed the critical review of thermodynamic data for Am compounds and species (Silva et al., 1993). A detailed discussion of original publications and the data selection process is available in the NEA-TDB review; thus, only a brief summary is presented here.

$\mathrm{AmCO}_{3} \mathrm{OH}$ has two structural forms: orthorhombic and hexagonal. Solubility studies have been made only for the orthorhombic form. At $25^{\circ} \mathrm{C}$, the equilibrium between $\mathrm{AmCO}_{3} \mathrm{OH}$ (orthorhombic) and $\mathrm{Am}_{2}\left(\mathrm{CO}_{3}\right)_{3}(\mathrm{cr})$ occurs at a $\mathrm{CO}_{2}$ equilibrium partial pressure of approximately 0.1 bar. Solubility product constants for $\mathrm{AmCO}_{3} \mathrm{OH}$ (orthorhombic) and $\mathrm{Am}_{2}\left(\mathrm{CO}_{3}\right)_{3}$ are well known near room temperature, but elevated-temperature solubility data, aside from the results of Nitsche et al., (1993a, 1995, 1993b) discussed previously, are not available.

$\mathrm{Am}(\mathrm{OH})_{3}$ may be significant in waters with very low carbonate concentrations. Both crystalline and amorphous forms of $\mathrm{Am}(\mathrm{OH})_{3}$ have been identified, and they have similar solubilities in aqueous media. At $25^{\circ} \mathrm{C}$, the equilibrium between $\mathrm{Am}(\mathrm{OH})_{3}(\mathrm{cr})$ and orthorhombic $\mathrm{AmCO}_{3} \mathrm{OH}$ occurs at a $\mathrm{CO}_{2}$ equilibrium partial pressure of about $10^{-4}$ bar. Solubility product constants for the $\mathrm{Am}(\mathrm{OH})_{3}$ solids are reasonably well known near room temperature, but elevated-temperature solubility data are not available.

The identity of Am solubility-limiting solids for expected Yucca Mountain repository conditions is known, and the solubility product constants at $25^{\circ} \mathrm{C}$ are relatively well understood. Detailed solubility behavior of the solid phases at elevated temperatures is not known. Thermodynamic functions for $\mathrm{AmCO}_{3} \mathrm{OH}, \mathrm{Am}_{2}\left(\mathrm{CO}_{3}\right)_{3}$, and $\mathrm{Am}(\mathrm{OH})_{3}$ solids could be determined by calorimetric measurements or solubility experiments at elevated temperatures.

\section{Technetium}

The $\mathrm{Tc}(\mathrm{VII}) / \mathrm{Tc}(\mathrm{IV})$ redox equilibrium has a critical effect on Tc solubility under expected repository conditions (Palmer et al., 1996). At $25^{\circ} \mathrm{C}$ and reducing conditions, the Tc concentration is limited to $<10^{-7} \mathrm{M}$ by the $\mathrm{TcO}_{2} \cdot x \mathrm{H}_{2} \mathrm{O}$ solid phase. Under oxic conditions Tc is very soluble as $\mathrm{TcO}_{4}^{-}$(Kerrisk, 1984).

The Tc(VII)/ Tc(IV) redox equilibrium has been investigated by emf measurements on $\mathrm{TcO}_{2} \cdot x \mathrm{H}_{2} \mathrm{O}(x=1.63 \pm 0.28)$ electrodes in contact with $\mathrm{TcO}_{2}^{-}$solutions at $24^{\circ}$ to $25^{\circ} \mathrm{C}$ (Cartledge and Smith, 1955; Cobble et al., 1953; Meyer and Arnold, 1991). The reaction can be written as

$$
\mathrm{TcO}_{4}^{-}+4 \mathrm{H}^{+}+2 \mathrm{e}^{-}=\mathrm{TcO}_{2} \cdot x \mathrm{H}_{2} \mathrm{O}(\mathrm{s})+(2-x) \mathrm{H}_{2} \mathrm{O}
$$


The Nernst equation for the reaction is

$$
E=E^{0}+\frac{2.303 R T}{3 F} \log a\left(\mathrm{TcO}_{4}^{-}\right)-4 \mathrm{pH}
$$

where $E$ is the potential, $R$ is the gas constant, $F$ is the Faraday constant, and $a\left(\mathrm{TcO}_{4}^{-}\right)$denotes the $\mathrm{TcO}_{4}^{-}$activity. The standard potential $E^{0}$ is $0.75 \pm 0.02 \mathrm{~V}$ (Meyer and Arnold, 1991). The Nernstian behavior has been verified as a function of $\mathrm{pH}$ and $\mathrm{TcO}_{4}^{-}$activity, but not temperature.

The solubility of $\mathrm{TcO}_{2} \times \mathrm{H}_{2} \mathrm{O}$ has been investigated in aqueous solutions at room temperature in the $\mathrm{pH}$ range 6 to 12 and under constant $\mathrm{pCO}_{2}$ conditions (Eriksen et al., 1992). The results were described by four different equilibrium expressions to take into account the principal aqueous species in each case:

- $\mathrm{CO}_{2}$-free solutions with $\mathrm{pH} 6$ to 9.5

- $\mathrm{CO}_{2}$-free solutions with $\mathrm{pH} 11$ to 12

- Carbonate-containing solutions with $\mathrm{pH} 6$ to 9.5

- Carbonate-containing solutions with pH 11 to 12

Tc solubility experiments have been conducted under ambient-temperature conditions relevant to a proposed radioactive waste repository in the UK (Pilkington, 1990). The waste is stored in steel containers, which are surrounded by a cementitious grout. This results in a $\mathrm{pH}$ above 10.5 and reducing conditions $(E h=-400 \mathrm{mV})$. Ammonium pertechnetate solution or solid hydrated technetium dioxide was added to cement-equilibrated waters. The experiments were equilibrated for only six to seven weeks. No accurate statements were given concerning the solubility-controlling solids formed during the oversaturation experiments. The measured Tc concentration in solution was about $10^{-7} \mathrm{M}$. An increasing Tc solubility by a factor of 10, if organic degradation products were present, was observed.

The NEA-TDB review of thermodynamic data for Tc compounds and species is in preparation.

Few or no data are available on the temperature-dependence of the solubility of Tc compounds. However, the $\mathrm{Tc}(\mathrm{VII}) / \mathrm{Tc}(\mathrm{IV})$ redox equilibrium has a strong effect on the Tc solubility and is the critical issue for Tc in a repository. The $\mathrm{Tc}(\mathrm{VII}) / \mathrm{Tc}(\mathrm{IV})$ redox behavior is relatively well understood at $25^{\circ} \mathrm{C}$. The validity of temperature extrapolations using the Nernst equation could be verified by emf measurements on $\mathrm{TcO}_{2} \cdot x \mathrm{H}_{2} \mathrm{O}$ electrodes at several temperatures. High carbonate or $\mathrm{Si}$ concentrations may alter the $\mathrm{TcO}_{2} \cdot x \mathrm{H}_{2} \mathrm{O}$ surface, and this could be checked by measurements in various electrolyte solutions. A small number of Tc solubility experiments in reference waters could also help verify the expected behavior. For performance assessment of a repository, it is probably not necessary to have a detailed understanding of the solubility behavior of individual Tc compounds, and undersaturation experiments with Tc compounds should be given relatively low priority.

Nickel

If $\mathrm{Ni}(\mathrm{OH})_{2}$ is assumed to be the solubility-controlling solid, then $\mathrm{Ni}$ is relatively soluble (to about $0.1 \mathrm{~m}$ ) in J-13 water at $25^{\circ} \mathrm{C}$ (Wruck and Palmer, 1997). The main aqueous species is $\mathrm{Ni}^{2+}$, and a small amount of $\mathrm{NiSO}_{4}{ }^{\circ}$ is also present.

The solubility of nickel oxocompounds has been reported by various authors at ambient temperatures (Balej and Divisek, 1993; Dibrov and Grigoreva, 1977; Gayer and Garrett, 1949; Jena and Prasad, 1956; Novak-Adamic et al., 1973; Pavlovic and Popovic, 1969). A few papers are also available on the solubility of nickel oxocompounds at elevated temperatures (Chickerur et al., 1980; Dinov et al., 1993; Tremaine and Leblanc, 1980). A brief summary is presented here. 
The solubility of $\mathrm{Ni}(\mathrm{OH})_{2}$ has been investigated at room temperature (Gayer and Garrett, 1949; Jena and Prasad, 1956; Novak-Adamic et al., 1973). For the reaction

$$
\mathrm{Ni}(\mathrm{OH})_{2}(\mathrm{~s}) \Leftrightarrow \mathrm{Ni}^{2+}+2 \mathrm{OH}^{-}
$$

the various solubility products reported are not consistent (see Table 6-13). Possible reasons for the different values obtained could be the preparation method and the age of the nickel hydroxide as well as the different electrolyte systems used.

Table 6-13 Summary of solubility product constants for $\mathrm{Ni}(\mathrm{OH})_{2}$ (from Eq. 6-11)

\begin{tabular}{|l|c|c|l|}
\hline \multicolumn{1}{|c|}{ lonic Medium } & $\mathbf{T}\left({ }^{\circ} \mathbf{C}\right)$ & $\log \boldsymbol{K}$ & Reference \\
\hline \hline $\mathrm{HCl} / \mathrm{NaOH}$ & 25 & -17.2 & $\begin{array}{l}\text { Gayer and } \\
\text { Garrett, 1949 }\end{array}$ \\
\hline $\mathrm{HCl} / \mathrm{CH}_{3} \mathrm{COOH} / \mathrm{NaCH}_{3} \mathrm{COO}$ & $28-30$ & -16.0 & $\begin{array}{l}\text { Jena and } \\
\text { Prasad, 1956 }\end{array}$ \\
\hline
\end{tabular}

The solubility of $\mathrm{Ni}(\mathrm{OH})_{2}$ has also been investigated up to $40^{\circ} \mathrm{C}$ (Chickerur et al., 1980). Dissolution of $\mathrm{Ni}(\mathrm{OH})_{2}$ is an endothermic process (i.e., the solubility product determined for the reaction in Eq. 6-11 increased with increasing temperature. The experiments were only conducted over a time of about 10 hours.

Thermodynamic values $\left(\Delta_{\mathrm{f}} \mathrm{H}^{0}, \Delta_{\mathrm{f}} \mathrm{G}^{0}\right.$, and $\mathrm{S}^{0}$ at $\left.298 \mathrm{~K}\right)$ for the system $\mathrm{Ni}$ / aqueous solution are available in Balej and Divisek (1993), Dibrov and Grigoreva (1977), Pavlovic and Popovic (1969), and Tremaine and Leblanc (1980).

The solubility of $\mathrm{NiO}$ in pure water has been investigated over the temperature range $373 \mathrm{~K}$ to $523 \mathrm{~K}$ in a special batch autoclave system (Dinov et al., 1993). The Ni solubility from $\mathrm{NiO}$ decreased with increasing temperature (at $373 \mathrm{~K},[\mathrm{Ni}]=2.099 \mu \mathrm{M} / \mathrm{kg}$; whereas at $573 \mathrm{~K}$, $[\mathrm{Ni}]=0.769 \mu \mathrm{M} / \mathrm{kg})$. The formation of $\mathrm{Ni}(\mathrm{OH})^{+}, \mathrm{Ni}(\mathrm{OH})_{2}(\mathrm{aq})$, and $\mathrm{Ni}(\mathrm{OH})_{3}^{-}$influences the dissolution of $\mathrm{NiO}$. The data reported in Dinov et al. (1993) are consistent with the data of Tremaine and Leblanc (1980). The dissolution process of $\mathrm{NiO}$ is described by the reaction

$$
\mathrm{NiO}(\mathrm{s})+2 \mathrm{H}^{+} \Leftrightarrow \mathrm{Ni}^{2+}+\mathrm{H}_{2} \mathrm{O}
$$

The solubility product constant for the reaction in Eq. 6-12 decreased from $\log K(\mathrm{~kg} / \mathrm{mol})$ $=12.16$ at $298 \mathrm{~K}$ to about 3.11 at $573 \mathrm{~K}$. The same trend was observed for $\mathrm{Ni}$ and $\beta-\mathrm{Ni}(\mathrm{OH})_{2}$.

The solubility of nickel ferrite $\mathrm{NiFe}_{2} \mathrm{O}_{4}$ at high temperatures $(273 \mathrm{~K}$ to $623 \mathrm{~K}$ ) is reported in Chung and Lee (1990) and Hanzawa et al. (1996). This compound may be relevant because of the use of stainless-steel alloys in the waste containers. Thermodynamic calculations were also described for predicting equilibrium phenomena at high temperatures in water-cooled nuclear reactors (Chung and Lee, 1990).

Although sulfate concentrations are low in J-13, nickel sulfate and mixed nickel hydroxide sulfate solids have low solubilities and may possibly be significant (Dobrokhotov, 1954). No data were found for the elevated-temperature solubility behavior of nickel sulfates.

The solubility of $\mathrm{NiO}$ and $\mathrm{Ni}(\mathrm{OH})_{2}$ are relatively well understood under expected repository conditions. There are little or no data on the high-temperature solubility behavior of $\mathrm{Ni}$ silicates, $\mathrm{Ni}$ carbonates, and $\mathrm{Ni}$ sulfates. $\mathrm{NiO}$ and / or $\mathrm{Ni}(\mathrm{OH})_{2}$ are generally assumed to be solubility-controlling in equilibrium with atmospheric $\mathrm{CO}_{2}$ at $25^{\circ} \mathrm{C}$ and $\mathrm{pH} 7$, but the increase in carbonate (Emara et al., 1987) and silicate concentrations with temperature may result in a change in the solubility-controlling solid at elevated temperatures. A small number of Ni solubility experiments in reference waters could help verify the expected behavior. If 
the limiting $\mathrm{Ni}$ concentrations are high [for example, as calculated when $\mathrm{NiO}$ or $\mathrm{Ni}(\mathrm{OH})_{2}$ is assumed to be the solubility-controlling solid], it may not be necessary to have a detailed understanding of the solubility behavior of individual Ni compounds.

\section{Zirconium}

The maximum $\mathrm{Zr}$ concentration in J-13 water at $25^{\circ} \mathrm{C}$ is estimated to be less than $10^{-10} \mathrm{M}$ and limited by the $\mathrm{Zr}(\mathrm{OH})_{4}$ solid phase (Kovalenko and Bagdasarov, 1961). $\mathrm{Zr}(\mathrm{OH})_{4}{ }^{0}$ and $\mathrm{Zr}(\mathrm{OH})_{5}^{-}$are expected to be the primary solution species for a $\mathrm{pH}$ range of 6 to 9 .

The solubilities of $\mathrm{Zr}(\mathrm{OH})_{4}$ and $\mathrm{ZrO}_{2}$ have been investigated at room temperature (Baes and Mesmer, 1981; Bilinski and Branica, 1966; Kovalenko and Bagdasarov, 1961). Very little data are available for the solubility of these solid phases at elevated temperatures (Bilinski et al., 1966). The dissolution reactions were given as

$$
\mathrm{Zr}(\mathrm{OH})_{4}(\mathrm{~s})+4 \mathrm{H}^{+} \Leftrightarrow \mathrm{Zr}^{4+}+4 \mathrm{H}_{2} \mathrm{O}
$$

and

$$
\mathrm{ZrO}_{2}(\mathrm{~s})+4 \mathrm{H}^{+} \Leftrightarrow \mathrm{Zr}^{4+}+2 \mathrm{H}_{2} \mathrm{O}
$$

The reported solubility products are summarized in Table 6-14.

Table 6-14 Solubility products for $\mathrm{Zr}(\mathrm{OH})_{4}$ and $\mathrm{ZrO}_{2}$

\begin{tabular}{|l|l|l|l|c|l|}
\hline \multicolumn{1}{|c|}{ Method } & \multicolumn{1}{c|}{$\begin{array}{c}\text { lonic } \\
\text { Medium }\end{array}$} & Compound & $\mathbf{T}\left({ }^{\circ} \mathbf{C}\right)$ & log $K$ & \multicolumn{1}{c|}{ Reference } \\
\hline \hline Solubility & 0 corr & $\mathrm{Zr}(\mathrm{OH})_{4}$ & 19 & 2.04 & $\begin{array}{l}\text { Kovalenko and } \\
\text { Bagdasarov, } 1961\end{array}$ \\
\hline $\begin{array}{l}\text { Tyndallometric } \\
\text { Method }\end{array}$ & $1 \mathrm{M} \mathrm{NaClO}_{4}$ & $\mathrm{Zr}(\mathrm{OH})_{4}$ & 20 & $\approx 3.8$ & Bilinski et al., 1966 \\
\hline $\begin{array}{l}\text { Tyndallometric } \\
\text { Method }\end{array}$ & Dilute & $\mathrm{Zr}(\mathrm{OH})_{4}$ & 20 & $\approx 4.6$ & Bilinski et al., 1966 \\
\hline $\begin{array}{l}\text { Tyndallometric } \\
\text { Method }\end{array}$ & Dilute & $\mathrm{Zr}(\mathrm{OH})_{4}$ & 40 & $5.05 \pm 0.18$ & Bilinski et al., 1966 \\
\hline $\begin{array}{l}\text { Tyndallometric } \\
\text { Method }\end{array}$ & $1 \mathrm{M} \mathrm{NaClO}{ }_{2}$ & $\mathrm{Zr}(\mathrm{OH})_{4}$ & 20 & $(-4.36 \pm 0.05)^{\star}$ & Bilinski et al., 1966 \\
\hline $\begin{array}{l}\text { Tyndallometric } \\
\text { Method }\end{array}$ & Sea water & $\mathrm{Zr}(\mathrm{OH})_{4}$ & 20 & $(-4.60)^{*}$ & Bilinski and Branica, 1966 \\
\hline Solubility & $\begin{array}{l}\text { NaOH: } 1 \text { to } \\
18.4 \mathrm{M}\end{array}$ & $\mathrm{Zr}(\mathrm{OH})_{4}$ & 25 & $(-3.98)^{* *}$ & Sheka and Pevzner, 1960 \\
\hline Solubility & Dilute & $\mathrm{ZrO}{ }_{2}$ & 25 & -1.9 & Baes and Mesmer, 1981 \\
\hline
\end{tabular}

* For reaction: $\mathrm{Zr}(\mathrm{OH})_{4}(\mathrm{~s}) \Leftrightarrow \mathrm{Zr}(\mathrm{OH})_{4}(\mathrm{aq}) . \quad$ ** $\quad$ For reaction: $\mathrm{Zr}(\mathrm{OH})_{4}(\mathrm{~s})+\mathrm{OH}^{-} \Leftrightarrow \mathrm{Zr}(\mathrm{OH})_{5}^{-}$.

In Kovalenko and Bagdasarov (1961), the solubility of freshly prepared $\mathrm{Zr}(\mathrm{OH})_{4}$ was measured in the $\mathrm{pH}$ range 1.54 to 2.02 at a temperature of $19^{\circ} \mathrm{C}$. The $\mathrm{Zr}$ concentration in solution was detected via a colorimetric method over a period of $24 \mathrm{hr}$. The solubility product constant determined in these experiments appears to be reliable. 
In Bilinski and Branica (1966) and Bilinski et al. (1966), the solubility of $\mathrm{Zr}(\mathrm{OH})_{4}$ was characterized using a tyndallometer. The turbidity of the solution was measured to determine whether precipitation of the assumed solid $\mathrm{Zr}\left(\mathrm{OH}_{4}\right.$ had occurred. The solubilities determined in these experiments are much larger than the solubility determined in Kovalenko and Bagdasarov (1961). Admittedly, the precipitate is difficult to detect by the tyndallometric method at low $\mathrm{Zr}$ concentration (below about $10^{-6} \mathrm{M}$ ). In addition, the nature of the solution species and the solid phases were not determined, and the ionic strength was not always well defined during the tyndallometric studies. Therefore, the solubility products reported in Bilinski and Branica (1966) and Bilinski et al. (1966) are unreliable.

The dissolution of $\mathrm{Zr}(\mathrm{OH})_{4}$ in different $\mathrm{NaOH}$ solutions ranging from 1 to $18.4 \mathrm{M}$ was investigated in Sheka and Pevzner (1960). A linear relation between the Zr concentration in solution and the $\mathrm{NaOH}$ concentration, with a slope of unity, was measured in the range of 1 to $8 \mathrm{M}$. It is assumed from the slope the solubility reaction $\mathrm{Zr}(\mathrm{OH})_{4}(\mathrm{~s})+\mathrm{OH}^{-} \Leftrightarrow \mathrm{Zr}(\mathrm{OH})_{5}{ }^{-}$for zirconium hydroxide in highly concentrated $\mathrm{NaOH}$ solutions.

There are little or no data on the high-temperature solubility behavior of $\mathrm{ZrSiO}_{4}$. The silicate phase has a low solubility at room temperature and may become solubilitycontrolling at elevated temperatures. Thermodynamic data for $\mathrm{Zr}$ have been tabulated in Wagman et al. (1982).

Phosphate concentrations are low in J-13, but Zr phosphates have low solubilities and may become significant. Thermodynamic data are available for $\mathrm{Zr}\left(\mathrm{HPO}_{4}\right)_{2}$ (Allulli et al., 1979)

A review of the chemical thermodynamics of $\mathrm{Ni}$ and $\mathrm{Zr}$ is currently being conducted by the Yucca Mountain project, and it will result in a database for $\mathrm{Ni}$ and $\mathrm{Zr}$ that is internally consistent with the NEA-TDB thermodynamic data for Tc, U (Grenthe et al., 1992), Np, Pu, and Am (Silva et al., 1993).

Little or no data are available on the temperature-dependence of the solubility of $\mathrm{Zr}(\mathrm{OH})_{4}$ and $\mathrm{ZrSiO}_{4}$. More reliable values for the solubility product constants of these compounds are needed to provide confidence in modeling calculations of zirconium solubilities. Solubility experiments could be performed from oversaturation in reference waters, and from undersaturation at ambient and elevated temperatures. Such experiments could help bound the maximum $\mathrm{Zr}$ concentrations at ambient and elevated temperatures, and solubilitycontrolling solid phases may also be identified. However, detailed experimental investigations of $\mathrm{Zr}$ solubilities are not of the highest priority because the $\mathrm{Zr}$ solubility is expected to remain low as the temperature increases.

\section{Concluding Remarks}

The goal of this review has been to present a summary of recent literature on the solubility behavior of selected elements (U, Np, Pu, Am, Tc, Ni, and $\mathrm{Zr}$ ) with a focus on solubility-controlling solids for expected conditions at Yucca Mountain, Nevada. An overview of likely solubility-controlling solid phases is given in Table 6-15. The solubilities of the uranium oxides, schoepite, $\mathrm{UO}_{2} \mathrm{CO}_{3}, \mathrm{PuO}_{2}$, and the $\mathrm{Ni}$ and $\mathrm{Zr}$ phases can be reliably predicted over the temperature range $20^{\circ}$ to $150^{\circ} \mathrm{C}$. For the other phases, there are little or no experimental data on the temperature dependence, and solubilities must be estimated at temperatures above $25^{\circ} \mathrm{C}$. 
Table 6-15 Summary of expected solubility-controlling solids

\begin{tabular}{|c|c|c|}
\hline & Low-Eh Conditions & High-Eh Conditions \\
\hline$U$ & $\begin{array}{l}\mathrm{UO}_{2} \\
\mathrm{UO}_{x}, 2<x<3 \\
\mathrm{USiO}_{4}(\mathrm{cr}, \mathrm{am})\end{array}$ & $\begin{array}{l}\mathrm{UO}_{2} \mathrm{CO}_{3} \\
\mathrm{UO}_{3} \cdot 2 \mathrm{H}_{2} \mathrm{O} / \mathrm{UO}_{2}(\mathrm{OH})_{2} \\
\mathrm{Na}_{2} \mathrm{U}_{2} \mathrm{O}_{7} \\
\mathrm{Na}_{4} \mathrm{UO}_{2}\left(\mathrm{CO}_{3}\right)_{3} \\
\left(\mathrm{UO}_{2}\right)_{2} \mathrm{SiO}_{4} \cdot 2 \mathrm{H}_{2} \mathrm{O} \\
\mathrm{Na}\left(\mathrm{H}_{3} \mathrm{O}\right)\left(\mathrm{UO}_{2}\right) \mathrm{SiO}_{4} \cdot \mathrm{H}_{2} \mathrm{O} \\
\mathrm{Na}_{2}\left(\mathrm{UO}_{2}\right)_{2}\left(\mathrm{Si}_{2} \mathrm{O}_{5}\right)_{3} \cdot 7 \mathrm{H}_{2} \mathrm{O}\end{array}$ \\
\hline $\mathrm{Np}$ & $\begin{array}{l}\mathrm{NpO}_{2}(\mathrm{cr}) \\
\mathrm{Np}(\mathrm{OH})_{4}(\mathrm{am})\end{array}$ & $\begin{array}{l}\mathrm{NaNpO}_{2} \mathrm{CO}_{3} \cdot \times \mathrm{H}_{2} \mathrm{O} \\
\mathrm{Np}_{2} \mathrm{O}_{5}\end{array}$ \\
\hline $\mathrm{Pu}$ & $\begin{array}{l}\mathrm{PuO}_{2} \\
\mathrm{Pu}(\mathrm{OH})_{4}(\mathrm{am}) \\
\mathrm{Pu}(\mathrm{IV}) \text { polymer }\end{array}$ & $\begin{array}{l}\text { Same as low Eh, but metastable phases } \\
\text { (amorphous solids, } \mathrm{NaPuO}_{2} \mathrm{CO}_{3} \cdot \mathrm{x}_{2} \mathrm{O} \text { ) } \\
\text { are more prevalent }\end{array}$ \\
\hline Am & $\begin{array}{l}\mathrm{AmOHCO}_{3} \\
\mathrm{Am}_{2}\left(\mathrm{CO}_{3}\right)_{3} \cdot 2 \mathrm{H}_{2} \mathrm{O} \\
\mathrm{Am}_{2}\left(\mathrm{CO}_{3}\right)_{3} \\
\mathrm{NaAm}\left(\mathrm{CO}_{3}\right)_{2} \cdot 4 \mathrm{H}_{2} \mathrm{O} \\
\mathrm{Am}(\mathrm{OH})_{3}\end{array}$ & Same as low Eh \\
\hline
\end{tabular}

\begin{tabular}{|l|l|l|}
\hline & \multicolumn{1}{|c|}{ Low-Eh Conditions } & \multicolumn{1}{c|}{ High-Eh Conditions } \\
\hline $\mathrm{Tc}$ & $\begin{array}{l}\mathrm{TcO}_{2} \\
\mathrm{TcO}_{2} \cdot \mathrm{xH}_{2} \mathrm{O}(\mathrm{x}=1-2)\end{array}$ & $\mathrm{NaTcO}$ \\
\hline $\mathrm{Ni}$ & $\mathrm{NiO}$ & Same as low Eh \\
$\mathrm{Ni}(\mathrm{OH})_{2}$ & $\begin{array}{l}\mathrm{ZrO} \\
\mathrm{Zr}(\mathrm{OH})_{4} \\
\mathrm{ZrSiO}_{4}\end{array}$ & \\
\hline
\end{tabular}

Specific recommendations for further work have been made in the discussion for each element in this report. The relative need for further solubility experiments is roughly in the order $\mathrm{Np}>\mathrm{U}>\mathrm{Pu}>\mathrm{Am}>\mathrm{Zr}>\mathrm{Tc}>\mathrm{Ni}$. The kinetics of precipitation and transformation of the solid phases are particularly important for $\mathrm{Np}$ and $\mathrm{Pu}$. An additional area for further experimental work is the possibility of mixed actinide solids. For example, if $\mathrm{Np}$ and $\mathrm{Pu}$ are both present, does formation of a mixed $\mathrm{Np}-\mathrm{Pu}$ solid result in lower solution concentrations than would be predicted on the basis of individual $\mathrm{Np}$ and $\mathrm{Pu}$ solids? 


\subsubsection{Alternative Modes of Radionuclide Transport in the NF/AZ}

\section{by A. Meike with contributions from R. Knapp, C. Wittwer, and A. Miller}

Solubility and speciation relations are used to estimate total dissolved concentrations for radioelements. These estimates are often regarded as upper bounds because retardation processes such as sorption and coprecipitation are not taken into account. However, this assumption may not hold if there is significant transport by radiocolloids and pseudocolloids (nonradioactive materials to which radionuclides are sorbed). In addition, bacteria that grow in the NFE, and fragments of associated biomass, may act as biocolloids with affinity for radioelements.

Colloids can be collected by filtering pumped groundwater and are therefore mobile in geologic media. They are readily produced by controlled chemical reactions in the laboratory and have been observed from interaction of water with vitrified high-level waste and with hydrothermally altered concrete. In general, the study of colloids of any origin is relatively difficult compared with the study of the transport of dissolved species, for several reasons:

- Colloids tend to be unstable when sampled and stored.

- Larger water samples are required.

- Processes that produce colloids can be complex and difficult to control.

- Additional methods of instrumental analysis are needed to characterize colloidal transport.

Accordingly, relatively few studies with direct bearing on colloidal transport in the NF/ AZ have been performed. Similar statements can be made concerning the study of biocolloids. Important studies in these areas include Story and Hersman (1995) and Hersman (1996), and the reader is referred to these reports for additional information.

The following discussion describes a generalized conceptual model for colloid and biocolloid generation and transport behavior. A few laboratory and field investigations of colloid transport at repository conditions have been undertaken recently, and results are discussed subsequently, where applicable.

Colloids are transported only by advective flow in groundwater and are probably transported only in fractures. Conditions for the formation of radiocolloids, or sorption of radionuclides to pseudocolloids, will vary along transport pathways; thus, the predominant modes of retardation and transport may change between the NFE and the biosphere.

\subsubsection{Colloids in Radionuclide Transport}

Colloids could play a significant role in the transport of radionuclides from a repository to the biosphere, but the significance of colloidal transport in partially saturated fractures of the repository host rock is a matter of considerable uncertainty. It is widely accepted that significant quantities of introduced materials, performing in a chemically and hydrologically dynamic NFE, are likely to increase the abundance of colloid-size particles well beyond that which is observed in the ambient geochemical environment.

Introduced materials will form pseudocolloids that have high affinity for radionuclides such as $\mathrm{U}$ and $\mathrm{Np}$ and are of principal interest for radionuclide transport. Interactions between introduced materials and groundwater can form metal oxides, hydroxides, and oxyhydroxides; clays and other polysilicates; and organic particles. Introduced materials such as cement will provide many of the anions required to form pseudocolloids (hydroxides, carbonates, phosphates, silicates, and fluorides). Colloidal particles produced from the degradation of introduced materials will have approximately the same range of sizes as do natural colloids. These ranges and the types of materials generally represented are the following: 
- Small $(<10-\mathrm{nm})$ hydrated metal ions, small organic particles, polyhydroxy-complexes, polysilicates, and fulvic acids

- Medium-sized (10-1000-nm) clays and metal-hydroxides

- Large (>1-nm) inorganic and organic particles

The following discussion summarizes the environmental conditions that contribute to generation and transport of colloidal particles. A more complete literature review is presented elsewhere (Meike and Wittwer, 1993a, 1993b).

The behavior of colloids can be complex, particularly in an environment strongly influenced by introduced materials. Substantial quantities of colloidal particles will be introduced initially along with materials such as the concrete liner. Hydrothermal degradation will cause further development of colloids because of the various physical and chemical processes that are active where interaction with groundwater occurs. The quantities of introduced materials such as cement and their proximity to WPs suggests that colloids could be a dominant mode of radionuclide transport in the NFE.

Introduced materials that could have relatively strong impact on the generation of colloidal particles in the NFE include the following:

- Inorganic complexants (e.g., certain components from batteries)

- Organic compounds as complexants and nutrients for microbes (e.g., alcohols, antifreeze, bituminous materials, diesel fuel, hydrocarbons, some gases, clothing, lubricants, plastic, wood)

- Groundwater $\mathrm{pH}$ modifiers (e.g., acids, concrete, grout, lime, plaster) The mechanism of radionuclide sorption to colloids depends on chemical speciation. Specific sorption behavior depends on $\mathrm{pH}$ and the redox state of the sorbate. Whereas the effects of reversible sorption can be readily simulated, desorption kinetics are not understood, and the literature contains many examples of laboratory tests in which slow desorption was observed. A comprehensive discussion of sorption to colloidal particles is given by Triay (1997).

Whether sorption of radionuclides to colloidal particles will increase or decrease the waste-isolation performance of a repository depends heavily on mobility and remains to be determined. Several conditions that favor long-range migration are known: low ionic strength, decreasing $\mathrm{pH}$, low temperature, anoxic conditions, high organic content, high flow velocities, high colloid concentrations, and radionuclide desorption caused by changes in water chemistry along transport pathways.

The effects of many potential repository construction materials on colloid generation and transport have yet to be characterized. Some effects are not well known, yet are important for predicting colloid effects on radionuclide transport:

- Release of radionuclides from the NFE as anions, or associated with inorganic (silicate, aluminate) and organic complexes

- Interactions between colloids that influence their stability

- Coupled effects of $\mathrm{pH}$, ionic strength, competing ions, temperature, and sorbate concentration (Sanchez et al., 1985)

Research is generally shifting away from laboratory column experiments and toward modeling and the interpretation of field data. A principal reason is that the conditions governing colloidal processes in the laboratory can be difficult to control in a way that is sufficiently representative of field processes. 


\subsubsection{Generation of Colloids}

Colloids exist naturally as fracture-lining materials, clays, bacteria, algae, and humic acid. They are also produced from degradation of introduced materials such as glass, fuels, grease, metal, organic waste, and biomass (see Meike and Wittwer (1993a). Colloids are released from bulk material by dissolution or other chemical reaction, hydrodynamic force, or mechanical stress. They are created by chemical precipitation and microbial activity (Agrawal et al., 1989; Ershov and Sukhov, 1990; McCarthy and Zachara, 1989). Each of these mechanisms may occur in connection with various repository materials.

The tendency for colloids to remain suspended is determined by fundamental characteristics such as size, bulk charge, density, chemical reactivity, and structure. These characteristics can be modified by redox conditions, $\mathrm{pH}$, temperature, ionic strength, organic content, water velocity, and colloid concentration (Mills and Liu, 1989).

A number of processes and sources have been identified as originators of radionuclidesorbing pseudocolloids in the WP environment (Olofsson et al., 1981; Olofsson et al., 1982):

- Naturally occurring colloids such as clays, organics, and precipitates

- Leaching of the waste form

- Corrosion of WP material

- Degradation of backfill, if present

- Introduced organic materials

- Formation of polymerized silica or similar inorganic species

- Cellular and extracellular microbial biomass

- Hydroxides and oxyhydroxides from other sources such as cement

Iron will be the most abundant introduced metal in the repository. The kinetics and stability of ferrihydrite, which is the amorphous precursor of the ferric oxyhydroxides, and its transition to goethite and hematite depend on the environment. Controlling factors include temperature, $\mathrm{pH}$, the presence of aqueous species such as fulvic acid and $\mathrm{Si}$, and even the timing of exposure to these species (Anderson and Benjamin, 1985; Carlson and Schwertmann, 1981; Cornell et al., 1987; Fox, 1988). Hematite particle coagulation in water depends on water chemistry (Liang, 1988). Metal sorption to oxyhydroxides is also controlled by a combination of surface properties that are sensitive to temperature, sorbate concentration, and ionic strength (Balistrieri and Murray, 1982; Benjamin and Leckie, 1981a, 1981b; Hsi and Langmuir, 1985; Hunter et al., 1988; Johnson, 1990). For example, the adsorption of organic acids onto goethite generally increases with decreasing $\mathrm{pH}$. However, the intrinsic affinities of various acids for goethite varies (e.g., oxalic $>$ phthalic $>$ salicylic $=$ lactic), so the resultant adsorption effects are expected to differ markedly (Balistrieri and Murray, 1987).

The metals $\mathrm{Cr}, \mathrm{Ni}$, and possibly $\mathrm{Co}$ will also be present in various forms. Formation of colloidal corrosion products from these metals has been described in detail (Matijevic, 1982a, 1982b, 1986). Actinides and hydrolyzable elements—-such as $\mathrm{Sr}, \mathrm{Pb}$, and $\mathrm{Cu}$-are also likely to form colloids (Olofsson et al., 1981). Colloidal manganese oxides can form by oxidation of $\mathrm{MnO}_{2}$ (Jenkins, 1973).

\section{Cement}

Degradation of cement phases (tricalcium and dicalcium silicates, termed $\mathrm{C}_{3} \mathrm{~S}$ and $\mathrm{C}_{2} \mathrm{~S}$ in cement nomenclature) leads to formation of calcium-silicate-hydrate colloidal gels (Ramsay, 1988). The calcium silicate phases make up a major part of the cement volume. Sorption of metals to cement degradation products is similar to sorption to cement (Gardiner et al., 1990), 
which suggests that pseudocolloidal transport of radionuclides could be significant. The rate of cement degradation therefore affects the rate of colloid generation and the potential for pseudocolloidal transport.

The presence of other ions and organic material in proximity to the cement will probably affect cement degradation, the contribution of chemical species to the aqueous state, and thus, the formation of colloids.

The major materials that make up cement and that will likely contribute to colloid generation include silica fume, blast-furnace slag and other sources of $\mathrm{Si}(\mathrm{OH})_{4}$, ash, iron hydroxides and oxyhydroxides from rebar and wire-mesh reinforcement, and cement impurities that include sulfate and iron. Other constituents that may also be important include salt solutions, aliphatic aromatics, and saccharides that are added to modify handling and curing properties. Cements are complex chemical systems, and relatively little is known about the long-term degradation characteristics.

\section{Organics}

Sorption of metals to colloidal organic matter can be an order of magnitude greater than that on soils and sediments (Reynolds, 1982), which could significantly enhance the mobility of certain radionuclides. Degradation of plastics, polyvinyl chloride, cellulose, rubber, and polyethylene under chemical and radiolytic attack will produce small water-soluble molecules (Ewart and Williams, 1986; Ramsay, 1988), which can participate in reactions that form and stabilize organic colloids. There is considerable uncertainty associated with prediction of metal sorption to organic colloids, even for humics, which have been studied most intensively (Grauer, 1989). Major questions arise with regard to stability constants for metal complexation, ionic strength and $\mathrm{pH}$ effects, competitive sorption, and the relation of anionic and cationic sorption. The behavior of other organic compounds such as EDTA, salicylic acid, and phthalic acid cannot be readily related to the behavior of humic and fulvic acids (Hayes and Weber, 1990).

Hydrocarbons that result from the thermal decomposition of grease, paint, and other organic substances can form ligands that may enhance radionuclide transport. The few data available on this subject suggest that such organic ligands complex radionuclides more effectively (i.e., result in lower dissolved concentrations and in more irreversible sorption) than do inorganic complexes (Raloff, 1990). Hydrocarbon compounds, to the extent that they exist in the NFE, may therefore significantly enhance radionuclide mobility.

The formation and stability of organic complexes involving radionuclides will depend on multiple factors that include the type and concentration of organic matter, the solution chemistry including $\mathrm{pH}$ and redox state, and the temperature (Boggs et al., 1985; Carlsen, 1984; Ewart and Williams, 1986; Grauer, 1989). When adsorbed to hydroxyl groups, organic matter can provide new functional groups (carboxyl, phenolic) for complexation with metals (Davis, 1982).

Organic films on inorganic surfaces have also been reported to modify trace-element mobility (Allard et al., 1990). Much of what has been reported on the interaction of metals with organics is related to common polymers. Metal adsorption sites on polypropylene are likely to be carboxyl and carbonyl groups formed as the surface degrades under the influence of oxygen, heat, or light and in the presence of catalysts, plasticizers, and fillers (Kim and Hill, 1993). Polypropylene is known to absorb significant amounts of lead from solution. A study that compared the sorption of lead with polypropylene and borosilicate glass found that at $\mathrm{pH}$ 7.0, polypropylene ". adsorbed significantly more lead than borosilicate glass, and that at $\mathrm{pH} 5.5$, polypropylene adsorbed less than the glass," indicating a fundamental change in the nature of the polypropylene surface (Kim and Hill, 1993). There have been many 
studies of metal chelates of polymers for high-performance and filtering applications, especially of epoxy. One study (Kurnoskin, 1992) found that synthesized metalliferous epoxy chelate polymers have high thermal stability and chemical resistance when compared with the polymer alone.

Russian studies have suggested that the sorption of various radionuclides from sea water on Teflon ${ }^{\mathrm{TM}}$ is time-dependent. Others have shown that, in the presence of tetraphenylboron, there is selective sorption of trace amounts of cesium from aqueous solution onto the hydrophobic surfaces of polyethylene and Teflon (Raber et al., 1983). This same study found that Teflon was the least sorptive of all the polymers tested.

Organic polymers do not readily sorb metals; however, many polymers have been chelated to promote the sorption of metals. The stereochemistry and the nature of the complexes are markedly dependent on the molar ratios of the reactants, the $\mathrm{pH}$ of the system, and the nature of the anions involved (El-Sonbati et al., 1994). Data also exist for metal complexes involving aliphatic alcohol compounds binding through deprotonated hydroxyl groups. These data indicate that deprotonated hydroxyl binding is strong compared to carboxylate binding, especially at higher $\mathrm{pH}$ values (Greenfield et al., 1993). An extensive project undertaken by Raber et al. (1983) to determine which materials would limit the effects of radionuclide sorption found that polypropylene and polyethylene sorbed the least.

\subsubsection{Influences on Colloid Formation and Sorption}

The formation and stability of colloids, and sorption of radionuclides to pseudocolloids are influenced by solution conditions including radionuclide complexation, $\mathrm{pH}$, redox state, and ionic strength (Bidoglio et al., 1985; Buddemeier and Hunt, 1988; Lieser et al., 1986; Maiti et al., 1989; Olofsson et al., 1981; Ramsay, 1988; Sanchez et al., 1985). These factors will be modified significantly by introduced materials in the NFE, especially if large quantities of concrete are used in repository construction. The current state of knowledge with respect to these factors and their implications for introduced materials is summarized in the following text.

\section{Gradients}

Transport of chemical species along gradients of chemical potential will lead to dissolution and precipitation of colloids and to altered sorption conditions. Such gradients will abound in the NFE because of the inhomogeneous distribution of introduced materials. Changes in solution composition, $\mathrm{pH}$, redox state, $\mathrm{CO}_{2}$ fugacity, temperature, radiation conditions, and bacterial activity can result in colloid formation. Microbial activity often occurs at zonal interfaces where such gradients exist. The effects of $\mathrm{pH}$ and redox gradients as water percolates from cementitious materials into the repository host rock are not well understood and may favor the formation of insoluble hydroxides. Changes in redox conditions resulting from introduction of reducing compounds (e.g., dissolved organic carbon) can also cause colloid formation.

\section{Redox State and $\mathrm{pH}$}

The relation between $\mathrm{pH}$ and the occurrence of colloidal silica is particularly important in the repository because of silica abundance (Stumm et al., 1967; Weres et al., 1981). Changes in $\mathrm{pH}$ changes associated with introduced materials may impact the formation of silica colloids. Elevated $\mathrm{pH}$ and chemically reducing conditions are expected to result from degradation of cement and corrosion of WPs. Reducing conditions may favor stability of radionuclides in reduced valence (e.g., $\mathrm{Pu}(\mathrm{IV})$ or $\mathrm{Np}(\mathrm{IV})$ ). However, gradient in redox potential may favor more oxidized states, thereby changing the predominant mode of colloidal transport from 
true colloids to pseudocolloids and possibly increasing the dissolved concentrations of these species. Changes in redox conditions during the repository thermal evolution need to be characterized, and the effects on radionuclide transport need to be assessed.

\section{Steric Chemistry}

Many chelating resins have been developed to separate metal ions from dilute solutions. It has been found that the hydrophobic character of the polystyrene backbone in many such resins limits the access of metal ions to chelating groups on the resin. The presence of hydrophilic ligands improves chelation (Chessa et al., 1991). The $\mathrm{pH}$ is also used in chromatographic separation because steric effects control the rate that soluble complexes form during desorption. Similar effects probably control the sorption of radionuclides to pseudocolloids.

\section{Temperature}

The effect of elevated temperature on the formation and stability of colloidal particles is also uncertain. The rate of temperature change will be very slow throughout much of the repository thermal evolution, but temperature changes will result when species are transported to or from the emplacement drifts. Retrograde solubility relations will probably contribute to colloid formation. The solubility behavior of radionuclides depends on solution conditions such as $\mathrm{pH}$, redox state, and major ion chemistry, which may vary along transport pathways (Boggs et al., 1985). For example, soluble oxidized species of $\mathrm{Pu}, \mathrm{Np}$, and Tc can form as a result of increased temperature (Weres et al., 1981), but decreasing temperature could cause hydrolysis and precipitation of a colloidal phase. The coupled effects of temperature $\left(25^{\circ}\right.$ to $\left.80^{\circ} \mathrm{C}\right)$, ionic strength, and $\mathrm{pH}$ have been reported for hematite sorbed on steel (Kuo and Matijevic, 1980). Sorption of U on hematite, over a range of temperature, was described in a previous section of this report (see Section 6.4).

\section{Time}

Analog studies are a useful resource for investigation of colloid behavior and transport over extended time periods. A few references exist for humic substances, but the results appear to be in conflict. No difference between fresh and mature humic substances was observed by Petersson et al. (1990), although Avogadro and deMarsily (1984) noted that aggregation and size distribution should be enhanced with time. Polymerization and reduction in carbonyl-group content during diagenesis were inferred to cause a reduction in solubility of humic substances (Longworth and Ivonovich, 1990). Desorption has also been observed in long-term experiments (Anderson and Benjamin, 1985). Colloid stability depends on physical adhesion, which is found to increase over time for some substances (Dabros and deVen, 1982). Data are needed not only for the chemical factors of colloid formation and stability, but also for rate-dependent factors.

\section{Radiolysis}

Only a few authors have considered the effects of radiolysis on colloid formation. Radiolysis can produce more soluble, oxidized states of some radionuclides (e.g., Pu and Np), which could significantly increase the solubility of radionuclides that might otherwise form colloids (Avogadro and deMarsily, 1984; Ewart and Williams, 1986). Other possible effects of radiolysis include degradation of organic substances and release of small water-soluble molecules from rubber, polyethylene, and polyvinyl chloride.

\section{Microbial Activity}


Microbial activity can cause dissolution, deflocculation, and colloid formation (Ramsay, 1988; Scholl and Harvey, 1990; Seyler and Martin, 1989). For example, organic substances such as kerogen, bitumen, and lignines that usually have low solubility can be solubilized by microbial activity. Formation of ferrous iron from the dissolution of iron in a reducing environment created by bacterial degradation has also been observed (Gschwend and Reynolds, 1987). Bacteria can also bind metals, secrete metal-solubilizing compounds, and transform metal ions to altered speciation states, thus affecting transport. These effects are presently being studied and models are being developed by Los Alamos National Laboratory, in collaboration with LLNL. Because knowledge of the processes that control the formation and behavior of biofilms is very scarce, the capability to predict radionuclide transport processes associated with generated biocolloids is limited (Hunt, 1990).

\subsubsection{Microbes in Radionuclide Migration}

Bacteria are important to repository performance because of their well-established capabilities to mediate chemical corrosion of metals and to adsorb radionuclides, thereby changing the mode of transport. Bacterial survival, growth, and transport in the NFE will be affected by radiological, thermal, hydrologic, and chemical conditions and by the intrinsic characteristics of the microbes.

The objective of this discussion is to provide an updated conceptual model for bacterial mobility in the NFE based on advances reported in the open literature and to discuss recent observations from the LBT. A basic understanding of bacterial and colloidal transport in porous media has emerged over the past $10 \mathrm{yr}$ (e.g., Ryan and Elimelech, 1996). Extension of this understanding to rough-walled fractures such as at Yucca Mountain will require formulating and experimentally validating an underlying conceptual model.

Much of the research on transport of bacteria through rocks and sediments has been conducted on porous media (e.g., Bales et al., 1989; Hanna and Taylor, 1996; Ryan and Elimelech, 1996; Tan et al., 1994). For this discussion, the detailed information in the literature is summarized into two main points:

- There are a limited number of sites on a mineral or grain surface to which bacteria can attach (Figure 6-43).

- Bacterial fluxes and attachment rates are predictable (Figure 6-44).

Figure 6-43 Maximum attached population density $\left(c_{\mathrm{s}}{ }^{\max }\right)$ at various ionic strengths

\section{Figure 6-44 Measures and predicted bacterial recovery in column effluent}

There is a dearth of observational data on the transport of bacteria and colloidal particles through individual fractures and fracture systems (Allen and Morrison, 1973; Bales et al., 1989; Reimus, 1993). Conceptual and analytical models have been developed from reported experiments, but the predictive power of these models is limited, and it has not been possible to produce quantitative predictions of bacterial transport in the repository or in the LBT.

In response to this situation, a preliminary set of experiments has been conducted on the transport of abiotic colloidal particles through smooth- and rough-walled fractures in Topopah Springs tuff (Figure 6-45). Abiotic particles are commonly used as proxies for bacteria because of their similar size and surface-charge density, their more easily controlled population density, and other characteristics.

Figure 6-45 Two types of fractures, a rough-walled fracture and a parallel-plate fracture, used in the laboratory experiments 
The surface topography of a rough-walled fracture in Topopah Springs tuff (Figure 6-46), has been measured using the PEAK device described in Durham (1997). The fracture aperture distribution can be classified into three groups: isolated pits where apertures exceed $1 \mathrm{~mm}$, a relatively large proportion of the area where the aperture is small and hydraulically insignificant, and an intermediate area. For the fracture surveyed, the mean and median apertures are 450 and $500 \mu \mathrm{m}$, respectively. These values are much greater than the typical bacterial size on the order of $1 \mu \mathrm{m}$. There is micron-scale texture on the fracture walls that could control the attachment of particles.

\section{Figure 6-46 A reconstructed image of the apertures and the aperture-probability plot for the fractured core}

Column experiments were conducted using the characterized natural fracture and an artificial sawcut fracture. Pulses of eluant laden with 1- $\mu \mathrm{m}$ latex microspheres, alternating with filtered eluant with a dye added, were introduced to the sample. Flow rates throughout the experiments were $0.154 \mathrm{~mL} / \mathrm{min}$ or about 1 fracture volume every $10 \mathrm{~min}$, and the background electrolyte was $10^{-2} \mathrm{M} \mathrm{KCl}$. Pulses of $10-\mathrm{mM}$ phenol red dye were alternated with pulses of about $10^{9}$ particles $/ \mathrm{mL}$. The sample was conditioned by flushing with the background electrolyte to remove reversibly attached particles; this was followed by a brief pulse of deionized water.

For the sawcut fracture (parallel plates), the observed particle transport was typical for open channel transport, as indicated by recovery curves (Figure 6-47). Tracer breakthrough occurred at about 1 fracture volume, and the shape indicates minimal dispersion. The latex particles and the dye tracer exhibited similar behavior. The maximum attached population was calculated from mass balance considerations to be $379 \times 10^{6}$ particles. The specific attachment was $55 \times 10^{9} \mathrm{~m}^{-2}$, which compares to the $30 \times 10^{9} \mathrm{~m}^{-2}$ observed in porous media experiments at the same ionic strength (Hanna and Taylor, 1996).

\section{Figure 6-47 Particle recovery curves for the parallel plate core}

Even though the breakthrough curves were similar, particle transport through the naturally fractured core was significantly different from that through the parallel-plate core (Figure 6-48). The particles showed rapid breakthrough and significant dispersion in the breakthrough curve. Rapid breakthrough indicates preferential pathways. Some parts of the fracture aperture system were apparently less accessible to the particles than to the dye tracer. The attached population density was calculated from mass balance to be $754 \times 10^{6}$ particles, or twice the value computed for the parallel-plate experiment. Calculation of specific attachment was complicated by the fractal nature of the surfaces. In addition, the particles may not have had equivalent access to all parts of the surface. Assuming that the particle diameter is the appropriate length-scale, then the specific attachment is $77 \times 10^{9} \mathrm{~m}^{-2}$, about $40 \%$ greater than specific attachment to the parallel-plate sample.

\section{Figure 6-48 Particle recovery curves for the fractured core}

Three conclusions can be drawn from these preliminary laboratory experiments:

- Preferential pathways for particle transport exist in the fracture aperture system.

- Preferential pathways produce rapid breakthrough of a large fraction of the injected particles.

- A significant fraction of injected particles are irreversibly captured. 
These experiments and the information available in the literature provide the starting point for a predictive model of bacterial mobility in the NFE. The following additional aspects can be investigated experimentally, and the results will support a more quantitative approach:

- The fracture capture capacity and the degree of irreversibility

- The effects of particle size

- The effects of temperature and saturation

- The effects of changes in fluid chemistry ( $\mathrm{pH}$ and ionic strength)

\section{Bacterial Movement During the Large Block Test}

The LBT is a large-scale field experiment with concurrent activities designed to evaluate coupled processes. The block was excavated in place, in the Topopah Spring tuff at the surface of Fran Ridge, near Yucca Mountain. The block dimensions are 4.5-m vertically and $3 \times 3 \mathrm{~m}$ horizontally (Lin et al., 1998). It was heated electrically for approximately 11 months in 1997 and early 1998. The block is insulated on its sides and top, has an impervious material wrapped around the sides, and has a heat exchanger on the top to impose a constant temperature $\left(60^{\circ} \mathrm{C}\right)$.

The block is extensively fractured (Figure 6-49); a subset of these fractures has been identified as most likely to contribute to transport (Figure 6-50), based on fracture aperture and persistence. Two fractures in this subset appear to be the major features of the block: a subhorizontal fracture is located in the upper part of the block (labeled 1 in Figure 6-50), and a subvertical fracture that intersects the heater holes into which labeled bacteria and microspheres were introduced (labeled 2 in Figure 6-50). Other subvertical fractures also intersect the heater holes.

\section{Figure 6-49 All fractures mapped on the five exposed surfaces of the large block}

\section{Figure 6-50 The major fractures of the large block fracture system}

Part of the LBT consists of evaluating the survival and migration of two strains of bacteria in response to TH activity and changes in the chemical environment. Transport of bacteria in the block will be controlled by fractures, which are also conduits for much of the mass flow. Qualitative predictions for microbial movement, based on laboratory experiments and assessment of the literature, included the following:

- Fluid flux will vary among different fractures, thus transport of labeled bacteria will likewise vary.

- Labeled bacteria will move mainly by advection along subvertical fractures. Owing to small pore sizes $(<<1 \mu \mathrm{m})$, matrix interactions will be minor.

- Channeling within individual fractures may lead to early arrival of bacteria.

- Irreversible capture of bacteria within the block fracture surfaces will be significant.

Mechanistic models for microbial transport in fractured geologic media have parameters (e.g., fracture-aperture distribution, flow velocity, and channeling characteristics) that cannot be readily evaluated in the block (or in the repository host rock). Accordingly, the time and location of breakthrough were not predicted quantitatively.

For the bacterial migration test, 2 separate 4-liter agar mixtures were prepared (Meike and Horn, 1997):

1. Bacillus subtillus, Arthrobacter oxydans and their drug resistant variants, (total concentration of $22 \mathrm{M}$ cells $\cdot \mathrm{mL}^{-1}$ and total number of 88 Gcells)

2. Fluorescent labeled B. subtillus and A. oxydans (total concentration of $15 \mathrm{M}$ cells $\cdot \mathrm{mL}^{-1}$ and total number of 59 Gcells) 
These two mixtures were uniformly distributed among the five heater holes about a month before the start of heating (see Figure 6-50). Sampling during the experiment was limited to three boreholes located below the heaters (boreholes E03, N01, and N02).

The heaters were energized at a total power of $2.2 \mathrm{~kW}$ for approximately 6 mos, then at lower power levels for an additional 4 mos. The temperature increased slowly as the thermohydrology of the block evolved (see Chapter 3). Evidence for episodic water reflux activity was observed as the temperature increased above boiling (Lin et al., 1998). Eventually the reflux subsided, and the temperature at the heater horizon approached the target $\left(140^{\circ} \mathrm{C}\right)$.

Sampling for labeled bacteria took place on August 5 and November 11, 1997, in the three sampling holes. The August samples tested negative for labeled bacteria, but the November samples were positive (Lin, 1998). It is unclear which fractures were involved in transport, nor is the timing of migration known. It would be of considerable interest to sample at additional points to evaluate fracture pathways and to assess whether bacteria have been transported upward. These objectives may be addressed during disassembly of the block at the conclusion of field activities. It should be noted that fractures directly intersecting heaters and boreholes are probably the major conduits for advective transport of particles; sampling of the block will be conducted accordingly.

The arrival of labeled bacteria at sampling locations in the LBT is consistent with results from laboratory experiments, which showed that bacteria are mobile and that considerable temporal and spatial heterogeneity of microbial transport can be expected. These results may eventually support a conclusion that microbial transport will be significant to repository performance.

Additional information is needed for this assessment, including characterization of the partitioning of dissolved radionuclides to bacteria and biomass, which is being investigated at Los Alamos National Laboratory. These results also indicate that indigenous bacteria, including those that can contribute to WP corrosion, are capable of movement into the NFE during cool-down or after the thermal period.

Further information that would support quantitative prediction of microbial and colloidal transport of radionuclides includes the following:

- Description of fracture fluid-velocity fields, in addition to the other results from $\mathrm{TH}$ modeling (see Chapter 3)

- Fracture-aperture distributions, for more quantitative description of arrival times and to describe the potential for irreversible capture of particles

- Fundamental, laboratory-derived experimental data on particle capture, flowchanneling, and the response of particle transport to changes in temperature, saturation, aqueous-phase chemistry, and particle size

The overall objective is to quantitatively bound the impact of bacterial and colloidal transport on radionuclide migration. A rigorous mathematical model of microbial behavior is probably unfeasible. Rather, an experimental approach, with emphasis on empirical models, is viewed as the most effective way to bound microbial effects in preparation for repository licensing.

\subsubsection{Summary of Alternative Modes of Radionuclide Transport}

Laboratory experiments consistently show that bacteria and colloids are transported faster than are conservative solute tracers in individual fractures. The wide range of apertures and the aperture correlation structure in natural fractures lead to preferential pathways for bacterial and colloidal transport. In addition, fracture surfaces appear to be able to irreversibly capture significant numbers of bacteria. 
Early results from the bacterial-transport experiment associated with the LBT show breakthrough of labeled bacteria near the base of the block. This observation is consistent with previous experiments and the basic understanding of bacterial transport, but quantitative prediction of the microbial contribution to radionuclide transport in the NFE requires more information. Specifically, the nature of the fracture-aperture distribution, fracture flow velocity, and irreversible capture are important questions. In addition, the response of microbial communities in the NFE to changes in environmental conditions will determine the population available for transport.

The formation, stability, and sorption of radionuclides by colloids in the NFE are matters of considerable uncertainty. The major introduced materials in the repository can serve as sources for colloidal particles with strong affinity for radionuclides. The chemical and physical environment is conducive to formation of colloids because of heterogeneous conditions, including composition, temperature, $\mathrm{pH}$, and redox state. There are few available data on colloid formation and mobility, particularly because colloidal processes are complex and difficult to control.

\subsection{Summary of Geochemical Conditions in the Near Field/Altered Zone}

This section describes modeling of chemical conditions in the NF/AZ. It emphasizes prediction of the water composition in the NFE (needed to assess the WP lifetime) and evaluation of the mobility of radionuclides that may be released from the WPs. The modeling approach begins with the interaction, at elevated temperature, of condensate water and the host rock in the $\mathrm{AZ}$ above the repository. A predictive model is presented for the chemical evolution of water in the host rock at temperatures from ambient to boiling. A second predictive model is presented for the chemical composition of water in contact with cementitious materials; this model is the basis for estimating the composition of water that contacts the WPs.

If radionuclides are released to the NFE, interaction with introduced materials will cause retardation of some species. Presented is a series of laboratory experiments that help define the retardation behavior of $\mathrm{U}$ and $\mathrm{Np}$ in contact with iron corrosion products and altered concrete. Also presented is a third chemical model that can be used to describe radionuclide speciation and precipitation at conditions ranging from an alkaline environment influenced by cement to the host rock environment at ambient temperature. Finally, interaction of radionuclides with introduced materials may cause colloidal transport, and this chapter provides a conceptual discussion of colloid transport issues. Further summary discussion on each of these topics is given in the following text.

This chapter summarizes model shat are available for use in repository design and performance analysis. Some calculations are provided as examples or to demonstrate model behavior over ranges of conditions. Additional calculations will be needed for viability assessment and repository licensing. The models are currently limited by the availability of laboratory experimental data, the completeness of chemical databases, and limitations on the chemical processes that are incorporated (e.g., boiling and coprecipitation).

\subsubsection{Altered Zone Water Composition}

Water that will interact with repository materials will be a mixture of ambient water similar to that from well J-13 and condensate that has interacted with rock fracture mineralogy. Evaporative concentration will produce significantly different effects, depending on the assumed chemical conditions (e.g., $\mathrm{CO}_{2}$ fugacity). The range of water compositions that is expected in the $\mathrm{AZ}$ will be bounded by the following: 
- During heat-up, the water composition will be dominated by evaporative processes, and composition will depend on the extent of evaporation. Most of this water will have been ambient percolation, but some may be refluxed condensate. Results presented in Section 6.2.2 bound the composition for this water, although some waters will be more concentrated as they are fully evaporated.

- During cool-down, the water composition will be dominated by condensate, and the actual composition will depend on the extent of rock-water interaction. The results presented in Section 6.2.2 summarize the range of expected compositions for these waters. It is expected that this water composition will interact most with repository materials.

- At the repository edges, and in the late stage of cool-down, the water composition will be dominated by ambient fracture flow percolating through the repository block. These waters are expected to be similar to J-13 well water as previously reported (Harrar et al., 1990).

- The actual water interacting with repository materials at various times and locations could be a mixture of these three types.

These estimates can be evaluated by comparing experimental and modeling results with observed natural-water compositions. The available data consistently show that fracture waters are dilute bicarbonate waters. However, significant discrepancies exist for some chemical components, suggesting that the modeling can be refined. Several specific areas for model refinement are identified, and they include chemical boundary conditions, dissolution rate kinetics, and minor mineral phases.

\subsubsection{Water Composition in Contact with Cement}

This chapter presents a model for water composition in the NFE that represents interaction with cementitious materials at different stages of maturation. The model differs from previous calculations in that additional cement phases are included in a new thermodynamic database to represent the observed products of hydrothermal alteration of Portland-type cement in the presence of $\mathrm{CO}_{2}$ (see Chapter 7). Cement mineral assemblages have been developed to represent different types of cement alteration. This new database is developmental; for TSPA-VA, it will be assessed along with previous data to produce estimates of water composition in contact with the WPs and interacting with released radionuclides.

Simulating the chemical environment when water first impinges on introduced materials after the thermal period is complicated by uncertainty about the nature and extent of cement alteration, the evolution of $\mathrm{CO}_{2}$ fugacity, and the chemical contributions from other concrete constituents. The alkaline $\mathrm{pH}$ effect from interaction with cement can be substantially moderated, depending on the $\mathrm{CO}_{2}$ pressure assumed. Using the new database, water compositions can be calculated for ranges of $\mathrm{CO}_{2}$ pressure and other variables.

\subsubsection{Concentrations of Radionuclides Leaving the Repository}

Iron oxides derived from the WP CAM have the potential to provide a sorptive barrier to radionuclide transport that could be equivalent to hundreds of meters of surrounding rock. Retardation will be strongly affected by fluid chemistry, particularly $\mathrm{pH}$ and $\mathrm{CO}_{2}$ fugacity, and by the nature of the flow path through the layer of corrosion products.

Model results indicate that, at high $\mathrm{pH}$, the presence of significant levels of $\mathrm{CO}_{2}$ can inhibit the sorption of $U$ and, by analogy, of Np. Simulations suggest that retardation will be significant for two scenarios that are likely to control fluid chemistry: (1) alkaline waters in 
which $\mathrm{CO}_{2}$ fugacity is controlled by calcite equilibrium (e.g., fluids within cementitious material) and (2) initially alkaline fluids in which $\mathrm{pH}$ is decreased by reaction with ambient $\mathrm{CO}_{2}$ (e.g., fluids that exit cementitious material).

Although the emphasis in these studies is sorptive behavior of near-field materials, simulations that consider precipitation and dissolution provide a more realistic assessment of radionuclide migration through corrosion products.

Hydrothermal alteration of concrete decreases the pore-fluid $\mathrm{pH}$ (relative to fresh concrete) and increases the relative abundance of crystalline Ca-Si-hydrates and clay minerals. Neptunium and $U$ were not detected in the effluent from a transport experiment conducted using a fractured core of concrete from the ESF that was hydrothermally altered to represent repository conditions. Altered concrete strongly retarded $\mathrm{U}$ and $\mathrm{Np}$ in these experiments, either by sorption or by causing precipitation of one or more solubility-limiting solid phases. The solubility hypothesis implies greater retardation performance than for sorption, but additional experiments are needed to establish the mechanism. Iodide was not retarded. Measurable fractions of the $\mathrm{U}$ and $\mathrm{Np}$ in batch experiments were associated with particulate matter; in the transport experiment, both the dissolved and particulate fractions were below detection.

\subsubsection{Radionuclide Transport in the Altered Zone}

It is expected that transport processes in the AZ below the repository will depart significantly from the unsaturated zone UZ site-scale transport model, which mainly considers the unaltered, ambient system. Thermally driven coupled processes could change the hydrologic and chemical characteristics of fracture pathways from the NFE in ways that are difficult to predict. However, chemical solubility can be used to bound the aqueous concentrations of radionuclides in the AZ.

The available data on the solubility of waste nuclides have been reviewed for use in repository performance analysis, with J-13 as the reference water composition. Solubilitycontrolling solid phases have been identified for $\mathrm{U}, \mathrm{Np}, \mathrm{Pu}, \mathrm{Am}, \mathrm{Tc}, \mathrm{Ni}$, and $\mathrm{Zr}$ for both oxidizing (high-Eh) and reducing (low-Eh) conditions. Solubilities of the U oxides, schoepite, $\mathrm{UO}_{2} \mathrm{CO}_{3}, \mathrm{PuO}_{2}$, and the $\mathrm{Ni}$ and $\mathrm{Zr}$ phases can be reliably predicted for J-13 water in the temperature range $20^{\circ}$ to $150^{\circ} \mathrm{C}$. For other radioelements, solubility-limiting phases have been identified (e.g., $\mathrm{NaNpO}_{2} \mathrm{CO}_{3} \cdot \mathrm{xH}_{2} \mathrm{O}$ and metastable phases containing oxidized $\mathrm{Pu}$ ), but there are no data for elevated temperatures. The relative need for further solubility experiments is reported to be $\mathrm{Np}>\mathrm{U}>\mathrm{Pu}>\mathrm{Am}>\mathrm{Zr}>\mathrm{Tc}>\mathrm{Ni}$. Development of a model for predicting coprecipitation effects has been initiated, but there are virtually no experimental data yet available for model testing. This is a potentially important mechanism for radionuclide retardation in the NF/AZ. An extension of this is the area of mixed-actinide solids, which will be important within the WPs and possibly within the NFE.

Laboratory experiments consistently show that bacteria and colloids are transported faster than are conservative solute tracers in individual fractures. The wide range of apertures and the aperture correlation structure in natural fractures lead to preferential pathways for bacterial and colloidal transport. In addition, fracture surfaces appear to be able to irreversibly capture significant numbers of bacteria.

Early results from the bacterial-transport experiment associated with the LBT show breakthrough of labeled bacteria near the base of the block. This observation is consistent with previous experiments and the basic understanding of bacterial transport, but quantitative prediction of the microbial contribution to radionuclide transport in the NFE requires more information. Specifically, the nature of the fracture-aperture distribution, 
fracture-flow velocity, and irreversible capture are important questions. The response of microbial communities in the NFE to changes in environmental conditions will determine the population available for transport.

The formation, stability, and sorption of radionuclides by colloids in the NFE is still a matter of considerable uncertainty. The major introduced materials in the repository can serve as sources for colloidal particles with strong affinity for radionuclides. The chemical and physical environment is conducive to forming colloids because of heterogeneous conditions, including composition, temperature, $\mathrm{pH}$, and redox state. There are few available data on colloid formation and mobility, particularly because colloidal processes are complex and difficult to control.

\section{Return to Table of Contents}

\subsection{References}

Agrawal, D.C., R. Raj, and C. Cohen (1989). "Nucleation of flocs in dilute colloidal suspensions." J. Am. Ceram. Soc. 72(11):2148-2153.

Ahn, T.M., and P. Soo (1995). "Corrosion of low-carbon cast steel in concentrated synthetic groundwater at $8-150^{\circ} \mathrm{C} . "$ Waste Manage. 15:471-476.

Albinsson, Y., K. Andersson, S. Borjesson, and B. Allard (1993). “Diffusion of Radionuclides in Concrete/Bentonite Systems." (SKB 93-29). Stockholm, Sweden: Swedish Nuclear Fuel and Waste Management Co.

Allard, B., I. Arsenie, H. Borne, J. Ephraim, G. Gardhammar, and C. Pettersson (1990). "Isolation and Characterization of Humics from Natural Waters." (SKB 90-27). Stockholm, Sweden: Swedish Nuclear Fuel and Waste Management Co. [NNA.19910123.0183]

Allen, M.J., and S.M. Morrison (1973). "Bacterial movement through fractured bedrock." Ground Water 11(2):6-10.

Allulli, S., M.A. Massucci, and N. Tomassini (1979). “The standard enthalpy of formation of anhydrous, monohydrated and dihydrated zirconium bis(monohydrogen phosphate)." J. Chem. Thermodynamics 11:613-618.

Anderson, P.R., and M.M. Benjamin (1985). "Effects of silicon on the crystallization and adsorption properties of ferric oxides." Env. Sci. Tech. 19(11):1048-1053.

Arthur, R.C., and W.M. Murphy (1989). "An analysis of gas-water-rock interactions during boiling in partially saturated tuff." Sci. Geol. Bull. 42(4):313-327.

Atkins, M., A.N. Beckley, and F.P. Glasser (1988). “Influence of cement on the near field environment and its specific interactions with uranium and iodine." Radiochim. Acta 44/45:255-261.

Atkins, M., and F.P. Glasser (1990). “Encapsulation of radioiodine in cementitious waste forms." In proceedings from Scientific Basis for Nuclear Waste Management XIII. V.M. Oversby and P.W. Brown (eds.). Mater. Res. Soc. 176:15-22. [203658]

Atkinson, A., N.M. Everitt, and R.M. Guppy (1989). “Time dependence of pH in a cementitious repository." In proceedings from Scientific Basis for Waste Management. Mater. Res. Soc. 127:439-446. [226315] 
Avogadro, A., and G. deMarsily (1984). "The role of colloids in nuclear waste disposal." In proceedings from Scientific Basis for Nuclear Waste Management VII (Mater. Res. Soc.). G.L.M. (ed.). Boston, MA: Elseview Science Publishing Company, New York. 26:495-505. [204393]

Badan, B., M. Magrini, and E. Ramous (1991). "A study of the microbiological-corrosion products of steel and cast iron pipes in fresh water." J. Mater. Sci. 26:1951-1954.

Baes, C.F., and R.E. Mesmer (1981). "The thermodynamics of cation hydrolysis." Am. J. Sci. 281:935-962.

Baker, S., R. McCrohon, P. Oliver, and N.J. Pilkington (1994). “The sorption of niobium, tin, iodine, and chlorine onto NIREX reference vault backfill." In proceedings from Scientific Basis for Nuclear Waste Management XVII. A. Barkatt and R.A. Van Konyenburg (eds.). Mater. Res. Soc. 333:719-724. [213541]

Balej, J., and J. Divisek (1993). "On the composition of higher solid nickel oxocompounds." Ber. Bunsenges. Phys. Chem. 97:929-933.

Bales, R.C., C.P. Gerba, G.H. Grondin, and S.L. Jensen (1989). “Bacteriophage transport in sandy soils and fractured tuff." App. and Environ. Microbiol. 55(8):2061-2067. [NNA.19930607.0065]

Balistrieri, L.S., and J.W. Murray (1982). "The adsorption of $\mathrm{Cu}, \mathrm{Pb}, \mathrm{Zn}$, and $\mathrm{Cd}$ on goethite from major ion seawater." Geochim. Cosmochim. Acta 46:1253-1265.

Balistrieri, L.S., and J.W. Murray (1987). "The influence of the major ions of seawater on the adsorption of simple organic acids by goethite." Geochim. Cosmochim. Acta 51:1151-1160. [NNA.19940509.0028]

Benjamin, M.M., and J.O. Leckie (1981a). “Competitive adsorption of $\mathrm{Cd}, \mathrm{Cu}, \mathrm{Zn}$, and $\mathrm{Pb}$ on amorphous iron oxyhydroxide." J. Colloid Inter. Sci. 83(2):410-419.

Benjamin, M.M., and J.O. Leckie (1981b). “Multiple-site adsorption of $\mathrm{Cd}, \mathrm{Cu}, \mathrm{Zn}$ and $\mathrm{Pb}$ on amorphous iron oxyhydroxide." J. Colloid Inter. Sci. 79:209-221.

Benjamin, M.M., R.S. Sletten, R.P. Bailey, and T. Bennett (1996). "Sorption and filtration of metals using iron-oxide-coated sand." Water Res. 30:2609-2620.

Bensted, J. (1989). “Oil well cements-A general review.” Chem. Ind. 20:100-105.

Bethke, C.M. (1994). The Geochemist's Workbench" ${ }^{\mathrm{TM}}$, Version 2.0. A Users Guide to Rxn, Act2, Tact, React, and Gtplot. Champaign, IL: Hydrology Program, University of Illinois.

Bethke, C.M. (1996). Reactive Transport and Basin Modeling. Champaign, IL: University of Illinois. (Short course workbook: Hydrogeology Program, May 29-31, 1996)

Bidoglio, G., A. Avogadro, and A.D. Plano (1985). "Influence of redox environments on the geochemical behaviour of radionuclides." In proceedings from Scientific Basis for Nuclear Waste Management IX. L.O. Werme (ed.). Stockholm, Sweden: Mater. Res. Soc. , Pittsburgh, PA. IX:709-716.

Bilinski, H., and M. Branica (1966). "Precipitation and hydrolysis of metallic ions in sea water. I. Iionic state of zirconium and thorium in sea water." Croatica Chemica Acta 38:263-267.

Bilinski, H., M. Branica, and L.G. Sillén (1966). "Precipitation and hydrolysis of metallic ions. II. Studies on the solubility of zirconium hydroxide in dilute solutions." Acta Chem. Scand. 20:853-861. 
Boggs, S., D. Livermore, and M.G. Seitz (1985). “Humic Substances in Natural Waters and their Complexation with Trace Metals and Radionuclides: A Review." (ANL-478). Argonne, IL: Argonne National Laboratory. [SRX. 19850420.0107]

Booker, C.L.C. (1976). “Nature of films, scales, and corrosion products on metals.” L.L. Shreir (ed.). Corrosion: Volume 1. Metal/Environment Reactions. London: Newnes Butterworths. pp. 1:22-31.

Borenstein, S.W. (1994). Microbially Influenced Corrosion Handbook. New York, NY: Industrial Press. [MOL. 19950314.0195]

Brady, P.V. (1994). "Alumina surface chemistry at 25, 40, and $60{ }^{\circ} \mathrm{C} . "$ Geochim Cosmochim. Acta 58:1213-1217.

Bruno, J., J. Depablo, L. Duro, and E. Figuerola (1995). “Experimental study and modeling of the $\mathrm{U}(\mathrm{VI})-\mathrm{Fe}(\mathrm{OH})_{3}$ surface precipitation coprecipitation equilibria." Geochim. Cosmochim. Acta 59:4113-4123.

Bruton, C. (1996). "Testing EQ3/ 6 and GEMBOCHS using fluid-mineral equilibria in the Wairakei geothermal system." D.G. Wilder (ed.). Near-Field and Altered-Zone Environment Report, Vol. II. Livermore, CA: Lawrence Livermore National Laboratory. (UCRL-LR-124998, Vol. II) [MOL.19961212.0121]

Bruton, C.J., W.E. Glassley, and B.E. Viani (1993). “Geochemistry.” Preliminary Near-Field Environment Report. Livermore, CA: Lawrence Livermore National Laboratory. Volume II: Scientific Overview of the Near-Field Environment and Phenomena:Section 3.0. (UCRL-LR-107476) [NNA.19920501.0002]

Bruton, C.J., and B.E. Viani (1992). "Geochemical modeling of water-rock interactions in the unsaturated zone." Y.K. Kharaka and A.S. Maest (eds.). Water-Rock Interaction 7. Netherlands: A.A. Balkema. pp. 705-708.

Bruton, C.J., and B.E. Viani (1996). “Ion Sorption onto Hydrous Ferric Oxides: Effect on Major Element Chemistry at Aspo, Sweden." (UCRL-JC-124329). Livermore, CA: Lawrence Livermore National Laboratory. [226334]

Buddemeier, R.W., and J.R. Hunt (1988). "Transport of colloidal contaminants in groundwater: Radionuclide migration at the Nevada Test Site." Appl. Geochem. 3:535-548. [NNA.19930701.0051]

Carlos, B. (1987). "Minerals in Fractures of the Saturated Zone from Drill Core USW-G4, Yucca Mountain, Nye County, Nevada." (LA-10827-MS). Los Alamos, NM: Los Alamos National Laboratory. (32 pp.) [HQS.19880517;1112]

Carlos, B. (1989). "Fracture-Coating Minerals in the Topopah Spring Member and Upper Tuff of Calico Hills from Drill Hole J-13." (LA-11504-MS). Los Alamos, NM: Los Alamos National Laboratory. (20 pp.) [202383]

Carlos, B. (1994). "Field Guide to Fracture-Lining Materials, Yucca Mountain, Nevada." (LA12803-MS). Los Alamos, NM: Los Alamos National Laboratory. (12 pp.) [MOL.19950117.0112]

Carlsen, L. (1984). Basic Geochemical Research for Migration Studies. Radionuclide-Soil Organic Matter Interactions. Denmark: Riso Laboratory.

Carlson, L., and U. Schwertmann (1981). "Natural Ferrihydrites in Surface Deposits from Finland and their Association with Silica." Geochim. Cosmochim. Acta 45:421429. 
Carroll, S., E. Mroczek, M. Alai, and M. Ebert (1996). “Kinetics of Rock-Water Interaction: Amorphous Silica Precipitation $\left(60\right.$ to $\left.120^{\circ} \mathrm{C}\right)$; Comparison of Laboratory and Field Rates." D.G. Wilder (ed.). Near-Field and Altered-Zone Environment Report, Vol. II. Livermore, CA: Lawrence Livermore National Laboratory. pp. 10.4-910.4-26. (UCRL-LR-124998, Vol. II) [MOL.19961212.0122]

Carroll, S.A., E. Mroczek, W. Bourcier, M. Alai, and M. Ebert (1995). “Comparison of Field and Laboratory Precipitation Rates of Amorphous Silica from Geothermal Waters at $100^{\circ} \mathrm{C}$." Milestone report for the CRWMS Management and Operating Contractor, U.S. Department of Energy. (MOL207;). Livermore, CA: Lawrence Livermore National Laboratory. (Also UCRL-JC-128486 [1997, Geochim. Cosmochim. Acta]) [MOL.19960415.0465]

Cartledge, G.H., and W.T. Smith (1955). "Revision of the electrode potential diagram for technetium." J. Phys. Chem. 59:1111.

Casas, I., J. Bruno, E. Cera, R.J. Finch, and R.C. Ewing (1994). “Kinetic and Thermodynamic Studies of Uranium Minerals: Assessment of the Long-Term Evolution of Spent Nuclear Fuel." SKB technical report. (SKB 94-16). Stockholm, Sweden: Swedish Nuclear Fuel and Waste Management Co.

Chessa, G., G. Marangoni, B. Pitteri, N. Stevanato, and A. Vavasori (1991). "Sorption and separation of palladium, platinum and gold chlorocomplexes by means of a dipicolinic acid polystyrene-based chelating resin." Reactive Polymers 14(2):143-150.

Chickerur, N.S., B.B. Sabat, and P.P. Mahaptra (1980). “Solubility and thermodynamic data of nickel hydroxide." Thermochim. Acta 41:375-377.

Chung, J.Y., and K.J. Lee (1990). “The solubility of magnetite and nickel ferrite in hightemperature aqueous solutions." High Temp. Sci. 30:51-67.

Cobble, J.W., W.T. Smith, and G.E. Boyd (1953). “Thermodynamic properties of technetium and rhenium compounds: II. Heats of formation of technetium heptoxide and pertechnetic acid, potential of the technetium(IV)-technetium(VII) couple and a potential diagram for technetium." J. Am. Chem. Soc. 75:5777.

Cornell, R.M., R. Giovanoli, and P.W. Schindler (1987). “Effect of silicate species on the transformation of ferrihydrite into geothite and hematite in alkaline media." Clays $\mathcal{E}$ Clay Min. 35(1):21-28.

Criscenti, L.J., and R.C. Arthur (1989). “The calculated effects of isothermal boiling on tuffwater interactions." Radiochim. Acta 52/53:513-517. [HQX.19891005.0043]

Dabros, T., and T.G.M.V. deVen (1982). "Kinetics of coating by colloidal particles." J. Col. Int. Sci. 89(1):232-244. [224945]

Daily, W., W. Lin, and T. Buscheck (1987). "Hydrological properties of Topopah Spring tufflaboratory measurements." J. Geophys. Res. 92(B8):7854-7864. (Also UCRL94363, Lawrence Livermore National Laboratory, Livermore, CA). [203030]

Daveler, S.A., and T.J. Wolery (1992). "EQPT, A Data File Preprocessor for the EQ3/6 Software Package: User's Guide and Related Documentation (Version 7.0)." (UCRL-MA-110662). Livermore, CA: Lawrence Livermore National Laboratory. (part 2) [NNA.19930129.0101]

Davis, J.A., and D.B. Kent (1990). "Surface complexation modeling in aqueous geochemistry." M.F.J. Hochella and A.F. White (eds.). Mineral-Water Interfacial Geochemistry, Reviews in Mineralogy. Washington, DC: Mineral. Soc. Am. (Volume 23) [NNA.19920501.0130; 224085] 
Davis, J.O. (1982). "Adsorption of natural dissolved organic matter at the oxide-water interface." Geochim. Cosmochim. Acta 46:2381-2393.

Delany, J.M. (1985). "Reaction of Topopah Spring Tuff with J-13 Water: A Geochemical Modeling Approach Using the EQ3/ 6 Reaction Path Modeling Code." (UCRL53631). Livermore, CA: Lawrence Livermore National Laboratory. (48 pp.) [NNA.871111.0114; HQS.19880517.2419]

Dibrov, I.A., and R.V. Grigoreva (1977). "Determination of the heat of formation of higher nickel oxides." Soviet Electrochem. 13:825-829.

Dinov, K., C. Matsuura, D. Hiroishi, and K. Ishigure (1993). "Solubility of nickel and cobalt oxides in high-temperature water." Nucl. Sci. E Eng. 113:207-216.

Dobrokhotov, G.N. (1954). "pH values during the processes of precipitation of metal hydroxides from sulfate solutions." J. Appl. Chem. USSR 27:995-1004.

Durham, W.B. (1997). "Laboratory observations of the hydraulic behavior of a permeable fracture from 3800-m depth in the KTB pilot hole." J. Geophys. Res. 102:1840518416.

Dzombak, D.A., and F.M. Morel (1990). Surface Complexation Modeling: Hydrous Ferric Oxide. New York, NY: John Wiley \& Sons. [224535]

El-Sonbati, A.Z., A.A. El-Bindary, M.A. Diab, M.A. El-Ela, and S.A. Mazrouh (1994). "Polymer complexes: 25. Complexing ability of poly(5-vinylsalicylidene-2aminopyridine) toward different metal (II) sales." J. Polymer 3:647-652.

Emara, M.M., N.A. Farid, and H.A. Shehata (1987). “Ionic association of transition-metal ions with bicarbonate using spectrophotometric method. 2. Nickel and cobalt bicarbonates in aqueous and aqueous-alcohol mixtures." J. Indian. Chem. Soc. 64:119-122.

Eriksen, T.E., P. Ndalamba, J. Bruno, and M. Caceci (1992). “The solubility of $\mathrm{TcO}_{2} \cdot \mathrm{nH}_{2} \mathrm{O}$ in neutral to alkaline solutions under constant pCO2." Radiochim. Acta 58/59:6770.

Ershov, B.G., and N.L. Sukhov (1990). "A pulse radiolysis study of the process of the colloidal metal formation in aqueous solution." Radiat. Phys. Chem. 36:93-97.

Evans, U.R. (1960). The Corrosion and Oxidation of Metals. London: Edward Arnold.

Ewart, F.T., and S.J. Williams (1986). "A Literature Survey of the Possible Effects of Humic and Fulvic Acids on the Disposal of LLW and ILW." (AERE R 12023). Harwell, UK: United Kingdom Atomic Energy Authority.

Felmy, A.R., D. Rai, J.A. Schramke, and J.L. Ryan (1989). "The solubility of plutonium hydroxide in dilute solution and in high-ionic-strength chloride brines." Radiochim. Acta 48:29-35.

Fox, L.E. (1988). “The solubility of colloidal ferric hydroxide and its relevance to iron concentrations in river water." Geochim. Cosmochim. Acta 52:771-777.

Fuentes, H.R., W.L. Polzer, J. Gruber, B. Lauctes, and E.H. Esington (1987). “Preliminary Report on Sorption Modeling." (LA-10952MS). Los Alamos, NM: Los Alamos National Laboratory. [NNA.19900206.0221]

Furet, N.R., C. Haces, F. Corvo, and C. Diaz (1990). “Corrosion rate determination using Fe-57 Mossbauer spectra of corrosion products of steel." Hyperfine Interact. 57:18331838. 
Fyfe, D. (1976). “The atmosphere.” L.L. Shreir (ed.). Corrosion Volume 1: Metal/Environment Reactions. London: Newnes Butterworths. pp. 2:26-37. [HQS.19880517.2536]

Gardiner, M.P., A.J. Smith, and S.J. Williams (1990). "Sorption onto colloidal corrosion product simulants." In proceedings from Manteo III: Concepts in Manipulating Groundwater Colloids for Environmental Restoration. Manteo, NC: U.S. Department of Energy.

Gayer, K.H., and A.B. Garrett (1949). "The equilibria of nickel hydroxide in solutions of hydrochloric acid and sodium hydroxide at $25^{\circ} \mathrm{C} . "$ J. Am. Chem. Soc. 71:29732975.

Glasser, F.P., M.J. Angus, C.E. McCulloch, D. MacPhee, and A.A. Rahman (1985). “The chemical environment in cements." Mater. Res. Soc. Symp. Proc. 44:849-858. [NNA.910207.0039]

Glassley, W.E. (1986). “Reference Waste Package Environment Report.” (UCRL-53726). Livermore, CA: Lawrence Livermore National Laboratory. [NNA.920506.0035; 20314]

Glassley, W.E., and S. Boyd (1995). “Preliminary Description of Small Block Mineralogical Features." Milestone report for the CRWMS Management and Operating Contractor, U.S. Department of Energy. (M0L062). Livermore, CA: Lawrence Livermore National Laboratory. [MOL.19950721.0246]

Glassley, W.E., and L. DeLoach (1997). “Second Quarter Results of Chemical Measurements in the Single Heater Test." Milestone report for the CRWMS Management and Operating Contractor, U.S. Department of Energy. (SP9240M4). Livermore, CA: Lawrence Livermore National Laboratory. [MOL.19971218.0917]

Goto, S., K. Suenaga, T. Kado, and M. Fukuhara (1995). “Calcium silicate carbonation products." J. Am. Ceram. Soc. 78:2867-2872.

Grambow, B., R.S. Forsyth, L.O. Werme, and J. Bruno (1990). “Fission product release from spent $\mathrm{UO}_{2}$ fuel under uranium-saturated oxic conditions." Nucl. Technol. 92:204-213.

Grauer, R. (1989). “The Coordination Chemistry of Humic Substances.” NAGRA Technical Report. (89-08E). Switzerland: NAGRA.

Greenfield, B.F., C.M. Linklater, A.D. Moreton, M.W. Spindler, and S.J. Williams (1993). “The effects of organic degradation products on actinide disposal." AEA Technol.

Grenthe, I., J. Fuger, R.J.M. Konings, R.J. Lemire, A.B. Muller, C. Nguyen-Trung, and H. Wanner (1992). Chemical Thermodynamics, Vol. 1: Chemical Thermodynamics of Uranium. Amsterdam: North Holland. [NNA.19940415.0009]

Grenthe, I., I. Puigdomenech, M.C.A. Sandino, and M.H. Rand (1995). “Corrections to the Uranium NEA-TDB Review." R.J. Silveet al. (eds.). Chemical Thermodynamics of Americium. Amsterdam: North Holland. (Appendix D)

Gschwend, P.M., and M.D. Reynolds (1987). "Monodisperse ferrous phosphate colloids in an anoxic groundwater plume." J. Contaminant Hydrol. 1:309-327. [NNA.19931005.0018]

Hanna, M.L., and R.T. Taylor (1996). “Attachment/ detachment and trichloreothylene degradation-longevity of a resting cell Methylosinus trichosporium OB3b filter." J. Biotech. E Bioengr. 51(6):659-672.

Hanzawa, Y., D. Hiroishi, C. Matsuura, and K. Ishigure (1996). "Solubility of nickel ferrite in high-temperature pure or oxygenated water." Nucl. Sci. Eng. 124:211-218. 
Harrar, J., J.F. Carley, W.F. Isherwood, and E. Raber (1990). “Report of the Committee to Review the Use of J-13 Well Water in Nevada Nuclear Waste Storage Investigations." (UCRL-ID-21867). Livermore, CA: Lawrence Livermore National Laboratory. [NNA.910131.0274]

Hayes, K.F., and W.J. Weber (1990). "Surfactant mobilization of NAPL residuals in subsurface systems." In proceedings from Manteo III: Concepts in Manipulating Groundwater Colloids for Environmental Restoration. Manteo, NC: U.S. Department of Energy. (platform and poster abstracts)

Heath, T.G., D.J. Ilett, and C.J. Tweed (1996). “Thermodynamic modelling of the sorption of radioelements onto cementitious materials." In proceedings from Scientific Basis for Nuclear Waste Management XIX. W.M. Murphy and D.A. Knecht (eds.). Mater. Res. Soc. 412:443-449.

Hersman, L.E. (1996). "Summary and Synthesis of Biological Sorption and Transport." Milestone report for the CRWMS Management and Operating Contractor, U.S. Department of Energy. (3663). Las Vegas, NV: Los Alamos National Laboratory. [MOL.19970822.0242]

Ho, C.H., and N.H. Miller (1986). "Adsorption of uranyl species from bicarbonate solution onto hematite particles." J. Colloid Inter. Sci. 110:165-171.

Hsi, C.-K., and D. Langmuir (1985). “Adsorption of uranyl onto ferric oxyhydroxides: Application of the surface complexation site-binding model." Geochim. Cosmochim. Acta 49:1931-1941. [NNA.19920922.0011; 224090]

Hughes, D.E. (1976). “The microbiology of corrosion.” L.L. Shreir (ed.). Corrosion Volume 1. Metal/Environment Reactions. London: Newnes Butterworths. pp. 2:73-82.

Hunt, J.R. (1990). “Moderator's overview on manipulating colloids to change aquifer permeability." In proceedings from Manteo III: Concepts in Manipulating Groundwater Colloids for Environmental Restoration. Manteo, NC: U.S. Department of Energy. (platform and poster abstracts)

Hunter, K.A., D.J. Hawke, and L.K. Choo (1988). “Equilibrium adsorption of thorium by metal-oxides in marine electrolytes." Geochim. Cosmochim. Acta 52:627-636.

Iler, R.K. (1979). The Chemistry of Silica. New York, NY: Wiley-Interscience.

Itagaki, H., S. Nakayama, S. Tanaka, and M. Yamawaka (1992). "Effect of ionic strength on the solubility of neptunium(V) hydroxide." Radiochim. Acta 58/59:61-66. [MOL.19950202.0003]

Jena, P.K., and B. Prasad (1956). “Determination of the solubility product of nickel hydroxide." J. Indian. Chem. Soc. 33:122-124.

Jenkins, S.R. (1973). "Effect of selected cation concentration on coagulation and adhesion to silica surfaces of $\partial-\mathrm{MnO}_{2}$." Environ. Sci. Tech. 7(1):43-47.

Johnson, B.B. (1990). "Effect of $\mathrm{pH}$, temperature and concentration on the adsorption of cadmium on goethite." Environ. Sci. Tech. 24(1):112-118.

Johnson, J., and S. Lundeen (1994). “GEMBOCHS Thermodynamic Datafiles For Use With the EQ3 / 6 Software Package." Yucca Mountain Site Characterization Project Milestone Report. (MOL72). Livermore, CA: Lawrence Livermore National Laboratory. [MOL.19950727.0017] 
Johnson, J.W., and W.E. Glassley (1996). "Formation of Flow and Transport Barriers Within the Altered Zone." D.G. Wilder (ed.). Near-Field and Altered-Zone Environment Report, Vol. II. Livermore, CA: Lawrence Livermore National Laboratory. (UCRL-LR-124998, Vol. II) [MOL.19960501.0271]

Kerrisk, J.F. (1984). "Solubility Limits on Radionuclide Dissolution at a Yucca Mountain Repository." (LA-9995-MS). Los Alamos, NM: Los Alamos National Laboratory. [NNA.19870519.0049]

Kim, J.I., and B. Kanellakopulos (1989). "Solubility products of plutonium(IV) oxide and hydroxide." Radiochim. Acta 48:145-150. [MOL.19950131.0021]

Kim, N.D., and S.J. Hill (1993). "Sorption of lead and thallium on borosilicate glass and polypropylene: Implications for analytical chemistry and soil science." Environ. Technol. 14:1015-1026.

Knauss, K. (1987). “Zeolitization of glassy Topopah Spring tuff under hydrothermal conditions." In proceedings from Mater. Res. Soc. Symposium. 84:737-745. (Also UCRL-94664, Lawrence Livermore National Laboratory, Livermore, CA) [NNA.920302.0050]

Knauss, K.G., and W.J. Beiriger (1984). "Report on Static Hydrothermal Alteration Studies of Topopah Spring Tuff Wafers in J-13 Water at $150^{\circ} \mathrm{C} . "$ (UCRL-53576). Livermore, CA: Lawrence Livermore National Laboratory. [HQS.19880517.2007]

Knauss, K.G., W.J. Beiriger, and D.W. Peifer (1985a). “Hydrothermal Interaction of Crushed Topopah Spring Tuff and J-13 Water at 90, 150, and $250^{\circ} \mathrm{C}$ Using DicksonType, Gold-Bag Rocking Autoclaves." (UCRL-53630). Livermore, CA: Lawrence Livermore National Laboratory. [NNA.19931005.0010]

Knauss, K.G., W.J. Beiriger, and D.W. Peifer (1987). “Hydrothermal Interaction of Solid Wafers of Topopah Spring Tuff with J-13 Water and Distilled Water at 90 and $150^{\circ} \mathrm{C}$ Using Dickson-Type, Gold-Bag Rocking Autoclaves: Long-Term Experiments." (UCRL-53722). Livermore, CA: Lawrence Livermore National Laboratory. [NNA.19870713.0081; 203013]

Knauss, K.G., W.J. Beiriger, D.W. Peifer, and A.J. Piwinskii (1985b). “Hydrothermal Interaction of Solid Wafers of Topopah Spring Tuff with J-13 Water and Distilled Water at 90, 150, and $250^{\circ} \mathrm{C}$, Using Dickson-Type, Gold-Bag Rocking Autoclaves." (UCRL-53645). Livermore, CA: Lawrence Livermore National Laboratory. [NNA.19900207.0282; HQS.19980517.2482]

Knauss, K.G., and S.A. Copenhaver (1995). “Progress Report on Hydrothermal Alteration of Vitric Tuff from Yucca Mountain." Lawrence Livermore National Laboratory, Earth Sciences Division: memorandum to Bill Glassley. June 1955.

Knauss, K.G., J.M. Delany, W.J. Beiriger, and D.W. Peifer (1986). “Hydrothermal interaction of Topopah Spring tuff with J-13 water as a function of temperature." In proceedings from Mater. Res. Soc. Symp. Proc. 44:539-546. (Also UCRL-90853 Abs, Lawrence Livermore National Laboratory, Livermore, CA)

Knauss, K.G., and D.W. Peifer (1986). “Reaction of Vitric Topopah Spring Tuff and J-13 Ground Water Under Hydrothermal Conditions, Using Dickson-Type, GoldBag Rocking Autoclaves." (UCRL-53795). Livermore, CA: Lawrence Livermore National Laboratory. [NAA.891102.0117]

Kobayashi, K., K. Suzuki, and Y. Uno (1994). “Carbonation of concrete structures and decomposition of C-S-H." Cem. Concr. Res. 24:55-61. 
Kohler, M., E. Wieland, and J.O. Leckie (1992). “Metal-ligand-surface interactions during sorption of uranyl and neptunyl on oxides and silicates." In proceedings from Seventh International Symposium Water-Rock Interaction. Park City, UT: International Assn. of Geochim. and Cosmochim. 1:51-54.

Kovalenko, P.N., and K.N. Bagdasarov (1961). "The solubility of zirconium hydroxide." Russ. J. Inorg. Chem. 6:272-275.

Kucera, V., and E. Mattsson (1987). “Atmospheric Corrosion.” F. Mansfeld (ed.). Corrosion Mechanisms. New York, NY: Marcel Dekker. pp. 211-284.

Kuo, R.J., and E. Matijevic (1980). "Particle adhesion and removal in model systems." J. Colloid. Interface. Sci. 78:407-419. [MOL.19950103.0112; 224881]

Kurnoskin, A.V. (1992). "Reaction mechanisms of the metal chelates with epoxy oligomers and the structures of the epoxy-chelate metal-containing matrixes." J. Appl. Polym. Sci. 46:1509-1530.

Langmuir, D. (1997). Aqueous Environmental Geochemistry. Upper Saddle River, NJ: Prentice Hall.

Lemire, R.J., G.D. Boyer, and A.B. Campbell (1993). “The solubilties of sodium and potassium dioxoneptunium $(\mathrm{V})$ carbonate hydrates at $30^{\circ} \mathrm{C}, 50^{\circ} \mathrm{C}$, and $76^{\circ} \mathrm{C} . "$ Radiochim. Acta 61(57-63).

Lemire, R.J., and F. Garisto (1989). “The Solubility of U, Np, Pu, Th and Tc in a Geological Disposal Vault for Used Nuclear Fuel." (AECL-10009). Atomic Energy of Canada Ltd. [213161]

Lemire, R.J., and F. Garisto (1992). "The effect of ionic strength, groundwater composition, and temperature on calculated radionuclide solubilities." Radiochim. Acta 58/59:37-44.

Lemire, R.J., and P.R. Tremaine (1980). “Uranium and plutonium equilibria in aqueous solutions to $200^{\circ} \mathrm{C} . "$ J. Chem. Eng. Data 25:361-370.

Liang, L. (1988). "Effects of Surface Chemistry on Kinetics of Coagulation of Submicron Oxide Particles $\left(\alpha-\mathrm{Fe}_{2} \mathrm{O}_{3}\right)$ in Water." Pasadena, CA: California Institute of Technology, Ph.D. Thesis.

Lieser, K.H., B. Gleitsmann, S. Peschke, and T. Steinkopff (1986). “Colloid formation and sorption of radionuclides in natural systems." Radiochim. Acta 40:39-47.

Lin, W. (1998). “Update of the Large-Block Test.” March 6, 1998. Biweekly electronic report. (UCRL-MI-129071). Livermore, CA: Lawrence Livermore National Laboratory.

Lin, W., and W.D. Daily (1984). "Transport Properties of Topopah Spring Tuff." (UCRL53602). Livermore, CA: Lawrence Livermore National Laboratory. [NNA.891026.0025; 203004]

Lin, W., and W.D. Daily (1991). "Variation of permeability with temperature in fractured Topopah Spring tuff samples." In proceedings from Second Annual International High-Level Radioactive Waste Management Conference. Las Vegas, NV: Am. Nucl. Soc., La Grange Park, IL. 988-993. (Also UCRL-JC-104765, Lawrence Livermore National Laboratory, Livermore, CA) [NNA.910523.0105; 201212]

Lin, W., J.J. Roberts, W.E. Glassley, and D. Ruddle (1995). “The Effect of Rock-Water Interaction on Permeability." (UCRL-JC-119574). Livermore, CA: Lawrence Livermore National Laboratory. [MOL.19960416.0275] 
Lin, W., D. Wilder, S. Blair, T. Buscheck, W. Daily, G. Gdowski, W. Glassley, K. Lee, A. Meike, A. Ramirez, J. Roberts, D. Ruddle, J. Wagoner, D. Watwood, T. Williams, R. Carlson, and D. Neubauer (1998). "An overview of progress on the large-block test of the ESF thermal tests." In proceedings from 1998 International High-Level Radioactive Waste Management Conference. May 11-14, 1998. Am. Nucl. Soc. (Also UCRL-JC-128796 Ext. Abs., October 1997 preconference print by Lawrence Livermore National Laboratory, Livermore, CA) [MOL.19980120.0041]

Linklater, C.M., Y. Albinsson, W.R. Alexander, I. Casas, I.G. McKinley, and P. Sellin (1996). "A natural analogue of high $\mathrm{pH}$ cement pore waters from the Maqarin area of Northern Jordan: Comparison of predicted and observed trace element chemistry of uranium and selenium." J. Contam. Hydrol. 21(1-4):59-69.

Longworth, G., and M. Ivonovich (1990). "Sampling, characterization and transport studies of groundwater colloids." In proceedings from Manteo III: Concepts in Manipulating Groundwater Colloids for Environmental Restoration. Manteo, NC: U.S. Department of Energy.

Ma, W.P., P.W. Brown, and S. Komarneni (1996). "Sequestration of cesium and strontium by tobermorite synthesized from fly ashes." J. Am. Ceram. Soc. 79:1707-1710.

Maiti, T.C., M.R. Smith, and J.C. Laul (1989). “Colloid formation study of U, Th, Ra, Pb, Po, $\mathrm{Sr}, \mathrm{Rb}$, and Cs in briny (high ionic strength) groundwaters: analog study for waste disposal." Nucl. Technol. 84:82-87.

Martin, S.I. (1994). “Synthesis of 1.1-nm tobermorite: A cement phase expected under repository conditions." In proceedings from Sixth International High-Level Radioactive Waste Management Conference. Las Vegas, NV: Am. Nucl. Soc., La Grange Park, IL. [MOL.19950501.0039]

Matijevic, E. (1982a). “Properties of Colloidal Corrosion Products and their Effects on Nuclear Plants. Volume 1: Executive Summary." (NP-2606 Vol. 1, Research Project 9661). Palo Alto, CA: Electric Power Research Institute.

Matijevic, E. (1982b). "Properties of Colloidal Corrosion Products and their Effects on Nuclear Plants. Volume 2." (NP-2606 Vol. 2, Research Project 966-1). Palo Alto, CA: Electric Power Research Institute.

Matijevic, E. (1986). "Properties of Colloidal Corrosion Products and the Effect on Nuclear Plants." (EPRI NP-4817). Palo Alto, CA: Electric Power Research Institute.

McCarthy, J.F., and J.M. Zachara (1989). "Subsurface transport of contaminants." Environ. Sci. Tech. 23(5):496-502. [NNA.19930625.0032]

McMurry, J. (1997). “Comparison of Thermodynamic Data in Three Uranium Databases." (TR-765, COG-960564-I, SDDO). Chalk River, Ontario, Canada: Atomic Energy Canada Limited.

Meijer, A. (1990). "A strategy for the derivation and use of sorption coefficients in performance assessment calculations for the Yucca Mountain site." In proceedings from DOE/Yucca Mountain Site Characterization Project Radionuclide Adsorption Workshop. Los Alamos, NM: Los Alamos National Laboratory: pp. 9-40. (LA-12325-C) [NNA.19920421.0117]

Meike, A. (1996). “Introduced (Man-Made) Materials." Near-Field and Altered-Zone Environment Report. Livermore, CA: Lawrence Livermore National Laboratory. Chapter 6.0. (UCRL-LR-124998) [MOL.19961108.0016] 
Meike, A., and W.E. Glassley (1997). “Chemical Modeling of Backfill Composed of Quartz Sand, Lime, and an Fe-Phase." (UCRL-ID-124631). Livermore, CA: Lawrence Livermore National Laboratory. [MOL.19971217.0351]

Meike, A., and J. Horn (1997). “Installation of Labeled Microbe Samples into Large Block.” Letter report for the CRWMS Management and Operating Contractor, U.S. Department of Energy. (SPLG1BM4). Livermore, CA: Lawrence Livermore National Laboratory. [MOL.19970507.0188]

Meike, A., and C. Wittwer (1993a). "Formation of colloids from introduced materials in the post-emplacement environment: A report on the state of understanding." In proceedings from Topical Meeting on Site Characterization and Model Validation: Focus '93. Am. Nucl. Soc., La Grange Park, IL. (Also UCRL-JC-114782, Lawrence Livermore National Laboratory, Livermore, CA)

Meike, A., and C. Wittwer (1993b). "Introduced materials and colloid formation: A report on the current state of knowledge." In proceedings from Boston, MA: Mater. Res. Soc. Fall Mtg. 333:783-789. (Also UCRL-JC-114122 [1994], Lawrence Livermore National Laboratory, Livermore, CA) [NNA.19940316.0115]

Meyer, R.E., and W.D. Arnold (1991). “The electrode potential of the Tc(IV)-Tc(VII) couple." Radiochim. Acta 55:19-22.

Mills, W.B., and S. Liu (1989). “Literature Review and Conceptual Model Development for Colloid/Metals Transport (COMET) Model in Porous Media." Contract No. 68-03-3513 for EPA and AQUA TERRA CONSULTANTS. Lafayette, CA: Tetra Tech, Inc.,.

Miuahara, K. (1993). "Sensitivity of uranium solubility to variation of ligand concentrations in groundwater." J. Nucl. Sci. and Technol. 30:314-332.

Moll, H., G. Geipel, W. Matz, G. Bernhard, and H. Nitsche (1996). "Solubility and speciation of $\left(\mathrm{UO}_{2}\right)_{2} \mathrm{SiO}_{4} \cdot 2 \mathrm{H}_{2} \mathrm{O}$ in aqueous systems." Radiochim. Acta 74:3-7.

Murphy, W.M., and R.T. Pabalan (1995). “Review of Empirical Thermodynamic Data for Uranyl Silicate Minerals and Experimental Plan." (CNWRA 95-014). San Antonio, TX: Center for Nuclear Waste Regulatory Analyses.

Nakayama, S., and T. Nagano (1991). “Dissolution of neptunium dioxide in aqueous solutions." Radiochim. Acta 52/53:9-11.

Nakayama, S., T. Yamaguchi, and K. Sekine (1996). "Solubility of neptunium(IV) hydrous oxide in aqueous solutions." Radiochim. Acta 74:15-19.

NEA (1992). Chemical Thermodynamics of Uranium. Amsterdam: North Holland. (Ingmar Grenthe [Chairman], Royal Institute of Technology, Stockholm, Sweden) [NNA.19940415.0009]

NEA (1995). Chemical Thermodynamics of Americium. Amsterdam: North Holland. (Robert J. Silve [Chairman], Lawrence Livermore National Laboratory, Livermore, CA)

Neck, V., J.I. Kim, and B. Kanellakopulos (1992). “Solubility and hydrolysis behavior of neptunium(V)." Radiochim. Acta 56:25-30. [MOL.19950202.0011]

Neck, V., W. Runde, J.I. Kim, and B. Kanellakopulos (1994). “Solid-liquid equilibrium reactions of neptunium $(\mathrm{V})$ in carbonate solution at different ionic strength." Radiochim. Acta 65:29-37. 
Neu, M.P. (1993). “Coordination Chemistry of Two Heavy Metals: I. Ligand Preferences in Lead(II) Complexation, Toward the Development of Therapeutic Agents for Lead Poisoning. II. Plutonium Solubility and Speciation Relevant to the Environment." Berkeley, CA: University of California, Berkeley, Ph.D. Thesis.

Nguyen, S.N., J.R. Silva, H.C. Weed, and J.E. Andrews Jr. (1991). “Standard Gibbs free energies of formation at the temperature $303.15 \mathrm{~K}$ of four uranyl silicates: soddyite, uranophane, sodium boltwoodite and soldium weeksite." J. Chem. Thermodynamics 24:359-376. (UCRL-JC-106032). [NAA.19940221.0163]

Nitsche, H. (1991). “Solubility studies of transuranium elements for nuclear waste disposal: Principles and overview." Radiochim. Acta 52/53:3-8. (LBL-27173). [NNA.19920421.0122]

Nitsche, H., and N.M. Edelstein (1985). “Solubilities and speciation of selected transuranium ions. A comparison of a non-complexing solution with a groundwater from the Nevada tuff." Radiochim. Acta 39:23-33. [NNA.19930326.0097]

Nitsche, H., R.C. Gatti, E.M. Standifer, S.C. Lee, A. Mueller, T. Prussin, R.S. Deinhammer, H. Maurer, K. Becraft, S. Leung, and S.A. Carpenter (1993a). “Measured Solubilities and Speciations of Neptunium, Plutonium and Americium in a Typical Groundwater (J-13) from the Yucca Mountain Region." Milestone report for the CRWMS Management and Operating Contractor, U.S. Department of Energy. (3010-WBS1.2.3.4.1.3.1, LA-12562-MS). Los Alamos, NM: Los Alamos National Laboratory. [MOL.19950621.0265]

Nitsche, H., K. Roberts, K. Becraft, T. Prussin, D. Keeney, S.A. Carpenter, and D.E. Hobart (1995). "Solubilities and Speciation Results from Over- and Undersaturation Experiments on Neptunium, Plutonium and Americium in Water from Yucca Mountain Region Well UE-25p \#1." Milestone report for the CRWMS Management and Operating Contractor, U.S. Department of Energy. (LA13017-MS). Los Alamos, NM: Los Alamos National Laboratory.

Nitsche, H., K. Roberts, T. Prussin, A. Mueller, K. Becraft, D. Keeney, S.A. Carpenter, and R.C. Gatti (1993b). "Measured Solubilities and Speciations of Neptunium, Plutonium and Americium in UE-25p \#1 Well Water from the Yucca Mountain Region." Milestone report for the CRWMS Management and Operating Contractor, U.S. Department of Energy. (33290-WBS1.2.3.4.1.3.1, LA-12562MS). Los Alamos, NM: Los Alamos National Laboratory. [MOL.19950621.0265]

Nitsche, H., K. Roberts, R. Xi, T. Prussin, K. Becraft, Al Mahamid, H.B. Silber, S.A. Carpenter, R.C. Gatti, and C.F. Novak (1994). "Long-term plutonium solubility and speciation studies in a synthetic brine." Radiochim. Acta 66/67:3-8.

Novak-Adamic, D.M., B. Cosovic, H. Bilinski, and M. Branica (1973). "Precipitation and hydrolysis of metallic ions. Nickel(II) in aqueous solutions." J. Inorg. Nucl. Chem. 35:2371-2382.

Ollila, K., M. Olin, and M. Lipponen (1996). “Solubility and oxidation state of uranium under anoxic conditions ( $\mathrm{N}_{2}$ atmosphere)." Radiochim. Acta 74:9-13.

Olofsson, U., B. Allard, K. Andersson, and B. Torstenfelt (1981). "Formation and Properties of Radiocolloids in Aqueous Solution-A Literature Survey." (Report Prav 4.25). Sweden: Chalmers University of Technology, Department of Nuclear Chemistry, Programrådet för radioaktvit avfall. [NNA.19920401.0110]

Olofsson, U., B. Allard, B. Torstenfelt, and K. Andersson (1982). "Properties and mobilities of actinide colloids in geologic systems." Scientific Basis for Nuclear Waste Management 5. (W. Lutze, ed.). [NNA.19920131.0322] 
Ortiz, T.S., R.L. Williams, F.B. Nimick, B.C. Whittet, and D.L. South (1985). “A ThreeDimensional Model of Reference Thermal/Mechanical and Hydrologic Stratigraphy at Yucca Mountain, Southern Nevada." (SAND84-1076). Albuquerque, NM: Sandia National Laboratories. [NNA.890315.0013; 201909]

Oversby, V.M. (1985). "The Reaction of Topopah Spring Tuff with J-13 Water at $150^{\circ} \mathrm{C}$ Samples from Drill Cores USW G-1, USW GU-3, USW G-4, and UE-25h\#1." (UCRL-53629). Livermore, CA: Lawrence Livermore National Laboratory. [HQS.19880517.2517]

Palmer, C.E.A., R.J. Silva, and J.J. Bucher (1996). “Thermodynamic Database Needs Modeling Studies of the Yucca Mountain Project." (UCRL-ID-125343). Livermore, CA: Lawrence Livermore National Laboratory. [MOL.19970123.0014]

Palmer, C.E.A., R.J. Silva, and C.W. Miller (1992). "Speciation Calculations of Pu, Np, Am, and U in J-13 Well Water: Effects of Anion Concentration and pH." Livermore, CA: Lawrence Livermore National Laboratory. (draft dated September 1992)

Pavlovic, Z., and R. Popovic (1969). "Analytical and thermodynamical aspects of nickel corrosion in aqueous solutions." In proceedings from Fourth International Congress on Metals Corrosion. N.E. Hammer (ed.). Houston, TX: Nat. Ass. Corros. Eng. pp. 517-526. (published 1972)

Petersson, C., J. Ephraim, B. Allard, and H. Boren (1990). "Characterization of Humic Substances from Deep Groundwaters in Granitic Bedrock in Sweden." (SKB Report 9). Stockholm, Sweden: Swedish Nuclear Fuel and Waste Management Co. [NNA.19910122.0281]

Pilkington, N.J. (1990). “The solubility of technetium in the near-field environment of a radioactive waste repository." J. Less-Common Met. 161:203-212.

Puigdomenech, I., J.A. Rard, A.V. Plyasunov, and I. Grenthe (1997). “Temperature Corrections to Thermodynamic Data and Enthalpy Calculations." Thermodynamic Modelling of Aqueous Systems. Livermore, CA: Lawrence Livermore National Laboratory. (UCRL-JC-126374; (preprint of chapter to be published))

Raber, E., J. Garrison, and V. Oversby (1983). “The Sorption of Selected Radionuclides on Various Metal and Ploymeric Materials." Radioactive Waste Management and the Nuclear Fuel Cycle. 4:41-52.

Rai, D. (1984). "Solubility product of $\mathrm{Pu}(\mathrm{IV})$ hydrous oxide and equilibrium constants of $\mathrm{Pu}(\mathrm{IV}) / \mathrm{Pu}(\mathrm{V}), \mathrm{Pu}(\mathrm{IV}) / \mathrm{Pu}(\mathrm{VI})$, and $\mathrm{Pu}(\mathrm{V}) / \mathrm{Pu}(\mathrm{VI})$ couples." Radiochim. Acta 35:97-106. [MOL.19950131.0020]

Rai, D., A.R. Felmy, and J.L. Ryan (1990). “Uranium(IV) hydrolysis constants and solubility product of $\mathrm{UO}_{2} \cdot \mathrm{xH}_{2} \mathrm{O}(\mathrm{am})$." Inorg. Chem. 29:260-264.

Rai, D., R.G. Strickert, and G.L. McVay (1982). “Neptunium concentrations in solutions contacting actinide-doped glass." Nucl. Technol. 58:69-76.

Rai, D., and J.L. Swanson (1981). “Properties of plutonium(IV) polymer of environmental importance." Nucl. Technol. 54:107-112. [NNA.19870406.0303]

Raloff, J. (1990). “The colloid threat.” Science News March 17, 1990: p. 1969. [NNA.19920131.0347]

Ramsay, J.D.F. (1988). "The role of colloids in the release of radionuclides from nuclear waste." Radiochim. Acta 44/45:165-170. 
Ramsay, J.D.F., R.G. Avery, and P.J. Russell (1988). “Physical characteristics and sorption behaviour of colloids generated from cementitious systems." Radiochim. Acta 44/45:119-124. [NNA.19931005.0015]

Reimus, P.W. (1993). “Transport of Synthetic Xolloids Through Single Saturated Fractures: A Literature Review." (LA-12707-MS). Los Alamos, NM: Los Alamos National Laboratory. [MOL.19951027.0220]

Reynolds, M.D. (1982). “Colloids in Groundwater.” Cambridge, MA: Massachusetts Institute of Technology, Ph.D. Thesis.

Robert, K.E., H.B. Silber, P.C. Torette, T. Prussin, K. Becraft, D.E. Hobart, and D.F. Novak (1996). "The experimental determination of the solubility product for $\mathrm{NpO}_{2} \mathrm{OH}$ in $\mathrm{NaCl}$ solution." Radiochim. Acta 74:27-30.

Runde, W., D. Janecky, J. Kaszuba, and C.D. Tait (1998a). “Impact of the Formation of $\mathrm{NpO}_{2}$ and $\mathrm{Np}_{2} \mathrm{O}_{5}$ on the NP solubility in Yucca Mountain Waters." Milestone report for the CRWMS Management and operating Contractor, U.S. Department of Energy. (SPU33M4). Las Vegas, NV: Los Alamos National Laboratory.

Runde, W., M.P. Neu, and D.L. Clark (1996). “Neptunium(V) hydrolysis and carbonate complexation-experimental and predicted neptunyl solubility in concentrated $\mathrm{NaCl}$ using the Pitzer approach." Geochim. Cosmochim. Acta 60:2065-2073.

Runde, W., P.D. Palmer, and D. Tait (1998b). “Synthesis and Characterization of $\mathrm{NpO}_{2}, \mathrm{NpO}_{2}$ $(\mathrm{OH})$, and $\mathrm{Np}_{2} \mathrm{O}_{5} . "$ Milestone report for the CRWMS Management and Operating Contractor, U.S. Department of Energy. (SP341W4). Las Vegas, NV: Los Alamos National Laboratory.

Ryan, J.N., and M. Elimelech (1996). “Colloid mobilization and transport in groundwater." Colloids and Surfaces A: Physiochemical and Engineering Aspects 107:1-56.

Saetta, A.V., B.A. Schrefler, and R.V. Vitaliani (1993). “The carbonation of concrete and the mechanism of moisture, heat and carbon dioxide flow through porous materials." Cem. Concr. Res. 23:761-772.

Sanchez, A.L., J.W. Murray, and T.H. Sibley (1985). “The adsorption of plutonium IV and V on goethite." Geochim. Cosmochim. Acta 49:2297-2307. [NNA.19920922.0019]

Sandino, A., and J. Bruno (1992). "The solubility of $\left(\mathrm{UO}_{2}\right)_{3}\left(\mathrm{PO}_{4}\right)_{2} \cdot 4 \mathrm{H}_{2} \mathrm{O}(\mathrm{s})$ and the formation of $\mathrm{U}(\mathrm{VI})$ phosphate complexes-their influence in uranium speciation in natural waters." Geochim. Cosmochim. Acta 56:4135-4145.

Scholl, M.A., and R.W. Harvey (1990). "Laboratory studies of bacterial sorption to sediments in a contaminated aquifer." In proceedings from Manteo III: Concepts in Manipulating Groundwater Colloids for Environmental Restoration. Manteo, NC: U.S. Department of Energy.

Schwertmann, U., and R.M. Cornell (1991). Iron Oxides in the Laboratory: Preparation and Characterization. New York, NY: VCH.

Serne, R.J., D. Rai, P.F. Martin, A.R. Felmy, L. Rao, and S. Ueta (1996). “Leachability of Nd, U, Th, and Sr from cements in a $\mathrm{CO}^{2}$-free atmosphere." Mat. Res. Soc. Symp. Proc. 412:459-467.

Seyler, P., and J.-M. Martin (1989). "Biochemical processes affecting arsenic species distribution in a permanently stratified lake." Environ. Sci. Technol. 23(10):1258-1263. 
Sheka, I.A., and T.V. Pevzner (1960). "Solubility of zirconium and hafnium hydroxides in sodium hydroxide solutions." Russ. J. Inorg. Chem. 5:1119-1121.

Silber, H.B., H. Nitsche, R. Gatti, and H. Gehmecker (1994). “The effects of radiolysis upon speciation and solubility of neptunium in brine solutions." Radiochim. Acta 66/67:15-18.

Silva, R.J., G. Bidoglio, M.H. Rand, P.B. Robouch, H. Wanner, and I. Puigdomenech (1993). Chemical Thermodynamics of Americium. Amsterdam: North Holland. [NNA.19931022.0023]

Silva, R.J., and H. Nitsche (1995). "Actinide environmental chemistry." Radiochim. Acta 70/71:377-396.

Smith, R.W., J.C. Walton, and M. Rahman (1994). "Effects of recrystallization on time variant sorption of radionuclides onto steel corrosion products." Mater. Res. Soc. Symp. Proc. 333:713-718.

Steefel, C.I., and S.B. Yabusaki (1995). “OS3D/GIMRT: Software for Modeling Multicomponent-Multidimensional Reactive Transport." Richland, WA: Pacific Northwest Laboratory, Battelle Memorial Institute.

Story, S.P., and L.E. Hersman (1995). “Desferriozamine B-Enhanced Transport of Iron in Volcanic Tuff During Unsaturated Conditions." Milestone report for the CRWMS Management and Operating Contractor, U.S. Department of Energy. (3410). Las Vegas, NV: Los Alamos National Laboratory. [MOL.19960813.9990]

Stumm, W., H. Hueper, and R.L. Champlin (1967). "Formation of polysilicates as determined by coagulation effects." Curr. Res. 1(3):221-227.

Tan, Y., J.T. Gannon, P. Baveye, and M. Alexander (1994). “Transport of bacteria in an aquifer sand: Experiments and model simulations." Water Resour. Res. 30(12):32433252.

Tang, D. (1997). “Emplacement Drift Ground Support." May 23, 1997. Yucca Mountain Project report. (BCAA00000-01717-0200-00003, Rev. 00B). Las Vegas, NV: prepared by the Civilian Radioactive Waste Management System, Management and Operating Contractor, for the U.S. Department of Energy.

Taylor, H.F.W. (1990). Cement Chemistry. London: Academic Press.

Ticknor, K.V. (1994). “Uranium sorption on geological materials.” Radiochim. Acta 64:229-236.

Tochiyama, O., S. Endo, and Y. Inoue (1995). "Sorption of neptunium (V) on various iron oxides and hydrous iron oxides." Radiochim. Acta 68:105-111.

Tremaine, P.R., and J.C. Leblanc (1980). “The solubility of nickel oxide and hydrolysis of $\mathrm{Ni}^{2+}$ in water to $573 \mathrm{~K} . "$ J. Chem. Thermodynamics 12:521.

Triay, I., B. Robinson, A. Mitchell, C. Overly, and R. Lopez (1993). “Transport of neptunium through Yucca Mountain tuffs." Mater. Res. Soc. Symp. Proc. 294:797-802.

Triay, I.R., C. Degeldre, A. Meijer, J. Conca, S. Kung, and R. Rundberg (1997). “Summary Report Geochemistry/Transport Laboratory Tests." Milestone report for the CRWMS Management and Operating Contractor, U.S. Department of Energy. (SP23QM2; LA-CST-7-TIP-97-016). Los Alamos, NM: Los Alamos National Laboratory.

TRW (1997). "The Site-Scale Unsaturated Zone Transport Model of Yucca Mountain." Prepared for the U.S. Department of Energy by the CRWMS M\&O Contractor, TRW Environmental Safety Systems, Inc. (SP25BM3). Las Vegas, NV: TRW Environmental Safety Systems, Inc. [MOL.19980205.0102] 
Turner, D.R. (1995). “A Uniform Approach to Surface Complexation Modeling of Radionuclide Sorption." San Antonio, TX: Center for Nuclear Waste Regulatory Analyses. (CNWRA 95-001)

Van Geen, A., A.P. Robertson, and J.O. Leckie (1994). “Complexation of carbonate species at the geothite surface: Implications for adsorption of metal ions in natural waters." Geochim. Cosmochim. Acta 58:2073-2086.

Van Luik, A., D. Stahl, and D. Harrison (1992). "Progress in waste package and engineered barrier system performance assessment and design." Mater. Res. Soc. Symp. Proc. 294:663-673.

Viani, B.E. (1995). “Scientific Investigation Plan for YMP WBS Element 1.2.3.10.3.1 Integrated Radionuclide Release: Tests and Model Development (Integrated Testing)."

(SIP-IT-01, Rev. 0). Livermore, CA: Lawrence Livermore National Laboratory.

Viani, B.E. (1996a). “Integrated Testing." Near-Field and Altered-Zone Environment Report, Vol. II. Livermore, CA: Lawrence Livermore National Laboratory. Chapter 7. [19961212.0121]

Viani, B.E. (1996b). "Materials to be used for Radionuclide Transport Experiments." Milestone report for the CRWMS Management and Operating Contractor, U.S. Department of Energy. (SPL3A1M4). Livermore, CA: Lawrence Livermore National Laboratory.

Viani, B.E., and C.J. Bruton (1991). "Modeling Fluid-Rock Interaction at Yucca Mountain, Nevada." (UCRL-ID-109921). Livermore, CA: Lawrence Livermore National Laboratory. [NNA.920805.0002]

Viani, B.E., and M.L. Carman (1996). "Transport of Soluble Species through Tuff Core.” Milestone report for the CRWMS Management and Operating Contractor, U.S. Department of Energy. (SPL7A1M4, MOL52). Livermore, CA: Lawrence Livermore National Laboratory. (Also UCRL-ID-129183, Lawrence Livermore National Laboratory, Livermore, CA) [MOL.19970117.0050]

Viani, B.E., and S.I. Martin (1994). “Core Flow Experiment Protocol.” Milestone Report for the CRWMS Management and Operating Contractor, U.S. Department of Energy. (M0L04). Livermore, CA: Lawrence Livermore National Laboratory. (Also UCRL-ID-129184, Lawrence Livermore National Laboratory, Livermore, CA) [MOL.19950727.0022]

Viani, B.E., and P.C. Torretto (1997). "Sorption and Transport of Uranium on Hematite." Milestone report for the CRWMS Management and Operating Contractor, U.S. Department of Energy. (SPL3BM4). Livermore, CA: Lawrence Livermore National Laboratory. [MOL.19980107.0394]

Viani, B.E., P.C. Torretto, and S.L. Matzen (1997). “Radionuclide Transport Through Engineered Barrier System Alteration Products." Milestone report for the CRWMS Management and Operating Contractor, U.S. Department of Energy. (SPL3FM4). Livermore, CA: Lawrence Livermore National Laboratory. [MOL.19980115.0871]

Wagman, D.D., W.H. Evans, V.B. Parker, R.H. Schumm, I. Halow, S.M. Bailey, K.L. Churney, and R.L. Nuttall (1982). "The NBS tables of chemical thermodynamic properties. Selected values for inorganic and $\mathrm{C}_{1}$ and $\mathrm{C}_{2}$ organic substances in SI units." J. Phys. Chem. Ref. Data 11(Supplement No. 2).

Weres, O., A. Yee, and L. Tsao (1981). “Kinetics of silica polymerization.” J. Colloid Interface Sci. 84(2):379-402. 
Wilder, D.G. (1993). “Preliminary Near-Field Environment Report, Volume I: Technical Bases for EBS Design." (UCRL-LR-107476, Vol. I). Livermore, CA: Lawrence Livermore National Laboratory. (NNA.19920501.0002)

Wilder, D.G. (1996). “Near-Field and Altered-Zone Environment Report, Vol. II.” (UCRL-LR124998, Vol. II). Livermore, CA: Lawrence Livermore National Laboratory. [MOL.19961212.0121; MOL.19961212.0122]

Wilder, D.G. (1997). “Near-Field and Altered-Zone Environment Report, Volume I: Technical Bases for EBS Design." (UCRL-LR-124998, Vol. I). Livermore, CA: Lawrence Livermore National Laboratory.

Wilson, C.N., and C.J. Bruton (1990). "Ceramic transactions." In proceedings from Nuclear Waste Management III. G.B. Mellinger (ed.). Westerville, OH: Am. Ceram. Soc. 9:423-441.

Wolery, T.J. (1992a). “EQ3/6, A Software Package for Geochemical Modeling of Aqueous Systems: Package Overview and Installation Guide." (UCRL-MA-110662). Livermore, CA: Lawrence Livermore National Laboratory. (part 1) [NNA.19921023.0028]

Wolery, T.J. (1992b). “EQ3NR, A Computer Program For Geochemical Aqueous SpeciationSolubility Calculations: Theoretical Manual, User's Guide, and Related Documentation." (UCRL-MA-110662). Livermore, CA: Lawrence Livermore National Laboratory. (Version 7.0) [NNA.19921218.0010]

Wolery, T.J., and S.A. Daveler (1992). “EQ6, A Computer Program for Reaction Path Modeling of Aqueous Geochemical Systems: Theoretical Manual, User's Guide, and Related Documentation (Version 7.0)." (UCRL-MA-110662). Livermore, CA: Lawrence Livermore National Laboratory. (part 4) [MOL.19980218.0570]

Wolery, T.J., C.E. Palmer, and K.G. Knauss (1995). “The Neptunium Solubility Problem in Repository Performance Assessment: A White Paper." July 24, 1995. White Paper. Livermore, CA: Lawrence Livermore National Laboratory, Yucca Mountain Site Characterization Project.

Wruck, D.A., and C.E.A. Palmer (1996). “Effect of Temperature on the Disproportionation Rate of Plutonium(V) in Near-Neutral Solutions." Milestone report for the CRWMS Management and Operating Contractor, U.S. Department of Energy. (WBS 1.2.3.10.3.2). Livermore, CA: Lawrence Livermore National Laboratory.

Wruck, D.A., and C.E.A. Palmer (1997). "Analysis of Elevated Temperature Data for Thermodynamic Properties of Selected Radionuclides." Milestone report for the CRWMS Management and Operating Contractor, U.S. Department of Energy. (SPL4B1M4). Livermore, CA: Lawrence Livermore National Laboratory. (UCRL-ID-128955) [MOL.19971210.0037]

Yamaguchi, T., Y. Sakamoto, and T. Ohnuki (1994). “Effect of the complexation on solubility of $\mathrm{Pu}(\mathrm{IV})$ in aqueous carbonate system." Radiochim. Acta 66/67:15-18. 
(a)

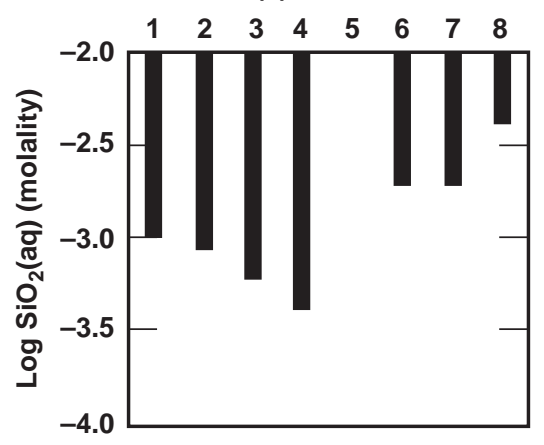

(b)
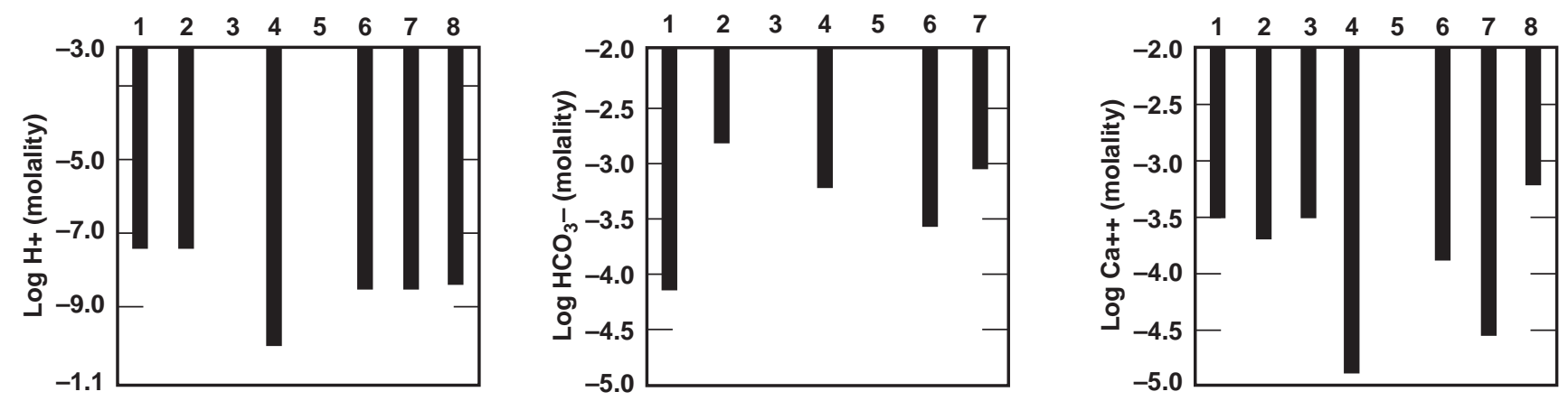

(c)
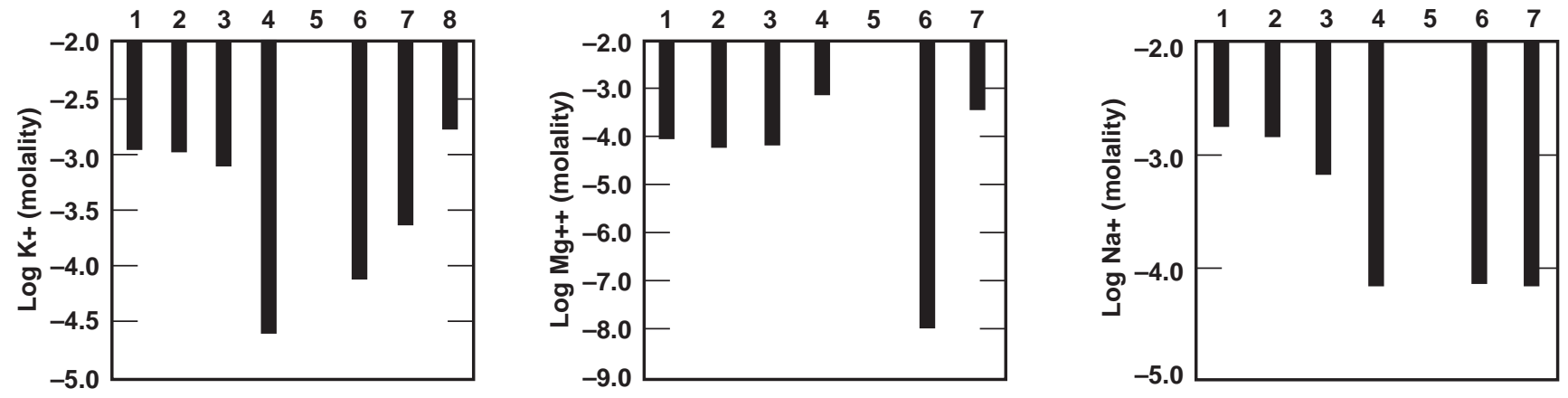

Figure 6-1. Bar graphs of water compositions from various fracture studies. The vertical axis on all graphs is the log molality of the indicated species. All values shown are negative, as indicated by the parentheses. Bar 1 is water from well J-13 (Harrar et al., 1990) shown for reference. Bar 2 is fracture water from Rainier Mesa (White and Peterson, 1990). Bar 3 is water collected from bore hole 16 from the SHT. Bar 4 is water composition for the $25^{\circ} \mathrm{C}$ model of condensate flow in a 15-m fracture. Bar 5 is blank. Bar 6 is water composition for the $95^{\circ} \mathrm{C}$ model of fracture flow in a $1.5-\mathrm{m}$ fracture. Bar 7 is water composition for the $95^{\circ} \mathrm{C}$ model of fracture flow in a $15-\mathrm{m}$ fracture. Bar 8 is the $104^{\circ} \mathrm{C}$ water composition for the fracture flow experiment (Lin et al., 1995). (a) Dissolved $\mathrm{SiO}_{2}$; (b) $\mathrm{H}^{+}, \mathrm{HCO}_{3}^{-}$, and $\mathrm{Ca}^{+2}$; and (c) $\mathrm{K}+, \mathrm{Mg}^{+2}$, and $\mathrm{Na}^{+}$. 


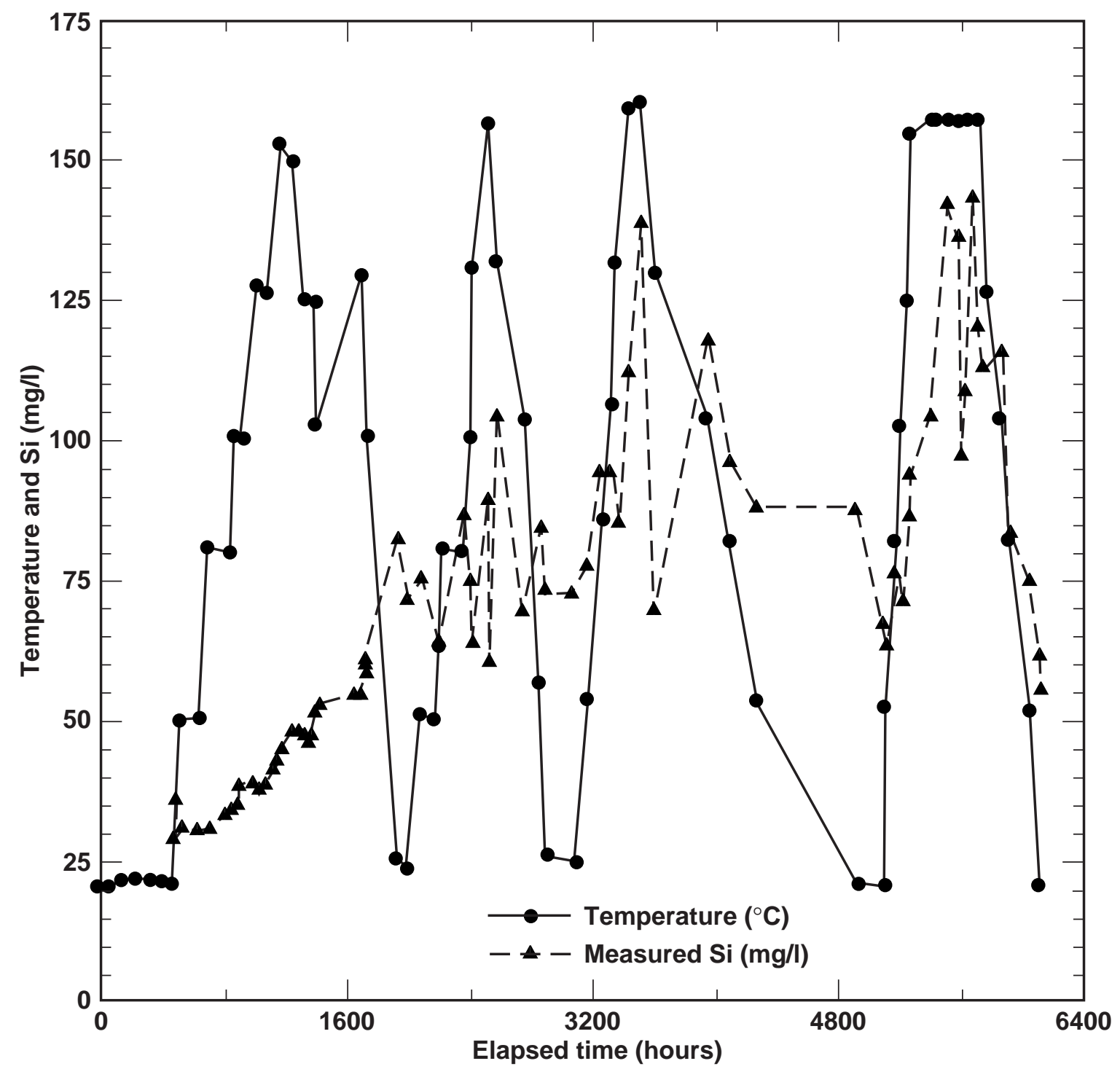

Figure 6-2. Temperature $\left({ }^{\circ} \mathrm{C}\right)$, outlet Si concentration $(\mathrm{mg} / \mathrm{L})$, and temperature-dependent theoretical saturation Si concentration values for amorphous silica and cristobalite as a function of time for the fracture flowthrough experiment (Lin et al., 1995). Temperature $\left({ }^{\circ} \mathrm{C}\right.$, circles) and outlet $\mathrm{Si}$ concentration $(\mathrm{mg} / \mathrm{L}$, triangles) are shown as a function of time for the fracture flow through experiment (Lin et al., 1995). Note the lack of correlation between temperature and Si concentration for the first heating-cooling cycle and the strong correlation between them for the third and fourth cycles. 


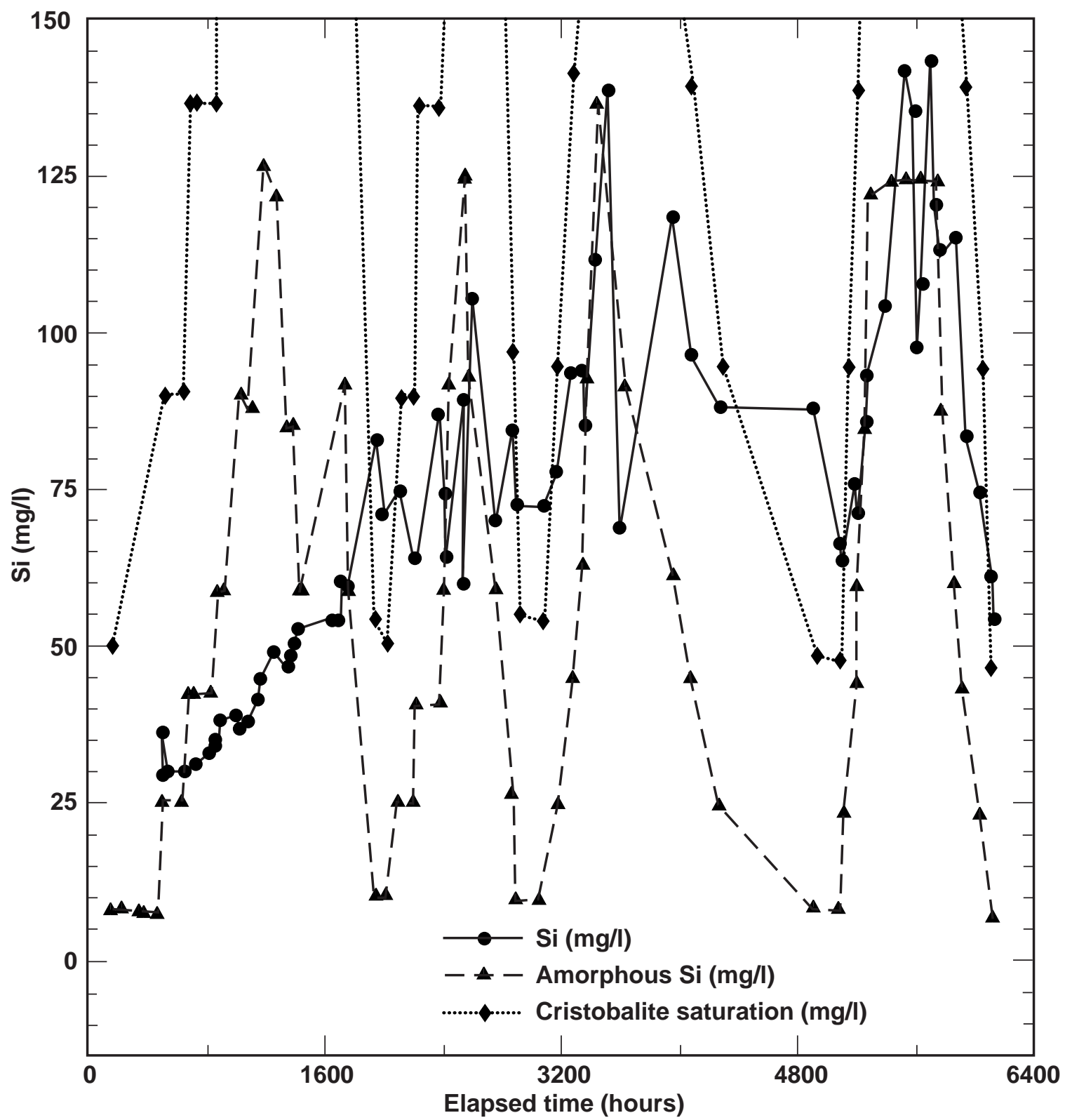

Figure 6-3. Temperature $\left({ }^{\circ} \mathrm{C}\right)$, outlet $\mathrm{Si}$ concentration $(\mathrm{mg} / \mathrm{L})$, and temperature-dependent theoretical saturation $\mathrm{Si}$ concentration values for amorphous silica and cristobalite as a function of time for the fracture flowthrough experiment (Lin et al., 1995). Si concentration (mg/L, circles) and the temperature-dependent amorphous silica (mg/L, triangles) and cristobalite $(\mathrm{mg} / \mathrm{L}$, diamonds) theoretical saturation concentrations are shown as a function of time. The saturation values were obtained from thermodynamic data, assuming nearneutral $\mathrm{pH}$. An actual concentration greater than a theoretical saturation concentration is supersaturated with respect to the corresponding phase. Note the supersaturation of the solution with respect to both amorphous silica and cristobalite at the temperature minima occurring at ca. 2000, 3000, and $4800 \mathrm{hr}$. 
(a)

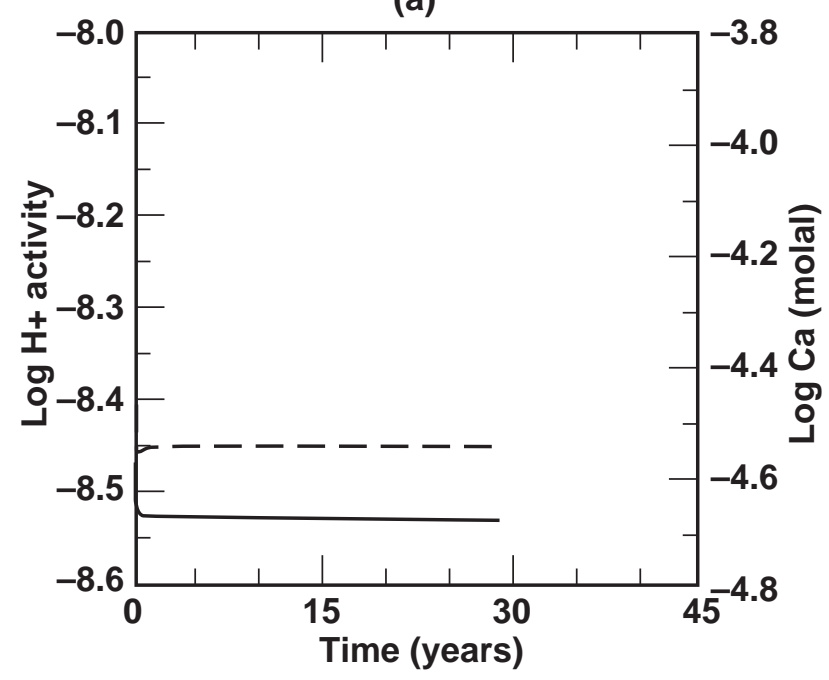

(b)

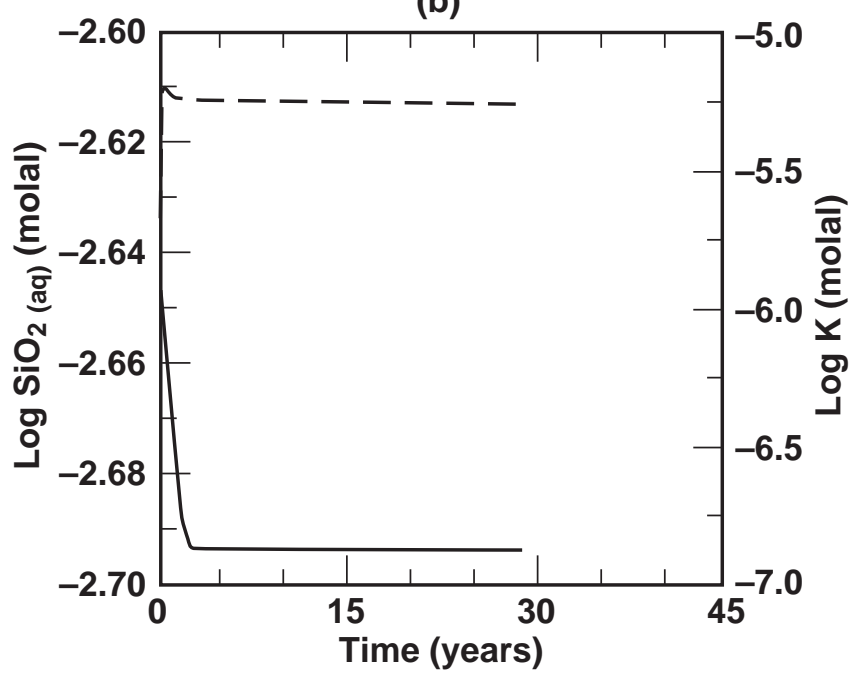

(c)

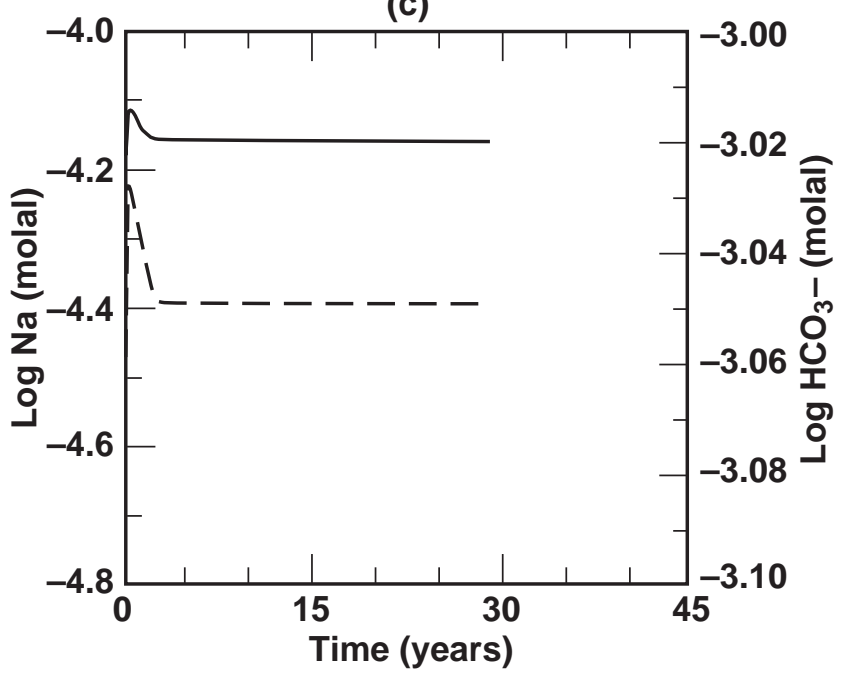

Figure 6-4. The $\log$ molality $[\log m(i)]$ of selected dissolved species in the outlet solution of the $95^{\circ} \mathrm{C}, 15-\mathrm{m}$ fracture flow model, as a function of time. All other species in the simulations had log molalities less than -7.0. For all figures, the solid line refers to the quantity on the left axis; the dashed line refers to the quantity on the right axis. (a) $\log m(\mathrm{H}+)$ and $\log m\left(\mathrm{Ca}^{+2}\right)$; (b) $\log m\left(\mathrm{SiO}_{2(\mathrm{aq})}\right)$ and $\log m(\mathrm{~K}+)$; and (c) $\log m\left(\mathrm{Na}^{+}\right)$and $\log m\left(\mathrm{HCO}_{3}{ }^{-}\right)$. 
(a)

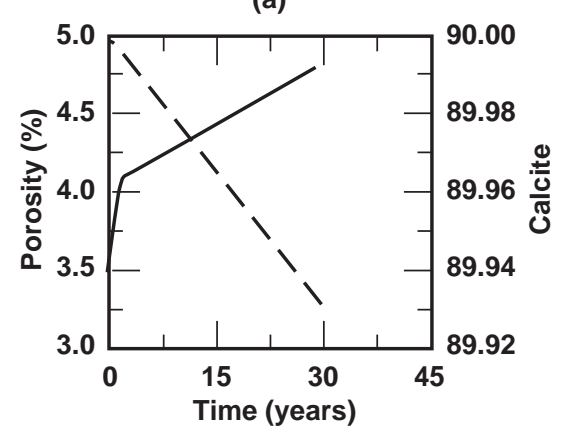

(b)

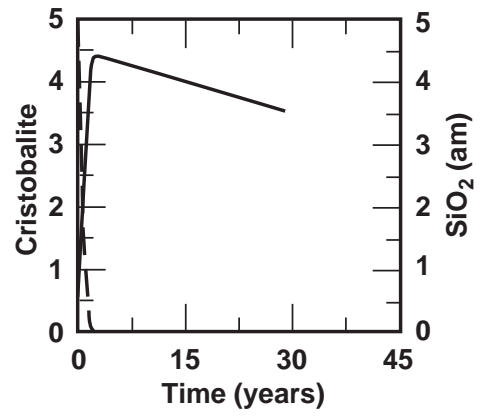

(c)

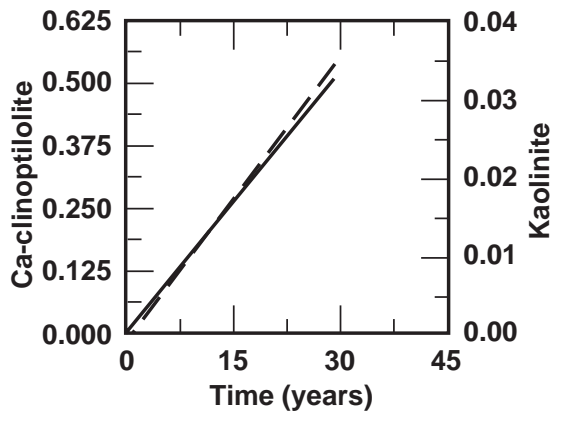

(d)

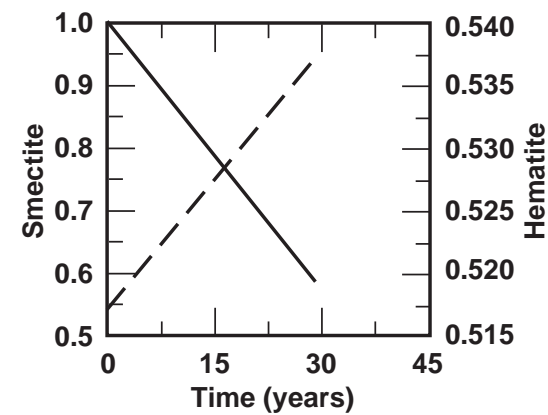

(e)

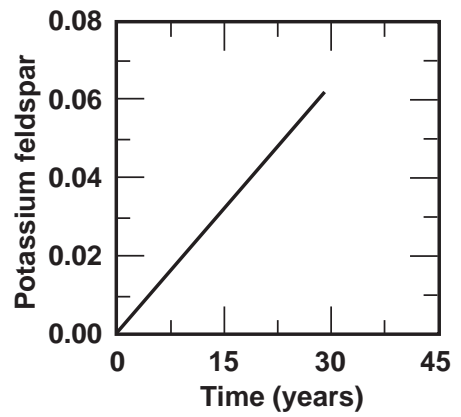

Figure 6-5. Porosity and mineralogy (all in volume percent) at the inlet position of the $95^{\circ} \mathrm{C}, 15-\mathrm{m}$ fractureflow model, as a function of time. For all figures, the solid line refers to the quantity on the left axis; the dashed line refers to the quantity on the right axis. (a) Porosity and calcite, (b) cristobalite and amorphous silica, (c) Ca-rich clinoptilolite and kaolinite, (d) smectite and hematite, and (e) potassium feldspar. 
(a)

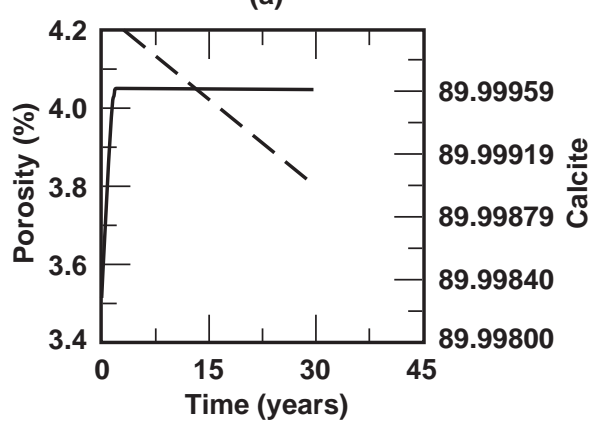

(b)

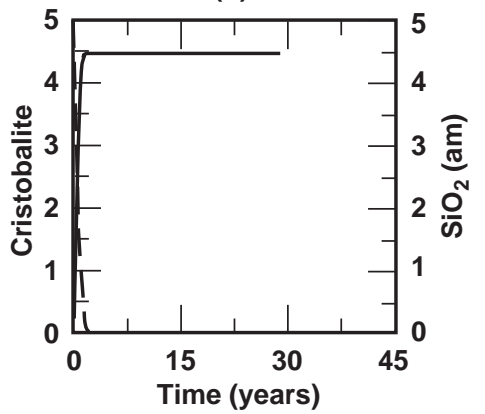

(c)

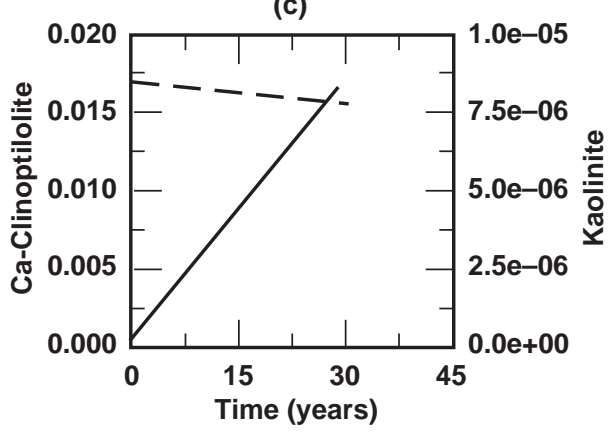

(d)

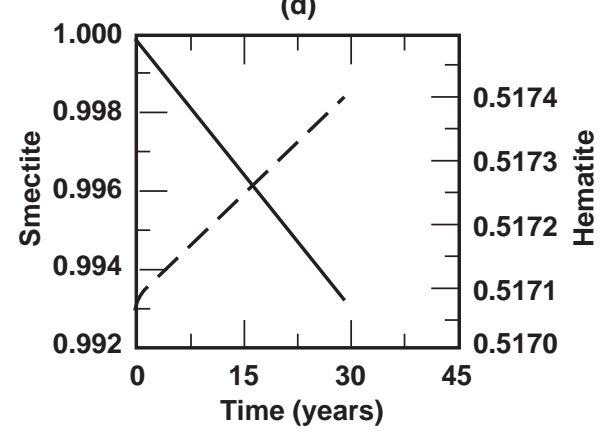

(e)

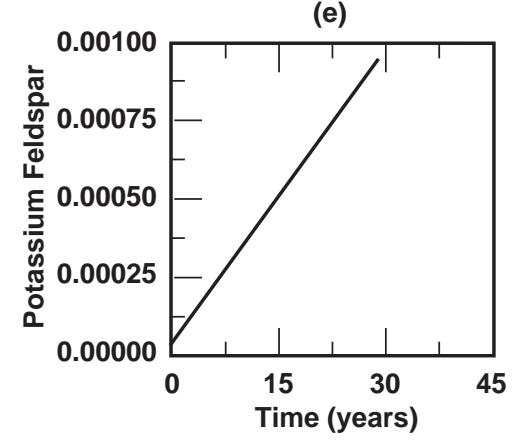

Figure 6-6. Porosity and mineralogy (all in volume percent) at the outlet position of the $95^{\circ} \mathrm{C}, 15-\mathrm{m}$ fracture flow model, as a function of time. For all figures, the solid line refers to the quantity on the left axis; the dashed line refers to the quantity on the right axis. (a) Porosity and calcite, (b) cristobalite and amorphous silica, (c) Ca-rich clinoptilolite and kaolinite, (d) smectite and hematite, (e) potassium feldspar. 


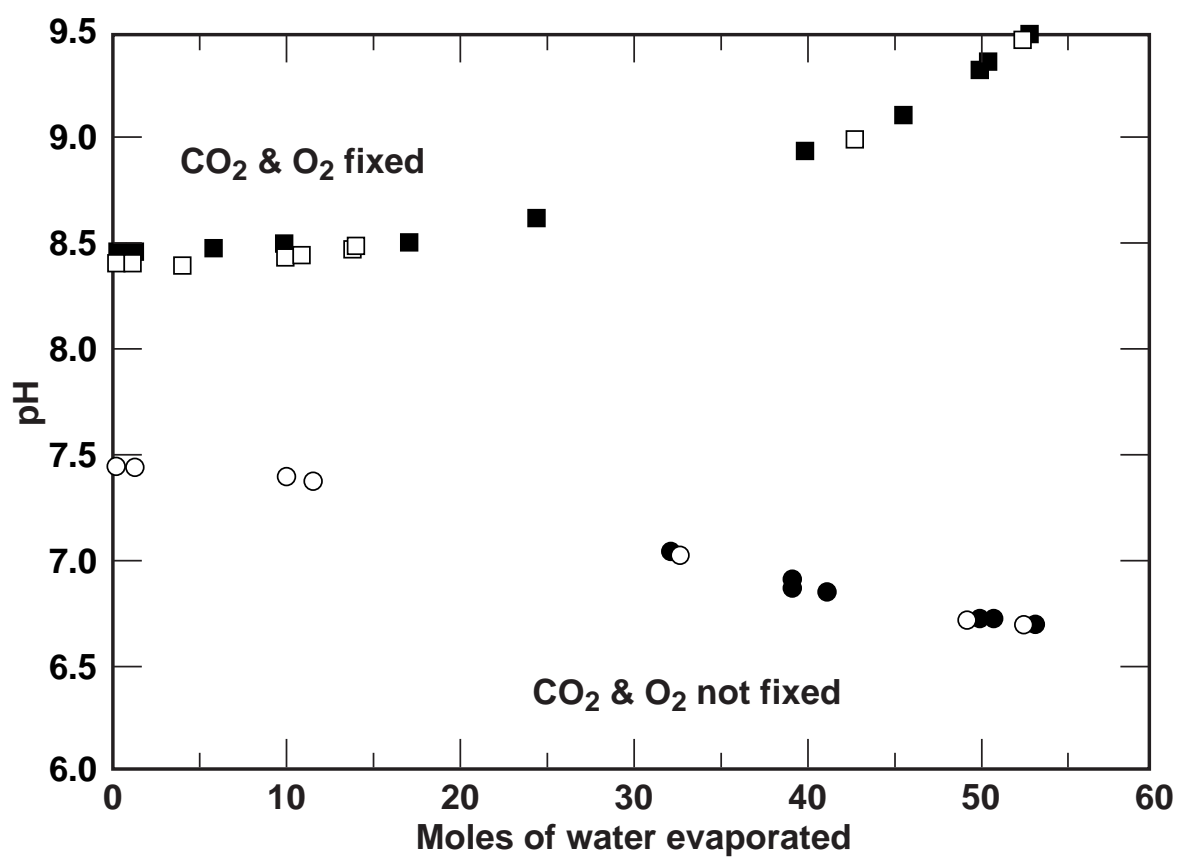

Figure 6-7. The $\mathrm{pH}$ as a function of moles of water evaporated. In this, and in Figures 6-8 through 6-17, circles represent results obtained from simulations in which $\mathrm{CO}_{2}$ and $\mathrm{O}_{2}$ fugacities were not fixed. Square symbols represent results obtained from simulations in which $\mathrm{CO}_{2}$ and $\mathrm{O}_{2}$ fugacities were fixed at atmospheric values. Filled symbols indicate runs in which precipitation of solids was not restricted. Open symbols indicate runs in which quartz, tridymite, and talc were suppressed.

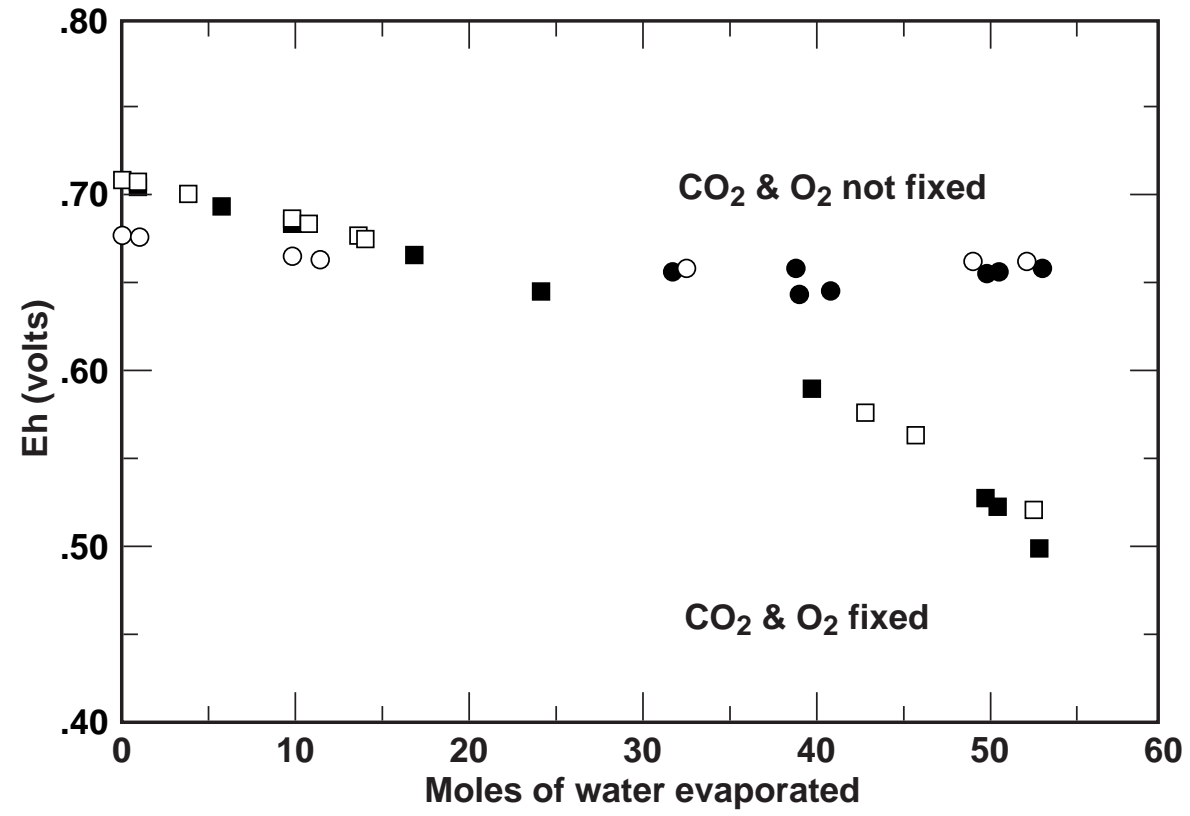

Figure 6-8. Eh (volts) as a function of moles of water evaporated. Symbols are as described in Figure 6-7. 


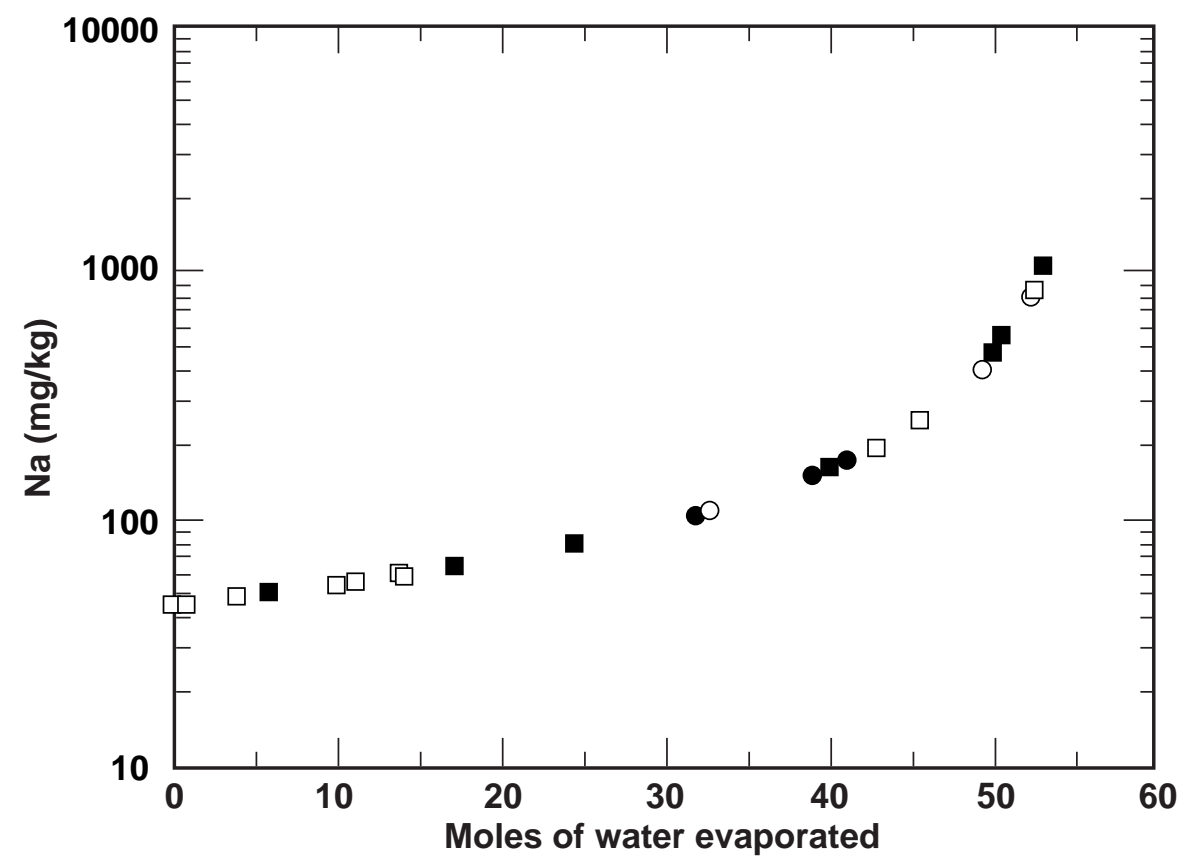

Figure 6-9. $\mathrm{Na}(\mathrm{mg} / \mathrm{kg})$, as a function of moles of water evaporated. Symbols are as described in Figure 6-7.

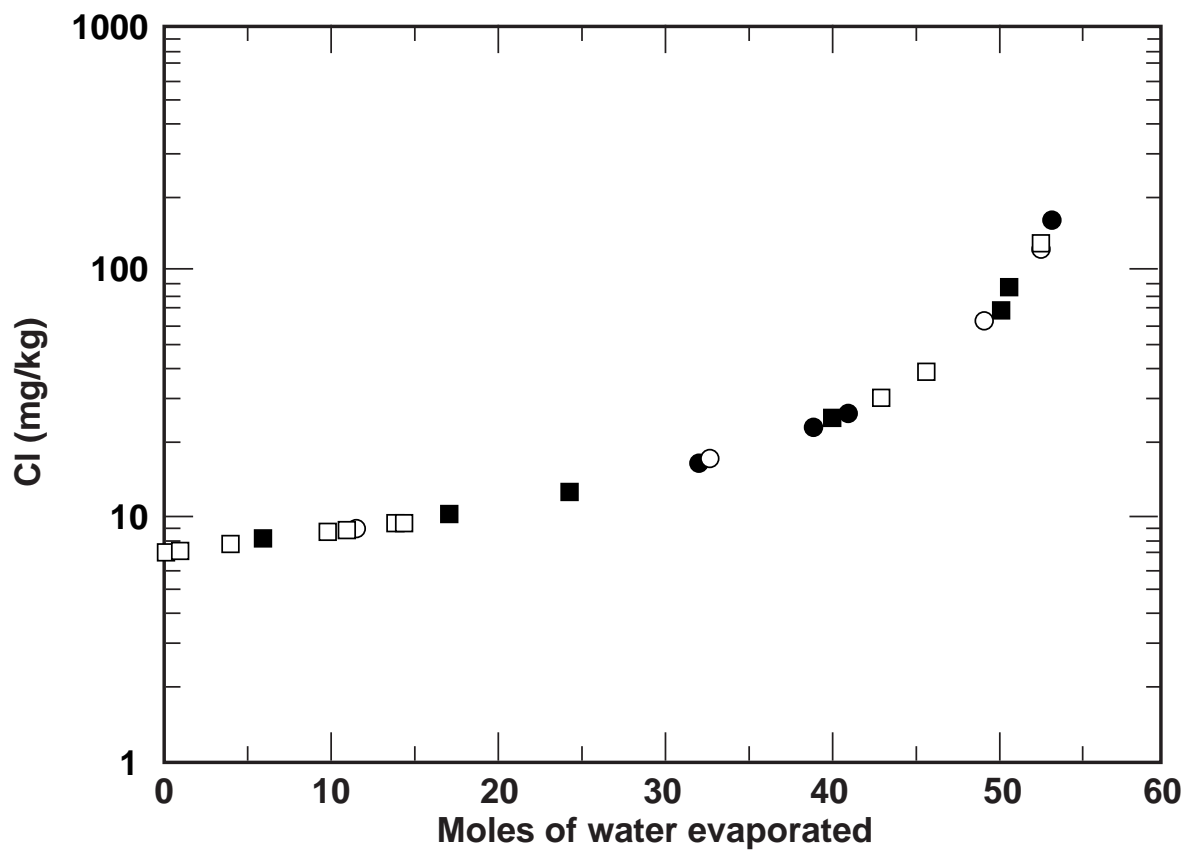

Figure 6-10. $\mathrm{Cl}(\mathrm{mg} / \mathrm{kg})$ as a function of moles of water evaporated. Symbols are as described in Figure 6-7. 


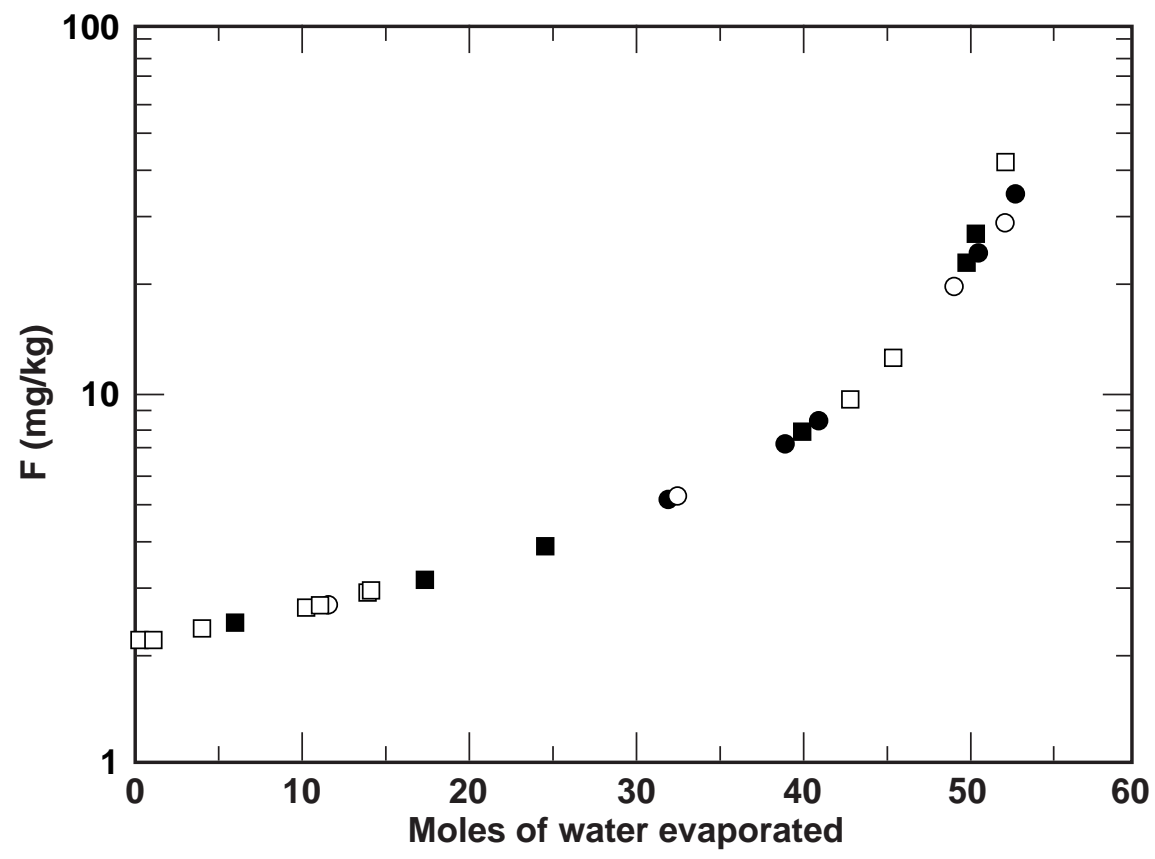

Figure 6-11. F (mg/kg) as a function of moles of water evaporated. Symbols are as described in Figure 6-7.

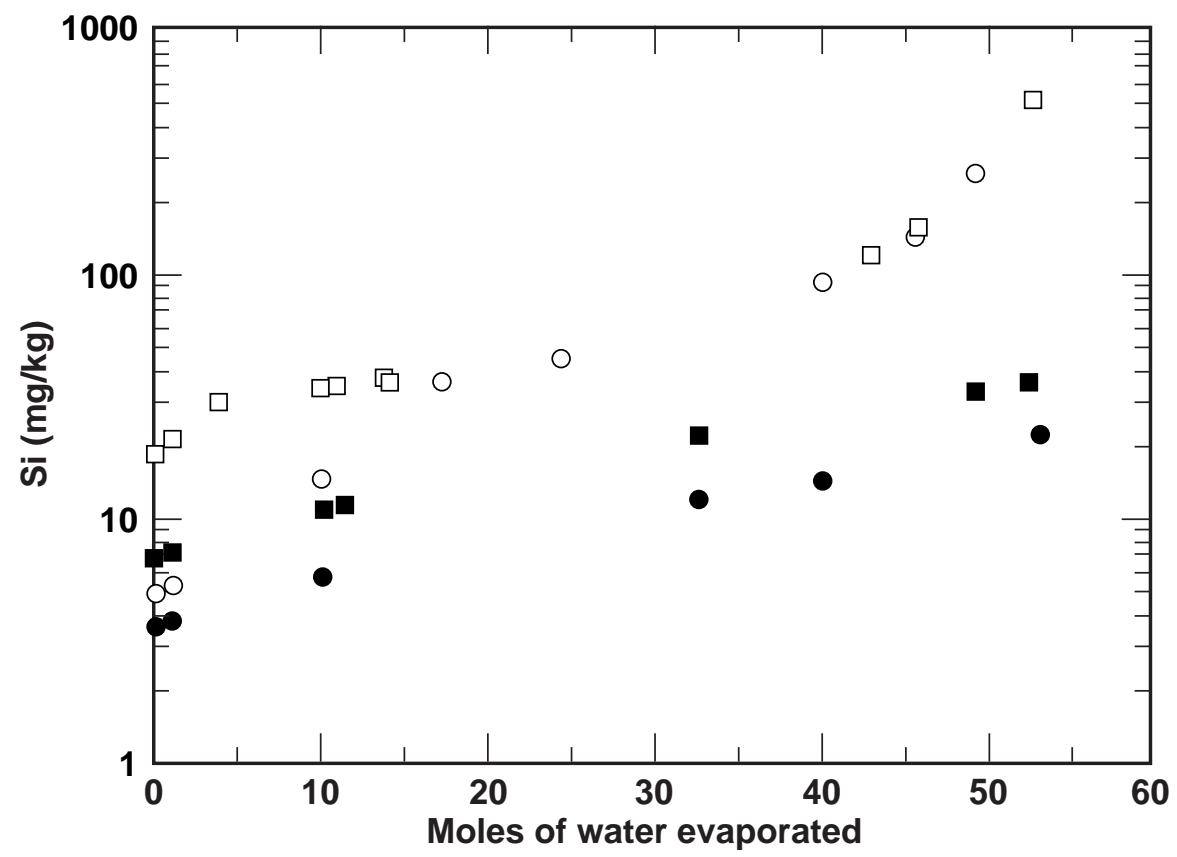

Figure 6-12. Si (mg/kg) as a function of moles of water evaporated. Symbols are as described in Figure 6-7. 


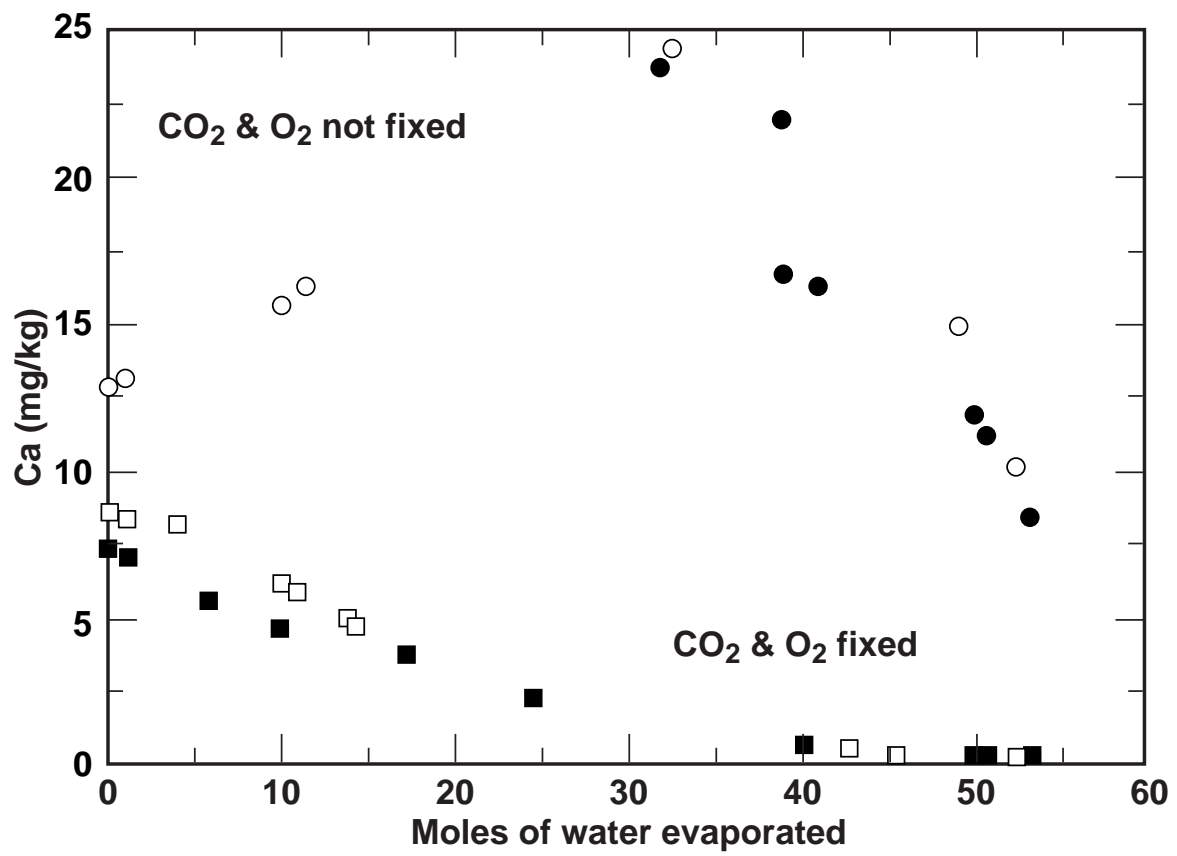

Figure 6-13. Ca $(\mathrm{mg} / \mathrm{kg})$ as a function of moles of water evaporated. Symbols are as described in Figure 6-7.

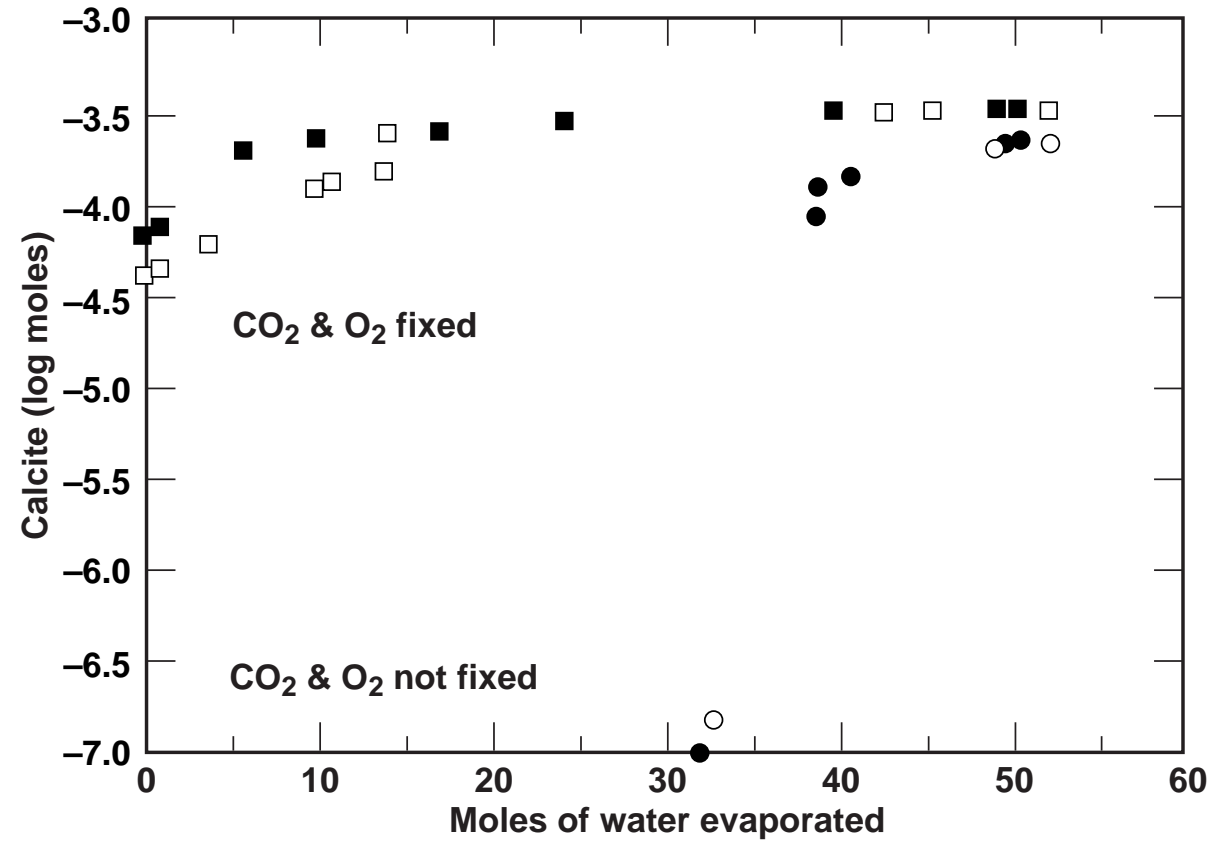

Figure 6-14. Log of the number of moles of calcite precipitated, as a function of moles of water evaporated. Symbols are as described in Figure 6-7. 


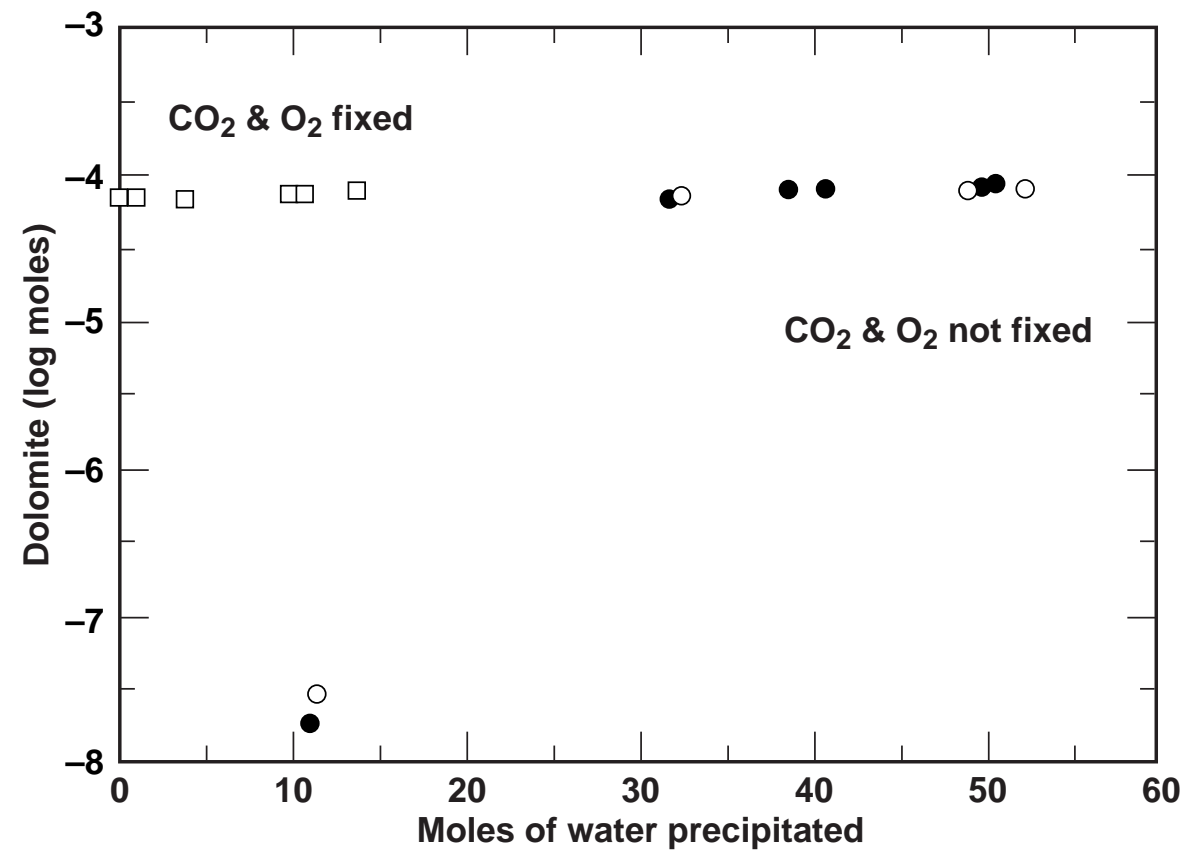

Figure 6-15. Log of the number of moles of dolomite precipitated, as a function of moles of water evaporated. Symbols are as described in Figure 6-7.

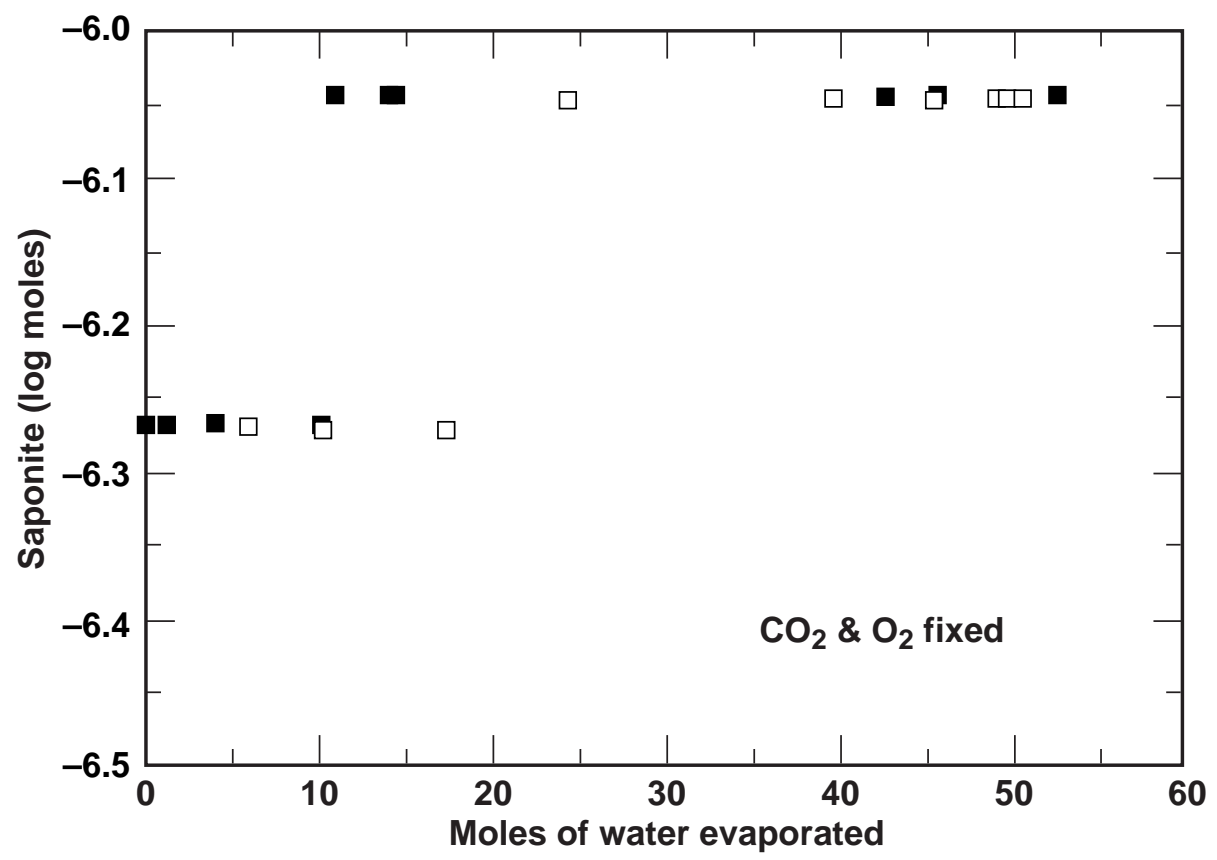

Figure 6-16. Log of the number of moles of saponite precipitated, as a function of moles of water evaporated. Symbols are as described in Figure 6-7. 


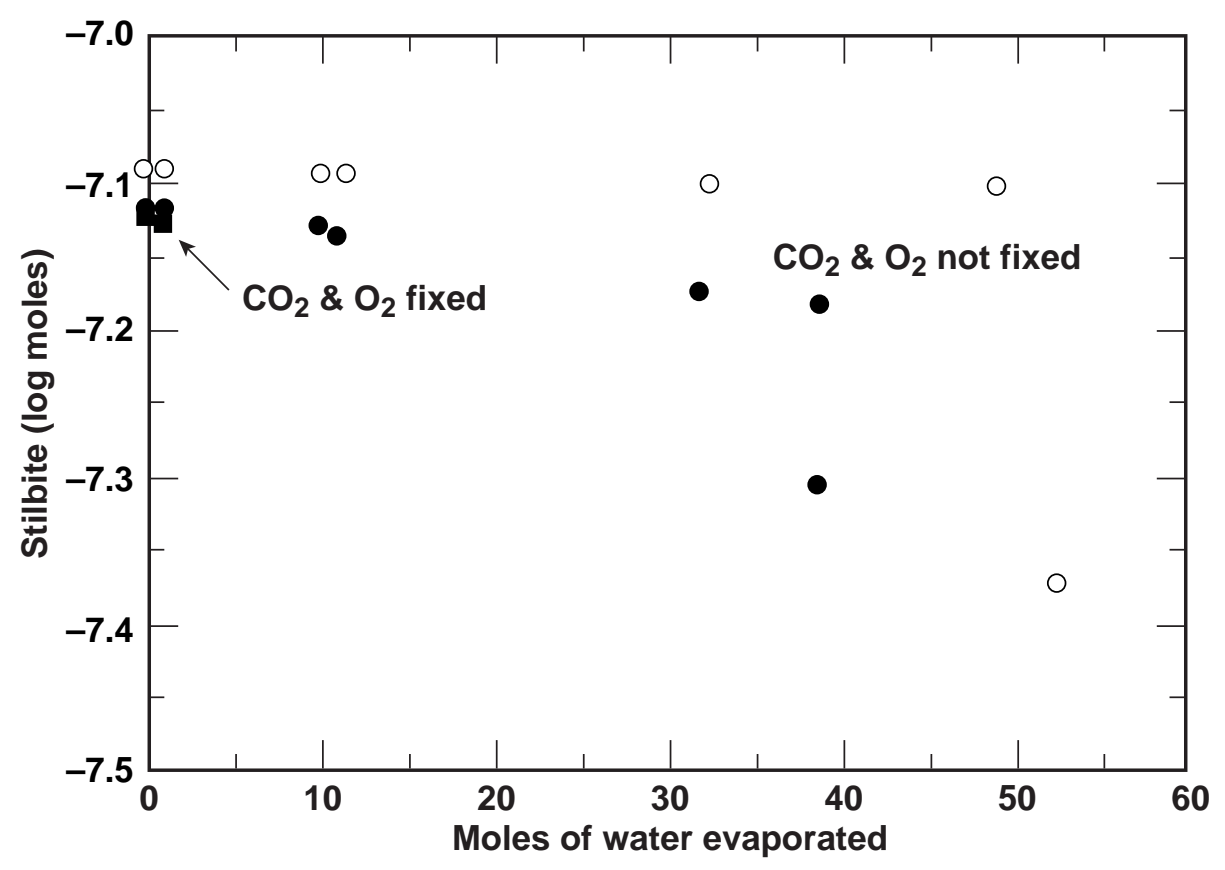

Figure 6-17. Log of the number of moles of stilbite precipitated, as a function of moles of water evaporated. Symbols are as described in Figure 6-7. 


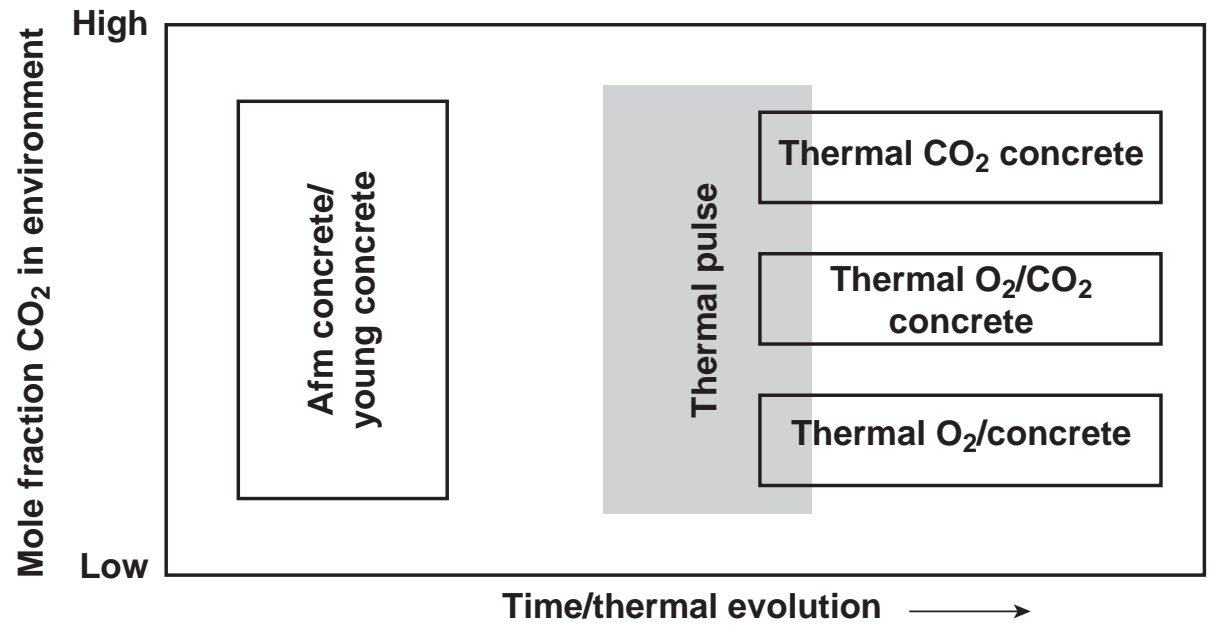

Figure 6-18. Schematic diagram of conditions under which the solid mineral assemblages described in Table 6.2 might develop from grout from an original Type V composition. See further discussion of composition in Chapter 7. 


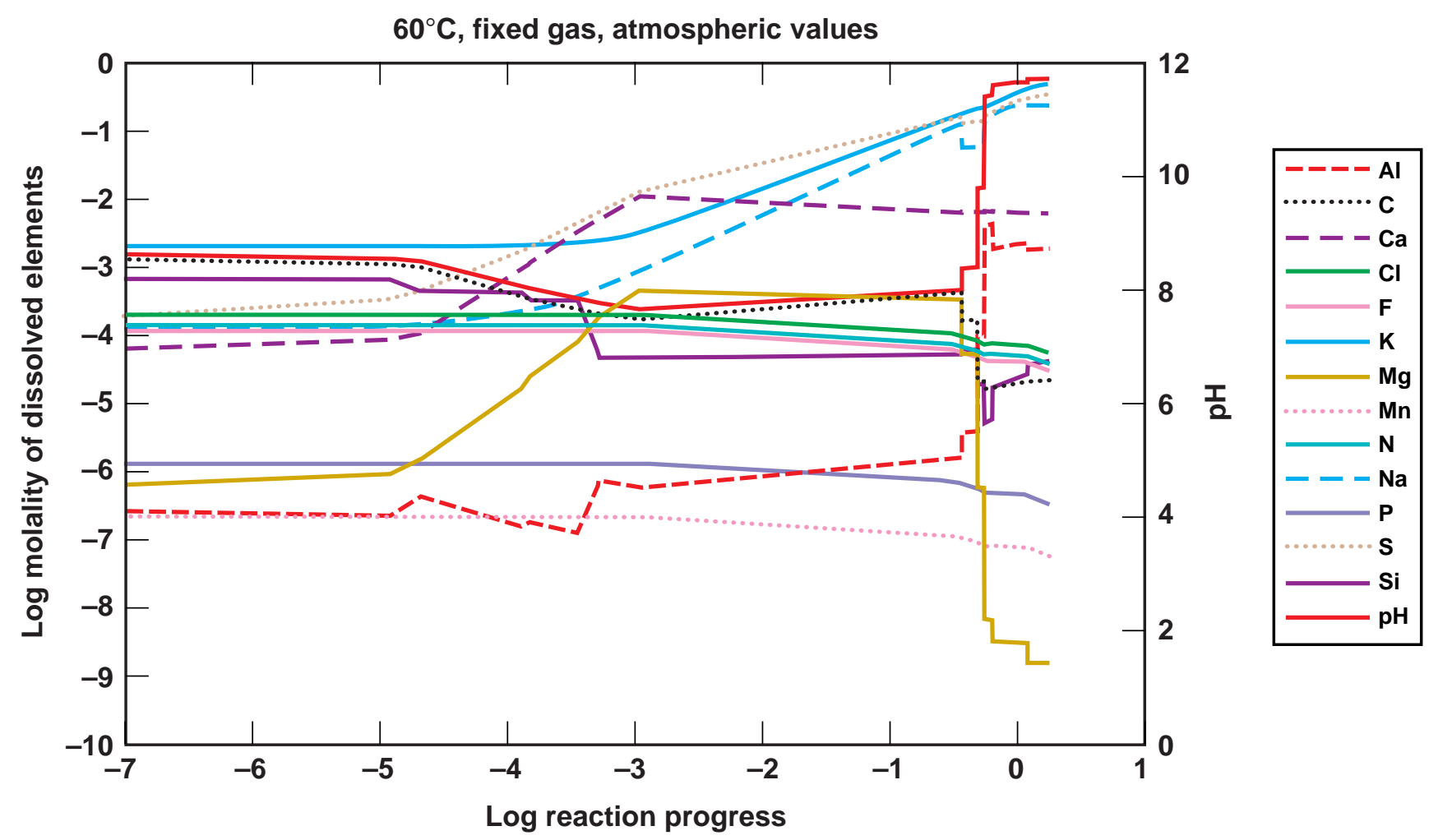

Figure 6-19. Plot of concentration of dissolved elements as a function of reaction progress for a simulation conducted at $60^{\circ} \mathrm{C}$, using a young concrete mineral assemblage and tuff aggregate composition and fixed $\mathrm{O}_{2}$ and $\mathrm{CO}_{2}$ compositions at atmospheric values 


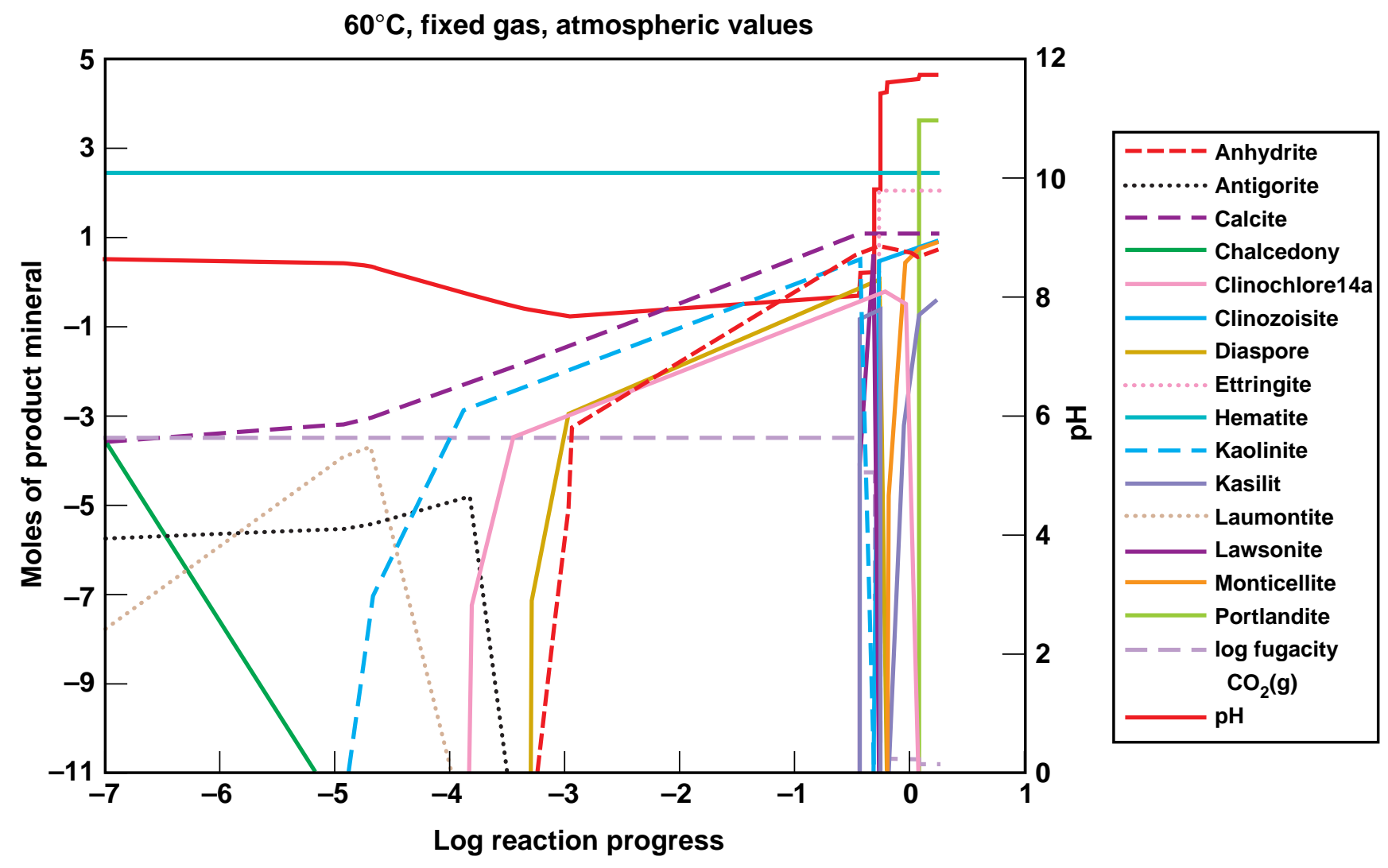

Figure 6-20. Plot of precipitated mineral phases as a function of reaction progress for the simulation described in Figure 6-19 


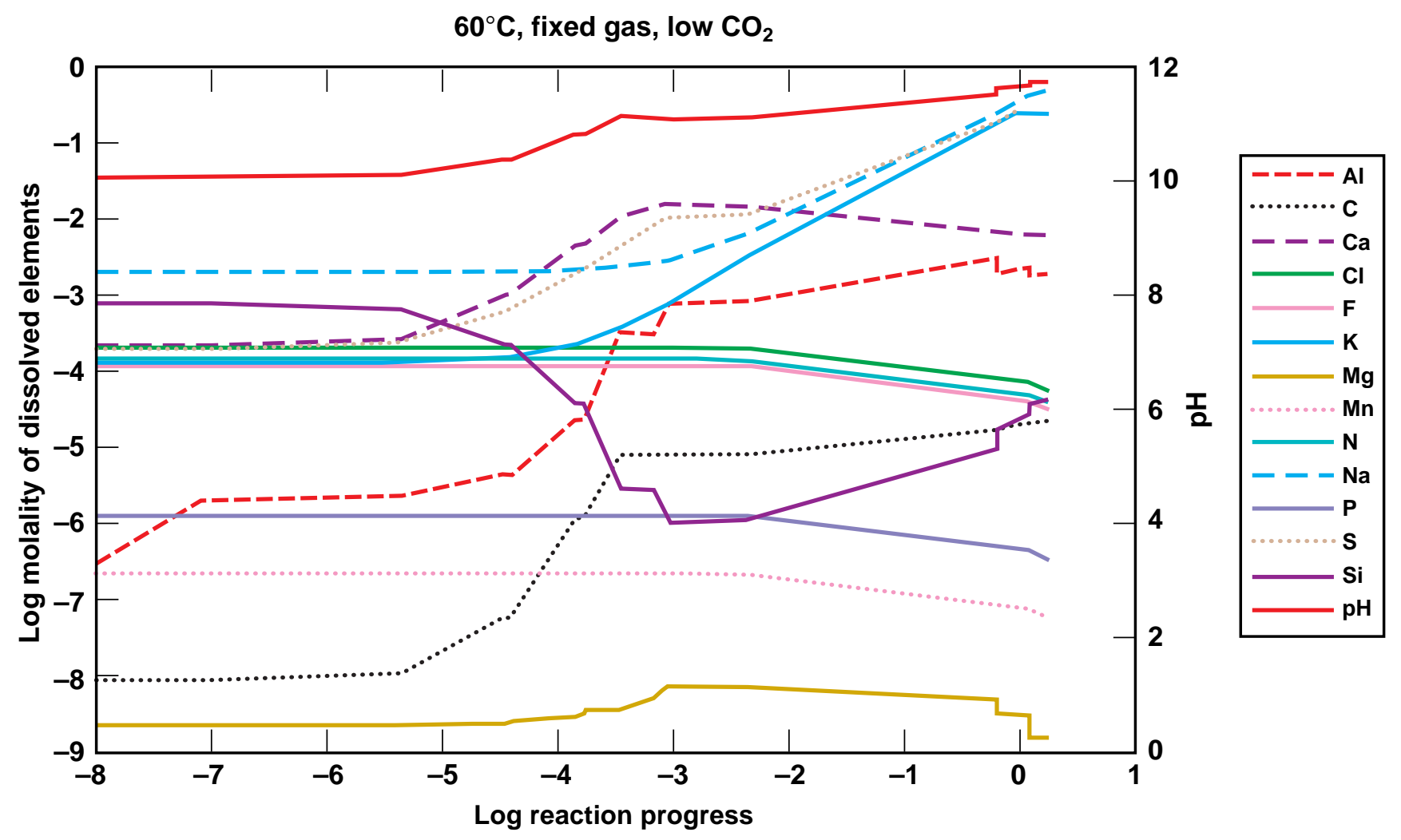

Figure 6-21. Plot of concentration of dissolved elements as a function of reaction progress for a simulation conducted at $60^{\circ} \mathrm{C}$, using a young concrete mineral assemblage and tuff aggregate composition and fixed $\mathrm{O}_{2}$ and $\mathrm{CO}_{2}$ compositions where $\mathrm{CO}_{2}$ is set at a low value (somewhat arbitrarily chosen) (10 to 10.5 bars) 


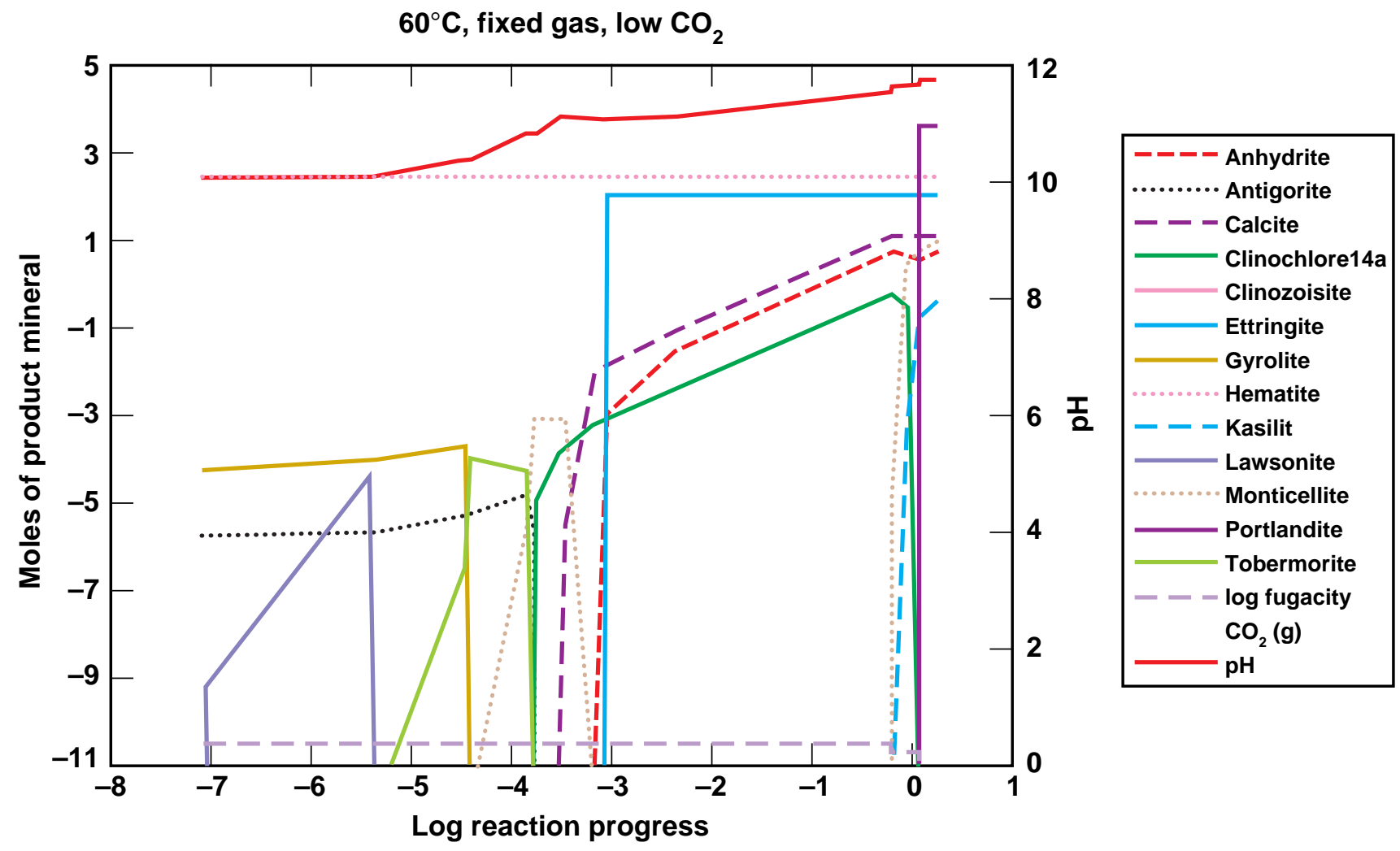

Figure 6-22. Plot of precipitated mineral phases as a function of reaction progress for the simulation described in Figure 6-21. 


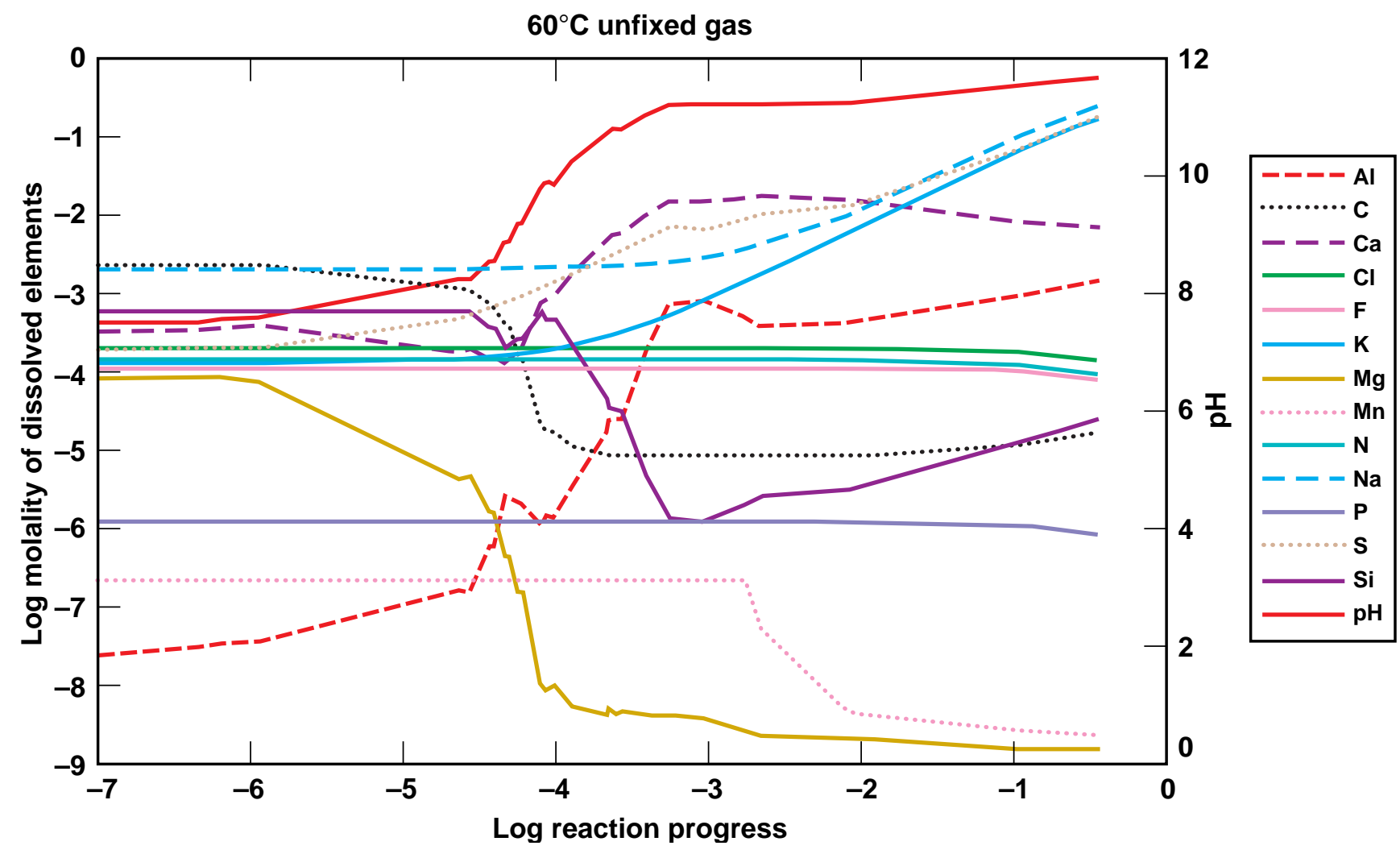

Figure 6-23. Plot of concentration of dissolved elements as a function of reaction progress for a simulation conducted at $60^{\circ} \mathrm{C}$, using a young concrete mineral assemblage and tuff aggregate composition and unfixed $\mathrm{O}_{2}$ and $\mathrm{CO}_{2}$ compositions. Thus, gas composition is allowed to evolve throughout the simulation. 


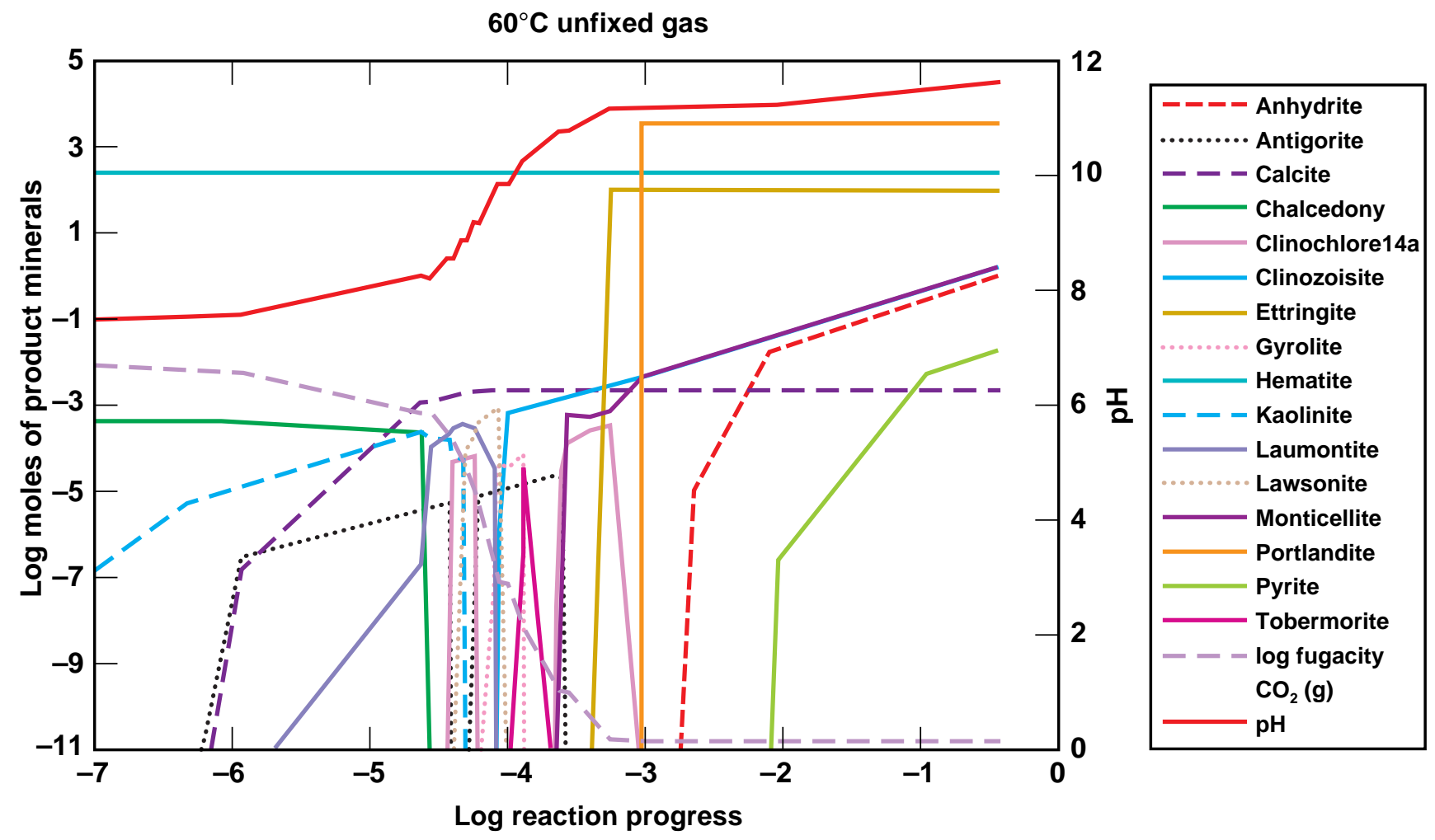

Figure 6-24. Plot of precipitated mineral phases as a function of reaction progress for the simulation described in Figure 6-23. 
(a)

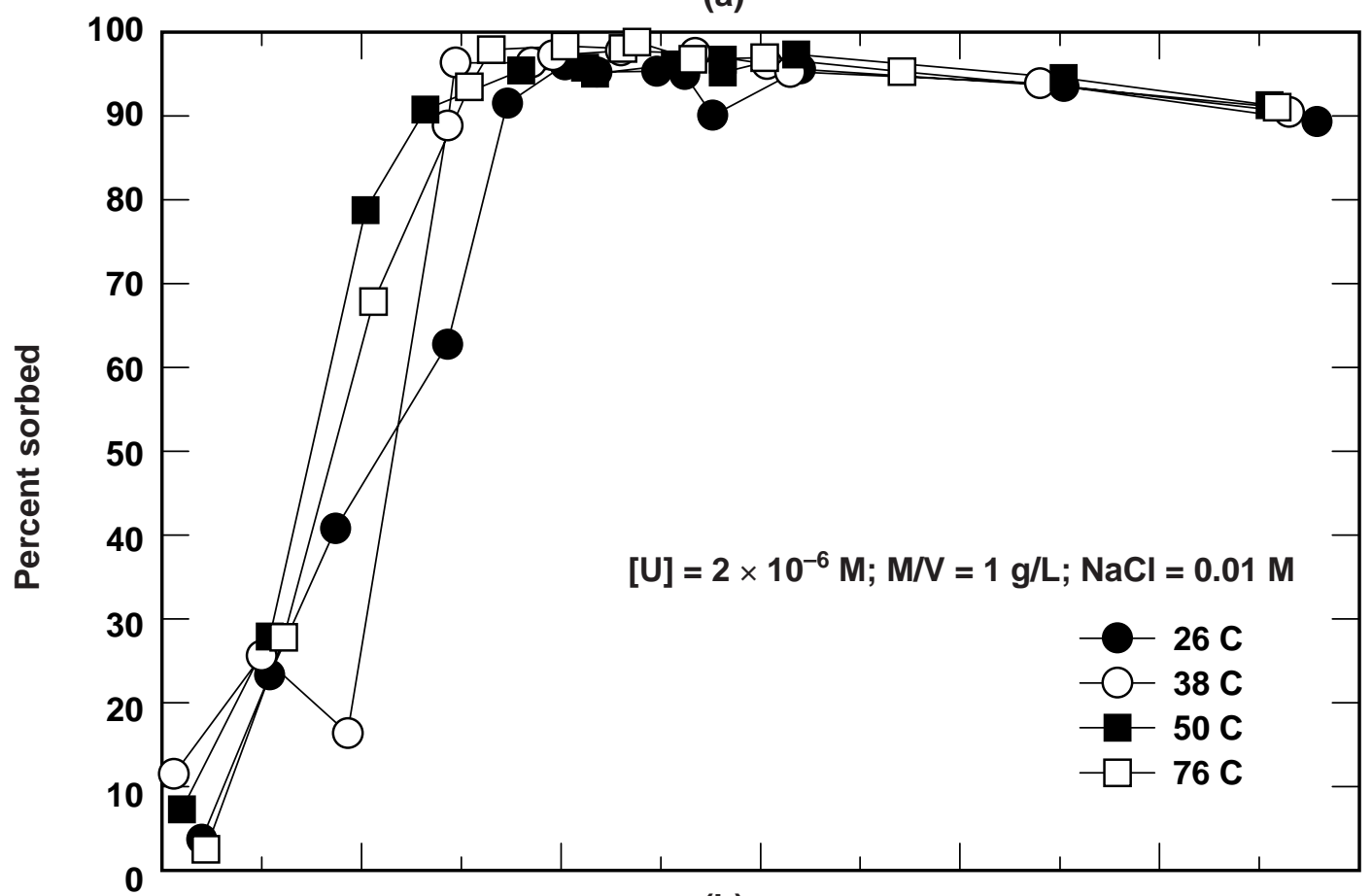

(b)

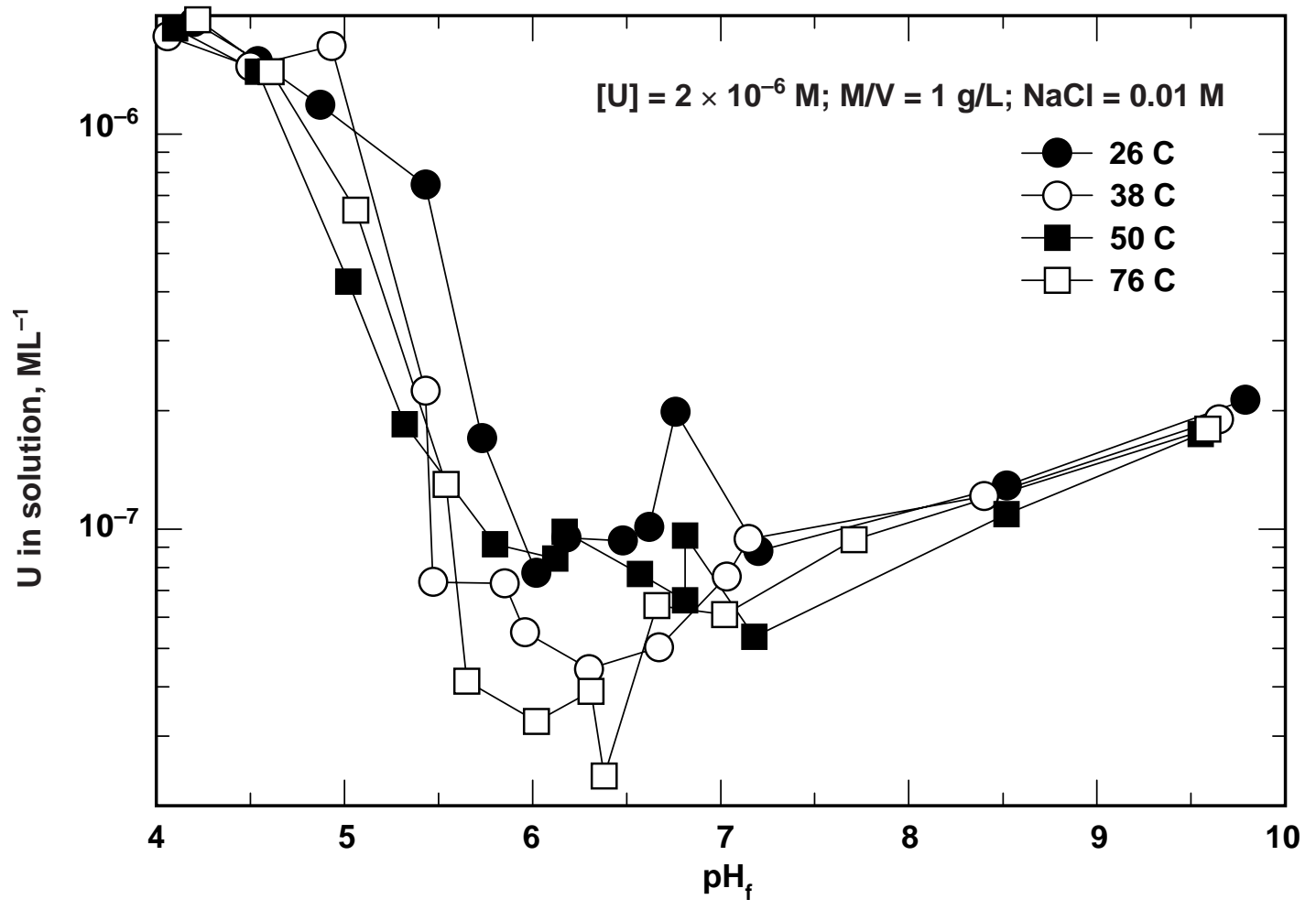

Figure 6-25. Variation in (a) percent sorbed and (b) concentration of $U$ in solution vs. final $\mathrm{pH}$ in four-day batch experiments. $[\mathrm{U}]=2 \times 10^{6} \mathrm{M} ; \mathrm{M} / \mathrm{V}$ of hematite $=1 \mathrm{~g} / \mathrm{L}$; and background electrolyte $=0.01 \mathrm{M} \mathrm{NaCl}$ 
(a)

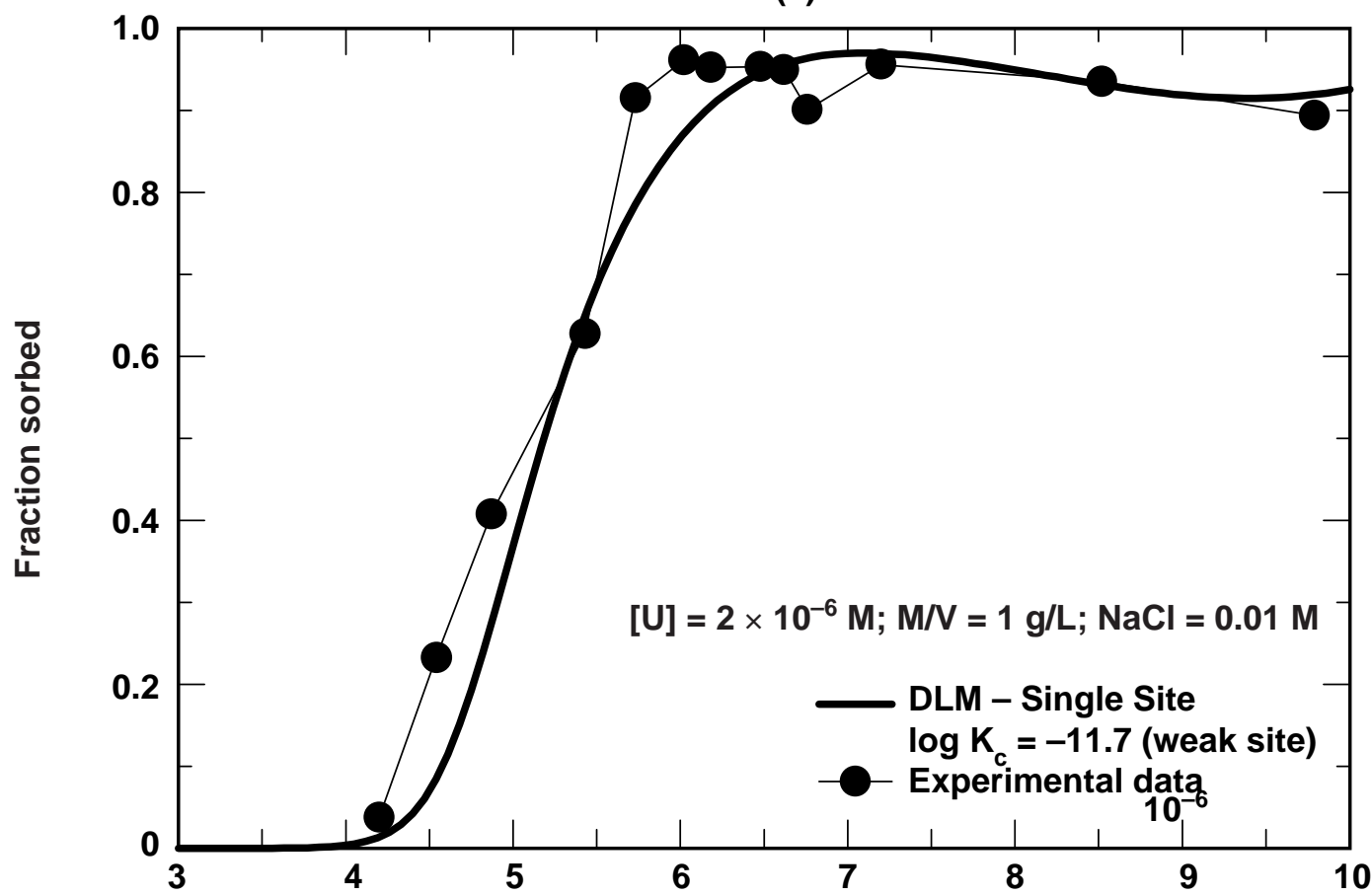

(b)

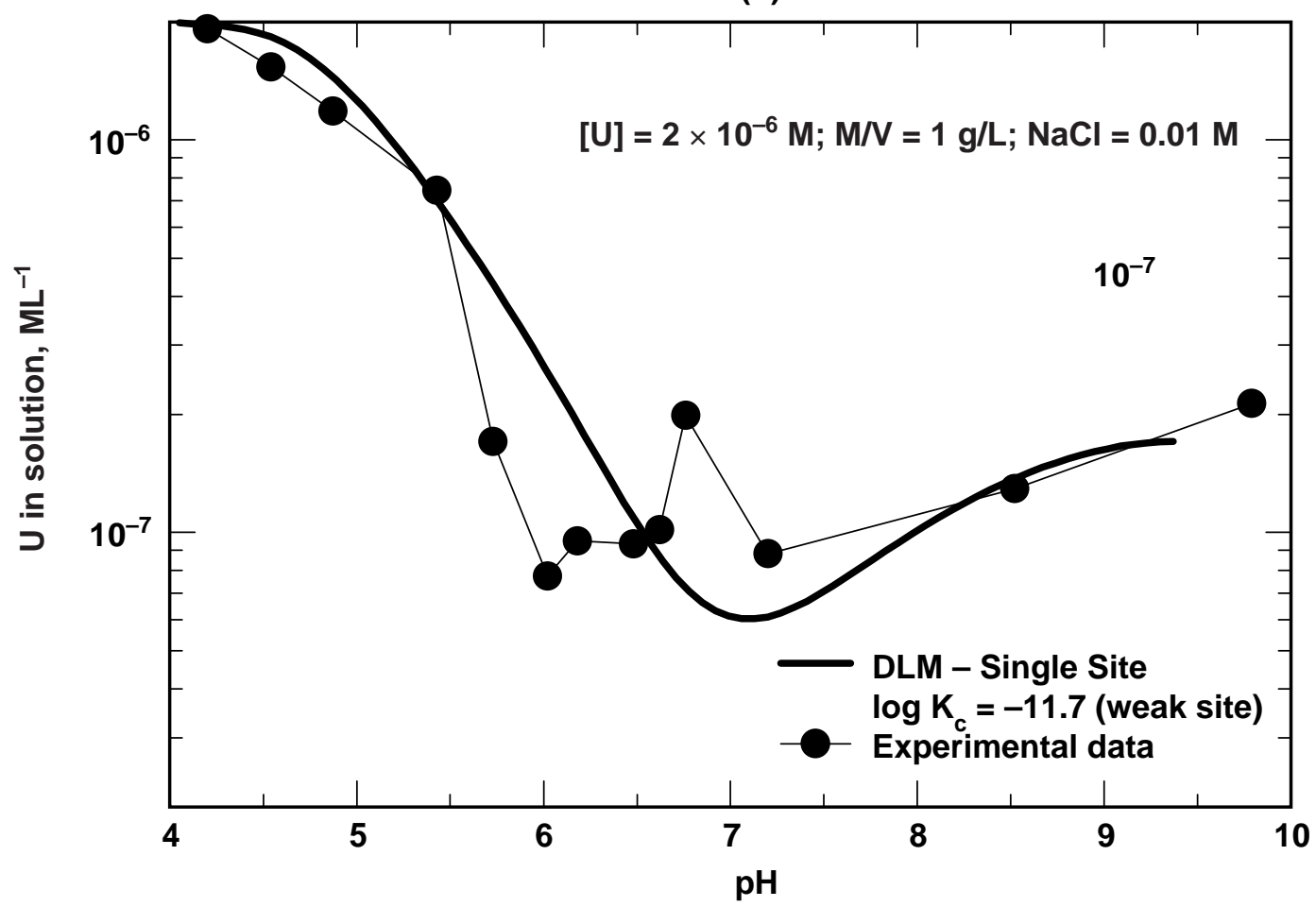

Figure 6-26. Comparison of DLM model (single-site) prediction. (a) Fraction of U sorbed, and (b) concentration of $\mathrm{U}$ in solution with experimental results at $26^{\circ} \mathrm{C}$. Model parameters used are listed in Table 6-3. 
(a)

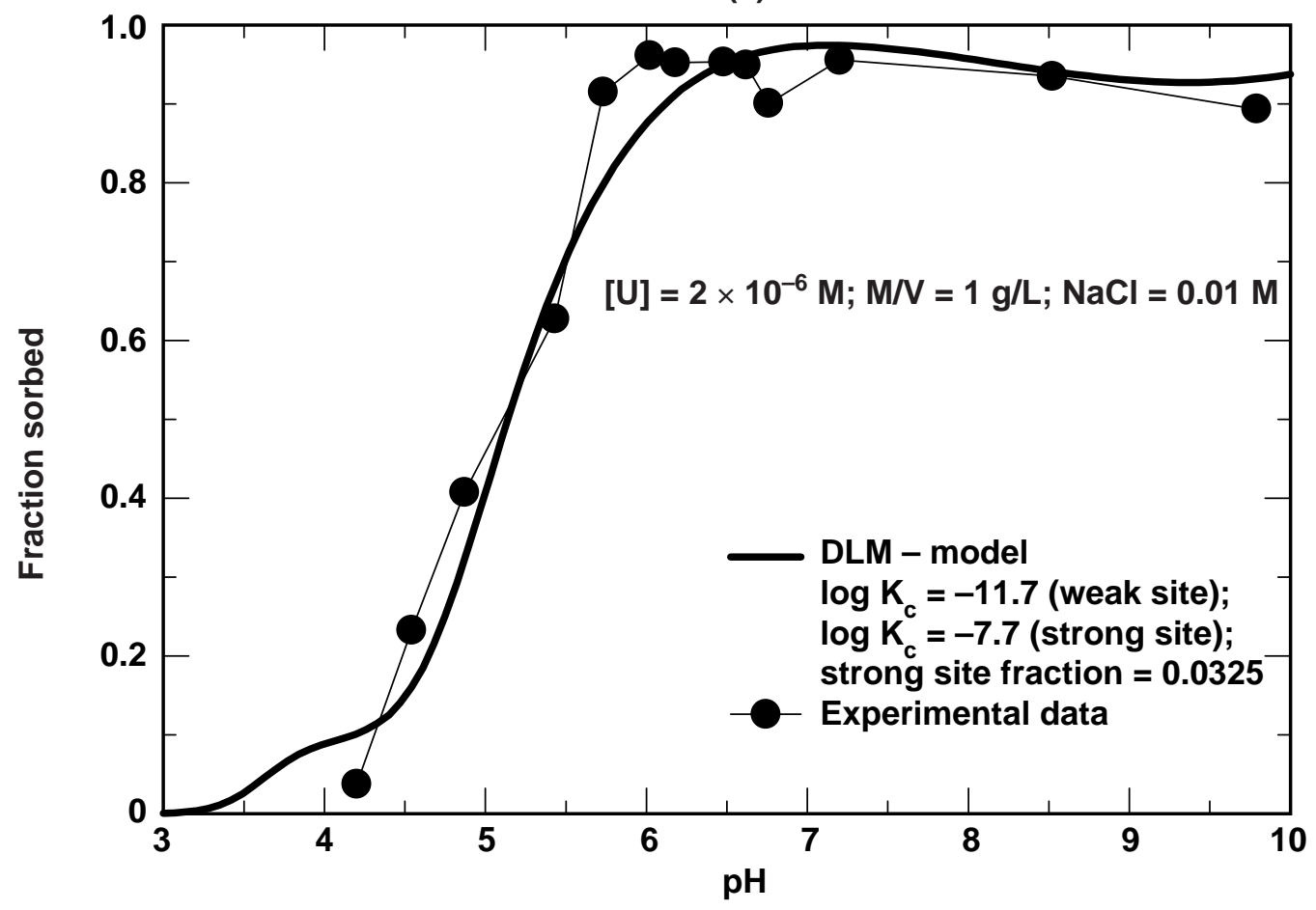

(b)

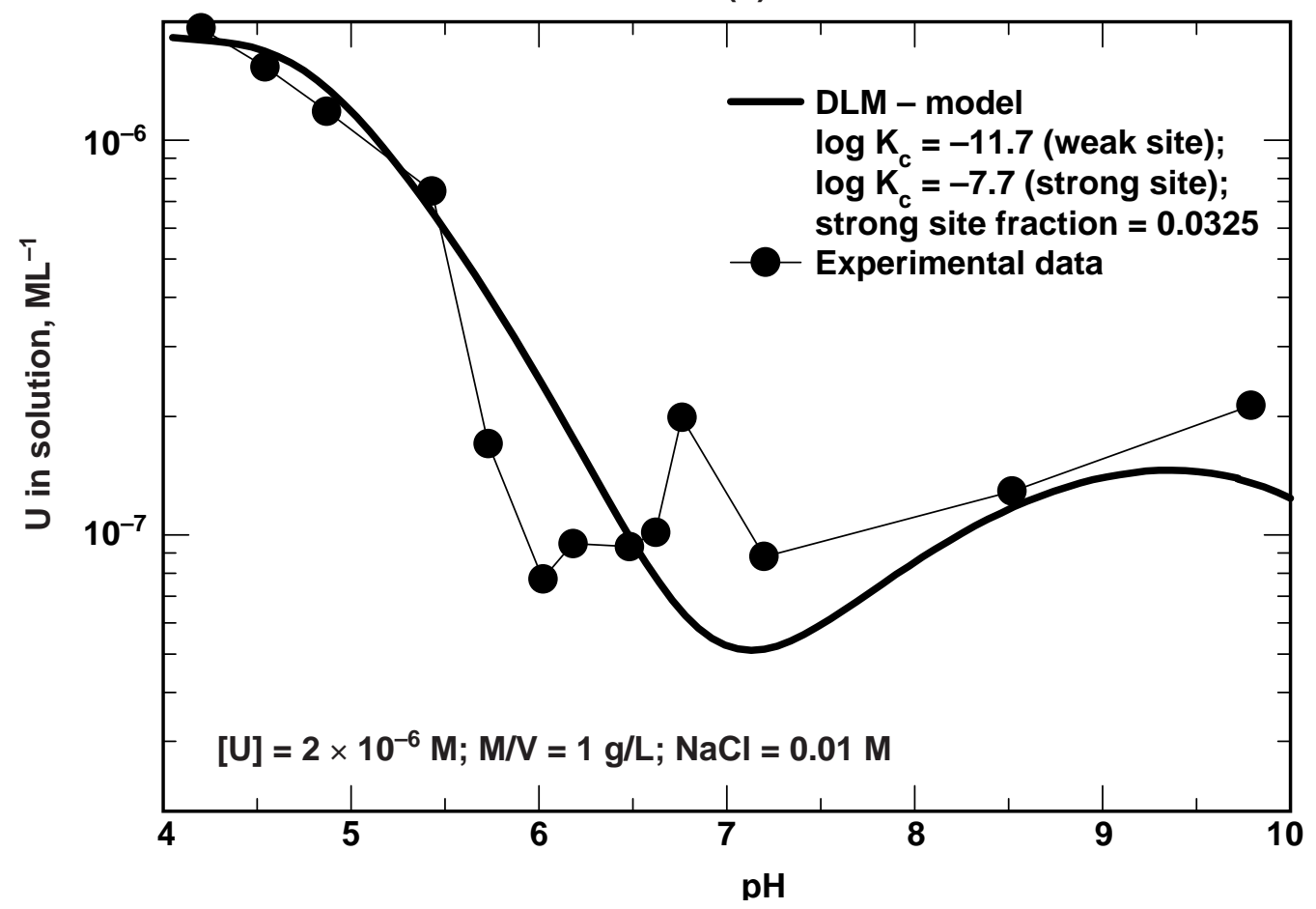

Figure 6-27. Comparison of DLM model (two-site) prediction. (a) Fraction of U sorbed and (b) concentration of $\mathrm{U}$ in solution with experimental results at $26^{\circ} \mathrm{C}$ Model parameters used are listed in Table 6-3. 


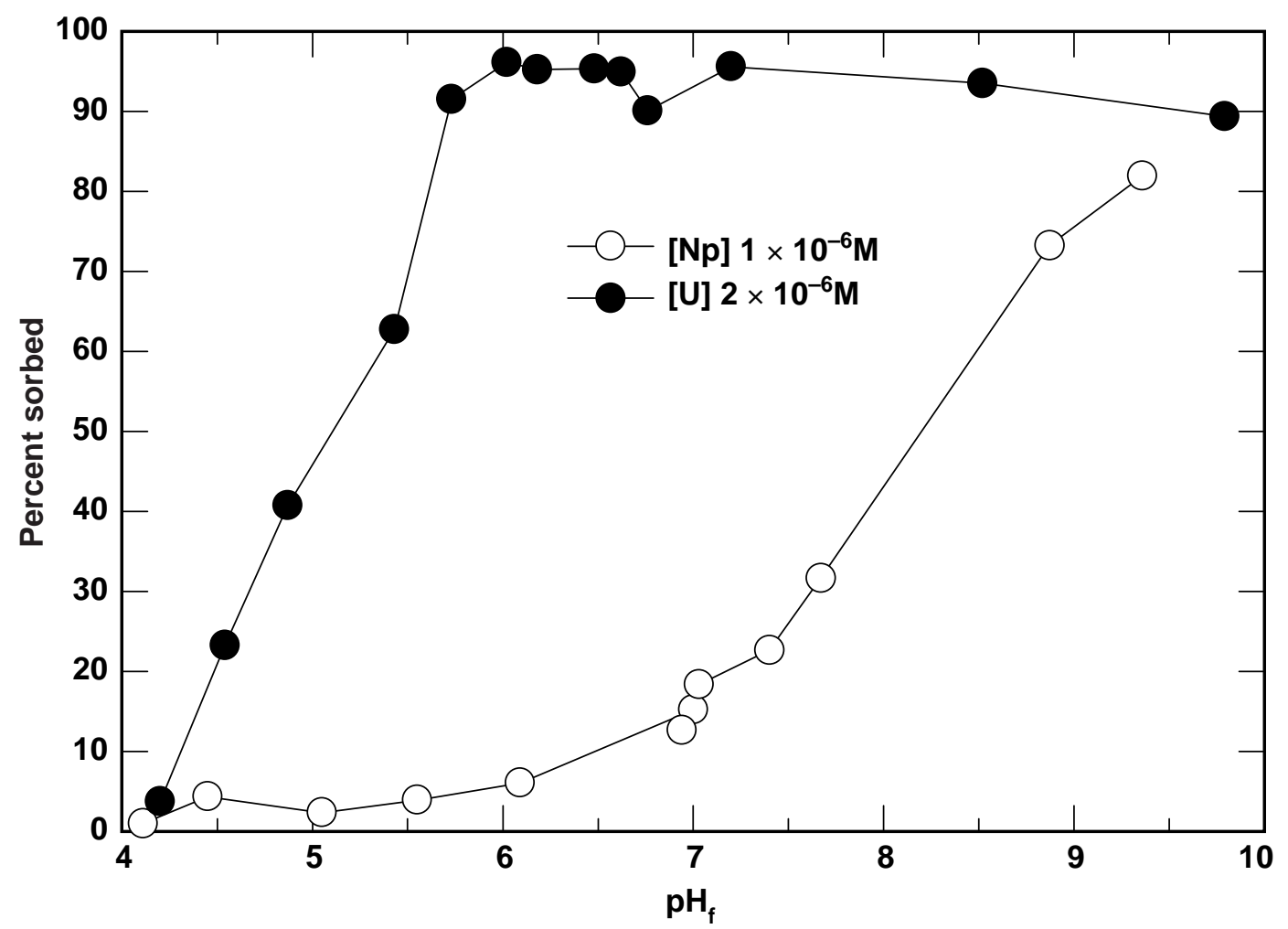

Figure 6-28. Percent $\mathrm{U}$ and $\mathrm{Np}$ sorbed vs. final $\mathrm{pH}$ in four-day batch experiments. $[\mathrm{U}]=2 \times 10^{-6} \mathrm{M}$; $[\mathrm{Np}]=1 \times 10^{-6} \mathrm{M} ; \mathrm{M} / \mathrm{V}$ of hematite $=1 \mathrm{~g} / \mathrm{L} ;$ and background electrolyte $=0.01 \mathrm{M} \mathrm{NaCl}$. 


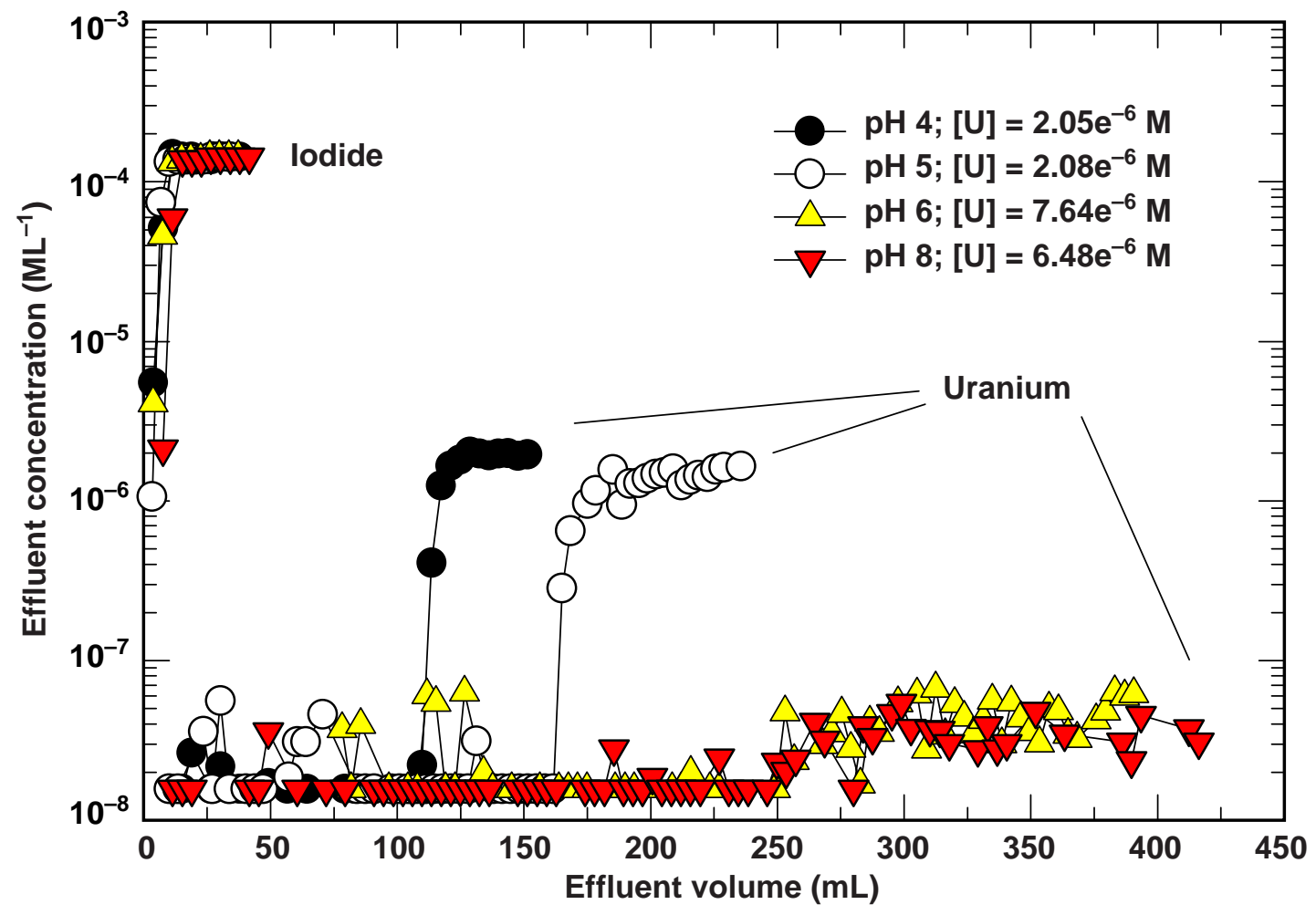

Figure 6-29. Variation in concentration of $\mathrm{I}^{-}$and $\mathrm{U}$ vs. volume of effluent at $26^{\circ} \mathrm{C}$ for hematite:quartz columns at four $\mathrm{pH}$ values. 


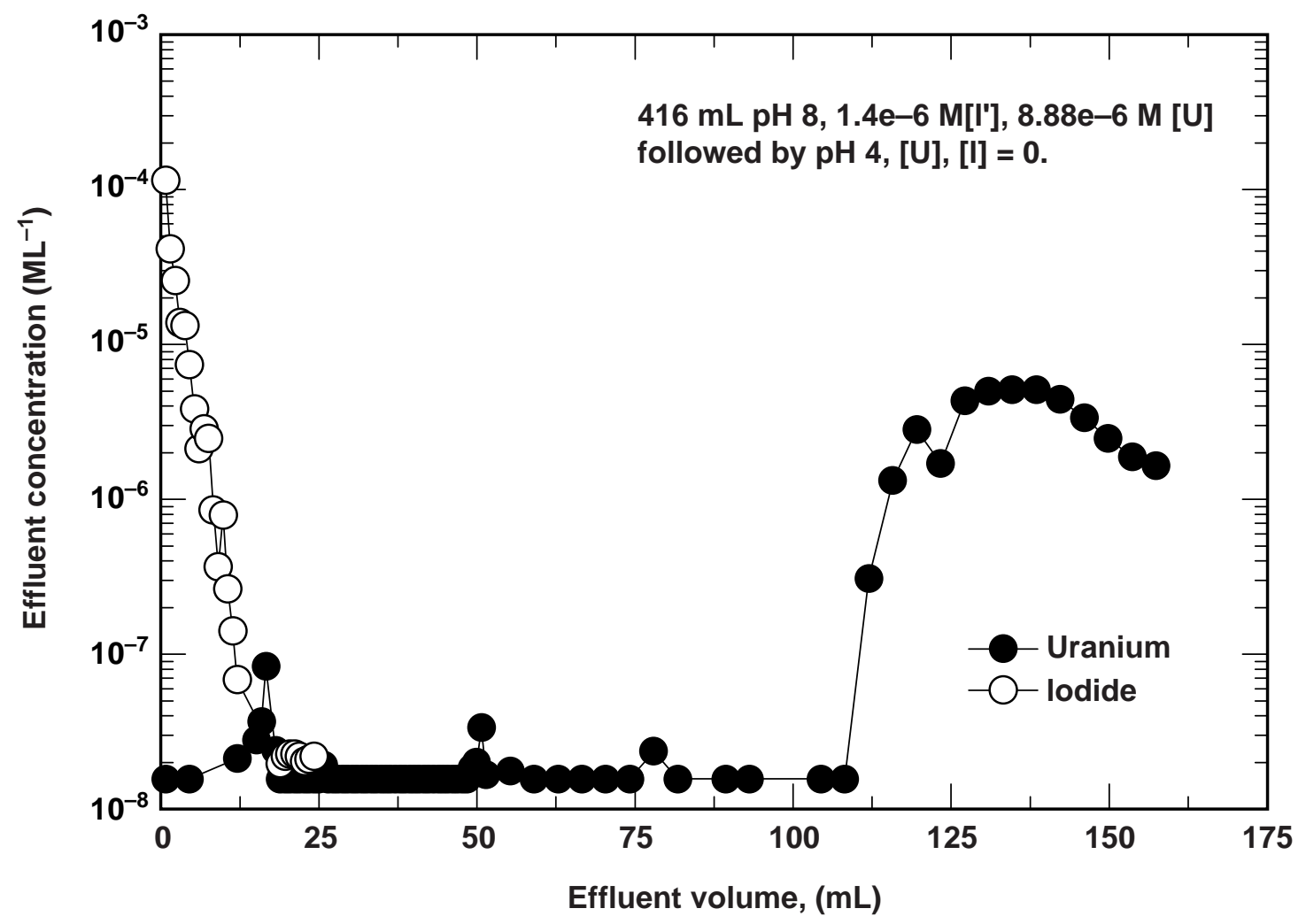

Figure 6-30. Variation in concentration of $\mathrm{I}^{-}$and $\mathrm{U}$ vs. volume of effluent in $\mathrm{pH} 8$ column subsequent to the input of $\mathrm{pH} 4$ tracer-free solution. 


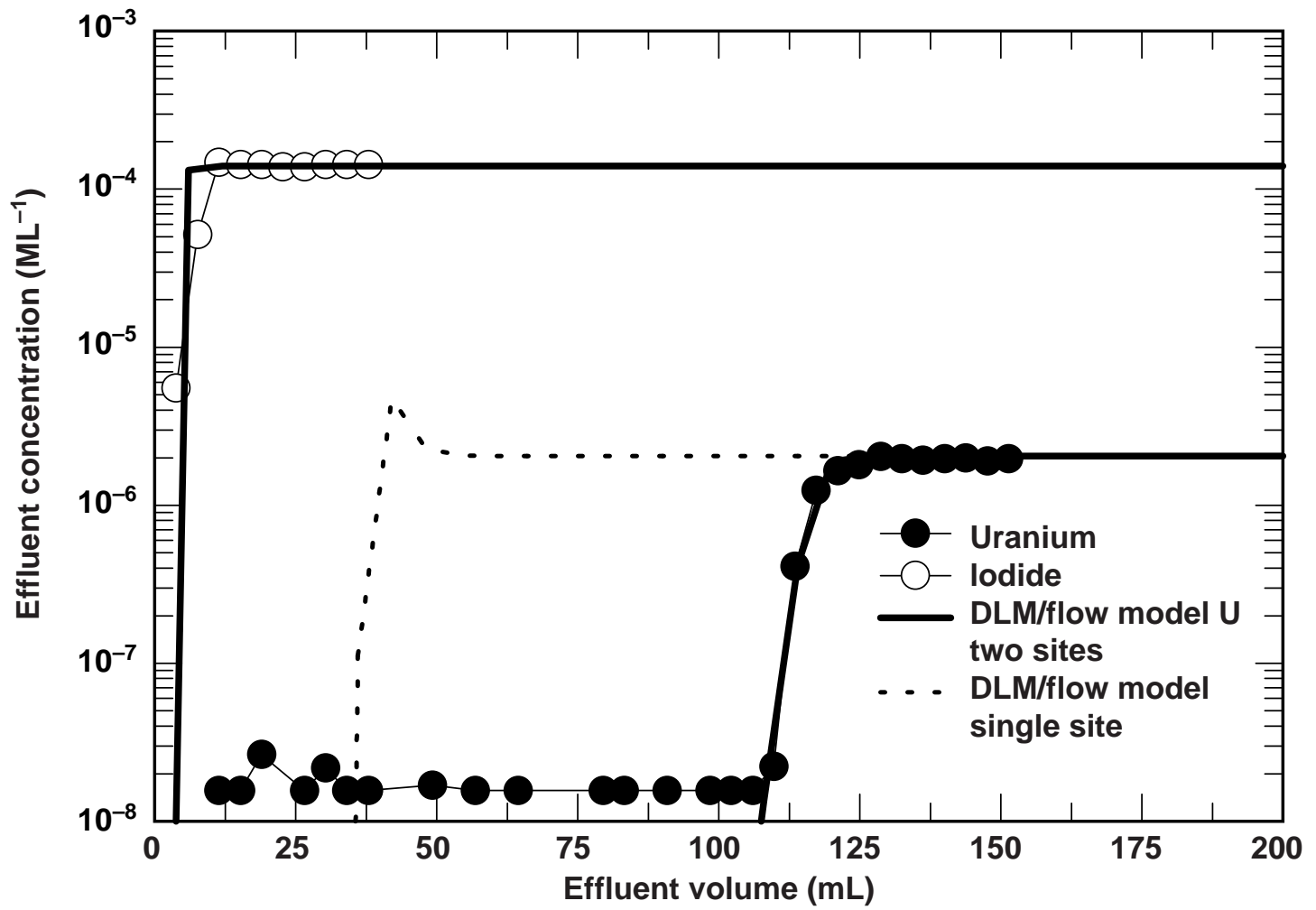

Figure 6-31. Comparison of X1t transport model (single- and two-site DLM) and observed $\mathrm{I}^{-}$and $\mathrm{U}$ concentrations in effluent from $\mathrm{pH} 4$ column. 


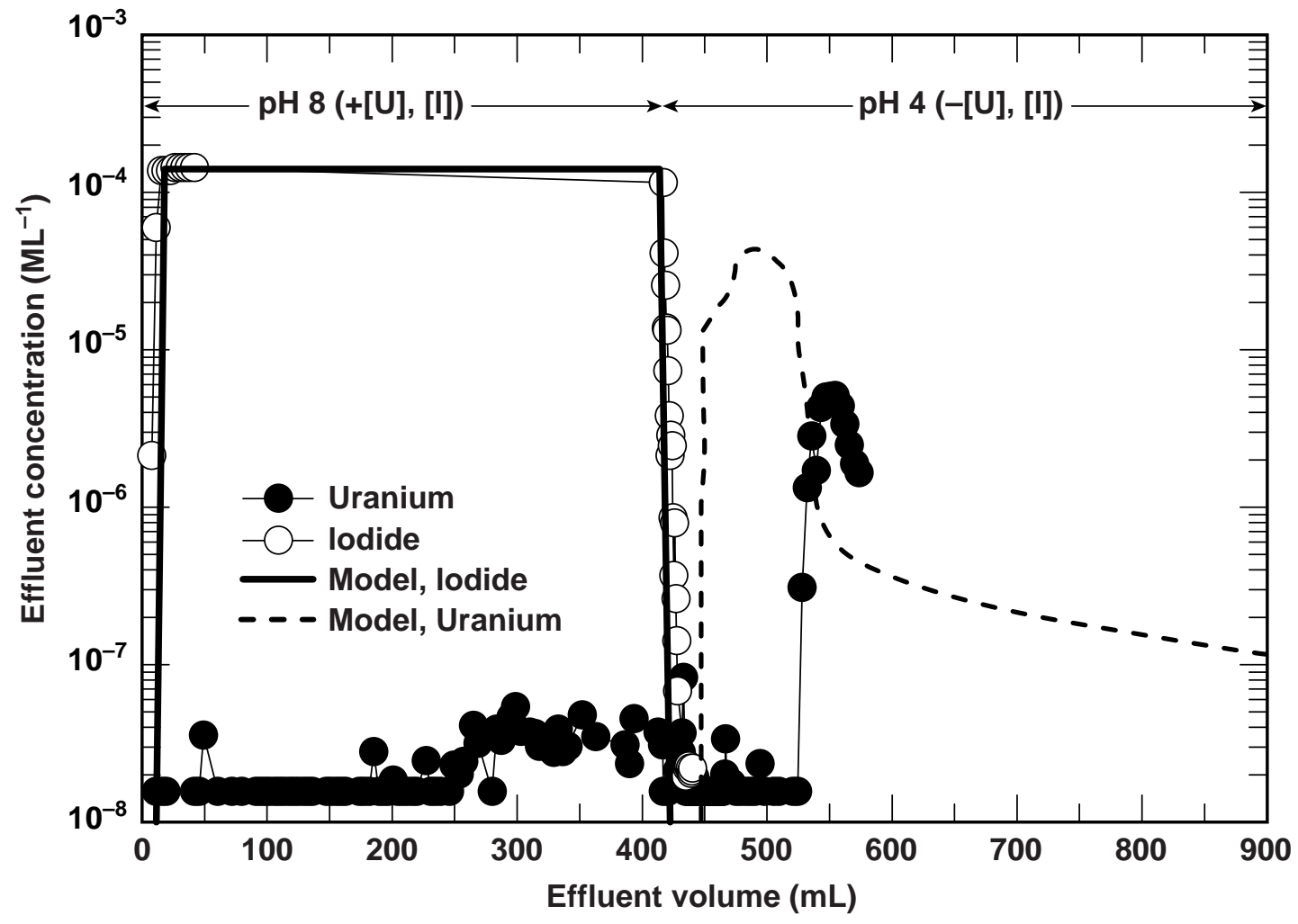

Figure 6-32. Comparison of X1t transport model (two-site DLM) and observed $\mathrm{I}^{-}$and $\mathrm{U}$ concentrations in effluent from $\mathrm{pH} 8$ column. 


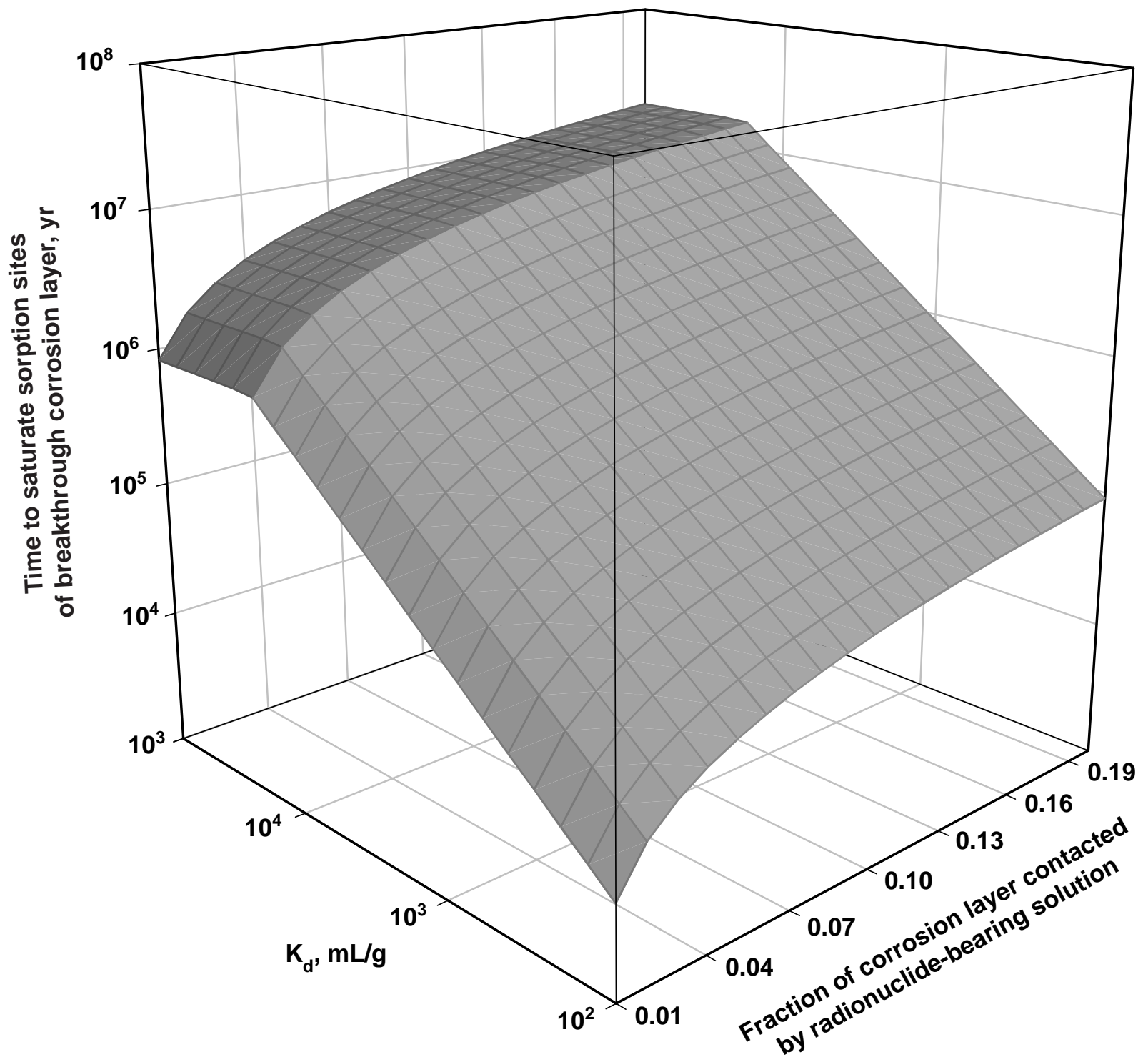

Figure 6-33. Calculated years to breakthrough of radionuclide-bearing groundwater through corrosion layer as a function of $K_{d}$ and volume fraction of the corrosion product from one waste canister $(\sim 30,000 \mathrm{~kg})$ contacted by the groundwater. (Assumptions: 6-L groundwater containing $1 \mathrm{mg} / \mathrm{L}$ radionuclide intercepts a fraction of the corrosion layer of each canister each year; the maximum adsorption is limited by an adsorption site density of 2.31 sites $/ \mathrm{nm}^{2}$ and a specific surface of $20 \mathrm{~m}^{2} / \mathrm{g}$; and sorption follows a linear isotherm.) 


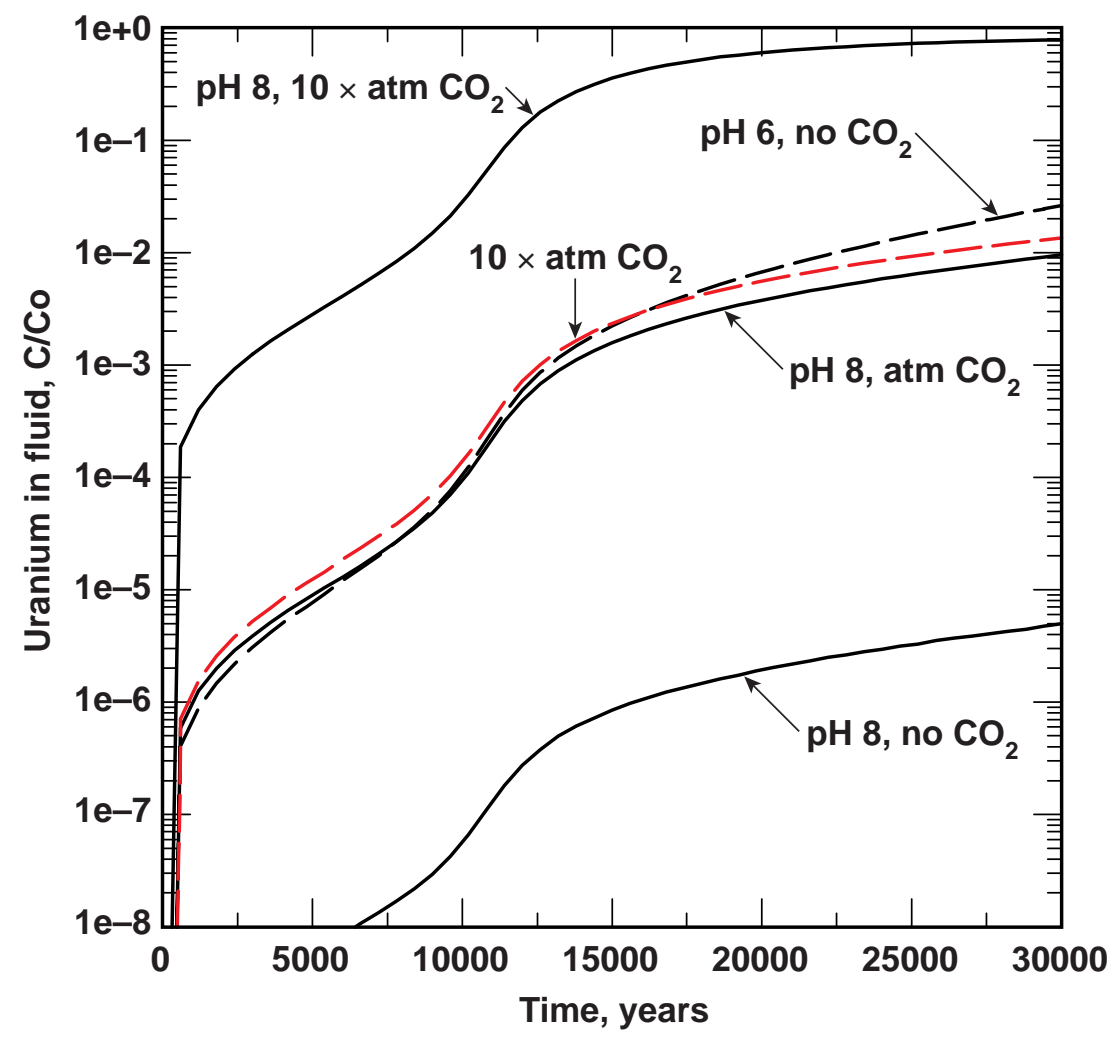

Figure 6-34. Variation in relative concentration of $U\left(C / C_{0}\right)$ with time at node $1,10 \mathrm{~cm}$ into hematite corrosion layer. 

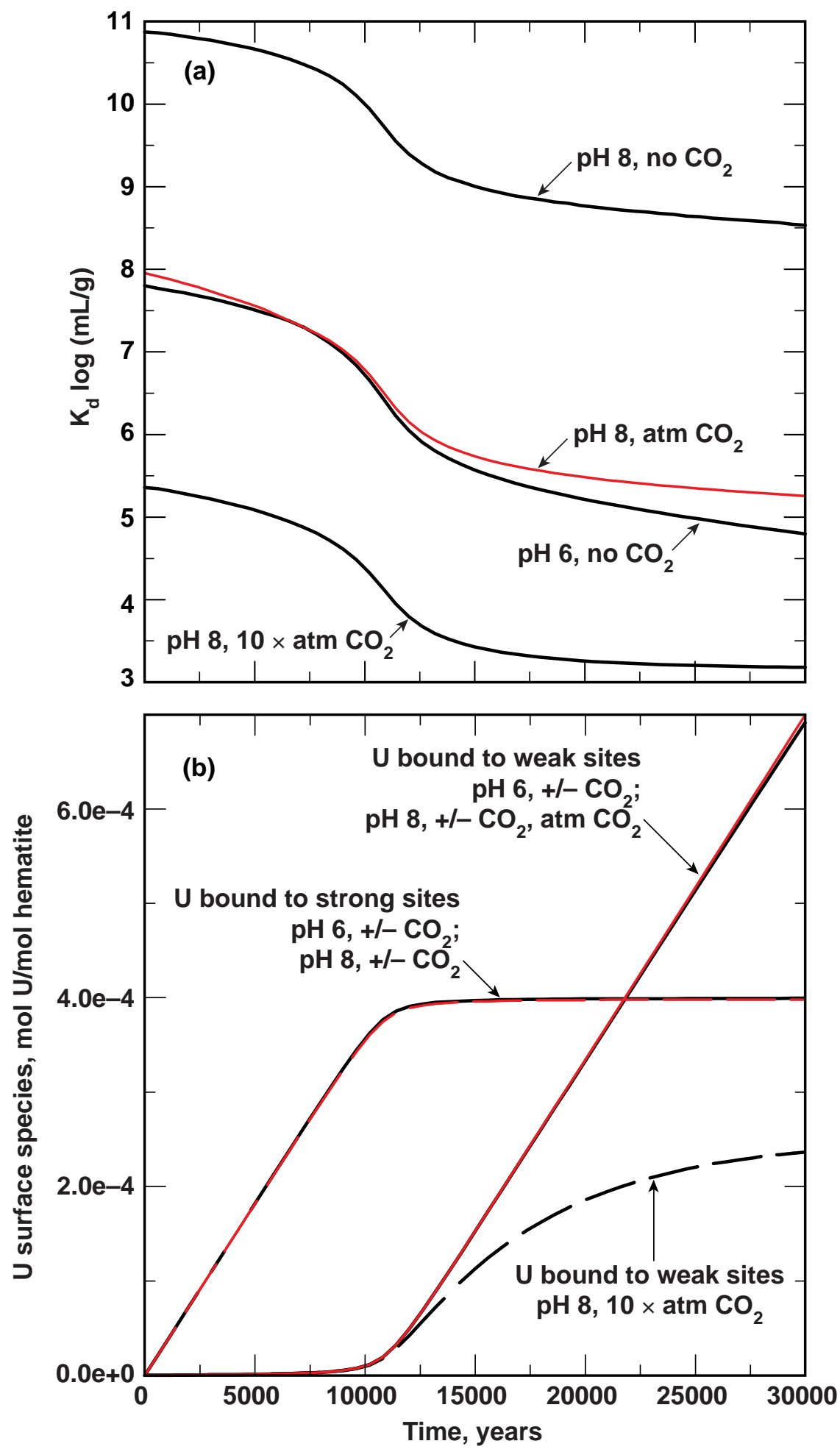

Figure 6-35. (a) Variation in $K_{d}$ vs. time at node $1(10 \mathrm{~cm})$, and (b) concentration of $U$ vs. time on strong and weak sites at node, $10 \mathrm{~cm}$ into hematite layer. 


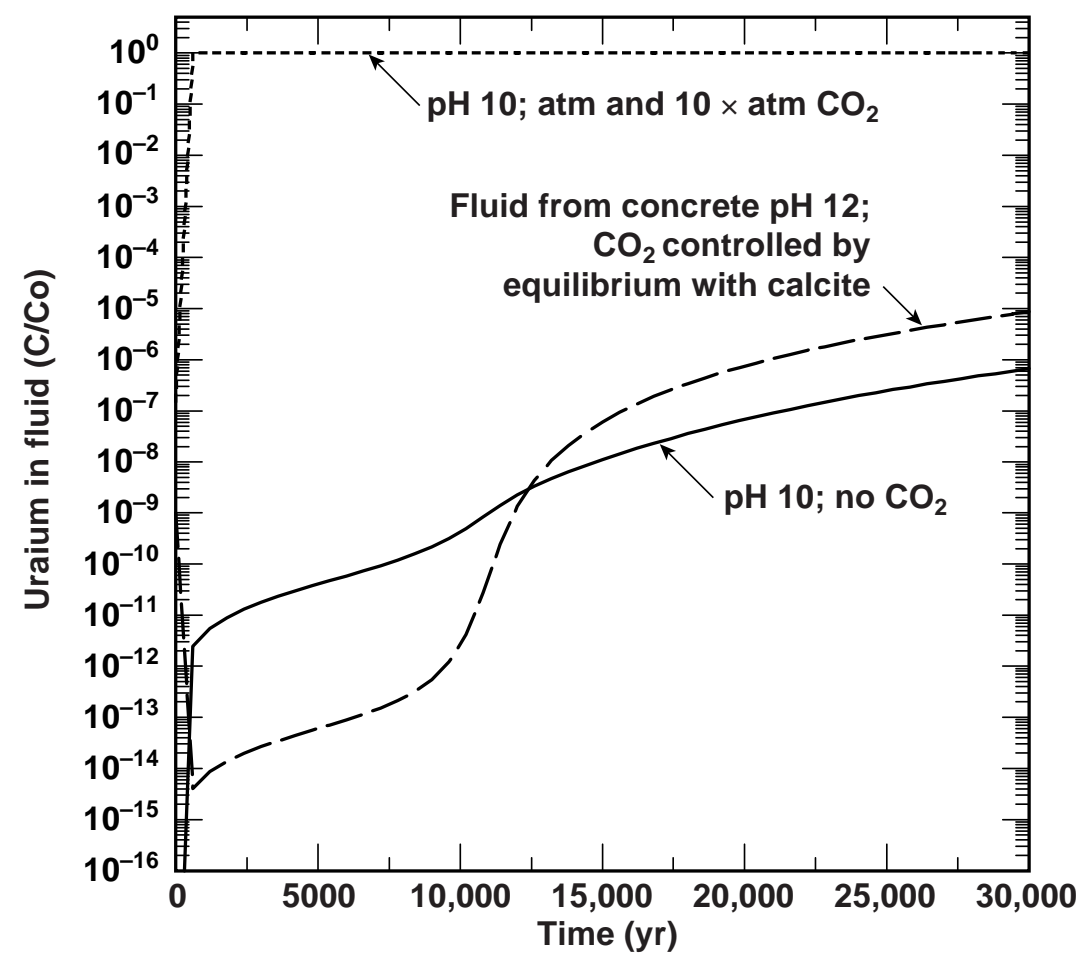

Figure 6-36. Variation in relative concentration of $U\left(C / C_{0}\right)$ with time at node $1,10 \mathrm{~cm}$ into hematite corrosion layer for $\mathrm{pH} 10$ and no $\mathrm{CO}_{2}$.

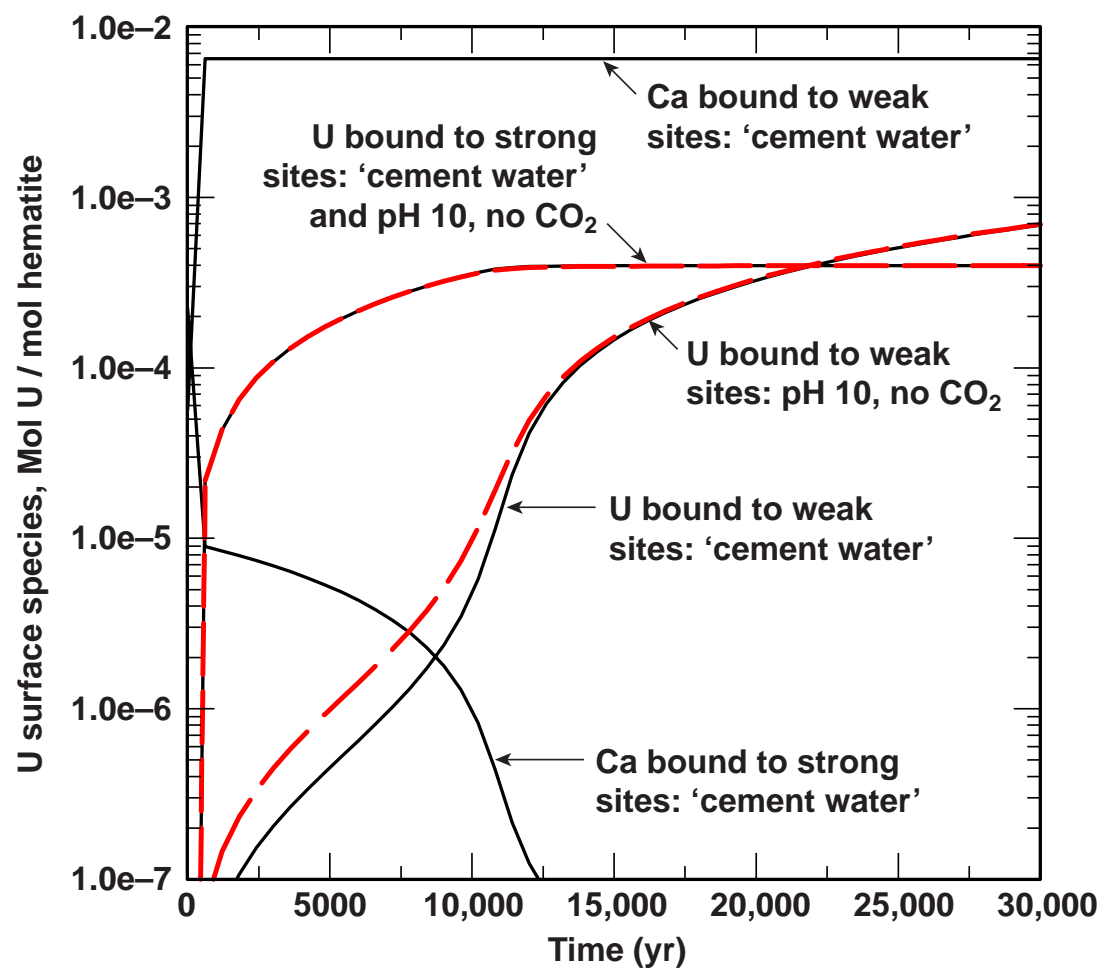

Figure 6-37. Variation in concentration of $U$ and Ca surface species with time at node $1,10 \mathrm{~cm}$ into hematite corrosion layer for $\mathrm{pH} 10\left(\right.$ no $\left.\mathrm{CO}_{2}\right)$ and cement-derived groundwaters. 


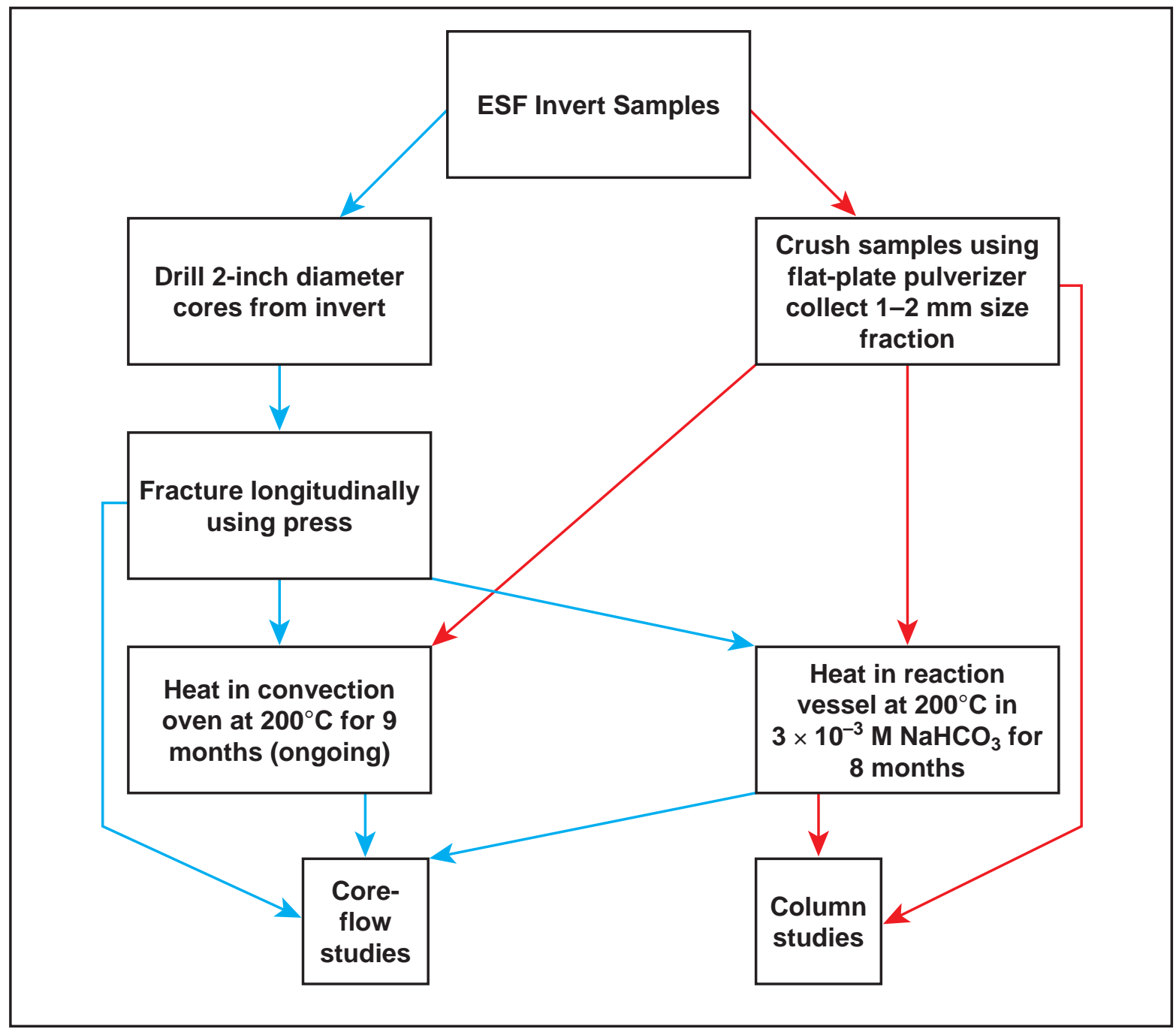

Figure 6-38. Flow chart showing the treatments applied to ESF concrete samples. 
(a)

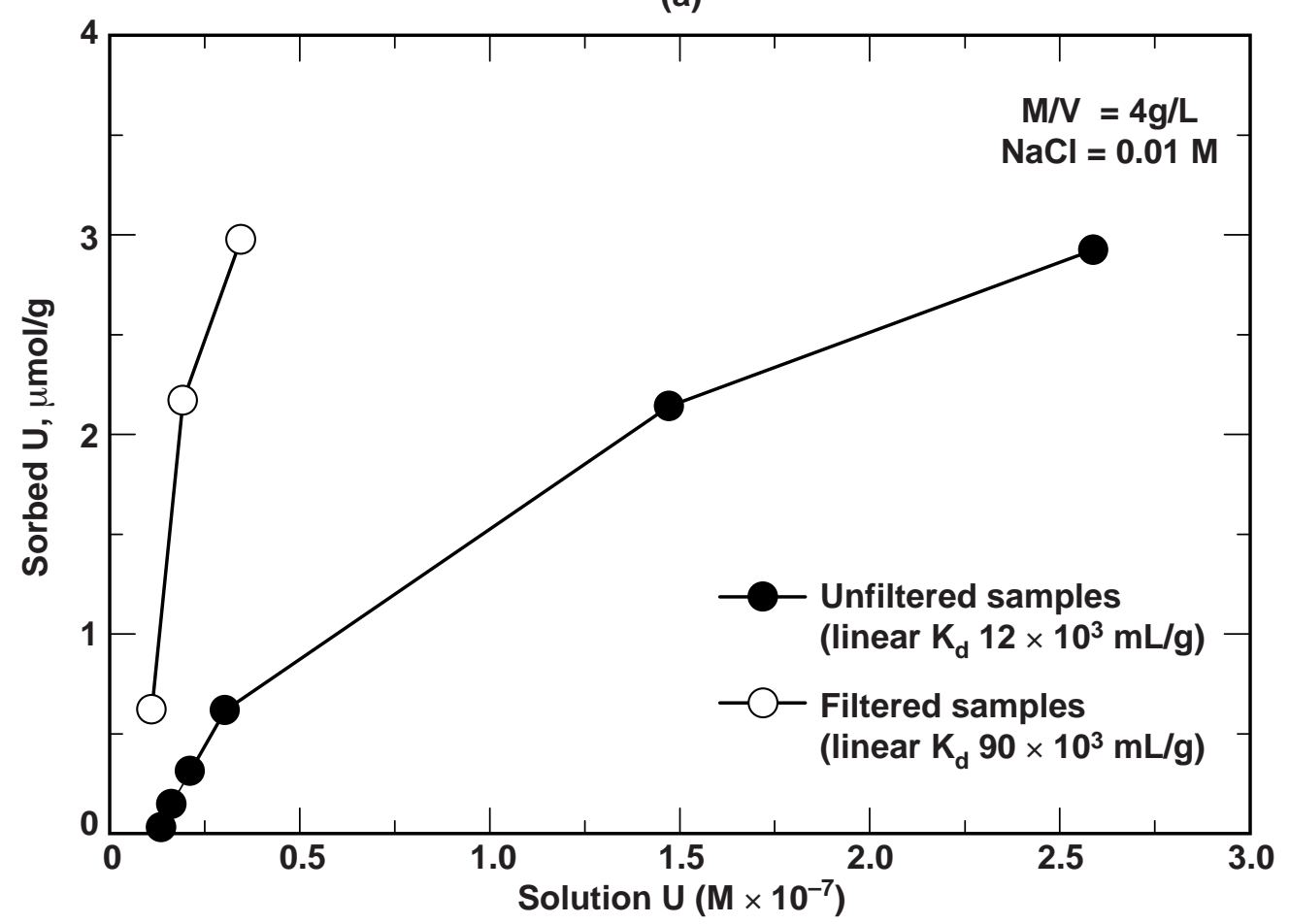

(b)

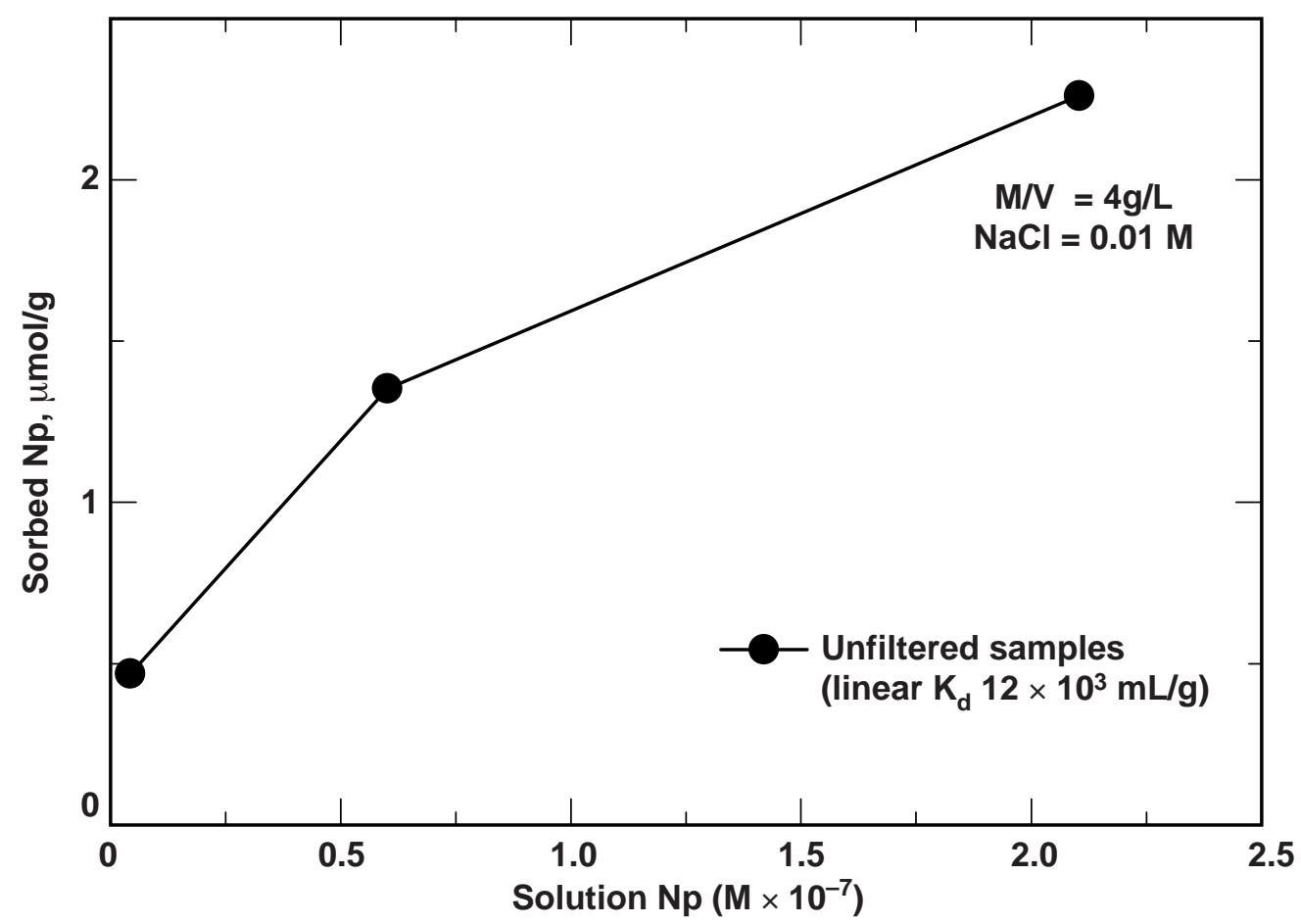

Figure 6-39. Batch sorption isotherms $\left(26^{\circ} \mathrm{C}\right.$; $\left.\mathrm{pH} 10.8\right)$ based on analysis of filtered (open circles) and unfiltered (closed circles) supernatants for (a) $U$ and (b) Np on hydrothermally altered crushed concrete. Linear $K_{d}$ represents the slope of the best fit line forced through the origin. Concentrations in filtered supernatants were at or below detection limits for three uranium samples and for all Np samples. 
(a)

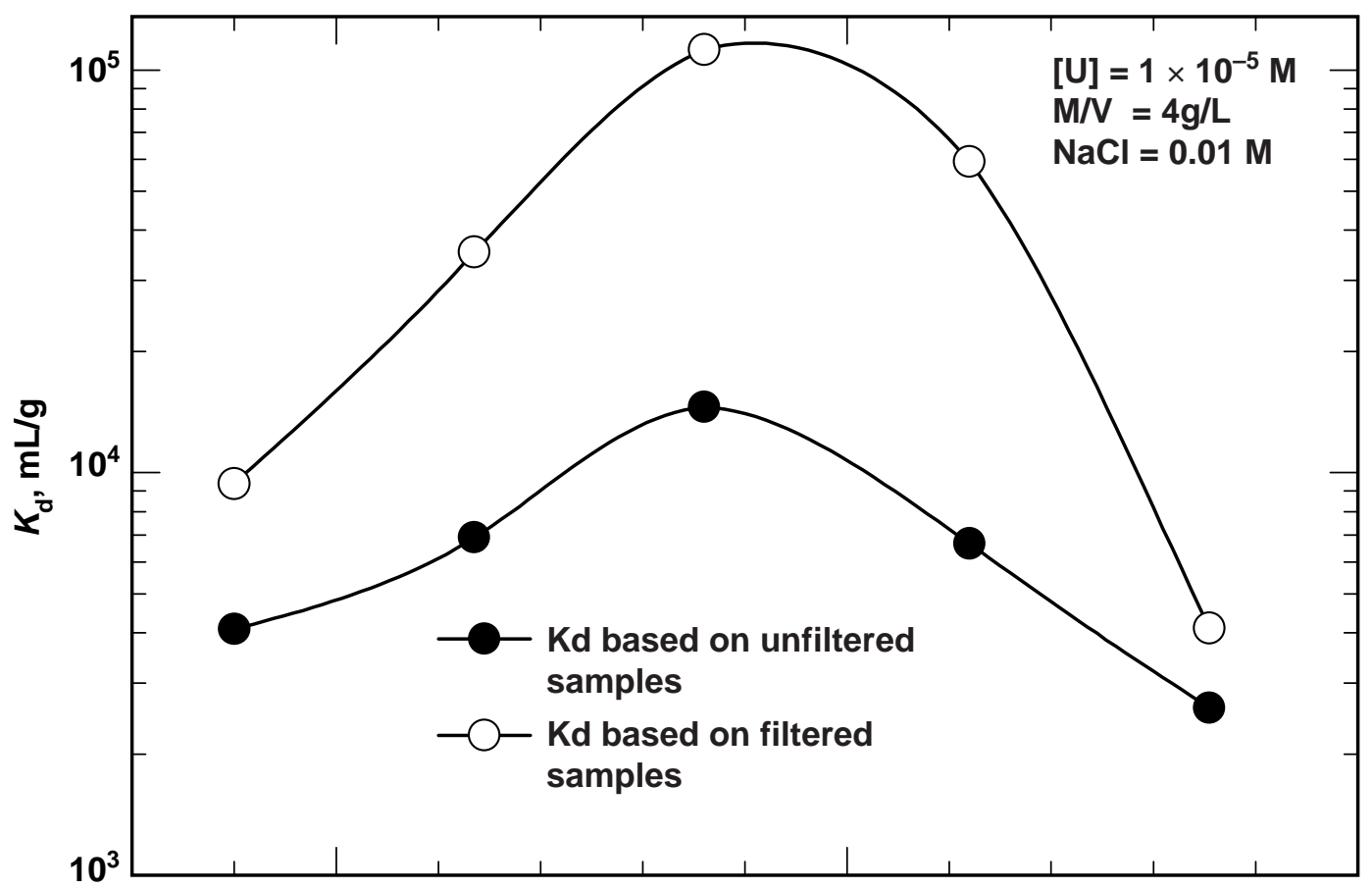

(b)

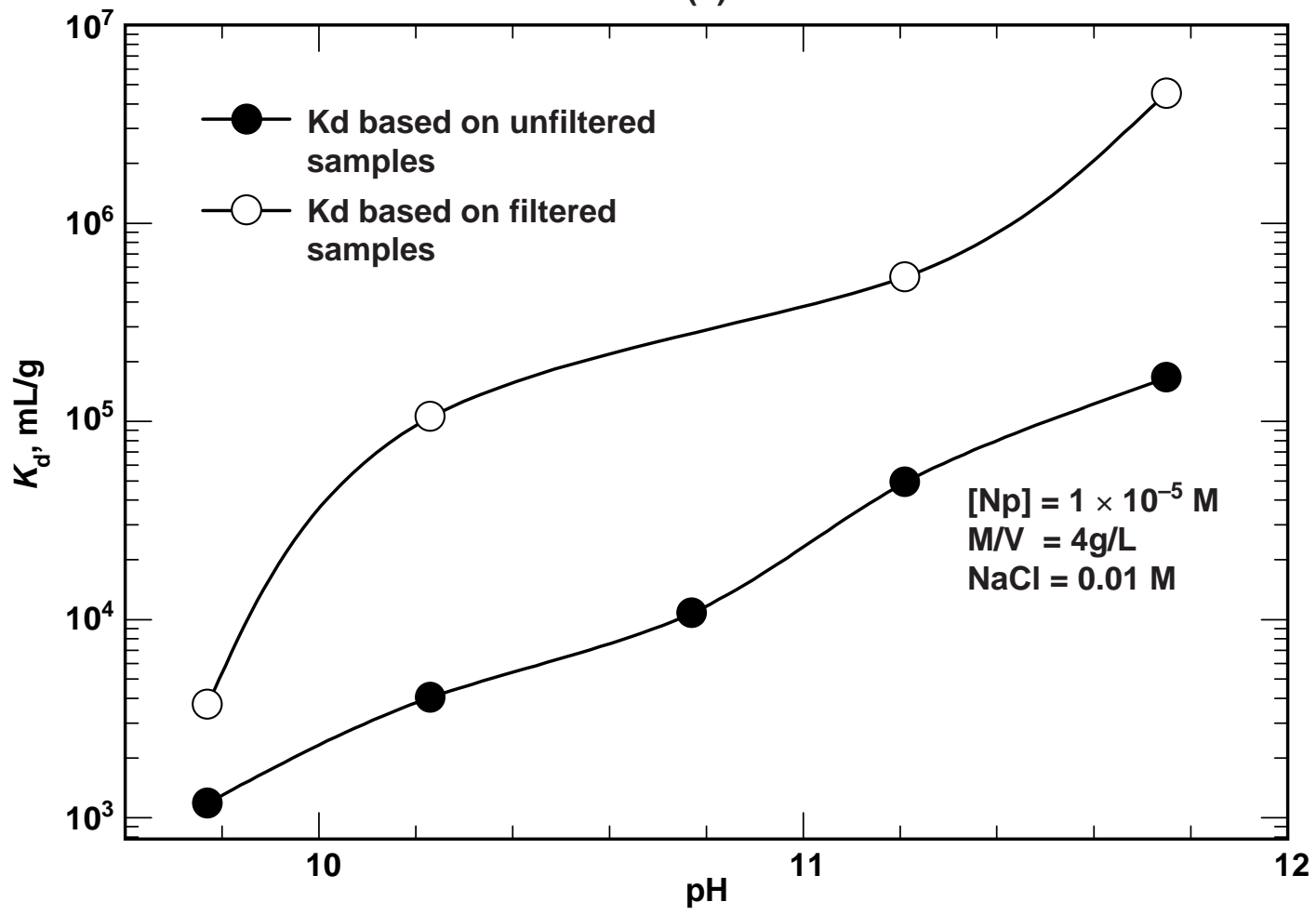

Figure 6-40. Partition coefficient $K_{d}$ vs. $\mathrm{pH}$ for (a) U and (b) Np on hydrothermally altered concrete. $K_{d}$ calculated from analysis of filtered (open circles) and unfiltered (filled circles) supernatants. 


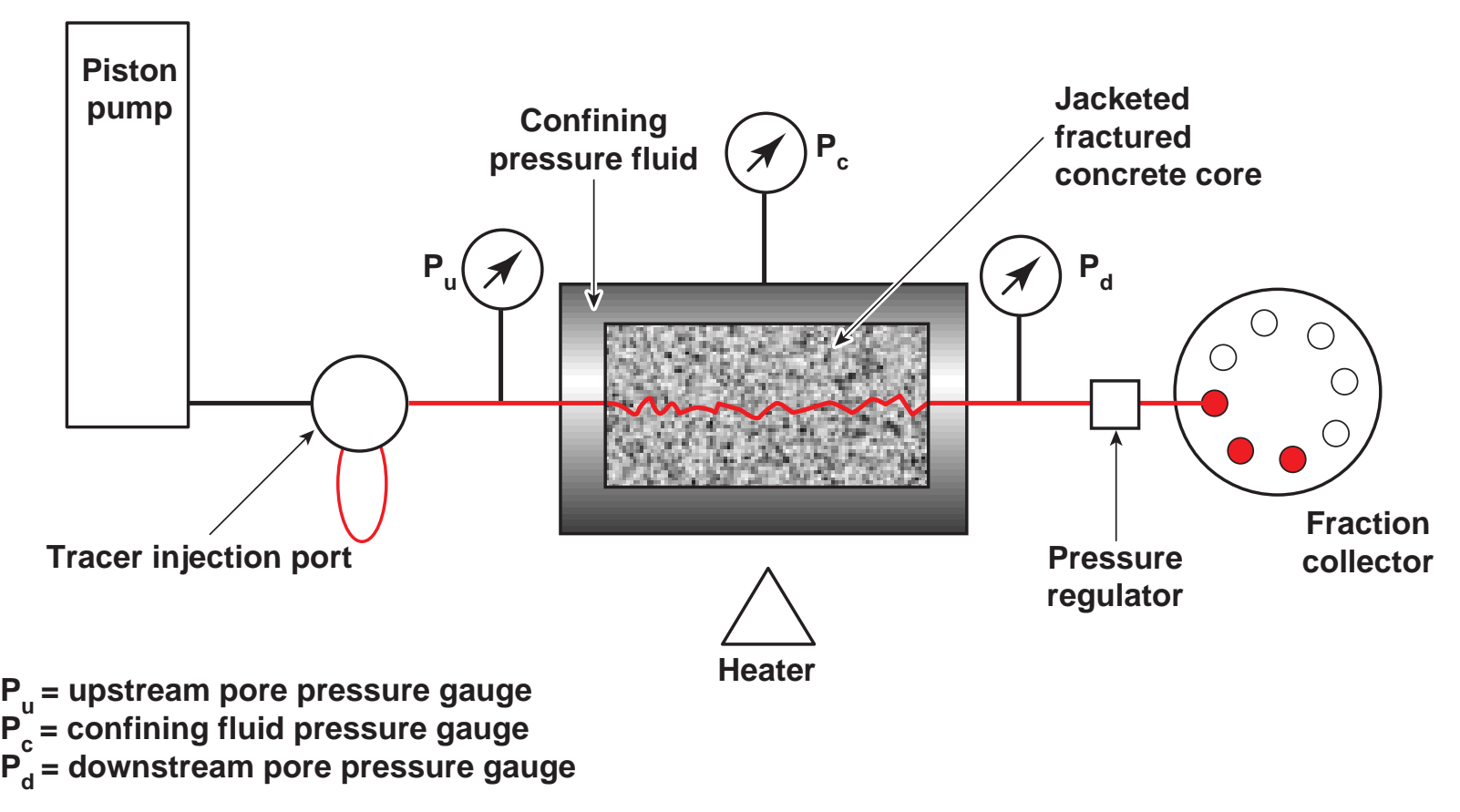

Figure 6-41. Schematic drawing of apparatus employed for experiments using intact, fractured, hydrothermally altered concrete core samples. 


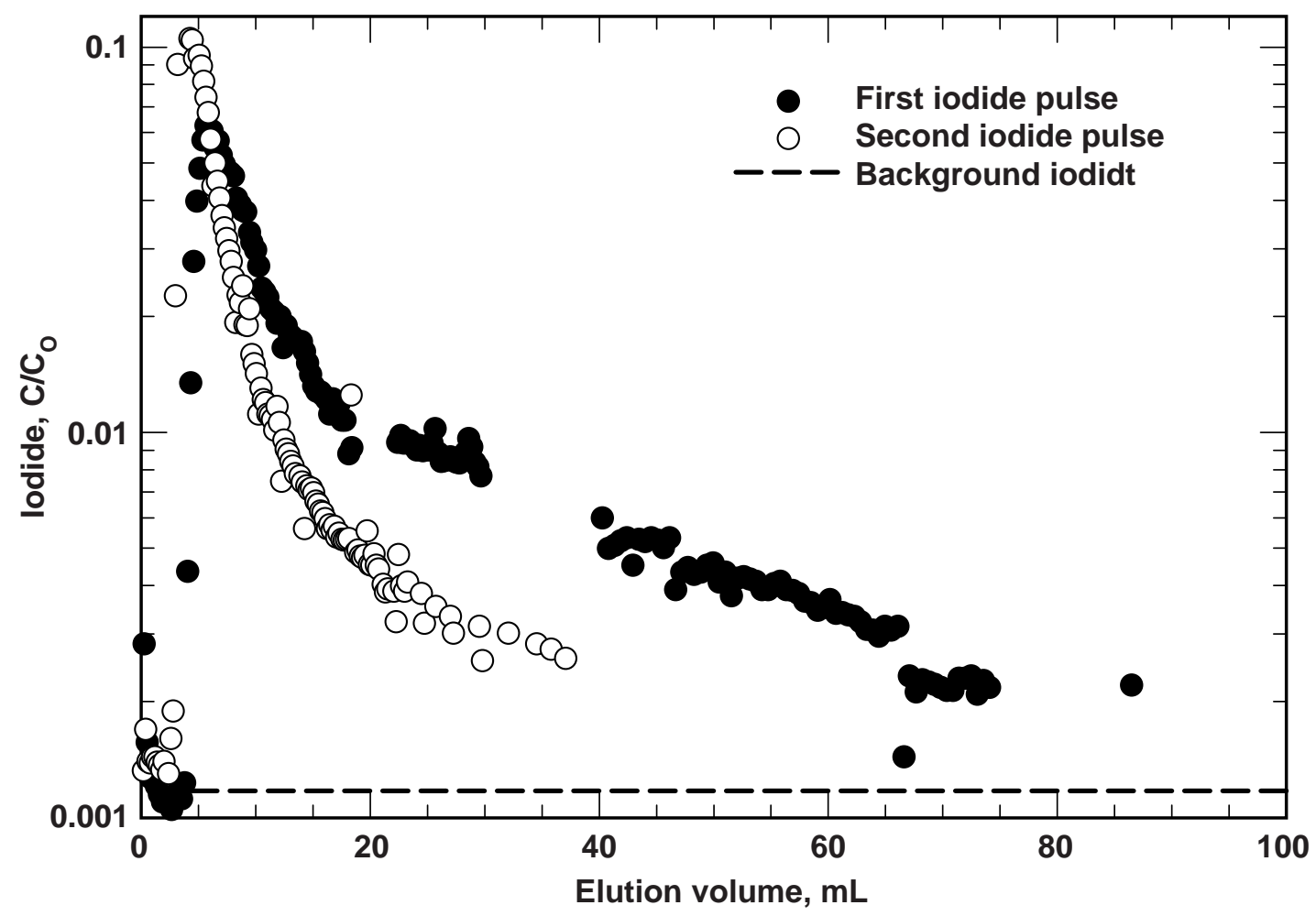

Figure 6-42. Relative concentration of iodide $\left(\mathrm{C} / \mathrm{C}_{0}\right)$ in $0.01 \mathrm{M} \mathrm{NaCl}$ effluent from fractured, hydrothermally altered concrete core for two pulses of $\mathrm{NaI}$ (circles). Pulses $(0.56 \mathrm{~mL})$ of $7.5 \times 10^{-4} \mathrm{M} \mathrm{NaI}\left(\mathrm{C}_{0}\right)$ were injected at 0 -mL elution volumes. The areas under the breakthrough curves, but above the background, equal 0.52 and $0.61 \mathrm{~mL}$ for the first and second iodide pulses, respectively. Solid line is iodide pulse observed for a core of Topopah Spring tuff containing a sawcut fracture with a $25-\mu \mathrm{m}$ aperture $\left(0.2 \mathrm{~mL} / \mathrm{hr}\right.$ flow rate, $92^{\circ} \mathrm{C}$ ) (Viani and Carman, 1996). 


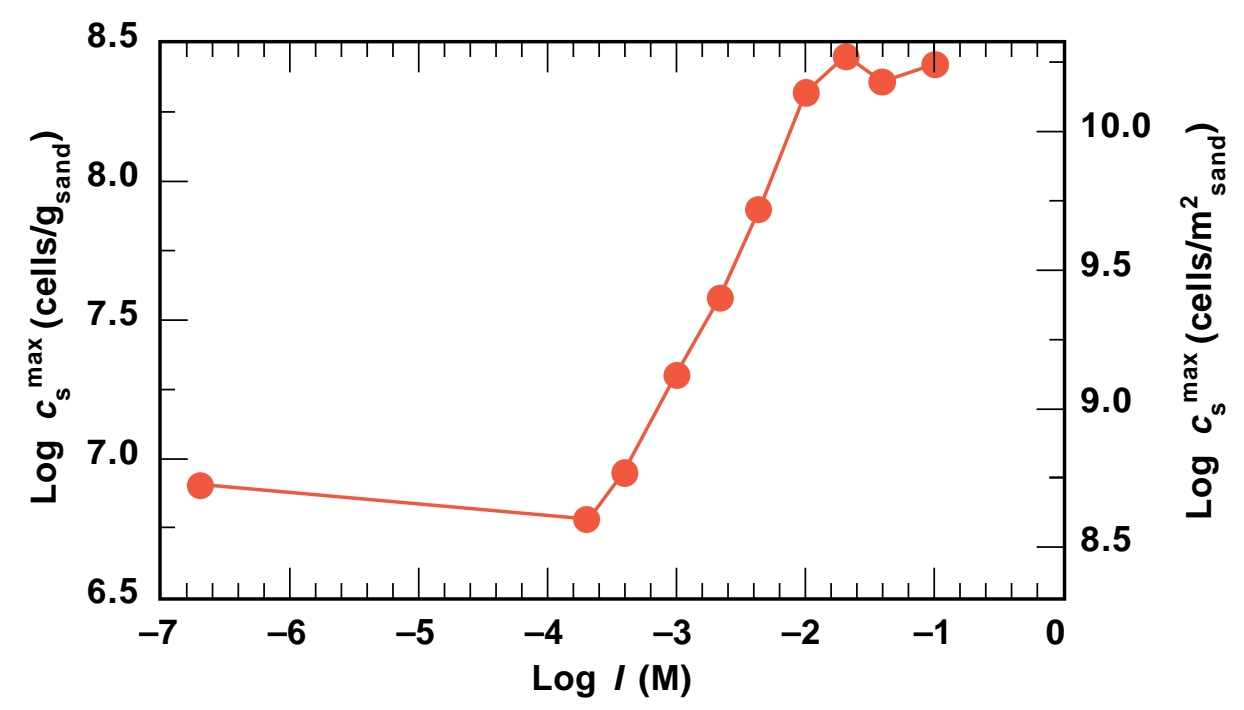

Figure 6-43. Maximum attached population density $\left(c_{\mathrm{s}}{ }^{\max }\right)$ at various ionic strengths. A limited number of bacteria can attach to a surface before the surface saturates.

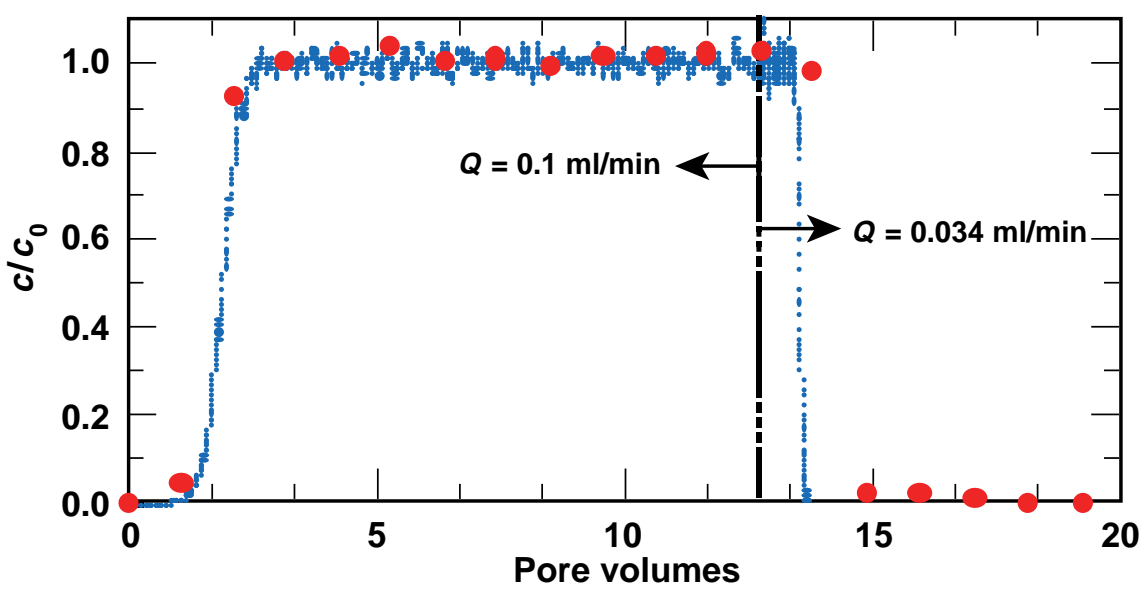

Figure 6-44. Measures (dots) and predicted (dotted line) bacterial recovery in column effluent. Predictions are from a particle-tracking numerical model that gives an excellent fit to observations. Particle transport in porous media is predictable. 


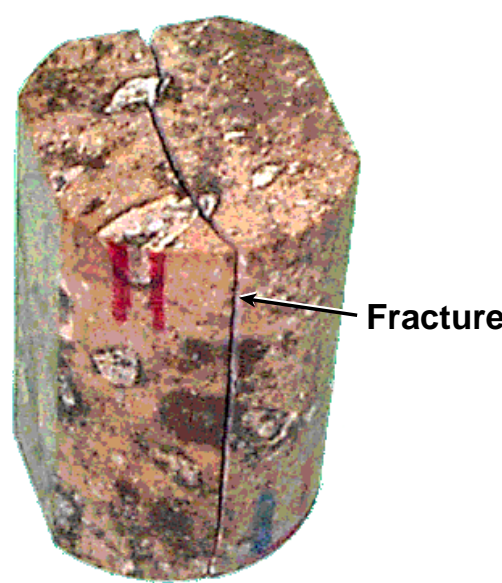

Tensile fractured core

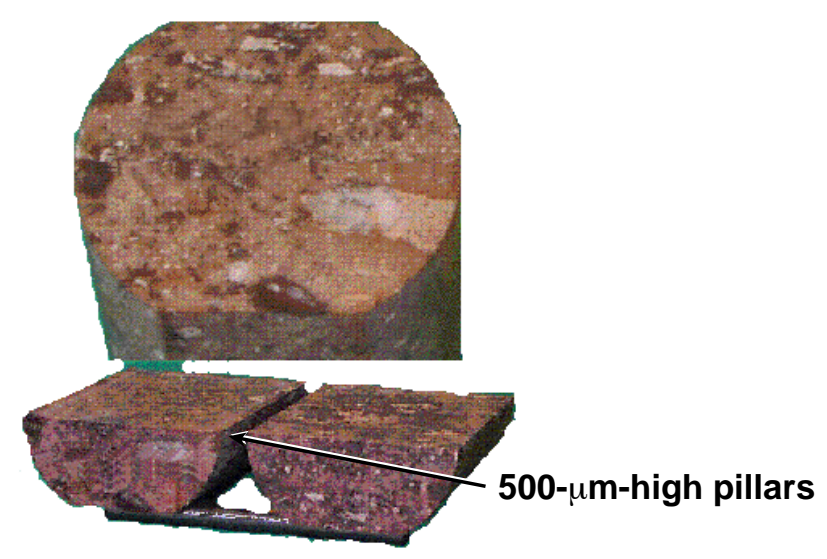

Ground parallel-plate core

Figure 6-45. Two types of fractures, a rough-walled fracture and a parallel-plate fracture, used in the laboratory experiments. The parallel-plate fractures serves as a good reference to explore the effects of fracture surface topography on transport and capture.

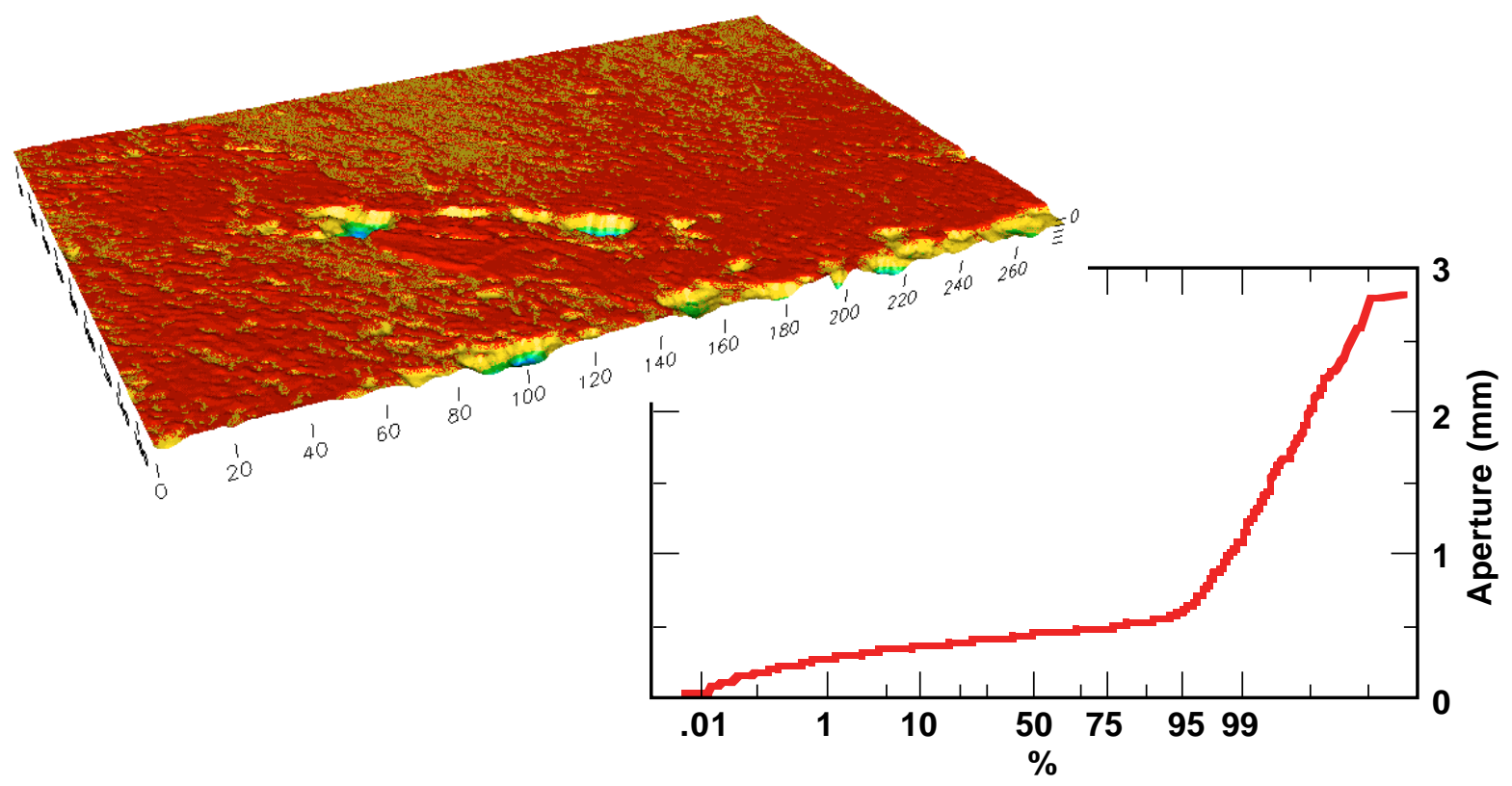

Figure 6-46. A reconstructed image of the apertures and the aperture-probability plot for the fractured core. 


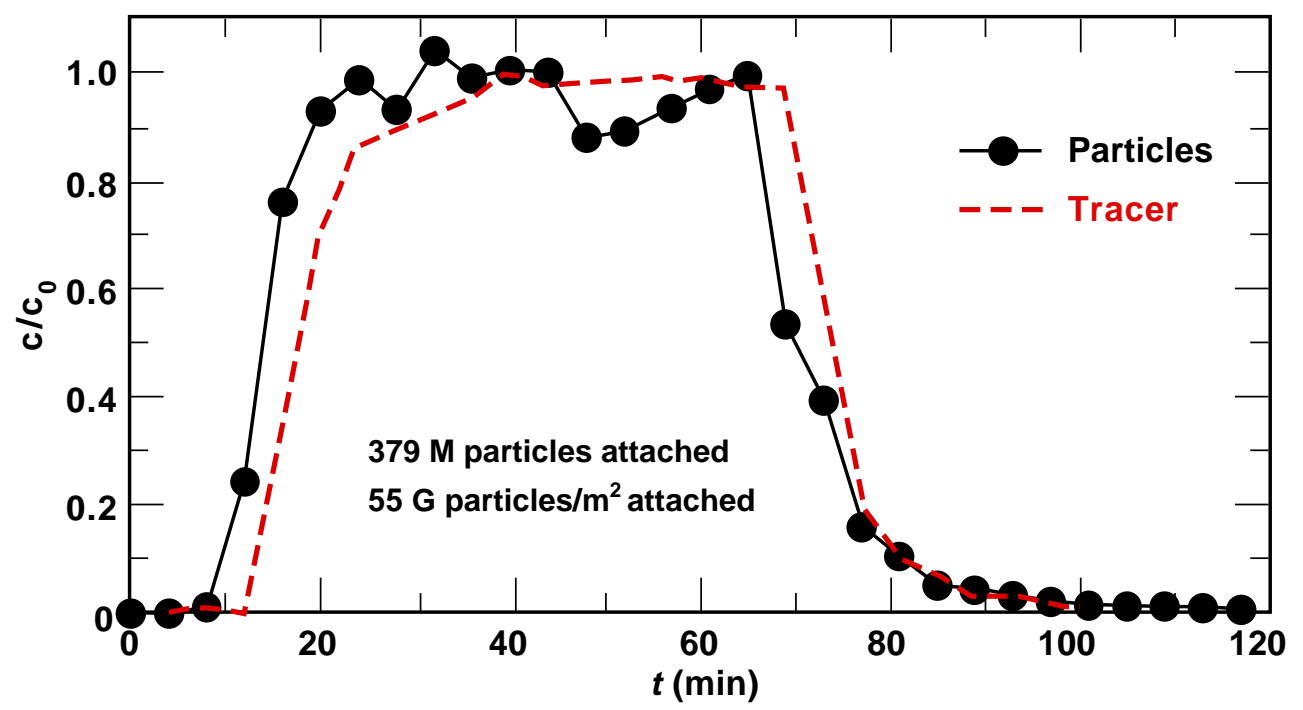

Figure 6-47. Particle recovery curves for the parallel plate core

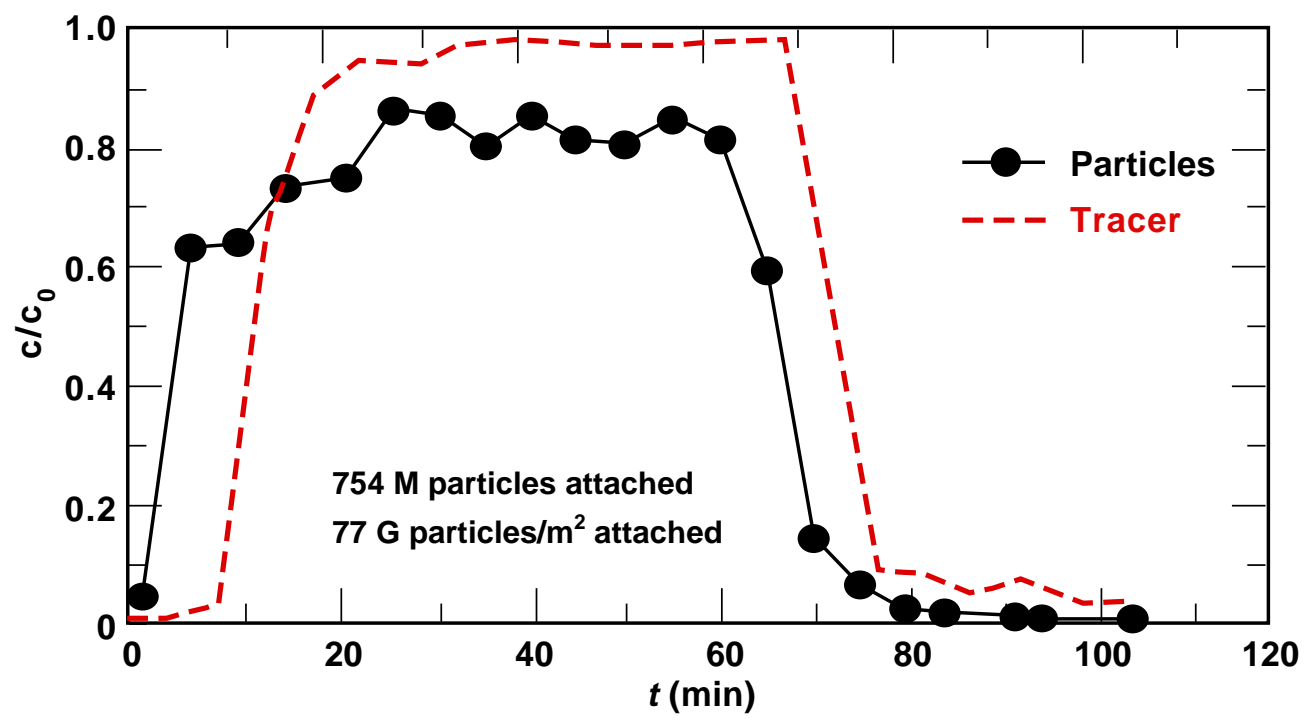

Figure 6-48. Particle recovery curves for the fractured core 


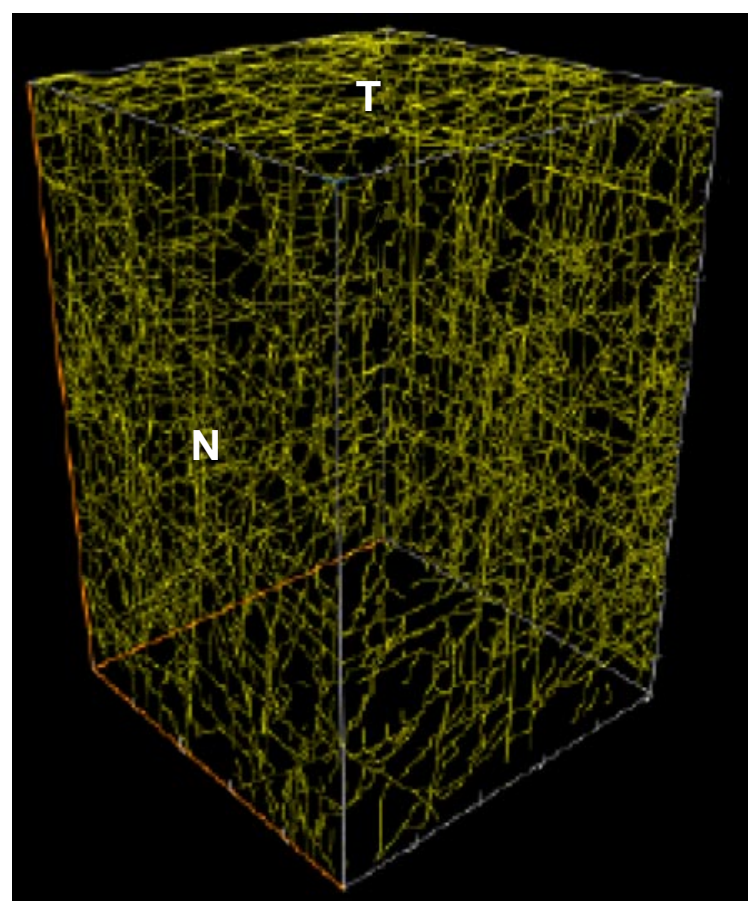

Figure 6-49. All fractures mapped on the five exposed surfaces of the large block. N (North) and T(top) denote the orientation of the outward-pointing normal to the surface. The block is $3 \times 3 \times 4.5 \mathrm{~m}$ vertically.

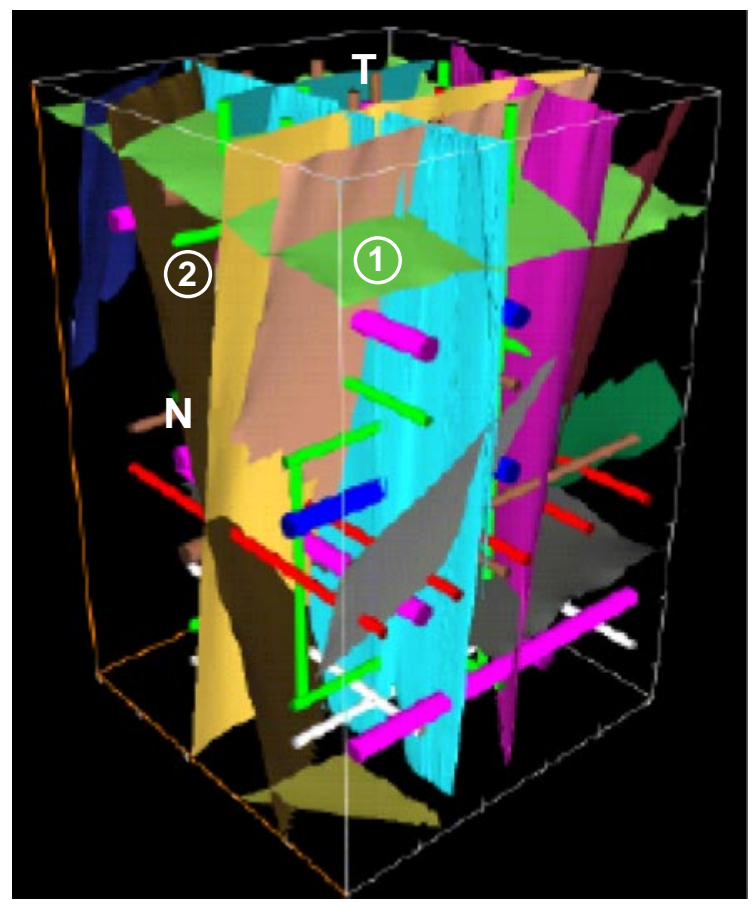

Figure 6-50. The major fractures of the large block fracture system. The 1 refers to the fracture that has the largest aperture; 2 denotes the fracture with the second largest aperture. The different colored rods depict the boreholes; red rods are the heater holes into which the bacterial cells were introduced about one month before heating commenced. 


\section{Modeling the Effects of Introduced Materials and Microbial Activity}

by Annemarie Meike with contributions from Katherine Myers, Maura K. Spragge, Angella Ann Barr, Marc W. Caffee, Angela Miller, William L. Bourcier, and Leah L. Rogers

This chapter provides supporting information about introduced materials that can be used in chemical models of the near-field environment (NFE). It identifies important classes of solid materials and identifies significant factors in the evolution of those materials under conditions that may be found in the proposed Yucca Mountain repository.

Section 7.1 discusses the quantities of steel and concrete that will be used in the reference repository design. It also discusses the introduction of microbes and organic materials into the NFE and the processes that will cause degradation of the introduced materials and will control the growth of microbes.

Section 7.2 discusses progress in modeling the aqueous chemistry of water in contact with these materials, particularly concrete. A database containing thermodynamic data for cementitious materials is developed in this section and is used to support calculations of cement-water interaction in Section 6.3. Section 7.2 also describes an approach to modeling the effects of microbial activity on near-field chemical conditions.

\subsection{Chemical Composition of Materials, Their Evolution, and Processes Operating in the Emplacement Drifts}

\subsubsection{Range of Introduced Materials That May Be Used in the NFE}

The purpose for studying introduced materials is to interact with the design effort and to identify and find alternatives for materials that could jeopardize the long-term wasteisolation performance of a Yucca Mountain repository. Ten years ago, a report by West (1988) pointed out that a wide range of materials is normally introduced into an undergroundtunnel construction site. That report showed that materials in an underground repository may be present in combinations, exposed to conditions (e.g., temperature), and need to be tracked over periods of time that have not heretofore been within the common experience of the designers. Therefore, it is possible that repository construction may require design restrictions and construction methods that are beyond normal construction practice. Since the inception of the introduced materials studies, the goal has been to predict and quantify materials usage, to predict the evolution of those materials over time as a function of repository conditions, and to determine whether their presence significantly impacts the processes that might be predicted in the absence of those materials.

Preliminary estimates of materials usage are outlined in the Introduced Materials Synthesis Report 1993-1996 (Meike, 1996). The materials include those introduced for excavation, mechanical support and stabilization, transportation, mucking, and dust control. In the absence of specific compositional information for the repository design, the Exploratory Studies Facility (ESF) was used as an analog for repository construction practice. This work led to some useful conclusions regarding the need for design flexibility, ancillary or implicit materials usage, and accidental spills as permanent materials.

Since publication of the synthesis report, additional information (Tang, 1997) has allowed estimation of bulk volumes and chemical compositions of the basic elements of the base-case repository design. Detailed construction drawings from the ESF were used where equivalent 
detail on the repository design was not available. These recent estimates include the chemical composition and design for two standard types of mechanical ground support under consideration for the repository: steel sets and concrete liners (Tang, 1997).

Metals

Metals introduced into the repository environment by construction will serve many different functions and are composed of many different alloys. The concerns of the introduced materials studies are different from those of waste-package (WP) material selection. Many of the alloys considered for WP corrosion-resistant materials are not used in construction. Conversely, the alloys used in construction are more common and, fortunately, have been used for many years, thus a foundation of knowledge regarding their behavior exists. For estimation purposes, a proportion of the repository ground support is assumed to be provided by steel sets, which are made up of materials conforming to ASTM A36. The other major contribution to metals inventory will be the WP, which also contains mild steel in a quantity comparable to the steel-set ground support.

\section{Concrete and Cement}

The standard concrete used in underground construction is made with ordinary Portland cement (OPC). Depending on engineering needs, other formulations can be used. The variations in the formulations are aimed primarily toward controlling the amount of $\mathrm{Si}, \mathrm{Al}$, and S. Different additives are used to obtain desired emplacement or cure characteristics. These are organic and inorganic compounds and can be present in a wide range of proportions. In addition, concrete contains aggregate, which is often specified primarily by size rather than by chemical composition. Thus, even though general expectations can be stated for the long-term impact of concrete (e.g., the potential for increasing the $\mathrm{pH}$ of water), the inherent uncertainty of this calculation is large because of the range of possible formulations. An explicit prediction of the long-term degradation of concrete and its impacts on aqueous, mechanical, and biotic processes depends on the original formulation. Conversely, if the potential long-term impacts of various aspects of concrete are made clear, it is possible that formulations can be modified somewhat to control or avert some negative impacts.

It is clear from examining the use of cementitious materials in the ESF (Meike, 1996; Meike and Spragge, 1995) that grout, shotcrete, and precast concrete are all standard materials of considerable utility, from rock-bolt emplacement to invert material to cast-inplace mechanical support. All of these materials are under consideration for repository design.

\section{Microbes}

Native microorganisms reside within the environment of the potential repository. Microbial communities have been characterized from a variety of deep subsurface environments, and ongoing work has already identified some of the native microbiota in the Yucca Mountain region (Russell et al., 1994). In addition, microbes will be introduced during the construction and monitoring period of the potential repository. Native and introduced bacteria can alter the chemical environment, radionuclide transport, and hydrologic properties of the surrounding engineered and natural barriers. Studies to assess potential impacts of microbial activity on the Yucca Mountain repository are focused on determining the significant microbial processes and on providing bounds for the effects of microbial activity (rates, biomass, material substrates, and products). 
Laboratory studies are intended to determine bounding conditions for various microbially mediated reactions. They focus on the chemical and hydrologic impacts of microbial communities rather than organisms, which leads in directions that are beyond the scope of traditional microbiology. These studies are conducted primarily using Yucca Mountain-derived microbial communities and isolates (representing both native and introduced populations), although carefully chosen analog studies also supplement characterization of in situ reaction rates to best predict long-term microbial effects.

Biomass can form colloidal particles or can modify fracture characteristics and thus modify hydrologic flow paths. Prediction of the chemical impacts of microbes is predicated on the ability to predict the nature and extent of biomass production. Preliminary determination of growth rates of whole Yucca Mountain microbial communities under varying conditions has been undertaken to support modeling efforts. Preliminary findings demonstrate that microbes present at the Yucca Mountain site are capable of survival and growth under conditions approaching those anticipated after waste emplacement (Meike, 1996, p. 184).

Pure strains of both aerobic and anaerobic bacteria were isolated from whole and aseptically crushed (1.7- to 2.4-mm) rock samples at room temperature. Identified and preserved Yucca Mountain bacterial isolates included representatives of the following genera: Bacillus, Arthrobacter, Cellulomonas, Corynebacterium, Pseudomonas, Staphylococcus, Xanthomonas, and Flavobacterium. These bacterial types collectively contain members that are capable of forming spores, producing acids, degrading a wide variety of organic compounds, and remaining active under both oxic and anoxic conditions. Some members of the total microbial community can grow in the absence of molecular oxygen and temperatures of at least $50^{\circ} \mathrm{C}$. Growth rates are measurable at ambient and elevated temperatures, and probable spore-forming organisms have been shown to be capable of growth after repeated exposure to $120^{\circ} \mathrm{C}$. Further in vitro determinations will aid in determining in situ rates of growth, which can then be correlated with hydrologic flow rates. Depleted nutrient conditions favor the growth of only a select group of community members, but these are capable of reaching high cell densities under aerobic conditions, even at $50^{\circ} \mathrm{C}$.

Additional information on microbial processes is provided in several reports that are not summarized here (Hersman, 1996; Story and Hersman, 1995).

\subsubsection{Range of Processes}

The product phases of material evolution, disintegration, biodegradation, and corrosion may include metal oxides, sulfides, carbonates, and silicates as well as organic compounds, alkali metals, and halogen elements. These materials have the potential to alter the $\mathrm{pH}$, ionic strength, and composition of water that may be present during the lifetime of the repository. In addition to considering aging, gamma-radiation effects must be considered in the WP environment, as discussed in Van Konynenburg (1984). The chemical effects of gamma radiation on human-made materials remain largely uninvestigated even though it is known that aggressive substances such as nitric acid can be a product of such processes.

A unique aspect of a Yucca Mountain repository, compared to other disposal concepts internationally, is that water will probably contact repository materials only after they have been exposed to elevated temperatures $\left(>100^{\circ} \mathrm{C}\right)$ for an extended period of time (hundreds or thousands of years). Thus, the mineralogical composition of many of these materials is expected to evolve, and the subsequent material-water interaction may be different than that with the original material. 


\section{Evolution of Metals}

Metals that may be introduced into the WP environment for construction purposes will be primarily iron and iron alloys, which can degrade through several mechanisms. Oxidation is one common degradation process. Such processes strongly depend on the Eh and $\mathrm{pH}$ of the environment within which oxidation occurs. According to corrosion test results (McCright, 1996), there will be a mixture of oxides that form from carbon steel. If conditions are very oxidizing, primarily hematite $\left(\mathrm{Fe}_{2} \mathrm{O}_{3}\right)$ is expected. If the conditions are less extremely oxidizing, the various $\mathrm{FeOOH}$ phases are expected. Under even less oxidizing conditions, magnetite is expected. According to corrosion studies (McCright, 1996), hydrated oxide, $\mathrm{Fe}_{2} \mathrm{O}_{3} \cdot \mathrm{xH}_{2} \mathrm{O}$, is also to be expected. Although $\mathrm{Fe}_{2} \mathrm{O}_{3} \cdot \mathrm{xH}_{2} \mathrm{O}$ may account for only a small fraction of the total oxide weight percentage, the hydrated oxide has a much lower density and thus may be significant with respect to volume changes during corrosion. At present, this aspect of corrosion and its relation to relative humidity $(R H)$ are being pursued (McCright, 1996). However, even a simple calculation, assuming that all of the iron is converted to hematite (representing a conservative bound) with a density of $5.28 \mathrm{~g} / \mathrm{cm}^{3}$, indicates that a major increase in volume will be associated with oxidation. A large volume increase is expected in conjunction with corrosion of iron and steel, regardless of the actual oxidation products yielded.

Iron, if present in large amounts, has the potential to consume significant amounts of oxygen during its oxidation and to influence the atmospheric chemistry around waste containers if the environment is sealed or semipermeable to gas. Another simple calculation, using the assumption that all iron is converted to hematite, indicates that approximately $300 \mathrm{~m}^{3}$ of oxygen (at $1 \mathrm{~atm}$ and $25^{\circ} \mathrm{C}$ ) will be consumed per linear meter of drift, for complete oxidation of the iron.

\section{Evolution of Cementitious Materials}

Reactions known to occur in cementitious materials over time at ambient temperature are expected to alter the cement mineral assemblage and to contribute to concrete degradation and reduction of mechanical strength. These reactions are chloride attack, the alkali-silica reaction, and delayed ettringite formation (DEF). Chloride attack works by the ingress of chloride-bearing water through permeable grout or cracks to contact and corrode the metal reinforcement (rebar). Expansion of the rebar due to corrosion causes the cementitious materials to crack and spall. One of the notable sources of chloride attack for the present application is the use of aggregate from desert climates that may contain evaporated salts (Taylor, 1990). Alkali-silica reaction occurs when silica-bearing aggregate reacts with alkali impurities in the cement paste. As happens with chloride attack, expansion occurs due to formation of the product phases, causing cracking (Taylor, 1990). DEF is also a cracking process due to the late formation of sulfate-bearing phases (Taylor, 1990). There is still much debate about the causes of DEF. However, some significant DEF has been related to the heatcuring of sulfate-bearing cements. A phenomenon similar to DEF may also occur through the formation of thaumasite, a sulfate-carbonate mineral.

Roy and Langton $(1983,1989)$ have studied ancient concretes to ascertain mineral stabilities and instabilities that may be applicable to modern Portland cements. The ancient concretes, which were made from lime formulations, are not completely analogous to modern concretes, which are formed from more reactive "alite" and "belite" phases that require higher temperatures for manufacture. Although made with unknown processes and varying starting materials and mixed using unknown water/cement ratios, ancient Roman concretes often incorporated pyroclastics, including tuffaceous material. A main conclusion that Roy and Langton drew from examination of ancient materials is that low-permeability 
cements, and particularly pozzolanic cements, have the greatest durability. A study of mortars from the Byzantine basilica of Hagia Sophia, Istanbul, suggests that a calciumsilicate-hydrate (C-S-H) phase is present. The degree of crystallinity is not well constrained, however, and the mortar appears to be dominated by a calcium carbonate phase (Livingston et al., 1992). A cursory examination of a Portland cement that had been submerged in water for $63 \mathrm{yr}$ at near room temperature (Rhoderick, 1981) suggested no "significant effect" on either composition or microstructure. The relevant experimental literature has been summarized in a synthesis report (Meike, 1996).

Notably scarce are studies of cementitious materials that have been exposed to elevated temperatures for extended periods of time. It is clear that elevated temperature will cause changes in both composition and microstructure, which will affect structural integrity and will ultimately affect the chemistry of the water in contact with the material. It is likely that the cementitious materials will dehydrate and transform to a more crystalline mineral assemblage. Many phases in the crystalline $\mathrm{Ca}-\mathrm{Si}-\mathrm{H}_{2} \mathrm{O}$ system can develop in cement exposed over long periods of time to temperatures greater than $25^{\circ} \mathrm{C}$. The phases themselves are found both naturally and in synthetic systems.

\section{Behavior as a Function of $R H$}

As part of an experimental and modeling program to characterize the effect that cement will have on the water budget at the potential Yucca Mountain repository, water sorption studies of crystalline phases are being performed. These experiments (Martin et al., 1996) demonstrate that many of the crystalline phases expected to form in concrete at elevated temperatures are sensitive to changes in $R H$. That is, they shrink and swell, sometimes within an hour after a change of $10 \%$ in $R H$. Thus, the crystalline phases that form at elevated temperatures may become increasingly hydrated and swell as the temperature decreases and $R H$ increases. Such behavior could significantly affect the mechanical stability of concrete after the thermal pulse.

\section{Experimental Results Relating to the Evolution of Concrete at Elevated Temperature}

Experiments were designed to provide data for a preliminary assessment of the microstructural, mineralogical, and (to a lesser extent) mechanical changes in hydrothermally altered concrete and changes in associated water chemistry (Meike et al., 1997b; Myers and Meike, 1997). In emplacement drifts, concrete will be subjected to elevated temperatures of at least $150^{\circ} \mathrm{C}$ and perhaps greater than $200^{\circ} \mathrm{C}$ if backfill is used (see Chapter 3).

The experiments were conducted with two types of cementitious materials: invert concrete and shotcrete, both from the ESF. Analysis of these materials showed that the invert concrete is composed primarily of carbonate aggregate, especially within the larger-size fraction. The shotcrete aggregate is composed primarily of quartz and other silicate minerals.

Three experimental suites of progressively longer duration were performed and analyzed (Myers and Meike, 1997). The experiments were designed so that vapor-phase and aqueousphase alteration could be observed at $90^{\circ} \mathrm{C}$. For each suite, sets of invert and shotcrete samples were exposed to four environments:

1. Water vapor

2. Aqueous solution of $1 \mathrm{M} \mathrm{NaCl}$ (invert only)

3. Aqueous solution of $1 \mathrm{M} \mathrm{NaHCO}_{3}$ (invert only)

4. A dry treatment in which deionized water was added for the last $2 \mathrm{wk}$ of the longestterm (8-month) suite 
Petrographic examination and X-ray diffraction analysis were performed on the altered samples. Some altered unaltered samples were subjected to destructive mechanical testing to evaluate changes in strength. Future work will compare the aqueous-phase compositions and mineralogical results from these experiments with chemical simulations to assess the adequacy of the available thermodynamic and kinetic data (Clodic and Meike, 1997).

The results demonstrate that concrete alteration in an aqueous environment can be very different from alteration taking place in a vapor-phase environment. Based on these and other studies, it appears that alteration in the vapor phase can depend on the composition of the gas phase. In the oxygen-rich, vapor-phase alteration case, hydrous minerals were formed; these can shrink and swell as a function of humidity, thus affecting mechanical stability. In contrast, vapor-phase alteration in a $\mathrm{CO}_{2}$-rich environment favored the formation of carbonates.

The compositions of the aqueous solutions were modified by the sample coupons of invert material, whereas the samples exposed to vapor did not affect the solution chemistry in similar vessels. The aqueous phase was analyzed periodically for $\mathrm{pH}$ and for concentrations of cement components. The $\mathrm{pH}$ of the $\mathrm{NaHCO}_{3}$ solution increased with time to a value of 9.5 at the end of 8 months. Apparently a quasi-steady state was achieved much sooner. The $\mathrm{pH}$ of the $\mathrm{NaCl}$ solution increased with time to a value of near 11.5, and a quasisteady state was apparently achieved within 4 months. For the "dry" samples, deionized water was added for the last $2 \mathrm{wk}$ of treatment at $90^{\circ} \mathrm{C}$, and a $\mathrm{pH}$ of 11.8 was measured. Additional detail on the evolution of aqueous-phase composition in these experiments is documented in the published reports (Meike et al., 1997b; Myers and Meike, 1997).

Thin-section and SEM evidence indicates that secondary calcite was precipitated in many of these experiments. Quantitative assessment of the amount of secondary calcite formation in these experiments is not straightforward and will require further work. However, preliminary work suggests that secondary calcite formation is more predominant in the samples from the vapor-phase environment of the $\mathrm{NaHCO}_{3}$ solution than in the samples from the vapor-phase environment of the $\mathrm{NaCl}$ solution or in the dry samples. This suggests some influence of $\mathrm{CO}_{2}$ gas partial pressure. Secondary calcite was found in all samples from the aqueous environment in all the experiments. The samples from the aqueous-phase experiments were all of invert composition and contained a large quantity of carbonate aggregate (calcite and dolomite). Clearly, the presence of carbonate minerals in the original aggregate affects the character of secondary mineralization that results from aqueous interaction.

An abundance of secondary crystalline C-S-H phases was observed in the samples from the vapor-phase environments. The secondary mineralization on these samples was more heterogeneous because of the localized nature of the reactions in the vapor-phase environment. The X-ray diffraction signal from these phases was so weak that, if present, it was masked by peaks in other minerals. Thus, some of these minerals have not yet been positively identified. Much of the reaction activity was located at boundaries between the aggregate and the grout, suggesting possible mechanical consequences. The samples from the aqueous-phase environments exhibited far less heterogeneous distribution of secondary phases and far fewer phases. Scawtite (a Ca-Si-carbonate-hydrate) and kilalite (a C-S-H) were observed fairly consistently in the long-term $\mathrm{NaCl}$ experiments. Neither of these mineral phases is represented in the available thermodynamic databases.

The formation of analcime is linked directly to the aqueous $\mathrm{NaHCO}_{3}$ treatment and is an example of how a particular phase depends not only on the concentration of the constituent ions in solution, but also on the $\mathrm{pH}$. 
The results of the mechanical tests exhibited strong variability, and interpretation has been deferred until the cause and statistics of variability are established. The trend appears to be toward increased mechanical strength in the samples that have experienced secondary carbonate formation, especially those samples subjected to $\mathrm{NaHCO}_{3}$ treatment. This interpretation is at least consistent with industry practice, in which precast concrete is carbonated for increased strength (Rubin et al., 1997). It is important to note that all the samples examined may have been subjected to greater-than-atmospheric $\mathrm{CO}_{2}$ fugacity because of large amounts of limestone in the aggregate.

For example, tobermorite, a cement phase expected under repository conditions, is relatively sensitive to the partial pressure of $\mathrm{CO}_{2}$ during synthesis (Myers and Meike, 1997). At $\mathrm{CO}_{2}$ partial pressures greater than ambient, compositional and microstructural changes are expected. The sensitivity of tobermorite and other crystalline C-S-Hs that may exist in OPC to the partial pressure of $\mathrm{CO}_{2}$ suggests that, in a repository environment, much of the cementitious material could evolve into $\mathrm{CaCO}_{3}$ phases such as calcite, vaterite, and other carbonates (e.g., scawtite). The availability of $\mathrm{CO}_{2}$ as a reactant will be limited to the amount initially present in the host rock plus that which is advected into the unsaturated zone (UZ) by thermal convection during the thermal period and by natural convective processes after the thermal period.

The consequences to the $\mathrm{pH}$ of water in the NFE are significant. Whereas a youngcement-water system could register $\mathrm{pH}$ of 11 or 12, a calcite-dominated aqueous system would have a significantly lower $\mathrm{pH}$. Alteration of cement in an aqueous environment leads to a more homogeneous secondary mineral assemblage than does alteration in the vapor phase. These observations provide the basis for more explicit description of solids as the repository evolves. Specific representative mineral assemblages are calculated subsequently.

\section{Calculation of the Evolving Concrete Solid for Simulations}

Based on information from the literature, plus hydrothermal experiments, and the repository design specifications, a composition was developed for one young grout and three old, thermally treated grouts. Each of the old-grout formulations represents a material that has been exposed to one of two different gas environments that represent extremes in concrete vapor-phase alteration: the oxygen-rich case and the $\mathrm{CO}_{2}$-rich case. Ultimately, it is intended that the simulations will use a full concrete formulation that will include the grout assemblages described subsequently, the appropriate aggregate, and the appropriate additives.

A first approximation of the quantity of cementitious material in the repository drift per linear meter is given in Tang (1997) and in Meike et al. (1997a). The aggregate, both coarse and fine, is specified as tuff for which an average composition is referenced to the Near-Field and Altered-Zone Environment Report, Vol. II (Wilder, 1996). This study's calculations use, as a basis for the original chemical composition, the Type V (Table7-1) cement composition supplied by Tang (1997). The chemical composition of the grout is given as charge-balanced oxides of the analyzed cation, so oxygen is not balanced in the calculations. It is noted that important compositional aspects of the concrete (e.g., sulfate) can vary from type to type and, to a lesser extent, between formulations that fit the criteria for a single type. 
Table 7-1 Calculation of the chemical composition of starting materials: grout per linear meter of repository emplacement drift

\begin{tabular}{|c|c|c|c|c|c|c|}
\hline \multirow{2}{*}{$\begin{array}{l}\text { Type V } \\
\text { Grout }\end{array}$} & \multirow[b]{2}{*}{ Wt\% Dry } & \multirow{2}{*}{$\begin{array}{c}\text { Total } \\
\text { Wt (lbs) }\end{array}$} & \multicolumn{4}{|c|}{ Amount per Linear Meter } \\
\hline & & & Lbs & $\mathbf{k g}$ & Molec. Wt & Moles Cation \\
\hline $\mathrm{SiO}_{2}$ & 25 & 3380 & 845 & 383.2 & 60.08 & 6377.8 \\
\hline $\mathrm{Al}_{2} \mathrm{O}_{3}$ & 3.4 & 3380 & 114.92 & 52.1 & 101.96 & 1022.3 \\
\hline $\mathrm{Fe}_{2} \mathrm{O}_{3}$ & 2.8 & 3380 & 94.64 & 42.9 & 159.69 & 537.5 \\
\hline $\mathrm{CaO}$ & 64.4 & 3380 & 2176.72 & 987.1 & 56.08 & 17602.6 \\
\hline $\mathrm{MgO}$ & 1.9 & 3380 & 64.22 & 29.1 & 40.30 & 722.60 \\
\hline $\mathrm{SO}_{3}$ & 1.6 & 3380 & 54.08 & 24.5 & 80.06 & 306.3 \\
\hline Total & & & & 2135.8 & & 60804.5 \\
\hline
\end{tabular}

In the following discussion, cement-mineral assemblages are postulated based on literature data, mineral-stability information, and stoichiometric considerations.

In the calculation of the "young-concrete" composition (Table 7-2), all water is conserved either as liquid or in solid form. Moisture is also conserved from the aggregate. The calculation thus derived may possibly overestimate the amount of liquid water in the pores because some small portion of the water is expected to be lost due to "bleeding" during the hydration of the concrete. For the case of precast concrete segments, the water lost due to bleeding would not be present in the repository. However, this is not the case for cast-inplace concrete, which would lose this water to the repository rock. In these calculations, it is assumed that $50 \%$ of the silicon from the silica fume is available for the formation of C-H-S gel.

The calculation of the three "old-concrete" compositions conserves only water bound in solid phases. It is assumed that pore water will be driven from the material during the thermal pulse. In these calculations, it is assumed that $100 \%$ of the silicon from the silica fume is available for the formation of C-S-H gel.

Table 7-2 Calculation of mineral assemblage per linear meter in young grout

\begin{tabular}{|c|c|c|c|c|c|c|c|c|}
\hline \multicolumn{9}{|c|}{ Ettringite as Sulfate Phase } \\
\hline & & $\begin{array}{c}g / \text { linear } \\
\mathrm{m}\end{array}$ & $\begin{array}{c}\text { Molec } \\
\text { Wt. }\end{array}$ & $\begin{array}{l}\text { Moles } \\
\text { Phase } \\
\end{array}$ & $\begin{array}{c}\text { Moles } \\
\mathrm{H}_{2} \mathrm{O} \\
\end{array}$ & $\begin{array}{c}\text { Moles } \\
\mathrm{Ca} \\
\end{array}$ & Moles Al & $\mathrm{Wt} \% \mathrm{H}_{2} \mathrm{O}$ \\
\hline C-S-H gel & $\mathrm{Ca}-\mathrm{Si}-\mathrm{H}_{2} \mathrm{O}$ & 549646 & 86.18 & 6377 & 12755 & 10842 & & 33 \\
\hline Ettringite & $\begin{array}{l}{\left[\mathrm{Ca}_{3} \mathrm{Al}(\mathrm{OH})_{6} \cdot 12 \mathrm{H}_{2} \mathrm{O}\right]_{2}} \\
\left(\mathrm{SO}_{4}\right)_{3} \cdot 2 \mathrm{H}_{2} \mathrm{O}\end{array}$ & 128163 & 1255.10 & 102 & 2654 & 612 & 204 & 7 \\
\hline Hydrogarnet & $\mathrm{Ca}_{3}\left[\mathrm{Al}(\mathrm{OH})_{6}\right]_{2}$ & 154729 & 378.29 & 409 & 409 & 1227 & 818 & 1 \\
\hline Hydrogarnet & $\mathrm{Ca}_{3}\left[\mathrm{Fe}(\mathrm{OH})_{6}\right]_{2}$ & 117186 & 436.02 & 268 & 268 & 806 & & \\
\hline \multirow[t]{3}{*}{ Portlandite } & $\mathrm{Ca}(\mathrm{OH})_{2}$ & 79046 & 74.09 & 1066 & 1066 & 1066 & & 2 \\
\hline & $\mathrm{Mg}(\mathrm{OH})_{2}$ & 42141 & 58.32 & 722 & 722 & & & \\
\hline & Total grout hydration & 1070914 & & & & & & \\
\hline
\end{tabular}


7. Modeling the Effects of Introduced Materials and Microbial Activity

\begin{tabular}{|c|c|c|c|c|c|c|c|c|}
\hline \multicolumn{9}{|c|}{ Ettringite as Sulfate Phase } \\
\hline & & $\begin{array}{c}\text { g/linear } \\
\mathbf{m}\end{array}$ & $\begin{array}{l}\text { Molec } \\
\text { Wt. }\end{array}$ & $\begin{array}{l}\text { Moles } \\
\text { Phase }\end{array}$ & $\begin{array}{c}\text { Moles } \\
\mathrm{H}_{2} \mathrm{O}\end{array}$ & $\begin{array}{c}\text { Moles } \\
\text { Ca }\end{array}$ & Moles Al & $\mathrm{Wt} \% \mathrm{H}_{2} \mathrm{O}$ \\
\hline \multirow[t]{2}{*}{ C-S-H gel } & $\begin{array}{l}\text { "From total } \\
\text { destruction of } 1 / 2 \\
\text { silica fume (excess } \\
\mathrm{CaOH}, \text { surface } \\
\text { reaction)" }\end{array}$ & 154486 & 86.18 & 1792 & 3585 & 3047 & & \\
\hline & $\begin{array}{l}\text { Total dry } \\
\text { components }\end{array}$ & 1225401 & & & & & & \\
\hline Liquid water & $\mathrm{H}_{2} \mathrm{O}$ & 230097 & 18.02 & 12772 & 12772 & & & \\
\hline \multirow[t]{2}{*}{ Liquid water } & $\begin{array}{l}\text { Add } \mathrm{H}_{2} \mathrm{O} \text { from } \\
\text { aggregate }\end{array}$ & 145845 & & & & & & \\
\hline & Total liquid water & 375943 & & & & & & 55 \\
\hline Grand total & & & & & 31768 & & & 100 \\
\hline \multicolumn{9}{|c|}{ Afm as Sulfate Phase } \\
\hline & & $\begin{array}{c}\text { g/linear } \\
\mathbf{m}\end{array}$ & $\begin{array}{c}\text { Molec. } \\
\text { Wt }\end{array}$ & $\begin{array}{l}\text { Moles } \\
\text { Phase }\end{array}$ & $\begin{array}{c}\text { Moles } \\
\mathrm{H}_{2} \mathrm{O}\end{array}$ & $\begin{array}{c}\text { Moles } \\
\text { Ca }\end{array}$ & Moles Al & Moles Fe \\
\hline C-S-H gel & 1.7Ca-Si- $2 \mathrm{H}_{2} \mathrm{O}$ & 549646 & 86.18 & 6377 & 12755 & 10842 & & \\
\hline $\mathrm{AFm}\left(\mathrm{C}_{4} \mathrm{ASH}\right)$ & $\begin{array}{l}\mathrm{Ca}_{3} \mathrm{Al}(\mathrm{OH})_{6} \mathrm{Ca}\left(\mathrm{SO}_{4}\right) \cdot 1 \\
2 \mathrm{H}_{2} \mathrm{O}^{*}\end{array}$ & 184291 & 601.59 & 306 & 4595 & 502 & 306 & \\
\hline Hydrogarnet & $\mathrm{Ca}_{3}\left[\mathrm{Al}(\mathrm{OH})_{6}\right]_{2}$ & 135415 & 378.29 & 357 & 2147 & 1073 & 715 & \\
\hline \multirow[t]{4}{*}{ Hydrogarnet } & $\mathrm{Ca}_{3}\left[\mathrm{Fe}(\mathrm{OH})_{6}\right]_{2}$ & 117186 & 436.02 & 268 & 1612 & 806 & & 537 \\
\hline & $\mathrm{Ca}(\mathrm{OH})_{2}$ & 98539 & 74.09 & 1329 & 1329 & 1329 & & \\
\hline & $\mathrm{Mg}(\mathrm{OH})_{2}$ & 42141 & 58.32 & 722 & 722 & & & \\
\hline & Total grout hydration & 1127221 & & & 23163 & 14555 & 1022 & 537 \\
\hline \multirow[t]{2}{*}{ C-S-H gel } & $\begin{array}{l}\text { Contribution from } \\
\text { silica fume (excess } \\
\mathrm{CaOH}, \text { surface } \\
\text { reaction) }\end{array}$ & 154486 & 86.18 & 1792 & 3585 & 3047 & & \\
\hline & $\begin{array}{l}\text { Total dry } \\
\text { components }\end{array}$ & 1281707 & & & 26748 & 17602 & 1022 & 537 \\
\hline \multirow[t]{3}{*}{$\begin{array}{l}\text { Liquid water } \\
\text { Liquid water }\end{array}$} & $\mathrm{H}_{2} \mathrm{O}$ & 134871 & 18.02 & 7486 & 7486 & & & \\
\hline & $\begin{array}{l}\text { Add } \mathrm{H}_{2} \mathrm{O} \text { from } \\
\text { aggregate }\end{array}$ & 145845 & 18.02 & & & & & \\
\hline & Total liquid water & 280717 & 18.02 & & & & & \\
\hline
\end{tabular}

* $\mathrm{Mg}$ is accounted for as solid solution in Afm phase.

** Destruction of $50 \mathrm{wt} \%$ 


\section{Young Concrete}

The formulation was balanced first on the available sulfate. In the first calculation, all available sulfate is accounted for in ettringite; in the second calculation, it is accounted for in a monosulfate (AFm) phase. The remaining aluminum is accounted for in hydrogarnet. Iron is also accounted for in a hydrogarnet phase. These are tabulated separately in Table 7-2; in reality the $\mathrm{Al}$ and Fe hydrogarnets would probably be present as a solid solution. The available silicon from the grout and half the silicon from the silica fume are calculated to become C-S-H gel. The chosen representative C-S-H composition is 1.7 Ca: Si: $2 \mathrm{H}_{2} \mathrm{O}$. Portlandite is formed from the remaining calcium, and magnesium hydroxide is formed from the remaining $\mathrm{Mg}$.

\section{Thermally Treated Concrete: Oxygen-Rich Environment}

This formulation is composed assuming the reactive gas composition is close to atmospheric (Table 7-3). The formulation was balanced first on the available sulfate. All available sulfate is accounted for in ettringite. The remaining aluminum is accounted for in gehlenite hydrate. The available silicon is converted to tobermorite. $\mathrm{Mg}$ is accounted for as solid solutions in tobermorite. Fe is not included in this calculation, but it could also be included in the tobermorite solid solution. The remaining calcium is converted to calcite.

Table 7-3 Calculation of mineral assemblage per linear meter in thermally treated concrete, $\mathrm{O}_{2}$-rich environment $\left(\mathrm{O}_{2}=\right.$ close to or above atmospheric)

\begin{tabular}{|l|l|r|r|r|r|r|r|r|}
\hline & & $\begin{array}{c}\text { g/linear } \\
\mathbf{m}\end{array}$ & $\begin{array}{c}\text { Molec. } \\
\text { Wt }\end{array}$ & $\begin{array}{c}\text { Moles } \\
\text { Phase }\end{array}$ & $\begin{array}{c}\text { Moles } \\
\text { H2O }\end{array}$ & $\begin{array}{c}\text { Moles } \\
\text { Ca }\end{array}$ & $\begin{array}{c}\text { Moles } \\
\mathbf{S i}\end{array}$ & $\begin{array}{c}\text { Moles } \\
\text { Al }\end{array}$ \\
\hline \hline Ettringite & $\begin{array}{l}{\left[\mathrm{Ca}_{3} \mathrm{Al}(\mathrm{OH})_{6} \cdot 12 \mathrm{H}_{2} \mathrm{O}_{2}\left(\mathrm{SO}_{4}\right)_{3} \cdot 2\right.} \\
\mathrm{H}_{2} \mathrm{O}\end{array}$ & 125408 & 1228.12 & 102 & 2654 & 306 & & 102 \\
\hline Tobermorite & {$\left[\mathrm{Ca}_{5} \mathrm{Si}_{5} \mathrm{O}_{16} \mathrm{H}_{2} \mathrm{O}\right] \mathrm{Ca} \cdot 4 \mathrm{H}_{2} \mathrm{O}^{*}$} & 1381675 & 726.97 & 1900 & 9502 & 8780 & 9502 & \\
\hline $\begin{array}{l}\text { Gehlenite } \\
\text { hydrate }\end{array}$ & $\mathrm{Ca}_{2} \mathrm{Al}_{2} \mathrm{SiO}_{7} \mathrm{H}_{16} \mathrm{O}_{8}$ & 192463 & 418.33 & 460 & 3680 & 920 & 460 & 920 \\
\hline Calcite & $\mathrm{CaCO}_{3}$ & 760253 & 100.09 & 7595 & & 7595 & & \\
\hline "Structural" $\mathrm{H}_{2} \mathrm{O}$ & & 285335 & 18.02 & & & & & \\
\hline \multicolumn{1}{|c|}{ Total } & & & & & 15838 & 17602 & 9963 & 1022 \\
\hline
\end{tabular}

Mg is accounted for as solid solution in tobermorite.

\section{Thermally Treated Concrete: Carbon-Dioxide-Rich Environment}

This formulation is composed assuming that the reactive gas contains more $\mathrm{CO}_{2}$ than does the normal atmospheric composition (Table 7-4). In this calculation, the sulfate is accounted for in the carbonate phase, thaumasite. The aluminum is converted into hemicarboaluminate. The silicon is converted into tobermorite and includes $\mathrm{Mg}$ in solid solution. Fe is not included in this calculation, but it could also be included in the tobermorite solid solution. The remaining calcium is converted into calcite. 
Table 7-4 Calculation of mineral assemblage per linear meter in thermally treated concrete, $\mathrm{CO}_{2}$-rich environment $\left(\mathrm{CO}_{2}\right.$ above atmospheric)

\begin{tabular}{|l|l|c|c|c|c|c|c|c|}
\hline & & $\begin{array}{c}\mathbf{g} / \text { linear } \\
\mathbf{m}\end{array}$ & $\begin{array}{c}\text { Molec. } \\
\mathbf{W t}\end{array}$ & $\begin{array}{c}\text { Moles } \\
\text { Phase }\end{array}$ & $\begin{array}{c}\text { Moles } \\
\mathbf{H}_{2} \mathbf{O}\end{array}$ & $\begin{array}{c}\text { Moles } \\
\mathbf{C a}\end{array}$ & $\begin{array}{c}\text { Moles } \\
\mathbf{S i}\end{array}$ & $\begin{array}{c}\text { Mole } \\
\mathbf{s} \text { Al }\end{array}$ \\
\hline \hline Thaumasite & $\begin{array}{l}{\left[\mathrm{Ca}_{3} \mathrm{Si}(\mathrm{OH})_{6} \cdot 12 \mathrm{H}_{2} \mathrm{O}\right]\left(\mathrm{SO}_{4}\right)} \\
\left(\mathrm{CO}_{3}\right)\end{array}$ & 190735 & 622.62 & 306 & 4595 & 919 & 306 & \\
\hline $\begin{array}{l}\text { Hemi- } \\
\text { carboaluminate }\end{array}$ & $\mathrm{Ca}_{3.5} \mathrm{Al}_{2} \mathrm{C}_{0.5} \mathrm{H}_{20} \mathrm{O}_{18}$ & 318183 & 622.50 & 511 & 5111 & 1788 & & 1022 \\
\hline Calcite & $\mathrm{CaCO}_{3}$ & 596587 & 100.09 & 5960 & & 5960 & & \\
\hline Tobermorite & {$\left[\mathrm{Ca}_{5} \mathrm{Si}_{5} \mathrm{O}_{16} \mathrm{H}_{2} \mathrm{O}\right] \mathrm{Ca} \cdot 4 \mathrm{H}_{2} \mathrm{O}^{*}$} & 1404028 & 726.97 & 1931 & 1931 & 8934 & 9656 & \\
\hline "Structural" $\mathrm{H}_{2} \mathrm{O}$ & & 209659 & 18.02 & & & & & \\
\hline \multicolumn{1}{|c|}{ Total } & & & & & 11637 & 17602 & 9963 & 1022 \\
\hline
\end{tabular}

$\mathrm{Mg}$ is accounted for as solid solution in tobermorite.

Thermally Treated Concrete: Carbon-Dioxide-Rich Environment $\left(\mathrm{CO}_{2}\right.$ Only)

This formulation is composed assuming that the only reactive gas present is $\mathrm{CO}_{2}$. In this calculation, the sulfate is accounted for in the carbonate phase, thaumasite (Table 7-5). The aluminum is converted into tricarboaluminate. Silicon is converted into an $\mathrm{SiO}_{2}$ phase, either chalcedony or microcrystalline quartz. The remaining Fe and $\mathrm{Mg}$ are converted to carbonates.

Table 7-5 Thermally treated concrete, $\mathrm{CO}_{2}$-rich environment $\left(\mathrm{CO}_{2}\right.$ only)

\begin{tabular}{|c|c|c|c|c|c|c|c|c|}
\hline & & $\begin{array}{c}\mathbf{g} / \text { linear } \\
\mathbf{m}\end{array}$ & $\begin{array}{c}\text { Molec. } \\
\text { Wt }\end{array}$ & $\begin{array}{l}\text { Moles } \\
\text { Phase }\end{array}$ & $\begin{array}{c}\text { Moles } \\
\mathrm{H}_{2} \mathrm{O}\end{array}$ & $\begin{array}{c}\text { Moles } \\
\mathrm{Ca}\end{array}$ & $\begin{array}{c}\text { Moles } \\
\mathrm{Si}\end{array}$ & $\begin{array}{c}\text { Moles } \\
\text { Al }\end{array}$ \\
\hline Thaumasite & $\begin{array}{l}{\left[\mathrm{Ca}_{3} \mathrm{Si}(\mathrm{OH})_{6} \cdot 12 \mathrm{H}_{2} \mathrm{O}\right]\left(\mathrm{SO}_{4}\right)} \\
\left(\mathrm{CO}_{3}\right)\end{array}$ & 190735 & 622.62 & 306 & 4595 & 919 & 306 & \\
\hline $\begin{array}{l}\text { Tri carbo } \\
\text { aluminate }\end{array}$ & $\mathrm{Ca}_{6} \mathrm{Al}_{2} \mathrm{C}_{3} \mathrm{H}_{60} \mathrm{O} \mathrm{O}_{45}$ & 567835 & 1110.93 & 511 & 15334 & 3066 & & 1022 \\
\hline Calcite & $\mathrm{CaCO}_{3}{ }^{*}$ & 1362887 & 100.09 & 13616 & & 13616 & & \\
\hline Chalcedony & $\mathrm{SiO}_{2}{ }^{* *}$ & 580213 & 60.08 & 9656 & & & 9656 & \\
\hline Siderite & $\mathrm{FeCO}_{3}$ & & & & & & & \\
\hline Magnesite & $\mathrm{MgCO}_{3}{ }^{*}$ & & & & & & & \\
\hline "Structural" $\mathrm{H}_{2} \mathrm{O}$ & & 359031 & 18.02 & & & & & \\
\hline Total & & & & & 19929 & 17602 & 9963 & 1022 \\
\hline
\end{tabular}

* Use as solid solution in modeling $\left(\right.$ moles $\left.\mathrm{CaCO}_{3}+\mathrm{MgCO}_{3}\right)=$ moles (high-Mg Calcite).

** Or micro-crystalline quartz

\section{Organic Materials}

The examination of potential chemical effects from organic materials has two goals: to assess the potential contamination of studies conducted in the ESF by introduced materials; and to examine organic materials in the context of repository performance. The following discussion provides a framework for investigating the interaction of organic materials used in concrete and other construction materials with the host rock, the WP, or the waste form. The discussion is limited mainly to polymers, which are considered to be the most abundant organic materials introduced to the NFE. 
At present, the available database for modeling aqueous chemistry of polymers includes some possible degradation products, but modeling capabilities are limited, as is the capability to predict the thermal evolution of the solids over long periods of time in response to elevated temperatures. The primary modes of degradation for synthetic polymers are thermal degradation, oxidation, photodegradation, and biodegradation. The degradation of a polymer cannot be followed by observing the behavior of a similar but simpler molecule. Models have been developed to predict specific degradation processes over times significant to repository performance (Burnay, 1990, pp. 524-533); however, most available models are suited for more typical, shorter-duration, normal-service conditions (Kenny et al., 1993). An activation energy must be reached for degradation to begin (Ravanetti and Zini, 1992); this energy can be derived from thermal analysis. The Arrhenius equation is often used to predict the chemical half lives of materials (Barr-Kumarakulasinghe, 1994). It has been found that the rate of thermal degradation is increased by higher temperatures, the presence of a radiation flux, oxidative agents, some chemical contaminants, and light. In addition, many polymers can exhibit dose-rate effects or synergism between radiation and temperature or chemical contamination (Burnay, 1990).

There are two types of thermal degradation. The first, depolymerization, involves the breaking of the main polymer chain backbone so that, at any intermediate stage, the monomer units can be recognized (Grassie and Scott, 1985). This is common for polymers that give high values of chain scission at ambient temperature (Garrett et al., 1990). The second, substitution reaction, involves the substituents attached to the backbone of the polymer molecules such that the chemical nature of the repeat unit is changed even though the chain structure may remain intact. In this case, volatile products will be unlike the monomer (Grassie and Scott, 1985).

Specific materials of interest to construction include epoxy resins. Leedy and Watters (1994) found that rock-bolt epoxy degrades at temperatures as low as $50^{\circ} \mathrm{C}$ and should thus not be used for long-term mechanical support in areas that will be exposed to elevated temperatures. However, a variation in degradation behavior exists and is probably dependent on the epoxy. It has been found that, at $125^{\circ} \mathrm{C}$, the primary degradation mechanism for epoxy resins is initiated by oxygen attacks rather than by a free radical mechanism. Many epoxy resin systems manifest significant oxidative degradation in air at temperatures as low as $100^{\circ} \mathrm{C}$ (Burton, 1993). A change in mass begins at just less than $250^{\circ} \mathrm{C}$.

\section{Radiation Effects on Polymers}

The effect of radiation on polymeric materials has been studied since the construction of the first nuclear power plants in the 1950s. When exposed to high-energy radiation, polymeric materials undergo chemical changes. The energy from the radiation excites the polymer molecules, leading to chemical change. Most energy is deposited in the substrate by Compton scattering, whereby the ejection of a valence electron is accompanied by deflection of the incident photon by the electron cloud around the atom. At lower energies, the incident photon is completely absorbed by the substrate atom to produce ionization. Radiation degradation can be measured from changes in the molecular weight, from which the degree of cross-linking and scission can be determined. After irradiation, polymers continue to undergo changes. For example, irradiation in air leads to the formation of peroxides, and these compounds have characteristic rate-versus-temperature relations for decomposition, usually with significant rates in the range $50^{\circ}$ to $150^{\circ} \mathrm{C}\left(\mathrm{O}^{\prime}\right.$ Donnell, 1990).

Radiation enhances degradation, especially thermal effects, which often occur in parallel with radiation exposure. As energy is added to a system, the temperature is raised. The reaction rates are often quite different in a glass and rubber of a given polymer and undergo 
changes at the transition temperatures. The deterioration in the properties of polymers may be markedly increased by relatively small rises in temperature (Garrett et al., 1990). This tends to be the case with most chemical reactions because of the energy provided by an increase in temperature. An increase in temperature, when combined with radiation, leads to an increase in chain scission. Radical-reaction kinetics are dependent on the polymer morphology, crystallinity, molecular weight distribution, and the main chain stability as well as on its higher structure (morphology) (Kaplan, 1991). Time-temperature-dose-rate models have been developed to predict the long-term aging of polymers exposed to radiation (Gillen and Clough, 1989).

Due to greater exposure, the surface effects of radiation tend to be much greater than the effects to the core of a sample. As a result of energy transfer, molecular components present in only small amounts may be the main sites of chemical change (O'Donnell, 1990). In addition, hydrogen atoms are often yielded and can lead to cross-linking. The penetration of low-molecular-weight liquids into polymers can lead to a decrease in the radiation yield of cross-linking (Smirnov and Dubova, 1992). Some correlations can be made between photo degradation and gamma radiation. Because absorption of radiation is an essential first step to photo degradation, strongly absorbed radiation will be attenuated as it passes through the polymer, and reaction will be concentrated in the surface layers. Thus, photolysis is often identified by the evolution of hydrogen, the development of insolubility, and discoloration. In addition, ultraviolet radiation often initiates oxidation. Photo degradation cannot readily be predicted from the chemical structure because of small impurities and abnormalities often present in polymer chains; it must be tested experimentally.

Thermosets are much more resistant to irradiation than are thermoplastics. Counterintuitively, perhaps, thermosets exposed to radiation in air degrade more because of the oxygen concentration in the interior of samples. The dissolved oxygen reacts with the radiation-induced radicals and builds peroxides. Thus, the thermoset becomes unstable and slowly decays by chain scission. The longer the irradiation time, the more complete is the breakdown of the peroxides and the damage to the material (Wilski, 1990).

\subsubsection{Water-Introduced-Material Interaction}

It is clear that the addition of human-made materials to the NFE may modify the chemical environment and influence geochemical reactions. The most significant of these interactions, based on mainly on the mass of material used, will probably involve cementitious materials and metals.

\section{Metals}

Much of the current understanding of the long-term chemical processes involving metals that may be used in the repository is based on historical analogs. Studies of metal artifacts from a variety of ages demonstrate that some phases that form cannot be predicted from present knowledge of material degradation. Some products of these human-made materials rarely occur naturally and are therefore not necessarily predictable from a geochemical database. For example, botallackite, a rare copper hydroxy-chloride, has been observed associated with the corrosion of a copper object exposed to chloride ion (Pollard et al., 1989). Stability fields and reactive sequences of the basic copper (II) chlorides have only recently been proposed (Pollard et al., 1989), on the basis of this and other historical data, as a complement to experimental data, where experimental data alone have previously failed. That diffusion-controlled phenomena should be expected over time periods of at least $2000 \mathrm{yr}$ is apparent from investigations of corrosion phenomena in ancient bronzes (Scott, 1985). Development of some phases appears to be mediated by the activity of microorganisms 
(McNeil et al., 1990). In addition, some information on the long-term corrosion of materials has being assembled from the New Zealand analog site (Bruton et al., 1995; Meike, 1996). Because these data represent materials and methods that are more similar to present-day construction practice, and more is known about the environment, the data from modern, natural analog studies may be more useful for chemical model confidence-building than are descriptions of much older archaeological artifacts.

\section{Cementitious Materials}

The presence of cementitious material may greatly alter the chemistry of water in the proposed repository. These materials provide a large reservoir of unstable Ca-silicate phases that will dissolve and reprecipitate at the rock-water interface. Chemical interactions between water and nonthermally treated grout at $20^{\circ}$ to $60^{\circ} \mathrm{C}$ may well be dominated by the dissolution kinetics of the unstable amorphous and crystalline phases and precipitation

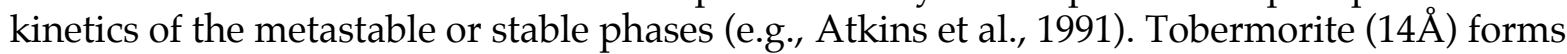
in water at temperatures less than $80^{\circ} \mathrm{C}$ (Fujii and Kondo, 1983), but it begins to lose interlayer water at $70^{\circ} \mathrm{C}$ in dry $\mathrm{CO}_{2}$-free air to yield $11 \AA$ tobermorite (Taylor, 1987). This type of low-temperature interaction has received much attention internationally and can be found in the radioactive-waste-disposal literature. It has also been discussed in Meike (1996).

The $\mathrm{pH}$ of water in contact with relatively young cement can be relatively high (10-12). Disintegration and dissolution of cementitious materials may change the $\mathrm{pH}$ of water to values as high as 11.5 , even at $100^{\circ}$ to $200^{\circ} \mathrm{C}$ (e.g., Myers and Meike, 1997). Portlandite $\left[\mathrm{Ca}(\mathrm{OH})_{2}\right]$ and alkali hydroxides are responsible for this chemical phenomenon.

Tests in which concretes were leached at $100^{\circ}$ to $200^{\circ} \mathrm{C}$ with either distilled water or Standard Canadian Shield Saline Solution in contact with a sodium-bentonite, a waste glass, or a silica fume additive have indicated that the identity and concentration of species in solution is time-dependent (Burnett et al., 1985; Heimann, 1988a, 1988b; Heimann and Hooton, 1986; Komarneni and Roy, 1983). The fate of C-S-H gels and the cement minerals and their interaction with the aggregate are a function of time, temperature, solid and aqueous solution compositions, and the availability of water. Of particular concern to the degradation of concrete is whether the concrete is exposed to air, $\mathrm{CO}_{2}$, or water; the aluminate and ferrite content of the cement; and the activities of carbonate and sulfate content of the water.

To some extent, physical properties (e.g., interconnected porosity) that can regulate the rate of, and the long-term susceptibility to, chemical attack are determined by the initial formulation. Porosity is affected by initial water:cement ratios and thus, potentially, by the method of emplacement. Leaching will preferentially dissolve some minerals such as portlandite from the set material and thus can increase permeability, which will influence the rate of degradation. Sufficiently high activities of sulfate or $\mathrm{CO}_{2}$ can react with concrete. At elevated temperatures, residual portlandite reacts with carbonates to form calcite (Milestone et al., 1987).

With regard to the potential survival of concrete, an important distinction is recognized between performance lifetime, which is linked to the mechanical stability of the structure, and chemical lifetime, which represents the duration of the chemical effects of the material long after the material has ceased to perform its function.

It is highly likely that, long after the mechanical performance lifetime of the cementitious materials has ended, these materials will serve chemical functions such as sorbing radionuclides, especially if the formulation contains zeolites or pozzolana. In fact, at the point in the evolution of the repository that sorption is required, an invert material that is considered to have poor long-term mechanical performance properties may be an asset 
because large surface areas predominantly determine the effectiveness of a sorbant. Thus, a cementitious material that becomes greatly fractured or disaggregated increases the effectiveness of the constituent sorbant materials.

\section{Water Stored in Solids}

Water will be conserved in several ways within concrete as a function of thermal evolution and of age. Water can be present in liquid form within concrete pores. It can be present in solid minerals with varying levels of binding energy such that different amounts of energy are required to release that water. For example, if one considers the two sulfatebearing phases used in these calculations, ettringite begins to lose water rapidly at approximately $50^{\circ} \mathrm{C}$ (Taylor, 1990), and thaumasite begins rapid weight loss at $110^{\circ} \mathrm{C}$ (Taylor, 1990). Other phases hold water in a different manner. C-S-H is an amorphous structure and holds its water loosely, unlike the higher-temperature crystalline C-S-H phases such as tobermorite. According to Lea (1971)), tobermorite is stable between approximately $100^{\circ}$ and $180^{\circ} \mathrm{C}$; beyond that, xonotlite is stable. For purposes of this study, tobermorite is allowed to experience slow, linear dehydration between $100^{\circ}$ and $180^{\circ} \mathrm{C}$. It is noted that tobermorite will lose water reversibly to a certain extent with increasing temperature and decreasing $R H$. Based on this information, five cumulative water-loss scenarios (Figure 7-1) as a function of temperature have been constructed for concretes with the idealized formulations calculated previously. For the purpose of elucidating the potential differences among these cases, several simplifying assumptions have been made that will require verification through experiment before they are used in any predictive sense. In these scenarios, it is assumed that liquid water is lost between $25^{\circ}$ and $100^{\circ} \mathrm{C}$. The thermally treated concrete, it is assumed, has already lost the liquid water that it may once have contained. It is important to note that rehydration scenarios would not simply reverse these plots in most cases. C-S-H loses water linearly between $30^{\circ}$ and $100^{\circ} \mathrm{C}$. The carboaluminates are dehydrated linearly between $100^{\circ}$ and $150^{\circ} \mathrm{C}$, as is gehlenite hydrate. The hydroxides, it is assumed, do not dehydrate.

\section{Figure 7-1 Idealized, cumulative water loss based on simplified mineral assemblages} calculated in Table 7-2, Table 7-3, Table 7-4, and Table 7-5

\section{Organic Materials}

Organic compounds take many forms, but the one class of compounds that is pervasive and deserves the most detailed scrutiny is polymers. Polymers are pervasive in the construction industry. For many polymers, temperatures within the range of potential repository conditions produce significant degradation, sometimes within hours rather than thousands of years. Because of potential thermal degradation effects, epoxy resins should not be included in the permanent structure without long-term tests. Caution will be needed because long-term effects are beyond the capabilities of testing that can be performed in a practical manner. Simulation may be useful for assessing the effects of polymer degradation for some modeling scenarios. For polymers that degrade over short periods of time, NFE chemical modeling may include products of the polymer degradation as reactants. The final products of most polymers are known and typically consist of monomer, hydrocarbons, and some volatile products such as toluene, xylene, and cresol.

Miscellaneous Organics - Observation of construction in the ESF makes it clear that certain needs may be met by the use of cellulose materials. These materials include excelsior (wood shavings) and rags. 
Organic Content of Concrete-Formulations for concrete and grout sometimes contain additives that improve the workability, curing time, or other characteristics. Some of these additives (e.g., superplasticizers) are organic.

Hydrous Pyrolysis of Diesel Fuel - The potential thermal degradation of diesel fuel at elevated temperatures was studied (Meike, 1996). These experiments were carried out at $200^{\circ}$ and $315^{\circ} \mathrm{C}$ to directly determine the rate and products of diesel-fuel degradation within a 2- to 3-month laboratory experiment. They thus provided an upper limit for the rate of abiotic degradation within the chemical system studied, which was largely depleted of $\mathrm{O}_{2}$ and other oxidizing agents. Recent work (Knauss et al., 1998) has distinguished two reaction-rate regimes for the hydrous pyrolysis of organic and halogenated organic materials in aqueous solutions: (1) a fast pyrolysis / oxidation regime where molecular (but dissolved) $\mathrm{O}_{2}$ is present, and the reaction rate is not dependent on $\mathrm{O}_{2}$ concentration, and (2) the reaction rate drops by several orders of magnitude once the $\mathrm{O}_{2}$ is depleted. If the initial $\mathrm{O}_{2}$-to-carbon ratio is low, analytical uncertainties might obscure the fast part of the reaction. However, it was found that thermal degradation of diesel fuel does not appear to be enhanced in the presence of cementitious material or at alkaline $\mathrm{pH}$. Similarly, diesel-fuel hydrous pyrolysis is not catalyzed by Fibercrete $^{\mathrm{TM}}$ or by Topopah Spring tuff and does not accelerate dissolution of silicate minerals found in these solids at $200^{\circ} \mathrm{C}$. These results agree with previous studies (see discussion in Meike, 1996). Secondary results support the understanding that the presence of cementitious materials will react and affect the chemistry of the water with which it is in contact, the repository rock, and its own porosity as well as its own permeability and mineral assemblage. These results provide a comparison to hydrothermal experiments (Meike, 1996).

\section{Microbial Processes}

Microbes vary widely in their types of metabolic activities and the consequent alterations to the surrounding environment that they can facilitate (Horn and Meike, 1996). Historically, bacteria (and the closely related Archea) have been classified according to their individual metabolic types. The potential reactions actually performed by bacteria are dictated by innate metabolic ability and by the conditions to which they are exposed. The types of metabolic activities that are possible are governed by the availability of substrates. The demands of describing and predicting microbial activity in the repository extend beyond the information that is presently available in the literature.

To model the impact of microbes, two types of parameters need to be established. First, the boundary conditions within which microbes operate (for example, water availability and temperature) must be established. These conditions are considered to be the "on and off switches" for microbial processes in the model. The second type of parameter relates to processes impacted by microbial activity. The purpose of such studies is to determine whether these metabolic activities are ongoing in both perturbed and unperturbed microbial communities, to assess the magnitude and rates of those reactions, and to establish boundary conditions for microbial survival and activity. Traditional biochemical assay techniques have been conducted and can be supplemented with more advanced molecular techniques to better predict the impact of microbial affects on geochemistry and repository components.

Organisms collected from the ESF have been cultured and assembled into a Yucca Mountain Site Characterization Project (YMSCP) library. These microbes were grown in lownutrient media with various amendments and are screened for various activities of significance to the long-term chemical and hydrologic properties of the NFE. Corrosionspecific bacteria, acid-producing bacteria, sulfide-producing bacteria, and slime-producing bacteria have all been isolated (Horn et al., 1996). 


\section{Biodegradation of Polymers}

The biodegradation of polymers is discussed in more detail by Meike (1996). The following summary is provided to demonstrate the complexity of developing chemical models to describe this area. Whereas natural polymers are readily broken down by microbes, synthetic polymers are more resistant. The enzymes required to break down these synthetic polymers are not found in nature. The biodegradation of polymers also depends on the intrinsic viscosity and the product structure. Many, but not all, degradation products of polymers are biodegradable (see discussion in Meike, 1996).

\section{Limiting Factors: Water, Nutrients}

A review of the microbiological literature and the preceding discussion make it clear that the impact and the character of microbial activity depend on nutrient conditions (see e.g., Horn and Meike, 1995). Microbes are capable of employing a wide range of organic compounds to serve as sources of carbon. Autotrophic organisms are capable of carbondioxide fixation to satisfy carbon requirements for synthesis of cellular materials. Energy can be derived from reduced organic or from inorganic compounds. For example, hydrogen gas, nitrogen, ammonia, nitrite, ferrous iron, and reduced sulfur compounds can all be used as energy sources by various microbial groups. Similarly, oxygen or a wide array of inorganic compounds may be used as a terminal electron sink. Nutrient supply, rate of nutrient transport, and the composition of the repository community will govern the specific types of metabolic activities that occur. Because little literature is available on the variability of chemical impact as a function of microbial activity, experiments are being performed to provide the needed modeling information.

In these flow-through experiments, a basic growth medium is modified such that the macronutrient $(\mathrm{C}, \mathrm{N}, \mathrm{P}, \mathrm{S})$ concentrations are varied to represent all possible combinations of a maximum value, a mid-range value, and a minimum value of each of the macronutrients. These trials are conducted at room temperature and at $50^{\circ} \mathrm{C}$. The bioreactor is inoculated with Yucca Mountain tuff that contains native microbes as well as those introduced by construction activities. Samples of efflux solution are collected on a regular basis and analyzed chemically until it can be demonstrated that a steady state has been achieved. The results of these experiments are being used in modeling efforts described in Chapter 6.

\section{Gradients of Chemical Potential, Concentration, Temperature}

At the drift scale, a radioactive waste repository is extremely heterogeneous. This heterogeneity is the basis for the existence of gradients in chemical composition, temperature, $R H$, and porosity. These gradients provide the basis of thermodynamic driving forces. As such, they can drive reactions and cause substances to behave in a manner that may not be predicted based on average values for the driving potentials. The formation of colloidal particles can fall into this category of processes.

The heterogeneous nature of the system also implies that processes that may be predicted based on average properties and attributes of a system may not drive reactions locally. For example, whereas the atmosphere in general around the drift could be oxic, puddles, crusts, crevices, and biomass could create anoxic subenvironments.

\section{Other Coupled Effects}

The number, identity, and significance of potential coupled interactions among humanmade materials have not yet been bounded, and the appropriate overall model has not been identified. For example, sorptive properties of zeolites could be degraded if the degradation products of human-made materials destroy the zeolite structure or provide preferentially 
adsorbed ions. Another example is the coupled effects inherent in the oxidation of rebar, if it is used in the concrete liner. Oxidation causes the rebar to expand and mechanically cracks the concrete, and also increases surface area, thus increasing the overall degradation rate of the concrete. Examples of this effect can be seen in many bridges and thoroughfares, but knowledge of the process remains qualitative.

\subsection{Modeling Activities}

This section describes modeling-related activities that address several different topics within the introduced materials area, particularly the development of a database for modeling cement interaction with water in the NFE.

\subsubsection{Abiotic Chemical Models}

To predict chemical behavior of cement and other introduced materials over long time periods, process-oriented models that use realistic physical and chemical approximations are used to represent the evolution of aqueous chemistry. These models are well developed in environmental science, where long periods of time are frequently addressed.

The chemical modeling approaches described in Chapter 5 are suited, in principle, to modeling the behavior of introduced materials. The discussion that follows is limited to modeling, with the EQ3 / 6 code, of cement-rock-water interactions.

\subsubsection{EQ3/6}

The EQ3 / 6 modeling package (Wolery, 1992a, 1992b; Wolery and Daveler, 1992) uses a database of fundamental thermodynamic parameters and reaction-rate constants to simulate the evolution of a chemical system (see Section 5.2). EQ3/ 6 consists of several executable programs and databases that are flexible in their application. The output from a typical EQ3/ 6 simulation includes the solution-composition parameters and associated mineral assemblages and gas-phase compositions as a function of reaction progress. These simulations provide important information about the following:

- The extent to which parts of the chemical system exhibit transient or steady-state behavior that may be abstracted for use in higher-level models (e.g., in repository total system performance assessment [TSPA])

- The chemical-system response to reactant availability and to chemical boundary conditions (e.g., gas fugacities)

- The final condition of heterogeneous chemical equilibrium between the aqueous phase and mineral assemblages, which represents the reaction path "endpoint"

\subsubsection{Modeling the Emplacement-Drift Gas Environment}

Physically, the exposure of the WPs to oxygen and other atmospheric gases is limited by two possibilities: (1) the drifts are ventilated by engineered measures or naturally through rock fractures or (2) the drifts are unventilated. This distinction is important because it can determine how gas fugacities remain evolve over time. These possibilities have been accommodated in EQ3/ 6 simulations by using two modeling options. The gas fugacity parameters are either "fixed," meaning the fugacity remains constant throughout the simulation (equivalent to a ventilated system), or "unfixed," which allows the fugacity to evolve (representing a closed system). Fugacity for the fixed case is set to atmospheric values. Simulations are conducted in "titration" mode, whereby aliquots of reactants are added to $1 \mathrm{~kg}$ of water and allowed to react, and the precipitated minerals remain in the system and are available as potential reactants (Wolery, 1992a; Wolery and Daveler, 1992). 
In this modeling strategy to predict chemical behavior of cement and other introduced materials over long time periods and to represent the likely evolution of aqueous chemistry, process models that use realistic physical and chemical approximations are needed. EQ3/6 simulations answer this need and can also be used to select the most important chemical components and mineral phases for reactive-transport modeling.

\subsubsection{Databases}

Five data files are available for use with EQ3/6. Three of these (COM, SUP, and NEA) may be used with either of two options for computing activity coefficients that are designed for dilute solutions (i.e., the Davies equation or the B-dot equation; see Section 5.2). In general, the use of these two options is restricted to geochemical solutions that have stoichiometric ionic strength less than that of sea water (i.e., $~ 0.7$ molal; Garrels and Christ, 1965). The two other data files (HMW, PIT) use Pitzer's equations (Pitzer, 1979) and are suitable for modeling higher-concentration solutions. These databases are outlined in the following list:

- SUP database-Based entirely on the SUPCRT92 program (Johnson et al., 1992), this database has a high level of internal consistency. The database covers a wide range of chemical species. However, it does not contain necessary data related to C-S-H species that might be formed in cements at temperatures between $60^{\circ}$ and $300^{\circ} \mathrm{C}$.

- NEA database-This database was produced by the Data Bank of the Nuclear Energy Agency of the European Community (Grenthe et al., 1992) and is specifically designed for uranium studies.

- HMW database-This database is based on the use of Pitzer's activity coefficient expressions that were used by Harvie, Moller, and Weare (Harvie et al., 1984) to model the solubility of geochemically significant compounds (Harvie and Weare, 1980). As currently constructed, it can be applied to dilute solutions and to concentrated brines at $25^{\circ} \mathrm{C}$. Where appropriate data exist, it can be extended to elevated temperature. It is consistent with solubility data and most mean molal activity coefficient measurements, but only treats the components present in the seasalt-water system. Important elements for the modeling of cement such as $\mathrm{Al}$ and $\mathrm{Si}$ are not included. This data set is not appropriate for extrapolation outside the compositional space defined by the regressions used to create it, nor can it be used in conjunction with other thermodynamic data.

- PIT database-This database is based primarily on data summarized by Pitzer (1979). This database is applicable to concentrated brines at $25^{\circ} \mathrm{C}$, and features of this database may be applicable at temperatures as high as $100^{\circ} \mathrm{C}$. It covers a larger set of species than does the HMW database, but it does not address the silica and inorganic carbon species that are necessary to model cement in a geologic repository. More details of the HMW and PIT databases are provided by Jackson (1988) and Jackson and Wolery (1985).

- COM database-This dataset represents a melange of data found in the SUP and NEA datasets. Other data in this database have been obtained by correlation or interpolation. This set therefore offers the least assurance of internal consistency. However, it is the only means available to model problems having a high degree of compositional complexity. 


\subsubsection{Data Availability and Modeling of Cementitious Materials}

Review of modeling capabilities shows that there is a gap in the capability to model aqueous systems that contain OPC (e.g., Bruton et al., 1994a; Meike et al., 1994). This gap has become more important as repository designers have begun to make extensive use of OPC materials in emplacement drifts.

Of the major reactant phases in cement powder-alite, belite, possible residual glass, gypsum, and ferrite $\left[\mathrm{C}_{2}(\mathrm{~A}, \mathrm{~F})\right]$ (see Taylor, 1990) for cement formula nomenclature)—only the thermochemical data for gypsum are complete. Knowledge of thermodynamic data for these phases, however, is only required to model the hydration and curing of concrete because the phases do not persist. The goal for NFE studies is to model interaction between water and well-cured concrete, which may have undergone extensive transformation in the solid state due to elevated temperatures. The following discussion reviews the phases of interest, which have been identified from experiments and natural and historical analogs.

Calorimetric data are lacking for most of the hydrous phases of cured cement and for the anhydrous and hydrous solid solutions. Of prime importance for modeling of cement at $25^{\circ} \mathrm{C}$ are calorimetric data for ettringite and tobermorite and for well-characterized solid solutions of these phases. Prediction of chemical reactions involving cement at greater temperatures requires more data than are presently available. The kinetics of transformation of C-S-H into other phases at elevated temperature are also significant to predictions of cement behavior in the repository.

Even in the absence of a complete set of thermodynamic or kinetic data for the solid phases in concrete, geochemical modeling codes may be used to conduct sensitivity analysis to explore the possible significance of solid solutions or end-member phases that are measured experimentally. The enthalpies of formation $\left(20^{\circ} \mathrm{C}\right)$ for $\mathrm{CaO}$ and silica gel and thermodynamic data for all $\mathrm{Ca}_{2} \mathrm{SiO}_{4}$ phases were evaluated by Haas et al. (1981). Qualitative rate information has been obtained for ettringite components (Majling et al., 1985). Other than an enthalpy of dehydration (Maycock et al., 1974) and $C_{\mathrm{p}}$ data (Ederova and Satava, 1979) obtained over the range of 273 to $333^{\circ} \mathrm{K}$, thermochemical data for ettringite for ettringite are calculated (Babushkin et al., 1985; Sarker et al., 1982). The only experimental data for "monosulfate" $\left(\mathrm{C}_{4} \mathrm{ASH}_{12}\right)$ presently known to the authors are $C_{\mathrm{p}}$ measurements from 273 to 353K (Ederova and Satava, 1979). As noted previously, the kinetics of the relevant reactions are even less well understood.

Comparisons of cement leachates with calculations performed using available data and standard thermochemical tables (Barnes and Roy, 1983) suggest the best agreement with the solutions buffered by tobermorite and possibly gyrolite. Calculated activity products were compared with 1) experimentally obtained solution compositions $\left(\mathrm{Ca}^{+2}, \mathrm{Na}^{+}, \mathrm{K}^{+}, \mathrm{pH}\right.$, and $\mathrm{SO}_{4}^{-2}$, but not $\mathrm{Al}$ or $\mathrm{CO}_{3}^{-2}$ ) from cement hydrated for as long as $3 \mathrm{hr}$ (Gartner et al., 1985) and 2) pure $\mathrm{C}_{3} \mathrm{~S}$. The comparisons suggest that, although no difference in supersaturation was observed with respect to portlandite, gypsum, and syngenite $\left[\mathrm{CaK}_{2}\left(\mathrm{SO}_{4}\right)_{2} \cdot \mathrm{H}_{2} \mathrm{O}\right]$, thermodynamic equilibrium is not achieved during early hydration (Moragues et al., 1987, 1988), and high ionic concentrations in the solutions result in activity-composition relations that deviate significantly from Debye-Huckel behavior. The most successful chemical models to date have been achieved by working with a limited number of equations that include C-S-H solid solutions, monosulfate solid solution, and ferrite solid solution. Glasser et al. (1985) analyzed a simplified cement system as the ternary $\mathrm{CaO}-\mathrm{SiO}_{2}-\mathrm{H}_{2} \mathrm{O}$. Barret and Bertrandie (1986) made a similar analysis of the system $\mathrm{CaO}-\mathrm{Al}_{2} \mathrm{O}_{3}-\mathrm{CO}_{2}-\mathrm{H}_{2} \mathrm{O}$. Incorporation of aggregate into repository concrete will increase the complexity of geochemical modeling. Calculations that include Portland cement, special cements, and concretes that incorporate 
bfs, fly ash, and silica fume (Berner, 1987) have achieved some success for equilibrium-solidphase and pore-solution-composition data obtained from experiment, but they do not readily take reaction progress into account.

The use of numerical simulations complements experimental and historical investigations. Coupled chemical effects that are difficult or inaccessible through experiment can be examined. Although the presently available chemical database for cement minerals is limited (Babushkin et al., 1985; Sarker et al., 1982), it can be manipulated to gain insight into some effects that may be expected because of human-made materials. The database can be enhanced in the future by incorporation of new thermodynamic data from experimental investigations.

\section{Development of a Database for Modeling Concrete Dissolution at $25^{\circ} \mathrm{C}$}

The qualified thermodynamic database normally used with EQ3/ 6 has been developed to study natural water-rock interaction and does not contain some of the thermodynamic data necessary for modeling cementitious systems (e.g., Bruton et al., 1994a; Meike et al., 1994). The goal is to develop this capability, which will involve extending existing thermodynamic data in addition to acquiring new data.

The objective for supporting total system performance assessment-viability assessment (TSPA-VA) is to develop two cement databases that reflect degrees of understanding for model systems that contain OPC and that produce results for other problems that are comparable to published results:

- Database 1-Three datasets for the Ca-Al-Si-S-Mg- $\mathrm{H}_{2} \mathrm{O}$ system were assembled by Atkins et al. (Atkins et al., 1992, 1994, 1991) for temperatures of $25^{\circ}, 50^{\circ}$, and $90^{\circ} \mathrm{C}$. They incorporated virtually all the available data for the minerals in a cement system at these temperatures. A database file compatible with EQ3/ 6 has been developed from these data to verify the reproducibility of the simulations reported by Atkins et al. (Atkins et al., 1992, 1994, 1991).

- Database 2-This database has been developed from a geochemical database (COM). It was first purged of all the chemical components outside the system $\mathrm{CaO}-\mathrm{SiO}_{2}-\mathrm{Al}_{2} \mathrm{O}_{3}$ $\mathrm{MgO}-\mathrm{SO}_{3}-\mathrm{Na}_{2} \mathrm{O}-\mathrm{K}_{2} \mathrm{O}-\mathrm{H}_{2} \mathrm{O}$. New minerals relevant to hydrothermally altered concrete were added one at a time, and the results were compared against available data in the literature. The benefit here was the inclusion of relevant data from the geochemical database. The database is incomplete and will require additional experimental data to simulate cement interactions at elevated temperatures. It is referred to as the cem.R27 database, which is equivalent to the cemcomp.R28 database.

Plans for future development call for new experimental data, for minerals relevant to hydrothermally altered concrete, to be added individually and the results compared against available data in the literature. The needed experimental data include solubilities, heats of formation, and heat capacities, which must be examined carefully to determine potential sources of error.

\section{The Structure and Chemistry of $\mathrm{C}-\mathrm{S}-\mathrm{H}$}

$\mathrm{C}-\mathrm{S}-\mathrm{H}$ is the major constituent of a cement paste and is characteristically poorly crystalline or nearly amorphous in a Young Cement. C-S-H can also be synthesized by reaction between $\mathrm{Ca}(\mathrm{OH})_{2}$ and silicic acid or between solutions of sodium silicate and a soluble calcium salt. In this manner, quasi-crystalline varieties can be obtained; two of them, known as C-S-H(I) $[0.8<\mathrm{Ca} / \mathrm{Si}<1.3]$ and $\mathrm{C}-\mathrm{S}-\mathrm{H}(\mathrm{II})$ [Ca/ $\mathrm{Si}>1.5]$, can be compared structurally to tobermorite and jennite, respectively. The relation between this important conceptual step and its thermodynamic significance has been discussed by Jennings (1986) 
and Gartner and Jennings (1987). From numerous values collected in the literature (e.g., Table 7-6) and for calcium and silica concentrations in the aqueous phase in contact with the gel, the authors established that the compositions cluster around one of two curves that correspond, respectively, to two different C-S-H structures. The points that cluster about one curve correspond to C-S-H gel prepared from $\mathrm{Ca}(\mathrm{OH})_{2}$ and silicic acid, by mixing solutions of sodium silicate and a soluble calcium salt, or by complete hydration of $\mathrm{C}_{3} \mathrm{~S}$. The structure of these gels can be associated with the C-S-H(I), which is structurally related to tobermorite. The other curve represents analyses of samples obtained by incomplete hydration of $\mathrm{C}_{3} \mathrm{~S}$ or $\mathrm{C}_{2} \mathrm{~S}$. It has been proposed that these gels are a mixture of stacked sheets of tobermorite-like and jennite-like units of C-S-H (Taylor, 1950). Most of the thermodynamic and compositional models found in the literature are developed assuming the existence of solid solutions. Jennings also developed a model to calculate the $\mathrm{Ca} / \mathrm{Si}$ ratio of this gel as a function of the thermodynamic properties of the aqueous phase, for which there is a state of metastable equilibrium of the system.

Table 7-6 Experimental compositions investigated by selected authors

\begin{tabular}{|c|c|c|}
\hline $\mathrm{Ca} / \mathrm{Si}$ & $0 \leq \mathrm{Ca} / \mathrm{Si} \leq \mathrm{x} 1^{*}$ & $\mathrm{x} 1 \leq \mathrm{Ca} / \mathrm{Si} \leq \mathbf{2 . 0}$ \\
\hline Atkinson et al., 1989 & $\begin{array}{l}\mathrm{C}-\mathrm{S}-\mathrm{H} \text { with } \mathrm{Ca} / \mathrm{Si}=0.8+\text { amorphous } \\
\mathrm{SiO}_{2}\end{array}$ & C-S-H with $\mathrm{Ca} / \mathrm{Si}=0.8$ \\
\hline $\begin{array}{l}\text { Berner, 1988; Berner, } \\
1990\end{array}$ & $\begin{array}{l}\text { C-S-H with } \mathrm{Ca} / \mathrm{Si}=1.0+\text { amorphous } \\
\mathrm{SiO}_{2}\end{array}$ & $\mathrm{C}-\mathrm{S}-\mathrm{H}$ with $\mathrm{Ca} / \mathrm{Si}=1.0+\mathrm{Ca}(\mathrm{OH})_{2}$ \\
\hline Fujii and Kondo, 1983 & & $\begin{array}{l}\text { Tobermorite } 14 \AA(\mathrm{Ca} / \mathrm{Si}=0.833)+ \\
\mathrm{Ca}(\mathrm{OH})_{2}\end{array}$ \\
\hline $\begin{array}{l}\text { Atkins et al., 1991; } \\
\text { Glasser et al., 1987b }\end{array}$ & $\mathrm{CaxH}_{6}-2 \times \mathrm{Si}_{2} \mathrm{O} 7 . \mathrm{nH}_{2} \mathrm{O}$ & $\mathrm{CaxH}_{6}-2 \times \mathrm{Si}_{2} \mathrm{O} 7 \cdot \mathrm{nH}_{2} \mathrm{O}+\mathrm{m} \mathrm{Ca}(\mathrm{OH})_{2}$ \\
\hline
\end{tabular}

* Where $\mathrm{x} 1=0.8$ for Atkinson et al., 1.0 for Berner, 0.833 for Fujii and Kondo

Alkalis are retained in the solid hydration products, notably C-S-H gels, to a lesser extent. However, even small concentrations can modify their solubility products significantly. The impact of alkali content on the mineralogy and morphology of cured cement is discussed in Suzuki et al. (1985). Solubility, chemistry, and aging of C-S-Hs in alkaline solutions is also discussed by MacPhee et al. (989), Glasser et al. (1985), and Damidot et al. (1994). The Bernertype model (see discussion in following text) has been modified empirically to take into account this influence of alkali on the C-S-H thermodynamic behavior (Atkins et al., 1992).

\section{Building a Cement Database (cem.R27) Compatible with EQ3/6}

Clodic and Meike (1997) describe the construction of Database 2 (cem.R27) by incrementally adding components and mineral phases to a chemically simple, threecomponent system. Each increment was tested for internal consistency and for its ability to simulate relevant experimental data from the literature.

The literature sources for two well-recognized models of cement-water interaction are compared in Table 7-7 and Table 7-8 and discussed further in Clodic and Meike (1997). The objective in building a new database is to combine features of these databases with other reported data and experimental observations of altered concrete composition, along with existing data describing other phases such as those present in tuff. The desired result is a database that can be used to model cement-water-tuff interactions with EQ3/ 6 and that can 
be used to assess the additional information needed for confidence in the predicted chemical evolution. The cem.R27 database is included among EQ3/ 6 files submitted in electronic form to the YMSCP record system (cemcomp.R28 file; see Appendix B).

Table 7-7 Sources of the Berner and Glasser et al. models

\begin{tabular}{|l|l|l|}
\hline & \multicolumn{1}{|c|}{ Berner (1987) } & \multicolumn{1}{c|}{ Glasser et al. (1987a) } \\
\hline \hline $\begin{array}{l}\text { Experimental data used } \\
\text { to build and fit the model }\end{array}$ & $\begin{array}{l}\text { Flint and Wells, 1934 } \\
\text { Fuji and Kondo, 1983 } \\
\text { Greenberg and Chang, 1965 } \\
\text { Kalousek, 1952 } \\
\text { Roller and Ervin, 1940 } \\
\text { Taylor, 1950 }\end{array}$ & $\begin{array}{l}\text { Fujii and Kondo, 1983 } \\
\text { Greenberg and Chang, 1965 } \\
\text { Roller and Ervin, 1940 } \\
\text { Suzuki et al., 1985 }\end{array}$ \\
\hline $\begin{array}{l}\text { Code used for the } \\
\text { modeling }\end{array}$ & MINEQL & Not identified in references cited \\
\hline $\begin{array}{l}\text { Database used for } \\
\text { aqueous species }\end{array}$ & MINEQL and PHREEQE & $\begin{array}{l}\text { Sillen and Martell, 1964 } \\
\text { Babushkin et al., 1985 }\end{array}$ \\
\hline
\end{tabular}

Table 7-8 Selected aspects of the Berner and Atkins et al.-Bennett et al. models

\begin{tabular}{|c|c|c|}
\hline & Berner $(1988,1990)$ & Atkins et al. (1992)-Bennett et al. (1992) \\
\hline System & $\mathrm{CaO}-\mathrm{Al}_{2} \mathrm{O}_{3}-\mathrm{SiO}_{2}-\mathrm{MgO}-\mathrm{SO}_{3}-\mathrm{H}_{2} \mathrm{O}$ & $\mathrm{CaO}-\mathrm{Al}_{2} \mathrm{O}_{3}-\mathrm{SiO}_{2}-\mathrm{MgO}-\mathrm{SO}_{3}-\mathrm{H}_{2} \mathrm{O}$ \\
\hline Solid phases & $\begin{array}{l}\text { portlandite } \\
\text { brucite } \\
\mathrm{C}_{3} \mathrm{AH}_{6}-\mathrm{C}_{3} \mathrm{FH}_{6} \\
\mathrm{C}_{\mathrm{x}} \mathrm{SH}_{\mathrm{x}}, \mathrm{x}<1.8 \\
\text { hydrotalcite } \\
\text { ettringite } \\
\text { AFm phase } \\
\text { calcite } \\
\mathrm{NaOH}, \mathrm{KOH}\end{array}$ & $\begin{array}{l}\text { portlandite } \\
\text { gypsum } \\
\text { silica gel } \\
\text { gibbsite or bayerite } \\
\text { brucite } \\
\text { hydrogarnet } \\
\mathrm{C}_{4} \mathrm{AH}_{13} \\
\mathrm{C}-\mathrm{S}-\mathrm{H} \text { gel } \\
\text { tobermorite } \\
\text { hydrotalcite } \\
\text { siliceous hydrogarnet } \\
\text { ettringite } \\
\text { monosulphate AFm } \\
\text { gehlenite hydrate }\end{array}$ \\
\hline Code & & PHREEQE \\
\hline Database & $\begin{array}{l}\text { MINEQL } \\
\text { PHREEQE (Parkhurst et al., 1980) }\end{array}$ & $\begin{array}{l}\text { Subset of CHEMVAL } \\
\text { (Read, 1991) }\end{array}$ \\
\hline $\begin{array}{l}\text { C-S-H thermodynamic } \\
\text { treatment }\end{array}$ & Berner's model & Adaptation of Berner's model \\
\hline Validation of the model & $\begin{array}{l}\text { Degradation of cement in natural marl } \\
\text { groundwater }\end{array}$ & $\begin{array}{l}\text { A series of pore-fluid analysis extracted } \\
\text { from } 5 \text {-yr-old pastes }\end{array}$ \\
\hline
\end{tabular}


Several software tools are available to build a database that can interact with EQ3/6 and are included in a database known as GEMBOCHS. The approach used was not to modify the actual GEMBOCHS database, but to use the same tools to create a new database for analysis of cement-water interaction.

Uncertainty in thermodynamic constants originates from several sources. First, uncertainty arises because the crystallized C-S-Hs are found to have various chemical composition depending on the source of the data. Second, and more fundamental, is the difference in the Ca:Si ratio of the formula unit. These differences reflect the limited state of knowledge of some of these phases at the structural level. Another uncertainty in thermodynamic values arises in that substitution of elements can occur, especially in natural samples. Thus, Al can substitute for some Si sites in a crystalline structure, with consequent changes to the thermodynamic values measured. This variability contributes significantly to uncertainty when the samples are not characterized with respect to these factors.

\section{Creation of the cem.R27 Database}

The initial goal for developing a cement database was to simulate OPC degradation in systems that contain only cement and water and in those larger systems that include OPC cement or concrete at $25^{\circ} \mathrm{C}$ and at elevated temperatures. The obvious choice as the starting point was the SUP database. However, because of the manner in which that database is constructed, this was not possible. A second option was to remove from the COM database chemical components and related species that were not present in the SUP data file.

The solution was to manually remove the species, except for certain aqueous species required to describe the solution composition, from the COM database. Next, all the minerals not present in the SUP database, with the exception of the cement-related minerals, were removed. These core data represent the heart of the new cem.R27 database. The new database was first checked by running a comparison simulation of the same reactant file against each of the two databases (SUP and COM) and comparing the results. As a consequence, some new aqueous species were added. Subsequently, data corresponding to new minerals were added one at a time. The results were tested incrementally by calculating equilibrium conditions for the appropriate chemical system. The following discussion explains these steps in more detail.

\section{Selection of Aqueous Species for cem.R27}

The first database to be constructed describes the Al-Ca-Cl-Fe-C-K-Mg-Na-S-Si chemical system (Clodic and Meike, 1997). One important distinction between the Atkins et al. (1992) database and the new cem.R27 database is that the latter includes inorganic carbon species. Carbon dioxide and the aqueous carbonate species are of major importance in the cement systems for a number of reasons. First, by lowering the $\mathrm{pH}$, aqueous carbonate species can lead to the dissolution of major phases such as the portlandite, can impact the solid-solution composition of phases such as the monosulphoaluminate and the calcium-aluminatehydrates, and also can impact the Ca/Si ratio of amorphous phase C-S-Hs.

To measure the achievements of this study, a few preliminary calculations were performed using a typical OPC cement-phase reactant file (Clodic and Meike, 1997) to compare the SUP and COM databases and with the new database. Comparison of the typical composition of a pore solution in a mature cement paste (data from Glasser and Marr, 1984), their Table 5) indicated that a good first-order approximation of the concentrations of elements in solution could be achieved using either database. The $\mathrm{pH}$, ionic strength, and electrical imbalance of these initial simulations, although not listed here, reflected the uncertainty in the carbonate speciation and concentration in the experimental example. More 
important at this point, the simulations using the two databases disagree on the aqueous species distribution. Accordingly, the relevant aqueous species from the COM database were returned to the new database to provide a more complete and accurate description of speciation in the aqueous phase (Table 7-9).

Table 7-9 List of the cement-related minerals present in the EQ3/6 COM data file (included in cem.R27): extrapolation algorithm, specific heat ( $C_{p}$ coefficients; (Johnson and Lundeen, 1994), and corresponding bibliographic references

\begin{tabular}{|c|c|c|c|c|c|c|}
\hline Minerals & $\begin{array}{c}\text { Extrapolation } \\
\text { Algorithm }\end{array}$ & $\begin{array}{l}\mathrm{T}^{\star \star 0} \\
\text { cal }\end{array}$ & $\begin{array}{l}\mathrm{T}^{\star \star 1} \\
\text { cal }\end{array}$ & $\begin{array}{c}\text { Coefficients } \\
\qquad T^{\star \star-2} \\
\text { cal }\end{array}$ & $\underset{{ }^{\circ} \mathbf{C}}{T_{\text {limit }}}$ & Source \\
\hline Afwillite & $\mathrm{C}_{\mathrm{p}}$ integration & $0.8154 \mathrm{E}+02$ & $0.4510 \mathrm{E}-01$ & $-0.1467 \mathrm{E}+07$ & 626.85 & $\begin{array}{l}\text { Barin and } \\
\text { Knacke, } 1973\end{array}$ \\
\hline \multicolumn{7}{|l|}{$\mathrm{C}_{4} \mathrm{AH}_{13}$} \\
\hline \multicolumn{7}{|l|}{$\mathrm{C}_{4} \mathrm{AH}_{19}$} \\
\hline \multicolumn{7}{|l|}{$\mathrm{CAH}_{10}$} \\
\hline \multicolumn{7}{|l|}{$\mathrm{C}_{2} \mathrm{AH}_{8}$} \\
\hline Ettringite & $\begin{array}{l}\text { Constant enthalpy } \\
\text { approximation }\end{array}$ & & & & & \\
\hline Gyrolite & $C_{p}$ integration & $0.7947 \mathrm{E}+02$ & $0.363 E-01$ & $-0.1755 \mathrm{E}+07$ & 726.85 & $\begin{array}{l}\text { Barin and } \\
\text { Knacke, } 1973\end{array}$ \\
\hline Foshagite & $C_{p}$ integration & $0.8795 \mathrm{E}+02$ & $0.395 \mathrm{E}-01$ & $-0.1348 \mathrm{E}+07$ & 726.85 & $\begin{array}{l}\text { Barin and } \\
\text { Knacke, } 1973\end{array}$ \\
\hline Gypsum & $\mathrm{C}_{\mathrm{p}}$ integration & $0.2184 \mathrm{E}+02$ & $0.76 \mathrm{E}-01$ & & 526.85 & $\begin{array}{l}\text { Barin and } \\
\text { Knacke, } 1973\end{array}$ \\
\hline Hillebrandite & $\mathrm{C}_{\mathrm{p}}$ integration & $0.4104 \mathrm{E}+02$ & $0.224 \mathrm{E}-01$ & $-0.7400 \mathrm{E}+06$ & 626.85 & $\begin{array}{l}\text { Barin and } \\
\text { Knacke, } 1973\end{array}$ \\
\hline Monohydrotalcite & $\begin{array}{l}\text { Constant enthalpy } \\
\text { approximation }\end{array}$ & & & & & \\
\hline Okenite & $\begin{array}{l}\text { Constant enthalpy } \\
\text { approximation }\end{array}$ & & & & & \\
\hline Portlandite & $\mathrm{C}_{\mathrm{p}}$ integration & 44.615 & $-5.23685 \mathrm{E}-03$ & -382.36 & 426.85 & $\begin{array}{l}\text { Robie et al., } \\
1978\end{array}$ \\
\hline Toberm.-9Å & $C_{p}$ integration & $0.14355 E+03$ & $0.747 \mathrm{E}-01$ & $-0.2082 \mathrm{E}+07$ & 726.85 & $\begin{array}{l}\text { Barin and } \\
\text { Knacke, } 1973\end{array}$ \\
\hline Toberm.-11Å & $C_{p}$ integration & $0.1106 \mathrm{E}+03$ & $0.1891 \mathrm{E}+00$ & & 726.85 & $\begin{array}{l}\text { Barin and } \\
\text { Knacke, } 1973\end{array}$ \\
\hline Toberm.-14£ & $\mathrm{C}_{\mathrm{p}}$ integration & $0.1322 \mathrm{E}+03$ & $0.17 \mathrm{E}+00$ & & 726.85 & $\begin{array}{l}\text { Barin and } \\
\text { Knacke, } 1973\end{array}$ \\
\hline Xonotlite & $\begin{array}{l}\text { Constant enthalpy } \\
\text { approximation }\end{array}$ & & & & & \\
\hline Wollastonite & SUPCRT92 & $0.2664 \mathrm{E}+02$ & $0.36 \mathrm{E}-02$ & $-0.652 \mathrm{E}+06$ & 1126.85 & $\begin{array}{l}\text { Johnson et al., } \\
1992\end{array}$ \\
\hline
\end{tabular}




\section{Addition of Data for Mineral Phases}

The next step in the development of the cem.R27 database was to assess the thermodynamic data already available in the GEMBOCHS database for the phases that might be formed in room-temperature and thermally treated cement. These phases in the chemical systems can form over long time periods at temperatures between $25^{\circ}$ and $250^{\circ} \mathrm{C}$. Some have been observed in experimental studies (see, e.g., Meike, 1996). The phases were analyzed component by component, proceeding toward increasing complexity. Five chemical systems were examined: $\mathrm{CaO}-\mathrm{SiO}_{2}-\mathrm{H}_{2} \mathrm{O}, \mathrm{CaO}-\mathrm{Al}_{2} \mathrm{O}_{3}-\mathrm{H}_{2} \mathrm{O}, \mathrm{CaO}-\mathrm{Al}_{2} \mathrm{O}_{3}-\mathrm{SO}_{3}-\mathrm{H}_{2} \mathrm{O}, \mathrm{CaO}-\mathrm{Al}_{2} \mathrm{O}_{3}-\mathrm{CO}_{2}-\mathrm{H}_{2} \mathrm{O}$, and $\mathrm{CaO}-\mathrm{Al}_{2} \mathrm{O}_{3}-\mathrm{SiO}_{2}-\mathrm{SO}_{3}-\mathrm{H}_{2} \mathrm{O}$. Future development will include calculations of solubility products for phases not already represented in the database.

\section{Summary}

Thermodynamic data from the extant literature on cement have been rendered into a form that can be used by the EQ3/ 6 modeling package. The new database (cem.R27 or file cemcomp.R28) will support advancement in modeling interaction between cement and water at $25^{\circ} \mathrm{C}$. However, some phases expected to participate in chemical reactions between cement and water at elevated temperature are not represented because thermodynamic data are not available.

\subsubsection{Calculation of Needed Thermodynamic Data}

As described by Meike et al. (1995), many of the experimental data needed to model cement interactions, with confidence similar to that associated with modeling of the natural geochemical systems, are unavailable. An approach has been developed to estimate the needed thermodynamic data.

To provide a means for verifying experimental data, and ultimately to estimate missing thermodynamic data, a program was initiated to conduct electronic structure calculations of C-S-Hs (Sterne and Meike, 1995). A principle reason for pursuing this approach was to better understand the relation between $\mathrm{RH}$ and the stability of $\mathrm{Ca}-\mathrm{Si}-\mathrm{H}_{2} \mathrm{O}$ phases. Given the difficulty inherent in direct measurement of thermodynamic parameters, a set of electronic structure calculations was undertaken.

Electronic structure calculations, and the Linear Muffin Tin Orbital theory in particular, are standard methods for approaching the physical properties of metals and metal alloys, semiconductors, and simple insulators (see, e.g., Anderson, 1975; Skriver, 1984). In the past, these methods have been confined to small systems having less than 20 atoms in a unit cell. Thus, calculations for wollastonite (which contains a 30-atom unit cell) and xonoitlite (which contains a 62-atom unit cell) were out of reach. However, recent developments in algorithms and computer power have brought larger systems within the range of these techniques.

The goal of this modeling effort is to determine the energetics of hydration for crystalline $\mathrm{C}-\mathrm{S}-\mathrm{H}$ phases. The work performed to date represents an initial step in this direction. The initial results represent the water-poor end members, wollastonite $\left(\mathrm{CaSiO}_{3}\right)$ and xonotlite $\left[\mathrm{Ca}_{6} \mathrm{Si}_{6} \mathrm{O}_{6} 17(\mathrm{OH})_{2}\right]$. The results, reported in Sterne and Meike (1995), are summarized in subsequent text.

The calculated ground-state properties of wollastonite and xonotlite are in very good agreement with experiments and provide equilibrium lattice parameters within $1 \%$ to $1.4 \%$ of the experimentally reported values. The roles of the different types of oxygen atoms, which are fundamental to understanding the energetics of crystalline C-S-Hs, examined in terms of their electronic-state densities, appear to be in good agreement with experiments for the lattice parameters and internally consistent when comparisons are drawn between the two structures. The exercise, completed with wollastonite and xonotlite, demonstrates the 
applicability of these electronic-structure methods in calculating the fundamental properties of these phases. The electronic-structure calculation methods are demonstrated to give reliable results, even for the relatively large wollastonite and xonotlite unit cells. Thus, the application of this new approach to the study of C-S-Hs appears to be fruitful in terms of the ability to calculate heats of formation and in the insight that it can provide into the nature of hydration and dehydration.

\subsubsection{Generation of Kinetic Data from Multicomponent Experiments}

The need for kinetic data is similar to that described for thermodynamic data, but experiments and modeling were also halted in November 1995. However, in the process of conducting other experiments described by Meike et al. (1995), some additional data were collected regarding the degradation of a cementitious material, specifically Fibercrete ${ }^{\mathrm{TM}}$. In all experiments containing Fibercrete), $11 \AA ̊$-tobermorite formed. Thus, $11 \AA$-tobermorite appears to be a stable, or at least metastable, phase at $200^{\circ} \mathrm{C}$. The precipitation of $11 \AA$-tobermorite, calcite, and cristobalite may control geochemistry and affect porosity and permeability for waters contacting both cements and Topopah Spring tuff. The importance of the dissolution and precipitation kinetics could be seen in the slow changes in solution $\mathrm{pH}$ and dissolved silica concentrations and the small fraction of the initial starting material dissolved to form secondary precipitates at the Fibercrete and Topopah Spring tuff surfaces. In the absence of Fibercrete, cristobalite appeared to be the dominant secondary mineral formed in Topopah Spring tuff experiments; this was in agreement with previous studies (Knauss et al., 1985a, 1987, 1985b; Knauss and Peifer, 1986).

Calculations using the current database (cem.R27) are not consistent with these observations. Aqueous chemical modeling results suggest that mesolite should precipitate from the solution. They predict that the chemical system is saturated with respect to quartz and calcite after 20 days of reaction and undersaturated with respect to $11 \AA$-tobermorite. Zeolites were not detected in any of the experiments, suggesting either that the thermodynamic data are not correct, that zeolite nucleation from solution has a large activation energy, or that zeolite precipitation rates are very slow even in supersaturated solutions. Given the lack of thermodynamic and kinetic data pertinent to aqueous degradation of $\mathrm{Ca}-\mathrm{Si}-\mathrm{H}_{2} \mathrm{O}$ phases, the first explanation is most likely (i.e., the thermodynamic data are incorrect). Thermodynamic data for cement minerals in the $\mathrm{Ca}-\mathrm{Si}-\mathrm{H}_{2} \mathrm{O}$ system are sparse and mutually inconsistent (Bruton et al., 1994b; Meike et al., 1994; Vieillard and Rassineux, 1992). Previous experiments that investigated the stability of these phases had typical durations of only a few days or weeks (Lea, 1971). For example, Atkins et al. (1991) determined the solubility of cement hydrate phases after four weeks of reaction at $25^{\circ} \mathrm{C}$. Clearly, longer reaction periods are required for the crystalline phases to reach equilibrium.

From the experiment reported by Meike et al. (1995), it is possible to calculate a solubility constant $(K)$ for $11 \AA$-tobermorite at $200^{\circ} \mathrm{C}$ to be $10^{39.7}$, using the experimental ion activity product (IAP) from the following solubility expression:

$$
\begin{aligned}
& \mathrm{Ca}_{5}\left(\mathrm{SiO}_{2}\right)_{6}(\mathrm{OH})_{10}-0.5 \mathrm{H}_{2} \mathrm{O}+10 \mathrm{H}^{+}=5 \mathrm{Ca}^{2+}+6 \mathrm{SiO}_{2}+10.5 \mathrm{H}_{2} \mathrm{O} \\
& \operatorname{IAP}\left(200^{\circ} \mathrm{C}\right)=\frac{\left[\mathrm{Ca}^{2+}\right]^{5}\left[\mathrm{SiO}_{2}\right]^{6}}{\left[\mathrm{H}^{+}\right]^{10}}
\end{aligned}
$$


assuming $a_{\mathrm{H}_{2} \mathrm{O}}=1$ and unit activity for the solid phase. This simplifying assumption is also taken that only crystalline tobermorite of pure composition is involved in the reaction. In the absence of other data, this IAP may prove to be a useful guide. However, the derivation does not allow for the interaction of complexes or other amorphous or crystalline Ca-Si- $\mathrm{H}_{2} \mathrm{O}$ phases that are known to precipitate under these conditions. This calculation thus requires verification using independent experimental checks for consistency.

\subsubsection{Biotic Models}

Although it is not possible to produce rigorous models of microbially mediated chemical systems at this time, much progress can be made toward understanding the relative significance of microbial effects using a simple box model. A box model treats microbial processes as a simplified chemical reactor. Microbial reactions can be viewed as enzymatically controlled chemical processes that are governed by the size, identity, and metabolic state of the microbial community and by the traditional abiotic chemical parameters such as $\mathrm{pH}$ and temperature. Each microbe must have an energy supply, a source of carbon, and a set of nutrients essential for life. The energy supply generally involves an electron acceptor-donor process; the carbon supply is usually some form of organic matter or carbon dioxide; and most microbes need sufficient amounts of nitrogen, phosphorous, and sulfur to live. Other elements are needed in trace amounts but are generally not limiting in terms of microbial growth, especially in a heterogeneous repository environment. Water is also essential, and temperature is an important limiting factor. For a review of these considerations with regard to a potential repository at Yucca Mountain, see Horn and Meike (1995). Although specific microbes exist over subsets of this range, it is possible to establish, for this first step, a range of temperatures in which microbes are active, as opposed to inactive (e.g., spore, dormant, or dead state).

The first step in using a box model to understand microbial effects in a repository is to establish limits to the total amount of microbial activity possible in the repository based on the microbial needs listed previously (e.g., energy, carbon, nutrients, water, appropriate temperature). This type of approach is described well by McKinley and Hagenlocher (1993) for the Swiss high-level waste and low/intermediate-level waste repositories. McKinley and Hagenlocher examined the energies available from all redox reactions possible for the materials present in the repository that can be utilized by microbes. This information is combined with an estimate of how much energy is needed to synthesize the compounds that make up biomass ( 0.1 mole of adenosine triphosphate [ATP] to produce one gram of dry cell mass, which is equivalent to $450 \mathrm{~kJ} / \mathrm{mol}$ if the efficiency of energy utilization is $10 \%$ ). With this approach, the total biomass that can be sustained can be related to the masses of redox species present. Finally, if one can assume an average biomass metabolic rate, the chemical effects of the biomass in terms of corrosion enhancement, ligand production, gas production, or any other rate of chemical change can be estimated and related to overall repository performance.

A similar simplified approach can be used to determine the maximum possible biomass based on the amounts of essential nutrients (phosphorous, nitrogen, and sulfur) present in the repository. An average biomass is defined in McKinley and Hagenlocher (1993) and in Grogan and McKinley (1990) to have the composition $\mathrm{C}_{160}\left(\mathrm{H}_{250} \mathrm{O}_{80}\right) \mathrm{N}_{30} \mathrm{P}{ }_{2} \mathrm{~S}$. The total amount of possible biomass is simply the total amount of accessible nutrient divided by its weight fraction in biomass. 
This determination is complicated somewhat by the fact that nutrient availability may be a function of corrosion rates of repository materials, whose corrosion rates themselves depend on active biomass. Some nutrients are available only if they are released during corrosion of repository materials. The overall process is therefore coupled and amenable to modeling provided that some quantitative information on the type of coupling is available.

Applying this approach to the Swiss high-level waste repository, McKinley and Hagenlocher (1993) and Grogan and McKinley (1990) found that, for a bounding calculation not limited by microbe mobility, the overall biomass was limited by energy availability and not by nutrient availability. In particular, the amounts of nitrogen, phosphorous, and sulfur available in the backfill are much higher than the total mass of electron donors needed to fuel microbial growth. The only exception is during the first $10 \mathrm{yr}$ of repository existence, in which the availability of oxygen is expected to be high; consequently, energy availability should also be high. The availability of $\mathrm{O}_{2}$ over time in a potential Yucca Mountain repository drift is still under discussion because the availability of air through the mountain's fracture system, the amount of time that the repository drifts will be ventilated artificially, and the amount of chemically conditioned backfill that may affect the $\mathrm{fO}_{2}$ have not yet been bounded. The proposed Yucca Mountain repository differs from the Swiss repository in many ways: for example, the former is an unsaturated environment. However, although the Swiss repository conditions are not necessarily analogous to those of the Yucca Mountain repository in many important aspects (including, for example, $\mathrm{fO}_{2}$ ), it is illustrative to follow through the calculation as an exercise.

The biomass production rates calculated for the Swiss high-level waste repository range decrease from an initial rate of approximately 300 grams dry biomass / year per WP to longterm rates of approximately $0.3 \mathrm{~g} / \mathrm{yr}$ per WP. These values can be used to constrain likely production rates of byproducts such as organic ligands and gas generation, which, in turn, affect radionuclide transport rates and repository performance. For this case, even at maximum biological activity, the total production of organic complexing agents is only approximately equal to the estimated release rates of radionuclides. The net effect of microbial activity in terms of solubilization of radionuclides is therefore small for this repository.

Because the conditions of the Swiss repository (e.g., granitic rock, repository design, saturated rock) are quite different from those of the Yucca Mountain proposed repository, the findings of McKinley and Hagenlocher (1993) and Grogan and McKinley (1990) cannot be directly applied to the Yucca Mountain site. Information gained from this simple box-model approach should be used to prioritize and guide more detailed work on microbial effects.

\section{Return to Table of Contents}

\subsection{References}

Anderson, O.K. (1975). "Linear methods in bond theory." Phys. Rev. B 12:3060.

Atkins, M., D. Bennett, A. Dawes, F. Glasser, A. Kindness, and D. Read (1992). “A Thermodynamic Model for Blended Cements." (DOE report DOE-HMIP-92005).

Atkins, M., F. Glasser, L.P. Moroni, and J.J. Jack (1994). “Thermodynamic Modeling of Blended Cements at Elevated Temperatures (50-90C)." (DOE-HMIP-94-001). Washington, DC: U.S. Department of Energy. 
Atkins, M., F.P. Glasser, A. Kindness, and D.E. MacPhee (1991). Solubility Data for Cement Hydrate Phases $\left(25^{\circ} \mathrm{C}\right)$. Washington, DC: U.S. Department of Energy. (DOE/HMIP/RR/91/032)

Atkinson, A., N.M. Everitt, and R.M. Guppy (1989). “Time dependence of $\mathrm{pH}$ in a cementitious repository." In proceedings from Scientific Basis for Waste Management. Mater. Res. Soc. 127:439-446. [226315]

Babushkin, V.I., G.M. Matveyev, and O.P. Mchedlov-Petrossyan (1985). Thermodynamics of Silicates: Springer-Verlag. (459 pp.) [NNA.19920401.0112]

Barin, I., and O. Knacke (1973). Thermochemical Properties of Inorganic Substances. New York, NY: Springer-Verlag.

Barnes, M.W., and D.M. Roy (1983). "The buffering mechanisms in leaching of composites of cement with radioactive water." Mater. Res. Soc. Symp. Proc. 15:159-166. [NNA.19920131.0250]

Barr-Kumarakulasinghe, S.A. (1994). "Modelling the thermal oxidative degradation kinetics of polyethylene film containing metal pro-oxidants." Polymer 35:995-1003.

Barret, P., and D. Bertrandie (1986). "Fundamental hydration kinetic features of the major cement constituents: $\mathrm{Ca}_{3} \mathrm{SiO}_{5}$ and b-Ca $\mathrm{SiO}_{4}$." J. Chem. Phys. 83:765-775.

Bennett, D.G., D. Read, M. Atkins, and F.P. Glasser (1992). “A thermodynamic model for blended cements II: cement hydrate phases; thermodynamic values and modelling studies." J. Nucl. Mater. 190:315-325.

Berner, U.R. (1987). "Modelling porewater chemistry in hydrated Portland cement." Mater. Res. Soc. Symp. Proc. 84:319-330.

Berner, U.R. (1988). "Modelling the incongruent dissolution of hydrated cement minerals." Radiochim. Acta 44/45:387-393.

Berner, U.R. (1990). "A Thermodynamic Description of the Evolution of Pore Water Chemistry and Uranium Speciation during Degradation of Cement." Villigen, Switzerland: Paul Scherrer Institut, PSI Bericht Nr. 62.

Bruton, C.J., W.E. Glassley, and W.L. Bourcier (1994a). "Field-Based Tests of Geochemical Modeling Codes Using New Zealand Hydrothermal Systems." (UCRL-ID118009). Livermore, CA: Lawrence Livermore National Laboratory. [MOL.19950314.0162]

Bruton, C.J., W.E. Glassley, and A. Meike (1995). “Geothermal Areas as Analogues to Chemical Processes in the Near-field Altered Zone of the Potential Yucca Mountain, Nevada Repository." October 1995. (UCRL-ID-119842). Livermore, CA: Lawrence Livermore National Laboratory. [MOL.19960408.0120]

Bruton, C.J., B.L. Phillips, A. Meike, S. Martin, and B.E. Viani (1994b). “Cement minerals at elevated temperature: Thermodynamics and structural characteristics." Mater. Res. Soc. Proc. 133:327-333. (UCRL-JC-115796). [NNA.19940318.0008]

Burnay, S.G. (1990). “A practical model for prediction of the lifetime of elastomeric seals in nuclear environments." In proceedings from ACS Symposium Series: Radiation Effects on Polymers. R.L. Clough and S.W. Shalaby (eds.). Washington, DC: American Chemical Society. 524-533.

Burnett, N.C., R.D. Hooton, R.B. Heimann, and M. Onofrei (1985). “The development of durable cementitious materials for use in a nuclear fuel waste disposal facility." Mater. Res. Soc. Symp. Proc. 50:461-468. 
Burton, B.L. (1993). “The thermooxidative stability of cured epoxy resins.” Int. J. of Appl. Poly. Sci. 47:1821-1837.

Clodic, L., and A. Meike (1997). “Thermodynamics of Calcium Silicate Hydrates Phase I: Development of a Database Dedicated to Modeling Concrete Dissolution at $25^{\circ} \mathrm{C}$." Milestone report for the CRWMS Management and Operating Contractor, U.S. Department of Energy. (SPLG1EM4). Livermore, CA: Lawrence Livermore National Laboratory. [MOL.19971217.0321]

Damidot, D., S. Stronach, A. Kindness, M. Atkins, and F.P. Glasser (1994). “Thermodynamic investigation of the $\mathrm{CaO}-\mathrm{Al}_{2} \mathrm{O}_{3}-\mathrm{CaCO}_{3}-\mathrm{H}_{2} \mathrm{O}$ closed system at $25^{\circ} \mathrm{C}$ and influence of $\mathrm{Na}_{2} \mathrm{O}$." Cem. Concr. Res. 24(3):563-572.

Ederova, J., and V. Satava (1979). "Heat capacities of $\mathrm{C}_{3} \mathrm{AH}_{6}, \mathrm{C}_{4} \mathrm{ASH}_{12}$ and $\mathrm{C}_{6} \mathrm{AS}_{3} \mathrm{H}_{32}$." Thermochim. Acta 31:126-128.

Flint, and Wells (1934). "Study of the system $\mathrm{CaO}-\mathrm{SiCO}_{2}-\mathrm{H}_{2} \mathrm{O}$ at $30^{\circ} \mathrm{C}$ and of the reaction of water on the anhydrous calcium silicates." J. Res. NBS 12:751.

Fujii, X., and W. Kondo (1983). "Estimation of thermochemical data for calcium silicate hydrate (C-S-H)." Am. Ceram. Soc. J. 66:C220-C221.

Garrels, R.M., and C.L. Christ (1965). Solutions, Minerals, and Equilibria. San Francisco, CA: W.H. Freeman \& Co. (450 pp.) [HQS.19880517.1981]

Garrett, R.W., D.J.T. Hill, T.T. Le, K.A. Milne, J.H. O'Donnell, S.M.C. Perera, and P.J. Pomery (1990). Temperature Dependence of the Radiation Chemistry of Polymers. Washington, DC: Am. Chem. Soc.

Gartner, E.M., and H.M. Jennings (1987). “Thermodynamics of calcium silicate hydrates and their solutions." Am. Ceram. Soc. J. 70:743-749. [NNA.19910128.0147]

Gartner, E.M., F.J. Tang, and S.J. Weiss (1985). “Saturation factors for calcium hydroxide and calcium sulfates in fresh Portland cement pastes." Am. Ceram. Soc. J. 68:667673.

Gillen, K.T., and R.L. Clough (1989). “Time-temperature-dose rate superposition: a methodology for extrapolating accelerated radiation aging data to low dose rate conditions." Poly. Degrad. Stab. 24:137-168.

Glasser, F.P., M.J. Angus, C.E. McCulloch, D. MacPhee, and A.A. Rahman (1985). "The chemical environment in cements." Mater. Res. Soc. Symp. Proc. 44:849-858. [NNA.910207.0039]

Glasser, F.P., S. Diamond, and D.M. Roy (1987a). "Hydration reactions in cement pastes incorporating fly ash and other pozzolanic materials." Mater. Res. Soc. Proc. 85:167-186. (This paper also appears in Mater. Res. Soc. Proc. 86).

Glasser, F.P., E.E. Lachowski, and D.E. MacPhee (1987b). “Compositional model for calcium silicate hydrate (C-S-H) gels, their solubilities, and free energies of formation." Am. Ceram. Soc. J. 70(7):481-485.

Glasser, F.P., and J. Marr (1984). “The alkali binding potential of OPC and blended cements.” Il Cemento 1985 82:85-94.

Grassie, N., and G. Scott (1985). Polymer Degradation and Stabilisation. Cambridge, UK: Cambridge University Press.

Greenberg, S.A., and T.N. Chang (1965). "Investigation of the hydrated calcium silicates II Solubility relationships in the calcium oxide-silica-water system at $25^{\circ} \mathrm{C}$." $\mathrm{J}$. Phys. Chem. 69:1151. 
Grenthe, I., J. Fuger, R.J.M. Konings, R.J. Lemire, A.B. Muller, C. Nguyen-Trung, and H. Wanner (1992). Chemical Thermodynamics, Vol. 1: Chemical Thermodynamics of Uranium. Amsterdam: North Holland. [NNA.19940415.0009]

Grogan, H.A., and I.G. McKinley (1990). An Approach to Microbiological Modeling: Application to the Near Field of a Swiss Low/Intermediate-Level Waste Repository. Baden, Switzerland: NAGRA, Baden. (NTB 89-06)

Haas, J.L.J., G.R.J. Robinson, and B.S. Hemingway (1981). “Thermodynamic tabulations for selected phases in the system $\mathrm{CaO}-\mathrm{Al}_{2} \mathrm{O}_{3}-\mathrm{H}_{2} \mathrm{O}$ at $101.32 \mathrm{kPa}(1 \mathrm{~atm})$ between 273.15 and 1800 K." J. Phys. Chem. Ref. Data 10:575-669.

Harvie, C.E., N. Moller, and J.H. Weare (1984). “The prediction of mineral solubilities in natural waters: the $\mathrm{Na}-\mathrm{K}-\mathrm{Mg}-\mathrm{Ca}-\mathrm{H}-\mathrm{Cl}-\mathrm{SO}_{4}-\mathrm{OH}-\mathrm{HCO}_{3}-\mathrm{CO}_{3}-\mathrm{CO}_{2}-\mathrm{H}_{2} \mathrm{O}$ system to high ionic strengths at $25^{\circ} \mathrm{C}$." Geochim. Cosmochim. Acta 48(4):723751. [NNA.19891006.0190]

Harvie, C.E., and J.H. Weare (1980). “The prediction of mineral solubilities in natural waters: The Na-K-Mg-Ca-Cl-SO $\mathrm{S}_{4}-\mathrm{H}_{2} \mathrm{O}$ system from zero to high concentration at 25ㄷ." Geochim. Cosmochim. Acta 44(7):981-997.

Heimann, R.B. (1988a). “Interaction of cement and radioactive waste forms in multicomponent systems tests at $200^{\circ} \mathrm{C}$. Part 1: Leaching and sorption of cesium, strontium, and actinides." Cem. Concr. Res. 18:389-400. [NNA.19920506.0048]

Heimann, R.B. (1988b). "Interaction of cement and radioactive waste forms in multicomponent systems tests at $200^{\circ} \mathrm{C}$. Part 2: Mineralogical changes of cement." Cem. Concr. Res. 18:554-560. [NNA.19920506.0049]

Heimann, R.B., and R.D. Hooton (1986). "Mineralogical changes of various cement formulations during reaction with groundwater in the presence of $\mathrm{CA}$ and Nabentonite at $150^{\circ} \mathrm{C} . "$ C. Can. Min. 24:289-302. (Also issued as AECL-8895.). [NNA.19920506.0050]

Hersman, L.E. (1996). "Summary and Synthesis of Biological Sorption and Transport." Milestone report for the CRWMS Management and Operating Contractor, U.S. Department of Energy. (3663). Las Vegas, NV: Los Alamos National Laboratory. [MOL.19970822.0242]

Horn, J.M., B. Economides, A. Meike, and R.D. McCright (1996). “Initial Studies to Assess Microbial Impacts on Nuclear Waste Disposal." (UCRL-JC-122587). Livermore, CA: Lawrence Livermore National Laboratory. [MOL.19960409.0170]

Horn, J.M., and A. Meike (1995). “Microbial Activity at Yucca Mountain, Part I: Microbial Metabolism, Adaptation and the Repository Environment." Position Paper resulting from the Workshop on Microbial Activity at Yucca Mountain, Lafayette, California, April 10-12, 1995. (UCRL-ID-122256). Livermore, CA: Lawrence Livermore National Laboratory. [222145]

Horn, J.M., and A. Meike (1996). "A program to assess microbial impacts on nuclear waste containment." In proceedings from 1996 High-Level Radioactive Waste Meeting. Las Vegas, NV. (Also UCRL-JC-122732, Lawrence Livermore National Laboratory, Livermore, CA) [MOL.19960409.0194]

Jackson, K.J. (1988). “Verification and Validation Studies of the Addition of Pitzer's Equations to the EQ3 / 6 Brine Model." (UCRL-53841). Livermore, CA: Lawrence Livermore National Laboratory. (65 pp.) [NNA.19910326.0060] 
Jackson, K.J., and T.J. Wolery (1985). “Extension of the EQ3/ 6 computer codes to geochemical modeling of brines." In proceedings from Scientific Basis for Waste Management VIII. Boston, MA: Mater. Res. Soc. 44:507-514. [NNA.19891006.0198]

Jennings, H. (1986). “Aqueous solubility relationships for two types of calcium silicate hydrates." Am. Ceram. Soc. J. 69:614-618.

Johnson, J., and S. Lundeen (1994). "GEMBOCHS Thermodynamic Datafiles For Use With the EQ3 / 6 Software Package." Yucca Mountain Site Characterization Project Milestone Report. (MOL72). Livermore, CA: Lawrence Livermore National Laboratory. [MOL.19950727.0017]

Johnson, J.W., E.H. Oelkers, and H.C. Helgeson (1992). “SUPCRT92: A software package for calculating the standard molal thermodynamic properties of minerals, gases, aqueous species, and reactions from 1 to 5000 bars and $0^{\circ} \mathrm{C}$ to $1000^{\circ} \mathrm{C}$." Comput. Geosci. 18:899-947. (UCRL-JC-107907). [234273]

Kalousek, G.L. (1952). "Application of differential thermal analysis in a study of the system lime-silica-water." In proceedings from Third International Symposium on Chemistry of Cements. London, UK: p. 296.

Kaplan, M.L. (1991). "Solvent Penetration in Cured Epoxy Networks." Poly. Eng. Sci. 31(10):689-698.

Kenny, J.M., L. Torre, and L. Nicolais (1993). "Short-term and long-term degradation of polymer-based composites." Thermochim. Acta 227:97-106.

Knauss, K.G., W.J. Beiriger, and D.W. Peifer (1985a). “Hydrothermal Interaction of Crushed Topopah Spring Tuff and J-13 Water at 90, 150, and $250^{\circ} \mathrm{C}$ Using DicksonType, Gold-Bag Rocking Autoclaves." (UCRL-53630). Livermore, CA: Lawrence Livermore National Laboratory. [NNA.19931005.0010]

Knauss, K.G., W.J. Beiriger, and D.W. Peifer (1987). “Hydrothermal Interaction of Solid Wafers of Topopah Spring Tuff with J-13 Water and Distilled Water at 90 and $150^{\circ} \mathrm{C}$ Using Dickson-Type, Gold-Bag Rocking Autoclaves: Long-Term Experiments." (UCRL-53722). Livermore, CA: Lawrence Livermore National Laboratory. [NNA.19870713.0081; 203013]

Knauss, K.G., W.J. Beiriger, D.W. Peifer, and A.J. Piwinskii (1985b). “Hydrothermal Interaction of Solid Wafers of Topopah Spring Tuff with J-13 Water and Distilled Water at 90,150 , and $250^{\circ} \mathrm{C}$, Using Dickson-Type, Gold-Bag Rocking Autoclaves." (UCRL-53645). Livermore, CA: Lawrence Livermore National Laboratory. [NNA.19900207.0282; HQS.19980517.2482]

Knauss, K.G., M.J. Dibley, R.N. Leif, D.A. Mew, and R.D. Aines (1998). “Aqueous oxidation of trichloroethene (TCE): A kinetic analysis." Geochim. Cosmochim. Acta(submitted). (Also UCRL-JC-129743, Lawrence Livermore National Laboratory, Livermore, CA).

Knauss, K.G., and D.W. Peifer (1986). “Reaction of Vitric Topopah Spring Tuff and J-13 Ground Water Under Hydrothermal Conditions, Using Dickson-Type, GoldBag Rocking Autoclaves." (UCRL-53795). Livermore, CA: Lawrence Livermore National Laboratory. [NAA.891102.0117]

Komarneni, S., and D.M. Roy (1983). "Hydrothermal interactions of cement or mortar with zeolites or montmorillonites." Mater. Res. Soc. Symp. Proc. 15:55-62.

Lea, F.M. (1971). The Chemistry of Cement and Concrete. New York, NY: Chemical Publishing Co. Inc. (727 pp.) [NNA.19890713.0195] 
Leedy, W.T., and R.J. Watters (1994). “Assessment of rock bolt systems for underground waste storage." In proceedings from High-Level Radioactive Waste Management Conference. Las Vegas, NV: Am. Nucl. Soc., Inc.

Livingston, R.A., P.E. Stutzman, R. Mark, and M. Eridik (1992). "Preliminary analysis of the masonry of the Hagia Sophia Basilica, Istanbul." In proceedings from Mater. Res. Soc. Symp. Mater. Res. Soc. 267:721-735.

MacPhee, D.E., K. Luke, F.P. Glasser, and E.E. Lachowski (1989). “Solubility and ageing of calcium silicate hydrates in alkaline solutions at $25^{\circ} \mathrm{C}$." Am. Ceram. Soc. J. 72:646-654.

Majling, J., V. Tomkova, and E. Istenikova (1985). "Calorimetric study of reactions in the system $\mathrm{C}_{4} \mathrm{~A}_{3} \mathrm{~S}-\mathrm{CH}-\mathrm{CS}-\mathrm{H} . "$ Thermochim. Acta 85:219-222.

Martin, S.I., B. Viani, and A. Meike (1996). "Water Adsorption by Okenite $\left(\mathrm{Ca}_{10} \mathrm{Si}_{18} \mathrm{O}_{46} \cdot 18 \mathrm{H}_{2} \mathrm{O}\right)$ at Ambient Temperature." Livermore, CA: Lawrence Livermore National Laboratory. [MOL.19960223.0336]

Maycock, J.N., J. Skalny, and R.S. Kalyoncu (1974). “Thermal decomposition of cementitious hydrates." R.S. Porter and J.F. Johnson (eds.). Analytical Calorimetry. New York, NY: Plenum Press. [NNA.19920506.0051]

McCright, R.D. (1996). “Engineered Materials Characterization Report.” (UCRL-ID-119564, Rev. 0). Livermore, CA: Lawrence Livermore National Laboratory. [MOL.19961022.0024]

McKinley, I.G., and I. Hagenlocher (1993). “Quantification of microbial activity in a nuclear waste repository." In proceedings from $I S S M^{\prime} 93$. Bath, United Kingdom: pp. $1-24$.

McNeil, M.B., D.W. Mohr, and B.J. Little (1990). “Correlation of laboratory results with observations on long-term corrosion of iron and copper alloys." In proceedings from Symposium on Materials Science in Archeology. San Francisco, CA: Mater. Res. Soc. (1990 Spring Meeting) [NNA.19920506.0038]

Meike, A. (1996). “Introduced Materials Synthesis Report 1993-1996." Yucca Mountain Project internal report for the CRWMS Management and Operating Contractor, U.S. Department of Energy. Livermore, CA: Lawrence Livermore National Laboratory. [MOL.19961108.0016]

Meike, A., W.L. Bourcier, M. Alai, D.L. Haldeman, P.S. Amy, and T. Lagadinos (1995). Potential Long-Term Chemical Effects of Diesel Fuel Emissions on a Mining Environment: A Preliminary Assessment Based on Data from a Deep, Subsurface Tunnel at Rainier Mesa, Nevada Test Site. Livermore, CA: Lawrence Livermore National Laboratory. (UCRL-ID-121046) [MOL19950406.0141]

Meike, A., K. Myers, M. Spragge, and A. Barr (1997a). “Calculation of Quantities, Masses, and Chemical Compositions of Steel Set and Concrete Liner Mechanical Support Components for Emplacement Drifts." Report for deliverable reports WP26809 and WP26815 for the CRWMS Management and Operating Contractor, U.S. Department of Energy. Livermore, CA: Lawrence Livermore National Laboratory. [MOL.19980109.0258]

Meike, A., M. Onofrei, C.J. Bruton, and B.E. Viani (1994). "Progress in understanding the structure and thermodynamics of calcium silicate hydrates." In proceedings from Fifth Annual International High-Level Radioactive Waste Management Conference. Las Vegas, NV: Am. Nucl. Soc., La Grange Park, IL. pp. 2590-2596. (Also UCRL-JC-116358) [NNA.19940517.0130] 
Meike, A., and M. Spragge (1995). "Progress Report \#1 on the Materials Identification, Characterization, and Evaluation Activity: Acquisition of Materials Data from the Exploratory Studies Facility." Milestone report for the CRWMS Management and Operating Contractor, U.S. Department of Energy. (MOL 132). Livermore, CA: Lawrence Livermore National Laboratory.

[MOL.19960415.0416]

Meike, A., M. Spragge, and C. Aracne-Ruddle (1997b). "Hydrothermal Alteration of Concrete: First Batch." Deliverable report for the CRWMS Management and Operating Contractor, U.S. Department of Energy. Livermore, CA: Lawrence Livermore National Laboratory. [MOL.19970507.01957]

Milestone, N.B., T. Sugama, L.E. Kukacka, and N. Carciello (1987). “Carbonation of geothermal grouts-Part 3: $\mathrm{CO}_{2}$ attack on grouts containing bentonite." Cem. Concr. Res. 17:295-306. [NNA.902506.0052]

Moragues, A., A. Macias, and C. Andrade (1987). "Equilibria of the chemical composition of the concrete pore solution. Part I: Comparative study of synthetic and extracted solutions." Cem. Concr. Res. 17:173-182. [NNA.19920506.0053]

Moragues, A., A. Macias, and J. Losada (1988). "Equilibria of the chemical composition of the pore concrete solution, part II: Calculation of the equilibria constants of the synthetic solutions." Cem. Concr. Res. 18:342-350. [NNA.19920506.0054]

Myers, K., and A. Meike (1997). “Hydrothermal Alteration of Concrete: Second and Third Batches." Milestone report for the CRWMS Management and Operating Contractor, U.S. Department of Energy. (SPLGDM4; SPLGEM4). Livermore, CA: Lawrence Livermore National Laboratory. [MOL.19980114.0113]

O’Donnell, J.H. (1990). “Chemistry of Radiation Degradation of Polymers.” In proceedings from Radiation Effects on Polymers. R.L. Clough and S.W. Shalaby (eds.). Washington, DC: Am. Chem. Soc.

Parkhurst, D.L., D.C. Thostenson, and L.N. Plummer (1980). “PHREEQE: A Computer Program for Geochemical Calculations." (Report 80-96). Reston, VA: Water Resources Investigation, U.S. Geological Survey. (210 pp.) [HWS.19880517.2817]

Pitzer, K.S. (1979). “Theory: Ion Interaction Approach.” R.M. Pytkowicz (ed.). Activity Coefficients in Electrolyte Solutions. Boca Raton, FL: CRC Press. [NNA.19891996.0194]

Pollard, A.M., R.G. Thomas, and P.A. Williams (1989). "Synthesis and stabilities of the basic copper (II) chlorides atacamite, paratacamite, and botallackite." Mineral. Mag. 53:57-563. [NNA.19920401.0111]

Ravanetti, G.P., and M. Zini (1992). "A study on the thermal-degradation kinetics of syndiotactic polystyrene by thermogravimetric analysis." Thermochim. Acta 207:53-64.

Read, D.e. (1991). “Chemval Project: Report on Stages 3 and 4. Testing of Coupled Chemical Transport Models." (DOE/HMIP/RR/91/003). London: UK DOE.

Rhoderick, J.E. (1981). “Examination of Samples of Grout After 63 Years Exposure Underground." (ONWI-248). Springfield, VA: U.S. Department of Commerce. (6 pp.) 
Robie, R.A., B.S. Hemingway, and J.F. Fisher (1978). “Thermodynamic Properties of Minerals and Related Substances at $298.15 \mathrm{~K}$ and 1 bar (19** Pa) Pressure and at Higher Temperatures." U.S.G.S. Bulletin 1452 (with corrections). U.S. Geological Survey. (1456 pp.) [NNA. 19900702.0002]

Roller, P.S., and G. Ervin (1940). "The system calcium oxid-silica water at $30^{\circ} \mathrm{C}$; The association of silicate ion in dilute alkaline solution." J. Am. Chem. Soc. 62:461471.

Roy, D.M., and C.A. Langton (1983). “Characterization of Cement Based Ancient Building Materials in Support Seal Materials Study." (MI/ONWI-523). Columbus, OH: Battelle Memorial Institute/Office of Nuclear Waste Isolation. (139 pp.) [SRX.19840127.0146]

Roy, D.M., and C.A. Langton (1989). "Studies of Ancient Concrete as Analogs of Cementitious Sealing Materials for a Repository in Tuff." (LA-11527-MS). Los Alamos, NM: Los Alamos National Laboratory. (101 pp.) [HQX.19890608.0033]

Rubin, J.B., J.W. Carey, and D.M.V. Taylor (1997). Enhancement of Cemented Waste Forms by Supercritical $\mathrm{CO}_{2}$ Carbonation of Standard Portland Cements. (LA-UR-97-1859)

Russell, C., R. Jacobsen, D.I. Haldeman, and P.S. Amy (1994). “Correlations to hydrogeological and geochemical parameters." Geomicrobiol. J. 12:37-51.

Sarker, A.K., M.W. Barnes, and D.M. Roy (1982). “Longevity of Borehole and Shaft Sealing Materials: Thermodynamic Properties of Cements and Related Phases Applied to Repository Sealing." (ONWI-201). Springfield, VA: U.S. Department of Commerce. (47 pp.) [NNA.19900316.0016]

Scott, D.A. (1985). “Periodic corrosion phenomena in bronze antiquities." Studies in Conservation 30:49-57. [NNA.920401.0108]

Sillen, L.G., and A.E. Martell (1964). "Stability Constants Spec. Publ." Chem. Soc. 17:144.

Skriver, H.L. (1984). The LMTO Method. Springer, NY: Springer Series in Solid State Sciences.

Smirnov, V.V., and E.B. Dubova (1992). "Characteristics of the radiation modification of highdensity polyethylene in the presence of solvents." High Energy Chem. 26(3):173-177.

Sterne, P.A., and A. Meike (1995). "Electronic Structure Calculations of Calcium Silicate Hydrates." (UCRL-JC-121437). Livermore, CA: Lawrence Livermore National Laboratory. (For submittal to Mater. Res. Soc. Fall Meeting, Boston, MA, November 27-December 1, 1995)) [235671]

Story, S.P., and L.E. Hersman (1995). “Desferriozamine B-Enhanced Transport of Iron in Volcanic Tuff During Unsaturated Conditions." Milestone report for the CRWMS Management and Operating Contractor, U.S. Department of Energy. (3410). Las Vegas, NV: Los Alamos National Laboratory. [MOL.19960813.9990]

Suzuki, K., T. Nishikawa, and S. Ito (1985). "Formation and carbonation of C-S-H in Water." Cem. Concr. Res. 15:182-188.

Tang, D. (1997). “Emplacement Drift Ground Support.” May 23, 1997. Yucca Mountain Project report. (BCAA00000-01717-0200-00003, Rev. 00B). Las Vegas, NV: prepared by the Civilian Radioactive Waste Management System, Management and Operating Contractor, for the U.S. Department of Energy.

Taylor, H.F.W. (1950). "Hydrated calcium silicates, Part I. Compound formation at ordinary temperatures." J. Chem. Soc.:pp. 3688-3690. 
Taylor, H.F.W. (1987). “Bound water in cement pastes and its significance for pore solution compositions." In proceedings from Scientific Basis for Waste Management. Mater. Res. Soc. 85:47-53.

Taylor, H.F.W. (1990). Cement Chemistry. London: Academic Press.

Van Konynenburg, R.A. (1984). "Radiation Doses in Granite Around Emplacement Holes in the Spent Fuel Test-Climax." (UCRL-535801). Livermore, CA: Lawrence Livermore National Laboratory. [NNA.19870903.0040]

Vieillard, P., and F. Rassineux (1992). "Thermodynamic and geochemical modelling of the alteration of two cement matrices." Appl. Geochem. Suppl. 1:125-136.

West, K.A. (1988). “Nevada Nuclear Waste Storage Investigations Exploratory Shaft Facility Fluids and Materials Evaluation." (LA-11398-MS). Los Alamos, NM: Los Alamos National Laboratory. [NNA.890926.0014]

Wilder, D.G. (1996). “Near-Field and Altered-Zone Environment Report, Vol. II.” (UCRL-LR124998, Vol. II). Livermore, CA: Lawrence Livermore National Laboratory. [MOL.19961212.0121; MOL.19961212.0122]

Wilski, H. (1990). "Radiation stability of polymers radiation physics and chemistry." Int. J. Rad. Appl. E Instrument. Part C 35(1-3):186-189.

Wolery, T.J. (1992a). "EQ3/6, A Software Package for Geochemical Modeling of Aqueous Systems: Package Overview and Installation Guide." (UCRL-MA-110662). Livermore, CA: Lawrence Livermore National Laboratory. (part 1) [NNA.19921023.0028]

Wolery, T.J. (1992b). “EQ3NR, A Computer Program For Geochemical Aqueous SpeciationSolubility Calculations: Theoretical Manual, User's Guide, and Related Documentation." (UCRL-MA-110662). Livermore, CA: Lawrence Livermore National Laboratory. (Version 7.0) [NNA.19921218.0010]

Wolery, T.J., and S.A. Daveler (1992). “EQ6, A Computer Program for Reaction Path Modeling of Aqueous Geochemical Systems: Theoretical Manual, User's Guide, and Related Documentation (Version 7.0)." (UCRL-MA-110662). Livermore, CA: Lawrence Livermore National Laboratory. (part 4) [MOL.19980218.0570] 



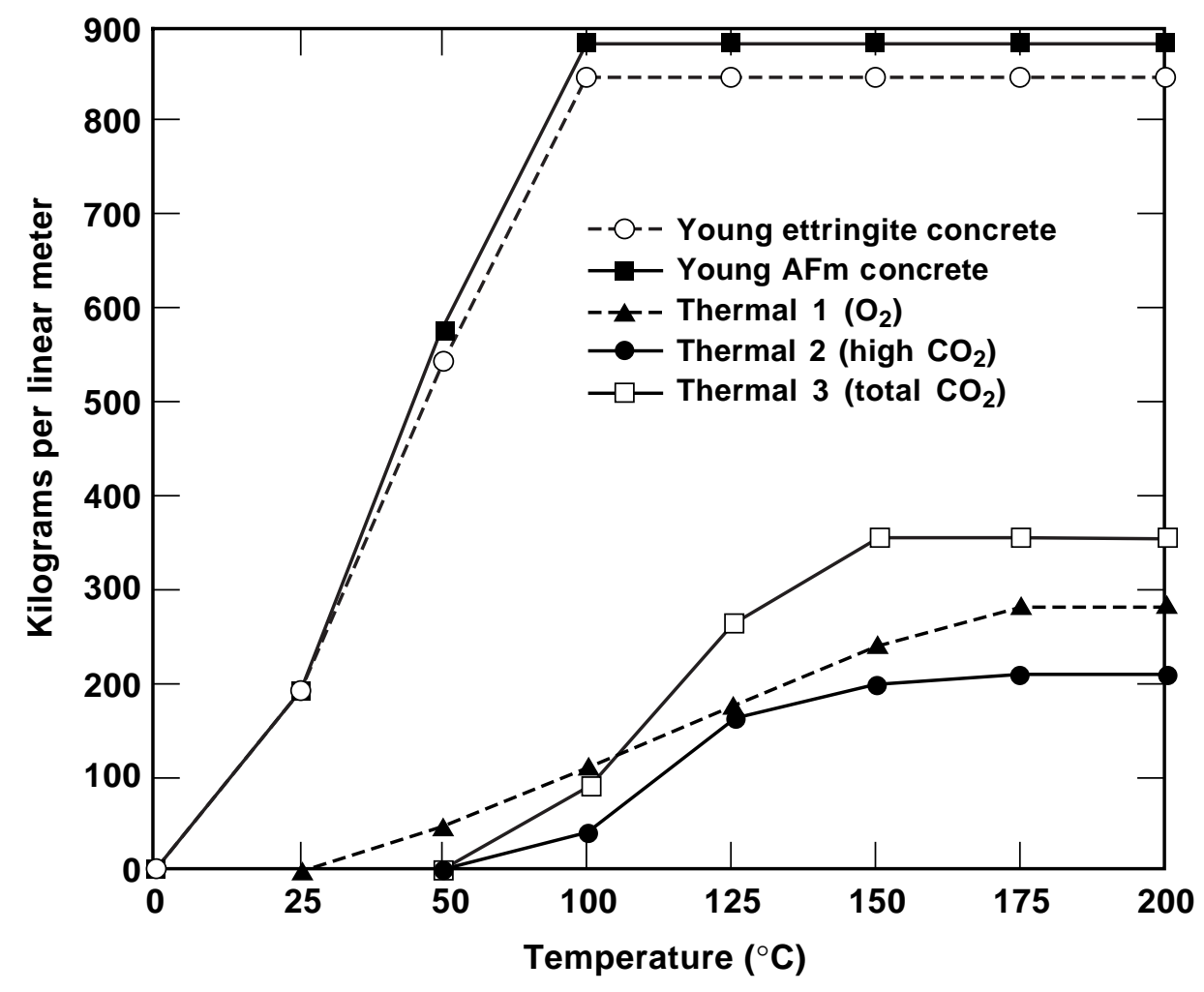

Figure 7-1. Idealized, cumulative water loss based on simplified mineral assemblages calculated in Table 7-2 through Table 7-5 



\section{Summary and Conclusions}

\subsection{Thermohydrologic Models}

Thermohydrologic (TH) models are used to calculate the evolution of liquid-water seepage flux, temperature, relative humidity $(R H)$, and air-mass fraction within the drifts as functions of time after repository closure. These are used directly as near-field environment (NFE) performance measures in assessing total system performance. TH models also serve as the starting point for other process model calculations that describe water chemistry, engineered-barrier performance, and radionuclide transport. These models compute distributions of temperature and flow velocity in the repository host rock, which are used with partially coupled models to evaluate whether more rigorous fully coupled process models are needed.

The physical principles of heat and mass flow in fractured porous media are well understood, can easily be expressed mathematically, and can be solved numerically. Available TH simulator codes (principally NUFT, VTOUGH, and TOUGH2) have been thoroughly tested and qualified, or are in the process of being qualified, under the Yucca Mountain Site Characterization Project quality assurance (QA) program. All of these codes can perform calculations using any of several different means for treating the coupling of heat and mass flow between matrix elements and fracture elements. The highest frequency of fracturing at Yucca Mountain occurs in densely welded units, including the principal host rock for the potential repository.

It is impractical to explicitly model a network of fractures or to rigorously model threedimensional (3-D) problems with spatial heterogeneity representing the spatial variability in the fracture system. Accordingly, several approximations have been developed, including the following:

- Equivalent continuum method (ECM)—By assuming there are no local differences in flow potential or temperature between a matrix block and the adjacent fractures, the bulk rock can be described as an equivalent continuum with averaged properties. This assumes instantaneous heat- and mass-transfer equilibrium to exist at each point in the model. ECM is computationally efficient, but may be inadequate if transient effects are important. Repository thermohydrology will be essentially transient, and episodic infiltration is the principal conceptual model for recharge; thus, ECM has been supplanted by other models in these applications.

- Dual-permeability method (DKM) - The rock is represented by two interpenetrating continua with different properties: one for fractures and the other for the rock matrix. Coupling between them is determined from the local difference in potential between fracture and matrix elements. The fracture-matrix coupling constant allows the model to more realistically represent nonequilibrium interaction by replacing the local equilibrium assumption of ECM with an assumption of local steady state. However, the coupling constant is not directly measurable and must be inferred by comparing model predictions with field data. 
- Discrete-fracture method (DFM)-This method requires explicit representation of fractures and matrix components by grid blocks, including the interblock transfer functions for heat and mass. Because of computational limitations on the numerical solution of the relevant equations, DFM can be applied only to simple geometry in which the fracture distribution has strong spatial symmetry. These problems can readily exceed the capacity of professional workstations and even the current generation of supercomputers.

\subsubsection{Activities for Building Confidence in Models}

Field tests in unsaturated tuffs are the principal means for building confidence in $\mathrm{TH}$ models. Although not discussed here in detail, the G-Tunnel prototype engineered barriers test was a key step in developing TH modeling capability. The Single-Heater Test (SHT) in the Exploratory Studies Facility (ESF) at Yucca Mountain is being conducted in welded tuff near the repository horizon, near the planned location of emplacement drifts; pretest characterization was more comprehensive for the SHT than for the G-Tunnel test. Modeling results for the SHT have focused on the extent to which capillary forces appear to control the distribution of temperature and liquid water. Results using six property sets have been compared, and those that produce temperature profiles that most closely resemble observed data also have the least capillarity.

The Large-Block Test (LBT) is still in progress, using a partially excavated 3-× 3-× 4.5-m block in an outcrop of the repository host rock at Fran Ridge near Yucca Mountain. One of the goals for the LBT is to produce a heat-pipe zone to observe the TH and chemical effects. Both ECM and DKM calculations have been performed with the same property sets used in modeling the LBT and have produced similar temperature fields. The LBT field-temperature data show several episodes in which measured temperatures within the superheated region suddenly decrease by as much as $40^{\circ} \mathrm{C}$, reaching the boiling point in much less than one hour. (See the "Large-Block Test Animation" on the CD-ROM accompanying this report.) These events probably resulted from episodic flow of condensate in fractures, something which has never been modeled by the available TH codes because they only simulate temporally and spatially averaged behavior.

\subsubsection{Reference Calculations for Evaluation of Coupled Processes}

The NUFT code was employed in a series of two-dimensional (2-D) models; these served as the basis for chemical and mechanical calculations used in evaluating coupled processes for this report. The infiltration conditions and stratigraphy chosen for these simulations correspond to a location near the center of the repository layout, where the percolation flux is approximately $16 \mathrm{~mm} / \mathrm{yr}$. The calculations have been repeated using two sets of hydrologic properties: a smaller infiltration rate and two thermal-loading options (i.e., point load and line load). Temperature and fracture liquid-flow fields were extracted for several time steps representing the repository thermal evolution and were used to compute thermomechanical (TM) and thermohydrochemical (THC) responses.

The reference calculations indicate that the thermally driven liquid flux near the emplacement drifts will initially be much greater than the ambient percolation flux and will gradually decline to the ambient flux within 100 to $500 \mathrm{yr}$. The maximum flux will depend mainly on the heat output rather than on the thermal-loading options. For both the point-load and line-load cases, the same fraction of heat output is transported as latent heat in the heatpipe zone. However, a larger dryout zone will develop for the line load because there is better condensate drainage through the wider pillars. 


\subsubsection{Drift-Scale Seepage}

Estimation of the temporal and spatial distributions of seepage into the emplacement drifts is arguably the most important problem for ambient and thermally driven unsaturatedzone (UZ) hydrology at Yucca Mountain. This estimation is difficult to treat rigorously because the location of seeps and their magnitude depend on the spatial heterogeneity of flow properties at scales smaller than typical grid-block dimensions used in site-scale models. Ideally, a model should have grid-block dimensions smaller than the corresponding correlation lengths. Using the NUFT code, models have been developed for estimating seepage into drifts under isothermal and nonisothermal conditions. Results to date show the sensitivity of seepage to model dimensionality, spatial heterogeneity of fracture properties, and episodic percolation conditions. These results confirm other, ongoing seepage studies (Tsang et al., 1997).

Results to date show that seepage increases with the degree of heterogeneity in hydrologic properties and is greater for 2-D than for 3-D models with similar properties. DKM simulations of transient, episodic percolation project greater seepage than do simulations of steady infiltration at the same average rate. For models with heated drifts, some TH property sets lead to seepage when the drift-wall temperature is below boiling; others do not.

Perhaps the most important result from seepage models is that the seepage flux is only a fraction of the ambient percolation flux in the host rock. When combined with the likelihood that only part of the seepage flow will actually drip onto the waste packages (WPs), a case can be made that very little water will contact the waste. It should be noted that, even though the spacing between fractures may be a significant fraction of the drift radius, this result is obtained using a continuum representation of the fracture network. This is an approximation, and there is considerable opportunity for reduction of uncertainty in this area.

\subsubsection{Total System Performance Assessment Multi-Scale Abstraction}

A multi-scale TH abstraction methodology has been developed to provide important input to total system performance assessment (TSPA). This abstraction tool calculates performance measures including the WP temperature and drift-air RH at the WP surface. The need for abstracting from $\mathrm{TH}$ simulations, rather than using $\mathrm{TH}$ simulations directly, arises from the limited number of simulation runs that can reasonably be conducted. Using the DKM problem formulation with radiative thermal coupling inside the drift and accommodating the expected variability of $\mathrm{TH}$ properties, percolation flux, and WP configurations in the repository block, the abstraction methodology provides useful estimates for TSPA TH performance measures.

The abstraction tool exploits several aspects of the repository $\mathrm{TH}$ problem to reduce computational effort. Scaling relations are used in which the repository is modeled as a "smeared-heat source" for calculating large-scale effects such as 3-D repository-edge effects. For regions of the host rock that are dried out or beyond the influence of heat-pipe activity, behavior is represented by thermal-conduction-only models. The abstraction tool has been tested by comparison to computationally intensive, full-TH simulations.

The methodology has made it possible to compare the effects of different TH property sets and percolation flux maps and to compare the performance of point-load vs. line-load repository designs. Comparisons are facilitated by calculating the TSPA performance measures over the entire repository layout. Results show that, depending on the values 
selected for capillary properties and infiltration flux, rewetting behavior can significantly prolong or shorten the period of reduced $\mathrm{RH}$ in the emplacement drifts. The magnitude of estimated infiltration is arguably the strongest influence on the calculated performance measures.

The abstraction methodology has been constructed so that refinements in the repository design can be readily analyzed for thermohydrologic effects. Backfill has already been incorporated as an option. Seepage models using spatial heterogeneity of fracture properties, and coupled THC and THM processes, can also be incorporated, although the computational effort will increase significantly.

\subsubsection{Improving Thermohydrologic Models to Reduce Uncertainty}

TH modeling has led the way in predicting NFE conditions and will continue to do so. Improved accuracy, flexibility, and reliability of $\mathrm{TH}$ predictions will contribute directly to success in licensing and to potential cost-saving features in the repository design. Following are some specific areas for improvement to reduce uncertainty:

- The multi-scale abstraction methodology is a significant accomplishment in modeling for NFE studies because it consolidates what is known about variability of infiltration and rock properties and optimizes the use of the available modeling tools. The full benefit of this methodology has yet to be realized for total system performance assessment-viability assessment (TSPA-VA). The methodology can be used to incorporate engineered barrier system (EBS) features such as backfill and drip shields and could be extended to efficiently estimate the TH effects associated with alternative repository development sequences. For each major advance in the application of the abstraction methodology, additional work will be needed to verify the method against computationally intensive, full-scale simulations for processes of interest.

- The usefulness of TH simulations depends critically on the property sets. Problems have been identified with simulation of field-scale heater tests, and these properties have been corrected. An approach to estimating parameters is needed that identifies which TSPA performance measures are most affected and then constructs the complete UZ hydrostratigraphy using heuristic principles that maximize confidence in the most affected properties.

- In the future, more emphasis will be given to TH models for evaluating EBS design alternatives that will control in-drift flow processes. Modeling of drip shield and backfill options will involve very finely gridded models, application of existing software for radiative heat transfer in drift openings, conceptualization of the properties of failed concrete and rockfall, and representation of the partial failure of EBS components. Modification of TH simulators to investigate these effects is underway.

\subsubsection{Conclusions Regarding Thermohydrologic Models}

Evolution of temperature and matrix saturation calculated for the reference cases and for other cases discussed in Chapter 3 makes possible some conclusions regarding the important dimensions of the TH regime. In addition, based on the observed reflux behavior in the LBT, repository design could be optimized to permit drainage while optimizing dryout. These points are discussed in more detail in the following bulleted items:

- The TH system has a tendency to achieve quasi-steady conditions because the duration and scale of repository heating are large compared to the characteristic time and length scales for thermal diffusion and convective heat transport (see Chapter 2). 
During this period, boiling behavior tends to be localized to the bottom of the heat pipe, whereas condensation occurs near the top. The heat-pipe zone is isothermal at the boiling temperature, and heat transfer occurs predominantly by liquid-vapor counterflow rather than by thermal conduction.

- Major features of the TH regime are determined by macroscopic factors such as heat output, repository depth, and the water-table boundary condition. Dryout zone thickness and heat-pipe thickness are controlled by thermal conduction and by the thermal conductivity of rock units overlying the repository. To a reasonable approximation, the average heat flux and the thermal conductivity of the dryout zone determine the dryout zone thickness required for the temperature to decrease to boiling. Similarly, the heat-pipe thickness depends on repository heat output, such that the average heat flux and the thermal conductivity of overlying strata determine the interval above the top of the heat-pipe zone that is required for the temperature to decrease from boiling to the average ground-surface temperature. The temperature of the NFE, and throughout the UZ, depends on the type of thermal boundary condition imposed at the water table.

- Conduction-only problems are similar enough to full-TH problems that useful information can be abstracted for estimating TSPA performance measures and substantially decreasing computational effort.

- Coalescence of dryout zones results if the sustained thermal output of the repository during the early thermal period exceeds the power required to (1) evaporate the average ambient percolation flux and (2) evaporate the matrix pore water in the region between drifts. Once the coalesced dryout region forms, maintenance of the quasi-steady state requires thermal power sufficient to evaporate the ambient flux.

- Results from the LBT show that the behavior of liquid condensate can produce episodic reflux events. Extrapolated to a thick heat-pipe zone, episodic reflux may be capable of penetrating into the emplacement drifts in significant quantity. Evaluation of this possibility appears to be beyond the capabilities of current TH simulators, which compute average behavior. In addition, when dryout zones from adjacent emplacement drifts coalesce, the heat-pipe zone may accumulate water because the ambient percolation flux does not drain. To avoid the potential for reflux into the drifts during the thermal period, design features are available that can optimize drainage between drifts while promoting dryout around the drifts. This can be done by increasing drift spacing and using a line-load or similar thermal option to optimize heat output and minimize "cold traps" between WPs.

\subsection{Thermomechanical Models}

Excavation and thermal loading will cause stress changes in the repository host rock, which will couple to changes in fracture permeability. Changes in permeability ranging over as many as three orders of magnitude are generally observed in fractured rock in response to excavation (e.g., Zimmerman and Finley, 1987; Zimmerman et al., 1986). When stress is reapplied, fractures close and permeability decreases, but the original permeability is not recovered. This indicates that both shear and normal displacement occur in response to excavation, causing mismatch in the mating fracture surfaces and, thus, permanent changes to fracture apertures. When normal stress is applied, fracture permeability can increase by an order of magnitude, but rapid stiffening occurs with diminished rate of aperture change. In a field-scale test, Zimmerman et al. (1986) cycled the applied load among stress conditions representing the range of horizontal stress that will be observed in the repository host rock. The observed changes in fracture permeability were substantially less than an order of 
magnitude. The largest changes in permeability are considered to be increases associated with shear displacement of fractures (e.g., Hardin et al., 1982), and these changes are also the most permanent.

An approach was presented for estimating changes in fracture permeability caused by deformation response to TM stress changes. This method is based on published approaches (Barton et al., 1985) and considers only effects from shear displacement, which has greater potential to permanently change permeability than does normal displacement. The approach provides the basis for estimating the magnitude of permanent changes in permeability associated with repository heating and can be tested by comparison to changes in permeability associated with the Drift-Scale Test (DST).

For this report, regions where permeability changes could occur in the repository host rock are evaluated using an elastic continuum model of rock-mass response. The simulators available (e.g., FRACROCK, FLAC, and UDEC) also support the use of ubiquitous fracture or discrete-element models.

\subsubsection{Drift-Scale Test}

For the DST, TM effects may cause significant enhancement of permeability in a significant volume of the rock mass. Permeability is predicted to increase where temperature gradients and the associated thermal-stress gradients develop. A zone of enhanced permeability is likely to accompany the outwardly diffusing thermal "wave" as it emanates from the heaters. Permeability is predicted to increase by a factor of two to four. Assuming that shear slip causes permanent changes in fracture apertures, the resulting permeability differences should be detectable after the affected region dries out. During the test and before dryout, aperture changes could be difficult to discern from changes in fracture saturation on the basis of pneumatic testing.

The guard-heater geometry used in the DST introduces TM effects that may differ significantly from repository drift-scale effects. The DST heater arrays were designed primarily to achieve representative TH conditions (Wilder, 1996). Additional work is needed to improve predictions of permeability changes in response to repository heating.

\subsubsection{Analysis of Reference Cases}

Smaller changes in permeability are projected for the reference cases used to assess coupled processes for this report than are projected for the DST. The stress fields based on TH-calculated temperature fields for all of the point-load cases (Cases 1 through 4) are similar and can be represented by Case 1. Slip on fractures resulting from induced shear stresses is expected to be limited to a few, small zones that are within a few drift diameters of the drift wall. Application of other conceptual models (e.g., discontinuum behavior) for the contribution of fractures to rock-mass deformability could further limit the extent of slip on fractures. For the reference cases, the zone of shear slip calculated for the DST is not present because the shear-stress effects from adjacent drifts tend to cancel. In practice, however, completion cancellation is unlikely because of constitutive heterogeneity and nonuniform development of the repository area; thus, durable increases in fracture permeability may result.

An important feature of the method for THM coupling presented in this report is the concept that permeability enhancement occurs as a result of shear offset due to Mohr-Coulomb slip on pre-existing fractures. This concept is widely accepted and can be tested by simulating the displacement measurements and permeability measurements made during the DST. Other experiments, including the LBT and the SHT (Lin et al., 1997), provide similar opportunities for model testing. 
Another important aspect of the TM calculations presented in this report is that they overestimate the distance to which shear slip may extend from the heated drift because they are linear-elastic. When fracture slip is incorporated, the effect will be to limit the distal extent of slip behavior, concentrating it in a smaller region within a few radii of the drift opening. This will occur because strain energy that would have contributed to rock-mass deformation will be consumed by fracture slip during the outward growth of the temperature field.

\subsubsection{Improving Thermomechanical Models to Reduce Uncertainty}

The uncertainties that attend predicting rock-mass response to the construction, operation, and postclosure performance of a Yucca Mountain repository are smaller than the uncertainties that are typically encountered in mine development, primarily because there are more data available. The following improvements could significantly reduce the remaining uncertainties:

- Interpret microseismic monitoring that is being performed as part of the DST, and for any future heater tests, to confirm joint slip events that are likely to be seen in other data sets such as rock-mass displacement, stress, and hydrologic testing.

- Assess the effects of alternative boundary conditions on TM models (i.e., which combinations of free-surface, fixed-displacement, and constant-stress boundaries best represent the transition from repository center to edge conditions).

- Perform ubiquitous fracture modeling (FLAC) and discrete-element modeling (UDEC) to examine the relative contributions from different joint sets, sensitivity to fracture parameters, etc.

- Measure additional laboratory-scale fracture properties (particularly the sheardeformation parameters), including dilation angle and frictional properties.

- Assess creep and pressure solution effects to determine whether THM changes caused by normal fracture displacements will be reversed during cool-down.

\subsubsection{Conclusions Regarding Thermomechanical Models}

Evaluation of the stress fields calculated for the reference cases makes possible some conclusions regarding important THM effects in the altered zone (AZ) around the repository:

- The thermal diffusivity and thermal expansion of the host rock control whether there is an expanding region in which shear stress tangential to the isotherms is maximal. Such a region is not seen in the simulations discussed in this report.

- The heat-pipe zone is isothermal, thus THM changes associated with shear deformation in this zone are limited to effects that occur during growth and collapse of the temperature field.

- Conditions favoring shear displacement of fractures are predicted to occur in the rock mass above and below the DST and, to a lesser extent, in the repository host rock. Accumulation of shear slip over time and the permeability effects of finite slip will be concentrated within a few diameters of repository emplacement-drift openings.

\subsection{Thermohydrochemical Models}

Chemical reaction models calculate the state of a reaction cell on the basis of equilibrium behavior and may also include nonequilibrium behavior expressed as relative reaction rates and controls on reactant availability. These concepts are embodied in the EQ3/6 modeling software package, which is widely used in geochemistry and is qualified for use in licensing. Reactive-transport models include reaction rates and a time variable and represent a spatial domain. Several reactive-transport simulators are available in the public domain and incorporate a wide range of chemical species and types of mass-transport behavior. The 
1DREACT simulator is typical. Its successor, the OS3D/GIMRT code, is used to evaluate reference cases in this report. Although this simulator handles chemical problems with multiple components and heterogeneous phases, it models only aqueous reactions and does not include a gas phase in mass or energy balances, as would be required to model $\mathrm{CO}_{2}$ transport.

Another category of reactive-transport simulators consists of TH codes such as FEHM and NUFT that have been modified to incorporate the chemical behavior of a small number of components. This approach has been useful for studying the effects of boiling processes on mineral precipitation, using silica as a single component.

Four modeling studies have been selected to represent significant progress in THC modeling. Two of these are recent Lawrence Livermore National Laboratory (LLNL) studies prepared for the Yucca Mountain project, and two are new work reported here. First, a reaction-path study is presented for water evolution in contact with the host rock (EQ3/6); this study examines the impact of uncertainty in the reaction-rate constants for water-rock interaction Next, reactive-transport of silica movement is simulated at the repository scale using FEHM. Fully coupled calculations are then presented that show reactive transport of silica at the drift scale (NUFT) and reactive-transport simulations involving aqueous mineral dissolution and alteration reactions in the AZ, using OS3D/GIMRT.

\subsubsection{Reaction-Path Study}

This study analyzes the impact of uncertainty, in relative reaction rates used in the EQ3/6 model, on the capability to simulate the evolution of J-13 water composition in the potential repository host rock. Results show that the evolution of recharge water to the J-13 composition depends on several rate reactions, principally those for silicate dissolution, for which reaction data are uncertain. Significant reaction progress and multiple mineral phases are required to achieve J-13 water composition. Uncertainty analysis of relative reaction-rate constants shows that the J-13 composition can be achieved using plausible assemblages of mineral phases, but that the relative amounts of particular phases and the time scale of water evolution depend on reaction-rate data about which there is considerable uncertainty. Depending on the problem formulation, the evolution time for water composition may vary by one or more orders of magnitude, corresponding to the uncertainty in reaction-rate parameters.

\subsubsection{Coupled Repository-Scale Calculation with Silica}

Fully coupled THC calculations were performed using the FEHM finite-element code, which was modified to incorporate the behavior of a single chemical component and to couple changes in fracture porosity with changes in permeability. The TH aspects of these calculations were done using the ECM model to represent fracture-matrix interaction (see Chapter 3). The entire repository block from the water table to the ground surface was modeled using a coarse mesh and simple constant temperature or no-flow boundary conditions. Ambient percolation flux was introduced to the top of the model at a low rate $(0.3 \mathrm{~mm} / \mathrm{yr})$, which represents the prevailing understanding of ambient UZ hydrology when the study was performed.

These calculations show that sufficient silica will be mobilized by repository TH processes to cause significant changes in local fracture porosity. Boiling occurs predominantly at the bottom of the isothermal heat-pipe zone, and the residual solute deposits from boiling accumulate there. Solute accumulation is not hindered in a simulation that couples fracture permeability and porosity (relative permeability and moisture characteristic relations were not coupled). Repository edge effects are reflected in the manner of silica dissolution and 
precipitation, and similar effects can be expected to result from repository loading scenarios. The low ambient percolation flux assumed in this study did not affect the redistribution of silica, which mainly involved the connate water.

\subsubsection{Drift-Scale TH Simulations with Silica}

Fully coupled THC calculations were performed using the NUFT code modified to incorporate the behavior of silica. The initial TH properties, 2-D geometry, and boundary conditions correspond to the reference cases developed for this report. Model input included coupling relations between porosity and permeability and between porosity and the water potential and relative permeability curves. Reaction rates and chemical equilibria are fully temperature-dependent in the model. The DKM model for fracture-matrix interaction is used for these calculations (see Chapter 3), and chemical dissolution/precipitation reactions are modeled in both the fracture and matrix continua.

Formation of a precipitation cap above the emplacement drifts is predicted by this model for all conditions considered, including ranges of the specific surface area available for silica dissolution and reaction constants corresponding to different silica phases (e.g., quartz, cristobalite, and amorphous silica). Significant precipitate accumulation takes place within a few years, or tens of years, after the start of heating. The simulations show that dissolution and precipitation reactions affect both the fractures and the matrix, depending on location. Because of the greater solubility, more dissolution occurs when the silica phase is assumed to be amorphous silica. The calculations extend to late time after the system has returned to ambient temperature, but dissolution of the precipitated material does not readily occur because of reaction-rate attenuation at cooler temperatures and because the ambient flux tends to equilibrate chemically with the host rock above the repository horizon.

Calcite dissolution, leading to deposition of additional species in the boiling zone, is not incorporated in this model. When included, the effect will likely increase the initial rate of precipitate formation because calcite dissolves 1 to 2 orders of magnitude faster than do silicates such as cristobalite. Incorporating more chemical components and reactions can also be used to investigate the accumulation of halide salts and other soluble compounds that are important in assessing the potential for WP corrosion.

The fully coupled NUFT THC simulations presented in Section 5.5 of this report were calculated for a range of reaction parameters corresponding to a 100-fold range in macroscopic reaction rates. From the reaction-path study in Section 5.3, which emphasized uncertainty analysis of reaction rates, a wider range of rates would be appropriate for sensitivity analysis.

To reflect the relations between permeability and heat-pipe formation, that is well established from TH simulations (see Chapter 3), a lower bound on permeability reduction in the boiling zone is incorporated in the model. When the bulk permeability decreases to a threshold value (100 millidarcy), throttling inhibits the penetration of liquid reflux. The lower end of the heat-pipe zone then migrates away from the heat source. Thus, although precipitation is concentrated at the bottom of the heat-pipe zone where boiling occurs, the zone of permeability reduction migrates upward with time. The rate at which this occurs will depend on the rate of solute precipitation and on the coupling relations between porosity and permeability. 


\subsubsection{Reactive-Transport Calculations}

Coupled reactive-transport simulations were performed using the OS3D/GIMRT code. Temperature fields and fracture-flow fields were taken directly from the 2-D TH reference cases (see Chapter 3) for several time steps representing the TH evolution. The GIMRT simulations are based only on fracture flow conditions extracted from DKM simulations, ignoring matrix processes. Fracture-matrix interaction is assumed to carry solute only from the fractures into the matrix. This conceptualization applies to steady-state hydraulic conditions in which the fracture flux is large, compared to the matrix flux, and/or the matrix porewater composition does not differ much from the that of the fracture water., Reactive processes were simulated for a few years at each selected time step to establish (1) the rates of dissolution and precipitation reactions relative to the availability of the affected mineral species and (2) the time to achieve steady state for a given set of initial conditions.

There is substantial uncertainty regarding the rate constants for silicate dissolution and precipitation reactions and regarding the inventory of fracture minerals present in the host rock. Reference values for rate constants are assumed that are consistent with the latest available experimental data, but are far from complete with regard to the number of silicates minerals represented, the representation of both dissolution and precipitation, and temperature effects. For these simulations, secondary calcite is assumed to be the predominant fracture-lining mineral phase, and dissolution progress is continued only for a few years at each time step as needed to establish the rates of reaction. The volume of mineral precipitates is assumed to be small when compared with the fracture porosity; thus, calculated changes to fracture permeability are not significant. In summary, the OS3D/GIMRT simulations were undertaken to examine the implications of what is known with relative certainty about the system behavior, without generating results that could be heavily influenced by uncertain parameters and mineral distributions.

These simulations show that dissolution of calcite will occur relatively rapidly. Aqueous dissolution and precipitation reactions involving silicates are likely to produce significant changes in fracture porosity, to do so but relatively slowly, over time periods on the order of tens of years. Later in the repository thermal evolution, $\mathrm{CO}_{2}$ will be displaced from the gas phase by water vapor as the air mass fraction approaches zero in the host rock (see Chapter 3). OS3D/GIMRT simulations show that decreased $\mathrm{CO}_{2}$ fugacity will have little impact on calcite and silicate reactions at near-neutral $\mathrm{pH}$. The presence of condensate that is less chemically aggressive to calcite will result in the physical spreading of zones of precipitation and dissolution.

Comparison of OS3D/GIMRT simulations with the silica distributions calculated using NUFT shows that the aqueous dissolution and boiling processes predicted by the two approaches may be incompatible above the emplacement drifts. Although the lower end of the heat-pipe zone is at relatively high liquid saturation and elevated temperature, dissolution may still be hindered because the concentrations of solutes are increased by evaporation. An integrated model that accommodates a full set of possible chemical reactions with evaporation, boiling, and gas-phase transport of reactive components such as $\mathrm{CO}_{2}$, is the next step for improved realism of THC simulations. 


\subsubsection{Conclusions Regarding THC Models}

The conclusions drawn from THC modeling presented in this report may be summarized as follows:

- THC effects will definitely cause redistribution of soluble components such as calcite and silica and will very likely produce a precipitation cap over the emplacement drifts. This is predicted for a range of values for the ambient percolation flux and reactive surface area present in the matrix and fractures. Precipitation will occur at the boiling zone. Accumulation will be limited by the liquid reflux rate and by the dissolution kinetics of minerals accessible on fracture walls.

- The bulk permeability of the rock mass making up the precipitation cap should reach a quasi-uniform value for a range of different model conditions (e.g., ambient flux, reactive surface). The existence of a lower-bound bulk permeability for heat-pipe effects has been shown from previous TH simulations. The result of this limit is that the precipitation cap will tend to move outward with time, increasing the zone thickness.

- Simulations show that, at later time, precipitation is likely to occur in the pillars; if capillary reflux is significant, precipitation can also occur below the drifts. This means that, depending on the TH properties, the emplacement drifts can become encircled by precipitation. This effect has adverse implications for repository performance because it implies that seepage may become trapped and accumulate in the emplacement drifts. Clearly, it is important, when simulating THC effects, to accurately represent the relative importance of gravity- and capillary-driven fracture and matrix flow imbibition and reflux processes.

- These simulations show that the TH properties of the fracture system directly above the drifts will change significantly and that analyses of post-thermal seepage should take these changes into account. Beyond the obvious decrease in seepage that could result from decreased permeability, the cumulative effect of precipitation may be to regularize the spatial heterogeneity of fracture properties of the host rock in this region.

- Other significant data uncertainties that affect THC models are the following:

- Reaction rates and specific surface areas for dissolving minerals and precipitation rates for reactions that will produce clays and zeolites at ambient and elevated temperature are poorly known.

- The mineralogical structure, the saturated and unsaturated hydrologic properties, and the bonding strength of fracture-filling precipitates that will form in the boiling zone are subject to considerable uncertainty.

- The $\mathrm{CO}_{2}$ fugacity is an important chemical uncertainty in the NFE throughout the thermal period and beyond. Existing TH or THC models need enhancement to represent the behavior of $\mathrm{CO}_{2}$ as it is mobilized in the host rock and is displaced by water vapor, then moves away from the repository, dissolves in condensate, and undergoes aqueous interaction with mineral phases. The $\mathrm{CO}_{2}$ fugacity in the host rock may eventually return to ambient conditions after satiation of the chemical demand from alkaline cementitious materials. A complete model for $\mathrm{CO}_{2}$ behavior can be tested against ambient conditions, including apparent ${ }^{14} \mathrm{C}$ age. 
- The different THC simulation approaches presented in this report have shown that conflicting results arise when simulations that consider only aqueous processes are compared with simulations that include boiling processes but do not incorporate a full suite of chemical components and reactions. Needed is a single, public-domain simulation code that includes all of these capabilities. Such a code does not presently exist.

- Following are additional areas of needed advancement in THC models:

- Sensitivity studies on THC models that incorporate silica redistribution should be conducted to focus laboratory investigation of the properties and reaction-rate parameters that are critical to precipitation cap formation.

- Simulation should be used to evaluate the postulated regularizing behavior of mineral precipitation, whereby all fractures are eventually filled.

\subsection{Geochemical Conditions in the Near Field/Altered Zone}

The approach to modeling geochemical conditions in the near field/altered zone (NF/ AZ) emphasizes water composition, which is needed to assess the WP lifetime, the release of radionuclides from the waste form, and the mobility of released radionuclides. Modeling of water composition begins with water in the $\mathrm{AZ}$ above the repository at ambient and elevated temperature conditions. Thermochemical modeling is presented that bounds the water composition with and without evaporative concentration. This water is then introduced to the NFE, where it interacts with introduced materials.

For this report, the near-field geochemical environment is assumed to be dominated by cementitious materials, although the WP materials will also contribute, and steel may be used instead of concrete for some ground-support components. The evolution of water composition in contact with cementitious materials is calculated for young concrete and for alternative compositions that represent concrete altered by heating in the presence of moisture and varying amounts of $\mathrm{CO}_{2}$.

If radionuclides are released to the NFE, interaction with introduced materials will cause retardation of some species. Presented is a series of laboratory experiments that have helped define the retardation behavior of $U$ and $\mathrm{Np}$ in contact with iron corrosion products and altered concrete. Preliminary results show that partition coefficients of at least $10^{4} \mathrm{~mL} / \mathrm{g}$, and probably higher, are likely. Modeling of radionuclide transport through a layer of degraded introduced materials shows that the retardation could be equivalent to that of hundreds of meters of the host rock.

A conceptual model for radionuclide mobility in the $\mathrm{AZ}$ is presented as a discussion of the available thermodynamic data for predicting solubility controls and as a conceptual discussion of colloid transport and what is being done to bound alternative modes of transport.

This chapter summarizes models that have been developed for use in repository design and performance analysis. Much of the chemical modeling in this area of investigation used the EQ3/ 6 modeling code with associated tools and databases (see Chapter 5). Some calculations are provided as examples or to demonstrate model behavior over ranges of conditions. Additional calculations are being performed for viability assessment. The chemical models are limited by the availability of experimental data, completeness of chemical databases, and limitations on chemical processes (e.g., boiling and coprecipitation) that are incorporated. 


\subsubsection{Altered-Zone Water Composition}

At ambient temperature, water in the host rock will have composition closely resembling that of J-13 water, whereas condensate will essentially be distilled water. Water that evolves in the AZ will have composition that represents a mixture of ambient water similar to that from well J-13 and condensate that has interacted with rock-fracture mineralogy. Evaporative concentration will produce different effects, depending on the $\mathrm{CO}_{2}$ fugacity and other factors. The range of water composition that is expected in the $\mathrm{AZ}$ will be bounded by the following:

- During heat-up, the water composition will be dominated by evaporative processes, and the composition will be represented by J-13 water concentrated to some degree. Most of this water will have originated as ambient percolation or matrix porewater. Results presented in Section 6.2.2 bound the composition for this water, although some waters may become even more concentrated as they approach full evaporation.

- During cool-down, the water composition will be dominated by condensate, and the actual composition will depend on the degree of completion of rate-dependent waterrock interaction. The results presented in Section 6.2.2 summarize the range of expected compositions for these waters. Waters of this type will enter the drifts and interact extensively with introduced materials.

- At the repository edges and in the late stage of cool-down, the water composition will be dominated by ambient percolation. These waters are expected to be similar to J-13 well water previously reported (Harrar et al., 1990).

These estimates can be evaluated by comparing experimental and modeling results with observed natural-water compositions. The available data consistently show that fracture waters are dilute bicarbonate waters. However, significant discrepancies exist for some chemical components, suggesting that the modeling can be refined. Several specific areas for refinement are identified and include chemical boundary conditions, dissolution rate kinetics, and treatment of minor mineral phases.

\subsubsection{Water Composition in Contact With Cement}

A model has been presented for NFE water composition, representing its interaction with cementitious materials at different stages of maturation. The model differs from previous calculations in that additional cement phases are included in a new thermodynamic database, using data from the open literature, to represent the observed products of hydrothermal alteration of Portland-type cement in the presence of $\mathrm{CO}_{2}$ (see Chapter 7). Cement-mineral assemblages have been developed to represent different types of cement alteration. This is a recently developed database that will be assessed along with previous data to produce, for TSPA-VA, estimates of water composition contacting the WPs and interacting with released radionuclides.

Simulating the chemical environment as water first impinges on introduced materials after the thermal period is complicated by uncertainty regarding the nature and extent of cement alteration, the evolution of $\mathrm{CO}_{2}$ fugacity, and the chemical contributions from different concrete constituents. These simulations show that the alkaline $\mathrm{pH}$ effect from interaction with cement can be substantially moderated, depending on the $\mathrm{CO}_{2}$ pressure assumed. The evolution of water composition is calculated as a function of reaction progress for different constraints on $\mathrm{CO}_{2}$ partial pressure; these constraints range from low to "unfixed" to sufficiently high to ensure complete carbonation. When sufficient $\mathrm{CO}_{2}$ is present, the $\mathrm{pH}$ is in the near-neutral range; however, when the $\mathrm{CO}_{2}$ is exhausted by reaction with portlandite, the $\mathrm{pH}$ increases to 10 or greater. Further specification of water composition in the NFE will require modeling the dispersal of $\mathrm{CO}_{2}$ in the TH regime and the redistribution of $\mathrm{CO}_{2}$ by gas-phase circulation processes in the post-thermal regime. 


\subsubsection{Concentrations of Radionuclides Leaving the Repository}

Iron oxides derived from the WP corrosion-allowance material (CAM) have the potential to provide a sorptive barrier to radionuclide transport that could be equivalent to hundreds of meters of surrounding rock. Partition coefficients of $10^{4} \mathrm{~mL} / \mathrm{g}$ and greater have been measured for $\mathrm{U}(\mathrm{VI})$ and $\mathrm{Np}(\mathrm{V})$ on hematite, and greater partitioning would probably be observed on the "lower" oxides of iron, which have greater specific surface area. Consistent results have been obtained from transport experiments conducted using these materials. Retardation will be strongly affected by fluid chemistry, particularly $\mathrm{pH}$ and $\mathrm{CO}_{2}$ fugacity, and by the nature of the flowpath through a layer of corrosion products. Whereas it is likely that colloidal particles will exist and be capable of transporting radionuclides through the corrosion layer, the magnitude of radionuclide transport via these mechanisms has not been determined. If corrosion products serve as a source for pseudocolloids, breakthrough at the boundary of the corrosion layer may occur sooner, and at greater total radionuclide concentrations, than discussed here.

Surface complexation modeling of uranium sorption to hematite indicates that, at alkaline $\mathrm{pH}$, the presence of significant $\mathrm{CO}_{2}$ can inhibit the sorption of $\mathrm{U}$ and, by analogy, $\mathrm{Np}$. Simulations indicate that retardation will be significant for two scenarios: (1) alkaline waters in which $\mathrm{CO}_{2}$ fugacity is controlled by calcite equilibrium (e.g., fluids within cementitious material) and (2) alkaline fluids that are neutralized by reaction with ambient $\mathrm{CO}_{2}$ (e.g., fluids that exit cementitious material).

Hydrothermal alteration of concrete decreases the pore fluid $\mathrm{pH}$ relative to fresh concrete and increases the relative abundance of crystalline calcium-silicate hydrates and clay minerals. Np and $\mathrm{U}$ were not detected in the effluent from a transport experiment conducted using a fractured core of concrete that had been hydrothermally altered to represent repository conditions. Altered concrete strongly retarded $\mathrm{U}$ and $\mathrm{Np}$ in these experiments, either by sorption or by causing precipitation of one or more solubility-limiting solid phases. The solubility hypothesis implies greater retardation performance than sorption, but additional experiments are needed to establish the mechanism. Iodide was not retarded in these experiments. Measurable fractions of the $U$ and $\mathrm{Np}$ in batch experiments were associated with particulate matter; however, in general, colloids did not retain a significant fraction of the radionuclides in these experiments. In the batch experiments, the partition coefficients were $>10^{4} \mathrm{~mL} / \mathrm{g}$ when colloids were analyzed with the liquid phase; in the transport experiments with altered concrete, both the dissolved and particulate fractions were below detection.

Although the emphasis in these studies is on sorptive behavior of near-field materials, simulations that also consider precipitation and dissolution would provide a more realistic assessment of radionuclide migration through corrosion products and would increase the retardation performance that can be attributed to degraded introduced materials.

\subsubsection{Radionuclide Transport in the Altered Zone}

It is expected that transport processes in the AZ below the repository will depart significantly from those in the unaltered, ambient system. Thermally driven coupled processes will likely change the hydrologic and chemical characteristics of fracture pathways from the NFE in ways that are difficult to predict. However, the current approach is to use chemical solubility to bound the aqueous concentrations of radionuclides in the AZ, while using an experimental approach to bound colloidal and microbial effects on transport.

The GEMBOCHS database associated with the EQ3/ 6 modeling code contains several data files that include qualified data on radionuclides of interest. An evaluation of the available thermochemical data for key elements has been performed, using J-13 water as the 
reference water composition. Solubility-controlling solid phases have been identified for $U$, $\mathrm{Np}, \mathrm{Pu}, \mathrm{Am}, \mathrm{Tc}, \mathrm{Ni}$, and $\mathrm{Zr}$ for oxidizing (high-Eh) conditions and for reducing (low-Eh) conditions. Solubilities for a range of $\mathrm{U}, \mathrm{Zr}$, and $\mathrm{Ni}$ phases can be reliably predicted over the temperature range of $20^{\circ}$ to $150^{\circ} \mathrm{C}$. For other radioelements, including $\mathrm{Np}$ and $\mathrm{Pu}$, the likely solubility-limiting phases in J-13 water have been identified, but there are no data for elevated temperature. The relative need for further solubility data is reported to be $\mathrm{Np}>\mathrm{U}>$ $\mathrm{Pu}>\mathrm{Am}>\mathrm{Zr}>\mathrm{Tc}>\mathrm{Ni}$. Coprecipitation is potentially important for radionuclide retardation in the NF/AZ, (e.g., actinide substitution in precipitating carbonates), but it has not yet been studied. A model for predicting coprecipitation effects is under development, but there are virtually no experimental data for model testing.

The formation, stability, and sorption of radionuclides by colloids in the NFE remain matters of considerable uncertainty. Introduced materials in the repository can serve as sources for colloidal particles with strong affinity for radionuclides. The chemical and physical environment is conducive to the formation of colloids and biocolloids. There are few data available on colloid formation and mobility, particularly because colloidal processes are complex and difficult to control.

Laboratory experiments consistently show that bacteria and colloids are transported faster than are conservative solute tracers in individual fractures. The wide range of apertures and the aperture correlation structure in natural fractures lead to preferential pathways for particle transport. Preliminary results from the bacterial transport experiment associated with the LBT show breakthrough of labeled bacteria near the base of the block, but additional information is needed to establish a model framework for interpreting this observation. Partitioning of radionuclides to microbes and biocolloids is being investigated at Los Alamos National Laboratory. Quantitative prediction of microbial transport of radionuclides will require information on fracture-flow processes and on capture properties of rock surfaces as well as ecological assessment of microbial population response to changes in NFE conditions.

\subsection{General Conclusions for the Near-Field/Altered-Zone Models Report}

Because of changes in the waste isolation strategy (Repository Safety Strategy; DOE, 1998) and related changes in the reference repository design, the need for detailed predictions of chemical conditions in the NFE has been elevated to a primary concern within the past year. Characterization of the site, natural processes, and conditions, especially those related to estimates of increased flux, has led to better understanding of how engineered barriers can be designed to assist the geologic setting in meeting post-closure performance objectives. At the same time, incorporation of large amounts of concrete into the reference repository design (for which steel was designated before) has significantly changed the conceptual model for the geochemistry of the NFE and, therefore, requires a different set of chemical data.

The extent of detailed predictions that can be made concerning long-term evolution of chemical conditions in the NFE is limited by the availability of experimental data on the properties and behavior of introduced materials, especially concrete and engineered backfill. Several types of concrete are listed as options in the reference repository design, which increases the amount of additional information needed for comparative analysis. With currently available information, comparison of the NFE conditions associated with alternative concrete formulations is approached with considerable uncertainty. 


\subsubsection{Understanding the Precipitation Cap}

The precipitation cap is very likely to form and will decrease fracture permeability while increasing fracture capillarity in the affected region. In regions immediately adjacent to the precipitation cap, fracture-matrix interaction will be inhibited by solute precipitation in the matrix. Although these simulations depend on reaction constants and coupling relations that are uncertain and have not generally been tested experimentally, it is certain that heat pipes will form, that dissolution will occur, and that solute will be precipitated at the boiling zones. Preliminary simulations performed for this report show the effect on permeability to be significant for a wide range of key input parameters.

Whether the precipitation cap will persist for thousands of years in the post-thermal performance period depends on whether the precipitates are dissolved by the ambient percolation flux and on whether vertical fractures reopen when the rock mass cools.

Dissolution can be addressed using the same THC simulator used to predict formation of the precipitation cap. More than one mineral phase (e.g., cristobalite and amorphous silica) may be needed if the concentration of silica in percolation is controlled by a mineral phase other than the precipitate. Evaluation of the TM effect will require additional information on fracture rheology and on the actual mode of fracture displacement that accompanies heating. Although the horizontal normal stress will certainly increase during the thermal period, shear displacement on vertical fractures may occur at the same time, causing fracture dilation instead of closure.

\subsubsection{Thermohydrology Issues for Repository Design}

The performance measures calculated for TSPA-VA using the abstraction methodology detailed in this report include the WP surface temperature, $R H$, and flux in the host rock above the emplacement drifts. The numerical TH (NUFT) simulations, which are the basis for these calculations, use constant properties within each hydrostratigraphic unit. From seepage calculations involving spatially heterogeneous fracture properties, it is evident that seepage is underestimated when unit properties are considered constant. The reasons for using constant properties are practical ones: the computational effort required to evaluate multiple realizations of property fields at many locations throughout the repository would be prohibitive.

The TH simulators described in this report actually simulate a spatially and temporally averaged response at each WP location in the model. Laboratory and field tests, including the LBT (as discussed in this report) and the lab-scale coupled process experiments (as described by Kneafsey and Pruess [1997], show that liquid reflux behavior in heat pipes is considerably more complex. In the LBT, unsteady refluxing behavior was observed whereby temperature fluctuated above boiling as water refluxed episodically. Similar results were observed in the laboratory. These experiments are evidently producing behavior that is not part of the conceptual models on which the simulations are based. By extrapolating these observations to the extensive heat-pipe zones simulated for the reference cases used in this report, it is plausible that unstable behavior could occur on a large scale above the repository. It is reasonable to postulate that a heat pipe that is $100 \mathrm{~m}$ high and that is separated from the emplacement drifts by a vertical distance of approximately $10 \mathrm{~m}$ could produce episodic reflux events capable of penetrating the dryout zone and cause drift seepage at elevated temperatures during the peak thermal period.

For some time after repository closure, WPs will be partly shielded from seepage by the drift liner. Due to the strength degradation associated with the thermal alteration of cement, combined with the effects of thermally induced stress in the host rock, liner collapse will 
probably occur within a few hundred years. With the current reference repository design, the liner is likely to collapse well before the end of the thermal period. The precipitation-cap effect is of special interest because it will provide shielding from seepage starting about the time of liner collapse. The consequences of possible penetration of thermally driven reflux into emplacement drifts can also be mitigated by engineered barriers such as backfill and/or a drip shield.

The repository design can be optimized to limit the possible effects from penetration of thermally driven reflux. By increasing the lineal power-loading within each emplacement drift, the NFE temperature is increased, and the dryout zone thickness is increased proportionately. In addition, by increasing the spacing between drifts, the pillars remain at sub-boiling temperature, and drainage of reflux and the ambient percolation flux can occur. Heat pipes will be smaller and function less efficiently because drainage through the pillar causes loss of mass and enthalpy from the reflux zone. This has been shown, in this report, in the analysis of reference Case 5, which represents a line-load design with twice the drift spacing of the reference design. The precipitation cap still forms, but the heat pipe will be smaller, and preferred pathways for condensate drainage will exist in the pillars.

\subsubsection{Near-Field/Altered-Zone Models}

Several areas have been identified for the future enhancement of predictive models used in NF/ AZ studies. The discussion of TM models described the various approaches available for modeling the effects of rock joints, including explicit-fracture models, ubiquitous-joint models, and discrete-element models. A continuum modeling approach was used to demonstrate that thermal-stress conditions in the host rock will be capable of causing significant permeability changes due to normal closure of fractures, and especially due to dilation associated with shear displacement. Methods of analysis that permit inelastic slip along fractures were not used for calculations, although it is recognized that the projected stress and displacement fields can be significantly different if joint-slip behavior is incorporated. In particular, two major differences would result: (1) shear slip would cause fracture dilation and increase permeability and (2) the softening that would result from slip would cause the rock-mass deformation response to be concentrated closer to the drifts. Computing significant slip might require the use of values for fracture-strength parameters that are somewhat different from those used in this report. Discrete-element calculations using commercially obtained modeling software are within current modeling capabilities.

Existing chemical modeling codes, such as EQ3/6, are capable of simulations involving redox reactions; with one exception (Meike and Glassley, 1997), these have not yet been incorporated into studies of the NFE. With abundant metallic iron present and with large quantities of reduced uranium $\left(\mathrm{UO}_{2}\right)$ in the waste form, there is the potential for reducing conditions in the NFE. Such conditions would give rise to lower solubility limits for several radionuclides, particularly $\mathrm{Np}$ and $\mathrm{Pu}$, of concern to performance. It is therefore conservative to ignore redox if the actual redox state is more reducing, and this is the approach that has been taken. Modeling of redox behavior would introduce additional complexity, and it is well known that redox disequilibrium often exists in natural waters. Plausible simulation of redox effects in the NFE would be difficult; if achieved, it could have a positive impact on radionuclide mobility. This is a matter for consideration in future experiments and modeling efforts.

The THC models presented in this report are limited with respect to the range of phenomena considered, particularly gas-phase transport of reactive components and reactions involving boiling. These limitations are common to all the thermochemical and reactive-transport models surveyed for this report and to other models for which detailed 
description were available. Incorporation of more chemical components, gas-phase transport, boiling reactions, etc., into an integrated model that will also simulate thermohydrology and may include coupled mechanical or TM effects is an active area of geochemical research.

\section{Return to Table of Contents}

\subsection{References}

Barton, N.R., S. Bandis, and K. Bakhtar (1985). "Strength, deformation, and conductivity coupling of rock joints." Int. J. Rock Mech. Min. Sci. and Geomech. 22(3):121-140. [217135]

DOE (1998). “Repository Safety Strategy: U.S. Department of Energy's Strategy to Protect Public Health and Safety After Closure of a Yucca Mountain Repository, Rev. 1." Washington, DC: U.S. Department of Energy, Office of Civilian Radioactive Waste Management. [MOL. 19980211.0250; MOL. 19980330.0239]

Hardin, E.L., N. Barton, R. Lingle, M. Board, and M.D. Voegele (1982). “A Heated Flatjack Test Series to Measure the Thermomechanical and Transport Properties of in situ Rock Masses." (ONWI-260). Columbus, OH: Office of Nuclear Waste Isolation, Battelle Memorial Institute. [NNA.19900827.0178; 232093]

Harrar, J., J.F. Carley, W.F. Isherwood, and E. Raber (1990). “Report of the Committee to Review the Use of J-13 Well Water in Nevada Nuclear Waste Storage Investigations." (UCRL-ID-21867). Livermore, CA: Lawrence Livermore National Laboratory. [NNA.910131.0274]

Kneafsey, T.J., and K. Pruess (1997). “Preferential Flow Paths and Heat Pipes: Results from Laboratory Experiments on Heat-Driven Flow in Natural and Artificial Rock Fractures." Milestone report for the CRWMS Management and Operating Contractor, U.S. Department of Energy. (SPL6A5M4). Berkeley, CA: Lawrence Berkeley National Laboratory. [MOL.19971204.0415]

Lin, W., S. Blair, M. Buettner, T. Buscheck, W. Daily, G. Gdowski, G. Glassley, K. Lee, A. Ramirez, D. Ruddle, J. Roberts, S. Trettenero, D. Watwood, and R. Carlson (1997). "Thermal-mechanical-hydrological-chemical responses in the Single Heater Test at the ESF." In proceedings from International Society of Rock Mechanics and Geomechanics Abstract. New York, NY: June 29-May 2, 1997. International Symposium of the Int'1 Soc. Rock Mech. (UCRL-JC-125611) [MOL.19971210.0044]

Meike, A., and W.E. Glassley (1997). “Chemical Modeling of Backfill Composed of Quartz Sand, Lime, and an Fe-Phase." (UCRL-ID-124631). Livermore, CA: Lawrence Livermore National Laboratory. [MOL.19971217.0351]

Tsang, C.-F., J. Birkholzer, G. Li, and Y. Tsang (1997). “Drift Scale Modeling: Progress in Studies of Seepage into a Drift." Milestone report. (SP331CM4). Berkeley, CA: Lawrence Berkeley National Laboratory. [MOL.19971204.0420]

Wilder, D.G. (1996). “Near-Field and Altered-Zone Environment Report, Vol. II.” (UCRL-LR124998, Vol. II). Livermore, CA: Lawrence Livermore National Laboratory. [MOL.19961212.0121; MOL.19961212.0122]

Zimmerman, R.M., and R.E. Finley (1987). "Summary of Geomechanical Measurements Taken In and Around G-Tunnel Underground Facility, Nevada Test Site." (SAND86-1015). Albuquerque, NM: Sandia National Laboratories. [NNA.19870526.0015; 202819] 
Zimmerman, R.M., R.L. Schuch, D.S. Mason, M.L. Wilson, M.E. Hall, M.P. Board, R.P. Bellman, and M.L. Blanford (1986). "Final Report: G-Tunnel Heated Block Experiment." (SAND84-2620). Albuquerque, NM: Sandia National Laboratories. [MOL.19961217.0085; 202721] 

Appendix A

Quality Assurance Information 



\section{Appendix A Quality Assurance Information}

The tables presented in this appendix address the acceptance criteria for the Level 4 deliverable SP3100M4 (Near-Field/Altered-Zone Models Report). Table A-1 is the required "roadmap" that indicates where the criteria are met in this report. Table A-2 is a list of the codes used for calculations discussed in this report, including the versions used and whether the codes are qualified. Some other codes are discussed in this report but do not appear in Table A-2 because they were not actually used for calculations by Lawrence Livermore National Laboratory for the Yucca Mountain Site Characterization Project.

Table A-3, Table A-4, Table A-5, Table A-6, and Table A-7 list the significant data used in Chapters 3 through 7 of this report, respectively, and the related data-tracking numbers (DTN) from the Technical Data Management System (TDMS). These tables emphasize input data that would be needed to replicate the model results presented in this report. Some data from various sources within and external to the Yucca Mountain Project are discussed in this report, but do not appear in these tables because these data have not already been submitted to the TDMS, and the suitability of the data for use in performance assessment or design was not established by the authors of this report.

The models presented in this report are summarized in Table A-8. A list of Yucca Mountain Site Characterization Project deliverables used in preparation of this report is given in Table A-9. Portions of some of these deliverables were used verbatim by the original authors who contributed to this report.

Table A-1 "Roadmap" table identifying where the deliverable (SP3100M4) acceptance criteria are met in the Near-Field/Altered-Zone Models Report

\begin{tabular}{|l|l|l|}
\hline \multicolumn{1}{|c|}{ Criteria Description } & Section & \multicolumn{1}{c|}{ Comment } \\
\hline \hline $\begin{array}{l}\text { Document conceptual and numerical } \\
\text { models used to describe the near field } \\
\text { and altered zone (NF/AZ) }\end{array}$ & $\begin{array}{l}\text { Chapters 3 } \\
\text { through 7 }\end{array}$ & \\
\hline $\begin{array}{l}\text { Incorporate singly and multiply coupled } \\
\text { models, as appropriate }\end{array}$ & $\begin{array}{l}\text { Chapters 3 } \\
\text { through 6 }\end{array}$ & $\begin{array}{l}\text { A set of reference 2-D cases has been generated; } \\
\text { this represents the NF/AZ at a typical repository } \\
\text { location and analyzes it with respect to } \\
\text { thermohydrologic (TH), thermohydromechanical } \\
\text { (THM), thermohydrochemical (THC), and thermal- } \\
\text { hydrologic-chemical-mechanical (THCM) effects. }\end{array}$ \\
\hline $\begin{array}{l}\text { Identify laboratory and field data that } \\
\text { serve as the basis for developing and } \\
\text { testing models }\end{array}$ & Chapters 3 & $\begin{array}{l}\text { To the extent possible, simulators are tested } \\
\text { through 7 } \\
\text { ased as the basis for models. Data used include TH } \\
\text { and TM data from the Large Block and Single- } \\
\text { Heater tests (Chapters 3 and 4); laboratory tests } \\
\text { on fractures (Chapter 4); qualified chemical } \\
\text { databases (Chapters 5 and 6); chemical-reaction } \\
\text { data from laboratory experiments (Chapters 5 and } \\
\text { 6); retardation data from experiments with } \\
\text { radionuclides (Chapter 6); field data on transport of } \\
\text { bacteria (Chapter 6); and literature data on colloidal } \\
\text { and microbial processes and on cement alteration } \\
\text { effects (Chapters 6 and 7). }\end{array}$ \\
\hline
\end{tabular}




\begin{tabular}{|c|c|c|}
\hline Criteria Description & Section & Comment \\
\hline $\begin{array}{l}\text { Reference any computer codes used } \\
\text { to implement models }\end{array}$ & $\begin{array}{l}\text { Chapters } 3 \\
\text { through } 6\end{array}$ & See also Table A-2 \\
\hline $\begin{array}{l}\text { Discuss model confidence-building } \\
\text { activities, model uses and limitations, } \\
\text { model sensitivities, and model } \\
\text { uncertainties }\end{array}$ & $\begin{array}{l}\text { Chapters } 3 \\
\text { through } 6\end{array}$ & $\begin{array}{l}\text { This is accomplished throughout the discussion of } \\
\text { NF/AZ models. Every effort is made to show } \\
\text { evidence of model suitability for projecting } \\
\text { conditions at a Yucca Mountain repository. }\end{array}$ \\
\hline $\begin{array}{l}Q \text { and non-Q data used and cited in } \\
\text { this deliverable will be appropriately } \\
\text { noted and clearly identified. }\end{array}$ & Appendix A & $\begin{array}{l}\text { Significant data are identified as Q or NQA in } \\
\text { Tables A-3 through A-7. The status of chemical } \\
\text { databases is indicated along with modeling } \\
\text { software in Table A-2. }\end{array}$ \\
\hline $\begin{array}{l}\text { Every effort will be made to ensure that } \\
\text { qualified data are used in this } \\
\text { deliverable. }\end{array}$ & $\begin{array}{l}\text { Chapters } 3 \\
\text { through } 7\end{array}$ & $\begin{array}{l}\text { The qualified unsaturated-zone site-scale model } \\
\text { has been used where possible and is the basis for } \\
\text { the reference cases used to compare simulators } \\
\text { and to assess coupled processes. Data from the } \\
\text { TDMS have been used where appropriate. }\end{array}$ \\
\hline $\begin{array}{l}\text { Technical data contained in the } \\
\text { deliverable and not already } \\
\text { incorporated in the GENISES will be } \\
\text { submitted, if appropriate, for } \\
\text { incorporation. Submittal compliance } \\
\text { will be demonstrated by including in } \\
\text { this report a copy of the technical data } \\
\text { information form (TDIF) and the } \\
\text { transmittal letter to the GENISES } \\
\text { administrator. }\end{array}$ & $\begin{array}{l}\text { Appendices } \mathrm{A} \\
\text { and } \mathrm{B}\end{array}$ & $\begin{array}{l}\text { Data actually used in this report are identified in } \\
\text { Appendix A. Where these data are not incorporated } \\
\text { in the automated data-tracking system, TDIFs } \\
\text { have been submitted, as demonstrated by the } \\
\text { records included in Appendix B. }\end{array}$ \\
\hline $\begin{array}{l}\text { Record accession numbers and } \\
\text { automated tracking numbers will be } \\
\text { included, as appropriate, for all data } \\
\text { used or cited in this deliverable. }\end{array}$ & $\begin{array}{l}\text { Chapters } 3 \\
\text { through 7; } \\
\text { Appendix A }\end{array}$ & $\begin{array}{l}\text { Accession numbers are provided in the reference } \\
\text { lists, for sources for which they are available. } \\
\text { Data-tracking numbers are provided in Appendix A } \\
\text { for data used. }\end{array}$ \\
\hline $\begin{array}{l}\text { Demonstrate close integration with } \\
\text { performance assessment (PA), } \\
\text { design, and other sciences; } \\
\text { completely satisfy all TSPA-VA } \\
\text { requirements and articulate the } \\
\text { uncertainties in models in a manner } \\
\text { that allows the CRWMS Management } \\
\text { and Operating (M\&O) Contractor to } \\
\text { plan a more focused set of future } \\
\text { activities }\end{array}$ & $\begin{array}{l}\text { Chapters } 3 \\
\text { through } 6\end{array}$ & $\begin{array}{l}\text { Coupled processes are a matter of considerable } \\
\text { uncertainty and concern for PA and design. The } \\
\text { reference cases defined for this report permit } \\
\text { direct assessment of partly coupled model } \\
\text { responses, consistent with the state-of-the-art in } \\
\text { modeling. The TH abstraction methodology } \\
\text { developed for PA is described in detail in Chapter } \\
3 \text {, and analysis of the potential for improvement is } \\
\text { provided. Reference cases in Chapters } 3 \text { and } 4 \text { are } \\
\text { analyses of the same type currently being } \\
\text { performed by the M\&O design team; these make } \\
\text { possible, for the first time, direct comparison of the } \\
\text { relation between stress effects and } \\
\text { thermohydrology. Chapter } 6 \text { is structured around } \\
\text { the PA approach to modeling the near-field } \\
\text { geochemical environment, and the included work } \\
\text { on water interaction with cement will provide } \\
\text { needed input for this part of the total system } \\
\text { performance assessment-viability assessment } \\
\text { (TSPA-VA). }\end{array}$ \\
\hline
\end{tabular}




\begin{tabular}{|l|l|l|}
\hline \multicolumn{1}{|c|}{ Criteria Description } & Section & \multicolumn{1}{c|}{ Comment } \\
\hline \hline $\begin{array}{l}\text { Ensure that the near-field environment } \\
\text { (NFE) model has used the most up-to- } \\
\text { date technology that is relevant to } \\
\text { creating coupled process models of } \\
\text { the NFE }\end{array}$ & $\begin{array}{l}\text { Chapters 3 } \\
\text { through 6; } \\
\text { accompanying } \\
\text { CD-ROM }\end{array}$ & $\begin{array}{l}\text { The state of the art in modeling approaches to } \\
\text { relevant problems in projecting NFE conditions, is } \\
\text { represented by the models in this report. The TH, } \\
\text { TM, and THC models used represent a full range of } \\
\text { available approaches. Virtually all the TH } \\
\text { calculations have been made using the dual- } \\
\text { permeability method (DKM) approach. In general, } \\
\text { 2-D models have been used to limit computational } \\
\text { effort, but all the approaches and most of the } \\
\text { codes used can be readily extended to 3-D. } \\
\text { Sophisticated computer graphics and presentation } \\
\text { of modeling results have been provided on the } \\
\text { accompanying CD-ROM. }\end{array}$ \\
\hline $\begin{array}{l}\text { Demonstrate that, as far as possible, } \\
\text { the report addresses the concerns of } \\
\text { the Nuclear Regulatory Commission } \\
\text { other regulatory bodies and other } \\
\text { parties with oversight responsibilities. }\end{array}$ & Chapter 1; \\
Chapters 3 & $\begin{array}{l}\text { Reports from oversight and regulatory bodies have } \\
\text { throen addressed in Chapter 1. Fully coupled THC } \\
\text { modeling capabilities involving silica redistribution } \\
\text { were developed for this report. Based on } \\
\text { comments in the letter, dated January 22, 1998, } \\
\text { from the PA Peer Review Panel, additional } \\
\text { modeling of water composition in the NFE was } \\
\text { performed and included in this report. To help } \\
\text { interpret this criterion, informal discussions were } \\
\text { held during preparation of this report. }\end{array}$ \\
\hline
\end{tabular}


Table A-2 Simulation codes and chemical databases used in the Near-Field/AlteredZone Models Report

\begin{tabular}{|c|c|c|c|c|c|c|}
\hline Section & Code & Version & Description & Application & Qualified? & Reference \\
\hline \multirow[t]{2}{*}{3.3} & \multirow[t]{2}{*}{ NUFT } & 1.0 & TH simulator & $\begin{array}{l}\text { TH simulation; } \\
\text { reference cases } \\
\text { for this report; } \\
\text { multi-scale } \\
\text { abstraction }\end{array}$ & $\begin{array}{l}\text { No } \\
\text { (in process) }\end{array}$ & $\begin{array}{l}\text { Nitao, 1993; } \\
1995\end{array}$ \\
\hline & & $2-8-98$ & THC features & $\begin{array}{l}\text { TH and silica } \\
\text { redistribution; } \\
\text { reference cases } \\
\text { for this report }\end{array}$ & No & This report \\
\hline 3.3 & YMESH & 1.48 & $\begin{array}{l}\text { Mesh generator } \\
\text { for NUFT }\end{array}$ & $\begin{array}{l}\text { All NUFT } \\
\text { simulations }\end{array}$ & No & \\
\hline $\begin{array}{l}3.3 \\
3.6\end{array}$ & RADPRO & 3.13 & $\begin{array}{l}\text { Preprocessor for } \\
\text { NUFT }\end{array}$ & $\begin{array}{l}\text { All NUFT } \\
\text { simulations with } \\
\text { cavities }\end{array}$ & No & \\
\hline 4.1 & FLAC & 3.22 & $\begin{array}{l}\text { Fast Lagrangian } \\
\text { Analysis of } \\
\text { Continua, a } \\
\text { geomechanical } \\
\text { simulator }\end{array}$ & $\begin{array}{l}\text { Multi-drift 2-D } \\
\text { thermoelastic } \\
\text { simulations }\end{array}$ & $\begin{array}{l}\text { No; } \\
\text { commercial } \\
\text { software }\end{array}$ & $\begin{array}{l}\text { Itasca } \\
\text { Consulting } \\
\text { Group Inc., } \\
1996\end{array}$ \\
\hline 4.1 & FRACROCK & 50.1 & $\begin{array}{l}\text { Boundary } \\
\text { element } \\
\text { geomechanical } \\
\text { simulator and } \\
\text { thermoelastic } \\
\text { preprocessor }\end{array}$ & $\begin{array}{l}\text { Symmetric drift- } \\
\text { panel 2-D } \\
\text { thermoelastic } \\
\text { simulations }\end{array}$ & No & Blair, 1994 \\
\hline $\begin{array}{l}5.1,5.3 \\
6.2,6.3 \\
6.4 \\
7.2\end{array}$ & EQ3/6 & $7.2 \mathrm{~b}$ & $\begin{array}{l}\text { Thermodynamic } \\
\text { and reaction-path } \\
\text { model }\end{array}$ & $\begin{array}{l}\text { Equilibrium water } \\
\text { composition and } \\
\text { reaction-path } \\
\text { modeling }\end{array}$ & Yes & $\begin{array}{l}\text { Wolery, } \\
\text { 1992a; 1992b; } \\
\text { Wolery and } \\
\text { Daveler, } 1992\end{array}$ \\
\hline $\begin{array}{l}5.1 \\
6.2 \\
6.4 \\
6.5 \\
\end{array}$ & GEMBOCHS & $\begin{array}{l}\text { EQ3/6 } \\
\text { V. } 6\end{array}$ & $\begin{array}{l}\text { Thermodynamic } \\
\text { database and } \\
\text { maintenance } \\
\text { software }\end{array}$ & $\begin{array}{l}\text { Derive, enter, } \\
\text { and test } \\
\text { thermodynamic } \\
\text { data files }\end{array}$ & No & $\begin{array}{l}\text { Wolery, } \\
\text { 1992a; 1992b }\end{array}$ \\
\hline $\begin{array}{l}5.1 \\
6.3 \\
7.2 \\
\end{array}$ & SUPCRT92+ & $\begin{array}{l}\text { EQ3/6 } \\
\text { V. } 6\end{array}$ & $\begin{array}{l}\text { Thermodynamic } \\
\text { database (see } \\
\text { text) }\end{array}$ & $\begin{array}{l}\text { Used in } \\
\text { derivation of } \\
\text { cement database }\end{array}$ & No & $\begin{array}{l}\text { Johnson et al., } \\
1992\end{array}$ \\
\hline 5.1 & NEA (92+) & $\begin{array}{l}\text { EQ3/6 } \\
\text { V. } 6\end{array}$ & $\begin{array}{l}\text { Thermodynamic } \\
\text { data for uranium }\end{array}$ & $\begin{array}{l}\text { Reviewed by } \\
\text { Nuclear Energy } \\
\text { Agency; NF/AZ } \\
\text { solubility limits }\end{array}$ & Yes & $\begin{array}{l}\text { Grenthe et al., } \\
1992\end{array}$ \\
\hline
\end{tabular}




\begin{tabular}{|l|l|l|l|l|l|l|}
\hline Section & Code & Version & Description & Application & Qualified? & Reference \\
\hline \hline 6.4 & REACT & 2.4 & $\begin{array}{l}\text { Reactive- } \\
\text { transport } \\
\text { simulator }\end{array}$ & $\begin{array}{l}\text { Radionuclide } \\
\text { sorption on iron } \\
\text { corrosion } \\
\text { products }\end{array}$ & No & Bethke, 1994 \\
\hline 6.4 & X1t & 1.1 -DEV & $\begin{array}{l}\text { Reactive- } \\
\text { transport } \\
\text { simulator }\end{array}$ & $\begin{array}{l}\text { Radionuclide } \\
\text { sorption on iron } \\
\text { corrosion } \\
\text { products }\end{array}$ & No & Bethke, 1996 \\
\hline 5.2 & $\begin{array}{l}\text { OS3D/ } \\
\text { GIMRT }\end{array}$ & 1.0 & $\begin{array}{l}\text { Reactive- } \\
\text { transport } \\
\text { simulator }\end{array}$ & $\begin{array}{l}\text { AZ water } \\
\text { composition; } \\
\text { reference cases } \\
\text { for evaluating } \\
\text { coupled } \\
\text { processes }\end{array}$ & $\begin{array}{l}\text { No; } \\
\text { commercial } \\
\text { software }\end{array}$ & $\begin{array}{l}\text { Yabusaki, } \\
\text { Steefel and }\end{array}$ \\
\hline 6.3 & CEM.R27 & $\begin{array}{l}\text { This } \\
\text { report }\end{array}$ & $\begin{array}{l}\text { Cement } \\
\text { thermodynamic } \\
\text { data for EQ3/6 } \\
\text { modeling }\end{array}$ & $\begin{array}{l}\text { Water } \\
\text { composition } \\
\text { contacting } \\
\text { cementitious } \\
\text { materials }\end{array}$ & No & This report \\
\hline
\end{tabular}


Table A-3 Summary of significant data used in Chapter 3

\begin{tabular}{|c|c|c|c|c|c|c|}
\hline Section & Data Description & $\begin{array}{l}\text { Interpretive } \\
\text { Report }\end{array}$ & $\begin{array}{l}\text { LLNL } \\
\text { Data? }\end{array}$ & Related DTNs & $\begin{array}{l}\text { QA/ } \\
\text { NQA }\end{array}$ & $\begin{array}{c}\text { TDIF } \\
\text { Enclosed? }\end{array}$ \\
\hline 3.2 & $\begin{array}{l}\text { Figure } 3-1 \text { a contour } \\
\text { map of repository } \\
\text { depth . . . }\end{array}$ & $\begin{array}{l}\text { Bodvarsson et } \\
\text { al., } 1997\end{array}$ & No & LB970601233129.001 & $\bar{Q}$ & No \\
\hline 3.2 & $\begin{array}{l}\text { Figure } 3-1 \mathrm{~b} \text { contour } \\
\text { map of infiltration } \\
\text { flux ... }\end{array}$ & $\begin{array}{l}\text { Flint et al., } \\
1996\end{array}$ & No & LB970601233129.001 & Q & No \\
\hline 3.4 & $\begin{array}{l}\text { Table 3-2 summary } \\
\text { of thermal and } \\
\text { hydrologic properties } \\
\text { of the host rock for } \\
\text { the SHT area (tsw34 } \\
\text { unit) }\end{array}$ & $\begin{array}{l}\text { Buscheck et } \\
\text { al., } 1997\end{array}$ & No & LB970601233129.001 & Q & No \\
\hline 3.4 & $\begin{array}{l}\text { Figure 3-7 calculated } \\
\text { and measured temp. } \\
\text { histories for the SHT, } \\
\text { compared at } \\
\text { thermocouple TMA- } \\
\text { TC-1A-9 and along } \\
\text { borehole TMA-TC- } 5\end{array}$ & This report & No & SNF35110695001.008 & NQA & No \\
\hline 3.4 & $\begin{array}{l}\text { Figure } 3-10 \\
\text { calculated and } \\
\text { measured temp. } \\
\text { histories for the LBT, } \\
\text { compared at } \\
\text { thermocouple TT-114 }\end{array}$ & This report & Yes & LL970803004244.036 & Q & No \\
\hline 3.5 & $\begin{array}{l}\text { Table 3-4 TSPA-VA } \\
\text { thermal properties } \\
\text { for the rock matrix }\end{array}$ & $\begin{array}{l}\text { Francis et al., } \\
1997\end{array}$ & No & $\begin{array}{l}\text { TDIF submittal in } \\
\text { preparation at Sandia }\end{array}$ & & No \\
\hline 3.5 & $\begin{array}{l}\text { Table 3-5 matrix } \\
\text { property values in } \\
\text { the } 7 / 97 \text { TSPA-VA } \\
\text { base-case property } \\
\text { set . . (Ix1) }\end{array}$ & $\begin{array}{l}\text { Bodvarsson et } \\
\text { al., } 1997\end{array}$ & No & LB970601233129.001 & Q & No \\
\hline 3.5 & $\begin{array}{l}\text { Table 3-6 fracture } \\
\text { property values in } \\
\text { the } 7 / 97 \text { TSPA-VA } \\
\text { base-case property } \\
\text { set . . (Ix1) }\end{array}$ & $\begin{array}{l}\text { Bodvarsson et } \\
\text { al., } 1997\end{array}$ & No & LB970601233129.001 & Q & No \\
\hline 3.5 & $\begin{array}{l}\text { Table 3-7 matrix } \\
\text { property values in } \\
\text { the } 7 / 97 \text { TSPA-VA } \\
\text { base-case property } \\
\text { set . . (I/5) }\end{array}$ & $\begin{array}{l}\text { Bodvarsson et } \\
\text { al., } 1997\end{array}$ & No & LB970601233129.001 & Q & No \\
\hline
\end{tabular}


Appendix A: Quality Assurance Information

\begin{tabular}{|l|l|l|l|l|c|c|}
\hline Section & Data Description & $\begin{array}{l}\text { Interpretive } \\
\text { Report" }\end{array}$ & $\begin{array}{c}\text { LLNL } \\
\text { Data? }\end{array}$ & Related DTNs & $\begin{array}{c}\text { QA/ } \\
\text { NQA }\end{array}$ & $\begin{array}{c}\text { TDIF } \\
\text { Enclosed? }\end{array}$ \\
\hline \hline 3.5 & $\begin{array}{l}\text { Table 3-8 fracture } \\
\text { property values in } \\
\text { the 7/97 TSPA-VA } \\
\text { base-case property } \\
\text { set . . (I/5) }\end{array}$ & $\begin{array}{l}\text { Bodvarsson et } \\
\text { al., 1997 }\end{array}$ & No & LB970601233129.001 & Q & No \\
\hline 3.5 & $\begin{array}{l}\text { Tables 3-9 through } \\
\text { 3-11; the 11/97 "TH" } \\
\text { property set for } \\
\text { TSPA-VA and } \\
\text { modifications } \\
\text { constituting the } \\
\text { "modified TH" } \\
\text { property set } \\
\text { developed for this } \\
\text { report. }\end{array}$ & This report & Yes & See Appendix B & Q & Yes \\
\hline
\end{tabular}

${ }^{*}$ Reports in which data are presented or interpreted in a form most applicable to discussion of $\mathrm{NF} / \mathrm{AZ}$ models; these may not be the same reports from which the TDIFs were generated. 
Table A-4 Summary of significant data used in Chapter 4

\begin{tabular}{|c|c|c|c|c|c|c|}
\hline Section & Data Description & $\begin{array}{l}\text { Interpretive } \\
\text { Report }^{*}\end{array}$ & $\begin{array}{l}\text { LLNL } \\
\text { Data? }\end{array}$ & Related DTNs & $\begin{array}{l}\text { QA/ } \\
\text { NQA }\end{array}$ & $\begin{array}{c}\text { TDIF } \\
\text { Enclosed? }\end{array}$ \\
\hline 4.1 & $\begin{array}{l}\text { Figure 4-1 } \\
\text { displacement data } \\
\text { from one of the } \\
\text { MPBX holes, } \\
\text { oriented in the E-W } \\
\text { direction, in the LBT }\end{array}$ & This report & Yes & LL970803104244.037 & $\bar{Q}$ & No \\
\hline 4.1 & $\begin{array}{l}\text { Table 4-1 LBT } \\
\text { expansion in the } \\
\text { E-W and N-S } \\
\text { directions }\end{array}$ & This report & Yes & LL970803104244.037 & Q & No \\
\hline 4.1 & $\begin{array}{l}\text { Table } 4-2 \text { values and } \\
\text { ranges of principal } \\
\text { stresses in the } \\
\text { potential repository } \\
\text { horizon ... }\end{array}$ & $\begin{array}{l}\text { Stock et al., } \\
1984 ; 1985\end{array}$ & $\begin{array}{l}\text { No } \\
\text { No }\end{array}$ & $\begin{array}{l}\text { GS900983115212.002 } \\
\text { GS900983115212.003 }\end{array}$ & $\begin{array}{l}\text { NQA } \\
\text { NQA }\end{array}$ & No \\
\hline 4.2 & $\begin{array}{l}\text { Table } 4-3 \text { mechanical } \\
\text { properties of } \\
\text { Topopah Spring tuff } \\
\text { and fractures }\end{array}$ & $\begin{array}{l}\text { Various; some } \\
\text { are LLNL }\end{array}$ & $\begin{array}{l}\text { Yes } \\
\text { Yes } \\
\text { Yes } \\
\text { No } \\
\text { No } \\
\text { No }\end{array}$ & $\begin{array}{l}\text { see Appendix B } \\
\text { LL980204504243.016 } \\
\text { LL960201104243.006 } \\
\text { SNL02120584001.001 } \\
\text { SNSAND92045000.000 } \\
\text { SNSAND86113100.000 }\end{array}$ & $\begin{array}{c}\text { Q } \\
\text { Q } \\
\text { NQA } \\
\text { NQA } \\
\text { NQA } \\
\text { NQA }\end{array}$ & $\begin{array}{l}\text { Yes } \\
\text { No } \\
\text { No } \\
\text { No } \\
\text { No } \\
\text { No }\end{array}$ \\
\hline 4.2 & $\begin{array}{l}\text { Table 4-3 thermal } \\
\text { properties of } \\
\text { Topopah Spring tuff }\end{array}$ & $\begin{array}{l}\text { Various; all are } \\
\text { non-LLNL }\end{array}$ & $\begin{array}{l}\text { No } \\
\text { No } \\
\text { No } \\
\text { No } \\
\text { No } \\
\text { No }\end{array}$ & $\begin{array}{l}\text { SN9510RIB00035.000 } \\
\text { SN9510RIB00036.000 } \\
\text { SNL01B05059301.006 } \\
\text { SNL22080196001.001 } \\
\text { SNL22100196001.001 } \\
\text { SNL22100196001.002 }\end{array}$ & $\begin{array}{l}\mathrm{Q} \\
\mathrm{Q} \\
\mathrm{Q} \\
\mathrm{Q} \\
\mathrm{Q} \\
\mathrm{Q}\end{array}$ & $\begin{array}{l}\text { No } \\
\text { No } \\
\text { No } \\
\text { No } \\
\text { No } \\
\text { No }\end{array}$ \\
\hline 4.2 & $\begin{array}{l}\text { Table } 4-4 \text { coefficient } \\
\text { of thermal expansion } \\
\text { in TSw2 unit at } \\
\text { different } \\
\text { temperatures }\end{array}$ & $\begin{array}{l}\text { Brodsky et al., } \\
1997\end{array}$ & $\begin{array}{l}\text { No } \\
\text { No } \\
\text { No } \\
\text { No } \\
\text { No }\end{array}$ & $\begin{array}{l}\text { SN9510RIB00035.000 } \\
\text { SNL01B05059301.006 } \\
\text { SNL22080196001.001 } \\
\text { SNL22100196001.001 } \\
\text { SNL22100196001.002 }\end{array}$ & $\begin{array}{l}\mathrm{Q} \\
\mathrm{Q} \\
\mathrm{Q} \\
\mathrm{Q} \\
\mathrm{Q}\end{array}$ & $\begin{array}{l}\text { No } \\
\text { No } \\
\text { No } \\
\text { No } \\
\text { No }\end{array}$ \\
\hline 4.3 & $\begin{array}{l}\text { Description of flow } \\
\text { experiment and } \\
\text { Table } 4-5 \text { values for } \\
\text { Young's modulus for } \\
\text { small block ( } 0.5-\mathrm{m} \\
\text { scale) experiment } \\
7195\end{array}$ & This report & Yes & LL980204504243.016 & $Q$ & No \\
\hline
\end{tabular}

${ }^{*}$ Reports in which data are presented or interpreted in a form most applicable to discussion of NF/AZ models; these may not be the same reports from which the TDIFs were generated. 
Table A-5 Summary of significant data used in Chapter 5

\begin{tabular}{|l|l|l|l|l|c|c|}
\hline Section & \multicolumn{1}{|c|}{ Data Description } & $\begin{array}{l}\text { Interpretive } \\
\text { Report* }^{*}\end{array}$ & $\begin{array}{c}\text { LLNL } \\
\text { Data? }\end{array}$ & Related DTNs & $\begin{array}{c}\text { QA/ } \\
\text { NQA }\end{array}$ & $\begin{array}{c}\text { TDIF } \\
\text { Enclosed? }\end{array}$ \\
\hline \hline 5.2 & $\begin{array}{l}\text { Table 5-6 provisional } \\
\text { dissolution rate constants } \\
\text { and activation energies for } \\
\text { important host-rock } \\
\text { minerals }\end{array}$ & This report & Yes & See Appendix B & NQA & Yes \\
\hline 5.3 & $\begin{array}{l}\text { Preliminary bounds on the } \\
\text { water composition and } \\
\text { mineral development that } \\
\text { may influence the NFE }\end{array}$ & $\begin{array}{l}\text { Whitbeck and } \\
\text { Glassley, 1996 }\end{array}$ & Yes & LL950813212011.003 & NQA & No \\
\hline 5.5 & $\begin{array}{l}\text { Tables 5-7 and 5-8 base- } \\
\text { case dissolution- } \\
\text { parameter values for } \\
\text { quartz and cristobalite }\end{array}$ & This report & Yes & See Appendix B & NQA & Yes \\
\hline 5.6 & $\begin{array}{l}\text { Table 5-9 carbon dioxide } \\
\text { extraction yield from } \\
\text { distillation of preserved } \\
\text { Yucca Mountain core } \\
\text { samples }\end{array}$ & This report & No & See Appendix B & NQA & $\begin{array}{l}\text { Yes } \\
\text { (TDIFS } \\
\text { submitted by } \\
\text { M\&O/SAIC) }\end{array}$ \\
\hline
\end{tabular}

${ }^{*}$ Reports in which data are presented or interpreted in a form most applicable to discussion of NF/AZ models; these may not be the same reports from which the TDIFs were generated. 
Table A-6 Summary of significant data used in Chapter 6

\begin{tabular}{|c|c|c|c|c|c|c|}
\hline Section & Data Description & $\begin{array}{l}\text { Interpretive } \\
\text { Report }^{*}\end{array}$ & $\begin{array}{l}\text { LLNL } \\
\text { Data? }\end{array}$ & Related DTNs & $\begin{array}{l}\text { QA/ } \\
\text { NQA }\end{array}$ & $\begin{array}{c}\text { TDIF } \\
\text { Enclosed? }\end{array}$ \\
\hline 6.2 & $\begin{array}{l}\text { Table 6.2-1 Single- } \\
\text { Heater Test borehole } \\
\text { 16-4 water analysis }\end{array}$ & $\begin{array}{l}\text { Glassley and } \\
\text { DeLoach, } \\
\text { 1997; } \\
\text { Glassley, } 1997\end{array}$ & $\begin{array}{l}\text { Yes } \\
\text { Yes } \\
\text { Yes } \\
\text { No } \\
\end{array}$ & $\begin{array}{l}\text { LL97010104244.027 } \\
\text { LL970409604244.030 } \\
\text { LL97003904244.034 } \\
\text { SNT05071897001.002 }\end{array}$ & $\begin{array}{c}\mathrm{Q} \\
\mathrm{Q} \\
\mathrm{Q} \\
\mathrm{NQA}\end{array}$ & $\begin{array}{l}\text { No } \\
\text { No } \\
\text { No } \\
\text { No }\end{array}$ \\
\hline 6.4 & $\begin{array}{l}\text { Table of } \\
\text { experimental } \\
\text { sorption ratios for } \\
\text { sorption of } U \text { on } \\
\text { hematite at ambient } \\
\text { and elevated } \\
\text { temperature }\end{array}$ & This report & Yes & See Appendix B & Q & Yes \\
\hline 6.4 & $\begin{array}{l}\text { Table of } \\
\text { experimental } \\
\text { sorption ratios for } \\
\text { sorption of } \mathrm{Np} \text { on } \\
\text { hematite at ambient } \\
\text { temperature }\end{array}$ & This report & Yes & See Appendix B & Q & Yes \\
\hline 6.4 & $\begin{array}{l}\text { Table of } \\
\text { experimental } \\
\text { sorption ratios for } \\
\text { batch sorption of } U \\
\text { and Np on } \\
\text { hydrothermally } \\
\text { altered invert } \\
\text { concrete from the } \\
\text { ESF }\end{array}$ & This report & Yes & See Appendix B & Q & Yes \\
\hline 6.4 & $\begin{array}{l}\text { Mineral species } \\
\text { identified in } \\
\text { hydrothermally } \\
\text { altered and unaltered } \\
\text { cement }\end{array}$ & $\begin{array}{l}\text { Myers and } \\
\text { Meike, } 1997\end{array}$ & Yes & $\begin{array}{l}\text { LL980108404245.010 } \\
\text { LL980108504245.011 }\end{array}$ & $N Q A$ & No \\
\hline
\end{tabular}

${ }^{*}$ Reports in which data are presented or interpreted in a form most applicable to discussion of NF/AZ models; these may not be the same reports from which the TDIFs were generated. 
Table A-7 Summary of significant data used in Chapter 7

\begin{tabular}{|l|l|l|l|l|l|l|}
\hline Section & Data Description & $\begin{array}{c}\text { Interpretive } \\
\text { Report* }\end{array}$ & $\begin{array}{c}\text { LLNL } \\
\text { Data } \\
?\end{array}$ & Related DTNs & $\begin{array}{c}\text { QA/ } \\
\text { NQA }\end{array}$ & $\begin{array}{c}\text { TDIF } \\
\text { Enclosed? }\end{array}$ \\
\hline \hline 7.2 & $\begin{array}{l}\text { Cement mineral } \\
\text { assemblages } \\
\text { developed for } \\
\text { chemical calculations } \\
\text { of water composition }\end{array}$ & This report & Yes & $\begin{array}{l}\text { Computer diskette } \\
\text { with EQ3/6 files }\end{array}$ & NQA & $\begin{array}{l}\text { Submitted to } \\
\text { Yucca Mountain } \\
\text { Site } \\
\text { Characterization } \\
\text { Project (YMSCP) } \\
\text { Records }\end{array}$ \\
\hline 7.2 & $\begin{array}{l}\text { Cement database, } \\
\text { including carbonate } \\
\text { phases, for } \\
\text { calculation of water } \\
\text { composition }\end{array}$ & This report & Yes & $\begin{array}{l}\text { Computer diskette } \\
\text { with EQ3/6 files }\end{array}$ & NQA & $\begin{array}{l}\text { Submitted to } \\
\text { YMSCP Records }\end{array}$ \\
\hline
\end{tabular}

* Reports in which data are presented or interpreted in a form most applicable to discussion of NF/AZ models. These may not be the same reports from which the TDIFs were generated.

Table A-8 Summary of models presented in the Near-Field/Altered-Zone Models Report

\begin{tabular}{|l|l|l|l|}
\hline \multicolumn{1}{|c|}{ Model } & \multicolumn{1}{|c|}{ Type } & \multicolumn{1}{c|}{ Section } & \multicolumn{1}{c|}{ Application } \\
\hline \hline Drift-Scale TH & Conceptual/numerical predictive & 3.5 & $\begin{array}{l}\text { PA and engineered barrier } \\
\text { system (EBS)/waste } \\
\text { package (WP) design }\end{array}$ \\
\hline $\begin{array}{l}\text { Drift-Scale Isothermal and } \\
\text { Nonisothermal Seepage }\end{array}$ & Conceptual/numerical predictive & 3.6 & PA and EBS/WP design \\
\hline Multi-Scale TH Abstraction & Conceptual/numerical predictive & 3.7 & PA \\
\hline Drift-Scale TM & Conceptual/numerical predictive & 4.4 & EBS/repository design \\
\hline Host Rock THM Response & Conceptual/numerical predictive & $4.5,4.6,4.7$ & PA \\
\hline AZ Water Composition & Conceptual/numerical predictive & $5.4,6.3$ & PA and EBS/WP design \\
\hline $\begin{array}{l}\text { Fully Coupled AZ THC/Silica } \\
\text { Partially Coupled AZ Reactive- } \\
\text { Transport/Aqueous Chemical } \\
\text { Reactions }\end{array}$ & Conceptual/numerical predictive & $5.5,5.6$ & PA \\
\hline $\begin{array}{l}\text { NF Water Composition/Introduced } \\
\text { Materials }\end{array}$ & Conceptual/numerical predictive & 6.4 & PA \\
\hline $\begin{array}{l}\text { NF Radionuclide Retardation/ } \\
\text { Introduced Materials }\end{array}$ & Conceptual/numerical predictive & 6.5 & PA and EBS/WP design \\
\hline $\begin{array}{l}\text { NF \& AZ Radionuclide } \\
\text { Retardation/Solubility }\end{array}$ & Conceptual/numerical predictive & 6.6 .1 & PA \\
\hline $\begin{array}{l}\text { NF Microbiological/Colloidal } \\
\text { Radionuclide Transport }\end{array}$ & Conceptual framework & 6.7 & PA \\
\hline
\end{tabular}


Table A-9 List of Yucca Mountain Site Characterization Project deliverables used in the Near-Field/Altered-Zone Models Report

\begin{tabular}{|c|c|c|}
\hline Deliverable ID & Title & Section \\
\hline SP342AM4 & Revised GEMBOCHS database & $5.2,6.5$ \\
\hline SP342FM4 & EQ3/6 Software Maintenance and Support Summary & 5.2 \\
\hline SPL2BM4 & $\begin{array}{l}\text { Models for the Thermohydrochemical Alteration of Flow Pathways Above and } \\
\text { Below the Repository }\end{array}$ & 5.8 \\
\hline SPL3BM4 & $\begin{array}{l}\text { Transport of Radionuclide-Bearing Fluids through Waste Package Corrosion } \\
\text { Products }\end{array}$ & 6.4 \\
\hline SPL3FM4 & $\begin{array}{l}\text { Radionuclide Transport Models: Comparisons of Reactive-Transport Models with } \\
\text { Laboratory Experiments }\end{array}$ & 6.4 \\
\hline SPL4B2M4 & $\begin{array}{l}\text { Analysis of Elevated Temperature Data for Thermodynamic Properties of } \\
\text { Selected Radionuclides }\end{array}$ & 6.5 \\
\hline SPL4C2M4 & Project Status of Solubility Data for Selected Radionuclides & 6.5 \\
\hline SPLA1M4 & Chemical Composition of Water Before Contact with Repository Materials & 6.2 \\
\hline SPLB1M4 & $\begin{array}{l}\text { Preliminary Bounds for Drift-Scale Distribution of Percolation and Seepage at } \\
\text { the Repository Level }\end{array}$ & 3.6 \\
\hline SPLB2M4 & $\begin{array}{l}\text { Models for Distribution of Percolation at Repository level \& Seepage Into Drifts } \\
\text { Under Pre-Emplacement Conditions }\end{array}$ & 3.6 \\
\hline SPLC2M4 & $\begin{array}{l}\text { Distribution of Post-Emplacement Seepage into Repository Drifts with } \\
\text { Parametric Variation of Intrinsic Properties }\end{array}$ & 3.6 \\
\hline SPLC3M4 & Models \& Bounds for Post-Emplacement Seepage into the Repository & 3.6 \\
\hline SPLD2M4 & $\begin{array}{l}\text { Bounding Models for the Temporal \& Spatial Variation of Temperature and } \\
\text { Relative Humidity in the Waste Package Environment }\end{array}$ & 3.7 \\
\hline SPLFA1M4 & Preliminary Results of a Coupled Fracture-Flow Test at the $0.5-\mathrm{m}$ Scale & 4.2 \\
\hline SPLF1M4 & $\begin{array}{l}\text { Evaluation of Models for Estimating Changes in Fracture Permeability Due to } \\
\text { Thermomechanical Stresses }\end{array}$ & 4.4 \\
\hline SPLF2M4 & $\begin{array}{l}\text { Bounding Models for Estimating Changes in Fracture Permeability Due to T-M } \\
\text { Stresses }\end{array}$ & $4.5,4.6$ \\
\hline SPLG1FM4 & $\begin{array}{l}\text { Bounding Models for Chemical Composition of Water Contacting Waste } \\
\text { Packages and the Waste Form }\end{array}$ & 6.3 \\
\hline SP3005M3 & Synthesis Report on Thermally Driven Coupled Processes & 2.1 \\
\hline SPLGBM4 & $\begin{array}{l}\text { Results from Hydrothermal Cement Alteration Experiment, First Batch of } \\
\text { Samples }\end{array}$ & 7.1 \\
\hline SPLGDM4 & $\begin{array}{l}\text { Results from Hydrothermal Cement Alteration Experiment, Second Batch of } \\
\text { Samples }\end{array}$ & 7.1 \\
\hline SPLGEM4 & $\begin{array}{l}\text { Results from Hydrothermal Cement Alteration Experiment, Third Batch of } \\
\text { Samples }\end{array}$ & 7.1 \\
\hline SPLG1BM4 & Installation of Labeled Microbe Sample Coupons in the Large Block & 6.5 \\
\hline SPLG1AM4 & Labeled Bacteria for the Large Block & 6.5 \\
\hline
\end{tabular}

\section{Return to Table of Contents}




\section{References}

Bethke, C.M. (1994). The Geochemist's Workbench ${ }^{\mathrm{TM}}$, Version 2.0. A Users Guide to Rxn, Act2, Tact, React, and Gtplot. Champaign, IL: Hydrology Program, University of Illinois.

Bethke, C.M. (1996). Reactive Transport and Basin Modeling. Champaign, IL: University of Illinois. (Short course workbook: Hydrogeology Program, May 29-31, 1996)

Blair, S.C. (1994). "Analysis of Compressive Fracture in Rock Using Statistical Techniques." Berkeley, CA: University of California at Berkeley, Dept. of Materials Science and Mineral Engineering. Ph.D. Thesis.

Bodvarsson, G.S., T.M. Bandurraga, and Y.S. Wu (1997). “The Site-Scale Unsaturated Zone Model of Yucca Mountain, Nevada, for the Viability Assessment." June 1997. (LBNL-40376, UC-814). Berkeley, CA: Lawrence Berkeley National Laboratory, in collaboration with the U.S. Geological Survey. [MOL.19971014.0232]

Brodsky, N.S., M. Riggins, and J. Connolly (1997). “Thermal Expansion, Thermal Conductivity, and Heat Capacity Measurements at Yucca Mountain, Nevada." Int. J. Rock Mech. Min. Sci. 34:3-4. (paper no. 40).

Buscheck, T.A., R.J. Shaffer, K.H. Lee, and J.J. Nitao (1997). “1997 Analysis of ThermalHydrological Behavior During the Heating Phase of the Single-Heater Test at Yucca Mountain." Supplemental submission of milestone report for the CRWMS Management and Operating Contractor, U.S. Department of Energy. (SP9266M4). Livermore, CA: Lawrence Livermore National Laboratory. [MOL.19980109.0262]

Flint, A., J.A. Hevesi, and L.E. Flint (1996). "Conceptual and Numerical Model of Infiltration for the Yucca Mountain Area, Nevada." Water Resources Investigation Report. (3GUT623M). Denver, CO: U.S. Geological Survey. (U.S.G.S. Survey Milestone Report) [MOL.19970409.0087]

Francis, N.D., C.K. Ho, and M.T. Itamura (1997). “UZ-Thermohydrology: Complete VA T/H Sensitivity Analysis." (SLX09F9). Albuquerque, NM: Sandia National Laboratories. [MOL.19980210.0914]

Glassley, W.E. (1997). “Third Quarter Report: Chemical Analyses of Waters Collected from the Single Heater Test." Milestone report for the CRWMS Management and Operating Contractor, U.S. Department of Energy. (SP9281M4). Livermore, CA: Lawrence Livermore National Laboratory.

Glassley, W.E., and L. DeLoach (1997). "Second Quarter Results of Chemical Measurements in the Single Heater Test." Milestone report for the CRWMS Management and Operating Contractor, U.S. Department of Energy. (SP9240M4). Livermore, CA: Lawrence Livermore National Laboratory. [MOL.19971218.0917]

Grenthe, I., J. Fuger, R.J.M. Konings, R.J. Lemire, A.B. Muller, C. Nguyen-Trung, and H. Wanner (1992). Chemical Thermodynamics, Vol. 1: Chemical Thermodynamics of Uranium. Amsterdam: North Holland. [NNA.19940415.0009]

Itasca Consulting Group Inc. (1996). FLAC, Fast Lagrangian Analysis of Continua, Version 3.3, Vol. I-IV, User's Manuals. Minneapolis, MN: Itasca Consulting Group, Inc. 
Johnson, J.W., E.H. Oelkers, and H.C. Helgeson (1992). “SUPCRT92: A software package for calculating the standard molal thermodynamic properties of minerals, gases, aqueous species, and reactions from 1 to 5000 bars and $0^{\circ} \mathrm{C}$ to $1000^{\circ} \mathrm{C}$." Comput. Geosci. 18:899-947. (UCRL-JC-107907). [234273]

Myers, K., and A. Meike (1997). "Hydrothermal Alteration of Concrete: Second and Third Batches." Milestone report for the CRWMS Management and Operating Contractor, U.S. Department of Energy. (SPLGDM4; SPLGEM4). Livermore, CA: Lawrence Livermore National Laboratory. [MOL.19980114.0113]

Nitao, J.J. (1993). “The NUFT Code for modeling nonisothermal, multiphase, multicomponent flow and transport in porous media." In proceedings from EOS. Washington, DC: American Geophysical Union. Supplement 73:31. (Also UCRL-JC-14769 Abs, Lawrence Livermore National Laboratory, Livermore, CA)

Nitao, J.J. (1995). Reference Manual for the NUFT Flow and Transport Code, Version 1.0. Livermore, CA: Lawrence Livermore National Laboratory. (UCRL-IC-113520)

Steefel, C.I., and S.B. Yabusaki (1995). “OS3D/GIMRT: Software for Modeling Multicomponent-Multidimensional Reactive Transport." Richland, WA: Pacific Northwest Laboratory, Battelle Memorial Institute.

Stock, J.M., J.H. Healy, and S.H. Hickman (1984). “Report on Televiewer Log and Stress Measurements in Core Hole USW G-2, Nevada Test Site." (OFR-84-172). Denver, CO: U.S. Geological Survey. [NNA.870406.0157; 208153]

Stock, J.M., J.H. Healy, S.H. Hickman, and M.D. Zoback (1985). “Hydraulic fracturing stress measurements at Yucca Mountain, Nevada, and relationship to regional stress field." J. Geophys. Res. 90(B10):8691-8706. [HQS.19880517.1509; 219009]

Whitbeck, M., and W.E. Glassley (1996). “Preliminary Bounds on the Water Composition and Secondary Mineral Development that May Influence the Near-Field Environment." Milestone report for the CRWMS Management and Operating Contractor, U.S. Department of Energy. (MOL205). Livermore, CA: Lawrence Livermore National Laboratory. [MOL.19960726.0040]

Wolery, T.J. (1992a). “EQ3/6, A Software Package for Geochemical Modeling of Aqueous Systems: Package Overview and Installation Guide." (UCRL-MA-110662). Livermore, CA: Lawrence Livermore National Laboratory. (part 1) [NNA.19921023.0028]

Wolery, T.J. (1992b). “EQ3NR, A Computer Program For Geochemical Aqueous SpeciationSolubility Calculations: Theoretical Manual, User's Guide, and Related Documentation." (UCRL-MA-110662). Livermore, CA: Lawrence Livermore National Laboratory. (Version 7.0) [NNA.19921218.0010]

Wolery, T.J., and S.A. Daveler (1992). “EQ6, A Computer Program for Reaction Path Modeling of Aqueous Geochemical Systems: Theoretical Manual, User's Guide, and Related Documentation (Version 7.0)." (UCRL-MA-110662). Livermore, CA: Lawrence Livermore National Laboratory. (part 4) [MOL.19980218.0570] 


\section{Appendix B \\ Technical Data Submissions}





\section{Lawrence Livermore National Laboratory}

LLYMP9802111

QA: N

February 26, 1998

Joanna Wiggins, GENISES Administrator

Yucca Mountain Site Characterization

Project Office

1180 Town Centre Drive

Las Vegas, NV 89134

SUBJECT: Tables of Data from the Near-Field/Altered-Zone Models Report, Deliverable SP3100M4. DTNs are: LL980208104243.019, LL980208751051.010, LL980208651051.009, LL980208851051.011, LL980209004242.026, and LL980204241.014 (WBS 1.2.3.12)

Enclosed are hardcopies of the subject data from the TDIFs. Also enclosed are a PC formatted disk with the data in tab delimited form (as well as in Word for Mac) and a copy of the TDIFs from the milestone.

The data review packages will be sent to the RPC in accordance with procedures. These data have been technically reviewed in accordance with 033-YMP-QP 3.6, "Collection, Review, and Submittal of Technical Data."

If there are any questions, please contact the author of the report, Ernest Hardin at (702) $295-4363$.

$\mathrm{WC} / \mathrm{BB} / \mathrm{bb}$

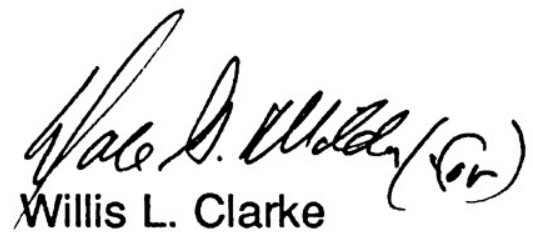

CRWMS LLNL Manager

cc (w/o enc):

C. Newbury, DOE/YMP 
YMP-023-R4

05/06/96

(Check one): $\square$ ACQUIRED DATA (complete Parts I and II)

Data Tracking Number (DTN):

$\square$ DEVELOPED DATA (complete Parts I, II and III)

Data Tracking Number (DTN):

LL980208104243.019

PART I Identification of Data

Title/Description of Data: TABLE OF MECHANICAL PROPERTIES OF TOPOPAH SPRING HOST ROCK, AND FRACTURES IN THE HOST ROCK, BASED ON LABORATORY EXPERIMENTS ON CORE SAMPLES AND SMALL (NOMINAL 0.5-M) TUFF BLOCKS AT LAWRENCE LIVERMORE NATIONAL LABORATORY.

Principal Investigator (PI): $\frac{\text { BLAIR, S C }}{\text { Last Name }}$ First and Middle Initials

PI Organization: LAWRENCE LIVERMORE NATIONAL LABORATORY

Are Data Qualified?: $\mathrm{X}$ Yes $\square$ No Governing Plan: SCP

SCPB Activity Number(s): $8 \cdot 3 \cdot 4 \cdot 2 \cdot 4.3 .3$

WBS Number(s): $\quad 1.2 \cdot 3 \cdot 12.3$

\section{PART II Data Acquisition/Development Information}

Method: THESE ARE LABORATORY-MEASURED DATA. THE TYPE OF MEASUREMENTS, AND THE SAMPLE CONDITIONS WHICH WERE USED, ARE INDICATED BY THE INVESTIGATORS IN THE CITED DTN.

Location(s): LAWRENCE LIVERMORE NATIONAL LABORATORY

Period(s): $\frac{10 / 1 / 96 \text { to } 9 / 1 / 97}{\text { From: MM/DD/YY }}$ To: $M M / D D / Y Y$

Sample ID Number(s):

\section{PART III Source Data DTN(s)}

LL970407204243.011

LL980204504243.016

LL980207304243.018

\section{Comments}

Checked by: M. Peanne Yalmer

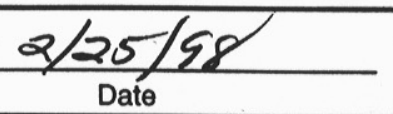


(Check one): $\square$ ACQUIRED DATA (complete Parts I and II)

Data Tracking Number (DTN): _ LL980208751051.010

DEVELOPED DATA (complete Parts I, II and III)

Data Tracking Number (DTN):

PART I Identification of Data

Title/Description of Data: TABLE OF EXPERIMENTAL SORPTION RATIOS FOR BATCH SORPTION OF NEPTUNIUM ON HEMATITE AT AMBIENT TEMPERATURE.

Principal Investigator (PI): VIANI, B E

PI Organization: LAWRENCE LIVERMORE NATIONAL LABORATORY

Are Data Qualified?: $\square$ Yes $\square$ No Governing Plan:

SCPB Activity Number(s): $8 \cdot 3 \cdot 5 \cdot 10.5 .1$

WBS Number(s): $1 \cdot 2 \cdot 3 \cdot 10 \cdot 3 \cdot 1$

\section{PART II Data Acquisition/Development Information}

Method: LABORATORY MEASUREMENTS USING CHARACTERIZED HEMATITE, IN A BACKGROUND ELECTROLYTE, WITH NEPTUNIUM IN THE (V) VALENCE STATE AT 26 DEGREES CELSIUS.

Location(s): LAWRENCE LIVERMORE NATIONAL LABORATORY

Period(s): $\frac{10 / 1 / 97 \text { to } 2 / 1 / 98}{\text { From: } M M / D D / Y Y}$ To: MM/DDNY

Sample ID Number(s):

\section{PART III Source Data DTN(s)}

\section{Comments}


(Check one): $\square$ ACQUIRED DATA (complete Parts I and II)

Data Tracking Number (DTN): $\quad$ LL980208651051.009

DEVELOPED DATA (complete Parts I, II and III)

Data Tracking Number (DTN):

\section{PART I Identification of Data}

Title/Description of Data: TABLE OF EXPERIMENTAL SORPTION RATIOS FOR BATCH SORPTION OF URANIUM ON HEMATITE AT AMBIENT AND ELEVATED TEMPERATURE.

Principal Investigator (PI): VIANI, B E

$$
\text { Last Name }
$$

PI Organization: LAWRENCE LIVERMORE NATIONAL LABORATORY

Are Data Qualified?: $\square$ Yes $\square$ No Governing Plan: SCP

SCPB Activity Number(s): $8 \cdot 3 \cdot 5 \cdot 10.5 .1$

WBS Number(s): $1.2 \cdot 3 \cdot 10.3 .1$

\section{PART II Data Acquisition/Development Information}

Method: LABORATORY MEASUREMENTS USING CHARACTERIZED HEMATITE, IN A BACKGROUND ELECTROLYTE, WITH URANIUM IN THE (VI) VALENCE STATE AT 26, 38, 50 AND 76 DEGREES CELSIUS.

Location(s): LAWRENCE LIVERMORE NATIONAL LABORATORY

Period(s): $\frac{10 / 1 / 96 \text { to } 9 / 1 / 97}{\text { From: } M M / D D / Y Y}$ To: $M M / D D / Y Y$

Sample ID Number(s):

\section{PART III Source Data DTN(s)}

\section{Comments}


YMP-023-R4

05/06/96
YUCCA MOUNTAIN SITE CHARACTERIZATION PROJECT

TECHNICAL DATA INFORMATION

(Check one): $\square$ ACQUIRED DATA (complete Parts I and II)

Data Tracking Number (DTN):

$\triangle$ DEVELOPED DATA (complete Parts I, II and III)

Data Tracking Number (DTN):

LL980208851051.011

\section{PART I Identification of Data}

Title/Description of Data: TABLE OF EXPERIMENTAL SORPTION RATIOS FOR BATCH SORPTION OF U AND NP ON

HYDROTHERMALLY ALTERED INVERT CONCRETE FROM THE ESF.

Principal Investigator (PI): VIANI, B E

$$
\text { Last Name }
$$

First and Middle Initials

PI Organization: LAWRENCE LIVERMORE NATIONAL LABORATORY

Are Data Qualified?: $\square$ Yes $\quad \mathrm{X}$ No Governing Plan:

SCPB Activity Number(s): 8 3.3.5.10.5.1

WBS Number(s): $1 \cdot 2 \cdot 3 \cdot 10 \cdot 3 \cdot 1$

\section{PART II Data Acquisition/Development Information}

Method: LABORATORY MEASUREMENTS USING PULVERIZED SAMPLES OF ESF INVERT CONCRETE, WHICH WERE HYDROTHERMALLY ALTERED UNDER LABORATORY CONTROLLED CONDITIONS, WITH U IN THE (VI) VALENCE STATE, AND NP IN THE (V) VALENCE STATE, AT 26 DEGREES CELSIUS.

Location(s): LAWRENCE LIVERMORE NATIONAL LABORATORY

Period(s): $\frac{10 / 1 / 97 \text { to } 2 / 1 / 98}{\text { From: MM/DD/YY }}$ To: $M M / D D / Y Y$

Sample ID Number(s):

\section{PART III Source Data DTN(s)}

LL980108404245. 010

LL980108504245. 011

\section{Comments}

DATA UNQUALIFIED BECAUSE INFORMATION EXTRACTED FROM SOURCES THAT ARE NON-QUALIFIED.

Checked by:

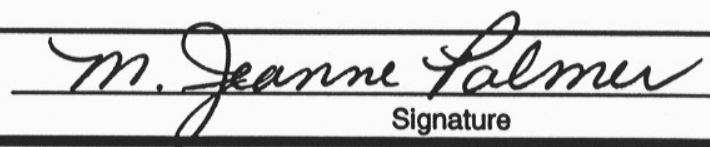


YMP-023-R4

05/06/96

\section{YUCCA MOUNTAIN SITE CHARACTERIZATION PROJECT}

TECHNICAL DATA INFORMATION

(Check one): $\square$ ACQUIRED DATA (complete Parts I and II)

Data Tracking Number (DTN):

$\square$ DEVELOPED DATA (complete Parts I, II and III)

Data Tracking Number (DTN): $\quad$ LL980209004242.026

\section{PART I Identification of Data}

Title/Description of Data: THREE TABLES (1,2, AND 3) DESCRIBING THE 11/97 "TH" PROPERTY SET FOR TSPA-VA AND MODIFICATIONS CONSTITUTING THE "MODIFIED TH" PROPERTY SET DEVELOPED FOR THE NEAR FIELD MODELS REPORT (SP3100M4/SP3100M3).

Principal Investigator (PI): $\frac{\text { BUSCHECK, T A }}{\text { Last Name }}$ First and Middle Initials

PI Organization: LAWRENCE LIVERMORE NATIONAL LABORATORY

Are Data Qualified?: $\mathrm{X}$ Yes $\square$ No $\quad$ Governing Plan: SCP

SCPB Activity Number(s): $8 \cdot 3 \cdot 4 \cdot 2.4 .2 .4$

WBS Number(s): $\frac{1.2 .3 .12 .2}{2}$

\section{PART II Data Acquisition/Development Information}

Method: THE 11/97 "TH" DUAL PERMEABILITY (DKM) PROEPRTY SET FOR THE TOTAL SYSTEM PERFORMANCE ASSESSMENT-VIABILITY ASSESSMENT (TSPA-VA) WAS REPORTED BY JENS BIRKHOLZER IN A MEMO TO G.S. BODVARSSON, BOTH OF LAWRENCE BERKELEY NATIONAL LABORATORY, ON NOVEMBER 19, 1997. SUBSEQUENT TO THAT MEMO MATRIX PROPERTIES FOR THE TSW36 AND TSW37 WERE MODIFIED BY LLNL TO DECREASE THE AMOUNT OF CAPILLARITY IN THOSE Location(s): PARAMETERS SPECIFIED FOR EACH HYDROSTRATIGRAPHIC UNIT LB970601233129.001

Period(s): $\frac{11 / 1 / 97 \text { to } 2 / 1 / 98}{\text { From: MM/DD/YY }}$ To: $M M / D D / Y Y$

Sample ID Number(s):

\section{PART III Source Data DTN(s)}

LB970601233129.001

\section{Comments}

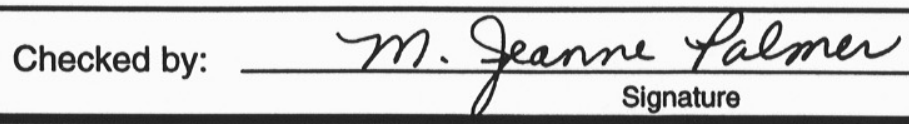
$\frac{2 / 25 / 98}{\text { Date }}$ 
Method (continued)

UNITS. (IN THE THREE TABLES, THE VAN GENUCHTEN "M" PARAMETER IS GIVEN INSTEAD OF THE "N" PARAMETER, BUT THESE ARE RELATED THROUGH N=1/[1+M].) REFERENCE: BIRKHOLZER, J.T. (1997) "T-H MODELING OF THE SINGLE-HEATER TEST WITH SITE-SCALE CALIBRATED PROPERTIES". LAWRENCE BERKELEY NATIONAL LABORATORY: MEMO TO G.S. BODVARSSON/LBNL/NOVEMBER 19, 1997. 
YMP-023-R4

05/06/96

YUCCA MOUNTAIN SITE CHARACTERIZATION PROJECT

TECHNICAL DATA INFORMATION

Page 1 of

(Check one): $\quad \mathrm{X}$ ACQUIRED DATA (complete Parts I and II)

Data Tracking Number (DTN): $\quad$ LL\$80208204241.014

$\square$ DEVELOPED DATA (complete Parts I, II and III)

Data Tracking Number (DTN):

\section{PART I Identification of Data}

Title/Description of Data: PROVISONAL DISSOLUTION RATE CONSTANTS AND ACTIVATION ENERGIES FOR IMPORTANT HOST ROCK MINERALS.

Principal Investigator (PI): $\frac{\text { KNAUSS, K G }}{\text { Last Name }}$

PI Organization: LAWRENCE LIVERMORE NATIONAL LABORATORY

Are Data Qualified?: $\square$ Yes $\quad \mathrm{X}$ No $\quad$ Governing Plan: SCP

SCPB Activity Number(s): $8 \cdot 3 \cdot 4 \cdot 2 \cdot 4 \cdot 1.10$

WBS Number(s): $\quad 1.2 \cdot 3 \cdot 12.1$

\section{PART II Data Acquisition/Development Information}

Method: ASSESSED FROM REACTION RATE DATA IN PEER REVIEWED PUBLICATIONS. REFERENCE DATA LOCATED IN NEAR-FIELD/ALTERED-ZONE MODELS REPORT UCRL-ID-129179DR.

Location(s): LAWRENCE LIVERMORE NATIONAL LABORATORY

Period(s): $\frac{1 / 1 / 89 \text { to } 1 / 1 / 96}{\text { From: } \mathrm{MM} / \mathrm{DD} / \mathrm{YY}}$ To: $M M / D D / Y Y$

Sample ID Number(s):

\section{PART III Source Data DTN(s)}

\section{Comments}

DATA NON-QUALIFIED BECAUSE INFORMATION EXTRACTED FROM SOURCES THAT ARE NON-QUALIFIED. 
LLYMP9802114

RECORDS PACKAGE TRANSMITTAL FORM TABLE OF CONTENTS

TO: YMP LOCAL RECORDS CENTER, L-217

DATE: $2-27-98$

$$
\text { [ ] QA } \begin{array}{llll}
{[} & ] & L \\
{[} & ] & N
\end{array} \quad[x] \text { QA: N/A }
$$

TOTAL NUMBER

OF PAGES:

3

TITLE/SUBJECT (include author and designator(s) (e.g., activity number, report number, WBS number):

Disk with data that can replicate the calculations in Section 6.3 of the Near

Field / Altered Zone Models Report, Milestone SP3100M4. Author is Annemarie

Meike. WBS. 1.2.3.12.1

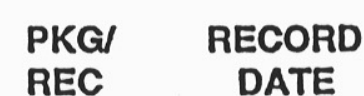

RECORD TITLE / SUBJECT

RECORD

PAGE

REC DATE

Special Instruction Sheet

COUNT

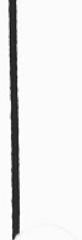

MOL.19980224.0294 ai ready assigned

Disk

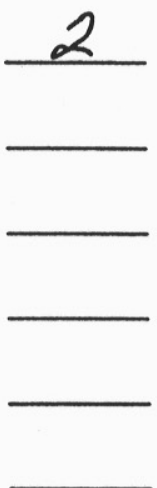

Runemaric Mike signature

$\frac{26 \text { Feb- } 1998 \text { SENDER: Annemarie Meike }}{\text { Dato }}$

This record has intentional spaces or blanks on page(s) 
May 5, 1998

Joanna Wiggins, GENISES Administrator

Yucca Mountain Site Characterization Project

1180 Town Center Drive

Las Vegas, NV 89134

SUBJECT: Technical Data Information Form (TDIF) Submittal for DTN TM000000001998.001

Enclosed are hard copies of the TDIF and the subject data table. The data table is also provided as a Word97 document file on the accompanying DOS formatted disk.

The data have been technically reviewed (QAP-SIII.2) and the review documentation will be transmitted under separate cover to the Records Processing Center in accordance with procedures.

If there are questions regarding this submittal, please contact Ernest Hardin, the $\mathrm{M} \& \mathrm{O}$ Natural Environment Program Operations representative responsible for the subject data (702/295-4363)

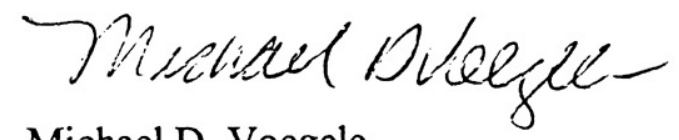

Michael D. Voegele

Operations Manager

Science Application

International Corporation

MDV/eh:jlm

Enclosure:

1. Electronic diskette

2. Technical Data Information Form 
YMP-023-R5

$04 / 02 / 98$

(Check one ): \ ACQUIRED DATA (complete Parts / and II)

Data Tracking Number (DTN): TM000000001998.001

DEVELOPED DATA (complete Parts I, II, and III)

Data Tracking Number (DTN):

\section{PART I Identification of Data}

Title/Description of Data: Preliminary data on carbon dioxide extraction yield from distillation of condensable gases from preserved Yucca Mountain core samples

Principal Investigator (PI): $\frac{\text { Davidson, G. R. }}{\text { Last Name }}$ First and Middle Initials

PI Organization: SAIC

Qualification Status:

$\square$ Q
$\square$ Non-Q
$\square$ Accepted

Governing Plan: SCP

Accepted

SCPB Activity Number(s): Study 8.3.1.2.2.2

WBS Number(s): WBS 1.2.3.3.1

\section{PART II Data Acquisition/Development Infomation}

Method: Vacuum distillation of condensable gases from preserved rock core, using the method detailed in the reference below.

Location(s): Distillations were performed by Dr. Davidson working at the University of Arizona.

\section{Period(s):}

06/01/97

$M M / D D / Y Y$
08/31/97

TO: $\quad M M / D D / Y Y$

Sample ID Number(s): SD-12/691.3-693.3, SD-7/1810.2-1814.0, SD-9/1360.1-1363.8, SD-7/1131.7-1133.3,

NRG-7/7A/960.2-961.5, NRG-7/7A/386.7-388.0, SD-7/409.7-412.6, and SD-12/1262.1-1263.8

\section{PART III Source Data DTN(s)}

none

\section{Comments}

Reference for extraction method: Davidson, G.R., E.L. Hardin, and R.L. Bassett (1995). "Extraction of 14C from Pore Water in Unsaturated Rock Using Vacuum Distillation." Radiocarbon 37:861-874.

Checked by:

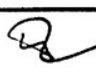

Q

Signature

$$
\frac{5 / 5 / 98}{\text { Date }}
$$

